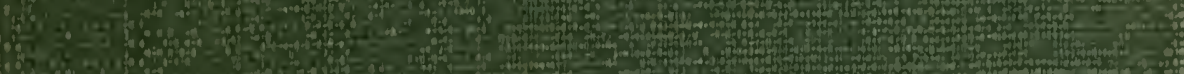

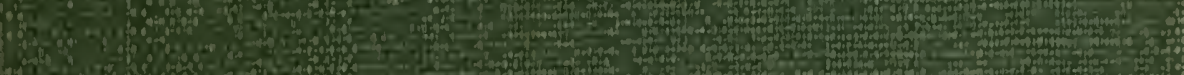
a to

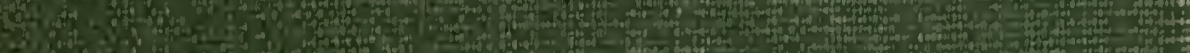
10.

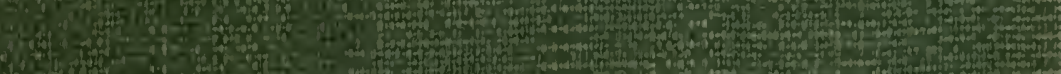

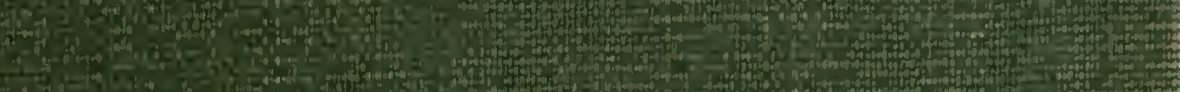

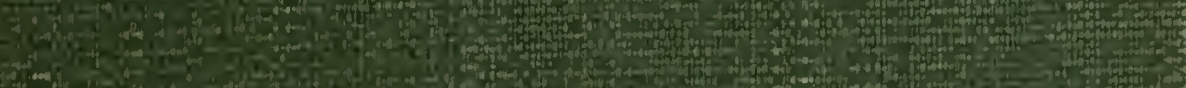

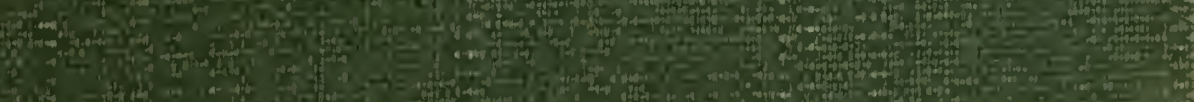

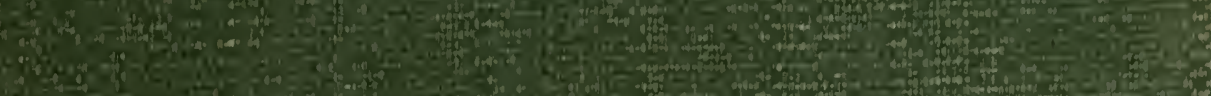

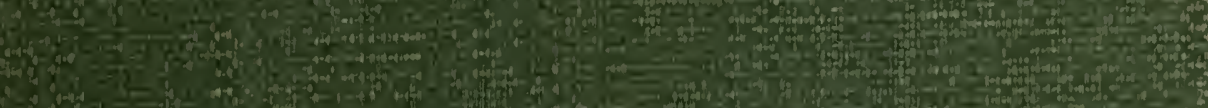

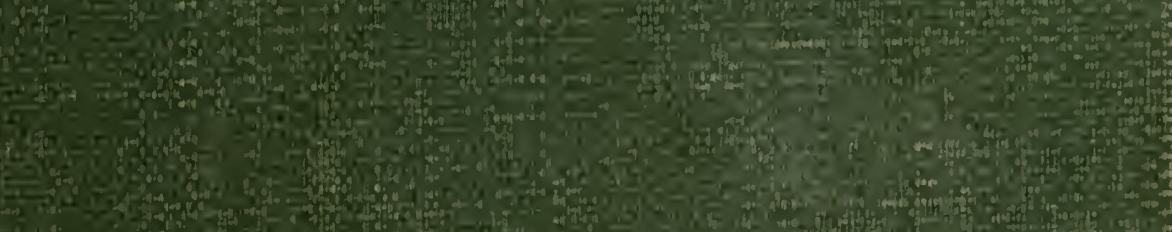

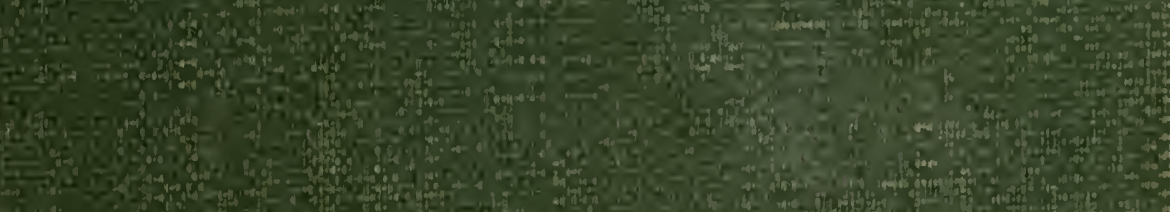

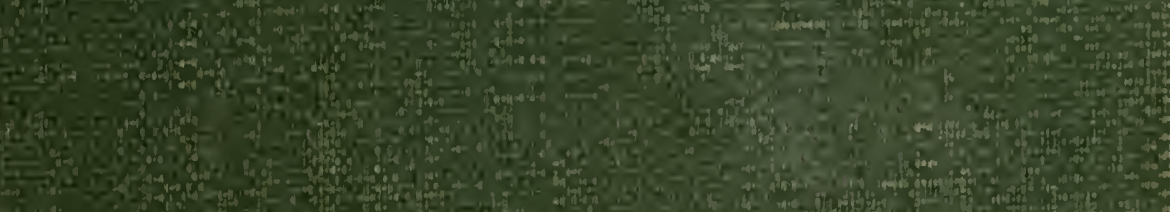

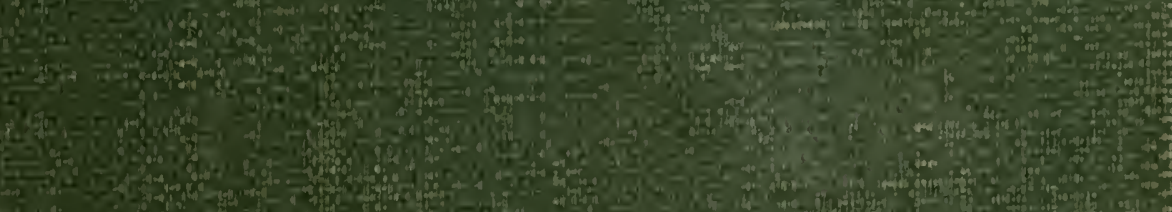
$2 \bar{w}^{*}$

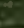

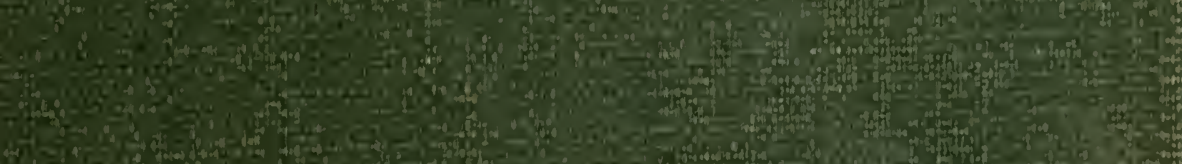
S.

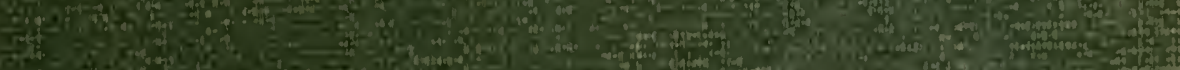
Q

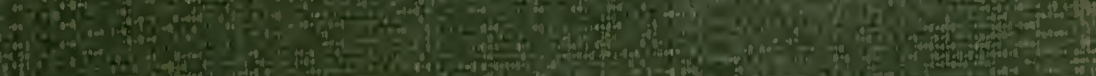

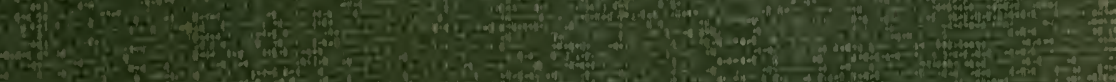

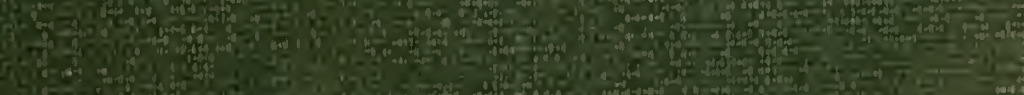
180 for

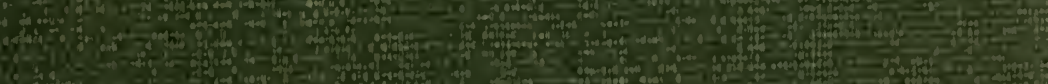
So $x^{2}$ 350 350 350 -

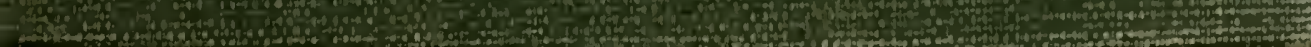





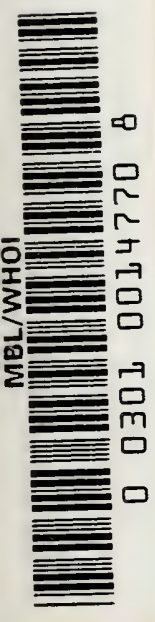







\section{A MANUAL OF ZOOLOGY}

BY

RICHARD HERTIVIG

PROFESSOR OF ZOOLOGY IN THE LXIVERSITY AT MUNICH

THIRD AMERICAN

FROM THE NINTII GERMAN EDITION

TRANSLATED AND EDITED BY

J. S. KINGSLEY

PROFESAOR OF ZOOLOGY IN TUFTS COLLEGE

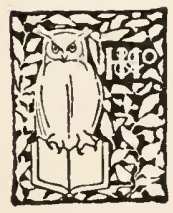

NEW YORK

HENRY HOLT AND COMPANY

1912 
Copyright, 1902

$\mathrm{BY}$

HENRY HOLT AND COO.

Copyright, 1912

$\mathrm{BY}$

HENRY HOLT AND COMPANY

897

THE. MAPLE,PRESS Y Y ORE PA 


\section{PREFACE TO THE THIRD EDITION.}

THE favor with which the first and second American editions of Hertwig's Zoology have been received has led to a thorough revision of the whole with a close comparison with the latest German edition.

In this there have been introduced many new features bringing the work up to date. These include a discussion of Mendelian inheritance, many modifications in the account of the theory of evolution, and a considerable enlargement of the Protozoa and especially of the pathogenic forms, making the volume of more value to the student of medicine.

To have included these without changes elsewhere would have resulted in a much larger volume. But the demand in American colleges has been for a smaller work and so a reduction has been made in two ways. There has been a condensation by the elimination of unnecessary words and phrases and by the omission of considerable matter of minor importance. Then there has been the recognition of the fact that the book has two uses, one in the class room the other as a reference work. The two classes of matter have been distinguished by differences of type.

No attempt has been made to bring the systematic names into accord with the latest vagaries of the systematists. No useful and could be served by changing or transferring the well-known names of Echidna, Coluber, Amia, Homarus, Unio, Holothuria, Amœba, etc., while the confusion this would introduce would be enormous.

It should be understood that while the revision is based upon the German edition of Professor Hertwig, he should not be held responsible for any changes introduced. The whole responsibility for these rests upon the American reviser.

Tufts College, Mass., June, igr2.

J. S. K.

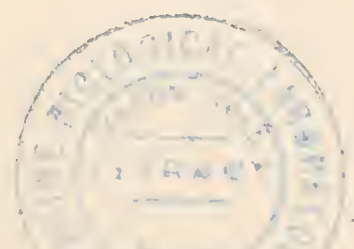





\section{PREFACE TO THE FIRST EDITION}

ON account of its clearness and breadth of view, its comparatively simple character and moderate size, Professor Richard Hertwig's 'Lehrbuch der Zoologie' has for ten years held the foremost place in German schools. The first or general part of the work was translated in 1896 by Dr. George W. Field, and the cordial reception which this has had in America has led to the present reproduction of the whole.

This American edition is not an exact translation. With the consent of the author the whole text has been edited and modified in places to accord with American usage. For these changes the translator alone can be held responsible. Some of the alterations are slight, but others are very considerable. Thus the group of Vermes of the original has been broken up and its members distributed among several phyla; the account of the Arthropoda has been largely rewritten and the classification materially altered; while the Tunicata and the Enteropneusti have been removed from their position as appendices to the Vermes and united with the Vertebrata to form the phylum Chordata. Other changes, like those in the classification of the Reptilia and the nephridial system of the vertebrates, are of less importance.

A large number of illustrations have been added, either to make clearer points of structure or to aid in the identification of American forms. Except in the Protozoa, American genera have in most cases been indicated by an asterisk. Numerous genera have been mentioned so that the student may see the relationships of forms described in morphological literature.

In the translation the word Anlage, meaning the embryonic material from which an organ or a part is developed, has been transferred directly. As our language is Germanic in its genius, there can be no valid objection to the adoption of the word.

As this work is intended for beginners, no bibliography has been given. A list of literature to be of much value would have been so large as materially to increase the size of the rolume. Experience has shown that beginners rarely go to the original sources. This omission it the less important since in all schools where the book is likely to be used other works containing good bibliographies are accessible. Refer- 
ence might here be made to those in the Anatomies of Lang and Wiedersheim, the Embryologies of Balfour, Korschelt and Heider, Minot, and Hertwig, and Wilson's work on 'The Cell.

The editor must here return his thanks to Dr. George W. Field for his kindness in allowing the use of his translation of the first part of the book as the basis of the present edition.

Tufts College, Mass., Sept. I9, I902

J. S. KingsLey. 


\section{TABLE OF CONTENTS.}

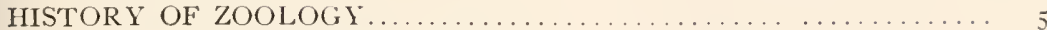

Developmext of Systematic Zoology..................... 6

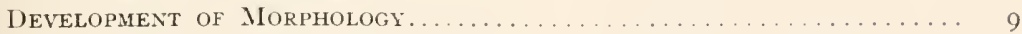

History of the Theory of Evolution....................... I4

THEORY OF THE ORIGIN OF SPECIES....................... I 8

GENERAL MORPHOLOGY AND PHYSIOLOGY .............. 50

General Anatomy................................ 50

The Morphological Units of the Animal Body................ 5 I

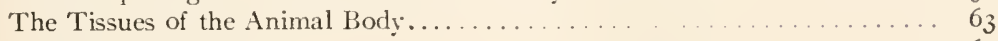

Epithelial Tissues.................................. 64

Connective Tissues.............................. 74

Muscular Tissues................... . . . . ........ so

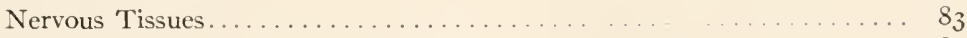

Summary .................................. 87

The Combination of Tissues into Organs............ 88

Vegetative Organs.......................... $9^{\text {I }}$

Organs of Assimilation..................... 9I

Digestive Tract..................... . . . . 93

Respiratory Organs...................... . . $9^{6}$

Circulatory Apparatus........................ 99

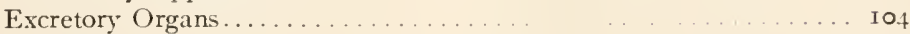

Sexual Organs......................... I0

Animal Organs........................ II

Organs of Locomotion..................... II

Nervous System...................... I I I

Sense Organs.......................... IIt

Summary ...................... 122

Promorphology ...................... 123

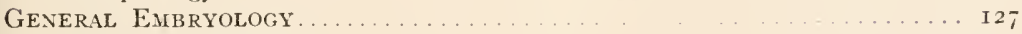

Spontaneous Generation..................... $\ldots \ldots$ 27

Generation by Parents...................... . . . . 28

Asexual Reproduction........................... I 28

Sexual Reproduction. . . . . . . . . . . . . . . . . . . . . I 20

Combined Methods of Reproduction .................... Ijo

General Phenomena of Sexual Reproduction.... . . . . . . . . . . I $3^{2}$

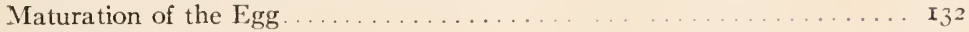

Fertilization..................... I34

Cleavage Processes. . . . . . . . . . . . . . . . . . . . .

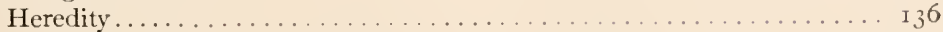

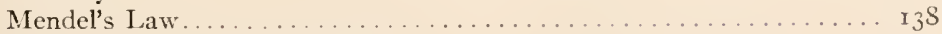

Formation of the Germ Layers. . . . . . . . . . . . . . . . I 45

Different Forms of Sexual Development................... I $\$$. . . . . . .

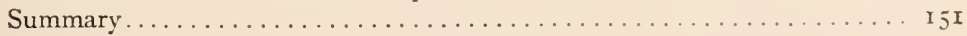




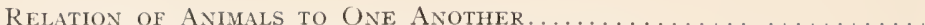

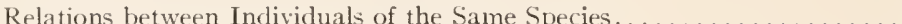

Relations between Individuals of I)ifferent Suecies........ .

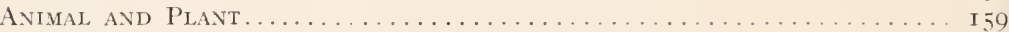

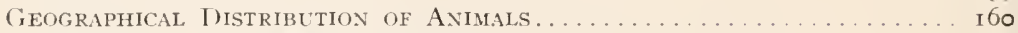

Distribution of ANimlals in TIME.................... I 64

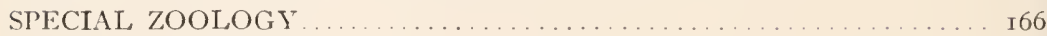

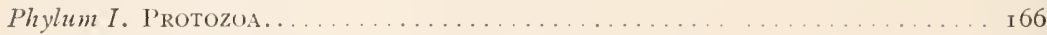

Class I. Rhizopoda.............................. I

Order I. Monera............................ I 72

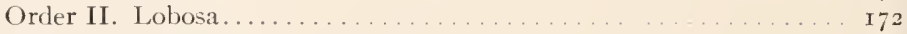

Order III. Heliozoa . . . . . . . . . . . . . . . . . . I73

Order IV. Radiolaria......................... . . 7 74

Order $V$. Foraminifera................. I 7 . . . .

Order VI. Mycetozoa.................... ISo

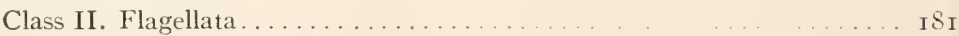

Order I. Autoflagellata......................... I $S_{\text {I }}$

Order II. Dinoflagellata.................... . . . . . . $s_{4}$

Order III. Cystoflagellata..................... . . $8_{4}$

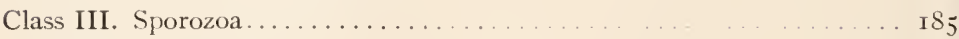

Order I. Gregarinida..................... . . . 56

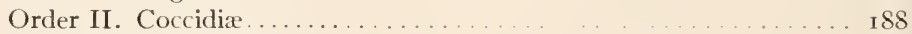

Order III. Hæmosporida ... . . . . . . . . . . . . . . . I 88

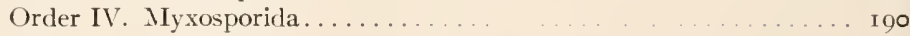

Order V. Sarcosporida....................... I9I

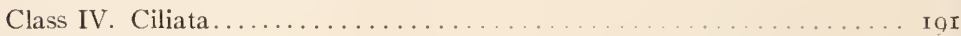

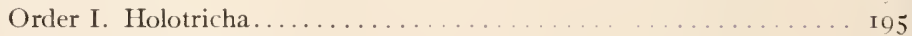

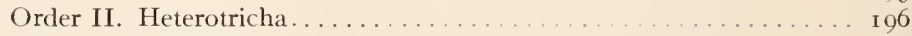

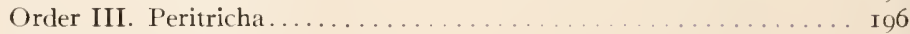

Order IV. Hypotricha.............. . . . . . . . . . I97

Order $\mathrm{V}$. Suctoria........................... 197

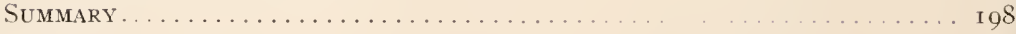

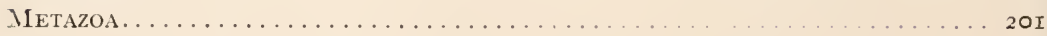

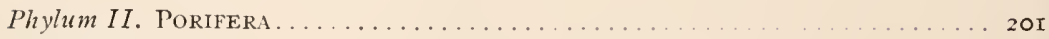

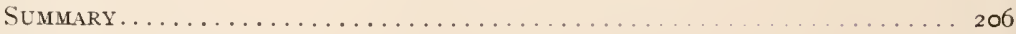

Phylum III. Ceqlenterata.......................... 206

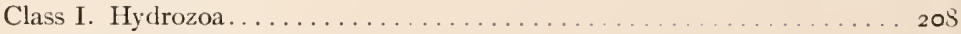

Order I. Hydraria......................... 2 I 7

Order II. Hydrocorallinæ.................... 2 I 7

Order III. Tubularix $=$ Anthomedusæ .............. 2 I 7

Order IV. Campanulariæ $=$ Leptomedusæ ............ 2 I 7

Order $V$. Trachymedusæ......................... 2 I 8

Order VI. Narcomedusæ......................... 2 I8

Order VII. Siphonophora.................... 2 I 8

Class II. Scyphozoa................................. 220

Order I. Discomedusæ. . . . . . . . . . . . . . . . . . 223

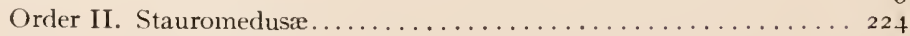

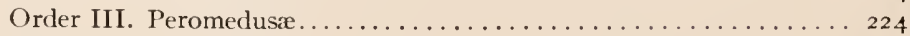

Order IV. Cubomedusæ. . . . . . . . . . . . . . . . . . 224 
PAGE

Class III. Anthozoa.............. . . . . 224

Order I. Tetracoralla ......... . . . . 230

Order II. Octocoralla.......... . . . . . 230

Order III. Hexacoralla......... .... . . . . . 230

Class IV. Ctenophora. . . . . . . . . . . . . . . . . . . . . . . 232

SuMuLRY. ..................... . . . . . 235

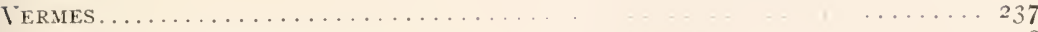

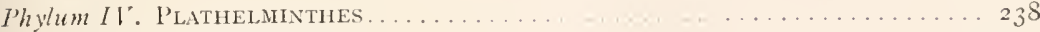

Class I. Turbellaria............................ 240

Class II. Trematoda . . . . . . . . . . . . . . . . . . . . 242

Order I. Polystomia........... . . . . . . . . . . . . . 244

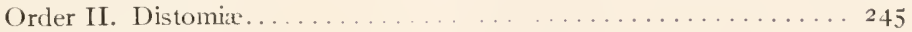

Class III. Cestoda . . . . . . . . . . . . . . . . . . . . . . 247

Class IV. Nemertini. . . . . . . . . . . . . . . . . . . . . . . . . 255

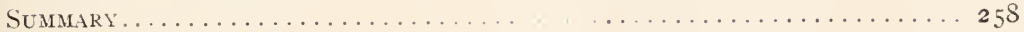

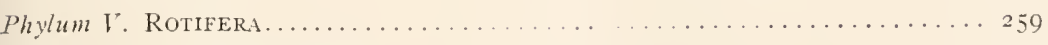

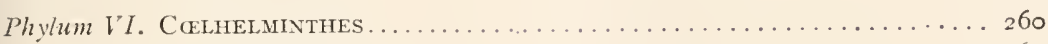

Class I. Chetognathi. . . . . . . . . . . . . . . . . . . . . . 262

Class II. Nemathelminthes. . . . . . . . . . . . . . . . . . . . . . $26_{3}$

Order I. Nematoda.... . . . . . . . . . . . . . . . . . . . . . . 263

Order II. Gordiacea............................... 268

Order III. Acanthocephala... . . . . . . . . . . . . . . . . . . . 268

Class III. Annelida . . . . . . . . . . . . . . . . . . . . . . . . . . . . 260

Sub Class I. Chetopoda...... . . . . . . . . . . . . . . . 270

Order I. Polychretre... . . . . . . . . . . . . . . . . . 276

Order II. Oligochreta..... . . . . . . . . . . . . . 278

Sub Class II. Gephyræa . . . . . . . . . . . . . . . . . . . 79

Order I. Chatiferi....................... . . . $8 \mathrm{Sr}_{\mathbf{r}}$

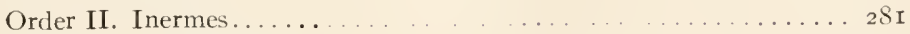

Order III. Priapuloidea ............................. 28 I

Sub Class III. Hirudinei. ... . . . . . . . . . . . . . . . $28 I$

Class IV. Polyzoa. ......... . . . . . . . . . . . . . . . . . . . . . . $28_{4}$

Class V. Phoronida........................ . . . . . $288_{7}$

Class VI. Brachiopoda. . . . . . . . . . . . . . . . . . . . . . . . . $28_{7}$

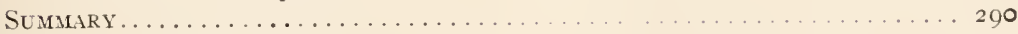

Phylum VII. Echinoderma............................. 291

Class I. Asteroidea. . . . . . . . . . . . . . . . . . . . . . . . 295

Class II. Ophiuroidea................. . . . . . . . . . . . . 298

Class III. Crinoidea ... . . . . . . . . . . . . . . . . . . . . . . . . 209

Sub Class I. Eucrinoidea. . . . . . . . . . . . . . . . . . . . . . . . 302

Sub Class II. Edrioasteroidea . . . . . . . . . . . . . . . . . . . . 302

Sub Class III. Cystidea . . . . . . . . . . . . . . . . . . . . . . 303

Sub Class IV. Blastoidea . . . . . . . . . . . . . . . . . . . . . . . $30_{3}$

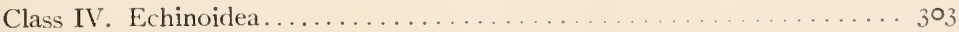

Class V. Holothuroidea... . . . . . . . . . . . . . . . . . . . . 306

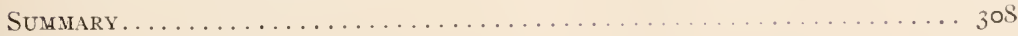

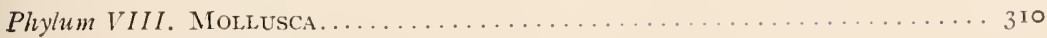

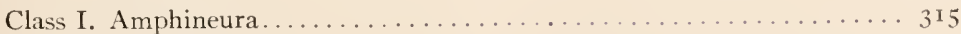

Class II. Acephala . . . . . . . . . . . . . . . . . . . . . $33^{17}$

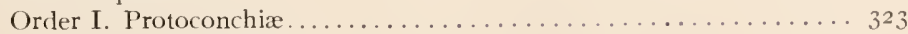

Order II. Heteroconchiæ............................ 324 


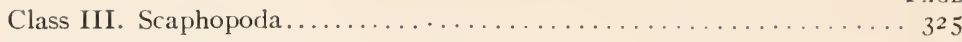

Class IV. Gasteropoda . . . . . . . . . . . . . . . . . . . . . . . . 325

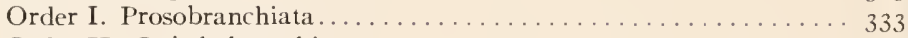

Order II. Opisthobranchiata . . . . . . . . . . . . . . . . . . 334

Order III. Pulmonata . . . . . . . . . . . . . . . . . . . . 335

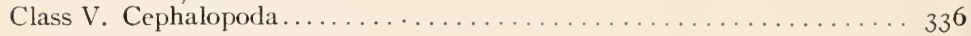

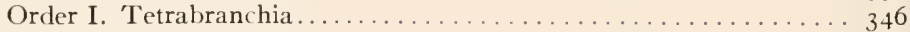

Order II. Dibranchia . . . . . . . . . . . . . . . . . . . 347

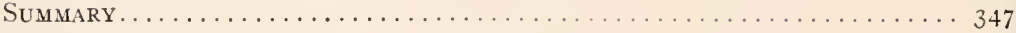

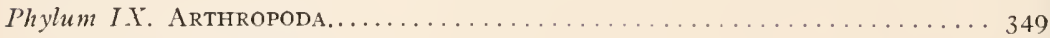

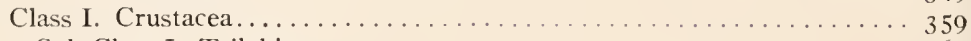

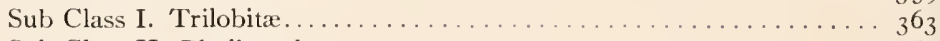

Sub Class II. Phyllopoda . . . . . . . . . . . . . . . . . . . . . . 365

Order I. Branchiopoda........................... 366

Order II. Cladocera............................ 366

Sub Class III. Copepoda. . . . . . . . . . . . . . . . . . . . . . . 366

Order I. Eucopepoda. . . . . . . . . . . . . . . . . . . . . . . . . 369

Order II. Siphonostomata . . . . . . . . . . . . . . . . . . . 360

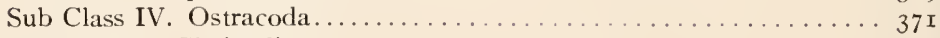

Sub Class V. Cirripedia ......................... 37 r

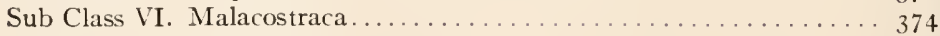

Legion I. Leptostraca . . . . . . . . . . . . . . . . . . . . . 375

Legion II. Thoracostraca . . . . . . . . . . . . . . . . . . . 375

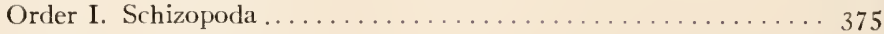

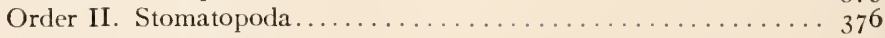

Order III. Decapoda ...................... 377

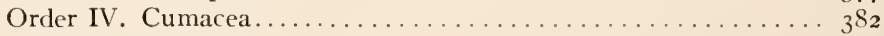

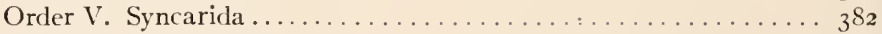

Legion III. Arthrocostraca . . . . . . . . . . . . . . . . . . . . . . . . . . . . . . . . . . . . . .

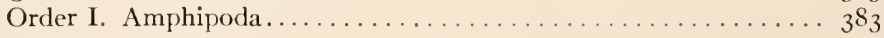

Order II. Isopoda . . . . . . . . . . . . . . . . . . . $3{ }_{3} 8_{5}$

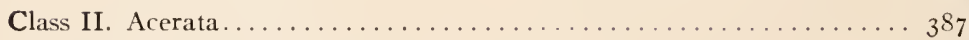

Sub Class I. Gigantostraca. . . . . . . . . . . . . . . . . . . . . . . . . . . . . . . . . . . . . . . . . . . . . . .

Order I. Xiphosura . . . . . . . . . . . . . . . . . . . . 388

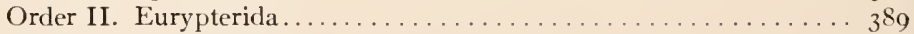

Sub Class II. Arachnida . . . . . . . . . . . . . . . . . . . . . . . 389

Legion I. Arthrogastrida........... . . . . . . . . . . . 39 I

Order I. Scorpionida.............................

Order II. Phrynoidea . . . . . . . . . . . . . . . . . . . . 392

Order III. Microthelyphonida . . . . . . . . . . . . . . . 393

Order IV. Solpugida . . . . . . . . . . . . . . . . . . . . . . . . . . . . . . . . . . . . . . . . 393

Order V. Pseudoscorpii. . . . . . . . . . . . . . . . . . . . . . . . . . . . . . . . . . . . . . . . . . . . .

Order VI. Phalangida . . . . . . . . . . . . . . . . . . . . . . 394

Legion II. Sphrerogastrida . . . . . . . . . . . . . . . . . . . 395

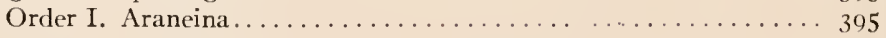

Order II. Acarina . . . . . . . . . . . . . . . . . . . . . . . . . . . . . . . . . . . . . . . . . . . 396

Order III. Linguatulida. . . . . . . . . . . . . . . . . . . . . . 397

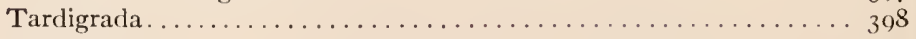

Pycnogonida. . . . . . . . . . . . . . . . . . . . . . . . . . . . . . . 399

Class III. Malacopoda. . . . . . . . . . . . . . . . . . . . . . . . . . . . . 399

Class IV. Insecta ... . . . . . . . . . . . . . . . . . . . . . 400

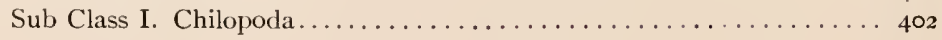


Sub Class II. Hexapoda.
Order I. Apterygota...

Order II. Archiptera

Order III. Orthoptera

Order IV. Neuroptera.

Order V. Strepsiptera.

Order VI. Coleoptera.

Order VII. Hymenoptera

Order VIII. Rhynchota.

Order IX. Diptera

Order X. Aphaniptera

Order XI. Lepidoptera

Class V. Diplopoda........................... 433

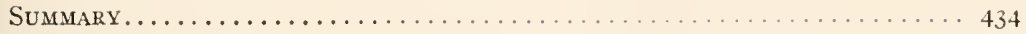

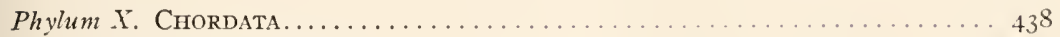

Sub Phylum I. Leptocardii............................ 439

Sub Phylum II. Tunicata......................... 44 I

Order I. Copelatæ.......................... 443

Order II. Tethyoidea........................... 443

Order III. Thaliacea ......................... 447

Sub Phylum III. Enteropneusta . . . . . . . . . . . . . . . . . . . 448

Sub Phylum IV. Vertebrata . . . . . . . . . . . . . . . . . . . . . . 449

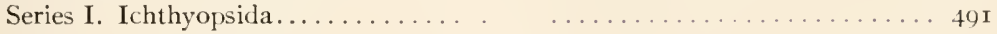

Class I. Cyclostomata . . . . . . . . . . . . . . . . . . . . 49 I

Sub Class I. Myzontes............................. 493

Sub Class II. Petromyzontes........................ 493

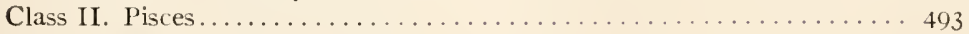

Sub Class I. Elasmobranchii....................... 504

Order I. Selachii. .......................... 506

Order II. Holocephali...... . . . . . . . . . . . . . . . . . 507

Sub Class II. Ganoidei........................... 507

Sub Class III. Teleostei......................... 50 o

Order I. Physostomi.......................

Order II. Pharyngognathi..................... $5^{10}$

Order III. Acanthopteri...................... $5^{\text {I I }}$

Order IV. Anacanthini..................... $5_{\text {II }}$

Order V. Lophobranchii....................... 5 I I

Order VI. Plectognathi........................... $5^{12}$

Sub Class IV. Dipnoi........................ $5^{\mathrm{I} 2}$

Class III. Amphibia . . . . . . . . . . . . . . . . . . . . $5^{\text {I } 3}$

Order I. Stegocephali....................... 5 I9

Order II. Gymnophiona ....................... 519

Order III. Urodela . . . . . . . . . . . . . . . . . . . 5 I9

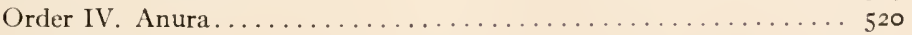

Series II. Amniota ............................ 520

Class I. Reptilia ............................ 52 I

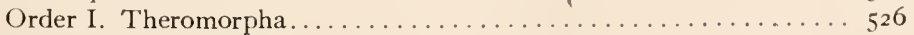

Order II. Plesiosauria ......................... 526

Order III. Ichthyosauria . . . . . . . . . . . . . . . . . 526

Order IV. Chelonia . . . . . . . . . . . . . . . . . . . . . 526

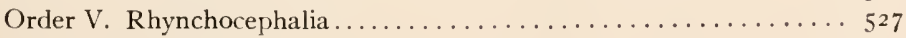

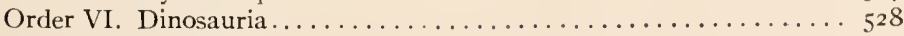

Order VII. Squamata........................... $5^{29}$ 
Order VIII. Crocodilia

Order IX. Pterodactylia

Class II. Ares

Order I. Saurure

Order II. Odontornithes

Order III. Ratitx.

Sub Class I. Monotremata. 545

SubC lass II. Marsupialia ........

Sub Class III. Placentalia ........... . . . . . . . . . 56 I

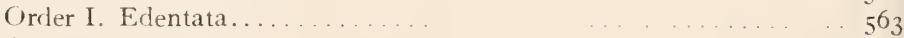

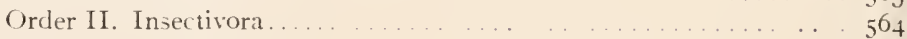

Order III. Chiroptera. ....................... 564

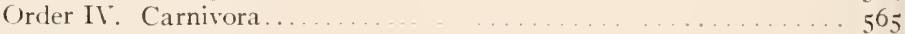

Order $\mathrm{V}$. Rodentia............................ . . . . 566

Order VI. Engulata..... . . . . . 566

Order III. Proboscidia... . . . . . . . . . . . . . . . . . 570

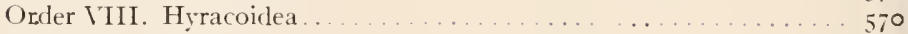

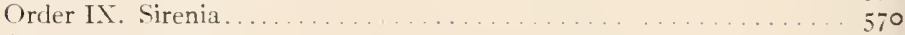

Order X. Cetacea ........................ $57^{\mathrm{r}}$

Order XI. Prosimix..... … . . . . . . . . . . . 572

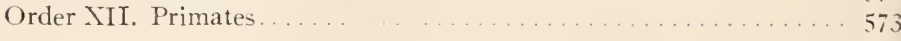

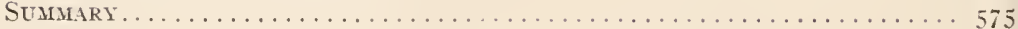




\section{GENERAL PRINCIPLES OF ZOOLOGY.}

\section{INTRODLCTION.}

Man's Relation to Other Animals.-The observant man sees himself in the midst of a manifold variety of organisms, which in their structure, and even more in their vital phenomena, exhibit a similarity to his own being. This similarity, with many of the mammals, especially the anthropoid apes, has the sharpness of a caricature. In the invertebrate animals it is softened; yet even in the lowest organisms it is still to be found: although here the vital processes which have reached such complexity and perfection in ourselves can only be recognized in their simplest outlines. Man is part of a great whole, the Animal Kingdom, one form among the many thousand forms in which animal organization has found expression.

Purpose of Zoological Study.-If we would, therefore, fully understand the structure of man, we must, as it were, look at it upon the background which is formed by the other animals, and for this purpose we must investigate their conditions. Apart from its relations to man, zoology has to explain the organization of animals and their relations to one another. This is a rich field for scientific activity; its enormous range is a consequence, on the one hand, of the well-nigh exhaustless variety of animal organization, and, on the other, of the different points of view from which the zoologist attacks his problem.

In the first half of the last century the conception was prevalent that the aim of zoology is to furnish every animal with a name, to characterize it according to some easily recognizable features, and to classify it in a way to facilitate quick identification. By Natural History was understood the classification of animals or systematic zoology; that is to say, only one part of zoology, which can pretend to scientific value only when it is brought into relation with other problems (geographical distribution, variation, evolution). This conception has become more and more subordinated. The ambition to describe the largest possible number of new forms belongs to the past. In fact there is a tendency to undue neglect of classification. Morphology and Physiology to-day dominate the sphere of the zoologist's work. 
Morphology, or the study of form, has first to describe all which can be seen externally, as size, color, proportion of parts. But since the external appearance cannot be understood without knowledge of the internal organs which condition the external form, the morphologist must make use of dissection, of Anatomy, and describe their forms and methods of combination. In his investigation he only stops when he has arrived at the morphological elements of the animal body, the cells. Therefore we cannot contrast Morphology and Anatomy, and ascribe to the former the description of only the external, and to the latter of only the internal parts; the kind of knowledge and the mental processes are the same in both cases. The distinction, too, is unnatural, since in many instances organs which usually lie in the interior of the body, belong in other cases to the surface of the body, and are accessible for direct observation.

Comparative Anatomy.-For morphology, as for every science, the mere accumulation of facts is not sufficient to give the subject the character of a science; an additional mental elaboration of this material is necessary. Such a result is reached by comparison. The inorphologist compares animals with each other according to their structure, in order to ascertain what parts of the organization recur every where, what only within narrow limits. He thus gains a double advantage: (I) an insight into the relationships of animals, and hence the foundation for a Natural System; (2) the evidence of the laws which govern organisms. Any organism is not a structure which has arisen independently and which is hence intelligible by itself: it stands in relation to the other members of the animal kingdom. We can only understand its structure when we compare it with the closely and the more distantly related animals, as when we compare man with the other vertebrates and with many lower invertebrates. We have to consider one of the most mysterious phenomena of the organic world, the path to the full explanation of which was first outlined by the Theory of Evolution, as will be shown in another chapter.

Ontogeny.-To morphology belongs, as an important integral part, Ontogeny or Embryology. Only a few animals are completely formed in all their parts at the beginning of their individual existence; most of them arise from the egg, a relatively simple body, and then step by step attain their permanent form by complicated changes. The morphologist must determine by observation the different stages, compare them with the mature animals, and with the structure and developmental stages of other animals. Then there is revealed to him the same conformity to law which dominates the mature animals, and a knowledge of this conformity 
is of fundamental importance as well for classification as for the causal explanation of the animal form. The developmental stages of man show definite regular agreements, not only with the structure of the adult human being, which in and of itself would be intelligible, but also with the structure of lower vertebrates, and even with many of the still lower invertebrate groups.

Physiology.-In the same way as the morphologist studies the structure, the physiologist studies the vital phenomena of animals and the functions of their organs. Formerly life was regarded as the expression of a special wital force peculiar to organisms, and any attempt at a logical explanation of the vital processes was thereby renounced. Nost modern physiologists have abandoned this theory of a vital force; they have attempted to explain life as the summation of extremely complicated chemico-physical processes, and thus to apply to the organic world those principles which prevail in the inorganic realm.

Developmental Physiology ("Entwicklungsmechanik").--Since each organism is the product of its development; since, further, the derelopment represents the summation of most complicated vital processes, the explanation of the organic bodily form is, therefore, in ultimate analysis a physiological problem; a problem whose solution lies still in the indefinitely distant future. It has to explain how the apparently simple fertilized egg is converted into the complicated adult organism with its many organs regularly arranged. The potentiality of the complexity of the adult must be contained in the egg. But it is still a matter of dispute as to how this potentiality is conditioned: whether as a mosaic of minute particles, each corresponding to a peculiarity of the adult organism, or as a substance of simpler structure, in which complexity only appears in the course of development. We can proceed experimentally in such a way that the conditions of development are artifically altered, and the results may be compared with the normal processes. It is also possible to study the modifications which one and the same developmental process undergoes in different species, modifications which are dependent upon the life conditions of the animals and of their young. Then, too, there are experiments of the same kind performed by nature and which have the same informing value as the artificially arranged conditions. Such researches have accomplished much in the last decade and have resulted in a deeper understanding of the developmental processes.

The potentialities contained in the fertilized egg are the hereditary elements which are transmitted from the parent to the next generation and which result in the resemblance of the offspring to the parents. The study of these elements and the way in which they are transmitted from 
one generation to another-in other words, the laws of heredity-has recently made a great advance by investigations in two directions: (I) through the biometric method or the statistics of variation, and (2) by the so-called 'Mendelian analysis' of the hereditary potentialities. Both lines of investigation (to be considered more in detail later) have opened up in an unexpected way the possibility of submitting to exact research the questions of variation and heredity, fundamentally important for the understanding of the living world.

Biology.-According as the relations of each organism to the external world are brought about through its vital phenomena, there belongs to physiology, or at least is connected with it, the study of the conditions of animal existence, Ecology, often called Biology in the narrower sense, the broader meaning being the science of all living things, both animals and plants. This branch has of late attained considerable importance. How animals are distributed over the globe, how climate and conditions influence their distribution, how by known factors the structure and the mode of life become changed, are questions which are to-day discussed more than ever before.

Paleontology.-Finally to the realm of zoology belongs also Paleozoology or Paleontology, the study of the extinct animals. For between the extinct and the living animals there exists a genetic connection: the former are the precursors of the latter, and their fossil remains are the most trustworthy records of the history of the race, or Phylogeny. 


\section{HISTORY OF ZOOLOGY.}

Methods of Zoological Study.-In the history of zoology we can distinguish two great currents, which have been united in a few men, but which on the whole have developed independently, nay, more often in pronounced opposition to each other; these are on the one side the systematic, on the other the morphologico-physiological mode of studying animals. In this brief historical summary they will be kept distinct from one another, although in the commencement of zoological investigation there was no opposition between the two points of view, and even later this has in many instances disappeared.

Aristotle, the great Greek philosopher, has been called the Father of Natural History. Equipped with the literary aid of an extensive library and pecuniary means, he pursued the inductive method, the only one capable of furnishing secure foundations in the realm of natural science. There have been preserved only parts of his three most important zoological works, "Historia animalium," "De partibus," and "De generatione," works in which zoology is founded as a universal science, since anatomy and embryology, physiology and classification find equal consideration. How far Aristotle, notwithstanding many errors, had a correct knowledge of the structure and embryology of animals, is shown by the fact that many of his discoveries have been confirmed only within a century. Thus Aristotle knew, though only lately rediscovered by Johannes Muiller, that many sharks are not only viviparous, but that also the embryo becomes fixed to the maternal uterus and there is formed a contrivance for its nutrition resembling the mammalian placenta; he knew the difference between male and female cephalopods, and that the young cuttlefish has a preoral yolk-sac.

The position which Aristotle took in reference to the classification of animals is of great interest; he mentions in his writings about five hundred species. Since he does not mention very well-known forms, like the badger, dragon-fly, etc., we can assume that he knew many more, but did not regard it necessary to give a catalogue of all the forms known to him, and that he mentioned them only if it was necessary to refer to certain physiological or morphological conditions found in them. 
This neglect of the systematic side is further shown in the fact that the

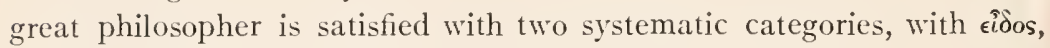

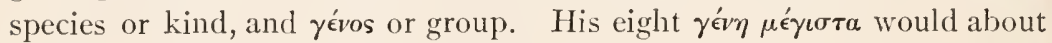
correspond with the Classes of modern zoology; they have been the starting-point for all later attempts at classification, and may therefore be

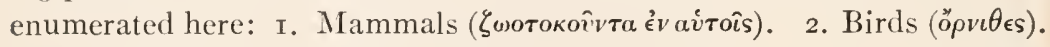

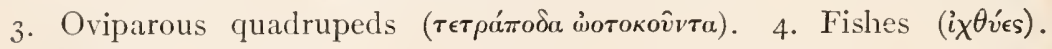

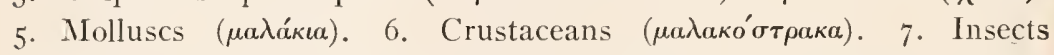

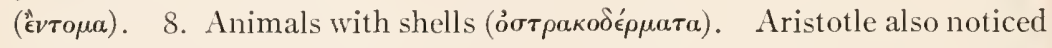
the close connection of the first four groups, since he, without actually carrying out the division, contrasted the animals with blood, "ैvou red blood), with the bloodless, ávaua (better, colorless blood or no blood at all).

\section{DEVELOPEMENT OF SYSTEMATIC ZOOLOGY.}

Pliny.- It is a remarkable fact that after Aristotle, an exclusively systematic direction should have been taken. This is explicable only when we consider that the continuity of investigation was interrupted by the decline and ultimate complete collapse of ancient civilization, and by the triumphant advance of Christianity. The decay is seen in the writings of Pliny. Although this Roman scholar was long lauded as the foremost zoologist of antiquity, he is now given the place of a not even fortunate compiler, who collected from the writings of others the accurate and the fabulous indiscriminately, and replaced the natural classification according to structure by the unnatural division according to the place of abode (flying animais, land animals, water animals).

Zoology of the Middle Ages.-The rise of Christianity resulted in the complete annihilation of science and investigation. Then came a time when answers to questions capable of solution by the simplest observation were sought by rummaging of the works of standard authors. How many teeth the horse has, was debated in many polemics, which would have led to bloodshed if one of the authors had not thought to look into a horse's mouth. Significant of this mental bias which prevailed throughout the entire Middle Ages is the 'Physiologus' or 'Bestiarius,' from which the zoological authors of the Middle Ages drew much material. The book in its various editions names about seventy animals, among them many creatures of fable: the dragon, the unicorn, the phonix, etc. Most of the accounts given of rarious animals are fables intended to illustrate religious or ethical teachings. There are indeed, exceptions to this ceneral characteristic of 
the Middle Ages, notably in the writings of the Dominican, Albertus Magnus and the Augustinian, Thomas Cantipratensis. In so far as he had opportunity, Albertus Magnus, endeavored to support his statements by personal observation. But that this beginning of the scientific method raised hardly an echo only emphasizes the general spirit of the time.

At the close of the Middle Ages, when the interest in science awoke anew, Aristotle's conceptions were taken up and elaborated by the Englishman Wotton. In 1552 he published his work "De differentiis animalium," in which he essentially copied the system of Aristotle, except that he admitted the new group of plant-animals or zoophytes. However, the title, 'On the Distinguishing Characters of Animals,' shows that of Aristotelian knowledge the systematic sile obtained the chief recognition, and thus Wotton's work inaugurated the period of systematic zoology, which in Ray, but even more in Linneus, found its most brilliant exponents.

Linnæus, the son of a Swedish clergman, was born in Rashult in 1707 . Pronounced by his teachers to be good for nothing at study, he was saved from learning the cobbler's trade through the influence of a physician, who recognized his fine abilities and turned him to medical studies. He studied at Lund and Upsala; at the age of twenty-eight he made extended tours on the Continent, and at that time gained recognition from the foremost men in his profession. In I74I he became professor of medicine in Upsala, some years later professor of natural history. He died in ${ }_{7} 78$.

Improvement of Zoological Nomenclature by Linnæus.-Linnæus's most important work is his "Systema Naturæ," which, first appearing in I735, up to I $766-68$ passed through twelve editions. 'This has become the foundation for systematic zoology, since it introduces for the first time (I) sharper divisions, (2) a definite scientific terminology, the binomial nomenclature, and (3) brief, comprehensive, clear diagnoses. Linnæus dirided the entire Animal Kindom into Classes, the Classes into Orders; these into Genera, the Genera into Species. The term Family was not employed. Still more important was the binomial nomenclature. Hitherto the common names were in use and led to much confusion; the same animals had different names, and different animals had the same names; in the naming of newly discovered animals there was no generally accepted principle. This inconvenience was entirely obviated by Linneus in the tenth edition of his Systema by the introduction of a scientific nomenclature. The first word, a noun, designates the genus to which the animal belongs. the following word, usually an adjectve, the species within the genus. 'The names Canis familiaris, Canis lupus, Canis vulpes, indicate that the dog, 
wolf, and fox are related to one another, since they belong to the same genus, the genus of doglike animals, of which they are different species. Linnaus's method was particularly valuable in the description of new species, inasmuch as it at the ouset informed the reader of the relationships of the new species.

In his characterization of the various groups Linnæus broke with the prevailing custom. His predecessors (as Gessner, Aldrovandus) had given a verbose and detailed description of each animal, from which the beginner was scarcely able to see what was specially characteristic for that animal, a matter which should have been emphasized in the definition. Linneus, on the other hand, introduced brief diagnoses, which in a few words, never in sentence form, gave only what was necessary for recognition, a matter of great importance, in view of the enormously increasing number of known animals.

Influence- of the Linnean System.-But in the great superiority of the Linnean System lay at the same time the germ of the one-sided derelopment which zoology came to take. The perfecting of the system, which undoubtedly had become necessary, gave that a brilliant aspect, and hid the fact that classification is not the ultimate purpose of investigation, but only an important and indispensable aid to it. In the zeal for naming and classifying animals, the higher goal, knowledge of the nature of animals, was lost sight of, and the interest in anatomy, physiology, and embryology flagged.

From these reproaches we can scarcely spare Linnæus himself, for while in his "Systema Nature" he treated of a much larger number of animals than any earlier zoologist, he brought about no deepening of our knowledge.' The manner in which he divided the animal kingdom is rather a retrogression than an advance. He recognized six classes: Mammalia, Ares, Amphibia, Pisces, Insecta, Vermes. The first four classes correspond to Aristotle's four groups of animals with blood. In the division of the invertebrated animals into Insecta and Vermes Linnous stands undoubtedly below Aristotle, who set up a larger number of groups.

But in his successors, we see the damage wrought by the systematic method. The diagnoses of Linnxus were for the most part models, which, mutatis mutandis, could be employed for new species with little trouble. There was needed only some exchanging of adjectives to express the differences. With the hundreds of thousands of different species of animals there was no lack of material, and so the way was opened for that spiritless species-making which in the first half of the last century brought zoology into such discredit. 


\section{DENELOPMENT OF MORPHOLOGY.}

Anatomists of Classic Antiquity.-Comparative anatomy-for this chiefly concerns us here-for a long time owed its development to the students of human anatomy. 'The disciples of Hippocrates sturlied animal anatomy for the purpose of obtaining an idea of human organization from the structure of other mammals. The work of classical antiquity most prominent in this respect, the Human Anatomy of Galen ( $13 \mathrm{I}-20 \mathrm{I}$ A.D.), is based chiefly upon observations upon $\operatorname{logs}$, monkeys, etc.; for in ancient times, and even in the Middle Ages, there was repugnance to making the human cadaver a subject of study.

Middle Ages. - The first thousand years, in which Christianity ruled the mental life of the people, held to the writings of Galen and the works of his commentators, and seldom took occasion to prove their correctness by observations. With the ending of the Middle Ages the interest in scientific research first made its way.

Vesal ( $\left.5^{1} 4^{-1} 5^{6} 4\right)$, the creator of modern anatomy, investigated the human cadaver and pointed out numerous errors in Galen's writings which had arisen through the extension to human anatomy of the discoveries made upon other animals. By his corrections of Galen, Vesal was drawn into controversy with his teacher, Sylvius, and with his renowned contemporary Eustachius, which did much for the development of comparative anatomy. At first animals were dissectect only for the purpose of disclosing the cause of Galen's mistakes, but later through a zeal for facts. It was natural that vertebrates were first studied, since they stand next to man in structure. Thus there appeared in the same century with Vesal drawings of skeletons by Coiter; the zootomical writings of Fabricius ab Aquapendente, etc.

Beginning of Zootomy.-But later attention was turned to insects and molluscs, even to the echinoderms, colenterates, and Protozoa. Here three men who lived at the end of the serenteenth century deserve mention, the Italian Malpighi and the Dutchmen Swammerdam and Leeuwenhoek. The former's "Dissertatio de bombyce" was the pioneer for insect anatomy, since by the discovery of the vasa Malpighii, the heart, the nervous system, the trachea, etc., an extraordinary extension of our knowledge was brought about. Of Swammerdam's writings attention should be called to "The Bible of Nature," a work to which no other of that time is comparable, since it contains discoveries of great accuracy on the structure of bees, Mayflies, snails, etc. Leeuwenhoek, finally, was most fortunate in the field of microscopic research. Besides other things he studied especially the minute inhabitants of the fresh waters, the 'infusion-animalcules.' 
The great service of the men named above consists chiefly in that they broke away from the thraldom of book-learning and, relying alone upon their own eyes and their own judgment, regained the blessing of independent and unbiased observation. They spread the interest in observation of nature so that in the eighteenth century the number of naturalhistory writings had increased enormously. There were busy with the study of insect structure and development, de Geer in Sweden, Réaumur in France, Lyonet in Belgium, Rösel von Rosenhof in Germany; the latter besides wrote a monograph on the indigenous batrachia, which is still worth reading. The investigation of the infusoria formed a favorite occupation for Wrisberg, von Gleichen-Russwurm, Schäffer, Eichhorn, and O. F. Müller. As a criterion of the progress made, a mere glance at the illustrations is sufficient. Any one will at a glance recognize the difference between the shabby drawings of an Aldrovandus and the masterly figures of a Lyonet or a Rosel von Rïsenhof.

Peroid of Comparative Anatomy.-Thus through the zeal of numerous men a store of anatomical facts was collected, which needed only a mental reworking; and this was brought about, or at least entered upon, by the great comparative anatomists who lived at the end of the eighteenth and the beginning of the nineteenth century. Among these the French zoologists Lamarck, Savigny, Geoffroy St. Hilaire, Cuvier, and the Germans Meckel and Goethe are especially to be named.

Correlation of Parts.-When the various animals were compared with one another with reference to their síructure there was obtained a series of fundamental laws, particularly the law of the Correlation of Parts and the law of the Homology of Organs. The former showed that there exists a dependent relation between the organs of the same animal; that local changes of one organ lead to corresponding changes at some distant part of the body, and that therefore from the structure of certain parts an inference can be drawn as to the constitution of another part of the body. Cuvier particularly made use of this principle in reconstructing extinct animals.

Homology and Analogy.-Still more important was the idea of the Homology of Organs. In the organs of animals a distinction was drawn between an anatomical and a physiological character; the anatomical character is the sum of form, structure, position, and mode of comnection of organs; the physiological character is their function. Anatomically similar organs in closely related animals will usually have the same functions, as, for example, the liver of all vertebrates produces gall; here anatomical and physiological characteristics go hand in hand. But this need not be the case; very often it may happen that the same function 
is possessed by organs anatomically different; as, for example, the respiration is carried on in fishes by gills, in mammals by lungs. Conversely, anatomically similar organs may have different functions, as the lungs of mammals and the swim-bladder of fishes; similar organs may also undergo a change of function from one group to another; the hydrostatic apparatus of fishes has come to be the seat of respiration in the mammals. Organs with like functions-physiologically equivalent organs-are called 'analogous'; organs of like anatomical constitution -anatomically equivalent organs-are called 'homologous.' It is the task of comparative anatomy to discover in the various parts of animals those which are homologous, and to follow the changes in them conditioned by a change of function.

Cuvier.-The foremost representative of comparative anatomy was Georges Dagobert Cuvier. His investigations extended to the coelenterates, molluscs, arthropods, and vertebrates, living and fossil. He collected his extensive observations into his two chief works "Le règne animal distribué d'après son organization" and "Leçons d'anatomie comparée." Of epoch-making importance was his little pamphlet "Sur un rapprochement à établir entre les différentes classes des animaux," in which he founded his celebrated type theory, and which introduced a reform of classification. The Cuvierian division, the starting-point for all later classifications, differed from all the earlier systems in that the classes of mammals, birds, reptiles, and fishes were united in a higher grade under the name, introduced by Lamarck, of 'vertebrate animals'; and the so-called 'invertebrate animals' were divided into three similar grades, each equal to that of the vertebrate animals, viz., Mollusca, Articulata, and Radiata. Cuvier called these grades standing above the classes, provinces or chief branches (embranchements). But still more important are the differences which appear in the structural basis of the system. Instead of, like the earlier systematists, using a few external characteristics for the division, Cuvier built upon the totality of internal organization, as expressed in the relative positions of the most important organs, especially the position of the nervous system, as determining the arrangement of the other organs. Thus for the first time comparative anatomy was employed in the formation of a natural system of animals.

Cuvier found prevalent the theory that all animals formed a single connected series ascending from the lowest infusorian to man; within this series the position of each animal was determined by the degree of its organization. On the other hand Cuvier taught that the animal kingdom consisted of several co-ordinated unities, the types, which exist inde- 
pendently side by side, within which again there are higher and lower forms. The position of an animal is determined by two factors: first, by its conformity to a type, by the structural plan which it represents; second, by its degree of organization, by the stage to which it attains within its type.

\section{Comparative Embryology.-Evolution vs. Epigenesis.-The} same results which Cuvier reached by the way of comparative anatomy were attained two decades later by C. E. von Baer by the aid of embryology. Embryology is the youngest branch of zoology. The difficulties of observation, due to the delicacy and the minuteness of the developmental stages, were lessened by the invention of the microscope and microscopical technique. Further, there was no idlea of developmental history in the present sense of the word; each organism was thought to be laid down from the first complete in all its parts, and only needed growth to unfold its organs (evolutio); either the spermatozoon must be the young creature which found farorable conditions for growth in the store of food in the egg, or the egg represents the individual and was stimulated to the 'evolutio' by the spermatozoon. This theory led to that of inclusion, which taught that in the ovary of Eve were included the germs of all human beings who have lived or ever will live.

Caspar Friedrich Wolff combated this idea (I 759); he sought to prove by observation that the hen's egg at the beginning is without organization, and that gradually the various organs appear in it. In the embryo there is a new formation of all parts, an epigenesis. This assault upon the evolutionist school was without result, chiefly because Albrecht von Haller, the most celebrated physiologist of the eighteenth century, suppressed the idea of epigenesis.

Von Baer.-Carl Ernst von Baer in his classic work, "Die Entwicklung des Hühnchens, Beobachtung und Reflexion" (I $8_{32}$ ), established embryology as an independent study. Baer confirmed Wolff's doctrine of the appearance of layerlike anlagen, from which the organs arose; and on account of the accuracy with which he proved this he is considered the founder of the germ-layer theory. Further, he came to the conclusion that each type had not only its peculiar structural plan, but also its peculiar course of development. Here we meet for the first time the idea that for the solution of the questions of relationship of animals, and therefore a basis for a natural classification, comparative embryology is indispensable; an idea which in recent years has proved exceedingly fruitful.

Cell Theory.-Of fundamental importance for the further growth of comparative anatomy and embryology was the proof that all organisms, as well as their embryonic forms, were composed of the same elements, the cells. This cell theory, was advanced by Schleiden and Schwann 
during the third decade of the last century and three decades later was completely remodelled by the protoplasm theory of Max Schultze. In the cell theory a simple principle of organization was found for all living creatures, and the wide rea $m$ of histology was open for scientific treatment. But the theory was of the greatest importance for developmental physiology, for only with the recognition of egg, spermatozoon, and clearage spheres as nucleated cells, was there a sound basis for the solution of the problems of fertilization, heredity, and embryonic differentiation and for the experimental proof of hypotheses.

With the establishment and systematic use of comparative anatomy, the cell theory and histology, the ground was prepared for the series of researches which characterized the second half of the nineteenth century: Great advances were made in vertebrate anatomy by the classic researches of Owen, Johannes Müller, Rathke, IIuxley, Gegenbaur and others; our conceptions of organization have been completely altered by the work of Dujardin, Max Schultze, Haeckel, and others, who have proved the unicellularity of the lowest animals. The germ-layer theory was further dereloped by Remak and Kölliker; and applied to the invertebrates by Kowalewsky, Haeckel, and Huxley. It is beyond the limits of this brief historical summary to go into what has been accomplished in other branches of the animal kingdom; it must here suffice to mention the most important changes which the Cuvierian system has undergone under the influence of increasing knowledge.

Changes in the System.- Of the four types of Cuvier the branch Radiata was the one of which he had the least knowledge; it was also the least natural, since it comprised, besides the radially symmetrical cœlenterates and echinoderms, other forms, which, like the worms, were bilaterally symmetrical, or, like many infusorians, were asymmetrical. C. Th. von Siebold introduced the first important change. He limited the type Radiata, or, as he termed them, the Zoophytes, to those animals with radially symmetrical structure (Echinoderms and the Plant-animals); separating all the others, he formed of the unicellular organisms the branch of 'primitive animals' or Protozoa; the higher organized animals he grouped together as worms or Vermes; at the same time he transferred a part of the Articulata, the annelids, to the worm group, and proposed for the other articulates, crabs, millipedes, spiders, and insects, the term Arthropoda.

Leuckart, about the same time ( 1848 ), divided the Radiata into two branches differing greatly in structure. The lower forms, in which no separate body-cavity is present, the interior of the body consisting only of a system of cavities serving for digestion, he called the Colenterata 
(essentially the Zoophyta of older zoologists); to the rest, in which the alimentary canal and the body-cavity occur as two separate cavities, he gave the name Echinoderma.

Thus there resulted seven classes: Protozoa, Colentera, Echinoderma, Vermes, Arthropoda, Mollusca, and Vertebrata. Still this arrangement does not meet the requirements of a natural system and is more or less unsatisfactory. Upon anatomical and embryological characters the Brachiopoda, the Bryozoa, and the Tunicata have been separated from the Mollusca; they form the subject of diverse opinions. The relationships of the first two groups have not yet been settled: of the Tunicata we know indeed that they are related to the Vertebrata, but the differences are such that they cannot be included in that group. The only way out of the difficulty is to unite vertebrates, tunicates, and some other forms in a larger division, Chordata. The Vermes, too, must be divided, as will appear in the second part of this volume.

In the last decade of the nineteenth century and the beginning of the present, physiological investigation has taken a place beside morphology. Its most important tool is experiment. While experiments have long been invoked to settle biological problems, they are now used in the most extensive and systematic manner; especially are methodical breeding and crossing experiments employed to solve the problems of variation and heredity. There are also investigations into the laws which regulate the animal form, in which the separate stages of embryonic and post-embryonic development are exposed to modifying influences (removal or transplantation of blastomeres or parts of the body, employment of different temperatures, chemical, mechanical, electrical stimuli), and the results are compared with those of normal conditions. An important aid in these studies is the mathematical statistical method by which the value of the results of observation and experiment is tested. The second half of the century just closed was especially characterized by the development of the theory of evolution, the history of which is given in a separate section.

\section{HISTORY OF THE THEORY OF EVOLUTION.}

The theory of evolution has developed into a question whose importance might, on a superficial examination, be underrated, but which has grown into a problem completely dominating zoological research, and has occupied not only zoologists, but all interested in science generally. This is the question of the logical value of the conceptions species, genus, family, etc. 
The Nature of Species.-In nature we find only separate animals: how comes it that we classify them into larger and smaller groups? Are the single species, genera, and the other divisions fixed quantities, as it were fundamental conceptions of nature, or a Creator's thoughts, which find expression in the single forms? Or are they abstractions which man has introduced into nature for the purpose of making it comprehensible to his mental capabilities? Are the specific and generic names only expressions which have become necessary, from the nature of our mental capacity, for the expression of relationships in nature, which in and for themselves are not immutable, and hence can undergo a gradual change? Practically speaking, the problem reads: are species constant or changeable? What is true for species must necessarily be true for all other categories of the system, all of which in the ultimate analysis rest upon the conception of species.

Ray's Conception of Species.- One of the first to consider the conception of species was John Ray. In the attempt to define a species he encountered difficulties. In practice, animals which differ little in structure and appearance from one another are ascribed to the same species; this practical procedure cannot be carried out theoretically; for there are males and females of the same species which differ more from one another than do the representatives of different species. Ray reached the genetic definition when he said: for plants there is no more certain criterion of specific unity than their origin from the seeds of specifically or individually like plants; that is to say, generalized for all organisms: to one and the same species belong individuals which spring from similar ancestors.

The 'Cataclysm Theory.'-With Ray's definition an uncontrollable element was brought into the conception of species, since no systematist usually could know anything, as to whether the representatives of the species before him sprang from similar parents. It was therefore only natural that the conception of species put on a religious garb, since by resting upon theological ideas it found a firmer support. Linnecus said: "Tot sunt species quot ab initio creavit infinitum Ens." Linnxus's definition showed itself untenable, as soon as paleontology began to make accessible a vast quantity of extinct animals preserved as fossils. Cuvier proved that these fossils were the remains of animals of a previous time. Just as the formation of the earth's crust by successive layers made possible the recognition of different periods in the earth's history, so paleontology showed how to recognize different periods in the regetable and animal world of life on our globe. Each geological age was characterized by a special world of animals; and these animal worlds differed the more from 
the present, the older the period of the earth to which they belonged. All these generalizations led Cuvier to his cataclysm theory: that a great revolution brought each period of the earth's history to an end, destroying all life, and that upon the newly formed virgin earth a new organic world of immutable species sprang up. As a believer in the immutability of species, Cuvier was forced to combat the idea of any genetic connection between the living and the fossil forms.

Cuvier's theory of cataclysms gave no scientific explanation of the origin of the successive populations of the earth. Such an explanation is only possible by the hypothesis that the later animal worlds have descended from the earlier. So it happened that the idea of the fixity of species was given up and replaced by that of mutability and descent-the theory of evolution.

Darwin's Predecessors.-Even in Cuvier's time there prevailed a strong current in favor of this theory. It found expression in England in the writings of Erasmus Darwin (grandfather of Charles Darwin); in Germany in the works of Goethe, Oken, and the disciples of the 'natural philosophical' school; in France the genealogical theory was developed by Buffon, Geoffroy St. Hilaire, and Lamarck. Its clearest expression was found in Lamarck's "Philosophie zoologique" (I809); its arguments will be considered in the following paragraphs.

Lamarck (born, 1744, died, 1829) taught that at first organisms of the simplest structure arose through spontaneous generation from nonliving matter. From these simplest living creatures have developed, by gradual changes in the course of an immeasurably vast space of time, the present species of plants and animals, without any break in the continuity of life upon our globe; the terminal point of this series is man; the other animals are the descendants of those forms from which man has developed. Lamarck regarded the animal kingdom as a single series grading from the lowest animal up to man. Among the causes which may influence the change of organisms, Lamarck emphasized particularly use and disuse; the giraffe has obtained a long neck because he was compelled to stretch, in order to browse the leaves on high trees; conversely, the eyes of animals which live in the dark have degenerated from lack of use. The direct influence of the external world must be unimportant; the changes in the surroundings must for the most part act indirectly upon animals by altering the conditions for the use of organs.

Evolution vs. Creation.-Lamarck's work remained almost unnoticed by his contemporaries. Later there arose a violent controversy between the defenders and the opponents of the evolution theory when [1830] Geoffroy St. Hilaire in a debate defended against Cuvier the thesis 
of a near relationship of the vertebrates and the insects. The conflict ended in the complete overthrow of the theory of evolution; the defeat was so complete that the problem vanished for a long time, and the theory of the fixity of species agrain became dominant. This was occasioned by many causes. The theory of Geoffroy St. Hilaire and Lamarck was rather a clever conception than founded on abundant facts; besides, it had in it as a fundamental error the doctrine of the serial arrangement of the animal world. Opposed to this stood Cuvier's extensive knowledge, making it easy for him to show that the animal kingdom was made up of separate co-ordinated groups, the types.

Lyell.- In the same year in which Cuvier obtained his victory, his theory of the succession of numerous animal worlds upon the globe received its first blow. Cuvier's cataclysm theory had two sides, a geological and a biological. Cuvier denied the continuity of the various terrestial periods, as well as the continuity of the fauna and flora belonging to them. In I830-32 appeared the "Principles of Geology" by Lycll, which, in the realm of geology, completely set aside the cataclysm theory. Lyell proved that the supposition of violent revolutions was not necessary to explain the changes of the earth's surface and the superposition of its strata; that rather the constantly acting forces, elevations and depressions, the erosive action of water, are sufficient to furnish a complete explanation. Very gradually in the course of time the earth's surface has changed, and passed from one period into the next, and still at the present day constant change is going on. The continuity in the history of the earth, here postulated for the first time, has since then become one of the fundamental axioms of Geology; on the other hand the discontinuity of living creatures, was for a long time regarded as correct.

Darwin.- It is the great merit of Charles Darwin that he took up the theory of descent anew after it had rested a decade, and later brought it into general recognition. With this began the most important period in the history of zoology, a period in which the science not only made such an advance as never before, but also began to obtain a permanent influence upon the general views of men.

Charles Darwin was born in I809. After studying at the universities of Edinburgh and Cambridge, he joined the English war-ship "Beagle," as naturalist. In its voyage from ${ }^{2} \delta_{3}$ I to 36 around the globe, Darwin recognized the peculiar character of island faunas, particularly of the Galapagos Islands, and the remarkable geological succession of edentates in South America; these facts formed the germ of his epoch-making theory. After his return to England Darwin lived, entirely deroted to scientific work, up to his death in 1882 . He was incessantly busy in 
developing his conception of the orgin of species, the fundamental ideas of which he communicated to friends, but not until 1858 did Darwin decide to make them public. In this year he received an essay by Wallace which in its most important points coincided with his own views. At the same time with Wallace's manuscript an abstract of Darwin's theory was published. In the next year ( $\left.{ }^{8}{ }_{59}\right)$ appeared the most important of his writings, "On the Origin of Species by means of Natural Selection," and in rapid succession a splendid series of works, the most important of which are: (I) "Upon the Variation of Plants and Animals under Domestication," (2) on "The Descent of Man."

No scientific work of that century has attracted so much attention in the whole educated world, as "The Origin of Species." It was generally received as something entirely new, so completely had the scientific tradition been lost. In professional circles it was stoutly combated by one faction, with another it found hesitating acceptance. Only a few men placed themselves from the beginning in a decided manner on the side of the great British investigator. There was a lively scientific battle, which ended in a brilliant victory for the theory of evolution. At the present time all our scientific thoughts are permeated with the idea of evolution.

Post-Darwinian Writers.-Among the men who have most influenced this rapid advance is to be mentioned, besides A. R. Wallace, the co-founder of Darwinism, above all Ernst IIaeckel, who in his "General Morphology" and his "Natural History of Creation" has done much towards the extension of the theory. Among other energetic defenders of the theory in Germany should be mentioned Fritz Müller, Carl Vogt, Weismann, Moritz Wagner, and Nägeli. Among the English naturalists are to be named particularly Huxley, Hooker, and Lyell. In America Gray, Cope, Morse, and Hyatt were early supporters. Darwinism was long in obtaining an entrance into France.

\section{DARWIN'S THEORY OF THE ORIGIN OF SPECIES.}

Before Darwin wrote the idea of fixity of species prevailed. It was recognized that all the individuals of a species are not alike, and that more or less variability occurs, so that it was possible to distinguish races and varieties within the species, but it was believed that the variations never transcended specific bounds.

Darwin begins with a criticism of the term species. Are the conceptions of species on the one side and that of race and variety on the other something entirely different? Are there special criteria for determining 
beyond the possibility of a doubt whether in a definite case we have to do with a variety of a species or with a different species? or do the conceptions pass into one another in nature? Are species varieties which have become constant, and are varieties species in the process of formation?

Morphological Characters.-A. Distinction between Species and I'ariety. For the settlement of these fundamental questions morphological and

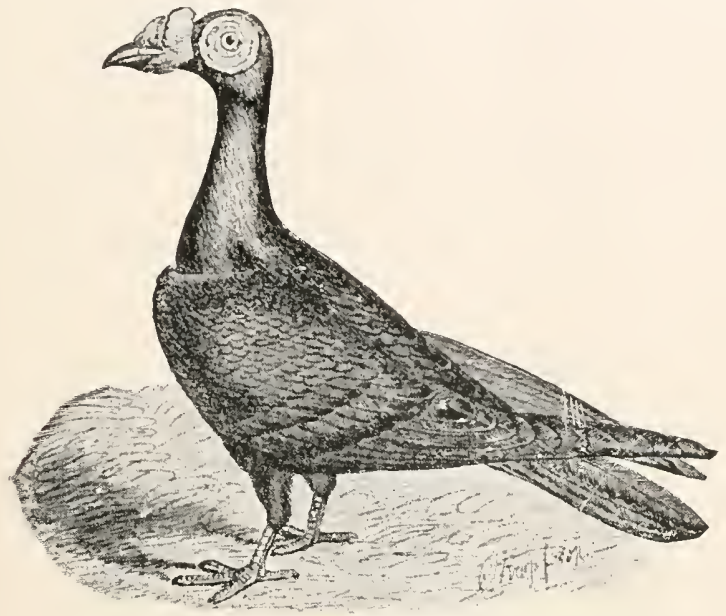

FIG. IA.-English carrier-pigeon (after Darwin).

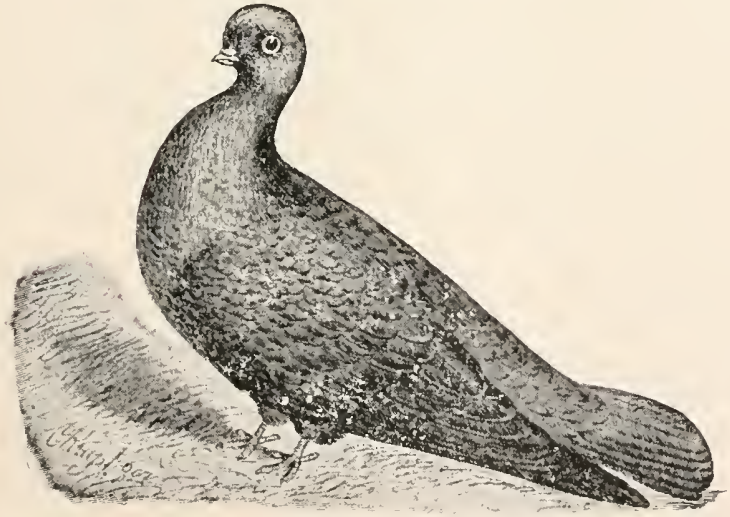

FIG. IB.-English tumbler-pigeon (after Darwin).

physiological characters can be considered. In the practice of the sýstematists usually the morphological characters prevail exclusively; and hence will be here considered first. If, among a great number of forms 
similar to one another, two groups can be recognized which differ considerably from each other, if the differences between them be obliterated by no intermediate forms, and if in several successive generations they remain constant, then the systematist speaks of a 'good species;' on the other hand he speaks of varieties of the same species when the differences are slight and inconstant, and when they lose their importance through the existence of intermediate forms. A definite application of this rule discloses great incongruities, many groups being regarderl by one set of systematists as good species, by another only as 'sports,' i.e., as varieties of the same species. The differences between the 'races' of our domestic animals are often so considerable that formerly they were regarded not only as sufficient for the foundation of good species, but even of genera and families. In the fantail pigeon the number of tail-feathers, originally only $\mathrm{I}_{2-\mathrm{I}} 4$, has increased to $30-42$ (fig. IC); among the other races of

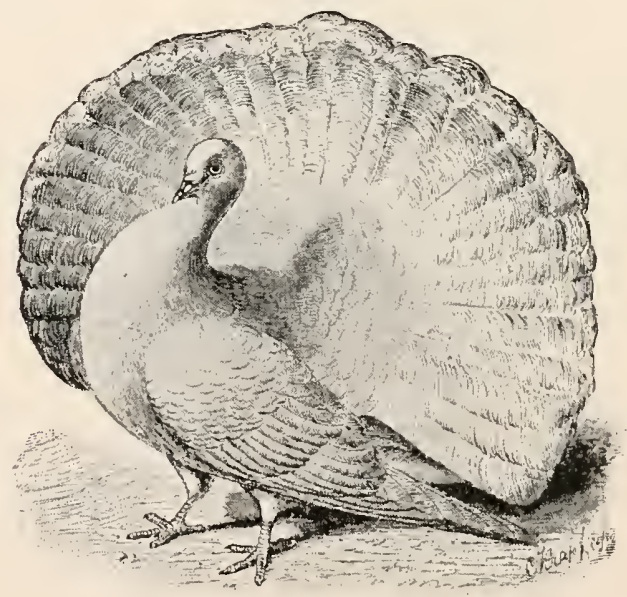

FIG. IC.-English fantail pigeon (after Darwin).

plgeons enormous variations are found in the size of the beak and feet in compariscn with the rest of the body (figs. IA, IB); even the skeleton itself participates in this variation, as is shown by the fact that the total number of vertebræ varies from 38 to 43 , the number of sacral vertebræ from I 4 to II.

B. Variation within the Species.-Now in respect to the occurrence of transitional forms and the constancy of differences, there is within one and the same 'good species' the greatest conceivable difference. In many very variable species the extremes are united by many transitions; 
in other cases sharply circumscribed groups of forms, or races, can be distinguished within the same species. In the race, the peculiar characteristics are inherited from generation to generation with the same constancy as in good species. This is shown in man, and in many pure races of domesticated animals.

Physiological Characters.-A. Crossing of Species and Varieties.A critical examination leads to the conclusion that morphology is incleed useful for grouping animals into species and varieties, but it leaves us in the lurch when called upon to show the disinctions between a species and a variety. Therefore there remains open to the systematist only one resource, i.e., to summon physiology to his aid. This has disclosed considerable distinctions in reproduction. We should expect a priori that the individuals of different species would not reproduce with each other; on the other hand the individuals of one and the same species, even though of different varieties or races, should be entirely fertile. One must beware of arguing in a circle in proof of these two propositions; so the question must read: does physiological experiment lead to the same distinctions as does the common systematic experience?

B. The Intercrossing of Species.-This field is as yet far from being sufficiently investigated; yet some general propositions can be set up: (I) that not a few so-called 'good species' can be crossed with one another; (2) that in general the difficulty of crossing increases, the more distant the systematic relationship of the species used; (3) that these difficulties are by no means directly proportional to the systematic divergence of the species. Thus hybrids have been obtained from species which belong to quite different genera, while very often nearly-related species will not cross. Among fishes we know hybrids of Abramis brama and Blicca björkna, of Trutta salar (salmon) and Trutta fario (trout); among sea-urchins the spermatozoa of Strongylocentrotus lividus fertilize with great readiness the eggs of Echinus microtuberculatus, but only rarely the eggs of Spharechinus grunularis, which is nearer in the system. It also happens that crossing in one direction (male of $\mathrm{A}$ and female of $\mathrm{B}$ ) is easily accomplished, but in the other direction (male of B and female of A) it completely fails; as, for example, the sperm of Strongylocentrotus lividus fertilizes well the eggs of Echinus microtuberculatus, but, conversely, the sperm of E. microtuberculatus does not fertilize the eggs of $S$. lividus; salmon eggs are fertilized by trout sperm but not trout eggs by salmon sperm. Eggs have been fertilized by sperm belonging to different families, orders, and possibly classes-eggs of Pleuronectes platessa and Labrus rupestris by sperm of the cod, frogs' eggs by sperm of two species of Triton, eggs of a starfish (Asterias forbesii) by milt from a sea-urchin, Arbacia pustulosa. In 
these extreme cases, it is true, the hydrids die during or at the close of segmentation, before the embryo is outlined.

In the case of animals where copulation is necessary the difficulties of experimentation increase, since of ten there exists an aversion between males and females of different species which prevents any union of the sexes. Yet we know crosses of different species; e.g., between the horse and the ass; our domestic cattle and the zebu; ibex (or wild buck) and she-goat; sheep and goats; dog and jackal; dog and wolf; hare and rabbit (Lepus darwini); American bison and domestic cattle; etc.; among birds, between different species of finches and of grouse; mallard and the pintail duck; the European and the Chinese goose (Anser ferts and A. cygnoides). Among the insects, especially the Lepidoptera, the cases are many, but the resulting eggs usually produce larve of slight vital force.

C. Fertility of Hybrids and Mongrels.-Since many hybrids, as the mule, have been known for thousands of years, the criterion is, as it were, pushed back one stage; if the infertility in cases of crosses in many species is not immediately noticeable, yet it may be apparent in the products of the cross. While the products of the crossing of varieties, the 'mongrels,' always have a normal, often an increased, fertility, the products of the crossing of species, the hybrids, should always be sterile. But even this is a rule, not a law. The mule (which only very rarely reproduces) and many other hybrids are indeed sterile, but there are not a few exceptions, although the number of experiments in reference to this point is very small. Hybrids of hares and rabbits have continued fruitful for generations; the same is true of hybrids obtained from the wild buck and the domesticated she-goat; from Anser cygnoides and A. domesticus; from Salmo salvelinus and S. fontinalis; Cyprinus carpio and Carassius zulgaris; Bombyx cynthia and B. arrindia.

Difficulties in Classification.-The final result of all this discussion may be summed up as follows: up to the present time, neither by physiological nor by morphological evidence has there been found a criterion which can guide the systematist in deciling whether certain series of forms are to be regarded as good species or as varieties of a species. Zoologists are guided rather in practice by a certain tact for classification, which, however, in difficult cases leaves them in the lurch, and thus the opinions of various investigators vary.

Change of Varieties into Species.-The conditions above discussed find their natural explanation in the assumption that sharp distinctions between species and variety do not exist; that species are varieties which have become constant, and varieties are incipient species. The meaning of the above can be made clear by a concrete case. Individuals of a 
species vary, i.e., compared with one another they attain greater or less differences. So long as the extreme differences are bridged by transitional forms we speak of rarieties of a species; if, on the other hand, the intermediate forms have died out, or were not present in the beginning, and the differences have in the course of time become fixed, and so intensified that a sexual union of the extreme forms results either in complete sterility or at least in a marked tendency towards sterility, then we speak of different species.

Species may be Related to each other in Unequal Degrees.-In favor of this riew, that varieties will in longer time become species, is the great agreement which usually exists between the two. In genera which comprise a larger number of species, the species usually show also many varieties; the species are then usually grouped in sub-genera, i.e., they are related to each other in unequal degrees, since they form small groups arranged around certain species. With varieties the case is similar. In such genera the formation of species is in active progress; but each species formation presupposes a high degree of variability.

Phylogeny. - It is clear that what has here been worked out for the species must also apply to the other categories of the system. Just as by divergent development varieties become species, so must species by continued divergence become so far removed from one another that we distinguish them as genera. It is only a question of time when these differences will become still greater, and give rise to orders, classes, and branches, just as the tender shoots of the young plant become in the tree the chief branches from which spring lateral branches and twigs. If we pursue this train of thought we reach the conception that all the animals and plants living at present have arisen by means of variation from a few primitive organisms. Inasmuch as thousands of years are required for the formation of new species through the variability of one, there must have been necessary for this historical development of the animal and regetable kingdoms a space of time greater than our mental capacity can grasp. Since for the individual development (embryology) of an animal the term ontogeny has been chosen, it has also proved convenient to apply to the historical development of animals the term History of the Race or Phylogeny.

Spontaneous Generation.-If we attempt to derive all living animals from a common ancestor, we must assume that this was extremely simple, that it was unicellular; for the simpler, the less specialized, the organization, so much the greater is its capacity for modification. Only from simple organisms can the unicellular organisms, the Protozoa, be derived. Finally, for the simple organisms alone can we conceive a natural origin. 
Since there was undoubtedly a time upon our earth when temperatures prevailed which made life impossible, life must have arisen at some time, either through an act of creation or through spontaneous generation. If, in agreement with the spirit of natural science, we invoke for the explanation of natural facts only the forces of nature, we are driven to the hypothesis of spontaneous generation, namely, that by a peculiar combination of materials without life, the complicated mechanism which we call 'life' has arisen.

Starting from a basis of facts, by generalization we reach a simple conception of the origin of the animal kingdom, but we have in equal maasure departed from the results of direct observation. Observations only show that species are capable of modifications. That this capacity for variation is a principle which explains to us the origin of the animal world, needs further demonstration.

Proofs of Phylogeny.-The evolution of the existing animal world has taken place in the thousands of years long past, but is no longer accessible for direct observation, and therefore it can never be followed in the sense that we follow the individual development of an organism. In regard to the conception of the evolution of animals we can merely prove the probability; yet all our observations of facts not only agree with this conception, but find in it their only simple explanation. Such facts are furnished to us by the classification of animals, paleontology, geographical distribution, comparative anatomy, and comparative embryology.

(I) Proofs from Classification.-It has long been recognized, that if we wish to express graphically the relationships of animals, their classes, orders, genera, and species, simple co-ordination and subordination are not sufficient, but we must have a treelike arrangement, in which the principal divisions, more closely or distantly related to one another-the branches, phyla, or types-represent the main limbs, while the smaller branches and twigs correspond to the several classes, orders, etc. This is, in fact, the arrangement to which the theory of evolution necessarily leads.

(2) Paleontological Demonstration approaches nearest to direct proof; for paleontology gives the only traces of existence which the predecessors of the present animal world have left. Even here a hypothetical element creeps in. We can only observe that various grades of forms of an animal group are found in successive strata; if we unite these into a developmental series, and regard the younger as derived from the older by variation, we connect the single observations by a very probable hypothesis. But the value of palecntological evidence is weakened much more by its extreme incompleteness. In fossils only the hard parts are generally 
preserved; the soft parts, which alone are present, or at least make up the most important portions of many animals, are almost always lost. Only rarely are they (muscle of fishes, ink-bag of cephaloports, outlines of meduse) preserved in the rocks. Even the hard parts remain connected only under exceptionally favorable conditicns. If further we take into consideration the fact that these treasures are buried in the earth, and are usually obtained only by accident, in quarrying and road-building, it becomes clear how little of the racial history is to be expected from paleontology.

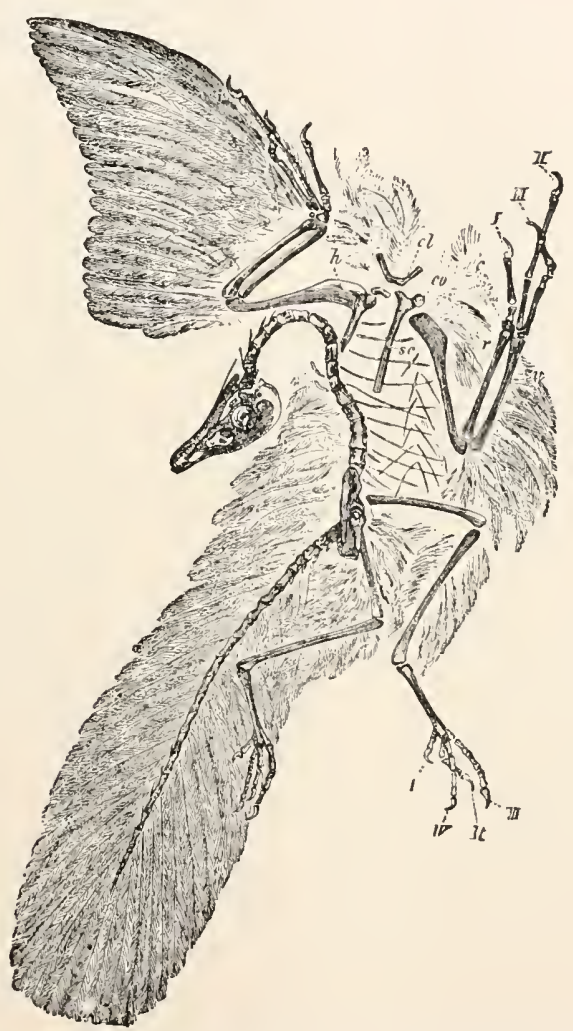

FIG. 2. Archroptervx lithographica (after Steinmann-Döderlein). cl, clavicle; co, coracoid; $h$, humerus; $r$, radius; $u$, ulna ; $c$, carpus; I-IV, digits; sc, scapula.

Examples of Paleontological Proof.-Yet paleontology has already furnished many importaint proofs of the theory of descent. It has shown that the lower forms appeared first, and the more highly organized later. Among animals in general the latest to appear were the vertebrates, and 
of these the mammals; among the mammals man. For smaller groups genealogical material has fortunately been found. Transitional forms connect the single-toed horse of the present with the four-toed Eohippos of the eocene; for all the hoofed animals a common ancestral form has been found in the Condylarthra. Transitional forms have also been found between the greater divisions, as, e.g., between reptiles and birds, the remarkable toothed birds, and the Archaopteryx (fig.2), a bird with a long, feathered, lizard-like tail.

(3) Morphological Proofs.-When we employ comparative anatomy and embryology in support of evolution, we find that the two have so much in common that they can best be treated together.

Cuvier and von Baer taught that the separate types of the animal kingdom are units, each with a special structure and plan of development peculiar to it; farther, that there are no similarities in structure or development forming a bridge from type to type. The first of these propositions is still regarded as correct, but the second, which alone is important for the theory of evolution, has become untenable. All animals have a common organic basis in the cell and are thereby brought ciose to one another; all multicellular animals agree in the principal points during the first stages of their development, during the fertilization, cleavage of the egg, and the formation of the first two germ-layers, and vary from one another from this time on only in such differences as may occur within one and the same type. Also the peculiarities which distinguish each type in structure and in the mode of development are not without intermediate phases. In some representatives of each type the structure and the mode of development are simpler, thereby approaching to the conditions which obtain in the other types. The existence of such transitional forms is one of the most important proofs in favor of the theory of evolution.

Fundamental Law of Biogenesis.-A fact that weighs heavily in favor of the theory of evolution is that the structure and mode of development of animals is ruled by a law which at present can only be explained by the assumption of a common ancestry. Each animal during its development passes through essentially the stages which remain permanent in the case of lower or better, more primitive animals of the same branch, as the following examples show: (I) In the early stages of development the human embryo (figs. 3, 609) possesses remarkable resemblances to the lowest vertebrates, the fishes. Like these it has gill-slits, a simple heart with auricle and rentricle; instead of a separation of aorta and pulmonary arteries (body and lung arteries) a single arterial trunk going from the heart: and aortic arches connecting this trunk with the descending aorta. All of these are structure adapted for branchiate respiration 
and are functionally intelligible in fishes but they are not compatable with a lung-breathing mammal and must undergo fundamental changes to become of use. They are, like so many other structures in the human being, not to be understood from present functions, but must have an historical meaning. (2) Frogs in their tadpole stage have an organization similar to that which is permanent in certain Amphibia, the Per-

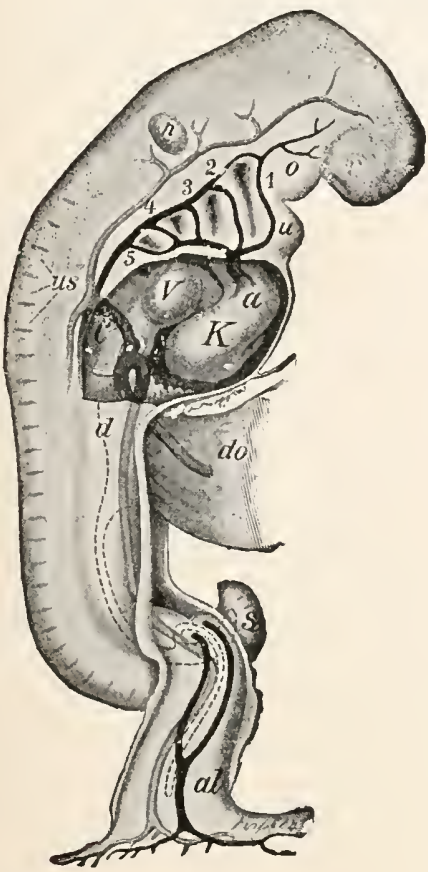

Fig. 3.

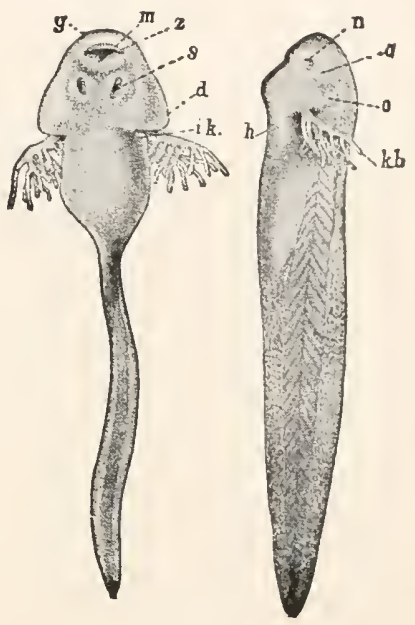

FIG. 4.

Fig. 3.- Muman embryo, $4.2 \mathrm{~mm}$. long (after His). Pericardium and lateral body wall opened, yolk-sac and allantois cut away, course of blood-vessels shown; $a$, arterial trunk; al, allantois stalk; $c$, precava, uniting with yolk and umbilical veins; $d$, intestine; $d o$, yolk stalk; $h$, ear vesicle; $K$, ventricle of heart; $o$, upper jaw; $r$, olfactory groove; $s$, tail; $u$, lower jaw; $u$, somites; $V$, atrium of heart; $I-5$, the five arterial arches.

FIG. 4.- Tadpoles of Rana temporaria. m, mouth; $g$, upper jaw; $z$, lower jaw; $s$, sucking-disc; $k b$, external gills; $i k$, region of the internal gills; $n$, nose; $a$, eyes; $o$, auditory vesicle; $h$, cardiac region; $d$, operculum.

ennibranchiata (fig. 5), which stand lower in the system; they have a swimming tail and tuft-like gills, which are lacking in the adult frog. (3) There are certain parasitic crustacea, which live upon the gills of fishes, and seem not at all like their relatives. They are shapeless masses which were formerly regarded as parasitic worms. Their systematic position was only determined by their embryology (fig. 6). Here it is 
shown that they pass through a nauplius-stage (fig. 6a), characteristic of most crustacea, and that they then assume the shape of small crustacea (fig. 6, b), like $C y c l o p s$ (fig. $7, A$ ), so widely distributed in fresh waters. Very often the males make a halt in the cyclops-stage while the female

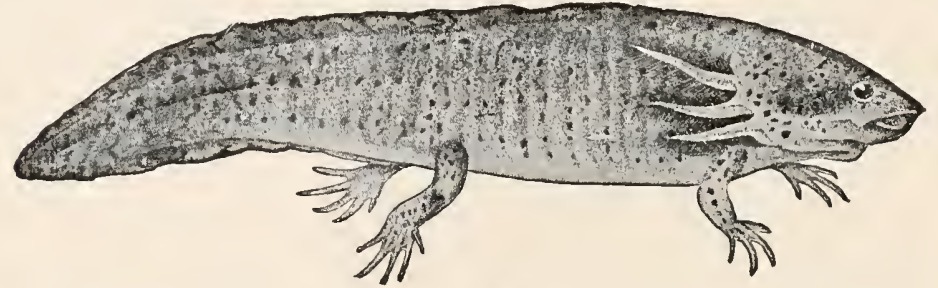

FIG. 5.-Siredon pisciformis (larva of Amblystoma tigrinum) (after Duméril and Bibron).

develops farther and assumes a shapeless form, so that there arises a very remarkable sexual dimorphism (fig. 8). All these examples, which can be multiplied by hundreds, can be explained in the same way. The higher

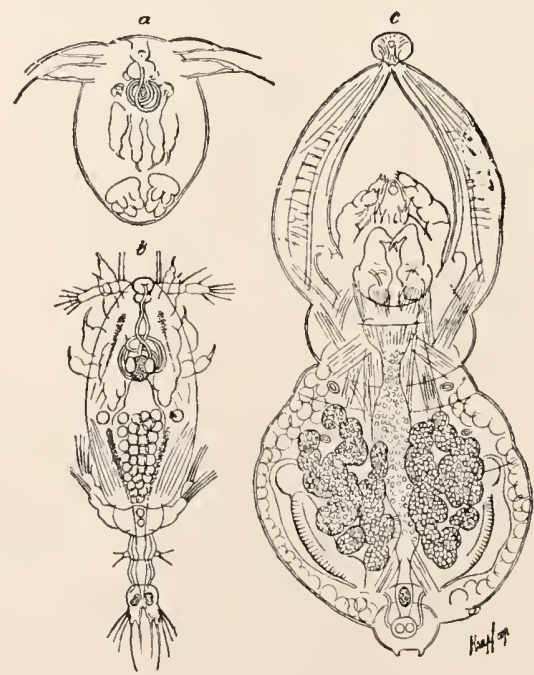

FIG. 6.-Achtheres percarum. a, nauplius-, b, cyclops-stage; $c$, adult female (after Claus).

forms pass through the stage of the lower, because they spring from ancestors which were more or less similar to the latter. Man in his embryological development passes through the fish stage, the frog the peremnibranchiate stage, the parasitic crustacean first the nauplius- 
and then the cyclops-stage, because their ancestors were once fish-like, perennibranchiate-like, nauplius- and cyclops-like. Here is expressed a general phenomenon which Haeckel has stated under the name of 'the Fundamental Law of Biogenesis.' "The development history (ontogeny)
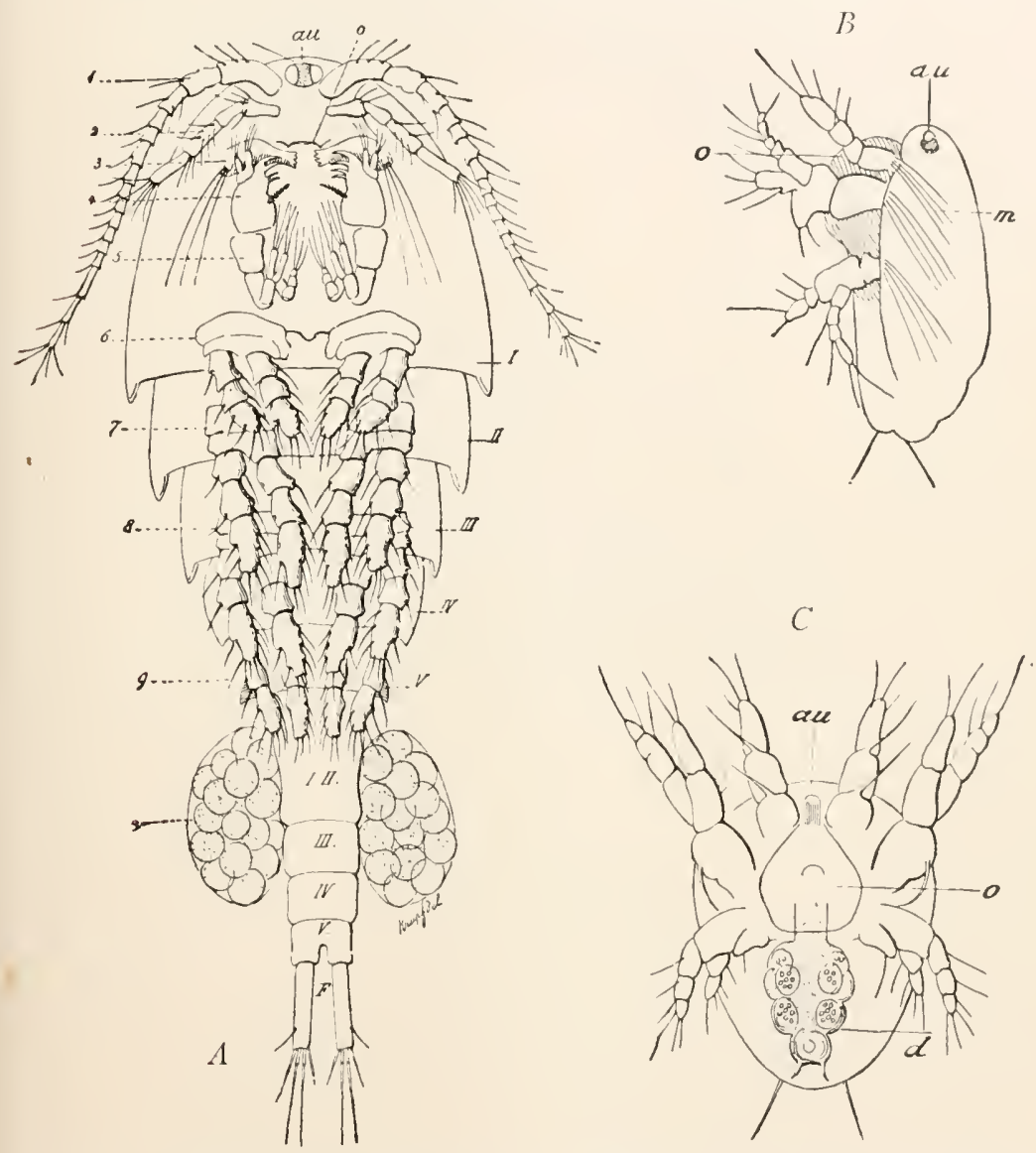

FIG. 7.-Cyclsps coronatus $(A)$ and also its nauplius in lateral $(B)$ and in ventral view $(C)$. $I$, head; $I I-Y$, the five thoracic, and behind these the five abdominal segments; $F$, furca; I, the first, 2, the second, antennæ; 3 , mandibles: 4 , maxilla; 5 . maxillipeds; 6-9, the first four pairs of biramous feet, while the rudimentary fifth pair are hidden; $a u$, eye; $o$, upper lip; $e$, egg-sacs; $d$, gut; $m$, muscle.

of an individual animal briefly recapitulates the history of the race (phylogeny); i.e., the most important stages of organization which its ancestors have passed through appear again, even if somewhat modified, in the development of individual animals." 
The Nervous System.-This law applies as well to single organs as to entire animals. The central nervous system of many lower animals (echinoderms, cœlenterates, many worms) forms part of the skin; in its
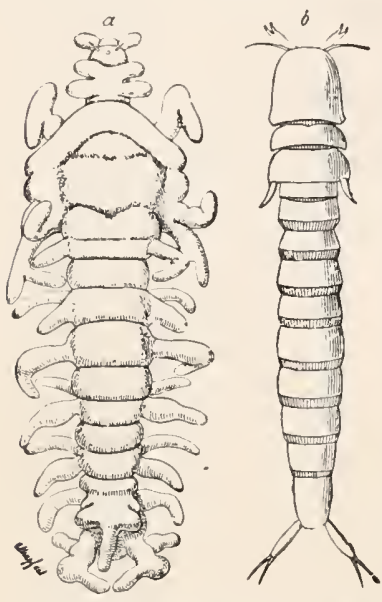

FIG. 8.-Philictlys xiphia. $a$, female (after Claus), $\times_{4} ; b$, male (after Bergsoe), $X_{13}$. first appearance it belongs to the surface of the body, because it has to mediate the relations with the external world. In the case of higher animals, e.g., the vertebrates, the brain and spinal cord lie deeply imbedded in the interior of the body; but in the embryo they are likewise laid down as a part of the skin (medullary plate) which gradually through infolding and cutting off from this comes to lie internally (fig. 9).

The Skeletal System.-The skeleton of vertebrates is a further example. In the lowest chordates, amphioxus and the cyclostomes, the vertebre are lacking, and in their place we find a cylindrical cord of tissue, the notochord. In the fishes and Amphibia the notochord usually persists; but it is partially reduced and constricted by the vertebre, which in the lower forms consist of cartilage, and in the higher of bone. Mature birds and mammals finally have a completely ossified vertebral column; their embryos, on the other hand, have in the early stages only the notochord (amphioxus stage); later this notochord becomes constricted by the vertebre (fish-amphibian stage) and finally entirely replaced; the vertebral column is in the beginning cartilaginous, only later becoming ossified. Comparative anatomy and embryology thus give the same developmental stages of the axial skeleton: (I) notochord, (2) notochord and vertebral column, (3) vertebral column alone, the latter at first formed of cartilage, then of bone.

We have spoken of a parallelism between the facts of comparative anatomy and of embryology. But we should expect a threefold parallelism; for according to the theory of evolution the systematic arrangement of animals is based upon a third factor-phylogeny. The fossils, should give the same progressive series in the successive geological strata as the stages of forms found by comparative anatomy and embryology. We know instances of such threefold parallelisms. Comparative anatomy teaches that the lowest developed form of a fish's tail is the diphyceral (fig. Io, $A$ ); that from this the heterocercal $(B)$, and from the heterocercal the homocercal form of tail-fin $(C, D)$ can be derived. Embryo- 

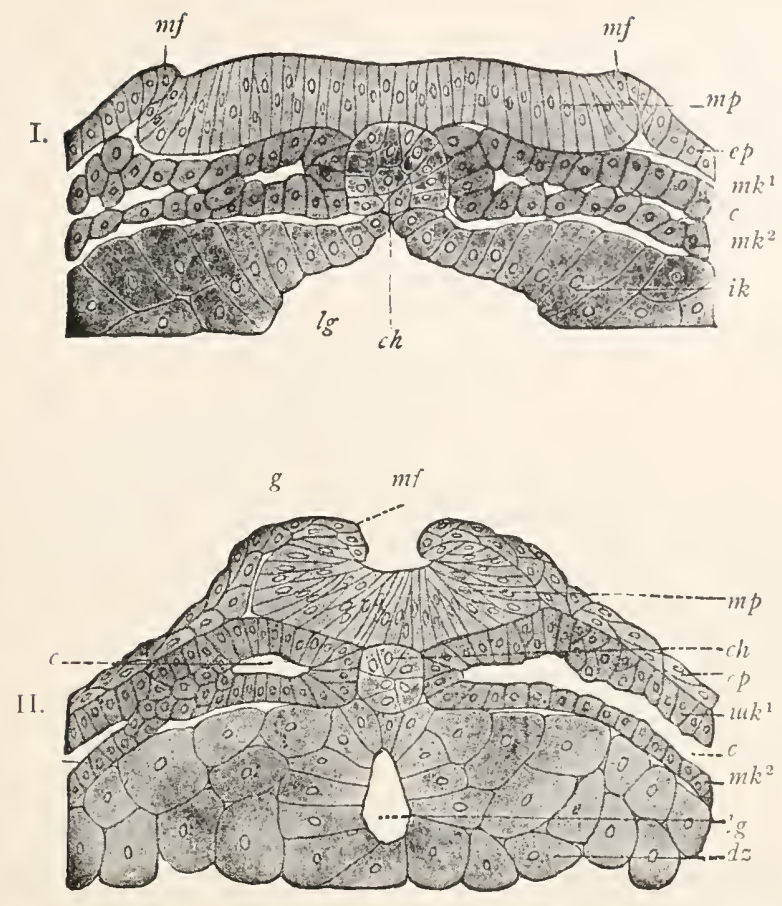

$i k$

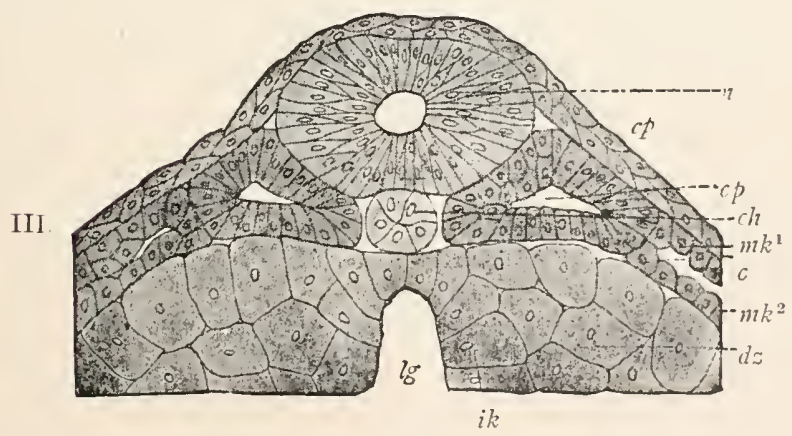

FIG. 9.-Cross-sections through the dorsal region of Triton embryos at different ages (from O. Hertwig). In $I$ the medullary plate (anlage of spinal cord) $m p$ is marked off from the skin (epidermis, $e p$ ) by the medullary folds ( $m f$ ). In $I I$ the medullary plate, by inrolling of the medullary folds, is converted into a groove. In $I / I$ the groove has closed into a tube $(n)$, the spinal cord, which has separated from the rest of the ectoderm (epidermis). c, body cavity (colom); ch, notochord; $c p$. cavity of primitive somite (myotome); $d z$, yolk-cells; $i k$, entoderm; $l g$, Iumen of gut; $m k^{1}, m k^{2}$, somatic and splanchnic layers of mesoderm; $n$, spinal cord. 
logically the most highly developed fishes are first diphycercal, later heterocercal, and finally become homocercal. Last of all, paleontologically the oldest fishes are diphycercal or heterocercal, and only later do homocercal forms appear.
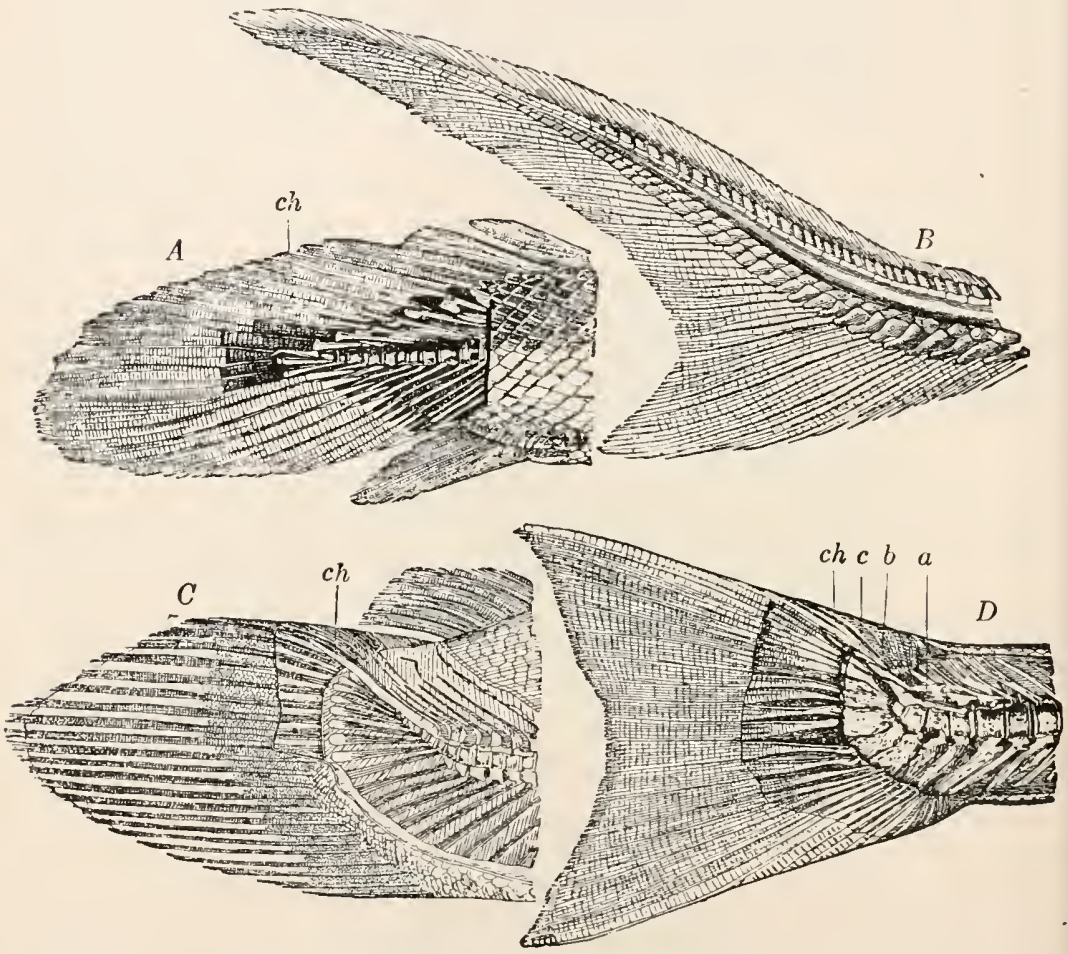

FIG. Io.-Tail-fins of various fishes (from Zittel). $A$, Diphycercal fin of Polypterus bichir. (Vertebral column and notochord divide the tail into symmetrical dorsal and ventral portions.) $B$, Heterocercal tail of the sturgeon. (As a result of an upward bending of the notochord and vertebral column the fin has become asymmetrical, the ventral portion much larger than the dorsal.) $C, D$, Homocercal fins, $C$, of $A$ mia calva; D, of Trutta salar. (By a still greater upward bending of the notochord and vertebral column the dorsal portion has almost entirely disappeared and the ventral portion almost alone forms the fin, externally apparently symmetrical, but in its internal structure very asymmetrical.) $c h$, chorda; $a, b, c$, cover-plates.

What has here been referred to is only a small fraction of the proofs which morphology offers in favor of evolution; it can only serve to show how morphological observations can be employed. For the reflecting naturalist the facts of morphology are a great inductive proof in favor of the theory of evolution. 
Distribution of Animals.-From Animal Geography we learn that the present distribution of animals is the product of the past. It will therefore be possible from this to figure out many of the earlier conditions of things.

If we assume that from the beginning all animal species were constituted as they now are, they would then have been placed by the purposeiul Creator in the regions best suited to them; their distribution would therefore have been determined by favorable or unfarorable conditions of life prevailing in the various regions, as the climate, food-supply, etc. If, on the other hand, we assume that the animal species have arisen from one another through variation, then there must have been, as an influence determining the manner of distribution, besides the conditions of existence, still a second factor, which we will call the geological. We know that the configuration of the earth's surface has changed in many respects in the course of the enormous time of the geological periods; that land areas which earlier were united, have become separated by the encroachments of the sea; that by the uphearal of mountains important barriers to the distribution of animals were also formed. On the other hand regions which were formerly separated have become connected; islands, for example, being united ly the emergence of land from the sea. From the fact that these two changes-the changes in the earth's surface and in the animal world established upon it-have gone on hand in hand there follows necessarily the consequence that greater differences in the faunal character of two lands must result, the longer the inhabitants have been separated by impassable barriers. For the rarious groups the character of the barriers is different; terrestrial animals, which cannot fly, are hindered in their distribution by the sea; marine forms by land barriers; for terrestrial molluscs mountain ranges, which are dry and barren, or constantly snow-capped, are effectual.

Instances of Proofs. - Since attention has been called to these conditions, many facts favorable to the theory of erolution have been ascertained: ( I) Of the various continents Australia has faunally an independent character; when discovered it contained almost none of the higher (placental) mammals, except such as can fly (Chiroptera), or marine forms (Cetacea), or such as are easily transported by floating wood (small rodents), or such as could be introduced by man (dingo, the Australian dog); instead, it had remarkable birdlike animals (with beak and cloaca), and the marsupials, which have become extinct in the Old World and, opossums excepted, in America as well. The phenomenon is explained by the geological fact that in the earth's history Australia, with its surrounding islands, was certainly the earliest to lose its connec- 
tion with the other continents. While in the other parts of the earth the higher vertebrates, which were developed from the marsupials and their lower contemporaries, came, by way of the lands connecting the various continents, to have a wide distribution, in isolated Australia this process of evolution did not go on, and its ancient faunal character was preserved. (2) As Wallace has shown, the Malay Archipelago is divided faunally into an eastern and a western half. The fauna of the first has a thoroughly Australian character; that of the latter recalls Farther India and the Oriental Province. Differences in climate and vegetation cannot be the cause of this, since in both there are islands with dry and others with moist climates, with sparse and with luxuriant vegetation. The only explanation is that the eastern Malay Islands have developed geologically in connection with Australia, the western with India. Wallace tried to draw a sharp line ('Wallace's line') between the two regions, passing between the islands of Bali and Lombok. Later studies have not confirmed this, but have shown that between the two regions is a zone of islands (including Celebes) in which a mixture of faunas occurs. (3) Long before Darwin, the geologist von Buch, from the distribution of plants on the Canary Islands, came to the conclusion of a change of species in to new species; viz., on islands peculiar species develop in secluded valleys, because high mountain-chains isolate plants more effectually than do wide areas of water. Moritz Wagner has collected many instances which prove that localities inhabited by certain species of beetles and snails have been sharply divided by wide rivers or by mountain-chains, while in neighboring regions related so-called 'vicarious species' are found. 'The peculiar character of the fauna and flora of isolated island groups also needs mention. The Hawaiian Islands have no less than 70 endemic birds out of a total of II6, the Galapagos 84 out of 108 .

Causal Foundation of the Theory of Evolution.-The Darwinian theory, so far as the above exposition shows, is fundamentally like the theories of descent advocated at the beginning of the last century by Lamarck and other zoologists; it is distinguished from these only by its much more extensive foundation of facts, and further in that it abandoned the successional arrangement and replaced it by the branched, tree-like mode of arrangement-the genealogical tree. But still more important are those advances which relate to the causal foundation of the descent theory. The doctrine of causes which has brought about the change of species forms the nucleus of the Darwinian theory, by which it is especially distinguished from Lamarckism. In order to substantiate causally the change of species, Darwin proposed his highly important principle of 'Natural Selection by means of the Struggle for Existence.' 
Artificial Selection.-In the development of this principle Darwin started with the limited and hence easily comprehended subject, the artifical breeding of domesticated animals. Whether these be the descendants of a single species or have arisen from crosses of two or more species (authorities are not in agreement in all cases) they behave like representatives of a single species. How have the rarious races and subrace of pigeons, horses, cattle, dogs, etc. arisen? Darwin finds the causes of these great differences in artificial selection, practised by man for thousands of years. The method is to choose from the stock individuals showing the tendency toward the desired ideal in even the slightest degree more than their fellows, and then pairing these. By repetitions of this selection and breeding, the desired goal is slowly reached.

This artificial selection depends upon three factors: (I) V'ariability; the descendants of one pair of parents have the capability of developing new characteristics, thereby differing from their parents. (2) Hereditability of newly-acquired characters; the tendency of the daughtergeneration to transmit the newly-developed characteristic to the succeeding generation. (3) Artificial selection; man selects for breeding suitable individuals, and prevents a new character which has arisen through variation from disappearing by crossing with animals of the opposite variational tendencies.

If we compare with the facts of domestication the conditions of animals living in the state of nature, we find again variability and heredity, as efficient forces, inherent in all organisms, though the former is not everywhere of the same intensity. There are many species which vary only slightly or not at all, and therefore have remained unchanged for thousands of years. But contrasted with these conservative species are in every group plastic species, which are in the process of rapid change, and these alone are of importance in causing the appearance of new forms. Since heredity is present in all organisms, there is only lacking a factor corresponding to artificial selection, and this Darwin discovered in the so-called 'natural selection.'

Natural Selection: Struggle for Existence.-Natural selection finds its basis in the enormous number of descendants which every animal produces. There are animals (e.g., most fishes) which produce many thousands of young in the course of their lives; not to mention parasites, whose eggs are numbered by millions. For the development of this multitude of germs there is no room on the earth. In order to preserve the balance of nature great numbers of unfertilized and fertilized eggs, as well as young animals and many that are mature but have not yet attained their physiological destiny, must perish. Many individuals will 
be blotted out by accidental causes; yet on the whole those individuals which are best protected will best withstand adverse conditions. Slicht superiority in structure will be of importance in this struggle for existence, and the possessors of this will gain an advantage over their companions of the same species, just as in domestication each character which is useful to man is of advantage to the possessor. Among the numerous varieties that appear the fittest will survive, and in the course of many generations the fortunate variations will increase by summation, while destruction overtakes the unfit. Thus will arise new forms, which owe their existence to 'natural selection in the struggle for existence.'

The 'Struggle for Existence.'--The expression 'struggle for existence' is figurative, for only rarely does a conscious struggle decide the question of an animal's existence; for example, in the case of the beasts of prey, that one which by means of his bodily strength is best able to struggle with his competitors for his prey is best provided in times of limited food-supply. Nuch more common is the unconscious struggle: each man who attains a more favorable position by special intelligence and energy, limits to an equal degree the conditions of life for many of his fellow men, however much he may interest himself in humanity. The prey, which by special craft' or swiftness escapes the pursuer, turns the enemy upon the less favored of its companions. It is noticeable that in severe epidenics certain men do not fall victims to the disease, because their organization better withstands infection. Here the term 'survival of the fittest,' which Spencer has adopted in preference to 'struggle for existence,' is better.

Instances of the Struggle for Existence.-Although the foregoing suffices to show that the struggle for existence plays a very prominent rôle, yet on account of the importance of this feature it will be illustrated by a few concrete examples. The brown rat (Mus decumanus), which swarmed out from Asia at the beginning of the eighteenth century, has almost completely exterminated the black or house-rat (Mus rattus) in Europe, and has made existence impossible for it in other parts of the world. Several European species of thistle have increased so enormously in the La Plata states that they have in places completely crowded out the native plants. Another European plant (Hypochæris radicata) has become a weed, overrunning everything in New Zealand. Certain races of men, like the Dravidian and Indian, die off to the same degree that other races of men, like the Caucasian, Mongolian, and Negro, spread. The more one attempts to explain that endlessly complicated web of the relations of animals to one another, the relations of animals to plants and to climatic conditions, as Darwin has done, so much the more does he 
appreciate the methods and effects of the struggle for existence. Islands in the midst of the ocean have a disproportionately large number of species of wingless insects, because the flying forms are easily carried out to sea. For example, on the Kerguelen Islands, remarkably exposed to storms, the insects are wingless; among them one species of butterfly, several flies, and numerous beetles.

Sympathetic Coloration.-V'Tery often, in regions which have a prevailing uniform color, the coat of the animals is distinguished by a similar hue; this phenomenon is called sympathetic coloration. Inhabitants of regions of snow are white, desert animals have the pale yellow color of the desert, animals which live at the surface of the sea are transparent; representatives of the most diverse animal branches show the same phenomenon. The adrantages connected therewith scarcely need an explanation. Every animal may have occasion to conceal himself from his pursuers; or it may be his lot to approach his prey by stealth: he is much better adapted for this the closer he resembles his surroundings. Natural selection fixes every advantage in either of these directions, and in the course of many generations these advantages increase. Among the most interesting are the cases of sympathetic coloration, of mimicry and of the development of secondary sexual characters as a result of sexual selection.

Mimicry is referable to the same principle, except that the imitation is not here limited to the color, but also influences form and marking. Frequently parts of plants are imitated, sometimes leaves, sometimes stems. Certain butterflies with the upper surfaces of the wings beautifully colored escape their pursuers by the rapidity of their flight; if they alight to rest, they are protected by their great similarity to the leaves of the plants around which they chiefly fly. When the wings are folded over the back, the dark coloring of the under sides comes into sight and the color on the upper side is concealed. The parts are so. arranged that the whole takes on a leaf-like form, and certain markings heighten the imitation of the leaf (fig. II). Among the numerous species of leaf-butterflies there are different grades of completeness of mimicry; in many even the depredations of insects are imitated; in others the form and marking are still incompletely leaf-like, the marking being the first to come into existence. Among the grasshoppers also there are imitations of leaves, like the 'walking-leaf,' Phyllium siccifolium, P. scythe, while other nearly' related forms more or less completely approach the appearance of dried, sometimes of thorny twigs (fig. I 2, $a$ and $b$ ).

Very often insects are copied by other animals. Certain butterflies, the Heliconias of warmer America, the Danaids of the Old World, fly heavily in large swarms, clumsy and yet are unmolested by birds, because 
they contain bad-tasting fat bodies. Another species of butterfly accompanies them (Pieridæ), which does not taste bad, and yet is not eaten, because in flight, in cut, and marking of the wings it imitates the Heliconiue so closely that even a systematist might easily be confused (fig. 13). In a similar way bees and wasps, feared on account of their sting, are imitated by other insects. In Borneo there is a large black wasp, whose

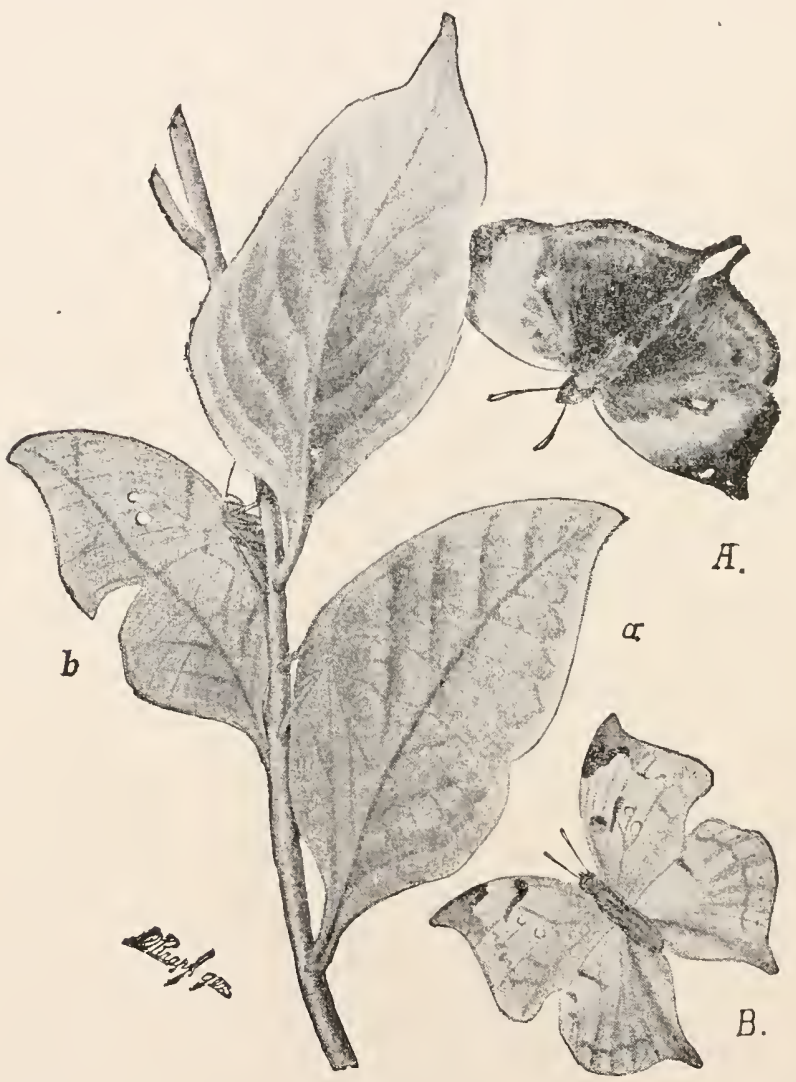

FIg. Ix.-Leaf-butterflies. A, Kallima paralecta, flying; $a$, at rest (after Wallace). $B$, Siderone strigosus, flying; $b$, at rest (after C. Sterne).

wings have a broad white spot near the tip (Mygnimia aviculus). Its imitator is a heteromerous beetle (Coloborhombus fasciatipennis), which, contrary to the habit of beetles, keeps its hinder wings extended, showing the white spot at their tips, while the wing-covers have become small oval scales (fig. I4). With many species the mimicry occurs only in the 

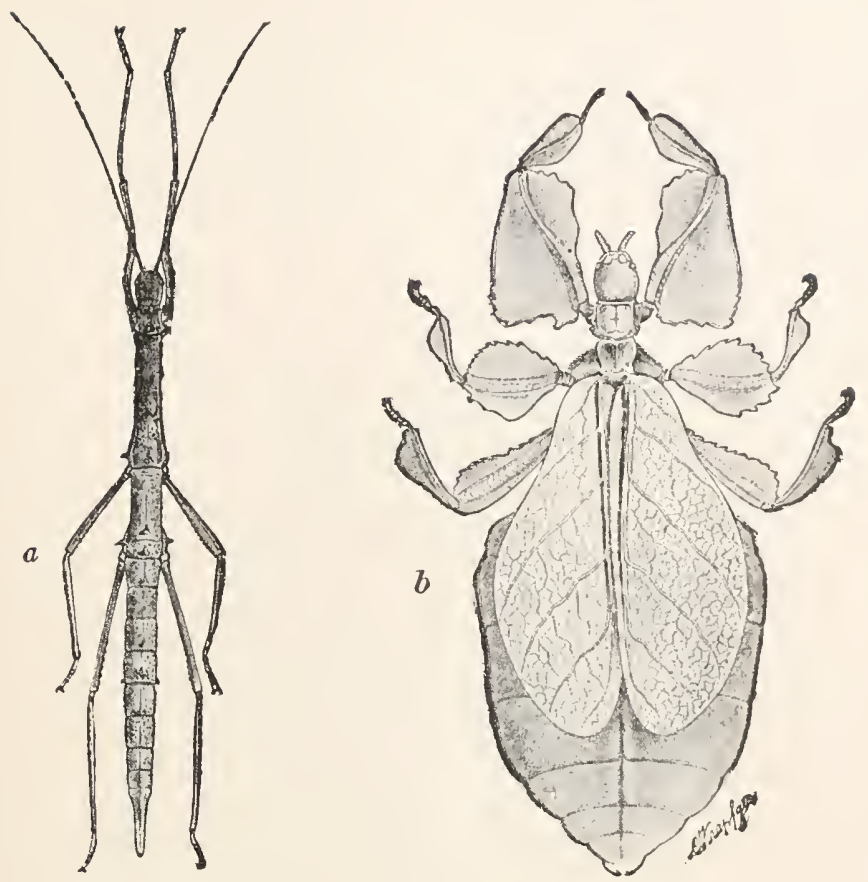

Fig. I2.-Grasshopper mimicry. $a_{t}$ Acanthoderus wallacei + +. $b$, Phyllium scythe $\stackrel{\circ}{+}$.
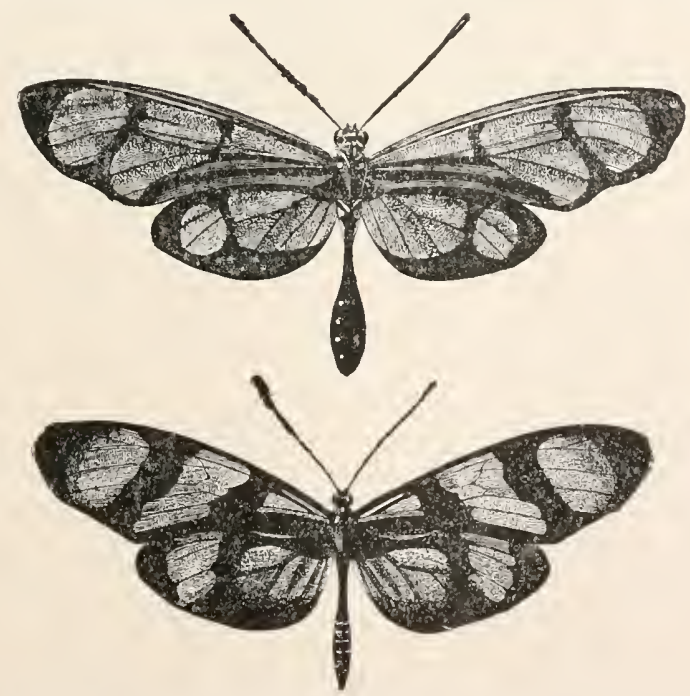

FIG. r3.-Methona psidii, a bad-tasting Heliconiid, copied by the Pierid, Leptalis crise. (after Wallace.) 
females, since these are less numerous and have heavier bodies than the males. So there arises a sexual dimorphism. If the mimicing species have a wide distribution, different bad flavored species may be mimicked in different parts of the range. The females of Papilio merops mimic, in different regions, Danais chrysippus, Amauris echeria and A. mavias, while the males have the same appearance throughout the whole habitat.

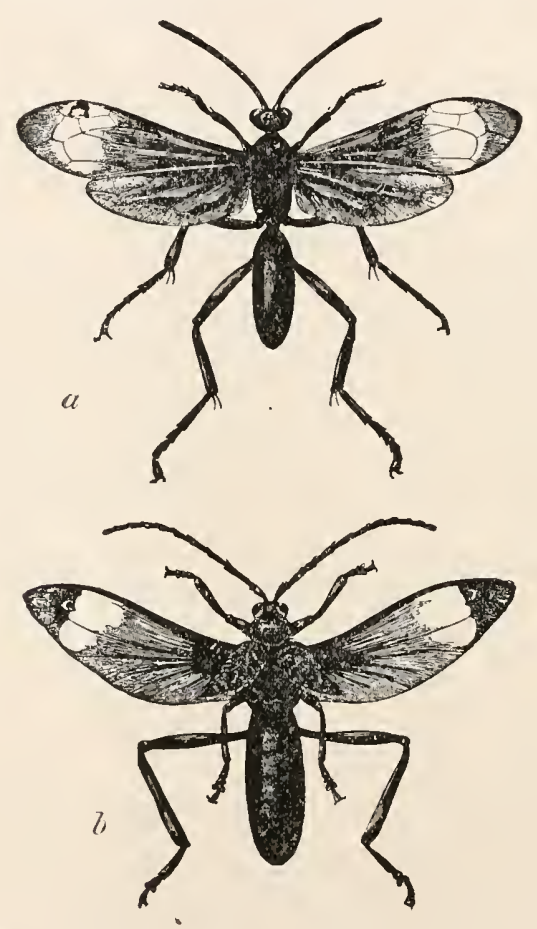

FIg. I4.- $a$, Mygnimia aviculus, a wasp imitated by a beetle, $b$, Coloborhombus fasciatipennis (after Wallace). Three-fourths natural size.

Sexual Selection is a special phase of natural selection, chiefly observed in birds and hoofed animals. For the fulfilment of his sexual instincts the male seeks to drive his competitors from the field, either in battle or by impressing the female by his charms. With strong wings and with spurs the cock maintains possession of his flock, the stag by means of his antlers, the bull with his horns. The male birds of paradise win the favor of the females by means of beautiful coloring; most singing-birds, by means of song; many species of fowl, by peculiar love-dances. Since all these characters belong chiefly to the male, and since only exceptionally are they inherited by the female (and even then are less pronounced), 
it is almost certain that in a great measure they have been acquired by the males through the struggle for the female. 'In the case of birds a second factor has undoubtedly co-operated to impress distinctly the often enormous difference between the feathers of the male and of the female - as is shown, for example, in the case of the birds of paradise (fig. 15);

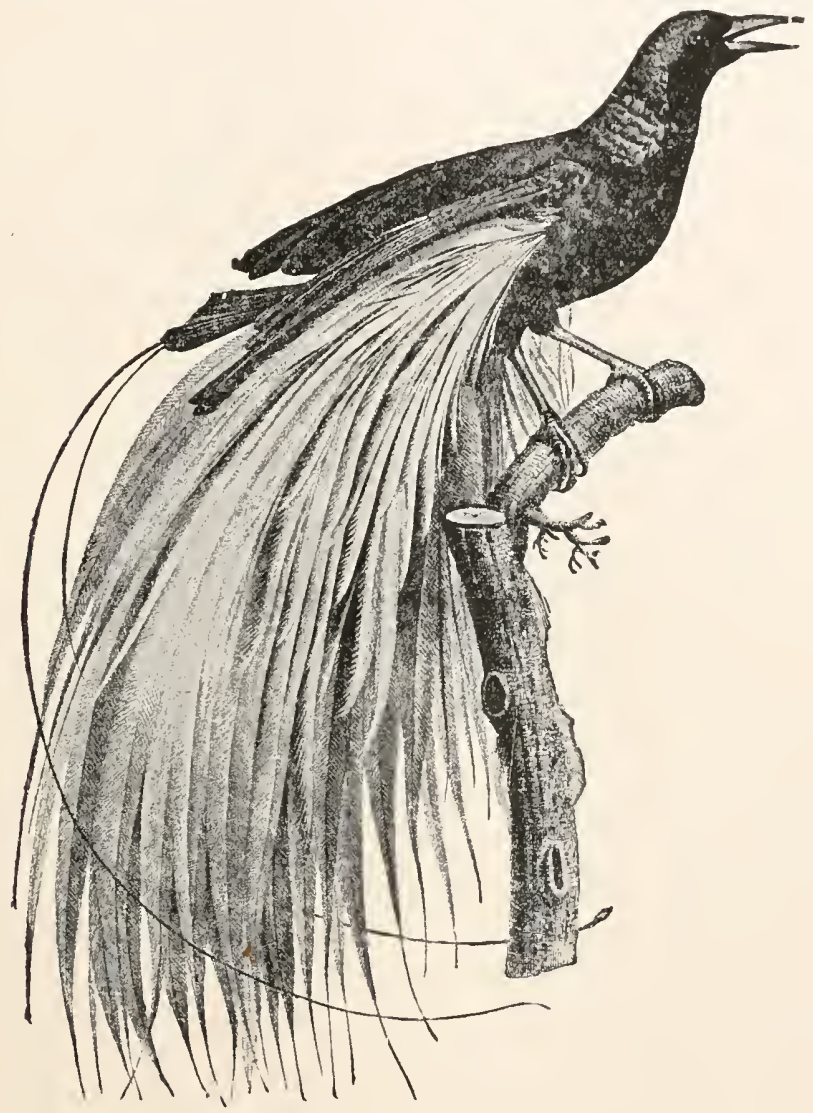

FIG. I 5A.-Paradisea a poda, male (after Levaillant).

for the nesting female inconspicuous colors and a close-lying coat of feathers are necessary in order that, undisturbed by enemies, she may devote herself to incubation.

On the Efficiency of Natural Selection.-In the course of the last twenty-five years there has been much controversy as to how far natural selection alone is a species-forming factor. A number of objectors dispute the possibility of fortuitous variations being utilized in the struggle for 
existence and thus fixed as permanent characters. It is not easy to see how many characters, especially those used in classification, can be of use to their owners. It can only be said that they have developed in correlation with other important characters. But useful characters must be considerable in order to be seized upon by natural selection. Fortuitous variations with which Darwinism deals are too inconsiderable to be utilized by the organism and so to be of value in the struggle for existence. In most cases, too, alteration in one organ alone is not enough to be of value; usually a whole series of accessory structures must be

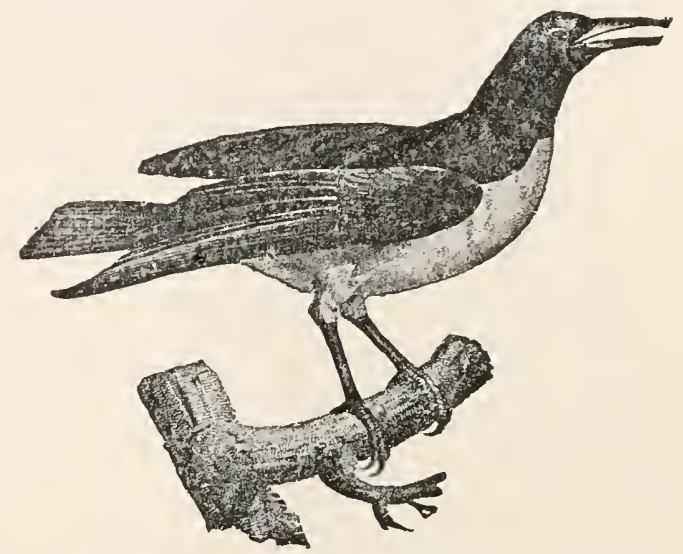

Fig. ${ }_{5}$ B.-Paradisea apoda, female (after Levaillant).

modified. In short, there must exist a harmonious co-adaptation of parts, which presupposes a progressive and well-regulated development extending through a long space of time, during which the struggle for existence could have exerted no directing influence. Thus, the wing of a bird in order to be used for flight must have already reached a considerable size; the muscles for moving it, the supporting skeletal parts, the nerves rumning to it must have a definite formation and arrangement. 'Then there are difficulties in that most animals are bilaterally or radially symmetrical, many in addition segmented. In all these cases the same organ is repeated two or more times. Organs which are repeated symmetrically and usually those which are segmental agree in general in structure. One must therefore admit that the alterations of chance must have occurred at at least two points simultaneously and in exactly the same way.

A further objection is that the action of natural selection would, under ordinary conditions, be negatived by unhindred crossing of the varying forms. If, for example, we do not isolate fantails from other pigeons, they will cross with these, and their descendants will soon resume the 
character of common pigeons. Finally, it has been claimerl that for the formation of new species a simple variation of form is not sufficient; it must reach still farther: ( 1 ) a variation in different directions, a divergent development of the individual members of a species; (2) the disappearance of the transitional forms which unite the divergent forms.

The objection that the struggle for existence cannot bring about the divergent development of individuals necessary for improvement is of least importance. Of the many rariations appearing at the same time in a species two or more may be equally useful; then one set of individuals will seize upon one, another set upon the other advantage, and that in consequence of this both sets will develop in different directions. Consequently the intermediate forms which are not pronounced in the one or the other direction will be in an unfarorable position, and must carry on the struggle for existence with both groups of partially differentiated companions of their species, and, being less completely adapted, must fall.

More important are the first two objections; they have led to the idea that the principle of selection alcne is insufficient to explain the origin and adaptive modification of new forms. So new theories have been advanced, older ones revamped, sometimes to replace that of natural selection, sometimes to strengthen certain links of its chain. Limited space permits only an outline of the more prominent of these and that without any attempt at a decision as to how far they complete the Darwinian hypothesis, are compatible with it, or replace it.

Germinal Selection.-According to its author, Weismann's 'germinal selection ' is only a completion of natural selection, the 'individual selection.' It presupposes a cletailed knowledge of modern investigations in the lines of fertilization and heredity (see chapter on Fertilization) and hence can only be outlined here. Weismann believes that all variations which are selected in the struggle for existence and are fixed in the successive generations must have their sources in the germ cells and since these arise from the fusion of male and female sex cells, they must, in the first instance, have been contained in these. Each germinal anlage consists of extremely numerous elements, the 'determinants' of the peculiarities of the organism. Accordingly as certain of these determinants develop at the expense of the others or are weakened or modified, the organism arising from the germ has special peculiarities or variations. If certain determinants tend constantly in a certain direction and these be present in large numbers, these will persist so that individual selection can have its influence.

Mutations.-The Mutation theory of de Vries is a considerable modification of the Darwinian theory. In rearing large numbers of the 
evening primrose, Enothera lamarckiana, besides plants of the true lamarckiana type there also arise a not inconsiderable number of others which are distinguished from the mother plant in noticeable ways and these may be arranged in sharply defined groups of forms which de Vries has named Oe. gigas, Oe.nanella, Oe. scintillans, etc. These groups of forms resemble 'small species' to the extent that, from the first, intermediate forms are lacking and, prevented from crossing, they produce only individuals with the same characteristics. These suddenly appearing and sharply marked and hereditary variations de Vries calls 1/utations. They have long been known in English as 'sports' and are Darwin's 'single variations.' While formerly these were regarded as merely special instances of general variation, de Vries regards them as different. The slight variations with which Darwinism had previously dealt, oscillatelike a pendulum about its point of rest- around a central point of greatest frequency and.result in no permanent modifications. Even in domestication it is not possible to advance by the continued selection of the slight differences of these 'fluctuating variations,' and to fix them as inheritances. On the other hand stable forms are produced by mutations and these persist if the mutants are better adapted to the conditions of life than is the parent stock. Just so far as the struggle for existence here plays a deciding rôle the mutation theory is a basis for the selection theory. It differs from Darwinism to the extent of being an 'explosive' method of species formation, by which several kinds, and these relatively constant, suddenly come into existence. For the proper valuation of the mutation theory it will be necessary in the future to separate two questions: Whether the sharp distinctions postulated by de Vries between mutation and variation actually exist; (2) Whether the mutation theory is able to explain the numerous adaptations of organisms to their-environment.

The mutation theory has shown anew how necessary it is to subject the phenomena of heredity and variation to exact examination. Thus there has developed in the field of botany an experimental direction which has contributed much to the solution of the problems and promises rich results for the future. Here come the studies of the statistics of variation, established by the mathematicians Galton and Pearson upon the old foundation of Quetelet, which more recently had received considerable modifications at the hands of the botanist Johannsen. This strives to show by the statistical method whether the characters arising by fluctuating variations can be made hereditary by selection.

If a study be made of the modifications of a single character in a 'population' (that is a large number of men, animals or plants living under similar conditions, but springing from different ancestors), it is seen-especially clearly if characters such as length, breadth, weight, number, capable of accurate quantitative statement be chosen-that the majority of individuals assume a middle point with regard to the development of this character, and that the variations from this mean, on either the positive or negative side (plus and mimus variants) are 
the fewer, the farther they be removed from the micldle valuc. From the figures of the statistics which express the relative frequency of each grade of peculiarity there can usually be constructed a curve (Galton's curve) with regular ascending and descending limbs.

If now the extreme plus or minus variants of such an animal or plant population be bred and the peculiarities under investigation be studied in the descendants, there is found a 'regression,' the descendants of the plus or minus variants approaching the mean fixed for the population. For example, very large parents have on the average, offspring smaller than themselves, diminutive ancestors children larger than they. But since the return to mean is not complete, there appears a possibility, by continued selection to establish the variation from the mean as a permanent character.

Again there are the cases where 'pure strains' are employed in lreeding, where the descendants from one and the same parents, or better, from a hermaphrodite plant, are used and the plus and minus variants are continually inbred. There then follows a complete reversion; the descentants of the plus variants give the same curve, with the same mean and the same limbs as the minus variants and the same as the pure strain. Within the pure strain there is therefore a certain 'genotypic character,' revealed by Galton's curve, equally applicable, whether plus or minus variants or mean forms be employed for breeding; against which selection is powerless. From these results, drawn certainly from insufficient empiric material, many have concluded that the fluctuating variations, on which Darwin's theory lays such stress, cannot be taken into account in the origin of new species.

The breeding of pure strains has given another important conclusion regarding the nature of variations: that mutations, that is pure-breeding, sharply circumscribed variations, are much more common than had been thought. 'There are mutations, which on account of the inconspicuousness of the character are easily to be regarded as fluctuating variations, and very likely some of these were regarded by Darwin as such. If different field crops (wheat, oats, retches, alfalfa) or many meadow grasses be cultivated in pure strains, there are found among the descendants of the same ancestors not a few varieties, which rliffer by such slight characters that the eye of the trained breeder is necessary to recognize them; yet, by prolonged pure cultures, they show themselves constant and furnish most favorable material for artificial and natural selection.

Mendelism. - In a third way these studies of pure strains have been remarkably fruitful. These are the researches which have followed the experiments of the Abbe Mendel upon inheritance, by crossing varieties, races and species. Since the explanation of the complicated phenomena involved will be given in a later section, it need only be said here that if in certain cases races be crossed and inbred for several generations, there arises a multiplicity of forms which have the appearance of newly arisen varieties. But more accurate sturly shows that these are not new forms but are only the grouping of manifold characters which were indeed present in the parents, often in a latent state. These 'analytic rarieties' called forth by crossing are in part constant and can furnish material for new varieties.

The importance of all of these researches cannot be estimated too highly; not because they have already given a final decision on the significance of the various kinds of variability but because they have brought the problem of species formation from the region of much sterile theoretical speculation into the clear light of exact experimental investigation.

Migration Theory.-To explain how characters formed by variation become fixed, and do not disappear again through crossing with differently 
modified individuals, Moritz Wagner has proposed the Theory of Geographical Isolation, or the Migration Theory. New species may arise if a part of the individuals of a species should wander to a new place, in which crossing with the others of their species who were left behind is not possible. The same might occur, if geological changes should divide the region inhabited by a species into two parts, between which interchange of forms would no longer be possible. The animals remaining under the old conditions would retain the original characteristics; the wanderers, on the other hand, would change into a new species. Direct observations support this. A litter of rabbits placed at the beginning of the fifteenth century on the island of Porto Santo has increased enormously and the descendants have taken on the characteristics of a new species. They have become smaller and fiercer, have acquired a uniformly reddish color, and no longer pair with the European rabbit. A further proof of the theory of geographical isolation is the peculiar faunal character of regions separated from adjacent lands by impassable barriers, broad rivers or straits, or high mountains (comp. p. 34); especially instructive is the peculiar faunal character of almost every island. The fauna of an island resembles in general the fauna of the mainland from which the island has become separated by geological changes; it usually has not only these but also 'vicarious species,' i.e., species which in certain characteristics closely resemble the species of the mainland. Such vicarious species have plainly arisen from the fact that isolated groups of individuals, scattered over the island, have taken on a development divergent from the form from which they started. With all due recognition of the migration theory, it will never be possible to explain the multiformity of the organic world by it alone. It must be assumed that formerly the earth's surface possessed an enormous capacity for change; but the more rescent investigations make it probable that the distribution of land and water has not varied to the degree that was formerly believed. The experience of botanists, too, teaches that several varieties can arise in the same locality and become constant.

Lamarckism.-While the migration theory agrees with Darwinism in this, that the new characters appearing through variation are to be regarded as the products of chance, yet it is just this part of the theory which has been subjected to searching criticism. Many zoologists have again adopted the causal foundation of the descent theory proposed by Lamarck and believe that the cause of species formation is to be found in part in the immediate influence of changing environment, in part in the varying use and disuse of organs, brought about by alterations in the conditions of life. Both principles, they say, are sufficient, even without the 
help of the struggle for existence, to explain the phylogenesis of organisms. (Neo-Lamarckism.)

Influence of Environment.-To what extent can the environment directly bring about a permanent change in the structure of plants and animals? To decide this is no simple problem, on account of the com plexity of the factors entering into the question.

In cases where the food-supply is altered, organisms change in a very remarkable manner and within a short time; but these changes (Nägeli’s 'Modifications through Nutrition') seem to have no permanence. Plants which, found in nature in poor soil, are transplanted into rich soil, or vice versa, soon acquire quite a different appearance, and preserve this through the following generations, so long as they remain in the rich soil but the plant quickly returns to its former appearance when replaced in its previous surroundings.

In general, a change seems to be the more permanent the more slowly it has developed. In researches upon the influence of environment, it is better to experiment with slowly-working factors, such as light and heat, dry or moist air, different intensities of gravitation, of stimuli, etc., which can be excluded from the environment of the organism. In this way positive results seem to have been attained. When pupe of l'anessa urtica and Arclia caja are reared in the cold (down to $-8^{\circ} \mathrm{C}$.) the butterily or moth which escapes is more or less conspicuously modified, the male the more. If these altered males and females be used for further breeding, a part of the male offspring will have the cold markings, even if reared in the normal conditions. It is however probable that in these cases the cold had a direct effect upon the germ cells from which the aberrant individuals arose. The results of Tower in breeding potato-beetles are in the same direction and are even more conclusive as to the modification of the germ cells.

Use and Disuse.-Regarding the efficiency of use and disuse, there is no doubt that an animal is influenced to a great extent by the manner in which the organs are used. The organs which are much used become strong and those which are not used become weak. The only question is whether these, in the strict sense of the word, newly-acquired characteristics are transmitted to the offspring, or whether the descendants, in order to attain to the same condition, must repeat in the same way use and disuse. In the latter case the cumulation of characteristics, and with it the possibility that these may become permanent, is excluder. It is to be regretted that accurate results are still lacking on a point so well adapted for experimental treatment. At this time rudimentary organs strongly favor the Lamarckian principle; for we see that cave animals, which for 
many generations have lived in darkness, are blind, either having no eyes, or only restiges of them, incapable of function. This seems to justify the view that this condition is attributable to lack of use, since it has brought about a functional and anatomical incapacity, which has increased from generation to generation. Now we must believe that what is true for disuse must express itself in the reverse sense in the case of use.

Nageli's Principle of Progression.-In conclusion, there is still to be considered the change of species from internal causes, which Nägeli has termed the 'perfecting principle,' or the 'principle of progression.' It cannot be denied that each species is compelled, by some peculiar internal cause, to develop into new forms, up to a certain degree independently of the environment and of the struggle for existence. In all branches of animals we see the progress from lower to higher going on, very often in a quite similar way, in spite of the fact that the plan of organization is-so different in the various phyla. We see how the nervous system, lying near the surface in the lower animals, becomes in the higher animals internal; how the eye, at first a simple pigment-spot, becomes in worms, arthropods, molluscs, and vertebrates, provided with accessory apparatus, as lens, vitreous body, iris, chorioid. Here we see an energy for perfection which, since it occurs everywhere, must be independent of the individual conditions of life, and must have its special explanation in the reaction of the living substance to light.

It is by no means justifiable to call an assumption, as here expressed, teleological, and to reject it as unscientific; rather the organism seems to be just as mechanically conditioned as a billiard-ball, whose course is determined not only by contact with the cushions of the billiard-table, but also in a large measure by its indwelling force, imparted to it by the stroke of the cue. An organism, too, is a store of energy which it must necessarily have developed from itself, but it is of more extraordinary complexity, and to an equal degree also is independent of the external world. A complete independence naturally never occurs. Instead there is always an 'action' of the external world, a modifying influence which is carried on by the external conditions of existence, either directly or by the mediation of use and disuse.

This outline of evolution has been given in a rather detailed way, because in the history of zoology it is the most important feature. No other theory has gained such a hold, none has propounded so many new problems and opened so many new fields for research. There is no other zoological theory which compares with it in value as a working hypothesis. To the objection that the theory is insufficiently grounded, 
it can only be replied that it is the only theory which agrees with our experiences and explains these in a simple way and on a scientific hasis. In this sentence is given the merit of the theory, and also a limitation of its applicability. For on the one side the statement attributes the merit in the applicability of the system to the necessity of the human mind for simple explanations of the facts of natural science, and on the other hand it makes the degree of correctness dependent upon the state, whatever it may be, of our knowledge. Many investigators see no necessity of reconciling paleontology and our knowledge of plants and animals. 'To such the Darwinian theory proves just as little as any opposing theory. Meanwhile thoughtful naturalists will keep in mind that our knowledge of nature is making considerable advances, and is visibly becoming wider and deeper. It is possible, even probable, that these advances will lead to many modifications of the theory. The conception of the way in which forms have developed from one another admits, as the mutation theory shows, of very different expressions. On the other hand, we can affirm with great certainty that the principle of descent, which first obtained credence through Darwinism, will be a permanent landmark of zoological investigation. 


\section{GENERAL MORPHOLOGY AND PHYSIOLOGY.}

General Zoology: Animal Morphology.-In vital phenomena a certain degree of similarity can be followed through the animal kingdom; the way in which animals are nourished and reproduce their kind, move, and gain experience, is essentially the same in great groups. Corresponding to this, the apparatus concerned with the above-mentioned functions, the organs of nutrition and reproduction, of motion and sensation in their grosser and finer structure, and in their ontogeny, must be similar to one another and show evidence of some fundamental characters which always or frequently recur.

Ecology or Biology.-When by means of anatomy and embryology we have learned the general character of the organism, we must then study its relations to the environment. In this study of the conditions of animal life, œcology or biology, we consider the geographical range of animals, their distribution over the surface of the earth and in the different depths of the sea; further, the reciprocal relations of animals and plants, and of beast to beast, as these find special expression in colony-building, symbiosis, parasitism, etc.

General Anatomy.-The synthesis of an organism, of which we can only gain an idea by general anatomy, actually takes place in nature during the development of every animal. Embryologically every organism is at some time a simple element, a cell; this divides and gives rise to tissues; from the tissues are formed organs, and from the organs the regularly membered whole of the animal body is composed.

\section{GENERAL ANATOMY.}

The Morphological Units.-The expression 'constituent parts of the animal body' can be used in a double sense. We can speak of the chemical units, the chemical combinations, which form the tissues; these are the subject of animal chemistry, and may therefore be passed over here. But we may also speak of the units of form (morphological units) of the animal body; these are the cells. These and their transformation into tissues, organs, and entire animals are for us of vastly greater importance. 


\section{The Morphological Lnits of the Animlal Body.}

The Cell.- The study of the morphological units of the organic body first found a firm foundation in the cell theory. Every scientific study of the anatomy of plants and animals must therefore take the cell as its starting-point.

The Cell Theory.-In order clearly to understand the conception of the cell and its name it is necessary to follow a little of the history of the theory of the cell. When the name was first given to the structures in plants it implied small chambers with firm walls and filled with air or fluid. Then came the discovery of a small body, the nucleus, insicle the cell. Next Schleiden made the generalization that the cell was the anatomical and physiological unit from which all plants are formed, but he held the erroneous view that in the development of cells, the nucleus was formed in a sort of matrix, then around it a nuclear membrane arose by precipitation, and then a larger membrane, the cell wall, around the whole. Then Schwann extended the generalization to animals, thus giving it an extension to all organisms.

In this Schleiden-Schwann cell theory the cell wall was all important, as through it diffusion currents must pass between the contents and the surrounding medium. Hence the wall and the contents must determine the character of the cell according to physical laws. Since the life of an organism is but the totality of the life of the cells of which it is composed, it was thought that the theory was a great advance in the problem of the physical explanation of the phenomena of life, and the origin of the cells themselves was as well explained as the formation of a crystal.

Our conception of a cell has completely changed. We know that they do not arise like crystals, but from preexisting cells. The cell is not merely a part of an organism; it is a physiological whole, which shows us all the enigmas of life. The membrane and cell sap, so important in the Schleiden-Schwann theory, have but a subordinate place, but all important is the previously disregarded substance, protoplasm. Now a cell may be defined as a small mass of protoplasm with one or more muclei. This change in the conception came so gradually, that the name cell has persisted, although it is an evident contradiction to call a solid lump without a membrane a cell.

These changes were due to many different lines of investigation. Thus the early discovery that the chlorophyl bodies in plant cells move and, later, that the motion is due, not to the bodies, but to the substance in which they are imbedded. This substance, to which the name protoplasm was given, acquired prominence when it was found that in the simplest 
algx, it, together with the chlorophyl, could leave the cell wall and swim freely in the water, eventually giving rise to a new plant, while the cell wall no longer showed signs of life. Then it was discovered that many animal cells had no cell membrane. These observations at once placed the cell wall in the background, while the protoplasm was recognized as all important. Here, too, should be mentioned the researches on the Protozoa, by which it was recogniced that these organisms had no true organs, but carried on all of the functions of life by means of a granular substance, at first called sarcode.

Then followed the recognition of the identity of the protoplasm of plants, the sarcode of the protozoa and the cell substance of animal cells. Equally important was the new idea as to the modifications of cells and the differentiation of tissues. These are not so much modifications of form and the like, based on osmosis and other physical phenomena as upon chemical changes. By means of its formative potentiality the protoplasm gives rise to non-protoplasmic structural parts, as, for example, connective-tissue fibrils, muscle fibrils, nerve fibres, etc. These give the various tissues their specific character and perform their functions. The tissues also retain as the source of life and formation the unemployed remnants of cells, the connective-tissue corpuscles, muscle corpuscles, etc.

Nature of the Cell.-The size of the animal cell varies; the smallest are the male sexual cells, the spermatozoa, whose bodies, in case of the mammals, may measure only $0.003 \mathrm{~mm}$; the largest, with the exception of the giant plasmodia of some Mycetozoa, are the egg cells. The yolk of the bird's egg, which alone forms the egg in the narrower sense, has for a time the value of a cell, and in the ostrich egg may reach a diameter of several inches. The form is likewise variable. Free cells, are usually spherical or oval as the egg cell shows; united into tissues, the cells, on the contrary, may be pressed together into polygonal or prismatic bodies, or may send out branching processes.

Protoplasm.- So there is left to characterize the cell only its substance: the cell is a mass of protoplasm with one or more nuclei. It is not known whether protoplasm is a definite chemical body, capable of infinite variation, or is a varying mixture of different chemical substances. So, also, we are not certain whether or not these substances (as one is inclined to believe) belong to the proteids. We can only say that the constitution of protoplasm must, with a certain degree of homogeneity, have a very extraordinary diversity. For if from the egg of a dog there comes always and only a dog with all his individual peculiarities; that a seaurchin's egg, under the most diverse conditions, produces always a sea- 
urchin; that a species of amœba always performs only the movements characteristic of that species, we must assume that the functioning part of this cell, the protoplasm, has in each case its peculiarities. We are driven to the assumption of an almost unlimited diversity of protoplasm, even if we concede an important share in the prominent difierences to the nucleus, of which we shall speak later.

General Properties of Protoplasm.-The similarity of protoplasm expresses itself in its appearance and in its vital phenomena. Under slight magnification, protoplasm appears as a faintly gray substance (sometimes colored yellowish, reddish, etc., by pigments) in which numerous stronglyrefracting granules are imbedded. The vital characteristics of this substance are movement, irritability, power of assimilation and of reproduction.

By using higher powers a finer structure can be seen in the 'homogeneous protoplasm' of earlier writers. It looks like a fine-meshed framework (filar substance, spongioplasm, cell reticulum) the interstices of which are filled with other material (interfilar substance, enchylema, ground substance). The question whether this framework is formed of threads and trabeculæ or whether the appearance is not formed by small chambers, bounded by fine fartition-walls (foam structure of protoplasm), such as results when two fluids which do not mix (like olive oil and soda solution) are shaken together until a very fine froth is produced. This view that protoplasm has a foam structure explains how it can be a fluid aggregate with a fine structure. Regarding the fluid aggregate condition $\mathrm{cf}$ protoplasm (long called a 'solid-fluid') there has been much dispute. Exact researches regarding its physical condition show that it behaves like a fluid.

Movement of Protoplasm.-Movement expresses itself first in changes of form of the whole body-amaboid movement-and secondly in the change of position of the small granules in the interior of the protoplasm-streaming of granules. Examples of amoboid movement (fig. I6) are found in many

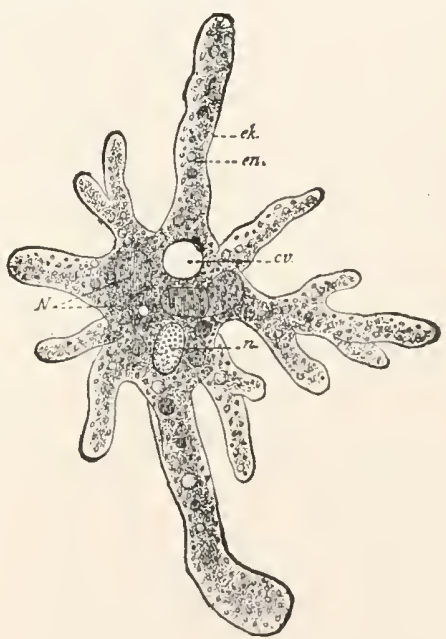

Fig. I6.-Amoba proteus (after Leidy). $e k$, ectosarc; $e n$, entosarc; $c v$, contractile vacuole; $n$, nucleus; $N$, food-vacuoles. Protozoa, and the colorless blood-cells (leucocytes) of multicellular animals; here the protoplasmic body sends out coarser and finer processes, which may be again withdrawn, serving for locomotion and hence called pseudopodia. The streaming of granules can be olserved in the interior of the cell-body, as well as in the pseudopodia extending from this. The pseudopodia may even be so fine as to be at the limits of visibility with our 


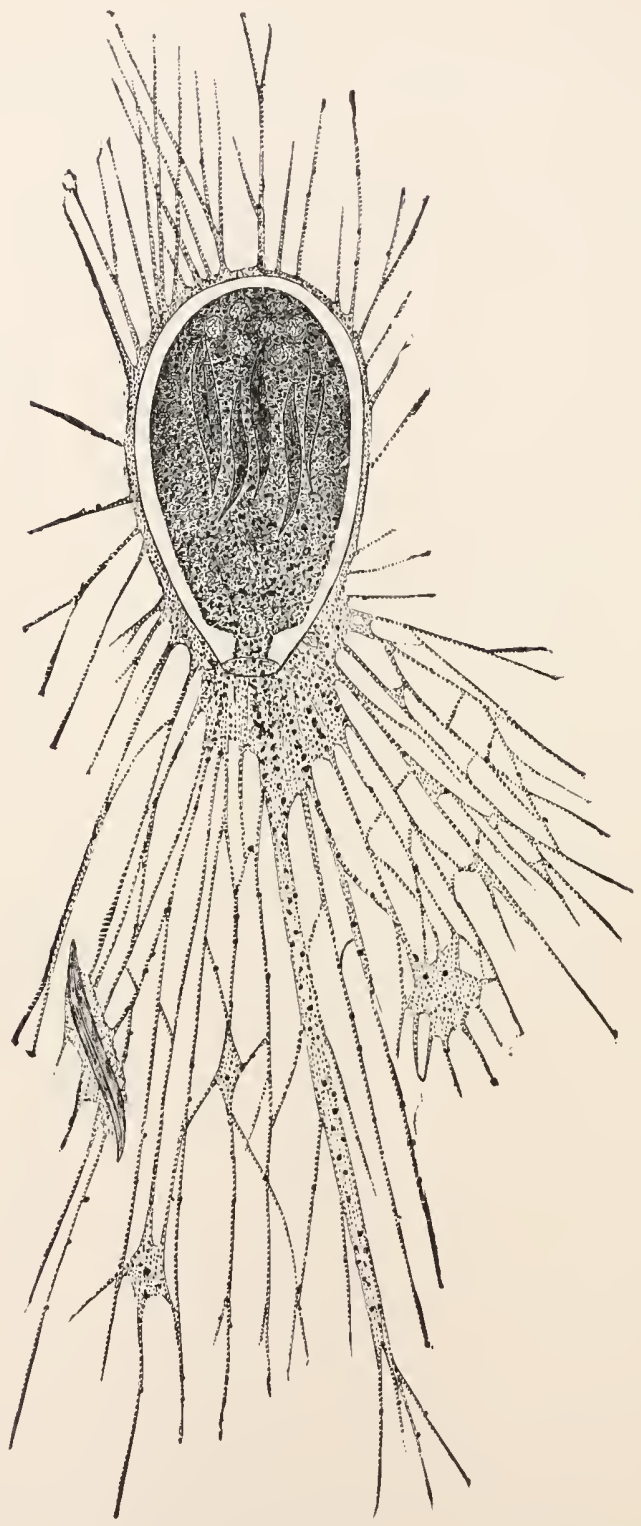

FIG. 17.-Gromia oviformis (from Lang, after M. Schultze). 
microscopes (fig. I 7 ), yet in them the granules wander hither and thither like people on a promenade, simultaneously centripetally and centrifugally, some with greater, others with less speed. 'The granules are moved by' the protoplasm, for if we feed the creature with finely-pulverized carmine, these granules show the same remarkable streaming. Indeed, nothing better illustrates the great complexity of protoplasm than these phenomena of motion in such narrow limits as pseudopodia in general.

Irritability of Protoplasm. - That amoeboid movements and streaming of granules can be induced, brought to a standstill, and modified by mechanical, chemical, and thermal stimuli, is a proof of the irritability of protoplasm. Most important are the thermal stimuli; if the surrounding melium rise above the ordinary temperature, the movements at first become more rapid up to a maximum: from that point begins a slowing, finally coming to a standstill-heat-rigor. If the high temperature continue much longer, or if it rise still higher, death results. The fatal temperature for most animals is between $40^{\circ}$ and $50^{\circ} \mathrm{C}$. ( $104^{\circ}-\mathrm{r}_{2} 2^{\circ} \mathrm{F}$.); its influence explains a part of the injurious effects which highfever temperatures have upon the human organism. Like the heatrigor, there is a cold-rigor, induced by a marked sinking of the temperature below the normal. This is accompanied by a gradual climinution of mobility; it results in death by freezing, which is, however, not so easily produced as death by heat. It is a remarkable fact that many animals, consequently their cells, may be frozen; and in this condition can endure still severer cold without dying. (For example: goldifish, a temperature of $-8^{\circ}$ to $-\mathrm{I} 5^{\circ} \mathrm{C}$.; frogs, to $-28^{\circ}$; 'blind worms', to $-25^{\circ}$ ).

Nutrition and Reproduction.-Irritability and power of motion are necessary for assimilation. Most animal cells, for example almost all the tissue cells, are not suitabe for studying assimilation, because they live upon liquid nourishment. But certain cells of higher animals, the leucocytes, and most unicellular animals can be fed with solicl substances; they take the food-particles into the midst of the protoplasm by flowing around them with the pseudopodia. They extract all the assimilable and reject the indigestible portions (fig. I6).

In the case of assimilation it is to be noted not only that the cells use the food which they have taken for their own growth and for replacing worn-out parts, but also that most of them have the power of producing substances other than protoplasm; for example, many Protozoa form shells or skeletons which are hardened with silica or lime. This formative power, the building of plasmic products, is the starting-point for tissueformation.

Cell Nucleus.-The reproduction of protoplasmic bodies is synony- 
mous with the division of the cell; but to understand this we must first consider the mucleus. This is a body enclosed in the protoplasm, whose form, though definite for each kind of cell, shows in general wide variations. Usually it is spherical or oval, but it may be elongated or rodshaped, bent into a horseshoe, with constrictions like a rosary, or even be branched or treelike (fig. I8); in many living cells it is but little different
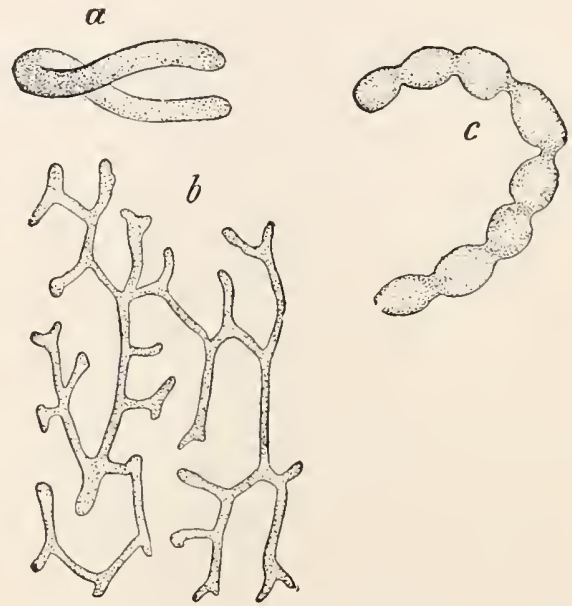

FIG. I8.-Various forms of nuclei. $a$, horseshoe-shaped nucleus of an Acinete; $b$, branching nucleus from the Malpighian vessel of a Sphingid larva; $c$, rosary-shaped nucleus of Stentor caruleus.

in appearance from the protoplasm and can only be seen with care and by employment of a special technique based upon the microchemical reaction of the nuclear substance.

The Nuclear Substance.-The nuclear substance is distinguished from protoplasm, among other ways, by its greater coagulability in certain acids, e.g., acetic and chromic, which therefore are often used for demonstrating the nucleus. In its minute structure the nucleus affords a wonderful variety of pictures varying according to the objects chosen. According to their reactions to stains two substances in particular are distinguished: chromatin or muclein (fig. I9, ch), which is easily stained by certain staining-fluids (carmine, hæmatoxylon, saffranin), and the achromatin or linin, which stains only under special conditions.

The achromatin forms a network or reticulum (according to another view a honeycomb structure) filled with a nuclear fluid, bounded externally by a nuclear membrane. If little nuclear fluid be present, and the reticulum consequently be narrow-meshed, the nucleus seems compact. 
If the fluid be abundant, the nucleus appears vesicular. This is especially the case when the lines of the framework are separated by considerable amounts of nuclear fluid (fig. I9, 4).

The chromatin enters into close relations with a less stainable element, also distinct from achromatin, the plastin, (paranuclein, $p$ ). In the protozoan nuclei plastin and chromatin are usually intimately united, the first forming a substratum in which the latter is imberleded (chp). The united substances are most frequently closely and regularly distributed as fine granules on the reticulum, so that the entire nucleus
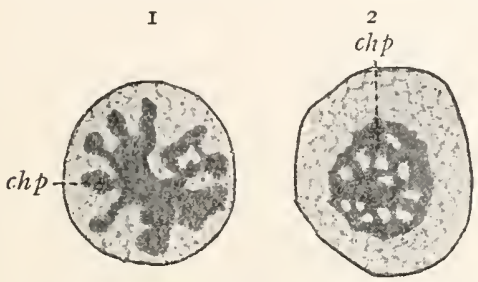

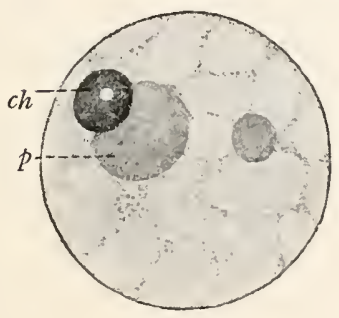

4

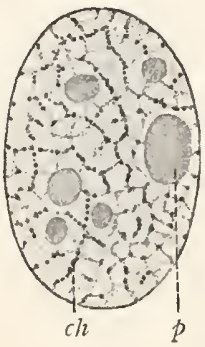

5
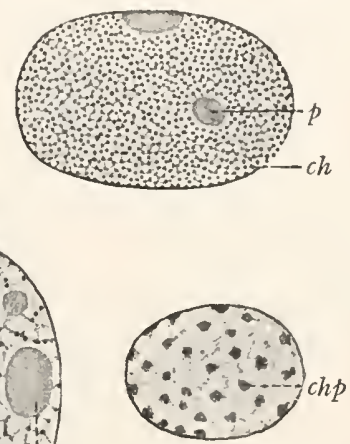

6

FIG. I0.-Vesicular nuclei with achromatic reticulum and different arrangements of the chromatin and nucleolar substance. p, plastin (nucleolar substance); $c h$, chromatin; chp, chromatin plus plastin. I and 2, nuclei of Actinospharium; 3 , of Ceratium hirundella (after Lauterborn); 4 , germinal vesicle of Unio (after Flemming); 5 , nucleus with many chromatin nucleoli.

appears uniformly chromatic (fig. I8). More rarely the mixture collects into one or more special bodies, the chromatic mucleoli (amphimucleoli, caryosomes, fig. I9, I, 2). The nucleolus is ordinarily a rounded body, more rarely branched (fig. 19, I).

In the nuclei of the Metazoa there may occur the same intimate mixture of plastin and chromatin (6). As a rule, however, the plastin (apparently not the whole, but a surplus) is separate from the chromatin. Thus there occur in the nuclei of many eggs nucleoli which consist of two distinguishable parts, the one containing chromatin, the other chromatin free (4). Usually in tissue cells only the plastin has the form of 
nucleoli (true or chromatin-free nucleoli), while the chromatin is distributed on the nuclear reticulum (chromatin reticulum). Nuch the same may occur in ihe Protozoa (fig. 19, 3).

Beside and outside of the nucleus there occurs in many Protozoa a 'chromidial apparatus,' a substance agreeing in its staining properties with the nuclear substance. Its pertinence to the nucleus is also shown by the fact that repeatedly it has been observed to arise from the nucleus (Actinospharium), as well as to be transformed into nuclei (Radiolaria, Monothalamia). 'The chromidial mass may surround the nucleus like a cortical layer (Euglypha, fig. 20, III, Radiolaria), or penetrate the protoplasm as a loose network (II), or form lumps or coiled threads. In this last shape the chromidial mass seems to be widely distributed in strongly functioning cells of Metazoa (I). Possibly the structures described as 'mitochondria' are identical with it.

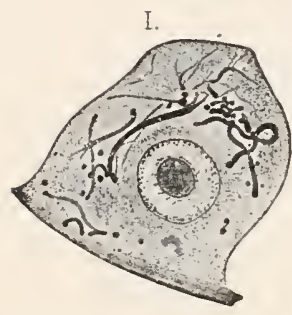

II.

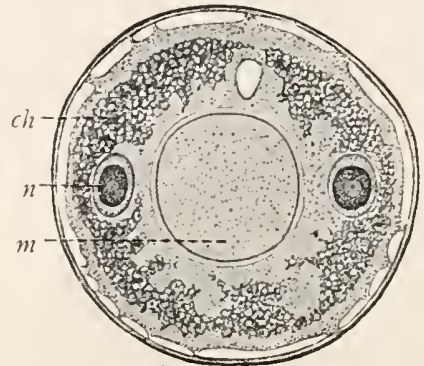

III.

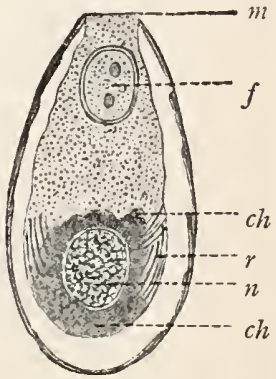

FIG. 20.-Cells with chromidial apparatus. I, muscle cell of Ascaris (after Goldschmidt). II, Arcella, with two nuclei and loose chromidial net. III, Euglypha with compact chromidial envelope of the nucleus. ch, chromidial mass; $f$, food body; $m$, mouth of shell; $n$, nucleus; $r$, reserve material for new shell.

Function of the Nucleus.-For a long time the function of the nucleus in the cell was shrouded in complete darkness, so that it was regarded, in comparison with the protoplasm, as of little importance. The evidence that the nucleus plays the most prominent rôle in fertilization has altered this conception. Then arose the view that the nucleus determines the character of the cell; that the potentiality of the protoplasm is influenced by the nucleus. If from the egg a definite kind of animal develop, if a cell in the animal's body assume a definite histological character, we are, at the present time, inclined to ascribe this to the nucleus. From this it follows that the mucleus is also the bearer of heredity; for the transmission of the parental characteristics to the children can only be accomplished through the sexual cells of the parents, the egg and sperm cells. Again, since the character of the sexual cells is determined by the nucleus, the transmission in its ultimate analysis is by the nucleus. This idea has a further support in experiments on Protozoa. If one of 
these animals be cut into nucleate and anucleate halves, the first lives and regenerates the lost parts; the anucleate portion moves about for a time, apparently as long as the stored energy lasts, but it cannot assimilate or reproduce the missing portions and so sooner or later it dies.

The Centrosome.-Besides the nucleus there frequently occurs a special body in the protoplasm, the centrosome, which, on account of its small size and a behavior similar to achromatin with reference to stainingfluids, was long overlooked. It is well distributed among the Metazoa, but is absent from most Protozoa, in many of which it appears only at certain times and then disappears. It is probable that it is a derivative of the nucleus, a part of the achromatin which has left the nucleus; in other cases possibly a second nucleus which by degeneration has lost the chromatin and retained only the motor nuclear substance, the achromatin. In its function the centrosome is a specific organ of cell division which controls both the division of the nucleus and that of the cell itself.

Multiplication of Cells. - Increase in cells occurs exclusively by division or by budding (gemmation). Most common is binary division in which a circular furrow appears on the surface of the cell, deepens and cuts the cell into two equal parts. Mutiple division is more rare and can only occur in multinucleate cells. Here the cell divides simultaneously into as many (sometimes hundreds) daughter-cells as there were nuclei present. In all forms of division the similarity of the products is characteristic, while in budding the resulting parts are unequal, one or more smaller daughter-

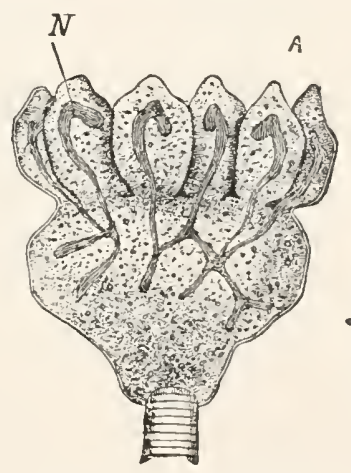

Fig. 21.-Cell budding. Padsphrya gemmipara with buds (a) which separate and form free young $(b) . \quad N$, nucleus. cells, the buds, being constricted from a large mother-cell (fig. 2I).

Direct Cell Division.-Every cell division is accompanied by nuclear division or nuclear division has previously occurred. Direct and indirect division are recognized. Direct division is most common in Protozoa, especially in nuclei with abundant chromatin (figs. 21, I20, I50, I55). The nucleus elongates and is divided by constriction, in the same way' that the cell itself constricts. Since the protoplasm has no special arrangement for dividing the nucleus (the latter besides protected by its membrane), we must conclude that the nucleus divides itself. The dividing force resides in the achromatic framework, which correspondingly often 
exhibits a certain arrangement, a fibrous structure in the direction of the elongating nucleus.

Indirect Cell Division, Karyokinesis.-Indirect cell division, karyokinesis or mitosis, is most beautifully shown in cells, poor in chromatin, which possess a centrosome. The process is introduced by a division of the centrosome (fig. 22). 'The daughter centrosomes migrate to two opposite poles of the nucleus, which now loses its membrane and becomes the nuclear spindle. The characteristics of the spindle are that

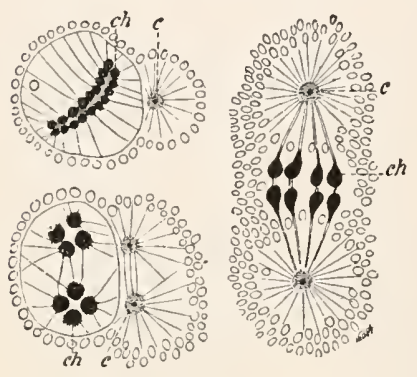

FIG. 22.-Spindle formation and division of the centrosomes in $A s$ caris megalocephala (after Brauer). $c$, centrosomes; $c h$, chromosomes. it is drawn out into points at two poles which are indicated by the position of the centrosomes, while from these poles fine threads, the spindle-fibres, run to the centre or equator of the nucleus. These fibres are in many cases certainly derived from the achromatic nuclear reticulum, while in others a greater or less part in their formation is taken by the protoplasm (fig. 22.) A debated point is the relations of the fibres in the equatorial plane of the spindle. Do all the fibres extend from pole to pole? Do all of them end in the equatorial plane, so that the spindle consists of two cones of fibres separated at the equator? Or, lastly, are fibres of both kinds present in the same spindle? It would appear that differences exist in these respects in different cells.

All of the chromatin of the nucleus lies in the equator, united in the 'equatorial plate,' but by this must not be understood a connected mass but a layer of separate bodies, the chromosomes (fig. 23, a). These develop at the beginning of nuclear division by the union of the chromatin granules (which are distributed diffusely over the reticulum of the resting nucleus) to strongly staining bodies, which are rarely spherical or rodlike, but usually have the shape of U-shaped loops. It is of the greatest theoretical significance, that their number is identical in all the cells of all the tissues of one and the same species.

The first step in the mitotic formation of the daughter nuclei is the division of the chromosomes, which is usually completed in the equatorial plate (division of the equatorial plate), but may be completed earlier. The division is an accurate halving (fig. $23, b$ ). The two halves of a mother-chromosome, the daughter chromosomes, now travel, under the influence of the spindle-fibres, towards the poles of the spindle. In this way, by a splitting of the equatorial plate, the lateral plates arise, the 
elements of each uniting and producing the daughter nuclei. The centrosomes remain separate as division organs for the next nuclear clivision (fig. $23, c, d, \epsilon)$.

What further distinguishes the indirect from the direct cell division is the active participation of the protoplasm. The centrosome is the centre of a marked radiation (aster) of the protoplasmic reticulum (fig. 22). When the centrosome divides a double radiation appears, the monaster becomes an amphiaster. Not only the spindle-fibres but the protoplasmic

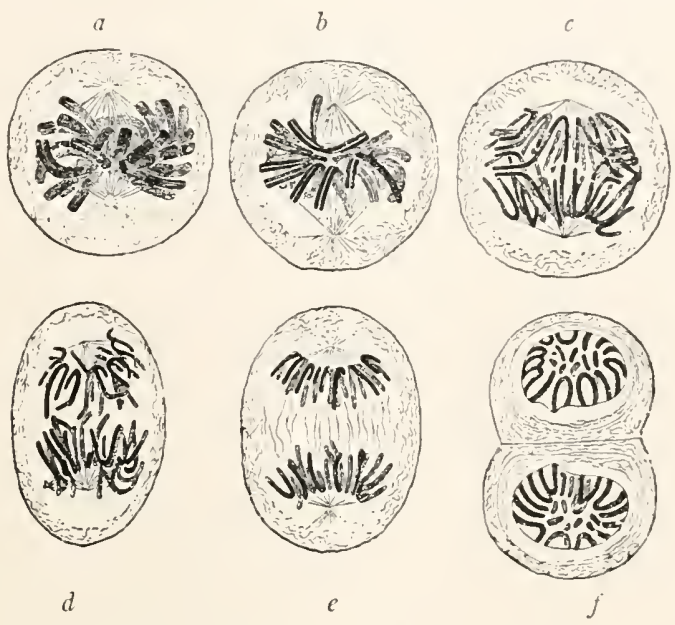

FIG. 23.-Cell division in the skin of Salamandra maculosa (after Rabl).

rays extend from the daughter chromosomes. Since the arrangement and degree of derelopment of the protoplasmic radiations stand in definite relation to the different phases of cell division we must recognize in them the expression of the forces (apparently contractile) in the protoplasm which cause cell division.

Between these two extremes of direct and indirect division are transitions which show how the mechanism of nuclear division has been completed step by step, first, by the fibrous arrangement of the nuclear reticulum (spindle structure); second, through the development of the centrosome by which the division obtains an influence on the protoplasm; and third, by the organization of the chromosomes. The irregular division of the chromatin mass in direct division is relatively crude in comparison with the complicated processes involved in the formation and division of the chromosomes. 'These become intelligilble if we regard the chromatin as the controller of the cellular processes and the bearer of heredity ( $c f$. fertilization, infra). The more highly organized the animal, the more its cells have to inherit and the more important it is that the physical basis of heredity should be accurately divided in amount and in quality between the daughter cells. This is accomplished by mitosis. 
Connected with this great functional importance of the chromosomes as the bearers of characteristics are two much disputed problems. (I) The 'individuality of the chromosomes.' 'This sees the persistent organization of the cell in the chromosomes, which persist between two cell divisions, but are not recognizable as such because their substance is vacuolated and distributed through greater space. Of course this view does not conflict with the fact that they, like all living substance, undergo a gradual renewal, in which effete parts are replaced by new and there is an increase of its substance without which a reproduction of chromosomes by division would be impossible. (2) The theory of the functional diversity of the chromosomes. If the chromosomes carry the characteristics, it is more probable that each one does not contain the germs of all the peculiarities of the organism; rather there is a division of labor by which the separate peculiarities are distributed among the different chromosomes. This view is supported by the fact that there is, in numerous instances, a morphological differentiation between them (differences in size, shape, staining qualities). That in the last analysis each category of characters consists of at least two

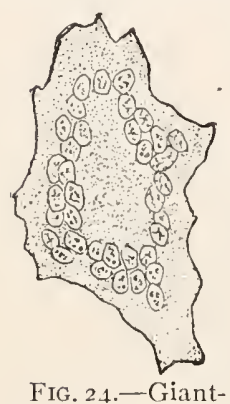

cell with many nuclei. lines (male and female) is shown by the fact that, as is explained in the section on fertilization, half of each nucleus is derived from the father, half from the mother.

Nuclear Fragmentation is to be distinguished from direct division; by it the nucleus becomes broken up into a few or numerous parts. Such nuclear fragmentation is not rare in the Infusoria but it occurs occasionally in Netazoa (giant cells of bone marrow-fig. 24-osteoblasts, certain stages of the genital cells). It is explained as follows. 'There normally exists a certain size relation between nuclear mass and protoplasmic mass. With greater cell activity, as with Infusoria which have long been well fed, the nucleus grows at the expense of the protoplasm until it reaches a size which makes further assimilation and increase impossible. This kind of animal (or cell) can return to the normal vital activities if the nuclear mass be reduced. This is begun by the fragmentation and is completed by the resorption of the nuclear substance. Many cases of nuclear fragmentation, formerly regarded as amitoses are really functional conditions of actively functioning cells and have erroneously led to the view that amitosis is beginning cell degeneration.

\section{Multinuclearity, Multicellularity.-Nuclear division and cell division} commonly constitute a well-arranged mechanical process, the separate phases of which follow one another according to a definite law. The plane of division is perpendicular to the long axis uniting the two poles of the spindle; usually also each phase of division of the nucleus corresponds to a certain phase of the protoplasmic division. But the interrelation of cytoplasm and nucleus is by no means an unchangeable and indissoluble one, for very often nuclear division takes place without participation of the cytoplasm. If this process be repeated several times, there results a mass of protoplasm with many nuclei (fig. 24), which now may become many cells, if subsequently the protoplasm divide according to the number of nuclei. Hence multinucleated protoplasmic masses are transitional stages between the simple mononucleated cell and a collection of several 
mononucleated cells, and in consequence of this are sometimes regarded as the equivalent of one cell, sometimes as equivalent to many cells, and are called sometimes multinucleated giant-cells, sometimes syncytia. In the following pages a multinucleated mass of protoplasm will be considered as a single cell, because a cell is a vital unit, has a physiological individuality, and in this respect a multinucleated mass of protoplasm behaves like a mononucleated. As the tissue cells and the Protozoa show, the plane of organization is not raised in the least by the multinuclearity. A change only begins at the moment when many particles of protoplasm are separated from one another, and many vital units are formed, i.e., when in place of multinuclearity a true multicellularity appears.

\section{The Tissues of the Animal Bodr.}

Definition of Tissue.-In the formation of tissues two processes are operative: (I) the multiplication of cells into cell-complexes, and (2) the histological differentiation of cells. I tissue, therefore, can be defined as a complex of differentiated cells histologically similar.

Histological Differentiation.-The chief result of the histological differentiation is that the cells have a definite form and definite relations to neighboring cells. In addition, there almost always occurs, as a more important feature, the histological modification of the cell. The fact has already been mentioned that the cell uses its food-material, no: only for its own growth, for increase of its protoplasm, but also for forming substances, plasmic products, either in its interior (internal plasmic products), or more often on its surface (external plasmic products). The histological differentiation is the formation of specific plasmic products. A cell in becoming a muscle fibre (fig. 25), continually secretes upon its surface new fibrillae of specific muscle substance until finally the remnant of the formative cell, the 'muscle corpuscle,' is contained in a mantle of
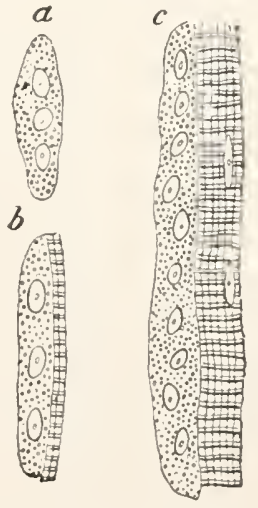

FIG. 25.-Formation of muscle fibrils in the frog (diagram). $a$, formative cell; $b$, formative cell with two transversely striated muscle fibrils; $c$, formative cell with numerous muscle fibrils. muscle fibrillæ. In the same way, each tissue, upon histological examination, is seen to be composed of cells and plasmic products. The former control the formation, the renewal, and the sustenance of the tissue; the latter are the agents of its physiological function. 'The advantages of tissue formation are far-reaching, since in general they are connected with division of labor. Solong as the cell unites in itself 
all the vital functions, these are incomplete because they mutually hinder each other in their free development; the plasmic product, on the other hand, has only the single function peculiar to it and can therefore perform this with greater completeness. Muscle fibrilla, the characteristic elements formed by the muscle cells, have preserved of the various properties of pro oplasm only the capability of contraction; but this contraction is much more energetic and stronger than the mere movement of protoplasm. Nerve fibrilia only transmit stimuli, but in far more rapid and orderly manner than does simple protoplasm.

Classification of Tissues.--Since in every tissue its function interests us most, it is natural to classify tissues by the function and the intimate structure connected therewith. The tissues are arranged in four groups: I. Epithelial tissue; 2. Supporting tissue; 3 . Muscular tissue; 4. Nervous tissue. Within these, however, certain parts of the animal body, to which indeed the term 'tissue' is scarcely applicable, find no shelter; these are the sexual cells, the blood, and the lymph. The first may be spoken of in connection with the epithelium, the others with the supporting substances.

\section{Epithelial Tissues.}

Morphology of Epithelial Tissues.-An epithelium is a layer of cells covering any free surface, external or internal, of the body. The epithelia must be considered first, because they are the oldest tissues; the first to appear in the animal kingdom, there being animals which consist only of epithelia. Further, every metazoan, during the first stages of embryonic life, consists only of epithelial layers, the germlayers. With this is connected the fact that epithelial cells have undergone the least degree of histological change, and that the formation of plasmic products is subordinated.

Function of Epithelium.-Epithelium forms a protecting and excluding covering over surfaces, equally valuable whether the surfaces are external (surface of the body) or in cavities in the interior of the body (the body cavity, lumen of the gut and blood-ressels). The importance of the epithelia in this respect is shown by the fact that if the protecting layer be removed, inflammation arises and continues until the epithelium is regenerated. Only exceptionally do areas occur which are free from epithelium; the teeth of vertebrates, the antlers of stags, on account of their hardness, can exist, at least for a time, without epithelial covering.

Glandular and Sensory Epithelia.-By their position epithelia are suited for two other functions: all substances which ought to be removed from the body-some because they have become useless, and consequently 
injurious (excreta), and others, as, for example, the digestive fluids, because they have to perform important functions (secreta - must pass the surface and are therefore exuded by the epithelia; these are the glandular cpithelia. Further, all external influences chiefly impress the surface of the body, causing sensations; hence certain epithelia are of the greatest importance for the reception of sensory stimuli, and serve for hearing, seeing, smelling, tasting, and touching. Such areas of epithelium are called sensory epithelia.

Covering Epithelium.-The covering epithelium consists of cells which are united by a small quantity of cementing substance. We speak of simple or of stratified epithelia, according as we find, in sections running perpendicularly to the surface, one or several superimposed layers of cells (figs. 26, 27, 28).

Simple Epithelium.-Only one-layered epithelia are found in all invertebrated animals and in Amplioxus; in the vertebrates, on the other hand, they are limited to the internal cavities of the body, and even here are occasionally, as always in the skin, replaced by a many-layered epithelium. According to the shape of the cells we distinguish cuboidal or pavement, flat, and columnar epithelium. In pavement epithelium (fig. $26, b$ ) the cells are developed about equally in all directions of space, and because they have become compressed by lateral pressure have the appearance of cubical blocks or paring-stones. In columnar epithelium the long axis, the distance from the deeper to the peripheral end of the cell, is especially great (fig. $26, c$ ); finally, in tlat or squamous epithelium this is greatly shortened (fig. 26, $a$ ) and the separate cells form thin plates.

Flagellated and Ciliated Epithelia.-Further differences in these three kinds of epithelium are caused by the presence or absence of processes (cilia, or flagella) on the peripheral end of the cells. These are fine threads which arise from the body of the cell, extend above the surface and maintain an extremely lively motion. In flagellated epithelium (fig. 26, d) each cell has only one vibratile projection, but this is strongly developed; in ciliated epithelium (fig. $26, e$ ), the surface of each cell is covered with a thick forest of shorter threads moring in unison.

Cuticle.--The majority of the one-layered epithelia are covered by a cuticle, a membrane secreted by the epithelial cells which hence very frequently shows the impression of the cells as polygonal markings. In many cases thin and inconspicuous, it may in other instances become thickened into a very considerable layer, much thicker than the matrix layer of epithelium which secretes it. The cuticle is composed of layers parallel with the surface, and forms a more effective protection for the body than does epithelium; it becomes a protective armor, as shown, 
among other examples, by the calcareous shells of molluses and the chitinous integument of insects (fig. 26, $f$ ).

Stratified Epithelia.-The protection furnished by the cuticle in the case of simple epithelium, may in the stratified be obtained immediately through a change of a part of the cells themselves. In the stratified epithelia the cells of the various layers can be distinguished by their form.
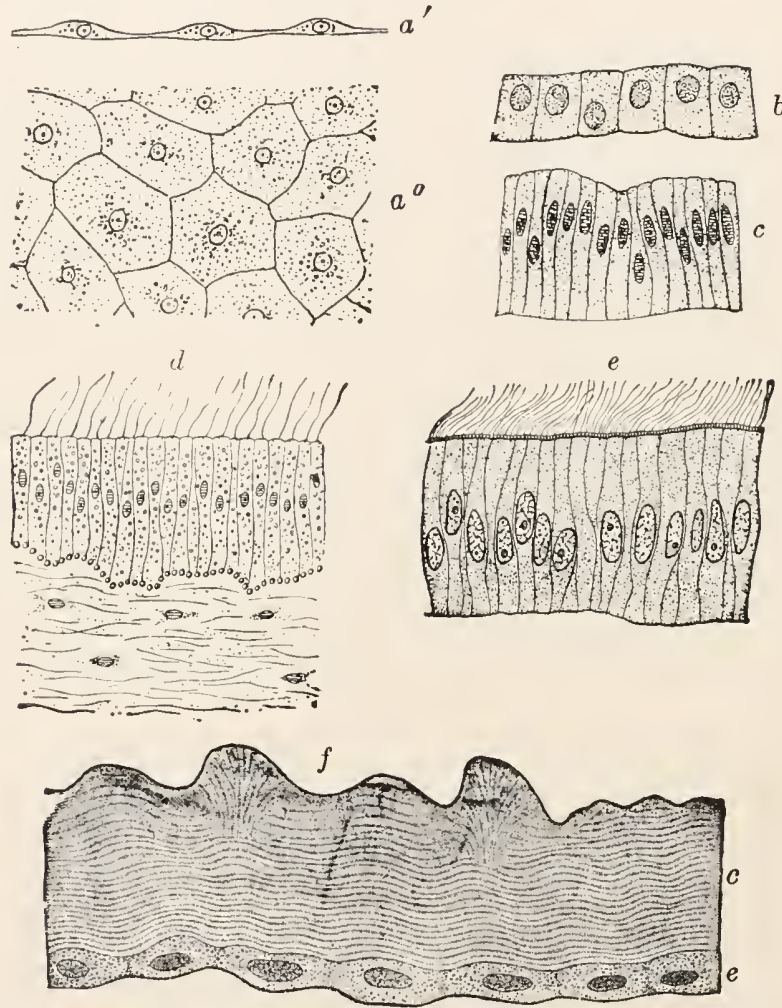

FIG. 26.-Various forms of epithelia. a, flattened epithelium of Sycandra raphamus, $a^{\prime}$ in cross-section, $a^{\prime \prime}$ in surface view; $b$ and $c$, cuboidal and columnar epithelium of a mollusc (Haliotis tuberculata); d, flagellated epithelium of an actinian (Calliactis parasitica); $e$, ciliated epithelium from the intestine of the fresh-water mussel; $f$, epithelium (e) with cuticle (c) of Cimbex coronstus ( a wasp).

The deepest layer consists of cylindrical cells; the superficial, of more or less flattened elements; between lie sereral layers of transitional forms, so that starting from the cylindrical cells we gradually pass through cubical cells to the flat cells of the surface. As this arrangement shows, there exists a genetic connection between the layers of cells: the lower cylindrical cells are in a state of active multiplication; their descendants, with gradual 
changes of form, become the superficial layers, here to replace an equal quantity of worn-out cells (ing. 27).

In the course of this change of position, the cells may undergo an alteration; in the reptiles, birds, and mammals (fig. 28) they became cornified, first the margins, then the inner part of the cell, changing into horn ('keratin'). 'The nucleus remains for some time, but at length this vanishes,

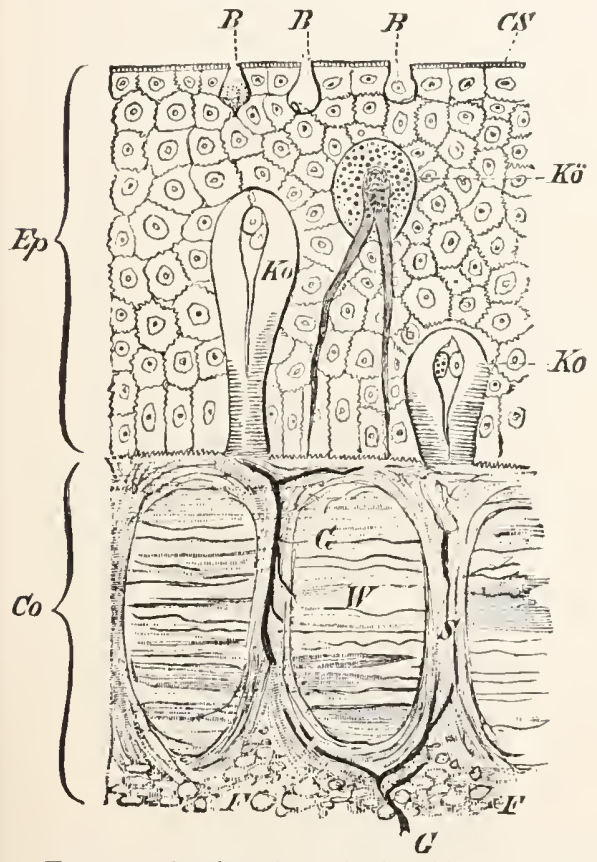

FIG. 27.-Section through the skin of Petromyzon planeri. Ep, the many-layered epithelium of the epidermis, including $B$, goblet cells; $K \ddot{o}$, granular cells; Ko, club-cells; Co, corium (with blood-vessels, $G$ ), consisting of bundles of fibrils running horizontally $(W)$ and vertically $(S)$ (from Wiedersheim).

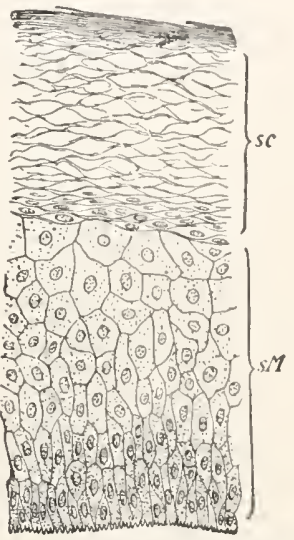

FIG. 28.-- Stratified epithelium of man. s. $M$, stratum Malpighi; sc, stratum corneum.

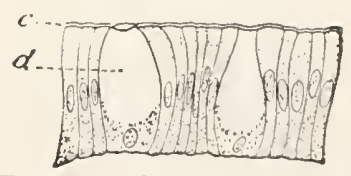

FIG. 29.-Single-layered epithelium of a snail. $c$, cuticle; $d$, goblet cells.

and then the cell becomes completely changed into a dead, horny scale. In the skin of the higher vertebrates the zones of the living protoplasmic, and the cornified cells, are sharply marked off from one another. In cross-section they are readily distinguished as the stratum corneum (sc) and the stratum Malpighi $(s M)$ of the skin (fig. 28 ). In the manylayered epithelia the cuticle has lost its importance, and it is either an inconspicuous boundary line or is entirely absent. 
Glandular Epithelium.-Glandular epithelium is distinguished physiologically from ordinary epithelium by the fact that it also produces secretions or excretions; anatomically it is recognizable by the presence of gland cells, which carry on the secretion and, to a greater or less extent, reveal their character by their structure. Characteristic glandular cells are, for example, the goblet cells; here the secretion, usually mucus, is collected as a clear mass in the interior of the cell, the cytoplasm being compressed into a thin external wall, reminding one of a goblet, containing the nucleus at its base (fig. $27, B, 29, d$ ). Other gland cells are granular cells, swollen bodies filled with secretory granules (fig. 27, $K \ddot{o}$ ). Naturally all grades between pavement and glandular epithelium occur. Commonly the latter name is only employed when the gland cells are especially numerous, thereby giving to the area a pre-eminently secretory character. This is especially the case with the structures which have the name of glands, among which we distinguish unicellular and multicellular glands.

Unicellular Glands.-Unicellular and multicellular glands increase the secretory surface by invagination. Invagination of a single cell

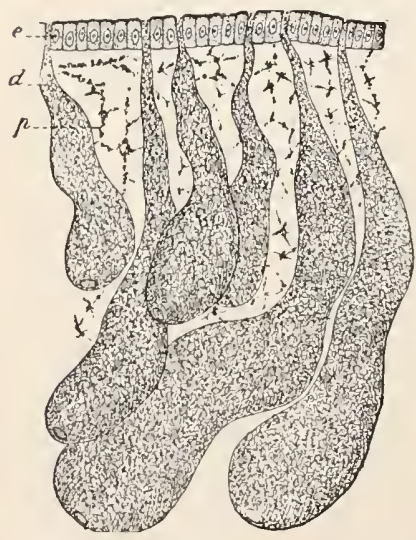

Fig. 30.-Unicellular glands from edge of the mantle of Helix pomatia. $e$, epithelium; $d$, unicellular glands; $p$, pigment cells. produces the unicellular glands which are chiefly found among the invertebrate animals (fig. 30); a gland cell here becomes so enormous that there is no room for it in the epithelium, but it is pushed into the deeper, the subepithelial layers, the nucleated cell body, distended by secretion, sending up a slender process, the duct, to the epithelial surface.

Multicellular Glands.-In the formation of multicellular glands a considerable area of glandular epithelium grows as a tube or duct from the surface down into the deeper tissues; this seldom remains simple; it usually branches and forms the compound glands, which may consist of hundreds or thousands of glandular sacs, all emptying into a common duct. Among the multicellular glands are to be distinguished tubular and acinous (racemose) forms. In tubular glands (fig. 3I) the simple or branched glandular pouches preserve the same tubular diameter from beginning to end; in the acinous glands (fig. 32), on the contrary, the blind end of the glandular pouch widens into a sac (acimus), largely composed of secretory cells, and related to the duct, as grapes to their stem. To the tubular glands belong the 
liver, kidney and sweat glands of man; to the acinous belong the salivary glands, not only of vertebrates, but also of arthropods and molluscs.

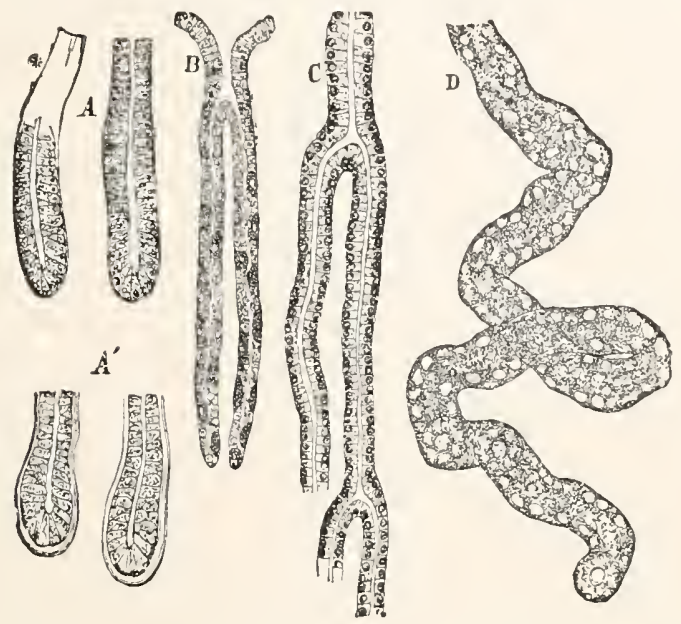

FIg. 31.-Tubular glands (after Toldt). A, glands of Lieberkühn from the human intestine; $A^{\prime}$, of the conjunctiva of the eye; $B$, of the cat's stomach: $C$, from the medullary pyramids of the dog's kidney; $D$, from the cortex of the rabbit's kidney.

Sexual Epithelium.-The sexual cells may be considered in connection with glandular epithelium. As the secretion of some glands must be expelled from the body, so the sexual cells are elements which must

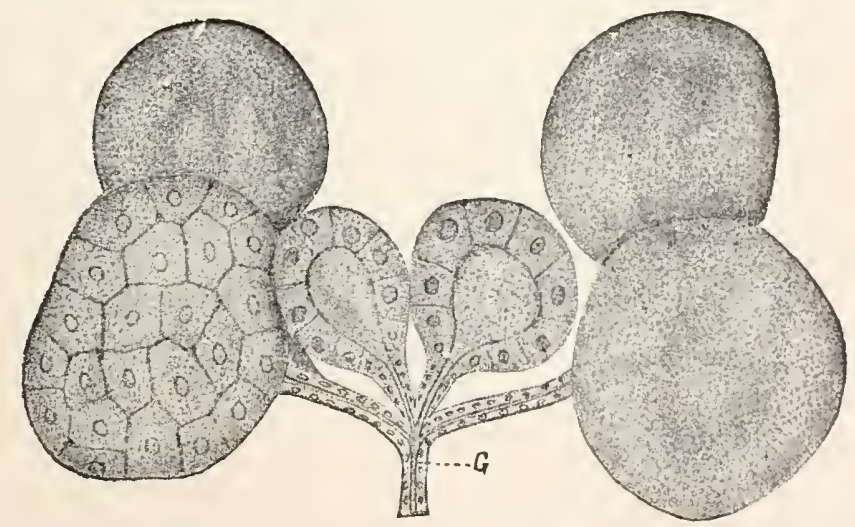

FIG. 32.-Acinous salivary gland of the aph'd Orthesia cataphracta (after List). In some acini the nuclei and boundaries of the cells are shown.

reach the exterior in order to perform their function. Just as the gland-cells are usually scattered among ordinary epithelial cells, so the sexual cells almost invariably lie imbedded in epithelium of the skin 
(fig. 33), of the gut, of the body cavity, or of parts cut off from this (fig. 34). This connection of the sexual cells with the epithelium has a deeper meaning since many organisms, particularly those of low structure, consist exclusively of epithelia and therefore must develop

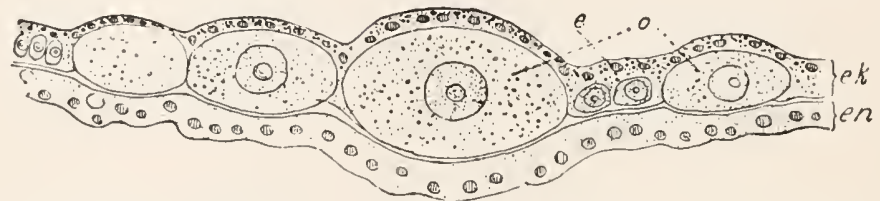

FIG. 33.-Germinal epittelium of a medusa. ek, ectoderm; en, entoderm; $o$, egg; $e$, epithelium.

their sexual products in epithelium. In other words, sexual and epithelial cells are the oldest elements of the animal body, and hence very early came into relation with one another.

Sexual epithelium (or germinal epithelium) like glandular epithelium has a tendency to grow inte the subepithelial tissues in the form of

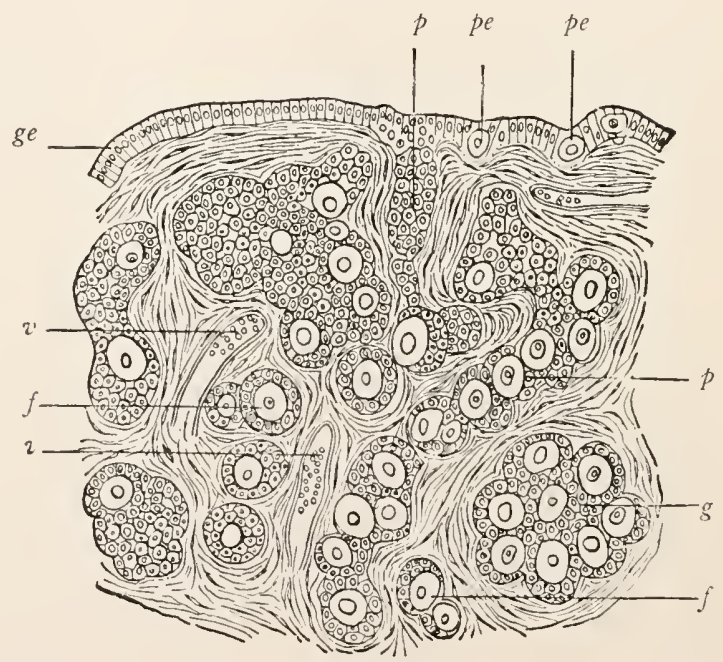

FIG. 34-Section through the ovary of a new-born child (after Waldeyer). ge, germinal epithelium; $p$, primitive egg in the germinal epithelium; $p$, egg-pouch; $g$, egg-nest constricted off from the pouchlike growth $(p) ; f$, single egg with follicle; '', blood-vessel.

isolated or branching tubes (figs. $34, p, 35$ ), and thus in many groups of animals the sexual organs resemble branched glands; hence one speaks as often of sexual glands as of sexual organs (fig. 34). The male and female cells, the specific elements of the germinal epithelia and of the 
sexual glands, differ from each other in the fact that the eggs are generally the largest, the spermatozoa the smallest, cells of the animal body.

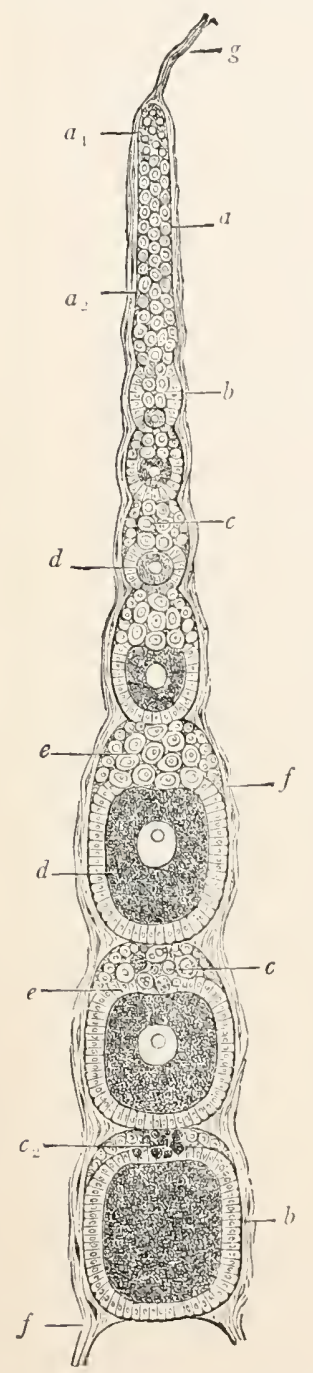

FIG. 35 .

Egg-cell.-The egg-cell or oocyle (fig. 36) as it is formed in the ovary varies in size according to the animal group: in case of the microscopic Gastrotricha it is less than $0.04 \mathrm{~mm}$., in man about $0.2 \mathrm{~mm}$., in the frog several millimetres, and in large birds often several inches: however, only the yolk of the bird's egg is the egg-cell, the white and the shell are structures formed outside of the ovary in the oviduct. These remarkable differences in size are caused less by the quantity of the peculiar cell-substance, protoplasm (primary yolk), than by the accumulation of deutoplasm (food or accessory yolk, also brietly called yolk). The deutoplasm is to nourish the embryo during derelopment, and hence consists of substances rich in fat and proteid, arranged in oil-drops, or in fine granules or polygonal bodies, the yolkgranules. Its quantity, and therefore the size of the egg, is in part proportional to the length of time which the egg is cut off from any other supply of nourishment. In general we find the largest eggs in the case of the highly organized oviparous animals, where a long development

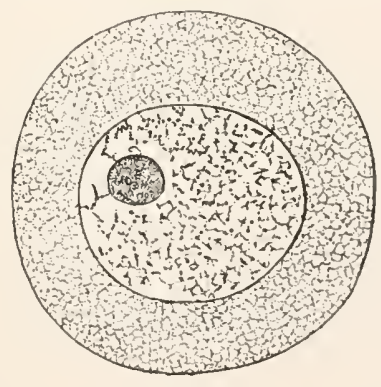

FiG. 36 .

FIG. 35.-Ovarian tube of an insect, Tanessa urtica. $a$, formative cell; $b$, follicular epithelium; $c$, nutritive cells; $d$, egg-cells: $f$, fibrous covering extencling out into the terminal fibres $(g)$ (after Waldeyer).

FIG. 36.-Immature egg-cell of Strongylocentrotus lividus.

inside of the shell is necessary to lay the foundation of the manifold organs. Besides the protoplasm and deutoplasm, a cell nucleus or 
germina! vesicie always occurs in the egg. Its contents are mainly the nuclear fluid, through which is distributed an achromatic network, and in addition the nucleolus (germinal spot).

The Spermatozoa, the morphological elements of the male reproductive product, are so small that their finer structure can be studied only with the strongest powers of the microscope (fig. 37, I and II). Easiest to rec-

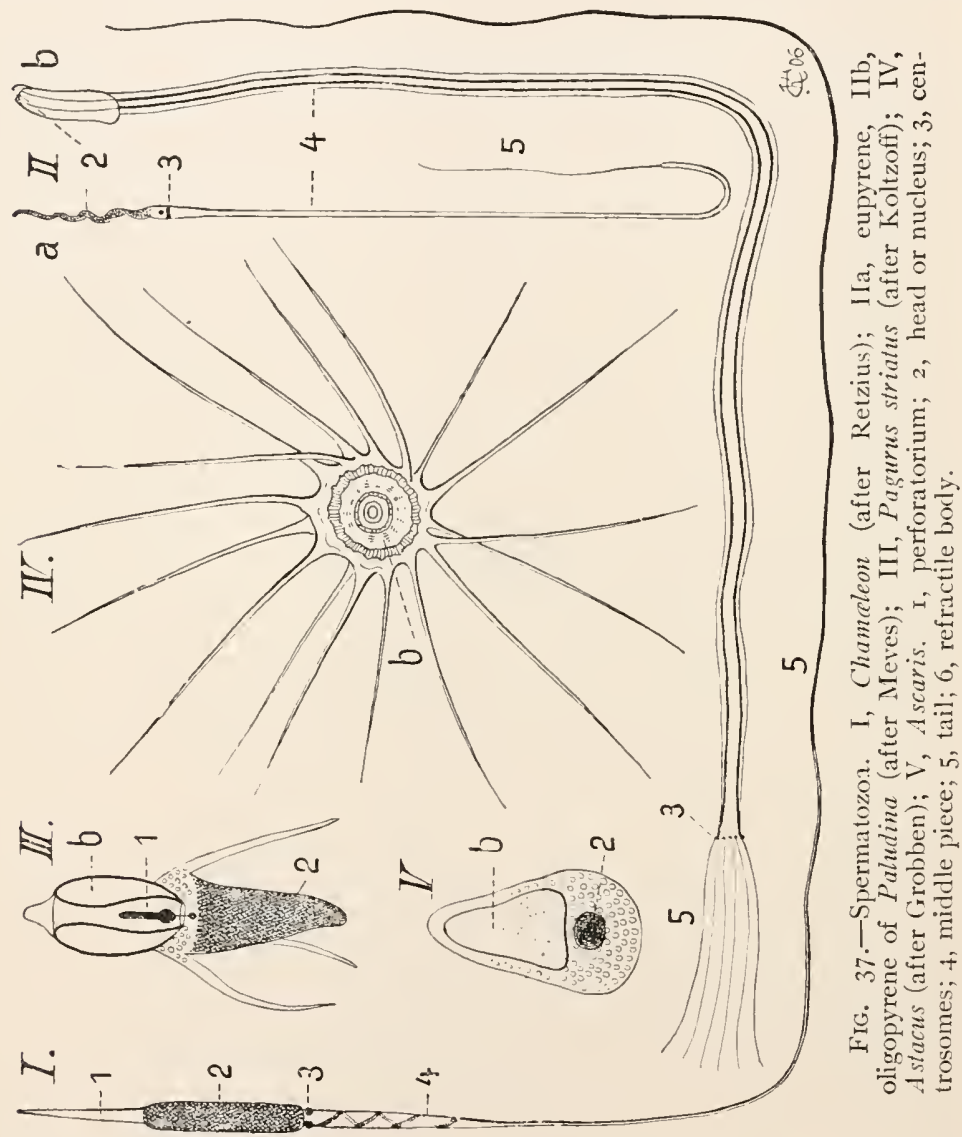

ognize is the head, which from its form--spherical, oval, sickle-shaped, etc.- often renders possible the specific determination of the spermatozoa. The hearl (2) is the closely compacted chromatic part of the nucleus, and hence colors very deeply in staining fluids. Often the head is continued in front as a sharp point, the perforatorium (I), which is apparently adapted to aid in the penetration of the egg in fertilization. Next comes 
an unstaining second part, the middle piece ( 4 ), and then the tail (5), a long flagellum, which causes the active motility of the ripe spermatozoon. Cytoplasm is present only in an extremely thin layer surrounding the nucleus. A centrosome (3) is nearly always present in the middle piece.

With the exceptions of the crustacea, nematodes and many myriapods, the spermatozoa are usually constructed after this type, often with complicated modifications. In the groups just mentioned the spermatozoa are large and immobile and contain a homogeneous body $(b)$ which is strongly refractive; its functions are uncertain. The spermatozoa of Ascaris ( $V$ ) are shaped like a sugar loaf, the broad, rounded end containing the nucleus. 'The spermatozoa of the decapod crustacea (III, IV ) have three or more stiff processes arising from the periphery of the cake-like or cylindrical body which contains the refractile body, and in this again a rod (III, I), possibly to be compared to the perforatorium. In other crustacea the spermatozoa are threarls, of ten of extreme length ( $7 \mathrm{~mm}$. in many ostracoda). It is noticeable that in some animals there are dimorphic spermatozoa. In Paludina viripara (the same is true of other Prosobranchs), there arise in the same individual hair-formed spermatozoa with cork-screw shaped heads (IIa) and others, worm-like and with a bundle of flagella at the hinder end (IIb). The first of these contains the normal chromatin mass (eupyreme spermatozoa); the others have very little chromatin (oligopyreme spermatozoa). In many spiders where a similar dimorphism occurs, the second type of spermatozoa is chromatin free (apyreme). The supposition that the dimorphism of the spermatozoa is connected with sex determination receives support in the study of the spermatozoa of some Hemiptera. Here half of the spermatozoa have one chromosome ('accessory chromosome') more than the other half. Eggs which are fertilized by the relatively oligopyreme spermatozoon probably produce male animals.

\section{Sensory Epithelium.-The last} modification of epithelium is sensory epithelium, characterized by the connection of certain of its cells, the sensory cells, with twigs of branching nerves which arise in the central nervous system. 'This connection may be of two kinds. In the first the cell is slender and filiform, the position of the nucleus being indicated

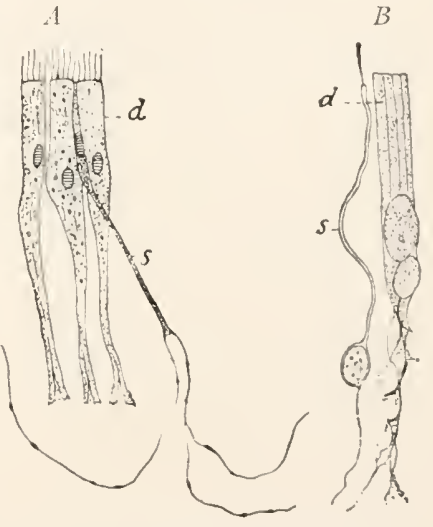

FIG.38.-Sensory epithelium. 1, of an Actinian; $B$, from the olfactory epithelium of man; $d$, supporting cells; $s$, sensory cells.

by a swelling. The peripheral end is concernerl with the reception of sensory stimuli, while the deeper end is continued directly into the nerve ends and correspondingly is branched into two or more extremely fine processes which take on the character of nerve fibrillix (fig. 38 ). In the second type the sensory nerve ends in a ganglion cell beneath the epithelium, sending processes into the latter, the ends of these being applied to 
the sensory cell, the connection being one of contact, not of continuity. In both the peripheral end of the cell bears appendages for sense perception; auditory and tactile hairs, stronger processes in olfactory and taste cells, conspicuous rods in visual cells. Almost without exception the sensory cells are part of the skin (ectoderm), or arise from it in development. This is true for sense organs like the eye and ear of vertebrates, which are separated from the skin by thick intermediate tissue, for in these the sensory epithelium (retina, crista acustica) is derived from the ectoderm. Recent studies seem to show that the taste organs in some forms are entodermal in origin.

Supporting Cells.-In sensory epithelium between the sensory cells are found still other epithelial cells, which are not connected with nerves, but have accessory functions: they serve as supports for the sensory cells; in the eyes they contain pigment; in the auditory organs they often bear the otoliths, etc. They have the general name of supporting cells.

\section{Supporting or Connective Tissues.}

From a histological point of view there is no greater difference than that between epithelium and connective tissue; the former belongs to the surface, the latter to the interior of the body; in the former the cells play the chief rôle, in the latter their importance is subordinate to the plasmic products, the intercellular substances which chiefly determine the character of the various kinds of connective tissue.

In spite of this contrast the connective tissues are genetically connected with epithelium. In embryos, which at first consist only of epithelia, the connection can be directly seen. The epithelia secrete a gelatinous substance from their deeper surfaces into which separate cells migrate. Thus arises the embryonic connective tissue, the mesenchyme (fig. Io8).

Function of Connective Tissue.-The primary function of connective tissue is to fill the spaces between the various organs in the interior of the body, thus connecting not only the single parts of the organs, but also the various organs themselves. In consequence of this the connective tissues contribute to the firmness of body, and are frequently employed in building up a skeleton. To accomplish this, substances which are usually firmer than protoplasm are formed on the surface of the cells, and, since they lie between the cells, these are called intercellular substances. In proportion as the intercellular substance increases in volume the cells themselves diminish and become inconspicuous connective-tissue corpuscles, or, as seldom happens, entirely disappear. Since in connective tissues, the intercellular substances are most important, it follows that the 
distinctions between the various kinds rest chiefly upon the differences of this intercellular substance. The following forms are clistinguished: (I) cellular connective tissue; (2) homogeneous connective tissue; (3) fibrous connective tissue; (4) cartilage; (5) bone.

Cellular Connective Tissue (which, strictly speaking, does not belong here, since it does not arise from mesenchyme lout directly from the metamorphosis of epithelium) shows the characteristics of the group least distinctly. It owes its name to the fact that the cells make up the chief

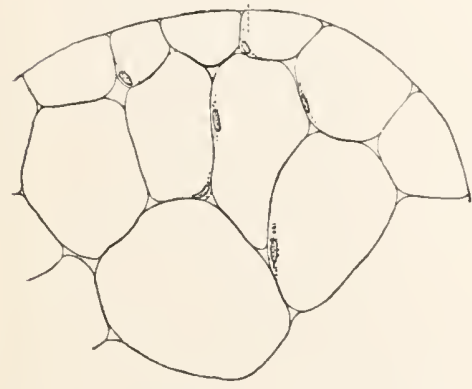

FIG. 39.-Cellular connective substance. Cross-section through the notochord of a newly hatched trout.

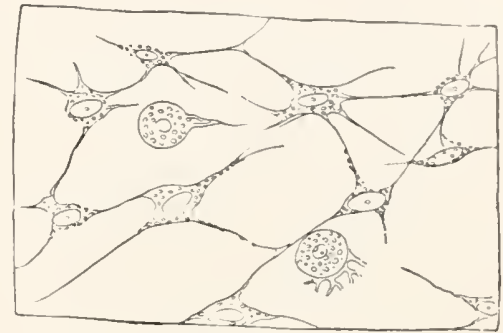

FIG. 40.--Homogeneous connective substance of Sycandra raphamus (after F. E. Schulze).

mass, while the cell-products are inconsiderable. The cells are large, vesicular bodies which are closely pressed together and are consequently polygonal (fig. 39). They have between them a firm but thin layer of intercellular substance.

Homogeneous Connective Tissue.-In homogeneous connective substance the intercellular substance (or matrix) is usually present in considerable quantity as a transparent mass, sometimes soft like jelly, often firmer (fig. 40). The cells lying in it are either spherical or send branching processes into the matrix. Such processes may unite to form meshes which, like a pseudopodial network, unite cell to cell. Frequently the matrix contains, in addition, isolated firm fibres or threads, which, on account of their physical characteristics, are called elastic fibres, and consist of a substance (elastin) exceedingly resistant to all reagents. Finally, in the matrix there may develop the finer connective-tissue filsrils characteristic of the next group; they may become so increased in number as to determine the character of the tissue.

Fibrous Connective Tissue is characterized by the rich supply of connective-tissue fibrillæ; these are fibres of extraordinary fineness, lying in a homogeneous matrix, which is the less evident the richer it is in 
fibres. The fibres may either cross in all directions, or may run essentially parallel and in a definite direction. Between them are found the rounded, spindle-shaped or branched connective-tissue corpuscles (fig. 4I). It is characteristic of vertebrates that the fibres are grouped into bundles, each bundle generally surrounded by connective-tissue corpuscles,

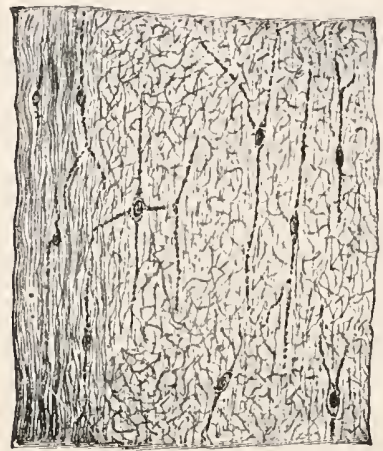

FIG. 4I.-Fibrous connective tissue of an Actinian.

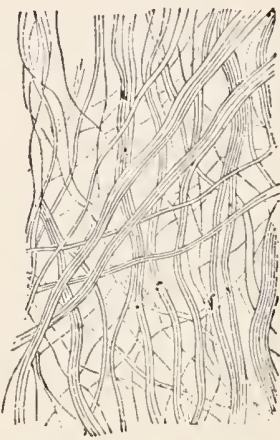

FIG. 42.-Areolar fibrous connective tissue (after Gegenbaur).

metamorphosed into flat cells. The bundles, loosely interwoven, run in all directions (areolar connective tissue, fig. 42), or they may be parallel, forming a compact mass of fibres (tendinous tissue fig. 43). The fibrils of the fibrous connective tissue of the vertebrates have the peculiarity not met with elsewhere, they are composed of glutin, and upon boiling become gelatine or glue.

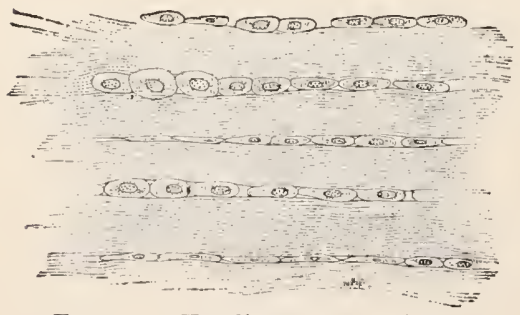

FIG. 43--Tendinous tissue (after Gegenbaur).

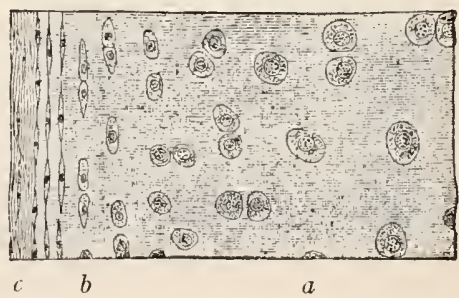

FIG. 44.-Cartilage (after Gegenbaur). $c$, perichondrium; $b$, transition into typical cartilage $(a)$.

Elastic Tissue.-In all fibrous connective tissue there may appear, as a further constituent, elastic fibres; they may indeed supplant the ordinary connective-tissue fibrils and become the predominant element of the tissue, which is then called elastic tissue. 
Cartilage.-Cartilage and bone are likewise tissues which find their characteristic development only in the vertebrates. In appearance cartilage is similar to the homogeneous connective tissue of many invertebrates; the matrix is homogeneous and, at first glance, appears structureless (fig. +4 ), but, under the action of certain reagents, assumes a fibrous condition. This, as well as the fact that the cartilage grows through changes of the perichondrium-a thin, fibrillar membrane covering its surfacemakes it more evident that it is homogeneously fibrillar; and it is thereby distinguished from homogeneous connective tissue since it is not, like the latter, a lower but a higher stage of tissue formation. The matrix of cartilage (chondrin) by cooking produces a kind of glue which differs from true or glutin glue in that it is precipitated by acetic acid. The cartilage cells lie in the matrix united in groups and nests, a mode of grouping pointing to their origin, since each group has arisen from a single mother-cell by successive divisions. In cartilage also, elastic fibres are found; if present in great number, these change the bluish, shiny, $h$ valine cartilage into the yellow-colored elastic cartilage.

The 'head cartilages' of the cephalopoda differ from vertebrate cartilage in that the cartilage corpuscles have branched processes.

Bone is the most complicated structure in the series of connective tissues. It consists of a matrix (ossein), closely allied to glutin, so intimately combined with inorganic constituents that it appears under the microscope as a homogeneous mass. The proportion of organic and inorganic substances varies according to the age and species of animal: in man there is $65 \%$ inorganic to $35 \%$ organic substance; in the turtle, $63^{\%} \%$ to $37^{\circ} \%$ Of the inorganic constituents, the most important is calcic phosphate, $8_{4} \mathrm{C}$; in smaller quantities, combinations of fluorine, chlorine, carbonic acid and magnesia. In compact bone the matrix is composed of the bone lamella (fig. 45), whose arrangement is determined by the surfaces present in and on the bone. In a hollow bone (like that of the upper arm) there is an outer surface to which a fibrous membrane, the periosteum, is closely applied; the presence of the marrow-cavity necessitates a second surface. Finally, the mass of the bone is permeated by the Haversian canals, which run chiefly in a longitudinal direction, united by cross or oblique canals, and serve for the passage of blood-ressels. Since the bone lamellix 
arrange themselves parallel to the surfaces mentioned, two systems may be distinguished in cross-section, the fundamental lameltce and the Haversian lamella. The former are arranged parallel to the surface of the periosteum and of the marrow-cavity and form a mantle of concentric layers around the marrow-cavity. Into this groundwork the Haversian canals with their lamellæ enter, destroying and superseding the fundamental lamellæ coming in their way. The Haversian lamellæ are concentrically arranged around the lumen of the Haversian canals just as the fundamental lamellie are around the marrowcavity and beneath the periosteum.

Formation of Bone.-The stratification of bone is caused by its mode of origin. Where the bone borders upon the Haversian canals, the marrow-cavity, and the periosteum, there is transiently or permanently an epithelial-like layer of cells, osteoblasts, which secrete the bone-substance on their surface, which, as with all similar conditions, gives the substance a stratification. Certain cells in the matrix participate in this secretion, and here give rise to the bone-corpuscles, which are distinguished from the cartilage-cells by their numerous processes ramifying through the matrix. The processes of a bone-corpuscle branch and unite with the neighboring cells through fusion of the processes, an arrangement most beautifully seen in dried bone, because here the cavities and the canals of the matrix are filled with air. In spongy bone the structure is simplified since the Haversian canals with their lamelle, and often the stratification of the ground lamellie, are lacking. Special modifications of bone are found in the substance of fish scales and in the dentine or ivory of teeth.

Blood and Lymph, here treated in connection with the connective tissues, are in reality not tissues at all, but nutritive fluids. Two kinds

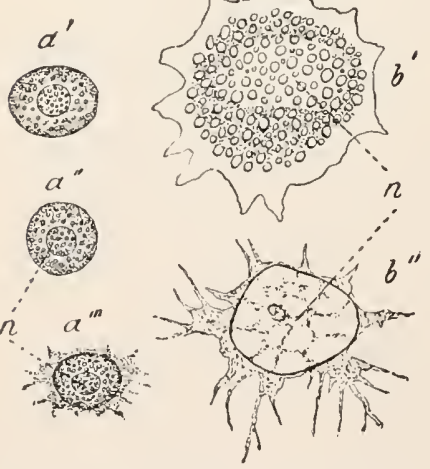

FIG. 46 - White blood-corpuscles $a$, of man; $b$, of crab $(n$, the nucleus). of nutritive fluids occur in the rertebrates, red blood and the colorless, weakly opalescent, or cloudy white lymph. The blood of man and other vertebrates consists of a fluid and the organized constituents. The fluid or blood-plasma is specially rich in proteids; after the removal of the blood from the blood-vessels a part of these separate by coagulation and form the blood-clot, made up of fibrin, leaving a fluid poor in proteids, the blood-serum. The organized constituents, the blood-cells, are distinguished as red and white blood-corpuscles. The latter, the leucocytes, are present in smaller numbers and have great similarity to the Amœba; they are particles of protoplasm, contain a nucleus, devour foreign bodies (for example, carmine granules injected into the blood), and move in the 'amoboid' manner by putting out pseudopodia (fig. 46). 
Red Blood-corpuscles.- In the mature condition the red bloorl-corpuscles of vertebrates (fig. 47 ) are circular or oval discs, which by external influences (e.g., pressure) may temporarily be bent or otherwise modified in form, but cannot actively change their shape, because they no longer consist of protoplasm. Embryologically they arise from true, nucleated, protoplasmic cells; gradually the protoplasmic cell-body changes completely into a plasmic product, the stroma of the blood-corpuscle. If the nucleus be retained in this metamorphosis, there is a slight swelling in the centre of the disc (fig. $47, d^{\prime}$ ); if, however, the nucleus degenerate, the bilateral convexity is replaced by a shallow concavity. In the latter case, one has, in reality, no right to speak of blood-cells, since the characteristic constituents of the cell-nucleus and protoplasm-have disap)peared. Systematically the red blood-corpuscles are of interest, since nonnucleate forms are found only in the mammals (fig. $47, a, b$ ), nucleated ones in

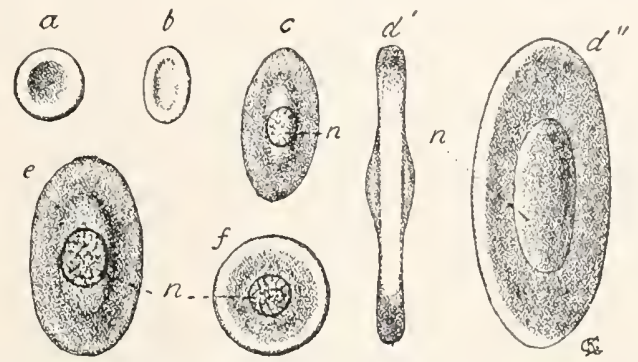

Fig. 47--Red blood-corpuscles. $a$, of man; $b$, of the camel; $c$, of the adder: $d^{\prime}$, of Proteus (seen from the edge); $d^{\prime \prime}$, surface view; $e$, of a ray; $f$, of Petromyzon; $n$, nucleus (all the blood-corpuscles are magnified 700 times, except $d$, which is magnified 350 times).

all the other vertebrates $(c, d)$. The mammals also have circular, the other vertebrates oval, discs. To this, however, exceptions occur, since among the mammals the Tylopoda (camel, llama) have oval, the cyclostomes have circular blood-corpuscles. Recent investigations tend to show that the corpuscles, at least in mammals, have a hat shape while in the living blood-vessels and that they become disc-like when the normal conditions are interfered with.

Hæmoglobin.- The red blood-corpuscles cause the color of the blood, and are the agents of one of its most important functions, the interchange of gases; both are connected with the fact that the stroma contains the coloring matter, hemoglobin, of the blood. Hæmoglobin is one of the few crystallizable proteids and is remarkable for the presence of a small, though extremely important, quantity of iron, and also for its affinity for oxygen. Hæmoglobin containing oxygen, oxy-hamoglobin, causes the carmine-like color of the so-called arterial blood; oxygen-free, 'reduced' hæmoglobin causes the dark bluish-red color of venous blood.

Lymph is distinguished from blood by the entire lack of red blood-corpuscles and the slight coagulability of its plasma. Lymph is accordingly a proteidcontaining fluid with leucocytes, which are here called lymph-corpuscles.

In the majority of invertebrates there is only one kind of nutritive fluid, and not even this in every class; the fluid is called blood, although it is usually colorless. Where color is present, it is generally, if not always, a yellowish-red or an intense red; this may, as in the vertebrates, be caused by himoglobin (some molluscs, annelids, and insects). Often other coloring matter occurs instead of hæmoglobin: in the cuttlefish, many snails, and in the lobster and Limulus, the 
oxygen is taken up by the bluish hamocyanin, which contains a trace of copper: in the Sipunculids by hamoerythrin, etc. The blood-plasma, as a rule, is the seat of the color (Chironomus, Hirudinea, earthworms, and most other annelids); only exceptionally do colored blood-corpuscles occur, as in the case of Arca, Solcn and some other molluscs, and also in Phoronis. Colored elements containing hæmoglobin, identical with blood-corpuscles, are found besides in the coelomic fluid of many annelids, and in the ambulacral vessels of some echinoderms. Most widely distributed in the invertebrates are the leucocytes, distinguished by their active amœboid movements; still even these may be absent, and then the blood is a fluid without any organized corpuscles.

\section{Muscular Tissue.}

Muscular Tissue is the agent of active movements in the animal body. Since active mobility occurs in protoplasm, it is important to notice the differences between the two kinds of movement. The distinctions lie in the direction and in the intensity of the movement. A mass of protoplasm has the capacity to move hither and thither in all directions, because in it there is a high degree of mobility between the smallest particles. Muscles and hence their separate elements, the muscle-fibres and muscle-fibrils, on the contrary, can shorten only by correspondingly increasing in diameter (fig. 48); they can therefore accomplish motion only in a definite direction, that of the axis of the muscle. The musclesubstance consequently is more limited in its movement than is protoplasm, but on the other hand it has the adrantages of greater energy and greater rapidity. One

FIG. 48. Four striped muscle fibres in resting and contracted condition (after Rollet). is able to decide with considerable accuracy, from the intensity and rapidity, whether in a given case a movement has been brought about by the agency of protoplasm or by muscle-substance.

Structure of Muscle Substance.-These physiological considerations show that protoplasm and the contractile substance are morphologically different, and that therefore one must distinguish sharply between formative cells, or muscle-corpuscles, and the product of these cells, the contractie substance, just as in the case of connective tissue, between the corpuscles and fibrils. There are recognized two kinds of muscle-substance, the homogeneous, or smooth, and the cross-striated. Since the former looks very similar to non-granular protoplasm, the boundary-line between it and the muscle-corpuscle is more difficult to recognize than in the case of the latter, which in its minute structure is quite different in appearance from protoplasm. In cross-striated muscles the contractile portion consists of two substances regularly alternating with one another in the direction of the contraction of the muscle, of which the one is doubly, the other singly, refractive (figs. 25, 48, 5 I). 
Smooth and Cross-striated Muscle Fibres.-The smooth musclesubstance represents a lower stage than the cross-striated, since it chiefly occurs in the less highly organized and more inactive animals. Interesting in this respect is the fact that in the two stages of development of the same animal the simple and inert polyp has smooth muscles, while the more highly organized and actively motile medusa has cross-striated muscles (fig. 49). The difference in their action has led in the vertebrates to a peculiar distribution of the muscle-substance, the smooth musculature being chiefly in internal organs, which are not under control of the will (involuntary muscles), while the musculature of the body, subject

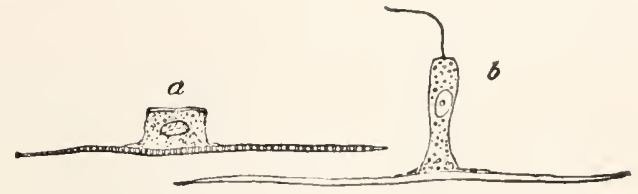

FIG. 49.-Epithelial muscle-cells. $a$, of a medusa; $b$, of an actinian.

to the will and hence demanding more rapid action, is cross-striated (voluntary muscles). We must not conclude that the difference between smooth and cross-striated musculature coincides with the distinction between visceral and body musculature; it should be noticed that the borly musculature of all molluscs is smooth, the visceral as well as the body muscles of many insects and crustacea, and the muscles of the heart of vertebrates are cross-striated.

It was pointed out above, in connection with epithelia and connective tissue, that these tissues differed fundamentally. 'This contrast has its bearing in dealing with the muscles, for both epithelial and mesenchymatous cells may form contractile substances and therefore there are two genetically different kinds of muscles, the epithelial and the mesenchymatous (contractile fibre-cell). Both kinds of muscle-cells can a priori form smooth as well as cross-striated muscle-substance; but the collection of connective (mesenchymatous) tissue around inner organs has caused most contractile fibre-cells to be smooth, while most of the epithelial muscle-cells are cross-striated.

Epithelial muscle-cells are cells of which one end extends to the surface of the body or the surface of an internal cavity (body cavity, lumen of the gut, etc.), and may here have a cuticle, cilia, or flagella, while at the opposite end it has secreted contractile substance in the form of musclefibrils (fig. 49). They combine the double function of epithelial and muscle cells. 
Contractile fibre-cells, on the other hand, are connective-tissue cells, which usually have surrounded themselves with a layer of contractile substance; corresponding to their origin, they have the form of connectivetissue cells, and are spindle-formed or branched; the branches arising from the ends of the cells (fig. 50). The similarity of form renders the distinction between ordinary connective-tissue cells and fibre-cells difficult; if the contractile layer on the surface be slightly developed, the distinction

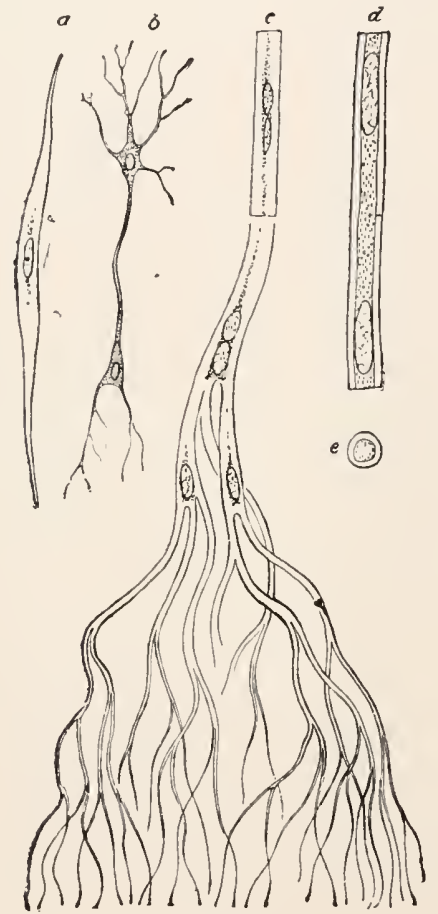

FIG. 50.

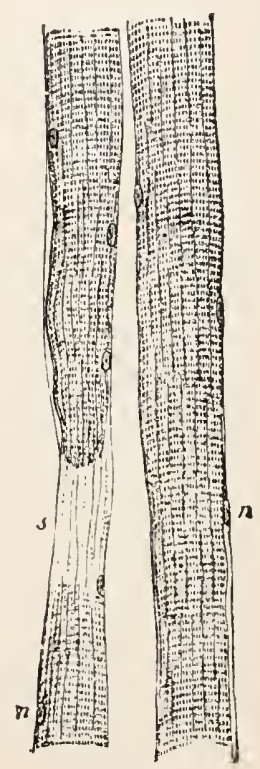

FIG. 5I.

FIG. 5o.-Contractile fibre-cells. $a$, of man; $b-e$, of Berö (a Ctenophore); $b$, young fibres; $c$, branched ends; $d$, middle portion of a fibre; $e$, cross-section.

FIG. 5I.-Cross-striated primary bundle (after Gegenbaur). n, nuclei; $s$, a point where the sarcolemma is plainly shown on account of the tearing of the fibres.

is impossible. To recognize the character of the elements, therefore, we must choose well-defined examples, in which the nucleated mass, the 'axial substance,' is sharply marked off from the muscle-mass, the 'cortical layer' (fig. 50, $c, d, e$ ). The regular arrangement of the epithelial cells side by side, gives the muscle fibres arising from them a parallel arrangement, so that a layer is formed, which becomes folded when much muscle 
substance is formed in a limited space (see figs. 193, 240, 24 I and their legends).

In vertebrates and arthropods the contractile fibre-cells occur in the vegetative organs as elements of the 'organic musculature'; on the other hand, we find here the epithelial musculature in the cross-striated primary bundles, separated from the epithelium, and only developmentally referable to the epithelium of the body cavity (fig. 5I). A primary bundle is cylindrical, bounded externally by a structureless envelope, the sarcolemma. Its contents consist of fine fibrils, which, closely parallel to one another and pressed closely together, run from one end of the mass to the other. Each fibril is formed of singly and doubly refractive parts, which alternate with one another in more or less complicated arrangement. Since now the doubly refracting parts of the fibrils within a bundle lie at about the same level, there is caused a cross-striation extending through the whole bundle (fig. 5x). Finally, scattered here and there between the muscle-fibrils are the muscle-corpuscles, spindle-shaped protoplasmic bodies with a nucleus, the remnants of the cells which have formed the musculature. Although the primitive bundles retain no epithelial characters, their epithelial nature is shown in their origin from the epithelium of the primitive body cavity (from that part of its wall known as the protovertebræ).

\section{Nervous Tissue.}

Function of Nervous Tissue.-As muscular tissue causes motion, so nervous tissue serves for the transmission of stimuli. It conveys the stimulations of the sense-organs at the periphery to the central nervous system, and here brings about perception (centripetal nerve tracts); further, it transmits the voluntary and reflex impulses to the periphery, particularly to the musculature and glands (centrifugal nerve tracts). By the nervous system, finally, the stimuli arising in various places are co-ordinated, thus furnishing the elements for that which we call independent psychic activity.

Elements of Nervous Tissue.-The agent of the transmission of stimuli is undoubtedly a specific nerve-substance different from protoplasm. The prevailing view is as follows: The elements of the nervous system are divided into ganglion cells and nerve-fibres, but it must be remembered that these are not independent of each other, but that the fil)res are enormously elongated processes of the ganglion cells. In both ganglion cells and nerve fibres there are extremely fine fibrillix which are connected with each other and are to be compared to the wires of a 


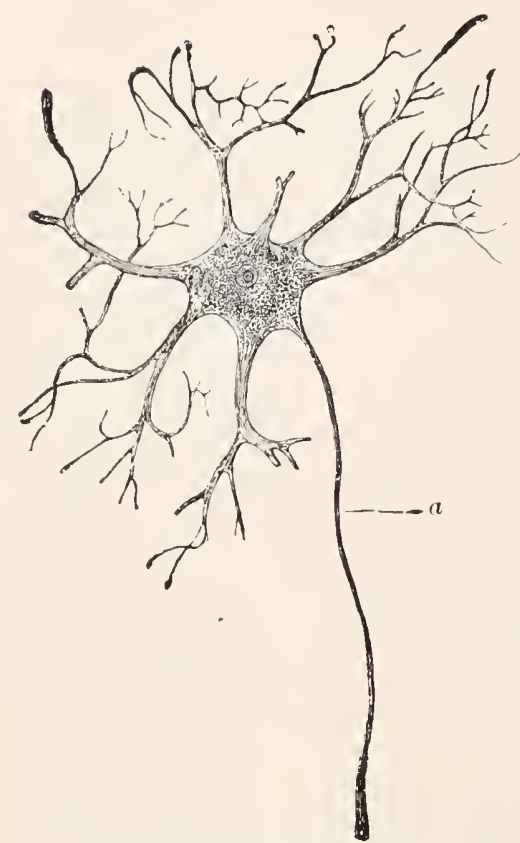

FIG. 52.-Multipolar gang'ion cell of man (after Gegenbaur). a, axon.

telegraph system. These are to be considered as the specific element of the nervous system.

In the vertebrates the ganglion cells vary greatly in size; besides small elements there are large cells, only exceeded by the eggs in size, which correspondingly have large nuclei recalling the germinal vesicles. Unipolar, bipolar, and multipolar ganglion cells are recognized, the differences depending upon the number of processes (nerve-fibres) which arise. In multipolar cells the number is large (fig. 52) and they are of two kinds, dendrites and axons or neurites. Dendrites are so called because they branch again and again, not far from their origin from the cell. The axons (of which there is usually but one to a ganglion cell) can extend a long distance without giving off branches, except here and there side twigs (coliaterals) which arise at right angles to the main fibre; they of ten continue into peripheral nerves. They branch at their tips (telodendra) so the morphological distinction from dendrites lies in the greater distance of the region of branching from the body of the ganglion cell. In bipolar ganglion cells both processes appear as neurites, but if one is to be regarded as a dendrite with its branching at some distance from the cell, the definite physiological distinction has to be invoked that the neurite carries the im-

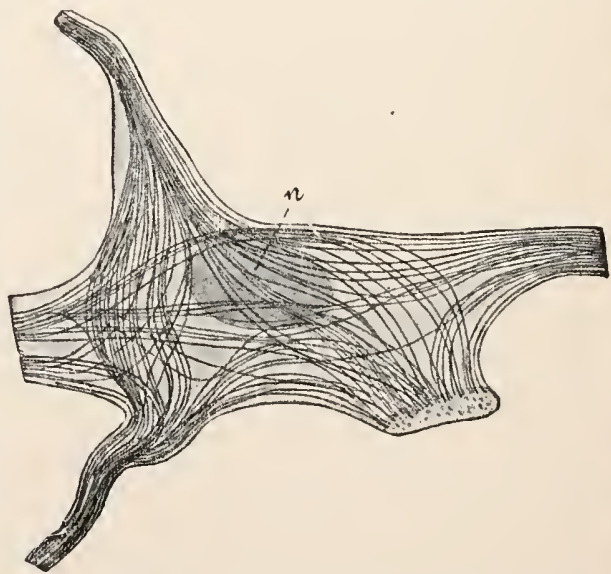

FIG. 53.- Motor ganglion cell from the thoracic region of the spinal cord of a dog (after Bethe). $n$, nucleus. 
pulse from the cell; the dendrite is the afferent tract. The unipolar nerve cell is also to be regarded as bipolar, its processes leaving the cell from a common point, running together a short distance and then branching like the letter $T$. This conception is intelligible if the fibrilla be regarded as the carriers of stimuli, each process consisting of a varying number of fibrilla. These enter the cell, where they cross similar fibril ix coming from other processes, and then are distributed to the various neurites and dendrites. The branching of a fibre is thus a gradual distribution of the constituent fibrilla, and the cell is the place where the exchange of fibrilla between the different processes occurs. Thus it is immaterial whether two bundles of fibrilla leave the cell at different points or whether they are united for a distance like a cable.

In the central nervous system of vertebrates the most minute elements are the nerve fibrillæ, distinguished from muscle fibrilla by the absence of cross-striation; from connective-tissue fibrilla by the ease with which they are injured; in preserved material they frequently swell and show varicosities (fig. 54). Many fibrillæe united in a bundle form a nerve-fibre (fig. 55, A) which is called a gray nerve-fibre in

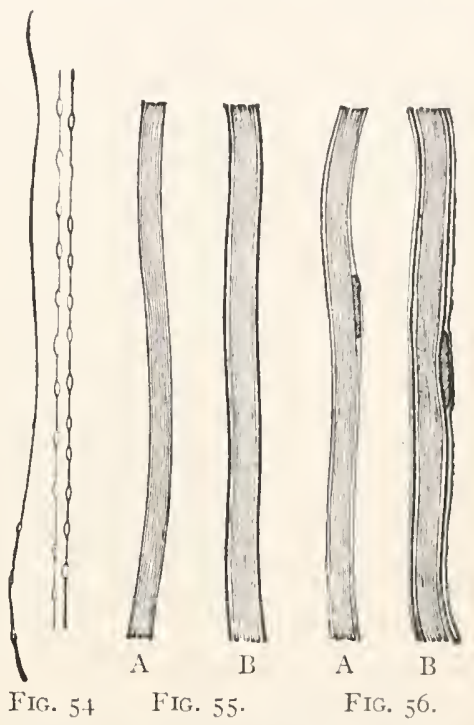

FIG. 54.-Nerve fibrillæ with varicosities (from Hatschek).

FIG. 55.- $A$, Non-medullated; $B$, nerve-fibres.

FIg. 56.- $A$, Non-medullaterl; $B$, medullated nerve-fibres, with sheath of Schwann (from Hatschek). distinction from the white or medullated fibres. In the latter the fibre or axis-cylinder is surrounded by a medullary sheath (fig. 55, B) composed of myelin, a strongly refractive fat-like substance, which appears to act as an insulator.

Both medullated and non-medullated fibres can be enclosed in a sheath of Schwann. This is a feature of the peripheral nervous system and is lacking in brain and spinal cord. It is a delicate envelope with nuclei here and there (fig. 56). At times it has constrictions which cut through the medullary sheath to the axis-cylinder (constrictions of Ranvier).

Multipolar and bipolar ganglion cells also occur in the invertebrates, most commonly in the colenterates (fig. 57), more rarely in worms, arthropods, and molluses, and then chiefly in the peripheral nervous 
system. In the ganglia (the nervous centres of the last three groups) the ganglion cell usually gives rise to a single strong process, which, however, is richly provided with lateral branches or dendrites (fig. 77). The medullary sheath and sheath of Schwann are usually absent in invertebrates even in the peripheral nerves. On the other hand, the true conducting elements, the nerve fibrillie, have been seen in invertebrate nervefibres, and have been followed into the ganglion cell in which the afferent and efferent fibrille are united in a lattice-like manner.

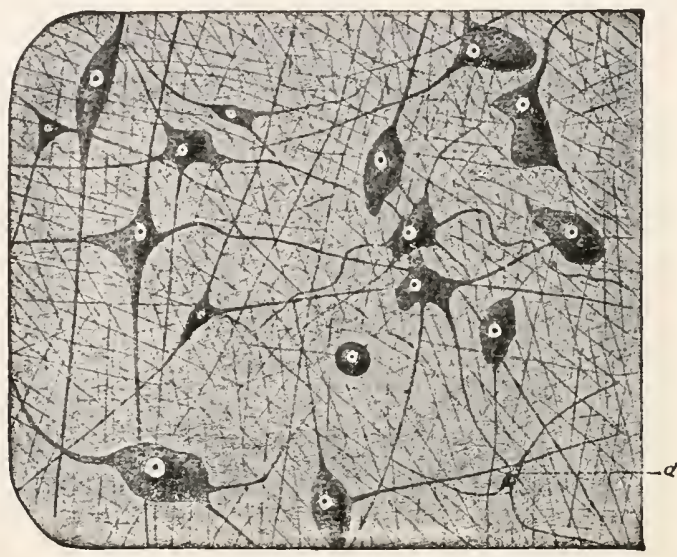

FIG. 57--Ganglion cells of an actinian.

A ganglion cell with its dendrites and neurites (the latter in large animals may be several feet long, since they extend from the central nervous system to the muscles) form a physiological unit, called a neurone. The advocates of the 'neurone theory' maintain that the processes of two neurones do not anastomose and that there is no continuity between them; they approach each other so closely that the nervous impulse may jump from one to another like an electric spark. The opponents of the theory assert that there is real continuity as seems to be certainly shown with the giant ganglion cells of the nematodes. A second disputed point is that the neurone theory holds that all processes, even the longest neurite, grows from the ganglion cell and throughout its length is a product of it. The opponents claim that the neurites which compose the peripheral nerve fibres are formed by special nerve-forming cells (neuroblasts) and these contribute to the length of the neurite starting from a ganglion cell. In many invertebrates (Hirudinei) such neuroblasts persist in the course of the peripheral nerves. Also the peripheral system of invertebrates can consist of a network of anastomosing neuroblasts (gang ionic plexus of medusæ and chætognaths). When we recall that the primitive cœlenterates (hydroid polyps) lack a central nervous organ and the whole nervous system is only a plexus of similar nerve cells, we may suppose that from this primitive condition the more highly developed forms of nervous systems have developed through division of labor, cells in a suitable position giving rise to the central system, while the remaining cells of the plexus form the conducting tracts. Recent experiments in growing nerve cells in nutrient fluids seem to support the view that the axons are exclusively products of the cell. 
SCMMARY OF HISTOLOGICAL FACTS.

Cells.-I. The most important morphological element of all tissues is the cell.

2. The cell is a mass of protoplasm which contains one or several nuclei (uninucleated, multinucleated cells).

3. The nucleus probably determines the specific character of the cell, since it influences its functions; accordingly it is alsc the bearer of heredity.

4. Cells and nuclei increase exclusively by division or budding, the cells of the Metazoa nearly always by mitosis, those of the Prctozoa frequently by direct division.

Tissues.-5. Tissues are complexes of numerous similar histologically differentiated cells.

6. Histclogical differentiation rests in part upon the fact that the cells assume a definite form and arrangement, in part upon the formation of plasmic products, which determine the character of the tissue (muscle-, nerve- and connective-tissue fibrils).

Classification of Tissues.- 7 . According to function and structure (I) epithelia, (2) connective tissue, (3) muscular tissue, (4) nervous tissue are distinguished.

8. The physiological character of epithelia is due to the fact that they cover the surfaces of the body; morphologically they consist of closely appressed cells united by a cementing substance.

9. According to their further functional character epithelia are divided into glandular epithelia (unicellular and multicellular glands), sensory, germinal, and protective epithelia.

I0. According to the structure are distinguished simple (cubical, cylindrical, squamous epithelia) and stratified epithelia, ciliated and flagellated epithelia, epithelia with or without cuticle.

II. The physiological characteristic of the connective tissues is that they fill spaces between other tissues in the body.

12. The morphological distinction depends upon the presence of the intercellular substance.

I3. According to the quantity and the structure of the intercellular substance the connective tissues are divided into (I) cellular (scanty intercellular substance); (2) homogeneous: (3) fibrous connective tissue; (4) cartilage; (5) bone.

I4. The physiological character of muscular tissue is its increased capacity for contraction.

15. The morphological characteristic is the fact that the cells have secreted muscle-substance. 
I6. According to the nature of the muscle-substance are distinguished smooth and cross-striated muscle-fibres.

I7. According to the character and origin of the cells (muscle-corpuscles) the muscles are divided into epithelial (epithelial muscle-cells, primary bundles) and connective-tissue muscle-cells (contractile fibrecells).

18. The physiological distinction of nervous tissue rests upon the transmission of sensory stimuli and voluntary impulses, and upon the co-ordination of these into unified psychic activity.

I 9. The conduction takes place by means of nerve-fibres (non-medullated and medullated fibrils and bundles of fibrils); the co-ordination of stimuli by means of ganglion-cells (bipolar, multipolar ganglion-cells).

20. Blood and $l y m p h$ are proteid-containing fluids; rarely without cells, they may contain only colorless amoeboid cells (white blood-corpuscles, leucocytes), or in addition to these also red blood-corpuscles.

$2 \mathrm{I}$. Red blood-corpuscles occur in vertebrates and cause the redness of the blood; they are absent in most invertebrates.

22. When invertebrates have colored blood (red, yellow, green), this is usually due to the color of the blood-plasma.

23. The red blood-corpuscles are non-nucleated in mammals, nucleated in all the other vertebrates.

\section{The Combination of Tissues into Organs.}

An Organ Defined.-Organs are formed from the tissues. An organ is a tissue complex, marked off from the other tissues, which has taken on a definite form for carrying on a special function. Thus a single muscle is an organ which consists of a certain amount of muscular tissue; with scalpel and scissors it can be removed as a connected whole from its environment and can still accomplish a definite movement.

Principal and Accessory Tissues.-In each organ there is a tissue which determines its function, and therefore its physiological character. This is called the principal tissue, for there may be accessory tissues present, which merely support or render possible the action of the principal tissue. In the muscle of the vertebrates we find, besides the musclefibres, connective tissue which unites the bundles of muscle; blood-vessels which provide nourishment; finally, nerves by which the muscles are aroused to action. In the human liver also, besides the functionally most important part (the liver-cells), blood-vessels, nervous and connective tissues occur. These accessory tissues are usually found only in the highly developed organs; in the case of the lower animals they may be 
absent; thus the digestive tract of coelenterates is only an epithelium; their nervous system consists merely of a plexus of nerve-fibres and ganglion-cells.

Effect of Use and Disuse.-It is of the greatest importance for the permanency of an organ that it be constantly in function. Living sulsstance is distinguished from the non-living by the fact that, if it be destroyed by use, it is immediately replaced, often by more than sufficient to make good the loss. Functioning tissues and organs under farorable conditions increase in volume; on the other hand, functionless parts undergo a gradual decrease, which finally leads to their disappearance. To the extent that functioning tissues grow at the expense of those not used there can be a 'struggle of the parts within the organism' (Roux). There is also the same struggle between the structural elements in one and the same tissue as is well shown in certain bones like the femur and tibia. These retain only those bony parts, the outer tube and the bony bars at the ends, which are necessary to support the body, all other parts being absorbed. These bony bars are in the position which can be mathematically demonstrated to be necessary to meet the strains. If the line of strain be altered, as when a leg is broken and badly set, the bars are altèred in position to meet the new strains.

Change of Function of Organs.-The two factors mentioned, that the permanence of the tissues depends upon continued use, and that usually several tissues enter into the structure of an organ, are important for the understanding of the principle of change of function which plays a prominent rôle in the metamorphosis of animal form. It may happen that an organ is brought under changed conditions and no longer has an opportunity to function as before. In that case the functioning tissue, from lack of use, gradually degenerates, but the organ may persist by means of its accessory tissues if the new conditions make it possible for one of them to attain to functional activity, and to give the organ a new physiological character.

A muscle, for example, may become functionless from many causes. Should the muscle-tissue disappear there are still left the accessory tissues, particularly connective tissue permeated by blood-vessels; this may remain intact and form a protecting band, a tendon, or fascia. We have then, morphologically, the same organ, changed in its physiological character; the muscle has undergone a change of function, and has become a ligamentous band. The visceral arches of fishes primarily are supports for the gills; if now by the acquirement of terrestrial habits the gills lue lost, the visceral arches become functionless and correspondingly undergo a partial degeneration; but a part persists by assuming a new function, 
and forms the jaws, the hyoid bone, and the small bones of the ear, which in spite of their quite different functions, are morphologically the same structures as the gill-arches.

Homology and Analogy.-In the History of Zoology (page Io) it was shown that comparative anatomy has caused a discrimination between homology or morphological equivalence, and analogy or physiological equivalence, i.e., between organs which appear in the same relative positions and relations, and organs which have the same function. What we have here learned of the structure of organs makes it evident that morphological and physiological characters do not necessarily coincide, that morphologically similar organs (lungs of mammals, air bladder of fishes) may have different functions, morphologically different organs (lungs of mammals, gills of fishes) the same functions.

Systems of Organs.-Organs identical, or, at least, functioning in an equivalent manner, may occur in considerable numbers in the same body. A man has many muscles, and many organs which carry on digestion. Hence we may group all organs which in the body have equivalent or similar functions into systems of organs. In all we recognize nine such systems: (I) skeletal, (2) digestive, (3) respiratory, (4) circulatory, (5) excretory, (6) genital, (7) muscular, (8) nervous, and (9) sensory systems. Not all are necessarily present; a skeleton, for instance, is frequently lacking. The various functions, which in man are divided among differeıt complicated and specialized systems, may be performed in a lower animal by one and the same apparatus. Yet according to the fundamental functions the following groups of organs may be recognized: I. Organs of assimilation (2-5); II. Organs of reproduction (6); III. Organs of motion (7); IV. Organs of perception (8 and 9).

Vegetative and Animal Organs.-The organs of assimilation and of reproduction (I and II) are grouped together as vegetative, the others (III and IV) as animal organs. The older zoologists used to say that assimilation and reproduction are functions common to animals and plants; that, on the contrary, sensation and motion are lacking in plants, and are exclusively characteristic of animals. The atom of truth in the fundamental idea needs reconsideration in the light of our present knowledge. We have seen that the protoplasm of plants and animals has not only the power of assimilation and reproduction, but also power of motion and of irritability. The latter characteristics consequently cannot be entirely lacking in all the plants, for they are found in their most important constituent. In fact many plants, as the mimosas, compassplants, insectivorous plants, show great irritability; the reproductive states of algæ move quite as actively as, or even more actively than, many of the lower animals. On the other hand, many animals are fixed in position like plants. Many Protozoa and worms, most polyps, some echinoderms like the crinoids, even many crustacea, the cirripedes (barnacles), can change their location only during the earlier stages of development, in later life being limited to movements of single parts of the body, the arms, tentacles, etc. In the sponges motions are 
so insignificant that they cannot be seen at all by the naked ere, and scarcely with the microscope.

Nevertheless the two terms, animal and vegetative, must be retainer. For although motion and sensation occur in the vegetable kingdom. still they reach no high development; they become more and more inconspicuous the higher the plants; in the animal kingdom, on the contrary, they are unfolded in extraordinary perfection and hie at the basis of its most characteristic features.

\section{SKIN AND SKELETON.}

With a few exceptions (cestodes, trematodes, nematodes) which are not fully explained, the outer surface of the Metazoan body is covered with a typical epithelium, commonly called cpidermis and occasionally, from its origin, ectoderm as well. In invertebrates and in Amplioxus it is one layered, but in all true vertebrates it is stratified. To this important part of the integument there is usually added the mesodermal part of the skin which is especially dereloped in rertebrates (corium or cutis, derma) and is derived from the connective tissue. The skin is frequently concerned in skeleton formation, as when in Coelenterates, molluscs and arthropods it secretes on the outer surface of the epidermis a cuticular armor, frequently hardened by lime. On the other hand there may be a calcification (echinoderms) or an ossification of the corium (scales of fishes, bony plates of reptiles and mammals). This dermal skeleton is in strong contrast to the axial skeleton of vertebrates which consists of cartilage and ossifications in the interior of the body.

\section{Vegetative Organs.}

\section{A. Organs of Assimilation.}

Assimilation Defined.-If the term assimilation be used in its widest sense, it includes all the contrivances in the animal body which render growth possible during the period of progressive development, and, during mature life, compensate for the loss of energy connected with each functional act, in order to preserve to the body its functional powers. With each functional act organic compounds are oxidized. Compounds which are especially rich in carbon and hydrogen (as well as some nitrogen and sulphur) and are poor in oxygen are changed by oxidation into carbon dioxide, water, and various nitrogenous products, like urea, uric acid, etc. A compensation takes place, for not only is the useless substance removed, but also compounds of oxygen and materials rich in carbon are furnished to the tissues to replace the material oxidized. An apparent exception is furnished by the 'anaerobic' organisms which live, move and perform work without oxygen. Aside from some bacteria, which are outside 
our scope, the entoparasites (cf. p. ' 157 ) take oxygen-containing compounds from their hosts, form from them carbohydrates and reduce them by a process analogous to fermentation into carbon dioxide and compounds poor in oxygen.

Assimilation in Animals.-In lowly organized animals all the processes connected with assimilative changes take place through the agency of one and the same organ, the digestive tract; but in the higher animals, through specialization, normal assimilation is a definite series of separate phenomena. Between the lower and the higher animals there are evidently intermediate conditions where specialization has halted at an earlier or a later stage.

Different Organs of Assimilation.-Assimilation begins with the presence of suitable food; the solid and liquid constituent parts of the body must digest and incorporate this, i.e., it must be altered so that it can be absorbed and distributed to the tissues. All this takes place through the agency of the digestive tract, which is provide i with accessory organs, the digestive glands; the digestive tract likewise removes all matter remaining undigested (the freces). The necessary oxygen, gaseous food, so to speak, is usually taken, however, by particular parts of the body, the respiratory organs, the gills or lungs. The oxygen and the digested (consequently liquefied) organic and inorganic compounds must further be distributed in the body to the organs and tissues according to their needs. Therefore there are usually blood-vascular or circulatory organs, which permeate the body. But the tissues need not only a means of obtaining new materials but also of getting rid of certain useless compounds. The accumulation of the oxidation products arising from functional activity is injurious to the organism; consequently they must be removed, and in a dissolved state they are taken up by the bloodvascular apparatus, and are brought to definite places for expulsion or excretion. Fluid wastes are expelled by the kidneys of vertebrates, the Malpighian vessels of insects, the water-vascular system of worms; these, together with their accessory apparatus, are embraced under the name excretory organs. Excreta are to be distinguished from faces; excreta are substances which have been a part of the tissues of the body itself, and, through oxidation, have become useless; while those sulstances which constitute the fres were useless from the beginning, and have never belonged to the body, but have remained separated from the tissues by the epithelium of the digestive tract. The gaseous oxidation product of the animal body, carbon dioxide, is removed by the blood-vascular apparatus through the agency of the respiratory organs. Since in the respiratory organs there takes place an exchange of the useless carbon dioxide for the 
oxygen necessary to life, these organs have a clouble function, being, at the same time, excretory organs for taking up food.

\section{The Digestive Tract.}

Archenteron or Primitive Digestive Tract.- Since the taking in of food and its assimilation are functions most important for the wellbeing of the animal, it is to be expected that of all the organs the digestive tract should be formed first. The fact that many worms (cestodes) and

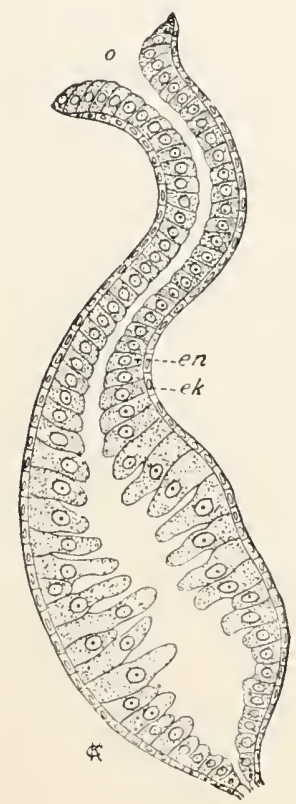

FIG. $5^{8}$.

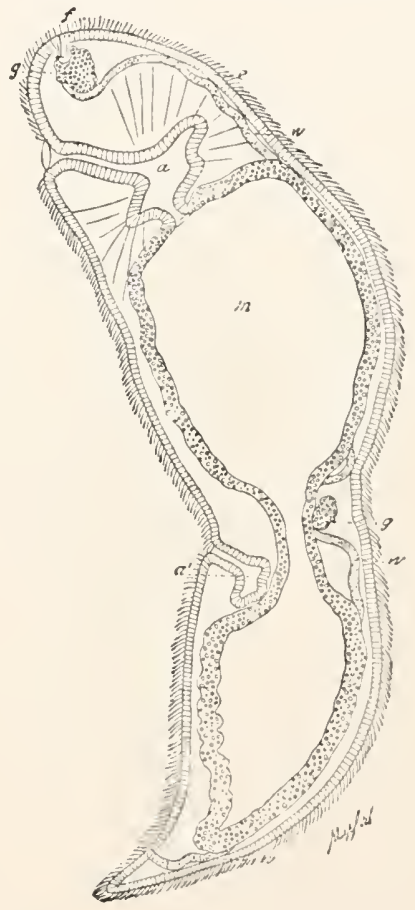

Fig. 50.

FIG. 58.-Longitudinal section through the nutritive polyp of a siphonophore (after Haeckel). o, mouth-opening; en, entoderm; $e k$, ectodern.

FIG. 59.- Stenostoma leucops, in division. $a$, ectodermal fore-gut, at $a^{\prime}$ forming anew for the hinder animal: $m$, the blindly ending entodermal mid-gut, $e$, ectorlermal ciliated epithelium; $g$, ganglion with ciliated pit; $w$, water-vascular canal; $g^{\prime}$, ganglion of the hinder animal.

crustacea (Rhizocephala) have no digestive tract does not alter this statement; for it can be definitely affirmed that, in adaptation to parasitism, the digestive tract has degenerated. The simplest multicellular, free-living animals are merely simple or branched digestive pouches 
which have only a single opening, functioning both as mouth and anus (fig. 58). Such an animal has necessarily at least two epithelial layers, one of which lines the digestive tract, the other covers the surface of the body. These two fundamental cell-layers are called entoderm and ectoderm. In many colenterates they are the only layers of the body. In most animals they are separated by intermediate tissues, called collectively mesoderm. The higher the animal, the more differentiated is the mesodermal layer. The primitive digestive cavity lined by entoderm is called the archenteron. In the case of meduse and polyps (fig. 58) it forms the entire digestive tract, but in most animals this is not sufficient for the needs of digestion and the alimentary tract is increased by invaginations of parts of the surface (ectoderm) of the body.

Stomodæum and Proctodæum.-Even in many cœlenterates and lower worms an invagination arises at the anterior end of the digestive tract, forming the ectodermal fore-gut or stomodcum (fig. 59). From the higher worms onwards, it is accompanied by a second invagination at the hinder end, the ectodermal hind-gut, or proctodcum (fig. 60); embryologically, this is formed as a blind sac whose closed end unites with the likewise closed posterior part of the archenteron (now called also mesenteron or mid-gut) until the separating wall disappears, whereupon mid- and end-gut communicate with each other, and the digestive tract becomes a canal extending through the entire body.

The part which the archenteron takes, in comparison with the ectodermal proctodieum and stomodæum in making up the completed digestive tract, is very different in the various groups. On one side the crustacea, on the other side the vertebrates, offer the strongest contrast; the crustacea have a very short mid-gut and consequently a long extent of fore- and hind-gut formed from the ectoderm; in vertebrates, on the contrary, the ectodermal portions are extremely short.

\section{Divisions and Appendages of the Digestive Tract.-The width} of the lumen varies in the course of the alimentary canal and renders possible the recognition of different divisions, which, so far as possible, have been provided with uniform names. Fig. 6I, drawn from a domestic fowl, illustrates the usual terms. The mouth-opening learls into a wider cavity, which is usually divided into an anterior division, the buccal cavity, and a posterior one, the pharynx. The narrow tube leading from this is the asophagus (a); here and there it may widen, or bear a pouchlike evagination, the crop or ingluzies (b), for the temporary reception of food. From the asophagus the food passes into a considerable enlargement, the stomach. Birds, like many other animals, have a double stomach, a thin-walled portion rich in glands, and a second part, the walls of which 
are remarkable for the thick masses of muscle; the former is the glandular stomach $(c)$, the latter is the grinding stomach or gizzard $(d)$, serving for comminution of the food. Behind the stomach the digestive tube narrows in to the small intestine $(h)$, following which is the hinder widened part, the

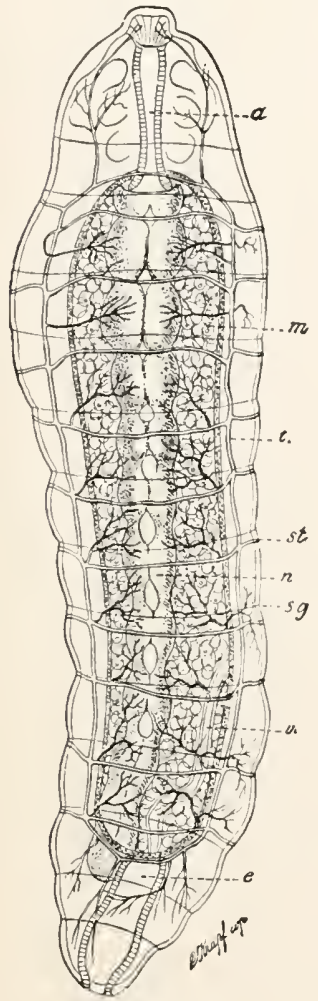

Fig. 6o.

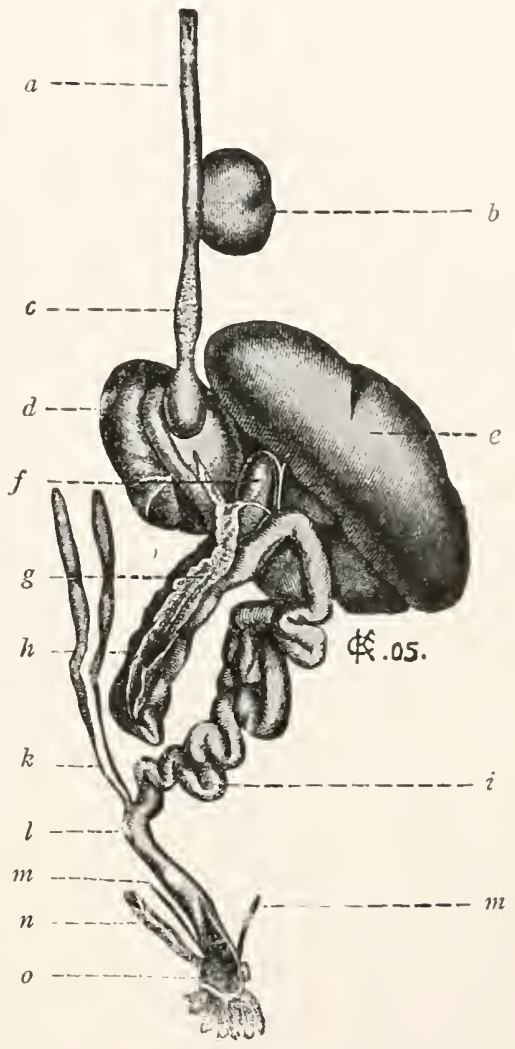

FIG. 6r.

FIG. 60.-Bee larva just after hatching: seen from the ventral surface. The digestive tract consists of three portions; $a$, fore-gut; $m$, mid-gut; $e$, hind-gut (not yet connected with the mid-gut); sg, limits of segments; st, stigma; $t$, trachea; $n$, ventral nervecord (after Bütschli).

FIG. 6I.-Digestive tract of the domestic fowl: $a$, oesophagus; $b$, crop; $c$, glandular stomach; $d$, gizzard; $e$, liver; $f$, gall-bladdler; $g$, pancreas; $h, i$, small intestine; $k$, crica; $l$, large intestine; $m$, ureters; $n$, oviduct; $o$, cloaca.

large intestine $(l)$, terminating in the anus. The limit of the small and large intestine is usually marked by blind pouches, the caca $(k)$. Connected with the anal gut also are the outlets of the kidneys $(m)$ and of the sexual apparatus $(n)$; hence the terminal portion, serving as the outlet for the urine and faces, and also for the sexual products, is called the 
cloaca (o). In the more highly organized animals there are accessory structures connected with the alimentary canal. Into the mouth empty the salivary glands; into the first part of the small intestine, close behind the stomach, the liver (e) and the pancreas ( $g$ ) (or a single glandular apparatus, whose secretion combines the characters of gall and of pancreatic juice, the hepatopancreas). Finally, in the hind-gut there sometimes occur glands which form a fetid secretion-the anal glands.

Digestive Functions.-Besides the comminution of the food which is often necessary, the alimentary canal has (I) to digest the food; that is to convert it into a solution; and (2) to resorb the digested food, that is to forward it to the tissues by the blood and lymph vessels. Digestion is effected by fluid ferments (enzymes), substances which by their presence can produce definite chemical changes without, apparently, being altered themselves. Thus the pepsin from the gastric glands of the vertebrates, in the presence of hydrochloric acid, can convert the proteid of the food into the soluble peptone; the trypsin of the pancreas has the same effect in alkaline media; the steapsin of the bile saponifies iats and makes them resorbable, while the ptyalin of the saliva converts starch into sugar. The resorbed substances are distributed to the tissues and are here assimilated; that is, so altered and appropriated that it becomes an integral part of the living, functioning structures-muscles, nerves, cells, etc.

In the vertebrates there is a division of labor, the glands functioning exclusively in furnishing the digestive fluids, the walls of the alimentary canal being chiefly resorbtive. In the invertebrates this distinction has not gone so far, so that the transfer of names from the higher group may lead to misconceptions as to the functions of the organs. When we speak of the liver of a crustacean, spider or mollusc, we must remember that this organ not only dissolves fat, and proteids and cellulose as well, but that it plays an important part in the resorbtion of nourishment. In the protozoa there is a cellular digestion, food particles being taken into the cell. A similar condition obtains in many colenterates, the individual entodermal cells eating the food particles; but there is also a true digestion in the archenteron, the walls of which secrete digestive fluids.

Even when the digestive tract has but little differentiation of its parts, it usually has a mesodermal layer added to its entodermal lining, the whole wall thus formed being called the splanchnopieure. The mesodermal additions take the form, not only of connective tissue but muscles as well (usually smooth, rarely of the striped variety). These muscles are important in effecting the (peristaltic) motion by which the contents of the canal are moved about independently of the body musculature. When these splanchnopleuric muscles are absent, the movement is caused by the contraction of the body muscles, or by the cilia which may cover the digestive epithelium.

The length of the digestive tract is chicfly influenced by the kind of food. In many groups of animals there is found a difference between herbivores and carnivores, the former having a very long and consequently convoluted digestive tract. That of a carnivore is about four or five times the length of the body, while in an herbivorous ungulate, on the other hand, it is twenty to twentyeight times. Similar, though not so great, are the differences between carnivorous and plant-eating beetles.

\section{Respiratory Organs.}

Sources of the Oxygen used in Breathing.-The oxygen which each animal must obtain in exchange for the carbon dioxide formed in the 
tissues is derived either from the air or from the water, according as the animal is terrestrial or aquatic. Less frequently it is the case that waterdwellers breathe air, and hence are compelled, from time to time to rise to the surface of the water for air; this is true for the large marine mammals, and for many insects, spiders, and snails found in fresh water. Airand water-breathing takes place exclusively through the skin, so long as this is delicate and readily permeable and no higher development of organization necessitates a more active interchange. If the demand for oxygen

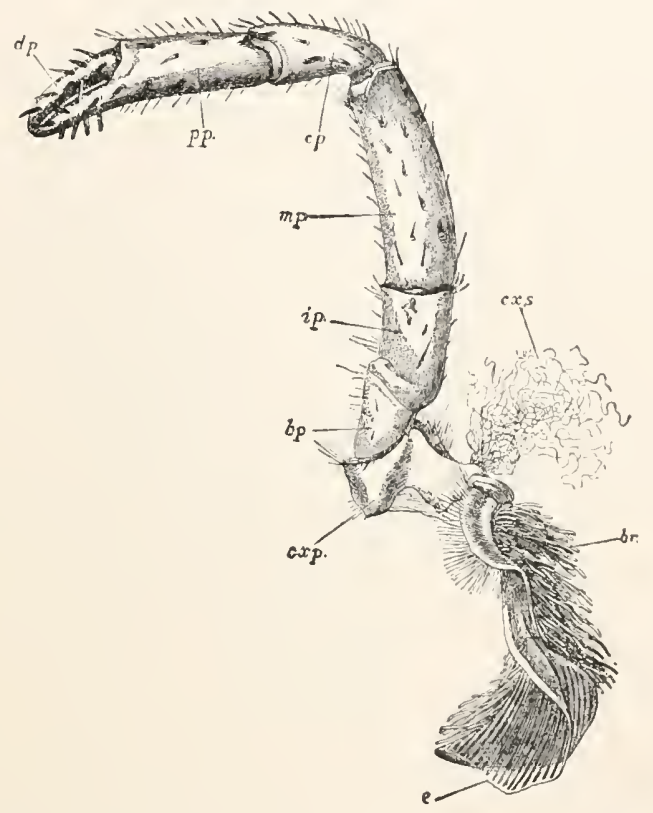

FIG, 62.- Left second foot of a crayfish with (br) attached gill (after Huxley). $c x p$, coxopodite; $b p$, basipodite; $i p$, ischiopodite; $m p$, meropodite; $c p$, carpopodite; $p p$, propodite; $d p$, dactylopodite; $c x s$, bristles of the coxopodite; $e$, lamina of the gill.

be greater, special breathing-organs are found-gills (branchia) for waterbreathing, lungs and trachece for air-breathing, in addition to which the skin functions as an accessory organ of more or less importance.

Gills are usually thin-walled, frequently ciliated areas of the skin which are abundantly supplied with blood-ressels, and where richly branched tuftlike projections or broad leaves have grown out, thus furnishing the largest possible surface for the interchange of gases: these occur in such a position as to be most exposed to fresh water; in the crayfish, for example, they are on the legs, where the motion drives fresh 
water constantly through them (fig. 62); in the swimming worms, on the back; in the tube-dwelling worms, at the anterior end, projecting out of the tube (fig. 63); in most amphibians (fig. 4), on each side of the neck. More rarely the digestive tract functions for water-breathing; in the fishes, Enteropneusta, and tunicates gill, have been formed in connection with the pharynx, its lateral walls being pierced by the gill-slits, which open to the exterior on the surface of the body. The water containing oxygen in solution passes out through the gill-slits, and bathes the gill-leaves which

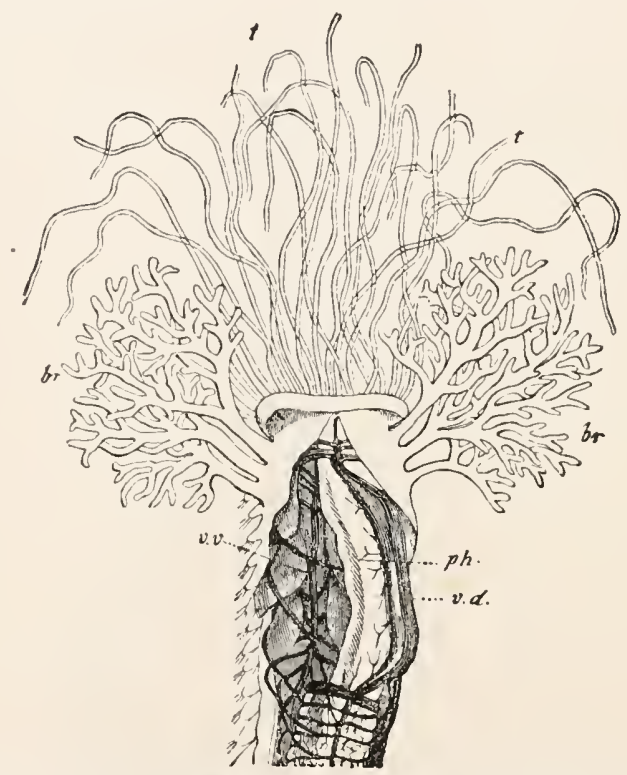

FIG. 63.-Anterior end of Terebella nebulosa (after Milne Edwards). ph, pharynx; $v d$, dorsal, $v v$, ventral, blood-vessel; $b r$, gills; $t$, tentacles.

are richly provided with blood-vessels. The hind-gut also in many fishes, insects, and worms may become an accessory respiratory organ, being filled from time to time with fresh water.

Aerial Respiration.-In the air-breathing animals the respiratory apparatus is derived either from the digestive canal or from the skin. With the vertebrates the former is the case, since the lungs, either directly or by way of the trachea and bronchi, are in connection with the lumen of the digestive tract. On the contrary, in snails and spiders when the term 'lung' is used, it refers always to an invagination or sac of the skin; the trachex of insects are similar tubes containing air, beginning at the surface of the body with a hole, the spiracle or stigma, and branching internally (fig. 6o, st). 


\section{Distinctions between the Respiratory Systems of Chordates} and Invertebrates.-In general, then, a distinction can be drawn between the respiratory systems of vertebrates and invertebrates: in the former, the digestive tract, or derivatives from it, are respiratory; in the latter, on the contrary, it is the skin. Of the vertebrates the only exceptions are most amphibians and a few fishes (Protopterus), in which the gills are tuftlike projections of the skin (figs. 4 and 5 ); while a mong the invertebrates some aquatic insects respire by the hinder end of the digestive tract.

\section{Circulatory Apparatus.}

In order that the oxygen, taken up by the respiratory organs, and the constituents of the food digested in the alimentary canal may reach the tissus, there is no need of special organs, so long as the body consists of only two thin epithelial layers, the ectoderm and entoderm. When, however, a third, a mesodermal, layer is interpolated between these, and the body consequently becomes more bulky, there is usually some apparatus for distributing the food. The simplest is when the digestive tract departs from the character of a straight tube and either gives off a few broad sacs (gastral pouches) or it branches, and by means of these branches extends into the various parts of the body. We speak then of a gastro-vascular system, because the alimentary canal itself takes on the function and the branching arrangement generally characteristic of the vessels or 'vascula' (fig. 64).

Cœlom.- The cœlom or enterocale is apparently derived from a pair of gastric diverticula which have become completely cut off from the archenteron (compare development of mesoderm, infra). It is a right and left cavity pushed in between the intestinal tract and the body-watl, is lined by a special epithelium, the peritoneum, and encloses most of the vegetative organs. If the two halves of the colom approach, without uniting, dorsal and ventral to the gut, the result is dorsal and ventral membranes, the mesenteries, which support the alimentary canal (fig. 241). In many invertebrates the colom plays an important rôle in nutrition since it contains a lymphoid fluid, rich in proteids and containing cellular corpuscles; it is also important for excretion since it may communicate with the nephridia (see p. 105) by the ciliated funnels. It loses this significance the more the blood system is developed, and in the vertebrates, so far as nutrition is concerned, it is a rudimentary organ.

A sharp distinction should be drawn between the cœlom and other cavities in the body. Not every 'body cavity' is a coelom, but frequently there occur large spaces which are entirely different in origin and in relations. Frequently, 
as in arthropods, these 'body cavities' contain blood and are in reality but expansions of the vascular system. To such cavities the term harmocele has been given.

Heart, Arteries, Veins, Capillaries.-The most complete method of food distribution is accomplished by the blood-vessels, which, therefore,

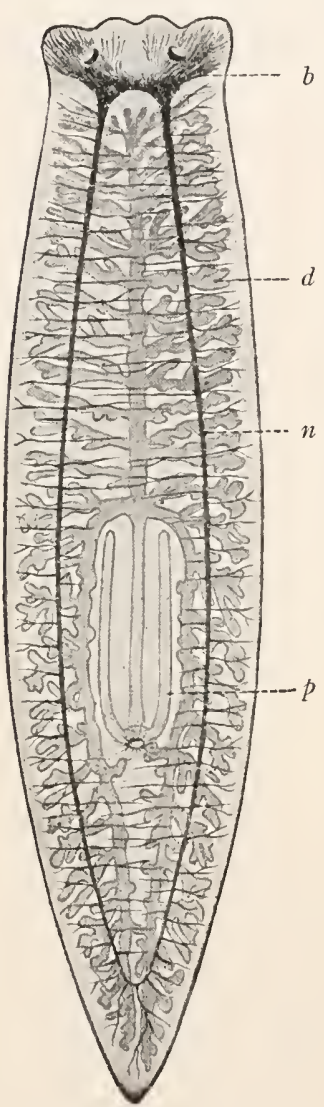

FIG. 64 . belong generally to the higher animals, and function, whether a body cavity be present or not. Blood-vessels are tubes containing the blood, which transports the oxygen received through the respiratory organs, as well as the food absorbed from the digestive tract, and later gives these up to the tissues. Since such an interchange of substances presupposes that the blood circulates in the vessels, definite parts of the blood-vessels are contractile; they are covered by muscles which by contraction narrow the tube and push the fluid forwards. In the lower forms wide areas are contractile; in higher

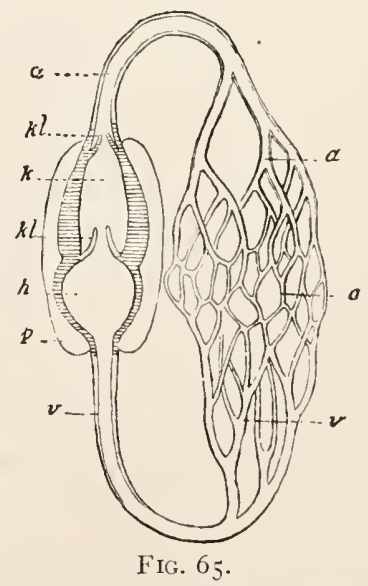

FIG. 64.-Dendrocclum lacteum (after Iijima). b, brain; $d$, digestive tract with cxcal branches: $n$, lateral nerve cords; $p$, pharynx with sheath and mouth.

FIG. 65 . - Schema of circulation of the blood. $a$, arteries; $c$, capillaries; $h$, auricle; $k$, ventricle; $k l$, valves; $p$, pericardium; $v$, veins.

animals a greater regularity of circulation is reached; a definite specialized muscular part of the course, the heart, alone propels the blood.

The Higher Development of the Heart.-A free motion of the heart is only possible when it is separated from the contiguous tissues and enclosed in a special cavity (fig. 65). Hence the heart always lies either 
free in the body cavity or enclosed in a special pouch $(p)$, the pericardium. The division of the heart into a part which receives the blood, the atrium or auricle $(h)$, and a part which drives the blood onward, the ventricle $(k)$, is of less functional importance; hence is not carried out in all cases. There are also valves $(\mathrm{kl})$, which, by closing, prevent the blood from flowing back when the walls relax at the end of the contraction.

Blood-vessels.- In order that the blood system may properly perform its function, in addition to circulation, it is necessary that the nutritive substances be rearlily taken up and given out again to the tissues. The part of the course of circulation concerned in this must have casily permeable walls, must be widely distributed in the body, ancl have a large superficial area. These demands are met by the capillaries (c), extremely fine, thin-walled and permeable epithelial tubes, which surround and penetrate all organs. Between the heart and the capillaries there exists, corresponding to their different functions, great differences in structure; they must therefore be united by special transitional vessels (1) ressels which begin large and thick-walled at the heart, and by branching, and thinning of their walls, pass gradually into the capillaries, the arteries (a) and (2) ressels (veins) which start from the capillaries and lead back to the heart, uniting to form larger and stronger vessels $(v)$.

Correlation of Respiratory Organs and Blood System.-It is a law that in all animals the blood-vascular system has been influenced in its arrangement and structure more by respiration than by nutrition in the narrower sense; there exists a correlation between the organs of respiration and of circulation. A double capillary region must be listinguished; besides the body capillary system already mentioned there is the respiratory capillary region, whose exclusive office is to remove the carbon dioxide from the blood and to furnish oxygen to it (gill and lung capillaries). A twofold capillary region makes necessary also a twofold system of arteries and veins (systemic arteries and systemic veins, respiratory artcries and respiratory veins). The accompanying diagram (fig. 66) of the blood circulation of fishes illustrates this. Veins lead from the capillary region of the tissues of the body to the auricle of the heart. The contraction or systole of the auricle drives the blood into the ventricle. While the auricle enlarges (diastole) and refills with blood from the veins, the systole of the ventricle forces the blood through the gill arteries to the gill capillaries. Since systole and diastole of a heart chamber alternate, the heart acts as a suction and force pump, and the systole of auricle and rentricle must alternate in time. From the gill capillaries the blood goes to the "gillveins' (efferent gill arteries), which unite into a single large trunk: this again gives off lateral branches passing into the capillary recion of the 
body. Since the branches of the main trunk formed by the 'gill-veins' lead again into a capillary region they must, like the main stem, be called arteries.

Arterial and Venous Blood.-During its course through the body the blood twice changes its chemical character and correspondingly its color. The blood which fiows from the body capillary region has given up

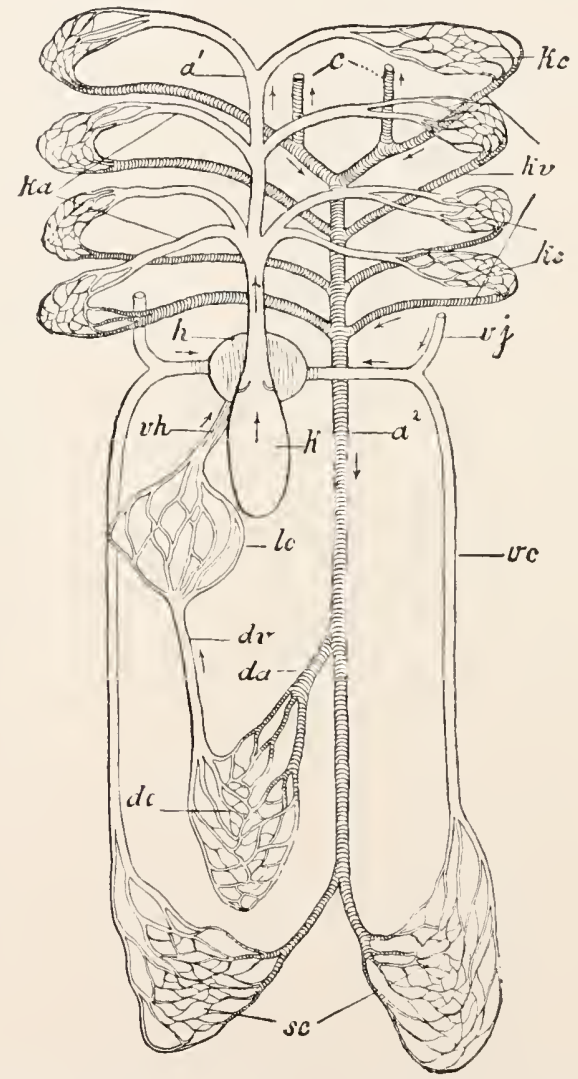

FIG. 66. - Scheme of circulation in a fish. $a^{\prime}$, ascending (ventral) oarta; $a^{2}$, descending (dorsal) aorta; $c$, carotid; $d a$, intestinal arteries; $d c$, intestinal capillaries; $d v^{\prime}$, intestinal veins; $h$, auricle; $k$, ventricle; $k a$, afferent gill-arteries; $k c$, gill-capillaries; $k z$, efferent gill-arteries; $l c$, liver-capillaries; $s c$, body-capillaries; $v c$, cardinal veins; $v h$, hepatic vein, $v j$, jugular vein.

its oxygen to the tissues, receiving in exchange carbon dioxide, and has become dark red. This character is maintained until, in the gill-capillaries, it again becomes oxygenated, giving up the carbon dioxide and becoming bright red. Since the different character of the blood was first known in 
the arteries and veins of the systemic circulatory system; the lark blood containing carbon dioxide is called venous, and the bright red, containing oxygen arterial blood, since the former flows in the reins, the latter in the arteries. These terms are entirely unsuitable, as can readily be seen from the diagram (fig. 66), because they easily lead to the false assumption that veins must always conduct blood containing carbon dioxide, and arteries always oxygenated blood. On the contrary, the diagram shows that, in the respiratory circulation, the conditions must be the reverse of those in the systemic circulation, since here the arteries contain 'venous' while the veins contain 'arterial,' blood.

Closed and Lacunar Blood-vascular Systems.- Such a bloodvascular system as has here been described is called a rlosed one, because the blood always flows in special tubes provided with their own walls. Opposed to the closed stands the lacunar blood-vascular system; here the bloodvessels lose, after a time, the character of tubes and become wide cavities, or simuses, which, without special walls, are enclosed between the intestines and other organs (hemocœle, supra).

Example of Lacunar Blood-vascular System. - The best example of a lacunar blood-vascular system is furnished by the insects and myriapods, which have only the heart and short arterial trunks: from the ends or the arteries the blood enters the hæmocœle, and from this through lateral slits (ostia) again enters the heart (fig. 67). In the arthropods and molluscs are found all transitions between so extreme a case of a lacunar system and the almost completely closed one. Here appears again a close correlation of the circulatory and respiratory organs, the latter determining the development of the former. If the respiration be diffusely distributed over or through the body, the circulatory apparatus is very simple; on the other hand, if the respiration be connected with definitely restricted areas, the apparatus is differentiated into heart, arteries, veins,

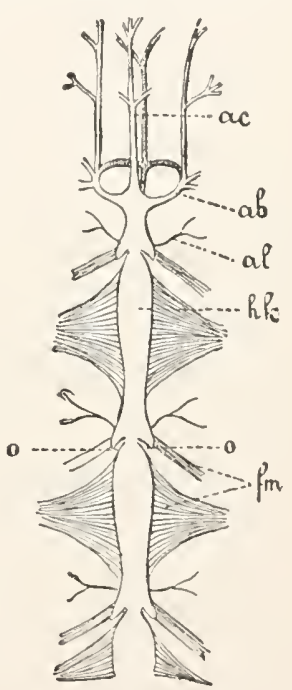

FIG. 67.- Anterior end of the heart of Scolopendra (af te $\mathrm{r}$ Newport). ac, cephalic artery: $a b$, arterial arch; $a l$, lateral artery; $f_{m}$, alary muscles (alix cordis); $h k$, chambers of heart; 0 , ostia. and capillaries. Details may be found in the sections on crustaceans, spiders, and insects, infra.

Lymph-vessels. - A special part of the rascular system is the lymfh system, known only in vertebrates. In the capillary region of the body proteids may pass into the tissues, but it is evident that an overtlow 
cannot re-enter the blood-vessels in the same way, on account of the higher pressure in the capillaries. This overflow is conducted back to the veins by the lymph-vessels. These begin with lacunæ in the tissues, and gradually pass into vessels with definite walls. The lymph-ressels of the digestive tract are particularly important since, during digestion, they become filled with the proteid and fatty constituents of the digested food; they are called chyle-vessels, because they contain the chyle, distinguished from ordinary lymph by its milky color.

Cold- and Warm-blooded Animals.--In connection with the bloodvascular system, two expressions are much used but not generally correctly understood, viz., cold-blooded and warm-blooded-or, more correctly, animals with variable and animals with constant temperatures. Under the head of animals with varying temperature (poikilothermal) or cold blood are placed forms whose temperature is largely dependent upon the temperature of the environment, rising and falling with it, but usually a few degrees above it. In our climate, where the atmospheric temperature is considerably lower than the temperature of the human body, such animals, for example the frog, feel cold to our touch, since they have a much lower temperature than we.

Such creatures as maintain about the same temperature, under any thermal condition are termed warm-blooded or constant temperatured, (idiothermal, homoiothermal) animals. Man in summer and winter under the equator and at the north pole, has approximately a temperature of $36^{\circ} \mathrm{C}$. $\left(98 \frac{2}{3}^{\circ} \mathrm{F}\right.$ ), showing higher temperatures only in fever. In order to maintain a constant temperature during the varying conditions, the animal must have the power to regulate the warmth of its body, either by limiting the production of heat, or by controlling its loss. If the environment be warmer than is suitable for the body temperature, then the production of heat must be limited to the smallest quantity compatible with the vital processes; but, if this does not suffice, the loss of heat must be increased by evaporation from the surface, usually accomplished by active perspiration. If, on the contrary, the environment be cold, then every unnecessary loss of heat must be avoided, while the production of heat must be increased. It is clear that idiothermy, since it requires complicated apparatus, can occur only in the highly organized animals.

\section{Excretory Organs.}

The excretory organs are tubes or glandular canals which open to the exterior either directly or by way of an end-gut (cloaca), and conduct substances which have become useless to the body to the outside. The presence of a blood-vascular system or a cœlom or both exercises an 
important influence on their structure. When neither are rleveloped the excretory tubules, in order to remove the excreta from the tissues, must branch and penetrate the body in all directions like a drainage system, being frequently connected in a network recalling the blood-capillaries (protonephridia or water-ìascular system of parenchymatous worms, fig. 68). The canals begin with closed tubes, which are provided internally at the end with a bundle of actively vibrating cilia, the 'flame' (fig. 7o). These flame cells are replaced in many protonephridia ('head kidney's' of many annelicl larre) by solenocytes (fig. 69), cells with a flagellum enclosed in a tube. One or more main trunks lead from the canal system to the exterior. A little before the external opening (excretory pore) there is frequently a contractile enlargement, the urinary bladder.

With the appearance of a coelom there is a central place for the collection of excreta. The nephridia or segmental organs are usually simple (rarely branched) tubes, open at both ends. One opening is external (fig. $7 \mathrm{I}$ ), the other communicates with the colom by means of a ciliated funnel, the neplurostome, a wide mouth with active cilia which connects with the canal of the tube. Through this the excretion is carried to the outside.

The excretory organs (kidneys) of vertebrates are derived from such nephridia. The fact that in the embryos (and frequently in the adults) these open into the colom by nephrostomes makes it probable that also in the vertebrates the colom was once important in excretion (fig. 72). The increasing import-

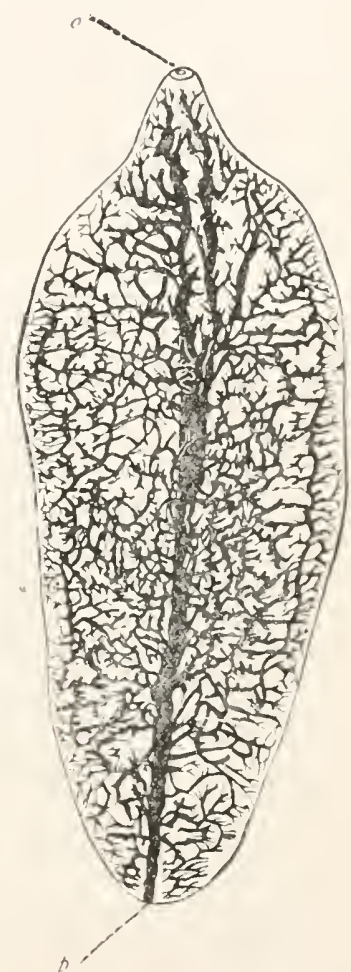

Fis; 68 -Distomum hepaticum with water-rascular system (from Hatschek). p, porous excretorius; $o$, mouth. ance of the blood-vessels which envelop the nephridial canals and bring to them the waste matter taken from the tissues is probably the cause of the loss of connection of the kidneys with the coelom by degeneration of the nephrostomata. The relation of the blood ressels to the nephridial tubes becomes specially close by the derelopment of the glomeruli (Malpighian corpuscles), bundles of capillaries carrying the walls of the canal before them and so projecting into the lumen of the tube. Since the nephridial tubules of the vertebrates open into a com- 


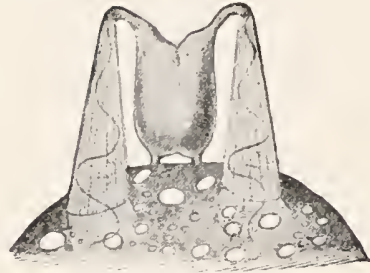

Fig. 69.

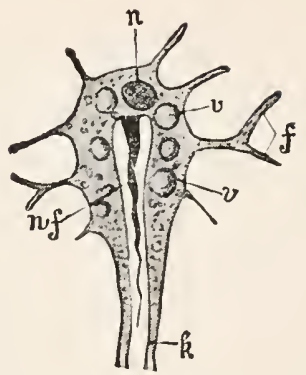

I. IG. 70 .

Fig. 69.-Blind end of an annelid protonephridium with two connected solenocytes which open with their flagellate tubes into the excretory duct (after Goodrich)

FIG. 70.-Blind end of one of the finest water-vascular canals $(k)$ of a Turbellarian (from Lang). $n$, nucleus; $f$, processes of the terminal cell; $w f$, 'Hame' of the terminal cell; r', vacuole.

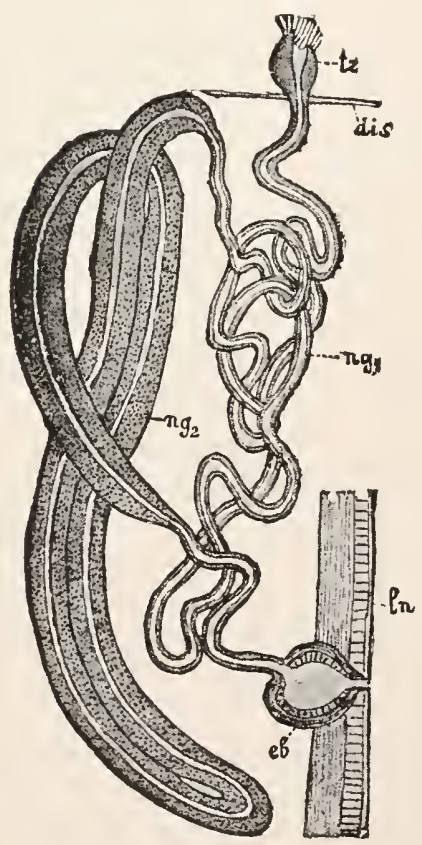

FIG. $\neg$ I.

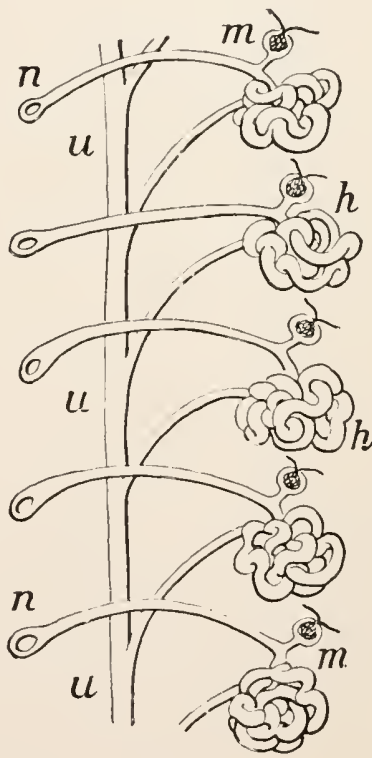

FIG. 72 .

FIG. 7I.-Segmental organ of an oligochæte (from Lang). tz, ciliated funnel; dis, septum; $n g^{1}$, non-glandular, $n g^{2}$, glandular, part of the canal; $e b$, terminal vesicle; ln, body-wall.

FIG. 72.- Scheme of a mesonephros of a vertebrate. $h$, nephridial tubules; $m$, Malpighian tubules with afferent and efferent blood vessels; $n$, nephrostomes; $u$, urinary duct. 
mon canal leading to the exterior (ureter) they are commonly aggregated into a compact mass, the 'kidney.'

\section{B. Sexual Organs.}

Sexual Glands and Ducts.- In the sexual apparatus are distinguished the areas where the germ cells are produced, the sexual glands or gonads, and the ducts for these. The former are present, temporarily or permaneintly, in all multicellular animals; the latter may be absent. If the sexual products arise in the skin or in the walls of the digestive tract, as is usual in the colenterates, then special outlets are superfluous, since the ripe elements can reach the exterior directly by rupture of their covering or by means of the digestive tract.

Germinai Epithelium and Germinal Glands.-Male and female sexual cells, as we have seen, originate from an undifferentiated incipient

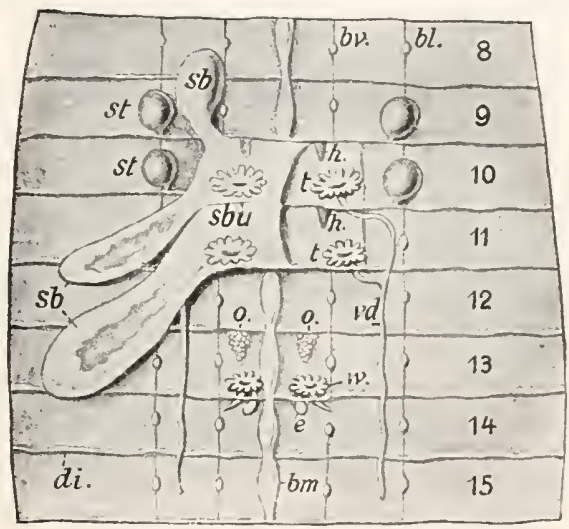

FIG. 73.-Sexual organs of Lumbricus agricala (from Lang, after logt and Yung). The seminal vesicles of the right side are removed. bm, ventral norve cord; $b v$ and $b l$, ventral and lateral rows of setæ; $s t$, receptacula seminis, $s b$, seminal resicles of the left side, connected with a median unpaired seminal capsule ( $\operatorname{lo} u$ ). Enclosed in the latter are the testes $(h)$, and the seminal funnels $(t)$. which lear into the vas deferen $=\left(\tau^{\prime} d\right) ; o$, ovaries; $w$, ciliated funnels learling to oviducts with egg capsule $(c)$; di, dissepiments; $S-I_{5}$, eighth to fifteeeth segments.

organ, or anlage, which is called the germinal epithelium. Usually it forms a part of the epithelial lining of the body carity, in many animals permanently, in others only temporarily; in the former case it separates. usually by constriction, and forms gland-like bodies, the gonads or sexual glands.

Gonochorism and Hermaphroditism.- In most animals the germinal epithelium produces either only female or only male sexual cells; 
such animals are called separate-sexed, diacious or gonochoristic, in opposition to the hermaphroditic forms, in which both kinds of sexual glands are contained in one and the same individual. Different degrees of hermaphroditism can be distinguished; commonly testes and ovary are contained in the same animal, some distance apart, as in the earthworm, in which two segments are male, while a third segment is female (fig. 73). More rarely there is a union of testes and ovary into a single hermaphroditic gland; land-snails have an hermaphroditic gland, which produces spermatozoa and eggs in the same follicle.

Occurrence of Hermaphroditism.-Hermarhroditism is, in general, of more frequent occurrence in the lower than in the higher animals. Insects and vertebrates are, almost without exception, dicecious; only among the teleosts is hermaphroditism not rare. It also occurs in the myxinoids. More commonly

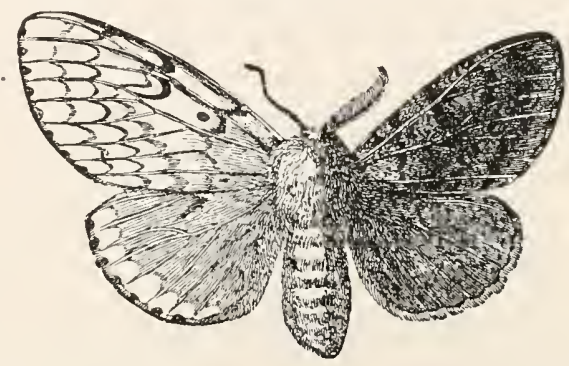

FIG. 74.-Lateral hermaphroditism of a gipsy moth (Ocncria dispar). Left female, right male (after Taschenberg).

hermaphroditism occurs as an abnormality; a striking form is lateral hermaphroditism, in which one half of the animal has only male, the other half only female, gonads. If the males and females of a species be distinguishable ty their appearance, then lateral hermaphroditism is expressed in their external form, since one half of the animal has the characteristic marks of the male, the other half those of the female (fig. 74). Still it must be noted that, in many instances where the external appearance pointed toward hermaphroditism anatomical investigation has disclosed either only male or only female sexual glands in a rudimentary condition (gynandromor phism). True hermaphroditism (the presence of both kinds of sexual glands in the same animal) is extremely rare in mammals and in man. What is described as hermaphroditism is usually gynandromorphism; rarely are both kinds of gonads present in the same individual, and then not in a functional condition.

The wide distribution of hermaphroditism among the lower Metazoa has led to the erroneous view that this was the primitive condition, from which the gonochoristic condition has been evolved. Studies on nematodes, crustacea and possibly molluses have shown that, on the contrary, hermaphroditism has followed a diøcious condition, since with the disappearance of males, the female animals may develop male sexual cells before the ovaries are mature. Contrasted to this 'protandry' a 'protogyncecy' is rare.

Genital Ducts.- Very frequently the excretory apparatus furnishes outlets for the sexual products. In the annelids and vertebrates portions 
of the nephridial system, either exclusively or in addition to their excretory function, become genital ducts. Hence we speak of a urogenital system. This connection of genital and excretory organs has a doul,le cause. Physiologically important is the fact that eggs and spermatozoa behave like excreta; substances which are no longer needed by the individual, but must reach the exterior in order to become of use. The morphological cause is the relation to the colom. A urogenital system occurs only in animals in which the germinal epithelium arises from the epithelium of the colom, and in which the kidneys or their rudiments are in connection with the body cavity and thus form the natural outlet for its products. Whether the accessory sexual parts are portions of the excretory organs or are independent structures, they have in the animal series a clefinite arrangement adapted to their function (figs. 73 and 75). Canals lead from the gonads to the exterior, oviducts in the female, iasa deferentia in the male (and the hermaphroditic duct from the hermaphroditic gland).

\section{Accessory Sexual Apparatus.-The} terminal portion of the vas deferens is often very muscular and is called the ductus ejaculatorius; it may be evaginated or project permanently beyond the surface of the body as a penis or cirrus. The terminal portion of the oviduct is often widened so that two portions may

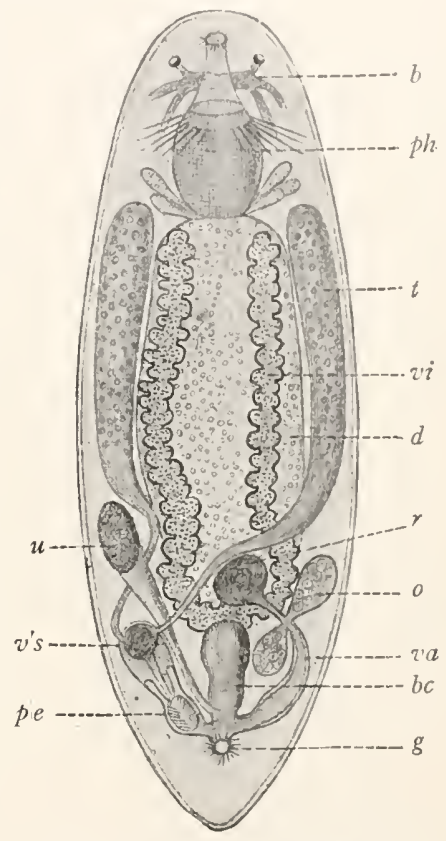

FIG. 75.-iortex zirdis (after Schultze and von Graff): $b$, brain with eyes; $b c$, bursa copulatrix; $d$, digestive tract; $g$, genital pore: 0 , ovary with oviduct; ph, pharynx; pe, penis: $r$, receptaculum seminis; $t$, testis with vas deferens; $u$, uterus; $z^{\prime} a$, vagina; $z^{\prime} i$, ritellarium; $z^{\prime}$, , resiculum seminalis. be distinguished, the uterus, which harbors the eggs during their development, and the ragina, which serves for copulation. In addition there may occur in both sexes other accessory glands of the most diverse character.

Occasionally, in the animal kingdom, a part of the eggs degenerate and are used for the nourishment of the others. This degeneration may take place in the uterus (Salamandra), in the egr cocoons (annelids), or in the ovary (many arthropoda, fig. $35, e$ ). In some cases a definite part of the ovary produces these 'yolk cells,' a condition that explains the fact that in many animals (Pla- 
todes) there are glands (vitellaria), distinct from the ovaries, which form the yolk cells (fig. 75).

Secondary Sexual Characters.-Often we can distinguish between the male and female of diœcious organisms only by the sexual products (medusæ, polyps, sponges). In other cases the sexual ducts are also characteristic. In the higher animals these primary sexual characters are associated with those of a secondary nature so that it is possible to recognize male or female at a glance. These secondary sexual characters are exemplified in many birds and mammals by the voice, the hair or feathers, strength of muscles and skeleton, presence of offensive or defensive weapons, etc.; in insects by structure and markings of wings, form of antennæ, etc. (fig. 74). This sexual dimorphism may become so marked that only careful study, especially of the development, shows that the male and female belong to the same species; dwarf males of Bonellia (fig. 268), Cirripedia, Copepoda (fig. 8).

A part of these secondary sexual characters are developed at the approach of sexual maturity and can be restricted or even suppressed when the gonads are destroyed or removed (castration). 'This leads to the conclusion that the development of the secondary sexual characters is correlated with the maturation of the gonads and is influenced by it. As a causal factor it is thought that 'internal secretions' (hormones) arise in the sex glands; these are passed into the circulation and cause the modification of distant parts like hair, larynx, mammary glands.

Yet this explanation must not be carried too far. Many secondary sexual characters, like those connected with the genital ducts, develop independently of the gonads. The peculiar developmental direction taken by the dwarf males just alluded to is begun before the maturation of the testes and apparently would appear even if the anlage of the gonads were removed in the embryo. We are on firmer ground with the corresponding modifications in the Lepidoptera. Here the secondary sexual characters clearly develop in the way laid down in the embryo, if the gonads be removed from the young larva; even if the testes are removed and replaced by ovaries taken from other individuals, or vice versa. The transplanted gonads become mature, while the rest of the sexual apparatus and the secondary sexual characters show the peculiarities of the original sex. All of these observations show that a correlation of gonads, genital ducts and secondary sexual characters exists to only a limited extent. The harmonious development of parts is rather regulated by a third factor, the peculiarities of the fertilized egg or its early developmental stages. It is these that prescribe, in a more or less striking manner, a certain developmental direction, not only for the gonads, but for the whole organism.

\section{Animal Organs.}

\section{Organs of Locomotion.}

Voluntary Locomotion.-The power to change their location voluntarily is a peculiarity so prominent in animals that usually it is sufficient for deciding whether an organism belongs to the vegetable or to the animal kingdom. On this account it is necessary to call attention to the fact that numerous animals, freely mobile in the larval stages, lose the power of locomotion, becoming fixed to the ground, to plants, or to other animals, and only retain the power to move parts of the body, as in the corals the crown of tentacles, the barnacles their feathery feet; many attached molluscs can actively close the shell. 
Locomotion among Lower Animals.-The lowest forms, the Protozoa, progress almost exclusively lyy processes of the cell: pseudopodia, cilia, or flagella. In the metazoa this is rarely the case. Amceboid movements of the epithelial cells, indeed, occur in the colenterates and in many worms, but do not suffice for change of position. More effective is the ciliated or flagellated epithelium, by which ctenophores, turbellarians, and rotifers swim; this occurs, besides, in many larve of animals which, in the mature state, are unable to change their location or do so only by the aid of muscles. Nearly all cœlenterates, echinoclerms, molluscs, and the majority of the worms leave the egg-membranes as larva swimming by means of cilia.

The musculature is alone adapted for energetic motions. The arrangement of this varies with and depends upon the constitution of the skeleton. Forms without a skeleton have commonly the dermo-muscular tunic, a sac of circular and longitudinal muscle fibres which is firmly united with the skin. If a skeleton be formed by the skin, as in the arthropods, where the epidermis secretes a firm cuticular skeleton, then the sac breaks up into groups of muscles, which find points of attachment upon it; if, on the other hand, as in the vertebrates, an axial skeleton be formed, fixed points are furnished for muscular attachment, so that the musculature obtains a new character, in particular a deeper position. A unique locomotor apparatus is the ambulacral system of the echinoderms, a system of delicate little tubes with protrusible portions which function as feet, described in connection with that group.

Electric Organs.-In several fishes the muscles at certain places are modified into electric organs, which, in Malapterurus, Torpedo, Gymmotus and dstroscopus can give energetic discharges; in Raia and Mormyrus the

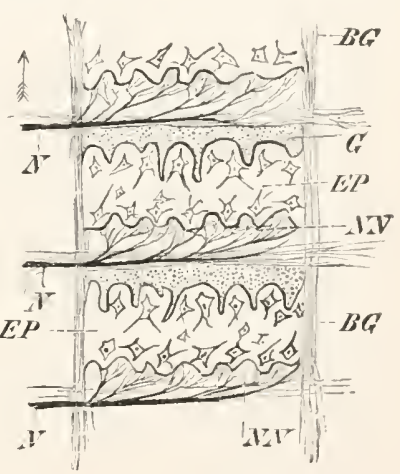

Fig. 76.--Diagrammatic section of electrical apparatus (from Viedersheim). The arrow points dorsally or anteriorly. $B G$, connective-tissue framework; EP, electrical plates; $G$, gelatinous tissue; $N^{\top}$, nerves entering through the septa; $N . T$, nerve terminations. discharges are weak and cannot be felt by man. Each organ is formed of columns of numerous superimposed plates separated by connective tissue. Each plate is a metamorphosed muscle fibre, the side to which the nerve is attached forming the negative pole.

\section{Nervous System.}

Scarcely a system of organs shows such a regular progression as the nervous system. The different stages may be termed the diffuse, the linear, the ganglionic, and the tubular types. 
Diffuse Nervous System.--The diffuse type is certainly the most primitive; it shows the two elements, nerve fibres and ganglion cells, distributed through the whole body, or, at least, through certain layers of it. The skin of the body, the ectoderm, is one of the fundamental elements in the nervous system, since it is related to the external world, and hence receives the sensory impressions, so important for the development of nervous tissue. The corals and hydroid polyps are examples,

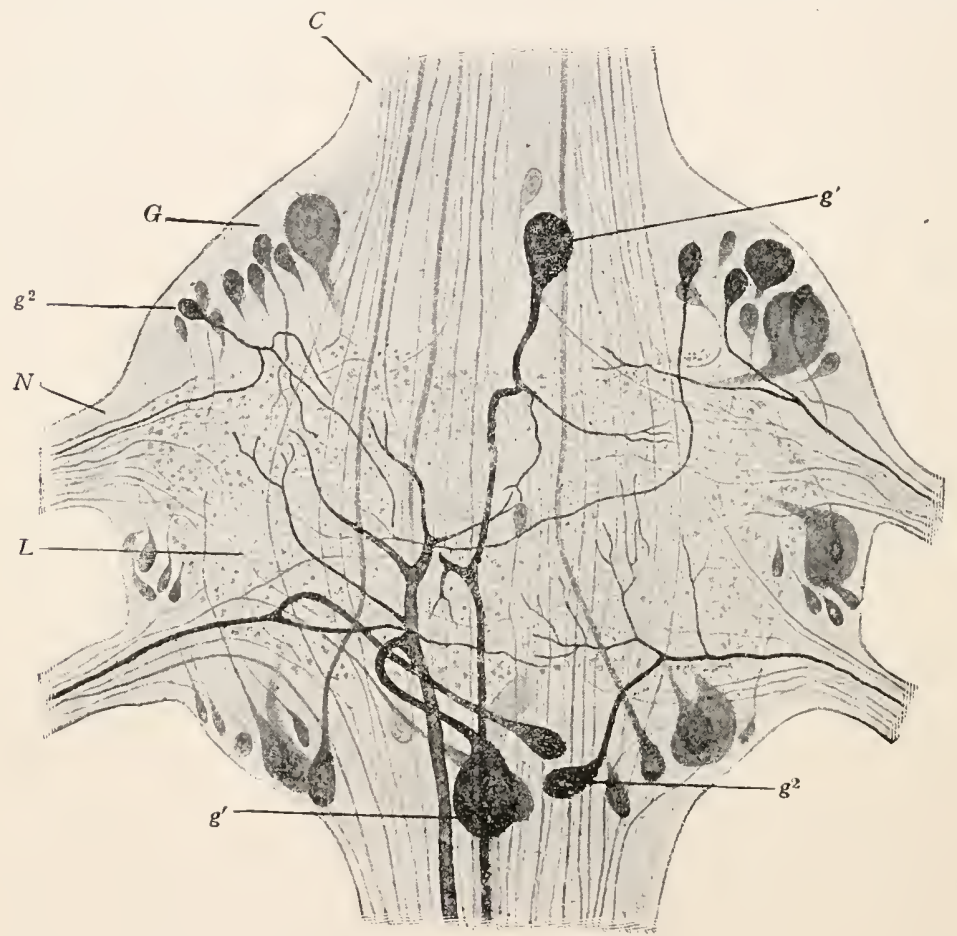

FIG. 77.-Third abdominal ganglion of a crayfish (after Retzius). $C$, connective or longitudinal commissure; $G$, ganglion cell layer; $g^{\prime}$, ganglion cells whose neurites enter the connective; $g^{2}$, ganglion cell whose neurites enter the peripheral nerve; $L$, granules, (Leydig's dotted substance); $N$, peripheral nerve.

since in them the ectoderm is permeated in all directions by a subepithelial spider-weblike network of nerve fibres and ganglion cells, which encroach even upon the entoderm (fig. 5 ) ).

Linear Nervous System.-From the diffuse type the other chief types can be derived through concentration, which is chiefly conditioned by the fact that there are a few points which are most advantageously located for the reception of sensory stimuli, and hence for the development 
of nervous elements. In the medusx such a place is the rim of the bell; consequently a stronger nerve-cord much richer in ganglion cells is found here. This, as well as the nerve-ring and the five radial nerves of echinoderms, may be called a central system, thereby distinguishing the rest of the nerrous network as the peripheral nerious system.

Ganglionic Central Nervous System.-Numerous transitional forms lead to the ganglionic central nervous system of the worms, molluscs, and arthropods (fig. 77). The central nervous system here consists of two or more ganglia; each ganglion being a bunch of regularly arranged nerve-fibres and ganglion-cells. The former constitute the centre of the mass, and, since they cross in all directions giving off branching dendrites, they appear like fine granulations. The ganglion-cells, on the other hand, collect in a thick layer around the granules. The peripheral nerves, and also the commissures, the cords connecting similar ganglionic masses, extend outwards from the ganglia.

\section{Supraœsophageal (or Cerebral) Ganglia.-} Since most animals are symmetrical, the ganglia occur in pairs; left and right ganglia correspond to one another and are connected simply by a cord of nerve-fibres, the transverse commissure. Of most constant occurrence are two ganglia, which lie dorsally above the pharynx, and hence are called the supra-a'sophageal or cerebral ganglia. If other ganglia occur, they lie ventra'ly ard below the digestive tract (ventral nerve-cord).

In the Ladder Nervous System of annelids and arthropods (fig. 78), numerous pairs of ganglia (in the example before us, nine) lie in serial order on the ventral side of the animal, and are connected by longitudinal commissures (connectives), and also by transverse commissures connecting the left and right ganglia. The first pair of the series is the infra-cesophageal ganglion,

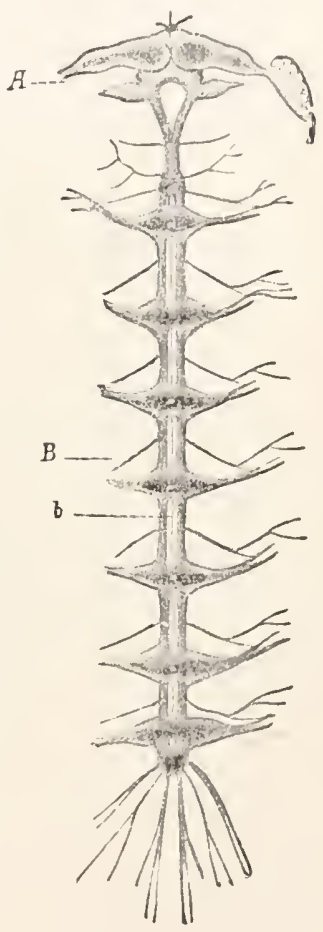

Fig. - - 8. - La al der nervous system of sowbug (Porcellio scaber) (after Leydig). $A$, brain: $B$, ventral cord, connected with the brain by the aesophageal commissures; $b$, a cord formerly regarded as sympatheticus. which sends out connectives right and left, surrounding the pharynx. to the supraœsophageal ganglion. The supra- and infra-œsophageal ganglia together with the osophageal connectives form the arsophageal ring, a nerve-ring surrounding the osophagus. 
The Tubular System is found only in the chordates (fig. 79). The vertebrate brain and spinal cord form a tube with greatly thickened walls. In the centre lies the extremely narrow central canal, which widens anteriorly into the ventricles of the brain. In a transverse section the nervous elements are seen grouped around the central canal in a manner almost the reverse of that of the ganglionic type. On the periphery lies a layer of nerve-fibres (the white matter); next is a central portion formed

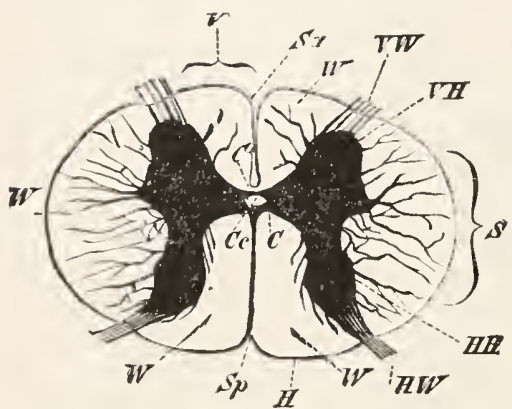

FIG. 79--Cross-section of the human spinal cord (from Wiedersheim). Black represents the gray, white the white substance of the cord; $C c$, central canal, surrounded by the anterior and posterior commissures $\left(C\right.$ and $\left.C^{\prime}\right) ; S a, S p$, anterior and posterior fissures; $\mathrm{IIT}^{\top}, H \mathrm{~W}$, anterior and posterior nerve-roots; $V H, H H$, anterior and posterior horns of gray matter; $V, S$, $H$, anterior, lateral, and posterior columns of white matter.

in the deeper layers of the body (fig. 9). In the vertebrates and some other higher animals, besides the body nervous system, there is a sympathetic system for the control of the vegetative organs which are not influenced by the will.

\section{Sensory Organs.}

What we know of the external world is founded upon experiences gained through our sensory organs, controlled by the judgment. If things exist outside of ourselves which have no influence upon our senses, we can form no conception of them. It follows from this proposition that we can gain knowledge of the capacity of the sensory organs of animals only by analogy with our own experiences. Hence the distinction of five senses, touch, taste, smell, hearing, and sight, based upon human physiollogy has been extended to the whole animal kingdom. A priori, however, it cannot be denied that sensations may occur in animals which we do not 
experience; following out this course of thought has lecl to the iclea of a 'sixth sense,' a designation which is no longer correct since mankind has more than five senses. The former sense of 'touch' really includes, besides true touch, the senses of temperature and pain. In adclition there are also the muscular and equilibrium senses. A still more important reason for our very fragmentary knowledge of animal sensations is the fact that, in regard to the function of the sensory apparatus, we can seldom depend upon experiments, and consequently must base our conclusions upon structure. But the anatomy of many sensory organs, like those of smell and taste, is by no means so characteristic that it alone is sufficient to determine the function.

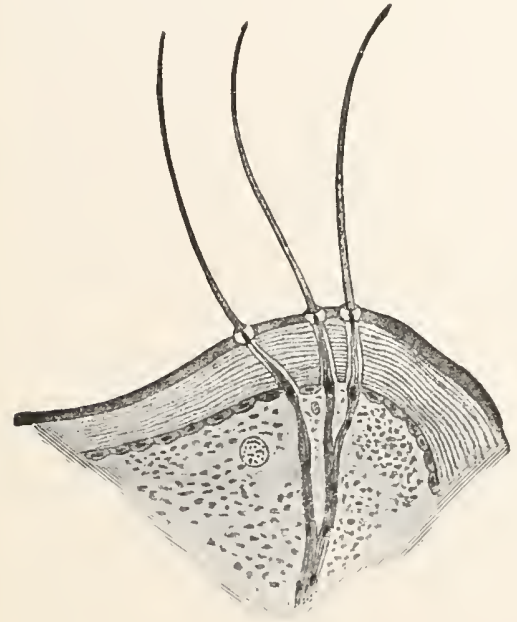

FIG. 80.

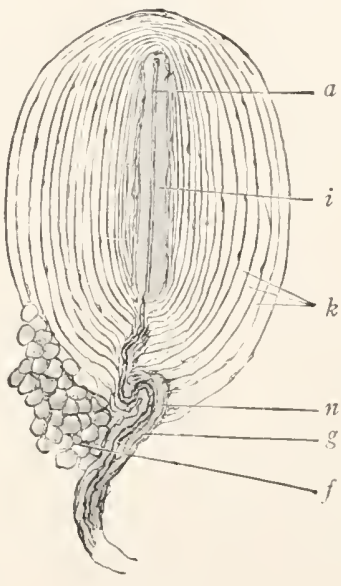

FIG. 8I.

Fig. So--Tactile hairs of a crab Cyrtomala (after Döflein).

Fig. $8 \mathrm{r}$ - - Vater-Pacinian corpuscle of the mesentery of a cat. a, axis cylinder: $f$, fat; $g$, blood-vessel; $i$, inner bulb; $k$, capsule with nuclei; $n$, medullated nerve-fibre.

Tactile Organs.--The skin is tactile, usually over the whole arra, although not everywhere with equal intensity. Prominent parts, like the tentacles of polyps and of many worms, the antenne of arthropods and snails, need only mention. Special epithelial cells with stiff hairs projecting above the surface, the tactile bristles or tactile hairs, are tactile (fig. 8o). Only in the vertebrates do the nerves of touch terminate in specially modified end organs (I'ater-Pacinian corpuscles, corpuscles of Meissner, etc., fig. 81); these usually lie under the epithelium.

Organs of Smell and of Taste are accurately known only in vertebrates. The olfactory organ of fishes consists of two pits in the skin, 
above or in front of the mouth. In the air-breathing vertebrates this pair of pits, which here also arise from the skin, are taken into the dorsal wall of the two respiratory canals leading from the outside to the mouth or pharynx. Now since the olfactory cells clistributed in these pits (fig. $38, B)$ are frequently characterized by bundles of olfactory hairs, while the surrounding epithelium is often ciliated, one is inclined to regard as organs of smell sensory organs of invertebrates, which have the form of ciliated pits or lie near the respiratory apparatus (e.g., the osphradium of molluscs). Yet there are exceptions. Experiments seem to show that in the arthropods the antennæ serve for smelling. Here the sensory perception can be connected only with certain modified hairs, the olfactory tubules of the crustacea and the olfactory cones of insects. In a similar way certain nerve end organs in the region of the mouth are considered as organs of taste, since the taste organs of vertebrates, the so-called taste buds, are abundant in the mouth cavity.

Organs of Hearing and of Sight are called the higher sense-organs, because they are of much greater importance than the other organs,

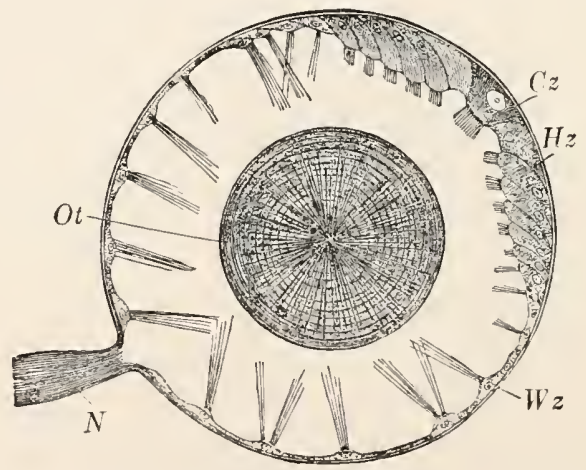

FIG. 82-Auditory vesicle of a mollusc (Pterotrachea). $N$, auditory norve; $H z$, auditory cells with the central cell; $C z, W^{\prime} z$, ciliated cells, $O_{i}^{\prime}$, otolith (after Claus).

since they furnish sensations which are quantitatively and qualitatively much more definite. Ears and eyes have therefore a complicated and characteristic structure, which renders them easily recognizable by the almost invariable presence of certain structures accessory to their functions.

The auditory organs of vertebrates and of most other animal groups can be traced back to a simple fundamental form, the auditory vesirle (fig. 82). This has an epithelial wall, a fluid contents, the endolymph, and an auditory ossicle or otolith, formed from one or from several fused concretions. In some instances the otoliths, to the number of 
thousands, may remain separate. In a definite region of the epithelial wall the sensory cells are developed into the crista acustica or the auditory ridge; they are in connection with the auditory nerve and bear the auditory hairs projecting into the endolymph. The otoliths are usually free in the centre of the vesicle, or are often held in place by bundles of cilia which project from the non-sensitive epithelial cells.

Every auditory vesicle develops from a pitlike invagination of the skin, and consequently is for a time an auditory pit. Therefore it is not surprising that in many animals the organ has stopped at the lower stage of development; for example, the crayfish has an open auditory pit (fig. 378). On the other hand, the auditory vesicle may develop a complicated system of carities as in mammals (fig. 83), where it is divicled by a constriction into the sacculus and the utriculus. The sacculus is pro-

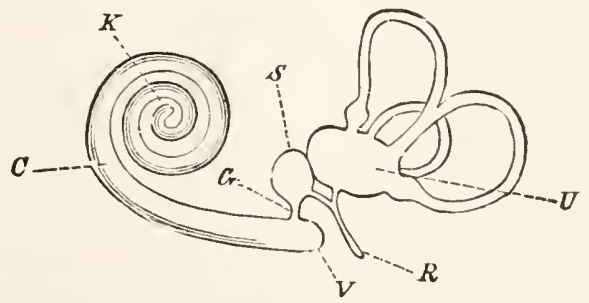

FIG. 83.-Diagram of the human labyrinth. $U$, utriculus with the semicircular canals; $S$, sacculus connected with the cochlea $(C)$ by the canalis reuniens; $R$, recessus labyrinthi; $V$, blind sac of the cochlea; $K$, apex of the cochlea.

vided with a spirally-wound blind sac, the cochlea, the utriculus with the three semicircular canals, the whole being called the labyrinth. In addition there is formed in most vertebrates, a sound-conducting apparatus, so that the auditory organ acquires a very complicated structure.

Other Forms of Auditory Organs.-Since there are animals without auditory vesicles which hear well, like the spiders and insects, we must assume that there are auditory organs of another type. Still we have no certain knowledge of these except in the case of the tympanal auditory organs of the grasshoppers (see p. 4IO).

Function of the Semicircular Canals.-Experiments upon representatives of the different classes of vertebrates have led to the conclusion. that the three semicircular canals, standing at right angles to each other, are organs of equilibrium, while the cochlea of the mammals and the homologous lagena of the other vertebrates is the seat of hearing. Corresponding to the poor development of the lagena, hearing is so poorly developed in the fishes that it was long believed to be absent and the labyrinth was regarded as a balancing organ, since when it was destroyed the 
animals stagger and lose their balance. Starting from this assumption, recent investigators have attempted to prove that the auditory vesicles of invertebrated animals are exclusively, or at least largely, organs of equilibration. This would explain the otoliths, for these bodies, of relatively high specific gravity, would affect the crista in different ways according to the position of the body. Statoliths is thus a better name.

Stimulation by Light is a phenomenon widely distributed among animals and plants; in its simplest form it is manifested by the organisms
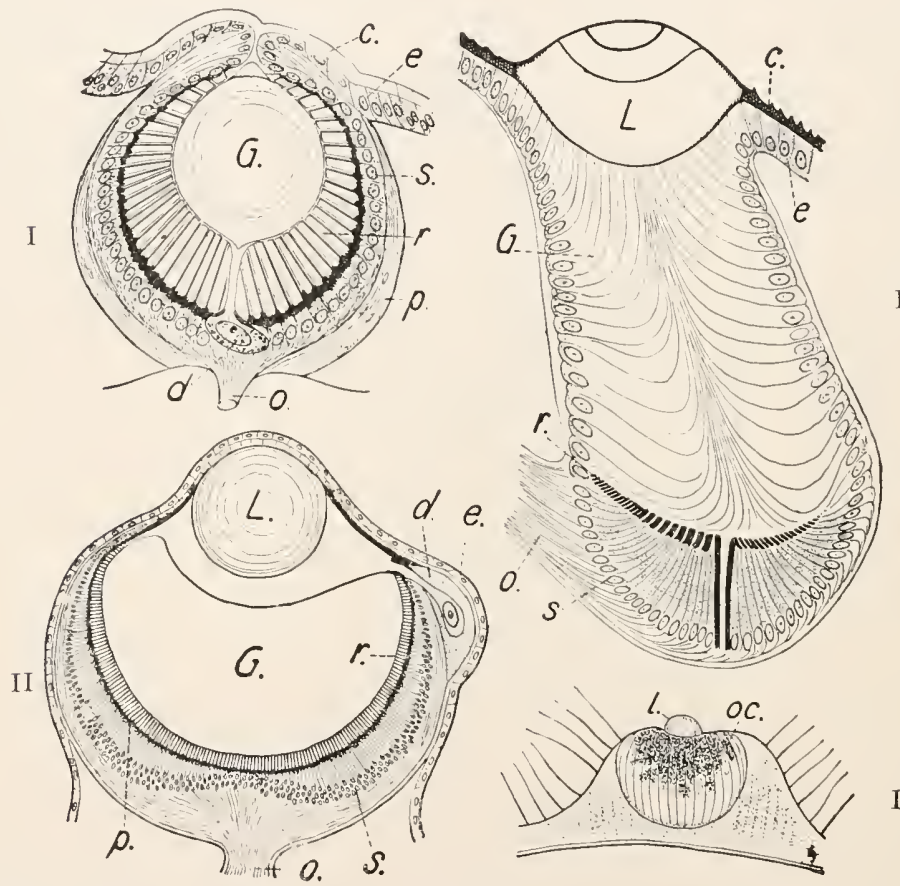

III

FIG. 84.- Invertebrate eyes. I, Phyllodoce (an annelid, after Hesse); II, Nauphanta (annelid, after Greef and Hesse); III, larva of a beetle, Acilius, after Grenacher; IV, a medusa, Lizzia; $c$, cuticle; $d$, gland cells which secrete the vitreous body; $e$, epidermis; $G$, vitreous borly; $L$, lens; $o$, optic nerve; $o c$, ocellus; $p$, pigment; $r$, rhabdoms of the retina; $s$, visual cells.

collecting in or shunning the lighted spot (positive and negative phototaxis). Phototaxis occurs, even when there are no special organs for the recognition of light (Infusoria, Hydra, many worms). It is increased when there are visual cells, that is light percipient spots connected with nerves. These may be on the surface, or deeper in position, if the overlying layers be translucent (earthworms, Amphioxus). If numerous visual cells be united into a layer this is called a retina. In the lowest developmental 
stages the visual cells are closely related to accumulations of pigment which occur either in or surrounding the cells. That this pigment is not absolutely essential for light perception is shown by the visual powers of albinos which are free from pigment, but it clearly must increase the sensitivity of the cells, for pigmentation is so common that the simplest eyes may be defined as sharply defined pigment spots, to which there is frequently added a lens to concentrate the light (fig. $8+$, III).

Eyes.-From such beginnings, which are evidently only intended to recognize light and darkness, there are all transitions to the image-forming eyes of the vertebrates and apparently the cephalopods. The retina is rendered more efficient by the development of rhabdoms on the peripheral ends of the visual cells, rod-like processes which aid in light perception, and in the vertebrates usually divided into rods and cones (fig. 85, 9).

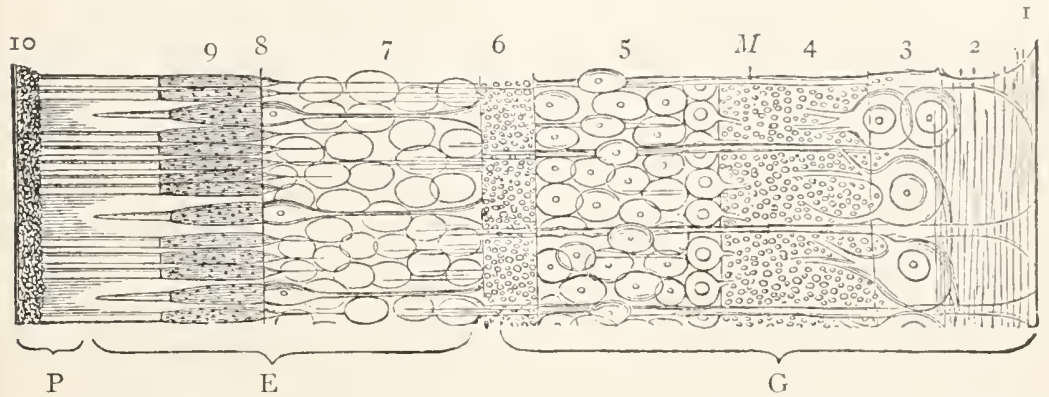

FIG. 85.-Human retina (after Gegenbaur). P, pigment-layer; $E$, layer of sensory cells; $\mathrm{G}$, optic ganglion; $\mathrm{I}$, limitans interna: 2, nerve-fibre layers; 3 , ganglion cells; 4 , inner reticular layer; 5 , inner granular layer; 6 , outer reticular layer; 7 , outer granular layer; 8 , limitans externa: 9 , rods and cones; Io, tapetum nigrum; .1 , Müller's fibres.

In the vertebrates and many invertebrates the retina contains a reddish pigment, the 'visual purple,' which is quickly bleached in the light and as quickly regenerated in darkness, and which apparently plays an important part in vision. In the course of the optic nerve there are numerous ganglion cells which form an optic ganglion (figs. 85 and 356 ), lying outside the eye in the invertebrates, in the vertebrates forming a number of layers (G, fig. 85), inside the retina proper (E), which is formed of the visual celis (outer granular layer) with the fibres of the rods and cones and the rhabdoms themselves.

Accessory Structures.-If a sharp image is to be cast on the retina, the light rays coming from a point without the eye must be brought again to a point on the retina by refractive substances (lens, cornea); therefore there must be a space between the dioptric apparatus and the retina. The 
eye is therefore developed as a camera obscura, the space between retina and lens being filled by the vitreous body (transparent cells or jelly-fig. 84 ). The amount of light is regulated by an iris, a pigmented membrane with circular opening, the pupil, the width of which is enlarged or contracted in accordance with the intensity of the light. Then nutrition is provided by a richly vascular coat, the chorioidea, and for protection there is a firm outer coat, the sclera. These accessory structures are developed and combined in the most diverse ways in the different classes of animals; eyes which are very similar in structure, like those of vertebrates and cephalopods (figs. 86, 349), have developed along entirely different ontogenetic and phylogenetic lines.

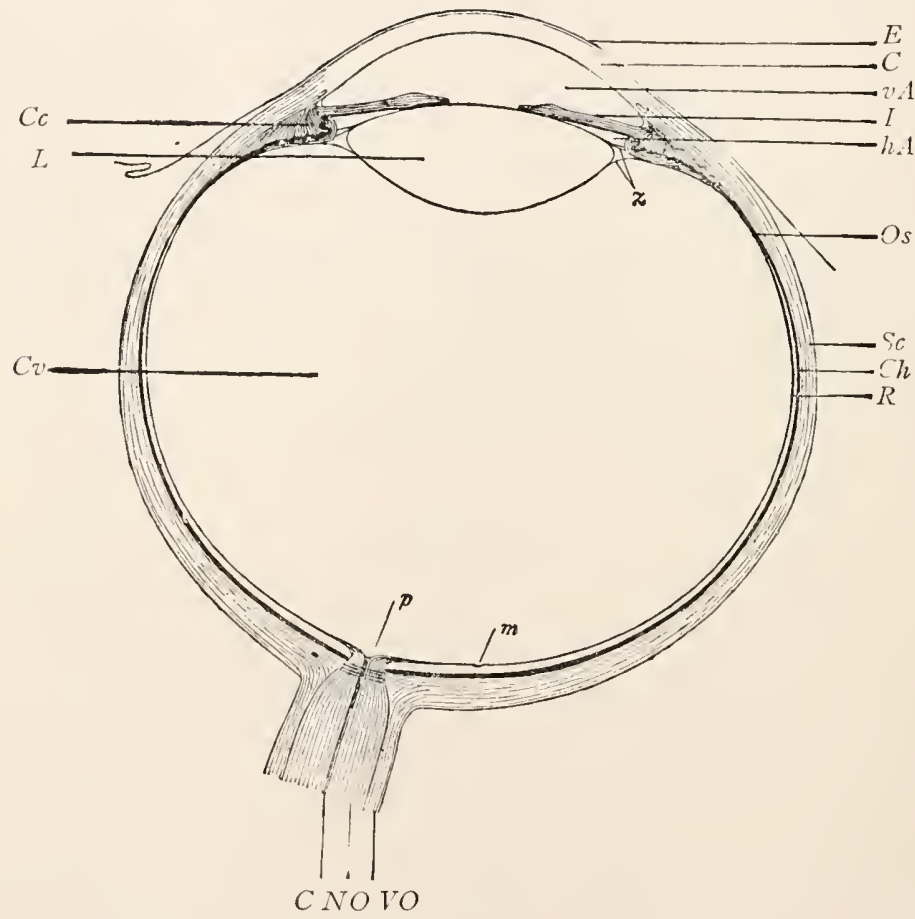

FIG. 86.-Horizontal section through the human eye (after Arlt, from Hatschek). $E$, epithelium of the cornea (conjunctiva); $C$, cornea; $v A$, anterior chamber of the eye; $I$, iris; $h A$, posterior chamber of the eye; $Z$, zonula Zinnii; $O s$, ora serrata; $S c$, sclerotic coat; $C h$, choroidea; $R$, retina; $p$, papilla of optic nerve; $m$, macula lutea, area of most distinct vision; VO, sheath of the optic nerve; NO, optic nerve; $C$, arteria centralis; $C c$, corpus ciliare; $L$, lens; $C v$, vitreous body.

The Eye of the Vertebrates.-The eye of the vertebrates usually is an approximately spherical body. Over the greater part of the circumference there is an opaque, fibrous or cartilaginous sclera, or sclerotica, transparent only in the most anterior part, where it forms a projecting portion like a watch-glass, 
the cornea. Internally to the sclera lies the chorioidea, which, at the junction of sclera and cornea, is changed into the iris. The iris, the seat of the color of the eye, is pierced by the pupil, which regulates the amount of hight. Next internal to the chorioid follows a layer of black cells, the tapetum nigrum (pigmented epithelium), and finally the retina itself, the expansion of the optic nerve which enters the eye at the hinder part. The tapetum nigrum and the retina arise together, and hence both end at the edge of the pupil, although the retina loses its nervous character at the ora serrata, some distance from the outer edge of the iris.

The cavity of the eye is completely filled by the vitreous body, aqueous humor, and the lens. For vision the lens is the most important, since, next to the cornea, it influences most the course of the rays of light. It lies behind the iris, fixed to the anterior wall of the chorioid, which here is changed into the ciliary process. In front of it is a serous fluid, the aqueous humor, partly in the so-called posterior chamber of the eve, between the lens and iris, partly in the anterior chamber, between the iris and cornea. The single, larger cavity behind the lens is filled up by a jelly-like vitreous body. The image formed on the retina is inverted.

Shining of Eyes.-In many vertebrates there is a tapetum. lucidum inside the chorioid which causes the so-called shining of eyes (cats). This is a layer, which reflects light so strongly that only a little light from the outside is necessary to illumine the back of the eye. There is no real production of light. The tapetum nirrum must be free from pigment in order that the tapetum hucidum may act. In many insects and spiders light is similarly reflected from the back of the eye.

Phosphorescent Organs.-For a long time eye-like organs have been known, especially in animals from the deep seas (fishes, cephalopods, crustacea). Many of these have been proved to be organs for the production of light, and the same is probably true of the others. Each is a spherical, eye-like body, arranged in a definite manner in the skin and of very

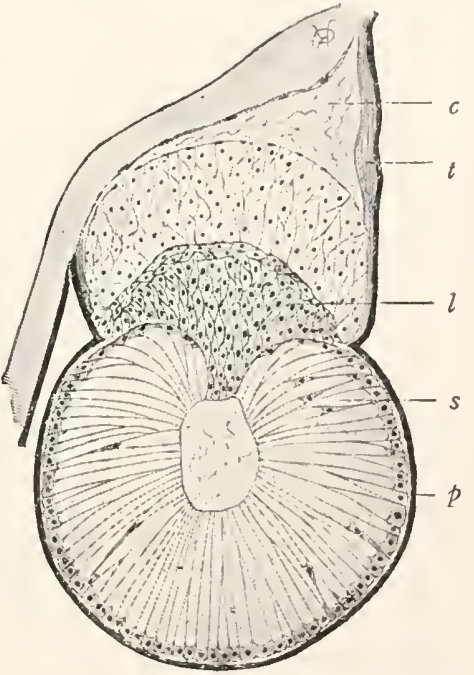

Fis. 87.-Phosphorescent organ of Chauliodus (after Brauer). c, cutis: $l$, lens; $p$, pigment layer; $s$, phosphorescent secretion cells; $t$, reflecting tapetum. varying structure. Many have a great resemblance to glands (fig. 87 ). The cells of the gland follicle are apparently to secrete the phosphorescent substance, its light being made more effective by a lens-shaped body of transparent cells and by a reflector (not always present) consisting of strongly iridescent cells, and all surrounded by a pigment layer, the whole being so eye-like that they were at first taken for visual organs. We have in these to do with highly specialized structures differing from the phosphorescent apparatus so common in marine animals of all classes, where (Noctiluca, medusæ, corals, etc.), the phosphorescent substance is widely distributed through the body. Perhaps the concentration of the phosphorescence in definite organs may serve to light the surroundings, to attract the prey and perhaps as an attraction between the sexes. In the latter case there would be an analogy with the phosphorescent organs of insects, which are formed in a totally different way. 
SUMMARY OF THE MOST IMPORTANT POINTS OF ORGANOLOGY.

I. Organs are tissue complexes, differentiated from the surrounding structures by a definite form and adapted to the performance of a peculiar function; consequently each organ can be classified morphologically (according to structure and relations) and physiologically (according to function).

2. Organs of different animals may be physiologically equivalent, analogous organs (i.e., with similar functions).

3. Organs of different animals may be morphologically equivalent, homologous (developing in similar relations).

4. In the comparison of the organs of two animals three possibilities become evident. $a$. They may be at the same time homologous and analogous. b. They may be homologous, but not analogous (swimbladder of fishes, lungs of mammals). c. They may be analogous, but not homologous (gills of fishes, lungs of mammals).

5. Organs are divided into animal and regetative according to function.

6. Animal functions are those which are only slightly developed in plants; in the animal kingdom, on the contrary, they undergo an increase and become characteristic.

7. Vegetative functions are developed with equal completeness, though in a different manner, in plants and animals.

8. Animal organs include the organs of motion and sensation, such as muscles, sense-organs, nervous system.

9. To the vegetative organs belong the organs of nutrition and reproduction.

Io. Under mutrition, in the widest sense, are included not only the taking in and digestion of food and drink, but also the taking in of oxygen (respiration), the distribution of food to the parts of the body, and the removal of matter which has become useless.

II. With nutrition, therefore, are concerned not only the digestive tract and its accessory glands, but also the organs of respiration, the blood-vascular system, and the excretory organs (kidneys).

I2. The male and female sexual organs serve for reproduction.

I3. The male and female organs may occur in different individuals (dicecious), or both may be found in one and the same animal (hermaphroditic).

I4. The highest degree of hermaphroditism is attained when one and the same gland (the hermaphroditic gland) gives rise to both eggs and spermatozoa.

15. Very often the sexual organs and the ducts from the kidneys are closely united; we then speak of a urogenital system. 


\section{Promorphology; the Fundamental Forms.}

The structure of the individual animal depends u on the definite arrangements of its organs, which are definite or only vary slightly in each group). Comparison shows that there are a few fundamental forms which play the same rôle in morphology as the crystal forms in mineralogy. But there is an important difference. A crystal is made up of similar parts, its form is the result of its physico-chemical composition. 'This condition cannot exist in animals as each organ is a complex of many chemical compounds. Nor, even where the symmetry is the most pronounced is there that mathematical accuracy found in cristals.

The form of an animal depends upon its extension in space, and accordingly we may pass through it three axes at right angles to each other, these defming the position of three planes. According to the relations of the body to these we may define five fundamental forms.

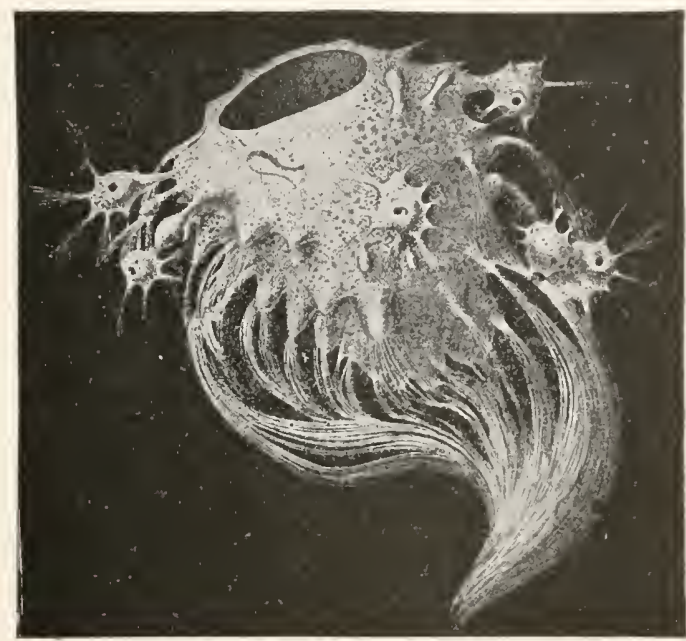

FIG. 88.- Sponge, Lophocalyx philippensis, with buds (after F. E. Schulze).

Asymmetrical (anaxial) animals (fig. 88) are such as have no definite arrangement of parts with regard to axes or planes, the body growing irregularly in any direction as in many sponges and protozoa.

Spherical (homaxial) animals have the parts arranged around a central point, through which innumerable axes and planes may be passed, each plane dividing the whole symmetrically as in some spherical protozoa, chietly radiolaria (fig. 89).

In radial (monaxial) symmetry there is a main or longitudinal axis which lies in the direction of growth. It may be longer, shorter or of the same length of the other axes, but it may be distinguished by the fact that it passes through parts, as the mouth, which are not found in other axes. Around this main axis the parts of the body are symmetrically arranged, like the spokes of a wheel, so that any plane passing through the main axis will divide the body into symmetrical parts. Most colenterates and echinoderms are more or less completely radially symmetrical (fig, 90). 
In the case of biradial symmetry (fig. 9I) there is the main axis, as in the last, and two other unequal axes at right angles to this, the inequality consisting in that organs occur in the line of the one that are not found in the other. One of these is called the sagittal axis, the other the transverse. Planes passing

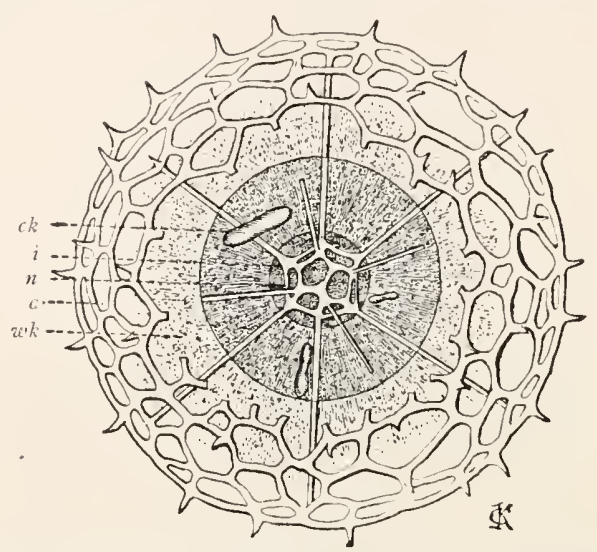

FIS. 89.- Haliomma erinaceus, a radiolarian. a, external, $i$, internal, latticed spherical skeleton; $c k$, central capsule; $w k$, extra-capsular soft parts; $n$, nucleus.

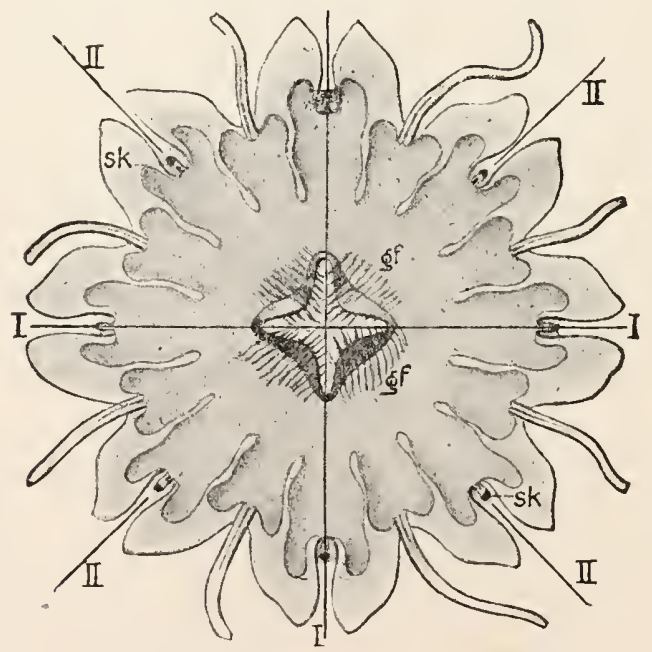

FIG. 90.-Young Chrysaora (after Claus). I, perradii; II, interradii; $g f$, gastral filaments; $s k$, sensory pedicels.

through the main axis and either of the others will divide the animal symmetrically. Corals, sea anemones and ctenophores belong here.

Bilateral symmetry has the same three axes, the two ends of the main or longitudinal axis being dissimilar, as well as those of the sagittal axis. These axes define three planes which have received names. That passing through the 
main and the sagittal axis is the sagittal or median plane and it divides the animal into symmetrical halves. A frontal or horizontal plane passes through the longitudinal and transverse axis, separating dorsal and ventral halves.

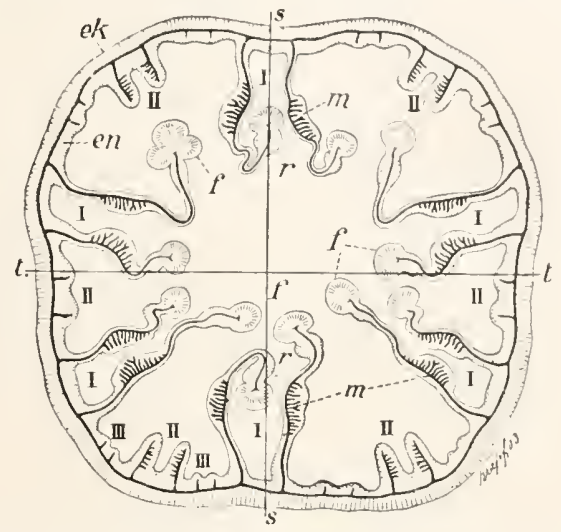

FIG. 9r.-Section of a young sea anemone (after Boveri). ss, sagittal plane; $t$, transverse axis; I, II, III, septa of first, second and third orders; $e k$, ectoderm; $e n$, entoderm; $f$, mesenterial filament; $m$, muscles; $r$, directive septa.

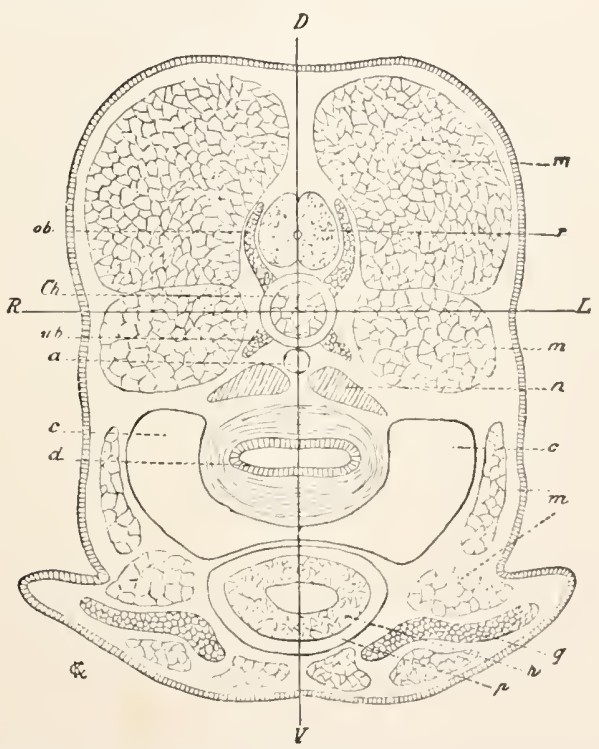

FIG. 92 - Cross section of a fish passing through the fore limbs. $D T$, sagittal axis; $R L$, transverse axis; $a$, dorsal aorta; $c$, body cavity; $d$, gut; $c h$, notochord; $g$, shouldergirdle; $h$, heart; $m$, muscles; $n$, anterior end of the kidneys; $p$, pericardium; $o b$, neural arch; $u b$, hæmal arch; $r$, spinal cord.

A transverse plane passes through transverse and sagittal axes, separating anterior and posterior parts of the body. The great majority of animals belong liere (fig. 92). 
Antimeres and Metameres.-The symmetrical parts of an animal are called antimeres; each antimere has organs which occur likewise in its adjacent antimere. The right arm of man is the antimere of the left, the right eye of the left, etc. Frequently there is also a repetition of organs in the direction of the long axis. Thus the body is made up not only of symmetrical parts, the antimeres, but also of similar parts placerl one behind the other, the metameres.

Metamerism or segmentation is spoken of when the body consists of numerous segments or metameres (consult fig. 60). Very often it is recognizable cxternally-when, for instance, the limits of the segments are marked on the surface by constrictions (arthropods and annelids). But this extemal metamerism may be entirely lacking, and the metamerism find expression only internally in the serial succession of organs. Man, for example, is segmented only internally; in his skeleton there are numerous similar parts, the vertebre, which follow one another in the long axis. In fishes the musculature also is made up of numerous muscle segments, as any one can readily see by examining a cooked fish. In the case of the externally segmented earthworm also, the ganglia of the nervous system, the vascular arches, the nephridia or segmental organs, the setæ, and the septa of the body cavity are repeated metamerically.

Homonomous and Heteronomous Metamerism.-The examples mentioned are well adapted for illustrating homonomous and heteronomous metamerism. The earthworm is homonomously metameric, because the single segments are much alike in structure, and only slight differences exist between them. Man and all rertebrates, on the contrary, are heteronomously metameric, because the successire segrments, in spite of many points of agreement, have become very unlike. The segments of the head have an importance, for the organism as a whole, quite different from those of the neck, the thorax, or the tail. A division of labor has taken place among the segments of an heteronomous animal.

Heteronomy and Homonomy.-The distinction between heteronomy and homonomy is of great physiological interest. 'I he more different the segments of an animal become the more dependent they are upon each other; so much has the whole become unified that the single parts can live only while the continuity is maintained. On the contrary, if the connection between the parts be less intimate, they are more similar, and the more able to exist after separation from one another. This is well shown in instances of mutilation. When many species of Lumbricidæ are cut in two each part not only lives, but it even regenerates the part which is lacking; if, on the other hand, the same thing is done to a heteronomously segmented animal, either death immediately ensues, as in the case of the higher vertebrates, or the parts live for a short time a hopeless existence, as can be seen in the case of frogs, snakes, insects, etc. There is always a certain capacity for regeneration, which is the more restricted, the more complete the organization. While crustacea, amphibia and reptiles 
can, for instance, regenerate lost appendages, the mammals have the regenerative powers reduced to the healing of wounds. In metamerism a phenomenon? is repeated which obtains widely in the animal kingdom, and contributes towards its higher development; first there is a reduplication of part (here the segments), then a division of labor, so that the final result is a whole composed of many parts, but a singly organized whole.

\section{GENERAL EMBRYOLOGY.}

Origin of Organisms.- Since the development of every individual begins with an act of generation, the ways by which new organisms may arise comes first. Admitting only that which has been actually observed, we must cling to the aphorism of Harvey, "Omne vivum ex ovo," altering it to Omne virum e vivo: every living organism is derived from another living organism. We must limit ourselves to the mode of origin which has been termed tocogony, or generation by parents. The great importance which the question of generation without parents, or spontaneous generation, has obtained through the evolution theory renders a consideration of this question necessary at this point.

\section{Generatio Spontanea (Archegony, Abiogenesis).}

The old zoologists, including Aristotle, believed that many animals, even frogs and insects arose spontaneously from the mud. This was not disproved until the seventeenth and eighteenth centuries, and even then the idlea of spontaneous generation still held, especially for parasites, for in the history of each animal there was a time when it contained none of these, and they were supposed to arise from the superfluous plastic material of the host. Later it was found how the eggs obtained entrance, and then the idea of abjogenesis persisted only for microscopic organisms. Water, which contained no living thing, after standing a while, was found to contain organisms. Lastly it was discovered that these do not arise de nov'o, but come from minute germs, carried by the winds, or distributed in other ways. If the fluids and the utensils are heated, and germs are prevented from entrance by proper means, no life will appear, even if the medium be kept for years. So it may be said, as the result of all recent experiment, that the present occurrence of spontaneous generation is not proved.

First Origin of Life.-If we adopt the view that our earth was at one time in a molten condition and has gradually cooled, we must assume that life has not existed on the earth from eternity, but at some time has had its beginning. If we wish to base our explanation, not upon a supernatural act of creation, nor upon hypotheses like that of the transference of living germs from other worlds by meteors, there is left only the hypothesis that compounds of carbon, 
oxygen, hydrogen, nitrogen, and sulphur have been brought together to produce living substance. This process is called spontaneous generation. If the carbon, oxygen, nitrogen, etc., which are now combined in a stable manner in organisms were formerly unstable, the conditions for the origin of compounds, through whose wider combination life would be possible, may have been more favorable. Thus the hypothesis of the first origin of life through spontaneous generation is carried to a logical postulate.

\section{Generation by Parents, or Tocogony.}

We deal here only with those methods of reproduction which have actually been observed, i.e., generation by parents. These methods fall mainly into two great groups, asexual and sexual generation, monogony and amphigony, to which may be added a third group, a combination of the two.

\section{a. A sexual Reproduction. Monogony.}

Monogony Defined.-The chief characteristic of asexual reproduction is the fact that only a single organism is necessary. But since, in certain modes of sexual reproduction (hermaphroditism, parthenogenesis), this also holds true, further explanation is necessary. Asexual reproduction must be a result of the growth of the organism, which has the peculiarity that it is not growth for an existing individual, but leads to the formation of new individuals. It is noteworthy in this connection that many animals can reproduce asexually before they have reached the normal size (budding in embryo and larval polyzoa and tunicates). This growth may be general and result in an equal growth of all parts; or it may be local and consequently lead to the formation of an outgrowth in the region of greatest increase. In the first case division takes place, in the latter budding.

Division.-In the case of division ( $c f$. figs. I20, I23, I50) an animal separates into two or more equivalent parts, so that it is not possible to distinguish the mother and the daughter animal, for the original animal has completely disappeared in the young generation. The division is commonly a transverse one, the plane of division being perpendicular to the long axis of the animal; less common is longitudinal division, rarest is oblique.

Budding.- In budding (fig. 93), the products are unequal. One animal maintains the identity of the mother, while the bud, the outgrowth caused by local increase, appears as a new formation, as the daughter individual. Yet the difference between division and budding is bridged by intermediate conditions. 


\section{b. Sexual Reproduction: Amphigony.}

Amphigony.-For sexual reproduction two animals are commonly necessary, a female and a male; the reproductive cells-the eggs-of one must be fertilized by the reproductive cells-the spermatozoa-of the other, and thus acquire the capacity of giving rise to a new organism. Now, since there are hermaphroditic animals and since with many of them the possibility of self-fertilization has been demonstrated, it becomes clear

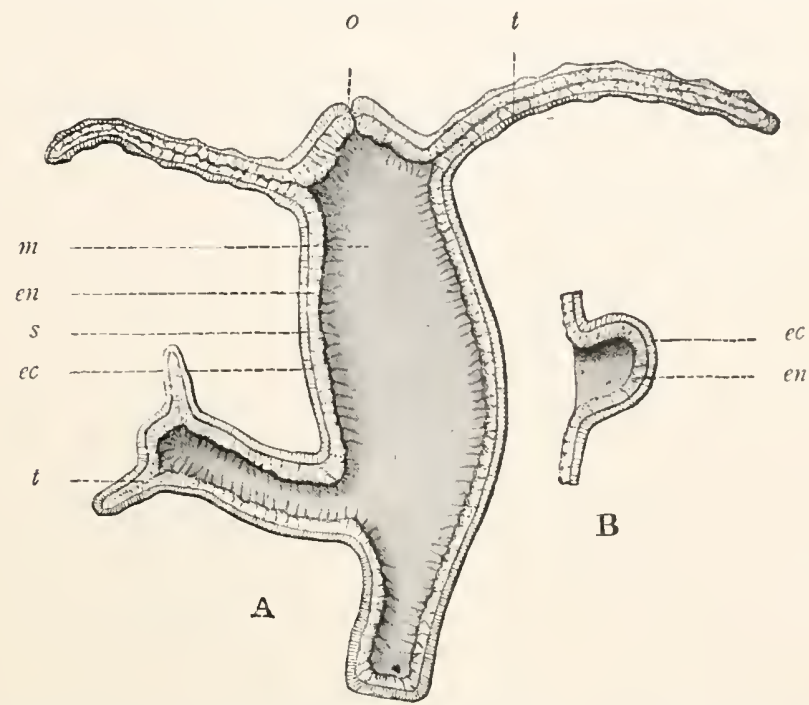

FIg. 93.-A, $H$ ydra grisea with a bud; $R$, first stage of bud. en, entoderm; er, ectoderm; $s$, supporting lamella; $t$, tentacle of mother and bud; $m$, stomach; $o$, mouth.

that the emphasis in the definition of sexual reproduction must be lairl, not upon the individual, but upon the sexual products. Consequently the essential point of sexual reproduction is to be sought in the union of male and female sexual cells.

Parthenogenesis and Pædogenesis.-This explanation is applicable to by far the greater majority of cases, namely, to all cases where the term sexual reproduction can be applied. Still, it has been demonstrated in many instances that two modes of reproduction formerly considered as monogony-parthenogenesis and predogenesis-must be regarded as modifications of sexual reproduction, although the conditions mentioned above are not strictly satisfied. In both cases the eggs develop because of some peculiar internal stimulus, without the occurrence of fertilization by spermatozoa. In case of padogenesis there is the addi- 
tional circumstance that reproduction is accomplished by animals which have not completed their normal development; for example, the larvæ of certain flies reproduce before they have passed through the pupal stage. Pædogenesis consequently is parthenogenesis in an immature organism.

Parthenogenesis and Typical Amphigony.-There is no absolute distinction between parthenogenetic eggs and those needing fertilization. On the other hand, their equivalency is fully shown in cases as the bees, where the queen decides at the moment of oviposition whether the egg shall receive a spermatozoan or not, this decision determining further whether the egg shall develop into a female (fertilized) or a male (unfertilized). Parthenogenesis is, therefore, not an asexual reproduction which was antecedent to sexual reproduction, but rather one which must have been derived from the sexual; it is a sexual reproduction in which a degeneration of fertilization has taken place. It is, therefore, more in accord with the natural relations to contrast reproduction by sex-cells with vegetative or growth reproduction (division, budding) rather than asexual with sexual reproduction.

Sexual and Somatic Cells.-The distinction of sexual cells from the asexual reproductive bodies, the parts arising by division and budding, is shown by their relations to the vital processes of animals. The cells of a bud had a share in the vital processes of the animal before the beginning of reproduction; they were functional or somatic cells. In the fresh-water polyp (fig. 93), when a bud arises, the cellular material employed is that which was previously related to the mother animal in exactly the same manner as the other parts of the body wall. The sexual cells of an animal, on the contrary, are excluded from the vital processes, remaining in a resting condition, and conserving their vital energies. Asexual reproduction is closely related to growth; sexual reproduction is not even a special form of it, but a complete renewal of the organism, a rejuvenescence of it. This explains the fact that asexual reproduction is most common in the lower animals (cœlenterates, worms), but is lacking from vertebrates, molluses, and arthropods. The higher the organization of the animal the more the energies of its cells must be employed to meet the increasing demands upon their functional capacity, and so the more necessary is sexual reproduction. It is farther noteworthy that fission and budding occur most frequently in attached, sessile, or slightly moving animals (cœlenterates, polyzoa, ascidians, oligochætes), an indication that the distribution of asexual reproduction may be determined by the method of life.

\section{c. Combined Modes of Reproduction.}

Very often two modes of reproduction occur in the same species side by side. Many corals and worms have the power of multiplying by division or budding, and also of forming sex cells; other animals have no asexual reproduction, but their eggs develop according to circumstances, either parthenogenetically or after fertilization. The appearance of two kinds of reproduction is very often governed by the fact that individuals with 
different modes of reproduction alternate with each other. This is called altemation of generations in the wider sense, and of this two special forms are distinguished: metagenesis (progressive alternation of generations), and heterogony (regressive alternation of generations).

Metagenesis.-Alternation of generations in the narrower sense, or metagenesis, is the alternation of at least two generations, one reproducing only asexually, by division or budding, the other exclusively, or at least to a great extent sexually. The first generation is called the nurse, the second the sexual animal. The reproduction of hydromedusx furnishes the best examples (fig. 94). The nurses here are the polyps, which

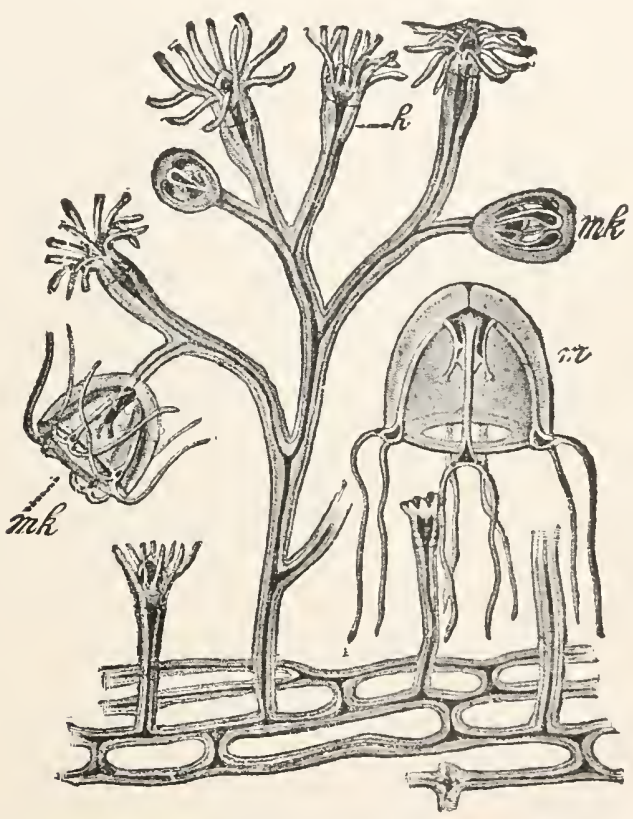

Fig. 94.-Bougainvillea ramosa (from Lang). h, hydranths (nurse) which have given rise to medusa-buds $(m k) ; m$, separated medusa, Margelis ramosa (sexual animal).

usually united into a colony, never produce sexual organs, but bud sexual animals, the medusa. The medusæ are unlike the polyps, being much more highly organized, and freely motile; only very rarely do they reproduce asexually; on the other hand, they develop eggs and spermatozoa, from which the non-motile nurses, the polyps, develop. This example shows that, in alternation of generations, there is not only a difference in the mode of reproduction, but usually in addition, a difference in form. 
and organization. Betwecn polyp and medusa the difference is so great that for a long time these two, though stages of the same species, were referred to different classes of the animal kingdom. In many cases the alternation of generations may be still further complicated by two asexual generations following each other, before the return to the sexual generation takes place.

Heterogony is distinguished from metagenesis by the fact that the asexual generation is replaced by parthenogenesis. Consequently there alternate animals of sometimes quite different structure, one arising from fertilized, the other from unfertilized, eggs. Certain crustacea, the Daphnidæe, show heterogony in a typical manner. During a large part of the year only females are found; these increase parthenogenetically by 'summer eggs'; then males appear for a short time; they fertilize the 'winter eggs,' yhich now are formed, from which again parthenogenetic generations arise. Very often heterogony has been insufficiently distinguished from metagenesis, parthenogenetic reproduction being regarded as an asexual mode, as was the case in the trematodes. The sexually ripe Distomum produces very peculiar sporocysts; these again give rise parthenogenetically to the larvæ of Distomum, the cercariæ. For a long time the erroneous view was held that the cells from which the cercarie arose were not eggs, but 'internal buds'. On the other hand there have been included under heterogony modes of reproduction in which no parthenogenesis whatever occurs, but in which only different forms and organization alternate. A hermaphroditic worm, formerly called Ascaris nigrozenosa, lives in the frog's lungs; it produces the separatesexed Rhabdonema nigrovenosum living in mud, from whose eggs the ascarid of the frog is again produced.

\section{GENERAL PHENOMENA OF SEXUAL REPRODUCTION.}

In sexual reproduction a series of developmental processes is observed which is repeated in an essentially similar manner in all multicellular animals. They are: (I) the maturation of the egg; (2) the process of fertilization; (3) the process of cleavage; (4) the formation of the germlayers.

\section{Maturation.}

The egg (oocyte) with the large vesicular nucleus cannot yet be fertilized; it must undergo a series of changes-the process of maturation, which consists in the replacement of the germinal vesicle by a much smaller egg-nucleus, and the formation at one pole of the egg of the 'directive corpuscles' or 'polar bodies' 
Formation of the Polar Bodies.-The germinal vesicle initiates these changes, its walls disappearing, its contents in part mingling with the cytoplasm of the egg, in part being employed in the formation of a nuclear spindle (directive spindle). The latter places itself with its axis in a radius of the egg so that one pole is turned towards the centre, the other being in the superficial layer of the egg (fig. 95, A). Now begins a regular cell-division, but the products of the division are of very unequal size; the larger part is the egg, the smaller quite insignificant part is the polar body (fig. $95, C)$. The latter projects above the surface carrying with it one half of the spindle, and when the globule is cut off half of the spindle is included in it.

The Second Polar Body.-The part of the directive spindle remaining in the egg immediately forms a new spindle; the cell-budding is repeater $(C, D)$ and leads to the formation of the second polar body. As a result two small cells (fig. 95, E) lie at one pole of the egg, in many cases even three, since during the formation of the second polar body the first may divide. The part of the directive spindle remaining after the second division becomes a vesicular resting nucleus, the eggnucleus or female pronucleus, the characteristic feature of the ripe egg capable of fertilization. In other words, by a double division there have been formed from the immature egg four (sometimes three) cells, of which one has retained by far the greatest part of the original mass of the cell and constitutes the ripe egg, while the others are small bodies like rudimentary eggs. The name directive corpuscles was given to them because in very many cases their position renders possible a definite orientation of the egg; i.e., a diameter, the main axis, can be passed through the egg, one end of which is marked by the directive corpuscles. With reference to later processes of development this end is called the animal pole of the egg, the opposite end the vegetative pole.

\section{Relation between Maturation and Fertilization.-}
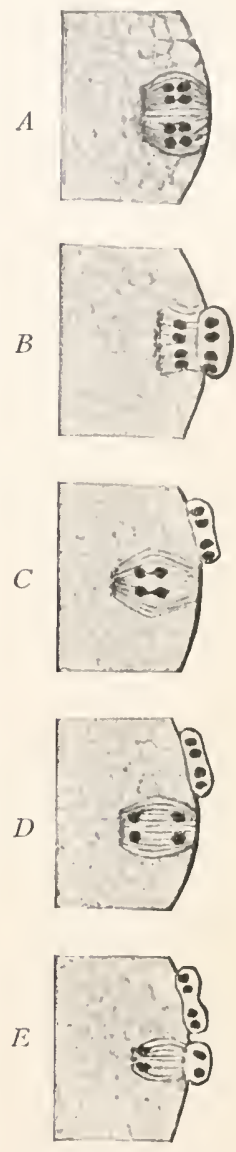

FIG. $95 .-$ Formation of polar globules in Ascaris megalocephala (diagrammatic, after Boveri). A, first directive spindle; $B$, culting off of first polar body; $C$ and 1 , two stages of the second spinale; $E$, separation of second polar body.

In many cases the maturation takes place before the entrance of the sperm, either in the ovary or at the beginning of the oviduct; in many animals, on 
the contrary, there ensues a pause after the first polar body has been formed, or the egg may remain in the oocyte stage; the egg then requires the entrance of a spermatozoon in order to complete the further changes, i.e., the formation of the second polar body and reconstruction of the egg-nucleus. This dependence of the last phenomena of maturation upon the beginning of fertilization led for a long time to the error that the formation of the polar bodies was a part of the fertilization process itself.

Spermatogenesis.-The maturation of the egg has its counterpart in the formation (maturation) of the spermatozoa-spermatogenesis. As the oocyte, by division, gives rise to four cells (the polar globules and the ripe egg), so the spermatocyte, a cell comparable to the oocyte, divides into four spermatids. Yet the two differ in that usually all four spermatids become spermatozoa. That three of the four sex-cells of the female remain rudimentary (polar globules) the fourth alone forming an egg, is explained by the need of the egg to contain all possible material for use in development.

Reduction Division. - In the maturation division of both male and female sex-cells agree in the chromosome reduction. This is due to the fact that the maturation spindles have but half the number of chromosomes characteristic of the species. Usually these chromosomes are distributed in four groups (tetrads) in the preparatory stages of the egg, the tetrads later being distributed among the four products of the divisions (fig. 95). The significance of this will be shown in connection with the phenomena of fertilization (p. I38).

\section{Fertilization.}

Copulation and Fecundation.-The term fertilization refers to the internal processes which, after the meeting of the egg and spermatozoon, go on in the interior of the former and end with a complete fusion of the two sexual cells; on the other hand, special expressions are necessary for those preparatory processes whose purpose is to render fertilization possible. Very often, but not in all cases, there is an active transfer of the sperm from the male to the female, a copulation. In many marine animals (most fishes, echinoderms, cœlenterates) the eggs and the spermatozoa are discharged into the water, and the union of these (impregnation or fecundation) depends upon chance.

Fertilization.-The process of fertilization begins with the entrance of the spermatozoon into the egg. Usually the egg is surrounded by a gelatinous envelope, the chorion, to which the spermatozoa adhere, and through which they bore until they reach the surface of the egg (fig. 96). But since the chorion, particularly in eggs laid in the air, may be hard and resisting, there exists very often a special arrangement, the micropylar apparatus, for the entrance of the spermatozoon; this may be a single canal extending through the chorion, as in the eggs of fishes, or a group of such canals, as in most insects.

Monospermy and Polyspermy.-Many spermatozoa may reach the egg but normally only one serves for fertilization. The spermatozoon which is in the slightest degree ahead of the others is met by a process of 
the protoplasm (fig. $96, A$ ) by means of which it enters the egg. The egg is now impervious to all others. Only in pathological eggs can two or more spermatozoa enter and then multiple impregnation (di-or polyspermy) occurs. There are means of protection against this abnormal fertilization; one, though not the only one, is the formation of the yolk-membrane, an impermeabte envelope which is suddenly secreted from the surface of the egg, as soon as the spermatozoon has entered. Within the yolk-membrane

A

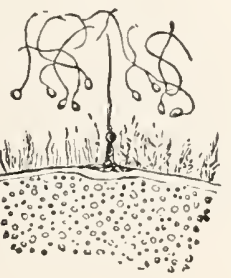

$B$

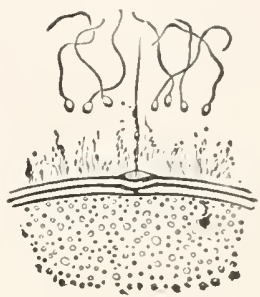

FIG. 96.-Egg of Asterias glacialis during fecundation (after Fol). A, entrance of the spermatozoon; $B$, the spermatozoon has entered; the yolk-membrane has formed.

the body of the egg contracts by discharging some of the more fluid constituents, so that between the yolk-membrane and the surface of the egr a cavity is formed, easily recognized in smaller fertilized eggs (fig. 96, B).

In the large yolk-laden eggs of many insects and vertebrates several spermatozoa may normally enter; but only one fuses with the egg-nucleus, the others degenerating sooner or later.

Essential Feature of Fertilization.-After the spermatozoon has penetrated into the egg, the head and the middle piece containing the centrosome can still be recognized, as the chromatic and achromatic parts of the spermatozoon or sperm-nucleus (male pronucleus), while the tail and the slight amount of protoplasm disappear in the yolk. 'The centrosome of the sperm-nucleus gives rise to rays in the cytoplasm of the egg, like those observed during division. Preceled by these rays the spermnucleus travels towards the egg-nucleus until it reaches (fig. 9i), and fuses with it to form a single cleavage nucleus. The centrosome now divides into two daughter centrosomes, which migrate to opposite poles of the cleavage nucleus and control its division. The cleavage nucleus changes to a clearage spindle, which divides and thus initiates the embryonic development, the successive divisions being known as the cliearage or segmentation of the egg. Since not until this point is fertilization complete, we arrive at the fundamentally important proposition that the essential featzre of fertilization consists in the union of egg and sperm nuclei. 
Part Played by the Two Nuclei.-In many cases an abbreviation of development may take place, the stage of the cleavage nucleus being omitted, and the egg and sperm nuclei, without uniting, pass directly into the cleavage spindle. This in no wise alters the above-mentioned proposition, but yet it is important, because it shows more plainly how the two nuclei
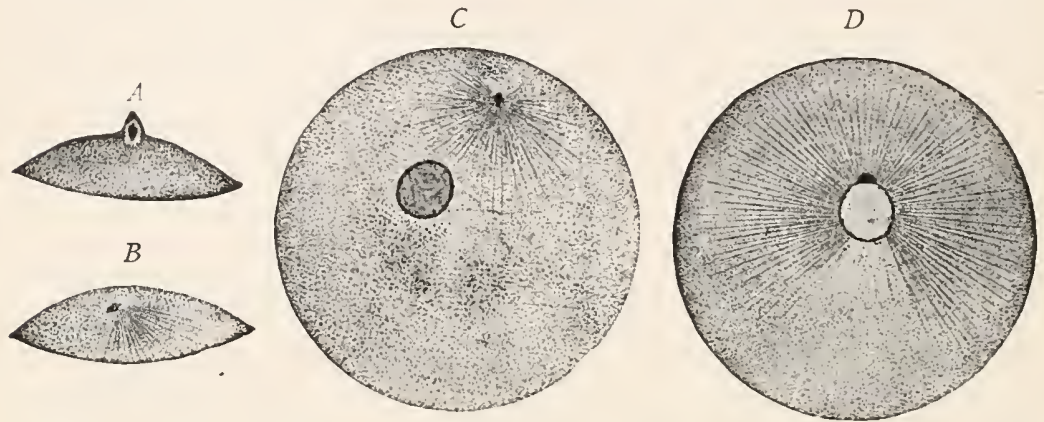

FIG. 07.-Four stages in the fertilization of Strongylocentrotus lividus (after Kostanecki). $A$, entrance of spermatozoon; $B$, turning of sperm nucleus; $C$, approach, and $D$, fusion of egg and sperm nuclci. (In $A$ and $B$ only a part of the egg is shown.)

participate in the formation of the cleavage spindle. It shows that of the chromosomes which form the equatorial plate exactly one-half are furnished by the egg-nucleus, the other by the sperm-nucleus. For, even before the spindle has been formed and the contour of the two nuclei has disappeared, the chromosomes destined for the spindle are completely developed in exactly the same number in each of these (fig. 98 ).
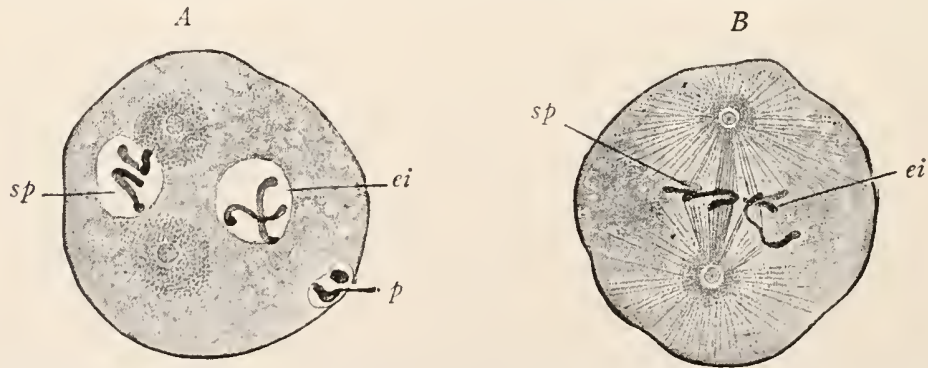

-FIG. 98.-Fertilization of Ascaris megaloccphala (after Boveri). A, the ends (centrosomes) of the spindle formed; $B$, the spindle completed; $s p$, sperm-nucleus with its chromosomes; $e i$, egg-nucleus; $p$, polar bodies.

Heredity.-Recent observations have furnished a certain basis for the theory of hereciity, the transmission of parental characteristics to the offspring. This transmission, on the whole, takes place with equal effect from the father's and from the mother's side; if we take the average of 
numerous cases, the child's peculiarities hold the mean between those of father and mother; or, in other words, male and female individuals on the average have an equal power of transmitting characteristics.

Physical Basis of Heredity.-Since in all animals with external fertilization a material connection between parents and offspring can cxist only through the sexual cells, these latter must contain the substances which render heredity possible; further, the two hereditary substances, in cases of equal capacity for transmission, must be present in the egg and in the spermatozoon in equal quantity. By this course of reasoning, the chromatin which forms the chromosomes has come to be regarded as the bearer of heredity; for we know that the egg contains a great quantity of cytoplasm, but the spermatozoon only the slightest trace of it; that, on the other hand, egg-nucleus and sperm-nucleus furnish equivalent substances, and especially the same number of chromosomes, to the cleavage spindles; hence only the chromatin can be regarded as the hererlitary substance (idioplasm). 'This supports the view expressed before $\left(p .5^{S}\right)$ that the nucleus is the bearer of hereditary qualities and determines the character of the cell.

Theory of Determinants.- These facts of the maturation of the egr and spermatozoa and of fertilization have become the starting point for further investigations and associated theories, which in the last few years have acquired great significance. Their relations have also been shown to the extremely important but long forgotten experiments on inheritance in plants by Miendel.

If we accept the sexual nuclei or their chomosomes as the bearers of heredity, it follows that certain constituents of the chromosomes must contain the anlagen of the characteristics, partly male, partly female, which later develop in the offspring. The simplest setting forth of the connection between the anlagen and the developmental product is Weismann's 'theory of determinants.' 'T his may be taken as a basis for the following discussion, although objections are brought against it. It represents an organism as a complex of innumerable peculiarities, as a sort of mosaic; and in a corresponding way, the anlagal substance, the chromosome mass (idioplasm) as a similar mosaic of anlagal particles, the determinants. There is a determinant for every paternal or maternal characteristic in the offspring, be it prominent or be it latent. The chromosomes must therefore consist of orderly groupings of innumerable determinants.

Merogony, Artificial Parthenogenesis.-Parthenogenesis shows that since an egg may develop, without the entrance of a spermatozoon, into a complete organism, the chromosomes of the egg are sufficicnt to produce all of the features necessary for life. This is more clearly shown by artificial parthenogenesis. Many eggs, which normally need fertilization for development, may be stimulated to develop by number of chemicals. Similarly the male chromosomes are sufficient for normal development, for if an egg from which the nucleus has been removed be fertilized by a single sperm, it likewise forms an organism with all the necessary features (merogony). A fertilized egg must therefore possess duplicate determinants, male and female, for each elementary character, a double assortment of chromosomes. Whether a certain characteristic shall appear purely maternal, or purely paternal in form, or in varying compromises between the two would depend upon the energy of the determinants concerned. 
If a determinant be so strong that its fellow cannot come to expression it is spoken of as dominant, the one that succumbs is recessive.

Mendel's Law.-Long before the phenomena of fertilization were known, Mendel had shown by experiments on plants, that if two individuals, easily distinguished by some peculiarity like color, be crossed, the cross so produced was, in many cases, not a blend of the two, but resembled exclusively one of the two parents. On crossing red and white flowered peas, no matter in which direction, the crosses were invariably red. The determinant for red was so dominant that the recessive determinant for white is powerless. But that the white determinant was present in the cross (according to the morphological conclusions regarding fertilization this must be so) was shown when the red-flowered crosses were self fertilized (fig. 99). Then one fourth of the offspring were

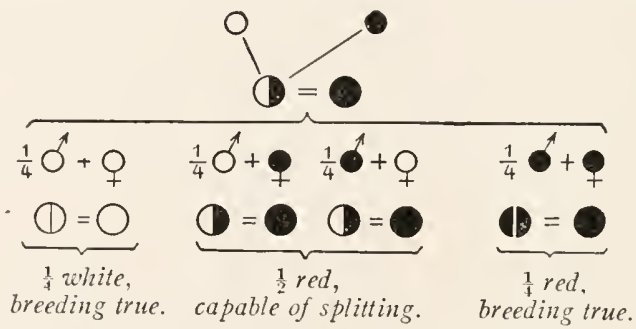

FIG. 99.-Diagram of Mendelian inheritance. Black and white representing red and white flowering kinds.

white and bred true; that is the white color, the recessive character, persisted in all the succeeding generations. Of the remaining three fourths, one fourth also bred true, but red, a sign that the recessive character was lost. Such pure breeding animals are called homozygous because the zygote, the fertilized cell from which they arise, contains, with reference to the peculiarity under investigation, only similar determinants. The remaining two fourths are heterozygotes; that is they possess determinants of two kinds and are thus like the first crossed generation, the red color alone appearing, owing to the dominance of the red determinant over the recessive white. If these be 'split' by breeding, the next generation will consist of one fourth red, one fourth white, each breeding true, and two fourths red with recessive white. If the process be continued, the percentage of homozygotes (pure-breeding forms) rapidly increases at the expense of the heterozygote forms.

Chromosome Reduction.-The results of Mendel's experiments may now be brought into harmony with the phenomena of maturation and fertilization, transferring for this purpose the rôle of the chromosomes to the hypothetical determinants. It has been found in numerous cases that the material of the egg and sperm nuclei-the two assortments of chromosomes-do not fuse with each other but remain distinct in all divisions of the somatic cells (P. I 30 ), a phenomenon apparently applicable to all organisms. Hence the similar determinants, the 'pairlings,' or as they have been called, the allelomorphs (in the example quoted, the determinants for red and white) remain separate from each other in the cleavage cell and in all of its descendants. They persist in the cells of the flower, where, according to their dominance, they determine the color of the blossom; they are also continued in the sex-cells, the oocytes and spermacytes, of the hybrid generation. Now the sex-cells, in the so-called growth period preceding the maturation divisions possess only half the number of chromosomes characteristic of the tissue cells. 
Conjugation of the Chromosomes.- This reduction in the number of chromosomes is explained by the plausible supposition (and this is supported by many observations) that the previously separated paternal and maternal chromosomes, and chromosomes with the same significance, have united (conjugation of chromosomes), and accordingly the allelomorphs, the equivalent male and female determinants unite (in our example the determinants for rerl and white flowers). If in this a complete fusion occur then there would result an inseparable compound of characters, but if it be but a juxtaposition, then in the further divisions of the sex-cells, the so-callerl maturation divisions, both kinds of determinants, paternal and maternal, would be separaterl again. The latter would occur in cases following Mendel's law. The white and red determinants, present but only juxtaposed in the male sex cells, would be separated again in one of the two maturation divisions (reduction division) and would be clistributed among the resulting cells so that two cells would contain only white, the other two only red determinants (fig. roo). The same would occur in the

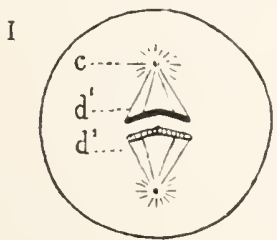

III

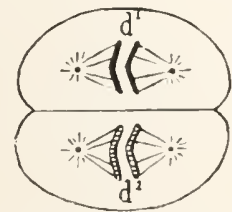

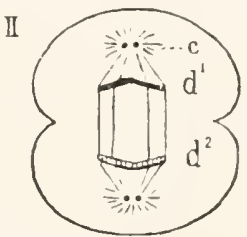

IV

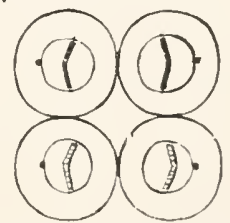

FIG. IOO.- Scheme of the maturation divisions of the sex cells to explain the splitting of hybrid characters. It is based on the idea that the splitting, the rerluction of the chromosomes, occurs in the first maturation division, while many regard the second as the reduction division. I and II, the first, III and IV, the second maturation division. $C$, centrosomes; $d^{1}, d^{2}$, centrosomes derived from different parents, which are distributed among separate sex-cells.

female sex cell, where at the end of maturation resulting in four cells-three polar globules and the ripe egg-two would contain only white, the other two only red determinants. Thus in the maturation divisions the sex cells lose their hybrid character, and we speak of 'purity of gametes.' Now if such material be used, as must be the case in close fertilization of crosses, there are four combinations possible, which according to the law of probabilitics (we know no reason favoring any particular combination) would occur in equal numbers; (a) one fourth red male with red female, producing a true-breeding, homozygote with red flowers, since no other determinant is present; $(b)$ one fourth white male with white female, producing homozygotes with white flowers; $(c)$ one fourth red males with white females; and $(d)$ one fourth white males with red females. The last two fourths would be red-flowered like the first, but according to the composition of their cells, they are heterozygotes like their parents, and like them, can be 'split' according to the same law.

Mendel's law, illustrated above by a single example of hybrid peas, has led to rich results, partly by Mendel himself, partly by his successors (Bateson, 
Correns, Cuénot, Tschermak, Castle, Davenport, DeVries, and others). Some of these matters must be mentioned here.

(I) Mendel's Law Applicable to Animals.-First applied to plants the law is applicable to animals as an interesting case lllustrates. There are two races of the snail, Helix hortensis, which breed true. The shell of one has five parallel bands, the other shell is unicolor and lacks bands. On crossing, the offspring is entirely without bands; bands are therefore recessive. The descendants of the crosses conform to the law and consist of one fourth banded and three fourths of bandless individuals, a third of the latter (a fourth of the whole) being homozygote and breeding true, the other two fourths being heterozygotes, capable of being split in the succeeding generations.

(2) It does not always occur that one character is dominant in crosses; intermediate conditions may arise. Crossing of white and dark red individuals of Mirabilis jalappa produces light red offspring, their descendants being one fourth dark red, three fourths light red, capable of farther splitting.

(3) Di- and Polyhybrids. - The examples given so far deal with only a single character in crossing, and if the varieties used in crossing differ by only a single character, the result is called a monohybrid. There may be di- and polyhybrids where two or more 'pairlings' are concerned. The latter condition is met in peas, which differ not only by color of flower, already mentioned, but by size, position of flowers, color of pod, color and form of peas. If all of these characters were indissolubly connected with each other, were only the external expressions of one and the same fundamental character, there would be, in polyhybrids, the simple relations found in monohybrids. The different characteristics would be inherited together and their relations to dominance would be the same. But usually this is not the case. More commonly each character of a polyhybrid is inherited independently of the others. In this lies the possi bility of taking a single character of a variety, even those which seem to be closely correlated, and by cross-breeding, to separate it and to cause it to be inherited in the most diverse combinations. This brings about very complicated relations in polyhybrids, which can only be made clear by extensive contrasts. Hence, to illustrate the principle, a case of dihybrid is given here, a crossing of two varieties of Indian corn (maize), one with only wrinkled ( $r$ ), blue (b) kernels, those of the other being white (w) and smooth (s). Smooth and blue are the dominating characters. Taking up each character separately, there would follow in the second generation:

For color, $\frac{1}{4}$ bb. $\frac{1}{4} b w=b, \frac{1}{4} w b=b, \frac{1}{4} w w ; a$ total $\frac{3}{4} b, \frac{1}{4} w$.

For form, $\frac{1}{4} r r, \frac{1}{4} r s, \frac{1}{4} s r=s, \frac{1}{4} s s ; a$ total $\frac{3}{4} s, \frac{1}{4} r$.

If now the two characters be combined, we have the following exposition, in which the recessive characters are designated by Italics, the homozygotes by capitals:
$\mathrm{bb}=\mathrm{B}$
$\mathrm{b} z \mathfrak{e}^{\prime}=\mathrm{b}$
$w b=\mathrm{b}$
$w w^{\prime}=\mathrm{IV}^{\prime}$

\begin{tabular}{|c|c|c|c|c|}
\hline $\mathrm{ss}=\mathrm{S}$ & BS & $\mathrm{l} \mathrm{S}$ & $\mathrm{bS}$ & WS \\
\hline $\mathrm{s} r=\mathrm{s}$ & $\mathrm{Bs}$ & $\mathrm{bs}$ & bs & $I^{\prime} \mathrm{s}$ \\
\hline $\begin{array}{l}r \mathrm{~s}=\mathrm{s} \\
r r=R\end{array}$ & $\begin{array}{l}\mathrm{Bs} \\
\mathrm{B} R\end{array}$ & $\begin{array}{l}\mathrm{bs} \\
\mathrm{b} R\end{array}$ & $\begin{array}{l}\mathrm{bs} \\
\mathrm{b} R\end{array}$ & $\begin{array}{l}W \dot{S} \\
W R\end{array}$ \\
\hline
\end{tabular}


In this scheme each character appears once from the father, once from the mother, the result being sixteen combinations, of which nine are blue and smoxth, three are blue and wrinkled, three white and smooth, and one white and wrinkled. Of these four groups only the last $\left(\frac{1}{16}\right)$ is homozygote with reference to both pairlings. Of the other groups there is only one homozygote in each, BS, $B R$, and $W S, \frac{1}{16}$ of each. Of these four homozygote, true breeding forms, two, BR and WS are reversions to the beginning type; the other two, BS and $W R$, are new and constant associations. They are the 'analytic species' to which allusion was made on an earlier page, which have no new characters but only a new combination of characters. Of the other, not true breeding forms, two W's ( $\frac{1}{3}$ of the whole) as to color, w, and two bR, as to shape, are homozygote. Among the blue forms there is the greatest variety: two bS are constant in shape, two I3s constant in color, four bs are heterozygotes in shape and color and are capable of splitting.

It is evident that with every new character entering, the number of combinations possible increases enormously, as does the number of new analytic species. Therefore in high polyhybrids many thousands of descendants are necessary to have all possible combinations actually realized. It is also apparent that species with a low rate of reproduction many combinations nay not appear, others only in distant descendants (atavism).

The numerical relations 9:7 (nine blue-smooth to seven of all others) is important, since it is characteristic of the second generation of dihybrids. Where it occurs it indicates that two pairlings are concerned, even when other facts seem to point to the presence of but one. Thus, in the crossing of white breeds of fowl, only colored animals appear in the second generation, but in the grandchildren there are nine colored, seven white. So one can say, with certainty, that the color depends upon the mutual action of two substances, which must be present simultaneously and as dominants. If these conditions be not fulfilled, the color is lacking and is replaced by white.

(4) Mendel's law is chicfly concerned with the crossing of varieties; it appears to have no significance for species, since with them there usually appear varying degrees of intermediate forms which remain constant in the next generation. But this cannot be proved in most cases, since hybrids are usually infertile. Still it does not appear that a fundamental distinction exists between species and varicties. Farther researches are necessary.

The great significance of the results of Mendel and his successors is apparent. There has been established a wide reaching harmony between the theoretical conclusions based upon the phenomena of maturation and fertilization and facts obtained by crucial experiments. Further, the extraordinarily complex phenomena of inheritance are now capable of expression in exact mathematical form. The knowledge of this regularity of inheritance has an enormous value for practical animal (and plant) breeding, since it affords the foundation for intelligent procedure.

\section{Cleavage Process.}

Arrangement of the Cleavage Planes.-The fertilized egg-cell divides in rapid succession into $2,4,8$, I6, etc., cells, which become continually smaller, since the mass of the egg does not increase. 'The cells are called cleavage spheres, or blastomeres, the whole process the cleavage process, or segmentation, because, at each division, furrows arise on the surface which continue to penetrate more deeply until finally the cells are 
cleft from each other (fig. Ior). As a rule each new plane of cleavage is perpendicular to the preceding. Hence the first three cleavage planes, which cause the division into 2,4 , and 8 parts, are similarly arranged in most animals. Using the globe for comparison, one speaks of a first and second meridional furrow (I, II), and calls the third the equatorial furrow (III). The intersections of the two meridional furrows form the poles of the egg, the animal and the vegetative, so called because the material of the one is used chiefly for animal organs (nervous system), that of the other for vegetative organs (digestive tract).
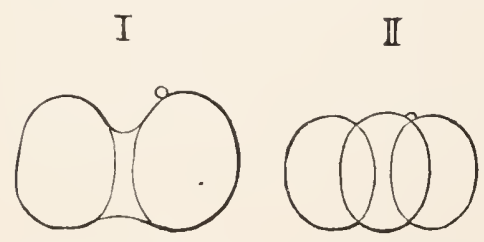

Fig. ror.-The equal cleavage of
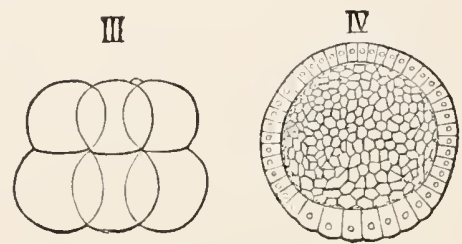
I, division into two (formation of the first meridional furrow); II, division into four (second meridional furrow) forming four cleavage spheres (fourth is hidden); III, division into eight (equatorial furrow; the seventh and eighth cleavage spheres hidden;) $\Gamma$, blastula, in optical section; a single layer of cells surrounds the cleavage cavity. In I, II, III, a polar body is shown.

Influence of the Yolk upon Segmentation.-Different kinds of cleavage are distinguished, the peculiarties of which depend upon two factors: (I) upon the quantity of material (food-yolk) serving for nourishment of the egg; (2) upon its arrangement. The food-yolk hinders the division, since it is incapable of active movement, and is only passively divided through the activity of the protoplasm. The more this increases in proportion to the protoplasm, the more slowly does the cleavage process proceed. Finally there comes a point where the resistance of the yolk becomes so great that the protoplasm is no longer able to carry out the work completeiy; then only the protoplasmic part of the egg is divided, that with much yolk remaining undivided. In this case we have a partial cleavage in comparison with the more primitive mode, the total cleavage; further, eggs which show a partial cleavage are called meroblastic, because only the segmenterl part of the egg is directly employed in the formation of the embryo ( $\beta \lambda a \sigma \tau o$ 's), while the undivided main mass serves merely as food-material. Eggs with total cleavage are called holoblastic.

The distribution of the yolk is connected with the position of the nucleus; either this is central and the yolk is concentric around it (centrolecithal eggs), or it is pushed, together with the greater part of the protoplasm, to one pole of the egg, while at the other pole the yolk predomi- 
nates (telolecithal eggs). Since the nuclear pole always becomes the animal pole, there can be distinguished in the egg an animal part rich in protoplasm and a vegetative part rich in yolk. In many telolecithal eggs the two regions pass gradually into one another, but in others a distinct boundary separates an almost purely protoplasmic animal portion from a yolk-containing regetative portion. This is well shown in the bird's egg (fig. I02). Here only the yolk is to be regarded as an egg in the embryological sense, while the white, egg-membrane, and calcareous shell are depositions upon the surface of the egg. The chief mass of the yolk is

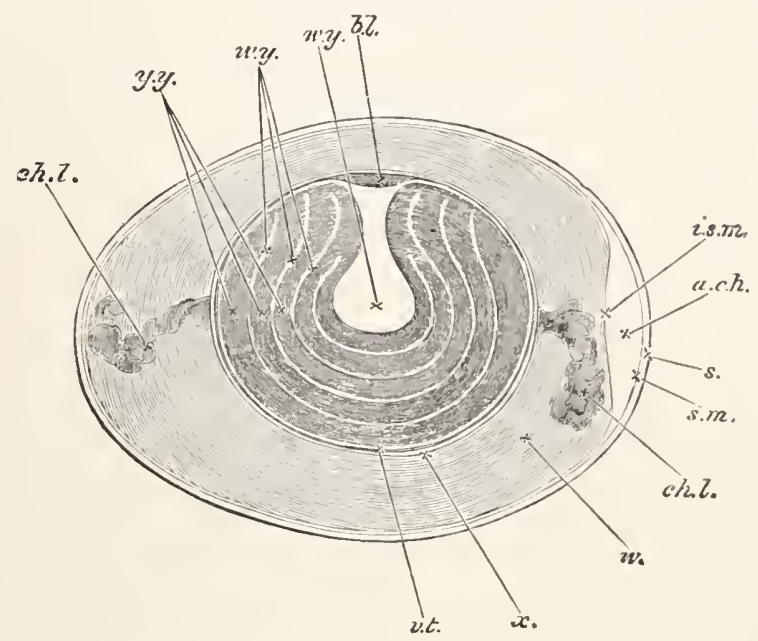

FIG. 102.-Diagrammatic longitudinal section through a bircl's egg (after Balfour). (I) The egg: b.l., blastoderm; $z \varepsilon^{\prime} . y_{\text {, }}$, white yolk; $y \cdot y^{\prime}$, yellow yolk. (2) Coverings of the egg: $v \cdot t$, yolk membrane (vitelline membrane); $x$ and $w$, inner and outer layers of white; ch.l., chalazæ; i.s.m. and s.m., inner and outer shell-membrane; between them at the right end is the air-chamber (a.ch.); s, shell.

deutoplasm, upon which rests a thin layer of protoplasm, the germinal disc, always uppermost in the bird's egg, whatever the position of the egg. The protoplasmic layer contains the egg-nucleus, and, after fertilization, by progressive development is separated (blastoderm) more and more sharply from the underlying yolk.

Types of Cleavage.-A brief explanation will now render the following figures of the various modes of clearage intelligible.

\section{a. Holoblastic Eggs with Total Cleavage.}

I. Equal Cleavage.-The yolk, present only in small quantity, is distributed equally through the egg; upon cleaving, the egg divides into parts of approximately the same size and equally rich in yolk (alecithal eggs, fig. IOI). 
2. Unequal Cleavage.-The yolk is abundant, but not in such a quantity as to prevent complete cleavage; it lies especially at the vegetative pole of the egg, causing the cleavage in this region to progress more slowly; here larger cleavage spheres are formed, because richer in yolk; hence the embryo, from the first, is composed of smaller animal cells poor in yolk, and larger vegetative cells rich in yolk (telolecithal, holoblastic eggs, figs. I03 and 104).
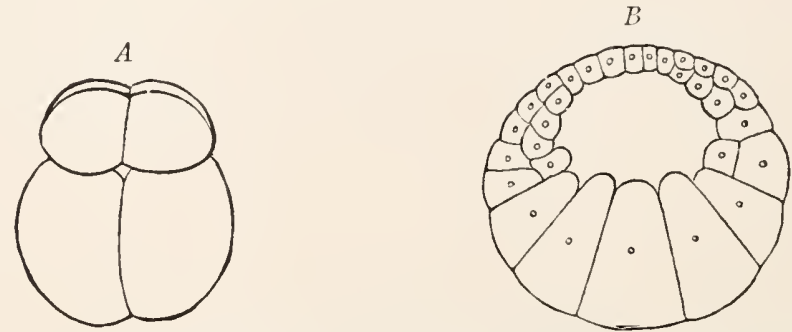

F1G. I03.- Unequal cleavage of the egg of Petromyzon (after Shipley, from Hatschek). $A$, stage of eight cleavage spheres; $B$, blastula in meridional section. The dissimilarity of the cleavage spheres begins with the equatorial furrow.
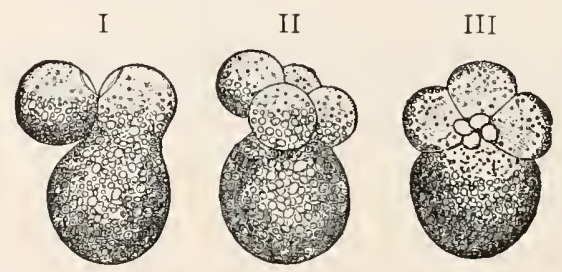

FIG. I04.- Unequal cleavage of a snail's egg, Nassa mutabilis (after Bobretzky). I, the first meridional furrow has divided the egg into unequal parts; II, the second meridional furrow has formed three smaller and one larger cleavage sphere (seein from the side): III, the equatorial furrow has formed four smaller animal and four larger but unequal vegetative cells (seen from the animal pole).

\section{b. Meroblastic Eggs with Partial Cleavage.}

3. Discoidal Cleavage.-The yolk is so abundant in the vegetative portion of the egg that it prevents cleavage; cleavage, therefore, is limited to the region around the animal pole and here forms a disc of small cells, the anlage of the embryo, or blastoderm (telolecithal, meroblastic eggs) (figs. I02, I05).

4. Superficial Cleavage.-The yolk is collected in the centre of the egg and prevents cleavage; consequently only the outer layer of the egg divides, forming a superficial layer of cells, enclosing the unsegmented central mass (centrolecithal eggs, fig. I06).

Distribution of the Types of Cleavage.-Of the four types of cleavage mentioned the superficial occurs exclusively in the arthropods. The others are distributed as follows: the discoidal in the majority of the vertebrates and in the most highly organized molluscs, the cuttlefishes and a few arthropods and tunicates, while the equal and the unequal cleavage can be found in all groups of Metazoa. 
Blastula.-Sometimes during the first stages of segmentation, sometimes later, there is usually formed a cavity, the cleav'age or segmentution cavity, (archicale) between the cells, in the interior of the egrg; with the progress of development this cavity becomes larger (figs. гог, IV; 103). Around it the cells lie in the form of a one- or many-layered epithelium
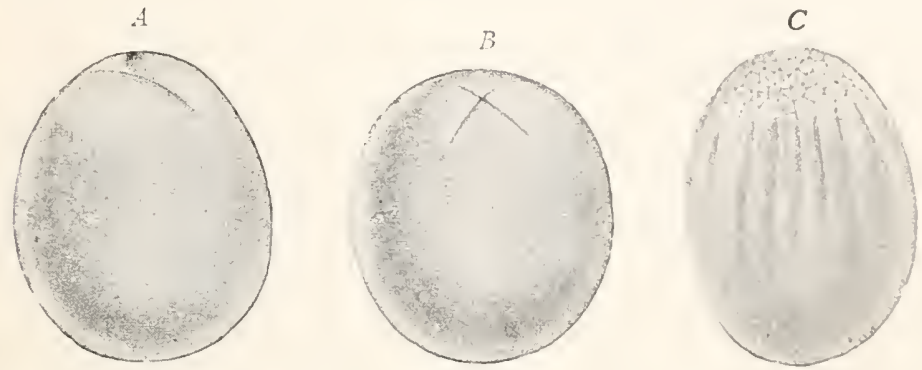

FIG. I05.-Discoidal cleavage of the egg of a cephalopod, Loligo pealii (afier Watase).
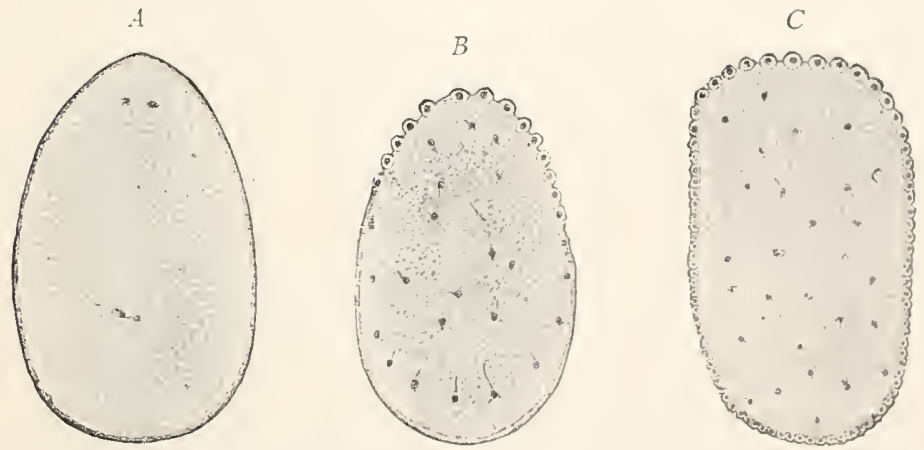

Fig 106.-Superficial clearage of an insect egg, Pieris cralcegi (after Bobretzky). $A$, division of the cleavage nucleus; $B$, movement of the nuclei to the periphery to form the blastoderm; $C$, furmation of the blastoderm.

and form the blastoderm; hence the name for this stage, blastodermic vesicle, or blastula. The more yolk present, the smaller is the cleavage cavity; in eggs with superficial cleavage it is entirely absent.

\section{Formation of the Germ-layers.}

Gastrula.-Besides the blastula there is a second stage of development, the gastrula or the two-layered embryo, common to all the Metazoa. This stage is understood easiest in eggs which have an equal clearage (fig. Iо7, B); here it has the form of a double-walled cup with a wider or 
narrower mouth. The cavity of the cup (the primitive digestive tract or archenteron) is the beginning of the most important part of the digestive system; the opening is the primitive mouth or blastopore. Of the two layers of cells forming the wall of the cup and uniting at the blastopore, the external is the ectoderm or outer germ-layer, the internal the entoderin

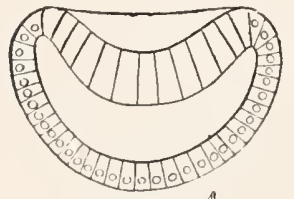

A

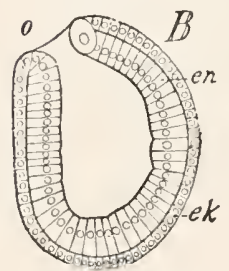

FIG I07-Gastrulation of Amphioxus (after Hatschek). The animal pole above, the vegetative below, in comparison with fig. IоI. In $A$ the cells of the vegetative pole are beginning to sink in; $B$, the invagination completed, the cleavage cavity reduced to a slit between the entoderm (en) and the ecto$\operatorname{derm}(e k) ; o$, blastopore.

the entodermal cells. In most coelenterata the gastrula is formed in another and apparently more primitive way. Isolated cells from the wall of the blastula migrate into the segmentation cavity and form a mass. In this a cavity is hollowed out and then a mouth breaks through, the result being structurally a gastrula.

Epiblast and Hypoblast.-For outer and inner germ-layer the terms epiblast and hypoblast, upper and lower germ-layer, have been much used. Other terms for the two germ-layers are entoblast andectoblast.

\section{Formation of Mesoderm. The Mesenchyme.-Many lower animals} e.g., most coelenterates, have in general only two germ-layers. When these are laid down there begins immediately the differentiation of muscle and nerve fibres and the other histological changes of the cells, as well as changes of form, by which the gastrula becomes the adult. In higher organisms, on the other hand, before further differentiation begins, there 
arises a third germ-layer, which owing to its position between the first two, is called the mesoderm, mesoblast, or middle germ-layer; this naturally can come only from the cell material of the existing germ-lavers, incleed only the entorlerm seems to participate in it. Two methods can he distinguished in its formation. In one the space between ectoderm and
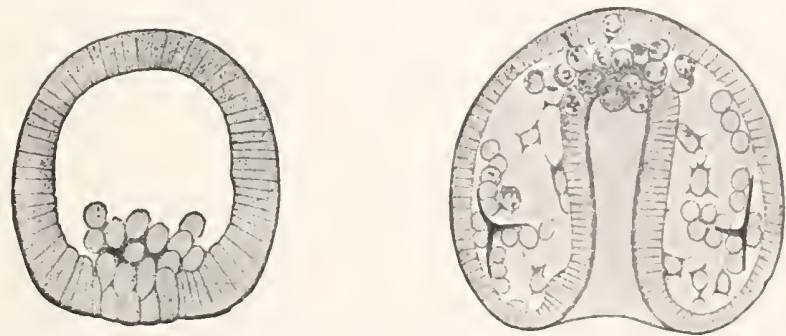

FIG. IOS.-Larve of Strongylocentrotus lividus (after Boveri). Left a blastula with mesenchyme formation; right a gastrula with differentiated mesenchyme.

entoderm becomes widened by the secretion of gelatinous sulsstance, and from the entoderm isolated cells push into this jelly; thus there arises a middle layer, the mesenclyme (fig. Io8), somewhat similar to gelatinous connective tissue, from which certain organs either wholly or in part take their origin. The mesenchyme can be formed before the beginning of gastrulation or after that process has been completed (fig. Ios; $c f . p .7 t$ ).

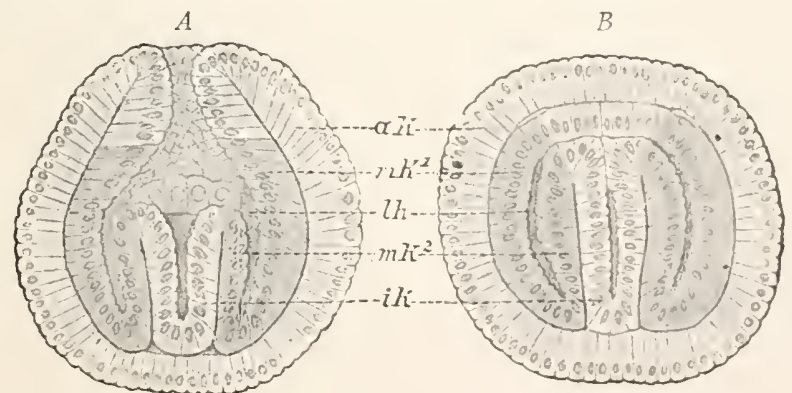

FIG. I09.-Formation of the mesothelium and colom of Sagit'u. A. From the bottom of the gastrula arise two folds, which divide the archenteron into the permanent digestive tract and the colomic diverticula. $B$. The separation is almost completecl by the pushing up of the folds. $a k$, outer, $m k$, middle, $i k$, inner germ-layer; $m k^{1}$, somatic layer; $m k^{2}$, splanchnic layer; $l h$, body-cavity.

Mesothelium.-In the second case the mesoderm may preserve the epithelial character of the two primary germ-layers, and is called mesothelinm. It is cut off from the entoderm, the mode of development leing shown in the worm Sagitta (fig. Io9). When the gastruta of Sagitta has been formed two folds arise from the archenteric walls opposite the 
blastopore ( 1 ), thus partially separating a pair of lateral chambers from the rest. The process continues; the blastopore closes, while the entodermal folds extend to the opposite side, where they fuse with the walls $(B)$. In this way a pair of colomic pouches are cut off from the rest of the archenteron which forms the lumen of the digestive tract and its derivatives, while the walls of the pouches form the mesothelium, that of the digestive region the secondary entoderm. In each colomic pouch two walls are recognizable, an inner or splanchnic layer which unites with the entoderm to form the wall of the digestive tract, the splanclinopleure, while the somatic layer unites similarly with ectoderm to form an outer body wall, the somatopleure. From the foregoing it is evident that the mesothelium is strictly not a single layer, but consists of two layers which pass in to each other, and that its origin is closely connected with the formation of the body cavity.

Occurrence of Mesenchyme and Mesothelium.-There are purely mesenchymatous animals, like the flat-worms, and purely mesothelial, like Sagitta, many annelids, and Amphioxus; there are also animals in which the mesoderm consists of mesenchyme and mesothelium: either the mesenchyme arises first and later the mesothelium, as in the echinoderms, or in the reverse order, as in most vertebrates.

Histological and Organological Differentiation.-All the organs of an animal arise from the three germ-layers. The details differ in the various groups; the following is the most general: from the ectoderm arise the skin with its glands and appendages, the nervous system, and the sensory epithelium; the entoderm gives rise to the most important part of the digestive tract with its glands; while muscles, blood, supporting and connective sulsstances, excretory organs, in whole or in part, arise in the mesoderm; the sexual organs are also usually mesodermal.

Relations of the Germ-layers in Budding.-The cuestion has been raised as to how far the germ-layer theory is applicable to the occurrences in asexual reproduction. At first one would expect that each organ of the daughter would arise from the corresponding organ of the maternal animal, or at least from a mass of tissue belonging to one of the same germ-layers. In many instances this is the case; in the budding of hydroids the entoderm and ectoderm of the bud arise from the corresponcling layers of the maternal body (fig. 93). But exceptions are known. In polyzoans and tunicates there are undifferentiated cells which are employed in cases of budding. In the regeneration of lost parts it is not necessary that the missing structure should be re-formed by the same layer from which it originally arose. The lens of Triton arises ontogenetically from the epithelium of the skin. If extirpated, it is regenerated from the pigmented epithelium of the iris.

Review of the Different Kinds of Reproduction.-The foregoing outline of reproduction is in accordance with the prevailing ideas. Although these are justified theoretically, they do not correspond to the actual relations, since the 
divisions of the Protozoa-cell divisions, and those of the Metazoa-divisions of cell complexes, are brought into the same category, in spite of their lifferent morphological value. Further, they accept a causal connection with fertilization in a form that does not agree with the actual conditions.

To make this clear we start with the fact that every reproduction depends upon an increase of cells. In unicellular organisms reproduction and multiplication of cells are one and the same thing. In multicellular organisms, on the other hand, two kinds of cell division may be distinguished, which may be called somatic and propagating coll divisions. Both are continuations of the cleavage process. The first is concerned with the growth of the inclividual, since it increases the formative material for the functioning organs; the other renders reproduction possible, since it furnishes the sex cells from which the new indivichal develops.

Fertilization enters in the course of the cell divisions which occur during life, in the Protozoa as the fusion of two individuals, in the Metazoa as the union of two sex cells which contain the anlage of the new individual. Both have in common the union (amphimixis) of two organizations to a comlsined organization. In the Protozoa the influence which fertilization exerts upon reproduction is very different in the different classes. In the Infusoria the rate of increase is not altered to any appreciable extent; in the Rhizopoda it is brought to a standstill, in which a period of rest (encystment) ensues; in Sporozoa it learls to a special form of increase and only in this group is there a sexual reproduction. The two others show that fertilization and reproduction are not necessarily connected, that the most important result of fertilization is not that it renclers increase possible, but that, in some way not yet clear but still fundamentally. important, it modifies the organism and influences the vital processes.

With these conditions an explanation is necessary of why, in the Metazoa in contrast to the Protozoa, there is such an intimate relation of fertilization to reproduction. It was held, indeed is the prevailing idea, that fertilization was introduced to render reproduction possible. The explanation lies in multicellularity. If it be necessary for the maintenance of the organism that the characters of two organizations be fused in one, the fusion can only take place when the characters of each or their anlagen are condensed into a single cell, as is the case with egg reproduction. But the union of two multicellular organisms to a single one is usually but a juxtaposition of parts; in only a few cases, not clearly understood (graft hybrids of many plants), is there a mingling of characters. Hence the inherited reproduction of the colonial Protozoa, which always arise from a single cell, is retained and combined with fertilization. On the other hand, the division and budding of the Metazoa are new acquisitions which have arisen independently in the different groups in adaptation to special conditions of life. They may be traced back to the phenomena of regeneration, to the capacity of parts of an organism to re-form, the whole. While typical regeneration results from a loss of a part from external causes, division and budding are apparently spontaneous, the result of the growth of the organism. That asexual (better 'propagative') reproduction occurs chicfly in lower animals is not a sign of its primitive character, but only the result of the fact that only in the simpler organisms is there the capacity of complete regeneration. This explains why the striking regularity which prevails in sexual reproduction is lacking in regeneration, division and budding.

\section{The Different Forms of Sexual Dezelopment.}

Embryonic and Postembryonic Development.- While the occurrences described (fertilization and cleavage of the egg, formation of the 
germ-layers) are going on the young animals are usually enclosed within a firm protective covering, or even in the maternal sexual apparatus (uterus), and are hence called cmbryos. Later stages, eren the formation of the most important organs, may occur during embryonic life, as we see in many vertebrates, worms and crabs, which, at the end of their embryonic existence, are complete in all their parts, and need only the maturity of the sexual organs, and growth of the body as a whole, in order to reach the climax of their development. On the other hand, there are animals, chiefly aquatic, which, after leaving the egg, undergo important changes, like the cœlenterates, echinoderms, insects, amphibians, etc. 'The cœlenterates, echinoderms, and many worms usually escape from the egg even before the formation of the germ-layers, and, as free-swimming ciliated planula, form the germ-layers and organs. Since there is here a more or less extensive postembryonic development, it is a misnomer to apply the term 'embryology' to both stages; it is necessary, rather, to limit the name to the developmental processes inside the egg, and, on the other hand, to speak generally of the history of the development of the individual, or ontogeny. As the undeveloped animal within its membrane is called an cmbryo, so the name lari'a is applicable to the free-living but not mature animal.

Direct and Indirect Development-Metamorphosis.-Larval development may be either direct or indirect. In direct development, as the term implies, the larva pursues a straight course towards the sexually mature animal, the lacking organs being outlined one after another; hence it is continually becoming more like the sexually mature animal. In indirect development, on the contrary, organs belonging only to the larval life, larval organs, are formed and later are destroyed. Therefore in the definition of indirect development, or metamorphosis, emphasis is laid upon the presence of larval organs. Thus caterpillars are distinguished from butterflies not only by the absence of compound eyes and wings, but by the presence of anal feet and spinning-glands, and further by the different shape of the jaws, antennæ, and legs, the different arrangement of the trachex and nervous system, etc. Tadpoles are distinguished from frogs not only by the absence of lungs and extremities, but also by the presence of gills and tail. The more numerous the larval organs, the more pronounced will be the metamorphosis.

Neoteny.-Occasionally the gonads of an indirectly developing animal become mature before the metamorphosis is complete, and as a result development is brought to a standstill (sexually mature, gill-breathing Triton larvæ, larvæ of Miastor, etc.). This peculiarity in which the gonads restrict the derelopment, is called 'neoteny'; and the attempt is made to regard a number of species as neotenic, that is sexually ripe in the larval stages. 
Oviparous and Viviparous Animals.-The time at which the exg leaves the mother's body is independent of that at which the embryoescapes from the egg membranes. Two extremes are known, the oviparous or egg-laying animals, and the viviparous or those which give birth to living young. Only those forms can be considered as strictly oziparous in which the egg at the time of laying is a single cell, as in the case of most fishes, sea-urchins, batrachians, insects, etc. In viriparous animals, on the contrary, birth and the rupture of the egg membranes occur quite; or almost, at the same time, and from the mother there emerges an animal which has completed its development or, at least, has progressed so far that it is able to live without protective coverings.

Ovo-viviparous Animals.-Varying degrees of orio-ziziparous derclopment connect these two extremes. What here appears at lirth at first impresses us, on account of its covering, as being an egg; but the first stages of development have already passed, so that, by artificial rupture of the egr membranes, an embryo more or less developed, but usually not yet capable of independent life, is exposed. Birds really belong in the category of oroviviparous animals, for their eggs are fertilized before they are laid, and have already completed the formation of the blastoderm. In the case of many snakes the egg-shell may contain, even at the time of laying, an animal all ready for hatching. Transitional forms of this kind show that no sharp line can be drawn between 'egg-laying' and 'bearing living young' and one must guard against attributing too much importance to the apparent distinctions. In many divisions of animals oriparous as well as viviparous forms are found. The majority of sharks are viviparous, but some lay eggs; in most bony fishes the eggs are laid before fertilization. Exceptions are the viviparous surf perches of the Pacific and many Cryprinodonts of fresh water. Most of the Amphibia, reptiles, and insects are egg-layers, but not a few forms are viviparous and even in the same genus (Lacerta and Chameleon) there may be viviparous and ovo-viviparous species. Even among the mammals, for which for a long time the 'bearing young alive' was regarded as diagnostic, it has been discovered that the monotremes lay eggs. Finally, exceptions to the rule occur in one and the same species. Adders are ovo-viviparous, but under unfarorable conditions they retain the eggs inside their body until ready to hatch.

\section{SUMMARY OF THE FACTS OF ONTOGFNY.}

I. The development of an animal begins with an act of generation; spontaneous generation and generation by parents are to be distinguisherl.

2. Spontaneous generation (abiogenesis) is the origin of living beings from lifeless matter (without pre-existing organisms).

3. The present existence of spontaneous generation is neither known nor is it, on the whole, probable; yet it is a logical postulate, in order to explain the origin of life on our globe.

4. Generation by parents, derivation of an animal from another of similar structure, can take place either by the sexual or the asexual mode.

5. Asexual generation may be either by division or by budding. 
6. In division an organism grows regularly in all its parts, and by constriction falls into two or more equivalent new pieces.

7. According to the direction of the plane of division in reference to the long axis of the animal we speak of longitudinal, transverse, and oblique division.

8. In case of budding a local growth occurs; the local outgrowth, the bud, separates from the mother as a smaller, usually incompletely formed animal.

9. According to the position and number of the buds we distinguish lateral, terminal, and multiple budding.

Io. Sexual reproduction occurs by means of special sexual cells, which have no part in the ordinary functions of the body.

II. In sexual reproduction two kinds of cells unite, the female egg and the male spermatozoon (fertilization).

I2. In rare'cases the egg develops without fertilization: parthenogenesis; this is a sexual reproduction with degenerated fertilization.

I3. Padogenesis is parthenogenetic reproduction by a young (i.e., incompletely developed) animal.

I4. Different modes of reproduction (asexual, sexual, parthenogenic, pælogenic) may occur in the same species; then these often occur in a regular order, and in such a way that individuals with different modes of reproduction alternate with one another: altemation of generations in the wider sense.

I5. Altemation of generations in the strict sense (metagenesis) is the alternation of two generations, one reproducing by division or budding, the other sexually. The former is called the nurse, the latter the sexual animal.

I6. The alternation of parthenogenesis or pædogenesis with pronounced sexual reproduction is called heterogony.

I7. Development which is inaugurated by sexual reproduction shows in nearly all multicellular animals a general agreement in the incipient stages: fertilization, cleavage, formation of germ-layers.

I8. The essential point of fertilization lies in the complete fusion of egg and spermatozoon, particularly in the fusion of the nuclei, egg and sperm nuclei, to form the cleavage nucleus.

I9. The cleavage of the egg is a cell division, a division of the fertilized egg into the cleavage spheres (blastomeres). The cleavage may be total (holoblastic egg) or partial (meroblastic egg); total cleavage is either equal or unequal, the partial either discoidal or superficial.

20. By repeated divisions of the cleavage spheres, and by the formation of a cleavage cavity, there arises a one-layered embryo, the blastula. 
2I. By the invagination of the blastula the gastrula or two-layered embryo arises.

22. The gastrula contains the primitive digestive tract or archenteron, opening to the exterior through the blastopore; it consists of two epithelial layers, the entoderm or the inner germ-layer, lining the archenteron, and the ectoderm or outer germ-layer.

23. Between the inner and the outer germ-layer still a third, the middle germ-layer, mesoderm, may be formed.

24. The middle germ-layer arises either by an infolding or a cutting off of a part of the entodermal epithelium: epithelial mesoderm, mesothelium; or by the migration of separate cells to form a gelatinous tissue (mesenchyme).

25. Many animals deposit their eggs before or shortly after fertilization (oviparous); others lay eggs which have already been fertilized in the maternal body, and at the time of laying have passed throurh some of the stages of development (ovo-viviparous). A third series of animals give birth to living young (viviparous).

26. The development of an animal is either direct or indirect (metamorphosis).

27. Indirect development or metamorphosis is where the young animal, as it comes from the egg, differs from the sexually mature animal in two points: (a) by the lack of certain organs which occur in the sexually mature animals; $(b)$ by the appearance of organs, larval organs, which are lacking in the sexually mature animals.

\section{RELATION OF ANIMALS TO ONE ANOTHER.}

Just as between the organs of an animal there exists a regular relation which is termed correlation of parts, so also the different individual animals stand in intimate reciprocal relations to one another. The conditions of existence of many animal species are altered, if other forms appear or disappear, or undergo an extraordinary reduction or increase in number of individuals. Such reciprocal effects are usually of a special nature and can be understood only by individual study; a few are of wide occurrence and are hence suitable for a general consideration; to such belong colony and society formation, parasitism, and symbiosis.

\section{Relations between Individuals of the SaMe Species.}

Colony Formation.-Colony and society formation are relations which exist between individuals of the same species. An animal colony is a union of individual animals by an organic bodily connection; the 
latter may arise in two ways: first, by animals, originally separate, partially fusing together; secondly, by individuals, formed by division and budding, remaining united with one another instead of separating. The first is extremely rare.

Colony Formation by Fusion.-Many Protozoa fuse with one another and form larger bodies in which the individual animals can still be recognized. Among the metazoa, Diplozoon paradoxum (fig. IIo) is the only case known where two animals (Diporpx), arising from different eggs, normally unite into a double animal, which recalls certain double monsters, as the Siamese twins.

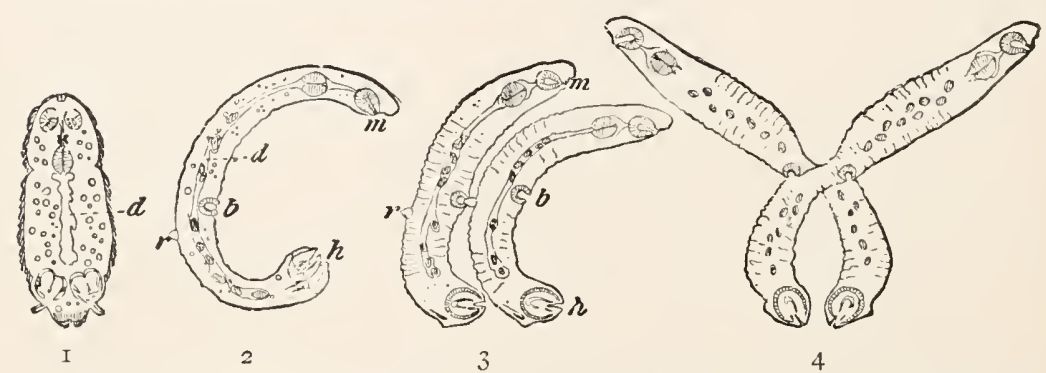

Fig. IIO.-Development of Diplozoon paradoxum (from Boas). (I) Larva, from which comes (2) 'Diporpa.' (3) Two Diporpæ uniting. (4) The Diporpe have united into Diplozoon. $m$, mouth; $d$, digestive tract; $h$, posterior adhering apparatus; $b$, ventral sucking-disc, which serves for attachment to the dorsal cone, $r$.

\section{Colony Formation by Incomplete Division and Budding.--In} general it can be said that colony formation rests upon incomplete asexual reproduction, since the new generation does not separate from the parent. The colonies of marine hydroids and corals (figs. 94, 205) may consist of thousands of individuals which, by repeated incomplete budding or division, have sprung from a single sexually produced mother animal.

Community of Functions.- - In the majority of cases the connection results in a considerable degree of community of functions. Stimuli which affect one individual are transmitted to the others of the colony; thus movements in common are rendered possible. In a similar way the food captured and digested by one animal serves for all. On account of the community of its functions, a colony appears like a unified whole, like an individual of a higher order; the same process which led to the formation of multicellular organisms is repeated. Just as there the elementary organisms, the cells (individuals of the first order) are united into a single animal (individual of the second order), so here the multicellular animals are united into a colony (individual of the third order).

Polymorphism.- When a whole is made up of numerous equivalent 
parts, the conditions for division of labor are present. Instead of the functions of the entire organism being distributed equally to the individual parts, many of the latter become employed more for this, others again more for that function, and açuire a corresponding structure. In such colonies one speaks of polymorphism. Polymorphism appears oftenest in connection with the regetative functions, leading to a distinction between sexual animals and nutritive animals, as in the case of most Hydrozoa, where often nutrition is accomplished by animals

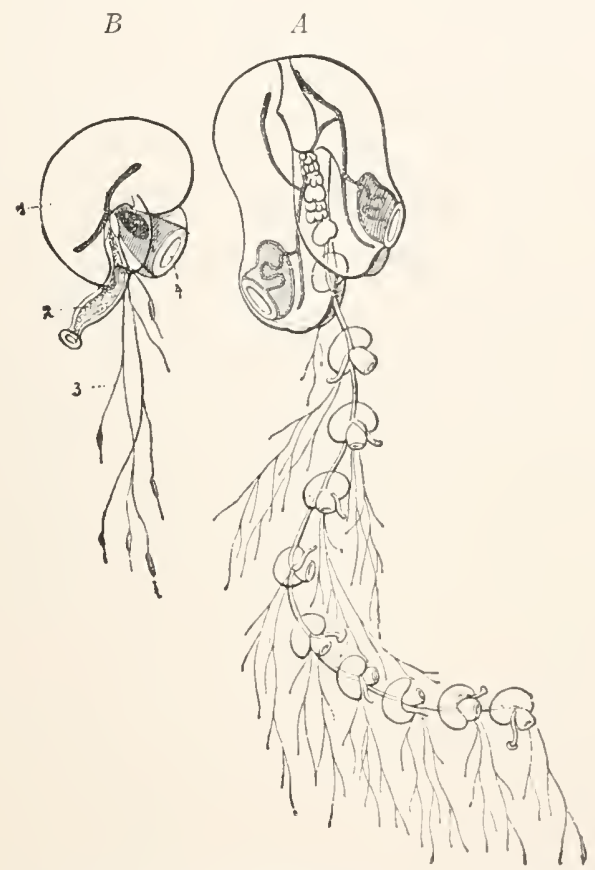

FIG. IIr.-Praya diphyes (after Gegenbaur). A, the entire animal; $B$, a single group of individuals greatly magnified (Eudoxia). I, covering scale; 2, nutritive polyp; 3 , nettle-threads; 4 , sexual bell.

without sexual organs, and reproduction is carried on by animals without a mouth. But other functions may also become specialized. Siphonophores are the classical examples of polymorphism (fig. III). Here united into a single body are locomotor animals, the swimming-hells, for locomotion only; covering scales, which serve only to protect the others; nutritive polyps, which alone take in and digest food; sexual animals and tactile polyps, concerned only with reproduction and with sensation. In regard to the other functions each animal is related to its brothers and 
sisters; its very existence therefore has become dependent upon these; the single individual can live only while a part of a whole. Thus division of labor leads to greater centralization; the more polymorphic a colony becomes, the more unified it is, the more it gives the inpression of being a single animal instead of an aggregation of single animals.

In Social Animals the reciprocal dependence of the individuals is much less, since there is no organic connection, only a voluntary communal life. As asexual reproduction is of importance in colonies, so here the sexual plays a prominent rôle. Under the influence of the sexual impulse, many animals, even some of the lowest organisms, flock together, either permanently or periodically; sea-urchins, sea-cucumbers, many fishes, collect near the coast at the time of egg-laying; it draws together herds of deer, elephants, etc. The care of the young further leads to a closer organization, to a society. All insect societies are built up on this basis. Consequently, since the sexual life is the starting-point of social life, in the different groups of individuals forming the community, the sexual organs may be influenced in their development. Besides males and females (kings and queens) there are other animals with degenerated sexual organs incapable of function, the workers; the latter are either only rudimentary females (bees and ants) or rudimentary females and males (termites). While the kings and queens give rise to the next generation, the workers care for the young, look after the hive, provide food, protection, and defence, if the latter be not delegated to a special class, the soldiers (termites).

\section{Relations between Individuals of Different Species.}

Where individuals of different species stand in close reciprocal relations to each other the cause is in the advantages which the one derives from the other, or which both furnish reciprocally; the former condition is called parasitism, the latter symbiosis.

Parasitism.-Parasites are organisms which dwell upon or in another organism, the host, and obtain nourishment from it. 'They have consequently come into a dependent condition and have undergone a more or less extensive change in their organization.

Degeneration Caused by Parasitism.-The degree to which a parasite has become dependent upon its host is determined by the extent to which the parasite has adapted itself to the organization of its host. Therefore it is necessary, in speaking of parasitism, to consider the modifications which the parasitic life has caused in the structure. These concern most immediately the organs of locomotion and nutrition. Since 
a parasite needs to fix itself as firmly as possible to the host, the locomotor apparatus more or less completely disappears and an apparatus for fixation becomes necessary; parasites of different groups are provided with hooks, sucking-discs, etc. 'The fluids of the host furnish nourishment to the parasite: these are substances in solution which scarcely need digestion. Usually, therefore, the digestive canal is simplified or disappears; among the parasites there are gutless worms as well as gutless crustacea. Very frequently the intestinal parasites live without oxygen; they are anaerobic (p. 92). The mode of life of a parasite is also simplified, since it is no longer compelled to seek its food; the nervous system and sense-organs undergo great degeneration; the former becomes limited usually to the most indispensable portion; the latter, except those of touch, may entirely disappear.

\section{Modification of the Sexual Apparatus} by Parasitism.-The sexual apparatus, on the contrary, undergoes a strong development. While' it becomes easier for the parasite to maintain itself, the existence of the species is more precarious. If a man die, then most of his parasites die with him, especially those in the interior of his body. In order that a parasitic species may not become extinct, it is necessary that the egrs be introduced into a new host. Since this is attended with difficulties, the parasites must produce an enormous number of eggs. The eggs, too, are distinguished by great resisting power and well-dereloped protective organs, such as strong shells, etc.; the eggs of Ascarids continue to develop for some time in alcohol, being protected by their impermeable shell.

\section{Ectoparasites and Entoparasites.-}

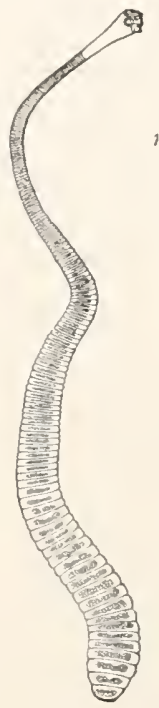

FIG. II 2 .

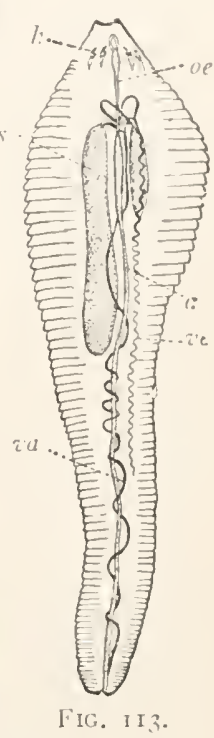

Fig. II2.-Tania nuna (after Leuckart).

Fig. II3. - Pentastomum tonioides female (after Leuckart). $h$, hooks right and left of mouth; ov, unpaired ovary, branching into two oviducts, which unite into the unpaired vagina $\left(z^{\prime} d\right)$; the latter receives the outlets of two receptacula seminis $(r s)$, and winds around the digestive tract $(d) ; a, \alpha$ sophagus.

All the above-mentioned phenomena are more conspicuous in the case of parasites which live insile of other animals, entoparasites, than in the case of the dwellers upon the skin or other superficial organs, the ectoparasites. In case of entoparasites the transforming influence of parasitism is so considerable 
that representatives of the most diverse groups take on a remarkable similarity of appearance and structure. Pentastomum (fig. II3), for example, belongs in the same class with the spiders, the Arachnida, but in external appearance it is entirely unlike them, resembling the tape-worms (fig. Iт2). Hence for a long time all entoparasites, on account of their similarity, were united into a single systematic group under the name of 'Helminthes,' comprising members of the crustacea, worms, and spiders. Only by embryology was the unnaturalness of this grouping recognized. Entoparasitism therefore is one of the best examples for illustrating convergent development, i.e., animals of different systematic position acquiring, under similar conditions of life, a great similarity of structure and appearance.

Symbiosis.-Less frequent than parasitism is symbiosis, or the association of animals for reciprocal advantages. Social animals frequently not only hold certain animals in bondage, but even seek to protect and serve them; as, for example, certain blind beetles, like Claviger or some species of plant-lice, (myrmecophiles) or even ants of other species and genera. But such cases of association correspond in part to the domestication of animáls, or to slavery, as carried on by man. The ants keep the plant-lice in order to lick the sweet juice ('honey dew') which is secreted in their honey-tubes; they steal the pupæ of other ants and rear them, to use them later as slaves. This state of things rests, consequently, not upon equal rights, since the one animal, in the present example the ant, brings about the association, while the other is passively led into it. Symphyly is close to true symbiosis. Besides Claziger, mentioned above,

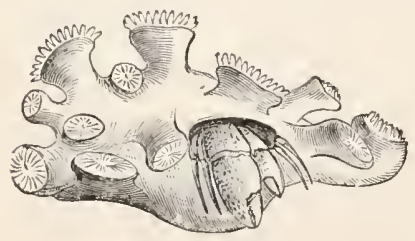

F1G. I 14.-A colony of Epizoanthus americanus on the shell occupied by a hermit-crab (from Verrill). many other insects, mostly beetles, which are cared for by the ants, are found in colonies of ants and termites, since they have a sweet secretion on special bundles of hairs, which the ants lick off. Frequently the beetles eat the younger stages of the ants.

An instance of most complete equal rights and true symbiosis is furnished us, however, by a hermit-crab and an actinian (fig. I I4), Eupagurus pubescens and Epizoanthus americanus. Like all hermit-crabs this also inhabits a snail-shell from the opening of which only his legs and pincers are protruded. Upon this sheli an Episoanthus becontes attached and by budding soon covers it with a colony of polyps. The advantage which the actinian derives from this symbiosis is clear: it gains a share of the food which the crab obtains. It is less clear what the crab gains; however, the polyp is perhaps a protection to him by its nettle cells, while by growth it increases the size of the 'house' occupied by the hermit and thus saves him periodic changes of abode.

Occurrence of Symbiosis.-That animals rarely live symbiotically with one another rests largely because the conditions of life of all animals to a certain point are similar or identical. They take in compounds rich in carbon and nitrogen, decompose them into carbon dioxide, water, and oxidation products containing nitrogen. All animals consequently are competitors in the strugrle for food. For the same reason, conversely, symbiosis between plants and animals is not so uncommon. There are certain lower algæ, the Zooxanthellæ, 
which often live in animals. The radiolarians so constantly contain greenor yellow-colored cells that for a long time these were regarled as constituent parts of the animal. Similar yellow and green cells inhabit the stomach cpithelium of many actinians, corals, and even of many worms. 'The Zooxanthellie are nourished by the carbon dioxide formed by the animal tissues, and loreathe out oxygen, which in turn is used by the animal; further, they form starch and other carbohydrates, and any surplus thus formed may be food for the animal. Thus there is on a small scale that cycle which exists on a grancl scale in nature between the animal and regetable kingdoms. By aid of chlorophyl and sunlight plants decompose water and carbon dioxide and form from them oxygen, which they respire, and compounds rich in carbon, which they store in their tissues: they are reducing organisms. On the contrary, animals give off carbon dioxide and water, but take their oxygen from the air, and carbon compouncls in their food; they use oxygen to break down the chemical combinations, to oxidize: they are oxidising organisms. This explains why the favorable influence of plants upon animals ceases immediately when they change the character of their metabolism. With the disappearance of their chlorophyl fungi and bacteria have lost the power of reducing carbon dioxide; they derive their food from other organisms and decompose this into carbon dioxide, water, etc.; like animals, they are oxidizing organisms, and consequently dangerous competitors and are the cause of many serious ailments.

\section{ANINAL AND PLANT.}

Distinction between Animal and Plant.- The consideration of symbiosis leads to the fact that a distinction exists between plants and animals in the morle of metabolism, which may be expressed thus: plants usually take in carbon dioxide and give off oxygen, while animals absorb oxygen and give out carbon dioxide. Hence it might be concluded that it is easy to discover universal distinctions between plants and animals. But the more one studies this question, the more difficult becomes its solution. The old zoologists believed that there are organisms which stand on the limits between the animal kingdom and the vegetable, and named these zoophytes or plant-animals. Now we know that these are true animals with but a superficial similarity to plants; but, by means of the microscope, we have become acquainted with numerous lower organisms, and it is still doubtful in which of the two realms some of these, like the Myxomycetes and many Flagellata, belong.

Physiological Distinctions. - In the search for distinctions both physiological and morphological characters may be considered. Starting from the physiological side Linnæus ascribed to plants only the capacity of reproduction and nutrition, but to a nimals the power of motion and sensation in addition. Now we know that vegetable, like animal, protoplasm is irritable and is capable of motion, as is shown by the

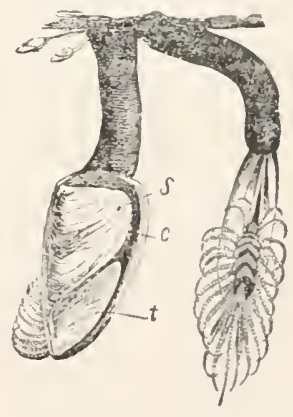

Fig. I 5.-Lepasanatifera (after Schmarda). $c$, carina; $t$, tergum; $s$, scutum. active movements of the lower Alga, the great sensitiveness of the Mimosa, and other plants; but further, we know that even many highly organized animals, e.g., crustacea (fig. I I5), lose the power of locomotion and become fixed, and many fixed forms, e.g., the sponges (fig. 88), appear immorable and unaffected by stimulation; thus the so-called animal functions cannot be regarded as affording accurate distinctions. 
Even the difference in metabolism is by no means sufficient. Every plant has a double exchange of material. In its movements and other vital functions the vegetable protoplasm produces carbon dioxide and consumes oxygen; at the same time there goes on, under the influence of sunlight and of chlorophyl, the reduction of carbon dioxide and the giving off of oxygen. In chlorophylcontaining plants the reducing process preponderates so considerably during the day that they give off a quantity of oxygen, and only at night, when the reducing process becomes interrupted on account of the lack of sunlight, does the production of carbonic-acid compounds become perceptible. But if the chlorophyl be absent the reducing processes disappear; chlorophylless fungi and bacteria have, therefore, the same metabolism, so far as carbon dioxide is concerned, as animals. So also it is incorrect to say that only plants have the power to make cellulose, for cellulose is found in many lower animals, the rhizopods, in the highly organized group of tunicates and even among the arthropods.

Morphological Distinctions.-Turning to the morphological characters, multicellular animals and multicellular plants are readily distinguished, since the former in the germ-layers have a principle peculiar to them; with the appearance of the gastrula each organism is undoubtedly an animal. But in unicellular animals the arrangement of the cells is lacking, and only the constitution of the single cell can guide us. Now are there unmistakable morphological differences between the animal and the vegetable cell?

Plant-cells have a Cellulose Membrane.-In the structure of plant and animal cells an important distinction is found in the fact that the former has a

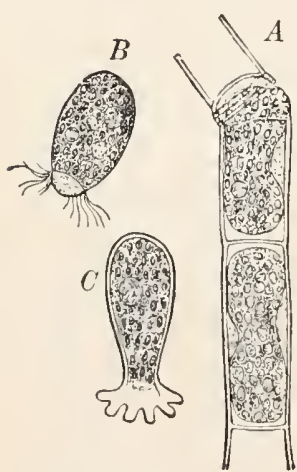

FIG. II6. - Edogonium in spore-formation (after Sachs). 1, a piece of the alga with escaping cell-contents: $B$, zoospore formed from the contents; $C$, zoospore fixed and germinating. cellulose membrane, but the latter is usually membraneless. To this distinction must be referred in the last analysis the widely different appearance of the two realms. Since the plant-cell is early surrounded with a firm coat, it loses a large part of its power of further changing its form; hence vegetable tissues and organs, in spite of the manifold intracellular differentiations, like the chlorophyl granules, are uniform in comparison with the inconceivable multiformity which animal structures disclose. The higher stages of organization which the animal kingdom reaches, even in its lower classes, is in great part the result of the fact that the cells of animals do not become encapsuled, but have preserved the capacity for more varied and higher development. But even here transitions are found between the lower plants and animals. In the lower Nlyre the cells can leave their cellulose membrane, and swim about freely (fig. I 6 ), before they enclose themselves anew. On the other hand, most unicellular animals encyst; they pause in their ordinary functions, become spherical, and surround themselves with a firm membrane, sometimes even of cellulose. Since in both cases an alternation between the encapsuled and the free condition occurs, only the longer duration of the one or of the other can lead to a distinction. But here occurs the possibility that indifferent intermediate forms exist; their actual existence prevents, even yet, a sharp distinction between the animal and vegetable kingdoms.

\section{GEOGRAPHICAL DISTRIBUTION OF ANIMALS.}

The Different Faunal Regions.--Even a superficial knowledge cf the distribution of animals shows that the animal population, the fauna, in different 
regions of the earth has an essentially different character. In part this is the immediate result of climatic differences. The polar hear, arctic fox, eirlerducks, are restricted to the polar zones, because they cannot endure more than a certain degree of warmth; on the other hand, the larger species of cats, the apes, the humming-birds, etc., occur only in warmer regions, because they are not sufficiently protected against cooler weather.

If climate were the sole factor determining distribution, the faunal character of two lands which have similar climatic conditions would be essentially the same; conversely, the separate regions of a continuous territory extencling through several climatic zones must have different faunas, according as they are nearer the equator or the poles. But such is not the fact; two tropical countries may differ more widely in their fauna than the hot and cold regions of one and the same country.

Factors in Distribution.-Modern zoology endeavors to explain these conditions by regarding the present distribution of animals as the product of two factors: the gradual changes of the animal world, and the gradual changes of the earth's surface on which the animals are distributed $(p .33)$. 'The history of the earth as disclosed by geology shows two facts: (I) that the connections between parts of the earth have varied greatly; that, for example, at a time when the Mediterranean had not yet reached its present extent, Morocco, Alyiers, Tunis, and Eirypt were more closely united with the European coast of the Mediterranean than with the southern part of the African continent separated from them by the Sahara; $(2)$ that considerable variations of climate have taken place: there prevailed in Europe in the tertiary period a subtropical clinate which rendered possible the existence of animals which now occur in Algeria (lions). But later a glacial period began, which introduced orer a large part of Europe arctic conditions, and consequently a fauna of northern animals (reindeer) which has left a few traces (Alpine hare) in the glacier regions. Hand in hand with the geological changes went changes in the animal world, the then existing species dying out under the change of conditions, or perhaps forming new species through gradual variations. Thus the distribution of animals constitutes an extremely complicated problem, for the solution of which it must be known how the climates and the comnections between the continents have changed, particularly in the later geological periods; further, not only how animals are distributed at present over the earth's surface but also how they were distributed in earlier times. Finally, we must have clear and detailed ideas of the relationships and interrelationships of animals.

It will be a long task to solve all the problems. What has been accomplished so far can only be regarded as showing that zoology, with its prevailing views of the changes of animals and of the earth, is on the right track. Two regions, separated early in the earth's history and never again connectech, must have greater differences in faunal characters than two lands still connected or only recently separated. We travel in the northern hemisphere and find in widely separated regions strikingly similar faunæ, while under the equator or in the southern hemisphere, under the same conrlitions for each reyion, striking differences are seen. This is explained on the hypothesis that in all past periocls as now the land masses of the northern henisphere have been closely connected, while the parts of the continents extending to the south have been seprarated through most of the earth's history.

Students of distribution have attempted to define the great faunal areas of the earth, the faunal provinces or regions, and within these the subregions. These provinces have been based chiefly upon the distribution of mammals, less upon that of birds and other animals; for the distribution of mammals is chiefly determined by those changes of the earth's surface which are best known geologically and possess most interest. Elevation or depression of the earth's 
surface often opposes impassable barriers to most mammals: rising, if it lead to the formation of glaciered mountain-chains; sinking, when arms of the sea are formed, which interpose straits or broader bodies, impassable to most mammals, between two land areas. Birds and strong-flying insects are less affected by all such changes; the majority of them can fly over arms of the sea and mountain-chains; there are birds which can even cross the Atlantic.

The Six Primary Regions.-Of the systems of animal geography proposed that advocated by Sclater and Wallace finds most favor. They distinguish six primary regions: (I) the palaarctic, comprising all Europe, northern Africa as far as the Sahara, and northern Asia to the Himalayas; $(2)$ the Ethiopian, Africa south of the Sahara; (3) the oriental, including upper and farther India, southern China, and the western Malay Islands; (4) and (5) the nearctic and the neotropical regions, which make up the American continent and are divided at about the northern border of Mexico; (6) the Australian, with, besides Australia itself, the larger and smaller islands of the Pacific Ocean and the Malay Islands, east of Celebes and Lombok.

(I) The Australian region is most sharply distinguished and by many is set apart as a distinct division called 'Notogra.' Its isolated geographical position together with the fact that it has long been separated from other countries (apparently since the beginning of the tertiary) explains why only the oldest mammals, the monotremes and marsupials, have entered the region, while the placental mammals have not been able to follow. The marsupials, which in the secondary period also inhabited the northern hemisphere, and were replaced there in tertiary times by the placentals, were able to develop farther in the Australian region. Australia and the adjacent islands are thus the land of marsupials, which have persisted elsewhere only in South America, the opossum ranging north into the United States. On the other hand, at the time of discovery Australia lacked all terrestrial placental mammals except those (bats) which were not restricted by water and the Muridæ, easily transported on floating wood. Two larger mammals, the wild $\log$ (Canis dingo) and the pig of New Guinea (Sus papuanus), may have accompanied man, this being probable for the dingo, although his remains occur in the pleistocene along with those of the giant marsupials. Further peculiarities of the Australian region are the birdsof-paradise in New Guinea, the egg-laying mammals (monotremes), and the cassowaries and the Australian ostrich (Dromaus novahollandia).

It is easily understood that the island groups of the South Sea (Polynesia) have developed many faunistic peculiarities, as well as that an exchange of forms may have taken place between the islands of the oriental province and those faunally related to Australia, and that 'Wallace's Line' (p. 34) is not so sharp a boundary as was once thought (extension of marsupials into Celebes, of placentals into the Moluccas). On the other hand the distinctness of New Zealand needs mention. It is distinguished from Australia by a large number of peculiar birds (Apteryx and the extinct Dinornithidx), reptiles (the ancient Splienodon), and molluscs. If the bats and mice be excepted, New Zealand lacks all native mammals, even marsupials.

(2) The Neotropical province (South and Central America) is, next to Australia, the most sharply characterized, and has also been set aside as a special division 'Neogxa,' partly in view of its geological history; during the cretaceous and early tertiary time it was separated from North America by the sea and had developed a peculiar fauna (e.g., gigantic edentates, no carnivores). These peculiarities disappeared towards the end of the tertiary by the entrance of carnivores and ungulates from the north and an extension of the edentates, marsupials, humming birds, etc., to the northern hemisphere. To Neogæa belong the platyrhine apes, the catarrhine to the Old World. Characteristic edentates are the armadillos, sloths, and ant-eaters; the marsupials are repre- 
sented by the opossums and Canolestes, nearly related to the Iustralian D)i. protodonts; among birds the humming-bircls, toucans, Cotingirle, 'Tanagriclic, Tinamous, Palamedida, Rhea, etc. The almost entire absence of insectivores and the considerable development of rodents (cavies, agoutis, chinchillas) are noteworthy.

The four remaining provinces are still closely connected geographically and form a third great division, 'Arctogea,' characterized by the entire alssence of platyrhine apes, monotremes, and, except the North American opossum, of marsupials. In the secondary and tertiary times the northern parts of these lands were connected and an interchange of faunas occurrecl, this being the easier on account of the extension of the warm climate to the far north. Hence many unite the palearctic and nearctic provinces into a 'holarctic' province, but when existing conditions are concerned it is better to retain them as distinct.

(3) The Nearctic region has three mammalian families, the prong-hornect antelope, the opossums, and the Haplodontx, peculiar to it; of the group of Amphibia, the Sirenide and Amphiumida. 'The Nearctic is distinguished from the nearly related paliearctic region through the crowding in of nentropical forms like the raccoon, opossum, humming-birds, ctc. The absence of the stag, badger, wild swine and all true mice is noticeable.

(4) The Palæarctic region covers the greatest area and consequently touches several others; hence climate and great distances have causerl important differences between the local faunas, but its contact with other regions explains the fact that it has no peculiar families. Deer, cattle, sheep, and camels have reached a great development; especially conspicuous genera are the chamois, squirrel, badger, and marmot.

(5) 'The Ethiopian region has many animals found only there; among these the hippopotamus, giraffe, the recently discovered Ocapia, the aardrark, and, if we include Madagascar, the lemurs are characteristic. To these are arlded a rich development of antelopes and zebras and the gorilla and chimpanzee. Equally noteworthy is the entire absence of striking families and genera, such as the bears, moles, deer, goats, tapirs, sheep, and the true swine.

Within the region the island of Madagascar occupies a remarkable position. This is the land of lemurs and Insectivora, the majority of the genera of lemurs living exclusively in Madagascar. On the other hand, the large beasts of prey, all the true apes, antelopes, elephants, and the various species of rhinoceros are absent. Consequently, since Madagascar is conspicuously distinguished from Africa, many zoologists separate the island from the Ethiopian region as an independent Malagassy province.

(6) The Oriental region contains, next to Madagascar, the most lemurs; among which the Tarsidx and Galeopithecidie are exclusively oriental. Remarkable inhabitants are the gibbons and orang-utans, musk-deer, numerous families and genera of birds.

Arctic and Antarctic Provinces.-Of late the view has gained ground that, besides these six, two other, circumpolar, provinces must be distinguished, the Arctic and the Antarctic. Both have a fauna consisting of few species but numerous individuals, of which the auks, polar bear, reindeer, and arctic foxes are characteristic of the northern or arctic region, the penguins and the entire absence of land mammals of the antarctic.

The Distribution of Aquatic Animals.-Since most seas are connecter, the faunal regions cannot be distinguished so sharply as in the case of the land faunas; conspicuous differences are present only when two oceans are separated by continents extending far to the north and south; such, for example, exist between the Red Sea and the Mediterranean, between the east and west coasts of North America, even where they are separated only by the isthmus of Panama. 
Then, too, considerable differences may exist where currents of greatly different temperatures meet.

Much more remarkable in the marine fauna are differences caused by the conditions of life in the different depths of the sea. A deep-sea fauna, a coast fiuna, and a pelagic fauna can be distinguished. The coast fauna embraces the animals which inhabit the plant-covered rocky or sandy shore to a depth of a few hundred feet. The deep-sea fauna swims, creeps, or is fixed at the bottom of the ocean at depths of Iooo to the greatest depth yet known, 9430 meters, $5^{1} 5^{6}$ fathoms; it is distinguished from the coast fauna in part by its archaic character, for here very often genera and entire groups of animals exist, like the Hexactinellidx, crinoids, etc., which long were chiefly known through fossils from earlier geological ages.

The Plankton.-The pelagic fauna comprises all forms which swim freely in the water, the plankton; here belong many colenterates, medusæ, and ctenophores, entire groups of Protozoa, like the radiolarians, many crustacea, the heteropods and pteropods. These animals live either at the surface of the sea itself or floating at greater or lesser depths, to 8000 meters or even more. Usually they are gelatinous and of glasslike transparency; this must be regarded as sympathetic coloring and adaptation to the transparency of the water. The plankton of the deep seas, extending up to about Soo meters, forms a special fauna characterized by the brownish-red color, which is also found in the bottom animals.

Distribution of Fresh-water Animals.-In fresh water two groups of animals must be distinguished, of which the one comprises mainly the more highly organized forms, the molluscs, fishes, and crustacea, the other the lower animal world. The distribution of the former is mainly determined by the same factors which influence terrestrial forms; they are therefore of great importance in matters of geographical distribution, yet it must be remarked that many fish at the breeding season ascend from the seas to the rivers (salmon, alewives, etc.) and on the other hand, others like the eels go from the rivers to the seas, so that the sea is not that sharp boundary for these animals that it is for land animals. The distribution of the lower fresh-water animals, however, is cosmopolitan. The same infusorians and rhizopods, copepods, fresh-water sponges and polyps which occur in America seem to be distributed over nearly the entire earth. This is connected with the fact that all these animals have resting stages in which they endure desiccation. The resting stage, be it as a hardshelled egg or as an encysted animal, may be borne about by the wind, or may be carried with the mud by aquatic birds, and upon again reaching the water resume its active state.

\section{DISTRIBUTION OF ANIMALS IN TIME.}

It is the province of paleontology or paleozoology, to treat of animals in the earlier periods of the earth's history, but since it is necessary to draw upon paleontological facts to understand the living forms, and especially the vertebrates, an outline of the geological periods with the characteristic animals may be given here.

\section{Azoic or Archean Fra.}

No organisms are certainly known from this age. The animal nature of Eozoon canadense of the Laurentian beds, once referred to the Foraminifera, is more than doubtful. 
II. PALAOzoIC ERA.
I. Cambrian.
4. Carboniferous.
2. Silurian.
5. Permian.

3. Devonian.

The oldest paleozoic period, the Cambrian, contains only invertelorate fossils: silicious sponges, the problematical graptolites, meclusi, trilobites, gigantostraca, cystoids, holothurians, brachiopods, nautiloids, gasteropodls, and a few lamellibranchs. Trilobices, cystoids, gigantostraca, and the blastoirls and tetracoralla, which appear in the Silurian, reach their culmination and become extinct in the paleozoic. Fishes appear in the Silurian, and acpuire a great development in the Devonian. The earliest Amphibia and reptiles come from the carboniferous.

\section{MEsozoIC ERA.}
I. Triassic.
2. Jurassic.
3. Cretaceous.

The mesozoic era was the age of reptiles, which were represented by numerous forms, some of gigantic size; most of them becoming extinct in the cretaceous. The first mammals appear in the triassic, the birds in the Jurassic. Among the invertebrates the ammonites, which appeared in the Devonian, reached their greatest development and became extinct in this era.

\section{Cenozoic Era.}

(a) Tertiary.

I. Eocene.

2. Oligocene.

3. Miocene.

4. Pliocene.

(b) Quaternary.
5. Pleistocene (Ice Age, Diluvium).
6. Recent.

In the tertiary all of the now living orders of mammals and birds appeared, among them probably man, whose remains have been traced with certainty to the pleistocene. 


\section{SPECIAI ZOOLOGY.}

SINCE comparative anatomy and the theory of evolution have made their impress upon systematic zoology one recognizes in classification not only a means of arranging the species, but also the possibility of expressing the relations which the larger and smaller groups bear to each other. The solution of these problems demands an accurate knowledge of comparative anatomy and embryology and a complete knowledge of animal forms based upon them. We are yet far from such a knowledge, farther with regard to some groups than others, and as a consequence systematic problems are not all equally advanced towards solution.

In general it may be said that certain natural groups are recognized: (I) Chordata; (2) Mollusca (after the elimination of the Brachiopoda); (.3) Arthropoda; (4) Echinoderma; (5) Colenterata (after the separation of sponges); (6) Protozoa. On the other hand, it is yet uncertain exactly how to regard the worms, brachiopods, polyzoa, and a few other forms. The general tendency is to distribute the worms in to at least three branches (flat worms, round worms, and annelids) and to unite the Polyzoa and Brachiopoda in a branch of Molluscoida. In this way groups poor in species and of little importance in a general account of the animal kindom are placed on the same basis as the large and exceedingly important groups of vertebrates, arthropods, and molluscs, and thus obtain, especially in the eyes of the beginner, an importance which does not belong to them. It therefore seems better in an elementary work to pursue a rather conservative course.

\section{PHYLUMI I-PROTOZOA.}

All of the Protozoa are small; some may be seen by a sharp eye as mere specks, but the majority are so minute as to be invisible except with a microscope. On the other hand, a few have a diameter to be measured by millimeters, especially where hundreds of individuals are united in colonies. This small size is a result of the fact that the Protozoa are single-celled animals. Like all cells they consist of protoplasm, and they have the further cell attribute, one or more nuclei. Being unicellular, it follows that they lack true tissues and true organs; alimentary canal, nerrous system, sexual organs, etc. The functions of nourishment, sensation, movement, and reproduction are performed more or less directly by the protoplasm. 
In nutrition, in so far as it is not produced by substances in solution, foreign particles pass into the protoplasm and are digested by it. "They usually lie during digestion in special collections of fluid, the food vacuoles (figs. I2I, I50, etc., $n a$ ), more rarely in the protoplasm itself. All indigestible portions are cast out after a time. 'This taking in and casting out of foreign matter can take place in the naked Protozoa at any point of the surface, while in the more highly organized species when the outer surface is hardened by a pellicle or a thin cuticula, there are lefinite openings which accorlling to analogy with many-celled animals are spoken of as mouth and anus, or more precisely, cytostome and cytopyge. The mouth may connect with a tibe, the oesophagus or cytopharynx, which ends free in the protoplasm.

Structures may occur within the protozoan cell which recall the organs of higher animals, and which are called cell organs. While motion is usually produced by the protoplasm and its processes-pseudopodia, flagella, and cilia-there are Protozoa, like Stentor and the Vorticellide which have muscular filsrillæ. 'The sensitiveness to light is often increased by an eye spot, a small pigment body in which eren a lens may occur. More constant of cell organs are the contractile z'acuoles (fig. I i 7 , etc., c'), rarely absent from fresh-water species, but commonly lacking from marine forms. These have a definite place in the cell; their number is approximately constant in most species; they exhibit extremely constant phenomena. The walls contract and empty the fluid contents to the exterior, often through a special duct. IThen one empties it completely risappears and is formed again anew in a short time, and is filled with fluid from the surrounding protoplasm. It thus resembles the contractile vacuoles in the water vascular system (excretory organs) of the worns to be described later. Apparently the contractile vacuoles are for the elimination of injurious substances in solution produced by the vital processes, among them possibly carbon dioxide, like a respiratory organ.

The occurrence of such diverse differentiations, recalling organs and tissues, gives such a complicated appearance and such a degree of specialization to the protozoan body, that it was questioned for a time whether all could belong to a single cell. Yet it was a mistake to doubt the unicellularity of the I'rotozoa, for according to our conceptions of the cell, there is the capacity to develop in many directions, to produce a kind of stomach, muscle fibres, sense apparatus, skeleton and the like; although in the organization of the higher animals it produces only a specific product (muscle cells, contractile substance, gland cells. secretion).

The vital phenomena of the Protozoa proceed from the protoplasm, but with a certain dependence upon the nucleus. If an infusorian or an Amcha be cut into nucleate and anucleate portions, only the first can 
live. The part without the nucleus loses the capacity for assimilation, for growth, and for regenerating the lost parts. For a time it can react to stimuli, move about. Sensibility and contractility persist only so long as the necessary elements, formed under the influence of the nucleus, are present. When they are used up the last manifestations of life are lost and death ensues. So it may be said that the chemism of the cell needs the participation of the nucleus.

The nucleus is also concerned in reproduction, of which the most primitive type is binary division (figs. I $20, \mathbf{I}_{50} \mathrm{O}, 15 \mathrm{I}$ ). Budding is rarer, its character being most evident when several buds are separated simultaneously from the mother animal (fig. 2I). The nuclear division occurs in different ways. Like the cell body, it may divide amitotically, but it can present the complicated phenomena of mitosis (formation of spindle and chromosomes). In not a few instances the specific organ of division, the centrosome, appears, so that all transitions from direct to extremely complicated division are present in the phylum.

Very frequently the nuclei multiply without a corresponding division of the protoplasm, so that large masses of protoplasm, with hundreds or even thousands of nuclei arise (multinucleate cells, syncytia); or both nucleus and protoplasm may grow, without division, to extraordinary size. In both instances, after an interval of time, there is a simultaneous division into hundreds or thousands of reproductive particles; the protoplasm, in the first case, dividing in accordance with the number of nuclei present; in the other following the division of the mother nucleus into a multitude of daughter nuclei. Many Protozoa divide in the free state while swimming or creeping about; others first encyst, that is, assume a spherical shape and secrete a protective envelope.

In the Protozoa may occur a fusion of individuals-conjugationwhich in many respects has much similarity to the process of fertilization in Metazoa and in plants. In some (conjugation of many Rhizopods) this does not correspond to true fertilization, since only the protoplasm unites (plasmogamy), while the fusion of nuclei (caryogamy) necessary to fertilization does not occur. In others a fusion of nuclei takes place. In the cases which have been accurately studied there has been seen, before the fusion of the nuclei, a process comparable to the formation of the polar globules in the egg, to this extent, that in each of the conjugating individuals the nucleus divides twice and of the products of division only one, the nucleus intended for caryogamy, persists, while the others (polar globules) degenerate.

These cases of true fertilization may differ greatly. The conjugating individuals may be equal in size, isogametes (most Infusoria, many Rhizo- 
poda), or there is a disparity in size (sexual dimorphism), in which smaller and consequently more molile 'males' (microgametes, zoospores) fertilize the larger fixed or slowly moving 'females' (macrogametes, oospores) as in Vorticellidae, most Sporozoa, and flagellates, forming with them a permanent zygote (copulation). The formation of a zygote can also occur by the permanent fusion of two isogametes, l)ut usually the union of isogametes is transitory (conjugation) and lasts only long enough for cross fertilization, gamete A fertilizing B, and in turn being fertilized by $B$, after which the two separate. A striking phenomenon is the not very rare 'autogamy' in which the mother animal divides into two daughter animals, which form polar globules and fuse to a zygote, an extreme case of inbreeding.

Thirty years ago it could be laid down as a universal fact that the Protozoa in contrast to the Metazoa lacked sexuality. Since then observations have so increased that the conclusion is that fertilization occurs in all Protozoa, although the rarity of the process in many species renders the demonstration difficult. Perhaps also in many groups fertilization has been lost through degeneration (similar to the apogamy of plants).

Still there remain certain interesting differences from the Metazoa. The Protozoa lack special sexual cells-egrs and spermatozoa. On the contrary, the whole body functions as a sexual cell. Further, the relations of fertilization to reproduction are not the same as in the Metazoa. (I) Protozoa may increase in the same way before and after fertilization, indeed somewhat more slowly after (Infusoria). (2) Sometimes fertilization brings nourishment and reproduction to a standstill, in which case encystment appears (many rhizopods and flagellates). (3) A third case is where division follows fertilization, occurring more rapidly and having another character (sexual reproduction, better 'metagamic division,' 'sporogamy') than the pre-fertilization divisions (asexual reproduction, 'schizogony,' better, 'metagamic reproduction'). These alternating pro- and metagamic reproductions have been called alternations of generations (most Sporozoa, many rhizopods).

Analysis of these phenomena leads to the conclusion that we may speak of fertilization but not of sexual reproduction in the Protozoa. As was said previously (P. I 49) these facts have great importance in the explanation of the existence of fertilization, since they show that it has not always the purpose of stimulating the reproductive processes and thus leading to the formation of a new individual. Fertilization has to accomplish other things for the organism; they must be of great importance, since they are so widely spread; but as yet their significance is not clea!

The Protozoa, with small and soft protoplasmic bodies, are but slightly protected against drying up, and therefore they are aquatic. Some, like Amoba terricola, are terrestrial, but these only occur in moist places. Salt and fresh water, of the latter stagnant pools rich in regetation, are the favorite places. The fresh-water forms are cosmopolitan, so that the species in all lands are very similar. This depends upon certain peculiarities. The fresh-water Protozoa can become encysted and in the encysted 
stage can endure unfavorable conditions such as lack of food, freezing, or complete evaporation of the water. When thus protected they may be blown about by the wind or carried far on the feet of birds. Hence one group bears the name Infusoria, for if dry earth or dry plants (e.g., hay) be soaked in water and this infusion allowed to stand for some time, a Protozoan fauna will develop in it. The encysted animals in the earth or on the plants are awakened by the moisture to new life and leave the cyst. Spontaneous generation, as was once beliered, does not occur here, for if one sterilize the materials and prevent the entrance of germs the water will remain uninhabited.

The protozoa are very important from the pathological standpoint. Each of the four classes includes numerous parasites, the Sporozoa being exclusively parasitic. Many cause serere infective diseases (malaria, relapsing typhus, sleeping sickness, etc.) especially in the warmer climates, while in the north, at least as far as man is concerned, the bacterial diseases predominate. Many protozoan diseases are 'inherited,' that is the egg cells are infected by the parasites. This is the case with the pebrine disease of silkworms, the Texas fever of cattle and others.

Historical.-On account of their invisibility the Protozoa were unknown until I 675; they were discovered in infusions by Leeuwenhoek, the discoverer of the microscope. Wrisberg called them Animalcula infusoria-infusion animals, and Siebold gave them the name Protozoa. Ehrenberg maintained that the Protozoa, like all animals, possessed alimentary canal, nervous system, muscles, excretory and sexual organs. Dujardin denied all this and recognized in them only a single homogeneous substance as sufficient to produce all vital phenomena. Siebold discovered that the Protozoa were unicellular. The fact that there are unicellular animals without organs and yet capable of existence was an extremely valuable addition to knowledge, for it not only broadens our conception of animal life, but it furnishes for the theory of evolution from simple organisms the most important link, the first of the chain.

The different appearances of Protozoa depend upon the degree of organological and histological differentiation. Since these are most prominent in the nourishing and locomotor structures, these become important in subdividing the group. In accordance with the motion and taking of food by pseudopodia, flagella or cilia, there are three classes: Rhizopoda, Flagellata and Ciliata (Infusoria, s. str.). To these are added the Sporozoa, modified in motions and mode of feeding by parasitism. Undoubtedly Rhizopoda, Flagellata and Sporozoa are much closer to each other than are the Ciliata; hence they are grouped as Plasmodroma or Cytomorpha in contrast to Ciliomorpha or Cytoida.

\section{Class I. Rhizopoda.}

First of the Protozoa are those organisms which lack permanent structures for locomotion and nourishment, the protoplasm of the body performing these functions. The term Rhizopoda refers to the fact that the protoplasm sends out root-like processes or pseudopodia forlocomotion and 
for taking nourishment. These differ from true appendages in that they are not constant, but are formed according to demand and again disappear. A pseudopodium arises when the protoplasm streams to one point of the body and extends as a process beyond the surface. Since the process becomes attached and draws the body after it, or since the protoplasm of the body may flow into it, a slow change of place occurs. In either case the process disappears in the organism, and new pseudopodia are formed at other places which are retracted in turn. This type of bocomotion is called amœboid after the $A m a b a$, in which it was first studied. When the Rhizoporla in their wanderings meet particles of nourishment, they enclose them with their protoplasm and take them into the interior of the body (fig. I1 $7, N^{\prime}$ ).

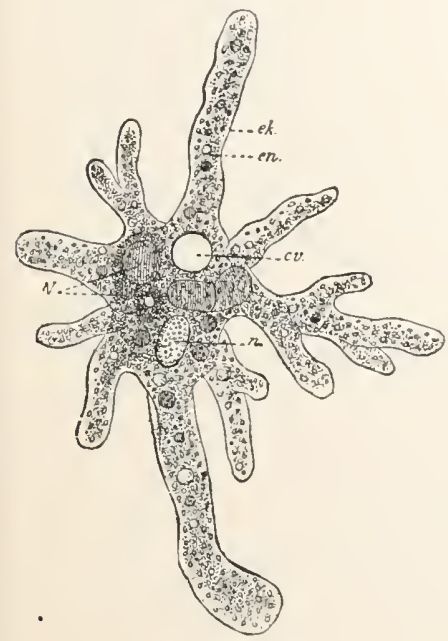

FIG. II 7 .

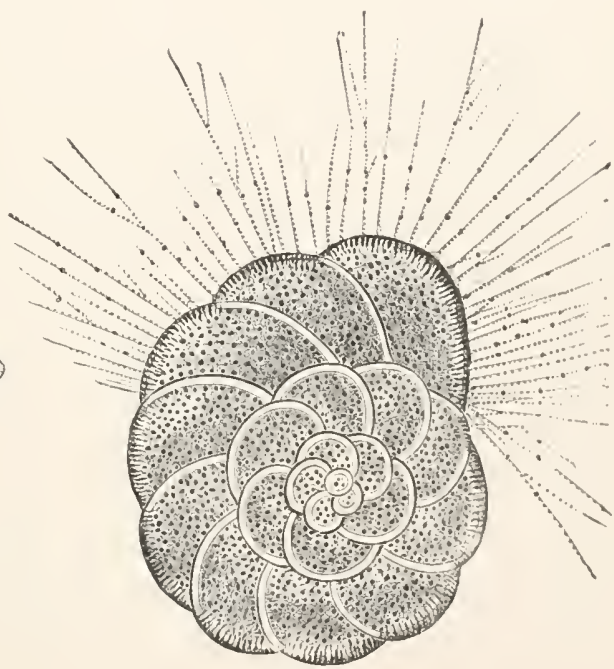

FIG. IIS

FIG. II7.-Amaba proteus (after Leidy). c2', contractile vacuole; en, entosarc; $c k$, ectosarc; $n$, nucleus: $N^{\top}$, food-body.

FIG. II8.-Rotalia freyeri (from Lang, after M. Schultze).

The shape of the pseudopodia is approximately constant for each species, but it varies so with different forms that it may be used not only for separating species but groups. On the one hand, there are finger-like pseudopodia (fig. II7), on the other, those of such delicacy that even under strong magnification they appear like fine threarls (fig. II8); and between these extremes many intermediate forms. Threal-like pseuclopodia usually branch, and when the branches meet they may fuse and form anastomoses, from which it follows that the pseudopodia are not 
covered by a membrane. The fine granules of the protoplasm usually enter the pseudopodia and produce here, as they move back and forth, the phenomenon of 'streaming.' Since foreign particles can participate in this streaming, it follows that the movements depend on the protoplasm itself. We have al ready used this fact (p. 55) to demonstrate the extraordinary complexity of protoplasm.

When Rhizopoda increase by division, the division products frequently become flagellate spores or zoospores. The body becomes oval and

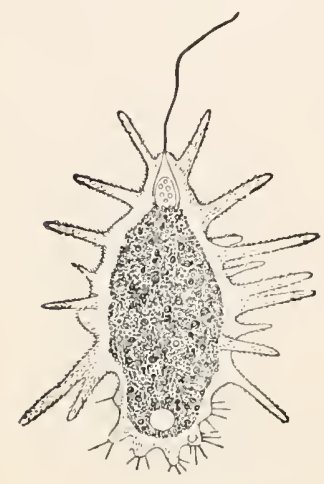

FIG. 1I0.-Mastigameeba aspera (afte: F. E. Schulze). develops, on the end which contains the nucleus, one or more flagella, which move more energetically than pseudopodia, and are permanent as long as the zoospore stage persists (fig. 122). Since many Protozoa possess flagella along with pseudopodia, the boundary between Rhizopods and Flagellates is not distinct (fig. I I g).

The Rhizopoda form an ascending series in which the systematic characters become more and more pronounced; such are the assumption of a definite form, as in the Radiolaria and Heliozoa, the formation of a skeleton of regular character, as in the Thalamophora, or the development of a peculiar reproduction, as in the Mycetozoa. At the bottom stand the Monera and the Lobosa whose characters are mostly negative, for neither form, skeleton, nor reproduction affords systematic distinctions.

\section{Order I. Monera.}

The most important character of the Monera is the lack of a nucleus. As with other negative characters this is somewhat uncertain. In many cases, especially when the protoplasm is filled with pignent granules, the nucleus is recognized with difficulty, and hence animals have been described as anucleate in which the nucleus was overlooked. So it is possible that, in the few forms now remaining in the group, the nucleus has merely escaped obscrvation; possibly it is functionally replaced by the chromidia (p. 58). There are several theoretical reasons favoring the idea of anucleate organisms. It is easier to suppose that with the appearance of life there were organisms consisting of but a single sulsstance than that these organisms had nucleus and protoplasm already differentiated. Several species of Protamaba are placed in the Monera.

\section{Order II. Lobosa (Amœbina).}

Lobosa are primitive Rhizoporla with one or several nuclei. The species of $A m c b a$, forms which owe their name to their constant change of shape, are typical (figs. I 7, I20). This change of form is due to the formation and disappearance of a few finger-like (lobose) pseudopodia. Body and pseudopodia consist of two layers, a soft granular inner entosarc $(e n)$ and a firmer, clear, outer ectosarc $(e k)$. In the entosarc is usually 
a single (sometimes several) nucleus ( $n$ ), which is vesicular, and contains either one large or several smaller nucleoli. A contractile vacuole is usually present. Reproduction occurs by binary or multiple division (fig. I 20), and encystment has been observed, the protoplasm dividing into many hundred small amobe, a phenomenon always connected with fertilization processes (autogamy?).

Most Lobosa occur in fresh water; A. terricola in moist earth. There are also parasites like $A$. coli, rare in colder climates, frequently observed in the tropics. According to recent researches two forms have been included under the name A. coli, one innocuous, Entamaba coli, and another (possibly several) pathogenic forms, including $E$. histolytica, which appears in enormous numbers in abscesses of the liver and ulcers of the colon of men ill with tropical dysentery For the first of these it is certain, for the other probable, that infection is caused by encysted forms, which arise as a consequence of fertilization and are passed out with the freces.

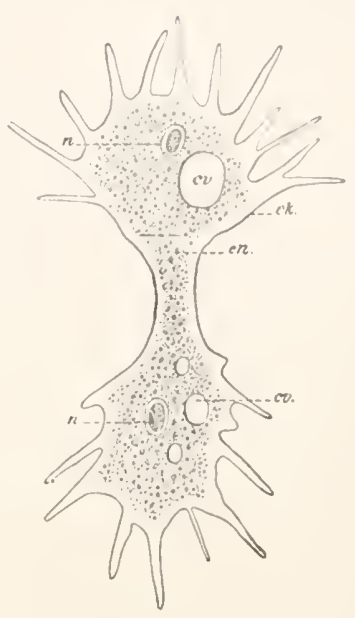

FIG. I20.-Ameba polypodia in division (after $\mathrm{F}$. E. Schulze). cr', contractile vacuole; $e k$, ectosarc; $e n$. entosarc; $n$, nucleus.

\section{Order III. Heliozoa, Sun Animalsules.}

The Heliozoa owe their name to the shape of the body, with the pseudoporlia arranged like rays. In each pseudopodium is a firm axial thread, forming a

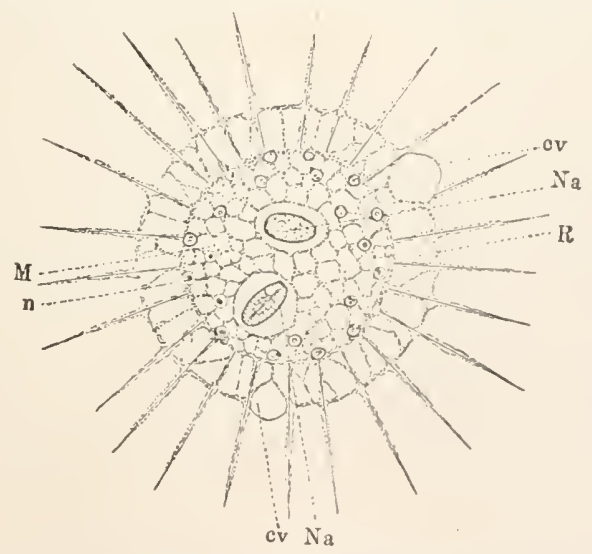

FIG. I2I.-Actinospherium eichhorni. $M$, medullary substance with nucleus $(n): R$. cortical substance with contractile vacuoles $\left(c 2^{\prime}\right) ; . \mathrm{T} a$, food-body.

skeleton, and a thin coating of protoplasm. Branching and anastomoses of the pseudopodia are rare. The axial threads frequently converge at the 


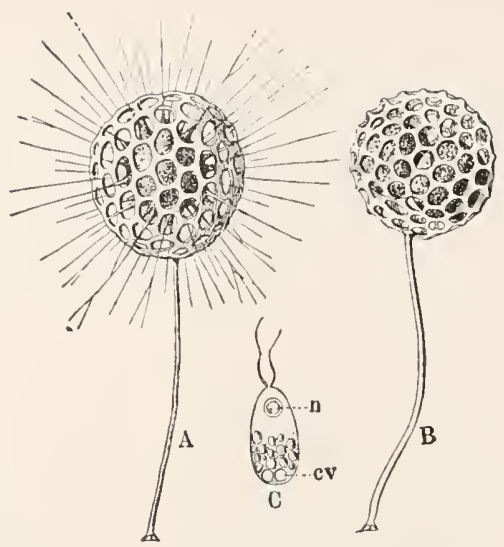

FIG. 122.-Clathrulina elegans. A, with extended pseudopodia; $B$, divided into two cysts; $C$, zoospore; $n$, nucleus; $c 2$, contractile vacuole.
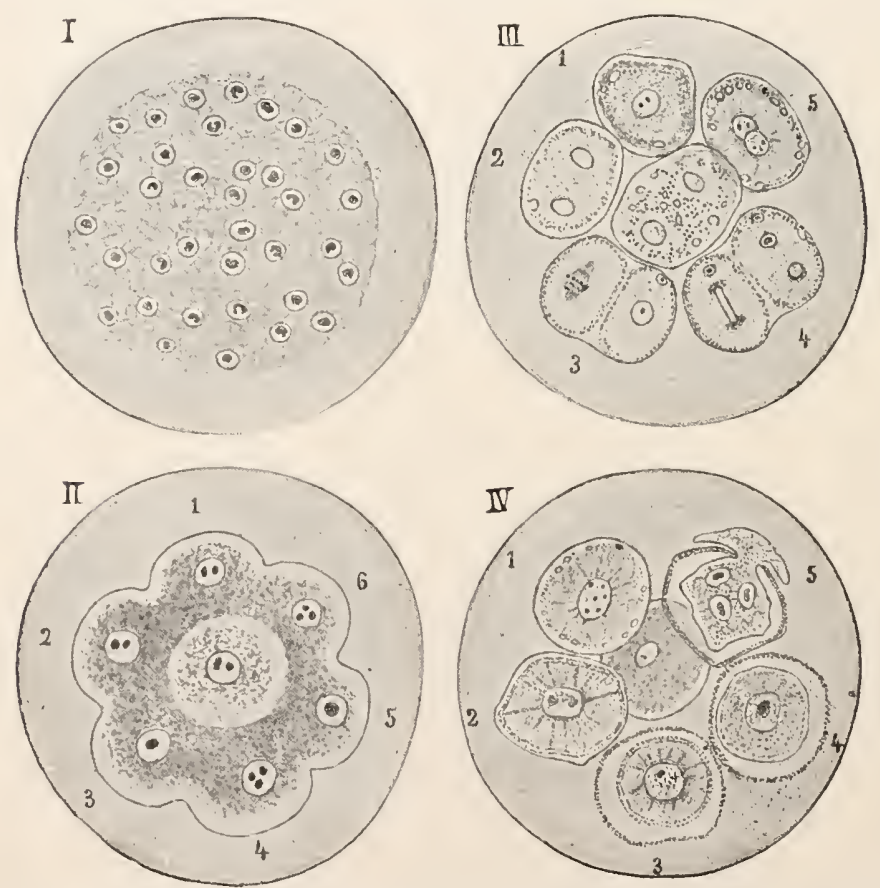

FIG. I23.-Encystment of Actinospharium, schematized to the extent that in III and IV, successive stages are shown in the same figure. I, just encysted animal; II, formation of the primary cysts, after resorption of most of the nuclei; III, I and 2, division of primary into secondary cysts; 3,4 , maturation of secondary cysts; 3 , formation of first, 4 , of second polar globule, 5, fusion of secondary cysts (autogamy); IV, I-4, stages of conjugated cysts; 5, escape of young Actinospharium. 
centre of the body. Here lies the centrosome separated from the nucleus ( $c f$. p. 59), which controls the division of nucleus and protoplasm. The londy consists of cortical and medullary portions (fig. 12I), distinguished by differences in the protoplasm, but not separated by membrane. In the cortex are the contractile vacuoles $(c v)$; the medulla contains the usually single nucleus. Reproduction takes place by division, and one or both moicties may become suirm spores, i.e., assume an oval form bearing at one pole one or two flagella (fig. I 22, C). These become widely distributed by the flagella before they resume the spherical shape, lose their flagella and form pseudopodia. It frequently occurs that several Heliozoa of the same species become connected by protoplasmic bricleses, and so form unions of from two to ten individuals (plasmogumy). 'True furtilization (karyogamy) has only been observed in Actinophrys and Actinosphorium.

Forms with skeleton and those without are distinguished. Among those with a silicious skeleton are Clathrulina clegans, with a spherical lattice-work skeleton supported on a stalk (fig. I22), and I canthocystis turfacea, skeleton of radial, branching needles. To the forms without a skeleton belongs Actinospharium cichhorni (fig. I2I), as large as a pinhead, milk-white, protoplasm foamy from the numerous vacuoles, the different sizes of which distinguish the cortical from the medullary parts. There are contractile vacuoles in the cortex, many nuclei in the medulla. In encystment the foamy appearance is lost and most of the nuclei are dissolved (fig. I23). Inside the grelatinous cyst the contents are divided into primary cysts, corresponding in number to the persistent nuclei, and these become enclosed in a silicious envelope (fig. I22, II). Wach primary cyst divides mitotically into wo daughter cysts (III, I, 2), which hecome mature by forming two polar globules (III, 3, 4) and then completely fuse with each other (III, 5). The germ cells arising in this autogamic manner enclose themselves in a resistent envelope (IV, I-4), from which, after a considerable period of rest, the young Actinospharia escape $(I V, 5)$.

\section{Order IV. Radiolaria.}

The Radiolaria, the most beautiful and most highly organized of the Rhizoporla, recall the Heliozoa. They are spherical, only rarely llattened into disks, or taking conical or lobular shapes. A second resemblance lies in the delicate pseudopodia, often with an axial filament. The most characteristic feature is the central capsule. This is the central portion of the body surrounded by a membrane, outside of which is the extracapsulum. If the central capsule be removed from the extracapsulum it not only lives but regenerates the lost parts, while the extracapsular portions die. Since the protoplasm of both parts is identical, the difference in regenerative powers can only depend on the nuclei, which are confined to the central capsule. The capsular memlorane is either perforated on all sides by numerous pore canals or by openings in certain places. The protoplasm passes through these and spreads itself in the cxtracapsulum. The chief part of this is a gelatinous mantle, through which the protoplasm extends as a fine network before it forms pseudopodia on the surface. In the larg 2 R Radiolaria it may be extensive, containing vacuoles (cxtracapsular aliecoli) developed in the protoplasmic net.

The central capsule may be uni- or polynucleate. In the first case the nucleus (fig. I24), a vesicle of appreciable size, lies in the centre of the capsule, in the others the capsule is thronged by hundreds of small homogeneous nuclei. The fact that certain species are nearly always multinucleate, others uninucleate is due to the fact that in them the primitively single nucleus divides early, while in the other it becomes considerably enlarged and forms in its interior material for the secondary nuclei, which escape, the remainder of the nucleus degenerating. 
With few exceptions the Radiolaria possess skeletons of wonderful beauty; latticed spheres, single or one within another, and bound together with radial rods (fig. 89), frequently ornamented on the outer surface with spines, or latticed discs, helmet-like or cage-like structures (fig. I 27 ). In other cases occur rings, tubes, spines, which meet in the central capsule (fig. I25), etc. In rare cases the skeleton is formed solely of organic substance (acanthin); usually it is silicious and resistant. Skeletons of Radiolaria occur in rocks of various ages, as in Sicily, the Nicobars (both tertiary), and the Barbadoes.

In reproduction there are numerous forms with a skeleton in which division begins with a cleavage of the central capsule and usually extends through the extracapsulum. If this latter does not divide a colony results, in which a jelly

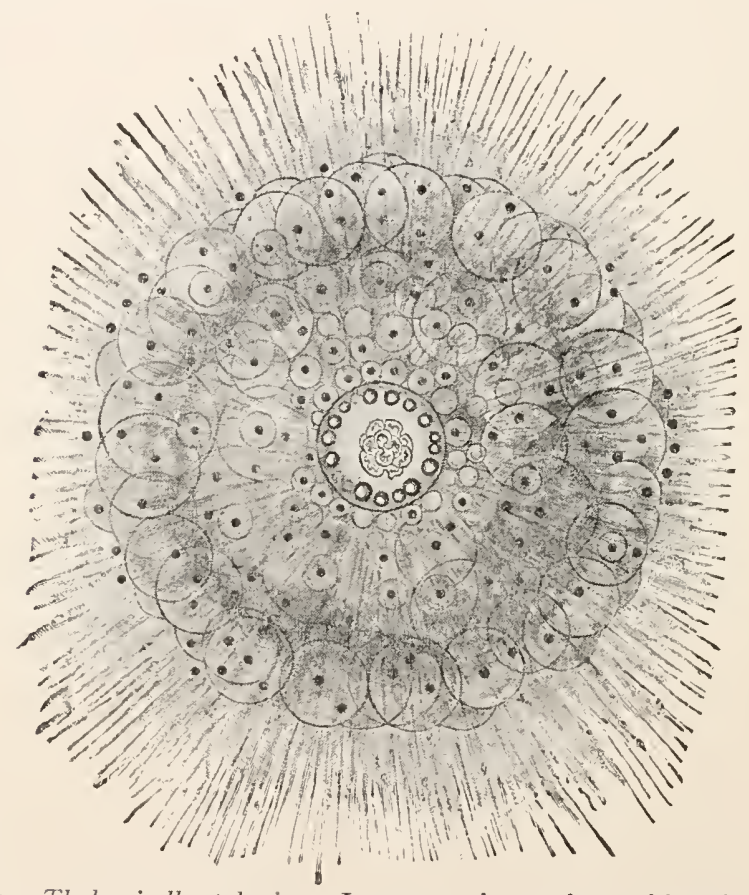

FIG. 124.-Thalassicolla pelagica. In centre the nucleus with coiled nucleolus, around it central capsule with oil globules: still outside the extracapsulum with vacuoles (extracapsular alveoli), yellow cells (black) and pseudopodia.

contains numerous central capsules, bound together by protoplasmic threads, which form the pseudopodia on the surface (fig. I28). A second type is reproduction by swarm or zoospores, which begins when the nucleus has divided into hundreds or thousands of daughter nuclei. The contents of the central capsule then divides into as many portions as there are nuclei, these become oval and develop two flagella (fig. I26), which soon begin to vibrate so that the central capsule is filled with a tumultuous crowd. With the breaking of the capsular membrane these swarm spores escape; here our knowledge of this type ceases. Since in many species there are macrospores and microspores it is probable that a copulation is necessary. 

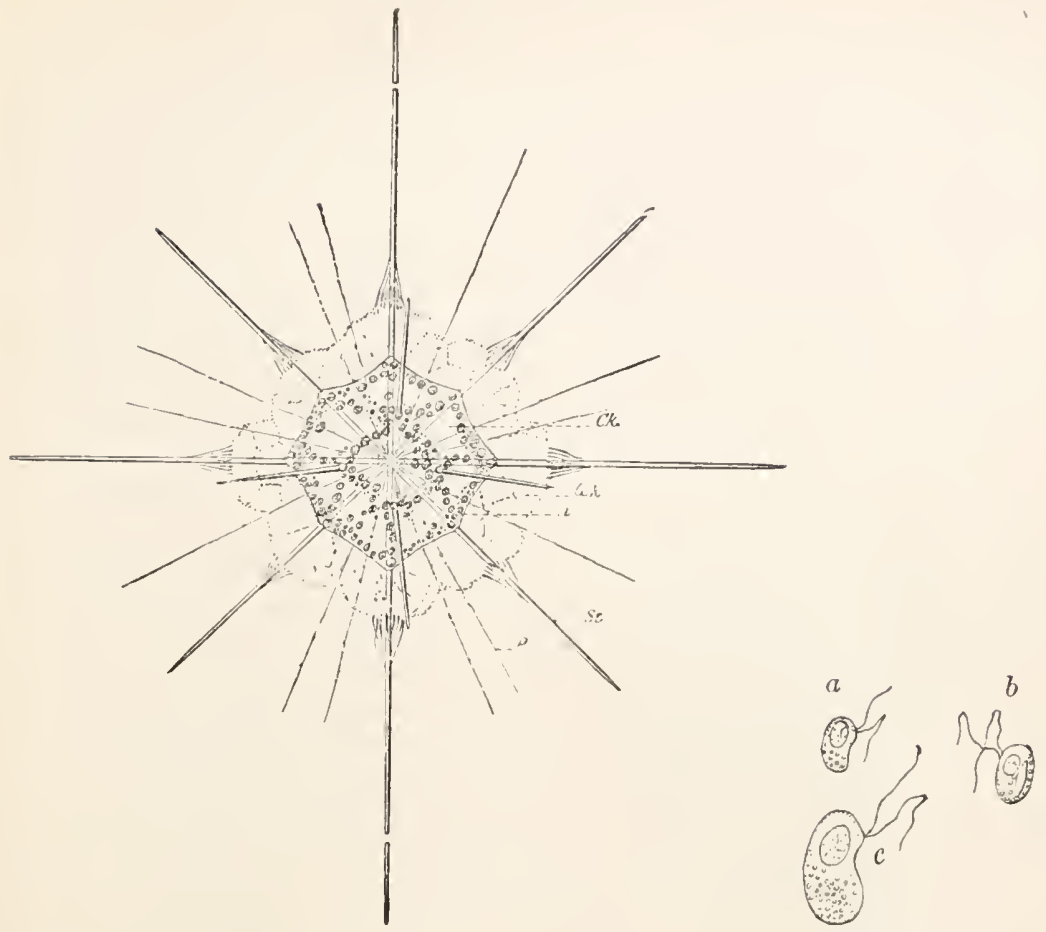

FIG. 125 .

F IG. 126.

FIG. I25.-Acanthometra elastica. ck, central capsule; $n$, nuclei; $p$, pseudopodia; St, spines; $W k$, extracapsulum.

FIG. I26.-Zoospores of Collozoum inerme. $a$, microspore; $b$, zoospore with fusiform body; $c$, macrospore.

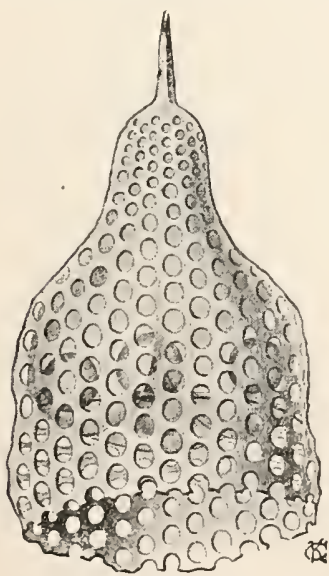

FIG. I27.

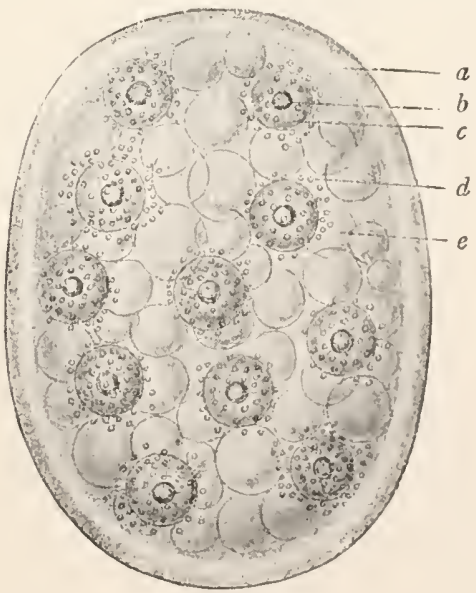

FIG. I2S.

FIG. I27.-Eucyrtidium cranioides (after Haeckel).

FIG. I28.-Collozoum inerme. $a$, jelly; $b$, oil globules in the central capsule; $c, d$. yellow cells; $e$, vacuoles. 
Common, if not constant, in the Radiolaria are the yellow cells, unicellular algæ (Zooxanthella), which are also present in other animals. (Thalamophora, actinians, sponges, etc.). They afford an instance of symbiosis, or the living together of different organisms for mutual good. The Radiolaria are exclusively marine. In fair weather they float at the surface, but sink in times of storm. Certain species and even large groups (Phæodaria) occur only at great depths (I 500-4000 fathoms); several thousand species known.

\section{Order V. Thalamophora (Foraminifera, Reticularia).}

The Foraminifera, though not equalling the Radiolaria in beauty and variety of forms, exceed them in numbers of individuals, and have a great importance in the history of the earth. No other group of animals has had so great a part in the formation of beds of rock.

The most prominent characteristic is afforded by the shell, which is closed at one pole, and usually open at the other, the pseudopodia passing through the aperture (fig. I 29). Accordingly as the axis connecting these poles is altered, the shell becomes disc-like, spherical, flask formed

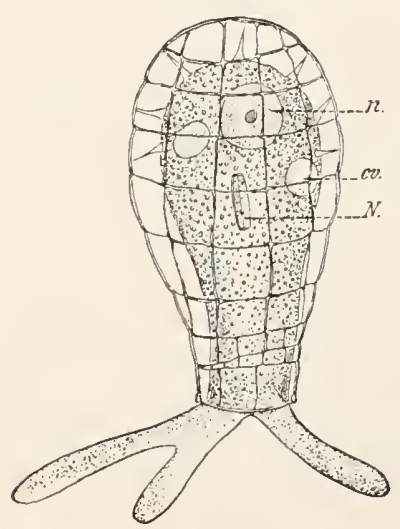

Fig. I29.- Ouadrula symmetrica (after F. E. Schulze). $c$ ', contractile vacuole; $n$, nucleus; $N$, food-body.

or even coiled in a spiral. The interior of the shell is frequently divided by transverse partitions into numerous chambers (fig. I3I). Such many-chambered shells (Polythalamia) are at first small, and consist of one or few chambers, but as the animal grows new chambers are added at the mouth of the shell. Openings (foramina) in the walls connect the adjacent chambers. The spiral shells with many chambers have a striking resemblance to the shells of the Nautilus (fig. 352).

In the fresh-water forms the shell is built of an organic substance which may be strengthened by silica or the incorporation of foreign particles. The more typical members, exclusively marine, have calcareous shells with but the slighest trace of organic matter. The presence of minute pores in the shell is of systematic importance, the group of Perforata (fig. I I ) being characterized by them.

The animal portions form a cast of the inside of the shell (fig. I30), and consist of as many pieces as there are chambers in the shell, connected by plasma bridges passing through the foramina of the partitions. In the protoplasm there is a large nucleus (figs. I 29, I30, $n$ ), which in some cases is early replaced by daughter nuclei. Contractile vacuoles usually occur only in the fresh-water forms. The pseudopodia project through 
the chief opening of the shell and in the Perforata probably through the pores in the shell wall. They are rarely finger-like (fig. I 29); usually they are thread-like, branched and anastomosing (figs. i 7, i is).

Reproduction is generally by fission, but presents many variations. Only rarely (fresh water MIonotha!amia) do both animal and shell divide; frequently the protoplasm protrudes from the mouth of the shell, a new shell is formed on the outgrowth and the protoplasm then divides, one of the resulting incividuals retaining the old shell. In the marine Polythalamia the following process is general: The polynucleate protoplasm divides into numerous uninucleate 'embryos' which frequently, while still within the mother, develop a shell.

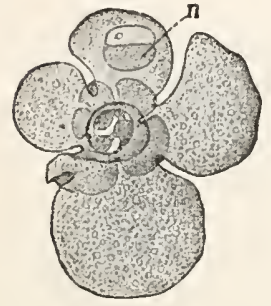

FIG, 130.-Protoplasm of Globigerina after solution of the shell. $n$, nucleus.

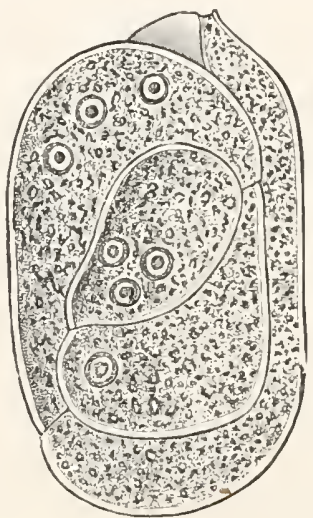

FIG. I3r.- Foung Miliola with several nuclei (from Lang).

A second kind of reproduction leading to a fertilization process appears to be common. Many swarm spores arise in the shells of the Polythalamia. These fuse in pairs with each other. Both of these reproductions alternate with each other, and with them is often connected a dimorphism of the individuals. The progamic generation, leading to the formation of gametes is distinguished by the long persistence of the chief nucleus and often by the structure of the shell (large central chamber, megasporic generation) of the metagametes arising from fertilization (polynucleate, microspheric gametes). I corresponding alternation has been observed in the Monothalamia.

Sub Order I. MONOTHALAMIA. Mostly fresh-water. They have onechambered shells of chitin or silica, often strengthened by foreign bodies. Contractile vacuole usually present. Pseudopodia lobose or filiform, branched or simple. A. Forms with finger-form pseudopodia: Arcella, Quadrula* (fig. I29); Diffugia* These forms are merely shelled Amabe and are frequently referred to the Lobosa. $B$. Forms with branching and anastomosing filiform pseudopodia. Euglypha, * Gromia (fig. I7). Sub Order II. POLYTHALAMIA. Exclusively marine; many-chambered shells. Thick beds of rock like the chalk, nummulitic limestone, and green-sand are largely foraminiferal in origin. The living species have an average diameter of about $\mathrm{I} \mathrm{mm}$. Rarely species have a diameter of several centimeters (Psammonyx zulcanicus, 5-6 cm.). The fossil nummulites reach a diameter of $10 \mathrm{~cm}$. 1. IMPERForata. Shell 
wall massive, the terminal opening the only communication with the exterior. Miliola (fig. I3I). B. PERForata. Shell perforated by many pores; terminal opening may be lacking. Polystomella, Rotalia (fig. ir8), bottom dwelling; Globigerina bulloides (fig. I30), pelagic. Among the fossils the Nummulites need mention.

Possibly the XENOPHYOPHORA of the deep seas belong near the For aminifera. These are large biconcave discs or fan-like plates 2 to $7 \mathrm{~cm}$. across, formed of delicate tubes, sometimes arranged in a network, sometimes branched like antlers, and between them a skeleton of foreign bodies, the 'xenophya.' The fine tubes (granellare) are filled with a polynucleate protoplasim; the larger tubes (stercomare) contain large balls, interpretated as frcal. The animals have not been studied in the living condition.

\section{Order VI. Mycetozoa (Myxomycetes).}

The Mycetozoa or slime animals are regarded by so me as animals, by others as plants (slime moulds). The first view is supported by the structure of the motile stage, the plasmodium, the second by the reproductive bodies, resembling those of many fungi. The plasmodia appear in damp weather as networks of brightred, orange or yellow slime on decaying wood. They are giant Amœbæ of reticulate protoplasm several centimeters in extent, containing many nuclei and

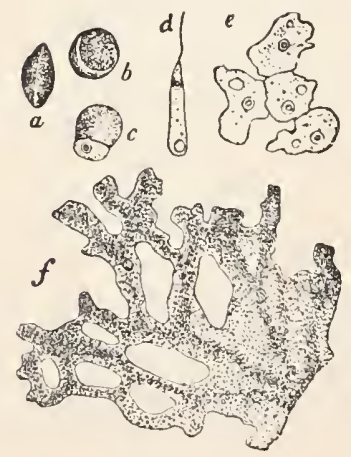

FIG. I32.

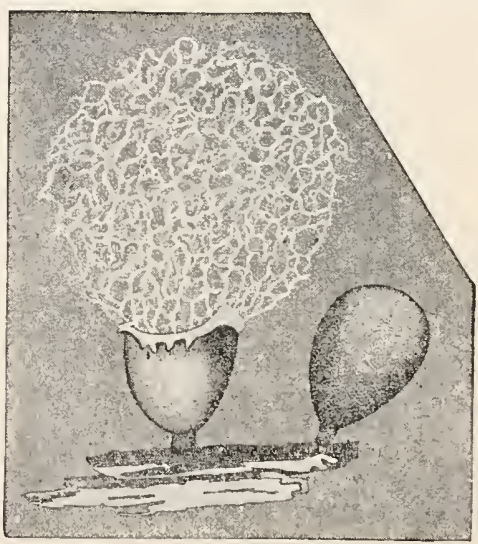

FIG. I33.

Fíg. 132.-Chondrioderma difforme (after Strasburger). $a$, dry spore; $b$, swollen in water; $c$, spore with escaping contents; $d$, zoospore; $e$, amoeboid modification of zoospores which are uniting to form a plasmodium; $f$, part of a plasmodium; in $d$ and $e$, nuclei and contractile vacuoles.

FIG. I33.- Spore-sacs of Arcyria incarnata (after de Bary). At the left the sporangium ruptured by the expanding capillitium, which has discharged the spores.

much foreign matter taken as food. They creep slowly by means of pseudopodia (fig. 1 $32, f$ ). On drying, the plasmodium encysts in a peculiar manner, and forms the reproductive bodies, the sporangia (fig. I33) and the 'carpome.' These are firm-walled vesicles, frequently stalked, the stalk sometimes extending into the axis of the sporangium as a columella. The space between the wall cf the sporangium and the columella is filled with fine powdery spores and an exploding apparatus, either a network of fine filaments (capillitium) or many 
spirally coiled threads (elaters). When wet, as by rain, the elaters or capillitium expand, rupture the sporangium and scatter the spores. The spores germinate in water or on moist surfaces, and from each comes out a small amoba-like embryo, frequently furnished with a flagellum (fig. 132). Several of these embryos fuse to form a plasmodium: Ethalium septicum, flowers of tan, plasmodium yellow, on spent tanbark; Arcyria (fig. I33), Plasmodiophora brassica, parasilic in cabloage.

\section{Class II. Flagellata (Mastigophora).}

In many Rhizopoda (P. I 72 ) the pseudopodia disappear at the time of reproduction and are replaced by one or two flagella; others have, besides pseudopodia, permanent flagella. Such flagellate spores and flagellate Rhizopods form the transition to the Mastigophora, which are permanently flagellate, the flagella serving as organs of locomotion and feeding. The three orders must be described separately.

\section{Order I. Autoflagellata.}

The Autoflagellata form a polymorphic group. The free forms are usually oval, with a nucleus in one end

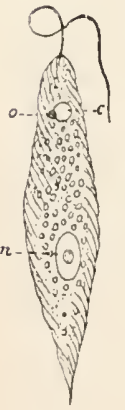

FIG. 134 .

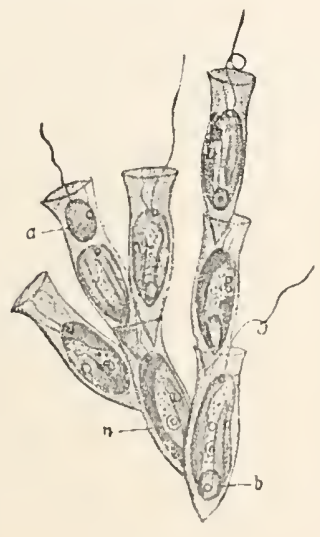

FIG. 135.

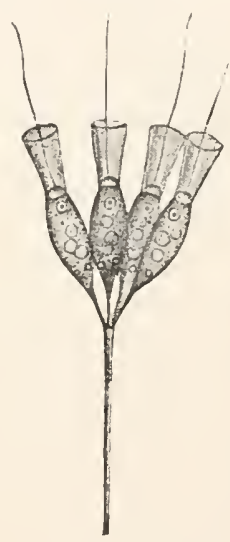

FIG. 136 .

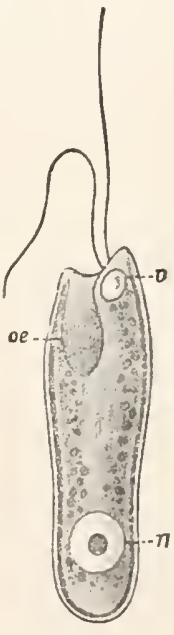

FIG. 137 .

FIG. I34--Euglena viridis (after Stein). c, contractile vacuole; $n$, nucleus; 0 , pigment (eye) spot.

FIG. 135.-Dinotryon sertularia (after Stein). a, a parasitic flagellate of ten found in the lorica; $b$, contractile vacuole; $n$, nucleus.

FIG. I36.-Conocladium umbellatum (after Stein).

FIG. I37-Chilimonas paramacium (after Bütschli). oe, cytostome; n, nucleus; v, contractile vacuole.

and a contractile vacuole in the other. It the anterior end there is often a red or brown eye-spot (fig. 134) which gives these forms great sensitiveness to light. In the parasitic forms the eye-spot and 
contractile vacuole are usually lacking (figs. I38, I39, I40, I4I). There are usually one or two flagella at the anterior end; when more occur (eight or more) they are distributed over the body. They differ in form and function so that chief, accessory and trailing flagella are distinguished. In the parasitic species there is usually a duality of the nucleus, recalling that of the Infusoria, there being, besides the principal nucleus, a second, the blepharoplast, at the base of the flagellum (fig. I $38, c$ ). The surface of the body is frequently naked, and then is of ten capable of amoboid motions. Again it may be covered with a more or less

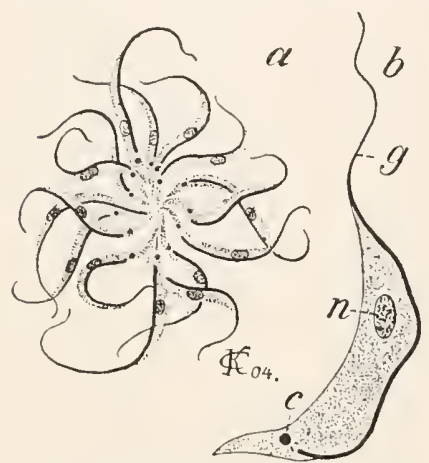

FIG. I38.--Trypanosoma levisi (after Laveran et Mesnil). $a$, agglomerate; $b$, single one enlarged; $g$, flagellum; $n$, nucleus; $c$, blepharoplast. evident cuticle. Closed gelatinous enrelopes or open, goblet-shaped cups (lorica, fig. I35) are common. There may also be slender stalks, to which he animals are attached in small groups (fig. I 36 ).

There are great differences in the feeding and in the organs connected therewith. Many feed like animals, taking solid food with pseudopodia like the Rhizopoda or with a mouth like the Infusoria. In the Choanoflagellata (fig. I36) there is a collar, a funnel-like process of the body protoplasm around the flagellum, to which foreign particles thrown by the flagellum adhere and are conveyed to the interior. Besides these animal forms are plant-like species which contain chlorophyl (Volvocine, Euglenidx) or brown chromatophores (Chromomonadinex), aiding in assimilation and enabling the organism to produce paramylum or even starch. It is noteworthy that vegetable and animal methods of nourishment appear in forms closely related anatomically. Indeed, there are species which possess a cytostome without taking solid nourishment, assimilating by means of chlorophyl or living on fluid food (fig. 137). All this shows that the Flagellata have relations with Rhizopoda, Infusoria, and the lower plants (bacteria and algæ).

Reproduction is nearly always by fission. Conjugation is known in many species. In the Volvocina two individuals fuse completely to form a resting spore. In the colonial Volvox globator the conjugating individuals are unequal, some animals of the colony growing to large immobile oospheres (macrogametes), while others by division form groups of minute active zoospores (microgametes). When fertilized by the zoospore the oospheres fall to the bottom, encyst, and enter a resting stage before they form a new colony. Young colonies reproduce 
asexually. While most of the individuals of a colony are concerned with nutrition and motion, others grow larger, and by rapid division, form new colonies.

There is yet uncertainty about the fertilization of the parasitic Autoflagellates. Encystment with autogamy has been described for the intestinal parasites, and in the Trypanosomes, parasitic in blood, conditions similar to those of the Hæmosporida seem to occur. 'The transfer of the parasite from host to host is
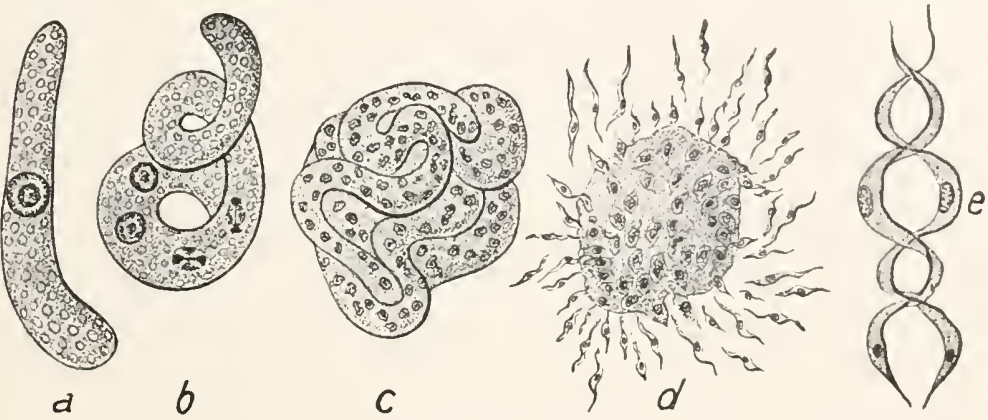

FIG. I39.-Leucozytozoon zicmanni (after Schaudinn). $a-c$, growth ancl multiplication of nuclei in ookinete, in intestine of mosquito; $d$, transformation of ripe ookinete into Spirochrte; $e$, division of Leucozytozoon.

accomplished by blood-sucking animals; insects (mosquitos, flies, lice, etc.) for mammals and birds; leeches (Piscicola) for fishes. Fertilization very probably takes place in the intermediate host. 'The following account, doubtful in several points, is given for Trypanosoma (Leucozytozoon) ziemanni. After the parasite has multiplied rapidly for some time in the blood of the owl, some

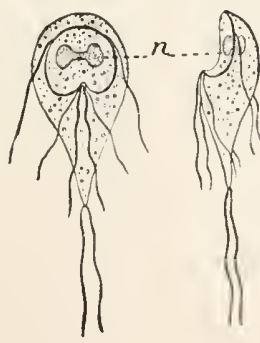

FIG. Ito.

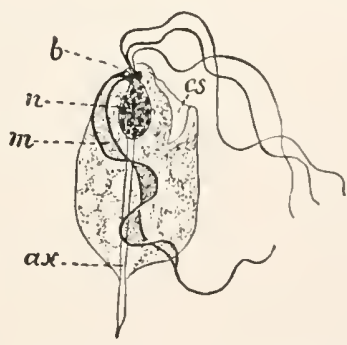

FIG. I4 I.

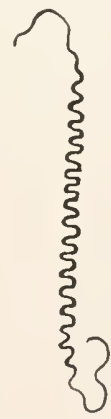

FIG. I 42 .

FIG. I 40.-Lamblia intestinalis (after Grassi). Front and side views. n, nucleus.

FIG. I 4I. - Trichomonas batrachorum (after Dobell). ax, axial rod; cs, cytostome; $b$, blepharoplast; $m$, undulating membrane; $n$, nucleus.

FIG. 142.- Spirochata pallida (after Schaudinn).

become macrogametes and others form eight microgametes. Macro- and microgametes unite in the intestine of the mosquito, Culex pipicns, to a zygote (ookinete), which grows to a large multinucleate body (fig. 139, $a-c$ ). From this, together with the formation of a residual body ( $c f$. Sporozoa) numerous young flagellates arise, which are transferred by the bite of the mosquito to a new host. 
Sub Order I. PHY'TOFLAGELLATA. Plant-like chlorophyl-bearing flagellates, mostly with eye-specks. Vouvocina: Volvox globator, * green sphere $0.2-0.7 \mathrm{~mm}$. in diameter, consisting of thousands of individuals. EugieNIDÆ: Euglena viridis* (fig. I34), solitary, coloring small pools by their immense numbers. Chrysomonadina, plant-like in nourishment: Dinobryon.* Sub Order II. CHOANOFLAGELLATA. With collars; mostly small colonial forms. Codosiga, * Conocladium (fig. I36). Sub Order III. EUFLAGELLATA. Animal flagellates, taking solid food by pseudopodia or by cytostome. Here belong, besides numerous free forms, several parasites of man: Lamblia intestinalis (fig. I40), also in rats and mice: Trichomonas hominis in small intestine. T. batrachorum (fig. I4I) is similar. Numerous flagellates are blood parasites, among them Trypanosoma brucei, which is introduced into horses and cattle in Africa by the tze-tze fly (Glossina morsitans), sometimes decimating the herds. Two other species, Tr. evansii and Tr. equiperdum attack horses. Tr. gambiense (castcllani) occurs in the blood and cerebrospinal fluid of man in tropical Africa, causing the 'sleeping sickness,' especially fatal to negroes, intermediate host Glossina palpalis. Tr. lewisii (fig. I38) in rat's blood.

Since the reproductive stages of $T r$. ziemanni show many resemblances to the Spirochcete, the latter, long regarded as Bacteria, may be flagellates. These

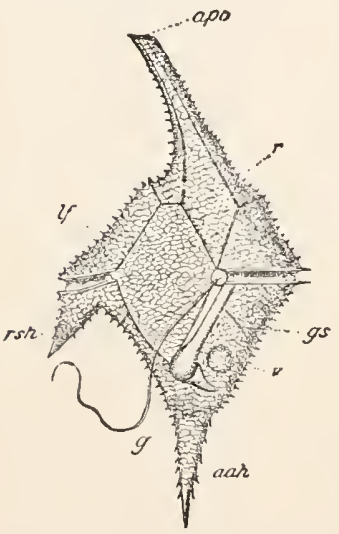

F1G. 143.-Ceratium cornutum (after Stein). apo, anterior horn with opening; aah, $r s h$, posterior and right horn; $g$, flagellum; gs, flagellar groove; lf, longitudinal groove; $r$, rhomboidal plate; v, vacuole. extremely dangerous organisms multiply by longitudinal division (the Bacteria by transverse), have a very motile corkscrew body, prolonged at either end into a flagellum. Sp. pallida (fig. I42) cause of syphilis; $S p$. recurrens, introduced by blood sucking arthropods (ticks, lice), causes recurrent typhus. Possibly Lieshmania, which causes splenomegaly and the Aleppo evil in man in warm climates, belongs near here.

\section{Order II. Dinoflagellata (Cilioflagellata).}

These forms, occurring in both fresh water and the sea, are placed near the plants because, with their brown chromatophores, their food relations are like those of plants, although the taking of solid food by a mouth opening has been observed. The armor formed of cellulose plates is also plantlike. This armor is divided by a groove into two parts which recall somewhat a box and its lid. There is also a longitudinal furrow which crosses the other. At the crossing are two flagella; the one in the transverse groove was regarded as a circle of cilia, whence the old name cilioflagellates given the order. Peridinium tabulatum and Ceratium cornutum, fresh water (fig. I43); Ceratium tripos, * marine.

\section{Order III. Cystoflagellata.}

The cystoflagellates, characterized by a gelatinous body surrounded by a membrane, include three or four species, all marine, which differ markedly in external appearance. Noctiluca miliars* (fig. I44), is remarkably phosphorescent. These spherical forms, about I $\mathrm{mm}$. large, sometimes occur in such numbers at night as to make the sea light at the slightest agitation. The phosphorescence is apparently caused by oxidation processes in the protoplasm. The body, a gelatinous sphere, is covered by a membrane, interrupted 
by a pit at one point, the cytostome, near which is the nucleus surrounded by an aggregation of protoplasm which sends branching threads into the jelly of the body. At the entrance to the cytostome is a flagellum, used in feeding but not in locomotion, and a band-like tentacle consisting of an outgrowth of the body membrane with a transversely banded muscular axis; it moves showly with a swinging motion. Noctiluca reproduces by simple fission (progamic reproduction) and by formation of swarm spores (? metagamic reproduction) (fig. I4t,

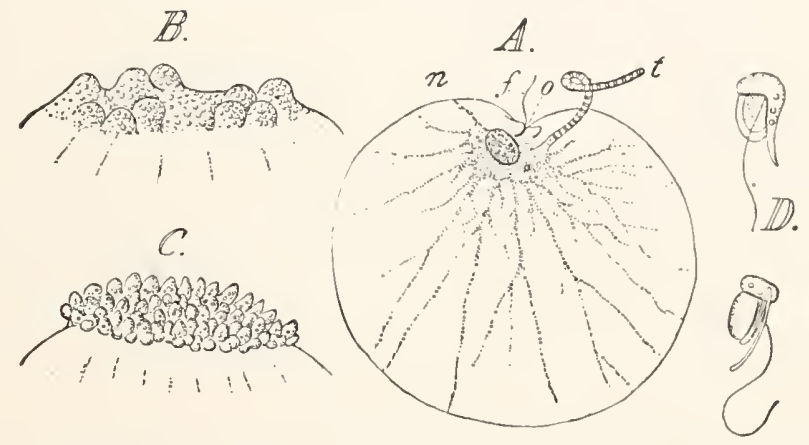

FIG. I H.-Noctiluca millaris (in part arter Cienkowski). A, entire animal; $f$, flagellum; $n$. nucleus; $o$, cytostome, beside it the 'tooth' and 'lip'; $t$, tentacle; $B, C$, upper end with two stages in the formation of zoospores; $D$, zoospores.

$B, C, D)$. In the latter two individuals lose tentacles, flagella, and cystostomes, and conjugate; after mutual nuclear fertilization (?) the animals separate, while the protoplasm in each collects in a disc which, by successive divisions, is converted into numerous uninucleate oval germs which at first project from the sphere, then separate and form small flagellate spores whose history is not known. Leptodiscus medusoides of Europe looks and swims like a small medusa. Craspedotclla.*

\section{Class III. Sporozoa.}

The Sporozoa are exclusively parasitic, and this has modified their feeding, movements and reproduction and in very similar ways. Many of them live as parasites in cells ('Cytosporida') as long as their size will permit; and after they have left the host cell, they live on fluid, not on solid food, hence they lack all arrangements for taking food, even in those cases where the body is covered with a cuticle. As a rule, there is also a lack of locomotor structures; but the occasional presence of amoboil motion or flagella indicates a near relationship with rhizopods and flagellates, so that it is difficult to draw sharp lines between the three classes. There are very close relations between the parasitic flagellates (Trypanosomes) and the Hæmosporida, and the Sporozoa must be regarded as rhizopods or flagellates modified by parasitism.

It is characteristic of the reproduction that the developmental stages before and after fertilization-pro-and metagamic-have different characters. The 
progamic development (schizogony) leads as a rule to autoinfection, to increase of the parasite in the tissues of the host. The parasite increases in size and breaks up into numerous young (merozoites), which grow in turn and divide. This process may continue many times until a sexually ripe form appears. Only in the gregarines is this replaced by a single large growth, which, in the period of preparation for fertilization, is divided into many parts. Fertilization usually precedes encystment, only rarely occurring in the cyst. Occasionally there is a fusion of isogametes; usually there are non-motile macrogametes fertilized by extremely active microgametes. In this the sexual dimorphism may be prepared long before and only be expressed in the generation (gamelocytes) from which the macro- and microgametes arise. The metagamic development (sporogony) requires encystment and serves to introduce the germs into a new host. Inside of the cyst spores are formed, which are rarely naked, usually surrounded with a firm enrelope. In the spore the sporozoite arises, the starting point for the progamic development. In all of the divisions which precede fertilization or immediately follow it, a part of the protoplasm, containing degenerating nuclei, remains behind as the residual body. From the development thus outlined the Myxosporida and Sarcosporida differ in some points, though they form spores.and sporozoites for new infections.

\section{Order I. Gregarina.}

The typical and longest known sporozoa are the Gregarines, parasites of oval or thread-like form (recalling round worms), usually somewhat flattened, which have only been found in the intestine or gonads, more rarely in the body cavity, of invertebrates. The protoplasm (fig. I45, A) is separated sharply into a clear ectosarc $(e k)$ and a granular entosarc $(e n)$. The ectosarc is covered by a cuticle $(c u)$, permeable by fluid food, for no cytostome exists. In many (perhaps all) there is a double striping of the body, a longitudinal recognizable by furrows on the surface and hence cuticular, and a transverse marking in the ectosarc, produced by circular or spiral muscle fibrille. These muscles explain the peristaltic motion and the occasional bending of the body, but not the peculiar gliding motion by which locomotion is usually effected. It may be that the gregarines secrete stiff gelatinous threads from the posterior end, and the elongation of these forces the body forward.

In many gregarines (Polycystidæ) the body is divided into a smaller anterior part, the protomerite, and a larger deutomerite (fig. I45, A). Internally this division is marked by a bridge of ectosarc across the entosarc. The vesicular nucleus (there is but one in any gregarine) lies in the deutomerite. All gregarines are parasitic in youth wholly inside of cells or with the anterior end imbedded in the host cell, which they leave in the developed stage. Many remain for a long time with a process of the protomerite in the cells. This process-the epimerite - is provided with threads or hooks for anchorage, and is usually lost when the animal gives up its connection with the host cell. Among the intestinal gregarines frequently occur 'associations' where two or more animals are fastened together head to tail in a row (fig. I 45, A). Perhaps these associations are preparations for conjugation which occurs in development. 
Reproduction typically occurs in an encysted condition (fig. I45, II). Usually two animals occur in a cyst. After each individual has become polynucleate by division of its nucleus, it divides at first superficially,

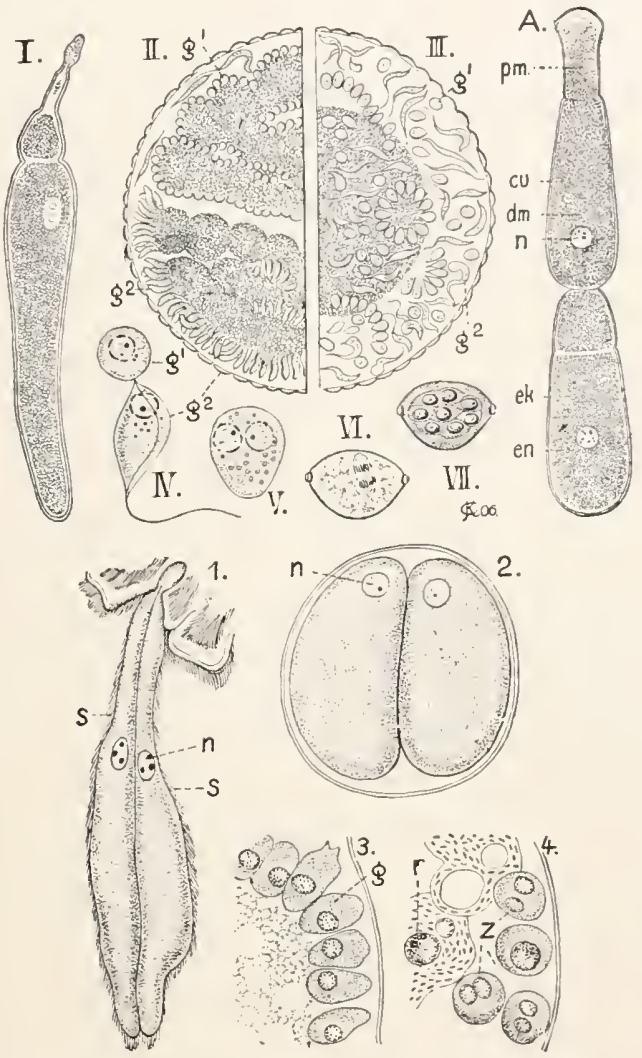

FIG. 145.-Different Gregarina. I-VII, development of Stylorhynchus; I, S. longicollis (after Schneider); II, encysted S. oblongatus (two animals) beginning gamete formation; III, same in later stage, the sexually differentiated gametes in copulation; IV-TII, formation and development of the zygote of S. longicollis more enlarged; IV, copulation of gametes; V, fusion; VI, beginning division; VII, 8, sporozoites formed. A. Clepsidrina blattarum. I-4, Monocystis magna (after Cuénot). I, two individuals in copulo in the spermatheca of an earthworm, surrounded by its spermatozoa; 2 , encysted; 3 and 4 , parts of cysts, formation and conjugation of the gametes, more enlarged (according to Brasil the gametes are slightly differentiated sexually). $c u$, cuticula; $d m$, deutomerite; $e k$, ectosarc; $e n$, entosarc; $g$, gametes; $g^{1}$, zoospores, $g^{2}$, oospore; $p m$, protomerite; $n$, nucleus; $r$, residual body; $s$, sperm of earthworm; $z$, zygote.

later internally into small spheres, the gametes (III). The gametes fuse in pairs to bodies which take a spinclle shape and become enclosed in a firm envelope, the spores, zygotes or 'pseudonavicellac' (fig. I $45,4, \mathrm{IV}-\mathrm{II}$ ). 
That always gametes of different origin fuse is shown by Stylorlynnchus where the gametes of one animal are flagellate, those of another are stationary. This dimorphism is so great in the Aggregatx that filiform spermatozoids occur, as in the Coccidia. After the formation of the gametes the movements of the residual body bring about the expulsion of the pseudonavicellæ; and in many Gregarines sporoducts are present for their escape. With repeated formation of a residual body, the contents of the pseudonavicella divides into (usually eight) sporozoites or falciform spores, which must leave the spores and pass anew into the tissue cells in order to form gregarines. This escape of the sporozoites depends upon entrance into the proper host. Often the transformation of the contents of the cysts into pseudonavicellæ takes place when the cysts have left the original host.

Best known are the Monocystis tenax of the spermatheca of earthworms, and Clepsidrina blattarum of the cockroach. The American species have scarcely been touched.

\section{Order II. Coccidiæ.}

Of all Sporozoa the gregarines are nearest the Coccidix, which are also cell parasites with a single nucleus, but without either cell membrane or division in to protomerite and deutomerite. Best known is Eimeria stieda (also called Coccidium cuniculi and oviforme), parasitic in the liver and intestinal epithelium of mammals. In the progamic development the fully grown parasite (fig. I 46 , 2) divides inside the infected cell into many cells $(3,4,8)$; these separate, infect other cells and begin growth and division anew (autoinfection). After this is repeated several times fertilization appears (5), some parasites giving rise to macrogametes, others by division forming small, actively swimming microgametes with one or two flagella. The fertilized macrogamete or zygote $(6,7$, 9 , ro) encysts, passes out and serves to infect a new animal. Beginning earlier or later, but only concluded in a new host, the contents of the cyst divide into several (in Eimeria, four) sporoblast-containing spores. Each spore (7, II) forms one or several (Eimeria, two) sporozoites, a residual body being lfft behind (r). Eimeria stieda produces cheesy granules in the liver of manmals. It is common in rabbits, rare in man. In cattle it is the cause of red dysentery.

\section{Order III. Hæmosporida.}

The Hæmosporida are very similar in structure and development to the Coccidia. They live in blood corpuscles, and on this account and from some analogies not sufficiently understood, they are regarded as related to the Trypanosomes. The IIæmamœbal forms parasitic in man cause malaria, there being in these a progamic reproduction with autoinfection and a metagamic in which the disease is transferred to another host. The parasites in the blood corpuscles (fig. I 47, I-3) grow and divide (daisy form, 2), characterized by little accumulations of pigment derived from the hæmoglobin of the corpuscle. These division products are set free by a breaking down of the corpuscle (period of chill) and infect other corpuscles. This autoinfection can continue a long time, until the Hæmamœbæ in the corpuscles grow, without dividing, to 'half moons' (4); these either become round and form macrogametes (5) or divide into eight microgametes (6). The conjugation of these seems only to take place 


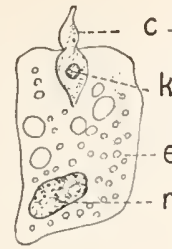

1.

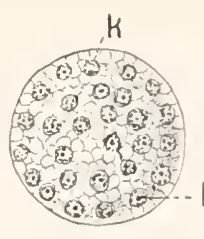

3.

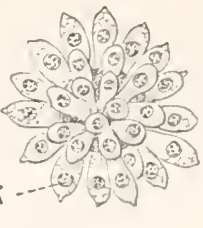

4.
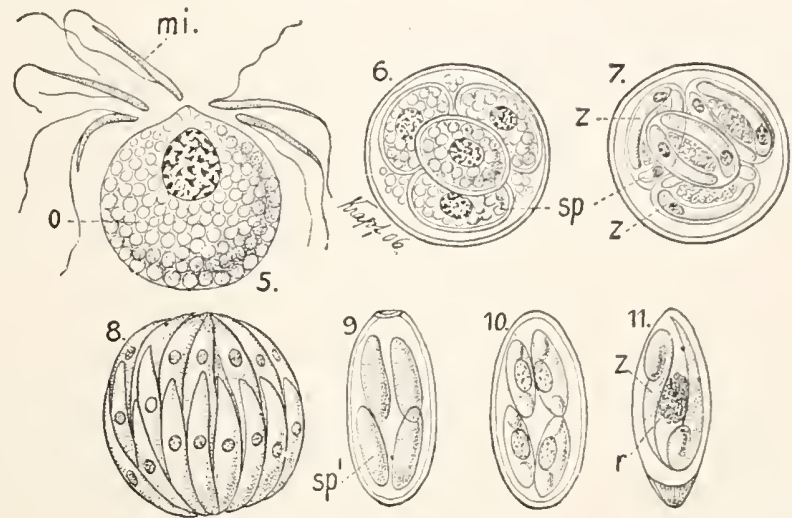

FIG. I46.-I-7. Development of Coccidium schubergi (after Schaudinn). I, entrance of sporozoites in cell; 2 , its growth; 3 , nuclear multiplication; 4 , division in to merozoites; 5, macro- and microgametes; 6 , zygote divided into four sporozoites. 8-II, Emeria stiedce (after Wasielewsky und Metzner). 8, autoinfection (progamic increase); 9, formation of sporoblists, Io, change of spores into sporozoites; II, spore with two sporozoites, more enlarged; $c, z$, sporozoite: $e$, epithelial cell; $k, n$, nucleus; $m i$, microgamete; $o$, macrogamete: $r$ residual body; $s p$, spore; $s p^{\prime}$, sporoblasts.

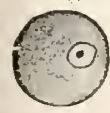

1

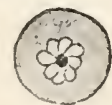

2

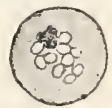

3

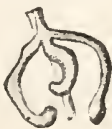

9
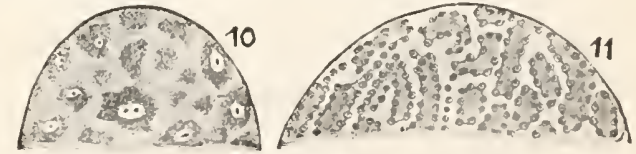
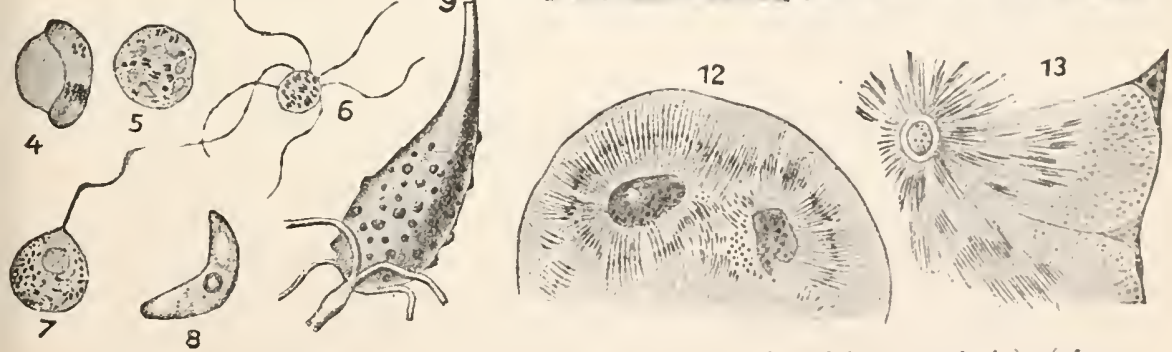

8

FIG. I47.-Development of Plasmodium pracox (pernicious malaria) (after Grassi). I, blood corpuscle with newly entered $H$ (emamoba; 2 , multiplication of parasite; 3 , young before the breaking down of corpuscle; $4-5$, formation of macrogametes; 6 , formation of microgametes; 7 , fertilization; 8 , fertilizer motile macrogamete (ookinet);9, digestive tract of mosquito, in front salivary glands, stomach covered with encysted parasites; IO-12, division of cyst; 10 , formation of sporoblasts: 11 and 12 , formation of sporozoites from sporoblast with the residual body; 1.3. part of salirary gland of mosquito infected with sporozoites. All figures except 9 greatly enlarged. 
when the gametes are taken into the digestive tract of a blood-sucking mosquito. The fertilized macrogamete, the ookinete, wanders into the intestinal wall, enlarges enormously, encysts and produces numerous naked sporoblasts. Each sporoblast gives rise to numerous sporozoites (II, I2) which wander into the salivary glands of the mosquito ( 13 ) and are transferred to the blood of man by the bite of the insect. For the transfer of human malaria apparently only mosquitos of the genus Anopheles will serve, not the more common Culex. Since a temperature above $20^{\circ} \mathrm{C}$. $\left(68^{\circ} \mathrm{F}\right.$.) is best for the development of mosquitos, and water is necessary for their development, the prevalence of malaria in warm climates is easily understood. The different kinds of malaria are caused by different parasites, the quartan fever being caused by Plasmodium (Hamameba malaria, pernicious malaria by $P$. precex. Allied to the HæmamœLæ and possibly also to the Trypanosomes, is Babesia (Piroplasma) bigemina, the cause of 'Texas fever in cattle. The tick, Boophilus bovis, serves as the intermediate host, the parasites being passed by the eggr ('inherited') to the next generation. Babesia bovis, intermediate host Ixodes reduvius, causes hæmoglobinuria in cattle.

\section{Order IV. Myxosporida.}

The Myxosporida (fig. I48) are mostly large (sometimes visible to the naked eye) and occur especially in fish and arthropods. When they occur in hollow

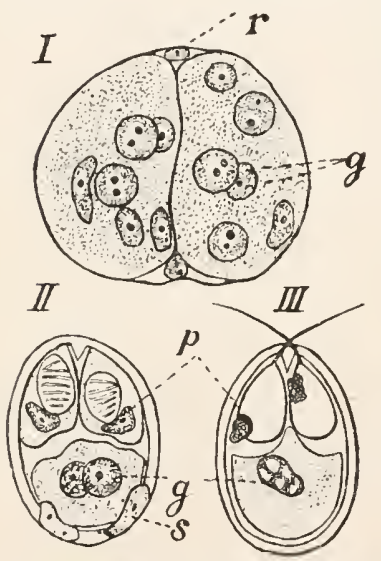

FIG. I48.-Development of Myxobolus pfeifferi, schematized (after Keisselitz). I, pansporoblast with envelope and residual nuclei, $r$, divided in to two sporoblasts; $I I$, sporoblast developing into spores; $s$, envelope cells; $p$, pole cells with pole capsule; $g$, amoboid germs with two nuclei; $I I I$, developed spore with extruded threads of the pole capsule, both nuclei of the amœboid germs fused. when they crawl into the tissues of the host. The number of pole capsules and of spores differs with the species. organs they are naked and have pseudopodia, but in parenchymatous organs like the heart, liver, brain, kidney, etc., they are usually enclosed in a membrane, and here they produce the greatest injury. At first binucleate, they soon become polynucleate, and apparently they can reproduce by fission. Even before the growth is ended they begin the process of sporulation, hence the name 'neosporida.' Reproductive bodies with one or two nuclei, the anlagen of the pansporoblasts, are differentiated in the protoplasm. In the best known forms each pansporoblast gives rise to two spores. By division there arise in all fourteen nuclei, two of which (fig. $148, r$ ), with the surrounding protoplasm, form the envelope of the pansporoblast. The others separate into two groups of six each. One pair in each group with their protoplasm form an 'amœboid germ;' they appear to be separated from each other early, but, though long separated, they at last unite (II, III, g), a case of caryogamy. Two other nuclei and protoplasm form the two-valved spore case, and the remaining pair furnish the 'pole capsules,' these being oval, and containing threads which under proper conditions, are protruded (III), the whole resembling a coelenterate nettle cell. The threads are for attaching the 'psorosperms' (as the spores were formerly called). The amœboid germs are set free, as experiments on fishes show, by the digestive fluids,
of the host. The number of pole capsules and 
The Myxosporida cause serious epidemics among fishes. The pebrine of the silkworm (the eggs are also infected) is caused by Tosemu (Glugea) bombycis. Rhinosporidium hominis, a parasite of the nasal mucous membrane of man in the tropics, is nearly related to the Myxosporidia.

\section{Order V. Sarcosporidia.}

The Sarcosporida (fig. I 49) - also called Rainey's or Miescher's corpusclesoccur in the voluntary muscles of vertebrates, especially mammals. 'They are oial cysts lying in sarcolemma sacs between the filorillæ. They have a cyst, tha wall of which is radially striped, and inside this, in the ripe condition, are spores, imbedded in a stroma, each spore containing numerous reniform or falciform sporozoites. Sarcocystis miescheriana in muscles of pig; S. muris in the mouse; S. lindemanni rare in human muscle.

At the end of the Sporozoa may be mentioned some much disputed bodies of very minute size, which are found in several infectious diseases (variola, trachoma, hydrophobia, etc.) and are regarded as their cause. They have been united under the common head of CHLAMYDOZOA.

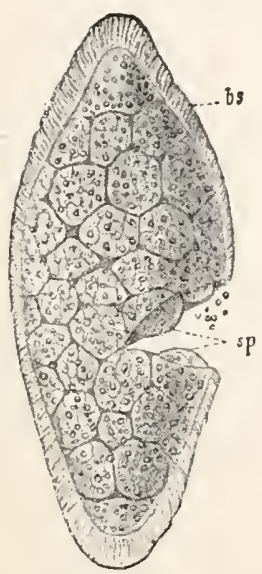

Fig. I49.
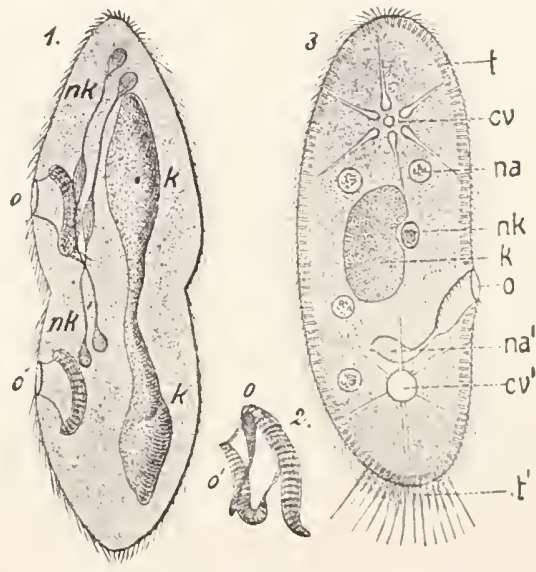

F1G. I50.

FIG. I49.--Sarcocystis miescheriana, from diaphragm of pig (after Butschli). bs, cyst; $s p$, spheres of spores.

Fig. I 50.-Paramecium aurelia in division; 2 , separation of cytostome of new individual from old cytostome, at an earlier stage; 3 , P. caudatum, flattened and schematic; $c v^{\prime}$, contractile vacuole, expanded and contracted; $k$, nucleus; $n a, n \iota^{\prime}$ food vacuole and one forming; $n k$, micronucleus; $o$, cytostome; $t, t^{\prime}$, trichocysts, $t^{\prime}$, discharged.

\section{Class IV. Ciliata.}

The Ciliata rival the Rhizopoda in numbers and variety of form. They are so complicated in structure that they were long he'd as multicellular. The form is definite for the species; and in the 'ametabolous' forms is unalterable, the 'metabola' can be temporarily pressed out of shape in passing through a narrow space. This constancy of form is due to a cu- 
ticle on the outside of the body, which in the 'ametabola' is firm; in the others very fiexible. The cuticle is covered with cilia-small vibrating processes which move together, and serve not only as organs of locomotion, but by creating vortices in the water bring food to the organism. They furnish the most important characterictic of the class (fig. I 50 ).

The presence of a cuticle necessitates a cytostome, except in the parasitic species, since food particles cannot be taken in at every point. At the cytostome the cuticle with its cilia forms a funnel-like food tube (cytopharynx) into the protoplasm. At the bottom the cuticle is interrupted so that water and protoplasm are in contact. By the action of the cilia food particles are taken into the cytopharynx and pressed against the protoplasm, forming a small enlargement which finally sinks into the substance as a food vacuole ( $n a$ ) which, by the streaming of the protoplasm, is carried about in the body. The digestible portions are absorbed, and those not capable of digestion are cast out of the body at a fixed point (cytopyge) usually not recognizable at other times (fig. I50.3). Contractile vacuoles $(c v)$ are lacking only in parasites and marine species. They are constant in number and position, and frequently have afferent ducts which empty into the vacuole, the vacuole in turn forcing the fluid to the exterior.

Trichocysts, nettle bodies, and muscular fibrillæ occur in some species. Trichocysts are minute rods vertical to the surface in the cortical layer, which under the influence of reagents (chromic acid) elongate into threads penetrating the cuticula. To these have been ascribed defensive functions; others recard them as tactile structures. They have no connection with the cilia. Nettle bodies are extremely rare. Muscle fibres lie between ectosarc and cuticle, and cause quick convulsive motions of the animal.

There are two nuclei physiologically unlike. The larger of these (nucleus of older writers, macronucleus) is a large oval, rod-like, or spiral body, deeply staining with microscopic stains, and surrounded with a membrane. It controls all the common vital functions of the animal (motion, feeding, etc.). Beside it or in a depression in it is the much smaller micromucleus (nucleolus or paranucleus of older authors) which stains less deeply. In all sexual processes it comes to the front and can be called the sexual nucleus.

Multiplication of Ciliata occurs by binary fission (fig. I50); more rarely, and then only in the encysted condition, by division into numerous parts. Buddling is known in the Peritricha and Suctoria. In fission first the micronucleus divides mitotically, and then the macronucleus separates by elongation and constriction. The old cytostome persists in the anterior offspring, but often an outgrowth from it $\left(2, o^{\prime}\right)$ passes into the posterior half and develops into a new mouth. 

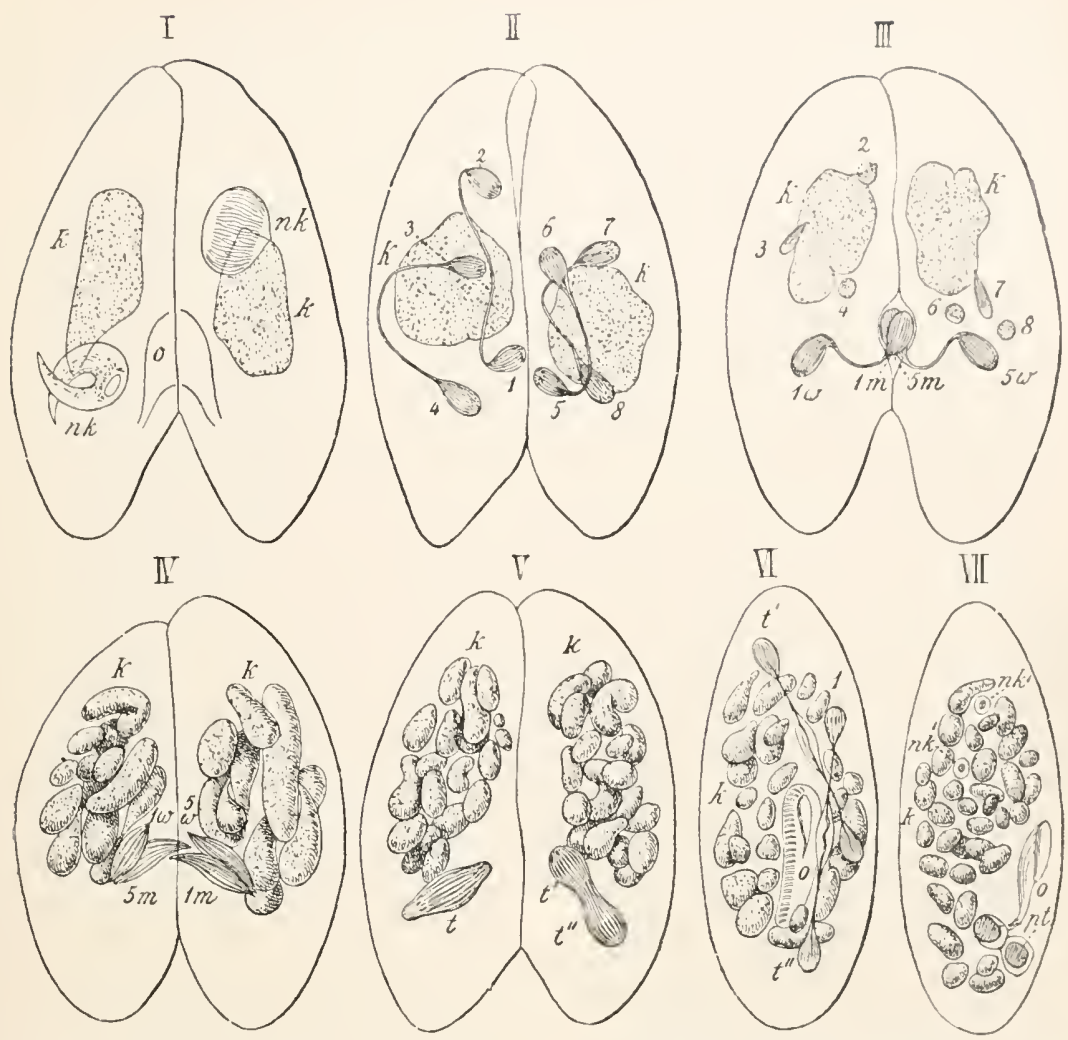

FIG. I5r.-Conjugation in Paramacium. $k$, macronucleus; $n k$, micronucleus; $o$, cytostomes.

I. Changes of micronucleus; left sickle stage, right spindle stage.

II. Second division of micronucleus; into primary spindles $(1,5)$ and secondary spindles $(2,3,4,6,7,8)$.

III. Degeneration of secondary spindles $(2,3,4 ; 6,7,8)$; division of primary spindle nto male $(\mathrm{I} m, 5 \mathrm{~m})$ and female spindles $(1 w, 5 w)$.

IV. Exchange of male spindles nearly complete (fertilization), one end still in the parent animal, the other united with the female spindle, Im, with $5 w$ and $5 m$ with $1 w$; macronucleus broken up.

$\mathrm{V}$. The cleavage spindle $t$ formed by male and female epindles dividing into the secondary cleavage spindles $t^{\prime}, t^{\prime \prime}$.

VI, VII. End of conjugation. The secondary cleavage spindle dividing into the anlage of the new micronucleus $\left(n k^{\prime}\right)$, and that of the new micronucleus, pt (placenta). The fragments of the old macronucleus begin to degenerate.

Since $P$. caudatum show's the earlier and $P$. aurelia the later stages better, these forms have been used, $P$. caudatum for I-III, $P$. aurelia for the rest. The differences consist in the existence of one micronucleus in $P$. caudatum, two in $P$. aurelia and that in the latter the nuclear degeneration begins in I. 
The periods of fission are interrupted from time to time by the sexual process of conjugation, which will be described as it occurs in Paramacium (fig. 15I). Two individuals touch by their whole ventral surfaces, so that their cytostomes come together. In the neighborhood of the latter a bridge of protoplasm connects the two animals. Later the individuals separate. While these easily observable external processes are occurring there is a complete modification of the nuclear apparatus in the interior. The macronucleus increases in size, and breaks into small portions which disappear within the first week after copulation (probably by absorption), and give place to a new nucleus derived from the micronucleus. At the beginning of copulation the micronucleus becomes spindle-shaped, divides and repeats the process, the result being the formation of four spindles in each animal, three of which break down, thus recalling the polar globules in the maturation of the egg (p. I33). The fourth or principal spindle places itself in the neighborhood of the cytostome at right angles to the surface and divides into two nuclei, the superficial being called the wandering or male nucleus, the deeper, the stationary or

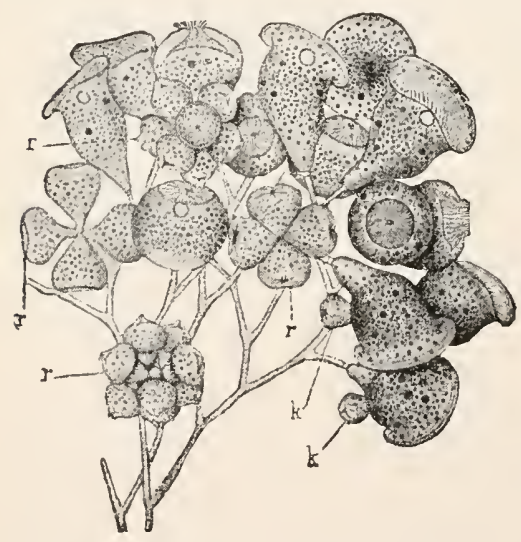

FIG. I 52.-Epistylis umbellaria (after Greeff). Part of a colony in 'bud-like' conjugation; $r$, microspores arising by division; $k$, microspore conjugating with a macrospore.

female nucleus. The male nuclei of the two copulating animals are exchanged, traversing the protoplasmic bridge in their course (III). Both male and female nuclei usually become spindle-shaped, and the immigrant male spindle fuses with the female spindle, forming a single spindle of division. At last, after processes which differ in the various genera, the division spindle produces (usually by indirect means) two nuclei, one of which becomes the new macronucleus, the other the new micronucleus.

In a comparison of the fertilization of the Metazoa, the female nucleus corresponds to the egg nucleus, the male nucleus to that of the spermatozoa. As the fusion of egg and sperm nuclei forms a segmentation nucleus, so here the division nucleus is formed in a similar manner. As the egg cell through fertilization acquires the capacity not only to produce sex cells but somatic cells-cells which carry on the common functions of the body -the fertilized micronucleus forms not only the new micronucleus, but 
also the macronucleus which controls the body processes, and hence is the somatic nucleus. In other words, fertilization in the Ciliates learls to a complete new formation of the nucleus and thus to a new organization of the organism.

In most Ciliata the conjugating individuals are similar, the fertilization is mutual, and the individuals separate later. In the Peritricha (mostly sessile forms, fig. I52), on the contrary, the resemblance to fertilization in the Metazoa is strengthened in that there is a sexual differ-

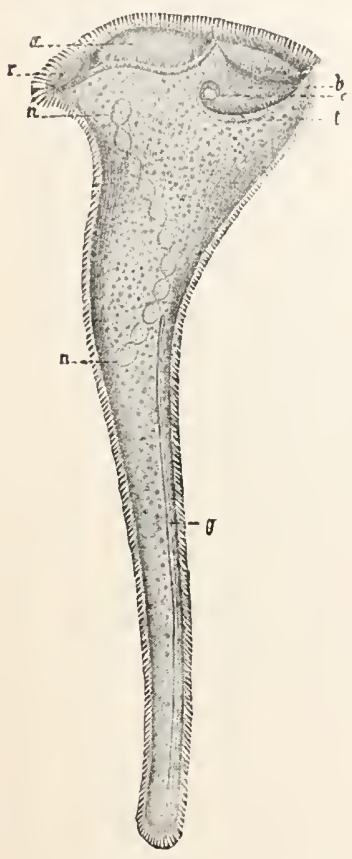

FIG. I53. entiation and a permanent fusion of the conjugating individuals. Some animals-the macrogametes-retain their size and sessile habits; others by rapid division prorluce groups of markedly smaller microgametes. The latter separate and fuse completely with the macrogametes. The nuclear phenomena are much the same as with Paramecium, allowance being made for the permanence of the fusion.

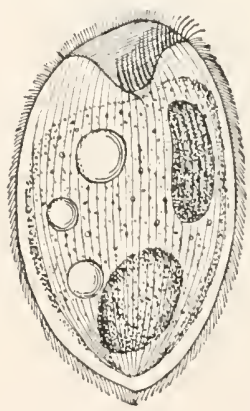

FIG. I54.

FIG. I53.- Stentor polymorphus (after Stein). $a$, peristomial area; $b$, roof of hypostome; $g$, contractile vacuole: $n$, nucleus; $o$, cytostome; $r$, adoral ciliated spiral; $t$, hypostome (excavation for mouth).

FIG. I54.-Balantiaium coli (after Leuckart).

\section{Order I. Holotricha.}

The Holotricha are the most primitive Ciliates, since the cilia on all parts of the body are similar; being at most slightly stronger at one end of the body or inside of the cytostome. Best known are the species of Paramacium* (fig. I50) occurring in stagnant water. Opalina ranarum* lives in the intestine of the frog. It lacks mouth, has numerous similar nuclei, no micronucleus and no conjugation. The small encysted Opaline pass out with the freces, and are eaten by the tadpoles, which thus become infected. 


\section{Order II. Heterotricha.}

Like the Holotricha the I'eterotricha are everywhere ciliated, but they have a tract of stronger cilia, the adoral ciliated spiral, beginning at some distance from the cytostome and leading in a spiral course into the mouth. It consists of rows of cilia united into membranelle placed at right angles to the course of the spiral. In the Stentor* (fig. I53), the peristomial area, surrounded by the spiral, forms the broader end of the body, which tapers toward the other end, by which the animal may attach itself. Muscle fibres running lengthwise immediately under the cuticle produce energetic movements. Balantidium coli (fig. I 54) appears in the large intestine of men ill with diarrhœea, it also occurs in swine without causing sickness. Other parasites of man are $B$. minutum and Nyctotherus faba.

\section{Order III. Peritricha.}

The Peritricha have a broad peristome area around the cytostome; the opposite end has a corresponding pedal disc or is narrowed like a goblet and ends in a stalk (fig. I55). Only the adoral ciliated spiral is constant. It arises from the swollen margin of the peristomial area, and continues on the 'operculum,' a
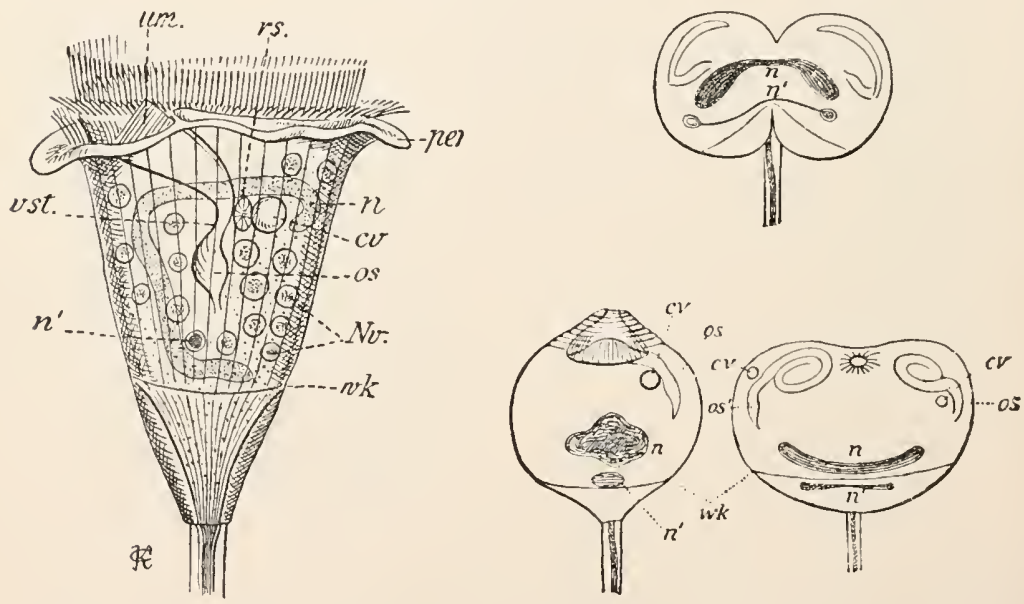

FIG. I55.-Carchesium polypinum (after Bütschli). Left, a single animal; right, three stages of division. $c v$, contractile vacuole; $n$, macronucleus; $n^{\prime}$, micronucleus; $N v$, food vacuoles; os, cytopharynx; per, peristome; $v$, reservoir of contractile vacuole; $u m$, undulating membrane; vst, vestibule; $w k$, ring on which a posterior circle of cilia may develop.

ciliated disc which projects free from the peristomial area, but in contraction is drawn close against it, the peristome lips folding over all. Besides, there may be a temporary or permanent circle of cilia near the hinder end. 'The nucleus is usually sausage-shaped, much bent, and with the small micronucleus in its hinder angle (fig. I $55, n^{\prime}$ ).

The VORTICELlide(figs. 152, 155 ), are attached by a long stalk which contains a slightly spiral muscle, dividing in the body into fine fibrillæ which extend under the cuticle to the peristome. When the muscle in the stalk contracts it becomes coiled into a corkscrew spiral, drawing back the animal, and 
folding in the anterior end. Torticella* is solitary; Carchesium* forms colonies with branched stalks; Zoothamnion, * colonies imbedded in a common jelly; Epistylis* (fig. 52), branched colonies with rigid stalks.

The fantastic Ophryoscolex, Cycloposthium, etc., are parasites in the stomach of ruminants.

\section{Order IV. Hypotricha.}

In this order the body is more or less flattened and rentral and dorsal surfaces are differentiated. The back lacks cilia, but of ten bears spines and bristles. On the ventral side are several longitudinal rows of cilia, and also straight spines and hooked cirri composed of united cilia, of use in creeping. The cilia are

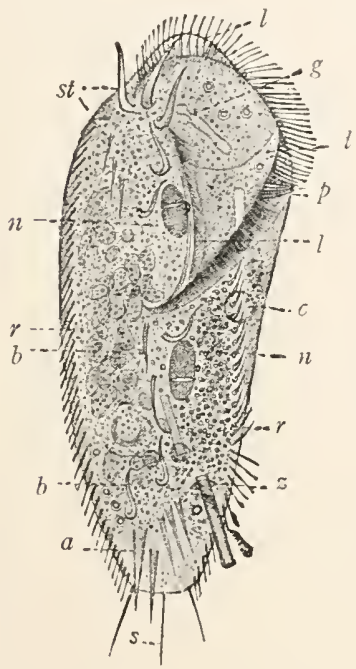

FIG. 156 .

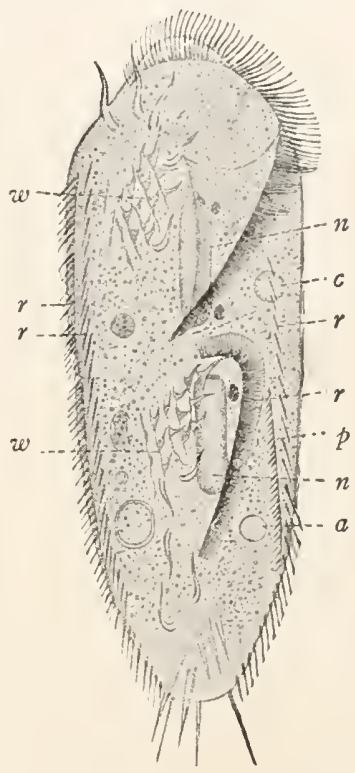

FIG. 157 .

FIG. I56.-Stylonychia mytilus (after Stein). $a$, anal hooks; $b$, ventral hooks; $c$, contractile vacuole; $d$, frontal ridge; $g$, canal leading to contractile vacuole; $l$, upper lip; $n$, nucleus with micronucleus; $p$, adoral ciliated spiral; $r$, marginal cilia; $s$, caudal cilia; st, frontal spines; $z$, anus (cytopyge).

FIG. I 57.-Division of Stylonychia mytilus (after Stein). $c, c^{\prime}$, contractile vacuoles of the two individuals: $n$, nucleus and micronucleus; $p, p^{\prime}$, adoral ciliated spiral; $r, r^{\prime}$, marginal cilia; $w, w^{\prime}$, ciliated ridges.

used in locomotion and producing vortices which bring food. The macronucleus is often divided into two oval bodies connected by a thread; the micronuclei vary in number from 2 to 4 in the same species. These are the best forms for studying the micronuclei. Stylonychia* (figs. 156, I57).

\section{Order V. Suctoria (Acinetaria).}

The Suctoria differ from other Infusoria in the absence of cilia from the adult and consequently have no means of locomotion. 'They are fixed to some support either by the base or by a slender stalk. The body is usually spherical 
and is covered with a cuticle, which in Acineta is produced into a cup-like lorica. There is no mouth, but in its place tentacles, very fine tubes with contractile walls which begin in the protoplasm and protrude through the cuticle (fig. I58, $F)$. The Acinetaria kill other animals, especially infusoria, with their tentacles, and then suck the substance through these tubes. The contractile vacuole, rarely lacking, lies near the compact macronucleus; micronuclei are generally present. The ciliated young (fig. $158, E$ ) are good swimmers. They arise either as buds from the surface of the mother (fig. 20) or as 'embryos' in her interior. This latter condition is only a modification of the other, part of the

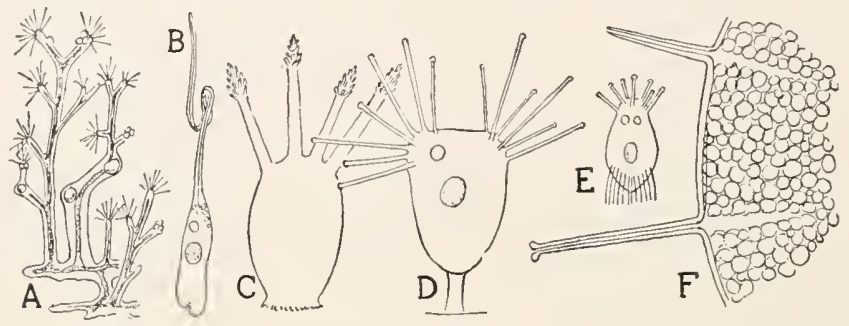

FIG. I58.--Suctoria (after various writers). A, Dendrosoma; B, Rhyncheta; $C$, Ophryodendron; $D$, Tokophrya; $E$, ciliated young of Spharophrya; $F$, diagram of capitate and styliform tentacles arising from ectosarc and canals in entosarc.

outer surface being pushed into the interior to form a brood cavity in which the embryos arise. After swimming for a while the young come to rest, lose the cilia, and develop the tentacles. Some species of Podophrya in fresh water, also Sphcerophrya, parasitic in Infusoria. Acineta and Podophrya gemmipara (fig. 20) are marine.

\section{Summary of Important Facts.}

I. The Protozoa are unicellular organisms without true organs or true tissues.

2. AIl vital processes are accomplished by the protoplasm, digestion directly by its substance, locomotion and the taking of food by means of protoplasmic processes (pseudopodia) or by appendages (cilia and flagella).

3. Excretion takes place by special accumulations of fluid, the contractile racuoles.

4. Reproduction is by budding or by fission. At intervals there is a true fertilization (caryogamy) sharply distinct from mere fusion of plasma (plasmogamy). Fertilization may be accomplished by a permanent fusion (copulation) or a transitory union (conjugation); it may be isogamic, anisogamic or autogamic.

5. Protozoa are aquatic, a few living in moist earth; they can only exist in dry air, surrounded by a capsule (encysted) which prevents desiccation.

6. Since encysted Protozoa are easily carried by the wind, the occur- 
rence of these animals in water which originally contained none is easily explained.

7. The mode of locomotion serves for division of the P'rotozoa into the classes Rhizopoda, Flagellata, Ciliata, and Sporozoa.

8. The Rhizopoda have temporary protoplasmic processes, the pseudopodia.

9. The Rhizopoda are subdivided into Monera, Lobosa, Heliozoa, Radiolaria, Foraminifera, and Mycetozoa.

ıo. The Lobosa and Monera have no definite shape. The Lobosa have a nucleus, the Monera are anucleate.

I I. Heliozoa and Radiolaria are spherical and have fine radiating pseudopodia and frequently silicious skeletons. They are distinguished by a central capsule in the Radiolaria which is lacking in the Heliozoa.

12. The Thalamophora (Foraminifera) have a shell, closed at one end, the other open for the extension of pseudopodia. The shell is chitinous or calcareous, one or several chambered, straight or spiral; the pseudopodia are occasionally lobular, but usually filiform, branching and anastomosing.

13. The Foraminifera are of great geological importance on account of their numbers and their shells, which have built and are still building extensive beds of rock (chalk, nummulitic limestone). 'The silicious skeletons of the Radiolaria are less important.

I4. Mycetozoa (Myxomycetes) are mostly enormous Amœbx with reticulate protoplasm (plasmodium). They form complex reproductive structures (sporangia), recalling those of the fungi.

I5. Flagellata have one or a few long vibratile processes-flagellawhich serve for locomotion and for the taking of food.

I6. The Autoflagellata have only flagella; they feed like plants by means of chlorophyl (Volvocinæ), or upon fluid food (parasites), or upon solid food, either by pseudopodia, by a mouth (cytostome), or by a collar.

17. Several are parasitic in man (Trichomonas i'aginalis, Lamblia intestinalis, and especially prominent Trypanosoma gambiense (cause of sleeping sickness). Perhaps Spirochete pallida (cause of syphilis) belongs here.

18. The Dinoflagellata have two kinds of flagella and usually an armor of cellulose.

I9. The Cystoflagellata have a gelatinous body enclosed in a firm membrane (Noctiluca).

20. Sporozon are parasitic Protozoa, usually without organs of locomotion or mouth. They take no solid food, but live by osmosis on tissue fluids. The encysted animals produce spores (beginning with fecunda- 
tion and accompanied by a change of host). The spores divide into sporozoites. Multiplication without change of host (autoinfection) can occur.

21. The Gregarinida are temporary or permanent parasites in cells. Coccidice, Hamosporida (cause of malaria, parasitic in blood corpuscles).

22. The Sarcosporida (Rainey's or Miescher's corpuscles of mammalian muscles) and II yosporida (psorosperm capsules of fishes, psorosperm $=$ spore) live in tissues or hollow organs.

23. The Ciliata have numerous vibrating processes, the cilia, a cuticle, and hence fixed openings for the ingestion of food (cytostome) and for extrusion of indigestible matter (cytopyge).

24. Of great interest is the occurrence of two kinds of nuclei, a functional macronucleus and a sexual micronucleus.

25. In conjugation portions of the micronucleus are exchanged and accomplish impregnation. The macronucleus degenerates and is replaced by part of the fecundated micronucleus.

26. The classification of the Ciliata is based on the structure and arrangement of the cilia.

27. The Holotricha have similar cilia over the whole body. The Heterotricha have, besides the total ciliation, stronger cilia in the neighborhood of the mouth (adoral ciliary spiral). The Peritricha have only adoral ciliation. The Hypotricha have the ciliary spiral and rows of cilia and coalesced cilia on the ventral surface. The Suctoria have cilia only in the young, later they become attached and feed through suctorial tentacles.

\section{APPENDIX.}

According to the evolution theory one should expect forms between the Protozoa and Metazoa. The CATALLACTA-spheres of ciliated cells which in reproduction break up into single cells-have been described as such. Other peculiar many-celled animals whose position in the system is difficult to decide are, Salinella salve, Lohmanella catemula, the OrTHONEctida and the Dicyemida. The Orthonectida and Dicyemida have a many-celled ectoderm, enclosing a solid mass of cells in the Orthonectida, a single giant cell in the Dicyemida. Salinella and Lohmanella consist of a single laver of cells enclosing a central digestive space. Since the Dicyemida live as parasites in the nephridia of cephalopods, the Orthonectida in worms and echinoderms, it is possible that their low organization is the result of degeneration. Trichoplax adharens, formerly placed here, is discoid, consisting of two epithelial layers separated by gelatinous tissue. It has recently been shown to be the larva of a medusa, Eleutheria. 


\section{METAZOA.}

Excluding the Protozoa, all the phyla of the animal kingdom are included under the Metazoa, i.e., higher animals. The point of union is that they consist of numerous distinct cells, arranged in several layers. At least two layers are present; one-the ectoderm-bounding the body externally, and a second-the entoderm-lining the digestive tract. Between these two a third may occur, frequently separated by a body cavity into an outer or somatic layer forming part of the loody wall, and an inner or splanchnic layer forming part of the intestinal wall. This middle layer is called mesoderm, no matter whether there be a body cavity or not.

The multicellular condition allows a higher organization, which appears in varying grades in the specialization of tissues and organs. No metazoan lacks a true sexual reproduction, that is one by sexual cells, but the possibility must not be overlooked that some species may have lost fertilization and may reproduce exclusively by unfertilized eggs in a parthenogenetic manner. Many species, especially the lower worms and coelenterates, also reproduce by budding and fission.

The segmentation of the egg is characteristic of all Metazoa. The fecundated egg divides into numerous cells which, as blastomeres, remain united and form the germ. No Protozoan has a true segmentation, division producing new individuals which either separate completely or remain in slight connection as a colony.

\section{PHYLUM II. PORIFERA (SPONGIDA).}

The Porifera, or sponges, the most familiar representative of which is the bath sponge (Euspongia officinalis), are, with few exceptions, marine. In fresh water occur but a few species of Spongilla. The animals have no powers of locomotion, but are attached to stones or plants, along the shores or at depths up to 4000 fathoms. They form spherical masses, thin crusts, small cylinders, or upright branching forms. Frequently the shape varies so that there is no typical form. Striking motions are rare; only with the microscope can one see the opening and closing of the pores and the currents of the gastrovascular system.

The simplest sponges, the Ascons (fig. I59), are thin-walled sacs, fixed at one end, and with an opening, the osculum (functional anus), at the other. The cavity of the sac, the 'stomach,' is a wide digestive cavity into which water bearing food enters through numerous small pores in the body wall. The basis of the body is a connective tissue permeated with branching cells (fig. I60) covered externally by a thin 
layer of pavement epithelium which is easily destroyed. This epithelium (earlier called ectoderm) and the connective tissue (mesoderm) are now regarded as a common layer, 'mesectoderm,' since the pavement epithelium is often genetically only connective-tissue cells which have spread over

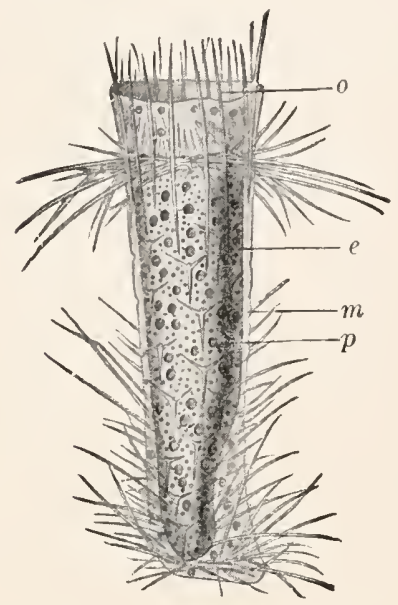

FIG. I59.

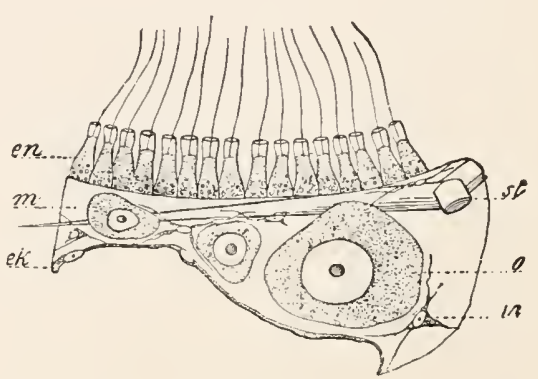

FIG. I60.

FIG. 159.-Ascon stage of Sycandra (after Maas). e, entoderm; $m$, mesectoderm; $o$, osculum; $p$, pores.

FIg. I60.--Section of wall of Sycandra raphanus (after Schulze). e, epithelium; $e n$, collared flagellate cells; $m$, mesoderm with connective-tissue cells; o, eggs; st, calcareous spicules.

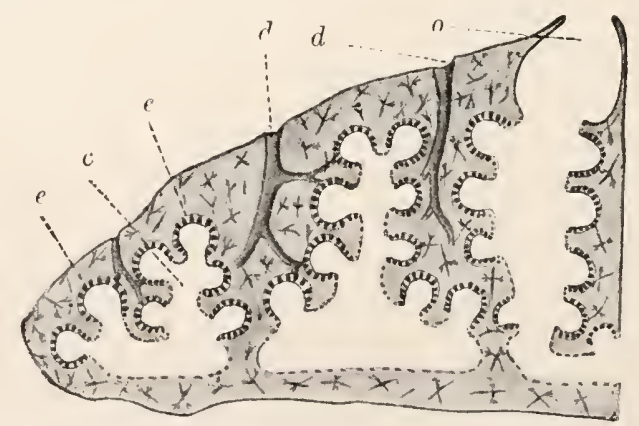

Fig. I6I.--Section of Plakina (after F. E. Schulze). c, canals leading from ampullæ to cloacal tubes; $\epsilon$, ampullæ; $d$, afferent canals; $o$, osculum.

the surface. On the other hand, there is a distinctly differentiated entoderm in the shape of a one-layered flagellate epithelium lining the stomach, the cells of which $(e n)$ recall the Choanoflagellata (p. I84), since they have collars surrounding the flagella. The taking of food is accomplished by the collared cells, its distribution by the amoboid cells. 
Sponges of this simple ascon type are few. As a rule sponges are more massive and have a more complicated canal system (figs. I61, i62). 'The first step towards complication is seen in the Sycon type, in which the gastral cavity consists of numerous radial chambers or ampulle which alone

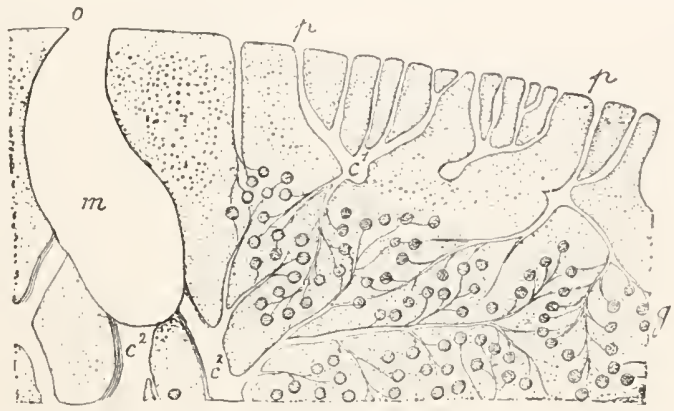

FIG. I62.-Section of cortex of Chondrilla mucula, the skeleton omitted (after Schuize). $c^{1}$, afferent canals; $c^{2}$, efferent canals; $g$, ampull $x ; m$, cloaca; $o$, osculum.

contain the collared cells, while the central cavity, now called cloaca, is lined with pavement epithelium. By increase of mesoderm and corresponding thickening of the body wall the ampullæ become separated from external and cloacal surfaces (Leucon type). They nevertheless retain their connection with both surfaces by means of cavities which may

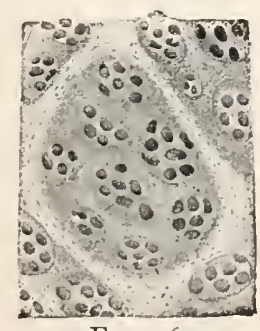

FIG. 163 .

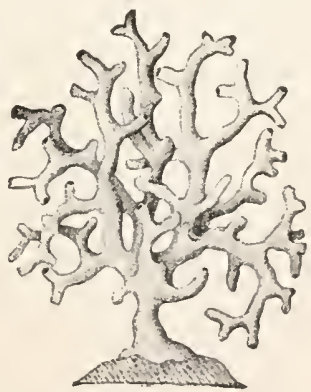

FIG. I64.

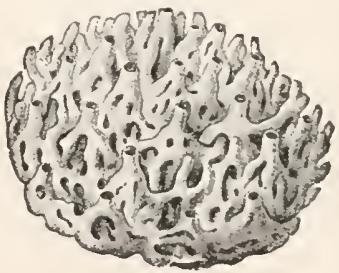

Fig. I65.

FIG. I63.- Surface view of dermal pores of $A$ plysina aërophoba (after Schulze).

FIG. I64.-A scyssa acufera (after Haeckel).

FIG. I65.-Leucetta sagittata (after Haeckel).

be lacunar (fig. I6I) or consist of a system of canals. The canal system is double; one part is incurrent and leads from the dermal pores to the ampullæ; the other or excurrent, from the ampulles to the cloaca, the two being connected by the ampullæ alone (fig. 162), the canals from the pores 
uniting in trunks and these in turn branching to go to the ampullæ. The excurrent canals also show a similar tree-like arrangement. Not infrequently extensive subdermal or subcloacal spaces occur. The relations may be more complicated by the development of several cloace, or by the branching of the sponge (fig.

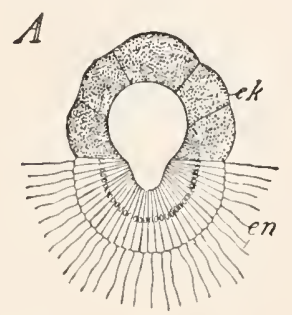
I64), while still further the branches may anastomose (fig. I65), giving rise to a netwolk.

Sponges may reproduce asexually, small portions separating as buds and producing new animals (fig. 88). Usually sexual reproduction prevails. The eggs, which like the spermatozoa arise from mesoderm cells (fig. I60), undergo segmentation and leave the parent as flagellate

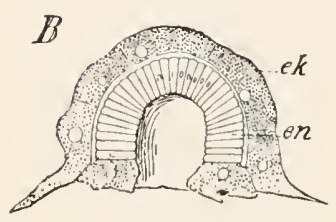

FIG. 165--Development of Sycandra raphanus (after Schulze). $A$ blastula; $B$, gastrula at the moment of fixation; $c k$, ectomesoderm; $e n$, entoderm. larvæ (fig. I66, $A$ ). At fixation a kind of gastrulation takes place, the blastopore $(B)$ closes, and the osculum, an entirely new formation, arises at the opposite pole.

The sponges are frequently regarded as Cœlenterata, but scarcely a single homology can te drawn between the two. The colenterate mouth is different from either pores or oscula. Indeed, it is disputed whether the collared cells are entoderm. Most sponges possess a skeleton secreted by special mesoderm cells, and this skeleton affords the means, according as it is composed of calcic carbonate or of silica, of dividing the sponges into two classes. Besides, there are two groups, Ceraospongiæ and Myxospongiæ, in which the skeleton is respectively of horny substance (spongin) or is lacking entirely. These seem to be descendants of the silicious forms.

\section{Order I. Calcispongiæ.}

The calc sponges are exclusively marine and mostly live in shallow water. They are grayish or white in color, of small size, rarely exceeding an inch in length. The skeletal spicules usually project through the epithelium, forming silky crowns in the neighborhood of the osculum. One-, three-, and four-rayed spicules are recognized, these ground forms presenting by unequal development a great variety of shapes.

Sub Order I. ASCONES. Thin porose walls and central 'stomach.' Leucosolenia:* Sub Order II. SYCONES. Cloaca present surrounded by a mpullæ radially arranged. Grantia,* Sycon,* Sycandra* Sub Order III. LEUCONES. A complicated system of branching canals in thick walls connects the ampullæ with outer surface and cloacal cavity. Leucetta, Leucortis.

\section{Order II. Silicispongiæ.}

The siliceous sponges are richest in species and occur at all depths of the sea, being frequently noticeable from their size and bright colors. They are subdivided into Triaxonia and Tetraxonia. In the Triaxonia the spicules composing the skeleton-appearing as if of spun glass (hence Hyalospongia, or 
glass sponges) -have three crossed axes (six threads radiating from a common point) -hence Hexactinellida. The mesoderm is scanty and in consequence the canals are loose-meshed, lacunar spaces and the ampulla large and barrelformed. In the 'Tetraxonia the mesoderm is usually abundant and the canal system well developed. The four-axial spicules of the Tetractinellicla must be regarded as the fundamental skeletal type. From this are derived the compact frameworks of the Lithistidæe and the monaxial spicules of the Monactinellidx.

In both groups the spicules may be united by secondary deposits of silica to an extensive framework; or the union is affected by spongin, which, if the spicules disappear, forms the whole skeleton (horny sponges); or, as in slime-sponges, the whole skeleton may be lost.

Sub Order I. TRIAXONIA. Hexactinellide, chiefly deep seas; Euplectella aspergillum, Venus' flower-basket. Hyalonema. Süb Order II. TETRANONIA. Typical are the largely extinct LiTHISTID (some generaDiscodermia-persist in deep seas) and Tetractinellide: Geodia.* Near here apparently belongs Oscarella, * without a skeleton (MYXospongLA). MoNACTINELLID£, spicules united by spongin (Cornacuspongia); can even be entirely replaced by that substance. Numerous marine forms, and the freshwater SPoxgillide (Spongilla, Ephydatia*), usually colored green by alga. They are distinguished by formation of gemmulce or statoblasts. At times the

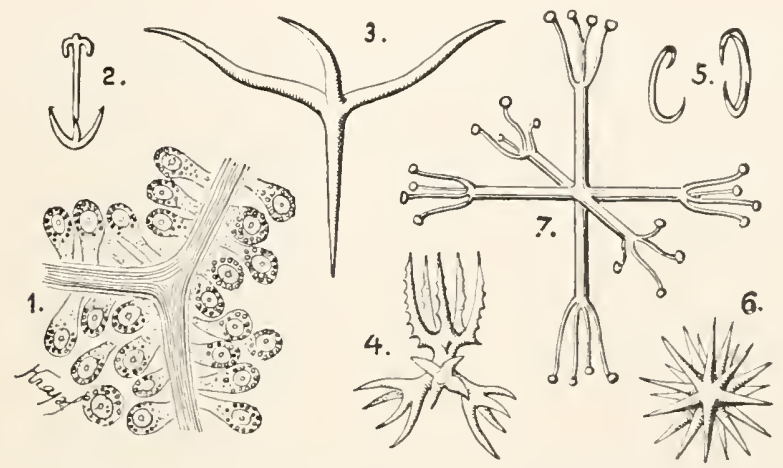

Frg. I67.-Skeletal structures of sponges (after Schulze and Maas). I, Horn fibre of bath sponge with spongioblasts; $2-7$, spicules of, 2, Esperia; 3,4 , Corticum; 5 , Mysilla; 6, Tethya; 7, Farrea.

protoplasm divides into round bodies, as large as the head of a pin and these become surrounded by a firm membrane often strengthened by co!lar-button-like spicules, the amphidiscs. These statoblasts survive times of freezing or drought On return of good conditions the contents escape and form small Spongilla, of ten utilizing the old skeleton. The spicules entirely disappear and nothing but the spongin fibres remain in the horny sponges, CERAOSPOxGIE. 'The skeleton consists of an organic substance, spongin, which differs chemically from true horn-keratin. This spongin is laid down by peculiar cells, the spongioblasts (fig. I67, I), and it always consists of concentric layers. The fibres interlace. branch, and unite. Best known are the bath sponges; Euspongia officinalis, occurring in the Mediterranean, West Indies, Florida, and other seas in many varieties. Best are the Levant sponges (var. mollissima). Sponges of commerce consist only of the skeleton, the animal parts being washed away: Less valuable are Euspongia simocca and Hippospongia equina,* the horse-sponge. 


\section{Summary of Important Facts.}

I. The sponge body is largely a mass of connective tissue covered externally with pavement epithelium (mesectoderm) and penetrated by canals.

2. An entoderm of collared flagellate cells occurs only in the ampullæ or flagellate chambers which are intercalated between incurrent and excurrent canals (in ascons in the central cavity).

3. The animals receive food through fine pores in the body wall; indigestible matter is cast out through one or more oscula.

4. Since nerves, muscles, and sense organs are lacking or very weakly developed, only inconspicuous movements occur.

5. Sponges are divided into Calcispongiæ and Silicispongiæ according to the character of the skeleton.

\section{PHYLUU III. CELENTERATA (CNIDARIA).}

The coelenterates, formerly called Zoophyta (plant-animals), were united by Cuvier with the Echinoderma to form the type Radiata, a union which Leuckart, the father of the name Colenterata, set aside because separate intestinal and body cavities occur in the Echinoderma, while in the Coelenterata there is but a single cavity in the body. Each name indicates certain important characters of the group.

(I) The name Zoophyta referred to the general appearance. Most cœlenterates, like plants, are fixed and by incomplete budding form bushlike or mossy colonies. This resemblance is but superficial, for there is not the slightest doubt of the animal nature of any colenterate. The name therefore does not imply that these are doubtful forms on the border between plants and animals. Besides, there are free-moving forms which swim with great ease.

(2) Most Colenterata are radially symmetrical. There is a main body axis, one end of which passes through the mouth and the other through the blind end of the digestive tract, and the organs of the body are radially arranged around this so that the body may be divided into symmetrical halves by numerous planes. In the higher Colenterata this may be replaced by a biradial symmetry or even by bilaterality (Ctenophora, many Anthozoa).

(3) The term Cœlenterata is given because these animals contain a single continuous calenteron or gastrovascular cavity. In its simplest form this is a wide-mouthed sac into which food passes for digestion. The single opening into it serves for both mouth and anus; the sac itself is the alimentary tract. Frequently lateral diverticula or branched canals 
are given off from the central sac which distribute the nourishment to the peripheral parts of the body, and thus functionally replace the vascular system of higher forms. Since this gastrovascular system is primarily for nourishment, it is not a body cavity and one cannot say that the colenterates are stomachless. On the other hand, the term 'coelenteron,' that is, a cavity at once gastric and colomic (P. I 48 ), is perfectly defensible, since in many higher animals which possess a true body cavity (cxilom) this arises in development as diverticula from the primitive stomach (enteron). Since such diverticula occur in cœlenterates without becoming inclependent, one can say that the gastrovascular system consists not only of intestinal portions but, in potentia, of the cœlom as well.

To even a superficial observation the Coelenterata are more clearly animals than are the sponges. The single animals, though of ten united in colonies and fixed to some support, are capable of quick and energetic motion. These movements are most striking in the tentacles-long tactile processes in the neighborhood of the mouth, which feel for food, grasp it, and convey it to the mouth. The means of killing the prey are the cnida (whence the name Cnidaria for the phylum), nematocysts, or nettle cells (fig. I68). These structures, of great systematic importance, are oval or elongate vesicles with fluid contents and firm membrane. Each is drawn out at one end into a long thread-like tube (hence an additional name, thread cells). In the resting stage the thread is spirally coiled inside the cell. On stimulation the thread is quickly extended ('explosion of cell') and produces a wound into which passes the irritating fluid contents. Some cœelenterates (e.g.,
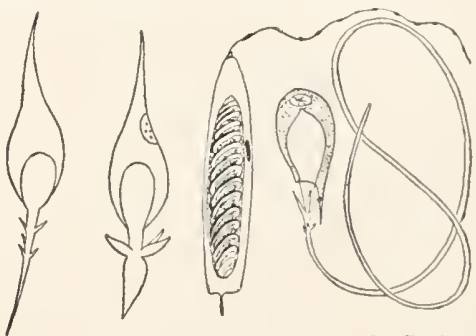

FIG. I68.--Nettle cells of Coelenterata (after Hertwig, Lendenfeld, and Hamann).

Physalia) can produce in this way very painful nettling even in man. The nettle capsule arises as a plasma product inside a cell. When fully developed the nettle cell extends to the surface and ends with a tactile process (cnidocill) which, upon contact, stimulates the protoplasm and causes the explosion, the thread being everted like the finger of a glove. The cell itself is frequently enclosed by a muscular sheath or a network of muscle fibres.

Among the cœelenterates both sexual and asexual reproduction may occur, the latter usually by budding, more rarely by division. Sexual and asexual reproduction can be combined in the same species, producing an alternation of generations. 
In comparison with the sponges the Coelenterata may be called epithelial organisms. A mesoderm (mesoglca) may be entirely lacking or may have but a subordinate development. The ectoderm and entoderm, on the other hand, are the important tissues-producing muscles, nerves, sense organs, sexual products and cnidæ. Hence the group is often called Diploblastica-two-layered animals.

\section{Class I. Hydrozoa (Hydromedusæ).}

According to varying standpoints the Hydrozoa can be placed either higher or lower than the Anthozoa in the system, since in the former group two forms frequently occur in the life history, one agreeing well in structure with the Anthozoa, the other standing on a higher grade. The first is the sessile and usually colonial polyp, the second the free-swimming medusa, well provided with sense organs. These are usually related to each other by an alternation of generations. The polyp is asexual and by budding produces medusæ; the medusa, on the other hand, is the sexual stage, and from its eggs polyps arise.

The polyp of the Hydrozoa is the hydropolyp, forming an important archetype from which all other conditions-medusæ, scyphopolyp, and coral polyp-may be derived. Our best example of this is the fresh-water Hydra. The body (fig. I69) is a sac, the closed end of which, the pedal disc, is used for attachment. The other end bears the mouth which leads to the gastrovascular (digestive) cavity. Around the mouth is a circle of tentacles used in capturing food. These are outgrowths of the body wall; the circle dividing the body into a peristome inside the circle and a column constituting the rest of the outer wall.

H ydra has but two body layers (fig. I 70), an entoderm of flagellate cells lining the gastrovascular space, and the ectoderm covering the outer surface. Between the two is the supporting layer (mesogloea), a membrane without cells and hence not a body layer. Both layers consist of epithelial muscular cells (cf. p. 8I), the basal ends of which are produced into smooth muscle fibres, those of the ectoderm running lengthwise, those of the entoderm around the body. The ectoderm further contains ganglion, nettle and sex cells. The nettle cells on the tentacles are crowded into small ridges or 'batteries.' The sex cells (at certain times) produce swellings on the column; a circle of male swellings close beneath the tentacles, the female cells farther down the column (fig. I69). Individuals reproducing by budding are more common than the sexually mature (fig. 93). Small elevations appear on the column, enlarge, form tentacles, and at last a mouth, after which they may separate from the parent. 
In the sea are numerous hydroid polyps which, while agreeing in the main with $H y d r a$, are distinguished from it in two important respects: (1) they do not directly produce sexual organs; (2) they reproduce asexually, and by incomplete budding form persistent colonies. In this a series of parts have arisen which require special designations (fig. I 7 r). The separate animals, hydranths, are connected by a system of tubes, the conosarc, which, like the hydranths, consist of ectoderm, entoderm, and mesoglœa, and since the gastrovascular space continues in them, these distribute food throughout the colony. The conosarc may creep over

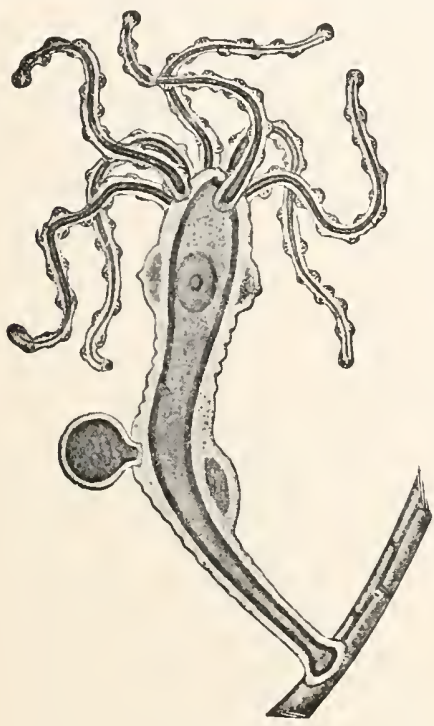

FIG. I69.

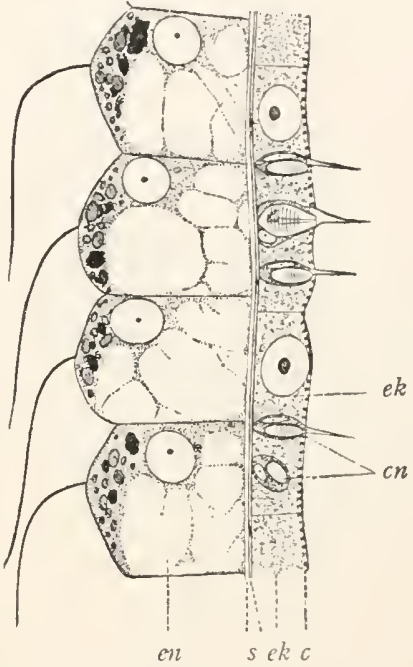

FIG. I7 0 .

FIG. I69.-Hydra viritis,* testes above; ovarizn enlargement and escaping egg below.

FIG. I70.-Body layers of Hydra (after Schulze, from Hatschek). c, cuticula; $n$, nettle cells; $e k$, ectoderm; $e n$, entoderm; $s$, supporting layer.

some support (stone, alga, snail-shell, etc.) and form a network, the hydrorhiza, or it may stand erect and free, forming a hydrocaulus. Usually both hydrorhiza and hydrocaulus occur in the same colony.

Usually the colony is strengthened and protected by the perisarc, a cuticular secretion of the ectoderm. In some (fig. 1 72 ) the perisarc stops at the base of the hydranth; in others (fig. 173 ) it expands distally into a wide-mouthed bell, the hydrotheca, into which the hydranth may retract. In rare cases this perisarc may be greatly increased and calcified, forming large coral-like masses with openings from which the hydranths may protrude (fig. I74). 


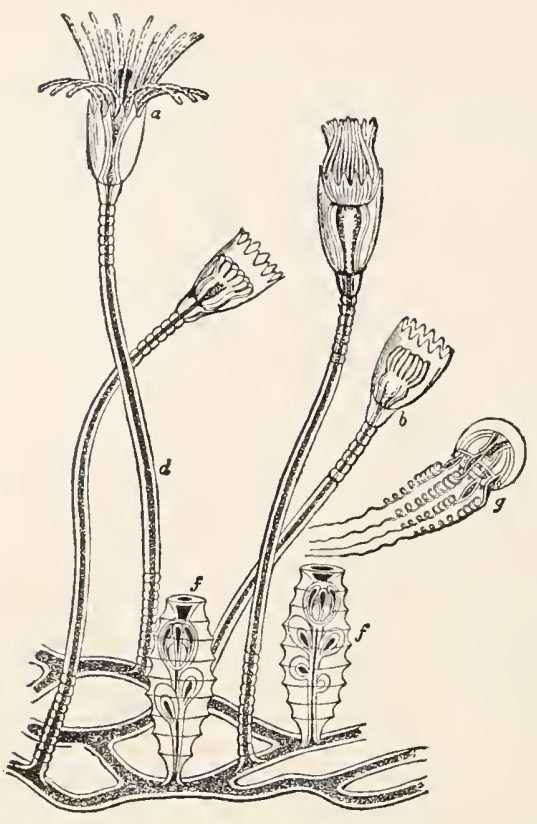

FIG. I7r-Campanularia jolnnstoni (after Allman). $a$, hydranth with hydrotheca; $b$, retracted; $d$, hydrocaulus; $f$, gonotheca, with blastostyle and medusa buds; $g$, free medusa. The hydrorhiza is shown as the creeping portion from which the hydrocauli and gonothecæ arise.

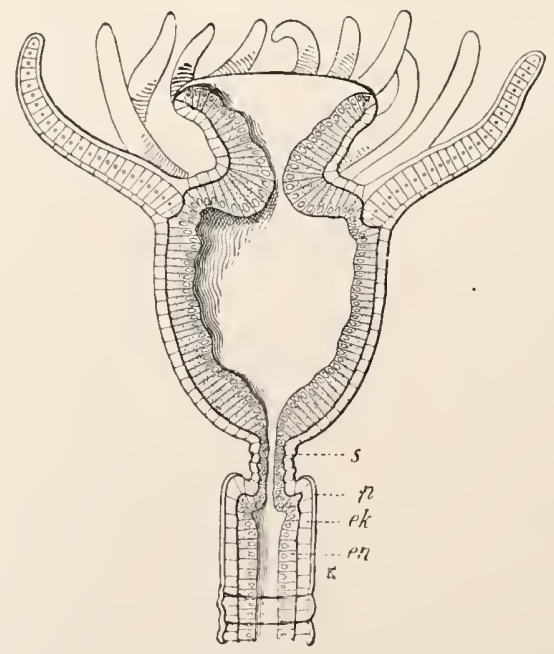

FIG. I72.- Section of Eudendrium ramosum. ek, ectoderm; en, entoderm; $p$, perisarc; $s$, supporting layer. 
The lack of sexual organs, which distinguishes most marine species from $H y d r a$, is due to the fact that sexual individuals of special form are produced from the colony by budding. These, the meduse, may separate early from the colony and swim freely. A medusa (figs. 175,176 ) has the form of a dome-like or disc-like bell and consists chiefly of very watery jelly. The bell or umbrella of the medusa is covered on both its surfacesthe concave or subumbrella, the convex or exumbrella-with ectodermal epithelium. At the margin of the bell the ectoderm is produced into a

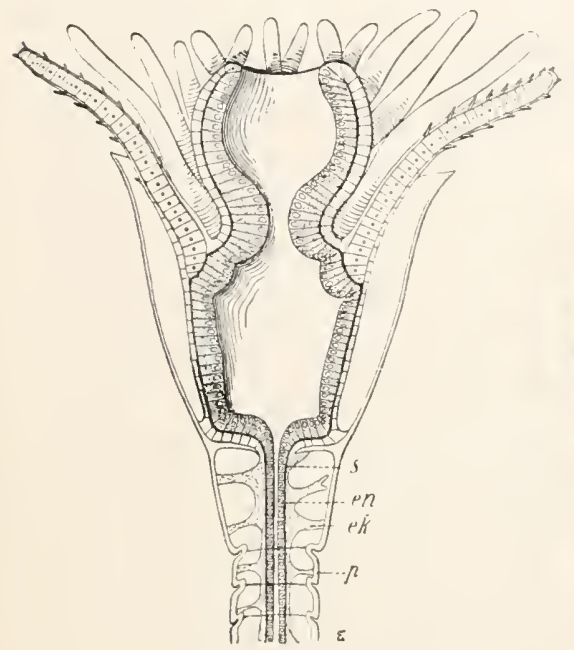

FIG. 173 .

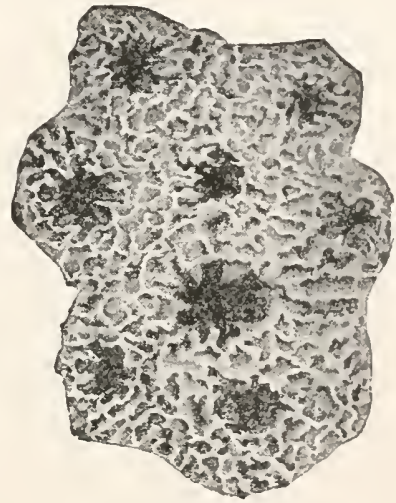

FIG. $17+4$.

FIG. I73.-Campanularia geniculata. ek, ectoderm; en, entoderm; p, perisarc, expanded around hydranth to a hydrotheca; $s$, supporting layer.

FIG. 174.- A bit of .Millepora alcicornis*, enlarged (after Agassiz).

two-layered sheet with a central opening, the velum or craspedon (fig. i 75 , $B, v)$ of systematic importance, since these medusx are often called ('raspedota. Tentacles (usually 4,8 , or multiples in number) also arise from the edge of the bell just outside the velum.

Comparable to the tongue of the bell or the handle of the umbrella is the mamubrium, hanging from the highest point of the subumbrella ancl bearing the mouth at its tip. It contains the chief digestive space, from which radial canals run on the subumbrellar surface to a ring canal in the margin of the umbrella. The radial canals are usually four in number, but in some species the number is increased during growth even to a hundred or more. Manubrium and canals are linel by entoderm, which also extends into the tentacles and forms their axes. 
All other important organs arise from the ectoderm. Gonads arise in many species (fig. I 76 from the ectoderm of the manubrium; in others
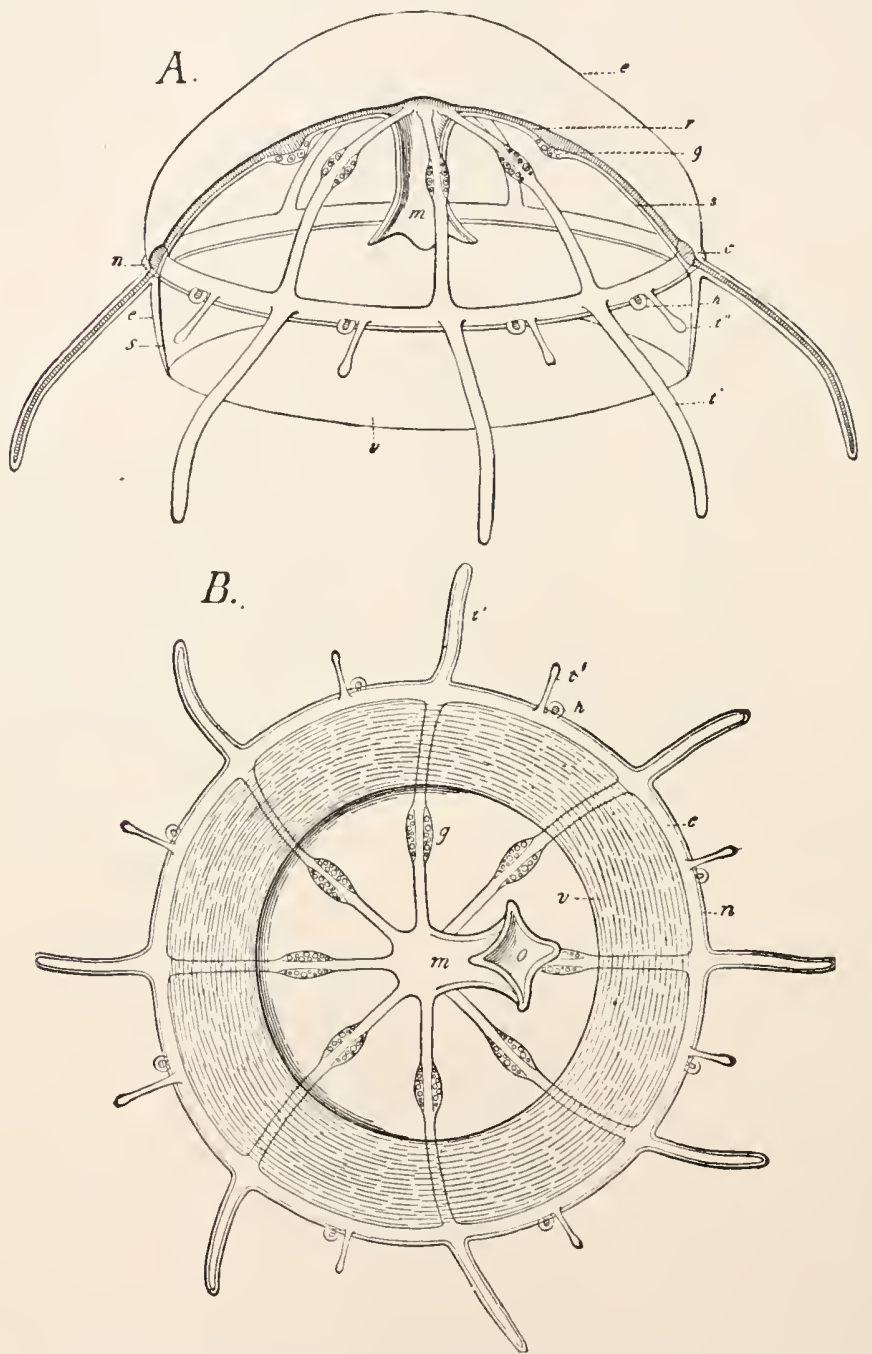

FIG. I75.-Rhopalonema relatum. $\quad c$, ring canal; $e$, exumbrella; $g$, gonarls; $h$, otocysts; $m$, stomach; $n$, nerve ring; $o$, mouth; $s$, subumbre:la; $t^{\prime}, v^{\prime \prime}$, tentacles of first and second order; $v$, velum.

from the same layer covering the subumbrellar surface of the radial canals (fig. I75), forming in either case conspicuous, often orange or red, thickenings. Longitudinal ectodermal muscles move the tentacles in a 
snaky fashion, whence the name medusa. Circular striped muscles run on the subumbrellar sirle of bell and velum, causing the characteristic motion. By their contraction the bell becomes more arched and narrowed, while the velum (which hangs down when at rest-fig. I75, A) contracts like a diaphragm across the mouth of the bell $(B)$. Since water is thus forced out through the opening the medusa is forced forward by the reaction. The circular muscles of the umbrella and velum are separated

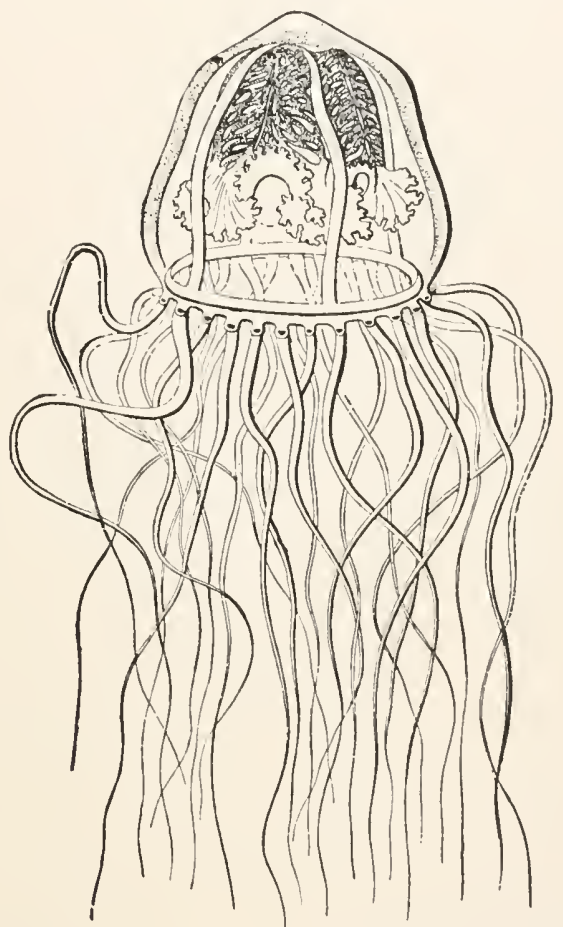

Fig. 176.-Tiara pileata (after Haeckel, from Hatschek).

by two nerve rings, one subumbrellar, the other cxumbrellar in position (fig. I $77, n^{1}, n^{2}$ ), the first supplying the muscle plexus, the other the sensory organs-eyes of the simplest type, red pigment spots with or without a lens; and open or closed statocysts ('ears'). Tactile hairs are abundant on the tentacles.

The statocysts are of two types, both beginning as open organs and reaching their highest development as closed vesicles. One type, the tentacular organs, occurs in the Trachymeduse (fig. I 77, I-4) the other, or velar organ, in the Leptomeduse $\left(5^{-6}\right)$. The tentacular organs are modified tentacles, the ento- 
dermal axis forming the statoliths and the ectodermal covering the sense cells. In the Eginidæ ( $\mathrm{I}$ and 3 ) the club-like tentacles, seated on an auditory cushion, project freely into the water; in the Trachynemidx (2) they are partially transformed into vesicles, and in the Geryonidx they are completely enclosed and are sunk in the jelly of the bell. The velar organs of the Leptomedusæ are placed on the subumbrellar surface of the velum. They may be either simple pits, or the mouths of the pits may close. In these both sense cells and statoliths are ectodermal. Eyes and statocysts occur in different forms, a fact which formerly led to a division of medusæ into ocellate and vesiculate groups.
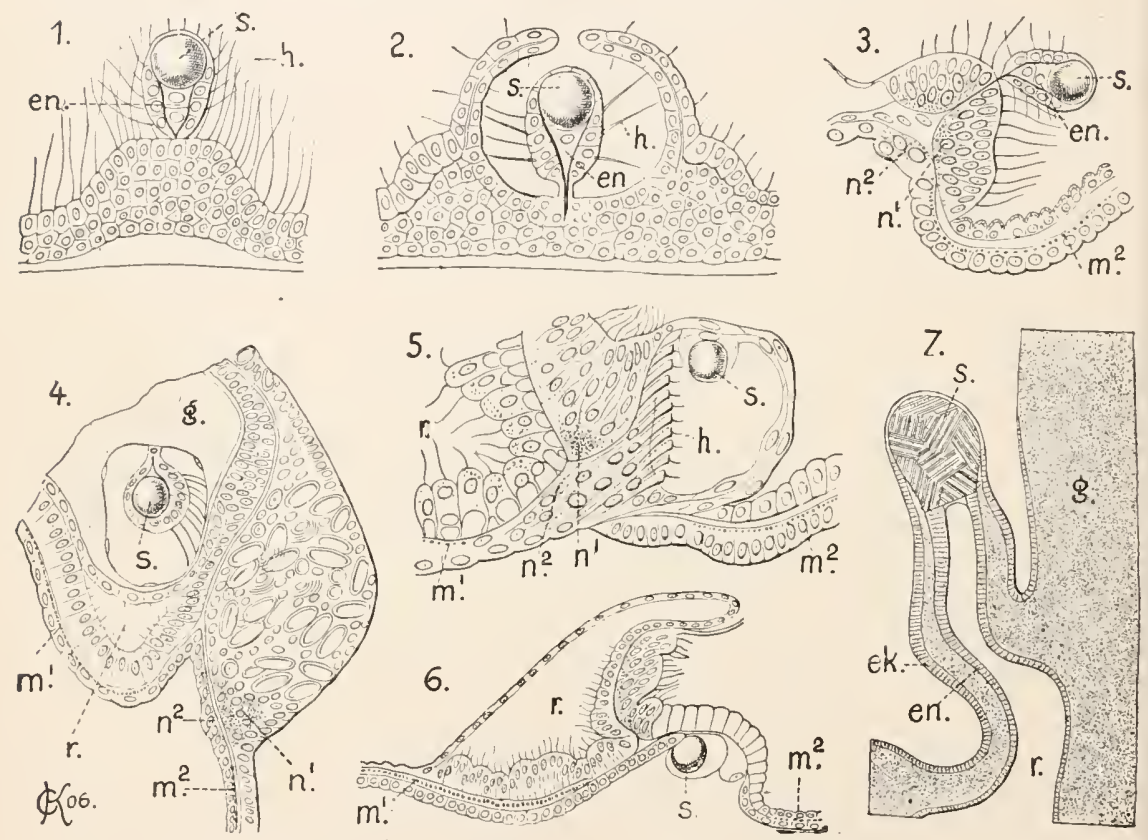

FIG. I77.- Statocysts (ear vesicles) of medusæ. I-4, tentacular statocysts of Trachymedusæ; 5, 6, velar of Leptomedusæ; 7, marginal body of Acraspedia. I and 3 , auditory clubs of Aeginopsis; 2, same of Rhopalonema, with beginning of ear vesicle; 4 , statocyst of Geryonia; 5, of Aequoria; 6, auditory pit of Mitrocoma anne; 7, marginal body of Aurelia. ek, ectoderm; en, entoderm; $g$, mesoglœea; $h$, auditory hairs; $m$, circular muscles cut across; $n^{2}, n^{2}$, upper and lower nerve ring; $r$, ring canal; $s$, statolith.

While polyps and medusæ apparently differ so greatly from each other, the medusæ are only highly modified polyps adapted to a swimming life. The long axis of the polyp has been greatly shortened (fig. I78) and the cylindrical body developed into a disc; the mesoglœa of column and disc thickened to a thick layer of jelly; while manubrial cavity, radial and ring canals are remnants of the large gastrorascular space of the polyp, obliterated in the other regions by the pressure of the mesoglœa. To the parts thus formed only the velum and sense organs are added. This comparison of medusa with polyp is important in understanding 
the development, which usually includes an alternation of generations. From an egg of a medusa a small ciliated embryo (plamula) escapes, which becomes attached, derelops mouth and tentacles, and, by budding, produces a hydroid colony. This colony lacks sexual organs. By budding
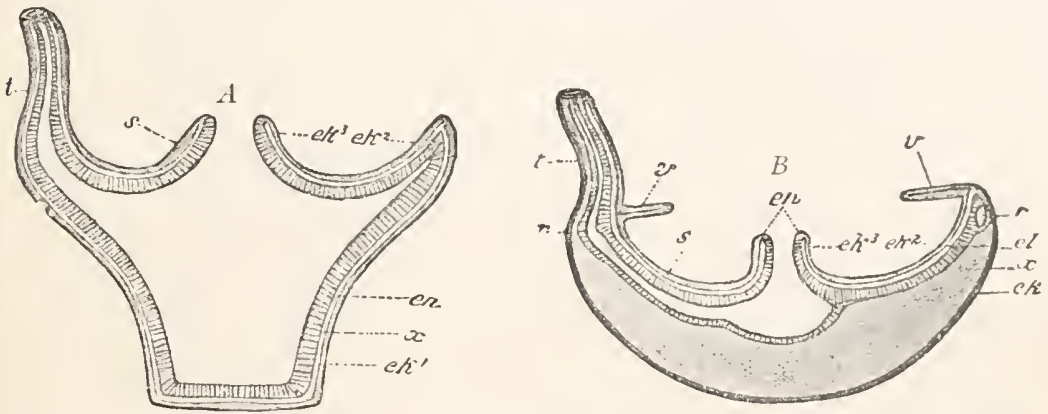

FIG. I78.-Diagram of sections of $(A)$ a polyp and $(B)$ a medusa. ek, ectoderm; $e k^{\prime}$, of exumbrella; $e k^{2}$, of subumbrella; $e k^{3}$, of manubrium; $e l$, endoderm (cathamnal) layer arising from obliteration of digestive space; $e n$, entoderm; $r$, ring canal; $s$, subumbrella; $t$, tentacles; $v$, velum; $x$, supporting layer (gela tinous in $B$ ).

it produces sexual individuals (medusæ) which separate and swim away. Since polyp and meduse are morphologically comparable, before the escape of the medus $x$ the colony is polymorphic, consisting of individuals (hydranths) which reproduce only asexually and of others which have taken over the sexual reproduction (medusx). Hence alternation of
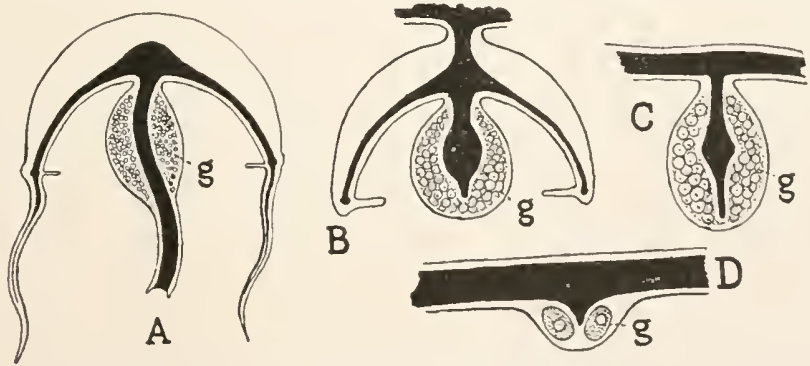

FIG. I79.--Comparison of a medusa and a sporosac (orig.). A, fully developed medusa; $B$, medusa with the manubrium closed, still attached to the blastostyle; $C$, medusa reduced to a simple manubrium (sporosac); $D$, last stage, eggs being produced in the body wall $(H y d r a)$.

generations has arisen here from a division of labor or polymorphism of individuals originally of equivalent value, in which some individuals (the sexual) have separated and acquired a peculiar structure.

While alternation of generation has arisen from polymorphism, it can again produce it. This occurs when the medusx, instead of separating, 
remain permanently attached to the colony. They then degenerate into 'sporosacs,' which always lack mouth, tentacles, and velum (fig. I 79), of ten also radial and ring canals, so that at last there remains only the manubrium (spadix) and the sexual organs, the latter enveloped by the rudiments of the umbrella. Since meduse and sporosac replace each other in closely allied species, a common name, gonophore, has been applied to both.

This developmental history may be modified in two ways: either the polypoid or the medusan generation may be suppressed. In the first case we have polyps which reproduce both sexually and asexually, in the other medusæ whose eggs develop directly into other medusæ. (A few medusie may bud new medusi.) Thus we can have four conditions: (I) Polyps which produce sometimes asexually, sometimes sexually, but always polyps; (2) Medusie which always produce medusx; (3) Polyps and medusx in alternating generations; (4) Polyps and sessile medusæ (sporosacs) united in a polymorphic colony.

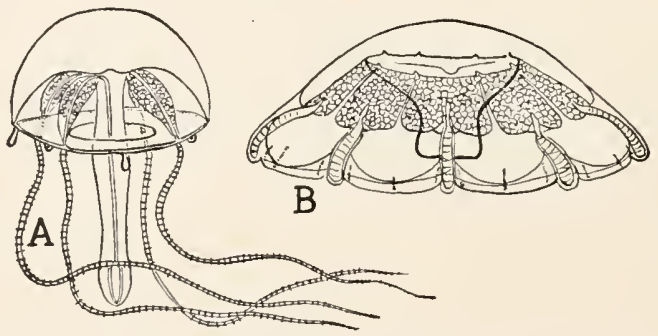

FIG. I80.-American Trachy- and Narcomedusa. A, Liriope scutigera (after Fewkes). $\dot{B}$, Cunocantha octonwria (after Brooks).

The Hydrozoa are almost exclusively marine. The colonial forms occur mostly on rocky coasts down to a depth of roo fathoms, but have been found in water 4000 fathoms deep. The medusæ belong to the pelagic fauna. For a long time the only fresh-water species known belonged to the cosmopolitan genus $H y d r a$, but more recently both hydroid and medusan forms have been found in various parts of the world.

The Hydrozoa may be classified according to characters, derived either from the hydroid or the medusan stage. The former gives four groups: (I) Hydraria. Polyps with asexual and sexual reproduction; no persistent colonies, no perisarc, no gonophores (fig. I69). (2) Tubulariæ. Mostly colonial, with perisarc but without hydrothece. Reproduction by gonophores (medusæ or sporosacs, figs. 94, I 72). (3) CAMpanularie. Colonial, with perisarc and hydrotheca. Reproduction by gonophores arising in special perisarcal envelopes, the gonotheca (figs. I7I, I73). (4) Hydrocorallina. Colonial, with massive, calcified perisarc, resembling coral. Reproduction by sporosacs or rudimentary short-lived medusæ (fig. I 74).

The characters derived from the medusæ give five groups: (I) ANTHomedus \& (Ocellatæ). Gonads on the manubrium; no statocysts; eyes usually present; polyp generation present. (2) Leptomedusæ. Gonads on radial canals; usually velar statocysts; polyp generation present. (3) Trachymeduse. 
Gonads on the radial canals; tentacular statocysts; develop directly to medusx (fig. I8o, A). (4) Narcomedusw. Gonads on the manubrium or gastral pouches; tentacular statocysts; no polypoid stage (fig. 180, B.) (5) SipłosoPHORA. Polymorphic, free-swimming colonies of Anthomeduse, no polyp generation.

As there are medusæ without polyp stages and polyps without medusæ, a natural system must take into account both these features. When the life histories are traced it is seen that the Anthomedusic and the Tubularia are connected by an alternation of generations, as in Leptomedusa and Campanulariæ. There are three groups-Trachymedusie, Narcomeduse, and Siphonophora-without a hydroid stage, and two in which the polyp plays the chief rôle, the medusa being rudimentary in the Hydrocoralline, lacking in the Hydraria. The hydroid polyps are usually but a few millimeters or fractions of a millimeter in size, but the huge Monocaulis imperator, of the deep seas, two yards in length, forms an exception. The colonies are usually only a few inches in extent. The medusæ have bells varying between a millimeter and a few inches in diameter (Equoria forskalea sixteen inches).

\section{Order I. Hydraria.}

Until recently only the cosmopolitan species of $H y d r a$ were known. During most of the year they reproduce by budding (fig. 93), only occasionally developing gonads (fig. I69). 'The eggs remain in connexion with the mother during segmentation, and later form an embryonal shell. In this 'encysted stage' they can be distributed by wind or water birds. These animals formed the basis of the celebrated researches of Trembley on regeneration. He showed that small portions which included both body layers could regenerate the whole animal. His experiments upon turning the animals inside out have not been fully confirmed; for in such cases the layers resume their normal positions. Hydra grisea* (fusca), brown; H. viridis, * green, from the presence of symbiotic algx. Protohydra ryderi, * without tentacles.

\section{Order II. Hydrocorallinæ.}

Exclusively marine, forming colonies of thousands of polyps whose calcareous skeletons so resemble true corals that they were associated with them until the animals were studied. Millepora alcicornis* (fig. I 74), stag-horn coral, in Florida. The rosy Stylasters in tropical seas.

\section{Order III. Tubulariæ $=$ Anthomedus (Gymnoblastea).}

As a rule these colonial forms with perisarc but without hydrotheca produce anthomeduse, but there are forms like Clava* and Hydractinia* which have sporosacs. Indeed, Corymorpha* and Monocaulis* differ only by meduse in the former and sporosacs in the latter. The medusx have the gonads on the manubrium, lack statoliths, and usually have a high-arched umbrella, and frequently eye spots. In the forms with alternation of generations different names are applied to the hydroid and medusan stages.

Amono hydroids are Pennaria,* Syncoryne, * Endendri,* Tubularia,* a mong medusæ Sarsia,* Turritopsis* Largelis* Nemopsis.*

\section{Order IV. Campanulariæ =Leptomedusæ (Calyptoblastea).}

These forms differ from the last in that they are always colonial and possess hydrothecæ, the medusi always being flattened Leptomedusæ (p. 2I6). A peculiarity is the existence of gonothece, closed perisarcal envelopes, inside 
which the gonophores arise from the blastostyle, a specialized polyp, without mouth or tentacles (fig. I 7 I $f$ ). The typical Campanulariæ produce medusæ, while some forms, like Thammantia* and Equoria* have no hydroid stage; on the other hand, Sertularia* and Plumularia* have no medusa stage. Other common genera, Clytia,* Diphasia,* and Aglaophenia* among hydroids;

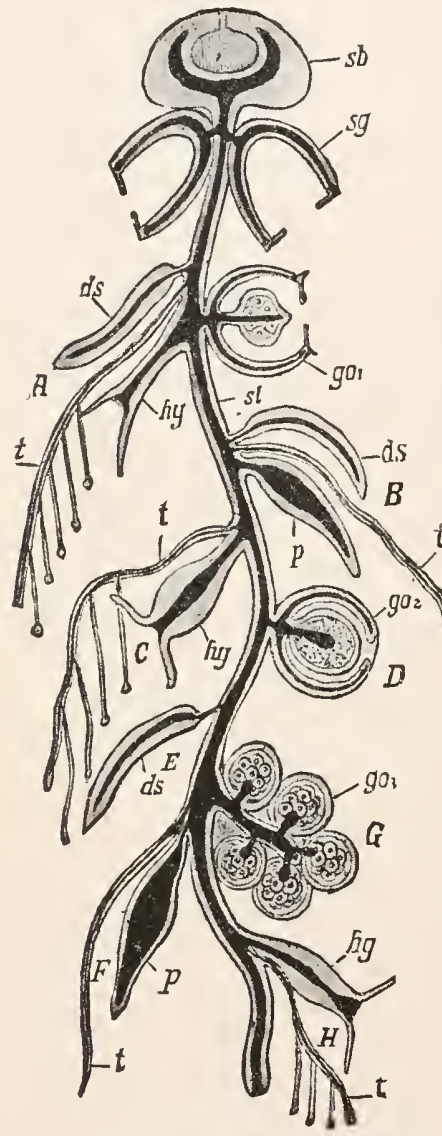

FIG. I8I.-Diagram of Siphonophore (from Lang). $A-H$, groups of different individuals; $d s$, covering scales; go, gonophores; $h y$, feeding polyps; $p$, 'feelers' (digestive); $s b$, float; $s g$, swimming bell (nectocalyx); st, stalk. Obelia,* Tima,* Rhegmatodes* among medusi. Possibly the fossil Graptolites belong near here. Only the perisarc is known; this has hydrothecre, in which it is supposed the hydranths occurred.

\section{Order V. Trachymedusæ.}

These meduse, mostly from warmer seas, have no hydroid stage. The characters are given on p. 2 I 6, Trachyncma, ${ }^{*}$ Liriope* (fig. I80), and Campanella* in our waters, Geryonia, etc., in Europe.

\section{Order VI. Narcomedusæ.}

In addition to the characters on P. 2I 7 may be added that the tentacles arise from the outside, above the rim of the bell. Cunocantha* (fig. r8o), Cunina*, Egina. The larva frequently live as parasites on other medusa, and they may be able to reproduce asexually, forming sacs in which new medusæ are budded.

\section{Order VII. Siphonophora.}

The Siphonophora are among the most beautiful of pelagic animals, some transparent, some brightly colored. Each (fig. r $8 \mathrm{I}$ ) consists of a colony of individuals springing from a common coenosarcal tube which is strongly muscular and contains a central canal, lined with entoderm, by which the members of the colony receive their nourishment. At one end the tube is usually closed by a float of invaginated ectoderm, filled with air, the pneumataphore, which keeps the colony vertical in the water.

The individuals, springing from the cœenosarcal axis, perform different functions and hence differ in structure. Close behind the float commonly come several swimming bells (nectocalyces) which retain only those medusan structures (bell, velum) necessary for swimming and those (ring and 
radial canals) for the distribution of nourishment received from the common tube. Then come, scattered through the colony, the cozering scales, for protection, firm gelatinous plates which have lost the ring canal, the muscles, and the bell shape of the medusix. Food is taken by widemouthed feeding tubes ( $h y$ ) which may be compared to polyps (fig. 58) or the manubrium of a medusa. They digest the food by means of large masses of glands ('liver bands,') and send it by the central tube to all the members of the colony. At the base are long muscular tentacles ( $t$ ) from which small lateral threads depend, each ending in a brightly colored swelling, the nettle head composed of large, closely packed nettle cells. These are the cause of the nettling, which in many species is so

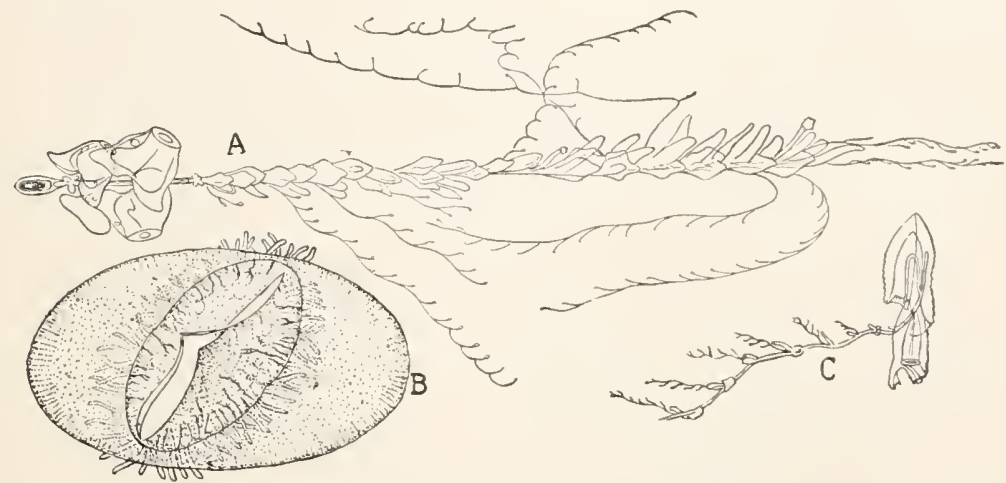

FiG. I82.-American siphonophores. A, Nanomia cara (after A. Agassiz). B, Velella meridionalis (after Fewkes). C, Diphyes praya (after Fewkes).

severe as to be feared by man. 'The 'feclers' $(p)$ recall mouthless polyps and manubria; they are very sensitive and mobile and, while tactile, apparently in some cases are digestive organs. Latest to develop in the colony are the sexual bells. They are usually brightly colored and resemble small mouthless Anthomedusie without tentacles. They but rarely (Chrysomitra) separate from the colony, but usually persist as more or less reduced sporosacs. From this it follows that the Siphonophora afford fine examples of division of labor and of the consequent polymorphism of individuals. This can indeed be carried so far that many convey the impression of being individuals with a multiplicity of organs. The Siphonophora are all marine, and occur most abundantly in tropical seas.

Sub Order I. PHYSOPHORÆ (Physonectr). Float present, small; next a large series of swimming bells; then the other members of the colony. $P / h y$ sophora, Agalmia, Nanomia* (fig. I82). Sub Orcter II. CALYCOPHOR.E (Calyconectæ). Float lacking; one or two large swimming bells; the other in- 
dividuals in groups which frequently separate before becoming mature, were once regarded as distinct animals. Praya, Diphyes* (fig. 182), in warmer seas. Sub Order III. CISTONECTÆ. Float greatly enlarged; the conosarcal tube reduced, the individuals (no covering scales nor swimming bells) attached to under side of the float. Physalia,* Portuguese man-of-war, stings severely. Sub Order IV. DISCONANTHÆ. Float a flattened disc; manubrium projects from centre of lower surface. Porpita,* disc. Velella* (fig. I82).

\section{Class II. Scyphozoa (Scyphomedusæ).}

The Scyphozoa parallel the Hydrozoa in frequently having an alternation of generations; the asexual generation being the scyphopolyp or scyphostoma, the sexual an acraspedote medusa. In contrast to the Hydrozoa the asexual stage plays a subordinate rôle; it is closely similar in all species, and can even be lost (Pelagia), while the medusæ are always well developed and present great variety of form.

The scyphostoma (figs. I83, I84) recalls $H y d r a$, but has a small perisarcal cup around the aboral end. Internally there are four longitudinal folds projecting into the gastral cavity and extending from the

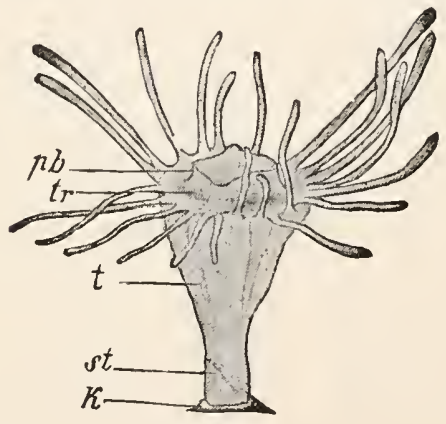

FIG. I $\delta_{3}$

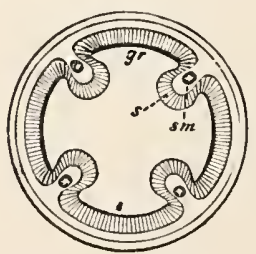

FIG. 184 .

FIG. I83.-Scyphostoma of Aurelia aurita (from Korschelt-Heider). k, perisarc cup; $p b$, proboscis; $s$, stalk; $t$, gastral folds; $t r$, ectodermal funnels.

Fig. IS4.-Section of Scyphostoma (from Hatschek). $g r$, gastric pouches; $s$, gastric septa; $s m$, muscles.

margin of the mouth to the opposite pole. These septa or taniolce (fig. $184, s$ ) appear in cross-section as small folds of entoderm supported by a process of the supporting layer containing a muscle band extending down from the peristome (fig. I84). They are important morphologically, since in budding they produce the gastral tentacles (phacella) of the medusx. Further, they are the first appearance of the septal system, so strongly developed in the Anthozoa.

The medusx are large (four inches to four feet or more in diameter) with a slightly arched umbrella, of ten of almost cartilaginous consistency. 
A knowledge of the development is necessary in order to understand the medusa. The young medusa (Ephyra stage, fig. I $8_{5}$ ) is eight lobed, each lobe with a sensory pedicel in a notch at the tip. These lobes indicate eight radii, the four passing through the angles of the mouth being the perradii, the others the interradii, the adradii being between the lobes (fig. I86). In most species the adradial regions increase with growth, and at last form a circular margin to the bell, divided by the notches of the oriçinal lobes (fig. I 87 ), tentacles occurring only in the adradial regions. The medusx difier externally from those of the Hydrozoa in the absence of a velum (hence acraspedote).

Instead of a nerve ring there are eight nerve centres connected with the sensory pedicels. Each pedicel is a modified tentacle (fig. I 77,7 ) its entodermal axis furnishing a statolith at the end, and usually a simple eyespot.

The gastrovascular system begins with a quadrate or $\mathrm{X}$-shaped mouth (fig. I86). The angles of the mouth are usually produced into long curtainlike oral tentacles of use in the capture

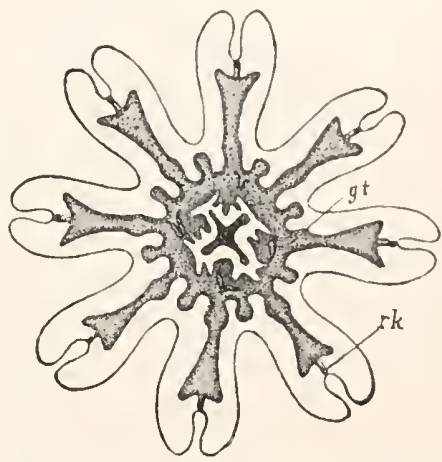

FIG. I85.-Ephyra of Colylorhiza (after Claus). git, gastral tentacles (phacella); $r k$, marginal (sensory) body. of food. The 'stomach,' which begins just inside the mouth, gives off four interradial pouches, the gastrogenital pockets, each containing a group of small gastral tentacles (phacelle), and the plaited folds of the gonads, these being, in contrast to the Hydrozoa, of entodermal origin. In this the Scyphomeduse show relationships to the Anthozoa. From the central digestive sac arise in the Ephyra stage (fig. I85) eight radial canals to the sensory pedicels, and most adult medusie have these same pouches and eight others, adradial in position. In some this primitive arrangement is complicated by a network of tubes (fig. I 86).

In the species with an alternation of generations the egg produces a ciliated larva, the planula (fig. IS8) which attaches itself and develops into a scyphostoma. This scyphostoma is capable of terminal, and often of lateral, budding. The lateral buds always produce new scyphostoma, the terminal, medusæ. In the latter the scyphostoma develops into a strobila, becoming divided by circular constrictions into a series of saucerlike discs, the young jelly-fish. As the successive discs become ready they separate from the pile and swim away as ephyra. At first the ephyre (fig. 185) have only four gastral tentacles, parts of the gastral 


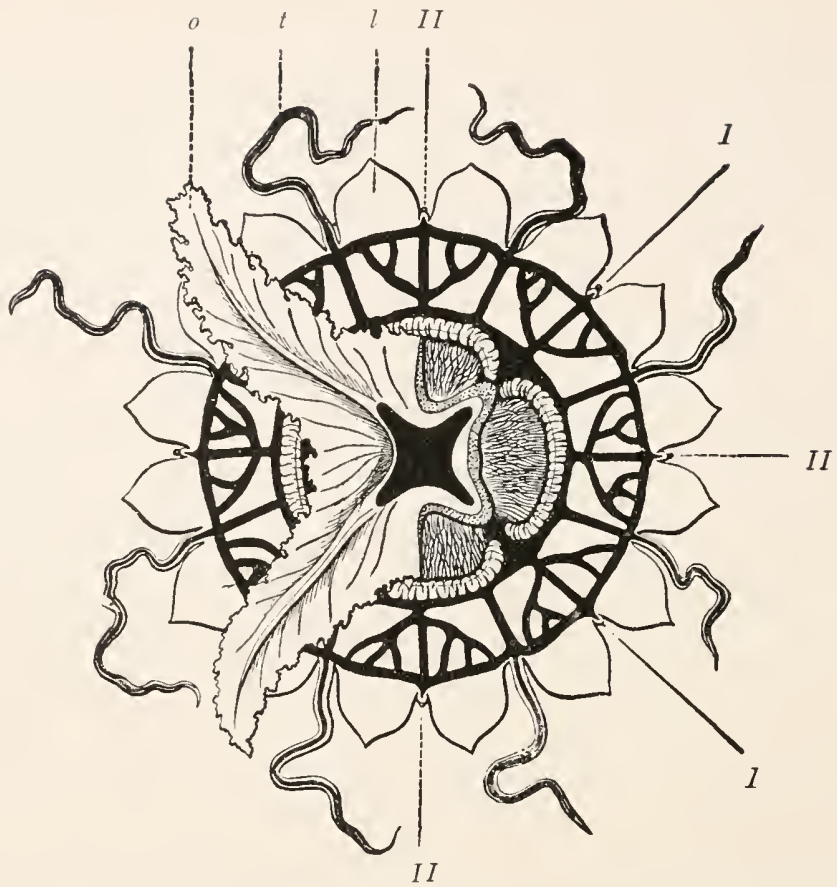

FIG. I86.-Ulmaris prototypus (from Hatschek). I, radii of first order (perradii); $I I$, radii of second order (interradii); $l$, marginal lobes; $o$, oral lobes (cut away on right side); $t$, tentacles (adradial); the gonads (right side) are interradial.
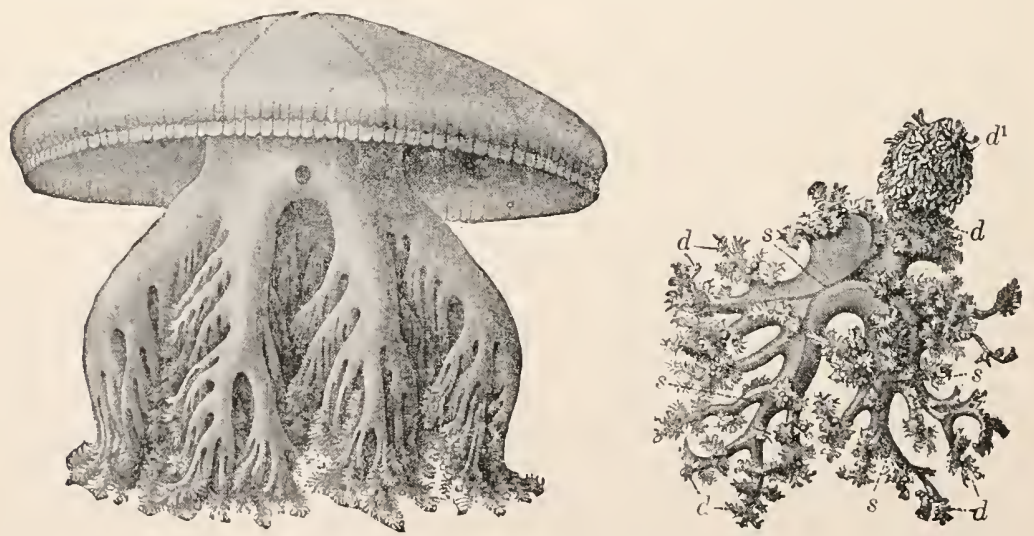

FIG. 187.-Polyclonia frondosa* and one of its branching oral lobes, showing the closed grooves (s) (after Agassiz). 
septa of the scyphostoma (p. 220). Since the ephyra differ markedly from the adult medusie and only gradually change into the sexual form, the alternation of generations is complicated by a metamorphosis. 'This metamorphosis persists in some cases (Pclagia noctiluca) where the alternation of generations is suppressed; the egg develops directly into an ephyra, which transforms into the adult jelly-fish.
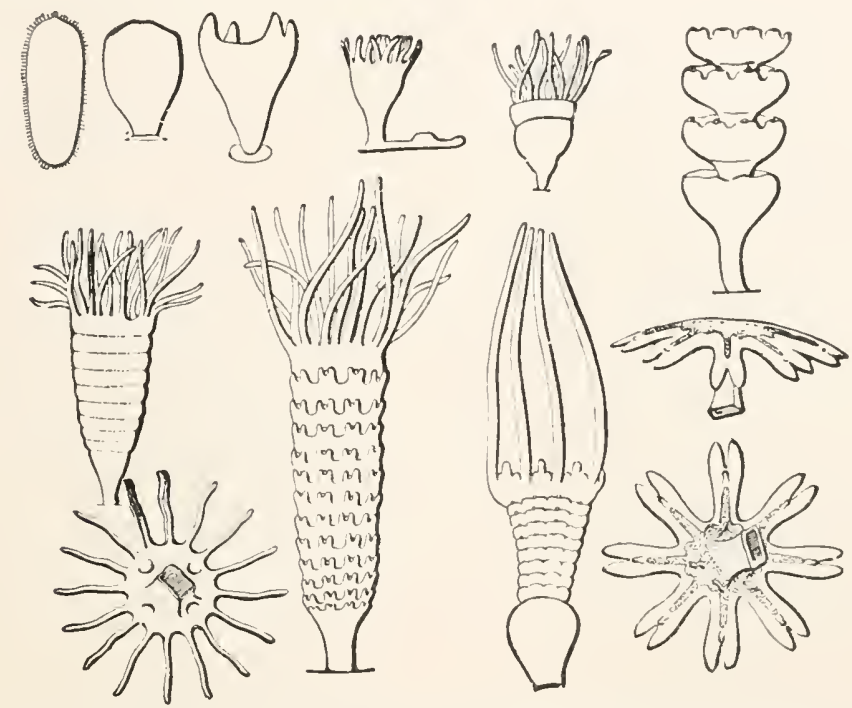

FIG. 188.-Development of Aurelia aurita (from Hatschek). First row, growth of planula to scyphostoma; below, strobilation (separation of ephyra): left, oral view of scyphostoma; right, two ephyræ.

\section{Order I. Discomedusæ.}

The foregoing account applies, as a whole to only the Discomedusx, the widest distributed and most abundant of the Scyphomedusie. The order is divided in to two suborders, I. SEMÆOSTOME and very mobile arms at the corners of the mouth. Aurelia flavidula ${ }^{*}$ and Cyanca arctica* ${ }^{*}$ common in north $A$ tlantic waters, the latter large, exceptionally seven feet in diameter; Pelagia,* Ulmaris (fig. I86). (2) Runzostomex, four oral arms which branch dichotomously; the mouth and grooves on the arms closed by union of their edges so that many small stomata remain through which food is taken. Stomolophus, * Polyclonia (fig. 187).

Certain Scyphomedusx are distinguished from the Discomerluse. Some of these are inhabitants of the deep seas and only recently known; others differ so from the Discomedusæ that the relationship was not seen at first. These have in common the rathamma, four partitions, homologous to the treniolic of the scyphostoma, which bear the phacelli and divide the peripheral part of the gastral cavity in such a way that the gonads are separated into cight groups. The marginal bodies vary in three ways. 


\section{Order II. Stauromedusæ.}

Best known are the Lucernarix (fig. I89) which lack marginal bodies, but usually have four small tentacles in their place, while the adradial regions are drawn out into arms, bearing bundles of tentacles. The aboral surface of the bell is produced into a stalk by which the animals are attached. The TesseRID止 (unknown in America) are free-swimming.

\section{Order III. Peromedusæ.}

Free-swimming, cup-shaped medusie, with four interradial sense bodies; mostly from the high seas. Pericolpa, Periphylla in Gulf Stream.

\section{Order IV. Cubomedusæ.}

Differ in the four perradial sense bodies. Tropical and subtropical; development unknown. Charybdea (fig. I90).

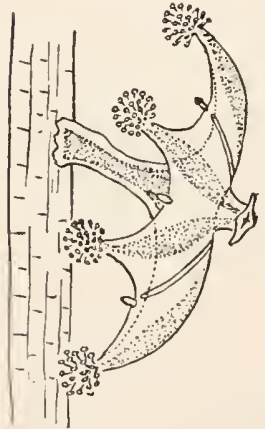

FIG. I89.-Halyclvstus auricu'aria: (after Clark).

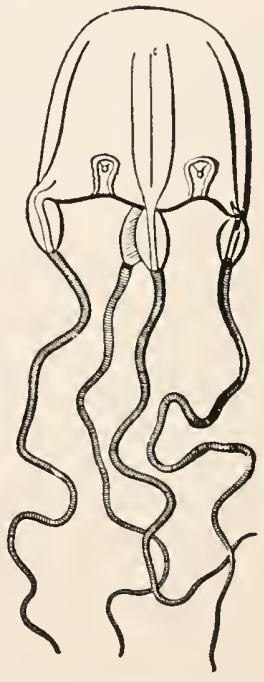

FIG. I90.-Charybdea marsupialis (from Hatschek).

\section{Order V. Coronata.}

A coronal furrow on the exumbrella; four to sixteen marginal sense bodies as in Discomedusæ, but eight gonads and presence of cathamma. Some of these formerly regarded as Discomedusæ (under the name of Cannostomex), because of eight sense bodies. Nausithoe albida arises by terminal budding from a scyphostoma (Stephanoscyphus mirabilis) parasitic in sponges. Atolla.

\section{Class III. Anthozoa (Actinozoa).}

The Actinozoa, including the sea anemones, sea pens, and corals, are exclusively marine. With few exceptions they are sessile and usually form colonies, often of enormous size. In this as in appearance (fig. I 92) 
they resemble the hydroid polyps. 'They have a pedal disc, column, tentacles, and peristome with central mouth. 'They are distinguished by their greater structural differentiation. 'The Anthozoan polyp has a well-developed mesoglœa, this being a layer of connective tissue with numerous cells, giving the animals a tough fleshy consistency. Still more important are the cesophagus and septx bearing mesenterial filaments and gonads.

The mouth, in the centre of the peristome, is usually oral or slit-like. Hence there is a biradial symmetry for there is a sagittal axis (fig. I $1, s, s$ )

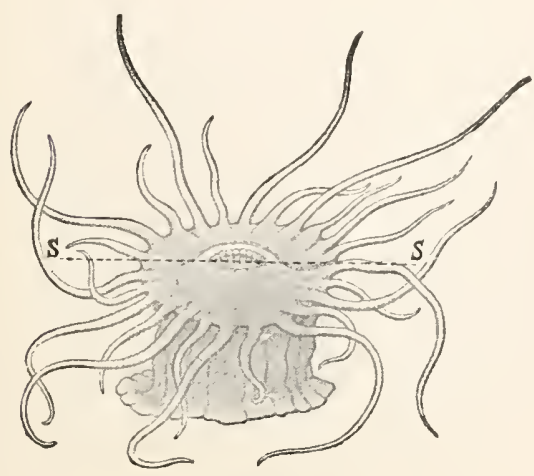

FIG. I9I.

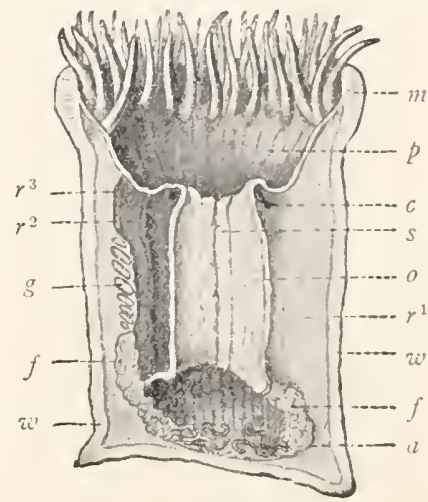

FIG. I92.

FIG. Igr.-Antheomorpla elegans. s, s, sagittal plane.

FIG. I92.- Sagartia parasitica split lengthwise. $a$, acontia; $c$, septal canal; $f$, mesenterial filaments; $g$, gonarls; $m$, sphincter muscle; $o$, asophagus; $p$, peristome; $r$, septa of different orders; $s$, siphonoglyphe; $w$, cut wall of column.

passing in the long axis of the mouth, and a transverse axis at right angles to it. From the mouth the œophagus hangs down into the body as a flattened tube and opens at its lower end in to the wille gastrovascular carity. This oesophagus is an inflected part of the peristome and hence lined with ectoderm, and its lower end alone can be compared with the mouth of the hydrozoan (fig. 192). It usually bears at either end a specialized groove, the siphonoglyphe $(s)$.

The oesophagus is held in position by radial partitions, the septa $(r)$, which stretch from base, column, and peristome to the osophagus, dividing the peripheral part of the gastral space into small pockets, the radial chambers, connected below the end of the asophagus with the central part. Above, these chambers continue into the tentacles. The tentacles therefore are outgrowths from the radial chambers and usually equal them in number. Besides the complete or primary septa which reach 
the osophagus, there may be others which do not reach the osophagus and belonging to secondary, tertiary or other series (fig. 194).

The septa support a number of important organs: the mesenterial filaments, gonads, and muscles. The mesenterial filaments are thick strands of epithelium, rich in glands and nettle cells, fastened like a hem on the edge of the septa. Since they are much longer than the peristomial-

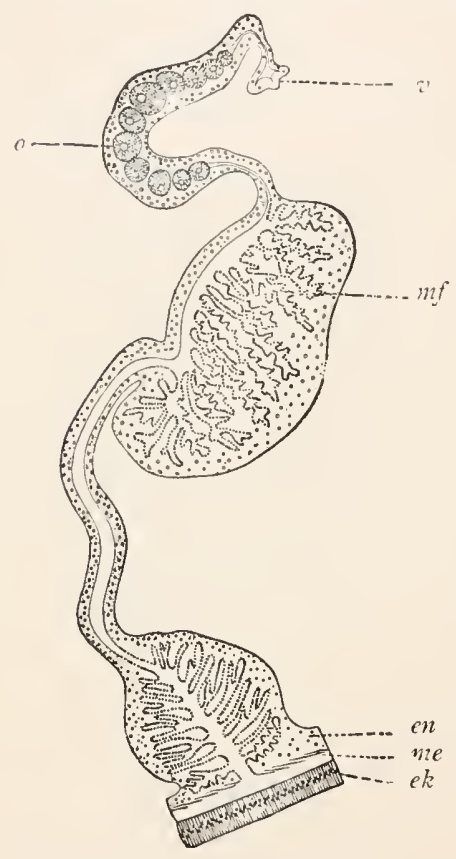

Fig. I93.-Section of septum of Edwardsia tuberculata. ek, ectoderm; en, entoderm; me, supporting layer; $m f$, septal muscle; $o$, ovary; $v$, mesenterial filament. pedal length of the septa, they cause these latter to wrinkle and fold, thus strikingly resembling the mesenteries of the mammals. They envelope the food and press it in, thus aiding the succeeding intracellular digestion. Lower down, in some species, the filaments become free and form long threads, acontia, rich in nettle cells which are protruded for defence, either through the mouth or pores (cinclides) in the column. The gonads -only exceptionally hermaphroditic -lie besicle the mesenterial threads as thickenings of the septum (fig. I92, g). The germ cells arise from the entoderm, but early migrate into the mesogloa of the septum $($ 193,o). The eggs, when ripe, escape into the gastrovascular cavity. The young leave the parent at various stages of development, sometimes as planule (fig. I97, A), sometimes as young with tentacles.

The muscles are very important, morphologically. Muscles and nerves occur in both ectoderm and entorlerm; but while the nerves are best developed in the ectoderm, especially around the mouth, and extend into the mesgolœa, the muscles of the ectoderm are weakly developed and are mostly confmed to the peristome and the tentacles. The entodermal musculature is much stronger. Just outside of the tentacles is usually a strong circular (sphincter) muscle $(m)$ which can close in the top of the column over the peristome. The septa also bear muscles, transverse on one side, longitudinal on the other, the latter producing ridges on the septa (fig. I93). 
In the Hexacoralla (fig. I94) the septa are in pairs, with the muscle ridres facing each other, except at the ends of the sagittal axis, where they face outwards. These are called the directives. Since the septa occur in pairs, two kinds of radial chambers occur, those between the pairs being called interseptal, those hetween the two of a pair being intrascptal. At first all Hexactinians have six pairs of septa-two pairs of dircctives, and four of lateral septa. With growth, septa of a secondary order may appear between these, giving twelve in all, then tertiary septa, the number of tentacles increasing with the septal chambers. The rule is not invariable, for some have modifier the plan of six to four or ten, without altering the primitive condition.

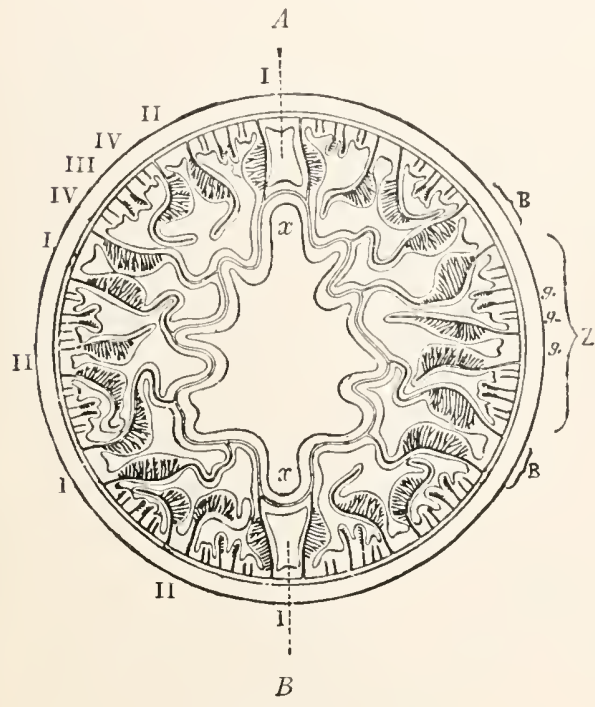

FIG. I94.

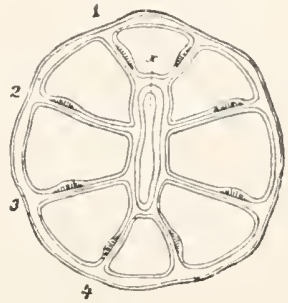

FIG. 195.

FIG. I94.-Transverse section of actinian (Adamsia diaphana) $A B$, plane of symmetry, a second lies at right angles. I-IV, septa of four orders.

FIG. 195.--Transverse section of an Octocorallan (Alcyonium). x, siphonoglyphe; $\mathrm{I}-4$, septa of one side, with their muscles on one side, symmetrical with those of the other side.

In the Octocoralla only eight septa are developed. These are disposed equally on either side of the cesophagus and may have (most octocorallans) all their muscles towards one end (fig. I95) or (Edwardsia, fig. I96, IV) have one pair reversed. It is to be noted that hexactinians pass through an Edwardsia stage. In Cerianthus new septa are always added at one end of the sagital axis (fig. I96, II), while in the extinct Tetracoralla (I), so far as one may judge from the hard parts, the septa have an arrangement with four as the basis.

Most Anthozoa reproduce by division or budding as well as by eggs. Occasionally the buds separate; usually they remain connected with the mother, forming colonies of hundreds or thousands of individuals, connected by a conosarc, consisting largely of mesogloea with a covering 
of ectoderm and penetrated by a network of entodermal canals. On disturbance the polyps retract into the cœnosarc.

Colonial Anthozoa, with few exceptions, have a skeleton (coral), secreted by the ectoderm, consisting of calcic carbonate or of an organic
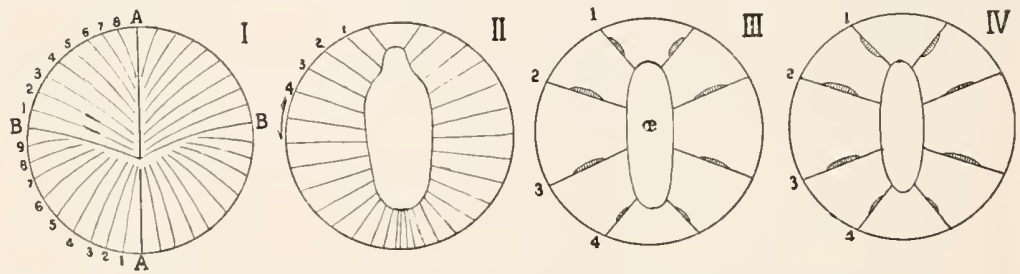

FIG. I96.-Arrangement of septa in various Actinozoa. I, Tetracoralla; II, Cerianthus; III, Octocoralla; IV, Edwardsia.

horny substance, the two sometimes occurring together. The skeleton may be internal (axial) secreted by the cœnosarc, or external (cortical), and formed by the polyps, repeating to a large extent their complicated structure (figs. 199, 200). In Fungia (mushroom corals) the cortical skeleton
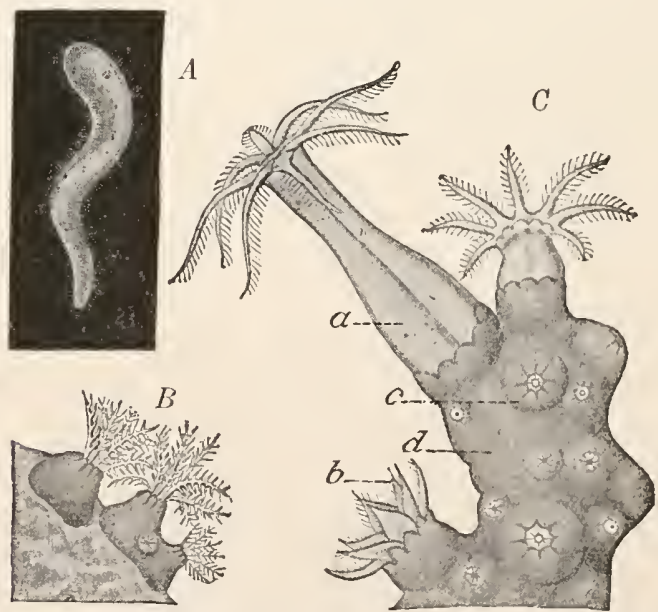

Fig. I97.-Corallium rubrum, red coral (after Lacaze Duthiers). A, ciliated young; $B$, young colony; $C$, part of colony with polyps in extension $(a)$ and contraction $(c) ; d$, coenosarc; $A$, greatly, $B, C$, slightly enlarged.

consists only of a base, with radiating ridges (sclerosepta) on the side towards the flesh. These alternate with the septa (sarcosepta) of the polyp. In most forms there is, in addition, a cup (theca) in the column of the polyp, the sclerosepta extending inward from this. 
The theca arises by a fusion of sclerosepta. If this fusion takes place some distance inside the peripheral ends of the sclerosepta, the distal ends of these project on the outer surface as coste. Still outside these may be a second cup, the epitheca. In the centre may occur a large calcareous column or sereral smaller ones, the columella. As the polyps grow they build the thecæ hicher and higher and consequently draw out from the deeper portions, which may become cut off by horizontal partitions, the tabula. Such tabula occur in some Madreporaria, Octocorallans, and Millepores ( $p$ ). 2I 7) which were formerly united in a group 'Tabulata.

It was once thought that the coral was a calcified portion of the soft parts and hence that sclerosepta were hardened sarcosepta, etc. This has been disproved. The sclerosepta are formed in the radial chambers between the sarcosepta, and the theca inside and at some distance from the column, the outer surface of which secretes only the inconstant epitheca (fig. I99). From the above it would appear that the sclerosepta

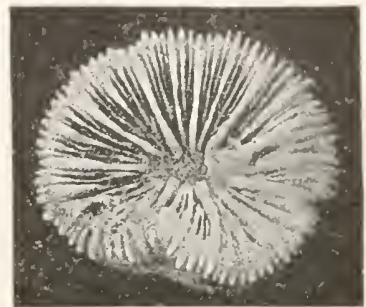

FIg. IoS.-Sclerophyllia margariticola (after Kilunzinger). correspond in number to the sarcosepta, but this is not always the case. Thus the Helioporida, which on the grounds of the skeleton were regarded as Hexacoralla, are shown by the soft parts to be undoubted Octocoralla.

By means of their skeletons the Anthozoa produce the well-known coral reefs. When the reef reaches the surface it produces an island, the most note-
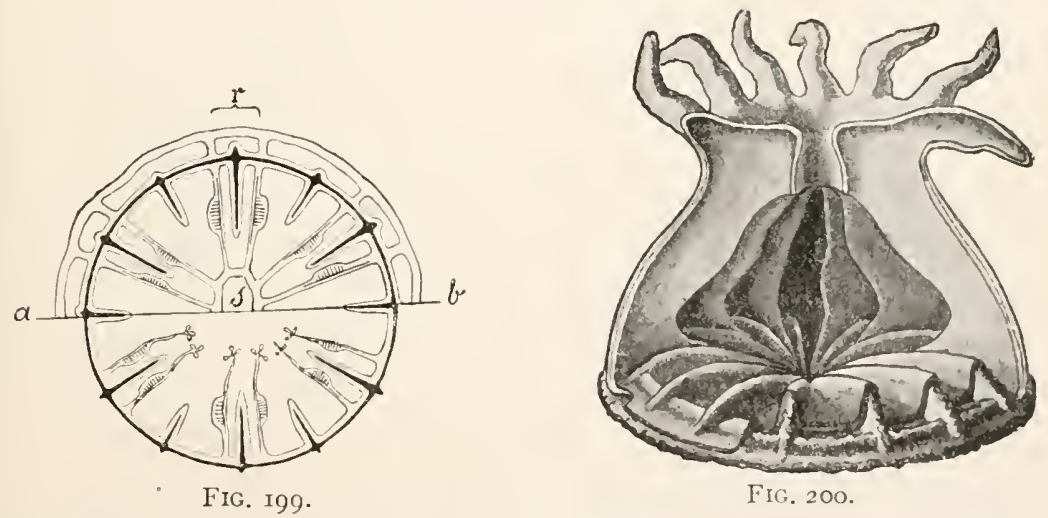

FIG. 199.-Diagrammatic section of the flesh and coral of a hexacorallan: above the line the section passes through the oesophagus, $s$; below the line it is lower lown; $r$, directives: coral black.

FIg. 20o.-Diagram of relations of soft parts to coral (after Pfurtscheller). Shows beginning sclerosepta and theca.

worthy form being the atoll, a ring-like structure with a central lagnon. The origin of these atolls, as well as that of fringing and barrier reefs, was for a long time explained by Darwin's and Dana's theory of coral reefs. Iater investigations, notably those of Mr. Agassiz, afford another explanation. 


\section{Order I. Tetracoralla (Rugosa).}

Extinct forms from the paleozoic rocks with the parts arranged in fours (fig. I96, I). The present tendency is to regard them as modified Hexacoralla.

\section{Order II. Octocoralla (Alcyonaria).}

These forms, which have eight single septa, are recognizable by their eight feathered tentacles (fig. I97). They occur in all seas from near the shore to great depths. In development there is a planula (fig. 20I) in which the oesophagus arises as a solid ingrowth which becomes perforated later. The eight septa arise simultaneously. Usually colonies are formed by budding and a polymorphism may occur, some individuals which have reduced septa and lack tentacles, taking in water for the colony. Many are phosphorescent.

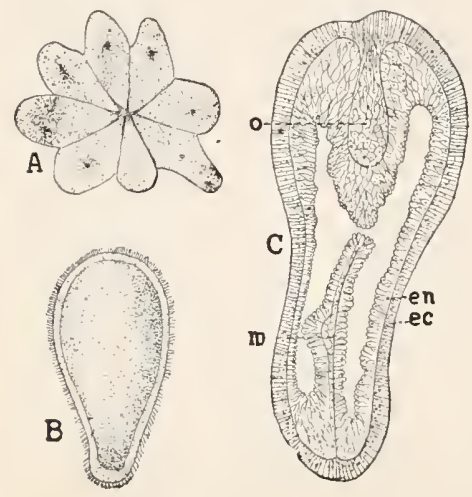

FIG. 20I.

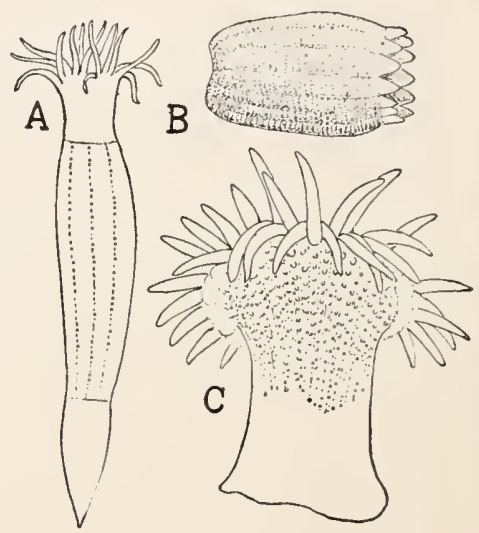

FIG. 202.

FIG. 20I.-Three stages in development of Renilla reniformis (after Wilson). $A$, cleavage of egg; $B$, planula; $C$, development of osophagus; $c c$, ectoderm; $c n$, entoderm; $m$, mesogloea; $o$, cesophagus.

FIG. 202.-American sea-anemones. A, Edwardsiella sipunculoides (after Stimpson). B, Bicidium parasiticum (after Verrill). C, Bunodes stella (after Verrill).

ALCyONIDE (Alcyonium*), axial skeleton is lacking, the flesh contains numerous calcareous particles (sclerodermites). The sea pens, Pexnatulide, have the basal part buried in the mud, the rest, expanded like a disc or feather, bears the polyps. An axial skeleton usually occurs in the stalk. Pennatula, Renilla*. The GorgoxidDE (sea fans, sea whips) have an axis of more firmness, which may be calcareous, and the colony may branch and the branches anastomose. Here belongs, besides many tropical genera whose names end in 'gorgia,' the precious coral (Corallium rubrum, fig. I97), the fishing for which at Naples amounts yearly to half a million dollars. TUBIPoride, organ-pipe corals. The HELIOPORID w were long regarded as Hexacoralla because of their massive skeletons with six sclerosepta. The paleozoic Syringopora belongs near Tubipora, while the FAvositide resemble the Alcyoniidæe.

\section{Order III. Hexacoralla (Zoantharia).}

The simple tubular tentacles are highly characteristic of the Hexacoralla, as is the arrangement of the paired septa in sixes as described above. Yet there are exceptions to this rule. On the one hand is Edwardsia* with sixteen or 
more tentacles and only eight septa (fig. 202), but which exhibits a condition through which the young actinians pass; on the other hand, in the Zoantharia, Cerianthix, and Antipatharia the rule of six has undergone extensive modification.

Sub Order I. AC'TINARIA (Malacoderma). The sea-anemones are mostly solitary, without skeleton; with numerous septa and tentacles. They occur in

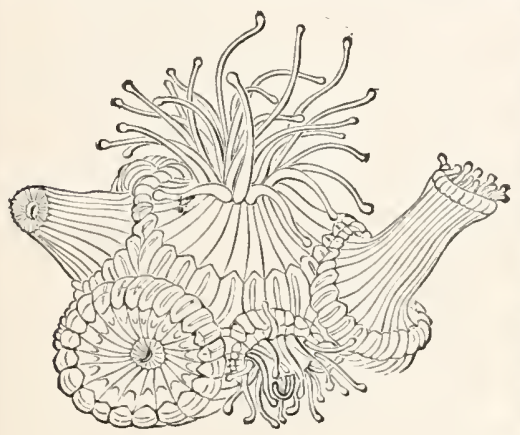

Fig. 203.-Astrangia dane*; five polyps in various stages of expansion.

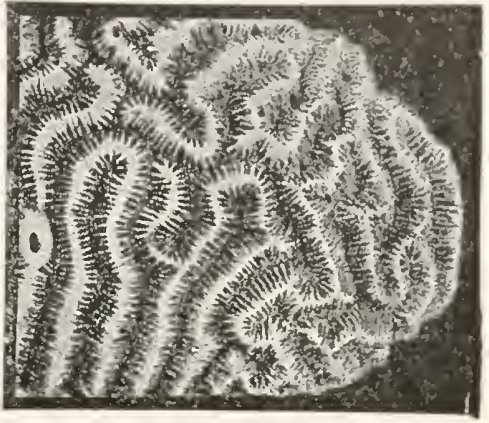

FIG. 204.-Caloria arabica (after Klunzinger).

all seas from tide marks to the greatest depth. A few are free, but most are sessile. Metridium,* Bunodes, * Sagartia,* Bicidinm* (parasitic on Cyanea), Halcampa*. ZOANTHE $a^{*}$ have two kinds of alternating mesenteries, individuals of the colonies usually incrusted with foreign matter. Epizoanthus* lives symbiotically with hermit crabs (fig. II 4 ).

Sub Order II. AN'TIPATHARIA. Six pairs of septa and six (Antipathes) or twentyfour (Gerardia) simple tentacles; colony with a black horny axis and no calcareous skeleton. Simulate the Gorgonids.

Sub Order III. MADREPORARIA (Scleroderma). This group, the richest in species of any, is characterized by the great development of the skeleton. Theca, septa, and usually columella are present, and frequently costæ as well. Solitary forms are few. Usually they form colonies, frequently of thousands of individuals, bound together by a coenosarc extending over the surface of the coral. A colony arises from a single animal by continued fission or budding. When the division is not complete the animals may form long series with numerous mouths but with the other parts united, the result being that the surface of the coral is

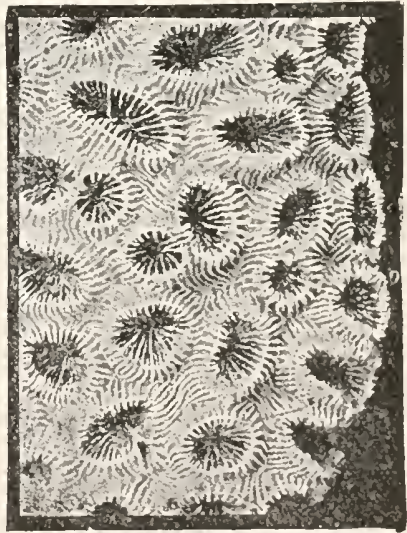

FIG. 205.-Faria caz'ernosa (after Klunzinger). marked by long winding grooves-incompletely separated theca-with srlerosepta, as in the brain corals (fig. 204). The fossil Tetracoralla (p. 230) are now regarded as modified Hexacorallans. (I) The Aporosi, a compact skeleton, the gastral canals running outside of the skeleton. Some, like Sclerophylla (fig. I98), are solitary. Others, like Oculina, * branch, and still others form 
compact masses. Astrangia dana (fig. 203), only coral in New England; A stroa; brain corals (Coloria, fig. 20.4, Manicina); Fazia (fig. 205). (2) FunglaceA, or mushroom corals, no theca. Some colonial, others (Fungia) solitary. A sort of strobilation in development. (3) POROSA, with skeleton porous like a fine sponge. Madrepora, ${ }^{*}$ deer's-horn coral (fig. 206), Porites, Astroides.

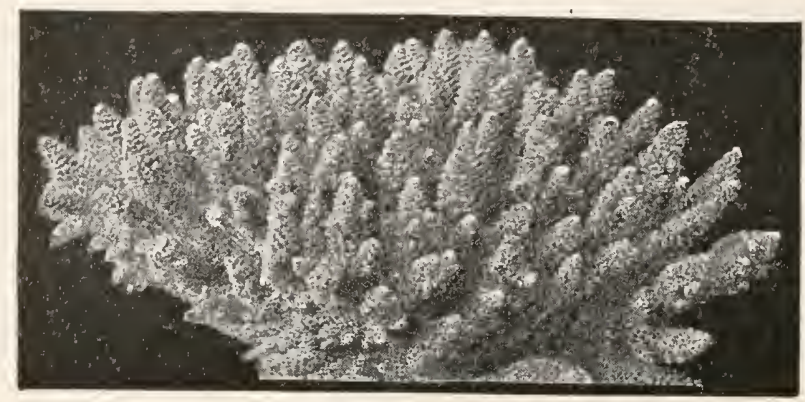

FIG. 206.- Kadrepora erythroa (after Klunzinger).

\section{Class IV. Ctenophora.}

The Ctenophores excel all animals, even the medusx, in transparency and delicacy of tissues; many are so soft that a strong current tears them, and no attempts to preserve them have been successful. The body is biradially symmetrical; i.e., is divided by both sagittal and transverse planes into symmetrical halves. Since the longitudinal axis is usually longer than the others, which are generally equal, the body is usually oval or pear-shaped. In Cestum the sagittal axis is greatly longer, giving the animal the form of a band, whence the name 'Venus girdle.'

The bulk of the animal is composed of a soft jelly with connectivetissue cells, penetrated in every direction by polynucleate muscle cells (fig. 50) branched at their ends and apparently innervated by special nerve cells. On the outer surface is a layer of ectoderm, while in the interior is a system of branched entodermal canals.

At the bottom of a depression (fig. $207, s$ ) at the aboral pole is a thickened patch of ectoderm, the sense body, a typical statocyst (fig. 208). The thick sensory epithelium forms a shallow groove, strong hairs which rise from the edge of the groove arch over it, enclosing a space to be compared to an incomplete vesicle. In the centre is a spherical mass of statoliths, supported on four bundles of S-shaped agglutinate cilia. From these bundles of cillia eight bands of thickened epithelium, at first in pairs (fig. 209, w's), later diverging, pass to the oral pole (fig. 207,r). These meridiona! bands (so called from their course) consist in part of ciliated epithelium, in part of the characterisic 'combs' which are the locomotor 


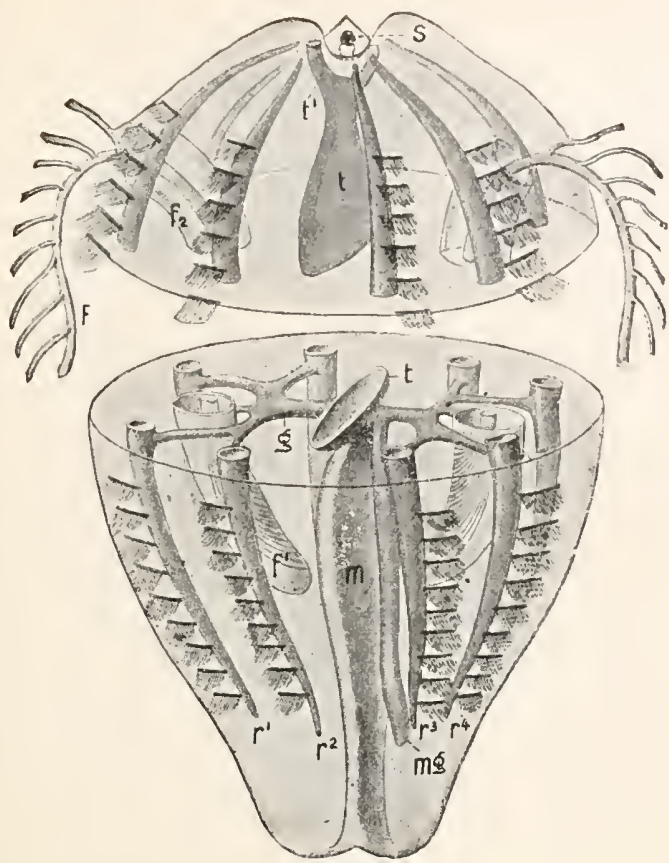

FIG. 207 .

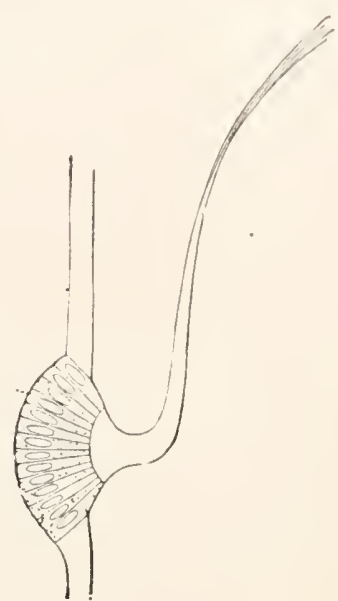

FIG. $207 \mathrm{~A}$.

FIG. 207.-Diagram of Hormophora, cut in two. $f$, tentacle; $f^{2-3}$, root and sheath of tentacle; $g$, main perradial vessel which divicles twice dichotomously to form the meridional vessels; $m$, stomach; $m g$, paragastric canals; $r^{t-4}$, rows of combs overlying meridional canals; $t, t^{1}$, funnel and funnel vessels; $s$, sense body.

FIG. 207 A.- Swimming plate and epithelial cushion (after Chun).

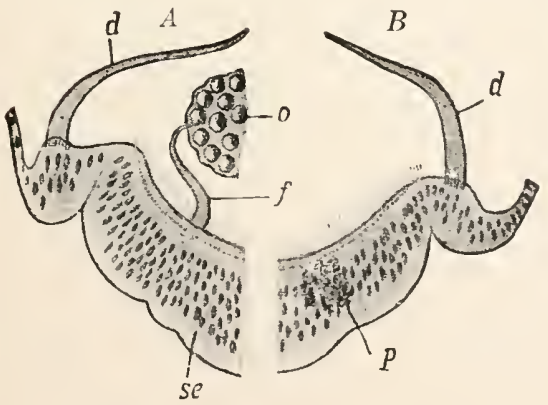

FIG, 208

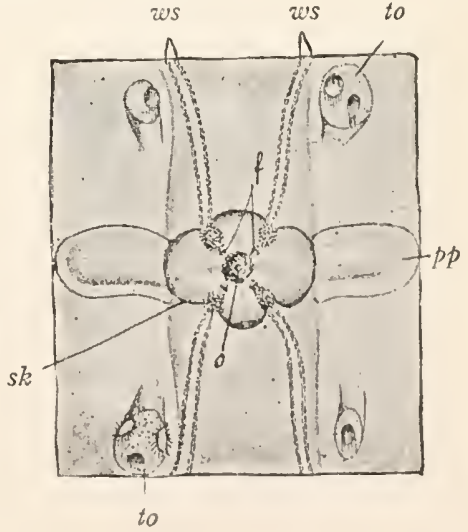

Frg. 200 .

FIG. 208. - Section of sense body of Callianira. $A$, through the centre; $B$, excentric; $d$, roof of sensory groove; $f$, support of statoliths, $o ; p$, pigment cell; se, sensory cells.

Fig. 209.-Aboral pole of Callianira (from Lang). $f$, supports of statoliths, $o ; p p$, pole plate; $s k$, sense body; to, openings of gastral funnels; ws, ciliated bands. 
organs, and which must be regarded as transverse rows of long agglutinated cilia. The combs (fig. 207 A) arise from thick epithelial ridges, transverse to the meridional bands, and are so far apart that the free edges of one comb overlap the base of the next like shingles. In consequence of their fibrous structure the combs are strongly iridescent and in motion cause a beautiful play of metallic red, blue, and green over the meridional bands These combs act like oars and row the borly about. Since the combs begin some distance from the aboral pole, they are connected with it by means of ciliated grooves following the line of the meridional bands. Experiment shows that the sense body is an organ of equilibration and for correlating the action of the different rows of combs.

The ectoderm gives origin to two other important organs, two pole fields and two tentacles. The pole fields (fig. 209, $p p$ ) are two epithelial patches extending a short distance in the sagittal axis from the sense body and possibly are olfactory or taste organs. The tentacles arise, in the transverse axis, from the bottom of deep tentacular sacs $\left(\right.$ fig. $\left.207, f^{2}\right)$ from which they project as long cords with numerous lateral branches, and into which they may be retracted. Tentacles and branches contain an axial muscle, while the ectodermal coating consists largely of adhesive cells. These are spherical bodies (fig. 2 Io) covered with a very sticky granular secretion,

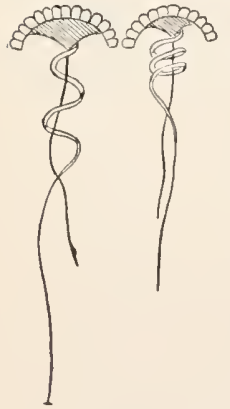

FIG. 2 10.-Arhesive cells of Ctenophora (after Samassa). and, like a Vorticella, supported on the end of a spiral stalk muscle. These are used in capturing prey, which adheres to them and is drawn inward by the muscles.

The ectoderm also forms part of the gastrovascular system. It turns inward at the mouth-situated at the lower end of the chief axis-and lines the large space commonly called stomach (fig. 207, $m$ ), but which corresponds to the œsophagus of the Actinozoa. At the aboral end of this stomach begin the true entodermal portions, the so-called funnels, and from them run canals distributed through the jelly to the various organs. Two (rarely four) funnel canals run to the aboral pole and empty (fig. 209, to near the sense body; a second pair, the paragastric canals (fig. 207, $\mathrm{mg}$ ), which run parallel to the oesophagus, end blindly. The perradial canals $(g)$ proceed outward from the funnel, and besides giving off a canal to the base of the tentacle, each divides dichotomously twice, first into interradial and then into adradial canals, each of these last connecting with a meridional ressel running just beneath a row of combs, nourishing them as well as the gonads. The gonads consist of two bands, one male, the other female, running in that wall of the meridional vessel nearest to the combs 
These gonads are regular in distribution, those of two vessels which are nearest each other being of the same sex. 'The eggrs and sperm pass out through the gastrovascular system.

The few species are divided into TENTACULATA, with tentacles, and NUD.A, without. To the first belong the CYDIPPID 2 , with pear-shaped bodies (Pleurobrachia*), Hormiphora (fig. 207); the LoBata (Mnemiopsis, Bolina*), with Iobes; and the band-like Cestid e (Cestum, Venus girdle). The BrRorde (Beroe, Idyia*), with wide mouth, belong to the Nuda. The small creeping Creloplana and Ctenoplana, are supposed by some to form a transition to the Turbellaria.

\section{Summary of Important Facts.}

I. The CELENTERATA and Echinoderma were formerly called Radiata because in most a radial structure is present; in the higher groups this is replaced by biradial or even bilateral symmetry.

2. The Colenterata are sometimes called Zoophyta (animal plants), from their appearance and their attachment. In many the resemblance is heightened by their formation of plant-like colonies by fission and budding.

3. The name Coelenterata was chosen because they have but one cavity, a simple or ramified digestive sac, representing at once the alimentary tract and the as yet undifferentiated body cavity.

4. This colenteric cavity is called the gastrovascular system because its branches distribute nourishment to all parts and so perform the function of blood vessels.

5. The reproduction is either sexual or asexual, very frequently cyclical (alternation of generations).

6. The animals are provided with nerves, muscles, and sense organs and possess marked sensibility and mobility.

7. Especially characteristic are the tentacles and small nettling organs, the cnidis, in special cells.

8. Nearly all histological differentiation proceeds from ectoderm or entoderm, since the mesoderm (mesogloea) plays but a subordinate rôle and usually functions only as a support (Diploblastica).

9. Four classes-Hydrozoa, Scyphozoa, Anthozoa, and Ctenophora are recognized.

Io. In Hydrozot and Scypiozos there are usually two alternating generations, the sessile asexual polyp and the free-swimming sexual medusa.

I I. The hydroid polyp and the craspedote medusa are characteristic of the HYDrozos.

I2. The hydroid polyp is a two-layered sac of ectoderm and entoderm, 
a supporting layer and a circle of tentacles. In the colonial forms there is usually a cuticular envelope, the perisarc, secreted by the ectoderm.

I3. The craspedote medusa is bell-shaped, with smooth bell margin, its aperture partially closed by a diaphragm-like relum; the gonads are ectodermal.

I4. The medusx arise by lateral budding from the hydroid.

I5. If the medusa remain attached to the parent as a sporosac, alternation of generations is replaced by polymorphism; it can entirely disappear with the total loss of either hydroid or medusa.

16. The scyphostoma and the acraspedote medusa are typical of the SCYPHOZOA.

I 7. The scyphostoma differs markedly from the hydroid polyp in the presence of four longitudinal gastric folds or septa (taniolx).

IS. The acraspedote medusa lacks a velum, has a lobed umbrella edge, gastral tentacles (phacella), and entodermal gonads.

I9. The medusa arises from the polyp by terminal budding (strobilation).

20. Alternation of generations rarely is lost, and then only by suppression of the scyphostoma.

21. The Anthozod have only one form, the coral polyp; it is distinguished from the hydroid polyp by the ectodermal osophagus, the radial septa reaching the œesophagus; the well-developed mesoglœa and the gonads which, arising from the entoderm, early migrate into the mesoglœa.

22. Most Anthozoa are colonial and produce a skeleton (coral) usually of calcic carbonate, but sometimes of 'horny' substance.

23. The skeleton may be either axial or it may be outside the individual polyps (cortical skeleton).

24. The living Anthozoa are divided according to the number of septa into Octocoralla and Hexacoralla. With the latter the fossil Tetracoralla are allied.

25. The Hexacoralla have numerous tubular tentacles and six, or a multiple of six, pairs of septa.

26. The Octocoralla have eight single septa and eight feathered tentacles.

27. The Ctenophora are always free-swimming and have a large mesoderm with numerous muscle cells.

28. Nettle cells are absent, and are replaced by adhesive cells.

29. Most characteristic are the eight meridional rows of 'combs' whose motions are controlled by a common organ, the sense body, a statocyst. 
3o. The digestive tract consists of an ectodermal oesophagus (stomach) and a branching system of entodermal ressels.

\section{VERMES.}

A large number of forms above the Colenterates are frequently grouped as Vermes, but there is little agreement as to what smaller divisions shall be included, some denying the existence of a natural group of worms, and separating the groups as phyla. Others include not only the flat-, round- and segmented worms, the Chatognaths and rotifers, but also the brachiopods, I'olyzoa and even the tunicates. Set such is the variety of form, structure and development that, no matter what limitation be accepted, it is impossible to frame a definition which shall include all of the species commonly known as worms. In taking the step from the diploblastic Colenterates to even the lowest 'worms' such adrances in structure are seen that a brief review of these is appropriate here.

The worms are distinguished from the Coelenterates by bilaterality, which is seen in the internal structure, in even those cases (round worms) where it is not visible on the exterior. 'There is also a higher degree of differentiation of organs - the development of a ganglionic nervous system, excretory organs, and frequently of a blood-rascular system. This advance is correlated with the appearance of a true mesoderm, the layer from which (the nervous system excepted) these organs and the muscles arise. Then there is the dermo-miscular $s a c$, the cause of the familiar 'worm-like' motions. This consists of an intimate connexion of the skin with the underlying muscles (figs. 2 I 2, 240, 24I). The skin, a one-layered epithelium, ciliated or covered with a cuticle, rests on eithes a structureless membrana propria or on a cellular connective tissue, to which the muscles are attached. Longitudinal muscles are always present, and frequently circular as well, while in the parenchymatous worms diagonal, crossed. and dorsoventral fibres may occur.

While certain worms (cestodes) lack an alimentary canal, or like many nematodes, may have a blind, functionless gut, these conditions are the result of parasitic habits. In the lower worms the digestive tract is like that of the Colenterates, consisting of archenteron and stomodeum, proctodeum and anus being absent. In most worms the tract is 'complete,' it being a tube with mouth and rent.

The digestive tract is either imbedded in the parenchyma and cannot be dissected out (fig. 212), or it is surrounded by the body carity (colom) which separates it from the body wall (figs. $238,2.4 \mathrm{I}$ ), the muscles of which are mesothelial in origin, in contrast to the mesenchymatous muscles of the flat worms. In the flat worms the excretory organs are protonephridia, and similar organs with solenocytes (p. I05) are common in the larva of the higher worms, being often replaced in the adult by true nephridia.

Here, too, a blood-rascular system first appears. Where it is lacking (flat worms) its place may be taken by the branches of the digestive tract, or in higher forms by the coelom with its albuminous fluids. The phylogenetic origin of the circulatory system may be considered here. Two explanations of the circulatory vessels have been advanced. (I) They are canals separated from the alimentary canal and developed farther independently; therefore they have an entodermal epithelium. (2) They are spaces filled with albuminous fluid, arising between entoderm and mesoderm (a modified form of this is that they are remnants of the segmentation cavity); hence at first they had no epithelium and obtained it later from the mesoderm. Lately the latter view is regarded as the more probable, since most olservers deny the existence of an epithelium in the blood vessels of worms and other invertebrates. Then in many worms 
a perigastric sinus, arising by a separation of the intestinal layers, forms a part of the circulatory system.

The nervous system of the 'worms' has in common a pair of supra-œsophageal ganglia ('brain') which sends out two strong longitudinal cords (to which others may be added) which must be regarded as part of the central nervous system since they bear ganglion cells. 'These cords may be lateral or on either side of the mid-ventral line. In the latter case those of the two sides may be united at regular intervals, thus giving the ladder type (p. I I 3 ), the ventral chain being connected with the brain by cords on either side of the nesophagus. This nervous system, always ectodermal in origin, may be epithelial, forming part of the skin, or it may sink to different depths in the other tissues.

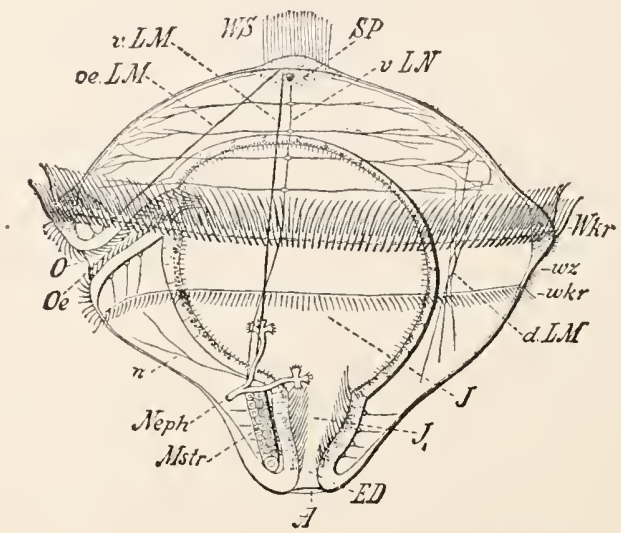

FIG. 2II.-Trochophore (Loven's larva) of Polygordius (from Hatschek). $A$. anus; $d L M$, dorsal muscles; $E D$, hind gut; $\mathrm{J}$, stomach; $\mathrm{J}_{1}$, intestine; 1 str , mesodermal band; $n$, nerves; $N e p h$, protonephridia; $O$, mouth; Oe, œsophagus; oeL $M$, œsophageal muscle; $S P$, apical plate; $v L M$, ventral muscle; $v \cdot L N$, lateral nerve; $W k r$, $w k r$, pre- and post-oral zones of cilia; $I V S$, apical cilia; wz, adoral cilia.

A peculiar type of larva appears in various groups of invertebrates, being recognizable in modified forms in echinoderms and molluscs as well as in worms. This is the trochophore (fig. $2 \mathrm{II}$ ) a gelatinous ball traversed by fore, mid and hind gut. At first the body is uniformly ciliated, but the cilia are later restricted to definite tracts. One of these ciliated bands is pretty constant, passing around the body in front of the mouth, thus marking off an apical region, in the centre of which is an epithelial thickening, the anlage of the brain, often marked by a bundle of cilia. Besides mesenchymatous muscle fibres, one or more pairs of protonephridia may be present. In the corresponding larva of many of the flat worms (the protrochula, fig. 216 ) hind gut and protonephridia are lacking.

\section{PHYLUM IV. PLATHELMINTHES (PLATODES, Flatworms).}

This group is well characterized by the names. With few exceptions (rhabdocœles, many trematodes) the nearly flat ventral surface and the slightly arched back pass with a more or less sharp margin into each other (fig. 2 I2). In many cases the ventral surface is distinguished by its lighter color. In all the bilaterally symmetrical body, without coelom, 
is composed of a solid parenchyma, a mass of connective tissue traversed by muscle fibres, in which the various organs-alimentary tract, nerves, excretory and reproductive organs-are imbedded. The digestive

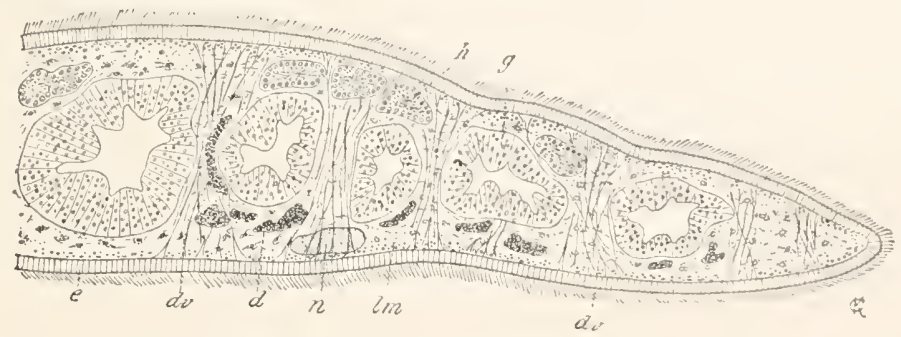

FIG. 2 I2.- Transverse section (right half) of a Planarian. d, vitellaria; $d v$, dorsoventral muscle fibres; $e$, ectodermal epithelium with cilia; $g$, gastric diverticula; $h$, testicular follicles; $l m$, longitudinal muscles (dots, in section); $n$, lateral nerve cord.

system has various shapes; in the lower groups it resembles that of the coelenterates in that there is but a single opening and this leads by an ectodermal œsophagus (stomodxum) to the interior (fig. 59). In

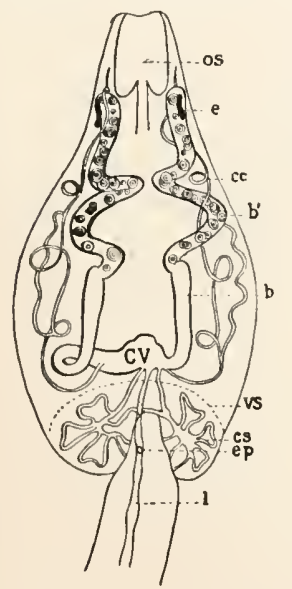

FIG. $2 I_{3}$. parasites the digestive tract may be lost. The skin is a one-layered epithelium, sometimes ciliated, sometimes protected by a thick cuticula. Inside this comes a muscular layer (fig. $2 \mathrm{I}_{2}$ ) in which longitudinal muscles are always present, and in addition frequently circular and oblique muscles, as well as those passing from dorsal to
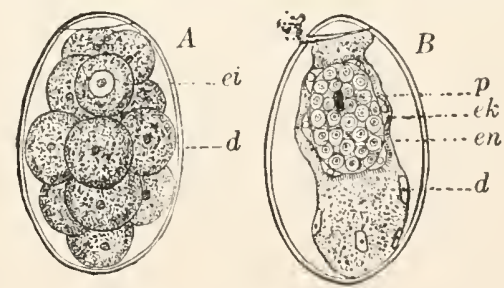

FIG. 2I. .

Fig. 2 I3.-Excretory system of Cercaria (after Albert Lang). b, limb of blacleler; $b^{\prime}$, same with urinary concretions; $c c$, collecting canal; $c s$, canals of ventral sucker; $c z^{\prime}$, collecting vacuole; $e$, eye; $e p$, excretory pore; $l$, lumen of tail; os, oral sucker; $v$, ventral sucker.

FIG. 2 14.-Eggs of Distomum nodulosum (after Schauinsland). A, before development; $B$, later, the yolk broken down. $d$, yolk cells; ei, egg cell; $e k$, ectorlerm; $e n$, entoderm; $p$, pigment spot.

ventral surfaces. The nervous system (fig. 215) consists of a pair of ganglia ('brain') in front of (i.e., above) the sesophagus, and longitudinal nerves leading backwards from it. The excretory organs (fig. 2I3) are 
composed' of a series of tubes, the protonephridia or 'water-vascular system,' which branch and ramify the parenchyma, and open to the exterior by one or more openings variously arranged. Most flatworms are hermaphroditic and the reproductive organs take up considerable space. There is a small paired or unpaired ovary and vitellaria, usually paired and branched. The eggs arise in the ovary, and to these are added nourishment in the shape of cells (abortive ova, 'yolk cells') rich in yolk from the vitellaria. At the point where oviducts and yolk ducts join, a single egg cell and several yolk cells are united into an oval body -the compound egg-protected by a shell, formed by the 'yolk cells,' which also have to do with the nutrition of the embryo. Of the 'compound egg' only the egg cell takes a direct part in the formation of the embryo and is the true orum.

The Platodes are usually divided into four classes. Of these the Turbellaria are the most primitive, and the others have come from them. The Trematodes and Cestodes have heen altered by parasitism, resulting in more or less degeneration. By some the Nemertini are not regarded as allied to the other flatworms, but as nearer the Annelids.

\section{Class I. Turbellaria.}

The Turbellaria are small, only a few being measured by inches, while many are almost microscopic in size. The name Turbellaria refers to the currents produced by the ciliated ectoderm which covers the body (fig. 59), and which serves at once for motion and for respiration. Most

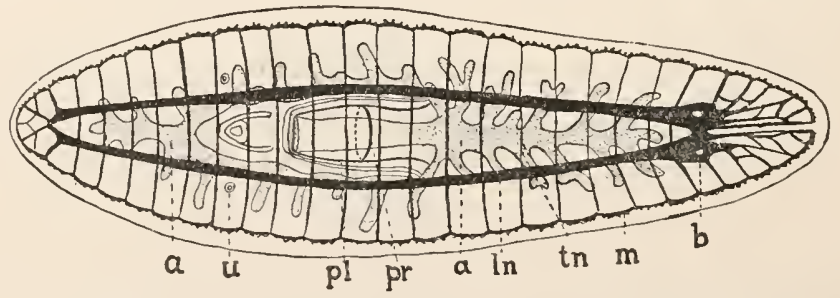

FIG. 2I5.-Digestive and nervous systems of Syncolidium pellucidum* (after Wheeler). $a$, alimentary tract; $b$, brain; lin, longitudinal (ventral) nerves; $m$, marginal nerve; $p l$, longitudinal nerve of pharynx; $p r$, ring nerve of pharynx; $t n$, transverse nerve; $u$, uterine ostium.

species are aquatic (fresh water or marine), only a few land planarians living in moist earth. In the water they either creep slowly over stones or plants, or they swim freely. The larger species swim by undulations of the body, the smaller by means of the cilia. Several are ecto- or entoparasites and often show adaptations to their life in the degeneration of 
part of the digestive tract (Fecampia) or by the development of anchoring structures. Appearance, ciliation, etc, often give the smaller turlsellaria a resemblance to the ciliate Protozoa, so that the beginner is often confused between them.

The alimentary canal (figs. 59, 215) consists only of osophasus (pharynx) and mesenteron, the latter terminating blindly since no anus is present. The mouth is on the lower surface, at some distance from the anterior end, being occasionally in the middle or even behind the middle of the body. It learls into the muscular oesophagus, which frequently can be protruded like a proboscis. The mesenteron, of entodermal origin, varies greatly in shape, its modifications being made the basis of division of the class into orders. In the Polycladidea there is a central portion from which numerous branched carca are given off; in the Tricladidea (fig. 2 I 5) there are three main trunks, each with lateral cacal diverticula; while in the Rhabdocolida the digestive tract is a simple rod-like sac, in some cases (Acola) without internal cavity. The supracesophageal ganglia always lie at the anterior end of the body, which is

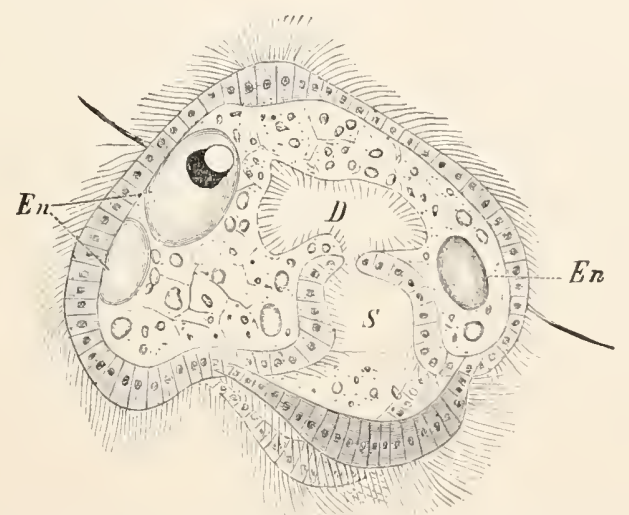

FIG. 216.-Larva of Stylochus pilidium (from Korschelt-Heider, after (sötte). I), enteron; En, remains of entoderm cells; $S$, asophagus.

most sensitive and may be producerl into feeler-like processes. 'This region usually bears two or more simple eyes, and in a few a single statocyst. Most of the turbellaria have numerous rhabdites and rhamnites, rod-like structures of varying shape and structure, in the skin. The nettle cells found in the skin of many species (Microstomirlic) are derived from the hydroids eaten.

The hermaphroditic sexual organs (fig. 75) and the excretory system vary considerably. The eggs are usually large and are fastened by a stalk 
to water plants. Many species form a sort of cocoon, containing a few eggs and numerous yolk cells. There are a few viviparous forms (summer generation of Mesostomidx). In the marine species a free-swimming larva (protrochula, fig. $2 \mathrm{I} 6$ ), with lobe-like processes may hatch from the egg. This larva, by a metamorphosis, is converted into the creeping adult. Not infrequently besides the sexual, asexual reproduction occurs. The Microstomide and some Planaria are capable of transverse division, and will form chains of individuals. For each posterior individual a new brain and a new œesophagus are formed (fig. 59). The Turbellaria possess a marked power of reproducing lost parts, making them favorites for regeneration experiments.

In a few Turbellaria the pharynx connects with a solid protoplasmic mass, in which, as in the protoplasm of a protozoan, the food is digested. This entoderm is hardly marked off from the mesoderm, but it is a question whether these 'Acœla' are primitive or degenerate, the latter being the more probable.

\section{Order I. Polycladidea.}

Marine species of considerable size, digestive cxeca springing from a central chamber. Leptopland,* Stylochus; * Thysanozoon, Europe.

\section{Order II. Tricladidea.}

Alimentary canal with an anterior unpaired and a pair of posterior branches, arising from the pharynx, and bearing lateral cæca. Marine, Bdelloura* and Syncolidinm* (fig. 215) (parasitic on Limulus), Gunda,* Polycharus; * freshwater, Dendrocahm* (fig. 64), Planaria,* and Polyscelis,* Phagocata* with divided pharynx. The tropical land planarians (Bipalium,* ${ }^{*}$ Io or I2 inches. long) have been introduced into greenhouses.

\section{Order III. Rhabdocœlida.}

Small, even microscopic, recalling in habits and appearance the Infusoria; alimentary canal rod-like, without branches. Vortex* (fig. 75), fresh water; Monops,* Monoscelis,* marine. The fresh-water Microstomide reproduce almost exclusively by fission.

\section{Class II. Trematoda.}

These are exclusively parasitic, some living on the skin or gills (ectoparasites) or in the interior of other animals (entoparasites). In structure they are closest to the triclad Turbellaria, from which they differ by characters, the direct result of their parasitic life. Thus they have lost the cilia or have them only in the larva. On the other hand, they are covered with a cuticle often with spines and with suckers and hooks for adhesion to the host. The suckers are shallow pits of columnar epithelium lined with cuticle and furnished with a thick layer of radial and circular muscles, which by their contraction increase the lumen of the sucker, the edges of which are closely applied to the host. At least one such sucker 
is present; if but one or two (entoparasites), one is at the anterior end (oral sucker) surrounding the mouth, while a second larger sucker may occur near the mouth (fig. $2 \mathrm{I}_{7}$ ), but may be ( 1 mphistomm) at the posterior end. In the ectoparasites there are a pair of anterior suckers ncar the mouth; at the posterior end a single sucker, or a number of suckers or hooks or both on a sucking disc (fig. 2 I9).

Other results of parasitism are the weak development of sense organs and brain and development of accessory ganglia near the adhesive organs. Eye spots (two to four) occur occasionally in the ectoparasitic species and in the larve of the entoparasitic, rarely in their adult condition. The alimentary tract is forked (fig. 2IS) and occasionally (fig. 2I 7 ) has dendritic blind sacs.

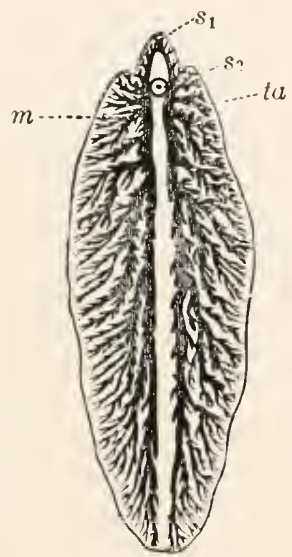

Fig. 217 .

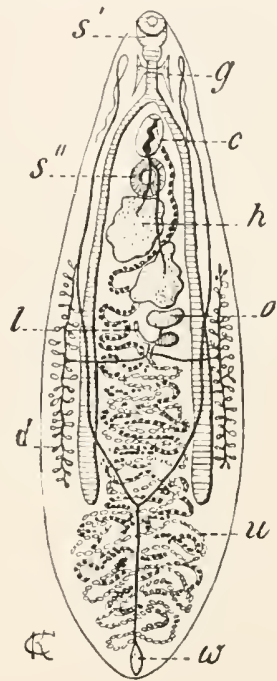

FIG. 2 IS.

FIG. 217.-Distomum hepaticum, liver fluke (from Boas). $m$, creca of $t u$, limbs of digestive tract; $s_{1} s_{2}$, anterior and posterior suckers.

FiG. 218.-Distomum lanceolatum. c cirrus, beneath it the opening of the oviduct; $d$, vitellaria, the ducts leading to the shell gland; $g$, ganglion; $h$, testes with clucts to cirrus; $l$, Laurer's canal; $o$, ovary, the shell gland behind it; $s^{\prime}, s^{\prime \prime}$, anterior and median suckers, the pharynx and the bifurcated digestive tract leading from $s^{\prime} ; u$, uterus; $w$, terminal vesicle of water-vascular (excretory) system.

To parasitism may also be attributed the great development of the sexual organs, which at maturity fill a great part of the body. Their features may be seen in fig. $2 \mathrm{I} 8$. Two vasa deferentia pass forward from the testes $(h)$, unite and form a seminal vesicle. The terminal portion of the united ducts can be protruded as a penis or cirrus $(c)$ armed with retrorse hooks. It is usually enclosed in a 'cirrus pouch.' 'The umpaired ovary $(0)$ is very small and produces small eggs, deficient in yolk; hence the paired vitellaria $(d)$ are well developed. The united ducts from these join the oviduct, producing the uterus (u), which receives the eggs, is much convoluted, and empties beside (in some 
species in a common antrum with) the male sexual opening. The first part of the uterus is called the ootype because here the eggs and yolk cells are formed juto eggs (fig. 2I4) and enclosed in a she!l with a lid or cover formed by the secretion of the yolk cells. A second duct-Laurer's canal-goes from the

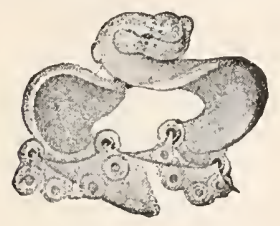
ootype to the dorsal surface. In the Distomes the canal is rudimentary or lacking. It apparently corresponds to vagina of the Polystomes, used in copulation. On the other hand, it may be homologous with the vitello-intestinal cana! which connects intestine and vitelline duct. In the Distomes ropulation occurs in the uterus, leading to self-impregnation.

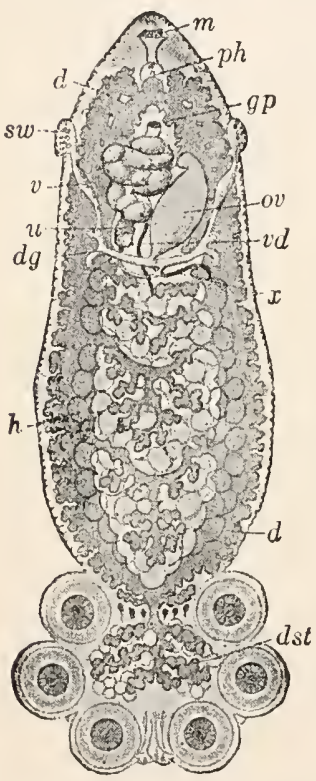

FIG. 2 I9.-Polystomum integerrimum (after Zeller). Above two individuals in copulation; below a single animal eslarred. $d$, digestive tract, distended with blood; $d g$, yolk duct; dst, vitellarium; $g p$, genital pore; $h$, testicular vesicles; $m$, mouth, ph, pharynx, ov', ovary; sw, openings of the paired vaginx; $u$, uterus: $\imath$, vagine; $z$, vas deferens; $x$, vitello-intestinal canal.
The Trematodes fall into two great groups, the Polystomex, largely ectoparasites, and the Distomex, exclusively entoparasitic, the distinctions in parasitism being correlated with differences in structure and development.

\section{Order I. Polystomeæ (Monogenea, Heterocotylea).}

Most Polystomes live on aquatic animals-usually fish, where they attach themsclves to the gills. Since they are exposed to more dangers, their adhesive organs are stronger than in the entoparasites. So while the anterior suckers are weakly developed or absent, the hinder end bears sometimes only a single sucker, but usually a large adhesive disc armed with many suckers and hooks (fig. 2I9). The transfer of Polystomes from one host to another is easy and the life history is without complications. The stalked eggs are attached near the mother and produce larve, which soon after hatching have the adult form (hence the name Monogenea).

Gyrodactylus, parasitic on the gills of the carp, brings forth living young which, even before kirth, produce a new generation in their interior, and these may contain a thind generation. More striking is Diplozoon paradoxum, in which, at the time of sexual maturity, two individuals become fused like Siamese twins (fig. IIo). The young, called Diporpa, escape from the eggs and only unite later. Each has a ventral sucker and a dorsal papilla. Each of the pair seizes the papilla of the other with the sucker, and then the two grow together so that the male opening of one comes opposite the ferale opening of the other. Polystomum integerrimum of the frog (fig. 219 ) affords a transition to en`oparasitism. At first it lives on gills of the tadpole, kut at the time of metamorphosis it is forced to leave this place and to pass, by way of the alimentary caral, to the urinary bladder. The monogenetic AspidoCOTYLID $£$ are internal parasites in turtles, fishes and molluscs. The TEMNOCEPHALID $£$ of warmer regions are parasitic on crustacea, molluscs, and turtles. American genera of Polystomeæ are Epibdella, Polystomum, Tristoma, Sphyranura, Microcotyle. 


\section{Order II. Distomeæ (Digenea).}

The entoparasitic Trematodes usually occur as sexual animals in the digestive tract and its appendages; more rarely in blood-vessels, urogenital

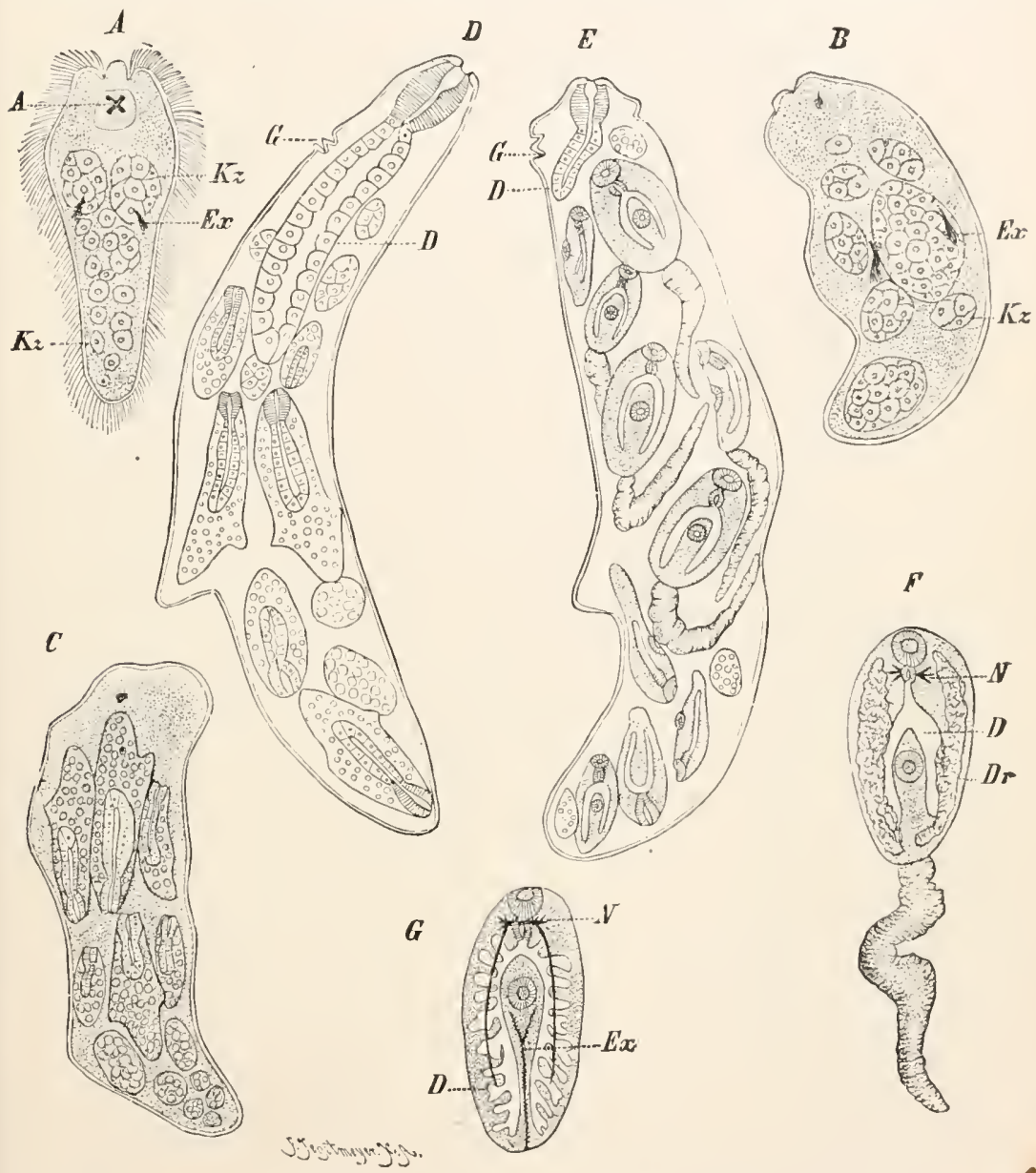

FIG. 220.-Development of Distomum hepaticum (from Korschelt-Heider after Leuckart). A, young larva; $B$. sporocyst from the lung of Limnca; $C$, older sporocyst with redix; $D$, redia which has produced redix internally; $E$, redia with cercarix; $F$, cercaria; $G$, encysted Distomum. A, eye spot; $D$, digestive tract; $D r$, glands; $E x$, ciliated lobules and main trunks of excretory system; $G$, birth opening; $K z$, germ cells; $N$, nervous system.

organs, and cœlom of vertebrates. As inhabitants of the dark they have, with few exceptions, lost the eyes, which appear in larval life, and not always then. Since not exposed to danger of being pulled from the host, 
they possess either the oral sucker alone (Monostomum) or this and a second ventral sucker, and only rarely other attaching apparatus. They are markedly separated from the Polystomes by their life history. The alternation of hosts necessitated by the endoparasitic life is complicated by an alternation of generations (heterogony, p. I32) with metamorphosis, hence 'Digenea.' To illustrate this the history of Distomum hepaticum, the liver fluke of the sheep is chosen (fig. 220).

The eggs leave the maternal uterus at the beginning of embryonic development, pass down the bile ducts and thence by the intestine to the exterior. They must come into water and remain here a while before the ciliated larva (miracidium, $A$ ) escapes by lifting of the lid of the shell. This larva bores its way into a small snail (sp. of Limnaa), where it grows into a sporocyst $(B)$. The sporocyst, a muscular sac with protonephridia but lacking all other organs, produces in its interior eggs which develop into a second reproductive sac, the redia $(D)$. These are distinguished from the sporocysts by the possession of pharynx and a tubular

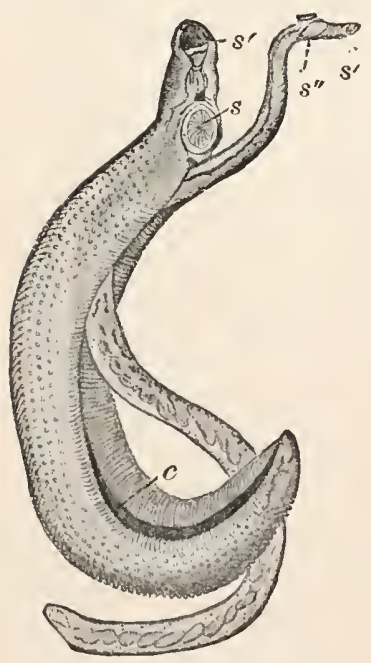

FIG. 22x.-Bilharzia hamatobia. Female in the gynæcophoral canal $(c)$ of the male; $s^{\prime}, s^{\prime \prime}$, anterior and posterior suckers. intestine as well as a birth-opening for the escape of the young produced inside. According to the season these young are either cercarice $(F)$, or another generation of rediæ may follow before the cercarix appear. The cercarix are adapted for an aquatic life, since each has, besides the characteristic organs of a Distomum (genitalia excepted), a strongly vibratile tail. The cercarix escape from the snail, swim about in the water until the tail drops off, when they encyst on water plants. When these encysted young are eaten by sheep along with the vegetation, infection follows.

In general it can only be said of the life history of other Trematoda that the miracidia must penetrate a mollusc, and that the different species have many modifications. Best known are the following: Distomum (Fasciolaria) hepaticum, the liver fluke (fig. 2 $\mathrm{I}_{7}$ ), about the size and shape of a pumpkin-seed, lives in the bile-ducts of sheep, cows, pigs, etc., and rarely of man. It causes a disease known as 'liver rot,' generally resulting in death. This history shows why sheep pastured in moist places are subject to the disease, and why wet seasons are times of epidemics. Thus in the rainy year of $18_{30}$ about one and a half miliions of sheep were killed in England; in I $8_{12}$, 300,000 in the neighborhood of Arles, France. It is frequently accompanied by $D$. lanceolatum, less than half an inch in length (fig. $2 \mathrm{I} 8$ ). 
Bilharia hamatobia is a human parasite, most common in hot climates, especially among the Fellahin of Egypt. The sexes are separate. The male, half an inch long, by inrolling of the rentral sicle (fig. $22 \mathrm{I}$ ) forms a groove in which the more slender female usually lies. 'These united worms occur in the portal vein and connected ressels, which they follow and lay their eggs in the mucous membrane of the ureters and urinary bladder, as well as in liver and intestine. Several other species occur in man, among them $D$. carnosum ${ }^{*}$ and $D$. westermanni*. Amphistomm is common in the intestine of Ungulates, one species, 1. hominis, occurring in man. With few exceptions the adult Distomes occur in rertebrates, the larval stares in molluscs. Aquatic birds are very apt to be infested with them.

\section{Class III. Cestoda (Tapeworms).}

The majority of the cestodes, and especially those of the human intestine, are sharply distinguished from the entoparasitic trematodes. But the boundaries disappear in forms like Archigetes, Caryophyllaus, and Amplilina, parasitic in lower vertebrates or invertebrates which are now assigned to the trematodes, now to the cestodes. The most important character of the cestorles is that as a result of their parasitic life they have lost the last traces of an alimentary canal, and are nourished by the juices or the partially digested food of the host, since the fluid nourishment is taken in through the skin. Two other characters are striking: (I) The differentiation of two developmental stages, the bladder worm, or cysticercus, living chiefly in solid organs (muscles, liver, brain), and the sexually mature animal, living in the alimentary tract; (2) the division of the body of the adult into the head or scolex, and following this a series of joints or proglottids. Since this last feature holds for

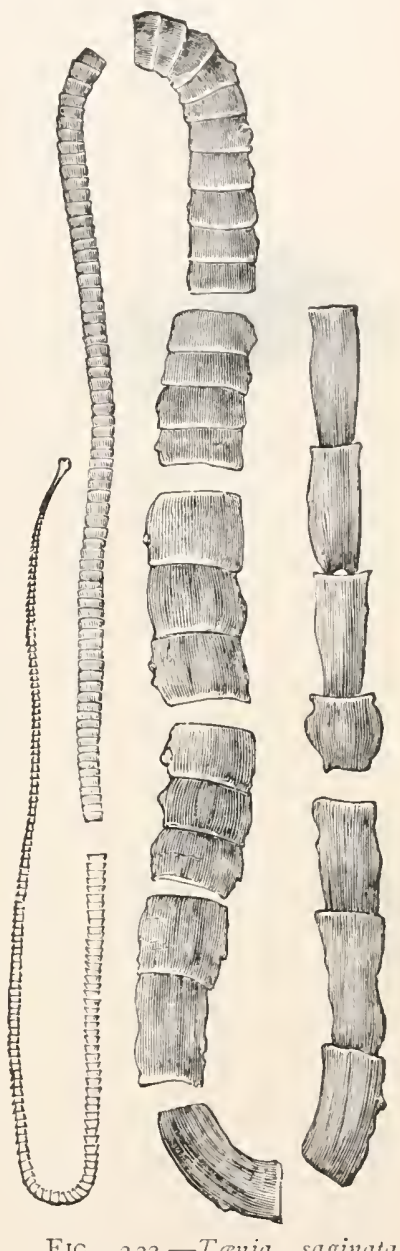

FIG. 222.-Tania saginata (from Boas, after Leuckart). Head with series of proglottids taken from various regions of the strobila. all human tapeworms, the best known species, the following description begins with these typical forms.

The sexually mature tapeworm or strobila (fig. 222) consists of a scolex in front, and behind this follow in a row the proglottids. The 
number of these varies from smaller forms (Tania echinococcus, fig. 232) with three or four to hundreds or even several thousands, a fact which speaks for the enormous size of some species. The proglottids arise from the hinder end of the scolex, by a kind of budding. This explains the well-known fact that one is not rid of the tapeworm, so long as the head remains in the host. It also explains the peculiar shape which is almost thread-like in front, increasing posteriorly to a broad band, whence the common name. At first the proglottids are small; they increase with age

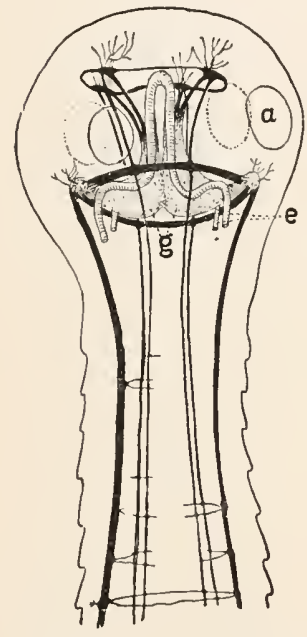

FIG. 223. - Nervous system of Moncria (after Tower). $a$, suckers; $e$, excretory tubes; $g$, cerebral ganglia. Nerves black. to considerable size, and separate from the hinder end of the chain and live independently when a certain development is reached. For example, the young proglottids of the human tapeworm, Tania solium, are $0.5 \mathrm{~mm}$. broad and $0.0 \mathrm{I} \mathrm{mm}$. long; the ripe proglottids are $5 \mathrm{~mm}$. broad and I $2 \mathrm{~mm}$. (half an inch) long.

Head and proglottids have certain common characters. Their connective-tissue parenchyma consists of cortical and medullary substance. The first contains most of the muscles, the latter the other organs. Nerves and water-vascular system extend the whole length of the worm. In the head is the paired cerebral ganclion (fig. 223), sometimes fused to a single mass by the great development of the commissure. From the brain two principal nerves run backwards, usually near the edge of the proglottids (fig. $228, N$ ). The water-vascular system begins with a capillary network richly provided with flame cells. It extends through head and proglottids; usually four main trunks are present, two being less developed and sometimes absent. The two chief trunks are frequently connected by a cross-trunk on the hinder margin of each proglottid (fig. 228). The system opens on the posterior edge of the last proglottid, but accessory mouths may occur on other proglottids.

Scolex and proglottids differ in that the proglottids contain the sexual organs, while the scolex bears the anchoring apparatus, for the latter has, besides producing proglottids, to fasten the worm in the intestines. Most important of the adhesive organs are the suckers (acetabula); less important are the numerous hooks, either arranged in a circle or borne on protrusible and retractile probosces (figs. 224-226). 
When a circle of hooks is present it is on the anterior end and is moved by a special apparatus, the rostellum, a plug of complexly arranged muscles (fig. 226) which can arch and flatten the central area. Each hook has its point outwards and its base with two roots, one of which rests on the rostellum; the protrusion of the rostellum forces the points outwards into the mucous membrane of the intestine. In some Tonia without hooks ( $T$. saginata) the rostellum is replaced by a sucker-like depression. Since the rostellum arises in development from a similar cup, it may be a modified apical sucker, but it is loubtful how far comparisons may be made with the oral sucker of the trematodes.

The animals are almost always hermaphroditic and the gonads are equal in numbers to the proglottids, so that these were formerly regarled as sexual individuals of a colony, each with its own reproductive apparatus. Two types must be recognized. In the one the presence of vitellaria and the separate openings of uterus and vagina recall the conditions in trematodes, while in the second the uterus ends blindly and the vitellaria are mudified into a small albumen gland. Since vagina and vas deferens almost always open together, self-jmpregnation is possible, but crossfertilization of separate proglotticls has been seen. The general features of the two types may be made out from figures 227 and 228 .

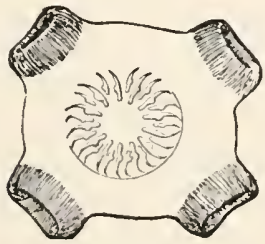

FIG. 224 .

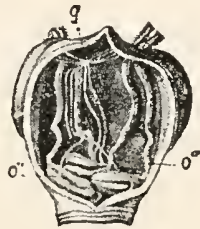

Fig. 225 .

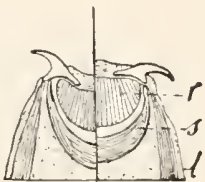

FIG. 226 .

FiG. 224.-Apical view of head of Tania solium (from Hatschek).

Fig. 225.-Head of Tetrarhynchus viridis (after Wagner). Dissected to show the internal parts of the proboscides $(o)$ and the ganglion $(g)$.

Fig. 226.- Schema of action of rostellum. On the right the hooks are exserted for adhesion, on the left retracted. $r$, rostellum; $s$, sheath; $l$, longitudinal muscles.

In the Bothriocephalidx numerous testes (fig. $227, h$ ) are scattered through the parenchyma. The small vasa deferentia unite repeatedly to a chief canal which opens in the middle line, near the anterior margin of the proglottid, the terminal portion, the cirrus being retractile into a cirrus pouch $(c b)$. The ovary is two-lobed $\left(o z^{\prime}\right)$ and is near the posterior margin of the proglotid. It forms eggs poor in yolk. The scattered vitellaria are voluminous. The unpaired oviduct and the vitelline duct unite to form the shell gland, in which each egg unites with a number of 'yolk cells' to form a compound egg (see p. 240). 'T he uterus (u) leads from the gland to the exterior. There is also a vagina (va) leading from the oviduct to the exterior. In distinction to the Trematodes, the vagina empties with the uterus apart from the male organs. The general differences in the Tæniida may readily be made out from fig. 228 , the chief points being the replacement of the vitellaria by an albumen gland, the blind uterus, and the termination of male and female ducts (vagina) at the side of the proglottid. 


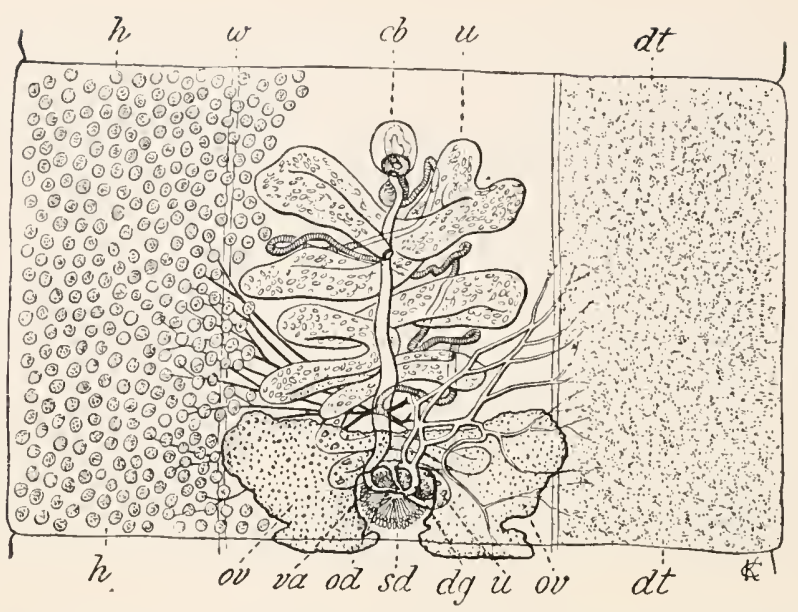

FIG. 227.--Proglottid of Bothriocephalus latus (after Sommer). Right only vitellarium, left only testes, shown. $c b$, cirrus sheath opening with the vagina; $d g$, vitelline duct: $d t$, vitellarium; $h$, testes; od, oviduct; $o v$, ovary; $s t$, shell gland; $u$, uterus; $v a$, vagina; $z^{\prime} d$, vas deferens (dark-lined); $w$, excretory canal.

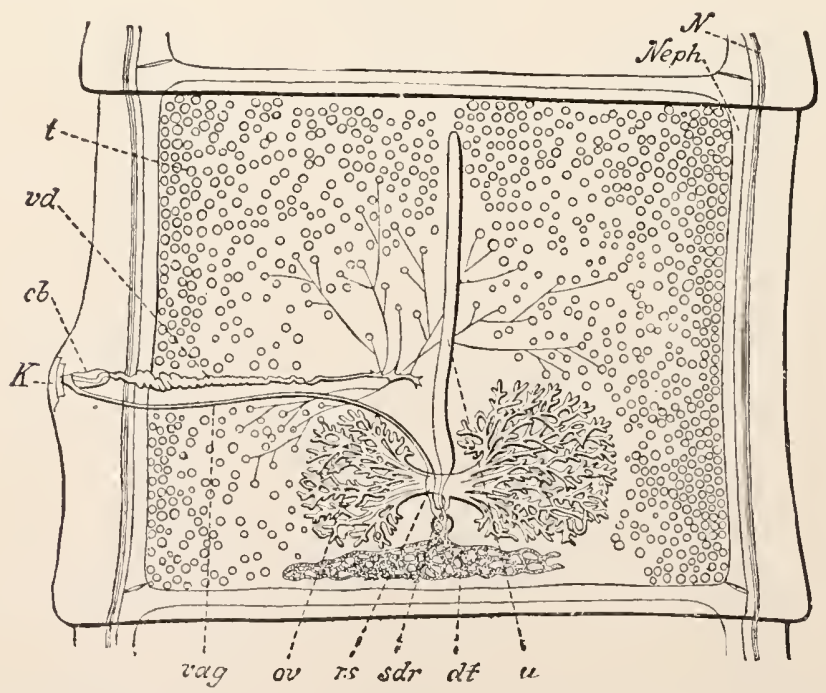

FIG. 228.--Proglottid of Tenia saginata, near maturity (after Sommer). cb, cirrus sheath; $d t$, vitellarium; $k$, genital pore; $\lambda$, nerve cord; $N^{\prime} p h$, excretory canal; $o z$, ovary; $r s$, receptaculum seminis; $s d r$, shell gland; $t$, testes; $u$, uterus; $v d$, vas deferens. 
The differences in the sexual apparatus influence the peculiarities of the egg. In Bothriocephalus it is large, has a tough shell with a lid, and encloses a small egg cell with numerous yolk cells. The eggs of Tienia are small, with a layer of albumen and a delicate shell which is lost early. It is replaced by a radially striped envelope secreted by the embryo in a somewhat advanced stage. It is in this condition that the eggs escape. A further consequence is a difference in development. In most Bothriocephalidx, as in the Trematoda, the egg must enter the water for its further development. Here a ciliated oval larva escapes which contains a six-hooked larva (oncosplucera, fig. 229). 'The ciliated envelope is temporary and is cast off like the ciliated coat of the trematode larva. The six-hooked larva enters a fish, becomes encysted (pleurocercoid) in muscles or viscera, and changes directly into the head of a Bothrioceplualus. This on being taken with food into the intestine of the proper host develops into the adult.

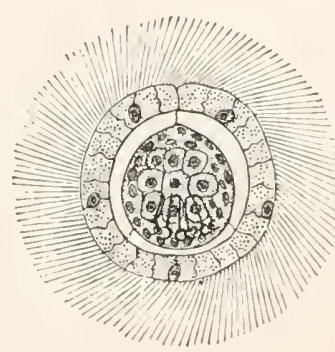

FIG. 229.-Ciliated embryo of Bothriocephalus latus enclosing the six-hooked larva.

The history of the Tenias differs considerably. The distinctions are early recognizable, since the six-hooked larva lacks the ciliated coat but is enclosed in its homologue, the envelope already alluded to. Since this cannot open of itself, the young are set free by its digestion in the stomach. Thus the eggs of Tania solium must pass into the stomach of the pig (embryos in frecal matter get mixed in the food) and after being freed from their shell in the stomach, the larve with their six hooks bore through the intestinal wall and migrate, using the blood-vessels in their course, into the muscles, or more rarely other organs. Here they develop into bladder worms (cysticerci), becoming oval and secreting a cyst to which the pig adds an envelope of connective tissue. The cysticercus grows by increase of cells, and by the infiltration of serous fluid, so that it becomes distended into a delicate translucent vesicle. In T. solium the microscopically small embryo can grow in three or four months to the size of a bean or pea; in some species as large as a hen's egg. By invagination the wall of the bladder produces the anlage of the scolex (fig. 230, c). This is at first sac-like but soon increases in length, its growth being confined by an envelope $(d)$, so that it is bent. The scolex appears like a white swelling through the wall of the blarkler.

At the apex of this blind sac arises the characteristic armature of the scolex which makes it possible to say what tapeworm will come from the cysticercus. Thus in $T$. solinm there are four suckers and a crown of 
hooks. These parts are at first inverted and only come to their definitive position on the outside of the scolex when the latter is protruded as one would turn out the finger of a glove. Rarely is the scolex protruded at this time (cysticercus of the mouse liver) and begins the formation of proglottids. Even in this case they do not become sexually mature until in the intestine of the cat.

The further development follows when the cysticercus is taken into the stomach of the new host When man, for instance, eats infected ('measly')
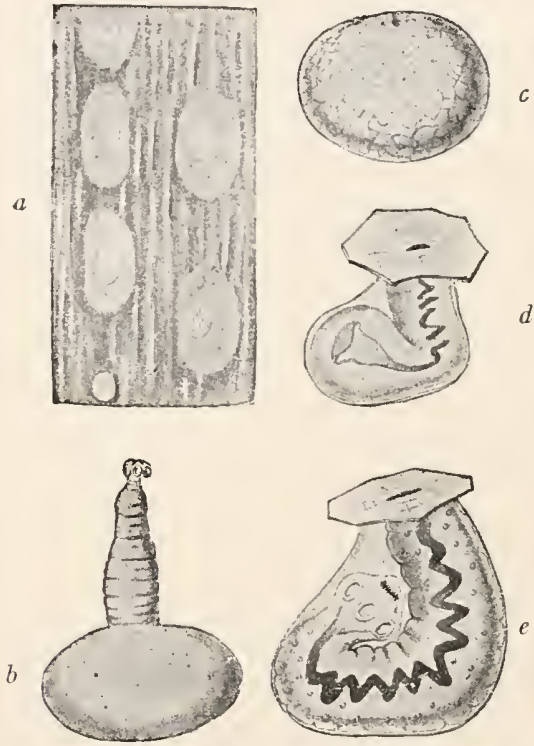

FIG. 230.- Structure and development of the cysticerus (C. cellulosæ of Trenia solium). $a$, measly meat, natural size; below an escaped cysticercus; $b$, cysticercus, with exserted scolex, enlarged; $c-e$, development of the scolex, more enlarged; $c$, young cysticercus with blastema of scolex (above) and water-vascular net; $d, e$, different stages of scolex in receptaculum, the cysticercal wail mostly removed.

pork; the cysticerci are freed by action of the digestive juices and the scolex is everted. The embryo passes to the intestine, becomes attached and, surrounded by nourishment, begins to grow, the bladder remaining attached to the hinder end. Soon the formation of proglottids begins in the piece connecting the bladder with the scolex So rapid is the growth that in ten or twelve weeks Tania solium begins to set proglottids free.

In cases where the bladder reaches a considerable size it may produce more than a single scolex. The bladder of Canurus cerebralis, which lives in the brain of sheep, produces hundreds of scolices. The number is even greater in Tania echinococcus, in which the bladder increases by budding, and by the 
formation of numerous daughter bladders produces marked tumors in the liver of man and domestic animals, before the formation of scolices begins. In the interior of each daughter vesicle appear a number of brood resicles, each of which produces numbers of scolices, so that from a single six-hooked eml.ryo thousands of scolices can arise (fig. 232). In contrast to this extreme case are others which connect with the development of Bothriocefhalus, in which the cysticercus is replaced by a cysticercoid (fig. 23I). Here there is no infiltration and the scolex is closely enclosed by an envelope comparable to the bladder wall. In several cysticercoids a caudal appendage recalls the cercaria.

The development of a tapeworm was earlier believed to be a complicated alternation of generations; the bladder to be a stage which by budding produced scolices; the scolex, in turn, a stage which by terminal budding produced the sexual animals, the proglottids, and the tapeworm itself a chain of individuals, a strobila. This view, so easy to learn, contains' two errors. The bladder is not an independent generation, but only the hinder end of the scolex. The tapeworm is not a colony, but a single animal; the proglottids are not individuals, but specialized parts of a whole. 'This view is confirmed by a comparison with other forms. The Caryophyllæidæ (fig. 233) are single bodies, the anterior end

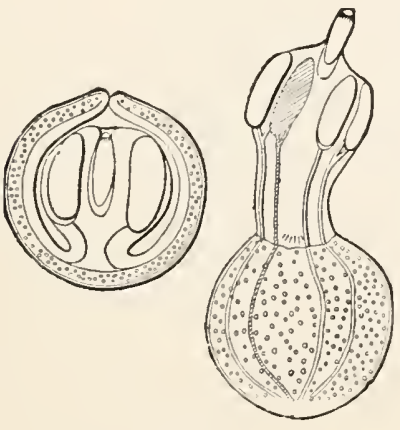

FIG. 23 I.

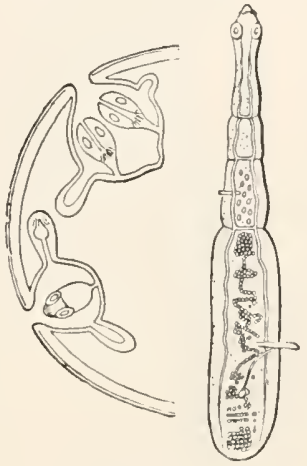

FIG. 232.

FIG. 23r.-Invaginated and extended cysticercoid from a slug (.1rion) (from Hatschek).

FIG. 232.-Tinia echinococcus (after Leuckart). Right sexually mature; left a part of an echinococcus with two brood capsules and their scolices.

elongate and taking the place of the scolex, while the broader hinder part contains a single hermaphroditic apparatus. In the Ligulida the body is still unjointed, but has increased in length and contains numerous sets of sexual organs. This duplication of the reproductive apparatus explains the appearance of proglottids. In this multiplication of the gonads, which is connected with the entoparasitic life and the necessity of increasing the fertility, lies the reason for the division into proglottids.

Family r. Caryophyllerde. No suckers, simple sexual apparatus, scolex and proglottis not differentiated. Distinguished from trematodes by absence of digestive tract. Larval stages in invertebrates, adults nearly always in fishes. Caryophyllaus (fig. 233); Archigetes in annelids (Scruris). Family 2. Ligulid æ. No suckers; numero's sexual organs, but no proglottids. The immature stages in fishes, the adults in birds. Ligula.* Family 3. Tetrarhyschid . $^{*}$ With scolex and proglottids, the head with four protrusible hooked prokosces 
(fig. 225). Immature and mature stages in fishes. Tetrarhynchus, * Rynchobolhrium.* l'amily 4. TETRAPHYLLIDE. Head with four very mobile suckers, often armed with hooks. Echinobothrium, Acanthobothrium.*

Family 5 Bothriocephalide. Scolex and proglottids present; head

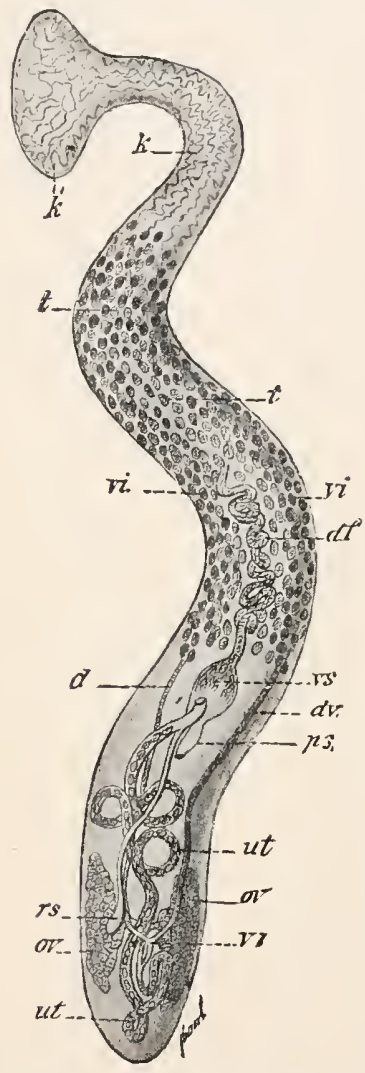

FIG. 233.-Caryophyllaus mutabilis (after M. Schultze). $d f$, vas deferens; $d v$, vitelline duct; $k$, scolex; ov, ovaries; $p s$, penis; 's, vagina with receptarulum seminis; $t$, testes; $u t$, uterus; vi, vitellarium; v's, vesicula seminalis. The connection of vagina with the crossing point of genital duct, vitelline duct, and uterus is lacking in the figure. the cysticercoid stage in its walls and then pass to the intestine to become the cysticercoid stage in its walls and then pass to the intestine to become
adult. $T$. diminuta, $*$ which has insects for its intermediate host, has been described from man. $B$. Forms passing the cysticercus stage in man. Besides the cysticercus narrower sides. Bothriocephalus latus* (fig. 234), the largest tapeworm in the human intestine (also dogs and cats), may reach a length of forty feet and consist of over four thousand proglottids. As said above, the pleurocercoid occurs in fishes, and man acquires the parasite by eating uncooked fish. It is abundant in Russia, Prussia, and Switzerland; rare in America.

Family 6. TæNIAD瓜. With scolex and separable proglottids; the scolex always bears four suckers and in many a rostellum with a circle of hooks (fig. 234). In the proglottids the genital pore occurs usually laterally in the proglottids, alternating right and left, rarely only on one side. It is rarely doubled in a proglottid. Intermediate stage a cysticercus or cysticercoid. The human tapeworms are here subdivided accordingly as the sexual animal or the cysticercus has been found in man.

A. Tanice sexually mature in the human intestine. Most noticeable are Tania solium* and $T$. saginata, * the differences between which are shown in fig. 234. In spite of the lack of hooks, the stronger suckers render $T$. saginata more difficult to expel. Tania solium is not rare in the cysticercus stage in man and occurs sometimes in places, like the brain and eyes, where it causes severe injury. These cases are in part explained by lack of cleanliness in the food, which may contain eggs, but possibly occur through internal infection; pieces of the worm passing the pylorus and entering the stomach, where they are digested, setting the embryos free.

Many other Tania, which are common to other mammals, occur occasionally in the human intestine. In mice and rats occur $T$. (Hymenolepis) nana* and $T$. diminuta. The first has recently been very abundant in human intestines in Italy and is probably common with us (fig II2). 'The worm, an inch or two long, may occur in thousands and cause severe injury. This species may derelop without an intermediate host; the egrs taken into the stomach pass

spatulate, with two sucking grooves on the 
cellulosæ of $T$. solium, found in man, more frequent and of more importance is the cysticercus of Tania echinococous (fig. 232), which lives as an adult in the dog, and is easily overlooked on account of its size. It is at most $\frac{1}{5}$ inch long and consists of a scolex and three or four proglottids. I'hen the eggs are taken in to the human stomach, as may easily happen by stroking and kissing infected dogs, the embryos are set free and wander into liver, lungs, brain, or other organs and produce here tumors which, in the case of the liver, may weigh ten or even thirty pounds. This extraordinary size is explained by the formation of daughter bladders (echinococcus) described above.
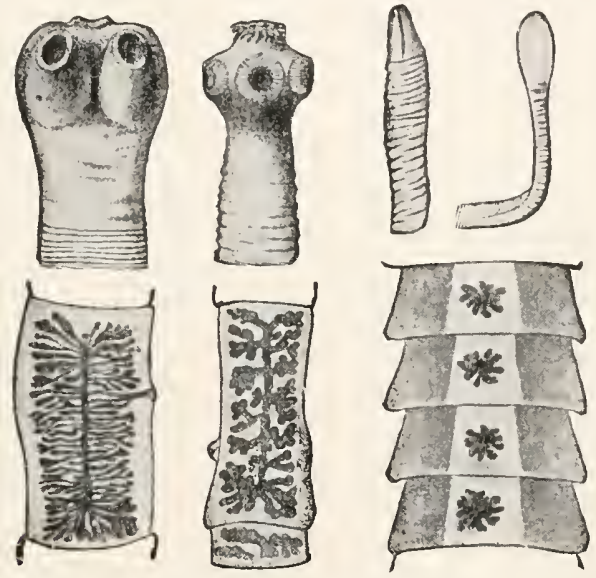

FIg. 234.- Heads and proglottids of three tapeworms of man. Left, Tænia saginata; middle, T. solium; right, Bothriocephalus latus, flat and side view of head. The heads enlarged about six times, the proglottids about $I_{\frac{1}{2}}$ (after Leuckart, Braun, and Schauinsland).

Common Tania of domestic animals are in the horse Anoplocephala plicata ( 4 to 30 inches), A. perfoliata ( $\frac{1}{2}$ to 3 inches), A. mamillana ( $\frac{1}{2}$ to 2 inches); in ruminants, Moniezia,* in the dog, Tania marginala* (cysticercus in sheep and swine), $T$. serrata* (cysticercus in rabbits), T. echinococcus (above), T. ccnurus (cysticercus in brain of sheep, causing the disease called 'staggers'), Dipylidium cucumerina* (most common, larva in the flea and dog-louse); in the cat, Tania crassicollis* (cysticercus in mice). Several species occur in domestic birds, one (Drepanidotenia infundibuliformis*), causing epidemics among chickens. Others in ducks and geese.

\section{Class IV. Nemertini.}

Most nemerteans are of appreciable size, some reaching a length of a yard or more (Lineus longissimus 90 feet!), and yet they are so contractile that our Cerebratulus lacteus, which can extend itself to fifteen feet, can retract to two. Nemerteans are rare in fresh water or moist earth, but are most abundant in the sea, where they burrow through the mud or lie rolled up beneath stones. Many are noticeable for their bright colors. Their systematic position is a problem. 
Like some flatworms they have a solid parenchyma bounded externally by a ciliated ectoderm rich in mucus cells, and inside this at least two muscular layers, an outer circular and an inner longitudinal layer. They differ from all other Plathelminthes in having a complete alimentary tract, beginning with a ventral anterior mouth and continuing as a straight tube, with, usually, pairerl diverticula, to the vent at the posterior end of the body (fig. 235).

Especially diagnostic is the proboscis, which lies dorsal to the alimentary tract and usually opens in front of the mouth. The proboscis is a

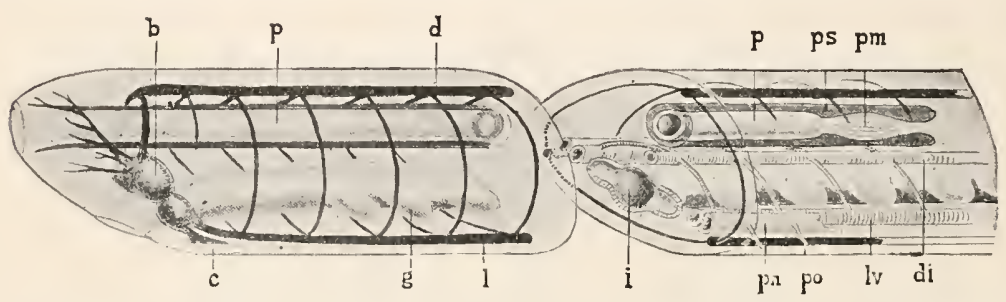

FIG. 235.-Diagram of Nemertean (orig.). $b$, brain; $c$, ciliated pit; $d$, dorsal nerve trunk; $d i$, dorsal blood-vessel; $g$, gastric cæca; $i$, intestine; $l$, lateral nerve trunk; $l v$, lateral blood-vessel; $p$, proboscie retracted; $p m$, proboscis muscles; $p r$, protonephridial tube; po, its opening; $p s$, cavity of proboscis sheat'.

muscular tube closed at one end and at rest is infolded like the finger of a glove inside a closed sac, the proboscis sheath, which extends far back in the body. Its tip is bound to the posterior end of the sheath by a retractor muscle. By contraction of the sheath the proboscis is everted, while it may be retracter again by the muscle. Nettle cells are not uncommon in the proboscis wall, while in some,forms (the older Enopla) the effectiveness of the organ is increased by the presence of a dart-like stylet at the tip (reserve stylets occur on either side, fig. 236), and at the base of the stylet is a poison sac.

The blood-rascular system consists of a pair of lateral tubes connected by transverse loops, and in most forms a third tube is present lying between the intestine and the proboscis sheath. The blood is colorless; it rarely contains red or green corpuscles.

The central nervous system (in some forms still in the ectoderm) consists of a supraœesophageal brain of a pair of ganglia, from which nerves run to the proboscis, and two lateral cords united on the ventral side by numerous transverse commissures. Connected with the brain, either directly or by means of a short nerve, are the cerebral organs or ciliated grooves, pits on the sides of the head, formerly regarded as respiratory, are now considered sense organs. Tactile organs and simple eyes are 
widely distributed; statocysts are very rare. The excretory system consists of two tubes lying beside the lateral blool-ressels and connecting

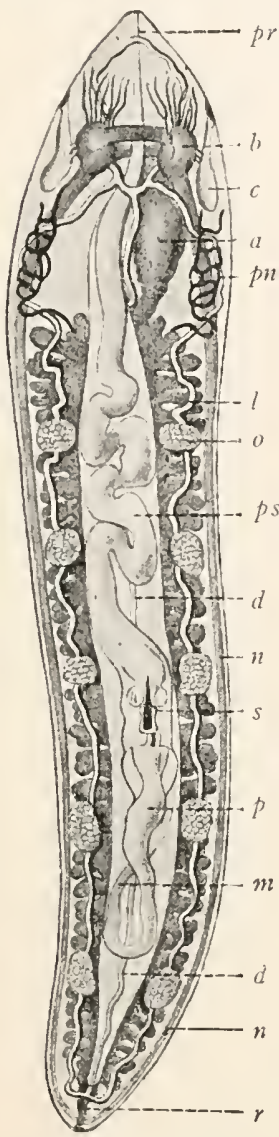

FIG. 236.

with branches terminating in flame cells, while they open separately to the exterior by one or several openings.

As a rule the nemertines are dicecious, the gonads forming a row of lateral sacs, alternating with the intestinal blind sacs and opening dorsally. The development is sometimes direct, but usually a metamorphosis occurs in which a larva, the pilidium (or a reduced form of it, Desor's larza), appears. 'The pilidium is a helmet-shaped larva with, right and left below, a pair of lappets (fig. 237). The margins of lappets and helmet are ciliated, while at the top a bundle of longer cilia project from a thickened patch of ectoderm, the

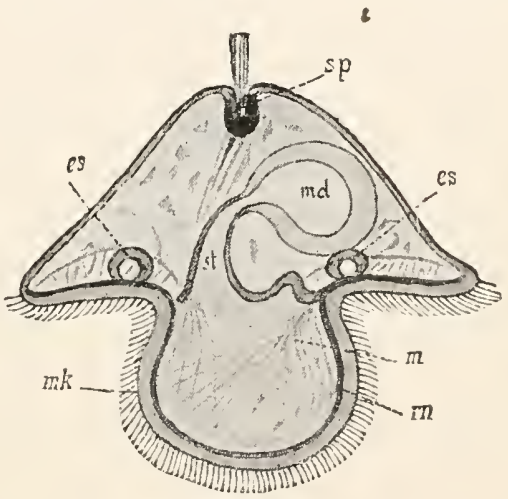

FIG. 237 .

FIG. 236.-Amphiporus pulcher (after Burger). a, alimentary canal; $b$, brain; $c$, ciliated groove; $d$, dorsal blood-vessel; $l$, lateral blood-vessel; $m$, retractor of proboscis; $n$, lateral nerve cord; $o$, ovary; $p$, poison sac; $p n$, protonephros; $p r$, proboscis; $p s$, proboscis sheath; $r$, rectum; $s$, stylet of proboscis.

FIG. 237.-Pilidium larva (from Lang, after Salensky.) es, invaginations which later give rise to the nemertine skin: $m$, oral lobes; $m d$, archenteron; $r m$, ring nerve; $s p$, apical plate; st, œesophagus; $m k$, ciliated band.

apical plate, which functions as a central nervous organ. Inside is the simple crecal archenteron, the mouth (blastopore) opening between the lappets. By a complicated process of growth and infolding this mesenteron becomes enclosed in a separate skin, produced from four inpushings (es); an anus is formed, and at the time of metamorphosis 
the worm thus produced escapes from the rest of the pilidium, which quickly dies.

\section{Order I. Protonemertini.}

Nervous system outside the muscles; no stylets in the proboscis; mouth behind brain. Carinella.*

Order II. Mesonemertini.

Nervous system in the muscles; mouth behind brain; no stylets. Cephalothrix.*

Order III. Metanemertini.

Nervous system inside the muscles, mouth in front of brain; proboscis as a rule with stylets. Geonemertes* and some species of Tetrastemm.a,* terrestrial. Amphiporus* (fig. 236, fresh watcr), Nectonemertes.* Malacobdella, * leechlike, with posterior sucker, parasitic in lamellibranchs.

\section{Order IV. Heteronemertini.}

Several muscular layers; nervous system in the muscles; mouth behind brain; proboscis unarmed. Lineus, * Micrura,* Cerebratulus.* Zygeufolia.*

\section{Summary of Important Facts.}

I. The PLATHELMINTHES are flattened bilateral animals without cœlom, whose nervous system consists of a supraœsophageal ganglion and lateral nerve trunks; the excretory system of branched protonephridia.

2. The Turbellaria are the most primitive; the Trematoda and Cestoda have descended from them.

3. The Turbellaria are ciliated externally. They have no anus and no circulatory system. The digestive tract consists of ectodermal pharynx and entodermal stomach, the latter many-branched in the Polyclads, with three main branches in the Triclads, and rod-like in the Rhabdoceles.

4. Polyclads and Triclads are often united under the name Dendrocola.

5. In the parasitic Trematoda the cilia are entirely lost or confined to the larval stages. Hooks and suckers are present for attachment to the host; several in the ectoparasitic forms; only one or two suckers in the internal parasites.

6. In the Distomice there occur heterogony and alternation of hosts. From the egg arises a sporocyst, always parasitic in molluscs, from the parthenogenetic eggs of which develop cercarix which become encysted Distomix in the second host, sexual Distomix in the third.

7. Best known of the Distoma are D. hepaticum and D. lanceolatum (rare in man, common in sheep) and D. hamatobium in the portal vein of man in warm climates. 
8. The CEstoda have no digestive tract; scolex and proglottids are usually developed.

9. The scolex is the organ of attachment, and as such is provided with suckers and frequently with hooks. It also produces the proglottids by terminal budding.

Iо. The proglottids contain an hermaphroditic sexual apparatus.

I I. The eggs produce a six-hooked embryo which must pass into an intermediate host, either by taking the eggs with the food, or the embryo must pass into the water, where it infects fishes.

I2. The embryo, in the intermediate host, becomes encysted and changes directly to a scolex (pleurocercojd) or into a bladder worm (cysticercus) which prcduces internally one or more scolices.

13. The scolex is freed from its cyst when taken into the stomach of the proper host, and then can develop into a tapeworm.

I. In man occur as cysticerci Tania echinococcus (adult in dog) and $T$. solium; as adults Tania solium (cysticercus in pigs), T. saginata (cysticercus in cattle), and Bothriocephalus latus (pleurocercoid in fish).

I5. The Nemertini have a complete alimentary canal with anus, blood-ressels and a proboscis dorsal to the digestive tract.

\section{PHYLUM V. ROTIFERA (ROTATORIA).}

The aquatic rotifers or wheel animalcules are among the smallest Metazoa, and can be distinguished from the Infusoria, which they resemble in habits, only by the microscope. The body is divisible into three regions, head, trunk, and tail. The trunk is covered by a tough cuticle into which head and tail can

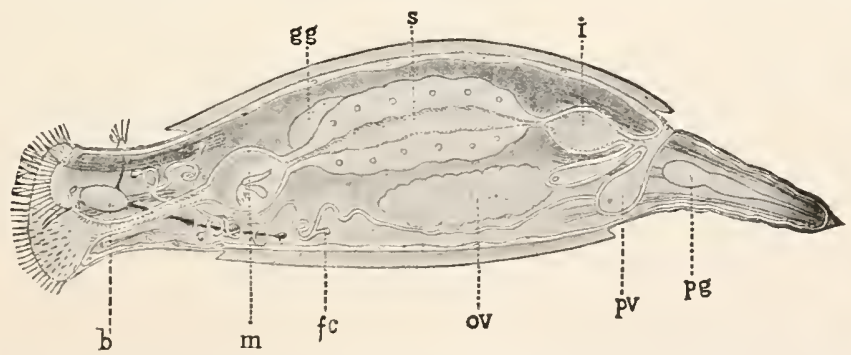

F1G. 238.-Diagram of rotifer (after Delage et Herouard). $b$, brain; $f c$, flame cell; $g g$, gastric gland; $i$, intestine; $m$, mastax; ov, ovary; $p g$, pedal gland; $p i$, pulsating vesicle of excretory system; $s$, stomach.

be retracted. The tail or 'foot' is often composed of rings which can be telescoped into each other. The last tail ring often bears a pair of pincer-like stylets by which, together with adhesive glands, the animal adheres to objects. The head is expanded in front to a trochal disc, an apparatus of varying shape, surrounded by a ring of cilia of use in swimming and in directing food to the ventral mouth. 'The alimentary canal consists of cesophagus, mastax (chewing 
stomach), glandular stomach, and intestine; all except the mastax ciliated. The mastax bears two chitinous jaws (trophi), which in life are in constant motion and comminute the food. Above the œesophagus is the cerebral ganglion with which simple eyes and peculiar sense organs, the cervical tentacles, are frequently connected. The usually single ovary and the paired protonephridia empty into the posterior part of the alimentary canal, which thus becomes a cloaca. The males are much rarer and smaller and have a much simpler structure (fig. $239, B$ ). Usually the alimentary tract is reduced to a solid cord in which the testes are imbedded.

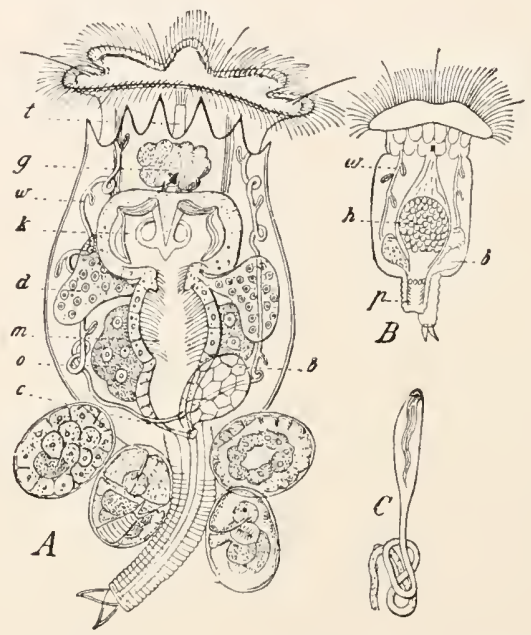

FIG. 239.-Brachionus urceolaris. $A$, female with four eggs in various stages; $B$, male; $C$, 'tlame' from protonephridia, greatly enlarged; $b$, urinary bladder; $c$, cloacal opening; $d$, gastric glands; $g$, ganglion, with eye; $h$, testis; $k$, mastax; $m$, stomach; $o$, ovary; $p$, penis; $t$, tentacle; $w$, protonephridia.

The Rotifers have large winter eggs enclosed in a thick shell and smaller thin-shelled summer eggs. The latter develop parthenogenetically and by their numbers and rapid growth aid in the distribution of the species. The winter eggs require fertilization, and have a long resting period, thus serving to tide over periods of cold or drought. The adults can withstand a certain amount of desiccation; and often occur in damp moss or in eave troughs in a sort of sleep from which they are awakened by water.

In structure the Rotifiers are much like the trochophore larvæ of annelids and molluscs to be described later. They are primitive forms, connected with the ancestors of these groups, and also, as shown by nervous system and excretory organs, with the flatworms as well. Most species are cosmopolitan and inhabitants of fresh water. Near the Rotifera may be placed the fresh-water GASTROTRICHA (Ichthydium,* Chatonotus*) and the marine ECHINODERID $\approx$, forms which are little understood.

\section{PHYLUM VI. CELHELMINTHES.}

The Colhelminthes are distinguished from all the forms which have gone before by a body cavity, separating the outer body wall from the 
intestine; but whether this cœlom be homologous in different groups, e.g., nematodes and annelids, is not settled. The body muscles are developed from the outer (parietal) epithelial wall of the colom and hence are 'epithelial muscle cells' (figs. 240, 24I). 'The pro-
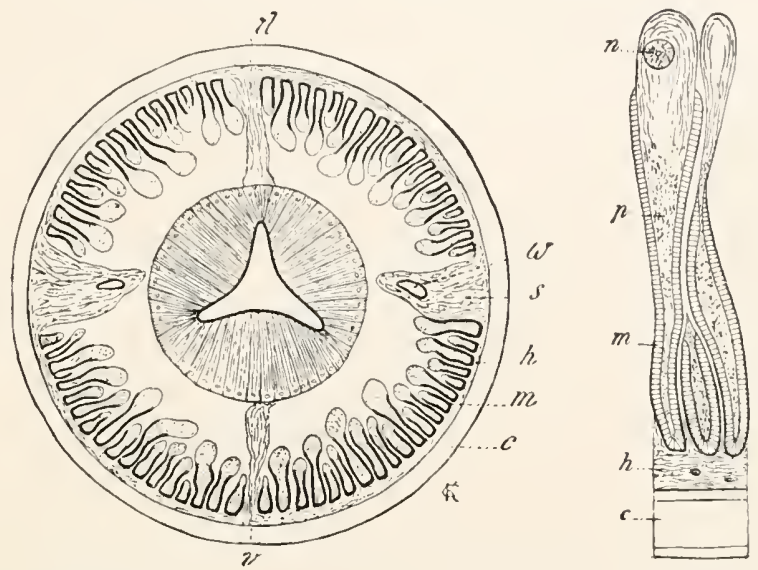

FIG. 240.- Section of Ascaris lumbricoides through the pharyngeal bulb; beside it a bit of the body wall more enlarged. $c$, cuticle; $d$, dorsal line; $h$, hypodermis; $m$, longitudinal muscle; $n$, nucleus of muscle cell; $p$, muscle cell; $s$, lateral line; $v$, ventral line: $w$, excretory canal.
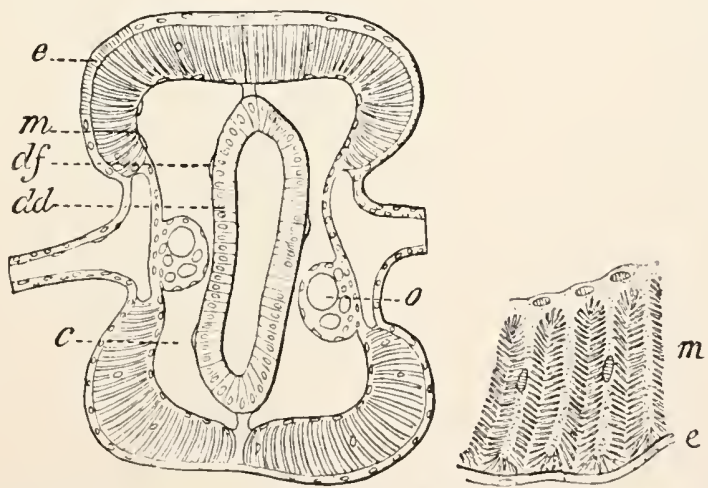

Fig. 24I.-Transverse section of Sagitta bipunctata and a bit of the body wall more enlarged (after $O$. Hertwig). $c$, colom; $d d$, entoderm; $d f$, splanchnic mesothelium; $e$, epidermis; $m$, somatic mesoderm (muscles and epithelium); o, ovary.

tonephridia of the larval stages are replaced by nephridia (fig. $7 \mathrm{I}$ ), connecting the body cavity with the outer world. Internally they begin with a ciliated funnel, the nephrostome, and continue as long coiled tubes, expanding just before the outer end to a kind of bladder. The gonads 
(fig. 24I, $o$ ) are specialized parts of the cœlomic epithelium and their products are usually carried to the exterior by the nephridia (more rarely by

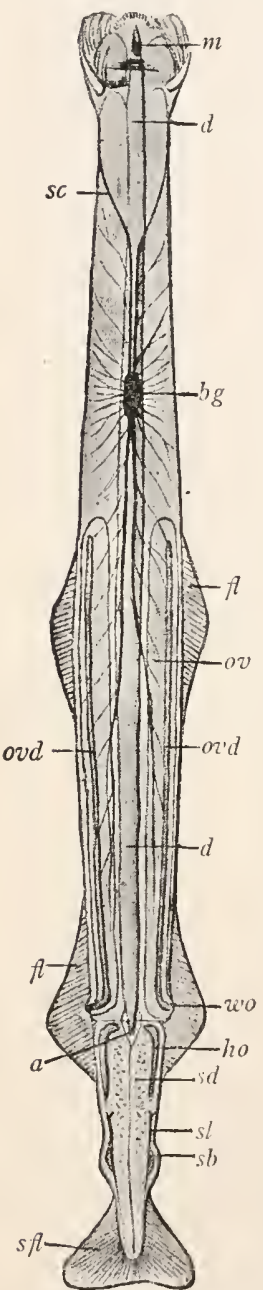

FIG. 242. special ducts), so that here, as in vertebrates, we can speak of a urogenital system. A closed blood system is now present, now absent. Little in general can be said of the nervous system; details will be given in connection with the separate classes.

\section{Class I. Chætognathi.}

These marine forms, a half to two inches long, perfectly transparent, live at the surface of the sea, preying on other animals, and from their shapes and rapid motions deserve the name Sagitta-arrow-given some forms. The animals swim by means of horizontal fins, one surrounding the tail and one or two pairs on the sides of the trunk (fig. 42). On either side of the mouth are strong bristles used in seizing prey (Chætognath, bristle-jaw). Internally the body is separated into head, trunk, and tail, by transverse septa which divide the colom into corresponding chambers. Each segment of the cœlom again is divided into right and left halves by a mesentery (fig. 24I), supporting the straight intestine, running lengthwise through it and terminating at the anus at the end of the trunk segment.

The nervous system is entirely ectodermal. In the head is a pair of fused cerebral ganglia (fig. 243), in the trunk segment a large ventral ganglion, and these are connected by long osophageal commissures. Of interest, because characteristic of nematodes and many annelids, are the relations of the musculature, which

F1G. 242.-Sagitta hexaptera, ventral view (after O. Hertwig). a, anus; $b g$, ventral ganglion; $d$, intestine; $f l$, fin; ho, testes; $m$, mouth; or, ovary; ovd, oviduct; $s b$, seminal vesicle; $s c$, œsophageal commissure; $s f l$, tail fin; $s l$, sperm; wo, female opening.

F1G. 243. - Head of Sagitta bipunctata, dorsal view (after O. Hertwig), an, nerve to $a u$, eye; $g$, brain; $g h$, bristles; $r m$, nerves to $r o$, olfactory organs; $s c$, ósophageal commissure.

consists of longitudinal fibres alone. The body cavity is lined with epithelium (fig. 24I), which, where it abuts against the alimentary tract, is 
called splanchnic mesoderm; that on the side of the cœlom towards the ectoderm is the somatic mesoderm. The muscles arise from the latter and are divided into four fields, right and left dorsal, right and left ventral. The sex cells also arise from the epithelium of the coelom, the eggs in the trunk segment (fig. 24I), the sperm in the tail. 'T he eggs are carried to the exterior by special ducts. The sperm-forming cells early fall into the coelom, where they develop the spermatozoa. These are carried out by canals which recall the nephridia of the annelids.

The development of Sagitta is significant from two points of view. The archenteron (fig. 109) is divided by lateral folds into an unpaired middle portion and two paired lateral chambers; the first is the definitive digestive tract, the latter the anlagen of the coelomic diverticula. In other words, the colom is an outgrowth from the archenteron, i.e., is an enterocole. Second: 'The gonads are derived from a pair of cells in the primitive entoderm, which later are carried into the colomic walls. Here each divides into anterior and posterior cells, the anterior developing into the ovary, the posterior into testes. Hence here the male and female sex cells are beyond doubt descendants of a common mother cell.

The few species are arranged in two or three genera, of which Sagitta, represented on our coasts by $S$. elegans, * is best known. Spadella.

\section{Class II. Nemathelminthes.}

Like the flatworms, the roundworms are characterized by their shape, they being thread-like or cylindrical animals whose form is the result of the existence of a body cavity in which the viscera are so locsely held that on cutting through the muscular body wall they will fall out (fig. 244). Since the Nemathelminthes share this colom with most annelids, the distinction between the two rests largely upon negative characters, the roundworms lacking the segmentation of the body cavity and the corresponding ringing or annulation of the body wall. The borly cavity apparently is different since the splanchnic wall is lacking, the space lying between mesoderm and entoderm (pseudoccrle). To the Nemathelminthes belong three orders, much alike in habits and appearance but differing considerably in structure. Of these the most important are the nematodes.

\section{Order I. Nematoda.}

The nematoda contain numerous species of thread-shaped worms varying from $0.00 \mathrm{r}$ to $\mathrm{I} . \mathrm{O}$ metre in length, many of which, through their wide distribution as parasites in plants, animals, and man, possess special interest. The outer surface is covered by a tough cuticle secreted by the subcuticula, a fibrous ectodermal syncitium (fig. 2.40), which in cross. section shows four thickenings, the dorsal, ventral, and lateral lines. In the lateral lines run the excretory vessels, two longitudinal canals, unitcr near the head by a transverse vessel opening on the ventral surface by an 
unpaired pore to the exterior. They are related to the cœlom by two giant cells on either side which send processes into the body cavity. These lateral and median lines divide the muscles (here only longitudinal) into four fields. These muscles are parts of the somatic epithelium, a layer of vesicular cells which by their size (fig. 240) so encroach upon the cœlom that scarce space is left for the alimentary canal and reproductive organs.

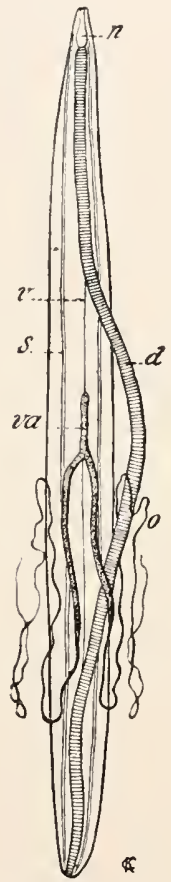

FIG. 244.

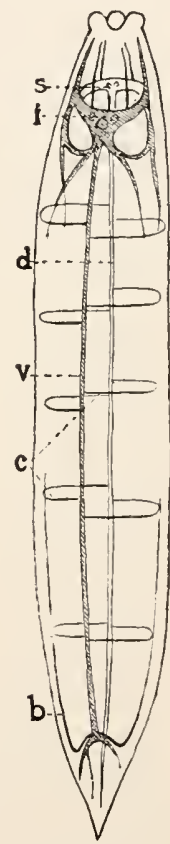

FIG. 245 .

FIG. 244.- Structure of young female Ascaris (based on a drawing by Leuckart). $d$, intestine; $o$, ovary; $p$, pharynx; $s$, lateral line; $v$, ventral line; $v a$, vagina.

FIG. 245. -Diagram of nervous system of a nematode (after Bütschli). $c$, commissures; $d$, dorsal nerve; $i$, infracesophageal, $s$, supraœesophageal part of nerve ring; i, ventral nerve.

The alimentary canal begins with a terminal mouth and ends with the ventral anus in front of the end of the body. The mouth connects with the muscular sucking œsophagus, which is expanded posteriorly to a pharyngeal bulb and is lined throughout with a cuticle. From this point to the anus the stomach-intestine is usually uniform (fig. 244). The œsophagus is surrounded by a nervous ring which sends forward and back a large 
number of nerves, those in the mid-dorsal and rentral lines being strongest. At points on these nerves are collections of ganglion cells, lut a formation of ganglia, as in the annelids, does not occur (fig. 245). 'The only sense organs are tactile papillæ near mouth and genital opening, and eyespots in a few free living forms.

The sexual organs of these rarely hermaphroditic forms are very simple. Males and females are easily distinguished, not only by the copulatory organs, but ly the openings of the genital ducts. These, in the male (fig. 246), are in the end of the alimentary canal, which hence is a cloaca. In the female (fig. 244) there is a special genital opening on the ventral surface between mouth and anus, the position varying with the species. In general the structure of the reproductive organs is alike in both sexes. These are long tubes coiled forward and back and ending in fine threads which produce eggs or sperm (ovaries, testes), while the rest serves as seminal vesicle, or receptaculum seminis, and ducts. In the male the genital tube is always single; in the female it is usually double, the right and left halves uniting a little before the external opening (fig. 244, va). Most common of copulatory organs in the male are spicula, bent spines, which lie in a sheath behind the vent and can be protruded through the cloacal opening. Besides there may be valves to right and left to clasp the female, or, as in Trichina, the whole cloaca is protrusible:

Since there is copulation, the eggs are fertilized in the uterus, after which they are either laid or retained for more or less of their development, many, like Trichina, being viviparous. The postembryonic development depends largely upon the mode of life. Free-living species grow by repeated molts without much change of form. In many Anguillulidx, which show how free life can be transformed into parasitic, there is an alternation of generations (heterogony) from a protandric hermaphroditic entoparasitic to a free diccious generation. The occasional suppression of the free generation which occurs in many Anguillulids leads to the Strongylidie, where the ofispring of the parasitic generation can live free for a time (rhabditis larra), but must return to parasitism to undergo a metamorphosis and become sexually mature. 'T he free life is shortened again in the Ascaridre, where the eggs must pass to the exterior for a longer or shorter time, but the embryos only escape when the eggs are taken into another host. Lastly, there are species like Trichina where the free life is entirely suppressed and transportation from host to host takes place in the encysted condition passively by food. This purely parasitic condition leads to species in which the rhabditis larva developed in water, enter a second host for encystment as the larva of Filaria medinensis in the Cyclopida.

Family I. Anguillulide; small thread-like nematodes which live in mud, organic fluids or plants, rarely in animals; male with two spicula. Anguillula aceti, vinegar eel, in vinegar and stale paste. Rhabditis (Rhabdonema) nigrovenosa, lives in mud and stands in heterogony with a second form which lives in the lung of frogs. Strongyloides intestinalis, which has recently appeared in southern Europe, has a somewhat similar history, the adult stage being reached in the human intestine. In the tropics one stage of this is passed in moist earth, but in colder climates the free-living generation drops out. Here belong 
Tylenchus tritici and Heterodera schachti, the first doing great damage to wheat, the second to turnips in Europe. T. devastatrix attacks rye and hyacinths.

Family 2. AsCarIDe. Mouth with three lips; males with two spicules. Numerous species in lower vertebrates, Ascaris lumbricoides, * the round worm of man (fig. 246), inhabits the small intestine, often in enormous numbers. The females are about $5^{-6}$ inches, the males 4 inches in length. $A$ female contains about $60,000,000$ eggs. Shortly after fertilization the eggs pass out with the fæces, but develop without intermediate host if, in the course of two or three
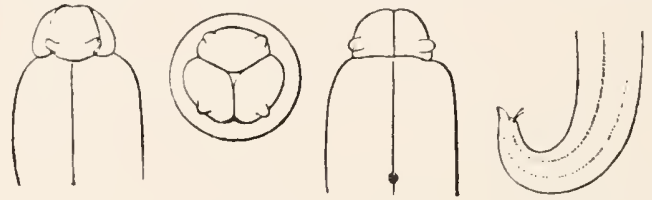

FIG. 246.-Dorsal, end, and ventral views of head and hinder end of male Ascaris lumbricoides (from Hatschek.)

months, when the embryo is formed, they are taken into the human intestine. The development of the pinworm, Oxyuris vermicularis, ${ }^{*}$ is somewhat similar except that the embryos are developed in the egg at the time of oviposition, and hence after a shorter stay outside the body are capable of infection. The white worm, not half an inch long, lives in the rectum, especially of children, and causes intolerable itching. Ascaris mystax* occurs in dogs and cats (occasionally in man). A. megalocephala* (a favorite animal for cytological researches) and Oxyuris equi in the horse, do little harm. Heterakis maculosa of ten destroys whole flocks of pigeons. Family 3. Strongylide.

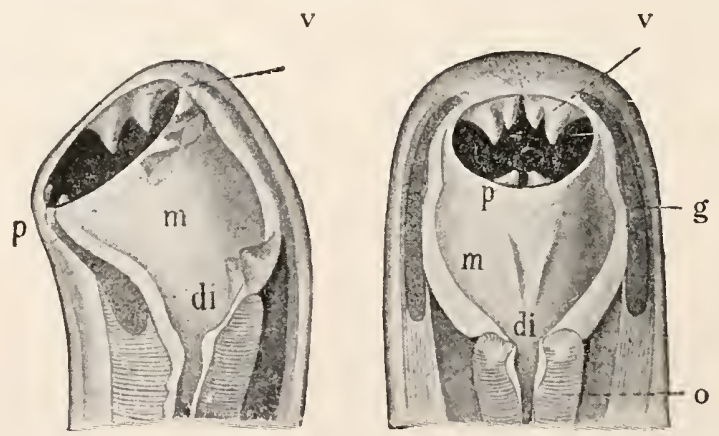

FIG. 247.-Anterior end of hook worm, Ankylostoma duodenale (after Looss). $d i$, lower teeth; $g$, lateral gland; $m$, oral capsule; $p$, dorsal tooth; $o$, osophagus; $v$, ventral teeth.

These are readily recognized by the bursa of the male, a broadening of the hinder end of the body by two wing-like processes, which contains two spicula. Frequent but not constant is a widened capsule surrounded by papillæ at the mouth. Strongy/us* in domestic animals. Syngamus trachealis, ${ }^{*}$ half to three quarters of an inch in length, the male and female always in pairs, cause the disease known as 'gapes' in fowl. Ankylostomum (Dochmius) duodenale* (fig. 247), about two fifths of an inch in length, lives in the small intestine of man, causing severe loss of blood. The eggs develop in moist earth, and hence people who 
drink muddy water (Fellahin of Egypt) or work much with clay (potters and brick-makers) are especially subject to infection. It was first known in Egypt; caused considerable trouble during the building of the St. Gotthard tunnel in Switzerland. More recently it has been recognized as frequent in our southern states, where it has become notorious under the common names of hook-worm and lazy worm. It has been thought that the Ankylostoma larve obtain entrance to man through the skin, as in bathing, etc.

Family 4. Trichotrachelid 2. These are called 'hair necks' because that part of the body which contains the pharynx is hair-like and elongate. Trichocephalus dispar* of man (fig. $248, A$ ), about an inch or an inch and a half in length, lives with its neck in the wall of the intestine near the crecum. Since it does not move, it causes little injury.

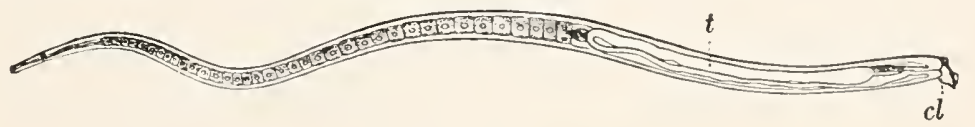

$B$

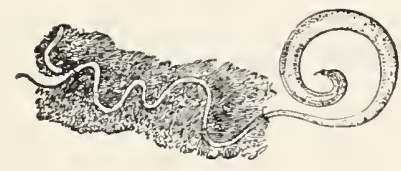

A

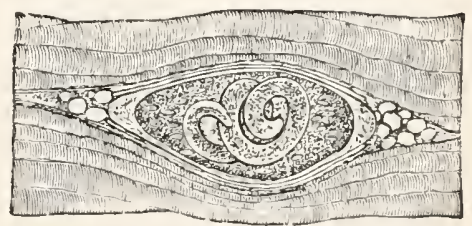

C

FIG, 248.-A, Trichocephalus dispar, male with anterior end embedded in intestinal wall (from Leuckart). $B$, Trichina spiralis, male (from Hatschek). $c l$, cloaca; t. testes. $\mathcal{C}$, Trichina in muscle (from Boas).

Trichina spiralis* (fig. $248, B, C$ ), is much smaller, but much more dangerous. 'Two stages are to be distinguished, the encysted muscle Trichina and the sexually mature intestinal Trichina. The first was discovered in a human body in 1835 ; the latter was not known until much later. In the encysted stage it occurs in the muscles of pigs, rats, mice, man, rabbits, guinea pigs, dogs, etc. (never in birds), enclosed in an oral capsule about $0 .+$ to $0.6 \mathrm{~mm}$. long and hence recognizable by a practised observer with the naked eye. Certainty in their recognition demands a low power of the microscope. Coiled up in the capsule is the worm, about I mm. long, which is not yet sexually mature. To attain this it must be transported into the intestine of another host. When, for instance, man eats trichinosed pork the worms are freed by the digestive fluids and, entering the small intestine, become sexually mature in a few days. The female $\left(3^{-4} \mathrm{~mm}\right.$. long, the male $1.5 \mathrm{~mm}$.) penetrates the intestinal villi and in course of a month gives birth to 1500 (some say ro,, 00 ) living young, after which she dies. The young enter the lymph vessels, are carried by way of the thoracic duct into the blood-vessels, and wander into the nuscles, especially those which are much worked, like the diaphragm, eve muscles, and muscles of the neck, and which consequently have a rich blood supply. They enter the sarcolemma of the muscle, destroy the muscle substance, and finally become enclosed by a capsule secreted by the host. The wandering takes place about the second or third week after infection, the encystment in about three months. A slight infection causes disagreeable symptoms; but where large numbers obtain entrance the cases are frequently fatal. The worst epidemic known was in 
Emmersleben, Saxony, in 188.4 , where 57 died in four weeks from infection from one pig.

Family 5. FilaritDe. Extremely elongate, hair-like worms. Dracunculus medinensis, the guinea worm (the female about a yard long, and about as large as stout packing twine), produces abscesses beneath the skin in which the worm is coiled up. The embryos break through the wall of the mother and must enter the water and penetrate a smali crustacean, Cyclops. It is apparently introduced into the human system by swallowing the crustacea with drinking water. The worm occurs in tropical America.

Filaria sangumis hominis, 3 to 6 inches long, lives in the lymphatic glands of man, the young escaping into the blood, often in immense numbers. They of ten pass through the kidneys, where they produce serious disturbance. There is possibly a connection between them and elephantiasis. The intermediate host is apparently the mosquito. As yet they are known only in the tropics. Other species occur in man and other animals.

Family 6. MERMithIDÆ. Elongate nematodes in the body cavity of insects; they pass into damp earth, where they become sexually mature. They share with the Gordiacea the name 'hairworms.' Mermis.*

\section{Order II. Gordiacea.}

The hairworms resemble the nematodes in general appearance, but differ greatly in structure. The body cavity has both splanchnic and somatic epithelium; the intestine is supported by mesenteries (fig. 249); there is an œsophageal

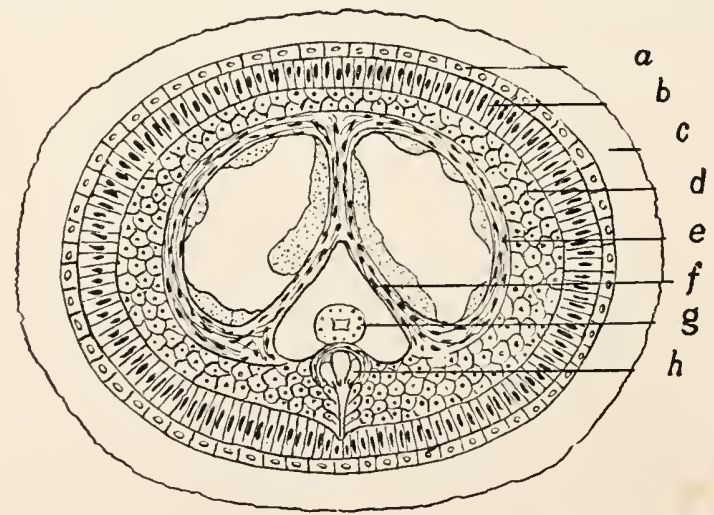

FIG. 249.-Transvers s section of young Gordius (after von Linstow). a, hypodermis; $b$, muscular layer; $c$, cuticle; $\dot{d}$, parenchyma; $e, f$, muscles and mesenteries; $g$, alimentary canal; $h$, nervous system.

nerve ring and unpaired ventral nerve cord, and the female genitalia open into the cloaca. 'The adults live in water, where they lay their eggs; the larva live in insects, there being in some cases an alternation of hosts. They are popularly believed to be horse hairs changed into worms. Gordius,* Chordodes,* Near the Gordiacea must be mentioned the marine Nectonema, young stages apparently passed in the mosquito.

\section{Order III. Acanthocephala.}

The adult spine-headed worms live in the alimentary canal of vertebrates. They resemble the Ascaridæ (p. 266), but are easily distinguished by the pro- 
boscis, which may be retracted by muscles and exserted by contraction of the muscular body wall. This proboscis bores into the intestinal wall and is held in place by numerous retrorse hooks (fig. 250). The entire absence of an alimentary canal marks them off from Nematodes and Gordiacea, as also the peculiar structure of the reproductive organs and a closed vascular system in the body wall which extends into two sacs, the lemnisci, lying beside the proboscis sheath. 'The unpaired ganglion lies on the proboscis sheath between the lemnisci. An intermediate host occurs in derelopment, the larva living in an arthropod. Thus the larva of Echinorhynchus gigas: of the pig lives in the larva of the 'June bug' (Melolontha), that of $E$. proteus of European fresh-water fishes in crustacea. E. hominis is extremely rare in man.

The Acanthocephali are dinecious. The oraries of the female early break up into groups of eggs which float in the body cavity. The ripe eggs have a peculiar method of escape from the body. There is a muscular uterus which connects by two narrow canals with the vagina and thus with the outer world. The uterus picks up immature and fertilized eggs indiscriminately by its wide mouth, but only those which are elongate, have a shell and contain embryos can pass the canals; the immature eggs are led through a ventral opening back to the colom. In $E$. gigas protonephridia open beside the genital opening.

\section{Class III. Annelida.}

The metamerism, which occurs in a slight degree in the Chretognathi, reaches its highest development in the Annelids, where it appears both in the outer ringing of the body and in the arrangement of the most important systems-excretory organs, nervous system, blood-vessels-internal segmentation. To this is added an extraordinary increase in number of 'body segments (somites, metameres), which can far exceed a hundred. The epithelial longitudinal muscles are reinforced by an outer layer of mesenchymatous circular filores. We can thus define the Annelids as worms with colom and with external and internal segmentation. In the development there frequently occurs a type of larva, the trochophore (p. 238 ).

The above account applies most closely to the

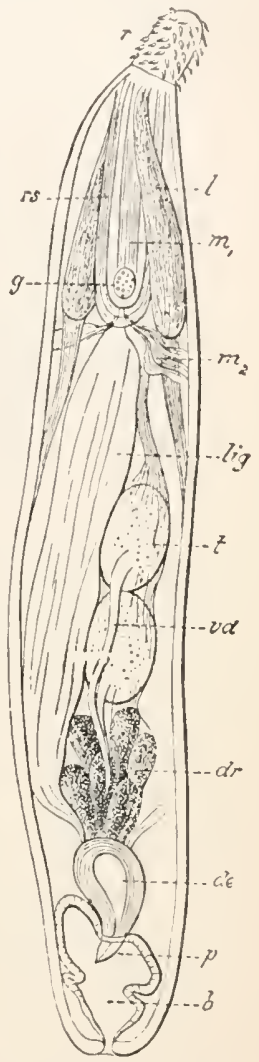

FIG. 250.-Male Echinorhynchus angustutus (from Hatschek). $b$, penis sac; de, seminal resicle; $d r$, glands; $g$, ganglion; l, lemnisci; lig, ligament; $m_{1} m_{2}$, retractors of proboscis and its sheath; $p$, penis: $r$, proboscis; rs, proboscis sheath; $t$, testes; r'd, ras deferens.

Chretopoda and Archianellida. In other forms one or more features may be lacking-in the Gephyraa segmentation of the body; in the Hirudinei most of the colom and the trochophore. Tet these are so 
closely related that they must be included under the common head; the missing characters have been lost during evolution.

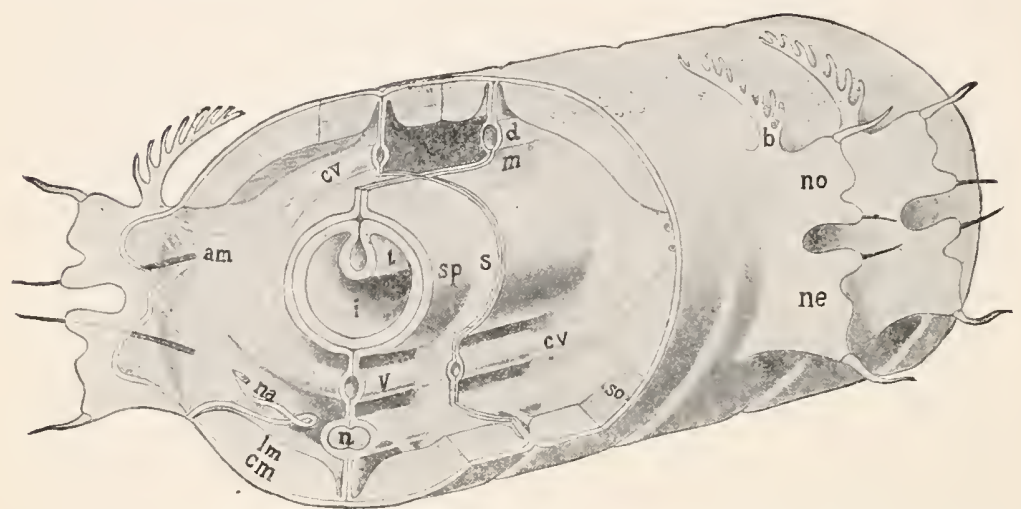

FIG. 25I.-Diagram of annelid somites (orig.). am, acicular muscles; $c$, coelom; $c m$, circular muscles; $c v$, circular blood-vessels; $d$, dorsal blood-vessel; $i$, intestine; $l m$, longitudinal muscles; $m$, mesentery; $n$, nerve cord; $n a$, nephridium; ne, no, neuro- and notopodia, forming parapodium; $s$, septum; so, somatopleure; $s p$, splanchnopleure; $t$, typhlosole.

\section{Sub Class I. Chatopoda.}

These, like the Nematoda, are cylindrical worms, but are at once distinguished by the segmentation. Deep circular constrictions (fig. 252)

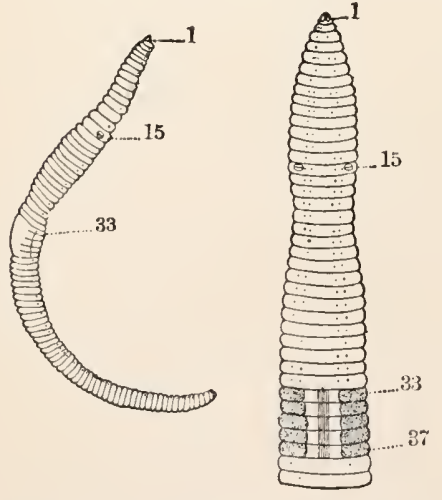

FIG. 252.-Earthworm, side view and anterior end enlarged (after Vogt and Jung). I, first segment with mouth and prostomium; i5, male sexual opening; 33-37, clitellum. begins with a supraœsophageal ganglion ('brain') in the prostomium, from which the osophageal commissures pass around the osophagus bound the somites externally. Internally the cœlom is divided by the septa-delicate double membranes which extend from the ectoderm to the alimentary canal-into as many chambers as there are metameres, while a longitudinal mesentery, also double, separates the cœlomic pouches of the right side from those of the left (figs. 25I, 253). The alimentary canal has a terminal anus, while the mouth is ventral and is overhung by the preoral segment, the prostomizm.

Nervous system, blood-ressels, and excretory organs are influenced by the segmentation. The nervous system is on the ladder plan (p. I I3). It 
to form the ventral chain, which consists of as many pairs of ganglia, united by longitudinal commissures, as there are somites present. 'These ganglia of the ventral chain are closely similar, since the segmentation of the body is homonomous. There is but the slightest division of labor

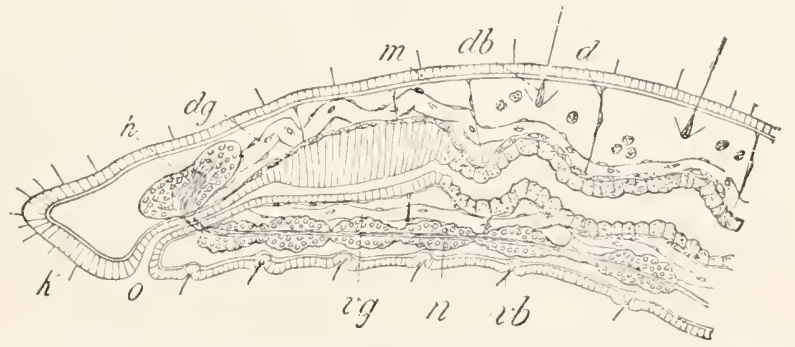

FIG. 253.-Anterior end of Nais elinguis. $h$, cerebrum, connected by commissure with ventral chain, $n$; $d g$, florsal, vg, ventral blood-vessel; $m$, muscular layer of skin; $d f$, vf, dorsal and ventral chætæ; $d$, septa; $k$, prostomium; $o$, mouth.

among the somites, and hence they differ but slightly among themselves. The prostomium always bears tactile organs and frequently eyes, which in many marine forms are hichly developed, wih lens, vitreous body,

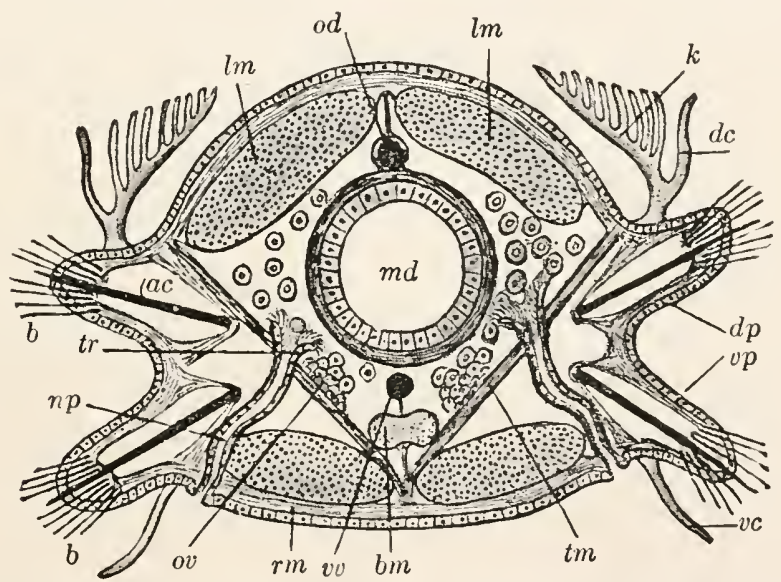

FIG. 254.--Schematic cross-section of an annelid (after Lang). ac, aciculum; $b$, chetx; $b m$, ventral nerve cord; $d c$, dorsal cirrus; $d p$, notopodium; $k$, gill; $l m$, longitudinal muscles; $m d$, digestive tract; $n p$, nephridium; ov, ovary; $r m$, circular muscles; $t m$, transverse muscles: $t r$, nephrostome; $v c$, ventral cirrus; $v d, v v$, dorsal and ventral blood-vessels; $v p$, neuropodium.

and retina (fig. 84, I, II). Statocysts are rare, but occur in diverse groups. Ciliated pits (olfactory ?) occur on the head, goblet organs (taste) on head and trunk, and lastly, lateral organs, sensory structures of unknown function, may be metamerically arranged. 
The blood-ressels usually are represented by two main trunks which frequentiy (as in earthworms) contain blood colored red by hxmoglobin. One trunk is dorsal, the other ventral, to the intestine, the two being connected by ressels (figs. $25 \mathrm{I}, 255$ ) in each segment. The blood passes forwards in the dorsal ressel, backwards in the rentral. It is propelled by contractile portions of the vessels; usually the dorsal ressel pulsates, but as in the earthworms, certain of the circular vessels in the anterior part of the body may function as hearts (fig. 255, c ). Rarely, as in the Capitellidx, circulatory organs may be lacking.

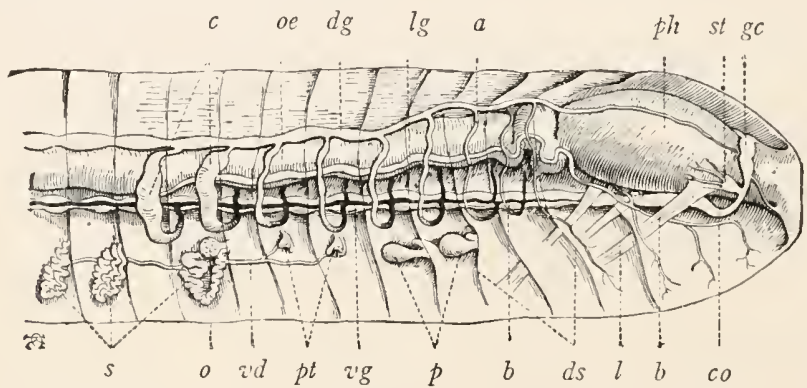

FIG. 255.-Anterior end of Pontodrilus marionis (after Perrier). a, vascular arches; $b$, ventral nerve chain; $c$, 'hearts'; $c o$, osophageal commissure; $d g$, dorsal blood-vessel; $d s$, septa; $g c$, cerebrum; $l$, retractors of pharynx; $l g$, lateral blood-vessel; $o$, ovary; oe, asophagus; $p$, receptacula seminis; $p h$, pharynx; $p t$, ciliated funnels of vas deferens; $s$, nephridia; st, pharyngeal ganglion; 2 ' , vas deferens.

The excretory organs (nephridia) were formerly known as 'segmental organs,' since they occur in pairs in each segment. Strictly speaking, each organ belongs to two segments. It usually begins in the anterior of the two with a ciliated funnel (nephrostome), passes through the septum, and, after convolutions, opens to the exterior in the second segment. The nephridial canal (usually lined with ciliated epithelium) often serves to carry off the sexual products, which in all chretopods, arise in the colomic epithelium.

The nephridia of Annelids seem to be derived from protonephridia (fig. 256 , I, II), which finally opened into the cœlom (III, IV). In many species they are simple or branched tubes, closed internally by solenocytes, large cells drawn out into a tube which empties into the blind end of the excretory tubule and has a flagellum in the interior (fig. 69, p. I06). With the development of the nephrostome the branched condition and the solenocytes are usually lost. The relations of the nephridia to the sexual products appear to be secondary, and are brought about by large ciliated grooves of the peritoneal epithelium. There are three possibilities. (I) The sexual products are emptied by dehiscence of the body wall; the ciliated organs are phagocytic. (2) The ciliated grooves at the time of sexual maturity open directly to the exterior and carry off the eggs and sperm (I and III). (3) They connect with the excretory tubules, be these nephridial or protonephridial (II or IV), the segmental 
organs thus becoming sexual ducts. The second of these conditions explains the coexistence of reproductive funnels and nephridia in the genital segments of many oligochætes. In the oligochetes there are other modifications: Opening of the nephridia into fore or hind gut, connection of the tubules into a network with several openings to the exterior in each segment (Megascolicida).

In many marine annelids there occurs a metamorphosis in which pelagic larva occur. 'These, in spite of many modifications, are comparable with 'Loven's larva,' the trochophore already described (p. 238 ).
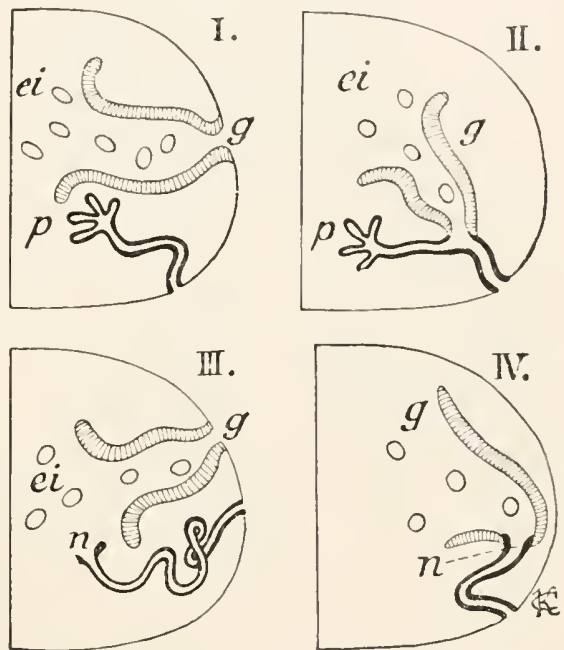

FIG. 256.-Different relations of nephridia and sexual ducts in chætopods (after Goodrich). I, hypothetical primitive condition; II, Phyllodoceids and Goniads; III, Dasybranchus; IV', Syllids, Spionids, etc. In I and III the ciliated grooves (sexual sacs, g) lead the sexual products (ei) direct to the exterior; in II and IV they empty into the nephridial canals, which are either protonephridia (I and II) covered with solenocytes, or (III and IV) are nephridia.

The differences largely consist of modifications of the ciliary apparatus; the number of bands may be increased (polytroche larvæ), or a single band may occur at the middle (mesotroche) or at the end (telotroche) of the body. The larva becomes a segmented worm by the hinder end of the larva growing out and dividing into segments (fig. $257, B$ ). In this growth new mesoderm develops as a pair of bands (usually from a pair of cells at the hinder end, the teloblasts). This mesoderm divides, from in front backward, into the primitive segments. Each of these become hollow, forming a colomic chamber. Since these, right and left, grow around the digestive tract, they give rise to the somatic and splanchnic mesothelium and form part of the rigestive and body walls. Where they come in contact above and below the intestine they form the mesenteries which frequently 
disappear in the adult. In many worms the septa between the somites also breaks down and the coelomic cavities unite into one. The nephridia also arise independently of the protonephridial system, which is often called head kidney because the chief part of the trochophore forms the head of the adult.

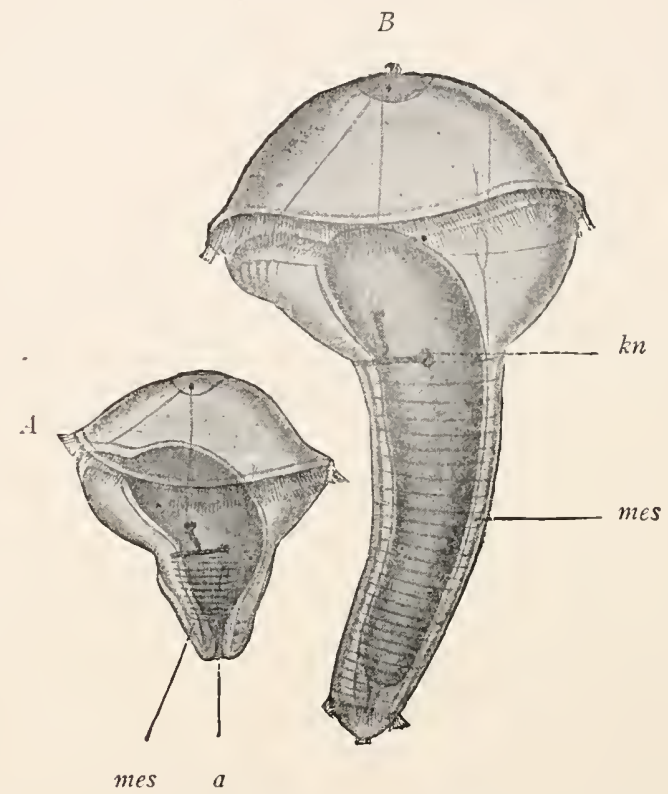

Fig. 257.-A, larva of Polygordius; $B$, same changing to segmented worm (after Hatschek). $a$, anus; $k n$, excretory organ; mes, segmented mesoderm.

The land and fresh-water annelids develop directly, but the embryos possess a reminiscence of a larva in that the head lobes are very apparent and contain protonephridia, which leads to the conclusion that these animals earlier had a metamorphosis. From the resemblance of the trochophore to the Rotifera the farther conclusion is drawn that the annelids have descended from rotifer-like ancestors, the body cavity, nephridia, blood-vessels, and ventral nerve chain being new formations.

Besides sexual reproduction many fresh-water and marine species reproduce asexually, this being possible from the great homonomy of the segmentation. By rapid growth at the hinder end as well as at a more anterior budding zone numerous somites are formed, which separate in groups from the parent to form young worms. In some cases the formation of new somites may take place more rapidly than the separation, the result being chains of worms (fig. 258) which in some instances branch.

By a combination of sexual and asexual reproduction a typical alternation of generations occurs, the origin of which receives light from the following facts: 
In many polychates which reproduce exclusively ly the sexual process the sexless, slowly-moving young (atoke) becomes so altered at sexual maturity as to have been described under another name. It becomes very active in its movements, and the hinder somites, which contain the gonads, develop special bristles and parapodia (fig. $26_{3}, 4$ ). 'Thus many species of $N$ ereis pass into the 'Heterancreis' stage. In other Polychrtes the sexual part (cpitoke) separates from the sexless atoke portion and swims freely, while the atoke produces new epitokes. In Samoa Eunice viridis reproduces in this way, the epitokes coming to the surface at certain times in incredible numbers, forming the 'palolo worm,' a delicacy in the Samoan diet. In still other species the epitoke regenerates the head and thus becomes an independent generation. Syllis and Heterosvllis are thus related. The Autolytidæ are most complicated. Here the atoke, by

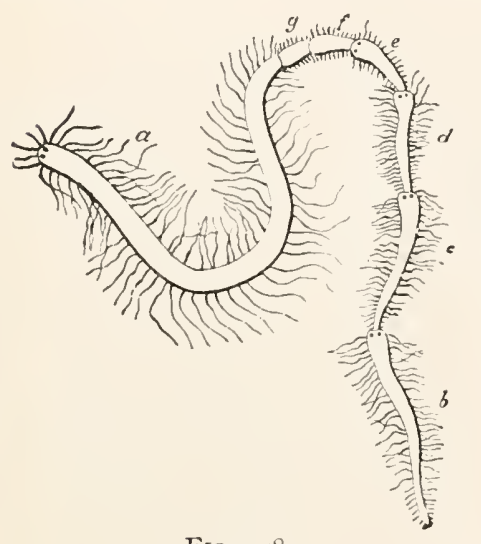

FIG. 258 .

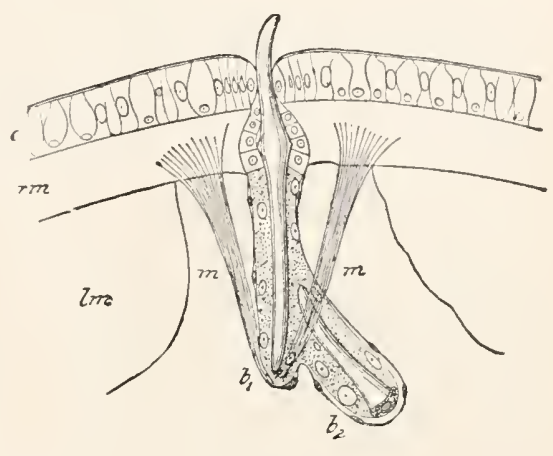

FIG. 259 .

FIG. 258.-Budding in Myrianida (after Milne-Edwards). The sequence of letters shows the ages of the individuals.

FIG. 259.-Arrangement of a bristle in an Oligochæte (after Vejdowski). $e$, epithelium; $r m, l m$, circular and longitudinal muscles; $m$, muscle of the follicle; $b_{1}$, chæta follicle, its chæta in function; $b_{2}$, follicle for replacement, the formative cell at its base.

budding as in Myrianida (fig 258), forms chains of dimorphic individuals which later separate. The individuals of male chains (fig. 263) were formerly described as 'Polybostrichus,' the females as 'Sacconereis.' 'This same homonomy explains the regenerative powers of many worms. Thus if certain earthworms be cut in two, they will live and reproduce the lost parts.

Another important character of the Chrtopoda is the possession of bristles or chata. These arise in special follicles, singly or several in a group, there usually being four groups-right and left, dorsal or lateral and ventral-in each somite. Each follicle (fig. 259) is a sac of epithelium opening to the surface and having at the base a special cell for the development of each bristle. The developed chrete project from the follicle and, moved by appropriate muscles, form small levers of use in locomotion. Their numbers, shape, and support are of much systematic importance. 


\section{Order I. Polychætæ.}

The Polychætæ owe their name to the fact that each group of bristles contains many chætæ; but more important is that the bristles of each side

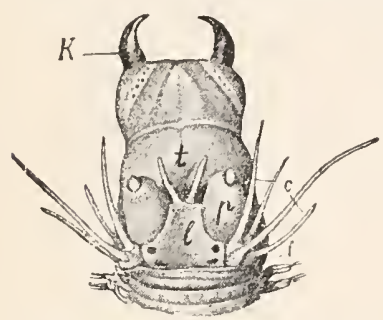

FIG. 260.--Head with protruded pharynx of Nereis versipedata (after Ehlers). c, cirri, $k$, jaws; $l$, head with eyes; $p$, palpi; $t$, tentacles. are supported by a fleshy outgrowth of the somite, the parapodium, in which two portions corresponding to the bunches of bristles-dorsal, notopodium; ventral, neuropodium-may be recognized (fig. 254). This is the first appearance of true appendages, but they differ from those of Arthropoda in not being jointed to the body nor jointed in themselves. On the dorsal surface may occur diverse outgrowths, known, according to position or function, as cirri, elytra, gills, etc.; on the head, palpi and tentacles. The cirri are long processes on the parapodia, and like palpi are tactile (fig. 254). Elytra are thin lamelle which cover the back like

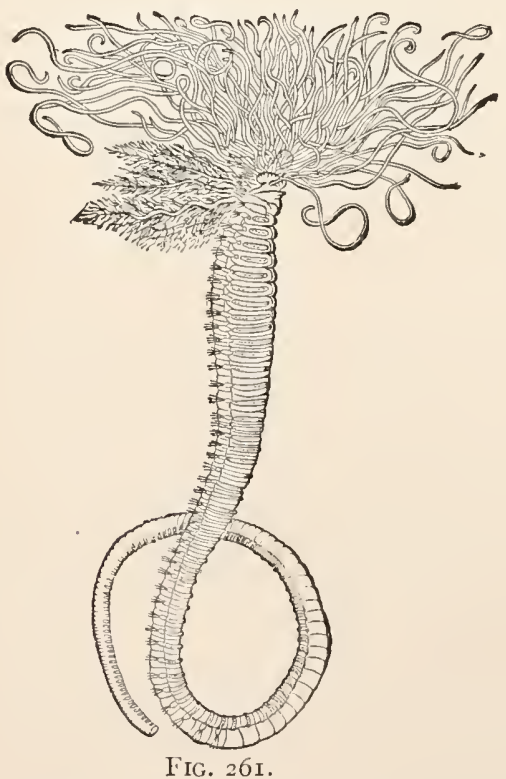
shingles and thus protect the body (fig. 262).

Nearly all Polychates are diœcious and undergo a more or less pronounced metamorphosis; with few exceptions (Manyunkia* from the Schuylkill, Nereis*in California) they are marine. They are usually

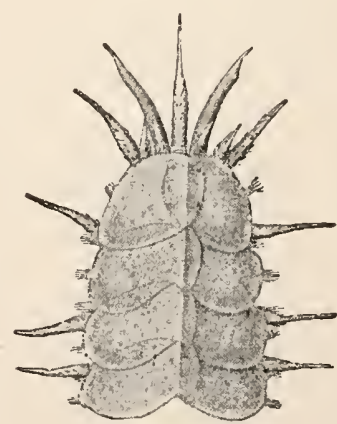

FIG. 262 .

FIG. 261.-Amphitrite ornata $*$ (from Verrill).

Fig. 262.- - Head of Polynoe spinifera (after Ehlers). Back entirely covered with elytra; cirri and parapodia projecting at the sides.

divided according to their habits into fixed (Sedentaria) and free forms (Errantia). 'The Sedentaria feed on regetable matter, usually form leathery 
organic tubes in which foreign matter may be incorporated or which may be calcified. The worms project their anterior segments from the tubes. 'The Errantia often burrow, but from time to time swim about preying on other animals. Correlated with habits are differences in structure. In the Errantia the head and trunk are not very different; the anterior part of the alimentary tract can be protruded as a proboscis, and, corresponding to their predaceous habits, is often armed with strong jaws (fig. 260). The Sedentaria have no such teeth, but there is a greater difference between anterior and posterior somites. In the latter the parapodia are weakly developed, and this part resembles the Oligochates in appearance. The head and beginning of the trunk (thorax) are richly provided with gills and tentacles for respiration and taking food (fig. 26I).

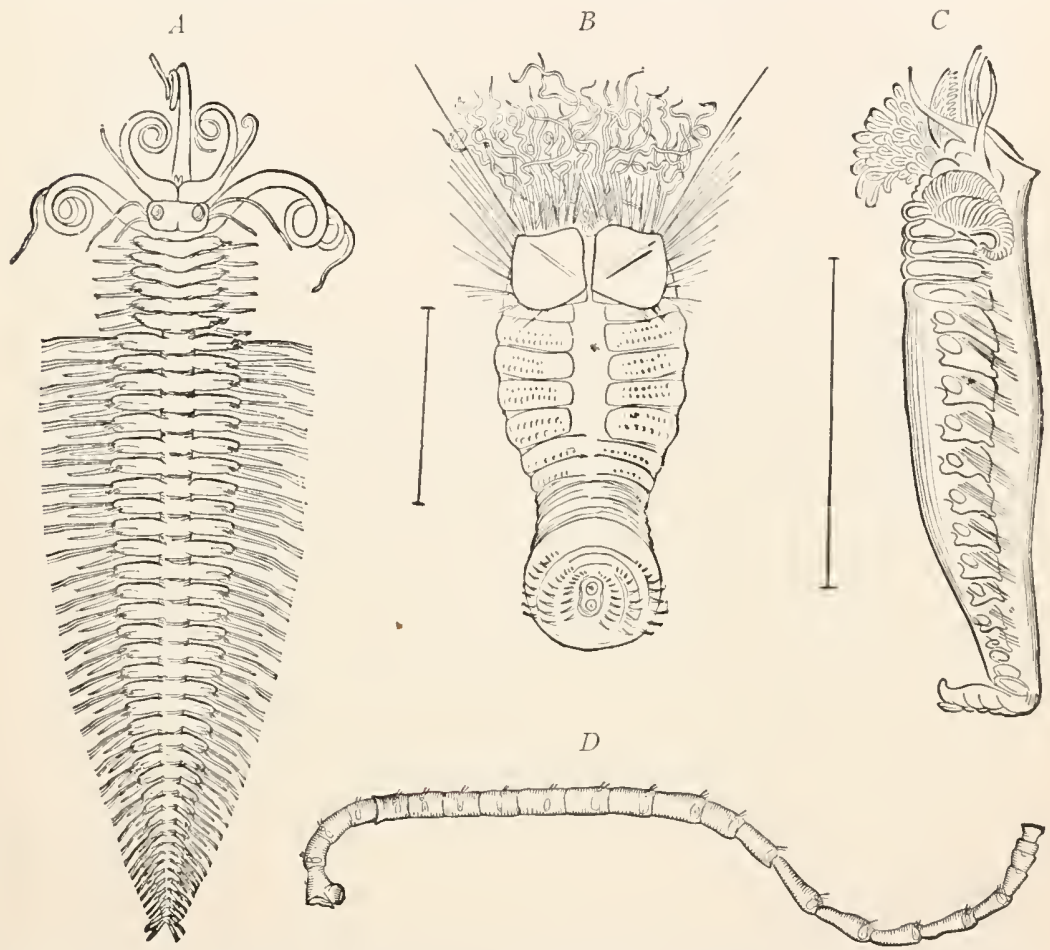

FIG. 263.-New England Annelids (from Emerton and Verrill). A, male Autolytus; $B$, Sternaspis fossor; $C$, Cistenides gouldii; $D$, Clymene torquata.

Sub Order I. ERRANTIA. Predaceous annelids with strongly armed pharynx. The EunICIDÆ, mostly represented on our shores by small species, contains some species a yard in length. Diopatra, Nothria** Alciopide, transparent, pelagic, with large, highly developed eyes (fig. 84). The SrLliD usually have three long tentacles; Autolytus* (fig. 263), Myrianida* (p. 275). The Polynoide* (Lepidonotus, * Polynoe* (fig. 262), are bottom forms with elytra. Nereide; Nereis virens, * clam worm of all northern seas.

Sub Order II. SEDENTARIA (Tubicola). These cannot wander, but live in tubes. SABELLID 2 , tube is membranous and there is a crown of gills; Mywicola, Chone, Manyunkia.* SERPUlide, tube calcified and closed by an 
operculum on one of the gills. Hydroides, Protula* Arenicolid $\approx$, * burrow in sand, have gills on the sides of body. MaLdanide (Clymene, fig. 263) have extremely long segments and build tubes of sand. TEREBELLIDE (Terebella, * Amphitrite (fig. 26I), numerous filiform tentacles and branched gills on the anterior end.

The ARCIIIANELLIDE, which lack bristles and parapodia, must be placed near the Polychætæ and are usually regarded as very primitive forms which in structure and development (fig. $25 \%$ ) are of importance in the phylogenesis of the Annelids. Polygordius.*

\section{Order II. Oligochætæ.}

The Oligochætes are almost as preeminently fresh-water and terrestrial forms as the Polychates are marine. They are in most respects simpler than their marine relatives, apparently the result of degeneration. Eyes are rudimentary or lacking, there are no palpi, cirri, or tentacles; gills are rare, but most striking

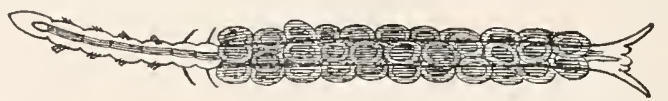

Fig. 264.-A Alophorus vagus, * in tube of Pectinatella statoblasts (after Leidy).

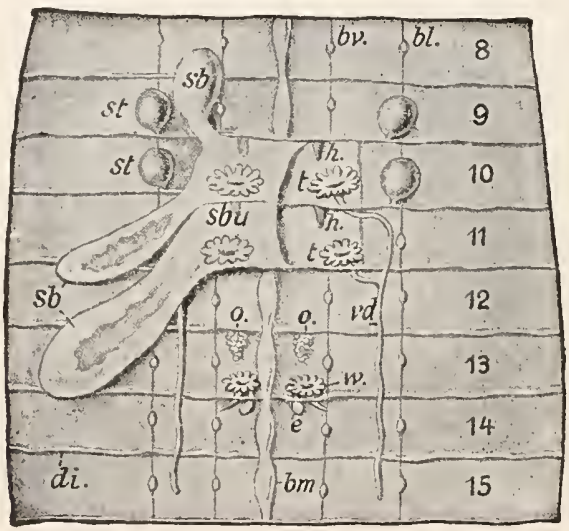

FIG. 265.-Sexual organs of Lumbricus agricola (from Lang, after Vogt and Yung). The seminal vesicles of the right side are removed. $b m$, ventral nerve cord; $b v$ and $b l$, ventral and lateral rows of setæ; $s t$, receptacula seminis; $s b$, seminal vesicles of the left side, connected with a median unpaired seminal capsule (sbu). Enclosed in the latter are the testes $(h)$, and the seminal funnels $(t)$, which lead into the vas deferens $(v d)$. o, ovaries; $w$, ciliated funnels leading to oviducts with egg capsule $(e)$; di, dissepiments; 8-15, eighth to fifteenth segments.

is the absence of parapodia, the bristles projecting directly from the body wall (fig. 259). The chrete may be regularly distributed around each somite (Pericheta) or gathered on the sides (Megascolex) or arranged in four groups so that in the animal four longitudinal rows occur. The animals are hermaphroditic, testes and ovaries lying in different somites. Usually the skin near the sexual openings is thickened by numerous glands, forming a clitellum (fig. 252), which secretes the egg cocoons and also functions in copulation, secreting bands which hold the animals together so that the sperm from one passes into the receptac- 
ulum seminis of the other. After impregnation the eggs are usually enclosed in cocoons.

Sub Order I. LIMICOLA (Microdrili). Mostly fresh-water. 'The TUBIFICIDE, in consequence of the red blood, when present in large numbers

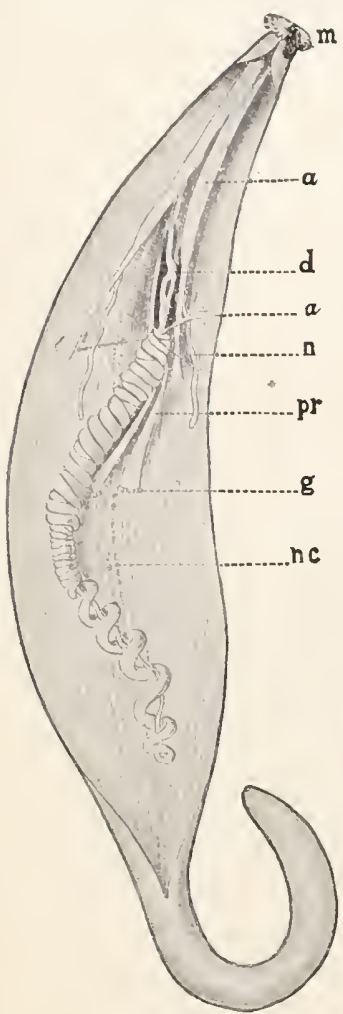

FIG. 266. color the bottom red. They form tubes in the mud. Tubifex,* Clitellio irroratus* common on seashore. NAIDID E, transparent forms living on water plants, reproduce asexually. Dero* and Aulophorus* (fig. 264) have gills around the anus. Exchytraide; Pachydrilus. Discodrilide (Myzobdella), parasitic. Sub Order II. TERRICOLA (Macrodrili). Terrestrial; the earthworms, our species of moderate size, in the tropics large (Megascolex australis four feet long). Lumbricus, * Allobophora*; Diplocardia* with double dorsal blood-vessel; Perichata* introduced from the

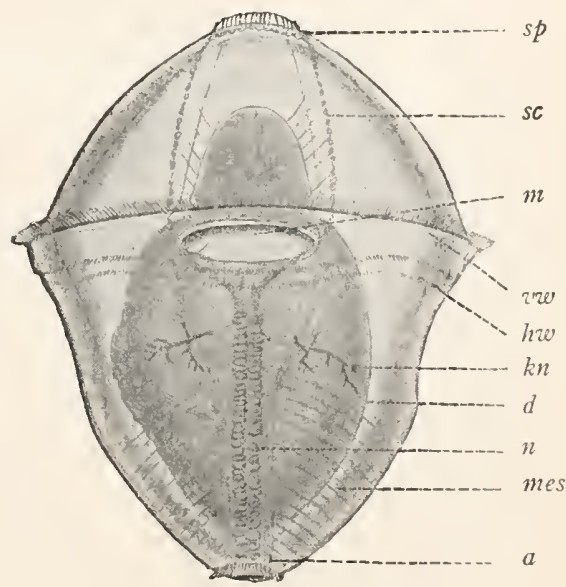

Fig. 267 .

Fig. 266.-Anatomy of Phascolosoma gouldi (orig.). a, anus; a, anterior retractors; $d$, digestive tract; $g$, gonads; $m$, mouth; $n$, nephridia; $n c$, ventral nerve cord; $p$ r, posterior retractors.

FIG. 267.-Larva (trochophore) of Echiurus (after Hatschek). a, anus; $d$, intestine; $h w$, postoral cilia; $k n$, protonephridia; $m$, mouth; mes, mesoderm bands with indication of segments; $n$, ventral nerve cord; $s c$, cesophageal commissure; $s p$, apical plate; vw, preoral ciliated band.

tropics. Most species burrow through the earth, swallowing the humus and casting the indigestible portions on the surface. 'They loosen the soil and continually bring the deeper parts to the surface. Details of the reproductive organs of one species in fig. 265. These vary and are used in classification.

\section{Sub Class II. Gephyrca.}

The exclusively marine Gephyraea are distinguished at the first glance from the Chetopoda by the absence of segmentation. The body is oval 
or spindle-shaped, circular in section. The mouth, at the extreme anterior end, is either surrounded by a circle of tentacles (fig. 266), retracted together with the anterior end of the body by internal muscles, or is overhung by a dorsal preoral lobe or proboscis which may be several times the length of the body and forked at its tip (fig. 268).

Internal segmentation is also lost, septa being entirely lacking. The nephridia are reduced in number, at most but three pairs being present, and in some but a single unpaired organ. They are the sexual ducts; the Chrtiferi have special excretory organs (fig. $268, g$ ) covered with branching canals opening to the body cavity by nephrostomes and
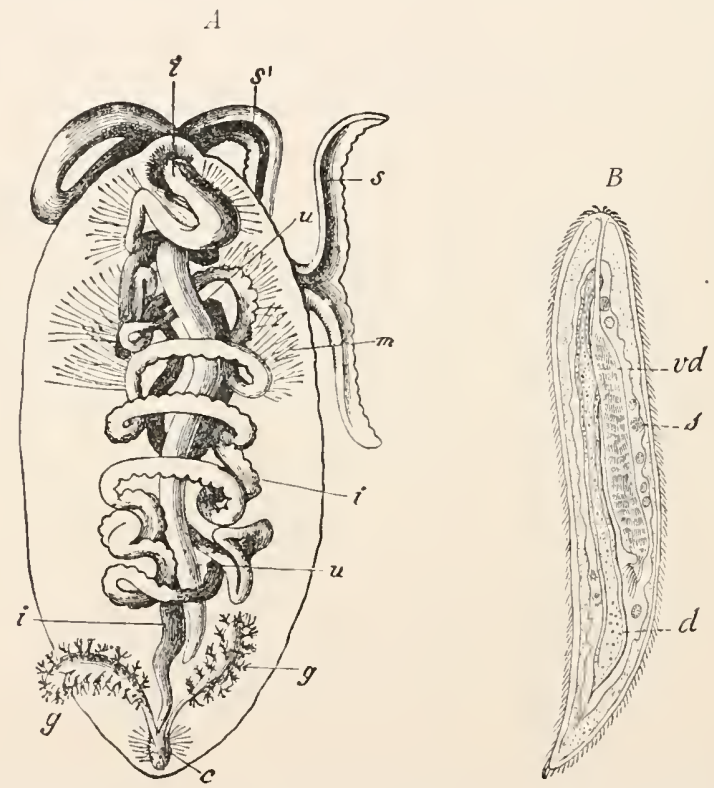

FIG. 268.-Bonellia viridis. A, female (after Huxley); $B$, male (after Spengel). $c$, cloaca $; d$, ruclimentary intestine: $g$, excretory organ; $i$, intestine; $m$, muscles supporting intestine; $s$, balls of spermatozoa in $B$, in $A$, proboscis (preoral lobe); $u$, single segmental organ, functioning as oviduct; $v d$, nephridium with ciliated funnel serving as vas deferens.

emptying into the intestine. These resemble somewhat the branchial trees of the holothurians (infra), and hence the gephyraa were formerly supposed to bridge the gap between holothurians and annelids, whence the name ( $\gamma$ '́申vpa, bridge). The vascular and nervous systems are more like those of other annelids. The vascular system consists of a sinus around the digestive tract and a dorsal and usually a ventral longitudinal trunk; the nervous system of a brain, œsophageal collar, and ventral cord, 
the latter without division into ganglia. The relations of the Gephyrea to the Chrtopoda are shown by the development. In some (Chetiferi) there is a trochophore (fig. 267) from which the worm arises, as in the Chrtopoda, by growth at the hinder end; this at first has a segmented colom and nervous system, the metamerism being lost later.

\section{Order I. Chætiferi (Armata, Echiuroidea).}

With spatulate preoral lobe, often forked at the tip; at least a pair of ventral setx; a trochophore in development. Echiurus, * northern, Thalassema.* In Bonellia there is a marked sexual dimorphism (fig. 268); female, 2 to 5 inches, has a proboscis a yard long. The male, only $\mathrm{x} \mathrm{mm}$. long, totally different in form and color, lives parasitically in the cesophagus of the female (fig. 268, $B$ ).

\section{Order II. Inermes (Achæta, Sipunculoidea).}

Distinguished by lack of chætæ, the mouth surrounded by tentacles, and the dorsal and anterior position of the anus. The larva is a modified trochophore without preoral ciliated band and without segmentation; only two, sometimes but one, nephridia. 'The vascular ring around the mouth, with its dorsal, heart-like prolongation, is not circulatory. It is a special part of the coelom for the protrusion of the tentacles and has no connection with the intestinal blood sinus. It is doubtful whether the Inermes are related to the Chætopoda. Some unite them with Brachiopoda and Polyzoa in a group Prosopygii, so called in allusion to the dorsal position of the anus. Phascolosoma* (fig. 266). Phascolion, * Sipunculus.*

\section{Order III. Priapuloidea.}

No tentacles, mouth with teeth, terminal anus, two protonephridia united with sexual organs and opening either side of vent. Priapulus.

\section{Sub Class III. Hindinei (Discophori).}

Three points of external structure distinguish the leeches from the chetopods. First, the absence of bristles (except in Acanthobdella) and the presence of two suckers; the one on the posterior ventral surface is used only for attachment and locomotion, the other, sometimes scarcely differentiated, surrounds the mouth and is used in sucking the food. In locomotion anterior and posterior suckers are alternately attached, the body being looped up and extended like that of a 'span worm.' The animals can also swim by a snake-like motion of the whole body.

A second point is the fine ringing of the body, there being usually many more rings than somites, the segments being divided by secondary constrictions, there being three, five, or even eleven rings to a segment. The middle or one of the anterior rings often bears strongly developed sense organs. As in earthworms, certain of the somites may develop a clitellum which secretes the egg cocoons. 
A third character is the marked dorsoventral flattening of the body (except in Ichthyobdellide and a few other forms), the animals thus recalling the flatworms. This may be the result of the very slight development of the colom. In most leeches there is a body parenchyma, traversed by muscles in which the organs are immediately imbedded (fig. 269).

The alimentary tract bears paired diverticula (fig. 270 ), varying in number in different species. Between the last and largest pair of these is the intestine, which opens dorsal to the posterior sucker. The jawed and jawless leeches show considerable differences in the pharyngeal region. In the first there are three semicircular jaws in the pharynx, the free edge of each armed with teeth (fig. $27 \mathrm{I}$ ). To these are attached two muscles, one to retract them, while the other exserts and rotates them, causing a triradiate wound from which the blood flows. This bleeding is difficult to staunch, since a secretion of glands on the lips and between the jaws

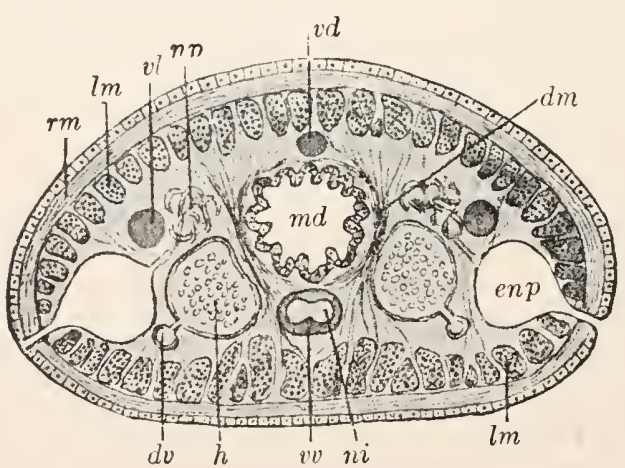

FIG. 269 .

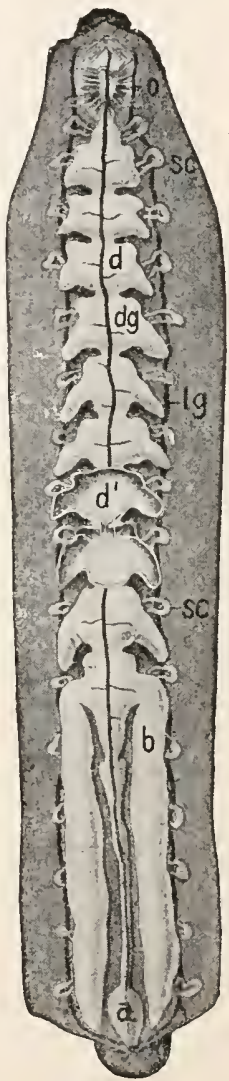

FIG. 270.

FIG. 260.-Transverse section of Hirudo medicinalis (from Lang). $d m, l m, r m$, dorsoventral, longitudinal, and cirular muscles; $v l, v d$, $v v$, lateral, dorsal, and ventral blood-vessels, the latter surrounding the ventral nerve cord, $n i ; h$, testes; $2 d$, vas deferens; md, midgut; $n p$, nephridial tubule; enp, urinary bladder.

FIG. 270.-Digestive tract of Hirudo medicinalis (after Hatschek). a, rectum and anus; $b$, last crecal pouches of intestine; $d$, intestine, opened at $d^{1} ; d g$, dorsal, $l g$, lateral blood vessel; o, cesophagus; sc, nephridia.

hinders the coagulation of the blood. In the jawless leeches a sharp conical process arising from the pharynx serves for wounding and sucking. The vascular system usually contains red blood, and consists, in the Gnathobdellidie, of four longitudinal trunks, a dorsal, two lateral, 
and a ventral, the latter surrounding the ventral nerve cord. These are connected by a complicated system of capillaries.

The nerrous system consists of lorain and ventral cord, the latter containing frequently twenty-three ganglia (the first of five fused, the last of seven). Nerves from the brain go to the eyes. Right and left of the ventral cord are the hermaphroditic sexual organs. In Himdo medicina-

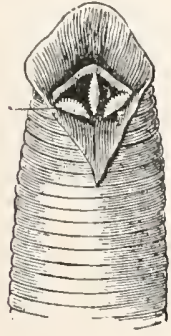

$a$

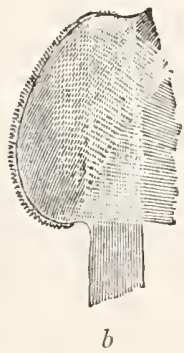

FIG. 27 I.

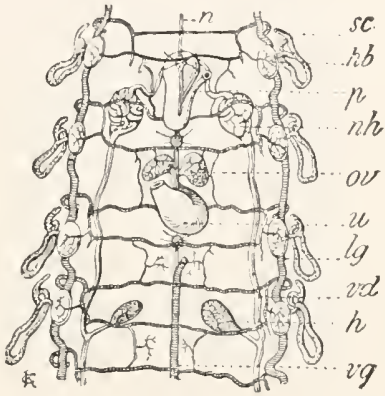

FIG. 272 .

FIG. 27 I.-Hirudo medicinalis, medicinal leech (after Leuckart). a, anterior end with three jaws $(k) ; b$, a single jaw with its muscles.

Fig. 272.- Nervous system, blood-vessels, sexual organs, and nephridia of a leech, ventral view. $h$, testes; $h b$, urinary bladder; $l g$, lateral blood-vessel; $n$, ventral nerve cord; $n h$, epididymis: $o v$, ovary; $p$, penis; $s c$, nephridia; $u$, uterus and vagina; $v d$, vas deferens; $v g$, ventral blood-vessel.

lis (fig. 272 ) there are nine pairs of testes $(h)$, the ducts of which unite to form a vas deferens on either side $\left(z^{\prime} d\right)$. These pass forward, form by coiling a so-called epididymis $(n h)$ and empty into the median unpaired penis $(p)$. In the jawless leeches the penis is lacking and the sperm, in pointed sprematophores, is inserted in the tissues of the leech. In the space between the epididymis and the first pair of testes are the ovaries (ov) and oviducts and an unpaired vagina $(u)$. The nephridia (I7 pairs in this species) are complicated canals and are provided with bladderlike expansions.

That the Hirudinei are true annelids and not segmented plathelminthes is based upon the view that their coelom is reduced by ingrowth of parenchyma and altered to canals connected with the vascular system. At any rate the ventral and lateral vessels are to be regarded as remnants of a colom. In Clepsine, etc., there are the dorsal and ventral blood-vessels of the Chætopoda and besides four longitudinal coelomic sinuses connected by lacunar spaces. The dorsal sinus encloses the dorsal blood-vessel, the ventral many of the viscera, among them the ventral nerve cord. These sinuses also have flagellated funnels which lead into lymphoid capsules, not, as was formerly thought, into nephridia. In the jawed leeches (apparently by degeneration as is the case in many polychetes) the true blood-vessels are replaced by a canal system derived from the coelomic sinuses, which in Nephilis has in part a lacunar character. For the 
origin of these vessels from the cœlom the following points are in favor.

The ventral cord is enclosed in the ventral blood sinus; (2) the flagellate funnels, just alluded to, lie in the blood lacunæ, usually in ampullar spaces between the ventral and lateral blood sinuses. Further relations are shown by Acanthobdella peledina, parasitic on fishes. This has both blood-vessels of the oligochætes, a body cavity divided by septa, and chætæ. On the other hand, it is leechlike in other features; two suckers and sexual apparatus on the Hirudinean pattern. Branchiobdella, parasitic on the gills of the crayfish, is a chætopod devoid of bristles and furnished with a sucker in correlation with its habits.

\section{Order I. Gnathobdellidæ.}

The jawed leeches include Hirudo medicinalis, once extencively used for blood-letting, now little employed. Hamadipsa includes land leeches of the tropics. Nephelis* soft jaws. Macrobdella* includes our largest species.

\section{Order II. Rhynchobdellidæ.}

Without jaws. ClepsPinide mostly feed on snails and fishes. Clepsine* Homentaria ghiliani of South America is poisonous. ICHTHYOBDELLIDe, ${ }^{*}$ cylindrical, occur in salt and fresh water, parasitic on fishes. Ichthyobdella,* Pontobdella, * marine; Piscicola, fresh water.

\section{Class IV. Polyzoa (Bryozoa).}

In cxternal appearance the Polyzoa closely resemble the hydroids,

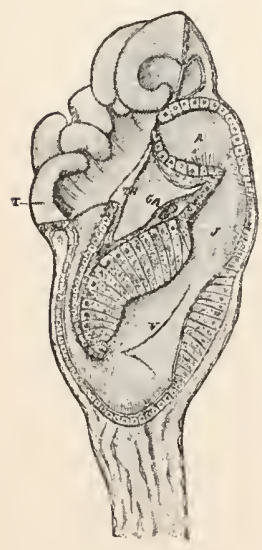

Fig. 273.--Loxosoma singulare (after Nitsche) in optical section. $A$, rectum; $G a$, ganglion; $J$, intestine; $T$, tentacles; $Y$, stomach. so that the inexperienced have difficulty in distinguishing them. Like them by budding they form colonies which are either incrusting sheets or assume a more bush-like character. Further they have a crown of ciliated tentacles which can be spread out or quickly retracted. In internal characters the two groups are greatly different. The Polyzoa have a complete alimentary canal, with its three divisions, which is bent upon itself so that the anus lies near the mouth. The central nervous system lies between mouth and anus, and the single pair of nephridia empty by a common opening. Beyond this it is difficult to go, since the two groups-Entoprocta and Ectoprocta-differ widely. The Entoprocta have no colom, resembling in this respect the Rotifera; the Ectoprocta are true Colhelminthes and by way of Phoronis show resemblances to the Sipunculoida ('Prosopygii,' p. 28I ) and also to the Annelida.

\section{Sub Class I. Entoprocta.}

The single individuals of the Entoprocta (fig. 273) are shaped like a wineglass and are placed on stalks which rise from (usually) creeping stolons. The 
circle of tentacles, arising from the edge of the cup, enclose the peristomial area in which are both mouth and anus, and between these the excretory and reproductive organs open. The space between the horseshoe-shaped intestine and the body surface is filled by a parenchyma containing muscle cells, and correspondingly the excretory organs are protonephridia. Urnatella, ${ }^{*}$ freshwater. Pedicellina, Loxosoma, marine.

\section{Sub Class 1I. Ectoprocta.}

The Ectoprocta have a spacious, often ciliated, cœlom between the alimentary canal and skin, so that these are separated and have a certain amount of independence (fig. 274). On this account has arisen a peculiar method (morphologically indefensible) of treating them as two individuals, polypid, the intestine and tentacles; cystid the rest, especially the body wall and skeleton.

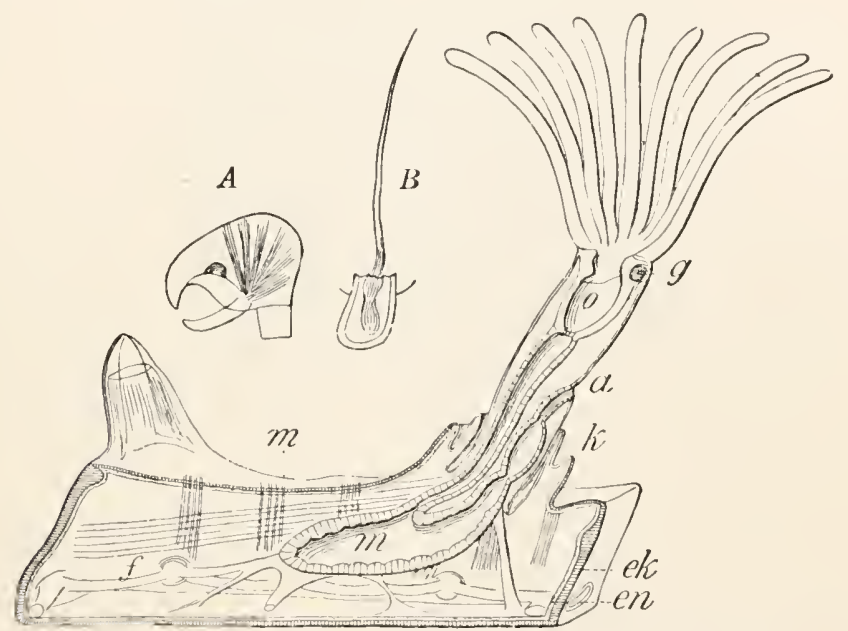

FIc. 274.-Flustra membranacea (after Nitsche), a single animal. $a$, anus; $e k$, ectocyst; $e n$, entocyst: $f$, funiculus; $g$, ganglion; $k$, collar which permits complete retraction: $m$, stomach, also dermal muscular sac; $o$, osophagus. $A$, avicularium; $B$, vibracularium of Bugula (after Claparède).

The cystid is cup-shaped or saccular. It consists of an endocystthe body wall-and an ectocyst-a cuticular skeleton, often strongly calcified, secreted by the ectoderm. The ectocyst covers only the base and side walls of the endocyst, leaving the outer end soft, thus forming a sort of collar into which the tentacles and adjacent parts of the cystid can be retracted. The opening thus formed in the ectocyst in many species (Chilostomata) can be closed by a lid (operculum). The circle of tentacles surrounds the mouth alone, while the anus is outside near the collar. The 
strongly bent alimentary canal extends into the colom and is bound at its hinder end by a cord, the funiculus, to the base of the cystid. Ganglion and nephridia lie between the mouth and anus. The gonads arise from the epithelium of the cœlom, the testes usually on the funiculus, the ovaries on the wall of the cystid.

Hundreds and thousands of individuals form colonies (fig. 275) in which cystid abuts against cystid. The cœlom of adjacent cystids may be distinct or a wide communication may exist. The colonies grow by budding; in the Gymnolæmata a part of a cystid becomes cut off as a

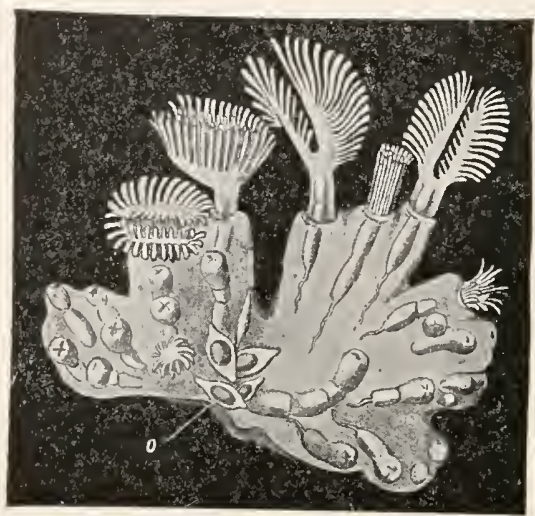

FIG. 275.-Small colony of Lophopus crystallinus (after Kraepelin), with young and old, some extended, others more or less retracted. o, statoblasts.

daughter cystid in which the polypid-alimentary tract and tentaclesarises by new formation; or (Phylactolæmata) the bud anlage of the polypid arises before the first appearance of the cystid.

Division of labor or polymorphism is common. Besides the animals already described, which are primarily for nourishment, three other individuals may occur, ovicells, vibracularia, and avicularia. All three are cystids which have lost the polypid The ovicells are round capsules which serve as receptacles for the fertilized eggs. The vibracularia (fig. 274, $B$ ) are long tactile threads: the avicularia (fig. $274, A$ ) are grasping structures which seize small animals and hold them until decay sets in; the fragments serve as food for the polypids. The avicularia have the form of a bird's head, the movable lower jaw being a modified operculum.

Under unfavorable conditions a polypid in a cystid may break down and be lacking for some time until better relations cause its new formation. Besides, in the depopulated cystids, there may appear statoblasts, internal buds enveloped in a firm envelope which form a resting stage (fig. 275). Each statoblast is surrounded by a girdle of chambers which by drying become filled with 
air, causing the statoblast to float when it again comes into water. From the statoblast a smaller polyzoon escapes which develops a new colony. 'The statoblasts are adaptations to the conditions of fresh-water life and occur only in the Phylactolamata, where they arise as a sort of internal burls on the fu. niculus, just before the degeneration of the polypids.

\section{Order I. Gymnolæmata (Stelmatapoda).}

Tentacles in a ring around mouth. Numerous species, almost exclusively marine. CHILOSTOMATA, cystids can be closed by an operculum: Gemmellaria, Cellularia, Bugula, Flustra* (fig. 274), Eschara** CYCLOSTONIATA, tubular cystids without operculum. Crisia, * Tubulipora, * Hornera.* CTENUSTOMATA, cystid is more calcareous, closed by a folded membrane. Alcyonidium, Vesicularia, Valkeria,* marine; Paludicella* (fresh-water).

\section{Order II. Phylactolæmata (Lophopoda).}

Tentacles borne on a horseshoe-shaped lophophore extending on either side of the mouth, the tentacles on its margins. All are fresh-water species. Pectinatella, * Lophopus (fig. 275), Plumatella.*

\section{Class V. Phoronidea.}

The single genus Phoronis* was first called a chætopod on account of its worm-like body situated in a chitinous tube like many sedentary annelids. Then it was placed in the Polyzoa, with which it is more nearly related. The young, described as Actinotrocha, is a modified trochophore with the mouth overhung by a large hood and with the postoral band of cilia drawn out into a series of fingers which become the tentacles of the adult; the anus is terminal. At the metamorphosis this larva becomes doubled on itself, so that the alimentary canal is $\mathrm{U}$-shaped, the anus near the mouth, while the tentacles are borne on a horseshoe-shaped basis around the mouth.

\section{Class VI. Brachiopoda.}

On account of the bivalve calcareous shells the Brachiopoda were long regarded as molluscs, but later the fact that the valves are not paired as in the lamellibranchs, but are dorsal and ventral, that the nervous system, the excretory and reproductive organs, the body cavity, and the development resemble those of the annelids rather than those of the molluscs, led to their recognition as a distinct class allied to the former group.

The body has a greatly shortened long axis (fig. 276) and in consequence a transversely oval visceral sac. In most a stalk (st) for attachment arises from the posterior end. From the anterior side two folds, the mantle lobes $(p)$, extend forward, one ventral, the other dorsal, their free edges bearing bristles. Each mantle secretes a calcareous shell. In a few the clorsal and ventral shells are similar, but usually the ventral valve (in Crania attached directly without the intervention of a stalk) is more 
strongly arched and has an opening at the posterior end for the passage of the stalk (figs. 277,278 ). The flatter dorsal valve frequently bears a characteristic feature in the calcareous skeleton of the arms (fig. 278) which, when present, has very different forms. When closed the valves completely

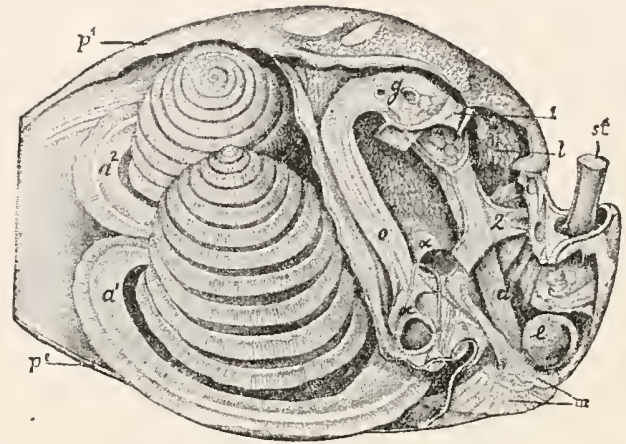

FIG. 276.-Anatomy of Rhynchonella psitlacea (after Hancock). $a^{\prime}$, left, $a^{2}$, right arm; $\alpha$, opening in to the cavity of the arm; $d$, intestine; $e$, blind end of the in testine; $g$, stomach with liver; $m$, adductors and divaricators of shell; 0 , cesophagus; $p^{\prime}, p^{2}$, dorsal and ventral mantle lobes; st, stalk; I, 2, first and second septum, on the second a nephrostome.

cnclose the body. When they open the gape is anterior, the posterior parts remaining in contact. Here, except in the Ecardines, a hinge is developed just in front of the posterior margin, consisting of teeth in the ventral valve which fit into corresponding grooves in the dorsal. Opening

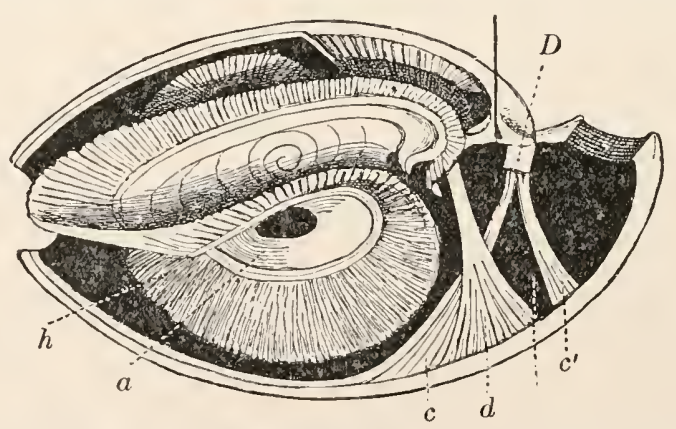

FIG. 277.-II'aldheimia flavescens (from Zittel). Shell with arms and muscles. $a$, arm with fringed border $(h) ; c, c^{\prime}$, divaricators; $d$, adductors; $D$, hinge process (the vertical line shows position of hinge).

and closing the valves are, contrary to what occurs in Lamellibranchs, active processes, accomplished by appropriate divaricator and adductor muscles (fig. 277). These produce scars on the shell, important in the study of fossil forms. 
The usually spirally coiled arms, which lie right and left of the mouth and which give the name to the class, fill most of the shell. On the outer side of each arm is a longitudinal groove, bounded by a row of small tentacles. By means of cilia on tentacles and groove food is brought to the mouth. These arms resemble the lophophore of a phylactoliemate Polyzoan, which only needs extension and coiling to produce this condition. In development the arms of the Brachiopod pass through a lophophore stage.

In the body there is a ciliated colom which extends into both arms and mantle folds. It encloses alimentary tract, gonads, and liver, and is

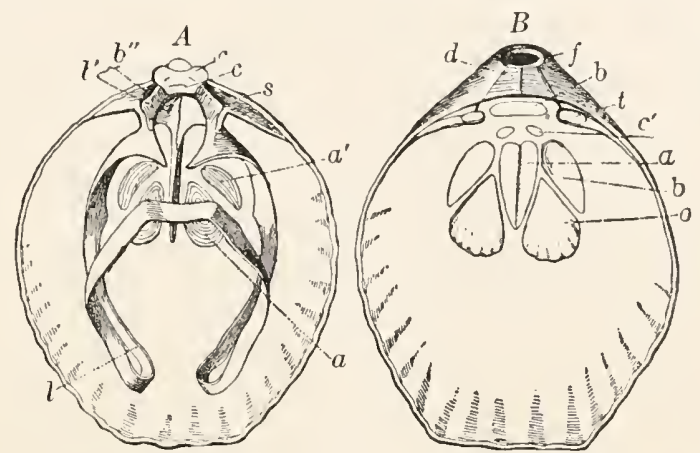

FIg. 278.-Waldheimia flavescens (from Zittel). A, dorsal, $B$, ventral valve; $a$, $b, c$, impressions of muscular insertions; $a$, adductors; $b^{\prime \prime}$, adjustors (stalk muscles); $c, c^{\prime}$, divaricators; $s$, hinge groove of upper valve in which the tooth $(t)$ of the lower valve passes; $l$, support of arms; $d$, deltidium; $f$, foramen for stalk.

divided into right and left halves by dorsal and rentral mesenterics supporting the intestine. Each half in turn is divided by incomplete septa into anterior, middle, and posterior divisions recalling those of Sagitta (p. 252). The arrangement of the septa is not so clear as in that form, the result of the shortening of the long axis and the twisting of the alimentary tract. This latter consists of œesophagus, stomach, which receives the liver ducts, and intestine, which in some species terminates blindly.

The gonads are chiefly in the mantle lobes. The sexual cells pass out through the nephridia, which begin in one colomic pouch with wide nephrostomata, perforate the septum, and open to the exterior in the next somite. Since usually there are two septa, two pairs of nephridia may occur, but one is usually degenerate. The nervous system consists of an œsophageal ring with weak dorsal ganglion, extending into the arms, and a stronger ventral mass representing the ventral chain. The heart lies dorsal to the stomach. 
In development the brachiopods recall both Sagitta and the Annelida. They resemble Sagitta in that in Argiope the colom arises by outgrowths from the archenteron (fig. 279), divided later by septa into three pairs of pouches. They are annelid-like in the form of larva and in the presence of chretre which are formed in separate follicles. Brachiopods were formerly so numerous that they are among the most important fossils in the determination of geologic horizons. Now there are but few species, some in habitants of the greatest depths of the sea.
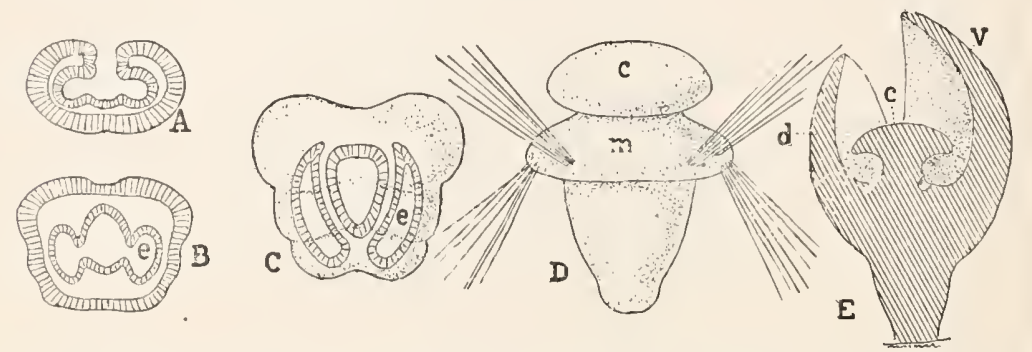

FIG. 279.-Development of brachiopod (after Kowalerskv). A, gastrula with early enterocolic pouches; $B$, closure of blastopore; $C$, colom separated, body annulated; $D$, cephalic disc and mantle developing, the latter with long setæ; $E$, attached embryo, the mantle lobes folded over cephalic disc (setæ omitted). $\quad c$, cephalic disc; $d$, dorsal lobe of mantle; $e$, enteroccle; $m$, mantle; $v$, ventral mantle lobe.

\section{Order I. Ecardines.}

Hinge absent: valves similar, the stalk passing between them (Lingula*),

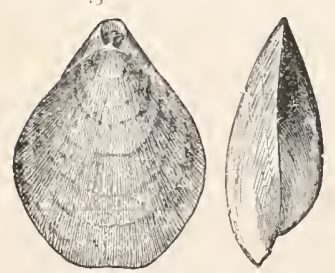

FIG. 2 So.-Terebratulina septentrionalis.* or unequal, the ventral perforated by the stalk (Discina), or the animal is directly attached by the shell (crania).

\section{Order II. Testicardines.}

Hinge present, valves unequal, the ventral perforated by the stalk; anus degenerate. Rhynchonella, * Terebratulina* in our colder waters. Spirifer, Orthis, Pentamerus, Atrypa, important fossil genera.

\section{Summary of Important Facts.}

(I) The CELHELMIINTHES have a well-developed body cavity (colom).

(2) The Chetognathi are hermaphroditic worms with three pairs of crelomic pouches, with fins, ard bristle-like jaws.

(3) The NemATODA are mostly dicecious, usually parasitic, elongate worms, with cylindrical unsegmented body, with nerve ring (no ganglia), paired excretory organs, and tubular gonads.

(4) The most important species parasitic in man are Ascaris lumbricoides in the small intestine, Oxyuris vermicularis in the large intestine, Ankylostoma duodenalis, and the notorious Trichina spiralis. In hot climates Filaria sanguinis hominis and Dracunculus medinensis.

(5) The Gordiaces have mesenteries and splanchnic mesoderm; they are parasitic in insects. 
(6) The Acanthocephal lack alimentary tract, have a spiny proboscis and a very complicated reproductive apparatus. The adults are parasitic in vertebrates, the young in arthropods.

(7) The CH.topod AxNelids have segmented bodies, the segmentation showing itself in ringing of the body wall and in the separation of the coelom into a series of pouches by transverse septa and the metameric arrangements of blood-ressels, ganglia, and excretory organs.

(8) The СнÆTOPODA are distinguished from other annelids by the chret (usually four groups in a somite) arising in special follicles. 'The chata are few in the hermaphroditic Oiigochæta, numerous and borne on special parapodia in the Polychietae.

(9) The GEPHYRæA are related to the Chretopoda. They are saccular, with tentacles or well-developed preoral lobe. They have largely or entirely lost the segmentation. Evidence of segmentation appears in some cases in development and in the ventral nerve cord and nephridia.

(10) The Hirudiver are hermaphroditic Annelida which lack chetæe, but have sucking discs. Their flattened bodies, ruciimentary coelom, and rich body parenchyma give them a certain similarity to the Plathelminthes.

(I I) The Hirudinei have either a protrusible pharynx (Rhynchobdella) or three toothed jaws (Gnathobdella). To the latter belongs the medicinal leech (Hindo medicinalis).

(12) The PolrzoA are like the Hydrozoa in being colonial and having a circumoral crown of tentacles. They are distinguished by the complete alimentary canal, the large colom, and the ganglionic nervous system.

(13) The Proronidea are closely like the Polyzoa.

(I 4) The BRACHIOPODA have a bivalve shell, the valves being dorsal and ventral.

(15) The body cavity is divided by two septa into three (paired) chambers, of which one, rarely two, are provided with nephridia.

(I6) Most brachiopods are attached by means of a stalk. They are divided into Ecardines, without a hinge and with anus, and Testicardines, with a hinge and no anus.

\section{PHYLUMI Y. ECHINODERMA.}

The Echinoderms differ from most other animals by their radial symmetry, but recall in this respect the Coelenterata, a fact which led to their inclusion in the 'Radiata' (p. 206), a view of their relationships which was set aside on account of their different structure, especially the presence of a colom. In fact the radial symmetry of the echinoderms has a different value, for while in the Colenterata the number four or six is fundamental, Echinoderma are, with few exceptions, five-radiate. Further, the radial symmetry of the Coelenterata is primitive, the echinoderms have descended from bilateral, possibly worm-like, ancestors, as is shown by the bilateral larva and many indications of bilaterality in structure, especially in the more primitive forms (crinoids). This primitive bilaterality is to be sharply distinguished from that resulting from modification of radially symmetrical organs like the sexual and ambulacral systems of highly differentiated echinoderms (bivium of sea 
urchins, trivium of holothurians). One pole of the axis of radial symmetry is marked by the mouth, which in the echinoids, starfish and brittle stars, is turned downward; the other is frequently indicated by the anus.

The structure of the integument gives these animals a characteristic appearance. Calcareous plates arise in the mesoderm, under the epithelium, which form a body armor or test, and since these are usually produced into spines, they give the name Echinoderma, spine skin, to the group. This skeleton at times becomes degenerate, as in the Holothurians (it rarely entirely disappears as in Pelagothuria), but even then shows itself as 'anchors' and 'wheels' of lime. The spharidia and pedicellaria, common in echinoids and asteroids, are characteristic appendages of the integument. The first are sense organs; the latter are usually stalked forceps-like grasping structures with calcareous skeleton. In life they

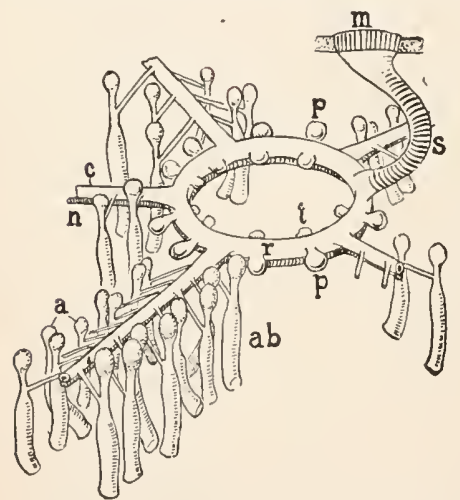

Fig, 28x. - Water-vascular system of starfish (orig.). a, ampulle; $a b$, ambulacra; $c$, radial canal; $m$, madreporite; $n$, radial nerve; $p$, Polian vesicle; $r$, ring canal, beneath it the nerve ring; $s$, stone canal; $t$, racemose vesicle. are active and apparently either clean the skin or are defensive. They are occasionally provided with poison organs.

Certain plates possess a morphological interest since they appear early in many larva, and in the adults of different classes can be recognized in similar positions. In the neighborhood of the anus are five basalia, interradial in position, farther five radialia ('apical skeleton') and five interradial 'oralia' around the mouth.

Not less characteristic than the s'ieleton is the ambulacral (or watervascular) system (fig. $28 \mathrm{I}$ ). This is a system of ciliated tubes which begins usually at the surface, ordinarily by a calcareous plate, the madreporite, perforated with fine pores for the entrance of sea water. The water passes into a tube which, on account of its calcified walls in the starfish is called the stone canal, and leads orally to a ring canal around the mouth. The ring canal bears usually several (up to five pairs) of Polian vesicles, which, with Tiedemann's vesicles of the starfishes, are now regarded as appendages which, like lymph glands, produce leucocytes. From the ring canal radiate five radial canals which give off, right and left in pairs, the ambulacral canals. These in turn connect with the ambulacra and ampulle, the highly characteristic locomotor organs of the echinoderms. An ambulacmm is a muscular sac which can be distended and lengthened by forcing in fluid from the ambulacral vessels, and is retracted 
and shortened by its muscles. The ampulla is a reservoir connected with the ambulacrum and projecting into the body cavity. In locomotion the animal extends its ambulacra, anchors them by the sucking disc at the tips, and then pulls the body along by contraction of the ambulacral muscles. In the sessile crinoids and the ophiuroils (which move by their snake-like arms) the ambulacra lack suckers and ampulle, and are not locomotor but tactile in function. So among the holothurians and sea urchins the ambulacra are often replaced by tentacles. Frequently each radial canal ends in an unpaired tentacle with olfactory functions.

The arrangement of the ambulacral system influences that of other organs. Alongside the stone canal is an elongate organ formerly called the 'heart,' but now regarded as a lymphoid gland or a collection of excretory lymphoid cells (ovoid gland, paraxon gland, septal organ). Ring and radial canals are accompanied by corresponding blood canals, with which are often associated two vessels to the alimentary canal. The blood system is surrounded by a perihæmal space, the oroid gland and stone canal by the axial sinus, which in the starfishes and urchins passes into an ampulla close beneath the madreporite; this in turn connects with the lumen of the stone canal and also with the exterior through the pores of the madreporite. When the axial sinus is lacking (crinoids, holothurians) the stone canal may open into the body cavity, water entering this in the crinoids by pores in the theca. There is a nerve ring and radial nerve, frequently in the ectoderm, to which may be added an 'apical' nervous system.

The courses of the radial vessels and nerves mark out five chief lines in the animal, the radii; between them come the interradii. The stone canal, madreporite, and lymphoid gland are interradial in position, as are the gonads, usually five single or five pairs of racemose glands; in some cases but one is present. Echinoderms are rarely hermaphroditic. The gonads are supported in the spacious cœlom by special bands, while mesenteries support the alimentary tract and its derivatives.

The five gonads develop from a single anlage which is genetically connected with the lymphoid gland. Except in crinoids and holothurians this anlage becomes modified into a perianal ring (rhachis) from which the gonads bud. The so-called blood-vessels hardly deserve the name, since they are fibrous cords with lacunar spaces. The perihæmal space, like the axial sinus and the space. around the gonads and the genital rhachis, are derived from the cœlom.

Respiratory organs are represented by very various structures: branchice, or thin-walled outpushings of the cœlom, either around the mouth, as in Echinoidea, or on the aboral surface, as in the Asteroidea, the burse of the Ophiuroidea, the branchial trees of the Holothoroidea and the various parts of the ambulacral system.

The Echinoderma are exclusively marine, occurring even in the deepest seas. Many groups, like the crinoids, are largely bathybial, others frequent rocky coasts. At the period of reproduction they pass their sexual cells into the sea, where fertilization occurs. In some, however, the young are carried about in brood cases until the earlier developmental stages are past. 
Where there is no brood pouch the young escape from the egg as larvie (fig. $282, I$ ) which swim at the surface, and are distinguishable from the adults by their soft consistency, transparency, and bilateral symmetry. By the development of lobe-like processes and slender arms supported by calcareous rods the larvæ assume the most different and bizarre shapes (plutei of echinoids and ophiuroids (II), brachiolaria (I $I I)$ and bipimaria $(I I)$ of asteroids, auricularia (III) of holothurians), all
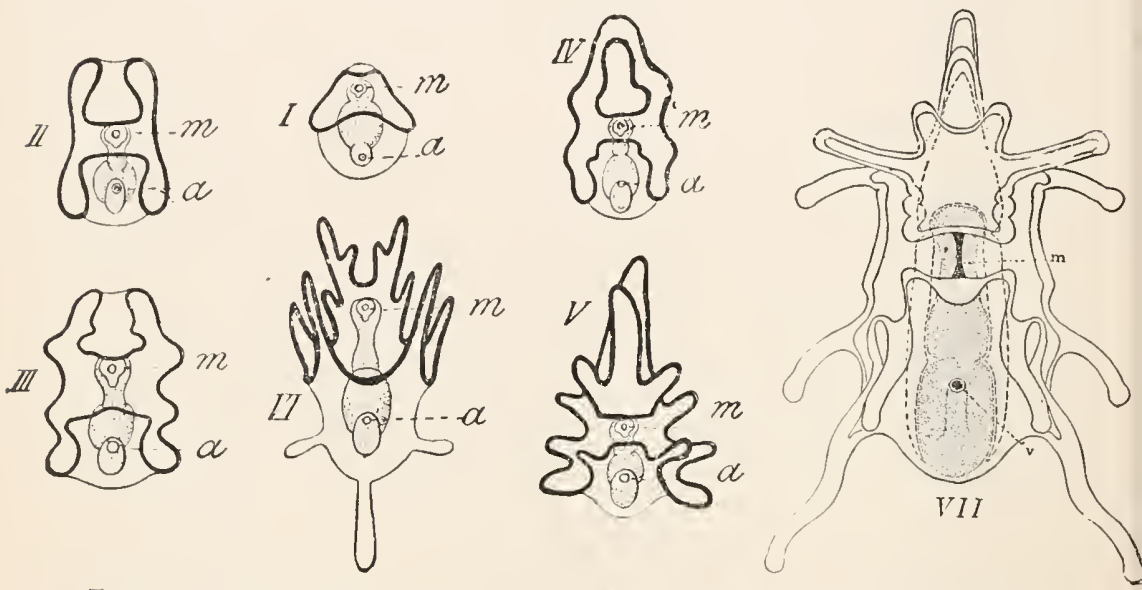

Fir. 282.-Echinoderm larvæ (after J. Muller). $a$, anus; $m$, mouth; the black line, the course of the ciliated bands. $I$, form common to all; $I I$, III, developmental stages of auricularia (Holothurian); $I T^{r}, V$, stages of the Asteroid bipinnaria; $V I$, pluteus of a spatangoid; VII, larva (brachiolaria) of Asterias (orig.). m, mouth; $v$,
vent.

of which can be referred back to a common type with tri-regional alimentary tract and a ciliated band around the mouth, strikingly resembling tomaria, the larva of Balanoglossus. The different appearances of the larve are due to the drawing out of the ciliated band into lobes and arms, and also to its becoming broken into parts which unite themselves in to complete rings $\left(I^{r}\right)$.

The metamorphosis of the bilateral larva into the radial adult is very complicated. It begins early with the formation of outgrowths from the archenteron (fig. 283), which become separated and form the anlagen of the coelom and ambulacral system. It is difficult to give a short summary of the development, partly from the differences in the separate groups, partly from the contradictions of authors. The following seems to be the most common. A vasoperitoneal diverticulum (fig. $28_{3}, h c$ ) arises from the bottom of the archenteron; this soon divides into right and left vesicles, the left acquiring a connection with the exterior (madreporic opening). Each vesicle separates into anterior $(h)$ and posterior (c) parts, the anterior forming the anlage of the water-vascular (hydrocale) system, the others the colom. The two cœlomic sacs expand and 
form the roomy body cavity of the adult, the membranes separating them furnishing the mesenteries. The right hydrocole remains rudimentary; the left, which has the external opening, separates into (I) a smaller anterior portion, the anlage of the ampulla and axial sinus; (2) the connecting duct or stone canal; and (3) a posterior cavity, the hydrocole in the narrower sense. 'The latter surrounds the oesophagus (ring canal) and sends off five radial diverticula, the anlagen of the radial canals, which form the basis of the conversion of the bilateral larva into the radially symmetrical adult (fig. 284). It is a question as to which group of Echinoderma is the most primitive, but ease of treatment makes it best to begin with the Asteroidea.
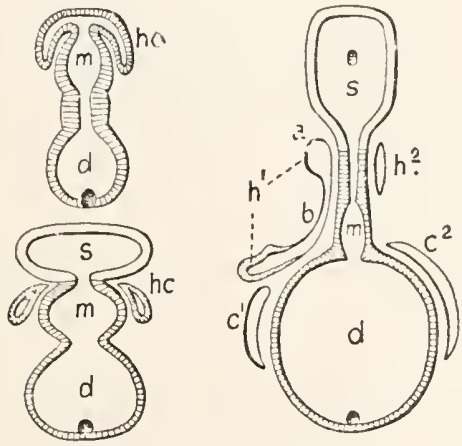

FIG. 283 .

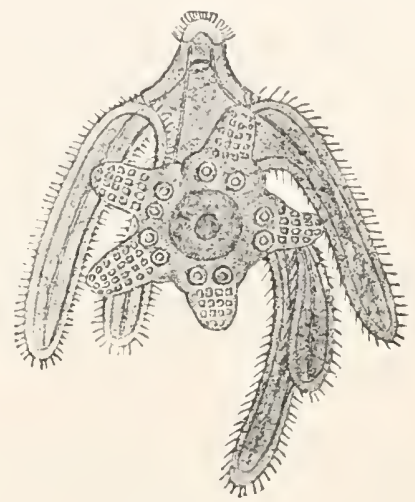

FIg. $2 S_{4}$.

Fig. 283.-Three stages in the development of the coelom and water-vascular system (after Bury and IcBride). $a$, ampulla; $b$, stone canal; $c^{1}, c^{2}$, left and right coelom sacs; $d$, hind gut with anus; $h^{\mathrm{i}}, h^{2}$, left and right (rudimentary) hydrocnele sac; $h c$, common anlage of hydrocoele and coelons: $m$, stomach; $s$, stomodeum and mouth.

Fig. $28+$. - Formation of Ophiuran from the pluteus larva after Müller, from Korshelt-Heider).

\section{Class I. Asteroidea (Starfish).}

Two parts can be recognized in the body of a starfish, a central disc and the arms, usually five in number, which radiate from it (fig. 290). The relations in which these stand to each other vary between two extremes. In many starfish the arms play the chief rôle and the disc appears as only their united proximal ends (fig. 285). On the other hand, the disc may increase at the expense of the arms, so that they form merely the angles of a pentagonal disc (fig. 286). In both arms and disc two surfaces are recognized, oral and aboral, which pass into each other, usually without a sharp margin. In the normal position the oral side is clownwards and has the mouth in the centre and radiating from it to the tips of the arms the five ambulacral grooves. Near the centre of the aboral surface is the anus (when not degenerate) and excentric from it in an interrarlius is the madreporite (in many-armed species two to sixteen interradii may have madreporites). 
A line passing through the madreporite and the opposite arm divides the body into symmetrical halves. This arm is called anterior, since in the irregular sea urchins (Spatangoids) the homologous area is clearly anterior, while the madreporic interradius is posterior. This plane of symmetry does not correspond with that of the larva. The two rays on either side of the madreporite form the bivium, the three others the trivium.

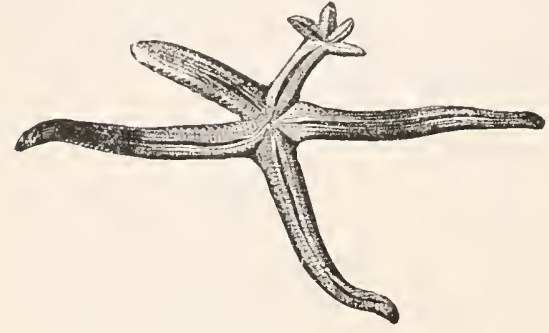

FIG. 285 .

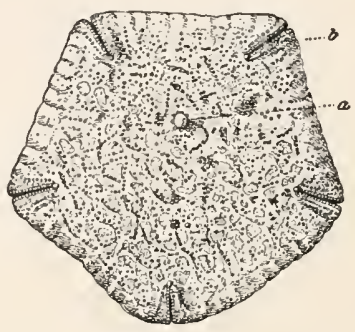

FIG. $2 \& 6$.

FIG. 285.-Comet form of Linckia multiflora (from Korschelt-Heider) One of the arms is producing a new animal by budding.

FIG. 286.-Culcita pentangularis, aboral view (from Ludwig). a, madreporite; $b$, reflexed end of ambulacral grooves.

The skin is everywhere protected by large and small plates jointed together. In life it is extremely flexible, the arms can be bent in any direction, and the animal can work its way through narrow openings. Of the skeletal pieces the ambulacral plates need special mention. These
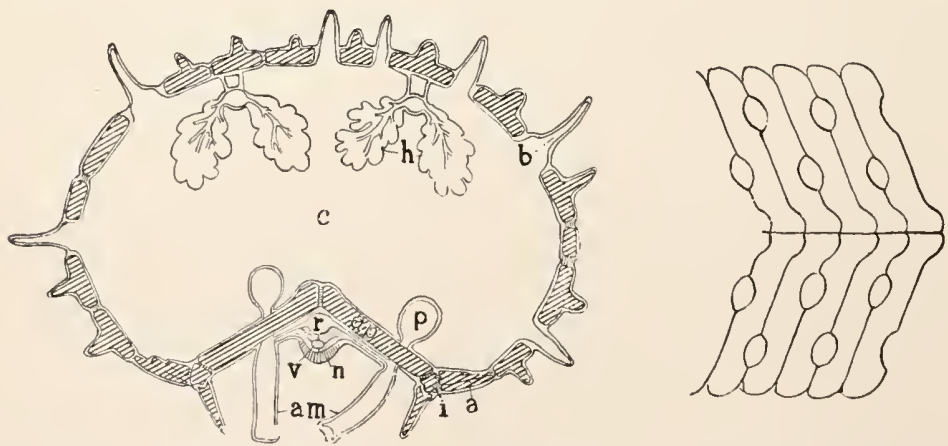

FIG. $2 S_{7} .-A$, cross-section of starfish arm (orig.). $a$, adambulacral plates; $a m$, ambulacra; $a p$, ambulacral plates; $b$, branchix; $c$, coelom; $h$, hepatic creca; $i$, interambulacral plates; $n$, radial nerve; $p$, ampulla; $r$, radial canal; $r$, radial blood-vessel. $B$, ambulacral plates, ventral view, showing the ambulacral pores between.

form the roofs of the ambulacral grooves, and between them are openings (fig. $287, B$ ), the ambulacral pores, through which connection is made between the ambulacra and ampulle. In each arm the pairs of ambulacral plates meet above the groove like the rafters of a roof. Laterally each 
ambulacral plate abuts against a small inferambulacral plate, bearing usually movable spines. Beyond these come the less constant adambulacral or marginal plates, and then those of the aboral surface. Each ambulacral area terminates at the tip of the arm with an unpaired ocular plate.

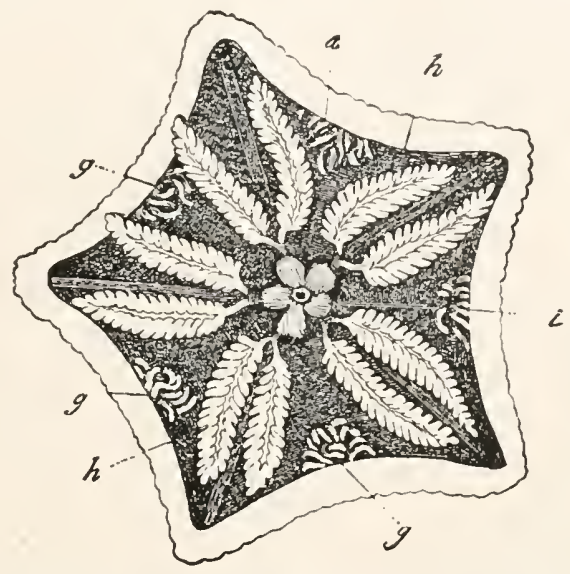

FIG. 288.-Asteriscus verruculalus, aboral surface removed (after Gegenbaur). g, gonads; $h$, hepatic cæca; $i$, stomach with anus.

The organs lie in part in the cœlom, in part in the ambulacral grooves. The alimentary tract is in the colom and extends straight upward from the mouth to the aboral surface, where it ends with an anus or is entirely

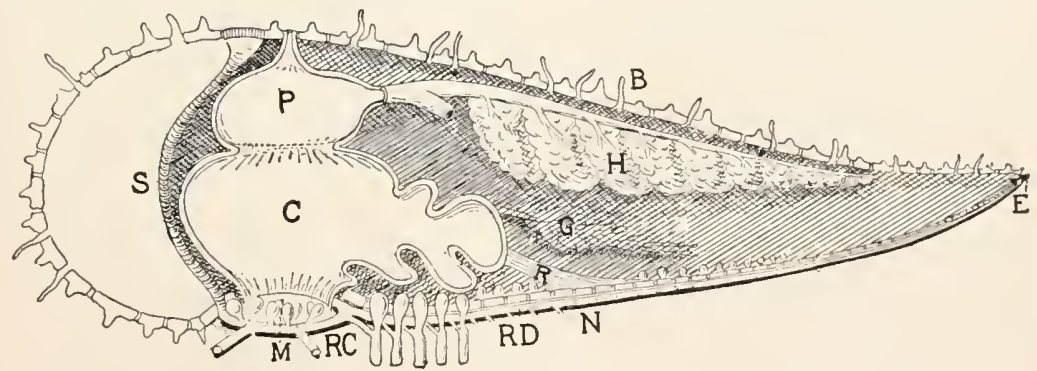

FIG. 289 .- Section through ray and opposite interradius of a starfish (orig.). B branchix: $C$, cardiac pouch of stomach; $E$, eye spot; $G$, gonad; $H$, 'liver';.$M$, mouth; $N$, radial nerve; $P$, pyloric part of stomach; $R C$, ring canal; $R D$, radial canal of watervascular system; $S$, stone canal.

closed (figs. 288, 289). By a constriction it is divided into a larger, lower cardiac portion and a smaller, upper pyloric division. From the latter arise five hepatic ducts which connect with five pairs of hepatic glands lying in the arms, while small creca arise from the intestine in some 
forms. The cardiac division gives origin to five gastric pouches which can be protruded from the mouth or retracted by appropriate muscles. The gonads are five pairs of racemose glands lying in the basis of the arms and opening interradially between the arms. Lastly, in the cœlom is the stone canal (acconpanied by the lymphoid glands, and with it enclosed in the axial sinus) extending from the aboral madreporite to the ring canal near the mouth.

The radial nerve, canal and blood-vessel, which start from the circumoral rings, lie in the roof of the ambulacral groove between the

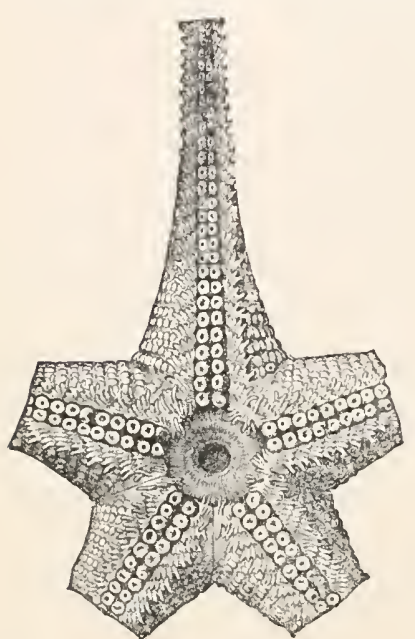

Fig. 200.-Pythonaster murray' (after Sladen). Oral view showing ambulacral grooves. a mbulacra. The nerve, lying in the ectoderm, ends at the tip of the arm in a compound eye spot colored with red or orange pigment which experiment shows is sensitive to light. A second nerve has been described lying in the colom of the arm. The ambulacral system corresponds with the foregoing description (p. 292), the ampullix as well as the five or more Polian and Tiedemann's (racemose) vesicles projecting into the cœelom.

Since the arms contain nearly all important organs, their physiological independence is easily understood. Arms broken off not only live, but regenerate first the disc and then new arms which appear at first like small buds (comet form, fig. 285). This separation of arms may occur through accident, or it not infrequently is produced by the animal itself.

Asteride, well developed arms, four rows of ambulacra; Asterias, ${ }^{*}$ Leptasterias, *

Heliaster* (numerous arms). SolASTERID E, two rows of ambulacra, arms sometimes numerous; Pythonaster (fig. 290). Asterinid.e, arms short or body is pentagonal, no large plates on the margins of the arms. Asteriscus (fig. 288). In other forms (Culcita, * fig. 286, Hippasteria,* Ctenodiscus*) the body is more or less pentangular, margin with large plates.

\section{Class II. Ophiuroidea (Brittle Stars).}

In these, as in the Asteroidea, there are disc and arms, the latter sometimes branched, but the internal anatomy is different. The ambulacral plates have been drawn inside the arm and each pair fused to a large 'vertebra' (fig. 29I). As a result the cœelom of the arms is greatly reduced, the hepatic ceca are lacking, and the alimentary canal, which lacks an anus, is confined to the disc. By the ingrowth of ventral plates 
the ambulacral grooves are closed, and the ambulacra, which lack sucking discs, are tactile, locomotion being effected by the snake like motion of the arms. 'The madreporite is on the ventral surface. Also on the ventral surface are five slits which connect with as many burse, thin-walled respiratory sacs into which the sexua? organs open. The gonads are attached to a genital rhachis which coils through the disc.

In many brittle stars, especially in young specimens, there is a kind of asexual generation (schizogony), the animal dividing through the disc, the halves regenerating the missing parts. OPHIURIDE, arms unbranched (Ophio-

pholis* (fig. 292), Ophioglypha,* Amphiura*); Eurvalid.e, the arms branched (Astrophyton,* fig. 293).

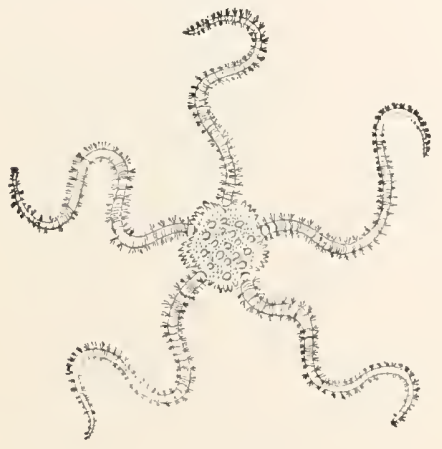

FIG. 292.-Ophiopholis aculeata* (from Morse).

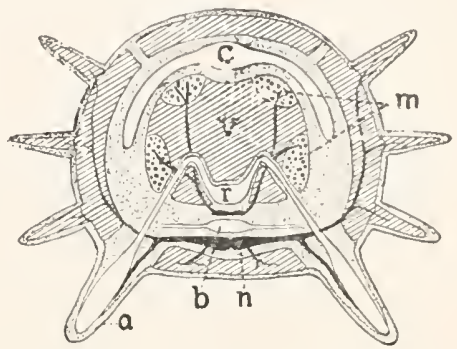

FIG. 291.-Section of Cphiuroid a rm (orig.). $a$, a mbulacrum; $b$, bloodvessel; $c$, coelom; $m$, muscles of arm; $n$, nerve; $r$, radial water tube; $v$, 'vertebra' (coalesced ambulacral plates).

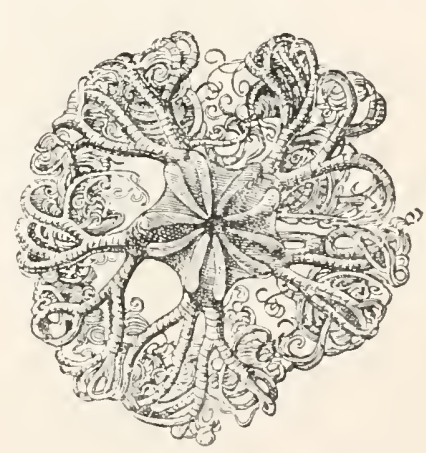

FIG. 293.-Astrophyton arborescens, basket fish (from Ludwig).

\section{Class III. Crinoidea (Pelmatozoa).}

The crinoids or sea lilies are on the road to extinction. In early times, especially in the paleozoic, they were very abundant, but to-day there are but few species, these mostly restricted to the greater depths of the ocean, only the Comatulidx occurring near the shore. The crinoids are attached to the sea bottom by a long stalk (fig. 294), composed of cylindrical discs which often bear five rows of outgrowths, the cirri. The young Comatulida (fig. 295) are similarly attached, having a Pentacrinus stage, but later they separate and live a free life, a proof that the attached condition was prinitive. When the separation takes place, one joint of the stalk 
with its cirri remains attached to the animal, as the centrodorsal united with the lowest cup plates, the infrabasals. On the upper joint of the stalk is a cup-shaped body (theca) the edges of which bear five or ten (usually branched) arms. The walls of the theca are covered with polygonal calcareous plates.

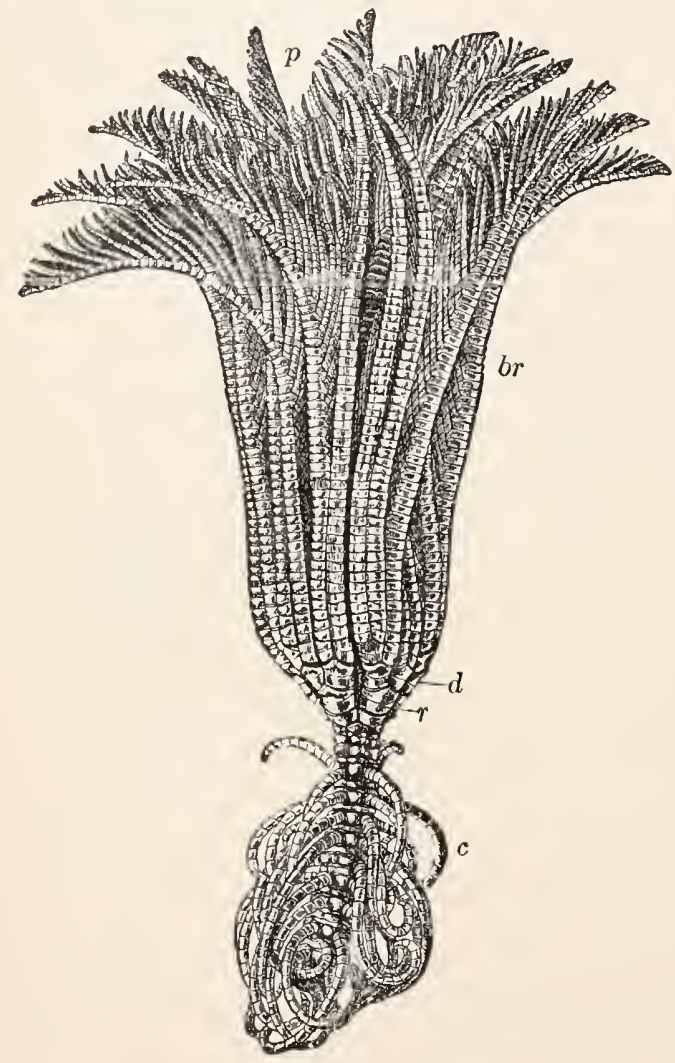

FIG. 294.

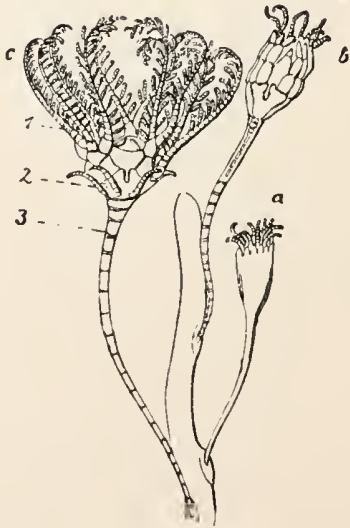

FIG. 295.

F1G. 294.-Pentacrimes marloayanus (after Wyvile Thompson). br, brachialia; $c$, cirri; $d$, distichalia; $r$, radialia; $p$, pinnule.

Fig. 295.-Different Pentacrinus stages $(a, b, c)$ of Antedon rosacea. I, arms; 2, cirri; 3 , stalk.

Usually the stalk bears five plates, the basalia, and then come five radialia, alternating in order with the basalia (fig. 296). In some there is a circle of infrabasalia in a line with the radialia. Frequently the elements of the arm, the brachialia, are directly attached to the radials (fig. 296). But of ten the arm branches once or several times dichotomously, and the first branching takes place at the base, so that the arms seem to spring from the theca. In these cases the first brachialia are considered as part of the theca and are called radialia distichalia (fig. 294). From the arms arise, right and left, a row of 
pinmula, lancet-shaped processes supported by calcarenus bodies in which the sexual products ripen until freed by dehiscence (fig. 293).

The mouth opening, in the middle of the oral disc which closes the theca, is frequently surrounded by five interradial plates, the oralia (fig. $205, B)$. The mouth, which in contrast to other echinoderms is directed upwards, connects with a spiral digestive tract in which sesophagus, stomach, and intestine can be distinguished. The anus is interradial and near the mouth (fig. 297). Five ambulacral grooves begin at the

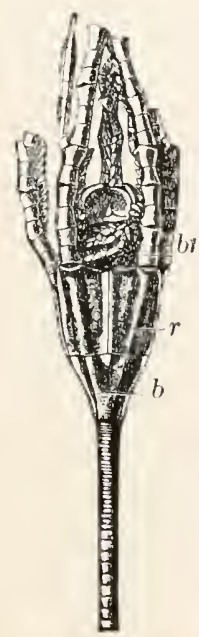

A

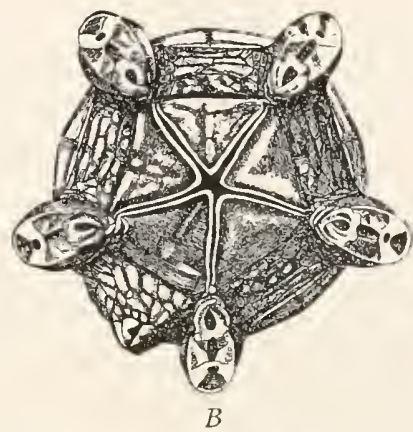

FIG. 296.-H yocrinus bethleyanus. A, upper end of stalk with cup, and the bases of the arms; $b$, basalia; $b r$, brachialia; $r$, radialia. $B$, oral surface of cup with mouth, five oralia, and the bases of the arms.

mouth and extend out on the arms, branching with them and extending to the tips of the pinnule. In the ten-armed species (fig. 297) the grooves fork near the mouth. These are ciliated and serve as conduits to bring food to the oral opening. Nervous, ambulacral, and blood systems begin with a circumoral ring. As in the asteroids, they follow the ambulacral grooves to the pinnulæ, but the ambulacra have no suckers nor ampulle and are merely tactile tentacles.

A typical stone canal is also lacking; in its place are five or several hundred tubules leading from the ring canal to the coxlom. Opposite their coxlomic mouths are fine pores in the theca through which water enters to pass through the tubules into the ambulacral system. The ambulacral nerrous system is weakly developed or may be absent. The apical system, on the other hand, is well developed and forms the axial cord running through the brachialia and 
radialia to unite in a complicated plexus in the centrodorsal. A problematical so-called dorsal organ also begins in the centrodorsal and extends up through the axis of the theca to the oral disc. It is apparently homologous with the 'heart' of other echinoderms. Its upper end lies in a cell complex from which the reticulum of genital cords extends into the arms, swelling in the pinnulæ to the gonads (fig. 298). The dorsal organ in the centrodorsal is enclosed in the chambered organ, a prolongation of the cœlom which extends into stalk ard cirri.

\section{Sub Class I. Eucrinoidea.}

The foregoing account applies entirely to the Eucrinoidea, which may bë divided into two groups:

Order I. TESSELLATA (Palreocrinoidea). Theca with its side walls composed of immovably united thin plates; the ambulacral grooves usually completely covered by calcareous plates. Exclusively paleozoic.

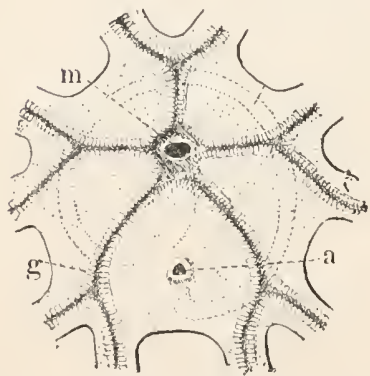

FIG. 207 .

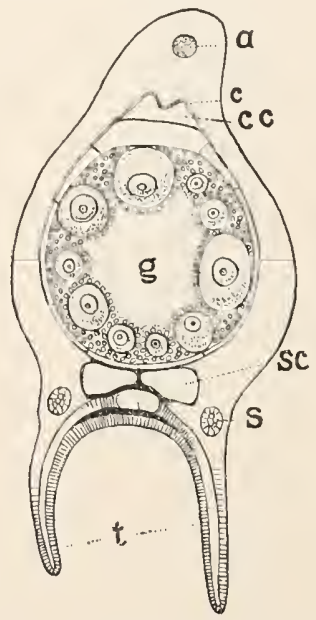

FIG. 298 .

FIG, 297.- Oral area of crinoid (.1ntedon), showing by dotted lines the course of the intestine from the mouth $(m)$ to the anus $(a) ; g$, ciliated grooves leading from the arms to the mouth (orig.).

FIG. 208.-Cross-section of pinnula of Antedon (after Ludwig). a, axial nerve cord; $c$, ciliated cups; $c c$, coeliac canal; $g$, gonad; $s$, sacculi; $s c$, subtentacular canal; $t$, tentacles.

Order II. ARTICULATA (Neocrinoidea). Ambulacral grooves open, theca with compact, in part movably articulated, plates. 'T'his order left the other in the mesozoic age, and some families have persisted until now. Rhizocrimus* (fig. 296) and Pentacrinus (fig. 29.4), deep seas; the Comatul.id.玉 of shallow water are fixed in the young, free in the adult. Antedon* (fig. 295).

\section{Sub Class II. Edrioasteroidea (A gelacrinoidea).}

Theca of irregular plates; arms unbranched and lying on the theca. Possibly the ancestors of the noncrinoid echinoderms. Paleozoic. Agelacrinus. 


\section{Sub Class III. Cystidea.}

Exclusively paleozoic; body spherical, composed of polygonal plates. Stalk and arm structures rudimentary, sometimes lacking. 'The AMpHORIDA are by some regarded as ancestral of all echinoderms. Holocystiles, Echinospharites (fig. 299).

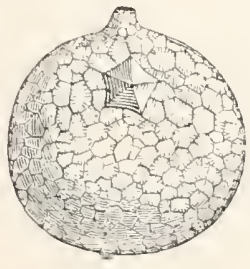

FIG. 299 .
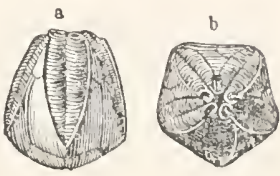

FIG. 300.

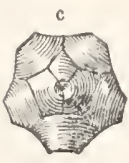

FIG. 209.-Echinospharites aurantiun (f rom Zittel).

FIG. 300.-Pentremites florealis (from Zittel). Lateral, oral, and aboral views.

\section{Sub Class Ir. Blastoidea.}

Arms lacking; the mouth surrounded by five petal-like ambulacral areas The group appears at end of Silurian and dies out with the carboniferous. Pentremites (fig. 300).

\section{Class IV. Echinoidea (Sea Urchins).}

The structure of the sea urchins is best understood in the spherical forms (figs. 30r, 303). Mouth and anus lie at opposite poles of the main axis, each opening immediately surrounded by areas covered by calcareous plates, the arrangement of which varies with the family. Around the anus is the periproct, around the mouth the peristome, the latter bearing sphiridia and in the Echinoids five pairs of interambulacral gills. Between peristome and periproct the body wall (corona) is composed of calcareous plates, which, except in the Echinothuridæ, are immovably united. Aside from the extinct Palachinoidea the plates are arranged in ten double meridional rows, two rows being always intimately associated together.

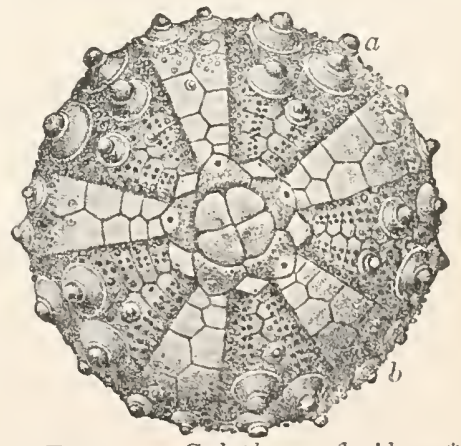

FIG. 301.-Colopleurus floridanus* (after Agassiz). Aboral view, the spines removed to show the ambulacral $(a)$ and $(b)$ interambulacral areas, ending respectively in the ocular and genital plates; in the centre the four plates of the periproct.

Five of these double rows are ambulacral, the alternating five interambulacral. Both bear small hemispherical articular surfaces on which 
are situated the spines, either long and pointed or swollen to spherical plates. These spines are moved by muscles so that they serve both as protecting and locomotor structures. The ambulacral plates are distinguished from the interambulacral by the ambulacral pores by which the ambulacra on the surface are connected with the internal radial canals and ampullæ. In most sea urchins the paired grouping of the pores results from the fact that a double canal extends from ampulla to ambulacrum.

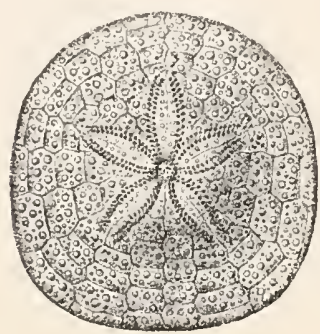

FIG. 302 .

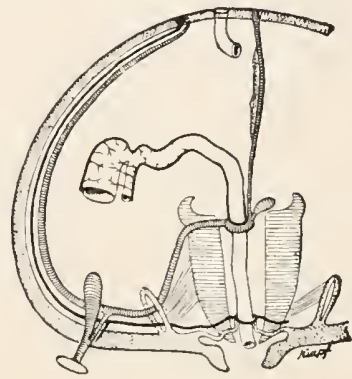

FIG. $3 \mathrm{O}_{3}$.

FIG. 302.- Clypeaster subdepressus (after Agassiz). Aboral view, showing the petaloid ends of the ambulacral a reas.

FIG. 303.-Diagrammatic longitudinal section through a sea urchin.

In the arrangement of the ambulacra two modifications, the band form and the petaloid, occur. In the first (Regularia) the ambulacra are equally developed from peristome to periproct (fig. 30I). In the second oral and aboral regions may be distinguished (fig. 302). In the oral region alone are locomotor feet always present, but these are irregularly arranged. In the aboral area the ambulacra are branchial or tentacular and are regularly arranged, their pores bounding five petal-like figures around the periproct, very distinct after removal of the spines (fig. 306). In the Regularia, the Cidaridie excepted, the interambulacral plates around the peristome show five pairs of notches for the gills, five pairs of thin walled branching extensions of the body cavity.

Ambulacral and interambulacral fields both end at the periproct with an unpaired plate, the five ambulacral plates (terminalia of morphology) being called ocular plates, since they often bear pigment spots formerly regarded as eyes. Each is perforated by the end of the radial canal and nerve. The five interambulacral plates (basalia) are called genital plates, since they usually contain the openings of the genital ducts. One is often madreporite as well.

Inside of the body is a spacious cœlom, to the walls of which the thinwalled alimentary tract is fastened by a mesentery. In the Clypeastroids this tract forms a simple spiral, but elsewhere it ascends from the mouth, turning once, and then, bending on itself, coils in the reverse direction to 
the anus (fig. 304). Usually the first portion of the canal is accompanied by a siphon, an accessory tube opening into the main tube at either end Except in the Spatangoids the mouth is surrounded by live sharp-pointed

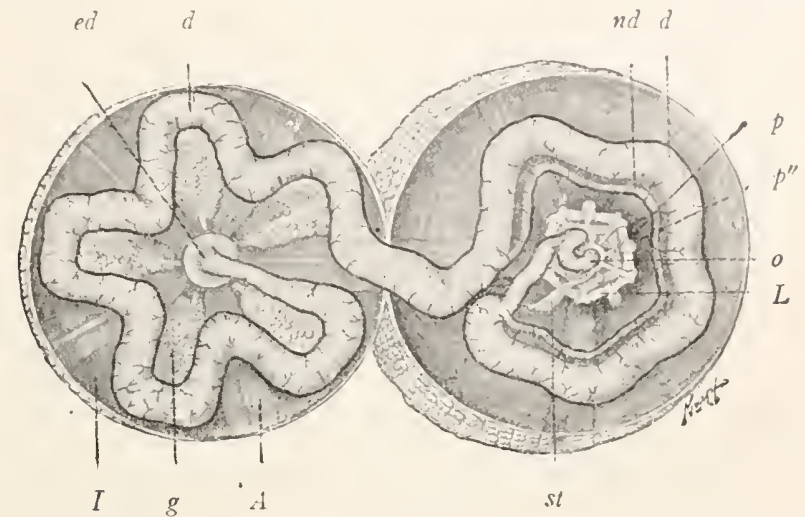

FIG. 304.-Sea urchin opened around the equator. $A$, ambulacral area; $I$, interambulacral area; $L$, lantern; $d$, intestine; $e d$, anal end of intestine: $g$, gonads; $n d$, siphon; oe, cesophagus; $p, p^{\prime}$, ring canal and Polian vesicles; st, stone canal.

calcareous teeth, which in the Regularia are supported by a complicated system of levers, fulcra, and muscles, the 'lantern of Aristotle' (fig. 305).

The ring canal and the ring of the blood system lie on the lantern, the stone canal and septal organ ('heart') extending upwards from them (fig. 303). The blood-vascular ring gives off two blood-ressels which run along the alimentary canal, while from the ring canal arise five ambulacral or radial canals which run on the inner side of the test, accompanied by nerves which, enclosed in a tube of infolded ectoderm, radiate from a nerve ring. The gonads are five (rarely four or two) unpaired organs in the aboral half of the test, opening through the genital plates, that is, interradially as in the starfish.

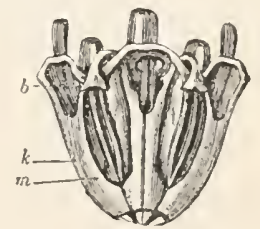

FIG. 305.-Aristotle's lantern of Strongylocentrotus lividus (after Schmarla). b, radulae; $k$, alveoli; $z$, teeth.

\section{Order I. Palæchinoidea.}

Palcozoic forms with five ambulacral areas, the interambulacral areas containing more than two rows of plates. Melonites.

\section{Order II. Cidaridea (Regularia).}

Ambulacral areas band-like, body more or less spherical, mouth and anus polar. Common urchins; Toxopneustes, ${ }^{*}$ Strongylocentrotus, * Arbacia, * Calopleurus* (fig. 30I). 


\section{Order III. Clypeastroidea.}

Flattened echinoids with central mouth and teeth; anus in the posterior interradius, sometimes marginal; five petaloid ambulacral areas. Clypeaster (fig. 302), Echinarachnius* (sand dollar, fig. 306), Mellita, with holes through the test.

\section{Order IV. Spatangoidea.}

Bilateral flattened forms more or less heart-shaped; mouth and anus excentric, no teeth; usually five petaloid ambulacral areas and four genital plates. From the forward position of the mouth it follows that only two ambulacral areas (bivium, p. 29I) are upon the lower surface. Warmer seas. Spatangus* (fig. 307), Echinocardium, Brissus.

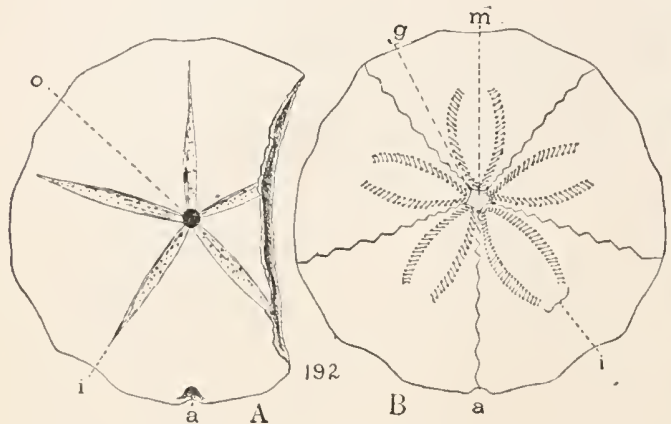

FIG. 306.

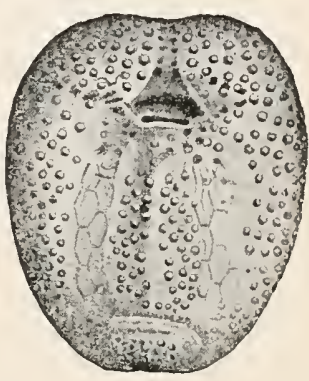

FIG. 307.

FIG. 306.-Oral (A) and aboral (B) surfaces of the sand dollar, Echinarachnius parma. $a$, anus; $g$, genital pores; $i$, ambulacral areas; $m$, madreporite; $o$, mouth.

Fig. 307.- Young Spatangus purpureus (after Agassiz), the spines removed, oral surface. In front, the slit-like mouth; behind, the anus. The bivium without tubercles.

\section{Class V. Holothuroidea.}

The sea cucumbers are most removed of any group from the typical echinoderm appearance. At the first glance, except in Psolus, the skin appears naked and the characteristic plates absent. Yet these are imbedded in the skin in the shape of plates, wheels, and anchors. The integument is leathery and muscular, with longitudinal and circular fibres. The saccular body gives these forms a worm-like appearance, strengthened by its elongation in the main axis, and with the mouth and anus at the poles. Unlike other echinoderms these move with the main axis parallel to the ground, a condition which, to a greater or less extent, leads to a replacement of radial by bilateral symmetry. One surface (trivium) becomes ventral, the bivium dorsal, and in many the trivial ambulacra alone are locomotor, those of the bivium being tactile or wholly absent.

The alimentary canal (fig. 308) (except in Synapta) is coiled in a uniform manner, although many minor convolutions may obscure this. 
It passes backwards in the median dorsal interradius, forward in the left ventral interradius, and then back in the right dorsal interradius to the anus. It is held in position by mesenteries (fig. 309), and near the anus by numerous muscular filaments. Into the terminal portion one or two

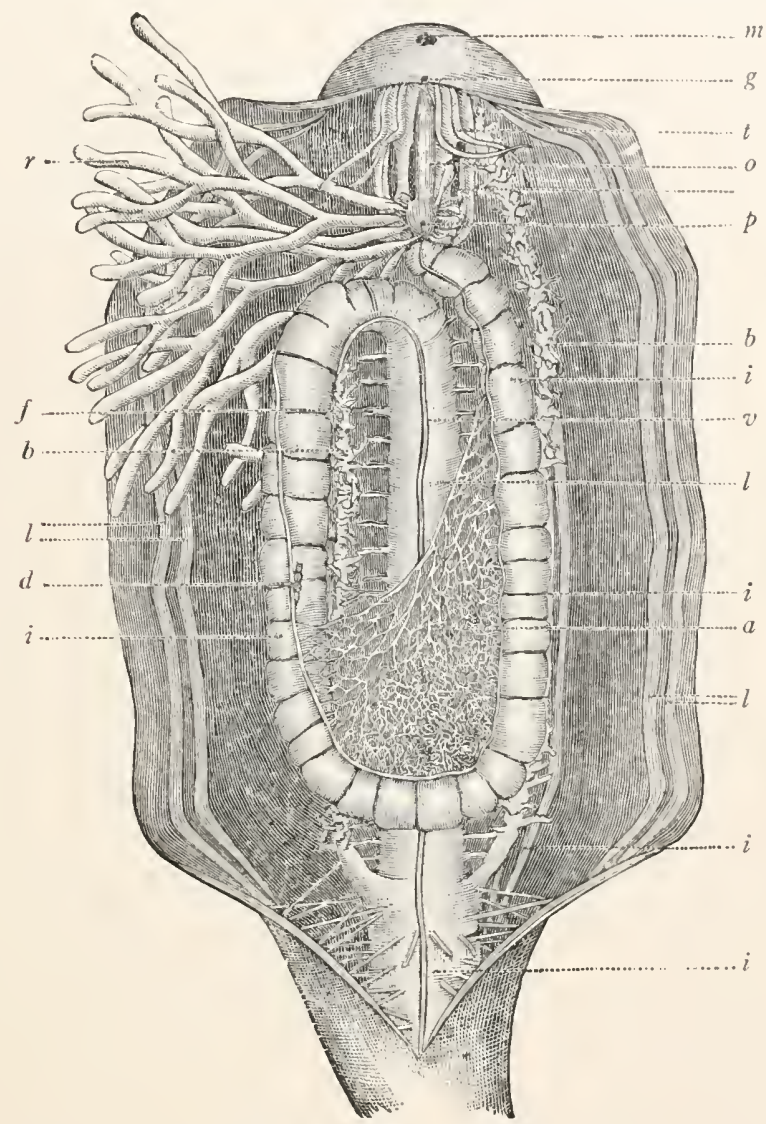

FIG. 308.-Anatomy of Caudina arenata (after Kingsley). a, anastomoses of dorsal blood-ressel; $h$, branchial tree; $d$, dorsal blood-vessel; $f$, mesenterial filaments; $g$, genital opening; $i$, alimentary canal; $l$, longitudinal muscles; $m$, mouth; $o$, genital duct; $p$, pharyngeal ring; $r$, gonads, cut away on right side; $t$, ampullie of tentacles; $v$, ventral blood-vessel.

branchial trees may empty. These are tubular sacs with small branched outgrowths which are filled with water. They are respiratory, and are periodically filled with fresh water. In many species 'Curicrian organs' occur; these are morphologically specially modified portions of the branchial tree and are either connected with them or separately with the 
cloaca. Many zoologists regard them as defensive structures because of their sticky nature and because they can be cast out through the anus.

The nsophagus is usually surrounded by a ring of five radial and five interradial plates which serve as points of attachment for the longitudinal muscles. Just behind it lie the ring canal, ring nerve, and the ring of the blood system,

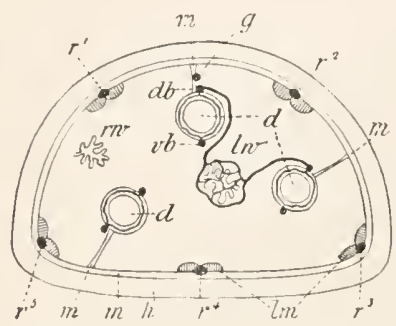

FIG. 309.-Transverse section of Holothuria tubulosa (after Ludwig). $d$, digestive tract; $d b$, dorsal blood-vessel; $g$, gonad duct; $h$, skin; $l m$, longitudinal muscles; $l w$, left branchial tree; $m$, mesenteries: $r^{1}, r^{2}$, ambulacral complex of bivium (ambulacral vessel) and nerve; $r^{3}-r^{5}$, same of trivium; $r w$, right branchial tree.

species are found a few parasites. One or two harbor a small fish (Ficrasfer) in their cloaca and branchial trees, while parasitic snails (Entoconcha, Entocolax, Enteroxcnus) live in several species and a mussel, Entovalva mirabilis, in another.

\section{Order I. Actinopoda.}

Radial canals present, sending branches to the tentacles and ambulacra when present. Divided into Pedata, with ambulacra, and Apoda without. The PEDATA include the HoLOTHURID w with peltate tentacles, (Aspidochirota). Holothuria* in warmer waters, one species forming the trepang of Chinese markets. Of the forms with branched tentacles (Dendrochirotæ) are the Cucumaride, Cucumaria, * Psolus, * Thyone.* The deep sea Elasipoda have statocysts and peculiar dorsal ambulacral processes. The APODA are represented by Caudina* and Molpadia.*

\section{Order II. Paractinopoda.}

No radial canals nor ambulacra. Tentacular canals arising from ring canal. Myriotrochus, * Synapta* with statocysts, Oligotrochus. * In Pelagothuria the anterior end is expanded to a disc with tentacular processes, used in swimming, like the bell of a medusa.

\section{Summary of Important Facts.}

I. The ECHINODERMA share the radiate structure with the Colenterata, but differ from them $(a)$ in the numerical basis of the symmetry (five); (b) in that, as embryology shows, they have descended from bilateral forms. 
2. Farther characters are the existence of a colom, the ambulacral system, and the mesodermal spiny skeleton, which has given the name to the phylum.

3. The ambulacral system is locomotor and occurs nowhere else. It consists of a sieve-like madreporite (not always present), which passes water to the stone canal, and from this to the ring canal and the five radial canals to fill the ampulle and ambulacra. Lateral branches supply the tentacles and cause their extension.

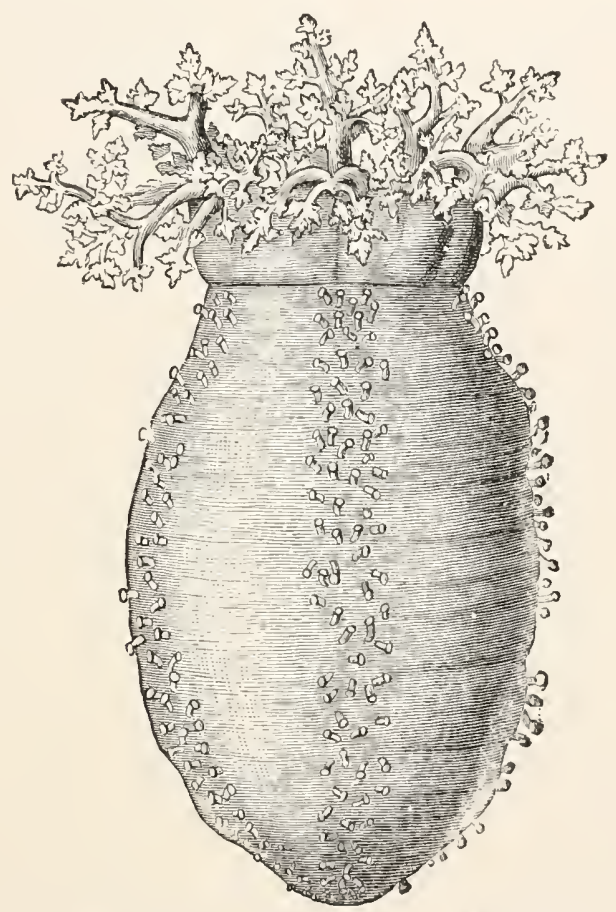

FIG. 310.-Cucumaria frondosa, sea cucumber (from Emerton).

4. Blood-vessels and nerve cords run in the same radii as the radial canals of the ambulacral system; stone canal, madreporite, septal organ and genital ducts are interradial.

5. The Echinoderma are divided into five classes: (I) Asteroidea, (2) Ophiuroidea, (3) Crinoidea, (4) Echinoidea, and (5) Itolothuroidea. 6. The Asteroidea have a disc and (usually) five arms into which the gastric pouches and hepatic cacca extend. The ambulacral groove open. 
7. The OpHitroidea also have disc and arms, but the ambulacral groove is closed and the hepatic cæca absent.

8. The CRINOIDEA have a cup-shaped body bearing arms, usually branching, with pinnulx, and a stalk, usually with cirri by which they are attached, either permanently or in the larval stages. In these latter free forms only the centrodorsal persists as the remains of the stalk. The Crinoidea are subdivided into Eucrinoidea, Edrioasteroidea, Cystidea, and Blastoidea.

9. The EchinoideA are usually spherical or oval, armored with calcareous plates which extend as five pairs of ambulacral and five of interambulacral meridional bands from peristome to periproct.

Io. The ambulacral plates end at the periproct with an unpaired ocular plate; the interambulacral with a similar genital plate. The madreporite is fused with one of the genital plates.

II. The regular sea urchins have the anus in the periproct, the mouth in the peristome; the ambulacral areas band-like.

12. The Clypeastroidea have a central mouth, the anus outside the periproct in the posterior interradius; the ambulacral areas petaloid.

13. The Spatangoidea are markedly bilateral, the mouth anterior, the anus posterior; ambulacral areas petaloid.

I4. The Holothuroidea are elongate and worm-like; the skeletal system greatly reduced; they are more or less bilaterally symmetrical and have usually a single gonad and one or two branchial trees. They are divided into Actinopoda, with radial canals, and Paractinopoda, without.

\section{PHYLUM VI. MOLLUSCA.}

At the first glance the molluscs, like the leeches and flatworms, appear like parenchymatous animals. A spacious colom is absent; what was formerly regarded as such is a system of blood sinuses surrounding the viscera, and is especially well developed in the snails. More recently it has been shown that the molluscs have descended from colomate animals, in which, by encroachment of connective tissue and muscles, the cœlom has been reluced to inconspicuous remnants, the pericardium and the lumen of the gonads.

Where the molluscan features are well developed, as in the snails, four parts may be recognised (fig. 3 I I,$B$ ). The visceral sac forms most of the body; it is less muscular than the rest and contains the alimentary tract, liver, nephridia, and gonads. In front it is continuous with the head, often separated by a neck, which bears the mouth and the most important sense organs, eyes and tentacles. Below, the visceral sac passes 
into the foot, a muscular mass, usually used for locomotion. The mantle or pallium, a dermal fold, extends downward from the body and encloses a part of the body. In the Acephala $(C)$ it has two halves, but in the snails $(B)$ and cephalopods $(A)$ it is umpaired, and either extends down on all sides (Chiton, Patella), or, like a cowl, covers the anterior side (most gasteropods), or envelops the posterior part of the body (pteropods, cephalopods). The mantle is important in two ways: its outer surface

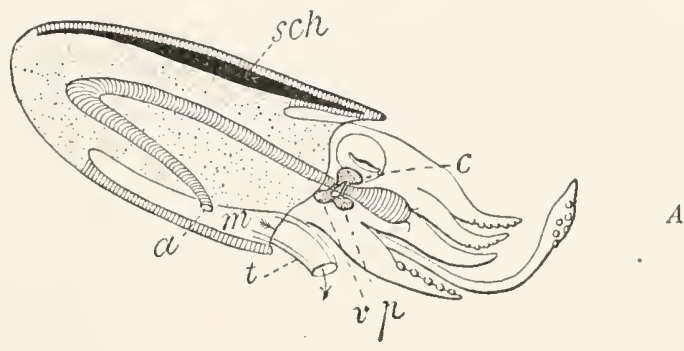

$A$

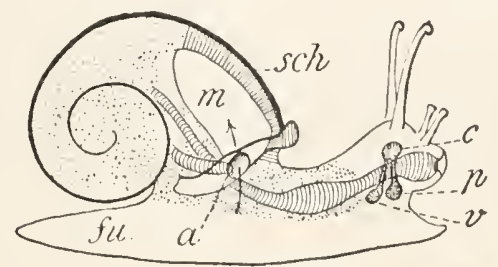

$B$

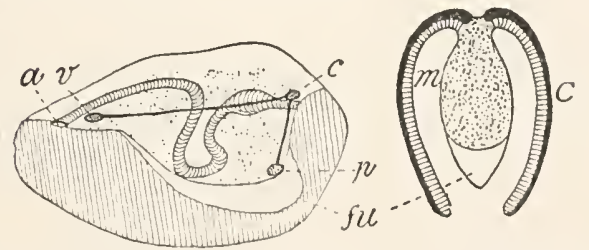

FIG. 3II.-Diagrams of three molluscan classes. $A$, a cephaloporl (Sopia); B, a gasteropod (Helix); $C$, an acephal (Anodonta). a, anus; $c$, cerebral ganglion; $f u$, foot; $m$, mantle chamber; $s c h$, shell; $t$, siphon; $v$, visceral ganglion. Visceral sac dotted; mantle lined, shell black.

is covered with epithelium which, like that of the adjacent surface, may secrete shell, a thick layer of organic matter (conchiolin) largely impregnated with calcic carbonate. The inner surface of the mantle, together with the outer surface of the body, bounds a space, the mantle cavity, which, from its most important function, is alṣo called the respiratory chamber. Since most molluscs are aquatic, special vascular processes of the body, the gills or branchice, lie in this space; in the terrestrial forms it contains air and with a richly vascular dorsal wall, serves as a lung. 
From the foregoing it will be seen that the mantle must exert an influence on the shell and on the respiratory organs. Paired mantle folds form two valves, right and left, to the shell; a right and left branchial chamber, and right and left gills. With an unpaired mantle the shell is always unpaired, while the gills may retain their primitive paired condition.

The gills in the mantle cavity are called ctenidia, from their resemblance to combs with two rows of teeth. Each consists of an axis (back of the comb), containing the chief blood-vessels, and two rows of branchial leaves. The whole is united to the wall of the branchial cavity by the axis (fig. 35 I). Many aquatic forms lack ctenidia, and then the respiration is either by the skin or by accessory gills which differ from ctenidia in structure and position (usually outside the mantle cavity).

Those parts of the surface which are not covered by the shell have a columnar epithelium which is frequently ciliated and which contains large unicellular mucus glands (fig. 29), especially abundant on the edge of the mantle. These give these animals the soft slippery skin which is implied in the name Mollusca (mollis, soft). Although head, foot, and mantle are very characteristic of the molluscs, they are not always present. In the Acephala there is no distinct head region; many gasteropods lack the mantle and hence the shell and mantle cavity; in the Cephalopoda the foot is converted into the siphon and arms.

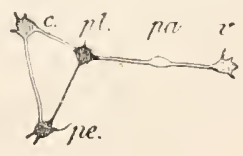

A

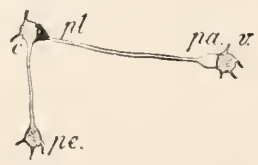

$B$

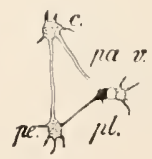

C

FIG. 312.-Nervous systems of Molluscs. A, most gasteropods; $B$, acephals; $C$, cephalopods and pulmonates. $c$, cerebral; $p a$, parietal, pe, pedal, pl, pleural, and $v$, visceral ganglia.

The nervous system has some highly characteristic features. As a rule it consists of three pairs of ganglia associated with important sense organs and connected by nerve cords. One pair lies dorsal to the œsophagus and corresponds to the supraœsophageal ganglion of the worms; it is the brain (cerebrum) and supplies the tentacles and eyes. A second pair lies ventral to the alimentary tract on the front part of the muscle mass of the foot; these are the pedal ganglia with which the statocysts are connected. The third pair, the visceral ganglia, are also ventral, and near them are the third sense organs, widely distributed through the Mollusca, and from position and structure are regarded as organs of smell cosphradia). They are thickened patches of ciliated epithelium in the mantle 
cavity. Pedal and visceral ganglia are united to the cerehrum by nerve cords, the cerebropedal and cerebrovisceral connectives respectively. Iccordingly as these connectives are long or short the ganglia are wide apart or united into a nerie mass around the oesophagus.

Primitive Mollusca (Amphineura) have a simpler condition. The cerebral ganglia are connected by a ring around the oesophagus (fig. 315, B). From it are given off two pairs of longitudinal nerve tracts, the ventral or pedal cords, and lateral or pleural cords, the latter united by a loop dorsal to the anus. By a concentration of ganglion cells in the higher molluscs the pedal cords give rise to the pedal ganglia, and similarly the pleural cords form three pairs of ganglia, the pleural and the parictal, as well as the visceral already mentioned, of the cerebrovisceral cord (fig. 3I2, A). The pleural ganglia are connected with the pedal by nerve cords; the parietal innervates the osphradium. When farther concentration takes place the pleural may unite with the cerebral, and the parietal with the visceral $(B)$, or both may fuse with the visceral $(C)$. In the latter case (pulmonates, cephalopods) the visceral ganglion (in the wider sense) is associated with the pedal by the pleuropedal connective; while in the other (lamellibranchs, scaphopods) the connective is fused with the cerebropedal. Although the statocyst receives its nerve from the pedal ganglion, the centre of innervation lies in the cerebrum. In the Nuculide the statocysts retain their connection with the parent ectoderm by means of a canal, which though closed, remains in part in the Cephalopods. Besides accessory eyes in various places, there are cephalic eyes, in general structure like those of the annelids. They are pits in the skin, the bottom differentiated to a retina. Usually they close to a vesicle, but only in the cephalopods do they reach a high development (fig. 349).

The heart, which lies dorsally, is arterial and consists of auricles and ventricles. The ventricle is al ways unpaired; there are two auricles where two gills exist from which the blood flows to the heart, but with the loss of one gill one auricle may disappear. Distinct arteries and veins occur; capillaries are found only in the Cephalopoda, while in the lower molluscs (especially Acephala), the smaller arteries open into lacunar spaces which were formerly regarded as the body cavity. A completely closed vascular system does not exist even in the Cephalopoda.

The heart is enclosed in a spacious sac or pericardium, which, with few exceptions, is connected with the nephridia by a ciliated canal (nephrostome), and in many molluscs (Cephalopoda, Solenogastres) is also related to the gonads. These facts support the view that the pericardium and the lumen of the gonads are the remnants of the colom; for here, as in the annelids, the nephridia open by ciliated nephrostomes into the cœlom, and the sexual cells arise either from the colomic walls or from sacs cut off from them.

Nephridia and sexual organs are primitively paired, but frequently are single by the degeneration of those of one side. The animals are either hermaphroditic or diøecious, but the gonads are always very large. 
Even more room in the visceral sac is demanded by the digestive tract in which osophagus, stomach, a coiled intestine, a voluminous liver, and usually salivary glands may be recognized. The liver is usually a paired tubular gland, emptying into the stomach. It not only digests fat and stores up glycogen, but forms an enzyme (cytase) which converts cellulose into sugar. The radula or lingual ribbon is also a characteristic organ, and its absence from the Acephala is probably the result of degeneration. It is a plate or band armed with teeth which lies on the floor of the pharynx on a ventral ridge, the tongue, and is used for the comminution of food (figs. 334,335 ).

Reproduction is exclusively sexual; budding, fission, or parthenogenesis being unknown. The eggs are usually united in large numbers,

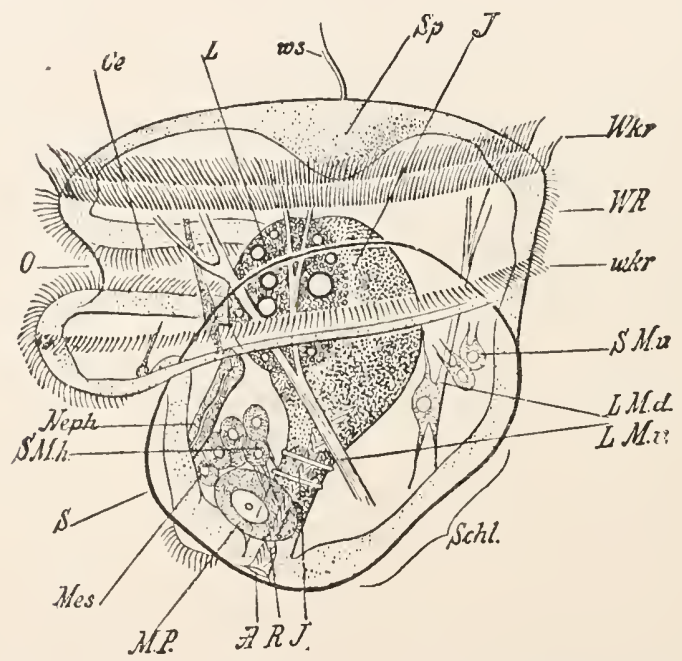

FIG. 313.-Veliger larva (trochophore) of Teredo navalis (from Hatschek). $A$, anus; $J$, stomach; $J_{1}$, intestine; $L$, liver; $L M . d, L M . v$, dorsal and ventral longitudinal muscles; $M e s$, primitive mesoderm cells; $M P$, teloblast; $N e p h$, protonephros; $O$, mouth; Oe, nesophagus; $R$, rectum; $S$, shell; $S c h l$, hinge; $S M . h, S H . v$, posterior and anterior adductors; $S p$, apical plate; II kr, wkr, pre- and postoral ciliated bands; ws, cilia of apical plate.

in a jelly and are either rich in deutoplasm or are enveloped in a nourishing albumen. A few molluses (e.g., Paludina rivipara) are viviparous. A metamorphosis is of wide occurrence, in which a veliger larva escapes from the egg (fig. 3 $\mathrm{I}_{3}$ ); in this can be recognized head, foot, and mantle, even when one or the other of these is lacking in the adult. 'This shows that the frequent absence of mantle, shell, or head is not a primitive condition, but can only be explained by degeneration. The name 
veliger arises from the velum, a strong circle of cilia, which surrounds a velar field in front of the mouth, and which serves as a locomotor organ for the larva. In some cases (fig. $3 \mathrm{I} 4, B$ ) it is loberl like the trochus of a Rotifer. The veliger recalls the annelid trochophore and serves for the distribution of the species; it is therefore of great importance for animals

A

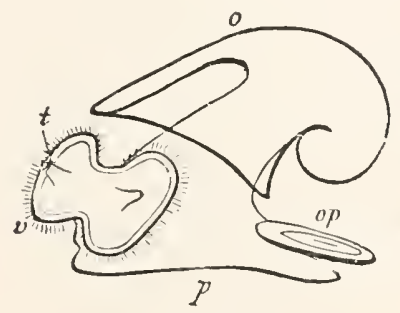

B

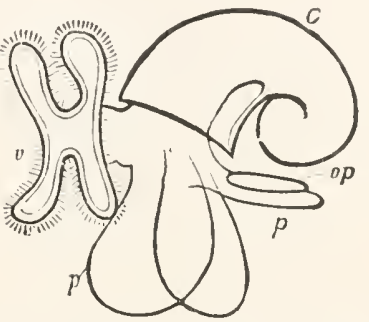

FIG. 314.-Veliger stages, $A$, of a snail; $B$, of a Pteropor (from Cregenbaur). $o$, shell; op, operculum; $p$, foot; $t$, tentacle; $v$, velum.

which, like most molluscs, are sedentary or slow-moving. In cases without metamorphosis (Cephalopoda, Pulmonata, etc.) the veliger stage is frequently indicated during embryonic development by a ridge of cells surrounding a preoral velar field

\section{Class I. Amphineura.}

These forms, some of which appear in the Silurian, are clearly the most primitive of molluscs, and are distinguished by a marked bilateral symmetry. The nervous system already described (p. 3 13) consists of pleural and pedal cords with scattered ganglion cells and no ganglia, these cords being connected by numerous commissures (fig. $3{ }^{1} 5, B$ )

\section{Sub Class I. Placophora (Chitonida).}

The chitons were formerly included among the gasteropods because of the presence of a creeping foot and a radula. They are at a glance distinguished from them by the rudimentary condition of the head (which lacks tentacles and eyes), and the peculiar shell. This last consists of eight transverse plates, overlapping like shingles which allows the animal to roll into a ball. The edge of the mantle extends beyond the shell and is covered with spines, while in the mantle cavity beneath are, right and left, a series of ctenidia. Nerves enter the shell and end with noticeable sense organs (asthetes and, in some, eyes, fig. 3 r6). There are no statocysts. The symmetry of the body is also expressed in the viscera. 
The anus is medial, and right and left of it are the openings of the nephridia and sexual organs. The sexes are separate, the gonads unpaired, while, corresponding to the paired arrangement of the gills, there are two auricles to the heart. Trachydermon,* Amicula,* Cryptochiton.*
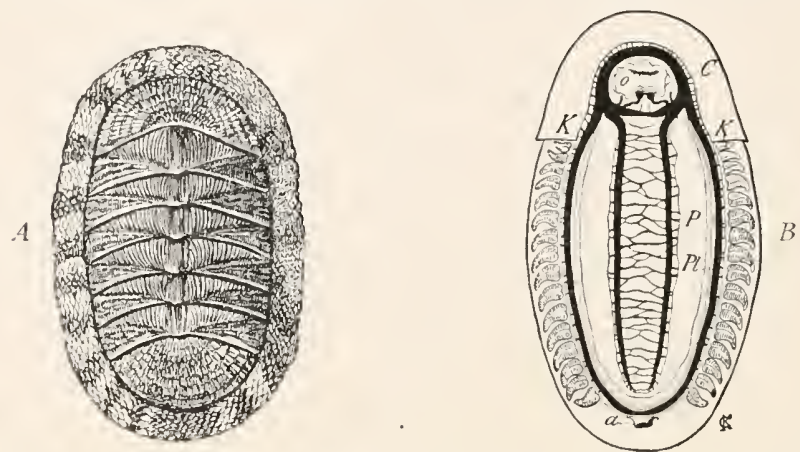

FIg. 315.- (hiton squamosus, dorsal view (after Haller). A, the entire animal; $B$, after removal of shell and viscera; $a$, anus; $C$, brain; $K$, ctenidia $a$, mouth; $P$, pedal nerve cord; $p l$, pleurovisceral nerve cord.

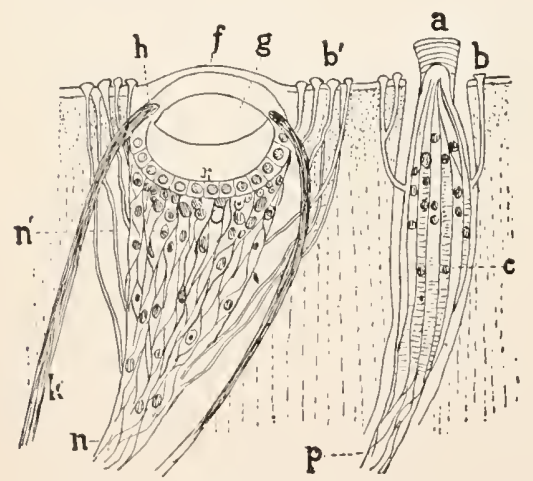

FIG. 3 I6.
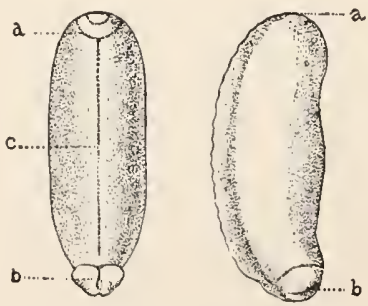

FIG. 317 .

F1G. 316.--Eye and esthetes of Acanthopleura spiniger (after Moseley). $a$, macræsthete; $b$, micresthete; $f$, calcareous cornea; $g$, lens; $h$, iris; $k$, pigmented capsule; $n, p$, nerves; $r$, retina.

FIG. 317.-Neomenia carinata, ventral and side views (after Tulberg). a, anterior; $b$, posterior; $c$, ventral groove.

\section{Sub Class II. Solenogastres (A placophora).}

Wormlike forms without a shell (occurs in the larva of Dondersia); instead of a foot there is a longitudinal ventral ciliated groove; the radula may be lost; in Conchoderma it bears but a single tooth. The gills are either small or wanting. The usually hermaphrodite animals have the gonads emptying into the pericardium and thence by the paired nephridia (?). Marine, living in ooze or sand. Chatoderma,* Neomenia, Dondersia. 


\section{Class II. Acephala (Lamellibranchiata, Pelecypoda).}

These have, among the molluscs, the least powers of locomotion. Some are fixed, the majority burrow slowly through sand or mud; only a few spring by means of the foot or swim by strokes of the valves. Hence they need more protection than other species, and this is afforderl by the strong shells in which the body can usually be completely enclosed. This shell recalls that of the brachiopod in that it consists of two halves or valves, but these valves are right and left and hence are usually similar in shape. Only when the animal rests permanently on one side is this symmetry lost (the extreme is reached in the fossil Rudistes), and then the symmetry of the soft parts is affected.

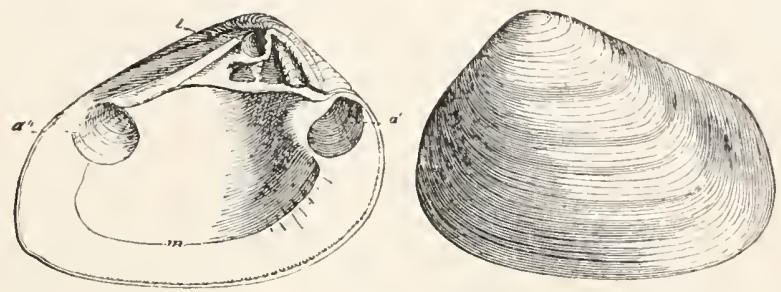

FIG. 3 IS.

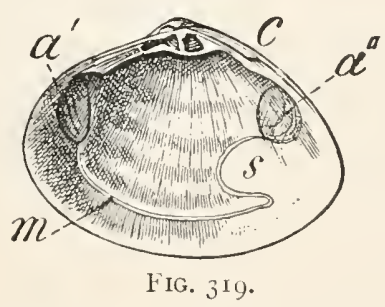

Fig. 3 18 .- - Left valve of Crassatella plumbea, inner and outer surfaces (from Zittel). The outer surface showing lines of growth; no pallial sinus.

FIG. 3I9.-Right valve of Mactra stultorum, with pallial sinus (from LudwigLeunis). Letters for both figures: $a^{\prime}$, anterior, $a^{\prime \prime}$, posterior adductor scar; $e$, hinge: $l$, internal ligamental groove; $m$, pallial line; $s$, pallial sinus.

The two lobes of the mantle which secrete the shell on their outer surface arise from the back of the animal and grow downwards, forwards, and backwards, so that they envelop the whole (fig. 322). Hence the oldest and thickest part of the shell, the $u m b o$, occurs near the back (fig. 318). Around this the lines of growth are arranged concentrically, lines which show how, by gradual growth of the mantle, the shell has increased in size. On the back the valves approach each other, and in the majority are movably connected by a hinge, which consists of projections or teeth on one valve fitting into depressions in the other. The valves 
are opened by an elastic hinge ligament usually placed dorsal to and behind the hinge. The shell is closed by adductor muscles which extend through the body from shell to shell, leaving their impressions on the inner surface (fig. 319). Usually there occur an anterior and a posterior adductor equally well developed (Dimyaria); less frequently the anterior is rudimentary (Heteromyaria) or entirely disappears (Monomyaria). When the muscles are relaxed the elastic ligament opens the valves.

The heterodont hinge is the typical form (fig. 3I9); each valve bears a group of teeth near the umbo, those of the left alternating with those of the right. Besides these cardinal teeth there are latcral teeth in front and behind, of ten produced into ridges. The ligament lies behind the hinge and is usually visible from the outside (external ligament), but is occasionally transferred to the interior (internal ligament, fig. $3 \mathrm{I} 8$ ). The so-called schizodont and desmodont hinges are modifications of the heterodont. Then there are Acephala of apparently primitive character which either lack the hinge (dysodont), or have one composed of numerous teeth in a series symmetrical to the umbo (taxodont), or of two strong teeth likewise symmetrical to the umbo (isodont). In these cases the ligament is developed in front of as well as behind the umbo, and may be either external or internal.

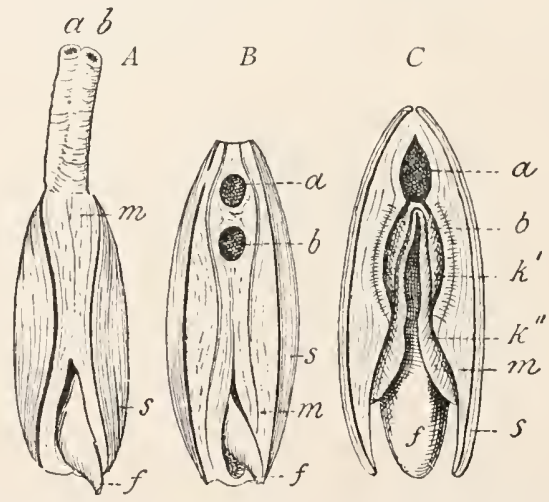

Fig. 320.-Ventral views of siphonate and asiphonate acephals. A, Anndonta cygnea; B, Isocardia cor; $C$, Lutraria elliptica. a, anal siphon; $b$, branchial siphon; $f$, foot; $k^{\prime}$, outer, $k^{\prime \prime}$, inner gill lamella; $m$, mantle; $s$, shell.

Since the secretion of shell takes place most rapidly at the edge of the mantle, both are closely united, the union being strengthened by small muscles. So the edge of the shell has a different appcarance from the rest, this part being marked off by a pallial line parallel to the margin (fig. 3I8). In many species (the Sinupalliata) the line at the hinder end makes a large bay (pallial simus) (fig. 3 I $9, s$ ). Since the mantle folds are membranes with free margins, it follows that when the shell is closed these edges are pressed together, which would prevent the free entrance and exit of water. To accommodate this each mantle has its margin 
excavated at the posterior end, so that when brought together two openings, an upper and a lower, result (fig. 320, C). The lower of these is the branchial opening by which fresh water passes into the mantle chamber; it flows out after passing over the gills, along with the fieces, through the upper or cloacal opening. In many bivalves the free edges of the mantle grow together, leaving three openings (fig. $320, B$ ), one for the protrusion of the foot, the others the two just described, now called the branchial and cloacal siphons. By further development the margins of these openings are drawn out into two long conjoined siphonal tulses $(A)$, which for their retraction need special muscles; these are attached to the valves and thus cause the pallial sinus referred to above.

In the shell there are three layers (fig. $32 \mathrm{I}$ ): on the outside a thin organic cuticula and below two layers largely of calcic carbonate. In many these two layers are distinguished as the prismatic layer and the nacreous laver, the first consisting of closely packed prisms; the nacreous layer of thin lamella generally

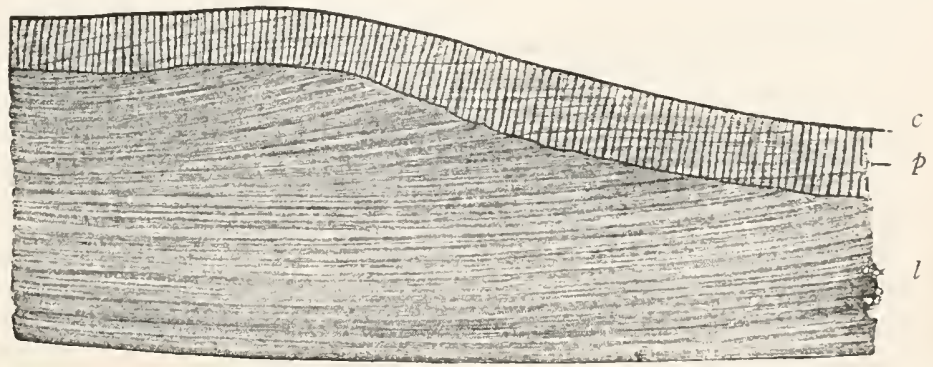

FIG. 32I.-Section of shell of Anodonta. $c$, cuticula; p, prismatic layer; $l$, nacreous layer.

parallel to the surface. These produce diffraction spectra and so the iridescent appearance of the shell; the finer the lines thus formed the more beautiful the play of colors. 'This is especially noticeable in the mother-of-pearl shell II cleagrina and many Unionidæ. When foreign substances get between mantle and shell they stimulate a greater secretion of nacreous substance and become surrounded by layers of it. Pearls are formed in this way.

The gills lie between the mantle and the body and from their lamellar character have given rise to the name Lamellibranchiata (figs. 322, 323). Two gill-leaves occur on either side, although occasionally the outer or both may degenerate. Frequently the inner gills of the two sides unite behind the body and produce a partition which divides the mantle cavity into a small dorsal cloaca and the larger lower branchial chamber. The anus and the water which has passed the gills empty into the cloaca which connects with the excurrent siphon. The incurrent siphon leads 
into the branchial chamber. In front of the gills are two pairs of leaf-like lobes, the labial palpi, between which is the mouth.

The Nuculidæ-the most primitive of living Acephala-have true ctenidia consisting of an axis grown to the body and an inner and an oucer row of gill leaves (fig. 326). From this the filibranch type is easily derived. The gill leaves grow out into long threads, each bent on itself so that it presents two limbs,

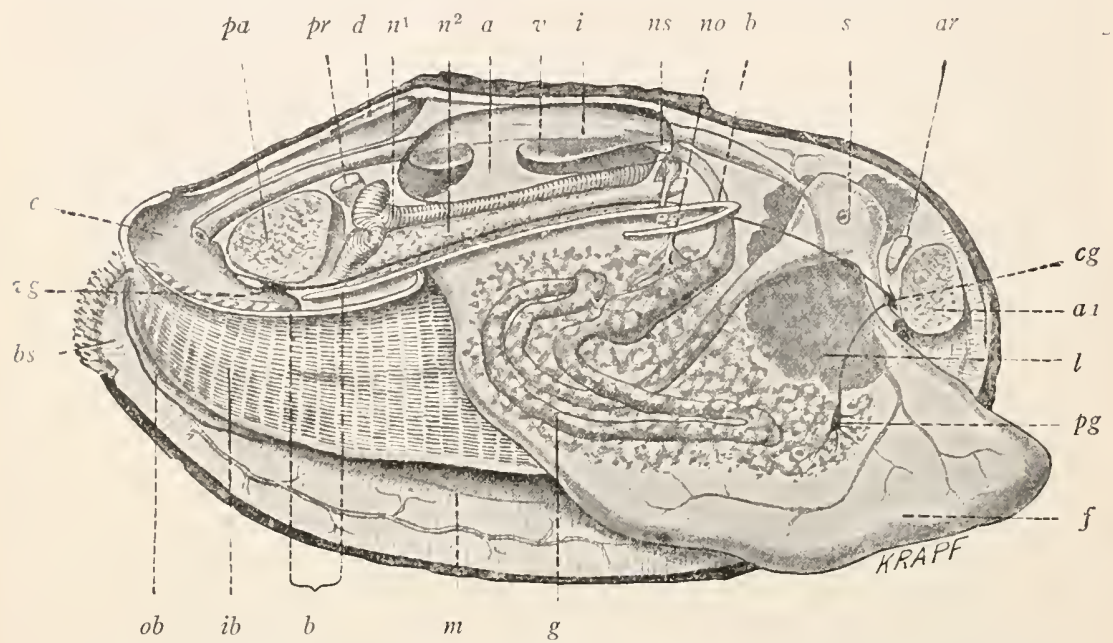

FIG. 322.-Anatomy of Anodonta, mantle, gills and liver of right side removed, pericardium opened. $a$, auricle; $a a$, anterior adductor; $a r$, anterior retractor; $b$, base of right gills, cut away; $b s$, branchial siphon; $c$, cloaca; $c g$, cerebral ganglion; $d$, dorsal mantle opening; $f$, foot; $g$, gonad; $i$, intestine; $i b$, inner gill; $l$, liver; $m$, mantle; $n^{1}, n^{2}$, parts of nephridium; $n o$, nephridial opening; $n s$, nephrostome; $o b$, outer gill; $p a$, posterior adductor; $p g$, pedal ganglion; $p r$, posterior retractor; $v$, ventricle; $v g$, visceral ganglion.

a descending and an ascending. These branchial threads are so hooked together that they give the impression of a continuous leaf. In the true lameilar gill the threads of the filibranch grow together at intervals, leaving openings, the gill slits. Since there is an ascending and a descending limb, it follows that each gill consists of an inner and an outer leaf (fig. 323), leaving a space between into which the gill slits open. This internal space in some serves to contain the young.

The complete enclosure of the body in the mantle folds has led to a degeneration of the head (acephala) and its normal appendlages (tentacles, usually the cephalic eyes, radula, and salivary glands). Hence there are only two divisions in the body, dorsally the visceral sac and ventrally the foot. The foot, degenerate in many, has a broad sole only in Pectunculus and the Nuculidæ; usually it is hatchet-shaped (Pelecypoda), that is, compressed with a rounded rentral margin. It may be enormously expanded and contracted again. This expansion is accom- 
plished by forcing blood from other regions into the foot. While this makes the foot on organ of locomotion, it often serves as a means of attachment. Inside is a large byssus gland which can secrete silky threads, the byssus (fig. 324), one end of which is fastened to foreign objects by means of a finger-like process of the foot, while the other end remains in connection with the foot. Molluscs with a byssal glind are found anchored by byssal threads to stones, etc.

The heart, surrounded by a pericardium, usually occupies the most dorsal part of the risceral sac. It consists of a rentricle and a pair of

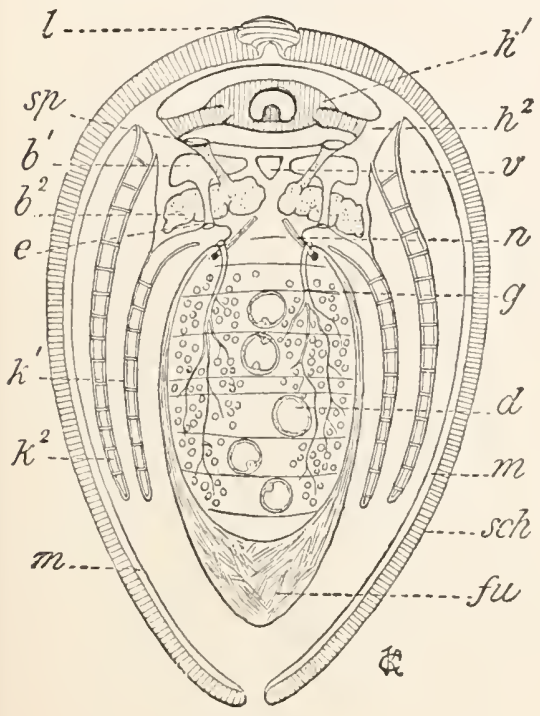

FIG. 323 .

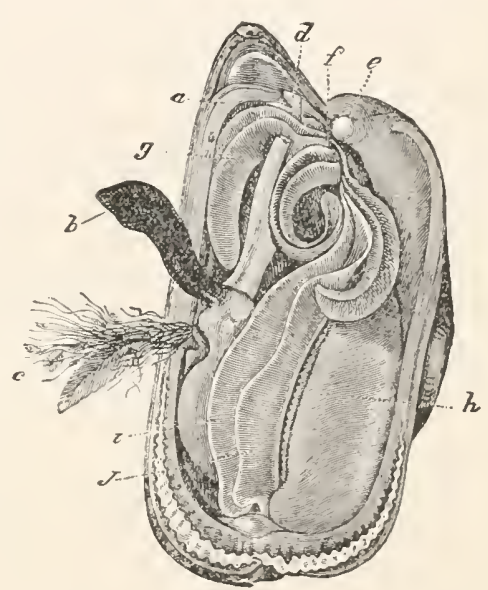

FIG. 324 .

FIG. 323.-Projection of section through foot and heart of fig. $322 . b^{1}, b^{2}$, upper and lower limbs of nephridium; $d$, intestine: $e$, nephridiopore; $f u$, foot; $g$, gonad; $h^{1}$, ventricle surrounding the intestine; $h^{2}$, auricle; $k^{1}, k^{2}$, inner and outer gill lamella; $l$, hinge ligament; $m$, mantle; $n$, cerebro-visceral commissure; $s p$, nephrostome; $v$, venous sinus.

FIG. 324.-1Mtilus edulis* (after Blanchard). $a$, edge of mantle; $b$, spinning finger of foot; $c$, byssus; $d, e$, retractors of foot; $f$, mouth; $g$, labial palpi; $h$, mantle; $i, j$, inner and outer gills.

auricles (figs. $322, a, v^{2}, 323, h^{1}, h^{2}$ ). The auricles receive the blood direct from the gills; the ventricle forces it out through anterior and posterior aortæ (fig. 322), the latter lacking in many species. The excretory organs (organs of Bojamus) lie immediately below the pericardium (fig $322, n)$. Each consists in fresh water mussels of a dorsal smooth-walled chamber and a lower portion traversed by threads, both connected behind but separated elsewhere by a thin partition. The lower chamber is con- 
nected in front with the pericardium by a ciliated canal, the nephrostome, while the upper opens to the outside by a short canal, the ureter, in the region of the inner cavity of the inner gill. In this way a connection is established from the pericardium to the exterior, the apparatus being apparently a true nephridium. In primitive forms it serves also as genital duct, but usually the genital and reproductive ducts are separate. The animals are usually diœcious, the gonads being acinose glands.

The digestive tract (fig. 322) begins with a short osophagus, widens out to a large stomach from which a slender intestine leads, with many convolutions, to the anus. In most Acephals the intestine passes through the pericardium and ventricle. The alimentary tract is enveloped by the gonads and the voluminous liver, the secretion of the latter emptying by two ducts into the stomach. Usually the stomach has a blind sac, in which lies the crystalline style, a rod-like structure of uncertain significance.

The three typical molluscan ganglia (p. 3I2) are uncommonly wide apart. The two brain (cerebropleural) ganglia lie either side of the mouth at the base of the labial palpi. They are very small, since cephalic sense organs are lacking, and are united by a transverse supraœsophageal

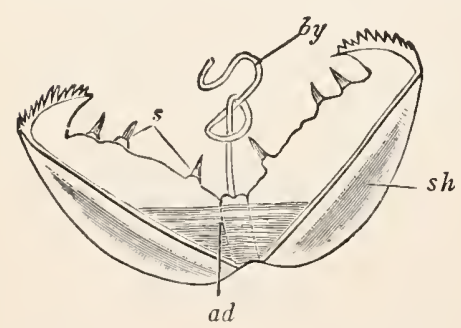

FIG. 325.-Glochidium of Anodonta (from Balfour). $a d$, adductor; $b y$, byssus; $s$, sense hairs; sh, shell. commissure. The posterior ganglia (united parietal and visceral ganglia) lie near the anus, ventral to the posterior adductor. The pedal ganglia, rather far forward in the muscles of the foot, are closely approximate. Of the higher sense organs only the statocysts near the foot are constant. The labial palpi are also highly sensory, while two small osphradia occur at the basis of the gills. When eyes occur they are, as in the scallops (Pectinidx), arranged in a row like pearls on the margin of the mantle. Their structure is different from that of the cephalic eyes of other molluscs. Small tentacles with sensory powers may occur on the margin of the mantle and the tip of the siphon.

Veligers (fig. 3 3 ) are very common in development. When this stage is lacking the history may contain a metamorphosis as in the fresh-water mussels. The young, known as Glochidia, live in the maternal gills and differ from the adult by a byssus thread, by only a single adductor, and by a hook or tooth on the free margin of the shell (fig. 325). After escape from the gills they attach themselves by means of the hooks to passing fish, where they produce an ulcer in the skin in which they grow, and by developing the adductor muscles attain the definitive form. After this metamorphosis they fall to the bottom, to live henceforth half buried in the mud. 


\section{Order I. Protochonchiæ.}

The primitive character of these forms is shown by the structure of the gills, which are either ctenidia (Protobranchiata) or filamentary (Iilibranchiata), yet here and there, as in the scallops and oysters ('seudolamellibranchiata), the fusion of gill filaments is already begun. Hinge and ligament are symmetrical with regard to the umbo, or vary little from symmetry. Hinge teeth may be lacking, and the ligament is wholly or in part internal. The mantle edges are free, and rarely is there the first trace of fusion to form a siphon.

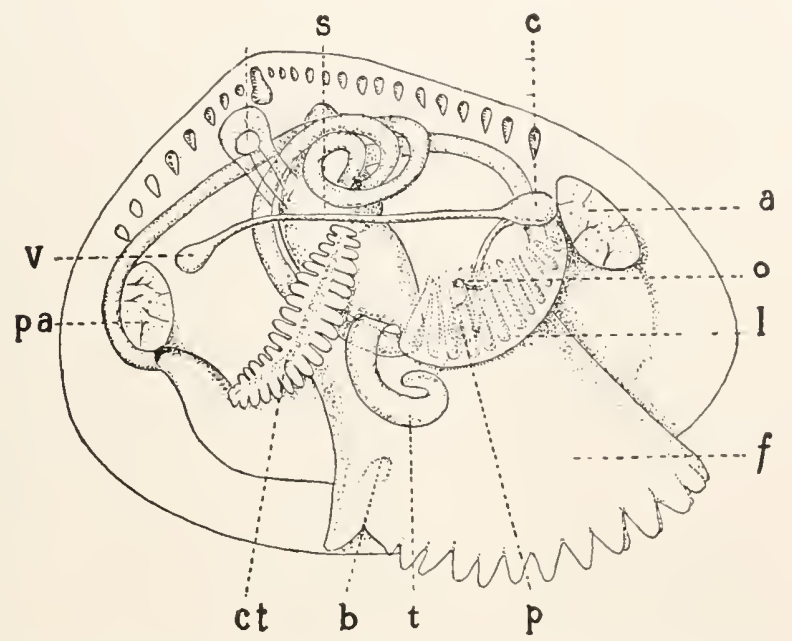

Fig. 326.-Anatomy of Nucula (after Drew). $a a$, anterior adductor; $b$, byssal gland; $c$, cerebral ganglion; $c t$, ctenidium; $f$, foot; $h$, heart; $l$, labial palpus; $o$, statocyst; $p$, pedal ganglion; $p a$, posterior adductor; $s$, stomach; $t$, appendage of palpus; $v$, visceral ganglion.

Sub Order I. DIMYARIA. Two equally developed adductors. The taxodont Nuculide have ctenidia, a broad foot, pleural and cerebral ganglia separate, and gonads emptying through the nephridia, all points which show them extremely primitive. Nucula,*Leda,* Yoldia.* The ARCID $\approx$ are also taxodont, but filibranch. Scapharca,*Argina.* Solemyide.

Sub Order II. ANISOMIYARIA. Anterior adductor rudimentary (Heteromyaria) or wanting (Monomyaria). With the exception of SpondyLide, all the families lack a hinge. To the Heteromyaria belong the MYliLidæ, or mussels, with strong byssus and shells pointed anteriorly. Modiola, * Pinna, * Mytilus edulis; * eaten in Europe, but occasionally poisonous. Lithodomus* bores into stone. The Aviculine includes the pearl oysters of the East and West Indies (1/eleagrina). OstrжID Ostræidæ, or oysters, usually attached by the right valve. The Pectinidæ, or scallops, are free-swimming and have highly developed green eyes on the edge of the mantle. 


\section{Order II. Heteroconchiæ.}

Gills always lamellar, their outer surface frequently plaited. Hingein rare cases (Anodonta) lost by degeneration-is heterodont or modified from a heterodont condition. The mantle edges but rarely free in their whole extent; siphons usually present, but in some so small (Integripalliata) as to cause no sinus in the pallial line; in others (Sinupalliata) large, the pallial line having a marked sinus. Anterior and posterior adductors equally developed.

Sub Order I. INTEGRIPALLIATA. UNIONIDÆ, freshwater mussels; hundreds of species in the Mississippi basin afford material for pearl buttons. In some pearls of value are occasionally found. Unio, ${ }^{*}$ Anodonta.* The tropical TRIDACNIDE includes the largest acephalan, Tridacna gigas, the shell of which may be four feet long and weigh three hundred pounds. The heart shells (CARDIID £-Cardium, * Serripes*) and AsTARTID瓜, marine, and the fresh-water Cycladide (Cyclas, Pisidium**), about the size of peas, belong here, as probably do the extinct RUDIsTID 2 of the cretaceous, in which the attached right valve is drawn out into a long cone, the left fitting like a lid over the small cavity.

Sub Order II. SINUPALLIATA. The VENERIDE with swollen shells, I'cmus inercenaria, q quahog, MACTRID王, hen

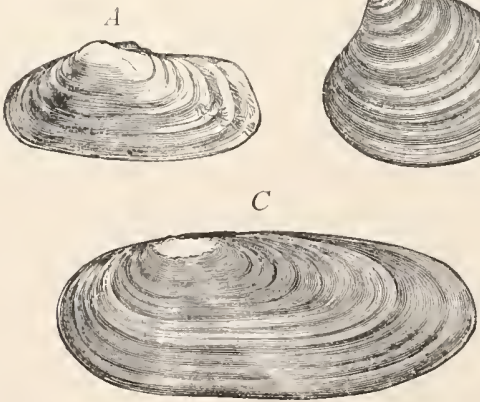

FIG. 327 .

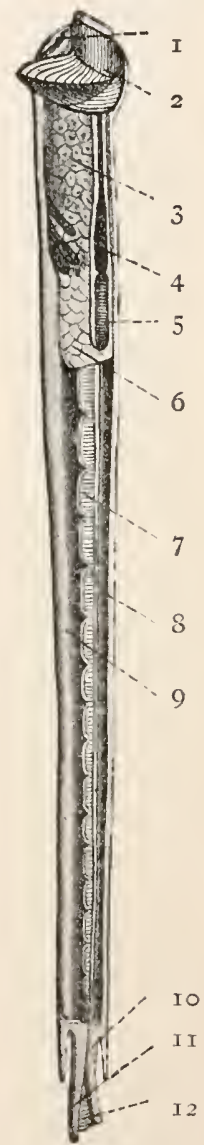

FIG. 328 .

FIG. 327.- A, Saxicava arctica; $B$, Astarte sulcata; $C$, Siliqua cosiata (from BinneyGould).

FIG. 328.-Teredo navalis, ship-worm (after Grobben). The mantle opened back to the siphons. I, foot; 2 , shell; 3 , liver; 4,5 , ventricle and auricle of heart; 0 , gonads; 7. gills; 8 , cloaca; 9, gill chamber; IO, I I, anal and branchial siphons; I2, pallets on siphons.

clams, and the Tellinide (Tellina* Macoma*), have short siphons. In others the siphons are so large that they cannot be entirely retracted within the shell, as in the MYIDE, represented in all northern seas by the long clam, $M y c$ arenaria, ${ }^{*}$ and in the razor clams (SolENide; Solen, Ensatella*). The allied 
SAXICAvide have burrowing species. These forms connect with others (Tered $0^{*}$ ) in which the united siphons far exceed the rest of the body in lengtl, giving the animal a worm-like appearance (fig. 328 ). Since the valves do not cover the whole body, they are supplemented by accessory shells, or the wormlike body secretes a tube in which the rudimentary valves are imbedderl. The PHoLadide (phosphorescent), burrow in wood, clay, or stone. The shell is well developed. In the ship worms (TEREDID E) the shells, on the other hand, are small, while some species line their burrows with lime. The species of Teredo* by their boring habits do much damage to wood in the sea, especially in the tropics.

Lastly, there should be mentioned the little-known SEPTIBRANCHIATA, in which the gills have the shape of a septum perforated by gill slits separating the branchial and cloacal chambers. Silenia, Cuspidaria.

\section{Class III. Scaphopoda (Solenoconchæ).}

The tooth shells resemble the Acephala in the paired liver and nephridia and in the nervous system (except that a buccal ganglion is present and the pleural ganglia are distinct from the cerebral). In some points they are primitive (persistence of jaws and radula), but in others they are considerably modified. They lack gills, have unpaired diøecious gonads, rudimentary heart (no auricle), and have two bunches of thread-like tentacles either side of the mouth. The mantle lobes, paired in the larva, unite below, forming a sac open at either end, and this secretes a shell shaped like the tusk of an elephant, from the larger end of which protrudes the long threelobed foot used for boring in the sand. Dentalium (fig. 329), Entalis.*

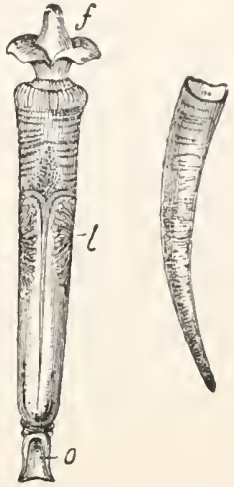

FiG. 329.-Dentalium elephantinum, tooth shell; left the animal, right the shell. $f$, foot; $l$, liver region; $o$, hinder opening of mantle

\section{Class IV. Gasteropoda.}

Although more highly organized than the Acephala, the snails are in some respects more primitive. The regions of the body-foot, visceral sac, head, and mantle-occur in all orders, although in each forms may occur in which one or another part is lost. As a rule, the foot is flattened ventrally to a creeping sole. In it may be distinguished anterior and posterior processes, the propodium and metapodium, a sharp lateral margin, the parapodium, and, above these, appendages or ridges, the epipodia. Inside the foot is usually a pedal gland.

The head bears (I) the tentacles, a pais of muscular lobes or hollow retractile processes; (2) a pair of primitive resicular eyes, which usually lie at the basis of the tentacles, but may rise even to their tips. In many snails the eyes are on special stalks which (stylommatophorous Pulmonata) form a second pair of tentacles. The protrusion of the hollow tentacles is caused by an inflow of blood, their retraction by muscles attached to the tip which draw them in like a finger of a glove. 
The mantle extends from the back forward over the body to near the beginning of the head. It covers the spacious mantle cavity, which in the water-breathing Prosobranchiata, etc., contains the gills (ctenidia) and opens outward by a large aperture under the margin of the mantle. The edge of the mantle may be produced into a long groove-like ciliated siphon, conveying water to and from the branchial chamber, and is of importance in determining the shape of the shell. In the terrestrial snails branchiate respiration is replaced by pulmonate (Pulmonata, Cyclostoma), which is retained in many forms (Basommatophora) which have returned to an aquatic life. In place of the mantle cavity there is a sac filled with air, with a rich network of blood-ressels in its dorsal wall, and with only a small opening, the spiracle, on the right side. This lung was formerly thought to be the mantle cavity in which the ctenidium had degenerated, but development shows it to be an evagination arising in the mantle groove.

The visceral sac, by the great development of the gonads and liver, becomes very large. Since growth downwards is prevented by the muscular foot, the organs press towards the back, carrying before them the dorsal wall at the origin of the mantle folds, the line of least resistance. Some organs, like hind gut, nephridia and heart, may be pressed into the roof of the mantle cavity. When the visceral sac, as often occurs, becomes enormous, it does not stand directly upwards, but coils, usually from left to right, in a spiral. The older the animal the more the spiral coils and the larger the last or body whorl.

From the foregoing the shape of the shell is easily understood. As a secretion of the mantle and the visceral sac it takes the form of the latter. With slight development of the visceral sac it forms a flattened cone (fig. $33 \circ, A$ ), or is slightly coiled at the apex, as in the abalone $(B)$. When the visceral sac is greatly elongate the shell is correspondingly an elongate cone. It is rarely irregularly coiled (Vermetida, $D$ ). It is usually coiled like a watch spring in one plane, or like a spiral staircase; in the latter case the shell is more or less conical $(C, E)$ and one can speak of its apex and base. In the middle of the base is usually a depression, the umbilicus. Sometimes the coils do not touch in the axis connecting umbilicus and apex, but usually they fuse into a calcareous pillar, the columella, around which the whorls pass $(E, c)$.

The shell increases by additions from the mantle edge; and since this determines the aperture, the shell is marked with parallel lines of growth. The pigment is produced on the edge of the mantle, and passes into the shell as formed, causing its color pattern. When the siphon is present the shell shows a corresponding process. Thus are distinguished 
holostome shells with entire mouths (C) and siphonostome shells, in which the anterior margin is drawn out in a groove $(E)$.

A simple conical shell without further evidence is not proof of primitive structure. It may arise from the spiral form by degeneration, if the visceral sac be reduced. Thus the shells of Fissurella and Patella are to be explainer, for the viscera here show the results of an earlier spiral twist.

In most places the union between shell and soft parts is not very firm, but the connection at the aperture is more intimate, while a muscle is attached to the columella at about its middle, the other end being inserted in the foot. This retracts the animal within the shell, the head and anterior part of the foot
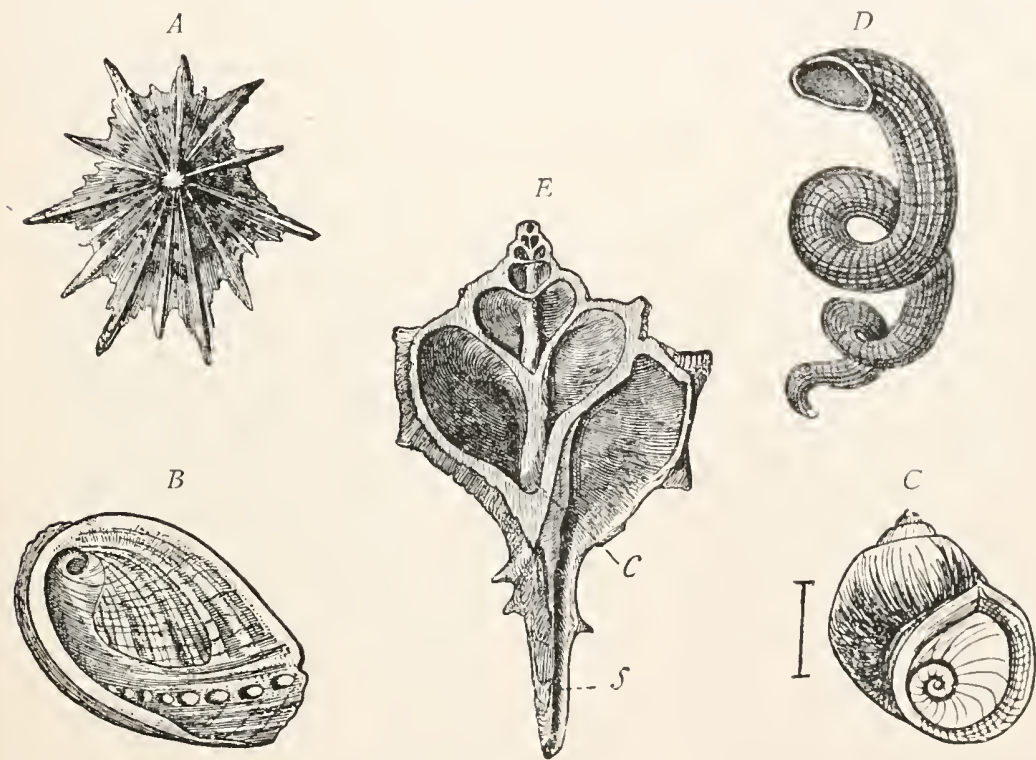

Fig. 330.-Various forms of shells (after Schmarda, Bronn, and Clessin). A Patella costata; $B$, Haliotis tuberculata; $C$, Lithoglyphus naticoides; $D$, Termetus dentiferus; $E$, shell of Murex opened to show $c$, columella; s, siphon.

going first and then the metapodium, with its dorsal surface towards the aperture. Hence in many spec:es this surface secretes a door, or operculum, which closes the aperture when the body retracts. Since the aperture increases in size with growth, the operculum enlarges in a spiral manner (fig. 330, C), some times forming a spiral line on the outside, or on both surfaces. Land snails are usually without opercula, but in hibernation they can close the shell by a calcareous plate, the epiphragm. In the spring this separates from the shell and is lost.

In most gasteropods the shell is coiled to the right, but in some species (fig. 33I) the whorls are constantly turned to the left, while reversed specimens occasionally occur in many species which are normally dextral.

There are usually two lavers in the shell, an inner lamellar laver (not always present), which sometimes is highly iridescent, and an outer opaque, porcellan- 
ous layer, contains the pigment and may be covered by a horny cuticle. In rare cases mantle and shell are lacking, or the mantle is present but the shell is rudimentary and not visible externally because the mantle folds have grown over it. In these cases the visceral sac is not prominent. Since the shelless forms possess a mantle and shell in the young, the adult conditions are explained by degeneration.

Only a few gasteropods are bilaterally symmetrical. Usually the spiral twist of the visceral sac has resulted in a torsion of other parts from left to

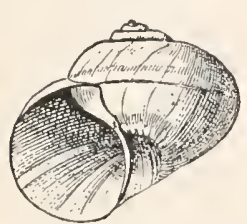

Fig. 331 .- Sinistral shell of Lanistes carinatus (f rom Leunis). right, in which alimentary tract, nephridia, gills, heart, and nervous system take part (fig. 332). The intestine is bent in this way, the anus opening into the mantle chamber on the right side $(B)$ or the twisting may be continued so far as to double the intestine on itself, the anus being in the middle line in front, near the head $(C)$. Nephridia, gills (with them the osphradia), and heart wander in company, so that the organs primitively belonging on the left side may be transferred to the right and vice versa. With this there is a tendency to asymmetry and the loss of the organs (usually of the primitively left side). When the nervous system takes part in the twist-
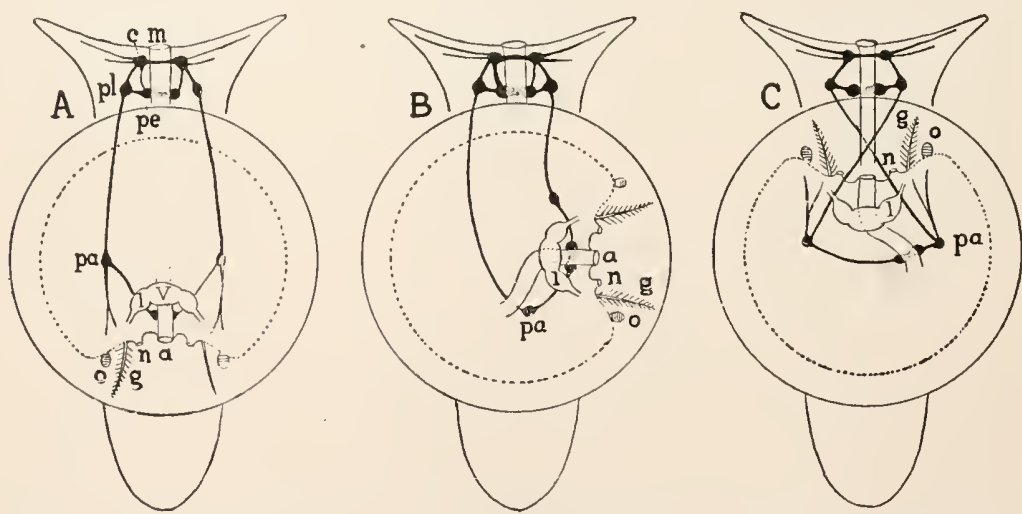

FIG. 332.- Three diagrams illustrating the torsion of the body and the twisting of the nervous system in gasteropods (after Lang). $A$, bilateral; $B$, asymmetrical; $C$, streptoneurous condition. The reference letters are placed upon the organs of the primitive left side. $a$, anus; $c$, cerebral ganglion; $g$, ctenidium; $l$, auricle; $m$, mouth; $n$, nephridial opening; $o$, osphradium; $p a$, parietal ganglion; $p \varepsilon$, pedal ganglion; $p l$, pleural ganglion; $v$, ventricle.

ing a crossing of the cerebrovisceral commissures takes place, known as streptoneury or chiastoneury $(C)$.

The alimentary canal begins with a muscular region which in some groups is dereloped into a large protrusible proboscis (fig. 333). The 
pharynx, which follows, contains the tongue, a rentral ridge supported lyy one or more cartilages and covered by the lingual ribbon or radula (odondophore). The upper surface of the radula is armed with sharp, backwardly directed teeth (fig. 334) arranged in transverse and longitudinal rows; these vary so in number, form, size, and arrangement that they are of value in classification. 'The radula is formed in the radula sac,

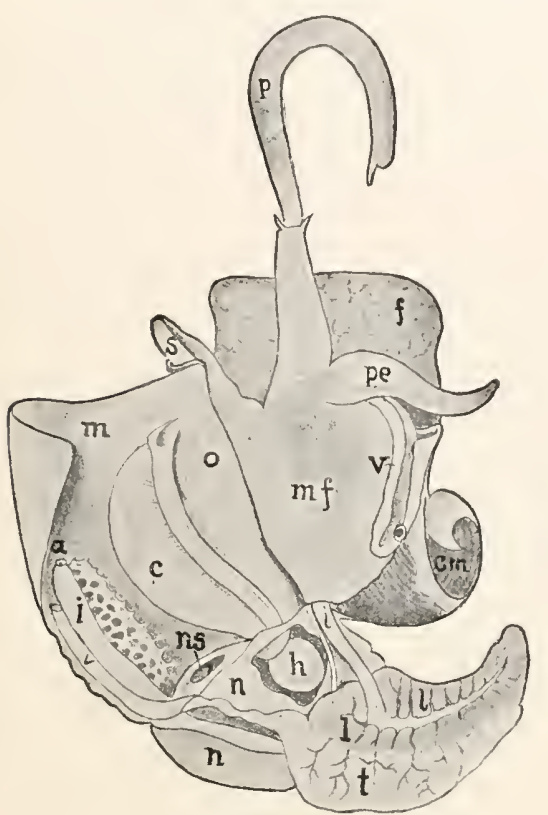

FIG. 333 . which lies behind the tongue (fig. $334, r s$ ). From this it grows forward like a nail over its bed as fast as it is worn out in front. It is opposed in eating by a single median or a pair of lateral jaws (lacking in carnivorous forms).

The rest of the alimentary canal is convoluted, the anus

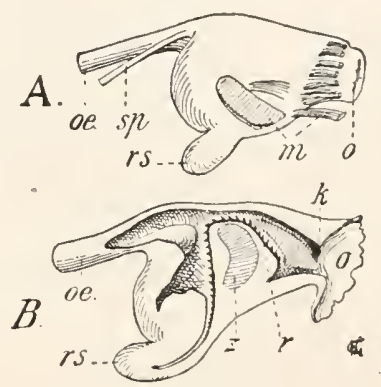

FIG. 334 .

FIG. 333.-Pyrula tuba, male (after Souleyet). The mantle has been cut on the right side and turned to the left, reversing the pallial organs. $a$, anus; $c$, ctenidium; $\mathrm{cm}$, columellar muscle; $f$, foot; $h$, heart in pericarlium; $i$, intestine; $l$, liver; $m$, mantle; $m f$, floor of mantle cavity; $n$, nephridium; $n s$, opening of nephridium; o, osphradium; $p$, proboscis; pe, penis; $t$, testes; $v$, vas deferens cut in two.

FIG. 334.-Pharyngeal region of Helix pomatia. A, side view; $B$, section. $m$, muscle; $o c$, osophagus; $r$, radula; $r s$, radula sac; $s p$, salivary duct, $z$, lingual cartilage.

being usually on the right side in front, in or beside the mantle chamber (figs. 337, 338, 339). Rarely it empties in the middle line behincl. Esophagus, stomach, and intestine are slightly marked off from each other. The convolutions of the intestine are enveloped by the liver, which forms the chief part of the visceral sac. A pair of salivary glands empty into the pharynx, these in the Doliidx secreting a saliva containing 5 per cent. of free sulphuric acid.

The nervous system usually differs from that of other molluscs in 
that the pleural and parietal ganglia are separate. If the commissures be short, the ganglia are collected near the pharynx and, thus freed from the body torsion, are symmetrical (orthoneurous, fig. $336, I I$ ). If the

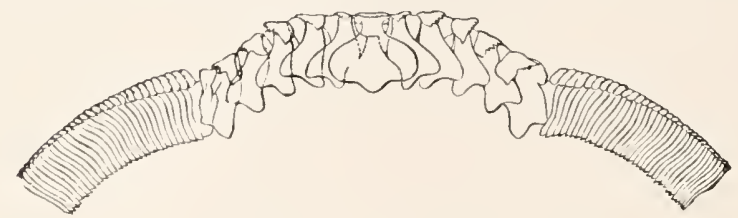

Fig. 335.-Row of teeth from the radula of Trochus cinerarius (after Schmarda).

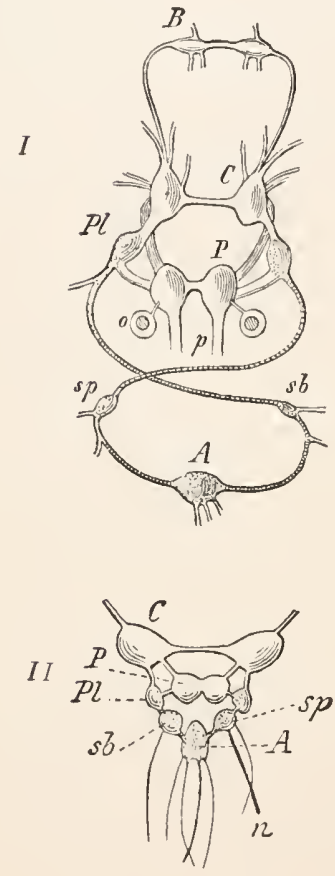

FIG. 336 .

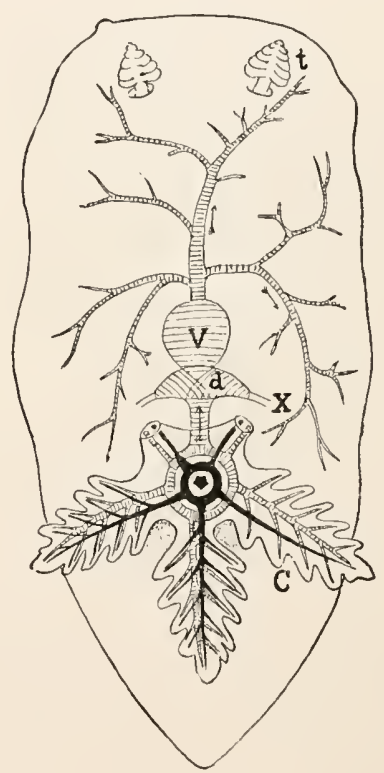

Fig. 337 .

FIG. 336.- $I$, streptoneurous nervous system of Paludina (after Hering, from Gegenbaur). II, orthoneurous system of Limnca (after Lacaze Duthiers). A. visceral; $B$, buccal: $C$, cerebral; $p$, pedal; $P l$, pleural; sh, $s p$, sub- and supraintestinal ganglia; $n$, olfactory nerve; $o$, otocyst.

FIG. 337--Diagram of circulation in Doris (after Leuckart). a, auricle; $c$, gills around anus; $t$, tentacle; $v$, ventricle; $x$, vessels returning venous blood from the body.

cerebrovisceral commissures be longer, the result is almost always streptoneury. Pleural and visceral ganglia hold their place, but the right parietal ganglion crosses above the intestine to the left side (hence called 
supraintestinal), while the left passes under the intestine to the right side (subintestinal), the cerebrovisceral commissures being twisted like the figure 8 . The strong development of the pharynx is accompanied by' buccal ganglia.

Gills, heart, and nephridia are best treated together. Certain genera (Haliotis, Fissurella, etc.) recall the Acephal a in having the heart traversed by the intestine, the paired ctenidia, nephridia and nephridial ducts, and two auricles to the heart. As a rule the asymmetry induced by the torsion of the body has resulted in the loss of the ctenidium, osphradium, and nephridium of the primitively left side, and with the loss of one gill there

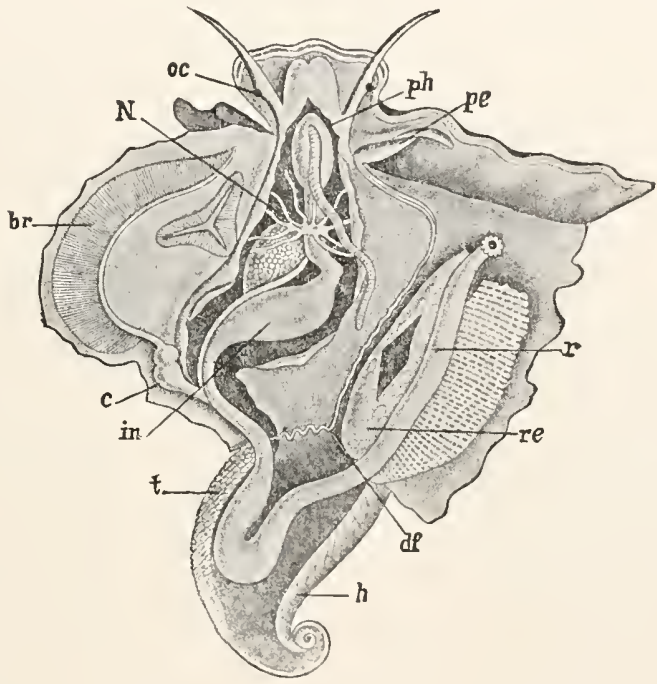

FIG. 338.-Anatomy of Cypraa tigris (after Quoy et Gaimard). br, ctenidium; $c$, heart; $d f$, vas deferens, $h$, liver; $m$, stomach; $N$, cerebral ganglion; oc, ere: pe, penis; $p h$, pharynx, the radula drawn out; $r$, rectum; $r e$, nephridium; $t$, testes.

is usually a loss of the corresponding auricle. Prosobranchs and Opisthobranchs are recognized accordingly as the gills are on the anterior or posterior part of the body. In the Opisthobranchs (fig. 337) the ctenidia have been lost and are replaced by secondary gills on the back. Here the heart is in front of the gills; it receives blood from behind and forces it forward to the head by an aorta. In the Prosobranchs the heart has loeen twisted so that the auricle and the ctenidium are in front (fig. 338). while the aorta leads backwards. The nephridium, which communicates with the pericardium by a nephrostome, is usually saccular, its duct empties beside the anus. 
The always unpaired sexual organs in some forms (Cyclobranchs and many Zygobranchs) empty into the nephridia and possibly the others utilize the rudiments of the right kidney. The sexual opening is almost always on the right side, beside the anus or in front of it on the head. Its position may be recognized in males and in hermaphroditic species by the grooved dermal fold, the penis (fig. $338, p e$ ). Occasionally this is separated from the genital pore, but is connected with it by a ciliated groove. The sexual organs are very variable in structure. They show two extremes. On the one hand are completely diœcious species, on the other there may be complete hermaphroditism

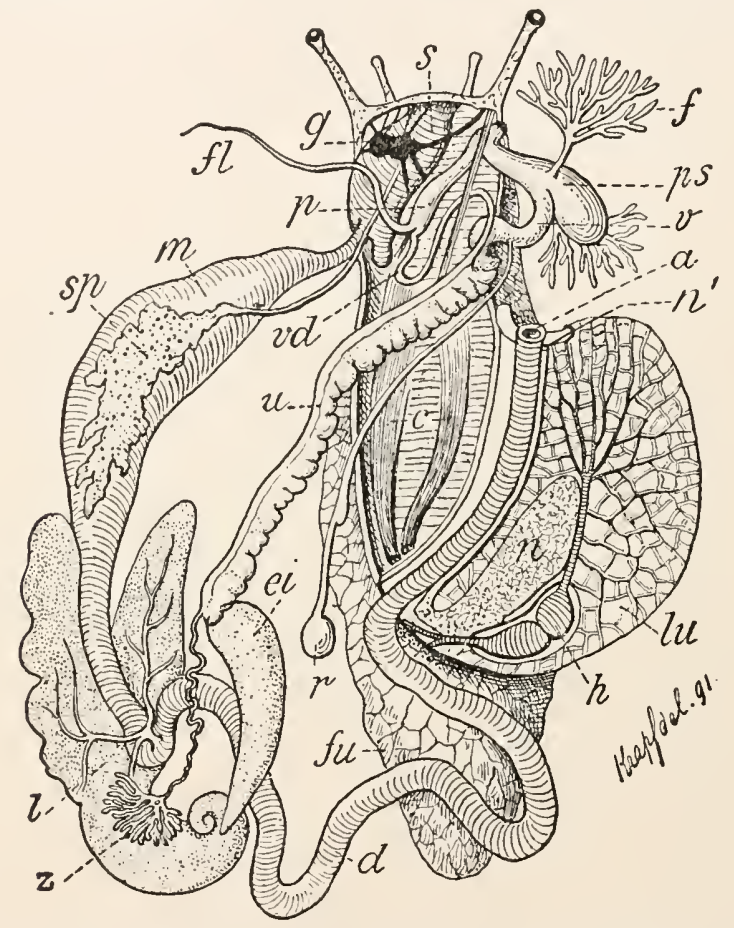

F1G. 339.-Anatomy of Helix pomatia, the roof of the pulmonary sac cut at the left sicle and turned to the right; the pericardium and visceral sac opened and the viscera separated. $a$, anus; $c$, columellar muscle; $d$, intestine; $e i$, albumen gland; $f$, finger-form gland; $f l$, flagellum; $f u$, foot; $g$, cerebral ganglion; $h$, heart; $l$, liver; $l u$, lung; $m$, stomach; $n$, nephridium; $n^{\prime}$, its opening; $p$, penis; $p s$, dart sac; $r$, receptaculum seminis; $s$, pharynx; $s p$, salivary gland; $u$, uterus; $v$, vagina; $v d$, vas deferens; $z$, hermaphrodite gonad.

(many Tectibranchs, Pteropoda), in which the male and female organs are united throughout their extent. Intermediate stages occur; that of the pulmonates is shown in fig. 339 .

The terrestrial snails lay their large tough-shelled eggs in damp earth; in the aquatic forms the eggs are laid in masses, usually gelatinous, each egg with a layer of albumen and a firm shell. Occasionally there is a kind of nest, as is the case with Ianthina which carries the mass of eggs, attached to the foot, about with it. A few gasteropods are viviparous. 
In the development the great constancy with which the veliger stage (figs. 3 I3, 314) appears is noticeable. Nost marine larve swim at the surface by their velum before creeping at the bottom. But in those cases where the snail leaves the egg in the adult form the velum is usually developed in embryonic life, sometimes so strongly that the embryo rotates in the surrounding fluid.

\section{Order I. Prosobranchia.}

In the prosobranchs the twisting of the body has brought the ctenidia and consequently the auricles in front of the ventricle, and hence the aorta leads backwards. It also results in the twisting of the nervous system like the figure 8 . The sexes are separate and the shell and mantle are usually well developed. Certain Prosobranchs are near the primitive Amphineura in the retention of both ctenidia, both auricles, and both nephridia, but in the great majority only one gill (the primitive right) is present and the corresponding auricle alone is well developed, although the other may exist in a rudimentary condition.

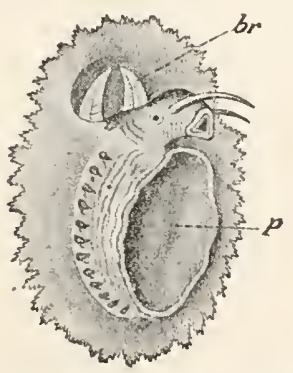

FIG. 3to.-Fissurella patagonica, ventral view (from Bromn). br, the paired gills; $p$, foot.
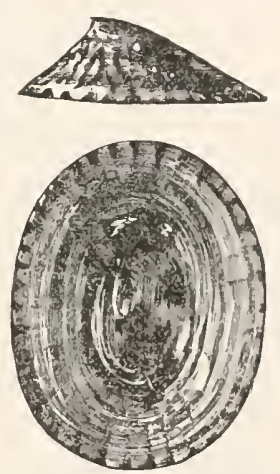

FIG. 3+1.--Acmaa testudinalis, * limpet (from Binney-Gould).

Sub Order I. ASPIDOBRANCHIA (Diotocardia), Ctenidia bipectinate (fig. 340) or absent. Usually two a uricles and two nephridia. DOCOGLOSSA (limpets), auricle single; intestine not passing through heart, shell conical.

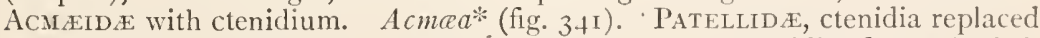
by a ring-like mantle gill. ZYGOBRANCHIA. Two ctenidia (fig. 3.0), shell with marginal slit or with holes; auricles and usually nephridia paired; heart traversed by intestine. Fissurellid e, keyhole limpets; shell conical, with apical opening. HALIOTID.玉, abalones (fig. 330, B). AZYGOBRANCHLA. One ctenidium, two auricles. TRochIDE, Trochus, Margarita** TuRBINID.s, top shells; Sub Order II. PECTINIBRANCHIA (Monotocardia). Ctenidium unipectinate, a single auricle, osphradium well differentiated (fig. 333), intestine not passing through the heart. Many groups based upon structure of lingual rib- 
bon. RHACHIGLOSSA; siphonostomate, predatory. MURICID (Iurex, Purpura, ${ }^{*}$ Urosalpin $x^{*}$ ) secrete a sulsstance turning purple by exposure to air. Tyrian purple was produced by Murex trunculus. Urosalpinx cinereus* drills into oysters. Buccinid.e, whelks, Volutide, and Olivide belong here. 'TOXIGLOSSA; CONIDA, with esophageal poison gland. TENIOGLOSSA; Naticide, Neverita, * Lunatia.* Littorinide; periwinkles. Cypræid. cowries; Cyprea moneta used as money in Africa. AMPUlLaRID part of branchial cavity acting as lung, part containing ctenidium. PaludivIDE, fresh water.

HETEROPODA. In details of gills, genitalia, heart, and nervous system these are true Pectinibranchs, but from an exclusively pelagic life have acquired peculiar modifications. Like most pelagic animals they are transparent. The head is elongate. Most characteristic is the division of the foot into pro- and

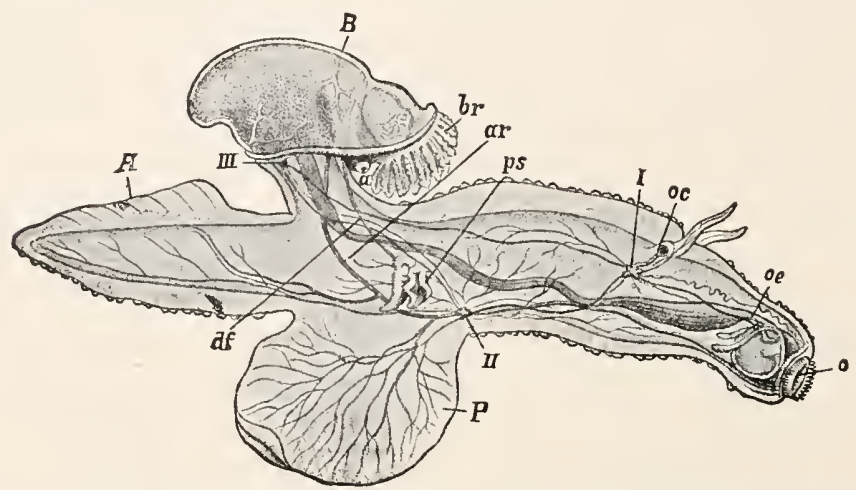

FIG. 342.-Carinaria mediterranea (after Gegenbaur), shell removed. $A$, metapodium; $a$, anus; $a r$, aorta; $B$, visceral sac; $b r$, branchiæe, the heart above; $d f$, vas deferens; $o$, mouth; $o c$, eye with tentacle; oe, oesophagus; $p$, propodium; $p s$, penis; $I, I I, I I I$, cerebral, pedal, and visceral ganglia.

metapodium (fig. 342), the latter forming a tail-like elongation of the body. The propodium is vertically flattened and serves as a swimming organ. The Heteropoda are predaceous and extremely voracious. ATLANTIDE and CARINARIIDE, with shells; PTERotraCHEIDe, without.

\section{Order II. Opisthobranchia.}

The Opisthobranchia have not varied from the primitive symmetry to such an extent as have Prosobranchs and Pulmonates. The anus is in or near the median line, although it may be far forwards. The nervous system is orthoneurous, the twist being straightened (except in Actronida). 'The heart also retains its primitive position, receiving blood from behind and forcing it forwards to the body through the aorta (fig. 337). In rare cases a (right) ctenidium, a poorly developed mantle, and a thin shell occur. Usually these have been lost and the place of the ctenidium is taken by accessory gills of various forms or a dermal respiration occurs. The larva have well-developed mantle and shell. Many of the Opisthobranchs afford fine examples, in form and coloration, of protective resemblance. All are hermaphroditic and marine.

Sub Order I. TEC'TIBRANCHIA. Mantle and usually shell and ctenidium present, Bulla,* Philine,* Aplysia. Sub Order II. PTEROPODA Transparent pelagic forms which in most points agree with the Tectibranchs. 
The head and usually eyes and tentacles are lacking, while the fins (greatly developed parapodia) are highly characteristic, giving the name 'wing-footed' to these forms. 'They have rarely a single ctenidium. The THECASOMATA have shells, LiLACINIDA, HYALEIDE. The shells of

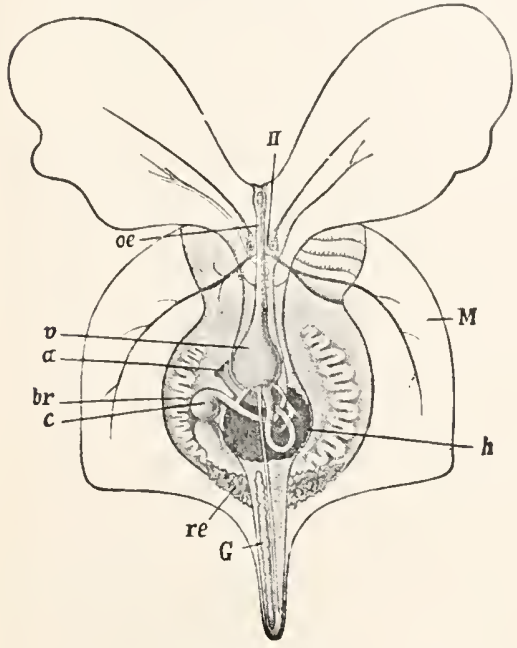

FIG. 343 .

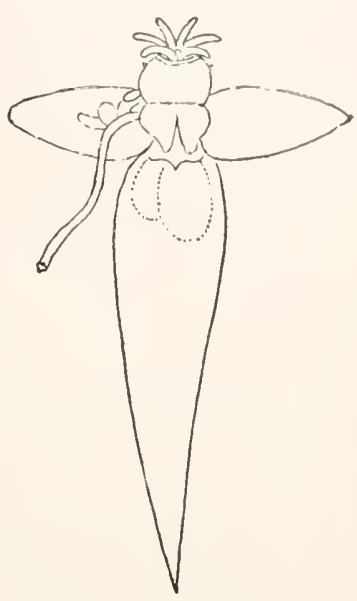

FIG. 344.

FIG. 343.-Hyalca complanata from above (after Gegenbaur). a, anus; br, gill; $c$, heart; $g$, gonad; $h$, liver; $m$, mantle; oe, aesophagus; re, nephridium; $v$, stomach; $I I$, pedal ganglion and otocyst.

FIG. $34+.-A$, Clione papilionacea.*

CAVolinid m make the 'pterpod ooze' of the deep seas. GYMNOSOMATA; shell lacking. Pneumodermon, Clione** Sub Order III. NUDIBRANCHIA. Shell, ctenidia, and osphradia lacking; most possessing accessory gills (cerata) of varying form and distribution. DORIDIIDE (Fig. 345). TRITONIIDE, EOLIDE

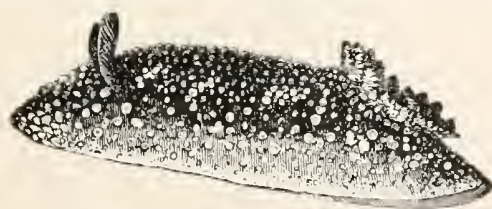

FIG. 345.-Doris bilamellata.*

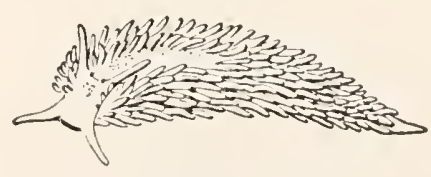

Fig. 346.- Eolidia papillosa (from Ludwig-Leunis).

(Dendronotus*); ELysmD压, cerata lacking. In Eolidæ branches of the digestive tract enter the cerata, expand distally to small sacs filled with nettle cells (p. 207) used for defense; they are derived from hydroids on which these animals feed

\section{Order III. Pulmonata.}

In several respects the Pulmonata are intermediate betwcen the Prosobranchs and Opisthobranchs. Like the latter they are orthoneurous and hermaphroditic (fig. 339). On the other hand, the respiratory organ is far forwards 
near the head, with the result that the auricle is forwards, the aorta behind. 'The Testacellidie have the lungs at the posterior end of the body. Occasionally streptoneurous conditions occur (Chilina). The lung, the most characteristic feature of the order, has already been mentioned (p. 326).

Many pulmonates are aquatic, but since they have no gills they must occasionally come to the surface to fill the lung with air, but some, which live at great depths in the Swiss lakes and consequently cannot reach the surface, use the skin and to some extent the lung for water-breathing. Several genera (Plunorbis, Pulmobranchia, Siphonaria) have formed secondary gills.

Sub Order I. STILOMMATOPHORA. Four retractile tentacles, eyes at the tips of the second pair. The Helicid w, a well-developed shell. Helix; * many hundred species. Pupa, * Achatina, Bulimus, many tropical species. Limacide. Shell reduced, completely concealed in the mantle. Limax,* Arion.* Sub Order II. BASSOMATOPHORA. Only one pair of nonretractile tentacles, eyes at their base. LmMNÆIDE, pond snails. Limnca, * Planorbis.*

\section{Class V. Cephalopoda.}

The Cephalopoda are distinguished among the molluscs by their size and high organization. The majority measure, including the arms, from

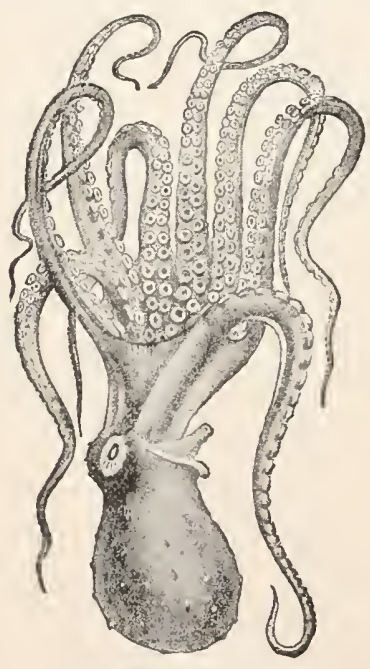

FIG. 347 .

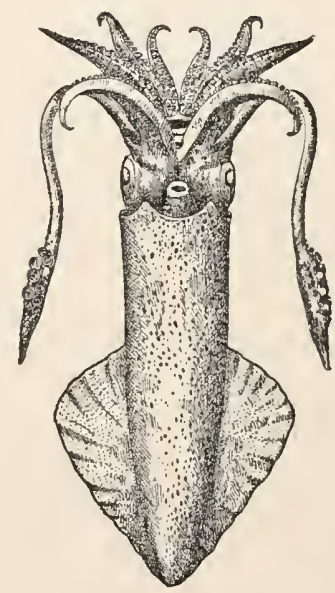

FIG. 348 .

FIG. 347.-Octopus tonganus from the side (after Hoyle). Funnel and mantle fold to the right; back and eyes on the left.

FIG. 348.-Loligo kobiensis, ventral view (after Hoyle).

eight inches to three feet in length, a few are smaller (two to seven inches), while especially rare are the giants, some of which may be over forty feet long. For a long time these large species were only known from the tales of sailors. In the last half-century some of these forms, belonging to the genus Architeuthis, have been stranded on the coasts of Newfoundland 
and Japan. One of the Newfoundland specimens had a body twenty feet long from head to tail, and one of the arms was thirty-five feet in length.

The body of a cephalopod is divided by a constriction into hearl and trunk. At the extremity of the head is the mouth, and around this a circle of arms or tentacles. Each tentacle is tapering and bears on its oral surface rows of suckers (in some species altered to hooks). 'The Octopoda have eight of these arms, all equal in size (fig. $3+7$ ), four on the right side, four on the left. The Decapoda (fig. 348 ) have in addition two longer arms between the third and fourth of the Octopoda, counting from the dorsal side. These pear suckers only on the enlarged tips and can be retracted into special pouches.

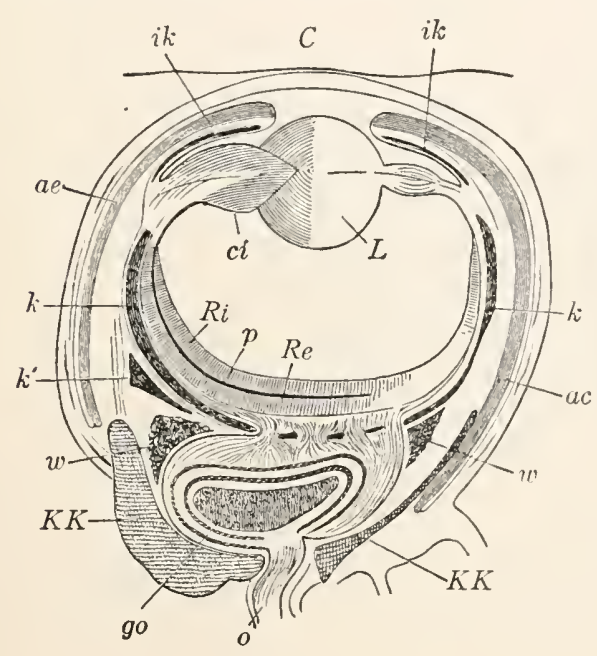

FIG. 349 .

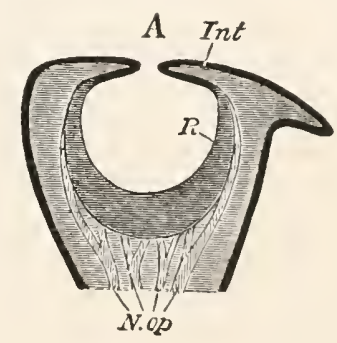

FIG. 350 .

FIG. 349-Diagrammatic section of Cephalopod eye (after Gegenbaur). ae, argentea (chorioid); $C$, cornea; $c i$, ciliary process; go, optic ganglion; $i k$, iris: $k$, cartilages; $L$, lens; $p$, pigment layer; $R e$, cellular layer of retina; $R i$, rod layer of retina; $w$, white borly.

FIG. 350.-Schematic section of eye of Nautilus (from Balfour). A, aperture of optic cup; Int, iris-like fold of integument, $N$ op, optic nerve: $R$, retina.

Behind the tentacles are the pair of large eyes which superficially closely resemble those of the vertebrates, since they have a transparent cornea and a large pupil surrounded by an iris. Internally the resemblance is not less pronounced (fig. 349). Behind the iris is a lens and a vitreous body, the latter being bounded by the retina and this in turn by a pigmented silvery layer, the argentca or chorioid, which contains cartilages 
recalling the sclerotic coat. Two striking peculiarities separate these eyes from those of the vertebrates and show that they have arisen independently and have an entirely different developmental history.

The cornea in most Decapoda has an opening by which water enters the anterior chamber; (2) the layer of rods in the retina abuts against the vitreous body and the ganglionic layer lies behind, while in the vertebrates the reverse is the casc.

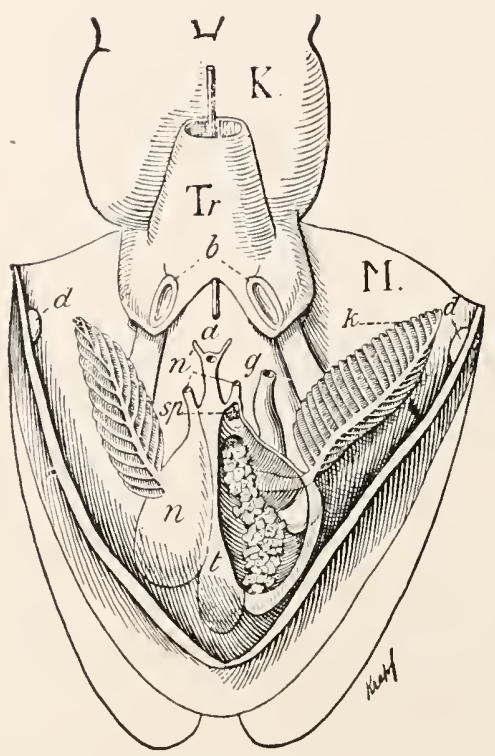

FIG. 351.-Sepia officinalis, the mantle and left nephridial sac opened to show the vena cava leading to the branchial heart. $a$, anus; $b, d$, lock of siphon and mantle; $g$, genital opening; $K$, head; $k$, ctenidium; $n$, nephridial sac; $n^{\prime}$, nephridial opening; $s p$, nephrostome; $t$, ink sac; $T r$, siphon.

The foregoing description applies to but part of the Cephalopoda. The very different Nautilide have, instead of tentacles with suckers, numerous shorter tentacles on lobular appendages, which are developed differently in the two sexes. The eyes are deep pits, opening to the exterior by a small aperture, the base of the pit being occupied by the retina, while lens, vitreous body, iris, and cornea are lacking (fig. 350). It is to be noticed that the other cephalopod eyes pass through a Nantilus stage.

In the trunk anterior and posterior regions are distinguishable, the two passing into each other on the sides. The anterior (which corresponds only in part to the ventral side of other molluscs) is wholly covered by the mantle, a strong muscular fold, which takes its origin from the periphery of the body, often encroaching upon the back and always terminating with free margins at the head. On opening the mantle by a 
ventral incision (fig. 351) the two ctenidia (four in Nautilus) are seen on the sides. Between them, in the middle line, is the anus, and right and left of this and a little behind are the nephridial openings (four in N'autilus, which also has osphradia). More lateral are the sexual openings of which one (usually the right) is commonly absent. It the head the mantle opens by a transverse slit to the exterior, but it can be closed and fastened by various locking contrivances (in Sepia, Loligo, etc., by buttonlike projections $(d)$ which fit into corresponding sockets $(b)$ on the trunk). When thus closed the communication with the exterior is by a special conical muscular tube, the funnel or siphon (Tr.), which is fastened to the body and opens widely to the mantle cavity. Since the cephalopods, ly contraction of the mantle wall, can drive the water from the mantle cavity through the siphon with great force, they can swim very rapidly by the

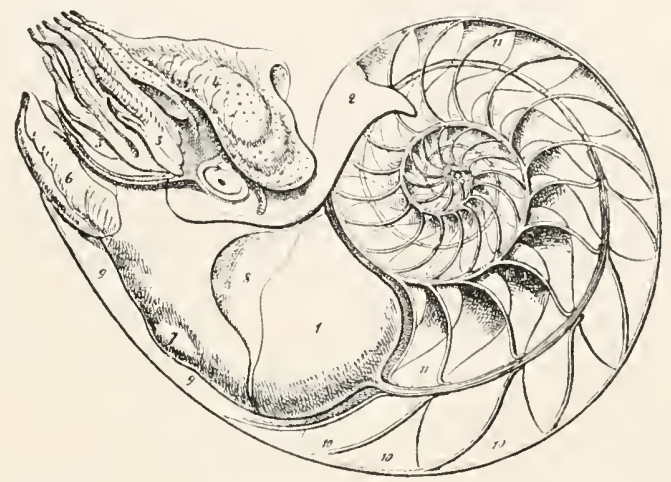

FIG. 352.-Female Nautilus, the shell bisected (from Ludwig-Leunis). I, mantle; 2, dorsal lobes; 3 , tentacles; 4 , head fold; 5 , eye: 6 , siphon; 7 , position of nidamental gland; 8 , shell muscle; 9 , living chamber; 10 , partitions between chambers; II, siphuncle.

reaction. Nautilus is peculiar in that throughout life the siphon is composed of two overlapped folds, which is significant since in the embryos of other forms the siphon (fig. 36r) arises as two separate folds which later unite to produce the definitive condition. A typical foot is lacking, but comparative morphology shows that the siphon is composed of a pair of epipodia, while many zoologists regard the arms as differentiations of the fused foot and head, since they are innervated from the pedal ganglia.

Head and trunk are covered with a thin mucous skin, which has the power of changing color in a marked degree. Loligo will quickly pass from a dark red to a translucent white; Octopus has an even greater gamut of color. 'These color changes are possible since in the corium there is a silvery layer over which are numerous different-colored pigment cells or chromat phores, in which radial muscle fibres are inserted. On contraction of these the chromatophores are 
flattened and thus influence the color; when the fibres relax the pigment cells contract to small spots. In deep-sea cephalopods phosphorescent organs have been observed.

Notwithstanding the soft bodies a well-developed shell occurs in living cephalopods only in Nautilus and Argonauta (figs. $35^{2}, 363$ ).

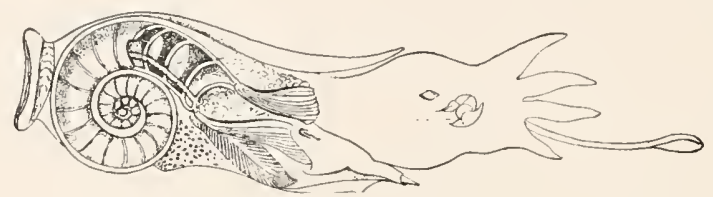

FIG. 353.-Spirula, with internal shell (after Owen).

Externally the shell of the former, coiled in a plane, resembles that of certain snails like Planorbis; but on section it is seen to be divided by partitions into numerous chambers which increase in size towards the aperture. The animal occupies only the last chamber with its back
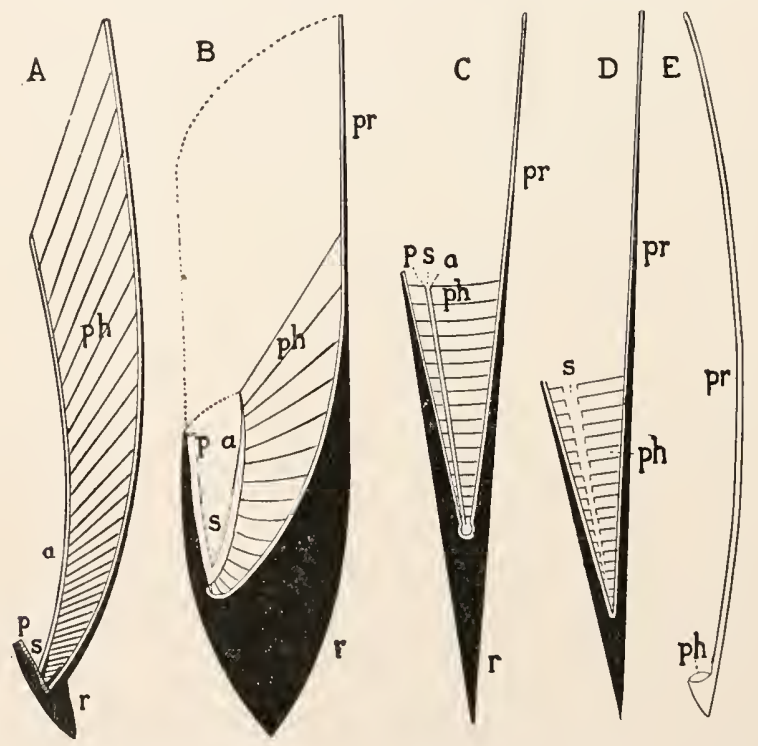

FIG. 354.-Diagram of shells, etc. of various cephalopods (after Lang). A, Sepia; B, Belosepia; C, Belemnites; D, Ostracoteuthis; E, Ommastrephes. a, anterior; $p$, posterior; $p h$, phragmocone; $p r$, proostracum; $r$, rostrum; $s$, siphon.

towards the centre of the shell (exogastric position); the other chambers are filled with air. Each partition has a small opening, and through these runs a stand of tissue, the siphuncle. Among the fossil cephalopods many forms-the Nautiloids and Ammonites-have similar 
chambered shells; but in other recent forms and in many extinct species the shell is more or less rudimentary. In Spirula (the animals of which are extremely rare, the dead shells common) there is a similar chambered shell, buried in the mantle (fig. 363). Its position (entogastric) is the reverse of that of Nautilus.

In the Decapoda the equivalent of the shell is completely concealed in the back of the animal. In the Sepias it is a lamellar calcareous structure, the well-known cuttle bone; in the Loliginicle it forms a 'pen' of purely organic nature (fig. 3 I I, $A$ ). Like true shell these dorsal structures are products of the external epithelium, but the epithelium, the shell gland which forms them, has become folded in and the walls have united over it.

The shell of Argonauta (fig. $3^{6}{ }_{3}$ ) is different. It occurs only in the female, is thin as paper, spirally coiled at the tip, and is only in part a secretion of the body, for a part of it is formed by two tentacles which are expanded for this purpose. Internal partitions are lacking, and this shell serves as a nest for the eggs. Most Octopoda also lack a shell. A word or two may be added to correlate the recent and fossil shells of the Dibranchiata, which are always internal and more or less rudimentary. The fossil Belemnites (fig. $354, C$ ) had a chambered shell (phragmocone) perforated for the siphuncle. In front this is prolonged ventrally into a thin broad plate, the proostracum, while behind it is inserted in a calcareous sheath, the guard or rostrum. From this, by comparison with the fossil Belosepia (B), it is seen that the cuttle bone of commerce $(A)$ is the anterior part of the chambered shell, its la minæ being the partitions, while in the animal the rostrum and siphuncle are in part retained. On the other hand, comparison with the fossil Ostracoteuthis $(D)$ shows that in Ommastrephes (E) we have but a remnant of the phragmocone, while the bulk of the pen is proostracum. In Loligo the phragmocone is entirely lacking.

The mouth, situated in an oval buccal mass, lies between two horny jaws, like the beak of a parrot (fig. 355 ); then follows a pharynx with a radula, and in turn a long œsophagus, often with a crop-like dilatation The œesophagus opens into a wider stomach, with which is connected a blind sac, frequently coiled. Here the tract doubles on itself and goes straight, or with one or two convolutions, to the anus (fig. $35^{6}$ ). One or two salivary glands (upper and

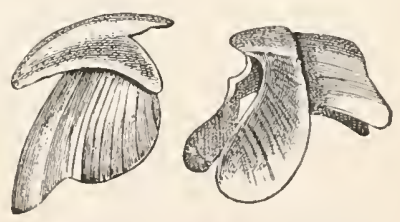

FIG. 355.-Jaws of Sepia officinalis. lower, the latter poisonous in Octopus) open into the osophagus, and a pair of liver sacs (frequently fused) open by two bile ducts into the gastric blind sac. 'These ducts may bear racemose glands called the pancreas. Lastly, the ink sac opens into the intestine near the anus. This gland secretes a brownish or blackish pigment. When alarmed the animal ejects this secretion and clouds the water so that it can escape unseen. This organ is best developed in Sepia officinalis, and 
its secretion forms the basis of the well-known color, sepia. Nautilus and some Octopoda have no ink sac.

Just behind the buccal mass are the closely united chief ganglia of the nervous system (fig. 357) surrounding the cesophagus. A single

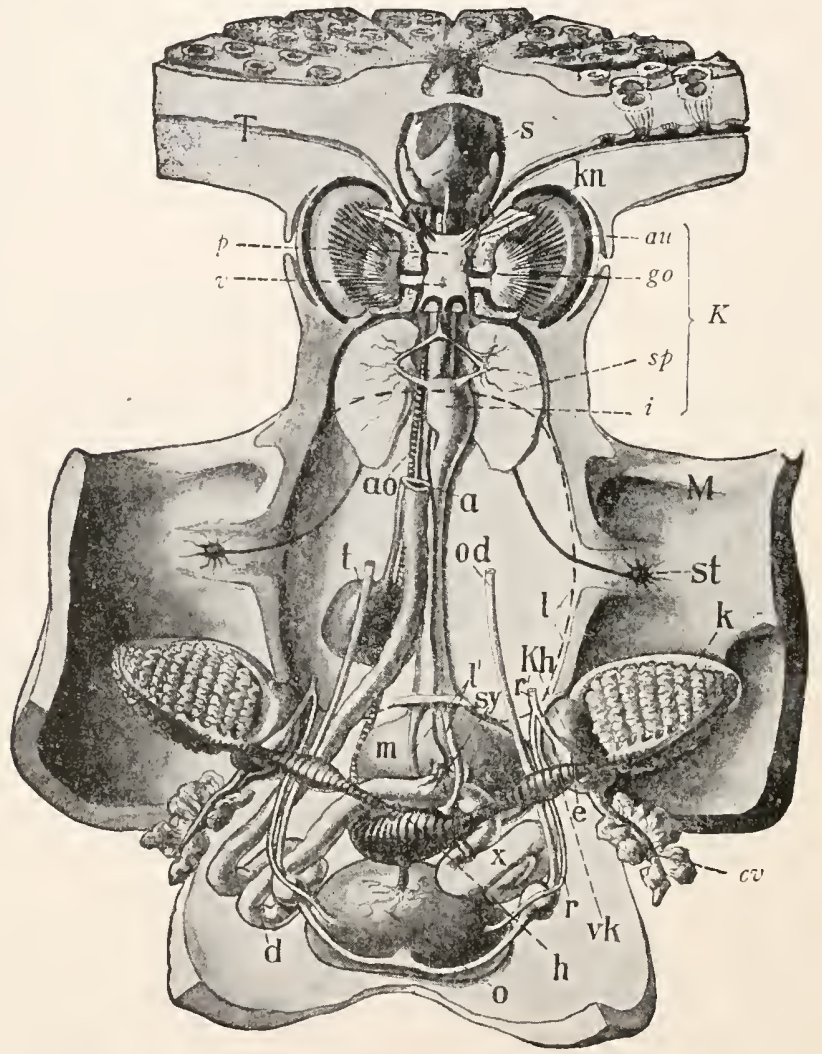

FIG. 356.-Anatomy of Octopus vulgaris. $T$, basis of tentacles; $K$, head; $M$, mantle split ventrally, opening visceral sac: liver and nephridia removed, venæ cavæ with appendages folded back; $a$, anus; $a o$, aorta; $a u$, eye; $c v$, vena cava with nephridial appendage; $d$, intestine; $e$, pericardial sac with nephridial opening; go, optic ganglion; $h$, systemic heart; $i$, crop (cesophageal appendage); $k$, gills; $k h$, branchial heart; $k n$, cephalic cartilage; $l$, liver and $l^{\prime}$, gall duct (position of liver indicated by dotted line); $m$, stomach; $o$, ovary; od, oviduct; $p$, pedal ganglion; $r$, passage connecting with ovary; $r^{\prime}$, mouth of pericardial sac in nephridial sac; $s$, cesophagus with dorsal salivary gland; $s p$, ventral salivary glands; $s t$, stellate ganglion; $s y$, sympathetic ganglion; $t$, ink sac; $v$, visceral ganglion; $z^{\prime}$, , auricle of systemic heart; $x$, spiral blind sac.

dorsal mass represents the cerebral ganglia; connected with this by broad commissures, the pedal and visceral (viscero-pleuro-parietal) ganglia lie close together ventrally. With these parts are associated 
upper and lower buccal ganglia. The large optic ganglia, in the optic nerve arising from the cerebrum and enclosed ventrally' in the "whitebody,' a lymphoid mass, are especially characteristic, as are the ganglia stellata, right and left at the anterior edge of the mantle (fig. $356, s t$ ), which owe their name to the radiation of fibres to innerrate the mantle. In unpaired sympathetic ganglion lies at the junction of stomach and intestine. Cerebral, pedal, visceral and optic ganglia are enclosed in the cephalic cartilage, which has the shape of a ring with wing-like processes. The complicated statocysts lie in the ventral arch of the ring. The sense of smell is highly developed. Apparently it resides in a pair of spots of skin between the eyes and the mantle which are richly supplied with nerves. In the Decapoda these are sunk in pits, in the Octopoda they form papillie. In Nautilus, which has also two pairs of osphradia, there is a papilla with a ciliated groove, beneath each eye, corresponding to the olfactory organ of the other groups.

Most noticeable of the circulatory structures is the presence of two kinds of hearts (fig. $35^{6}$ ). The systemic heart consists of two (four in $\mathrm{Nau}$ tilus) auricles receiving arterial blood from the gills, and a median ventricle from which arise anterior and posterior aorta. Then there is a branchial heart at the base of each ctenidium which receives the blood from the vena cava and pumps it into the gill. Of vene cave there are an anterior unpaired and two posterior paired trunks, the former dividing and sending a branch to each branchial heart. These trunks are connected with the nephridia. The nephridial openings (p. 339) lead to two spacious sacs through which the veins pass obliquely. This part of the blood vessels bears venous diverticula which pro-

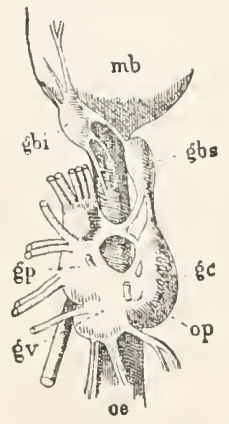

FIG. 357--Nervous system of Sepia officinalis from the side. $g b i$, inferior buccal ganglion; $g b s, \quad$ superior buccal ganglion; $g c$, cerebral ganglion: $g p$, pedal ganglion; $g r$, visceral ganglion; $m b$, buccal mass; oe, o'sophagus; op, optic ganglion. ject into the nephridial sac and are covered with an epithelium of excretory cells. Near its mouth each nephridial sac communicates by a nephrostome with the (usually large) cœlom.

In the Octopoda the colom is reduced to the gonads and narrow canals leading from the nephrostome to the gonads and branchial hearts, but elsewhere there is a well-developed system of connected cavities, consisting of the pericardium around the systemic and branchial hearts and the thin-walled genital sac, one wall of which bears the genital ducts, while on the other the sexual cells arise or the ducts of a separate sexual gland open (fig. $35^{8}$ ). 
The gonads of the always diøcious Cephalopoda are unpaired and lie far back in the visceral sac. The ducts in the female Octopoda (rarely in the males) and in some Decapoda (Oigopsida) are paired. In Nautilus

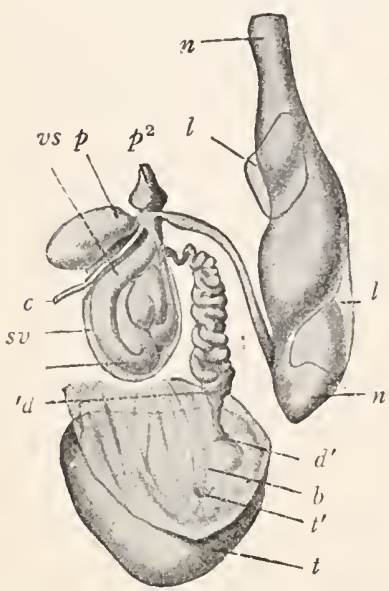

Fig. 358.- Male sexual organs of Sepia officinalis (after Grobben). $a$, coelomic sac passing to the left and above into the pericardium; $c$, colomic canal to the vas deferens; $d$, vas deferens; $d^{\prime}$, its opening to coelom; $l$, portions of colom; $n$, Needham's pocket; $n^{\prime}$, its mouth; $p^{\prime}, p^{2}$, prostates; $t$, testis; $t^{\prime}$, its opening to coelom; is, seminal vesicle. only the right duct is functional in either sex, although the left is well developed. Elsewhere there is only the left duct. The oviducts are saccular with glandular walls; independently of them two pairs of glands open to the exterior, the accessory glands and the large nidamental glands. The vas deferens (fig. 358) is more complicated. It has swellings known as seminal vesicle, prostate, and Needham's sac, in which the spermatophores are stored. These latter have such a complicated structure and show such motions when swollen with water that they were long regarded as parasitic worms (fig. 359).

The spermatophores are conveyed to the female by means of more or less modified (hectocotylised) tentacles of the miale. In a few genera the whole tentacle becomes a 'Hectocotylus' (fig. 360). It swells at its base to a sac in which the peripheral end is enclosed. This part, which contains a canal for the spermatophores, cuts loose from the male, and can creep about for days in the mantle chamber of the female. Since it appears like an independent animal, it was first described as a parasitic worm under the name Hectocotylus. In others the hectocotylization is not carried so far.

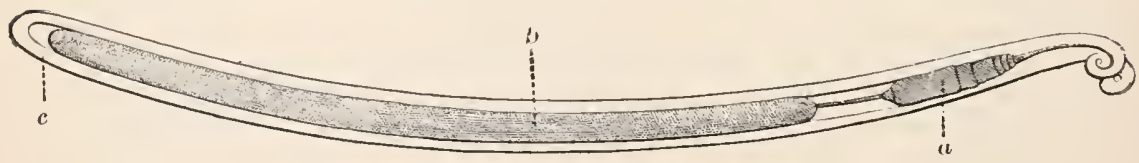

F1G. 359.-Spermatophore of Sepia (from Hatschek, after Milne Edwards). a, discharging apparatus; $b$, packet of spermatozoa; $c$, envelope.

The eggs are either fastened singly to aquatic plants or are laid in large gelatinous masses. They are rich in yolk, and in consequence undergo partial discoidal segmentation (fig. I05). The blastoderm, on the end of the oval egg, forms the anlagen of the separate organs (eyes, arms, siphon, and shell gland) as flattened projections. Later the embryonic body becomes distinct from the 
yolk, which, enclosed in a cellular envelope, remains attached to the rest, near the mouth, until it is absorbed in the growtli of the voung and the animal is ready for hatching (fig. $36 \mathrm{I}$ ).

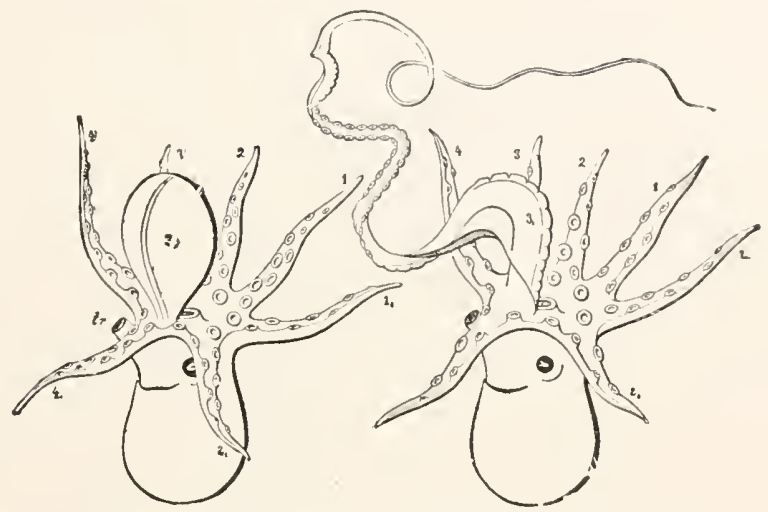

FIG. 360.-Male of Argonauta argo (after Müller, from Hatschek). I-4, arms of right side: $\mathbf{I} .-4$., arms of left side; 3 , hectocotylised arm, at the left in its sac, at the right protruded.
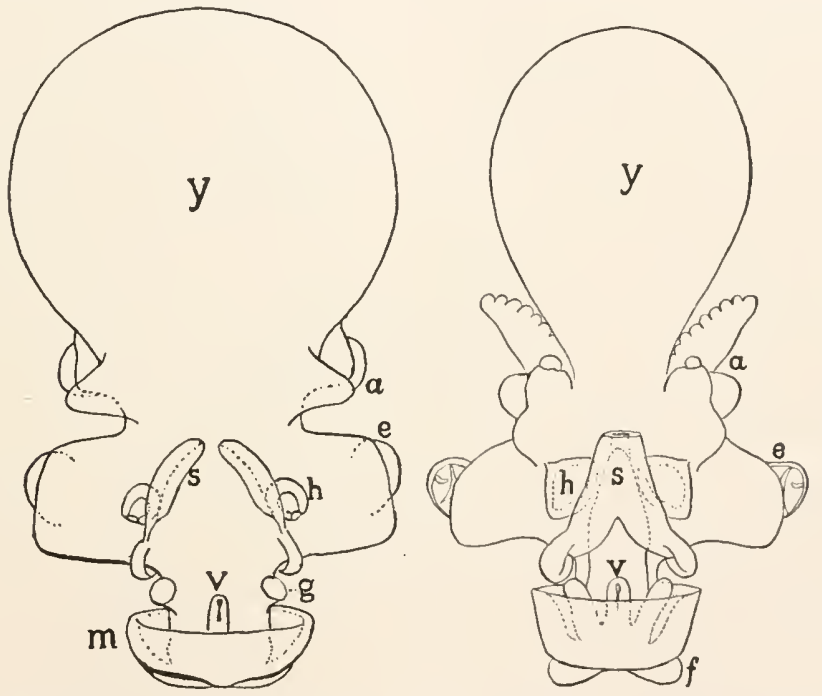

FIG. 36r.-Embryos of Loligo pealei (orig.). $a$, arms; $e$, eyes; $f$, fin; $g$, ctenidia; $h$, statocyst; $m$, mantle; $s$, siphonal folds and siphon; $v$, anus; $y$, yolk sac.

The Cephalopoda are exclusively marine. Some inhabit rocky shores, others the high seas. All are carnivorous and in turn are preyed upon by fishes, etc. Classification is based upon the number of gills and number and character of the arms. 


\section{Order I. Tetrabranchia.}

With four gills, four auricles, and four nephridia; numerous tentacles without suckers, a well-developed chambered shell (fig. 352), siphon of two separate epipodia, and simple eyes (fig. 350). Four living species, all belonging to Nautilus.

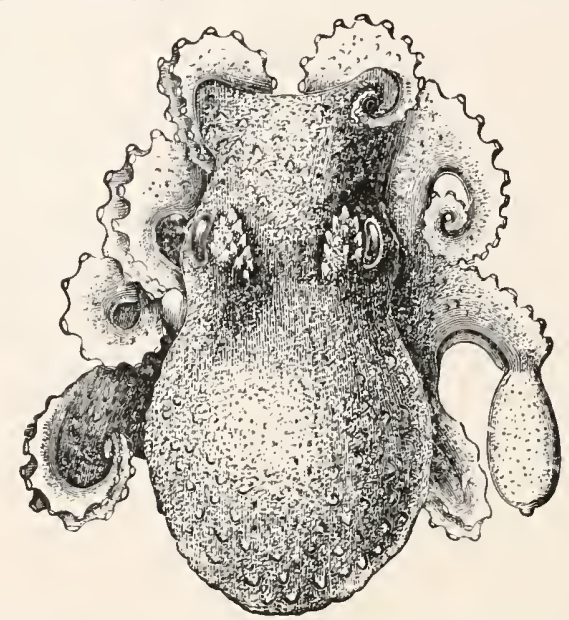

FIg. 362.-Octopus bairdii* (from Verrill). A hectocotylised arm on the right side.

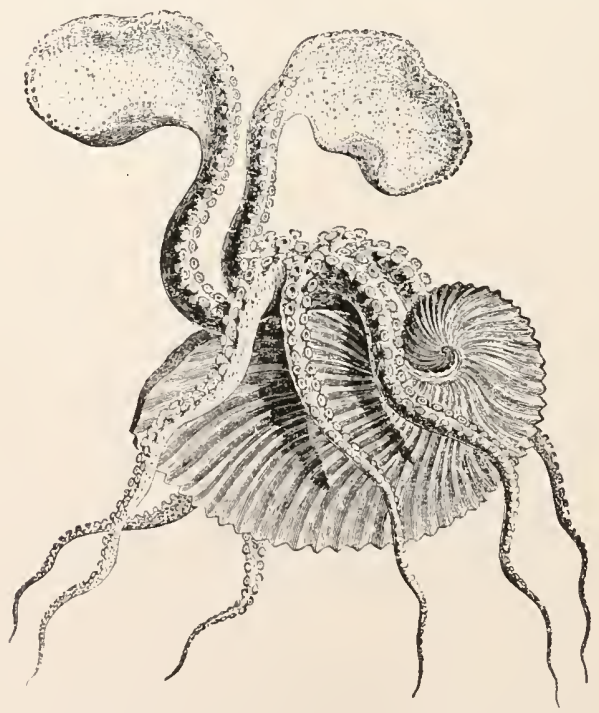

FIG. 363.-Argonauta argo, paper sailor, female (after Rymer Jones).

The animals, which live in the Malaysian regions, are rare, but their shells are abundant. In past time the tetrabranchs were very abundant; NAUTILIDE, with straight (Orthoceras) or coiled shells (Goniatites, etc.), paleozoic. They 
had simple septa. Ammoxitid., folded septa, largely mesozoic. Their pertinence to the tetrabranchiates is assumed from the character of the shell.

\section{Order II. Dibranchia.}

With two nephridia, two gills, and two auricles; eight or ten arms with suckers; highly organized eyes; shell rudimentary or absent.

Sub Order I. DECAPODA. Ten arms, body with lateral fins. Rudimentary shell usually present. SPIRULIDE, with internal chambered loose-coiled shell. Spirula (fig. 353). Orgopsid.1, with perforated cornea (p. 338) and two oviducts. Ommastrephes*; Architeuthis, * the giant squid (p. 336). Mropsid. Oviduct single (left); cornea unperforated. Loligo, * common squid; Rossia*; Sepia, cuttle fish, furnishing the 'cuttle bone' fed to cage birds, and the pigment sepia. Sub Order II. OCTOPODA. Eight arms, webbed at their base; shell very rudimentary, sometimes fragmentary or wanting; oviducts paired. ОстоPODIDE, Octopus* (fig. 362), Alloposus** Argonautidæ, female with boatlike shell (fig. $36_{3}$ ), males much smaller and without shell. In Argonautida and Philonexid.玉 the hectocotylus separates.

\section{Summary of Important Facts.}

I. The MOLLCSCA are parenchymatous animals with reduced cœlom. They consist of head, visceral sac, mantle, and foot.

2. The head bears eyes and tentacles.

3. The foot is an unpaired muscular mass used in locomotion.

4. The mantle bounds the mantle cavity which is connected with respiration; it either functions as a lung or covers the gills (ctenidia). It secretes the shell from its outer surface.

5. Foot, head, mantle, and with the latter the shell, may be lost in many groups.

6. The molluscs agree in the nervous system. Three pairs of ganglia, connected with three pairs of sense organs, occur: $a$, cerebral ganglia and eyes; $b$, pedal ganglia and statocysts; $c$, visceral ganglia and osphradia (olfactory).

7. The heart is dorsal and arterial; it is enclosed in a pericardium (reduced colom) which connects with the nephridia by nephrostomes.

8. There is always a single ventricle and, according to the number of respiratory organs, one, two, or four auricles.

9. The alimentary canal is well developed; the liver large; salivary glands usually present. In most there is a pharynx or buccal mass with radula and jaws.

ro. A veliger stage is common in development.

I r. The Mollusca are divided according to the respiratory organs and appendages of the body into five classes: (I) Amphineura; (2) Acephala; (3) Scaphopoda; (4) Gasteropoda; (5) Cephaloporla.

I2. The Auphineura have an extremely simple nerrous system in which the ganglia are replaced by nerve tracts. 
I3. The AcephaLa lack head and cephalic appendages.

I 4. They are bilaterally symmetrical and have paired organs: mantle folds, bivalve shell, gills, nephridia, and gonads.

I5. In many Acephala (Asiphonia) the mantle folds are completely separated ventrally.

16. In the Siphonata the lower edges of the mantle are united, leaving three openings: ( $\mathrm{I}$ ) in front for the foot; (2) behind and below, the branchial siphon for the ingress of water and nourishment; (3) behind and above, the anal or excurrent siphon for the water used by the gills and the fixces.

I7. There are two pairs of gills (ctenidia), which may be comb-like, filiform, or most commonly lamellar (lamellibranchs).

i 8. Correspondingly the heart has two auricles; the unpaired ventricle is usually traversed by the rectum.

19. The foot is a compressed muscular mass frequently provided with a byssus gland.

20. The shell consists of cuticular, prismatic, and nacreous layers. It is closed by one or two adductors and opened by an elastic ligament.

2I. Some Acephals (Protoconcha) have primitive gills and hinge; others (Heteroconcha) are more highly developed.

22. The SCAPHOPODA are primitive forms with tubular shells.

23. The Gasteropoda (Cephalophora, or snails) have a distinct head bearing eyes and tentacles; a creeping foot, an unpaired mantle (occasionally absent), and a univalve shell or none.

24. The mantle cavity contains one or less frequently two ctenidia, or these may be degenerate and a lung may occur.

25. Nephridia and auricles are rarely paired (with paired gills); the gonads, always unpaired, are hermaphroditic or diøcious.

26. The shell is always umpaired; it is usually coiled in a (right-hand) spiral, and is frequently closed by an operculum.

27. According to character of nervous system, sexual organs, heart, and respiratory organs the Gasteropods are divided into (I) Prosobranchia; (2) Opisthobranchia; and (3) Pulmonata.

28. The Opisthobranchia are hermaphroditic; orthoneurous; have secondary gills (or none), and have the auricle always behind the ventricle; shell and mantle reduced or absent.

29. The Pteropoda are pelagic Opisthobranchs with wing-like processes of the foot and frequently reduced shell or none.

30. The Prosobranchia have the gills (ctenidia-occasionally paired) far in front, and in consequence the auricle in front of the ventricle; they are streptoneurous and diœcious; the mantle and shell well developed. 
3I. The Heteropoda are pelagic Prosobranchia with foot divided into fin and tail, shell rudimentary or absent.

32. The Pulmonata are in some respects (orthoneurous and hermaphroditic) Opisthobranch-like; in other respects-as in position of heart, development of shell and mantle-like the Prosobranchs; the mantle cavity connects with a lung.

33. The Cephalopoda have no true foot; but its homologues are to be found in the siphon and in the tentacles, usually provided with suckers, on the head; they have an unpaired mantle and mantle cavity and a single shell or none.

34. The mantle cavity contains one or two pairs of ctenidia. Water is forced from the mantle cavity through the siphon.

35. The number of auricles and nephridia corresponds with the number of ctenidia; besides the systemic heart there are one or two pairs of branchial hearts, elsewhere unknown in molluscs.

36. The sexes are separate.

37. The ink sac is peculiar to Cephalopoda.

38. The eye is (usually) highly developed (with retina, chorioid, iris, cornea, vitreous body, and lens), as is the nervous system, which has, in addition to the usual centres, optic, sympathetic, and stellate ganglia.

39. The eggs have a discoidal segmentation.

40. The Cephalopoda are divided into Tetrabranchia and Dibranchia.

4I. The Tetrabranchia (extinct save for Nautilus) have four gills, a chambered shell, primitive eyes, and finger-like cephalic lobes in place of tentacles.

42. The Dibranchia have two gills, eight or ten tentacles with suckers, and the shell is reduced or absent.

\section{PHYLUM VII. ARTHROPODA.}

Under the term Arthropoda are included the crabs, spiders, insects, and myriapods, which, together with the annelids, were united by Cuvier in his sub-kingdom Articulata. Annelids and arthropods agree in many features. They are, as the term articulates implies, segmented animals, and they differ from the vertebrates, which are also segmented, in the extension of the segmentation, the ringing of the body, to the external surface. The boundaries between the successive segments, which cannot be recognized in the skin of the vertebrate, are marked in the articulates by constrictions of the body wall, whence the old names Evvora and Insecta, applied to these forms. The articulates are further characterized by a ladder-like nervous system (fig. 78 ), in which the brain is supple- 
mented by a ventral chain composed of ganglia metamerically arranged. The most erident distinctions between the annelids and the arthropods are (I) the character of the segmentation and (2) the presence of jointed appendages.

In superficial appearance the lines between the segments are constricted more deeply in the arthropods than in the annelids. The cause

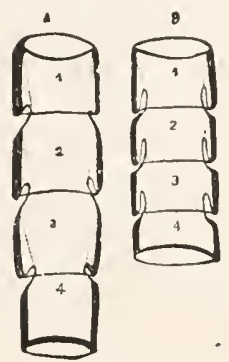

FIG. 364 - Diagram of Arthropod jointing; $A$, in expanded, $B$, in contracted condition; $I-4$, rings with connecting membranes, the muscles indicated by dotted lines, (after Graber). of this lies in the character of the integument (fig. $26, f$ ), which is developed as a hard armor, in which two layers are recognizable, the epidermis ('hypodermis') and the chitinous layer. The epidermis is a thin epithelium, while the chitinous layer is of greater thickness and, since it is secreted by the epidermis, is stratified parallel to the surface. Its firmness is due to chitin, which is unlike most organic substances in its resistance to acids and alkalis; only under the action of sulphuric acid and heat is it broken up into sugar and ammonia. Frequently (Myriapods, Crustacea) the chitinous armor is strengthened by the deposition of calcium carbonate and phosphate. A firm coat would render the animal incapable of motion were there not joints between the parts. While the segments themselves are heavily armored, the cuticle between them is reduced to a delicate articular skin, and this is so protected by a kind of telescoping of the segments that injury in these softer regions is nearly impossible (fig. 364).

Since the ringing of the body is connected with this armoring, it disappears with the need for such protection. The hermit crabs (fig. 406) illustrate this. These animals live with the abdomen inserted in a snail shell. That part of the body which projects from the shell is armored, while the abdomen is softskinned and without traces of external ringing. The hardened cuticula causes the periodic molting (ecdysis or exuviation). When once hardened it is incapable of distention and so would prevent farther growth. Hence when the body has completely filled the shell, the latter splits in definite places and the animal crawls out of the old 'skin' (exwria) and rapidly increases in size, while the new cuticula is yet soft and distensible. Another result of the cuticula is seen in the peculiar relations of both ordinary and sense hairs. These are cuticular structures, each usually secreted by a single epidermal cell and renewed after each molt. Each hair has a ball-like base situate in a socket in the surrounding chitin, and hence is movable; it is traversed by a canal in which is a process of the underlying matrix cell. In the case of sensory hairs these structures are connected with a nerve (fig. So). The sense cell has.two processes; one peripheral, which enters the axis of the hair, the other central, which runs as a nerve fibre to the central nerrous system. The cell itself may be in the epithelium or situated deeper and interpolater as a ganclion cell in the sensory nerve.

The muscles which are inserted on the integument are segmental in character 
and are arranged in metameric muscle groups. Frequently they are inserterl on the chitin by special tendons, portions of the chitin drawn inwards. 'Through such infoldings there arises in many arthropods an 'entoskeleton.'

Another important character is the heteronomous segmentation, which, in the lowest forms (Peripatus and myriapods), is little pronounced, but elsewhere leads to a marked inequality of the regions of the body. A few segments at the anterior end always fuse and form a head (fig. $36_{5}, C$ ) ; behind this there is usually a second segment complex, the thorax (percion) $(T)$, and then a third, the abdomen (pleon) $(A)$. An apparent

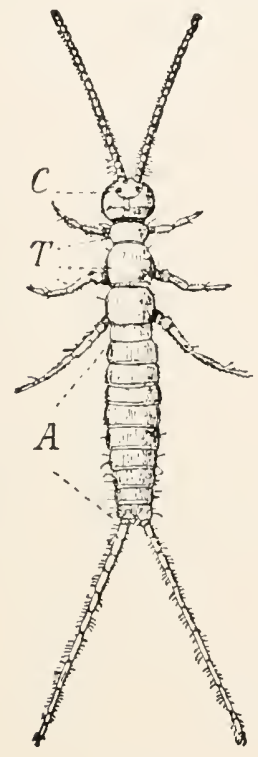

FIG. 365 .

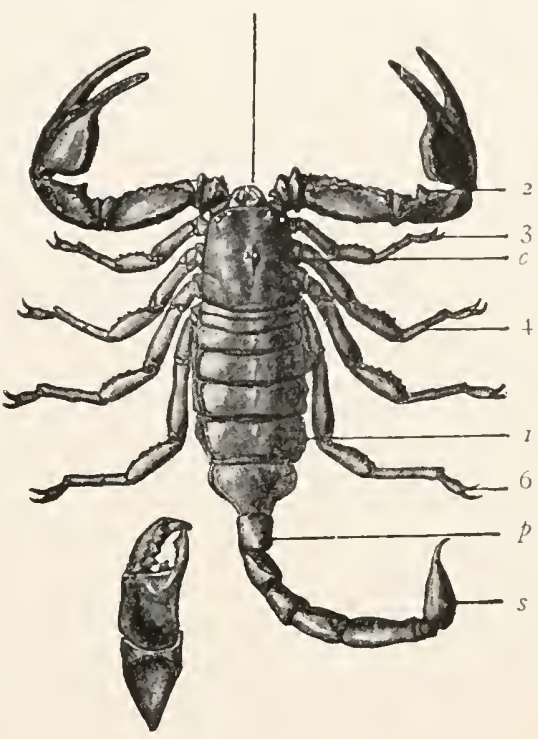

FIG. 366.

FIG. 365.-Campodea staphylinus (from IIuxley). A, abdomen; $C$, head; $T$, thorax.

FIG. 366.-Euscorpius italicus. a, abdomen; $c$, cephalothorax; $p$, post abdomen; $s$, sting; r, cheliceræ; 2 , pedipalpi; $3-6$, walking legs. Below chelicera enlarged.

reduction of regions occurs when the head and thorax unite (fig. $367, C t$ ) to form a cephalothorax; or the number of regions may be increased (fig. 366) by a division of the abdomen into abdomen proper $(a)$ and postabdomen $(p)$. Finally, in many arthropods (e.g., the mites or acarina, fig. 368$)$ it is impossible to recognize regions or somites because internal fusion of parts has obliterated the external evidences of segmentation.

In order clearly to understand what is meant by head, thorax, etc., requires a consideration of the second character distinguishing the arthropods from the annelids, the jointed appendages, which give the name to the former group. The arthropodan appendages are highly developed 
parapodia, differing in being jointed to the body, in consisting of a series of joints themselves, and in having their intrinsic musculature. There is but a pair of appendages to a somite, and this belongs to the ventral surface. Hence it follows (Savigny's law) that although a region may show no external signs of segmentation, if it bear more than one pair of appendages, we conclude that it is a complex of at least as many somites as there are pairs of appendages. Thus the unsegmented head of an insect consists of four somites, the cephalothorax of a lobster of thirteen, for the one bears four, the other thirteen, pairs of appendages. Ontogeny supports this, for in the embryo the somites are clearly visible. This statement is not exactly correct, for in certain insects and in the lobster there is one more

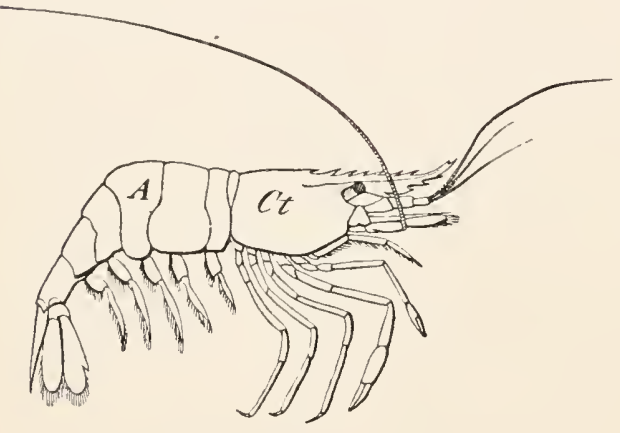

FIG. 367 .

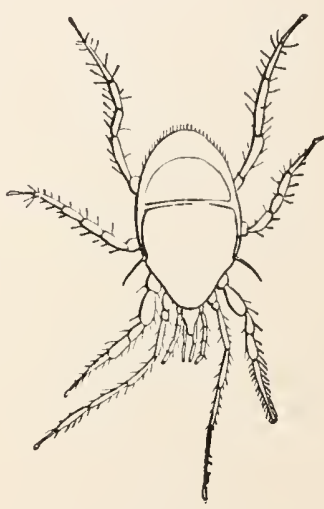

FIG. 368 . thorax.

FIG. 367.-Palamon serratus (from Ludwig-Leunis). A, abdomen; $C t$, cephaloFIG. 368.-Gamasus coleoptratorum (from Taschenberg).

somite which is entirely lost in the adult. It is not necessary that each somite in the adult should bear appendages, since these may disappear in growth without leaving a trace.

While originally all were locomotor, the appendages subserve many functions (fig. 369). Locomotor appendages (pereiopoda, feet or legs) are long and consist of a number of joints which may form flattened oars or may be provicled with claws for creeping (8). Besides locomotor appendages there are tactile appendages or antennce (I), chewing appendages (jaws, mandibles, maxilla, 2-4), false feet or pleopoda (9) of varying functions, and forms-maxillipeds $\left(5^{-7}\right)$-transitional between jaws and legs.

Aside from being tactile, antennx are characterized by position and innervation. They are always in front of the mouth and receive their nerve supply from the supraœsophageal ganglion, while all other appendages 
are innervated from the ventral chain. In their elongate shape antenna are not unlike legs, but they lack the terminal claws.

The form of the jaws is strikingly modified. One or two basal joints serve for the comminution of food, and these parts are strong and are covered, especially on the medial sicle with a hard, toorhed chitin (figs. 369,$2 ; 374, I I I, I^{\prime}$ ). The other joints may entirely disappear, or may form a more or less leg-like appendage, the palpus. Since several appendages may be modified into jaws, the first are called mandibles, the next maxillix, and second maxillie may follow. The maxillipeds may have more the appearance of jaws, at other times are more leg-like (fig. $369,5^{-7}$ ). The false feet (pleopoda) are small and inconspicuous appendages which have various functions: they may serve as gills or supports for gills, places for the attachment of eggs, organs for the transfer of sperm, or as swimming or creeping organs.

These appendages have constant positions in the body. First on the head come the antennæ and then, in the region of the mouth, the jaws and, so far as they are present, the maxillipeds. Third come the true feet, and lastly, when they exist, the false feet. Those somites which bear antennx or jaws belong to the head, those bearing walking feet to the

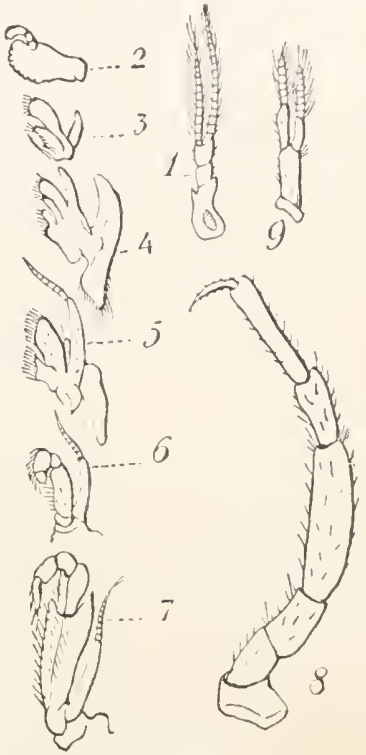

FIG. 369.-Appendages of the crayfish. r, first antenna; 2, mandible; 3 , 4, first and second maxillix; 5, 6, 7, maxillipeds; 8, walking leg; 9, pleopod.

thorax, while the somites of the abdomen bear either false feet or lack appendages. As a sequence the cephalothorax is that region of the body which bears, besides antennæ and jaws, legs as well.

The somites of Arthropoda have given rise to various disputes. Many zoologists speak of a pre-antennal somite and a pre-antennal appendage, referring to the eye stalk of some Crustacea, which, however, differs markedly in its development from the true appendages. Those who accept an ocular somite must add one to the number of somites as stated in this volume. A second theory regards the antennæ as ventral appendages inmervated from the ventral chain which secondarily become dorsal and receive their nerves from the brain. This view is firmly grounded for the second antennæ of the Crustacea. Other questions are as to the possible loss of both segments and appendages.

The union of somites to body regions has had an influence upon the internal structure and especially upon the nerrous system (fig. 370). A 
ladder-like nervous system consists, as was pointed out (p. II3), of a dorsal brain (supraœsophageal ganglia) and a ventral chain of ganglia, all connected by longitudinal nerve cords, the brain being connected with the rest by cords or commissures passing on either side of the œsophagus. The ventral chain should contain as many pairs of ganglia as there are somites, but this is not the case except in the embryo. The tendency is rather towards a fusion of ganglia, especially of those somites which unite or fuse. This fusion of ganglia occurs to a varying extent in different species, the extreme being reached in the spiders and crabs (fig. 402),

A

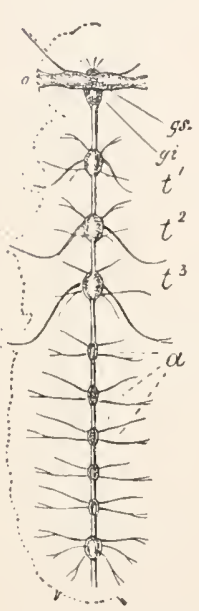

B

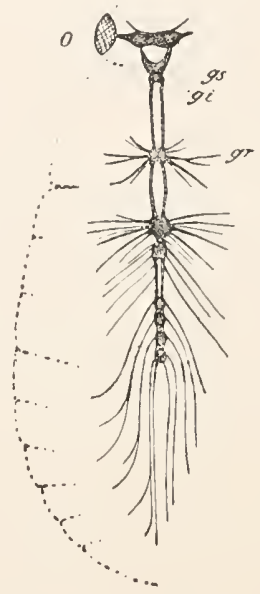

C

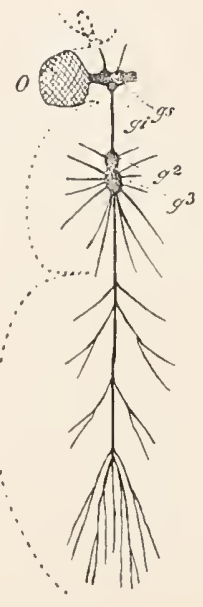

D

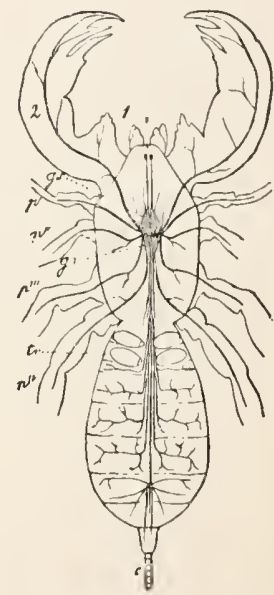

FIG. 370.-Different degrees of concentration of the ventral cord of Arthropods (from (iegenbaur). A, Termite (after Lespès). $B$, water beetle (after Blanchard). $C$, fly (after Blanchard). $D$, Thelyphonid (after Blanchard). $a$, abdomen; $g^{2}, g^{3}$, ganglia of ventral cord: $g i$, infraœsophageal ganglion; $g s$, supraœsophageal ganglion; $o$, eye; $p^{\prime}-p^{\prime \prime}$, walking feet; $t r$, lung books; I, cheliceræ; 2 , pedipalpus.

where the whole ventral chain forms a single ganglionic mass. In all cases, however, the brain remains distinct from the rest, its position dorsal to the oesophagus precluding its fusion with the ventral chain.

Two types of eyes are recognized, the simple (ocellus, stemma) and the compound (faceted). The ocelli are small. In their highest development, as in spiders (fig. $37 \mathrm{I}$ ), they are composed of lens, vitreous body, and retina. The lens is formed by the cuticula, the rest from the epidermis. The lens differs from the rest of the cuticle in being transparent, and is usually thickened to a biconcave body (I) which converges the light upon the retina. Only exceptionally (larve of Ephemerida) is the 
lens cellular, being formed by a thickening of the epidermis. Beneath the lens comes a layer of transparent cells, the vitreous body (2), and

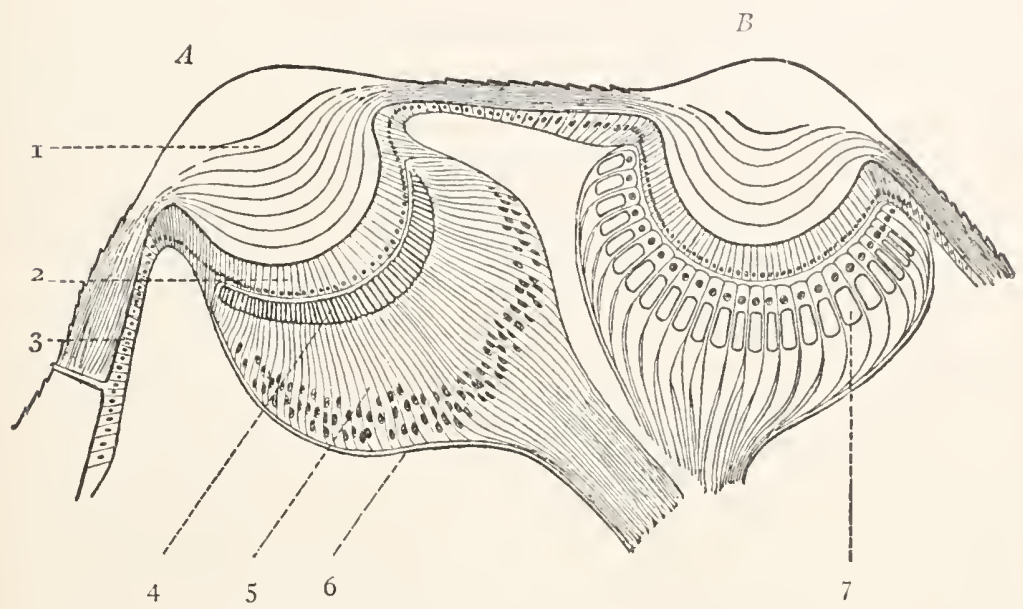

FIG. 37I.-Diagrammatic section through anterior $(A)$ and posterior $(B)$ eyes of Epeira diadema (after Grenacher). The hinder eye shows the inverted retina; I, lens; 2, vitreous body; 3, epidermis, outside this, chitinous layer; 4, rhabdomes; 5, retinal cells; 6 , capsule of eye; 7 , rhabdomes of inverted eye.

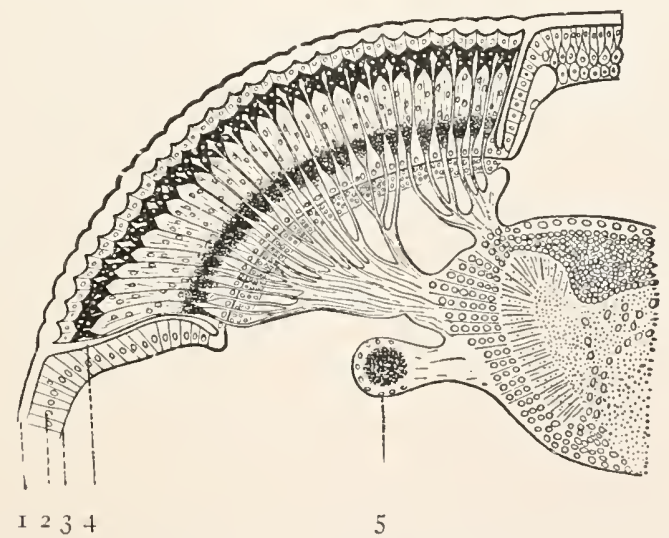

FIG. 372.-Section of compound eye of Forficula (after Carrière, from Hatschek). I, cuticula, producing the cornea of many lenses over the eye; 2 , epidermis, which in the eye forms the ommatidia; 3 , basal membrane; 4 , reentrant chitinous fold ('sclerotic'); 5 , rudimentary larval eye.

behind this, in turn, the retina, consisting of visual cells which, at the one end, bear 'rhabdomes' ( 4 and 7 ), at the other pass into nerve fibres. The retina and vitreous body, surrounded by pigment, form a spherical 
thickening sharply marked off from the rest of the epithelium. These eyes, like those of rertebrates, form inverted images.

Spiders have two kinds of ocelli (fig. $37 \mathrm{I}$ ), the chief eyes and the accessory. In the first $(A)$ the rhabdomes are next the ritreous body and in front of the nuclei of the cells. In the other $(B)$ the relations are reversed, the structure recalling the inverted eyes of vertebrates. Apparently the first are for distant vision, the others for near objects.

The compound eyes are much larger. They owe their name faceted eyes to the fact that the cuticle over them is divided into polygonal (usually

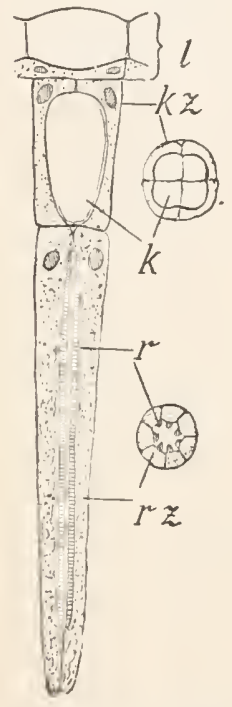

FIG. 373.-A single ommatidium (with sections) of a compound eye. $k$, crystalline cone; $k z$, cone cells: $l$, lens with hypodermis; $r$, rhah)domes; $r z$, retinular cell. hexagonal) areas or facets. Each facet corresponds to a small chitinous lens (the number of which varies, in different species, between a dozen and several thousand), and bounds the eye externally, whence this layer is called the cornea (fig. 372). The part of the eye beneath the cornea consists of radially arranged pyramidal parts or ommatidia which correspond in number and position to the facets, their broader ends being placed beneath the facets, their narrower internal ends connecting with fibres of the optic nerve which go to the brain. Each ommatidium (fig. 373) has essentially the structure of an ocellus: (I) the lens ( $l$ ) with its epithelium; (2) the virtreous body $(k z)$; (3) the retinula $(r z)$. The vitreous body is usually composed of four cells which, in the so-called euconous eyes, surround a transparent body, the crystalline cone $(k)$, secreted by these cells. The retinular cells are almost always seven in number, each bearing on its inner surface a rhabdome $(r)$, the seven rhabdomes frequently fusing into a common mass. Each ommatidium is surrounded by a pigment sheath, isolating it optically from its fellows.

There are really two pigment zones, an anterior which corresponds functionally to the vertebrate iris, and a posterior comparable to tapetum and chorioid. The iris pigment lies in special cells and is most abundant at the line between the vitreous body and retinula. The posterior pigment is in the retirular cells.

From this it appears that the compound eye may be regarded as a complex of ocelli. This anatomical conception must not, however, ol)scure the physiological. The whole compound eye forms but a single erect picture composed of separate images of small area formed by the 
separate ommatidia. This 'mosaic theory' has completely replacerl the view that each ommatidium formed a complete inverted picture.

While the number of ocelli varies, the compound eyes are almost invariably two in number, but can be constricted into four or eight. 'The single eye of Daphnids is the result of fusion. There is always a large optic ganglion in the optic nerve of each compound eye. As in vertebrates both ocelli and compound eyes can shine in the dark. 'This is the result of the 'tapetum lucidum,' formed in the crustacea and arachnids of modified pigment cells, in the insects by a rich tracheal network.

The tactile organs, consisting of tactile hairs (fig. 80), are uniform in structure. On the other hand the senses of hearing, taste, and smell are subserved by varying organs. We know but little of these senses in arthropods, although they are frequently well developed. The sense of smell resides chiefly in the antennx and in the palpi of the jaws. The organs are olfactory tubules or cones (modified hairs) which frequently lie in pits in the skin. Similar organs in the mouth are probably connected with taste. As organs of hearing (? equilibration) besides the statocysts of the Podophthalmata and the tympanal organs of the Orthoptera, the widely distributed 'chordotonal' nerve ends of insects are to be mentioned.

The larger proportion of the alimentary canal is formed of ectodermal stomodeum and proctodeum, while the entodermal portion (mesenteron) usually forms but one-third of the total length. At ectysis the chitinous lining of the ectodermal parts, including the large chewing stomach, is cast with the rest of the integument. The entire absence of ciliated epithelium is noteworthy. Ciliated cells have never been found in arthropods.

The most constant portion of the circulatory system is the heart, which lies immediately beneath the back, enclosed in a more or less distinct sac which, although called pericardium, is not a part of the cœelom. From the pericardium blood passes into the heart by openings (ostia) right and left. Since the margins of the ostia project far into the lumen of the heart and so form folds functioning as valves, the heart itself may be divided into a series of chambers (fig. 67 ). The chambers disappear when the heart is reduced to a sac. In small arthropods the heart together with the whole vascular system may be lost. Since the Annelida have a well-developed circulatory system, the loss in the arthropods is secondary rather than primitive, and is explained by the fact that with reduction in size the organization is simplified. The heart can thus be lost in small crustacea (many ostracods, copepods, and barnacles), in the smaller arachnids (mites), and in insects (many aphids), while it is present in allied forms. 
The blood may pass from the large arteries either directly into the large blood sinuses of the body (hrmocole, p. I Io), erroneously called the body cavity, or by a more complicated course through capillaries and veins as well as through the respiratory organs. There are, on this account, great differences in the development of the vascular system, but even in the highest forms the system is not entirely closed, the blood passing to the hxmocole and thence to the pericardium (probably arising from the coalescence of veins), from which it is sucked through the ostia into the heart. The variations in the circulation depend chiefly upon the modifications of the respiratory organs, which can be described adequately only in connexion with the various groups. In general it can be said that the more respiration is localized in regions and organs the more nearly complete is the circulation, while with respiration diffused over or through the whole body, the vascular system, including even the heart, may be reduced.

Anatomy and embryology show that the cœlom is greatly reduced in the arthropods and is represented by the cavities of the reproductive and nephridial organs, and in the decapods by a pair of thin-walled sacs connected with the green glands. Sinus-like enlargements of the blood-ressels like the hæmocœle occur in several annelids (.Magelona).

The excretory organs are of two different types. The segmental organs of Peripatus, the shell and green glands of crustacea, the coxal glands of Acerata, and the head glands of insects are modified nephridia in which the nephrostomes have been converted into small sacs. The other type includes the Malpighian tubules of insects, diverticula of the alimentary canal, opening at the junction of intestine and rectum.

The sexual organs, which empty through ducts which are apparently modified nephridia, are rarely hermaphroditic. In the bisexual species one can usually distinguish males and females by external characters, such as size, coloration, or form of appendages, especially those used in copulation. The eggs are usually large and rich in yolk, and consequently but rarely undergo total segmentation. Instead there is a superficial segmentation (fig. I06), in which the surface of the egg divides into the cells which form the blastoderm; the central yolk long remains undivided--a condition of systematic interest since it is not known to occur outside the Arthropoda. The cases of discoidal and unequal segmentation are apparently derived from the superficial.

In accordance with the high organization, reproduction by fission or budding never occurs, but parthenogenesis and padogenesis do. In some parthenogenesis has a certain relationship to the life history. In lower crustacea and in Aphides (plant lice) it allows the species to spread 
rapidly in large numbers over suitable feeding grounds. Among the lees parthenogenesis has a relation to the sexes, since males are only procluced from unfertilized eggs. Along with parthenogenesis-there may be rare exceptions-sexual reproduction occurs, so that asexual alternates with sexual generation (heterogony), though not in such a typical manner as in the worms.

Latreille divided the Arthropods into four classes: Crustacea, Myriapoda, Arachnida, and Insecta. Later the discovery that Peripalus possesses trachea led to the creation of a new class, Protracheata, and the grouping of all arthropods into Branchiata and Tracheata, the branchiates including the crustacea alone. Later researches have shown that these divisions are not natural and that trachere have had different origins, the spiders being nearer to the crustacea than to the insects, and that crustacea and insecta have come from the annelids through different lines.

\section{Class I. Crustacea.}

The Crustacea are so called because their chitinous cuticle is usually rendered hard and firm by deposits of carbonate and phosphate of lime and, in contrast to that of other arthropods, has become 'crusty.' A carapace, recalling the mantle of the molluscs, is widely distributed in the crustacea. It arises as a fold from the head, which may extend backwards as a shield, completely covering some or all of the thoracic segments (fig. 376), or it extends right and left on the sides of the body (fig. 389) and produces two valves strikingly like those of a lamellibranch, the resemblance being strengthened in the cirripeds and ostracodes by the extensive calcification. Another important characteristic is the habitat of the group; the Crustacea are typically aquatic and hence breathe by means of gills. This branchial respiration persists, as in the case of crayfish, when the animals are taken from the water, for they retain water in the gill chamber and hence for a long time the gills are wet by this fluid. There are but few exceptions to this rule, as some land crabs and the sow bugs; these breathe air, either by means of the gills or by special structures in the gill chamber to be mentioned later.

The branchix or gills occur where a rapid change of water is possible. The appendages afford such a position, and hence one finds the gills as thin-skinned rascular plumes or plates (figs. 62, 398) either on the appcndages or on the body near by; or the whole appendage may take a leaflike, thin-skinned shape and thus serve as a gill (fig. 375). Again, the whole body surface may be respiratory and in small forms may entirely replace the gills, so that these organs become rudimentary or entircly disappear, there being a diffuse respiration with corresponding effects in the circulatory system. With a localized respiration heart, arteries, 
capillaries, and veins are well developed, but with the diffuse respiration only the heart persists as a reduced structure, or with its disappearance the last traces of a circulatory system are lost.

Locomotion is also related to the aquatic life and these animals usually possess appendages of the biramous or schizopodal type, which at once

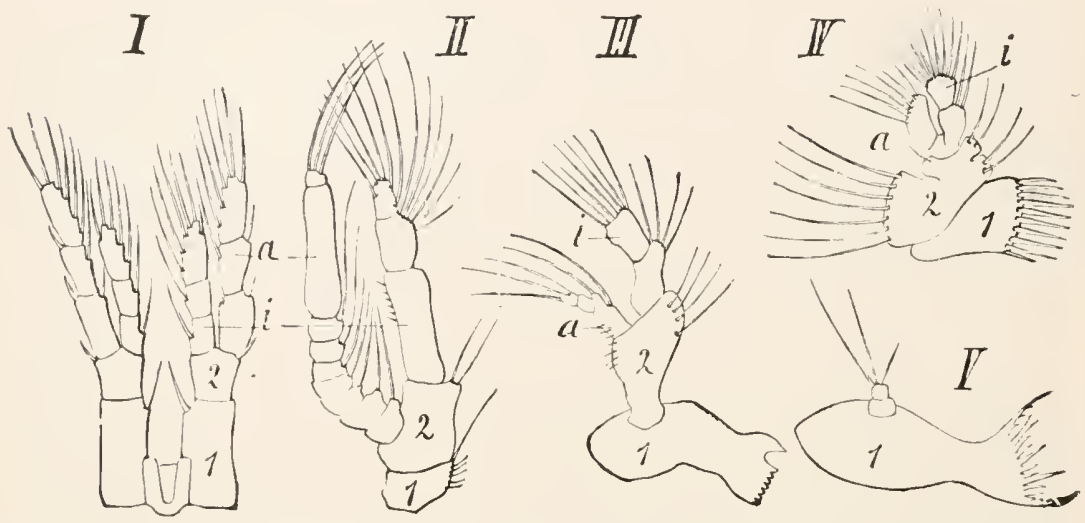

FIf. 374 -- Copepod appendages. I-IV, Diaptomus castor; $I$, a pair of schizopodal feet; $I I$, second right antenna; $I I I$, right mandible; $I V$, right maxilla; $V$, right mandible of $C$ yclops coronatus. $\quad \mathrm{I}, 2$, joints of basiopodite; $i$, endopodite; $a$, exopodite.

differentiates these forms from all other arthropods. While in the latter, as every insect shows, the joints of the limb are in a single row, the crustacean appendage has a two-jointed base (basiopodite), followed by two many-jointed branches (fig. $374, I$ ), an inner or cndopodite and an outer or exopodite.

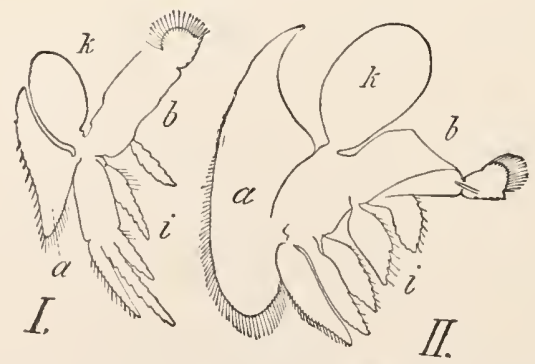

FIG. 375.-I and II first and sixth legs of Branchipus grubei (after Gerstäcker). $a$, flabellum; $b$, basis; $i$, axon and its endites; $k$, gills.

The schizopodal appendage occurs only when the limb is used for swimming; when it is used for walking, as in crayfish and crabs, the exopodite is lost and only the endopodite persists as the functional limb, which then closely resembles the appendages in the insects. This loss rarely occurs on all the appendages; 
ustually the abdominal feet and the mouth-parts retain the two-branched condition. Embryology further shows that even in the crabs all the feet are at first schizopodal and that the walking legs lose the exopodite during growth. 'There is evidence to show that the schizopodal foot is not the primitive type. 'This is furnished by the phyllopod foot (fig. $375, I I$ ), which consists of a median axis, $b$, hearing on the inside six cndites, $i$, and on the outer side two exites, a flabellum or epipodite, $a$, and a gill, $k$. This furnishes the schizopodal form by a loss of the four basal endites (those nearest $b$ ) and the development of the two terminal endites into exoporlite and endopodite. Still the schizopodal condition is so nearly universal among Crustacea that it must be accorded great weight in classification.

The appendages furnish a further diagnostic character in that two pairs of antenne are present in the crustacea (see, however, Tribbita). Antennx, it will be remembered, are preoral appendages innervated from the brain. In some cases, as many Entomostraca, the second pair may lose their sensory functions and become mere swimming organs.

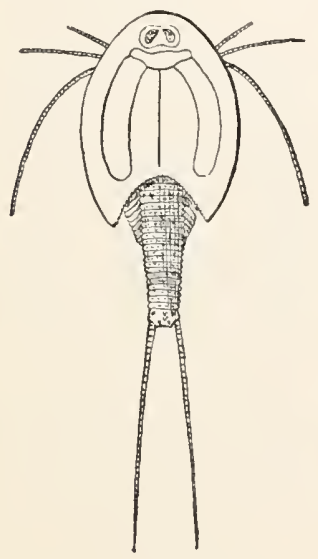

FIG. 376 .

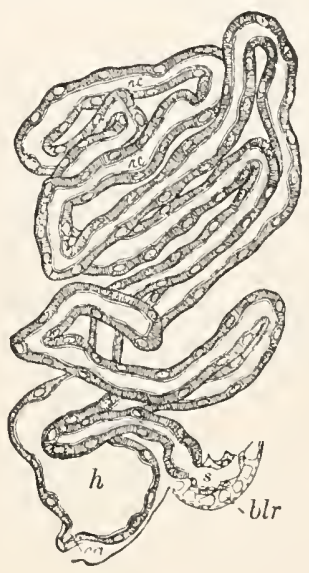

FIG. 377 .

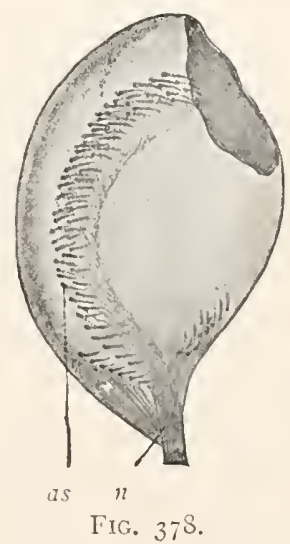

FIG. 377.-Antennal gland of Mysis (after Grobben). hlr, blood lacuna; ea,

FIG. 37 6.-A pus equalis * (after Packard). external opening; $h$, bladder; $r c$, canal; $s$, internal vesicle.

FIG. 378.-O Otocyst of crayfish. as, crista acustica; $n$, nerve.

Concerning the internal organs but few general remarks can be made. Salivary glands are wholly absent; on the other hand, the stomodeum is usually widened into a strong chewing 'stomach,' and behind this empty the ducts of the so-called liver. This varies between the two simple blind sacs of the Daphnida (fig. $38_{3}$ ) and the enormous livers of the Decapoda (fig. 400, 1). It is not exclusively glandular (hepato-pancreas), but is an expansion of the digestive tract, and like it partakes in the resorption of nourishment. Excretory organs are represented by so-called green glands 
(antennal glands) and shell glands. The latter (so called from the erroneous idea that they produced the shell) open to the exterior on either side at the bases of the maxillie (fig. $38_{3}, s$ ). The green gland opens similarly on the bases of the second antenne. Both have essentially the same structure (fig. 377); they begin with a terminal vesicle, which passes into a slender, greatly coiled tulse. Both occur together only in the larva; in the adult one or the other is suppressed. In some amphipods there are excretory diverticula developed from the intestine (fig. 409), which resemble the Malpighian tubes of insects, but differ from them in being of entodermal origin. In some decapods caca occur in the same region, but nothing is known of their function.

Besides compound eyes there may be a 'nauplius eye' situated on the brain and consisting of an $\mathrm{X}$-shaped pigment mass in which are placed three lens-formed groups of visual cells, connected with nerves. These are distinct from ocelli, but recall the eyes of Platodes. Both kinds of eyes may coexist in the same species.

Auditory (equilibration) organs (otocysts) occur only in the Malacostraca, either in the base of the first antennx or (Schizopoda) in the endopodite of the last abdominal feet (fig. $396, o$ ) with a large statolith of calcium fluoride. The antennal ears of Decapoda (fig. 378) are sacs, the opening to the exterior protected by strong hairs, and each internally with a row of chitinous sense hairs, the crista acustica, connected below with an auditory nerve, while their free ends extend between a cluster of statoliths.

At ecdysis these otocysts with their sensory hairs and statoliths are cast off. If a crayfish which has just molted be placed in perfectly clean water, the otocyst will remain without statoliths; but if some easily recognizable substance, like uric acid crystals, be placed in the water, some of these will soon be found in the sac, thus proving that the statoliths are introduced from the outside. Experiment shows that these organs are statocysts, but apparently they are also auditory, since some forms which have well developed otocysts have no statoliths.

Crustacea are only exceptionally hermaphroditic. The spermatozoa are noticeable for their great size, in many ostracodes equalling the body in length (Pontocypris paradoxa, 8 times as long as the body- $5-7 \mathrm{~mm}$.). Except in the Cirripedia the spermatozoa lack a flagellum and (Ostracods excepted) are immobile. Their round or elongate body is covered with rigid processes (fig. 37, III, IV). They are frequently enclosed in spermatophores (fig. 385).

The typical development of a crustacean includes a metamorphosis, and where direct development occurs the metamorphosis is either suppressed or the corresponding stages are passed in the egg. Two of the larral stages are especially important, the nauplius and the zoea. The nauplius (figs. 7,393 ) consists of three segments covered by a dorsal 
shield and bearing three pairs of appendages. The first pair, developing later to the first antenna, are simple; the others, corresponding to the second antenne and mandibles, are schizopodal. Internally there is a three-regional alimentary tract, a supraœesophageal ganglion on which is an unpaired eye, and a ventral chain. The nauplius is almost universal among the lower crustacea, and some believe that it represents an ancestral form from which the crustacea have descended, a view open to much objection.

The zoea consists (fig. 379) of cephalothorax and abdomen, the latter without appendages, the former with several pairs of schizopodal swimming feet. There are two large compound eyes and, dorsal to the intestine, a heart. Frequently the carapace is armed with very long spines projecting from front, back, and sides, which are intended as protection from enemies.

Both nauplius and zoea rarely appear in the life cycle of one individual. The nauplius is characteristic of the lower crustacea-the 'Entomostraca,' and appears in only a few Malacostraca, like the schizopods and Peneus, and there precedes the zoea stage. The zoea, on the other hand, has never been noticed in the Entomostraca, but occurs in many Malacostraca. It must not be forgotten that many forms among both Entomostraca and Malacostraca have no zoeal or nauplius stage. Frequently the lower crustacea are united under the name Entomostraca, but, aside from the nauplius stage and the possession of a shell gland, the only characters of the group are negative.

\section{Sub Class 1. Trilobitce.}

The most important crustacean fossils are the Trilobites which appeared in the Cambrian and died out in the Permian, being extremely 
abundant in the Silurian. The body (fig. 380) consists of head and trunk, the latter segmented. In the young the segments are very few, but increase in number (10-29, according to the species) with age. The hinder segments frequently differ from the rest and form an abdomen or pygidium. Dorsally the animal is divided by two grooves into three lobes, marking off in the head a glabella and two gena; in the trunk rhachis and two pleurce. On the head there are usually a pair of compound eyes, which in the young were frequently ventral, but are brought to the dorsal surface with growth. For years little was known of the ventral side, but lately specimens of Triarthrus bccki (fig. $3 \delta_{1}$ ) from the Utica slate have revealed

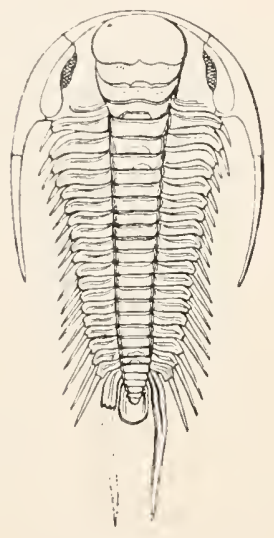

FIG. $3^{80}$.

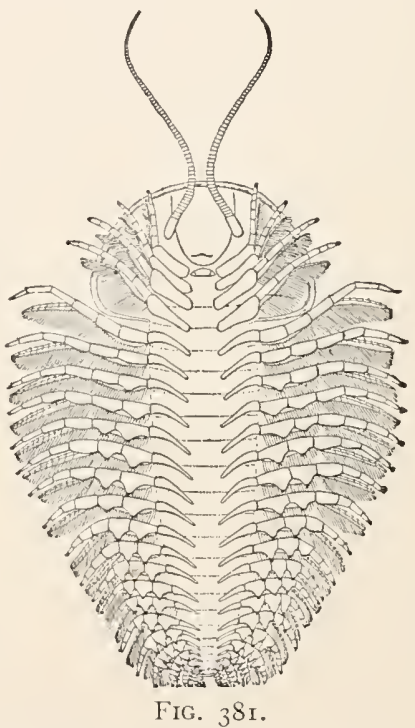

Fig. 380.-Paradoxides bohemicus (from Zittel).

FIG. 38I.-Triarthrus becki, ventral surface, restored (after Beecher). The head bears one pair of antenna and four pairs of biramous feet, the basal joints serving as maxillæ. Trunk with biramous feet.

the appendages. On the head are a pair of simple antennx, and four pairs of schizopodal feet, the bases of which acted as jaws. It is a question whether the first pair of jaw feet correspond to the second antennx or whether these have been lost in the group. The trunk segments hear biramous feet. In some respects the trilobites resemble the Xiphosura (infra), but the possession of antenne and biramous feet place them among the crustacea. Here their position is very uncertain. We have little knowledge of but one species, and this with its single pair of antenne differs from all recent crustacea. 


\section{Sub Class II. I'hyllopoda.}

The Phyllopoda are the most primitive crustacea. The name is derived from the leaf-like feet (fig. 375), which occur upon the thoracic region. The anterior appendages are schizopodal, the second pair of antennx often being efficient swimming organs. The number of somites varies between wide limits, there being less than a dozen in the Cladocera, while, if Savigny's law (p. 352) hold true, there are over sixty in scme Apodidie. Most forms (the Branchipodidie excepted) have a carapace. This forms a broad oval shell corering most of the body in the Apodidie (fig. 376); in the Estheriide and Cladocera it is divided into right and left halves hinged together in the mid-dorsal line, thus giving these animals the appearance of bivalve molluscs.

These forms have, besides the nauplius eye, a pair of compound eyes which in the compressed forms are frequently fused, although distinct in the young and retaining the double optic nerve throughout life. 'T he liver is present in the shape of simple ceca; the heart, elongate, chambered. and with many ostia in the Branchioporla, a short sac with only a pair of ostia in the Cladocera (fig. $38_{3}, h$ ), lies dorsal to the intestine. The shell gland is well developed.

In development summer and winter eggs are distinguished. The summer eggs form a single polar globule and develop parthenogenetically. The winter eggs form two polar globules and require fertilization. The thin-shelled summer eggs are carried by the mother in a brood pouch and soon hatch. The thick-

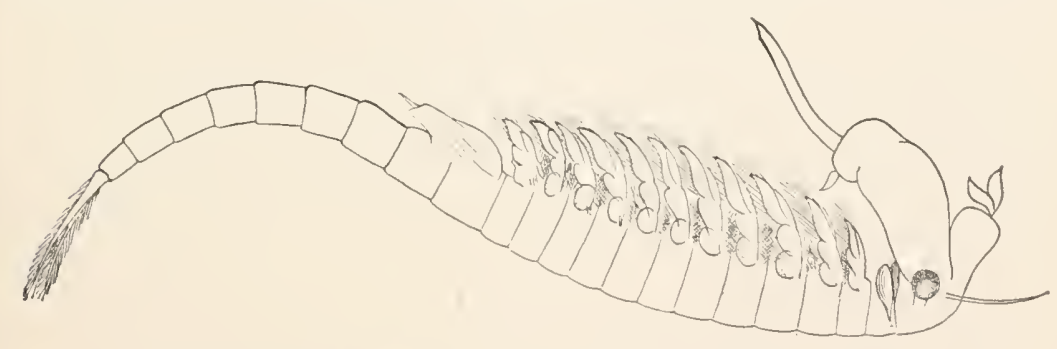

FIG. 382.--Branchipus zernalis,* fairy shrimp (after Packard).

shelled winter eggs fall to the bottom, where they require a long time for development. They may be dried or frozen without injury, and in some cases drying is necessary for development. This explains the appearance in early spring of large numbers of Branchipus and Estheria in pools which are dry in summer. The phyllopods are largely inhabitants of fresh water. The winter eggs preserve the species through times of dirought and cold; the summer eggs are for the rapid increase of the species during the wet season. The same relations also explain the fact that males are rare and only appear at intervals, indeed are not known in some species. 


\section{Order I. Branchiopoda.}

The Branchiopoda are relatively large with numerous segments, leaf-like appendages, long, chambered heart, and lack swimming antenna. With few exceptions they are inhabitants of fresh water. According to the development of the carapace they are subdivided into three families.

I. APODID.x. Body depressed, with large oval undivided carapace. Eggs carried in brood capsules formed by a pair of appendages. Apus* (fig. 376), Lepidurus. * Protocaris of the Cambrian is apparently an Apodid. 2. BRANCHIPID E. Body without carapace, the second antennx of the male large and modified for clasping the female. The female carries the summer eggs in a wide 'uterus' in the abdomen. Branchipus* (fig. 382), fresh water; Artemia, * in brine; one has been transformed into the other by changing the water from fresh to salt or the reverse. 3. EstheriID瓜. Body laterally compressed and enclosed in a bivalve shell, compound eyes fused; males very rare. Estheria,* Limadia, fresh water.

\section{Order II. Cladocera.}

Like the estheriids the small Cladocera have the body enclosed in a bivalve carapace, which in some is small and reaches back only over the first trunk segments, in others is large, enclosing the body, with a notch for the protrusion of the head, while behind it terminates in a sharp spine. The head bears a pair of large swimming antennæ and a much smaller first pair bearing olfactory bristles and, in the male, hooks for clasping the female. The body consists of few segments, the heart is a simple sac, and the fused faceted eyes are capable of motion in a special optic capsule. The young eggs in the sexual organs always occur in groups of four (fig. $38_{3}$ ). Of these but one grows into an egg, the others serving this as nourishment. Larger eggs with more yolk occur when several groups fuse to form a single egg. The summer eggs arise from a single group, the winter eggs from several groups of primordial ova. The space between the back of the animal and the shell serves as a brood pouch. The larger winter eggs-one or two in number-frequently remain for a while in the brood chamber and are there enveloped in a peculiar shell, the ephippium, consisting of two chitinous plates, like watch crystals, their edges closely appressed.

DAPHNide. Shell well developed; Daphnia* (fig. $3^{8} 3$ ), Bosmina. * PolyPHEMIDÆ. Shell small, only functioning as a brood case; head with an enormous eye and large swimming antenna; no phyllopodous feet; marine and lacustrine. Leptodora hyalina* appears at night, sometimes in great numbers, in some of our lakes. Evadne* (fig. $3^{84}$ ), marine.

\section{Sub Class III. Copepoda.}

A general description of the copepods can only apply to the nonparasitic forms, since many of the parasites are so degenerate (figs. 6, 388) as to be recognized even as arthropods only by a knowledge of the development. The sixteen somites of the body are nearly equally divided among the three regions-head (6), thorax (5), and abdomen (5)-of the animal. (In Cyclops the first thoracic segment is fused with the head, the first two abdominal segments are fused-fig. 7.) The last abdominal segment is two-forked, forming the furca. While the abrlomen lacks appendages, the thorax bears typical biramous appendages, consisting of a two-jointed 


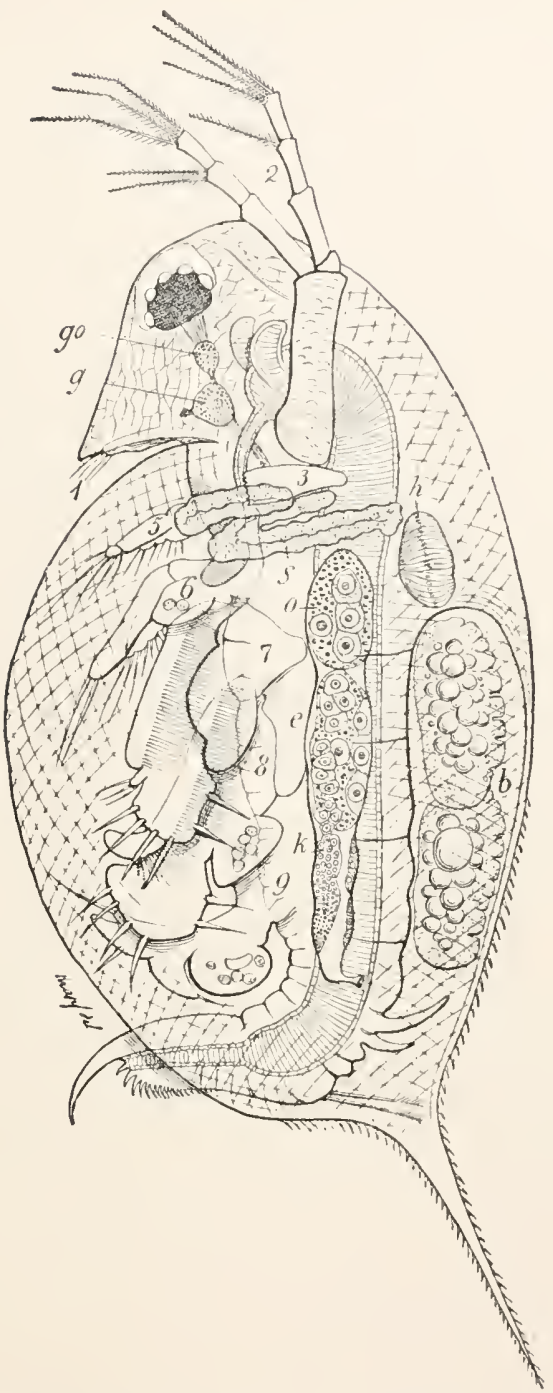

Fig. 383.-Daphnia pulex. $b$, brood chambers with embryos; $g$, brain with nauplius eye; go, optic ganglion; $h$, heart; $o$, ovary; $s$, shell gland. The eggs arise at $k$, anıl separate, forming in groups of four, as at $e$, of which one becomes the egg, while the others abort $(0)$ and form food. The egg then passes to the brood chamber. I, 2, first and second antenne: 3 , mandible (maxilla rudimentary and invisible); 5-9, legs. Alimentary canal cross-lined.

basiopodite, the basiopodites of a pair being frequently united for common motion (fig. $374, I$ ). Exopodite and endopodite, usually three-jointed, are fringed with bristles. Usually the fifth pair of thoracic appendages 
is not so well dereloped, and in some cases is represented by two bunches of bristles.

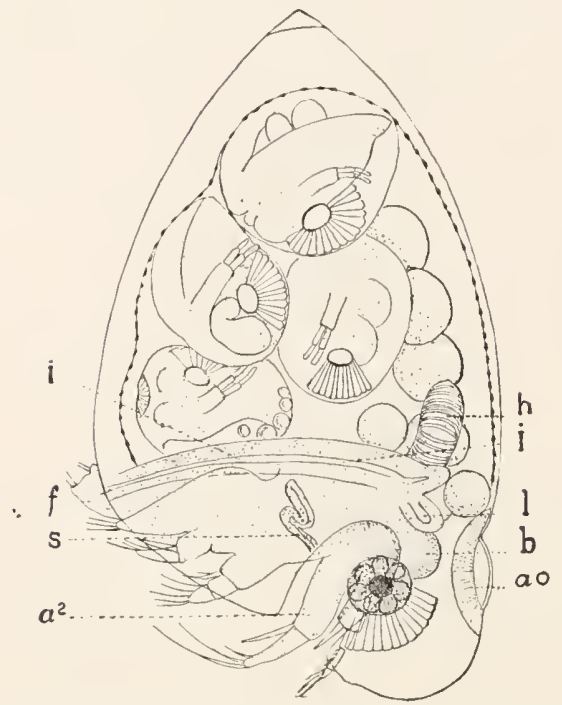

Fis. 384.-Ev'adne (orig.), showing the brood pouch filled with eggs and young. $a^{2}$. second antenna; $a o$, adhesive organ; $b$, brain; $f$, furca; $h$, heart $i$, intestine; $l$, liver; $s$, shell gland.

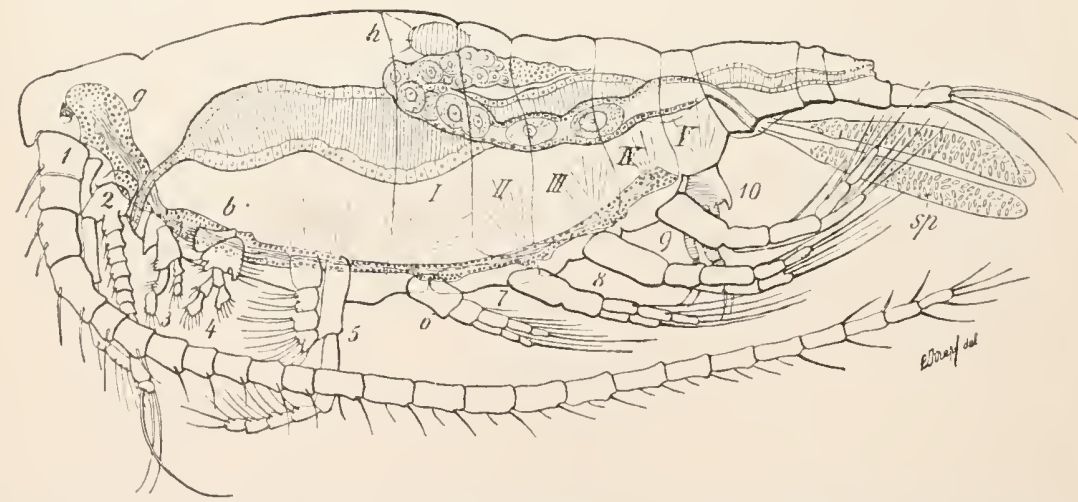

FIc. 385.-Dioptomus castor. $h$, ventral nerve corll; $g$, brain with nauplius eye; $h$, heart, beneath it the orary and digestive tract; $s p$, spermatophores; I, 2, first and second antennx; 3 , mandibles; 4 , maxilta; 5 , maxilliped; 6-10, swimning feet.

The first pair of antenne in the males may be hooked near the base for clasping; the second are sometimes biramous (fig. $374, I I$ ). The mandible (fig. $374, I I I, V^{\prime}$ ) is instructive, since a study of several species shows that it is derived from a schizopodal condition and that the first basal joint alone is used for chewing, the rest being reduced to a palpus of varying development. Both 
basal joints of the maxilla (fig. $374, I V^{\circ}$ ) can be used in eating. Two maxillipeds (formerly regarded as the separated branches of an appendage) mark the termination of the head (fig. $38_{5}, 5$ ).

The internal anatomy is simple. There is no liver, and the straight intestine (fig. $3 S_{5}$ ) runs without marked changes in size to the anus between the branches of the furca. The visual organ is the unpaired nauplius eye (which has given the name to one genus, Cyclops). It lies directly on the brain. The ventral chain has its ganglia irregularly distributed. Gills are always absent, as are usually the heart and bloodvessels. The gonads are unpaired, but the sexual ducts, which open at the base of the abdomen, are paired. 'I'he females possess a receptaculum seminis distinct from the oviducts, to which the male attaches spermatophores packed with sperm (fig. $38_{5}, s p$ ). As the eggs leave the oviduct they are fertilized by the sperm issuing from the spermatophores, and are enclosed in a gelatinous substance, thus producing the so-called eggsacs, attached to the abdomen, by which one can easily recognize the females (fig. 7). A nauplius hatches from the egg, and by budding segments and appendages at the hinder end, and by a change of the nauplius appendages into antenne and mandibles, passes through a 'cyclops-stage' into the adult. 'The Copepoda have descended from some phyllopodlike form. The poorly developed ventral chain, the loss, partial or complete, of a circulatory system, and the absence of gills are all against the view which would consider them primitive.

\section{Order I. Eucopepoda.}

The forms to which the foregoing description will apply are the Eucopepoda, and include many species, which often occur in enormous numbers in both fresh and salt water, forming the larger proportion of the plankton. They thus furnish the most important food supply not only for fishes but for the giant baleen whales. Cetochilus septentrionalis occurs at times in such myriads that the sea for long distances is colored red.

The CyClOPID E, no heart and paired egg sacs, fresh-water; Cyclops $s^{*}$ (fig. 7). Calanide, fresh water and marine; heart present, single egg-sac. Diaptomus, * fresh water (fig. 385 ); Cetochilus, * Pontilla, * marine. HARPACTIDE, creeping forms, mostly marine; Canthocamptus, * fresh water. The CORvC.iD.玉, half parasitic, include the wonderfully iridescent Sapphirina* and the NotodeLPHIDE, parasitic in ascidians, form a transition to the next order.

\section{Order II. Siphonostomata (Parasita).}

There are aiso Copepoda to which the account in large type will not apply, animals of such strange appearance that many of them were long regarded as parasitic worms (figs. 6, 386, 388). Their mandibles are altered to piercing bristles and enclosed in a piercing proboscis formed of upper and lower lips. With this sucking organ they bore into the skin or gills of fishes. They liave cylindrical forms or bodies of the most bizarre shapes, in which frequently no 
segmentation is visible, while the appendages are rudimentary or even entirely lost. Indeed one would not recognize them as arthropods save for the following features:

(I) Most of them have the typical Copepod egg-sacs attached to the hinder end. (2) A complete series of intermediate forms allows one to trace, step by step, the alterations of form from the free-living species to the most modified parasites. (3) Ontogeny is convincing. Most parasitic Copeporla leave the egg as a nauplius and pass through a Cyclops-stage before attaching themselves

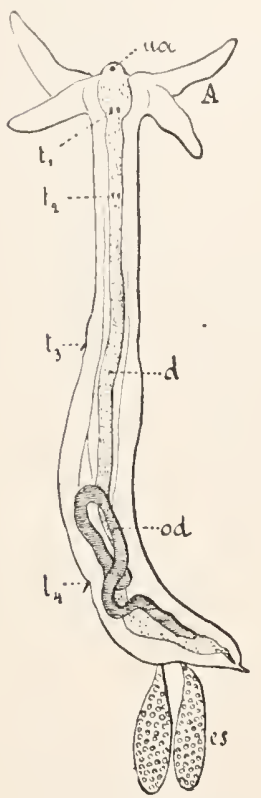

FIG. 386.

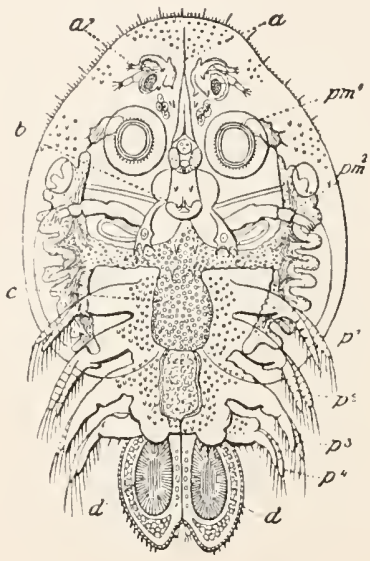

FIG. $3 s_{7}$.

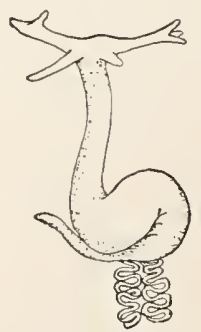

FIG. 388 .

FIG. 386.-Female Lerncocera esocina (from Lang, after Claus). A, armlike processes of anterior end; $d$, digestive tract; es, egg-sacs; od, oviduct; $t_{1}-t_{4}$, rudimentary thoracic appendages.

Fig. 387.-Argulus foliaceus (from Ludwig-Leunis). $a$, sting; $a^{\prime}$, antenna; $b$, mouth; $c$, intestine with liver; $d$, abdomen; $p m^{1}, p m^{2}$, first and second maxillipeds; $p^{1}-p^{4}$, biramous feet of thorax.

FIG. 388.-Lernca branchialis* (orig.).

to fishes and becoming the highly degenerate parasites. These parasites are always females. The males scarcely pass the Cyclops-stage, copulate with the females and then die, or if they pass through the metamorphosis, they remain small and different in appearance (fig. 8). They occur attached to the female near the genital openings. There is thus here a marked sexual dimorphism.

ARGULIDE (sometimes made a distinct order, Branchiura), fresh-water forms with compound eyes, liver lobes, and second maxillipeds metamorphosed into

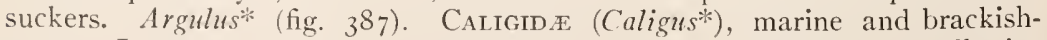
water. LERN EOPODID E. Fish parasites with maxillæ united into an adhesive organ. Achtheres* (fig. 6), perch. LERNAID 388); Lernaocera* (fig. 3S6); Penella.* 
Sub Class II. Ostracoda.

Like the Cladocera and the Estheriidx the Ostracoda are enclosed in a bivalve shell, which, when closed, includes not only the borly but the head and appendages as well, these being protruded when the shell is opened. The valves are closed by an adductor muscle, opened by a hinge ligament like that of lamellibranchs. This resemblance to the molluscs is heightened by lines of growth upon the shell. The antennie,

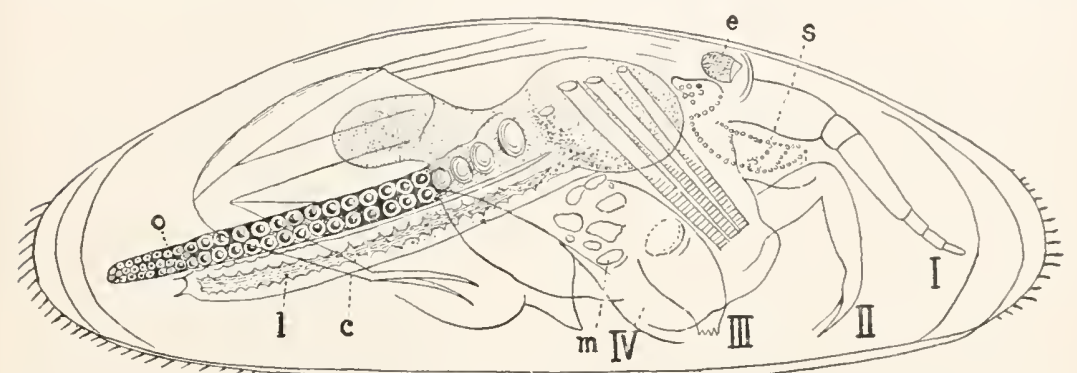

FIG. $389 .-C y p r i s$ fasciatus, adult female (after Claus). $I-I V$, appendages; $c$, furca; $e$, eye; $l$, liver; $m$, adductor muscle of shell; $o$, ovary; $s$, shell gland.

the first simple, the second frequently two-branched, are used for swimming and creeping. The mandibles, maxillæ, and three pairs of legs vary greatly from genus to genus. The internal structure is also variable. The Ostracoda are bottom forms and live in fresh and brackish water as well as in the sea.

Cypridinide. First two pairs of legs maxillary in character, the last dereloped into a hook for cleansing the shell; heart present; marine; Cypridina* CYPRIDID.e. First pair of legs maxillary in character; heart lacking; fresh water. Cypris,* Candona.*

\section{Sub Class V. Cirripedia.}

The barnacles differ from all other crustacea in that they have lost their locomotor powers and live attached to rocks, floating timber, and the like. In some cases they attach themselves to other animals, as crabs and molluses, or, as in the case of Coronula and Tubicinella, to whales. This leads in Anelasma and the Rhizocephala to a true parasitism, the barnacle not only attaching itself to an anjmal but sucking its juices as food.

The attachment is by the dorsal surface in the neighborhood of the head, and is initiated by the first antenne, in which is a cement gland secreting a rapidly hardening cement. The flat region of fixation in the Balanidie 
(fig. 390) is drawn out in the Lepadidx into a long muscular stalk (fig. I I 5). To this attached life are related all the peculiarities of structure. A fixed animal has greater need of protection than one which can flee from its enemies, therefore we find right and left mantle folds capable of complete closure, like those of an ostracode, each with two calcified plates the scuta and terga (figs. I I5, 390, $s, t)$, the first cephalic, the other posterior, in position. Between the pairs of these is the gap through or which the feet are protruded.

Besides there are other calcified portions, one of which, the carina (c), corresponds to the hinge-line of the ostracode and in some Lepads is supplemented by a farther unpaired piece,

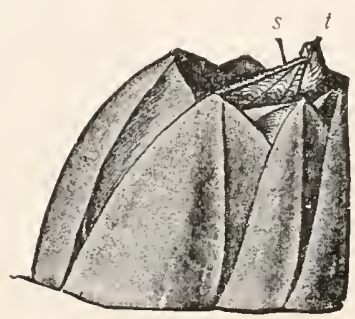

FIG. 390 .

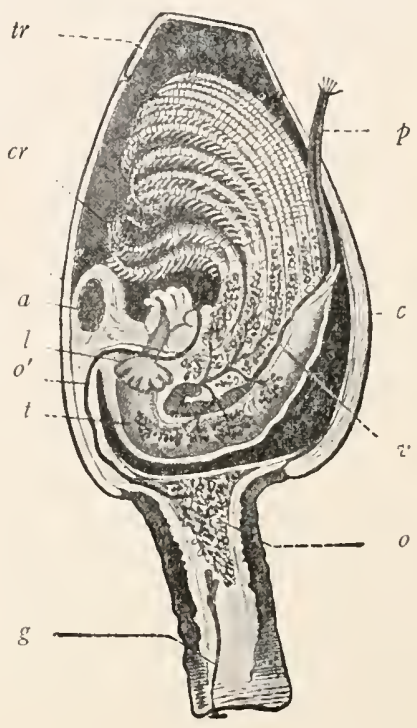

FIG. 39 I.

FIG. 370.-Balanus hameri,* acorn barnacle (from Lang, after Darwin). Formed of rostrum, lateralia, and carina, the operculum of scuta $(s)$ and terga $(t)$.

Fig. 391.-Anatomy of Lepas (after Claus). a, adductor muscle; $c$, carina; $c r$, cirri (feet); $g$, cement gland; $l$, liver; $o, o^{\prime}$, ovary and oviduct; $p$, penis; $t$, testis; tr, tergum; $v$, vas deferens.

the rostrum. In the Balanidæ the rostrum and carina are much stronger, while between them other paired pieces, the lateralia, are intercalated. Lateralia, rostrum, and carina arise from a base (usually calcareous) and form a capsule, closed above by a double valve formed of the paired scuta and terga, between which, when open, the animal can be seen (fig. 390).

The body in both lepads and balanids has essentially the same structure. It is flexed ventrally, so that mouth and vent are near each other, and bears six pairs of feathered feet, or cirri, which, when extended, become widely separated and form a most efficient means of straining small organisms from the water and conveying them to the mouth. These feet are biramous, with their branches ringed and thickly haired. Behind them is a rudimentary abdomen and an elongate penis; while the mouth is surrounded by a pair of mandibles and two pairs of maxilla.

In internal structure the most noticeable feature is that the animals 
with few exceptions, in contrast to most other arthropods, are hermaphroditic, a condition possibly correlated with their selentary life and the consequent need of self-impregnation. The testes lie in the sides of the body; the ovaries in the Lepadids are in the stalk, in the Balanids in the basal plate. In cases of several solitary hemaphrodite species complementary dwarf males occur. These are very small, purely male forms, with extremely simple structure (fig. 392), which live inside the mantle carity near the genital openings. The unsegmented body is enclosed in a sac (a

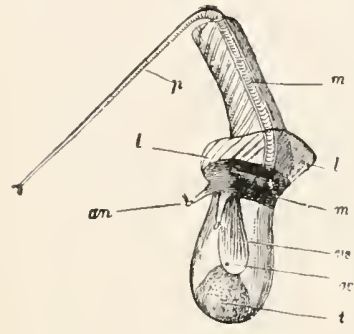

F1G. 392 .

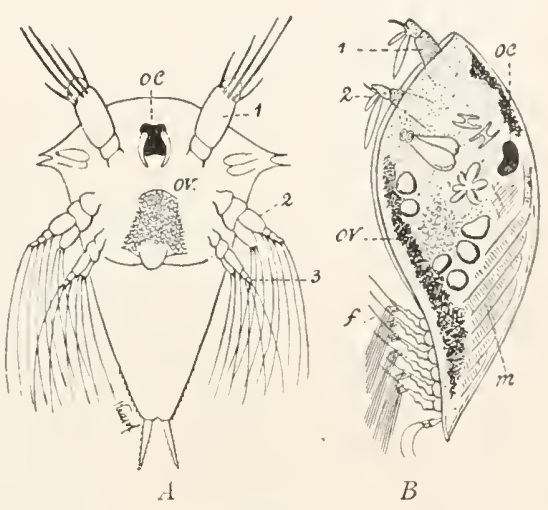

F1G. 393.

Fig. 392.-Male of Alcippe lampas. an, antenna; $l$, mantle lobes; $m$, muscles; oc, ocellus; $p$, penis; $t$, testis; $v s$, seminal vesicle.

Fig. 393.- Nauplius $(A)$ and Cypris $(B)$ stages of Sacculina carcini. (after I)elage). I, 2 , antennx; 3 , mandible; $f$, cirrous feet; $m$, muscles; $o c$, nauplius eye; $o v$, anlage of ovary.

soft-skinned shell), and anchored by the antenne. The long penis protrudes from the mantle. In the genus Scalpellum there are purely hermaphroditic species, hermaphroditic species with complemental males, and purely diøecious species.

Since the hard shells of the barnacles resemble those of the molluscs, it is not to be wondered that these forms were long regarded as belonging to that group. It was not until the development (fig. 393) was studied that the error was corrected. A large nauplius comes from the egg and later is metamorphosed into a second larval stage with bivalve shell which, from its appearance, is called the cypris-stage. This becomes fixed and develops into the adult, losing the compound eyes and retaining the nauplius eye.

\section{Order I. Lepadidæ.}

Stalked cirripeds, with shell largely formed of scuta, terga, and carina; other parts may be added. Lepas anatifera* (fig. II 5), the goose barnacle, owes its common name to a mediæval myth which claimed that the Irish (or bernicle) goose developed from these animals. Anelasma squalicola, thin-skinned, parasitic on sharks, forms a transition to the Rhizocephala. 


\section{Order II. Balanidæ.}

Sessile cirripeds with calcareous shell formed of carina, rostrum, and la ${ }^{2}$ eralia; scuta and terga forming the valves (fig. 390). Balanus, ${ }^{*}$ Coronula, attached to whales.

\section{Order III. Rhizocephala.}

These differ greatly from other cirripeds. They are parasitic on the abdcmens of decapod crabs and consist of a stalk which penetrates the body of the host and a body which remains outside (fig. 394). The stalk branches in a root-like manner, penetrates the cephalothorax and absorbs its juices. Since the stalk furnishes the food, an alimentary canal is absent. The body lacks all appendages, is enclosed by a soft-skinned mantle, and is almost entirely

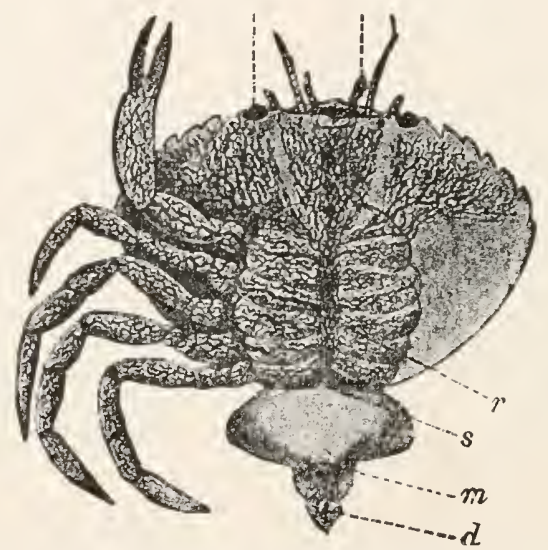

Fig. 394.--Sacculina carcini attached to Carcinus manas, whose abdomen (d) is extended. $m$, sex ofening of Sacculina; $r$, network of roots ramifying the crab; $s$, stalk.

filled with the gonads. Since the adult parasites lack all arthropodan features, their position is only settled by their development (fig. 393), which is like that of other cirripeds. These forms are rare on the American coast. Sacculina, Peltogaster.*

Two more orders, ABDOMINALIA and APODA, parasitic in the mantle and shells of molluscs and other cirripeds, scarcely need mention.

\section{Sub Class I. Malacostraca.}

The Malacostraca are sharply marked off from the other Crustacea by having a body which consists of twenty segments, of which seven are abdominal (Nebalia has twenty-one, eight abdominal). The excretory organs are represented by the antennal glands, and shell glands are lacking except in the larve and some Isopoda. The male genital ducts open on the thirteenth, the female on the eleventh, segment. 


\section{Legion I. Leptostraca.}

The Leptostraca connect the Phyllopoda with the higher groups. Their twenty-one somites (eight abdominal, eight thoracic, and five cephalic), and the openings of the genital ducts ally them to the Malacostraca. On the other hand, the bivalve carapace covering the cephalothorax and part of the abdomen, and the leaf-like thoracic feet, are phyllopodan. They have an antennal gland and a rudimentary shell gland; an elongate heart which extends through cephalothorax and abdomen; and stalked compound eyes. The few species are all marine and belong to the genus $N^{\top}$ ebalia* (fig. 395).

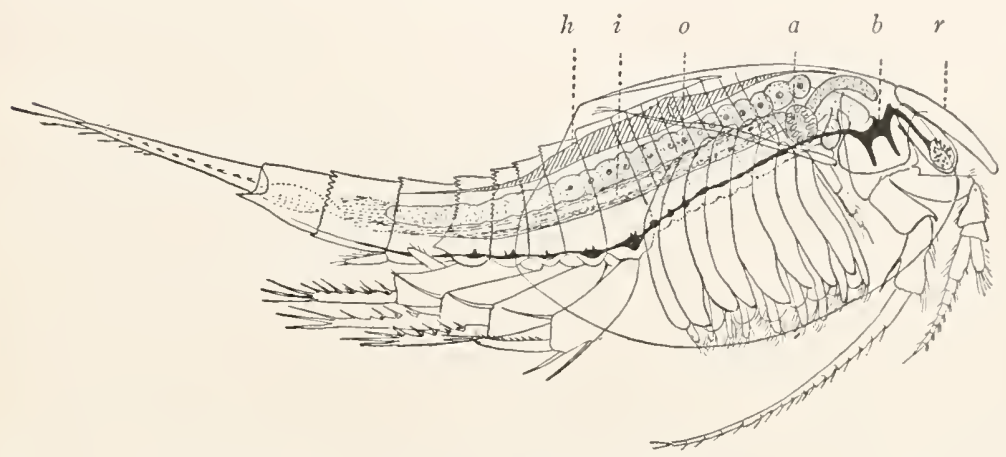

FIg. 395--Nebalia bipes* (after Sars). $h$, heart; $i$, intestine; 0 , ovary; $a$, adductor of carapace; $b$, brain; $r$, rostrum.

\section{Legion II. Thoracostraca (Podophthatmia).}

The names given this division have reference, first, to the fact that the head and some of the thoracic segments are immovably united and covered by a firm carapace; second, that the compound eyes (except in Cumacea) are placed on movable eye stalks. The first five appendages are always two pairs of antennæ, a pair of mandibles, and two pairs of maxilla. The remaining pairs vary greatly and from one to three may be modified into maxillipeds, while the abdominal somites except the last (telson) usually bear appendages, at least in the female. There is usually a metamorphosis in which a nauplius stage may appear, most frequently in the lower forms (schizopods), but even in the decapods (Peneus).

\section{Order I. Schizopoda.}

These are small forms (fig. 396), mostly marine, in which the cephalothorax is covered by a carapace with which some or all of the thoracic somites are firmly united. The eight thoracic feet are biramous throughout life and are used in swimming. The posterior pair of abdominal feet together with the telson form a caudal 'fin' by means of which the animal can swim backwards. The delicate skin permits of diffuse respiration, and gills are frequently lacking. In some genera plates from the legs of the female enclose a brood case beneath the cephalothorax, thus giving these forms the common name of opossum shrimps. 
The Mysidide are widely distributed, several species of $M$ ysis (fig. 396) occurring on our coasts and one in the Great Lakes. In these the endopodite of the sixth abdominal appendage contains a otocyst, with a calcic fluoride statolith. Other families are the EupHausinde and LopHOgastride of the high seas.

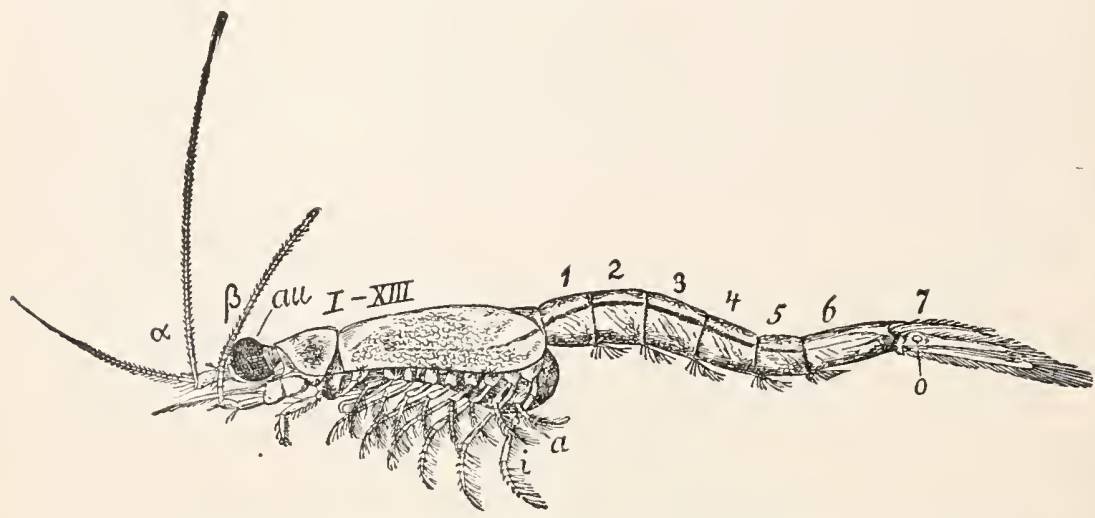

FIG. 396.-Mysis elongata (from Gerstäcker). $\alpha, \beta$, first and second antennæ; $a$, expodite; $a u$, eye; $i$, endopodite; $o$, otocyst; I-7, abdominal somites.

\section{Order II. Stomatopoda.}

In structure of the cephalothorax these forms, known as mantis shrimps (from a resemblance to the insect, the praying mantis), are lower than the schizopods, since the last three or four thoracic somites are free and are not covered by the carapace. The appendages, however, are more developed, since only the three posterior of the thoracic feet are biramous and natatory. The four in front of these are prehensile and bear a pincer formed of the last two joints, the last being slender and usually toothed and closing in a groove of the penult joint like a knife blade in the handle. The first of these raptorial

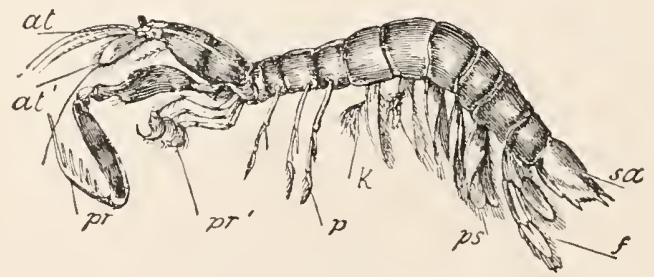

FIG. 397.- Squilla mantis. at, at', first and second antennæ; $f$, sixth abdominal feet; $k$, gills; $p$, schizopodal thoracic feet; $p r$, $p r^{\prime}$, raptorial feet; $p s$, pleopoda; $s a$, telson.

feet are the largest and are used in capturing fishes, etc. Since the thoracic feet are of little service for locomotion, the abdomen is long and stout, especially the caudal fin. The five anterior abdominal feet bear the gills, and correspondingly the elongate heart with many ostia extends into the abdomen. The transparent pelagic larve were formerly regarded as adults and described as Alima and Erichthus. Squilla,* Gonodactylus.* They are burrowing animals and deposit their eggs in their holes. 


\section{Order III. Decapoda.}

The Decapoda is the most important group of Crustacea, since it contains the shrimps, lobsters, crayfish, and crabs. It agrees with the Schizopoda in having a cephalothorax composed of thirteen fused somites, but differs in the structure and function of the thoracic extremities. Only the last five pairs (whence the name Decapoda) are locomotor. These lose the exopodite during development (Peneida excepted) and become strong walking legs, terminated either with claws or pincers (chele). Usually the first pair is distinguished from the others by its size and by being chelate, and is a grasping organ. In the development of a chela the penult joint sends out a strong process, the 'thumb,' which extends as far as the last joint (the 'finger'), which closes against it.

The mouth parts-a pair of mandibles, two pairs of maxillæ, and three pairs of maxillipeds (fig. 369) - lie in front of the first pair of legs. 'The maxillipeds $(7,6,5)$ show a biramous condition, while the maxillæ $(4,3)$ retain considerable of the phyllopod character. In the mandibles (2) there is always a strong basal joint, which serves as a jaw, while this may bear additional joints, the palpus. Behind the mouth are a pair of scales, the paragnaths or metastoma, which are not appendages. The antennæ are usually distinguished as antenne (second pair) and antenmule (first pair, fig. 369). They have large basal portions, which in the antennulæ bear two many-jointed flagella, while the antennæ proper have but a single though usually much larger flagellum. On the basal joint of the antennulie is the otocyst (p. 362), while the green gland opens on the basal joint of the antennæ (fig. $400, g d$ ).

When the abdomen is not rudimentary (as in the crabs) the appendages of the sixth abdominal segment together with the telson form a strong caudal fin (fig. 400); the other appendages (fig. $369, I$ ) are small, biramous organs to which, in the female, the eggs are attached. In the female the first pair is reduced, but in the male (except in Palinuridx) this pair is well dereloped, curiously modified, and serves as a copulatory (intromittent) organ. The shape of these appendages and the openings of the genital ducts-on the base of the third walking foot of the female, the fifth in the male-serve at once to distinguish the sexes. Frequently also the males have the larger pincers.

The thickness of the integument prevents diffuse respiration and accounts for the numerous gills (fig. 398) which are attached to the bases of the maxillipeds and walking feet or to the sides of the body near them. (In the Thalassinidae the gills are on the abdominal appendages). These gills are not visible externally, for the carapace extends down on the sides of the body as a fold (branchiostegite) over them, thus enclosing them in a branchial chamber. A process of the second maxilla-the scaphognathite-plays in this hranchial chamber and pumps the water over the gills, the water flowing out near the mouth. All decapods can live some time out of water; they retain some water in the gill chamber, which keeps the gills in a moist condition. In some tropical crabs which live almost exclusively on land there is a true aerial respiration, the lining of the 
gill chamber being modified into a kind of lung traversed by numerous blood-vessels. In the palm crab (fig. 399) the gill chamber is divided into two portions, the upper part being pulmonary, the lower containing the reduced gills.

Correlated to this localized respiration is the nearly closed circulatory system (fig. 400, $A, B$ ). The heart ( $h$ ), a compact pentagonal organ, receives its blood from the pericardial sinus $(p c)$ through three pairs of ostia, and forces it out through five arteries to the capillary regions of the body. The venous blood collects in a large venous sinus at the base of the gills (i), passes thence through gills, and is returned by several branchial veins $(\imath b r)$ to the pericardium.

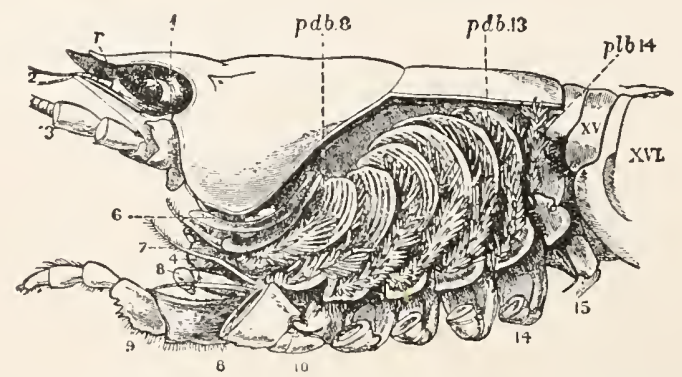

FIG. 398.--Gills of Astacus exposed by cutting away the branchiostegite. $p d b, p l b$, podo- and pleurobranchia of the corresponding segments; $r$, rostrum; I, stalked eyes; 2,3 , antennx; 4-6, mandibles and maxilla; $7-9$, maxillipeds; $10,1_{4}$, bases of thoracic eet; I 5, first pleopod.

The alimentary canal is straight and has only one conspicuous enlargement, the so-called stomach (fig. $400, A, m$ ), divided into two portions, an anterior (cardiac) sac, lined with chitinous folds and teeth and serving to chew the food and bearing in its walls the 'crab-stones,' masses of calcic carbonate stored up to harden the armor rapidly after the molt. The second (pyloric) portion of the stomach is guarded by hairs and serves as a strainer, allowing only food sufficiently comminuted to pass. The two liver lobes-voluminous masses of branched glandular tubes (l) open just behind the stomach.

The two antennal glands $(C, g d)$, each provided with a large urinary bladder $(b l)$, are dirty green in color, whence the name green glands often given them. The gonads (fig. 40I) lie close beneath the heart, those of the two sides being united behind, while their ducts remain separate. The structure of the nervous system is in part dependent upon that of the abdomen. In the Macrura (fig. 400, C) the ventral chain consists of six ganglia in the thorax, six in the abdomen, but in the Brachyura 
(fig. 402) these all flow together in a common mass, connected with the brain by two long œsophageal commissures.

The development of most decapods is interesting from the number of larval forms. As a rule a zoea (fig. 379 ) is hatched from the egg; this passes next into a Mysis stage (fig. 403) in which head, thorax, and abdomen are distinct, the thorax bearing biramous feet like those of schizopods-a proof of the origin of the simple feet from the biramous type. In the crabs (Brachyura) the Mysis stage is replaced by a Megalops (fig. 404), in which the abdomen is well developed, but the feet have lost their biramous character. In some prawns

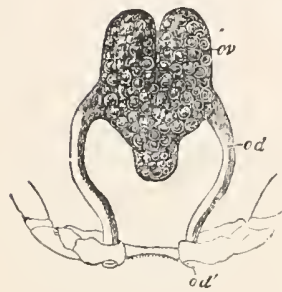

$A$

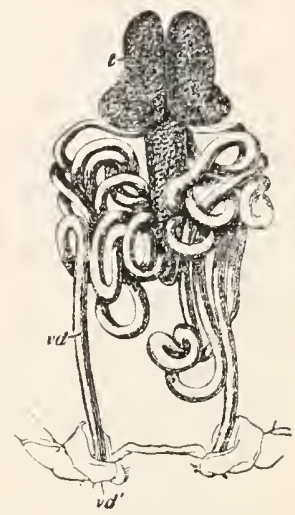

FIG. 40I.

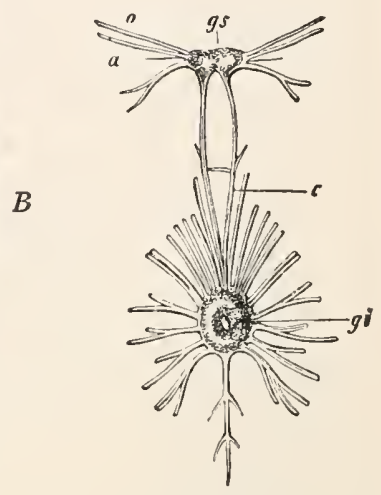

FIG. 402 .

Frg. 40I.-Reproductive organs of $(A)$ female and $(B)$ male crayfish (from Huxley). $o d$, oviduct; $o d^{\prime}$, its opening on I I th appendage; $o v$, ovary; $t$, testes; $v d$, vas deferens; $v d^{\prime}$, its opening on $\mathrm{I} 3$ th appendage.

FIG. 402.-Nervous system of crab, Carcinus (from (iegenbaur). a, antennal nerves; $c$, œsophageal commissures; gi, fused ventral chain perforated for sternal artery; $g s$, brain; $o$, optic nerve.

(Peneus) the series is rendered more complete by the appearance of a nauplius and a metanauplius with many appendages, before the zoeal stage. In the crayfish and many land crabs the metamorphosis has been lost, but the lobster leaves the egg in the Mysis stage. Differences may occur even in the same species; thus in the European Palamonetes varians the embryo, in the sea, leares the egg as a zoea; in fresh water in the Mysis-stage.

Sub Order I. MACRURA. Abdomen large; antennæ long; ventral nerve chain elongate; no megalops in development. CARIDEA. Body compressed; no sutures on carapace; feet weak, external maxillipeds pediform; a large scale on second antennæ. Peneide, weak exopodites. Peneus, ${ }^{*}$ Sicyonia.* PALÆMonid Crangonide, mandible simple. Crangon,* Sabinea.* ASTACOIDEA. Carapace crossed by a transverse groove. Astacide have well-developed chelæ. Cambarus* includes the crayfish of the eastern states; those of the Pacific coast and Europe belong to Astacus.* Homarus, * lobsters. Palinuride (Loricata), no chelæ, body with heavy armor; larvæ leaf-like (fig. 403). Palinurus, * spiny lobster. PAGURIDEA, hermit crabs; abdomen reduced, soft-skinned, and hidden in a snail shell which the animal carries about, which has resulted in a spiral abdomen. Some hermits carry sea anemones or hydroids on their shell, 
cases of symbiosis (p. I 58) Eupagurus, * Clibanarius.* Allied is Birgus, palm crab of the islands of the Indian Ocean; its respiratory organs referred to on p. 378. Sub Order II. BRACHYURA. Body depressed; abdomen rudi-

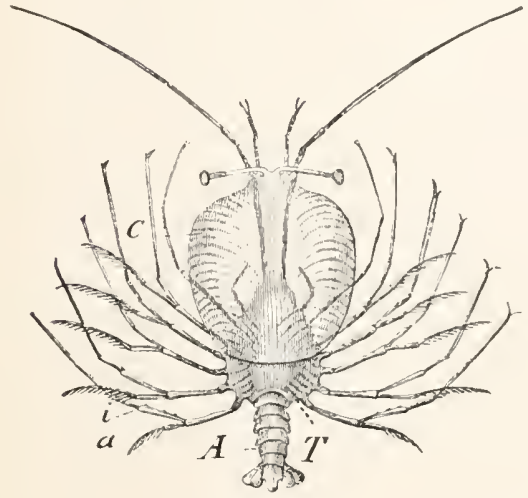

FIG. 403.

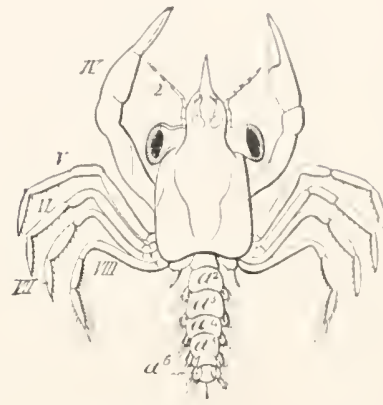

FIG. 404 .

Fig. 403.-Phyllosoma larva (Mysis-stage) of Palimurus (after (ierstäcker). $A$, abdomen; $C$, head; $T$, thorax; $a$ and $i$, exopodites and endopodites of thoracic feet.

FIG. 40.4.-Megalops larva of Portunus (from Lang, after Claus). 2, antenna; I I'$V I I I$, thoracic appendages; $a^{2}-^{6}$, abdominal somites $\left(a^{6}\right.$ is the seventh).

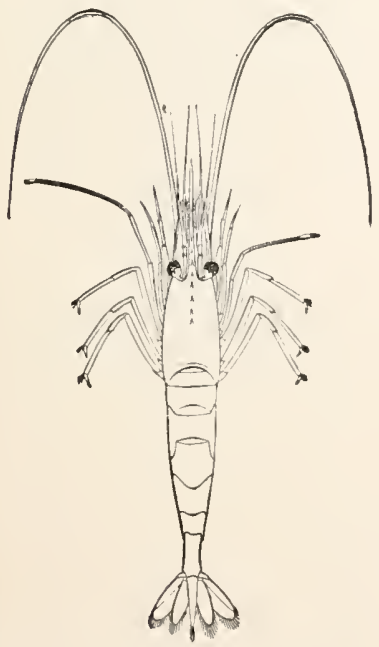

FIG. 405.-Pandulus montagui.*

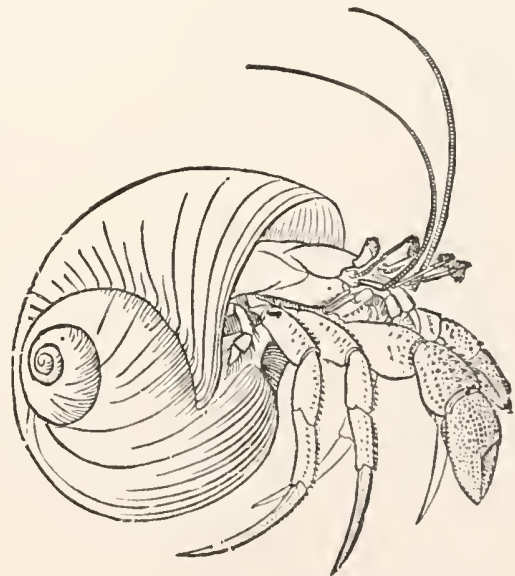

FIG. 106.- Eupagurus bernhardus, hermit crab (from Emerton).

mentary and folded in a groove under the cephalothorax; antenne short; never more than one pair of feet chelate; ventral nerve cord concentrated (fig. 402). Some inconspicuous groups like the PoRCELlanide, the HIPPID.E, and the 


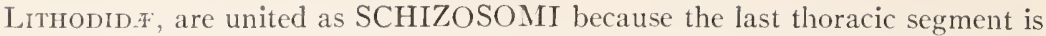
free from the carapace and its appendages are rudimentary. LEUCOSOIDEA (Oxystomata). Body oval or triangular, area of mouth parts triangular. Calappa, Hepatus.* OXYRHYNCHA (Maioidea). Cephalothorax triangular, narrowed in front; mouth area (as in the following tribes) quadrilateral.

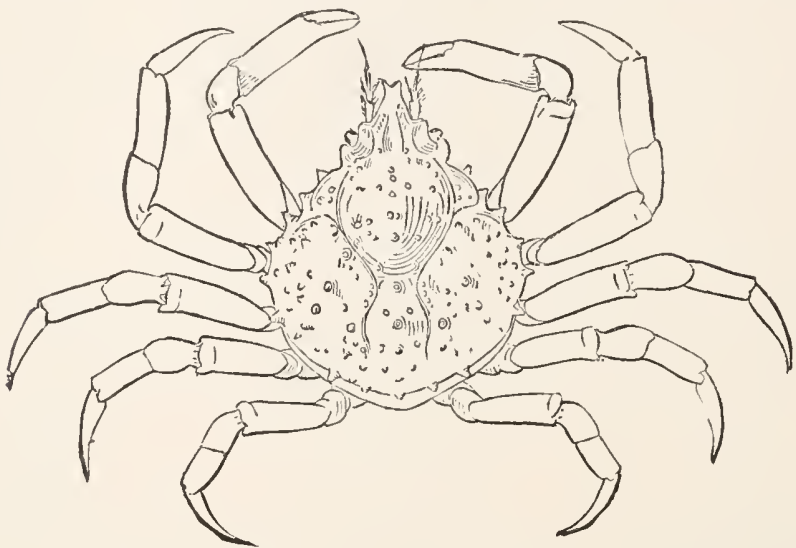

FIG. 407.-B, Libinia emarginata,* spider crab (from Emerton).

Mostly tropical. Myas, ${ }^{*}$ Libinia,* Pugettia,* spider crabs. CYCLOMETOPA. Body broader than long, arcuate in front. CANCRID $\approx$, with last pair of feet pointed. Cancer, * shore crab; Panopeus, * mud crab. PorTunid ${ }^{*}$, with last pair of feet flattened paddles. Neptumus hastatus, ${ }^{*}$ when thin-skinned after molting, is 'soft-shell crab.' CATOMETOPA. Front of carapace nearly straight; body from above nearly quadrilateral; Gelasimus, * fiddler crabs, Pinnotheres ostreum,* common in oysters; GECARCINID五 ( $U c a$, etc.), land crabs of the tropics, which only go to the sea at the reproductive season to lay their eggs.

\section{Order IV. Cumacea.}

Small marine forms with sessile eyes, three or four free thoracic somites; appendages biramous; a brood sac beneath the cephalothorax. Of interest because combining arthrostracan and thoracostracan features. Diastylis.*

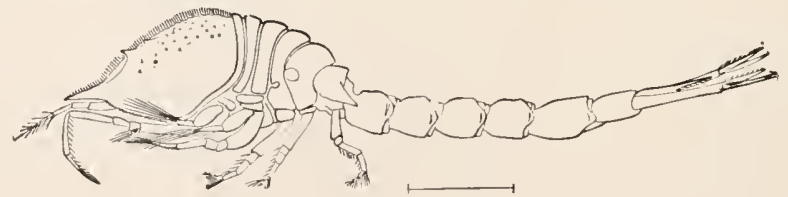

FIG. 403.-Diastylis quadrispinosus.*

\section{Order V. Syncarida.}

Especial interest also centres in Anaspides tasmanic (and a few other forms) from lakes in Tasmania, which unite schizopod and amphipod characters. They have the stalked eyes, caudal fin, and biramous feet of a schizopod; otocysts in the antennulæ like a decapod; but agree with the amphipods in shape 
of body and in free thoracic segments. The epipodial plates are paralleled elsewhere only in carboniferous species, with which these forms apjarently are closely allied.

\section{Legion III. Arthrostraca (Edriophthalmata, Tetradecapoda).}

Although the Arthrostracan head consists of six segments, it is very short. It bears six pairs of appendages, one of the normal thoracic pair being added to it as maxillipeds. Eyes, when present, are clusters of ocelli on the sides of the head. There are seven thoracic segments, the appendiages of which are walking feet without exopodites. The abdominal appendages, when present, are always biramous; the telson never bears appendages, and in the Amphipods is greatly reduced, sometimes being split nearly its whole length. The nervous system (figs. 78,409 ) is of the ladlder type. The alimentary canal is straight and has an anterior enlargement, the chewing stomach, behind which empty one or more pairs of long liver

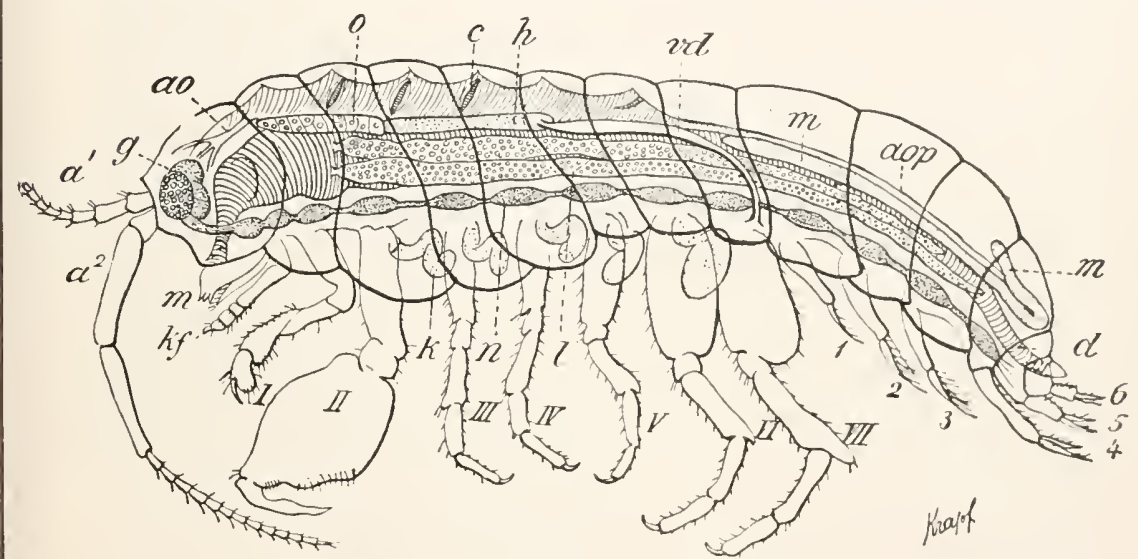

FIG. 409.-Male Orchestia cavimana (after Nebeski). $a^{\prime}, a^{2}$, antennx; ao, aop, anterior and posterior aortæ; $c$, heart; $d$, digestive tract; $g$, brain and eye; $h$, testes; $k$, gills; $k f$, maxilliped; $l$, liver; $m$, excretory organ and mandible; $n$, ventral nerve cord; $o$, rudimentary ovary; $v d$, vas deferens; $I-V I I$, thoracic feet; $1-3$, anterior, $4^{-6}$, posterior abdominal feet.

tubes, while in a few Amphipods a pair of excretory tubes ( $m$ ), the so-called Malpighian tubules, empty into the intestine near its end. Respiratory and circulatory systems vary so that they are best described in connection with two orders.

\section{Order I. Amphipoda.}

The Amphipods are almost exclusively aquatic, a few species living on the shore near high-tide mark. $\Lambda$ few live in fresh water (Gummarus, * Allorchestes*), the majority being marine. On land they move by a 
leaping motion, whence the common name, beach fleas. In swimming the abdomen is alternately bent against the breast and then forcibly straightened.

The body is usually strongly compressed from side to side. The

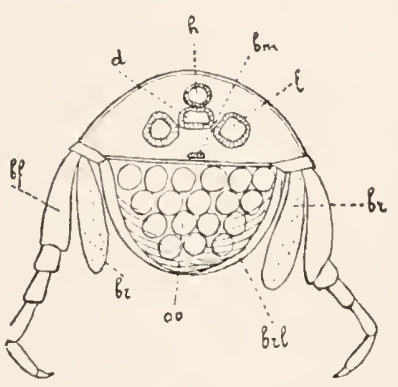

Fig. 410.-- Cross-section of Amphipod Corophizm, (from Lang, after Delage). bf, thoracic leg; bim, ventral nerve cord; $b r$, branchix: brl, brood lamella; $d$, intestine; $h$, heart; $l$, liver; or', eggs in brood chamber.

gills explains why the abdominal part of the heart is degenerate and only the anterior thoracic portion with three pairs of ostia persists.

Sub Order I. HYPERINA. Large head and eyes; strong prehensile feet. Live attached to other pelagic animals on which they feed; Hyperia, * Phronima.* Sub Order II. GAMMIARINA. Head much smaller; abdomen well developed; mostly free swimmers. Numerous species in the sea. Gammarus* in shallow water, some fluviatile; Orchestia* above tide marks. Chelura terebrans*

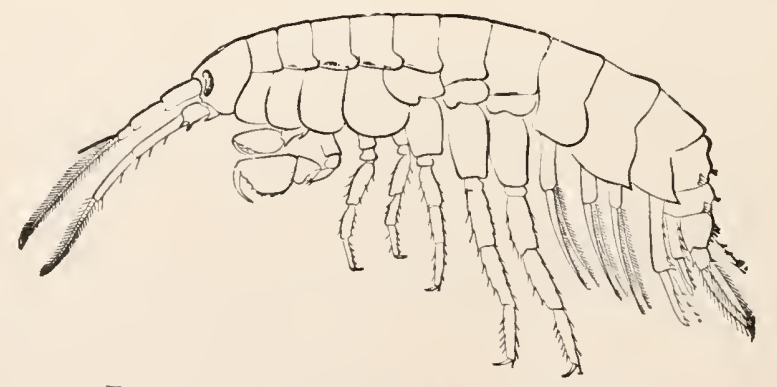

FIG. 4II.-Gammarus ornatus* (from Smith)

destroys submerged wood. Sub Order III. LÆMODIPODA. Parasitic or semi-parasitic; second thoracic somite is fused to head; appendages lacking from some thoracic segments, abdomen reduced. Caprella,* on hydroids. Cyamus ceti, parasitic on whales. 


\section{Order II. Isopoda.}

The Isopoda are readily distinguished from the Amphipoda by their depressed (horizontally flattened) ledies. The feet are adapted for creeping, and a brood pouch is formed as in the Amphipoda; gills are lacking on the thorax. In the abdomen, the somites of which exhilit a

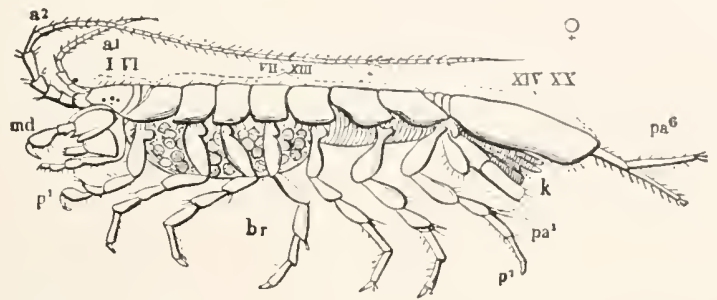

Fig. 4I2.-Asellus aquaticus (from Ludwig-I.eunis). $a^{2}, a^{2}$, antennx; $l$ r, brood pouch; $k$, pleoporla modified to gills; $m d$, mandibles; $p^{1-} p^{7}$, thoracic feet; $p a^{1-} p a^{6}$, abdominal feet (pleopoda); I-VI, head; VII-XIII, thoracic segments; XII-XI, abdominal segments partly fused.

great tendency to fusion, the sixth somite bears, in the walking forms, long forked appendages (fig. 4I2); in the swimming species (4I.4, C) they are flattened and, with the telson, make a swimming organ. The five anterior pairs of pleopoda are modified for respiration (Fig. +12, $k$ ), the endop-

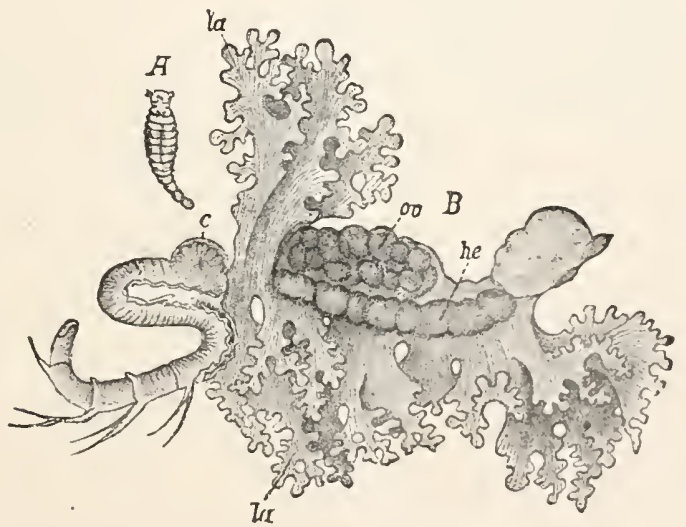

FIG. 4I3.-Entoniscus porcellance (from Gerstäcker, afier Müller). A, male; $B$, female; $C$, heart; he, liver; la brood lamella; or', ovary.

odites being thin-walled plates, while the exoporlites and the whole first pair serve as opercula or gill corers. As a result of this position of the gills the heart (usually with two pairs of ostia) is abdominal in position. 
In the terrestrial species the gills are adapted for breathing damp air. In Porcellio and Armadillidum the first or first and second opercula are permeated with a system of air tubes, which physiologically, though not morphologically, are comparable to the trachea of insects.

In the Isopoda the tendency to parasitism is greater than in the Amphipoda. Many swimming forms attach themselves to fishes and feed by boring with their modified mouth parts into the skin. The Bopyridæ live in the branchial chamber of shrimps. Cryptoniscus is a shapeless sac which attaches itself to the stalk of Sacculina (p. 374), and, after causing the death of this parasite, uses its network of 'roots' for its own nourishment. The Entoniscidæe (fig. 4I3) attack Decapoda and, pressing the skin before them, penetrate the interior. Their strange shape is largely due to the lobe-like brood lamellæ. They are usually hermaphroditic, but have besides complemental dwarf males (fig. 4 I $3, A$ ).
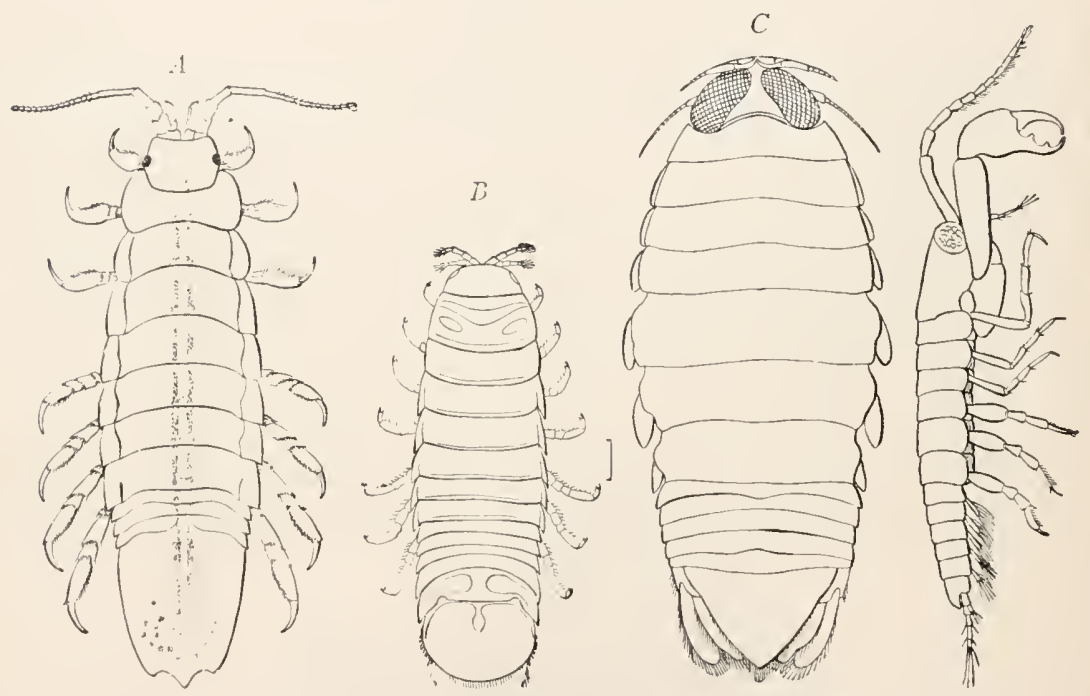

FIG. 4I4.-A, Idotea irrorata*; B, Limnoria lignorum*; C, Egga psora* ('salve bug'); $D$, Leptochela algicola $*$ (after Harger).

Sub Order I. ANISOPODA. Six free thoracic segments; heart thoracic; first thoracic foot (on head) chelate; abdomen with swimming feet; intermediate between Amphipoda and other Isopoda. Tanais, ${ }^{*}$ Leptochela* (fig. 4I 4). Sub Order II. EUISOPODA. Seven free thoracic segments. ONISCIDE; terrestrial, 'sow bugs'; Porcellio, * Oniscus, * Armadillidum, * 'pill bug.' AsELLIDE (fig. 4I2), fresh water. SPHÆROMID Spharoma.* Limnoria lignonm* (fig. 4I.4), gribble, destructive to submerged wood. Inoterd. Cacidotea, * Bopyrnde, parasitic on Caridea; body of female disc-like, asymmetrical, without eyes; Bopynus.* Crmothorde, parasitic on fishes or in their mouths. Cymothoa,* Ega, Cirolana.* Sub Order III. ENTONISCIDA, general features are described above. Entsmiscus. 


\section{Class II. Acerata.}

The animals comprising this group were formerly divided among the tracheates (p. 359) and the Crustacea, but although differing widely in respiration, the forms included are closely allied in structure and development and present many differences from both Crustacea and Insecta. The former views were based upon the view that trachee wherever found were homologous structures.

In the Acerata the body is usually divided into cephalothorax and abdomen, though in some cases (mites) the two regions become fused.

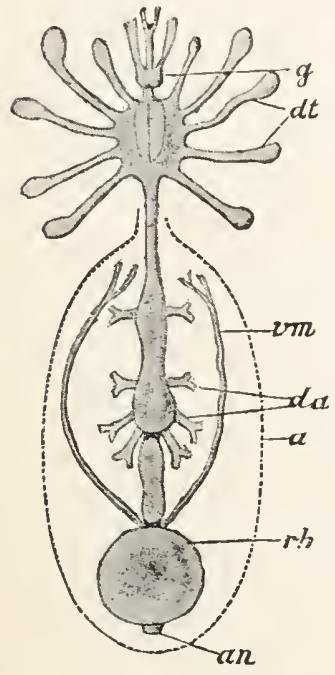

F1G. 4I5. The cephalothorax consists of six somites which always bear appendages, arranged in a circle around the mouth, the basal joints of one or more pairs frequently serving as jaws. None of these appendages are like antennix (whence

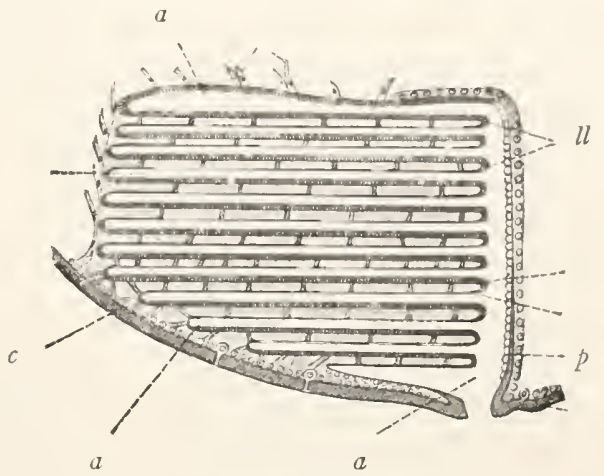

FiG, 4 I6.

FIG. 4I5-Digestive tract of Ctenida camentaria (from Lang, after Dugès). a, abdomen; $a n$, anus; $d a$, $d t$, diverticula ('liver') of midgut; $g$, brain; $v b$, rectal bladder (stercoral pocket); $v m$. excretory tubules.

FIG. 4I6.- Schematic long section of lung of spider (after Mac Cleod). a, air space; $c$, chitin layer; $l l$, lung leaves; $p$, posterior wall of lung sac.

the name of the group). The abdomen consists of a varying number of somites, all of which may be free, or may be fused. These abdominal somites bear appendages in the embryo, but in the adults (except the Xiphosura) these are usually lost or so modified that their existence is only recognized by a study of development.

The alimentary canal is straight, without marked enlargements, and lacks a chewing stomach. The liver is large and opens into the intestine by two or more pairs of ducts. The nervous system has some or all of its ventral ganglia arranged in a ring around the osophagus, and in many 
forms is enclosed in the ventral artery. Excretory organs, in the shape of nephridia, are frequently present and open to the exterior at the base of the second or the fifth pair of appendages. Malpighian tubes may occur, but these, unlike those of other tracheates, are entodermal in origin and hence not homologous with them

The respiratory organs are either gills, lungs or tracheæ. The gills are borne on some of the abdominal appendages. The lungs are sacs on the anterior abdominal somites opening by narrow slits (fig. $42 \mathrm{I}$ ) to the exterior. The anterior wall of each lung sac is made up of thin plates arranged like the leaves of a book (fig. 4 I6), and embryology shows that these lung books are gill books drawn into the ventral surface of the abdomen. The trachere replace one pair of the lungs and are apparently homologous with them. They penetrate all parts of the body. The reproductive openings are on the basal somite of the abdomen. The spermatozoa are motile. The development is direct, there being no metamorphosis.

\section{Sub Class I. Gigantosiraca.}

Marine forms with gills cn the 2-6 abdominal appendages; bases of five pairs of cephalothoraciac feet masticatory; a pair of median ocelli and a pair of compound eyes on the cephalothorax.

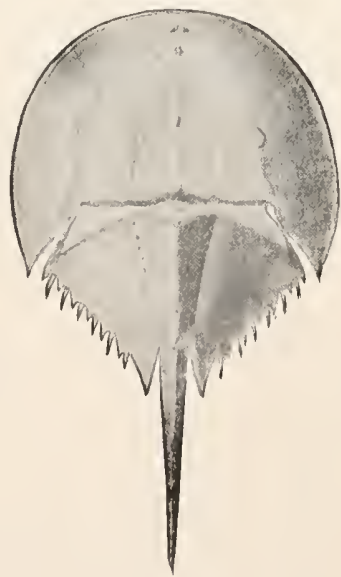

FIG. 417 .

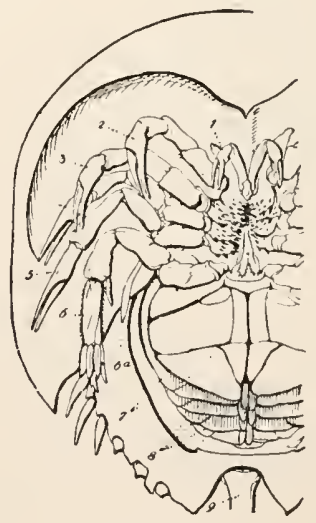

FIG. 4 I 8 .

FIG. 4I7.-Limulus polyphemus,* horseshoe crab (orig.).

FIG. 4I8.- Ventral surface of Limulus moluccanus (from Ludwig-Leunis). I, chelicere; $2-5$, walking feet; 6 , pushing foot; $6 a$, flabellum; 7 , genital operculum: 8 , gills (there should be five); 9 , base of telson.

\section{Order I. Xiphosura.}

Cephalothorax large; abdomen with joints fused, terminated by a long spiniform telson. Limulus polyphemus, * king crab or horseshoe crab. Other species on eastern shore of eastern continent. 


\section{Order II. Eurypterida.}

Extinct Silurian and Devonian forms with small cephalothorax and large twelve-jointed abdomen; intermediate between xiphosures and scorpions. Eurypterus; Pterygotus, some species seven feet long.

\section{Sub Class II. Arachnida.}

Under this name are included a number of orders of greater or less extent which can be arranged around the spiclers, or Aranea, as a centre. There is considerable modification of form, and the following account applies only to the more typical groups. In these the cephalothorax and abdomen are separated by a distinct line, and since the abdominal appendages almost entirely disappear in the adult, the number of abdominal somites can only be ascertained where their boundaries are evident. The number varies between six in the phalangids and thirteen in the scorpions.

The cephalothorax is, except in the Solpugida, a single piece which bears six pairs of appendages; the four posterior pairs, each typically seven jointed, are locomotor, so that eight legs are as characteristic for an arachnid as ten for a decapod or six for a hexapod. The first pair of appendages, the chelicere (fig. 4I9), are preoral, the second, or pedipalpi, beside the mouth. The chelicera are short and consist of two or three joints, the terminal joint either folding back upon the other or, pincer-like, meeting an opposable thumb. In the spiders the last joint or claw is forced jnto the prey, introducing poison from a sac in the basal joint. The pedipalpi are elon-

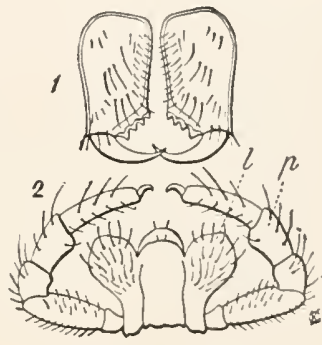

FIG. 419.-Mouth parts of Epeira. I, chelicera: 2 , perlipalpi; $p$, palpus; $l$, basal plate. gate, leg-like, their basal joints often forming a lip, the other joints forming the palpus, which may end with a claw or a pincer.

The question has often been discussed as to whether the chelicerx are the homologues of the antennæ of other arthropods. 'I he embryological evidence is in favor of their equivalence to the second antenna of the crustacea, and to the mandibles of insects. In the cephalothorax is a fibrous entosternite to which most of the muscles are attached, a structure paralleled in Limulus.

Since the Arachnida usually suck their food, the oesophagus is frequently widened to a sucking stomach, behind which comes the true stomach, with which, as well as with the intestine, a number of so-called liver tubes may arise (fig. $4 \mathrm{I} 5, d a, d t$ ). These may be restricted to the abdomen, as in the scorpions. The hinder part of the intestine is often 
enlarged into a stercoral pocket, just in front of which the excretory tubules empty. These resemble the Malpighian tubes of insects in function, but differ in being entodermal in origin. Besides there are also coxal glands (modified nephridia), of which only one pair comes to development, and this may lose its external opening on the base of the first or third leg.

The œesophagus is always closely surrounded by a nerve ring composed of brain above and of part of the ventral chain on the sides and below, the thoracic and more or fewer of the abdominal ganglia entering into its composition (fig. $370, D$ ). Of sense organs, besides tactile hairs, only the eyes (fig. $37 \mathrm{I}$ ), 2-1 2 in number, are well known. The large number of rods in the retina makes it probable that these eyes see well. Hearing is well developed, but it is uncertain whether certain hairs on the legs and palpi are the auditory organs. The function of the lyriform organs, which occur in the skin of body and legs in several groups, is unknown.

The respiratory organs already alluded to (p. 388) have their spiracles, always few in number, on the anterior ventral part of the abdomen and,

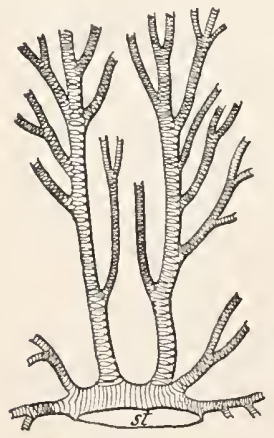

FIG. 420.-Beginning of paired trachere of Anyphana accentuata (after Bertkau). st, unpaired spiracle. it is stated, sometimes on the cephalothorax. The internal organs are the lungs and the trachex. A lung is a rounded sac just inside the spiracle and consists of numerous leaves on the anterior wall of the lung sac. Each leaf contains a blood space in its interior, while between the leaves are flattened spaces into which the air enters (fig. 4I6). The trachex are branched tubes arising from the abdominal spiracles and penetrating the abdomen (fig. 420). These are lined with chitin, and to strengthen them without undue thickness this lining is thrown into folds, usually arranged in a spiral. In the scorpions and tetrapneumonous Araneina only lungs occur. In other spiders one pair of lungs is replaced by trachex, while in most other arachnids only trachee occur. (The smaller mites and parasites lack specialized respiratory organs and circulatory organsas well.) These facts show that lungs and trachex are morphologically equivalent. The localization of respiration in the abdomen has resulted in having the heart in the same region. It is noticeable that, as the trachex are developerl, the circulatory vessels are reduced. In the scorpions, which have only lungs, the circulation is most nearly complete. 
In development the arachnidan trachere arise in connection with the abdominal appendages, as do the lungs. (In the Solpugide and some mites cephalothoracic trachea occur.) This shows that the arachnidan trachea are entirely different in origin from the trachea of insects.

The gonads (only the Tardigrades are hermaphroditic) are aldominal in position and open by paired ducts (sometimes with a single mouth) on the first abdominal somite. In most cases the animals are oviparous, but the scorpions and many mites bear living young. In many instances the mothers care for their eggs and young, the scorpions carrying their families on their bodies. Only rarely is there a metamorphosis, and then in the aberrant forms like the Linguatulida and Acarina, where the young have but two or three pairs of appendages, accuiring the others later.

\section{Legion I. Arthrogastrida.}

Arachnida in which the abdominal somites are distinct.

\section{Order I. Scorpionida.}

The scorpions bear a superficial resemblance to crayfish and for a long time were associated with them, since (figs. 366, $42 \mathrm{I}$ ) they have four pairs of walking feet $\left(3^{-6}\right)$, while the pedipalpi (2) are large and bear pincers.

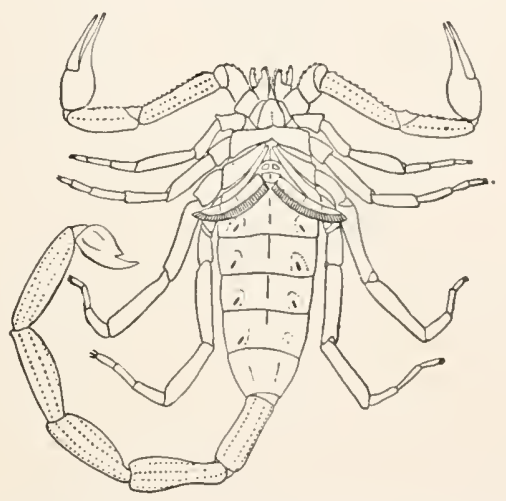

FIG. 421.-Under surface of scorpion, showing the combs and the outlines of the lung sacs with their spiracles (orig.).

The chelicera are also chelate. The pedipalpi and the two anterior pairs of legs have the basal joint expanded for chewing. The peculiarities of the abdomen mark the group off from all other arachnids. It consists of seven broader somites attached by their whole width to the cephalothorax and six narrower somites behind, forming a postabdomen. 'The last somite is produced into a sharp spine and contains two large poison 
glands. It is the 'sting' of the animal which causes painful wounds in man, and in the large tropical species is, perhaps, fatal. Usually scorpions feed upon insects, which they seize with the pincers and kill with the sting. On the ventral surface of the second abdominal somite (fig. 42 I) are a pair of appendages, the combs or pectines, rods with teeth on one side, of uncertain function. They are clearly appendages with modified gill leaves, and from their nearness to the sexual opening and their rich nerve supply are supposed to be stimulating organs in copulation. The next four segments bear spiracles which lead to four pairs of lung sacs. The

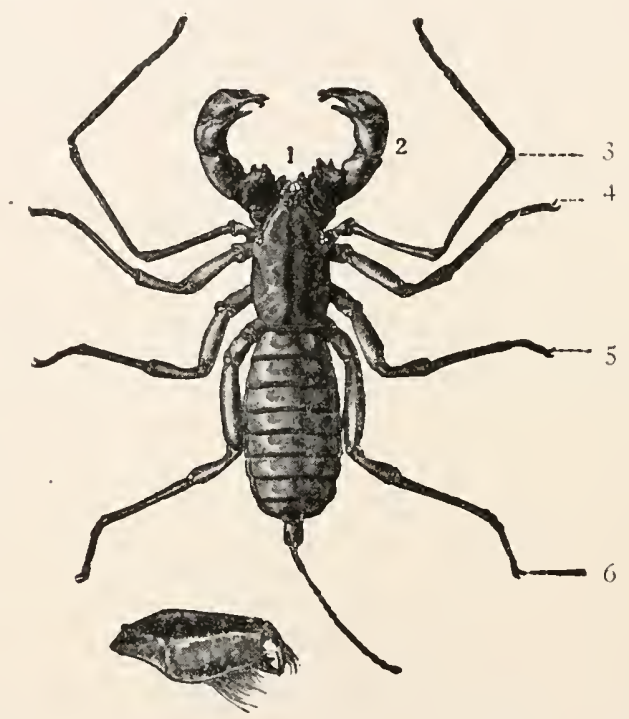

FIc. +22.-Thelyphonus caudatus. I, chelicera; 2, pedipalpi; 3, flagellate third leg; $4^{-6}$, walking feet. Below, chelicera enlarged.

heart is abdominal and the liver diverticula are confined to the same region. The large number of abdominal ganglia distinct from the œesophageal ring is also characteristic. From three to six pairs of eyes occur.

The scorpions are inhabitants of warm regions, ranging north with us to the Carolinas and Nebraska. Buthus, ${ }^{*}$ Centrurus.*

\section{Order II. Phrynoidea (Pedipalpi, Thelyphonida).}

The thoracic segments are fused, and of the appendages only the last three are walking feet, the third pair having the last joint (tarsus) developed into a long many-jointed tactile flagellum. The chelicere are strong and spined, but end in a pincer in some species. The chelicere are also clawed and are possibly poison organs, since the bite of these animals is feared. The abdomen consist 
of eleven or twelve somites and contains two pairs of lungs. There are eight eyes-two large ones in the middle of the cephalothorax, and three small ones on either side. 'The species are tropical. Phrynus, simple alodomen; Thelyphomus* (fig. 422), short postabdomen which bears a long, many-jointed thread.

\section{Order III. Microthelyphonida.}

Small animals only known from Texas, Sicily, Paraguay, and Siam. They have a general resemblance to a scorpion; the chelicerae are three-jointerl and chelate, the pedipalpi simple; neither these nor any of the legs having chewing

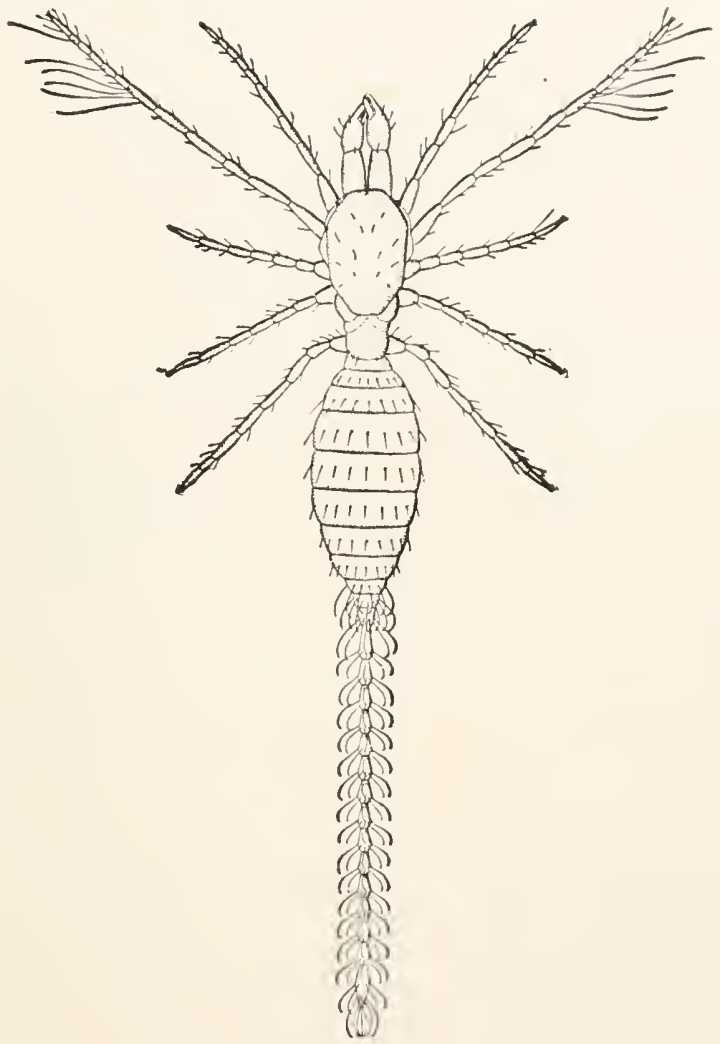

FIG. 423.-Kinenia wheleri (from IIheeler).

lamellæ. The head is distinct from two 'thoracic segments,' the abdomen is eleven-jointed and is terminated by a long many-jointed caudal flagellum. Lung sacs, which are true appendages without lung leaves, occur on abdominal segments four to six, and are eversible. The ovary is unjaired, the testes paired. There is a circumcesophageal nerve ring and a single ablominal ganglion. No Malpighian tubes occur. Kenenia.*

\section{Order IV. Solpugida (Solifugæ).}

In these the cephalothorax is broken up into a head bearing the chelicere, pedipalpi, and the first pair of legs; and three posterior free somites, each bear- 
ing a pair of legs, thus giving these forms a certain resemblance to the Hexapoda (infra). The cheliceræ are strong and chelate, the pedipalpi are simple and are used in walking, while the first pair of legs are tactile. Respiration occurs by four pairs of trachex, the first of which opens between the first and second 'thoracic' somites, a condition which deserves embryological investigation. 'The abdomen consists of nine or ten somites, and the head bears two ocelli. As the name implies, the Solpugidæe are nocturnal, living by day in holes in the sand and searching for their prey at night. In the Old IIorld they are reputed as poisonous, but no poison glands occur. Solpuga, * Galeodes, *Datames* (fig. 424).

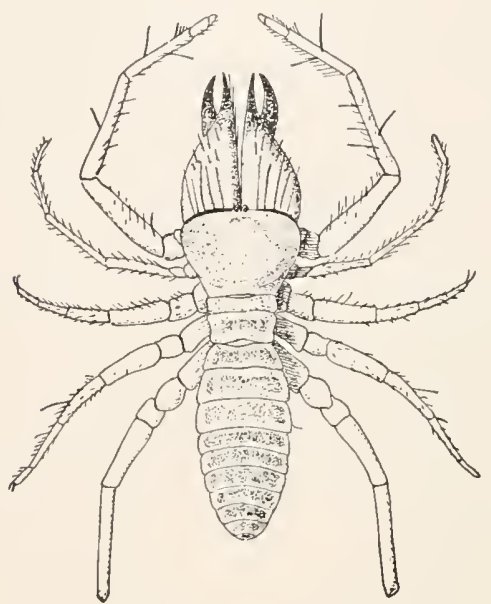

F1G. 424 .

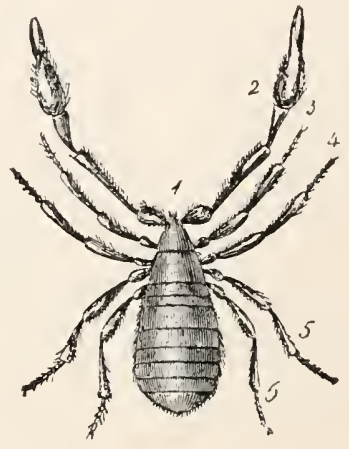

FIG. 425 .

FIG. 424.-Datames formidibilis* (after Putnam). FIG. 425.-Chelifer braz'aisi (from Schmarda).

I, chelicere; 2, pedipalpi.

\section{Order V. Pseudoscorpii.}

These small flattened forms resemble the true scorpions in the chelate cheliceræ and pedipalpi (fig. 425), and in the abdomen joined by its whole breadth to the thorax. They differ in the lack of postabdomen and sting. They breathe by tracher; have from two to four ocelli, and spinning glands opening on the second abdominal somite. These animals, 2-3 $\mathrm{mm}$. long, live in moss, etc., and among dusty books, feeding on mites and minute insects. Chelifer, * Obisium, * Chernes.*

\section{Order VI. Phalangida.}

The abdomen in the harvestman, or 'daddy long legs,' is less evidently segmented than in the forms already mentioned, nor is it sharply distinct from the cephalothorax. The small body bears four pairs of exceedingly long legs; the cheliceræ are drawn out in long horny processes; the pedipalpi are tactile organs as in the true spiders. The males possess a long penis, and the females a long ovipositor. 'They have two or four ocelli and breathe by tracheæ. 'These largely nocturnal animals are predaceous, feeding upon small mites. In structure they form in some ways an approach to the Acarina. Phalangium,* Liobunum.** 


\section{Legion II. Spharogastrida.}

Arachnida with the abdominal somites fused so that no traces of segmentation remain.

\section{Order I. Araneina.}

In the spiders the soft-skinned body is divided by a deep constriction into cephalothorax and abdomen (fig. 426). 'The four pairs of legs are adapted for springing or for walking, the hinder pair being also accessory to the spinning. It bears a comb-like claw with which several threads are combined into a stronger cable. 'The chelicera bears a sharp claw (fig. 419), traversed by the duct of the poison gland with which the prey is killed, although but few (species of Latrodectes, fig. 427 , the tarantula, and the bird spiders, Mygalidæ) can injure man. The pedipalpi are used as feeling organs and with the basal maxillary process to comminute the food. In the male the pedipalpi have the terminal joint swollen to a pearshaped structure (fig. 428) by which

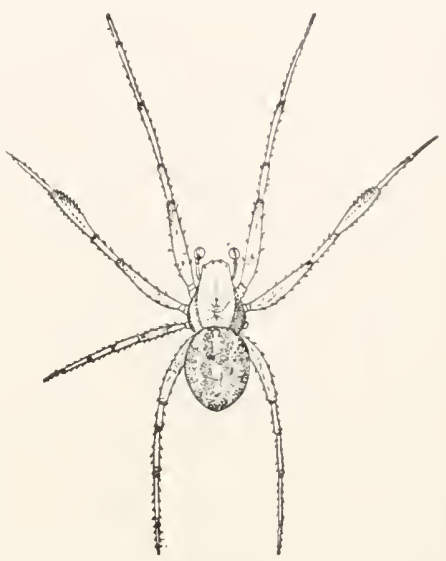

FIG. 426.-Epeira insularis, * roundweb spider (after Emertc 1 ). the sexes are easily distinguished. This is used to convey the spermatozoa to the female, a rather dangerous process, as the male is apt to be killed by the much stronger mate.

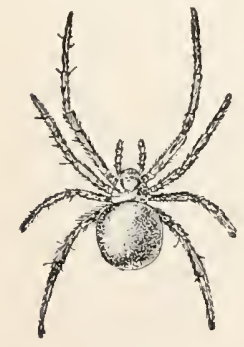

FIG. 427 .

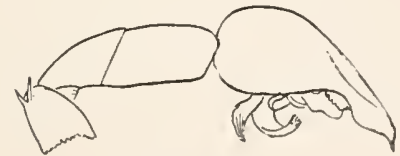

FIG. 423.

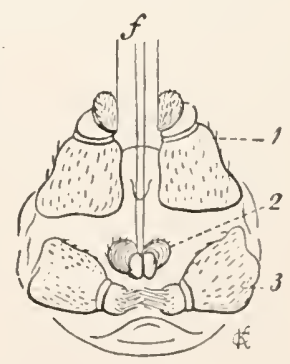

FIG. 429.

FIG. 427.-Latrodectes mactans,* poison spider (after Marx).

Fig. 428.- Pedipalp of Pardosa uncata (after Emerton).

FIG. 429.-Spinnerets of Epeira diadema (after Warburton). I, 2, 3, first, second, and third spinnerets; $f$, threads.

At the hinder end of the abdomen, just in front of the anus, are the spinnerets, which are reduced appendages, as is shown by their paired ar- 
rangement and their jointing (fig. 429), as well as by development. They are truncate and have at the tip a 'spinning field' from which numerous minute, two-jointed spinning tubes, resembling hairs, arise, each of which is the end of a duct of a silk gland. Different kinds of glands, producing silk for different purposes, occur. The number of spinnerets varies between two and three pairs, and in front of these may be an unpaired spinning region, the cribrellum, so that hundreds or even thousands (Epeiridx) of glands may be present.

The secretion of the glands hardens in contact with the air, and the single threads are united by the combs of the hinder feet into a larger cord which can be regulated in size according to the number of glands which are active. Yet the largest cord is finer than the finest silkworm silk, hence it is often used for the cross-hairs of telescopes. The spider silk has many uses; it is used to line the nests, to form cocoons for the eggs, as a means of descent from high places, and to form the well-known webs.

The nervous system consists of a brain and a circumosophageal ring, and, in the Mygalidx, a single abdominal ganglion. The arrangement of the six or eight ocelli and the relative lengths of the legs are matters of systematic importance. Trvo pairs of respiratory organs occur. In the Tetrapneumones there are two pairs of lungs, but in the Dipneumones the hinder pair are replaced by trachex, which may open by separate spiracles (Tetrasticta) or by a common opening (Tristicta, fig. 420). Rarely both lungs are replaced by trachea.

Sub Order I. TETRAPNEUMONES. Four lungs, four spinnents and eight eyes in two rows. MYGaLiD\&, large forms which spring upon their prey, capturing even small birds and mice. To I/ ygale* belong the spiders (erroneously called tarantulas) which occur in banana bunches, and the trapdoor spiders, Cteniza,* of the southwest, which excarate burrows in the soil, line them with silk, and close them with a hinged lid. Atypus.* Sub Order II. DIPNEUMONES. One pair of lungs, one of trachea; at most six spinnerets. Here belong most of the native and numerous tropical species. Some (VAGABUND $Æ$ ) use their webs only to line the nests and enclose the eggs, which are either hidden away or carried about attached to the body; they spring upon $\mathrm{r} r$ chase their prey. SEDENTARIA are the web builders, their webs varying widely in structure. Of the first group the SALtigrada include forms which jump upon their prey (Attus,* Phidiptus,* Habrocentrum*), and the Citigrada (Lycosa, ${ }^{*}$ Dolomedes, $*$ Trochosa*), which run their prey down. Among these is the true Tarantula, T. apulice of Italy, whose bite was once believed to cause a frenzy only to be cured by peculiar music ('Tarantella'). The Sedentaria are divided according to the web-building habits. The OrBITELARIE or orb weavers (Epeira, Argiope*) form vertical webs which in many instances are complete circles. The RetITElaria (Theridium,* Erigone*) build irregular webs. Latrodectes,* reputed poisonous to man (fig. 427 ). The Tunitelaries build horizontal webs with a tube to the margin in which they lay in wait for insects.

\section{Order II. Acarina.}

The mites, partly from parasitism, partly from other conditions of life, have become, in some instances, considerably modified. With the fusion of cephalothorax and abdomen the last traces of segmentation in the body are lost. Yet they retain the six pairs of appendages - four pairs of legs, which at once distinguish them from the parasitic hexapods; and two pairs of mouth parts, modified 
into a sucking beak. This consists of a tube formed by the basal joints of the pedipalpi, in which the chelicere, either chelate, clawed, or stylet-like, play Since the mites are small and half or wholly parasitic, they are much simplificel in structure. Frequently heart and trachea are lacking. The larva as it escapes from the egg lacks the last pair of legs and then closely resembles certain imperfectly segmented parasitic insects like the lice.

The red mites (TROMBIDIDE) and water mites, HY'DRACHNIDE (Hydrachna, * Ata $\left.x^{*}\right)$, are free-living as adults, but parasitic as young. The IxoDiD.Eor ticks (Ixodes*), attack man and other mimmals, burrowing beneath the skin, sucking

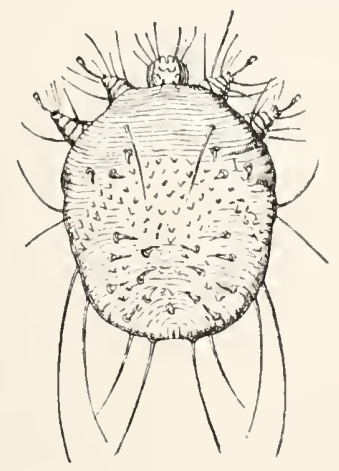

FIG. 430 .

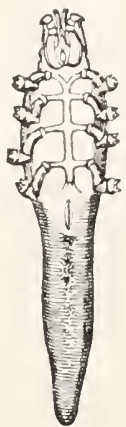

FIG 43 I.

FIG. 430.-Sarcoptes scabei, female itch mite (after Leuckart).

FIG. 431.-Demodex folliculorum, follicle mite (from Ludwig-Leunis).

the blood. Their relation to disease is referred to on p. I9o. The much smaller males are attached to the females and take no food. Argas persicus, of eastern lands, with habits like a hedbug, is poisonous. Gamasid on beetles, Dermanyssus* on bats. The ACARID E include permanent parasites like Sarcoptes scabei* (fig. 430), the cause of the 'itch,' and closely allied cheese mite The follicle mite, Demodex folliculorm,* lives in the sebaceous glands of various mammals, including man (fig. 43I).

\section{Order III. Linguatulida.}

Elongate mites like Demodex lead to the Linguatulida, which as adults live in the frontal sinuses of carnivorous mammals, as encysted young in the liver of herbivorous forms, especially rodents. 'The body is long, flattened and ringed, and somewhat tapeworm-like (fig. I I3). The adults have the mouth at the base of a chitinous capsule, and on either side are two hooks regarded as the claws of the first and second legs. Inside the body is a spacious cavity traversed by the alimentary canal which is without appendages. 'The nervous system is largely a circumcesophageal ring; the sexual organs are very complicated, the males having the openings in front, the females at the hinder end. 'The presence of these parasites causes a profuse catarrh, and the eggs pass out with the mucus. Falling on regetation, these are liable to be eaten by various animals. The larva (fig. 432) have a boring apparatus in front and two pairs of legs, the latter lost in the metamorphosis except for the hooks. It is by no means certain that these are degenerate arachnids. Pentastomum.

Usually associated with the Arachnida are two other groups of very doubtful position, which until more definite knowledge is obtained, may remain near them. 


\section{Tardigrada.}

These are minute fresh-water forms, known to microscopists as 'water bears' (fig. 433), which owe their name to their slow motions. They have four pairs of short, hooked legs, their sole Arachnidan character. The genital ducts empty

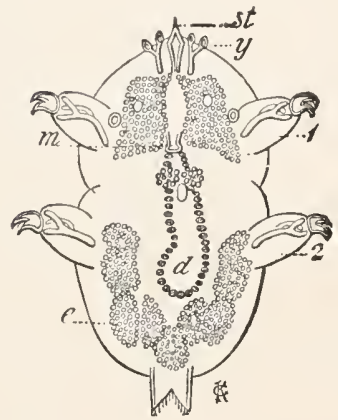

FIG. 432 .

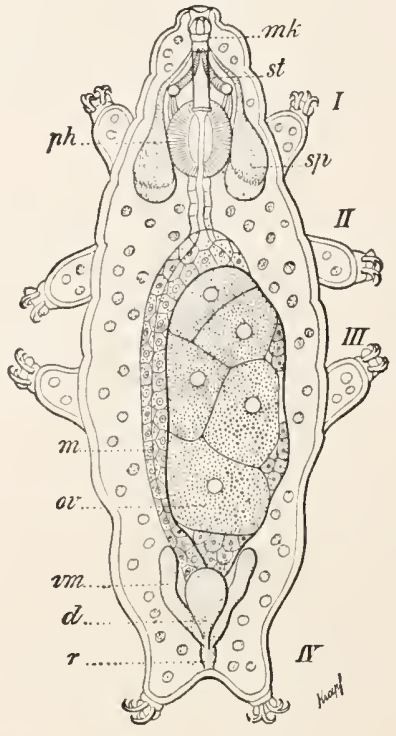

FIG. 433 .

FIG. 432.-Larva of Pentastomum proboscideum (after Stiles). d, stomach; $e$, gland cells; $m$, mouth; st, stylet; $y$, posterior larval hooks; I, 2, legs

FIG. 433.-Macrobiotus hufelandi, water bear (aiter drawings by Greef and Plate). $I-I V$, legs; $d$, accessory glands; $m$, stomach; $m k$, mouth capsule; $o$, ovary; $s p$, salivary glands; st, stylets; $v m$, excretory tubules; blood cells in the body.

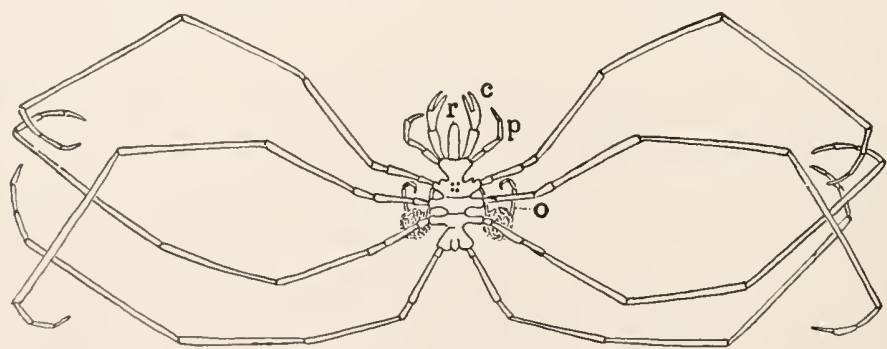

FIG. 434.-Nymphon stræmii* (orig.). $c$, cheliceræ; $o$, ovigerous legs; $p$, pedipalpi; $r$, rostrum.

into the rectum; the nervous system has four ventral ganglia; heart and respiratory organs are lacking. In development they are remarkable for the large colomic pouches. In the feet are glands recalling nephridia in their history. It is possible that these animals are to be placed among the Coelhelminthes. Macrobiotus.* 


\section{Pycnogonida (Pantopoda).}

These marine animals have a cylindrical body, with a tubular proboscis in front and an abdominal appendage behind, and four pairs of very long legs. In front of the legs is a pair of small chelate appendages and usually a pair more like pedipalpi. In the male there is an additional pair of 'origerous legs' to which the eggs are attached after being deposited by the female, thus giring a total of seven appendages, a number not reached in any arachnid. Diverticula of the stomach extend into the legs; a heart is present, but respiratory organs are lacking. The Pycnogonids, which creep slowly over seaweeds and hydroids, may be (I) a distinct group of arthropola, or (2) modified arachnids, or (3), and less probable, Crustacea. Nymphon, * Phoxichilidum, ${ }^{*}$ Colossendcis.*

\section{Class III. Malacopoda (Protracheata).}

These forms, including only a single family PERIPATID strange mixture of annelid and arthropodan (or 'tracheate') characters, so that they are usually regarded as representatives of the stock, early separated from the annelids, from which the Insecta have descended.

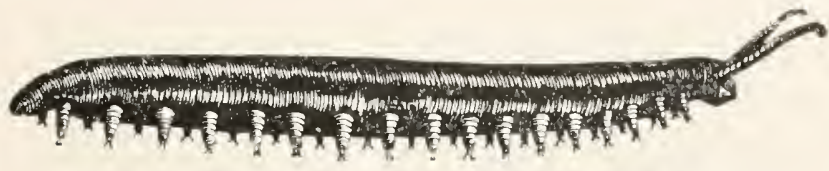

Fig. 435.-Peripatus capensis (from Balfour, after Moseley).

They recall the annelids by the nephridia, which begin by a closed vesicle (reduced coelom), pursue a short course, and expand into a urinary bladder before opening at the bases of the legs (fig. 436, so). On the other hand, they possess trachex, long unbranched tubes which arise in numbers from the spiracles, which are irregularly distributed in each somite $(t r)$.

Each segment of the soft-skinned body, which shows no external ringing, bears legs, each terminated by claws. These legs resemble the annelidan parapodia in not being jointed and not sharply separated from the trunk. The head is provided with three pairs of appendages: a pair of ringed antennx, a pair of mandibles, which lie in the oral carity, and a pair of mouth papillæe, at the tips of which are the openings of the slime glands, the sticky secretion of which is squirted out and serves to capture insects $(s d)$.

The nervous system consists of a pair of cerebral ganglia $(o g)$, supplying the antennx and a pair of very primitive eyes; and a pair of ventral cords $(b m)$, swollen slightly in each segment, which connect dorsal to the anus and are connected in the trunk by numerous non-segmental commissures. The muscles are of the smooth variety. 
The description may be completed by saying that the straight alimentary canal ( $p$ and $d$ ) bears only salivary glands $(s p)$; that it is accompanied throughout by a dorsal heart; that the gonads (the sexes are separate) open just in front of the anus $(g o)$, their ducts being modified nephridia. The animals are viviparous, live in decaying wood, hide by day and hunt their prey at night. The several species have a wide but discontinuous distribution (South America, Cape of (rood Hope, New Zealand, etc.), an indication of great antiquity. Recently the forms have been divided into several genera, Peripatus, Peripatopsis, Opisthopatus, etc.

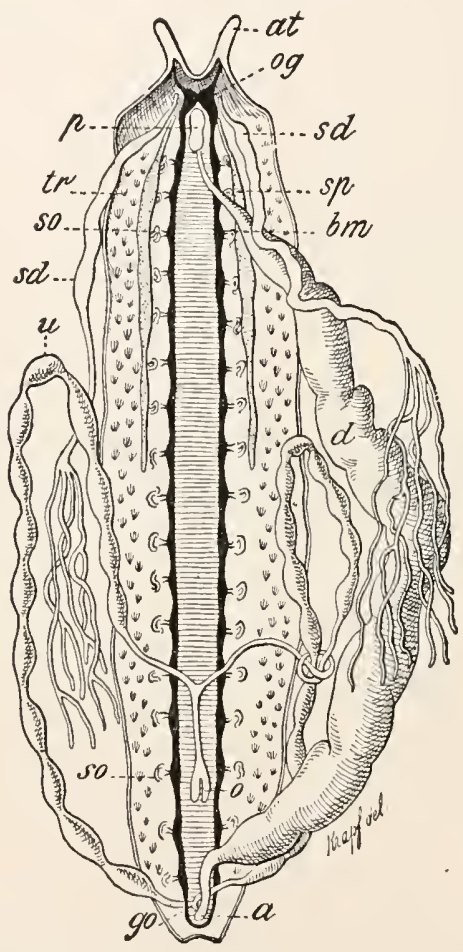

Fig. 436.-Anatomy of female Peripatus opened dorsally (from figures of Moseley and Balfour). $a$, anus; $a t$, antennæ; $b m$, ventral nerve cords; $d$, digestive tract: $g o$, genital opening; $o$, ovary; $o g$, brain; $p$, pharynx; $s d$, slime gland; $s o$, nephridia; $s p$, salivary gland; $t r$, trachex; $u$, uterus.

\section{Class IV. Insecta.}

The Insecta is a distinct group marked off from all other arthropods by several important characters. The appendages show no signs of a schizopodal condition. The head is always a distinct region, bearing a single pair of antenne, a pair of mandibles, and two pairs of maxillæ, the posterior pair often being fused into a lower lip or labium. 
The respiratory organs are trachea (figs. 437,438 ) which resemble the trachea of man only in that they are tubes filled with air, and kept from collapse by firm walls. 'They open to the exterior by openings (spiracles, stigmata) on the sides of the body. They are inpushings of the skin and consequently have the same structure, an epithelium and an

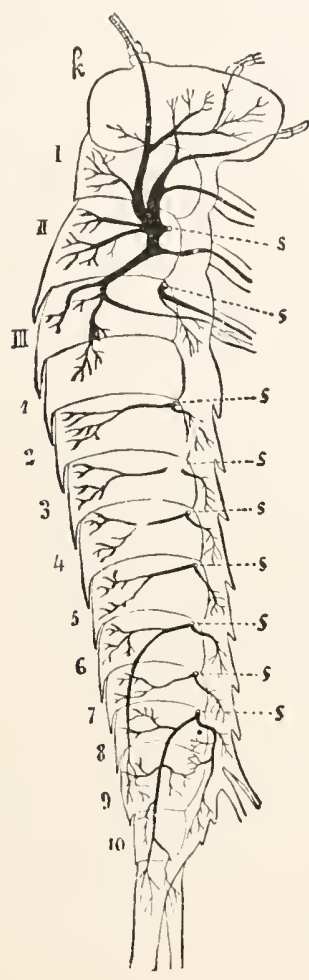

FIG. 437 . outer chitinous layer. 'The latter lines the lumen of the tubes, and since it must be thin to permit the passage of gases (oxygen, carbon dioxide), and at the same time firm, to keep the tubes open, it is thrown into folds which usually pursue a spiral course. The turns of the spiral are so close that it gives the tulses a

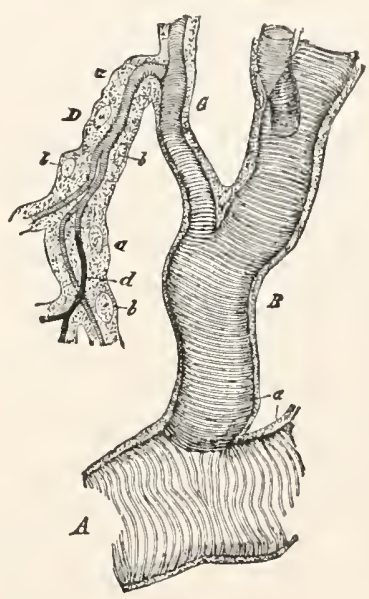

FIG. 438 .

FIG. 437-Tracheal system of Machilis (from Lang, after Oudemans). $k$, head; $I-I I I$, thoracic somites; $s$, spiracles; I-IO, abdominal somites.

FIG. 438.-Portion of trachea of caterpillar (from Gegenbaur). A, main trunk; $B, C, D$, branches; $a$, epithelium with nuclei, $b ; d$, air in tracheal tube.

ringed appearance (fig. 438). Inside the spiracles the trachee branch repeatedly until they end in fine tracheal capillaries in the tissues. In general it may be said that each segment has a pair of spiracles and corresponding tracheal systems (fig. 60), but this scheme is complete in no known species, for there are always some segments (especially in the head) which lack these organs and are supplied from adjacent segments (fig. 437). Again, the tracher may be connected by 
longitudinal trunks (fig. $45^{2}, b$ ), so that spiracles occur in only a part of the segments, these supplying the whole body. Although the tracheie are for aerial respiration, there are aquatic insects, but these also breathe air, which they carry about with them entangled among the hairs surrounding the spiracles. Then aquatic larve often have tracheal gills, thinwalled processes of the integument which project into the water and are penetrated by numerous tracheal twigs (fig. 453).

The alimentary tract always has excretory organs, the Malpighian tubules, connected with it. These vary in number between wide limits, but are always placed at the junction of the rectum with the rest of the tract. They differ from the physiologically similar tubes of the Arachnida in being of ectodermal origin, so that no homology can be traced between them. The gonads are always paired and placed dorsal to the intestine, while the ducts (at least in some cases modified nephridia) open ventrally at the hinder end of the body. The spermatozoa are motile.

In the subdivision of the 'tracheate' arthropods a group of Myriapoda is usually recognized, containing the centipedes and 'galley worms.' These two types are in reality very different. The centipedes (Chilopoda) show in all structural features close relationships to the Hexapoda, while the Diplopoda differ in almost every respect, except the presence of numerous walking legs, from the Chilopoda. Hence, since the object of classification is to show resemblances and differences, the Myriapoda has been dismembered, the Chilopoda being considered here, the Diplopoda as a distinct class at the end of the group of Arthropoda.

\section{Sub Class I. Chilopoda.}

The most striking characteristic of the chilopods is their long, flattened borlies, each of the numerous similar somites bearing a pair of six- or seven-jointed limbs. The head bears a pair of long antennæ and usually

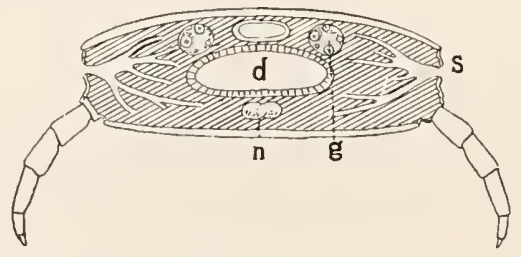

F1G. 439.-Diagram of transverse section of a centipede (orig.). d, digestive tract; $g$, gonad; $n$, nerve cord; $s$, spiracle and trachea.

numerous ocelli, which only in Scutigera show a tendency to become compound. The mouth parts (fig. 440) are a pair of mandibles and two pairs of maxillie, both united in the median line, the first pair forming a 'gnathochilarium,' the second the lower lip. Besides, the first pair of legs (5), with their fused bases, extend forward beneath the head and form the 
poison claws. Their terminal joints are sharp and contain the ducts of poison glands. The spiracles (at least a pair to every other somite except those of the head) are lateral in position in the soft integument between the dorsal and rentral plates (tergum and scutum) (fig. 439). The heart is elongate, with chambers in each somite (fig. 67 ); there are two large Malpighian tubes, and the nervous system is elongate, with ganglia in each somite. The unpaired gonads are dorsal to the intestine, while the single duct opens ventrally in the preanal somite.

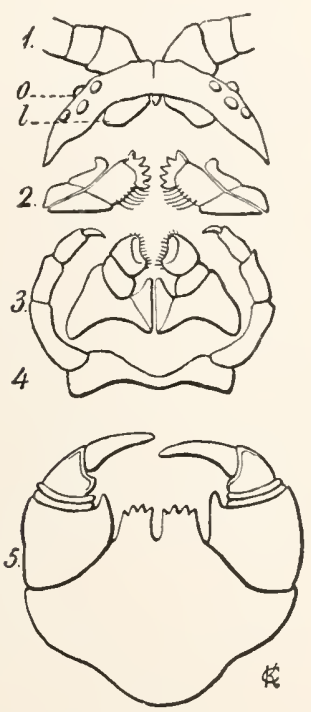

FIG. 440.

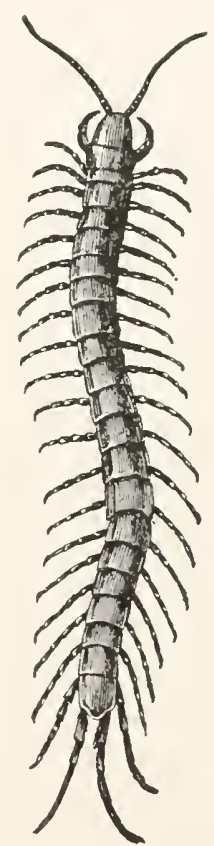

FIG. 44 I.

FIG. 440.--Nouth parts of Scolopendra morsitans. I, antenne; 2, mandibles; 3, maxillæ (gnathochilarium); 4 , second maxille (labium); 5 , poison feet.

FIG. 4.1.--Scolopendra morsitans, centipede (after Schmarda).

Liтновігде, I5 leg-bearing somites; certain dorsal plates enlarged and orerlapping the succeeding somites; Lithobius, ${ }^{*}$ etc. SCOLOPENDRIDæ, centipedes; at least 17 legs and 5 ocelli; Scolopendra* (fig. 4.4 I). GEopHILIDE, not less than 30 pairs of legs, spiracles 2 less than legs. Geophilus.* Scutigirid.F, legs very long, I 5 leg-bearing segments, but only 8 dorsal plates. Siutigera.*

\section{Sub Class II. IIexapoda.}

The Hexapoda is by far the largest division of the Arthropods, containing at least ten times as many known species as all the rest. The 
number is so large that it cannot be given with accuracy; an estimate is 250,000 . Since the tropics, which have not been exhaustively studied, are very rich in insects, it is conceivable that there are at least a million different species in the world. On the other hand, great uniformity of structure exists, all adhering with great fidelity to plan, regional divisions, and number of appendages, so that the difference between the most extreme forms is far less than that in Crustacea or Arachnida. But while hexapods thus lose in morphological interest, they gain in their life relations, in the way that they are injurious or beneficial to man, in their breeding habits, and in their intellectual and social relations. From the evolutionary standpoint they show marked arlaptations to environment, and the large number of species is only possible by taking advantage of every opportunity in nature.

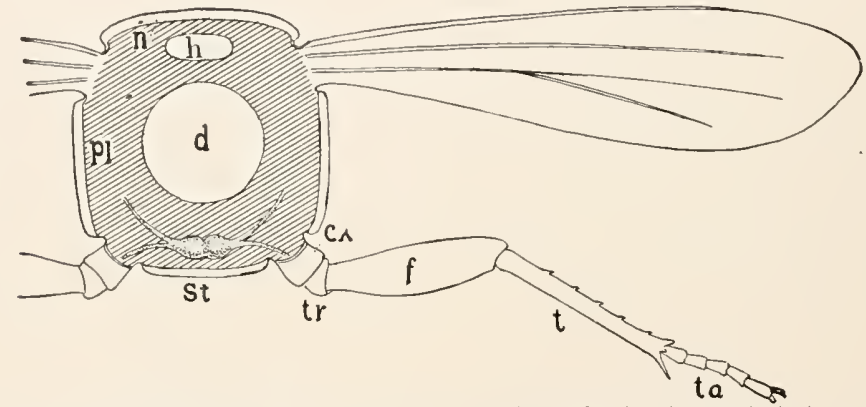

FIG. 442.-Schematic section of a hexapod through the thorax (orig.). $c x$, coxa; $d$, digestive tract; $f$, femur; $h$, heart; $n$, notum; $p l$, pleuron; st, sternum; $t$, tibia; $t a$, tarsus; $t r$, trochanter.

Of systematic importance are the regional division of the body and the number and character of the appendages. In the body three regions are distinguished, of ten separated by marked constrictions: head, thorax, and abdomen. The number of abdominal somites varies with the order and even with the family, ranging between eleven (in some larvæ and embryos twelve) in the Orthoptera and five in many Diptera. Each cuticular abdominal segment consists of two plates, tergite (dorsal) and sternite (ventral), united on the sides by a softer membrane which contains the spiracles. Head and thorax, on the other hand, have a constant number of somites. (See, however, Hymenoptera.) The thorax is plainly divided into three segments, pro-, meso- and metathorax, each composed of three elements, an unpaired dorsal portion, notum; a pair of lateral plates, pleura; and an unpaired ventral sternum (fig. 442). For simplicity one speaks of pronotum, mesosternum, etc., to indicate the por- 
tions of the separate segments. The head (fig. 443) is a continuous capsule in which the following parts are recognized: in front and dorsal clypeus and frons; dorsal and posterior a vertex and an occipul; laterally genc, ventrally a gula. The appendages show that the head is composed of at least four somites.

The view that the head consists of six somites is based on the existence of two more segments without appendages in the embryo, a preantennal and a postantennal (premandibular), as well as the fact that the brain consists of three pairs of ganglia (proto-, deuto-, and trito-cerebrum).

The three thoracic segments bear three pairs of legs, whence the name Hexapoda. The legs (fig. 442) are inserted between pleura and stema and begin with a short coxa $(c)$, followed by a trachanter (tr), also short. The two following joints are long, the first, the femur $(f e)$, being large and containing the muscles; the next, tibia $(t)$, being more slender; the foot, or tarsus (ta), is composed of several joints, the last bearing a pair of claws.

The first of the cephalic appendages, the antenna, are the most leg-like. 'They spring from the frons above the mouth and are innervated from the brain. The number and shape of the antennal joints vary with the group, often with the sex, and accorling as the single joints are lengthened or shortened, narrowed or expanded, or provided with appendages, etc., different kinds of antennx-knobbed, club-shaped, toothed,

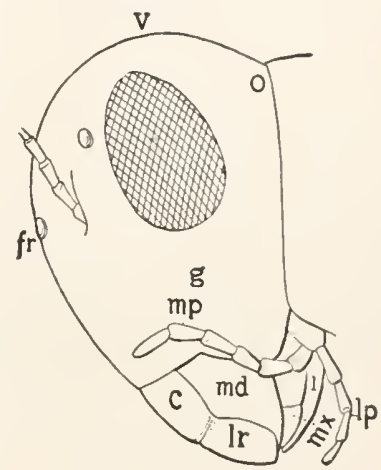

FIG. 443.-Head of a grasshopper. $c$, clypeus; $f$, frons: $g$, gena; $l$, labium; ip, labial palpi; $l r$, labrum; $m d$, mandible; $m p$, maxillary palpi; $m x$, maxilla; $o$, occiput; $v$, vertex. feathered, etc.-are recognized, distinctions of great value in classification.

The morphology of the three pairs of mouth parts, the mandibles ( $m d$ ), maxilla (mx), and second maxillx, or labium (la, figs. 443-447), is more interesting. The labium, formed of united right and left appendages, lies behind the mouth and forms the lower lip, and is in contrast to the upper lip, or labrum (lr), which, however, is not appendicular in character. Both labium and labrum may bear unpaired processes on their oral surfaces, an epipharynx above, a hypopharynx below the mouth, neither of them true appendages.

The different kinds of food necessitate differences in the character of the mouth parts-chewing, licking, sucking, or piercing-all referable back to the chewing kind, and these in turn are modified legs. In the description of the chewing type it is well to begin with the maxille (fig. 444), because of their easy comparison with the other mouth parts and with the legs as well. 'These begin 
with a triangular joint, the cardo $(c)$, which is followed by a larger stipes $(s t)$. The stipes in turn supports two chewing lobes, the inner, or lacinia (li), and an outer, or galea (le). The galea may either form a sheath for the lacinia, or, as in many beetles (fig. 470), it may be tactile and jointed again. The stipes also bears the maxillary palpus (pm), consisting of from three to six joints, and is the most ley-like part of the appendage. The labium arises as a pair of appendages which early approach each other and fuse behind the mouth. All the parts of the maxilla may be recognized, only it must be remembered that the basal parts of the two sides are fused. The united cardines form an under chin, the submentum, the stipites a chin or mentum, cleft in Orthoptera, a result of

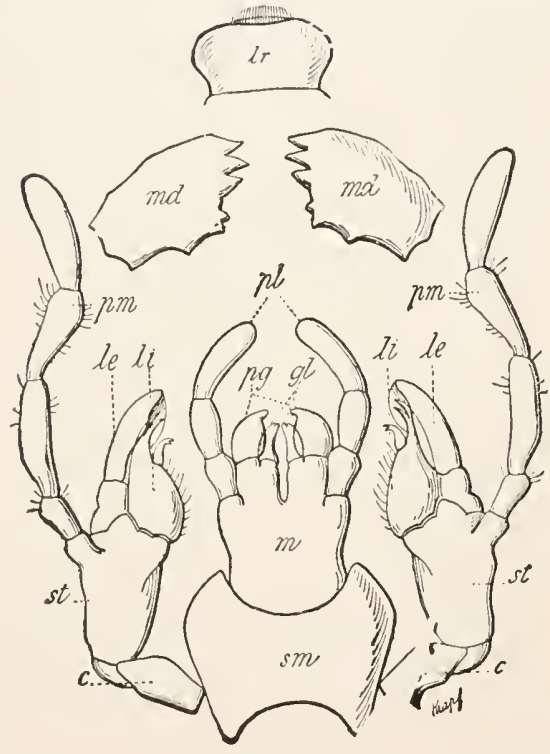

FIG. 444 .

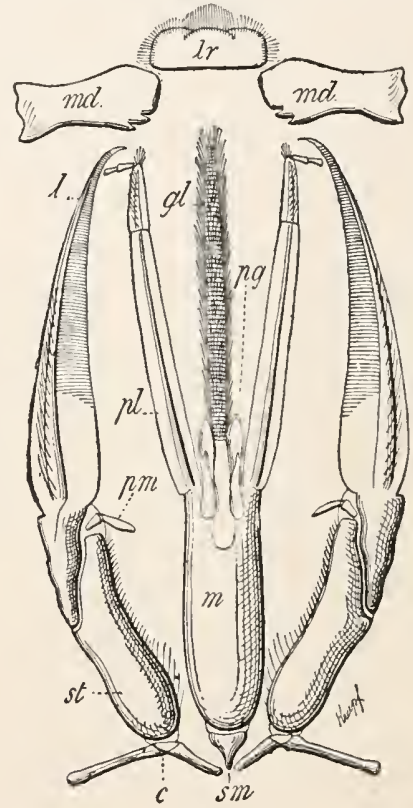

FIG. 445 .

F1G. 444.-Chewing mouth parts of cockroach (Periplaneta orientalis). The lettering is the same in figs. $444-447 . c$, cardo; $g l$, glossa; $h y$, hypopharynx; $l$, lobe; $l e, l i$, external and internal lobes of maxilla; $l r$, labrum; $m$, mentum; $m d$, mandible; $m x$, maxilla; $p$, pm, maxillary palpus; $p g$, paraglossa; $p l$, labial palpus; $s m$, submentum; st, stipes.

FiG. 445.-Licking mouth parts of bumble bee (Bombus terrestris).

incomplete fusion. This may bear inner and outer processes, the glossa $(\mathrm{gl})$ and the paraglosse $(p g)$ respectively, and the labial palpus. The mandible consists of merely the basal joint, altered for biting, while the rest of the appendage, common in crustacea as the mandibular palpus, is lacking.

The licking mouth parts, like those of the bees (fig. 445), stand next to those already described, there being many transitional stages. Labrum and mandibles retain their primitive condition, while maxillie and labium are greatly elongate, are connected at the bases, and can be folcled away beneath the head or extended at will. The small submentum is followed by an elongate mentum which bears the unpaired tongue or glossa $(\mathrm{gl})$, which corresponds to the fused glossæ (or to the hypopharynx?) of the first type and which is used for sucking 
honey and hence has the form of a nearly closed tube. Beside it lie the rudimentary paragloss $(p g)$ and the well-dereloped palpi. Similarly the maxillie have small cardines and palpi, while the stipites and the undivided lobe $(l)$ are long and well developed.

The piercing mouth parts of the flies (Diptera) and buess (Rhynchota) can be compared with those of the bees in so far as the labium forms the groundwork of the whole (fig. 446). The beak (rostrum, haustellum) of these animals curresponds to the labium; it is a grooved structure, either fleshy and llexible, or

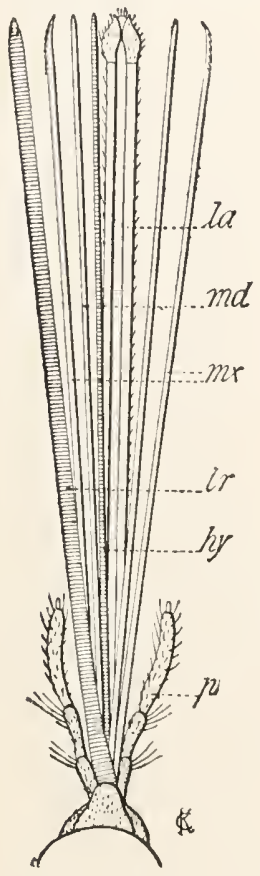

FIG. $4+6$.

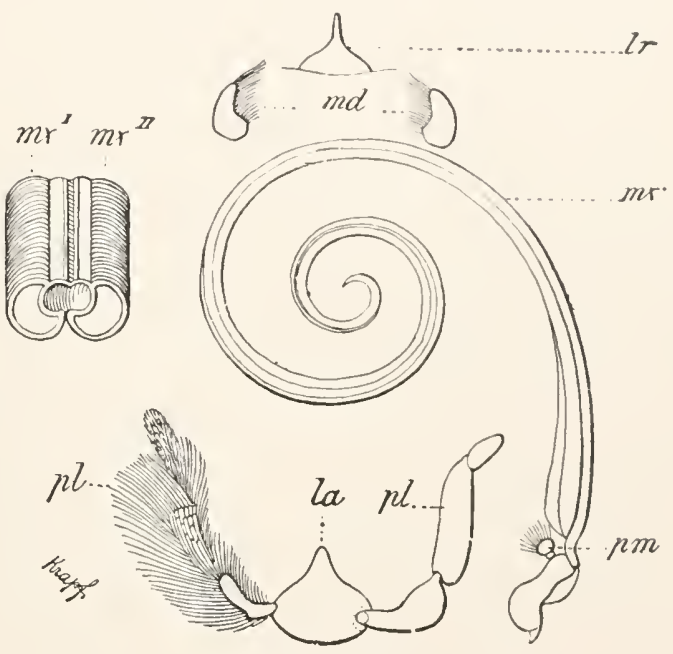

FIG. 447 .

FIG. 446.- Sucking mouth parts of mosquito, Culex pipiens (after Muln). The groove of labium opened by removing labrum; the stylets separated.

FIG. 47.- Sucking mouth parts of a butterlly (after Savigny). mx', mx.", shows how right and left maxille unite into a tube; right labial palpus $(p l)$ with hairs removed.

stiff and jointed. The edges of the groove are inrolled so that there remains a narrow dorsal slit, which can be closed by the slender upper lip (Ir). The tube formed of these parts contains four stylets, toothed or with retrorse hooks at the tip. These are the mandibles and maxilla, and a fifth stylet, the hypopharynx $(h y)$ can be present. The palpi, which only occur in the Diptera, belong to the maxille $(p)$. Reduction in number of stylets to four or three, or their complete absence (some flies), is brought about by fusion or by degeneration. The haustellum serves as a case for the sucking tube, which in the Rhynchota is formed by the united maxillx, in the Diptera by labrum and hypopharynx.

The proboscis, or haustellum (the so-called tongue), of the Lepidoptera 
(fig. 447) is a long tube coiled like a watch spring beneath the head. It consists of two long grooved maxillary galea firmly united by their edges. The maxillary palpi are well developed in the moths; elsewhere they show all stages of reduction to complete disappearance. Labium and labrum are reduced to small triangular plates at the base of the proboscis, the labium bearing a pair of hairy palpi $(p l)$. The mandibles are represented by small plates or bunches of hair. These conditions gain in interest when we remember that in the larva the mandibles are strong biting organs, while the maxillæ are small hooks, and the labium is better developed only in those parts connected with the silk glands, a beautiful example of relations of structure to life conditions.

In contrast to the other regions, the abdomen lacks appendages in the adults. Only in the Thysanura are small lobes present, behind and in the same line with the thoracic feet, which may be abdominal feet. Apparently, too, the appendages of the last segment, the stylets and cerci, are modified limbs, but the parts (gonapophyses) used in copulation and oviposition are different in character. False feet, or pro-legs, occur on the abdomen of the larvæ of the Lepidoptera and the Tenthredinidx, but since these are fleshy unjointed processes, it is doubtful whether these are true abdominal limbs, or are structures independently acquired.

Besides ventral appendages the insects usually have two pairs of dorsal outgrowths upon the meso- and metathorax, the wings. They are lateral folds of the chitinous coat of the notum and contain on their interior extensions of the blood sinuses and of the tracher, which are protected by thickenings of the chitin, causing the network of 'xeins' or 'nervures' in the wing. Both wings may be elastic, flexible, and adapted for flight, or the hinder pair may alone partake of this character (true wings or alce), while the first pair may be thick and parchment-like wing covers, or elytra, under which the true wings are concealed when at rest. When only the base of the wing is thus thickened hemelytra result. Between the bases of the anterior wings is frequently a chitinous plate, the scutellum, between the hinder wings a similar postscutellum. In many insects one pair of wings is lacking, the anterior pair being retained in the Diptera (fig. 486), the posterior in the Strepsiptera (fig. 469). The entire absence of wings may occur from two causes; wings have apparently never been developed in some (primary lack of wings of the Apterygota), while there are others in which wings once present have been lost, because nearly related forms -bugs, male cockroaches, sexual ants and termites-are winged (figs. $464,482,483$ ). The prothorax of all recent insects is wingless, but some Archiptera of the coal period had wing rudiments on this somite.

As a result of differences in food the alimentary canal (figs. 448, 449) varies greatly. 'The ectodermal stomodæum begins with a pharynx, which in the sucking insects is a sucking apparatus with radial muscles. The œsophagus, which follows, may be widened to a crop (ingluvies), or it may have a crecal outgrowth which in the butterflies and flies may take the shape of a stalked vesicle (falsely 'sucking stomach').

Also ectodermal 
is the gizzard $(k m, p v)$, or proventriculus, the chitinous lining of which is toothed for grinding the food. 'The true stomach, of entodermal origin $(m, c d)$, frequently bears blind sacs or gastric caca $(a p)$; in general it is short and its junction with the hinder ectodermal portion, the proctorleum, is marked by the entrance of the Malpighian tulbules $(2 \mathrm{~m})$. The latter, excretory in function, arise from the proctodeal region. The proctodeum is usually differentiated into a small intestine and a two-regional (colon and rectum) large intestine. The rectum may have enlargements called rectal glands. True glands, however, occur only at the beginning and end of the alimentary tract; from two to four salivary glands $(s p)$ empty into the mouth; at the anus are defensive anal glands with malodorous secretions of a protective character. The alimentary tract with the other viscera is enveloped in the fat body, a soft mass which contains, besides fat cells and connective tissue, concretions of uric acid.

The nervous system (fig. 370) has the ventral cord, especially in primitive forms (Apterygota, Archiptera, Orthoptera, fig. 449), and nearly all larve (fig. 60 ), long and composed of numerous separate pairs of ganglia. In beetles, moths, bees (fig. 452), and flies the cord is shortened and the ganglia are in part fused. The brain arises by the fusion of three pairs of ganglia (proto-, deuto-, and tritocerebrum), and is, especially in. colonial species, very complex. It is connected on cither side with a large optic ganglion, the size of which is correlated to that of the eyes. In the adult condition the Hexapoda have a single

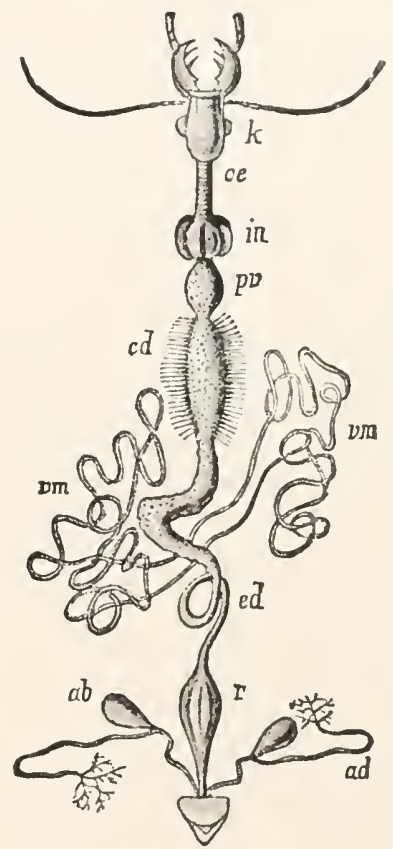

Fig. 448.-Alimentary tract of Carabus alvatus (from Lang, after Dufour). av', anal resicle; ad, anal gland; $c d$, stomach with ceca; $e d$, hind gut; in, ingluvies (crop); $k$, hear; oe, aesophagus; $p r$, proventriculus (gizzard); $r$, rectum; $v m$, Malpighian tubules. pair of highly developed compound eyes (fig. 372), (each occasionally divided into two), which not infrequently occupy nearly the whole of the top of the head. Between and in front of these, small ancl simple ocelli, usually three in number, frequently occur, especially in insects which are strong fliers. These are different from the more numerous simple eyes of the larva of holometabolous insects (e.g., butterllies and 
beetles) in the position of the later compound eyes. Of other sense organs only the tactile hairs of the skin are known with certainty, while similar hairs on the antennie and about the mouth are supposed to be organs of smell and taste, since these senses are known to be well developer. The tympanal organs of the Orthoptera are the only structures which can be

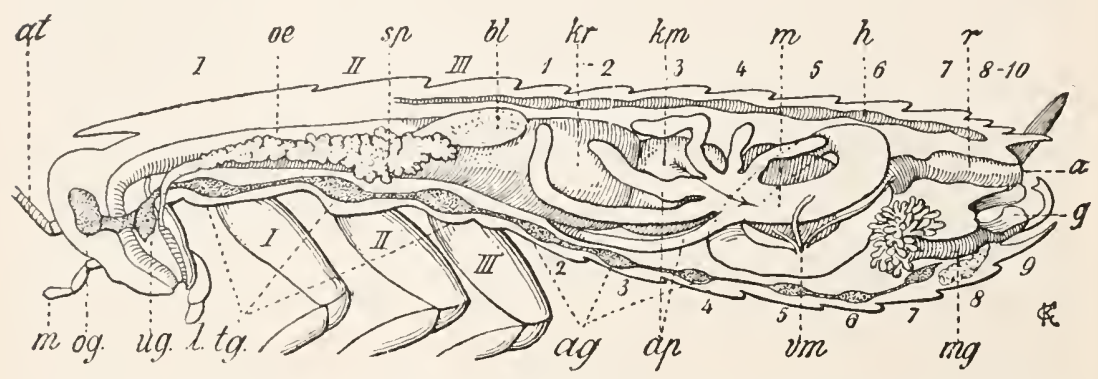

FIG. 449.- Viscera of male cockroach (Periplaneta orientalis) (partly after Huxley). $I-I I I$, segments of thorax and corresponding legs; I-IO, abdominal segments; $a$, anus $a g$, ventral ganglia; $a p$, gastric ceca; $a t$, antenna; $b l$, salivary bladder: $g$, sexual opening $h$, heart; $k r$, crop; $k m$, gizzard; $l$, labial palpus; $m$, stomach (the arrow shows the con nection between $m$ and $k m$ ), also maxillary palpus; $m g$, male genitalia; oe, œsophagus $o g$, brain; $r$, rectum; $s p$, salivary gland; $t g$, thoracic ganglia, $u g$, infracesophagea ganglion; vm, Malpighian tubules.

with much probability connected with hearing. These are thin drum-like parts of the chitin, framed in thicker portions (figs. 450, 45I), beneath which is a tracheal vesicle, with a nerve ending in a 'crista acustica.' End organs similar to those of the cristie acustica occur elsewhere than in the tympanal organs and are regarded as auditory ('chordotonal' sense organs).

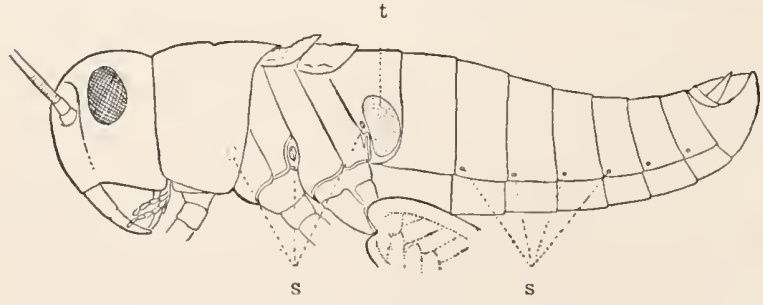

FIG. 450 .

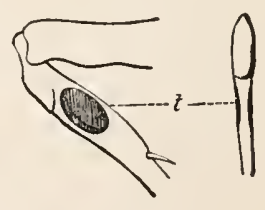

FIG. 45 I.

FIG, 450.-Side view of grasshopper. $s$, spiracles; $t$, tympanal organ.

FIG. 45I.-Anterior tibia of a Locustid with tympanum, $t$; (from Hatschek, after Fischer).

The power of producing sound is widely distributed and often highly developed, the organs for this purpose varying widely in character. Stridulating organs are formed by ridges on wings and legs, which are rubbed against each other or against similar ridges on the body. Humming is produced by the action of the wings or by the passage of air 
through the spiracles, which are often provided with vibrating membranes which also serve to close these openings.

The trachea (figs. 437,452) are usually united, just inside the spiracles, by longitudinal trunks from which fine branches extend, enveloping and penetrating all the organs with delicate silvery threads. This connection of trachex renders it possible for the spiracles of some segments to disappear, leaving but a single pair in the aquatic larrie of some Diptera.

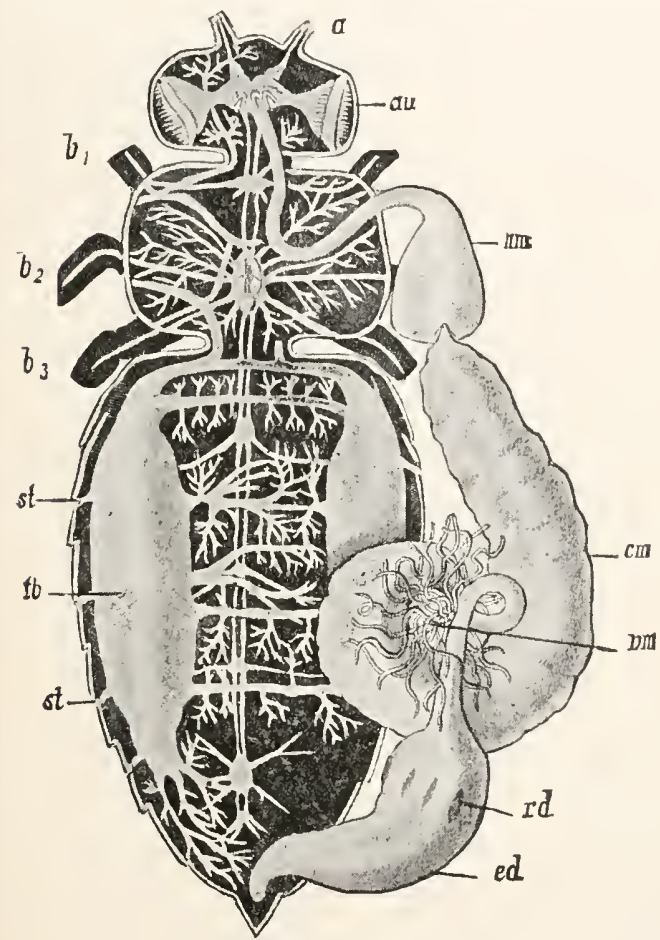

FIG. $45^{2}$.

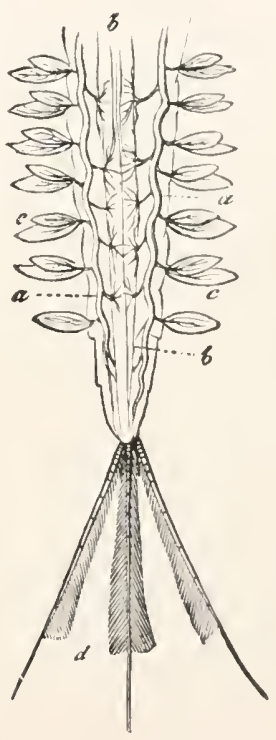

Fig. 453 .

FIG. 452.-Anatomy of honey bee (from Lang, after Leuckart). a, antenna; $a u$, eye; $b$, legs; $c m$, chyle stomach; $e d$, rectum; $h m$, honey stomach (proventriculus); $r d$, rectal glands; $s t$, spiracles; $t b$, tracheal chambers with trachet; $\tau^{\prime} m$, Malpighian tubules.

FIG. 453.-Abdomen of Ephemera larva (from Gegenbaur) with tracheal gills, $c$; , tracheal trunks; $b$, intestine; $d$, caudal bristles (cerci).

The spiracles of the abdomen are the most constant, usually occurring in or near the soft membrane between the sternites and tergites; the thorax at most has but two pairs, the head none. In insects with good powers of flight many of the tracheal trunks are expanded to large air sacs, which may be of value as reservoirs of air, so that the ordinary respiratory motions are less necessary during flight. 
An interesting adaptation of the tracheal system to acquatic life occurs in the larvie of many Archiptera (dragonflies and Nayflies) and Neuroptera, and even among Lepidoptera (Paraponyx) and Coleoptera (Gyrinidæe). The spiracles here are usually closed, and oxygen is taken either through the skin or by so-called tracheal gills-bushy or leaf-like appendages of the surface or the rectum, richly permeated by tracheal branches (fig. 453). In such cases the tracheal system has two portions, one which receives oxygen from and gives off carbon dioxide to the water; the other which supplies the tissues with oxygen and receives carbon dioxide.

Since the trachee, with their fine branches, supply the tissues directly with oxygen, the blood-vascular system is rudimentary. Directly under the back lies the elongate tubular heart in a special pericardial sinus. This is a part of the hemocœle cut off from the gastric portion of this space

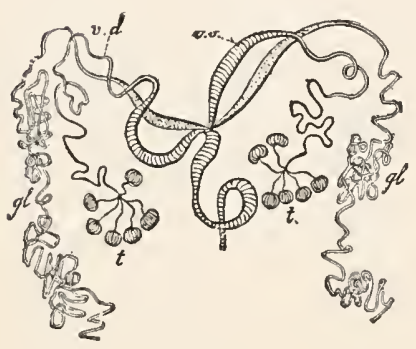

A.

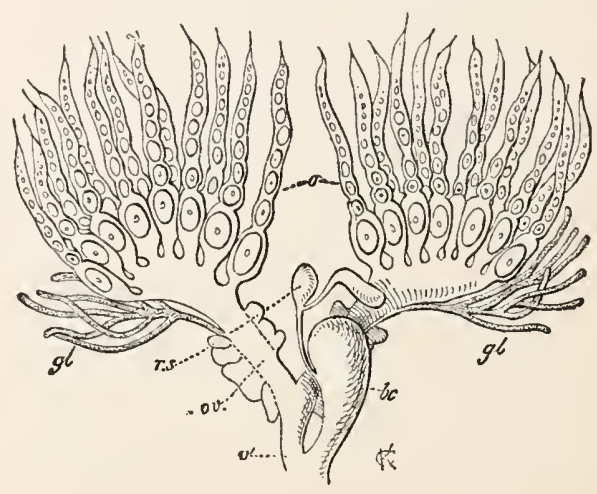

B.

FIG. 454.- A, Male genitalia of Melolontha (from Gegenbaur, after Fabre). $g l$, accessory glands; $t$, testes; $v d$, vas deferens; $v s$, seminal vesicles. $B$, genitalia of female Hydrobius (from Gegenbaur, after Stein). $b c$, bursa copulatrix; gl, tubular glands; $o$, ovarial tubes; $o v$, oviduct with glands; $r s$, receptaculum seminis; $v$, vagina.

by an incomplete partition in which, right and left, are the lateral muscles (alce cordis) of the heart. Since folds from the margins of the ostia extend into the cavity of the heart, and in the systole, which proceeds from behind forward, not only close the ostia, but prevent any back flow of blood into the posterior part of the heart, there is an appearance of a chambering of the heart. The blood passes forward through an anterior aorta into the hamocole and from this back to the pericardial sinus, the alary muscles aiding by moving the viscera, and enlarging the sinus. The arrangement of the viscera, fat bodies, and muscles gives a certain regularity to the circulation, especially in the appendages. Accessory pulsating ampulle in the bases of the antennæ (Orthoptera) help in the flow of the blood. Many beetles (Meloidx and Coccinellidæ) squirt blood contain- 
ing an irritating substance (cantharidin) through the jointing membranes of the legs as a means of defense.

The Hexapoda are dixcious. 'The paired gonads consist of a few or many ovarial or testicular tubules (fig. 454), in the abdomen. Their paired ducts (oviducts, vasa deferentia) open separately in the Ephemerida and young Apterygota, but all other Hexapoda have a single ventral unpaired sexual opening just in front of the anus. invagination of the ectoderm (hence lined with chitin), which extends inwards and meets the genital ducts (modified nephridia). The receptaculum seminis, a sac connected with the female genitalia, has a special biological interest. In insects which copulate but once during life it retains the spermatozoa for a long time (four years in bees) in a living condition. As the eggs are laid they may be impregnated by spermatozoa from it. Since a firm shell or chorion is developed around the egg in the ovary, entrance of spermatozoa is only possible by a micropylar apparalus, a system of tubes penetrating the chorion at one end of the egg.

Oviposition occurs in many insects by means of an ovipositor which may be developed in two ways. In beetles, flies and butterflies the last somites of the body are small, and are normally retracted into the body but can be protruded as a long tube for oviposition. In Hymenoptera, Hemiptera, Orthoptera and dragonflies the ovipositor (terebra) is formed by special appendages, the gonapophyses, four to six in number, which arise from the ventral This arises as a median

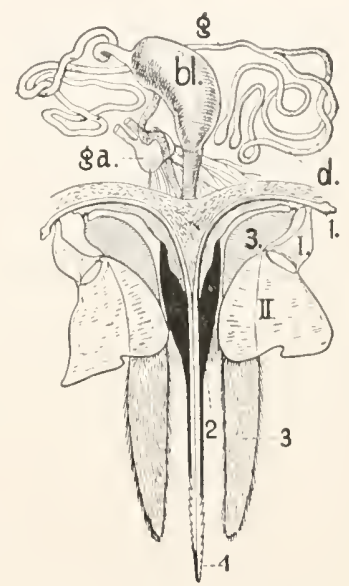

FIG. 455--Ventral view of sting of bee (after Packard and Cheshire). $b l$, poison sac; $d$, poison gland; $g$, its duct; ga, terminal ganglion, beside it accessory gland; I, stylet; 2, groove of sting (black); 3 , sheath of sting; $l$, angle piece; $I I$, quaslrate plate for attachment of muscles. side of the eighth and ninth abdominal segments. In the Orthoptera two pairs of gonapophyses of the eighth and ninth somites form a sheath in which two other parts, the egg-guide, also derived from the ninth, are enclosed. In the Hymenoptera (fig. 455) the latter are fused to a tube in which both parts of the eighth somite play as a piercing stylet, while two other parts lie at the sides as the sheath. In the wasps and bees these parts can be withdrawn into the body, and have frequently been converted into a sting (aculeus) provided with a poison gland, which is confined to the female. In the males there is usually a protrusible penis which is frequently composed of the same parts as the ovipositor; in others of metamorphosed somites. Further sexual differences lie in the form of the antennæ, shape and color of the wings, modifications of the eyes, etc. (fig. 74 ).

In many insects the eggs may develop parthenogenetically. Plant licc and scale insects reproduce for generations asexually, and parthenogenesis is widely distributed among Hymenoptera, Lepidoptera, and Ncuroptera. The condi- 
tions among the bees are especially interesting, since here the determination of sex rests with the existence or non-existence of fertilization (Pp. I30, I34). Much rarer than the ordinary parthenogenesis is pædogenesis (p. I 29), which occurs only in certain Diptera like Miastor. In the female Miastor larva (fig, 456) the eggs develop before the appearance of the ducts, so that the young can only escape by rupture of the mother. After several pædogenetic generations there appear at last larve which pupate and produce adult male and female flies.

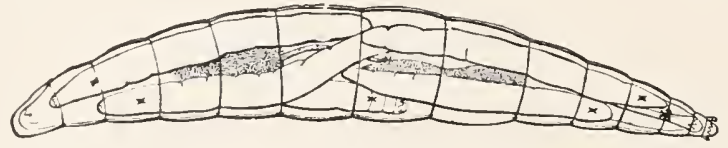

FIG. 456.-Larva of a Cecidomyid with pædogenetic daughter larvæ (from Hatschek. after Pagenstecher).

With the exception of these pædogenetic forms, the Pupipara, many Aphidæ and a few other viviparous species, the Hexapoda are oviparous. The development begins, after oviposition, by a superficial segmentation of the egg. Later there appear two embryonic structures, the yolk sac and the amnion; the first, in contrast to the vertebrate structure with the same name, is dorsal. The amnion is a thin layer of cells which covers the ventral surface and arises in a manner similar to the vertebrate amnion; folds arising from the blastoderm in front and behind, right and left of the embryo, fuse with one another and produce a double envelope, an inner amnion, an outer serosa, enclosing the germinal area.

The postembryonic development presents two important features. I. As in other arthropods grow th is possible only by periodic ecdy'ses so that

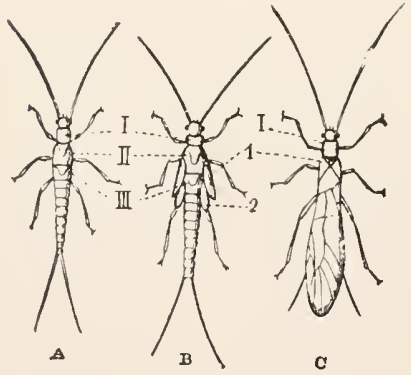

Fig. 457-- Hemimetabolous development of Perla nigra (from Huxley). $A$, wingless larva; $B$, larva with wing pads, $\mathrm{I}, 2 ; C$, adult; $I, I I, I I I$, thoracic segments. the life cycle consists of several periods separated by molts of the cuticle. 2. No insect, as it escapes from the egg, has wings. If present in the adult they must arise during the larval stages. This postembryonic development of the wings is the starting-point of the metamor phosis, and forms the basis of a division of the development into ametabolous (no metamorphosis), hemimetabolous (incomplete metamorphosis) and holometabolous (complete metamorphosis). An ametabolous development is possible only in wingless insects, the postembryonic development consisting only of periodic molts. Some wingless forms (fleas, wingless moths, ants, etc.), have a metamorphosis, because they have inherited it from winged ancestors and have not lost it with the wings.

Hemimetabolous development is marked by a gradual change from the newly hatched animal, the larva, to the sexually mature adult or imago (fig. 457). There often appears with the second molt, the 
anlagen of the wings as small folds in the chitinous coat of the meso-and metathorax; these increase with each successive molt, until, with the last, they become functional wings in size, form and motion. Inside of each wing pad ( $B$, I and 2) there is the anlage of the wing of the next stage. Since the larve, from lack of wings, are forced to live under diflerent conditions from the adults, conditions which demand special structures, the differences between the larve and the adults are emphasized ly the P.esence of specific larval organs. Thus the aquatic larve of fragonflies and Mayflies, are distinguished, not only by the alsence of wings, but by different form, different shaped mouth parts, and especially l,y the tracheal gills (fig. 452), usually lost at the last molt.

Increase in larval characters leads to complete metamorphosis. In order to profit as much as possible by its adaptation to its environment the larva retains its shape as long as possible; the gradual change to the adult is suppressed and the alteration in form is postponed until the end of the larval life, to the period between the last two molts. In this interval there is such an energetic transformation of the organism that ordinary vital functions, especially motion and feeding, are interfered with or rendered impossible. This last stage therefore becomes a period of rest
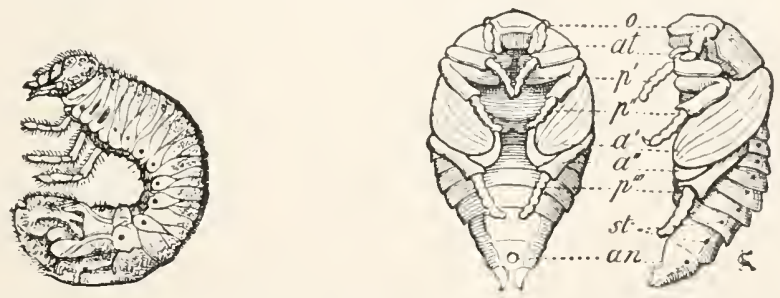

FIG. 458.-Larva and free pupa of May beetle. $a^{\prime}, a^{\prime \prime}$, fore aut hind wings; an, anus; $a t$, antennx; $o$, eyes; $p^{\prime}-p^{\prime \prime \prime}$, legs; st, spiracles.

the pupal stage, the existence of which is important in the definition of complete metamorphosis. The more complete the condition of rest the more pronounced is the holometabolous development. From this point of view different types of pupx are distinguished: pupa liberx, pupie obtectix, and pupa coarctate. In a free pupa (pupa libera) the appendages stand out from the body (fig. $45^{8}$ ), so that not only the segmentation of the body but the antenna, legs, wings, and often the mouth parts of the imago are visible. Such pupx have a certain power of motion, as, for instance, the pupe of many Neuroptera and mosquitos, the latter rising and falling in the water. The covered pupx (pupa obtecte) at the moment of pupation have free appendages which with the hardening of the chitin become closely. 
appressed to the body, so that only indistinct contours can be seen (fig. 459). Motion is confined to bending of the whole body, as is familiar in the pupe of moths and butterflies. The pupe coarctate are without motion becatse here the pupa (in structure a pupa libera) is enclosed in a larger coat, the last larval skin (some flies).

The variations among larva are even greater than with pupx. Here structure is so completely under the influence of environment that with similar or different conditions larve widely remote, from the systematic standpoint, may closely resemble each other, while those of closely related species may differ extremely. The leaf-feeding larve of Lepidoptera (fig. +60 ) and 'Tenthreds are brightly colored, the thoracic appendages remaining small and reinforced by the fleshy ventral prolegs. The pre-

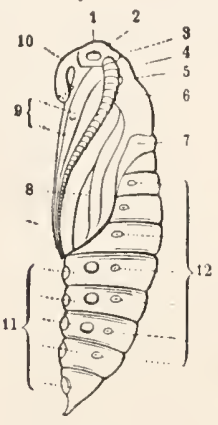

FIG. 459 .

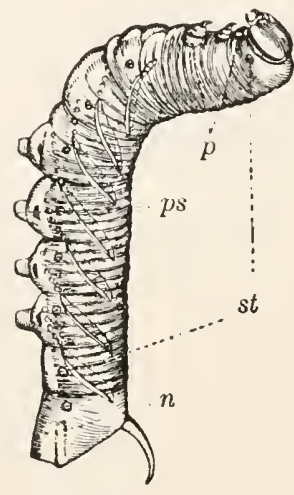

FIG. 460 .

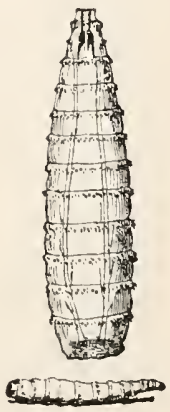

FIG. 46 I.

FIs. 459.-Pupa of Sphinx ligustri (after Ludwig-Leunis). I, eye; 2, head; 3, antenna; $4^{-6}$, thoracic somites: 7 , hind, 8 , fore wing; 9 , legs; Io, probos is; 1 I, abdominal somites; I2, spiracles.

FIG. 460.-Larra of Sphinx ligustri (after Ludwig-Leunis). n, caudal disc; $p$, thoracic feet; $p s$, prolegs.

Fig. 461.- Larva (maggot) of blowfly, Musca vomitoria (after Leuckart).

daceous larve of many beetles and Neuroptera have long thoracic legs, strong mandibles, and no prolegs. Other beetle larra, which burrow in wood or live in the earth, often have the legs rudimentary or wholly lacking. These lead to the maggot-like larve, in which the mouth parts are inconspicuous and the distinction between head and thorax may vanish. Such soft-skinned annulated sacs occur in the bees (fig. 59) and other Hymenoptera, as well as in many flies (fig. 46I); that is, larre which live in an abundance of food either because of parasitism or because the mother has provided plenty. 
From the outer appearance one woukl conclucle that these holometabolous larve not only lacked the wings, but that the appendages of the imago were entirely absent or had an entirely different form; farther, that wings, and frequently antenna, legs, and mouth parts, come into existence at the moment of pupation, and then in remarkable size and completeness. In fact, the anlagen of all these structures are formed long before pupation, often at the first molt. The wings of a butterfly are present in the caterpillar as small folds or processes of the surface which increase in size with each molt. They are not visible externally because they are pushed into the body and enclosed in sacs opening to the exterior. Such anlagen are called imaginal discs (fig. 462); with their recognition the distinctions between complete and incomplete metamorphosis in
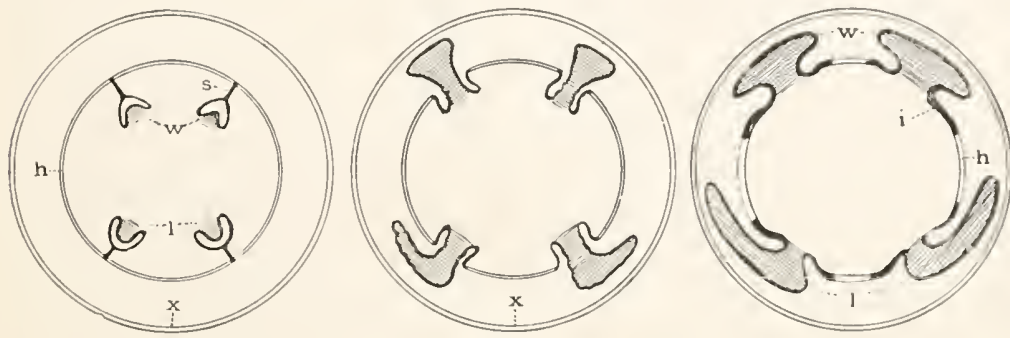

Fig. 462.-Diagram of development of wings and legs from the imaginal dises of a fly during metamorphosis (after Lang). $h$, larval hypodermis; $i$, imaginal hypodermis; $l$, w, imaginal discs and legs and wings formed from them; $s$, connection of discs with hypodermis; $x$, chitinous larval skin.

part disappear, since in the first the structures of the imago, even if in a modified shape, are outlined very early. Still there remains much to be remodelled during the pupal rest. The muscles must be adapted to the new locomotor organs, the digestive tract to the altered food, the nervous system re-formed. Since a great part of the larval structures must be broken down to afford material for the reconstruction of the organs, the pulpy nature of the insicle of the pupa is easily understood. With a rapid degeneration of the tissues this material is so homogeneous that it was formerly thought that the pupa returned to the indifferent condition of the egg.

With the sexual life is connected, in termites and Hymenoptera (bees, wasps, ants), a community formation or social state, consisting in the association of the sexual animals ('kings' or 'drones' and 'queens'), which have only reproduction as their function, with 'workers' which care for and protect the young and form the complicated nests, but which, on account of their undeveloped reproductive organs, can take no part in the perpetuation of the species. In the Hymenoptera these 'neuters' are rudimentary females; among the termites there are rudimentary males as well (fre. 464). With the termites, wasps and bees, this rudimentary character of the sexual organs appears to be the result of insufficient food in the larral stages. Some think this true of the ants as well; others deny it. With the ants the distinction between sexual animals and workers is frequently obliterated by the presence of intermediate forms ('ergatoids' and 'ergatomorphs'). But even where the distinction is the sharp)est, as in bees, the workers, by proper feeding, can be marle to produce eggs. Not inf requently there is a polymorphism among the neuters, the most frequent condition being small-headed workers and large-headed 'soldicrs' (termites, ants). 
The communities of termites and ants are complicated farther by the introduction of other insects of various kinds (mostly beetles) as 'guests' or 'symphila.' These, together with their young, are fed and cared for by the ants on account of the sweet fluid which they secrete. The ant communities are farther enlarged by other species kept as slaves. In the nests of many tropical termites and ants there are 'mushroom gardens' in the nests, cultures of fungi upon a layer of organic material formed of leaves chewed by the ants.

The highly developed capacities of these communal insects, their ability to recognize the members of their own colony from the same species of another colony, the working together for the common good, for a long time led to the idea of a high grade of moral and intellectual development, especially in the bees and ants. This view was erroneous. Since the behavior of the animals is much the same when they are separated from their fellows in the pupal stages and are reared so that they have no chance to learn how to work from others, it follows that their acts are innate complex reflexes and are not the result of a conscious education. Yet they have a certain ability to learn, an ability to modify their acts under strange conditions. It is noteworthy that the strikingly similar communities of ants and termites have developed independently of one another, and that the same is true of wasps and bees is shown by the existence of both solitary and social species in both families.

In the classification four points are of special importance: (I) The segmentation of the body; whether the segments of thorax and abdomen follow without change of form, or whether the thorax is sharply marked off from both head and abdomen. (2) The character of the wings, which in the lower forms are either lacking or are delicate chitinous structures, with numerous veins, the wings of the two pairs similar. In the higher forms a degeneration of the wing veins or a leathery consistence of the membrane, together with a divergent development, partial reduction of anterior and posterior wings may occur.

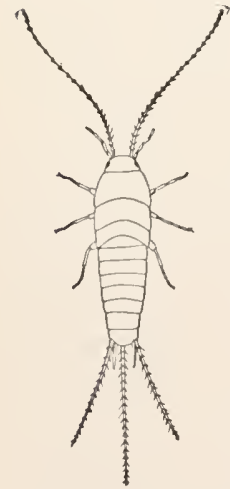

Fig. 463.-Lepisma saccharina,* silver fish (after Packard).

(3) The structure of the mouth parts, and (4) the type of development, both described above. With these characters it is easy to differentiate six orders: Lepidoptera, Diptera, Aphaniptera, Rhynchota, Hymenoptera, and Coleoptera. The remaining species were formerly divided among the Orthoptera and Neuroptera, but these groups are not natural and they have been divided into more or fewer groups. Here the Pseudoneuroptera or Archiptera are separated from the Neuroptera, the wingless forms or Apterygota from the Orthoptera.

\section{Order I. Apterygota.}

At the bottom of the Hexapoda come ametabolous forms which lack wings and which show no evidence of having descended from winged ancestors. They are regarded as slightly modified descendants of the ancestral Hexapod. Besides the lack of wings they show many primitive characters; compound eyes are poorly developed or lacking; the tracheal system-degenerate in Collembola-consists of isolated tracheal bushes, rarely connected by longitudinal trunks (fig. 437); the mouth parts are biting or piercing, though frequently rudimentary. Especially to be noted is the existence of abdominal appendages, possibly indicating a relationship to certain 'myriapods.' 'Thus in Scolopendrella (p. 434) there are small stylets on the feet and beside them protrusible sacs. These reappear in the Thysanura and Campodea, and Camapodea has in addition a rudimentary pair of appendages on the first abdom- 
inal somite; two or three additional pairs appear in other genera. The cerci or large 'bristles' are also regarded as abdominal appendages.

Sub Order I. THISANURA (Bristle-tails). Body elongate, with long bristles (cerci) at the hinder end. Lepisma saccharina, silver fish, common among old books and papers, does considerable damage. Campodea* (fig. 365). Machilis, ${ }^{*}$ Iapyx,* with caudal forceps. Sub Orier II. COLLENBOIA (Spring-tails). Compressed forms in which two jointed ajpendages bent under the body serve as a spring, throwing the animals (one to three mm. long) forwards. Podura*; Anurida maritima, ${ }^{*}$ in tide pools; Achoreutes nizalis, * snow flea.

The recently discovered group of PROTLRA may be mentioned here. They lack antenne, have the first leg directed forwards and tactile, twelve abdominal somites, appendages on first three, two thoracic spiracles. Europe and India. Acerentomon.

\section{Order II. Archiptera (Pseudoneuroptera).}

These represent the primitive forms of winged insects. The elongate lody usually bears the cerci of the Thysanura. The wings are delicate and transparent, supported by a close network of nervures, both pairs being very closely alike. The mouth parts are of the typical biting kind, the labium frequently deeply cleft. These points of primitive structure are correlated with a primitive, usually hemimetabolous development. The distinction between larva and imago is largely one of presence or absence of wings, although larval organs like gills (Amphibiotica) may occur. Frequently the development is direct when the adults, as in some Termites and the Psocida, are wingless.
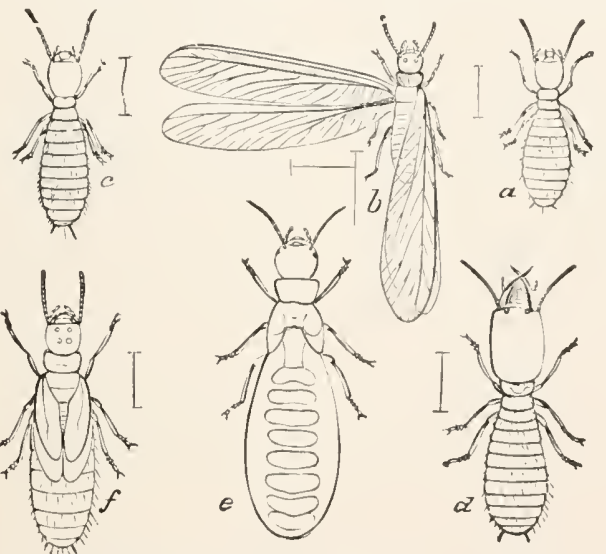

FIG. 464.-Termes flavipes,* white ant from Riley). $a$, Iarva; $b$, winged male; $c$, worker; $d$, soldier; $e$, queen; $f$. pupa.

Sub Order I. CORRODENTIA. Larva distinguished from the imagines by difference in size and, in the winged forms, by lack of wings. Best known are the TERMITIDE (Isoptera), or white ants, which must not le confused with the true ants (Hymenoptera). Like the true ants, they have a well-developed social state, their communities resembling each other in many details as the 'guests' (termitophiles), the mushroom gardens, etc. 'They differ from the ants in the similar segments of the body, the character of the mouth parts, the hemimetabolous development and by the fact that the workers include both males 
and females. A colony of termites, consisting usually of thousands of individuals, forms a nest with numerous chambers and passages. They are nocturnal, and they burrow, without coming to the surface, through old wood (timbers of houses, furniture, picture frames, dead wood in the forest, etc.). They line these chambers with a cement-like substance composed of refuse which has passed through the alimentary canal. Many species build dome-like nests, ten or fifteen feet high, of chewed earth. In a colony are winged and wingless indivirluals, the latter with ametabolous development (fig. 464). The wingless forms have the sexual organs rudimentary, luut, in contrast to ants and bees, may belong to either sex. 'They are frequently blind, have strong mandibles,

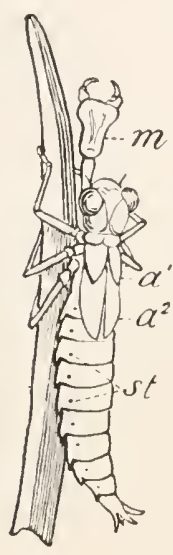

FIG. $40_{5}$. and are of two kinds, the workers $(c)$ and the largeheaded soldiers $(d)$. The winged forms are sexually functional $(b)$. Shortly after the metamorphosis they swarm, and then the wings are bitten off at the base and 'king' and 'queen' either form a new colony or enter one already in existence. After copulation the abdomen of the queen, by the formation of numerous eggs, swells to an enormous size $(e)$. Since the swarming individuals form the prey of birds and other animals, it often happens that a colony is left without a royal couple. In such cases the line is perpetuated by reserve males and

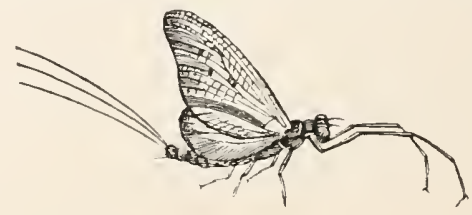

FIG. 466.

lig. 465 .--Larva of Eschna grandis (after Rösel von Rosenhof). $\quad a^{1}, a^{2}$, wing pads; $m$, mask; st, spiracles.

FIG. 466.-Ephemera vulgata (from Schmarda). The caudal bristles incomplete.

females, sexual animals which have not completed the metamorphosis, but are in the wing-pad stage. The termites are able, by quantity and quality of food, to modify the development of the larva and to determine which type of individual shall be produced.

Allied to the 'Termites are the often wingless Psocid $ઋ$, or book lice, Troctes.* Other species are winged. Near here belong the Mallophaga, bird lice, which live upon mammals and especially on birds. Like true lice they are wingless, but have biting mouth parts. Trichodectes, * dog, ox, etc; Goniodes, * Nirmus, * etc, on birds. Sub Order II. AMPHIBIOTICA. The three families differ in structure, but agree in having aquatic larvæ with tracheal gills (fig. 453). All of these larve are predaceous; Odonate larvæ have a peculiar apparatus for capture of prey. The mentum and submentum of the labium are greatly elongate and when folded bring the tip like a mask beneath the mouth. 'The structure can be suddenly extended (fig. 465) and grasps the food. PERLIDE (Plecoptera); hind wings larger. Perla,* Pteronarcys.* EPHEMERID fore wings large, hinder small or absent; May flies. Ephemera* (fig. 466), Batisca.* Odonata (Libellulidæ), wings nearly equal, hinder slightly larger; dragon flies, reritable insect hawks destroying numberless mosquitos. Libcllula, ${ }^{*}$ Eschna, ${ }^{*}$ grion.* Sub Order III. PHYSOPODA (Thysanoptera). Wings slender, fringed with hairs; tarsi bladder-like at tip; mouth parts bristlelike, probably used for sucking. Position uncertain. Thrips.* 


\section{Order III. Orthoptera.}

Like the Archiptera these are hemimetaloolous (a few ametaloolous) and the mouth parts (fig. 444) are fitted for biting, the mentum cleft. On the other hand, the wings have lost the delicate membranous character and have become more parchment-like, the fore wings being smaller and serving as covers for the larger, softer, and folded hind wings, which are the organs of flight; the condition in these respects recalls somewhat the Coleoptera. The abdomen bears cerci and frequently stylets. In internal anatomy the large number of Malpighian tubules is noticeable (iig. 449).

Sub Order I. CURSORIA. With rather long legs fitted for rapid running. Only cockroaches (BLATTIDE) belong here. Wings may be absent, according to species, in either sex; more frequently in females. Blatta,* Periplaneta.* Sub Order II. DERMATOPTERA (Euplexoptera). Front wings short elytra; hind wings folded crosswise and packed beneath them, or rudimentary; cerci developed to a forceps-like structure; Forficula, * earwigs. Sub Order III. GRESSORIA. Legs long, slender, adapted to walking. MANTIDE, long prothorax bears a pair of long raptorial feet; praying Mantes. Phasmomantis.* PHAsulde, with short prothorax, almost exclusively tropical, Diapheromera, * walking stick. This family is noted for mimicry of twigs and leaves (fig. I2). Sub Order IV. SALTATORIA. Hinder legs long, strong, and for jumping; other pairs much smaller. Hinder femora large and muscular, tibiæ elongate and spined. Wings usually functional. Males produce sound (stridulate) by rubbing the anterior wings together (Locustidæ, Gryllidx) or against the legs (Acridiidæ). 'Tympanal apparatus (p. 4Io) on the anterior tibix (Locustide, fig. 45I, many Gryllidx) or on first somite of abdomen (fig. 450). Females readily recognized by the ovipositor. ACRIDIID.æ; antenna and ovipositor short; tympani abdominal. Acridium*; Melanoplus*; (Edipoda.* * Locustide; antennæ long; tympani on first tibiæ; ovipositor long, flattened. Hadenceus, * Anabrus,* wingless; Conocephalus*; Cyrtophilus* and Microcentrum, * katydids, GRYllide, Crickets: antennæ long; ovipositor long, cylindrical; tympani on first tibia. Cryllus*; Cecanthus, * tree crickets; Gryllotalpa $*$ mole crickets.

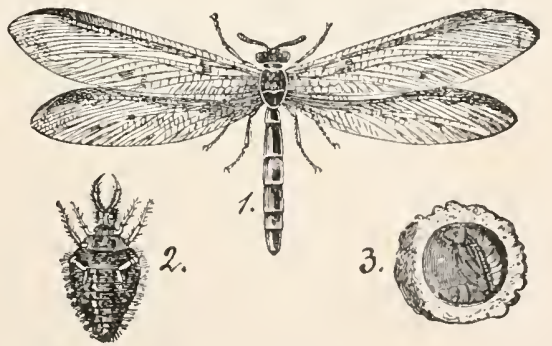

FIG. 467.-Myrmeleo formicarius (from Schmarda). I, imago; 2, larva; 3, pupa in its cocoon.

\section{Order IV. Neuroptera.}

The Neuroptera and Archiptera were formerly united, since they have the same wing structure and show in general appearance great similarities. 'Thus 
the ant lions (fig. $46_{7}$ ) recall the dragon flies; the Chrysopinæ, the Perlidæ. The Neuroptera, however, are holometabolous and have a resting stage, although the pupe (pupe libere) are capable of some motion.

Sub Order I. PLANIPENNIA. Biting mouth parts. Stalide, wings well developed, larvæ aquatic. Corydalis,* hellgrammite (fig. 468 ); Sialis.* Hemerobind, lace wings; wings well developed; larræ with sucking mouth parts, predaceous. Chrysopa* feeds on plant lice; Hyrmeleo, ${ }^{*}$ ant lions (fig. 467); larve dig pits and capture ants, etc., which fall into them. PANORPIDE

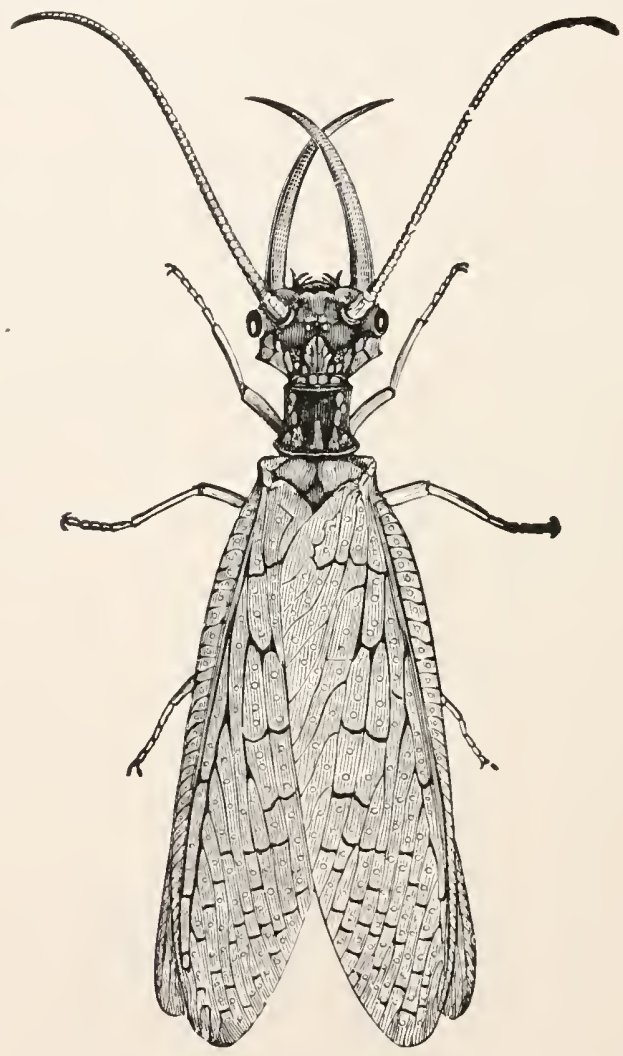

Fig. 468.-Corydalis cornutus,* hellgrammite, male (from Riley).

(Mecoptera); mouth prolonged into a rostrum; Bittacus.* Sub Order II. TRICHOPTERI (caddis flies). Wings usually large; mouth parts rudimentary, forming a short surcking tube which, with the wings covered with hair-like scales recalls the Lepidoptera; larve aquatic with tracheal gills; build cases of foreign matter, stones, sticks, etc., in which they live like a hermit crab. Phryganea, * Hydropsyche.*

\section{Order V. Strepsiptera.}

STYLOPID a are parasitic on Hymenoptera. The six-legged larvæ (fig. 469, 3) press in between the ventral abdominal plates of bees or wasps and pupate 
there. The quickly flying male (2) escapes from the pupal skin; it reca!ls somewhat a beetle; has rudimentary fore wings and large hinder ones. 'The wingless, legless female (I) remains in the pupal skin and is fertilized there; she is viviparous. Insects infested with these parasites are 'stylopized.' The affinities of the order are doubtful; they are frequently included with the beetles. Slylops, * Yenos.*

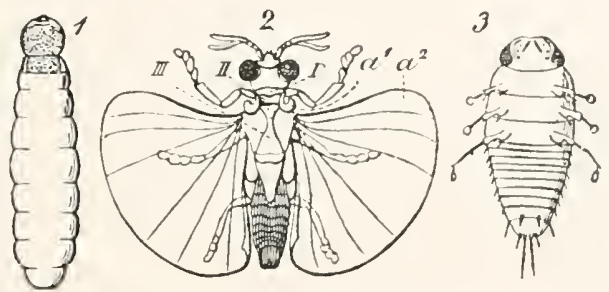

FIG. 469.-Xenos rossi (after Boas). I, female; 2 , male; 3 , larva; $I-I I I$, thoracic somites; $\mathrm{a}^{1}$, rudimentary fore wing; $a^{2}$, hind wing.

\section{Order VI. Coleoptera.}

The beetles are the highest Hexapoda with biting mouth parts. They are closest to the Orthoptera, as is shown by the structure of mouth parts and wings. The mandibles are strong; the maxilla (fig. 470) have lacinia and galea; the labium consists of a submentum (often called mentum), behind which the rudimentary mentum with its palpi, para-

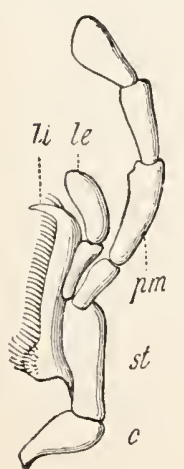

FIG. 470 .

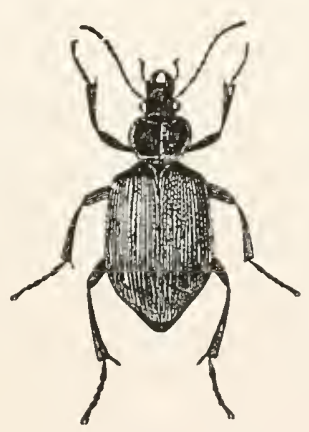

Fig. 47 I.

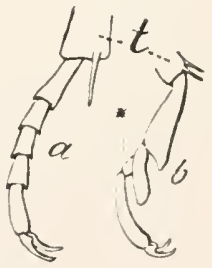

FIG. $47^{2}$.

Fig. 470.- Maxilla of Procrustes coriaceus. c, cardo: le, galea; li, lacinia: $p m$, palpus; st, stipes.

FIG. 47 I.-Calosoma sycophanta (after Ludwig-Leunis).

FIG. $472 .-a$, pentamerous tarsus of Dytiscus; $b$, cryptopentamerous tarsus of Coccinella; $t$, tibia; $*$, reduced tarsal joint.

glosse, and glosse (the latter frequently fused) are retracted. The group is distinguished from the Orthoptera by the holometabolous development with pupx libere, while the larve (fig. 458) show many modifications corresponding to the mode of life. Inother character is afforded by the 
wings. The anterior pair, separated at the base by a scutellum, are hard ely:ra not fitted for flight, and from these comes the name Coleoptera, sheath wings. Under the elytra are protected the delicate much folded hinder wings, the organs of flight. Since the second and third thoracic rings and those of the abdomen are covered by the elytra, these are soft above. Externally the relations of the elytra cause a regional division peculiar to the beetles (fig. 47I): head, prothorax, and a third division composed of meso- and metathorax plus abdomen covered by the elytra.

The numerous species of beetles-over 100,000 described-are divided into normal forms and Rhynchophora, the normal forms being subdivided upon characters derived from the tarsi as follows:

Sub Order I. PENTAMERA. Tarsus five-jointed, the last club-shaped and bearing the claws; the other four are short and somewhat heart-shaped (fig. $472, a$ ). This largest sub order contains the tiger beetles (CICINDELID.玉), the predaceous Carabide (fig. 47I); water beetles, HYDrophilide and DYTISCIDE; LAMELLICORNIA Or SCARABEIDE, represented by the 'June bugs,' Melolontha*; fire flies, Lampyride; rove beetles, Staphylinide, etc. Sub

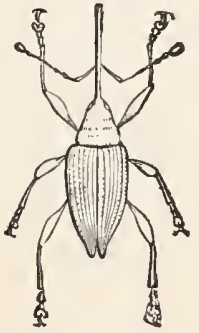

FIG. $473 .-B a-$ laninus nasicus, * hazel-nut weevil. Order II. HETEROMIERA. First and second legs pentamerous, third apparently four-jointed; few species; 'oil bottles' (MELOIDe) and the blister beetles, Cantharide, both containing a peculiar substance, cantharidin, which renders the 'Spanish flies,' an important ingredient of blistering plasters. TENEBRIONIDÆ.

Sub Order III. TETRAMERA (Cryptopentamera). Tarsi with the penult joint rudimentary, giving the impression of four joints (fig. $472, b$ ). The families numerous in species, are injurious to vegetation. The larvæ of CERAMBYCIDE bore in wood. The CHRYSOMELIDA. (Colorado potato beetle, Doryphora*) feed on leaves. Sub Order IV. TRIMERA; tarsi with penult and antipenult joints rudimentary, so that they appear three-jointed. CoccinelLid.e, lady birds, whose larva, because of feeding on plant lice, etc., are of value to man. Sub Order V. RHYNCHOPHORA, snout beetles; head produced into a long snout with mouth parts at apex. Here beleng weevils, which damage grain, nuts, timber, etc. Curculio,* Conotrachelus, * Calandra,* Balanimus* (fig. 473).

\section{Order VII. Hymenoptera.}

The Hymenoptera, of which bees, wasps, and ants are well-known representatives, have biting mandibles, while the other mouth parts are elongate and in a minority of the group converted into a sucking organ (p. 406). Since mouth parts vary, the wings and body segmentation have great value in defining the order. The wings are membranous and are supported by few nervures (fig. 474), and in light they act as one pair, since the two are usually connected by hooked bristles on the hind wing, which engage in a groove on the hinder margin of the front wing. The fore wings are the larger and, correspondingly, the mesothorax 
exceeds the other thoracic somites, so that these, especially the prothorax, seem but parts of the strong mesothorax. Besides, the first aldominal ring unites to the thorax so intimately in the Entophaga and Aculeata as to seem part of it. The constriction which then separates thorax and alsdo men comes between the first and second abdominal somites, and when the second (petiole) is elongate the stalked abdomen, familiar in the wasps, results.

The sexes are distinguished by the genital armature. The female is provided with the ovipositor already described (p. 413), which when used for this purpose only (terebra) permanently projects from the hinder end of the body (fig. 474), but when used as a sting (aculeus), can be drawn into the body when at rest. The sting, naturally lacking in the male, is connected with a

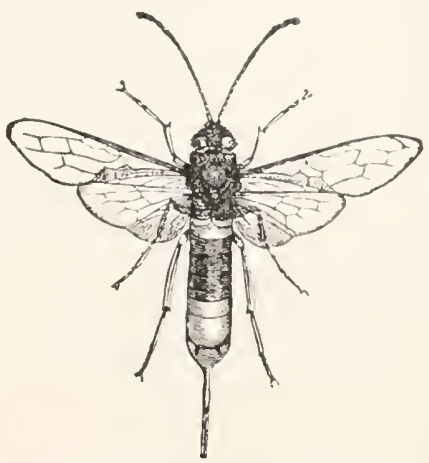

FIG. 474.-Sirex gigas, saw fly (after Taschenberg).

poison gland, the secretion of which owes its effect not, as once believed, to formic acid, but to a little known basic substance, possibly secreter in smaller accessory glands.

The distinction between terebra and aculeus affords characters of systematic importance; others are furnished by the development, which is always holometabolous. The pupæ, in all important points, are similar (pupæ liberæ), but two kinds of larvæ are distinguished. Some have well-developed legs. Others have footless larve (fig. 60). The first occur where the larva must shift

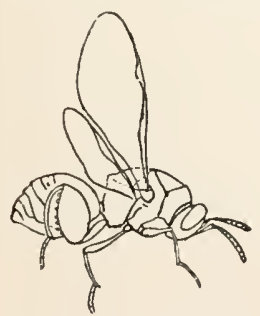

FIG. $475 .-$ Chalcis Alavipes* (after Howard). for itself, the second where it is surrounded by an abundance of food, either provided by the parents or by the host in which it is parasitic.

Sub Order I. TEREBRANTIA. Terebra present; larvæ with feet at least on the thorax; eggs laid on leaves or in wood, usually without gall formation; the larse therefore must move in order to feed. TENTEREDINID.E, saw flies, feed on leaves, larvæ caterpillar-like. Cimbex,** Nematus.* Siricide (Uroceridæ, fig. 47.4), horn tails, larve bore in wood. Sub Order II. ENTOPHAGA. Terebra present; larve legless, parasitic in galls or in animals. Some use the ovipositor to lay their eggs in plants. Galls are then produced, diseased structures by which the larva are nourished. Others lay their eggrs on or in other insects. The young feed on the host and at last cause its death, often before the completion of the metamorphosis. Gallproducing forms are the CYNIPIDÆ; some afford examples of heterogony (p. I32), the alternating generations distinguished by different structure, lyy sexual and parthenogenetic reproduction, and by different kinds of galls, and have frequently been described as different genera. The inquilines lay their eggs in the galls of other species. The insect parasites are divided among 
several families, the more prominent being the ICHNEUMONIDÆ, BRACONIDÆ,

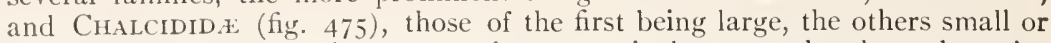
minute. These are of immense value to agriculture, as they keep down injurious forms as no economic entomologists or insecticides can.

Sub Order III. ACULEATA. Females with stings, larvæ footless, maggotlike. The digger wasps (Fossores) excavate tubes in earth or wood which they store with insects paralyzed by the sting, to serve as food for the larva. Some true wasps have similar habits. Most wasps (VESPARIE) and bees (APLARIE) build wonderful homes of chewed wood or leaves, earth, etc., or of wax which the animals (bees) secrete between the joints of the abdomen. The nests for the young, are either small tubes or hexagonal cells which are united to 'combs;' the food is either honey, pollen, or chewed fruits. The fact that the
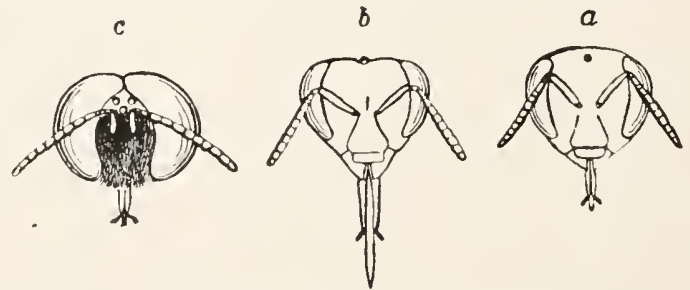

FIG. 476.-Heads of Apis mellifica (after Boas). $a$, queen; $b$, worker; $c$, drone with the compound eyes meeting above.

offspring are better protected when numerous individuals guard them has apparently led to different grades of social states. The honey bees ( $A$ pis mellifica*), which live in a colony, consist of three kinds of individuals distinguished by structure of the head (fig. $47^{6}$ ) and other features: a single queen, some hundred drones, and ten to thirty thousand, just before swarming even sixty thousand, workers. These last are females and hence have stings, but have rudimentary functionless sexual organs; their work being to build the home, to protect the young, and provide food for the winter, and for the young broodhoney and pollen. The queen copulates but once, at the beginning of her reign, when she and a drone take a wedding flight. For the four years of her life the sperm retains its vitality in the receptaculum seminis. In laying the eggs she can permit entrance or not of the spermatozoa at will and thus produce males or females. A queen who has not been fertilized can only lay drone eggs. The further fate of the eggs depends upon the food of the larvæ; with a small amount of bee bread (pollen) workers are produced, but the same larva placed in a larger cell and fed with the 'royal jelly' will develop into a sexually mature queen. Seven or eight days before the escape of a new queen from the roya! cell, the existing queen with a part of the hive, swarms to found a new colony. This operation may be repeated once or twice, but if there be danger of depleting the hive the remaining queen larve are killed. Wasp and bumble-bee colonies last but a year with us and are reformed by a fertilized female which has lived through the winter. In the tropics there are perennial colonies, like those of the bees.

The ants (ForvICARIÆ) have gone beyond the bees in the social organization. They have also departed most from the other Hymenoptera in that the workers, sometimes the sexual individuals, are wingless and the sting is rudimentary or entirely lacking. Only the Poneridæ and Myrmicidæ sting like bees and wasps; the others bite and squirt the secretion of the persistent poison gland (formic acid) into the wound. 'The homes of the ants are less wonderful 
than those of the bees, but their social organization is frequently more complicated. In the colony occur the wingless workers (rudimentary females with wing pads in larval life which are lost in pupation), and of these frequently there are different kinds, large-headed soldiers and small-headed workers; 'honey sacs' in $1 /$ yrmecocystus (fig. 477,1 ); and the sexual animals, queens and drones, which copulate in a marriage flight. The queens, after the flight found new colonies, in which, after biting off the wings, they enclose themselves in a royal chamber. 'They may (Dorylina), swell like the ternite queens so enormously that they were once regarded as different genera. There may lue a number of queens in a colony, and as swarming is not a necessity, a colony may
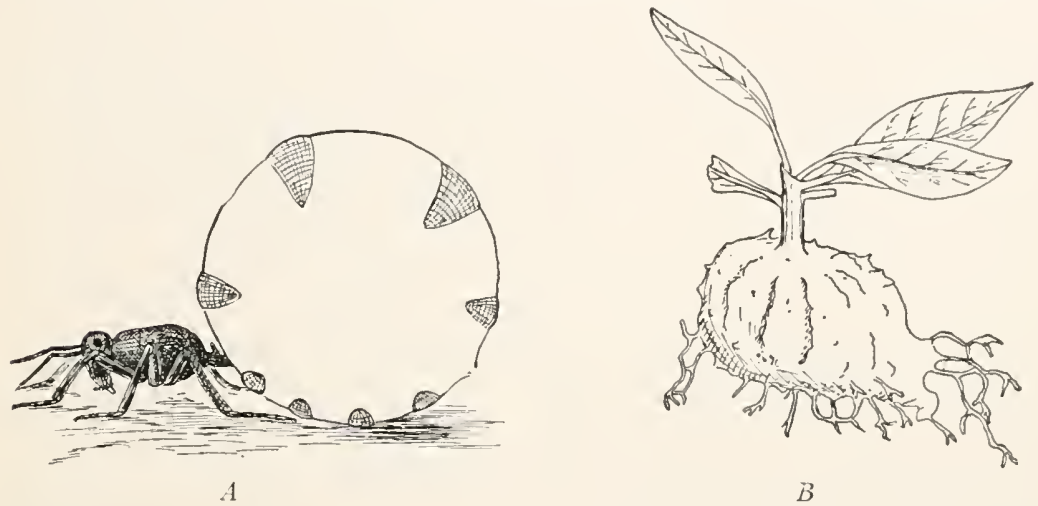

FIG. 477.- A, Myrmecocystus melliger,* honey-sac ant. (orig.) B. Plant of Hydnophyton (after Forbes) showing the bulb occupied by ants.

be enormous. It may send out other colonies which may retain relations with the mother colony, or may found a distinct state. Frequently other insects, like the Aphides, are kept for the honey dew they produce. IIany ants steal the pupx of others and, when the adults emerge, keep them as slaves. In Polyergus rufescens this has gone so far that the masters cannot care for themselves and must be fed by the slaves. The ants possess extreme interest on account of their carefully planned wars (Ecitons); on account of their relations to plants, some species making nests in the growing plant (fig. $477, B$ ) and protecting it by their bites; the leaf-cutting ants carry leaves into their underground nests for the cultivation of fungi on which they feed, the agricultural ants from their plantations and stores of grain, and the honey ants from the fact that certain workers (fig. $477, A$ ) act as reservoirs of honey, these 'honey sacs' swelling up to enormous size.

\section{Order VIII. Rhynchota.}

The Rhynchota, or bugs, in external appearance are nearest to the Archiptera and Orthoptera. The head, thorax, and abdomen are joined in the same way; the development is hemimetabolous (in the wingless species ametabolous). Confusion with the Orthoptera has led to the Cicadas with their membranous wings being called locusts, on the other hand, the delicate-winged Aphides resemble the Archiptera. 
Yet all Rhynchota may be recognized by the sucking proboscis (fig. 478), consisting of the grooved labium in which the needle-like mandibles

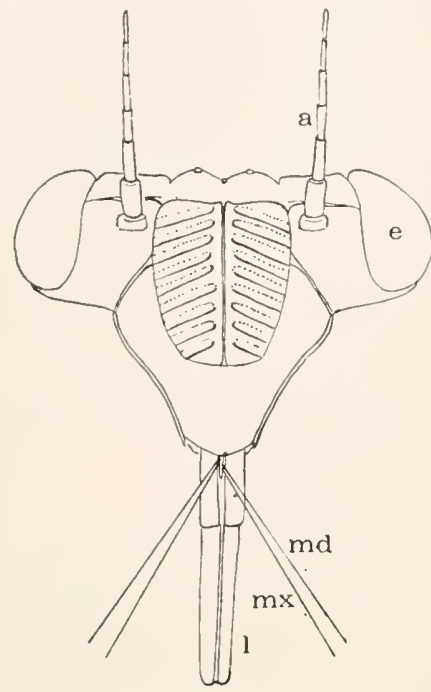

FIg, 478.-Head of Cicada septendecim, the mouth parts separated (orig.). $a$, antenna; $e$, compound eye; $l$, labium; $m d$, mandible; $m x$, maxilla. and maxilla play. The wing structures afford the basis of division into three sub orders.

Sub Order I. HEMIPTERA (Heteroptera). Anterior wings hemelytra, i.e., leathery at base, soft and elastic at tip (fig. 479); between the hemelytra is a conspicuous triangular scutellum $(s)$ covering more or less of the dorsal surface. Hemelytra and scutellum occasionally disappear. A further characteristic is the presence of stink glands, which open in adults ventrally on the metathorax; in larve dorsally on the abdomen. According to habits families nay be grouped into the aquatic HYDROCORES and the terrestrial GEOCORES Of the first the Belostomide are noticeable from their size, Belostoma amcricana* being nearly $2 \frac{1}{2}$ inches long. Other families are NEPID E (Ranatra,* water scorpion), Notonectide, HydroBATIDA, etc. Of the Geocores the REDUVIIDE, which feed on other insects; ACANTHHDE (Acanthia lectuaria, * bed bug); LyGæIDE, chinch bug, Blissus leucopterus, * injurious to grain; and PENTATOMID $£$, stink bugs, may be mentioned. Sub Order II. HOMOPTERA. Wings, when not degenerate, similar in texture throughout, although often differing in size. They are either parchment-like or delicate membranes. Frequently wax-like substances are secreted from dermal glands and cover the suiface like a down. The CICADID $\approx$, Cicada,* are noticeable from their shrill notes, produced by a drum on abdomen vibrated by muscles. CERCopide, the spittle bug (Aprophora*) causes drops of foam on grass. The leaf hoppers, or JASSIDA, contain some injurious forms, Erythrmura vitis* damaging the grape; the
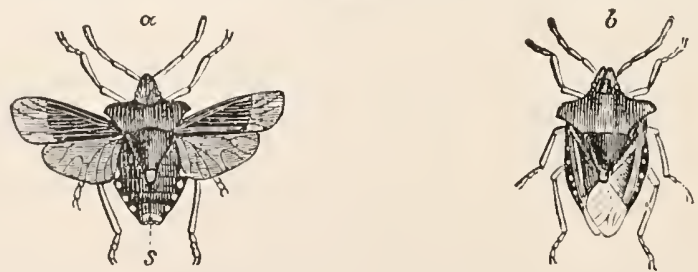

Fig. 479.-Pentatoma rufipes (from Iiajek). s, scutellum.

tree hoppers, MEMBracide (fig. $48 \mathrm{I}$ ), are scarcely less injurious. None are such serious pests as plant lice and scale insects. In the Coccide, or scale insects, the wingless female dies after laying the egrs and covers them with her dead scale-like body. Here belong the cochineal insects, Coccus cacti, * which furnish carmine, the lac insects, and a host of injurious forms, 

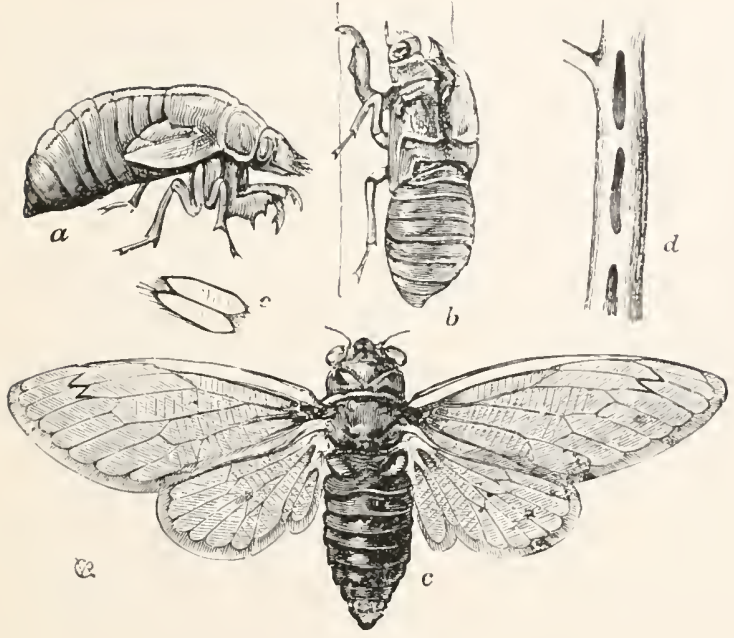

FIG. 4 So.

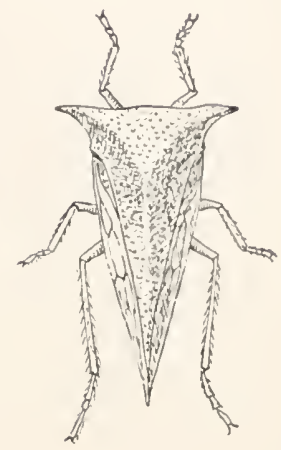

$48 r$.

FIG. 480 -Cicada septendecim,* seventeen year locust (from Riley). a, pupa; $b$, pupa case from which the imago, $c$, has escaped; $d$, twig bored for oviposition.

Fig. $4^{\mathrm{S}} \mathrm{I}$-Ceresa b: balus, * buffalo leaf hopper (after Marlatt).
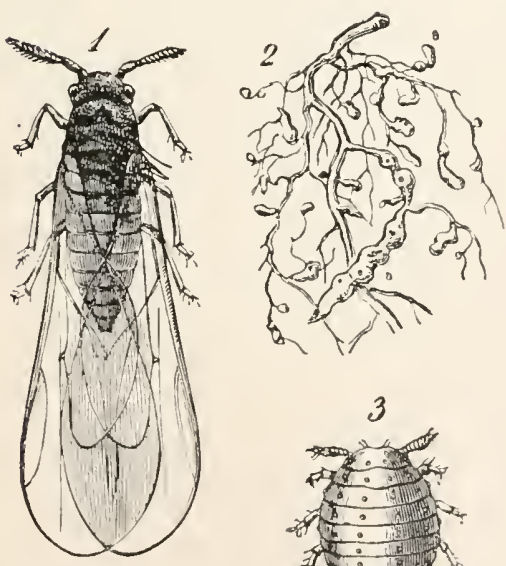

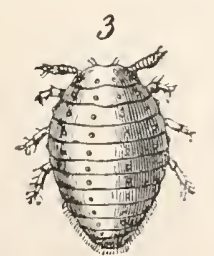

FIG. 482.

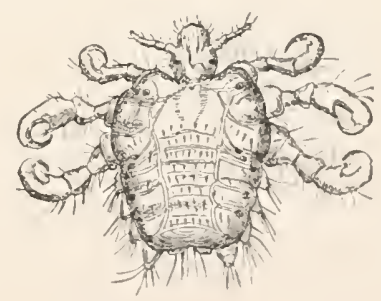

FIG. 483 .

FIs. 4S2.-Phylloxera vastatrix (from Ludwig-Leunis). I, winged generation; 2, grape root, with nodules $(a)$ caused by Phylloxera; 3 , wingless root-generation.

FIG. 483.-Phthirius inguinalis, crab louse (after Leuckart). 
like the orange scale, Aspidotus aurantii,* and the worse San José scale, $A$. ferniciosus, * recently spread through this country. The APHIDE, or plant lice, are soft-skinned and with their honey-containing excrement form a substratum for the growth of injurious fungi. 'They reproduce largely by parthenogenesis, but their spread is not rapid, since the usually viviparous females are wingless. At times winged females appear and spread the pests. Winged males appear in the autumn; the fertilized eggs endure the winter. None is more injurious than Phylloxera vastatrix* of the grape, which with us does slight damage, but in Europe has destroyed whole vineyards. Sub Order III. AP'TERA. Wingless bugs with direct development, commonly known as lice; three species attack man, one living in the hair (Pediculus eapitis*), the others ( $P$. vestimentorum* and Phthrmus inguinalis*) upon the body. Other species on other mammals.

\section{Order IX. Diptera.}

Like the Rhynchota, the Diptera, or flies, are sucking insects, but the haustellum is different, consisting of a tube, formed of labium and labrum, containing stylets which include, besides mandibles and maxilla (often rudimentary), the hypopharynx (fig. 446). Only the anterior wings (hence Diptera) are developed, the hinder wings being replaced by

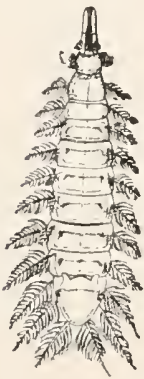

Fig. $484 .-$ Larva of Anthomyia canicularis(after Leuckart). the halteres or balancers, small drumstick-like structures richly supplied with nerves and functioning as organs of equilibration (fig. 486). The thorax is, as in the Hymenoptera, sharply marked off from head and abdomen, its somites frequently fused. 'The development is holometabolous, two kinds of larve and pupe occurring. The larve are always apodal, but have either a distinct head with biting mouth parts or they are headless and have a rudimentary sucking apparatus (fig. 484). The pupe are correspondingly either free with powers of motion, or are pupæ coarctate (p. 4i6). Development thus affords characters of systematic importance, and these are supplemented by differences in length of legs, antennæ, haustellum, and in body form. In number of species the Diptera stand next to the Coleoptera; in number of individuals they far exceed them.

Sub Order I. NEMOCERA. Elongate with long, many-jointed antennæ, long proboscis, long legs. The larvæ live in damp places or in water, where, lacking legs, they swim by movements of the body, capturing their prey with their strong mouth parts. The pupe also can swim well. The aquatic larve have two respiratory tubes at the end of the abdomen; in the pupæ they are on the back. Best known are the innocuous crane flies (TiPULID 2 ) and the mosquitos (CUl1C1D $\approx$ ) with their numerous species affecting man, among them, Stegomyia,* which carry yellow fever, and Anopheles, ${ }^{*}$ which distribute malaria. The CECIDOMYiD E include the injurious Hessian fly, Cecidomyia destructor, ${ }^{*}$ and the pædogenetic Miastor (fig. 456). Sub Order II. TANYSTOMA. Resemble the Muscariæ in the short stout bodies, short antennæ and legs. They resemble 
Nemocera in their long proboscis and in development. The larve and pupae live in damp places or in water and move rapidly, the larvae having liting mouth parts. Black flies, Simulidde, excel the mosquitos in viciousness; the horse flies, TABANide. Sub Order III. MLSCARIA (Brachycera). Body short, stout; antennæ three-jointed with a bristle (arista) (fig. $4 \delta_{5}$ ); legs short, cnding in an adhesive organ (pulvillus); larve headless, living in decaying sulstances or parasitic in other animals. Muscide; house flies ( $1 /$ uscu domestica* and

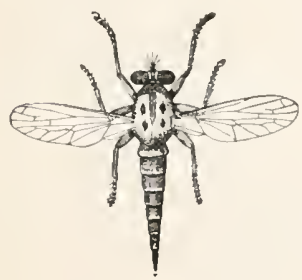

Fig. 485 .

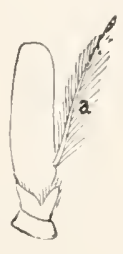

F1G. 485 .-Left, Erax bastardi, robber fly; right, antenna of Muscid showing

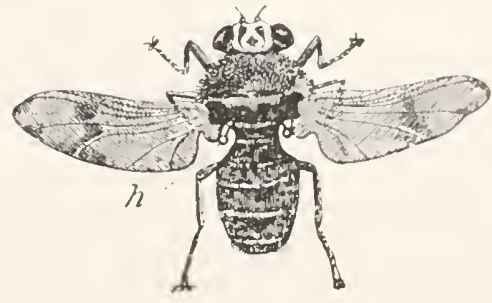

Fig. 486. arista at $a$.

FIG. 486.-Gastrophilus equi,* bot fy (from Hajek). $h$, halteres.

other species), blow fly (Calliphora vomitoria*), the flesh fly (Sarcophaga carnaria, ${ }^{*}$ viviparous). Many Muscida convey disease, the house fly carrying the germs of typhoid fever on its feet, while the tropical species of Glossina are responsible for the nagana disease of cattle and horses and for the sleeping sickness of man (p. I 84 ). AsIL1D E, robber flies (fig. 485) prey on other insects, as do some SYRPHIDÆ: Eristalis.* CESTRIDÆ, bot flies; the larvæ always parasitic; those of the sheep bot (Estrus ozis*) in the frontal sinuses of the sheep, those of the ox warble (Hypoderma lineata*) just beneath the skin of cattle; those of Gastrophilus equi* (fig. 486), in the stomach of horse. In the tropics Dermatobia noxialis lives as a larva in the human skin. Sub Order IV'. PUPIPARA. Very active, often wingless forms living as parasites on mammals and insects; Iarval development inside the mother; pupation occurring soon after birth. Melsphagus ovinus, * sheep tick; Braula caca, bee louse.

\section{Order X. Aphaniptera (Siphonaptera).}

In spite of the lack of wings the fleas are closely related to the Diptera, since they have doubtless descended from winged forms, as they have a holometabolous development. The larve, long and footless, live in decaying wood or dust in cracks in the floor, etc., and give rise to pupx, both without traces of wings. Yet fleas and flies differ in that fleas have similar body somites and lack the haustellum, the sucking tube being formed of labrum and mandibles, while the sharp maxilla puncture the skin. Besides Pulex irritans, * the flea that attacks man, many other species occur

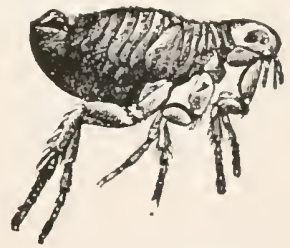

F1G. 487.-Pulex irritans, * Hea (from Blanchard).

on other animals. Fleas are now known to carry diseases, among them the bubonic plague. In warm countries the jigger or chigoe, Sarcopsylla 
penetrans,* attacks man, the female boring into the skin and there laying the eggs.

\section{Order XI. Lepidoptera.}

This group of butterflies and moths is the most sharply limited of any oider of Ilexapods. The wings, both pairs of which are well developed (rarely lacking, as in many female Psychidæ and some Geometridæ), are covered with scales (flattened hairs), and to these are due the frequently brilliant color patterns. Frequently the fore and hind wings are united by hooks. The mesothorax is large and the smaller pro- and metathorax are closely united to it, giving the region a distinctness from head or abdomen. The mouth parts are peculiar (fig. 44 $)$ ), although foreshadowed in the Phryganids, and not fully dereloped in the Microlepidoptera. The mandibles are rudimentary or absent, while the fused maxilli, greatly elongate, form the proboscis. The development is holometabolous; the

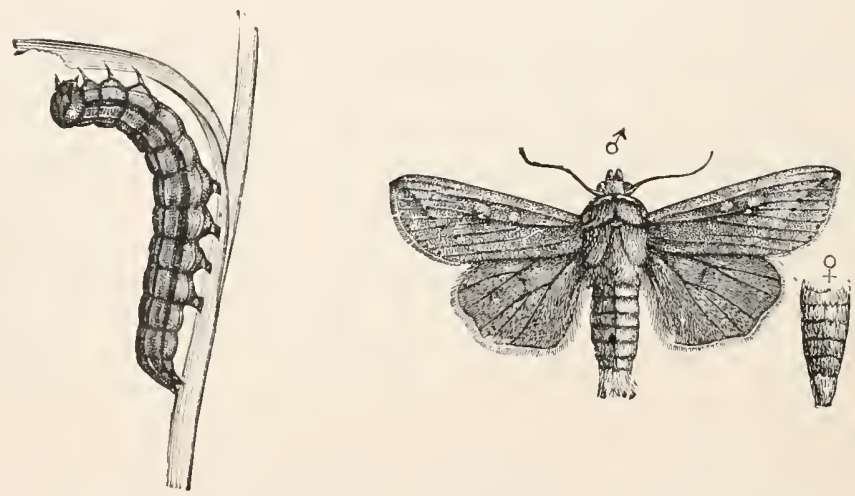

FIG. 483.--Leucania unipunctata, army-worm and moth (from Riley).

larve, frequently called caterpillars (fig. 460), have biting mouth parts, the mandibles very strong; and also a pair of silk glands (sericteria), which open on the labium and produce a secretion hardening to silk; besides the thoracic legs, prolegs (two to five pairs) are present. The pupx are usually pupx obtectax, sometimes ornamented with golden spots, whence the name chrysalides often applied to them.

Sub Order I. MICROLEPIDOPTERA. Small, inconspicuous; at rest holding the wings horizontally over the back. TiNEIDE; the larvæ form a tube of the food material which they carry around with them. Tinea pellionella, * the clothes moth. TORTRICIDE; the larve roll leaves into a tube. Carpocapsa pomonella, ${ }^{*}$ the codlin moth of apples. Sub Order II. GEOMETRINA. Moths slender, wings in pattern and shape recalling those of butterflies, but held horizontally when at rest; 'tongue' (proboscis) small; larve with two, rarely 
three, prolegs, known as measuring worms from their gait. Species numerous. Canker worms (Paleacrita vernata, 1 los shila pometaria, ${ }^{*}$ females wingless).

Sub Order III. NOCTUIN. . Owlet moths; with short bodies; fore wings usually gray and ornamented by two spots and zigzag lines which at rest cover the frequently (as in Catocula ${ }^{*}$ ) brightly colored hind wings; 1800 species in U. S. IH ypena humuli, hop worm: Aletia argillacea, cotton worm; Leucania unipunctata, * army worm; cut worms. Sub Order IV. BOMB lCINA, silk worms. Body large, woolly, usually broad dull-colored wings; occasionally lacking in females; proboscis frequently rudimentary; antenn long, pectinate; larva with well-developed spinning powers. Most important is the silk worm (Bombyx mori*). native of China; others, like Telea polyphemus, * furnish silk of

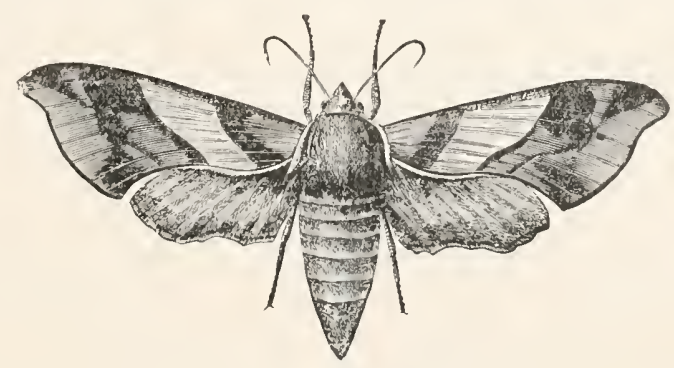

FIG. 4S9.-Lveryx myron (from Riley).

value. Many damage forest trees, among them the tent caterpillars (Clisiocampa*) and the imported gipsy moth Ocneria dispar (fig. 72 ). Sub Order V. SPHINGINA. Hawk moths (fig. 489 ), body long, stout; fore wings long, slender, hind wings shorter; proboscis very long; antennæ short; larvæ naked, with a caudal spine (fig. 459). Phlegethontius, * tomato and tobacco worms. SESIIDe, 'clear wings,' resemble bees and wasps.

Sub Order VI. RHOPALOCERA, butterflies. Body slender; wings held vertically when at rest, proboscis long; antennæ clubbed at the tip; larra usually spiny; punæ hung by a thread, never a cocoon. Species numerous. I'anessa antippa* lives over winter; Pieris* attack cabbages, etc.; Papilio, * swallow tails.

\section{Class V. Diplopoda (Chilognatha).}

The Diplopoda are usually united with the Chilopoda in a group of Myriapoda; but while they agree in having a head followed by numerous foot-bearing segments, they differ so greatly that no union is possible. The body is nearly cylindrical, although in Polydesmids lateral outgrowths give it a flattened appearance; the legs are close together on the ventral surface, with the tracheal openings near them, while on the sides of the body are other openings of defensive glands, the foramina repugnatoria (fig. 490). Each segment of the body except the first four or five bears two pairs of appendages, which, with a similar duplicity in chambers of the heart, trachex, ganglia, etc., shows that a fusion has occurred. The antcrior somites bear at most but a single pair of legs; both legs and antennix are short. The head bears, besides the antennx, but two pairs 
of appendages, a pair of several-jointed mandibles (fig. 49I), and a pair of rudimentary maxillie fused to a single plate, the gnathochilarium.

The gonads lie ventral to the intestine far back in the body, those of the right and left sides enclosed in a single sac; the ducts open separately on the second

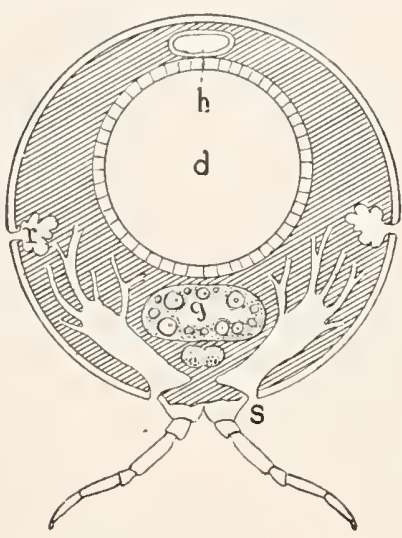

FIG. 490.

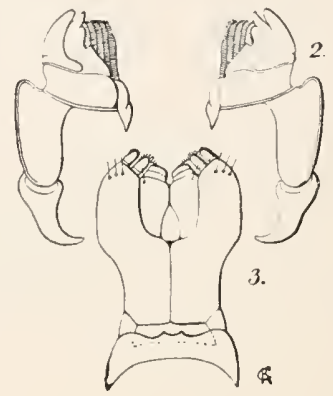

F1G. 49I.

Fig. 490.-Schematic section of Diplopod (compare with fig. 439). (l, digestive tract; $g$, gonad; $h$, heart; $r$, repugnatorial gland; $s$, spiracle and tracher.

Fig. 49I.- Mouth parts of Iulus (after Latzel). 2, mandibles of I. molybdinus; 3, gnathochilarium (fused maxillæ) of I. luridus.

somite of the trunk. The spermatozoa are not motile. The legs of the serenth segment of the male are used in copulation. The young escape from the egg with three pairs of leys, a point once thought to show resemblances to the Hexapoda, but which does not, for these legs are on the fourth, sixth, and seventh somites of the body. IULID s, elongate cylindrical bodies; Spirobolus.*

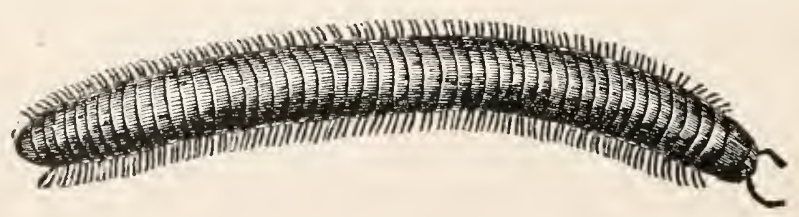

F1G. 492.-Iulus maximus (after Schmarda).

Glomeride short, capable of rolling into a ball; Polydesmide. Pauropoda: minute; body with twelve segments, tending to fuse to six. Pauropus.* More uncertain in position are the SrMPHYLA (Scolopendrella*); from the position of the genital opening they are placed here.

\section{S.ummary of Important Facts.}

I. The ARTHROPODA are animals with evident internal and external segmentation (metamerism).

2. The metamerism is expressed internally in the muscles, in the ladder-like nervous system, in the structure of the heart, and in the arrangement of segmental organs and tracher so far as these are present. 


\section{ARTHROPODE, SLMMARY OF IMPORTANT FACIS, 43j}

3. The outer segmentation is expressed in the rings of the chitinous coat of the body as well as in the metameric arrangement of the appendages.

4. From the similarly metameric Annelida the Arthropoda are distinguished by the presence of jointed appendages, at most a pair to a somite. The appendages may be divided according to function into antenne, jaws, accessory jaws, feet, and swimmerets.

5. A further distinction is the grouping of the somites into regions of which usually head, thorax, and abdomen are recognized.

6. The head bears the tactile and eating appendages; the thorax those used in locomotion (pereiopoda), the abdomen the swimmerets (pleopoda), or it lacks appendages.

7. By fusion of head and thorax a cephalothorax is produced; a postabdomen may be separated from the abdomen.

8. The eyes are either ocelli or compound eyes.

9. Hermaphroditism is rare; reproduction is by eggs; frequently there is parthenogenesis, rarely padogenesis. The eggs usually have a superficial segmentation.

ı. The Arthropoda are divided into Crustacea, Acerata, Malacopoda, Insecta, and Diplopoda.

I I. The CRUstaceA respire by gills; they usually have two pairs of antennx, and usually biramous feet; the reproductive ducts open near the middle of the body.

I 2. The Crustacea are divided into Trilobitæ, Phyllopoda, Copepoda, Ostracoda, Cirripedia, and Malacostraca.

I3. Phyllopoda, Copepoda, Ostracoda, and Cirripedia are frequently called Entomostraca; they have a shell gland and the nauplius as a larval stage.

I4. The Trilobita are extinct forms with one pair of antennæ, and the body divided by longitudinal grooves into three regions.

I5. The Phyllopoda have variable segments and primitive leaflike feet recalling the parapodia of the annelids.

I6. The Copepoda are without shells and have biramous feet.

I7. The Ostracoda have reduced bodies enclosed in a bivalve shell.

I8. The Cirripedia are usually hermaphroditic and are sessile.

I 9. The Malacostraca have 20 (2I) segments, of which 7 (8) are abdominal; the male sexual openings are on the 13 th, the female on the IIth, segment; the excretory organ is the antennal gland; the larra is a zoea, rarely a nauplius.

20. The Malacostraca are divided into Leptostraca, Thoracostraca, and Arthrostraca. 
2I. The Leptostraca have twenty-one somites; they are closely related to the Phyllopoda.

22. The Thoracostraca or Podophthalmia (Schizopoda, Stomatopoda, Decapoda) have stalked eyes and some or all of the thoracic somites fused with the hearl to a cephalothorax.

23. The A rthrostraca or Edrioph thalmia have sessile eyes and have seven free thoracic segments. They are divided into Amphipoda and Isopoda.

24. The ACERATA lack antenne; the body is divided into cephalothorax and alylomen; the cephalothorax bears six pairs of appendages; the genital ducts open on the seventh somite; the respiratory organs-gills, lungs, or trachea-develop, in connection with the abdominal appendages.

25. The Acerata are divided into Gigantostraca and Arachnida.

26. The Gigantostraca are large, and breathe by gills. The only living forms are Xiphosures.

27. The Arachnida breathe by lungs or by trachex derived from lungs, the openings to which are on the abdomen; they have a pair of cheliceræ, a pair of pedipalpi, and four pairs of legs; they have in addition several pairs of highly developed ocelli.

28. The Arachnida are divided into nine orders: Scorpionida, Phrynoidea, Microthelyphonida, Solpugida, Pseudoscorpii: Phalangida, Araneina, Acarina, and Linguatulida.

29. The Scorpionida have chelate pedipalpi and a postabdomen terminated by a sting.

30. The Phrynoidea have the first pair of legs tactile and not used in walking, and a continuous cephalothorax.

3r. The Microthelyphonida and the Solpugida have three 'thoracic' segments free. The Microthelyphonida have a long, jointed postabdomen, lacking in the Solpugida.

32. The Pseudoscorpii resemble the Scorpionida, but lack the postabdomen and sting.

33. The Phalangida have very long legs and spider-like bodies.

34. The Araneina have an unsegmented abdomen, bearing four or six spinnerets and numerous spinning glands. They are divided into Tetrapneumones, with four lungs, and Dipneumones, with two lungs and two trachea.

35. The Acarina have cephalothorax and abdomen fused and the mouth parts for sucking. Several species are parasitic on man.

36. The Linguatu? ida are complete parasites, ribbon-like and without legs; the young live in the lungs and liver.

37. The Tardigrada and Pycnogonida agree with the Arachnida in the number of walking legs. Their position is very uncertain. 
38. The Malacopoda are intermediate between Annelida and Insecta. 'They have indistinctly segmented bodies with parapodialike feet, segmental organs, and tracheæ.

39. The INSEcta breathe by tracheac; the head bears four pairs of appendages: antennæ, mandibles, maxillæ, labium; since trachex are present the circulatory system is reduced; the reproductive organs open at the hind end of the body.

40. The Insecta are divided into Chilopoda and Hexapoda.

4I. The Chilopoda have numerous body segments with a pair of legs on each; behind the head are a pair of poison feet.

42. The Hexapoda have the body divided into head, thorax, and abdomen.

43. The abdomen consists of a varying number of somites and lacks evident appendages.

44. The thorax consists of three segments, pro-, meso-, and metathorax, each bearing a pair of legs, and meso- and metathorax usually a pair of wings each.

45. The head bears, besides the mouth parts and antenna, an unpaired upper lip (labrum); two compound eyes, and usually one to three ocelli.

46. The structure of the mouth parts varies with the food; they are either biting, licking and sucking, or piercing in function.

47. Wingless insects usually have a direct (ametabolous) development with numerous ecdyses.

48. Winged insects (and many without wings have descended from winged forms) have a metamorphosis in which the larva differs more or less from the imago (metabolous insects); the larva never has wings.

49. An incomplete metamorphosis (hemimetabolous development) occurs when the larva with each molt becomes more like the adult, the wing pads becoming larger with each ecdysis.

50. In complete metamorphosis (holometabolous development) the changes occur in the last molting stage, which is a stage of rest, the pupa.

51. Classification of Hexapoda is based upon structure of mouth parts and wings as well as upon regional relations and development.

52. The Apterygota are wingless, ametabolous Hexapoda with biting mouth parts.

53. The Archiptera have biting mouth parts with incompletely fused labium, net-veined wings, and incomplete metamorphosis.

54. The Orthoptera resemble the Archiptera in mouth parts and development, but have parchment-like wings. 
55. The Neuroptera have net-veined wings and a holometabolous development; the mouth parts are modified.

56. The Coleoptera are biting insects with the fore wings changed to elytra; they differ from the somewhat similar Orthoptera by the complete metamorphosis.

57. The Strepsiptera are parasitic forms allied to the Coleoptera.

58. The Hymenoptera have partly biting, partly licking mouth parts; membranous wings with few nervures and holometabolous development.

59. The Rhynchota are hemimetabolous or ametabolous, with piercing mouth parts; the bed bugs and the Pediculina are parasitic.

6o. The Diptera are holometabolous, with piercing mouth parts and not more than one pair of wings. The larve of the Estridie are parasitic.

6. The Aphaniptera are holometabolous, wingless, parasitic, with sucking mouth parts.

62. The Lepidoptera have the wings corered with scales; labium and labrum rudimentary, the maxillæ altered to a sucking tube; the development holometabolous.

63. The Diplopoda have a head with three pairs of appendages; the trunk with double segments, each bearing two pairs of legs, the genital openings anterior.

64. The term Myriapoda is frequently used to include Chilopoda and Diplopoda.

\section{PHYLUM VIII. CHORDĄTA.}

Within recent years it has been realized that a number of animals, formerly distributed among various groups, possess structural features of great importance which ally them to the vertebrates; but they lack the vertebra and many other features characteristic of that group, so that the name cannot be extended to embrace them. Yet since all possess, as a temporary or a permanent feature, a structure known as the chorda dorsalis or notochord, the term Chordata has been adopted to include them. The notochord is an elastic rod arising from the entoderm and coming to lie between the digestive tract and the nervous system (fig. 9).

In all Chordates the anterior (pharyngeal) portion of the alimentary canal develops several pairs of pockets which grow outwards and fuse with the ectoderm. The fused portion then breaks through, and the pockets become converted into gill slits (branchial clefts), which, in the lower forms, allow the passage of water over the gills which line them.

The central nervous system lies on one side of the alimentary canal, 
there being no such nervous ring (Enteropneusta excepted) around the nesophagus, as is common in the invertebrata. This nervous system arises as a medullary plate on the dorsal side of the embryo around the blastopore. The edges of this plate are rolled inwards, converting it into a tube with nervous walls and a central canal. From this, as will readily be seen, when the blastopore remains open behind (fig. 500, ne), a temporary communication, the neurenteric canal, exists between the neural and alimentary canals.

On the other hand the chordates share with the annelids and arthropods a segmentation of the body which, however, is internal and only exceptionally is visible from the surface.

The Chordates include the Leptocardii, the Tunicata, doubtfully a group of Enteropneusta, and the Vertebrata.

\section{Sub Phylum I. Leptocardil (Cephalochordia, Acrania).}

The Leptocardii contains a few very similar forms. One of these, originally described as a mollusc, is comparatively simple in structure. The fish-like body, pointed at both ends (whence Amphioxus) lacks paired appendages but has a median fold, developed into a fin at the caudal end (fig. 493), and there is a ventral longitudinal fold on either

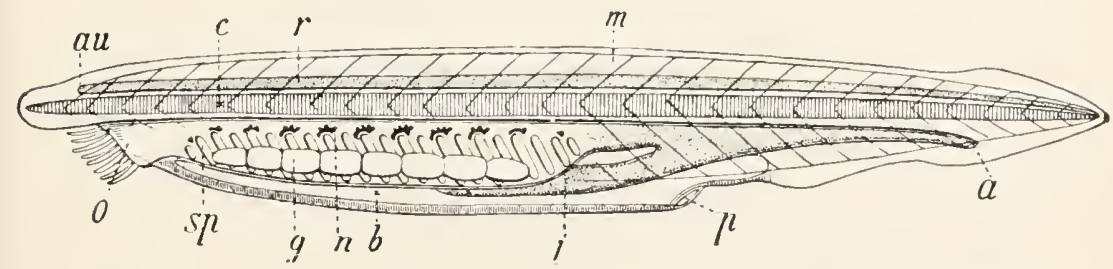

FIG. 493.-Amphioxus lanceolatus (diagram after Boveri). $a$, anus; $a$, eye; $b$, peribranchial space; $c$, notochord; $g$, gonads; $l$, liver; $m$, muscles; $n$, nephridia; $o$, mouth; $p$, atrial opening; $r$, spinal cord; $s p$, gill slits.

side of the peribranchial chamber (fig. 494). The epidermis is but a single cell in thickness, paralleled only in invertebrates, and through it the underlying muscle segments can be seen. Amphioxus differs from the fishes in the lack of skull (Acrania), vertebræ, brain, heart, and kidneys, although excretory organs and the rudiments of a brain are present. Connective tissue is scanty; the body is largely a much folded epithelium, separated by thin gelatinous layers, into which cells wander, the beginnings of a mesenchyme.

The notochord, which extends the length of the body (fig. 493, c) is the axial skeleton. Above it is a tubular spinal cord, expanded in front 
to a rudimentary cerebral vesicle, with a pigment spot, the rudimentary eye $(a u)$, but other places in the spinal cord are sensitive to light. The neural canal long remains open in front, the opening (neuropore) being at the bottom of a pit, once regarded as an olfactory organ.

The alimentary canal begins with the oval mouth $(o)$, surrounded by cirri; then comes a fold (velum), followed by the pharynx, perforated by numerous gill clefts, which extend over a third of the alimentary canal. Between the clefts are elastic gill arches (fig. $494, k b$ ) to support the walls. In the young the clefts open directly to the exterior; then a fold grows

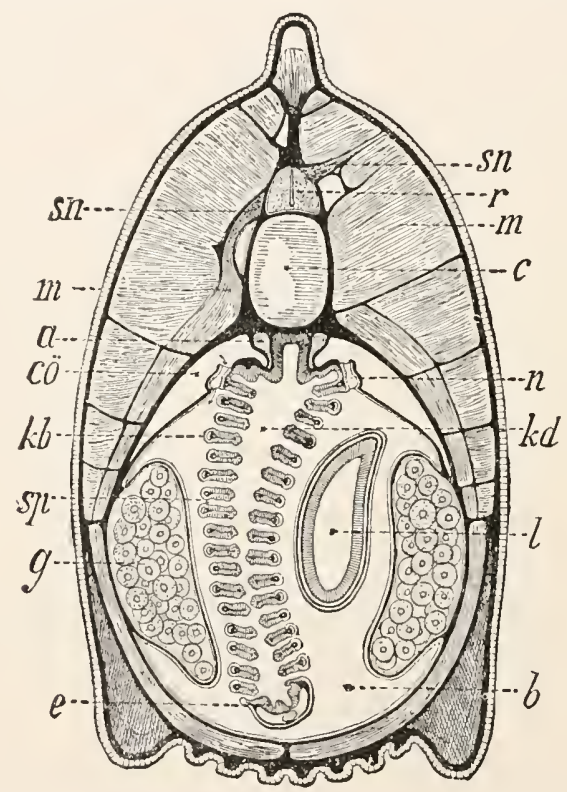

Fig. 494.-Section of the gill region of Amphioxus (after Lankester and Boveri). $a$, aorta descendens; $b$, peribranchial space; $c$, notochord; $c \ddot{o}$, colom (branchial body cavity); $e$, hypobranchial groove, beneath it the aorta ascendens; $g$, gonad; $k b$, gill arches; $k d$, pharynx; $l$, liver; $m$, muscles; $n$, nephridia, on the left with an arrow; $r$, spinal cord; sn, spinal nerve; $s p$, gill slit.

down on either side, the two folds uniting below to enclose a peribranchial chamber $(b)$ from which the water escapes by an opening, atriopore (493,), behind the middle of the body. On the floor of the pharynx is the hypobranchial groove, a ciliated tract which conducts food to the digestive part of the canal. This ends at the anus (a), on the left side of the body and has a liver connected with it in front, extending into the peribranchial chamber (figs. 493, 49., b). The vascular system, with colorless blood, consists of a dorsal arterial $(a)$ and a ventral venous trunk connected by lateral loops 
or arches. The ventral trunk begins as a subintestinal vein under the intestine, branches as a portal vein over the liver and, reuniting again in a ventral vessel, and joined by the paired veins (jugulars, cardinals and Cuvierian ducts) continues forward, as the aorta ascendens, below the gills. From this the gill arteries pass up between the gill slits and form the dorsal ressel, the aorta descendens. A true heart is lacking, but various parts of the ressels-a part of the ventral trunk and the bases of the gill arteries-are contractile, whence the name Leptocardii.

The digestive portion of the tract lies in a true colom, which extends forward (fig. 494, cö) into the gill-walls (branchial calom) and into the outer walls of the peribranchial chamber (peribranchial colom). In a distinct part of the peribranchial colom are the gonads $(g)$, a series of pouch-like cell folli-
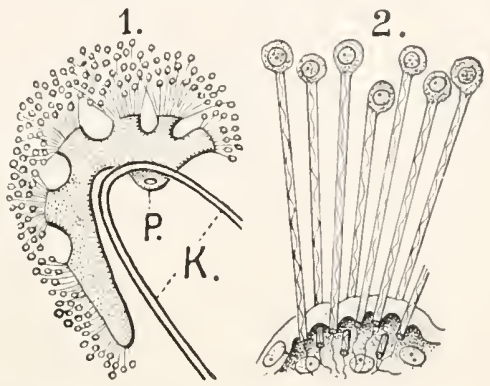

FIG. 495.-Excretory tubules of $\mathrm{Am}$ phioxus (after Boveri and Goodrich). I, a whole canal with several nephrostomes and the connected bunches of solenocytes. $K$, upper end of gill cleft; $P$, opening of canal into peribranchial chamber. 2 , a bit of canal wall with several solenocytes. cles which allow their products to escape into the peribranchial chamber. Into this chamber also empty the excretory organs $(n)$, a series, on right and left sides, of ciliated canals. Each canal begins with at least one ciliated nephrostome in the cœlom and opens separately and has the characteristic solenocytes like an annelid nephridium (fig. 495).

Like the structure, the development is comparatively simple. The following points deserve special mention: (I) The eggs have a nearly equal segmentation (fig. Ior). (2) A typical invaginate gastrula (fig. 107) occurs. (3) The mesoderm arises as a series of pouches, right and left, from the mesenteron, which later separate and form the primitive segments. Hence these are clearly mesothelial in nature. From the cavities of these arises the body cavity, which is conse quently an enterocœle. (4) The dorsal surface of the entoderm between these cœlomic pouches separates from the rest and forms the notochord, which lies between the digestive tract and the nervous system. (5) The nervous system arises from a longitudinal groove which becomes folded into a tube and is connected for a while with the digestive tract by a neurenteric canal.

Amphioxus,* Asymmetron,* Heteropleuron. The animals bury themselves in the sand, with only the mouth above the surface.

\section{Sub Phylum II. Tunicata (Urochorda).}

The adult Tunicata, or sea-squirts, bear some resemblance to the clams in the possession of a mantle and incurrent and excurrent orifices, usually close together. Hence they were long called molluscs; later they 
were grouped with the worms, but their development shows them to be more nearly related to the vertebrates.

The name is due to the tunic or mantle-lacking in the Copelatran envelope (fig. $+96, t$ ) formed, like a cuticle, by the epithelium of the skin, but distinguished from ordinary cuticula by its structure. It resembles connective tissue in that cells from the mesoderm wander into the ground substance, which is sometimes fibrous, sometimes homogeneous, and has an interesting chemical nature. It has the same chemical composition

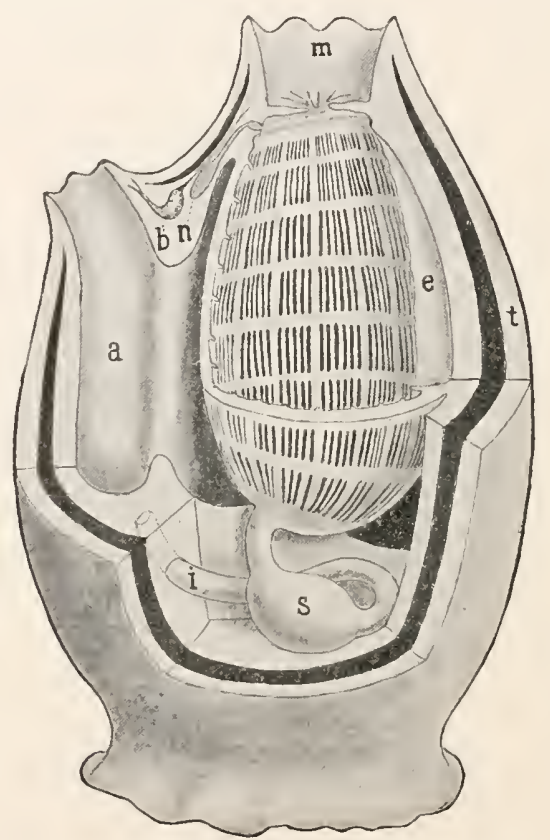

FIG. 496.- Diagram of a tunicate (orig.). $a$, atrium; $b$, nervous ganglion; $e$, endostyle; $i$, intestine; $m$, mouth; $n$, subneural gland; $s$, stomach; $t$, tunic. In the centre the branchial basket with the gill slits communicating with the peribranchial space, and this in turn with the atrium.

$\left(\mathrm{C}_{6} \mathrm{H}_{10} \mathrm{O}_{\overline{5}}\right)$ as cellulose and agrees with this substance, so characteristic of plants, in its reactions. No other animals have so much cellulose.

The anterior part of the digestive tract is modified into a pharynx or branchial chamber, the walls of which are perforated with a varying number of gill slits, these leading either directly to the exterior or, more usually, into a peribranchial chamber, and from this to a cloaca or atrium (a), before reaching the outside world. While the respiratory water passes through the gill slits the food particles which it contains are re- 
ceived by a ring-shaped ciliated band (peripharyngeal band) and, enveloped by mucus, are led to the osophagus. This mucus is formed by a ciliated glandular groove, the endostyle (e) or hypobranchial groove, on the ventral surface of the pharynx.

Between the end of the endostyle and the stomach lies the ventral heart enclosed in a pericardium. It has the peculiarity, met nowhere else, of changing the direction of its contractions at frequent intervals; after the heart has driven the blood for a time to the gills it stops and then forces the blood in the opposite direction, pumping it from the gills towards the stomach. If we add to the foregoing that a dorsal ganglion and a usually hermaphroditic gonad are present, the striking features of the group are enumerated. The extreme forms, the Copelatx and the Thaliacea, are rather remote, but they are connected by intermediate forms, the Ascidie and Pyrosomas.

\section{Order I. Copelatæ.}

These small forms-one or a few centimeters in length-are pelagic; they have the anterior end inserted in a gelatinous envelope or 'house' which replaces the lacking tunic and which they may leave without injury. They swim like a tadpole by means of a tail which arises from the hinder end of the trunk. The alimentary canal (fig. 497) is bent on itself, and both it and the two large gill slits, in contrast to all other tunicates, open directly to the exterior. The nervous system consists of a cerebral ganglion, with beside it a very simple statocyst and a ciliated groove, and farther a chain of ganglia extending into the tail. The gelatinous notochord, enclosed by a sheath of cells, forms the skeletal axis of the tail ventral to the nerve cord and gives attachment to muscles. Oikopleura, * Appendicularia.*

\section{Order II. Tethyoidea (Ascidiæformes).}

With the exception of the pelagic Pyrosomidx all of the ascidians are attached to rocks, etc., in the sea. The necessity for protection caused by this sedentary life has resulted in a great development of the cellulose tunic or test, which gives these animals a swollen, somewhat shapeless appearance. Two openings, mouth and atrial opening, lead into the interior, and the water which issues from these, when the animals are taken from the ocean, has given them the common name of 'sea-squirts.'

On removing the tunic, which is but slightly attached to the other parts except at mouth and atrial opening, a muscular sac is seen (fig. 498), the fibres running circularly and longitudinally. Inside this sac are the viscera, the pharyngeal region by far the most conspicuous. The mouth leads to a short tube with tentacles $(t)$, and then to the pharynx, a wicle sac which lies in a large peribranchial chamber, the walls of the pharymx and the enclosing space uniting on the ventral side (fig. 496). The pharyngeal walls are perforated like a net by small ciliated gill slits, 
4

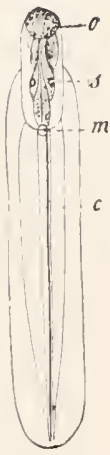

$B$

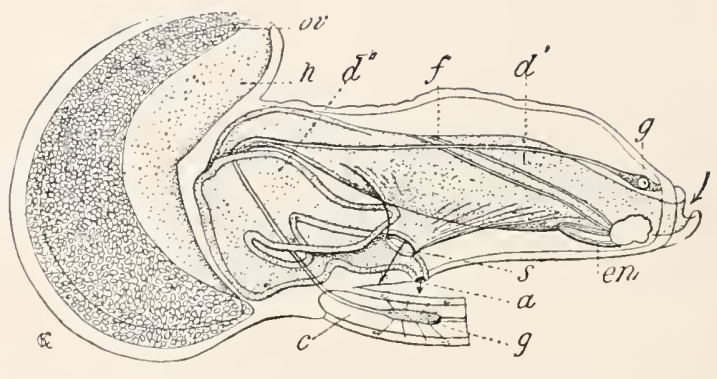

Fig. 497--Oikopleura cophocerca (after Fol.). $A$, the whole animal, removed from its 'house,' dorsal view; $B$, bodv, side view with base of tail. $a$, anus; $c$, notochord; $a^{\prime}$, branchial region; $d^{\prime \prime}$, stomach; $e n$, endostyle; $f$, ciliated peripharyngeal bands; $g, g^{\prime}$, brain and first ganglion of tail; $h$, testis; $m$, mouth; $o$, ov, ovary; $s$, gill slits.

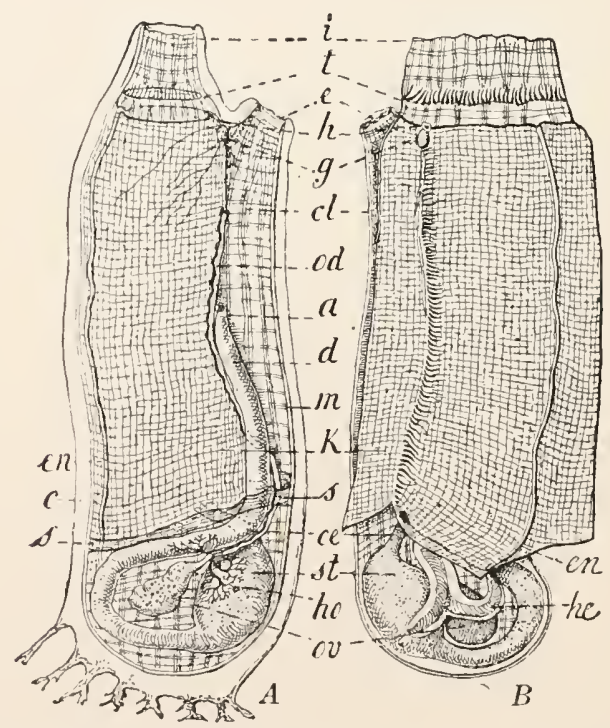

FIG. 498.-Ciona intestinalis. A, from the left side, the cellulose tunic and dermal muscular sac removed; $B$, from the right side, the tunic entirely removed, pharynx opened from the mouth. $a$, anus; $c$, cellulose tunic, below with adhesive processes; $c l$, cloaca; $d$, rectum; $e$, atrial opening; $e n$, endostyle ending above in the peripharyngeal band; $g$, ganglion; $h$, mouth of the 'hypophysis'; he, heart, with pericardium; ho, branched testes; $i$, oral opening; $k$, gill sac; $m$, muscular sac; oe, cesophagus; $o d$, oviduct, the black line beside it the vas deferens; ov, ovary; $s$, partition between atrium and body cavity; st, stomach; $t$, crown of tentacles. 
arranged in longitudinal and transverse rows (fig. 499), through which the water received from the mouth passes into the peribranchial chamber, thence to the atrium, and so to the external world.

While the respiratory water thus passes out in a nearly direct course, the food particles which it contains pass into the digestive tract. By means of the peripharyngeal band just inside of the tentacles and surrounded by mucus secreted by the endostyle the food is carried back to the œesophagus $(o c)$ at the base of the gill chamber, and thence to the stomach (usually provided with liver glands), and on to the intestine. The anus is at the base of the special portion of the peribranchial chamber, which also receives the genital ducts and hence is known as the cloaca or atrium.

In the body cavity, which is greatly reduced in the species with compact bodies, occur the digestive tract, the sexual organs, and the heart; the latter ( $/ l e)$ frequently S-shaped, extends between the stomach and the endostyle. Opposite to the endostyle is the ganglion $(g)$ in the dorsal wall between oral and atrial openings. Near it is a branched subneural gland which has been compared to the vertebrate hypophysis. In many there exist special excretory organs, numerous blind vesicles filled with excreta.

From the eggs are hatched small swimming tadpole-like larva (fig. 500), resembling Appendicularia and, like it, consisting of trunk and tail, in which the chordate features are strongly marked. The digestive tract is confined to the trunk; dorsal to it lies the tubular nervous system in which can be recognized a vesicular brain with a simple eye and a statocyst imbedded in its walls; farther back a narrower portion ('medulla oblongata'); lastly, a spinal cord extending into the tail. In the axis of the tail is a notochord which extends forward a short distance into the trunk between digestive tract and nervous system. In the metamorphosis of the free larvæ into the sessile ascidians four processes are important: (I) 'The larvæe attach themselves by means of three ventral anterior papilla; (2) The tail is retracted and absorbed; (3) 'The body becomes more or less spherical by development of the tunic; (4) 'Two dorsal invaginations ore formed, these envelop the pharyngeal resion, fuse and form the atrium and peribranchial chamber. It is to be noted that this arises from the dorsal surface and extends ventrally, while the peribranchial chamber of Amphioxus arises by folds which grow ventrally over the pharynx. Besides sexual reproduction many ascidians reproduce by budding. This results in the formation of colonies, a matter of systematic importance.

Sub Order I. MONASCIDIE. Simple ascidians of considerable size. The Claveldinide produce small colonies by basal budding, each individual with its own test; Perophora.* Cyntuinde, oral and atrial openings four-lobed; 


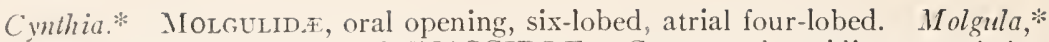
Lugyra * Sub Order II. SYNASCIDIF. Compound ascidians consisting of numerous small individuals imbedded in a common tunic. Usually (fig. 502) the individuals of a colony are divided into small groups, the oral openings of a group forming a rosette around a common central atrium. Distaplia, Lepto-
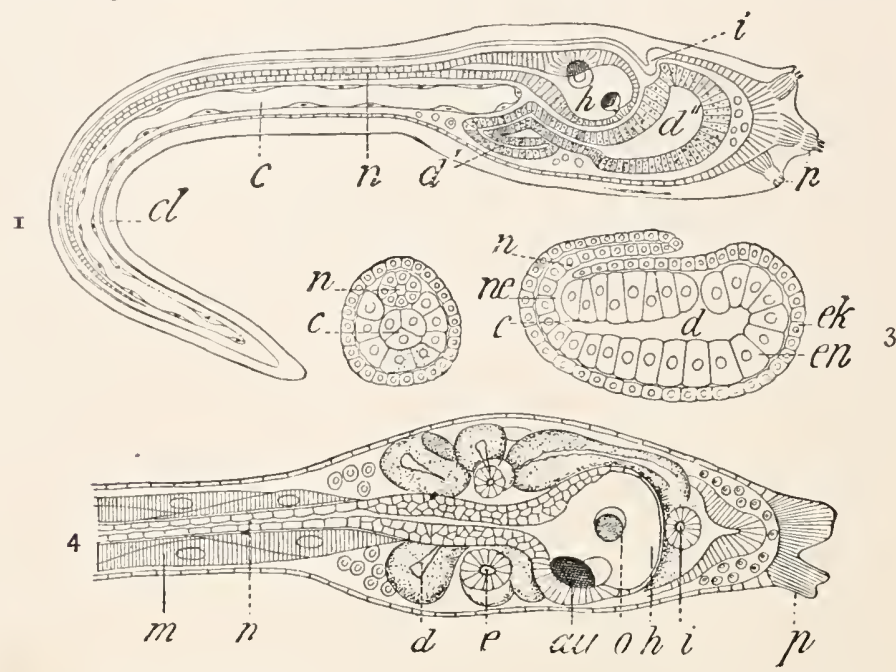

FIG. 500.-Development of an Ascidian (after Kupffer and Kowalevsky). I, larva, just hatched; 2 , cross-section through the tail of a slightly younger larva; 3 , much younger stage, formation of notochord and nervous system; 4 , anterior end of a larva just before attachment. (2, Phallusia mentula; 3, 4, Ph. mammillata.) au, eye; $c$, notochord; $c l$, tunic; $d$, digestive tract; $d^{\prime}$, its nutritive, $d^{\prime \prime}$, its respiratory division; $e$, atrial vesicle; $e k$, ectoderm; $e n$, entoderm; $h$, brain; $i$, oral invagination; $m$, muscles of tail; $n$, neural tube; $n e$, neurenteric canal; $o$, otocyst; $p$, adhesive processes.

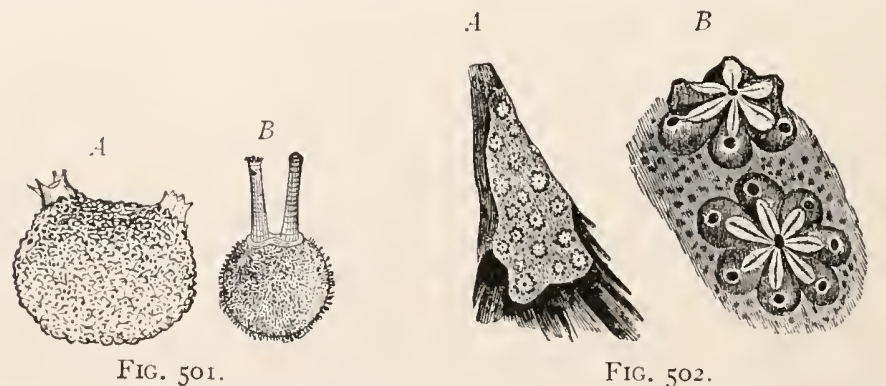

FIG. 50r.-A. Molgula manhattensis*; B, Eugyra pillularis* (from Verrill).

FIG. 502.-Botryllus violaceus (after Carpenter). A, small colony of eighteen individual groups; $B$, two inaividual groups somewhat enlarged.

clinum,* Polyclinum,* Amaroucium,* Botryllus.* Sub Order III. LCCIÆ. Free-swimming pelagic synascidians, having the form of a hollow cylinder closed at one end; the animals vertical to the axis of the cylinder, oral apertures on the outside, atrial in the central cavity. Pyrosoma, very phosphorescent, tropical, some species four feet long. 


\section{Order III. Thaliacea (Salpæformes).}

These, like the Lucie and Copelate, are pelagic, and play an important parin the plankton. In form a Salpa may be compared to a barrel consisting extert

A

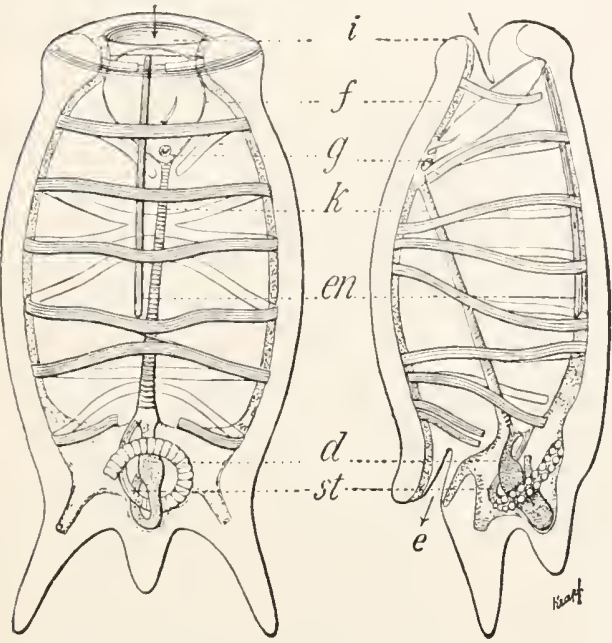

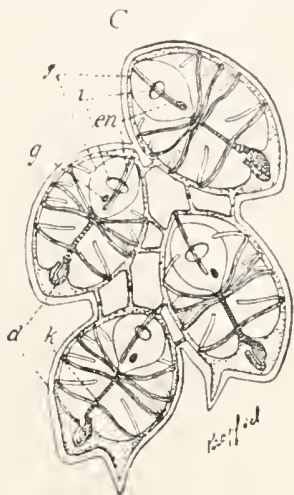

F1G. 503.- A, B, Salpa democratica with stolon, ventral and lateral views; $C$, Salpa mucronata, part of a young chain not yet separated. $a$, anus; $c$, tunic; $d$, digestive tract; $e$, atrial opening; $e n$, endostyle; $f$, peripharyngeal groove; $g$, ganglion with horseshoeshaped eye, and near it the tentacle and hypophysial groove; $h$, testis; $i$, mouth; $k$, gill; $m$, muscle hoops; st, stolo prolifer.

nally of a cellulose tunic, lined internally with six or eight circular muscles, not always closed rings, lise hoops. By their contraction the water is expelled through the posterior or atrial end of the body, while on their relaxation fresh water enters the other or oral aperture. By the reaction the animals swim through the water with the oral end forward. The cavity of the barrel corresponds to pharyngeal and peribranchial chambers of the ascidian. In the Dolioliidæe the two chambers are separated by a partition perforated by gill slits (fig. 504); in Salpa the partition is reduced to a bar with transverse rows of cilia so that branchial and peribranchial chambers are not distinct; yet the endostyle and the peripharyngeal band are retained.

The viscera lie in the muscular sac, where the branchial bar and the endostyle meet and are usually compacted into a mass, the 'nucleus' (intestine, liver, gonads, heart). The ganglion is distinct and lies dorsally opposite the endostyle, just in front of the branchial

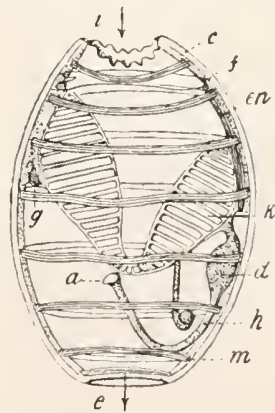

Fig. 504.-Doliolum denticulatum. (For explanation of letters see fig. 503.) bar. Associated with it is a horseshoe-shaped eve.

For a long time two kinds of Salpe have been known, one solitary, the other consisting of numerous individuals connected together like a chain or a rosette 
(fig. $503, \mathrm{C}$ ). At the beginning of the last century the poet Chamisso discovered that the chain salps were produced by the solitary individuals, and that these in turn came from the chain form, the first instance of alternation of generations. The solitary salp is asexual; gonads are lacking, but near the hinder end is a budding cone or stolo prolifer from which colonies of salps bud one after another. When the first is separated a second matures and a third begins. These colonial forms, the chain salps, are sexual, and each produces a single egg from which a solitary individual is formed.

Since both the solitary and the chain forms have received names, the species of Salpa* now have double names like Salpa democratica-mucronata, democratua

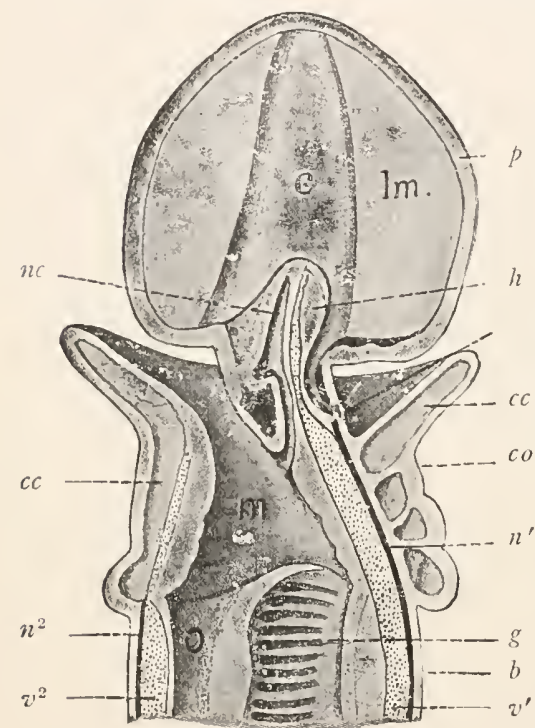

FIG. 505.- -Sagittal section of Gilosisbalanus minutus (diagram after Spengel). $c$, proboscis coelom; cc, colar coelom; co, collar; $h$, so-called heart; $l m$, long inuscles; $n^{1}, n^{2}$, dorsal and ventral nerve cords; $o$, œsophagus; $p$, proboscis; $n c$, so-called notochorel; $g$, gill slits; $v^{1}, v^{2}$, dorsal and ventral blood-vessels; $m$, mouth. being the asexual, mucronata the sexual, individual, etc. From the true Salpe Doliolum* is distinguished $1 \mathrm{y}$ the better developed gills, the complete muscular bands, and a more complicated alternation of generations.

\section{Sub Phylum Iit. Enteropneusta (HEMICHORDIA)}

The few marine forms here included are decidedly worm-like, and, like many worms, they burrow in the mud. The body consists of three parts-proboscis, collar, and trunk (fir. 506). The proboscis, which sits in the collar like an acorn in its cup, whence Balanoglossus, contains a cavity opening to the exterior by a dorsal pore, while two similar cavities i. the collar open separately. These can be filled with water, and by alternately enlarging and contracting these parts the animal is able to burrow. The mouth (fig. 505) lies ventral and in front of the collar and leads into a digestive tract, which in its anterior part is perforated by numerous paired gill slits, whence the name Enteropneusta, while the part behind it is covered with hepatic cæca. 'The intestine is supported in the colom by dorsal and ventral mesenteries, and is accompanied by a dorsal and a ventral blood-vessel, to which are added lateral canals and numerous anastomoses. A contractile vesicle on the dorsal vessel in the proboscis is called the heart. The nervous system is rery peculiar. There is a dorsal portion lying in the collar region, which is produced by inrolling, as is the central nervous system in the Chordates, and a ventral part, as yet lying in the ectoderm, the two being connected by nerves in the collar. The gonads are numerous follicles lying between gill and liver regions and opening to the exterior.

The systematic position of the Enteropneusta is uncertain. In the possession of gill slits and in the formation of the dorsal nervous system it closely resembles the other chordates, and the resemblance is strengthened by similarities in details of structure of the gills. The advocates of this view recognize the notochord in 
a blind tube, surrounded by tough membrane and thickened beneath, which extends from the pharynx into the proboscis. Embryology throws little light on the problem. Some species have a direct development (fig. $507, B, C$ ), while others have a larra (Tornaria, 1) which so resembles the larra of certain echinoderms that it was long held to belong to that phylum. The chief resemblances are in the relations of the ciliated bands to the alimentary tract and in the presence of the proboscis cavity, which, like the ambulacral system, opens to the

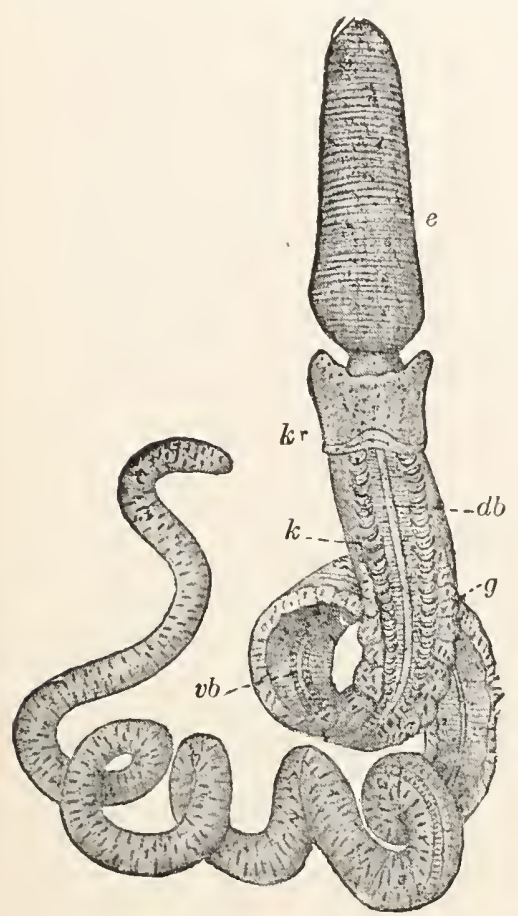

FIG. 506 . exterior. The olk genus Balanoglossus* has recently been subdivided. Two deep-sea forms, Cephalodiscus and Rhabdopleura, have the same type of 'notochord,' and the first has a pair of gill slits; in other respects these are strikingly Polyzoan in appearance.

\section{Sub Phylum IV. Vertebrata.}

In the vertebrates only the internal segmentation occurs. This is shown most clearly in the lower Vertebrata, in the muscles (myotomes, myomeres), the myosepta which separate them, and the protovertebra from

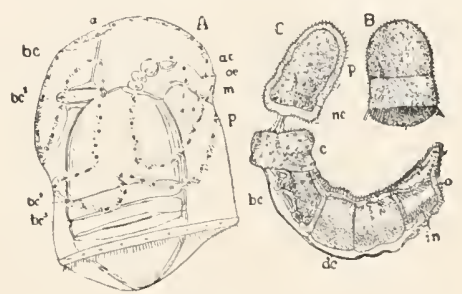

FIG. 507.

FIG. 506.-Balanoglossus kowalewskii* (from Korschelt-Heider, after A. Agassiz). $d b$, dorsal blood-vessel; $e$, proboscis; $g$, sexual region; $k$, gill region; $k r$, collar; $2 \cdot b$, ventral blood-vessel.

FIG. 507.- A, Tornaria larva of Balanoglossus (after Morgan). a, apical plate; $a c$, preoral part of ciliated band; $b c^{1}, b c^{2}, b c^{3}$, coelomic pouches; $m$, mouth; $p$, postoral part of ciliated band $B, C$, two stages of Balanoglossus with direct development (after Bateson). $a$, anus, , $b c$, branchial clefts; $c$, collar; $d c$, digestive part of alimentary canal; in, intestine; $n c$, 'notochord'; $p$, proboscis.

which they arise; in the nerves (neurotomes), the skeleton (sclerotomes), the blood-vesscls, and in the excretory organs (nephrotomes). In the higher vertebrates this metamerism is best seen in the embryonic stages. In part the absence of external segmentation has its cause in the heteronomy (p. 1 26) of the body and the obliteration of segmental boundaries, consequent upon the union of somites into regions, of which at least three 
-head, trunk, and tail-at most six-head, neck (cervical), thorax, lumbar, pelvic (sacral), and tail (caudal)-occur. Not less important in this respect is the character of the skeleton. The cuticular skeleton, the cause of the annulation of the arthroporl, is entirely lacking. The skin remains soft, or contributes to a subordinate degree, more for protection than for support, to the formation of hard parts (dermal skeleton of fishes, alligators, turtles). The firmer tissue is formed in the axis of the body, which, in the lowest vertebrates and the embryos of the higher, appears as the notochord already mentioned, but in the higher is supplemented by the vertebral column and skull.

The skin of the vertebrates is distinguished from that of all invertebrates by (figs. 27,28 ) the many-layered epidermis, and the thickness of the corium. The epidermis is rarely covered by a delicate cuticle (fishes, fig. $27,(s)$; usually such a protection is unnecessary since, especially in the land forms, the superficial layers become cornified and hence furnish the necessary resisțance without a cuticle. There are two epidermal layers, the deeper stratum Malpighii and the superficial stratum corneum (fig. $27, s M$ and $s c ;$ ).

The second constituent of the integument, the corium (cutis, derma), arises from the mesenchyme. It consists of many layers of close connective tissue, and is usually separated from the underlying structures, especially the muscles, by a loose tissue rich in lymph spaces, the subcutaneous tissue. Both of these constituents of the skin, aside from their own firmness, can give rise to protective structures. The horny layer of the epidermis in places becomes greatly developed and thus forms the tortoise shell of the turtles, the scales and scutes of the snakes and lizards, the feathers of the birds, the hair and horns of the mammals. Other epidermal products are the claws, nails, and hoofs of the terrestrial vertebrates. The corium is often the seat of ossifications which, in contrast to the deeper bones, are called the dermal skeleton.

The firmness of the vertebrate skin may be increased in three ways: I. Bony scales develop in the corium which project into the epidermis and receive from it. a horny outer coat, the horny scale (fig. $509, I I$ ). 2. The bony scales are lacking but the horny scales are formed (fig. 509, I). 3. The bony scales are developed but the epidermis remains soft, no horny scales being formed.

I Ioofs, claws and nails (fig. 508) are epidermal structures to be traced back to horny scales, one on the upper, the other on the lower side, enclosing the end of the digit. The first, the claw plate $(p)$ is the more important. In the mammals it grows back more and more into a pocket $(w)$ the root of the plate, from which it extends distally over the upper side of the digit, the claw bed. In claws (ungues) the claw plate is curved in both directions, longitudinally and transversely (fig. 473,III) reducing the lower claw sole (s). In the hoof (ungula) the claw plate is curved transversely $(I)$, the claw sole $(s)$ being reduced to a 
band, following the contour of the plate. 'The nail (lamna) is nearly flat, and since the sole is reduced, the nail appears as a purely dorsal structure (II).

Of great importance in understanding the dermal ossifications is the fact that all scales of fishes are derived from the placoid scale of the selachians. These are rhombic plates, bearing in the milllle pointerl spines, called dermal teeth from similarity in structure and development to the teeth of the mouth cavity (fig. 510). They consist of dentine $(d)$ and have a large pulp cavily $(p)$, with numerous blood-vessels in the interior.

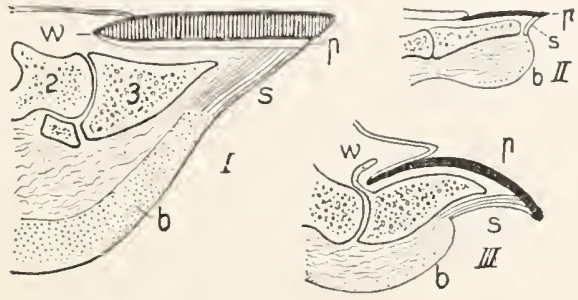

FIG. 508 .

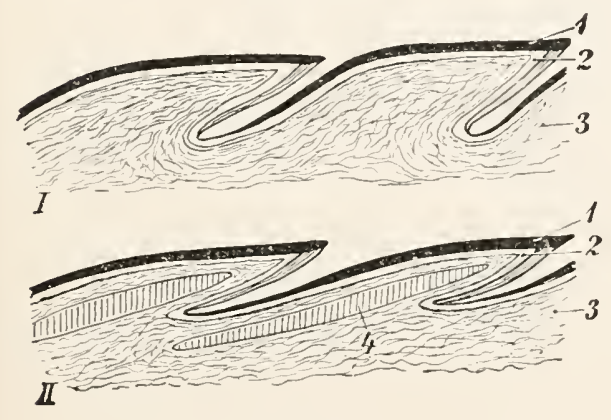

FIG. 509.

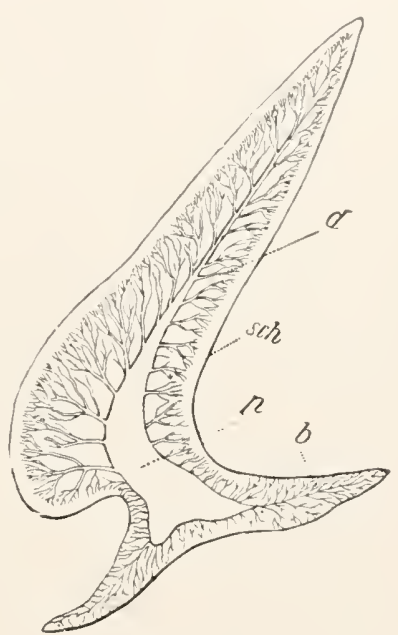

FIG. 5 IO.

FIG. 508.-Long sections through the toes of $I$, horse; $I I$, ape; $I I$, dog. $b$, ball of toe; $p$, claw plate; $s$, sole plate; $w$, root of claw; 2,3 , second and third phalanges.

FIG. 509.- -Sections (schematic) of scales of reptiles. I, snake; II, 'blind worm.' I, corneum; 2, Malpighii; 3, corium; 4, bony scale.

FIg. 510.- -Sagittal section of a scale of Scyllium stellare (after Hofer). b, basal plate; $d$, dentine; $p$, pulp cavity; sch, enamel.

Whether the thin layer $(s c h)$ covering the tip can be called enamel is disputed. Dermal teeth and true teeth are identical structures which, from different position and consequent difference of function, have developed differently.

The scales of fishes have a further anatomical interest, since from them have arisen, besides the bony plates which form the armor of the turtles, alligators, and many mammals (armadillos), important parts of the axial skeleton, the secondary or membrane bones. A membrane bone is a bony 
plate which has arisen from a fusion of clermal ossifications, becomes transferred to a deeper position, and contributes to the completion of the axial skeleton. From what was said above about the relations of dermal and true teeth it is rearlily seen that the lining of the mouth cavity is a source of membrane bones.

In describing the axial skeleton, the notochord comes first. This has already been mentioned in connexion with lower Chordates. It persists in the cyclostomes, but from them upwards it is gradually replaced by the vertebræ arising around it. It is of entodermal origin (fig. 9), arising as a

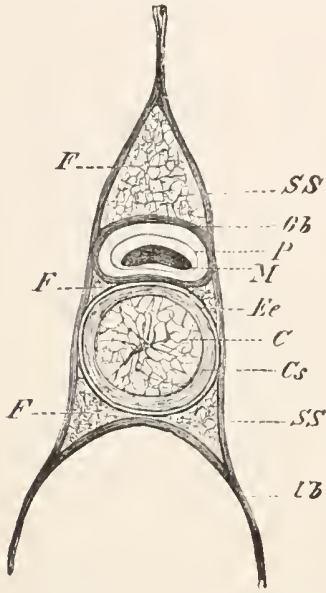

Fig. 511.-Transverse section of axial skeleton of Petromyzon (from Wiedersheim). $C$, notochord; $C s$, notochordal sheath; Ee, elastica externa; $F$, fatty tissue; $M$, spinal cord; $P$, its meninges; $O b$, upper process of skeletogenous tissue; SS, skeletogenous tissue; $U b$, lower process of (skeletogenous tissue.) longitudinal band of the epithelium of the archenteron $\left(I, c_{l}\right)$, and, becoming cut off, comes to lie in the long axis of the loody between digestive tract and nervous system (II, $I I I)$; here it forms a cyclindrical rod consisting of a connective tissue which, as already said, resembles plant tissues because of the vesicular nature of its cells (fig. 39).

In transverse section (fig. 5II) the notochord is surrounded by three layers, internally by a fibrous notochordal sheath, then an elastic layer (not always present), the elastica externa, so called because an elastica interna sometimes occurs inside the notochordal sheath; and lastly a skeletogenous layer $(S S)$. This last is a connective-tissue layer and is therefore connected with the other connective tissues which surround muscles, nerves, etc. It deserves special mention because in it the cartilages and bones arise from which the vertebre and skull are formed. Cells from it can penetrate the notochordal sheath, converting it into fibrous cartilage, thus enabling it to participate in the formation of the vertebre.

Since the notochord and its envelopes are elastic and give under the strain of the muscles, they are unsegmented. The segmentation of the axial skeleton begins with the appearance of firmer tissue as cartilage and bone. Then there is a separation of successive parts, and with this the gradual formation of vertebral column and skull. In both there is a connected series of modifications, whether studied ontogenetically or comparatively, from the lower to the higher forms.

The first parts of the vertebral column to appear are the upper 
(Cyclostome) and lower (figs. 5II, 512), or neural and hamal arches. These consist of paired parts in the skeletogrenous layer which abut against the notochord, and which are usually a pair to the somite, although occasionally two or more pairs, the arches proper and the intercalaria, may occur. The neural arches enclose a spinal canal surrounding the

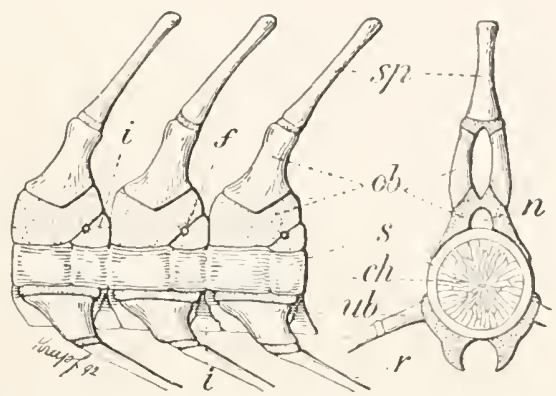

FIG. 5I2.--Vertebre of sturgeon. ch, notochord; $f$, exit of nerve; $i$, dorsal and ventral intercalaria; $n$, neural canal; $o b$, neural arch; $s$, chordal sheath; $r$, rib; ub, hemal arch. Bone white, cartilage dotted.

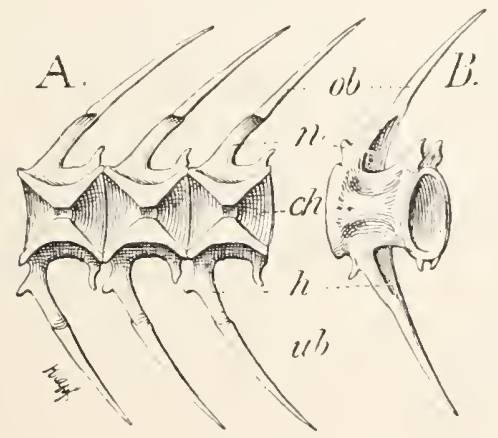

FIG. 513 .

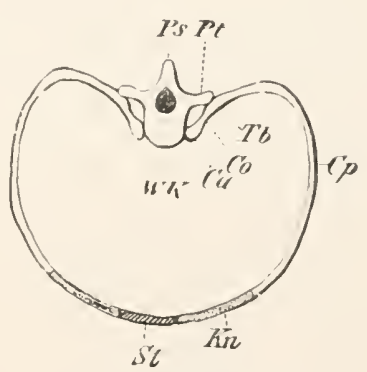

FIG. 5I 4 .

FIG. 5I3.-Caudal vertebræ of a carp, section (A) and nearly side view $(B)$. ch, space filled by notochord; $h$, hemal arch; $h$, neural arch; $o b$, neural sjine; $u b$, hixmal spine.

FIG. 5I4.-Thoracic vertebra, ribs, and sternum of a mammal (from Wieclersheim). $C a$, capitular hearl of rib; $C o$, neck of rib; $C p$, bony rib; $K n$, cartilaginous rib; $P s$, spinous process: $P t$, transverse process (diapophysis); St, sternum; $T b$, tubercular head of rib; $\amalg K$, vertebral centre.

spinal cord, the parts of the arch, neurapophyses, uniting above the cord to form the spinous process (frequently independent). In the caudal region, in the same way, hamal arches may be formed of hamapopliyses and hamal spine, the arches surrounding the blood-ressels of the tail (fig. 513). In the trunk region the ventral arch behaves differently. 
Since the large body cavity with its viscera is here, the hæmapophyses extend outwards and downwards and are divided into two parts, a basal apophysis and a lower movable portion, the rib (fig. 512). Also the union of hiemapophyses with hæmal spine does not occur; the ribs are either free (fishes) or are (at least in part) connected ventrally by a breast bone or sternum (amniotes, fig. 5 I4). The sternum is a derivative of the ribs. In development the ventral ends of the ribs of a side fuse and then these fused tracts of the two sides unite to form the sternum.

The hæmal arches lie medial to the longitudinal muscles of the body, and in the trunk region they lie in the same position just beneath the peritoneum. These are homal ribs and are found only in teleosts and ganoids. 'The ribs of all other vertebrates are morphologically different and are called lateral ribs. They develop in a horizontal connective-tissue septum which extends out through the longitudinal muscles from the axial skeleton to the skin, dividing the musculature into dorsal (epaxial) and rentral (hypaxial) portions (fig. 92). In the elasmobranchs these lateral ribs are attached to the hæmapophyses, in the others to diapophyses, which arise from the neuropophyses, and parapophyses, which arise from the vertebral centres. In the caudal region, often also in the cervical, lumbar, and sacral regions, the lateral ribs and dia- and parapophyses fuse to form transzerse processes. These occur together with hæmal arches in the tails of many Amphibia and reptiles and some mammals, the hæmal arches forming the chevron bones which, as in fishes, enclose the caudal blood-ressels. The presence of intercalaria in cyclostomes, sharks, and ganoids indicates that primitively a double vertebra arose in each somite. Paleontological and embryological researches on reptiles support this view.

In most vertebrates either the basal ends of the arches broaden out around the notochord and fuse with one another, or perichordal cartilages arise independently, furnishing in either case firm supports, the vertebral bodies, or centra, for the system of arches. These increase in size at the expense of the notochord on the inside, sometimes leading to its alm.ost complete obliteration, as in the mammals; in others, as the fishes, the reduction is less complete. The fishes have am phicole vertebre (fig. $5^{1} 3$ ), that is, the centra are hollow at either end. In these cups the notochord exists even in the adult, and when small connecting portions extcnd through the centra the notochord takes the form of a rosary with alternating enlargements and contractions.

Histologically the vertebral column may be either cartilage or bone; usually it is first formed in cartilage, which later may be replaced by bone. If the ossification do not occur, the column remains cartilaginous; if incomplete, cartilage and bone appear together. Since these histological differences are combined with varying degrees of persistence of the notochord and with modifications in the form of the rertebre and their processes, there results an extraordinary variety in the appearance of the vertebral column. 
In Amphibia, reptiles and birds intervertebrat cartilages develop between the centra, also constricting the chorda. In order that the column shall have the necessary flexibility, joints arise in the intervertebral cartilage in different manners: (a) Opisthocale vertebre have a socket on the hinder surface which receives the convex anterior end of the succerling centrum, forming a ball-andsocket joint. (b) Procolous vertebre have these relations reversed, the socket being in front. (c) The vertebre may articulate by a 'saddle joint' (birds). If the intervertebral cartilage become rudimentary, the amphicoclous condition reappears. (d) Between two successive vertebre an elastic intervertebral ligament may occur (mammals). The neurapophyses may bear, in arldition to the transverse processes, anterior and posterior articular processes (zygupophyses) connecting the separate vertebre.

The skull, the anterior continuation of the axial skeleton, occurs in all vertebrates; it appears before the vertebre, for it is found in the cyclostomes, which lack these. It surrounds the brain as the vertebre do the spinal cord: and, like them, its first stages are formed in the skeletogenous layer surrounding the anterior end of the notochord. It is so related to the surrounding parts that it may in general be said to be equivalent or homodynamous with the vertebre, although we cannot agree with Oken and Goethe, the founders of the 'vertebrate theory of the skull,' that it has arisen by the fusion of vertebre. On the other hand skull and vertebre are parts arising in the common basis of the skeletogenous layer, but which have developed in different directions. The vertebral column is metameric since the segmental muscles attached to it would otherwise be ineffective. The cranium is a continuous capsule, because the most important sense organs are on the head and they prevent the development of locomotor muscles. Many facts of anatomy and development, especially the relations of the nerves $(\mathrm{p} .+72)$, tend to show that one part of the skull, the palcocranium, has no relation to vertebre. This alone is found in cyclostomes. In other vertelorates this is joined by the occipital region (neocranium) of vertebre secondarily fused with the palacocranium.

Three stages are recognized in the development of the skull: the membranous, the cartilaginous cranium (chondrocranium), and the bony skull. The first, which consists of connective tissue, occurs only in the early embryonic stages, scarcely a trace of it persisting in the adults. It is early replaced by the cartilaginous skull, which may persist unaltered throughout life in the lower fishes (elasmobranchs, sturgeon). In most vertebrates, however, ossification sets in, embracing a part (tishes, amphibians) or practically the whole of the cartilage (birls, mammals), converting it in the latter case into a bony capsule. In the bony skull two kinds of bone, primary and secondary, are recognized, these varying in their origin. The primary or cartilage bones levelop from the cartilage itself. The secondary or membrane bones are, in their origin, foreign to the 
axial skeleton and arise from the ossifications in the skin (scales) or in the mouth (teeth), already referred to (p. 45I). They sink into the deeper portions and apply themselves to the axial skeleton, especially to those parts where, from lack of cartilage, no primary bones can be formed. It is not settled how far these distinctions are valid. According to Gegenbaur all ossification arose primarily in the skin or mucous membranes, and primary bones are merely membrane bones which have entered the

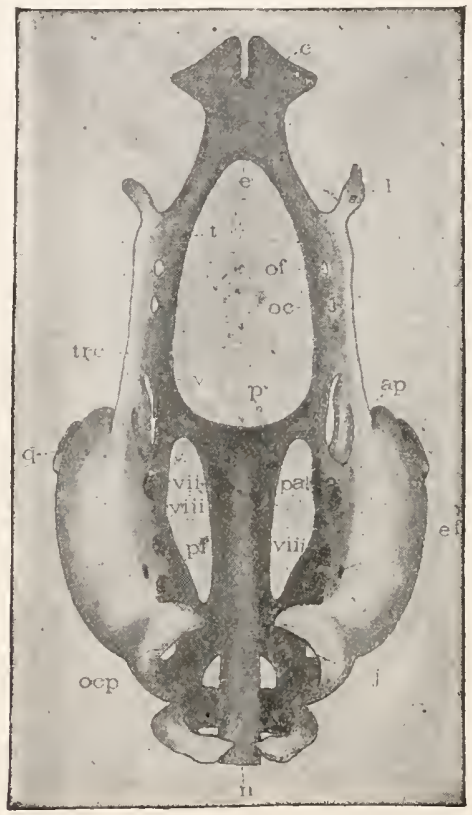

FIG. 515.-Chondrocranium of Amphiuma. anp, antorbital process; $a p$, ascending process of quadrate; $c$, cornu trabeculx; $e$, ethmoid plate; $e f$, endolymph foramen; $j$, jugular foramen: $l$, lamina cribrosa; $m$, Meckel's cartilage; $n$, notochord; $o c$, oculomotor foramen; ocp, occipital process: of, optic foramen; $p$, parachordal; $p a l$, palatine foramen; $p f$, perilymphatic foramen; $q$, quadrate; $s$, stapes; $s p$, stapedial process; $t$, trabecula; $\operatorname{trc}$, crest of trabecula; $V, V I I, V I I I$, foramina for $V, V I I$, VIII nerves.

cartilages and replaced them. Accordingly it is conceivable that the same bone in one animal may arise as a membrane bone and in another as a primary bone, a point of importance in deciding the homologies and nomenclature of many bones. It is but just to say that this view is not universally accepted.

The chondrocranium is most complete beside and beneath the brain (fig. 5r5). This basal portion is a direct continuation of the vertebral column, and a part of it (the parachordals) embraces the anterior end of the notochord, while parts (the trabecula) extend in front of the 
end of the notochord. The side walls of the skull are increased by the cartilaginous envelopes of the two sense organs, the nasul and ol ic capsules, around the nose in front and ear behind. Between these is a hollow for the eye which, although its capsule (sclera) may le cartilaginous or even ossified in part, needs to be movable and hence it contributes nothing to the skull. In only a few forms is the chondrocranium completely closed; usually gaps (fontanelles) occur in its roof, and frequently in its floor. 'The higher the animal intellectually and the larger its brain the more the connective tissue (primordial cranium) is called upon to roof in the chondrocranium. Hence it is that in the reptiles, birds, and mammals, where it is also confined to embryonic life, the chondrocranium is relatively the smallest. Since it only closes above in the occipital (hinder) region, while it gaps widely in front, it follows that the membrane bones play an important part in the completion of the skull.

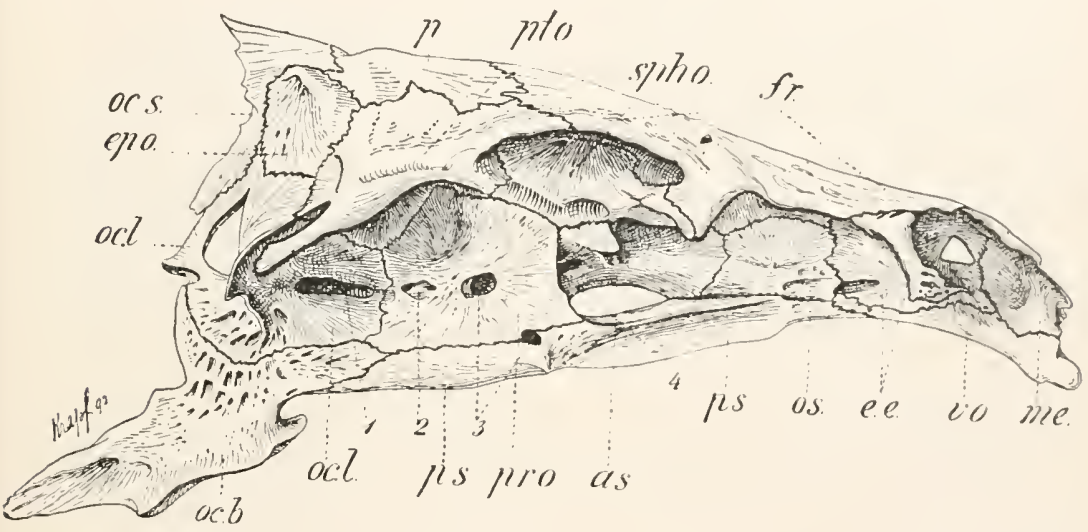

FIG. 5I6.-Skull of carp, the visceral skeleton removed. (A) Cartilage hones: $o c b$, ocl, ocs, basi-, ex-, and supraoccipitals; epo, epiotic; pto, pterotic; spho, sphenotic: pro, prootic; as, alisphenoid; os, orbitosphenoid; me, mesethmoid; ee, ectethmoid. (B) Ventral membrane bones: $p s$, parasphenoid: vo, vomer. (C) Lorsal membrane bones: $p$, parietal; $f r$, frontal; $1-4$, exits of nerves.

The bony skull presents great difficulties from the standpoint of comparative anatomy, in part from its varying appearance in the different groups, in part on account of the number and complicated arrangement of the constituent bones. It may be said that in general the same bone reappears in the different classes, and that the difficulties are connected with the fact that certain bones may fail to develop (Amphibia), or they may fuse to larger elements (mammals). A further complication results from the intimate union of bones of the visceral arches with the cranium, which, strictly speaking, do not belong to it. 
The cartilage bones can be divided according to the cranial regions into four groups: (I) bones of the hinder part of the head-occipitalia; (2) bones of the ear region-otica; (3) bones near the eye-sphenoidalia; and 4) of the nasal capsule-ethmoidalia. The occipital a-four in number (figs. 516-5 I8)-united in the higher mammals to a single occipital bone, surround the foramen magnum, the opening through which the spinal cord passes to connect with the brain. They are a pair of $e x$ occipitals, right and left, a supraoccipital above and a basioccipital below. The otica depend in their development upon the extent of the otic region. In the fishes (fig. 516) where this part is large, several bones may present: epiotic, pterotic, sphenotic, prootic, and often opisthotic. In the mammals, on the other hand, these are fused to a single petrosal bone (figs. 5 $17-5$ I9) of small size.

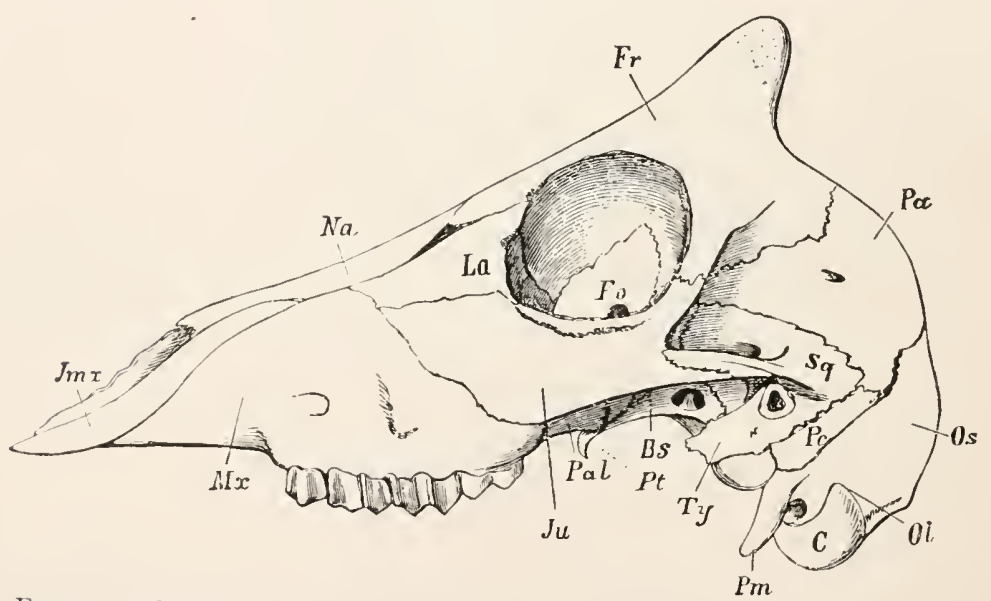

Frg. 517.-Skull of goat (from Claus). Als, alisphenoid; $B s$, basisphenoid; $C$, occipital condyle; Elh, mesethmoid, covering the ectethmoid; $F$ ), optic foramen in orbitosphenoid; Fr, frontal; Imx, premaxillary; $I p$, interparietal; Ju, jugal (malar);
$L u$, lacrimal; I I $x$, maxillary; $N a$, nasal; $O b$, basioccipital; Ol, exoccipital; Ors, orbitosphenoid; $P a$, parietal: $P a l$, palatine; $P e$, petrosal; $P m$, paramastoid process; $P s$, pre-

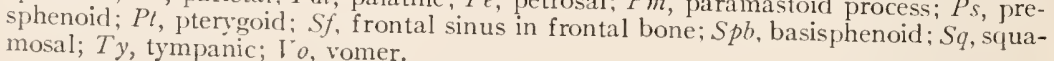

The line of the basioccipital is continued forward by the unpaired elements of the sphenoidalia, a basisphenoid behind and a presphenoil in front. With each is connected, right and left, a pair of bones; with the basisphenoid the alisphenoids, with the presphenoid the orbitosphenoids, just as the exoccipitals flank the basioccipital. In the region of the nasal capsule there is an unpaired mesethmoid with a pair of ectethmoids. Hence the cranium of primary bones may be described as a chain of four median 
basal bones, (fig. 519), basioccipital, basisphenoid, presphenoid, and mesethmoid; right and left of this a row of exoccipital, alisphenoid, orbitosphenoid, and ectethmoil. The position of the otic capsule results in the sum of the otic bones, the petrosal, being wedged in between the exoccipitals and the alisphenoid. Only behind is there a dorsal element, the supraoccipital.

The skull is roofed in by membrane bones, and of these three pairs are almost constantly present. These are, from behind forwards, a pair of parietals, a pair of frontals, and a pair of nasals, the latter covering the

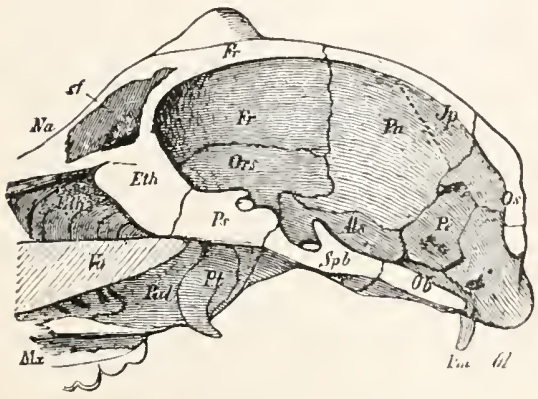

FIG. 5 I 8 .

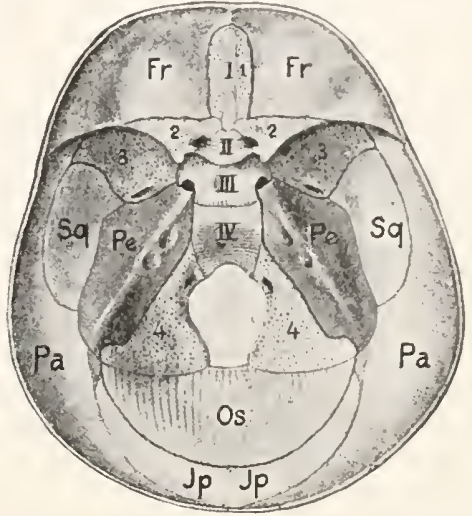

FIG. 519

FIG. 518.- Sagittal section of hinder part of goat skull (from Gegenbaur). For lettering see fig. $5 \mathrm{r} 7$.

FIG. 5ro.-Diagram of floor of skull of infant. I, mesethmoid; I, ectethmoid; IT, presphenoid; 2, orbitosphenoid; $I T I$, basisphenoid; 3 , alisphenoid; IV, basioccipital; 4, exoccipital. Other letters as in fig. 517 .

nasal capsules. The lower vertebrates have a large membrane bone on the floor of the skull, the parasphenoid, which reaches from the basioccipital to the mesethmoid.

The scheme of the cranium thus outlined undergoes the most modifications in the sphenoidal region. Parasphenoid, on the one hand, and basi- and presphenoid, on the other, may be substituted for one another, so that when the parasphenoid is present (fishes, Amphibia) the others are small or absent and vice versa (mammals). In the mammals, besides, the alisphenoids fuse with the basisphenoid (greater wings), the orbitosphenoids with the presphenoirl (lesser wings), so there arise here an anterior and a posterior sphenoid, fused in man and some other mammals to a sincle sphenoid bone. Mesethmoid and ectethmoids likewise fuse in the mammals to an ethmoid bone. 'The existence of these four complexes was the basis of the view $(p .455)$ that the skull is composed of vertebræ.

The brain case, or cranium, is developed into the complete skull by the addition of the visceral skeleton, a series of arches which embrace the begin- 
ning of the alimentary tract and are related to the cranium, much as are ribs to the vertebre. These must be considered as parts of the skull, although in part they are shoved backwards and lie under the anterior end of the vertebral column. As the ribs arise in alternation with the musculature (myomeric), so the visceral arches are similarly related to the gill formation (branchiomeric). Like the cranium the visceral skeleton has a cartilaginous and a bony stage. The visceral skeleton is entirely cartilaginous only in Elasmobranchs, and here it is so loosely connected with the cranium as to be easily separated from it. It consists in these forms usually of seven (rarely nine) arches (fig. 546); these are, from in front backwards, the large mandibular arch, the hyoid arch, and five (rarely seven) gill or branchial arches. The mandibular arch consists, on either sicle, of two pieces which bear teeth and oppose each other in biting; the upper half, attached to the skull in front and behind, is the pterygoquadrate (is not the upper jaw of higher forms). The lower part, which is hinged to the other, is the mandibular or Heckel's cartilage. In the same way the hyoid arch is divided into an upper, or hyomandibular, and a lower hyoid proper on either side, the hyomandibular being fastened to the otic capsule. The hyoids are united below by an unpaired piece, the copula. A copula also exists between the halves of the branchial arches, each of which consists of four parts on either side. Hyoid and gill arches bear gills. Certain features (existence of rudimentary gills and a rudimentary gill cleft, the spiracle) indicate also that the mandibular arch was once gill-bearing and that it lost its original function upon being converted into all organ of mastication.

In front of the mandibular arch, in the Elasmobranchs, are two or three labial cartilages, but it is doubtful if they are visceral arches. Recently they have been regarded as remnants of a support for tentacles around the mouth like those of Amphioxus and Myxine, which reappear in the barbels of bony fishes.

By ossification, the visceral arches of the higher fishes and all higher vertebrates produce a great modification of the skull, this being increased by a progressive change of function of the arches, which depart more and more from their relations to the respiratory apparatus. From this standpoint they may be divided into two groups, an anterior, consisting of labial cartilages, mandibular arch, and the hyomandibular; and a posterior, of the hyoid, the gill arches, and the copulix. The hinder arches are well developed as long is branchial respiration persists. With the loss of gills they largely disappear, but what remains forms the hyoid or tongue bone (not to be confused with the hyoid proper), its body being composed of the copula, its anterior horns of the hyoid, and its posterior horns of the rem- 
nants of a gill arch. Other gill arches contr.hute to laryngeal cartilages, the epiglotis and the cartilages of the auditory meatus.

The anterior members of the visceral skeleton (pterygoquarlrate, Meckelian, and hyomandibular) become developed further, but lose more and more their individuality and unite with the cranium; in the mammals forming the 'bones of the face.' It is therefore a source of adclitional bones which are difficult to follow from class to class, since they change in their functions and consequently in shape and relative size.

All vertebrates with bony visceral skeleton (figs. 51 $7,5+7$ ) have two pairs of membrane bones, right and left, in front of the pterygoquadrates, the premaxillaries (intermaxillaries) and maxillaries. They usually bear, in toothed vertebrates, the marginal row of teeth, which are distinguisherl from the palatopterygoid teeth in that they are opposed by the teeth of the lower jaw. The pterygoquadrates are thus forced backwards and form a second series of bones, parallel to the maxillary series, which likewise may bear teeth. This row of bones consists of an anterior palatine portion and a posterior quadrate part. The cartilages of the palatine part largely disappear and are replaced, in front, by a pair of z'omers followed by a pair of palatines, while farther back are a pair of pterygoids. The quadrate portion ossifies in to the quadrate bone, which, except in mammals, afford the articulation for the lower jaw. The ossifications for the lower jaw occur in a similar way; in front a series of membrane bones, of which the dentary is most important, surrounding Meckel's cartilage, while the hinder part of the Meckelian ossifies into the articulare, so called because it articulates with the quadrate. The hyomandibular forms only one constantly present bone known ly the same name.

All vertebrates with terrestrial habits have a sound-conslucting apparatus in connection with the ear. This is composed of elements which, in the fishes, lie in the neighborhood of the otic capsule, the hyomandibular, the quadrate, and the articulare, to which is added another element, the stapes, which occupies the fenestra ovalis (p. 479) and is derived from the otic capsule itself. In Anura, reptiles, and birds the hyomandibular apparently gives rise to an element, the columella, which abuts against the stapes. In the mammals stapes and columella are possibly fused, while quadrate and articulare undergo a change of function, losing their position in comnexion with the articulation of the jaws and being converted into part of the sound-conducting apparatus, the quadrate forming the incus, and the articulare the malleus (figs. 533, 534). Since the lower jaw in this way loses its articulation, a new one is formed by a process from the membrane bones which articulates with a membrane bone, the squamosal, to be mentioned below. 
According to this view the lower jaw of a mammal is not exactly equivalent to the lower jaw of a bird, since in the latter the hinge is furnished by the quadrate-articulare joint. It should be said that there is another view, though not so well supported, which considers the ear bones as exactly homologous throughout terrestrial vertebrates.

In conclusion three other bones, widely distributed, must be mentioned -the squamosal, the tympanic, and the jugal. The squamosal is a membrane bone arising at the boundary of quadrate and otic capsule (petrosal), and hence with relations to both these bones. It increases in size as the quadrate diminishes in changing to the incus, and in the mammals fuses with the petrosal to form the temporal bone. In common with the tympanic, which in mammals also fuses with the petrosal, it forms a frame for the attachment of the tympanic membrane of the ear. The jugal (malar), belongs to the maxillary series. In many vertebrates the maxillary bone is articulated only in front, its posterior end terminating freely in the soft parts, but when the jugal occurs it forms a jugal or zygomatic arch which bridges the gap between the maxillary and the quadrate region of the skull. When the quadrate becomes modified to the incus, the jugal articulates with its companion, the squamosal, which extends a zygomatic process forward for this purpose.

Difficulties in ascertaining the morphological relations of bones arise where the visceral and cranial parts join and where primary and secondary bones touch. Thus the pterotic, sphenotic, and ectethmoid of fishes are often replaced by secondary bones in the Amniotes; the pterotic by the squamosal; the sphenotic and ectethmoid by two membrane bones in front of and behind the frontals, the prefrontals and postfrontals of reptiles and other forms.

Just as skull and vertebral column form a firm axis for the body, the appendages are supported by axial skeletal structures. Two kinds of appendages are recognized, paired and unpaired, which generally occur together only in fishes. The unpaired consist of a fold of the skin beginning in the sagittal plane behind the head, running back around the tail and forward on the ventral surface to the anal region. This continuous fold is nearly always divided into three parts, a dorsal fin (often subdivided into smaller fins), a caudal fin, and an anal fin. In a similar way, apparently, the paired appendages-an anterior or thoracic and a posterior or pelvic (abdominal or ventral) pair-have arisen from a pair of continuous folds, by development of the appendages themselves and suppression of the intermediate regions. Of these the unpaired are possibly the oldest, since they occur not only in the cyclostomes, but in Amphioxus and the tunicates as well, where paired appendlages are lacking; on the other hand they disappear in the higher forms. Since they are of service only in an aquatic life, they are lost in Amphibia, in which a continuous fin, unsup- 
ported by skeletal elements, occurs only in larval life. On the other hand the paired appendages (arms and legs) gain in importance with terrestrial habits.

In the fins of fishes two kinds of skeletal elements occur which, in the Elasmobranchs (fig. 520), are distinguished by their histological structure, since the one, the fin supports (basalia and radialia), consist of cartilage, the others (actinotrichia, dermal skeleton) are of horny consistency. Since in the teleosts both kinds of supports may ossify, the distinction is here less striking, yet the basalia and radialia arise from cartilage and lie in the basal part of the fin, while the others

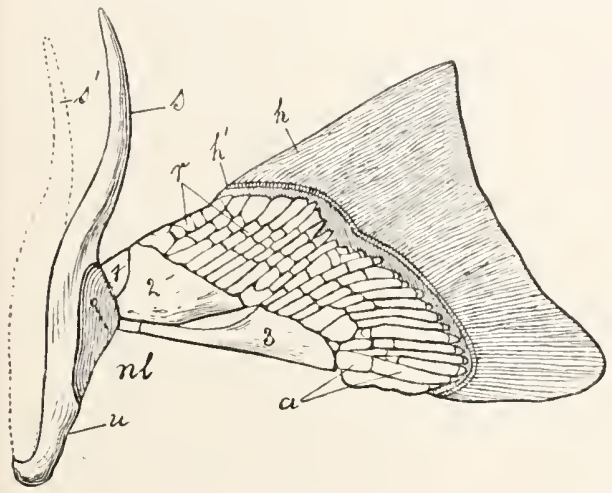

FIG. 520 .

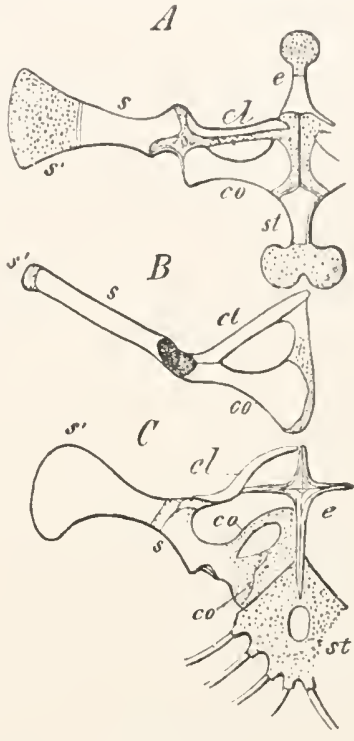

FIG. $5^{2}$ I.

Fis. 520.-Pectoral girdle and left fin of Heptanchus (after Wiedersheim). $a$, principal row of radialia; $h$, actinotrichia cut across at $h^{\prime} ; n l$, foramen for nerve; $r$, radialia; $s, s^{\prime}$, scapula; $u$, coracoid portion of girdle; $\mathrm{I}, 2,3$, basalia; $I$, propterygium; 2 , mesopterygium; 3 , metapterygium.

FIg. 52 I. - Right half of shoulder girdles of $(A)$ frog, $(B)$ turtle, $(C)$ lizard (after Gegenbaur, slightly modified). $c l$, clavicle; $c o$, coracoid; $e$, episternum; $s$, scapula; $s^{\prime}$, suprascapula; st, sternum, in $C$ with bases of ribs.

are never cartilaginous and occur in the distal portion. These distinctions are of importance, since the actinotrichial portions play no part in the development of the extremities of the higher groups. These arise from the cartilage supports of pectoral and pelvic fins, which therefore alone need further mention.

The skeleton of the paired appendages, preformed in cartilage, consists of two parts, the girdles lying in the lateral walls of the trunk, and the skeleton of the limbs themselves. A girdle-a shoulder or pectoral girdle in front, a pelvic girdle for the hind limbs-is in its simplest form an arch with risht and left halves, each half with an articular surface for 
the limb, dividing it into dorsal and rentral portions (fig. 520). The dorsal portion is the scupula (shoulder blade) in the pectoral, ilium in the pelvic girdle. With the development of bone the lower portion is usually split into anterior and posterior parts (fig. 52I). The anterior of these is the clavicle in the pectoral girdle, pubic bone in the pelvis; the hinder part is the coracoid or the ischium in the two girdles respectively. These parts are most constant in the pelvic girdle. In the pectoral girdle either coracoid or clavicle may be lacking, at times both are absent; but novertebrate with fore limbs lacks a scapula. In the clavicle there is frequently an element, preformed in cartilage, the procoracoid, to be distinguished from a membrane bone, the clavicle in the strict sense.

In the fishes the girdles are largely or entirely held in position by muscles; in most terrestrial vertebrates there is a more intimate connexion with the vertebral column. In the case of the pelvic girdle the connexion is direct, since the ilium is articulated with one or more sacral vertebre (in reality not with the vertebre themselves, but by the intervention of sacral rilss). The connexion of the pectoral girdle is less direct and is looser. This is effected by clavicle and coracoid. The latter connects with the sternum, which in turn is connected to the vertebral column by the rilss; the clavicle articulates with a bone, the episternum, which rests upon the breast bone, the morphological relation of which is doubtful, since under this term have been included different structures.

Since only the free portions of the appendages are concerned directly in locomotion, and since the various modes of motion--swimming, flight, running, leaping, climbing-demand special modifications, the skeleton of the limbs shows great variety. It is usually believed that all these forms are to be traced back to an ancestral type, the archipterygium. In this (fig. 520) are numerous skeletal parts which vary little in size and form and are arranged in many closely appressed rows. One of the rows has acquired prominence and is called the principal row; it begins with a larger piece, the metapterygium, which articulates with the girdle and bears either on both sides (archipterygium biseriale) or only on one (archipterygium uniseriale) the lateral rows of skeletal elements. Usually most of the lateral rows are not attached to the principal row, but arise independently from the girdle, and may begin with larger parts, the propterygium and mesopterygium.

From this archipterygium can be derived a primary form, the pentadactyle appendage, which serves for all terrestrial vertebrates from the Amphibia onwards (fig. 522). In tracing this from the archipterygium the following modifications must be supposed. First a reduction in the number of rows to five, a principal row and four accessory rows. The 
terminal portions of the principal row produce the bones of the fifth, the accessory rows those of the other fingers or digits. Then there is an unequal growth of parts; the metapterygium, already in Elasmol)ranchs a considerable element, increases in size and forms in the fore limb the humerus, in the hind limb the femur. In like manner the second element of the principal row and the first of the first accessory row increase and form respectively ulna and radius in front, fibula and tibia behind. Then follow parts which remain small and somewhat cubical, carpal bones in the fore limb, tarsals in the hinder extremity; they bear in turn slender bones, the metacarpals or metatarsals, and these at last the phalanges. (For the nomenclature of carpals and tarsals see the explanation of fig. 522.) A process on the upper end of the ulna is the olecranon; in the hind limb there is an analogous 'knee-pan' or patella. in the tendon passing over the knee.

The third and most important modification is brought about by the development of joints. So long as the appendage serves as an oar it must act as a single plate with its parts firmly held. On the other hand, when it must act as a system of levers to support and move the body, as is necessary in a terrestrial animal, it must be divided into sections, jointed to each other. By this there are leveloped two joints of importance in both fore and hind limbs; the ellow (knee) joint between humerus (femur) on the one hand and radius and ulna (tibia and fibula) on the other; and the wrist joint (ankle) between the bones of the fore arm (shank) and the carpals (tarsals). Less important are the joints of the fingers and toes.

If the limbs of terrestrial vertebrates be compared with this primary form, variations are seen in two directions. Rarely are there more bones than in the

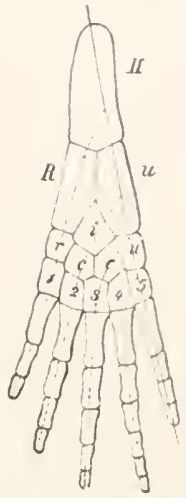

Fig. 522.-Schema of a pentadactyle appendage (after Gegenbaur). The dotted lines indicate the lateral rays; the names for the hinder extremities in jarentheses. $H$, humerus (femur); $U$, ulna (fibula): $R$, radius (tibia). Carpus (tarsus) consisting of two rows and two central portions: Row I: $r$, radiale (tibiale); $i$, intermedium; $u$, ulnare (fibulare:) $c$, centralia. Row II: $\mathrm{I}-5$, carpalia (t a $\mathrm{r}-$ salia); the metacarpals (metatarsals) and phalanges not lettered. schema; then there occur remnants of a sixth or even a seventh row or finger. More frequently there is a reduction in the number of parts, either by fusion or by absolute loss. Fusion accounts for the fact that with complete pentadactyly the number of carpalia is usually less than the ten, to be expected from the schema. Degeneration and loss explain the existence of animals with four, three, two, or even one digit, and one can say with certainty that the missing parts are in most cases 
lost, though a fusion of digits is not unknown. Paleontology, for example, teaches that the one-toed horse has descended, by gradual reduction, from five-toed ancestors.

The completeness and character of the skeleton thus outlined has a great influence on the rest of the organism. As already pointed out, the external appearance of vertebrates has been altered by it, since the skin is no longer a supporting structure and has consequently lost its segmentation. More immediate is its influence upon the arrangement of the

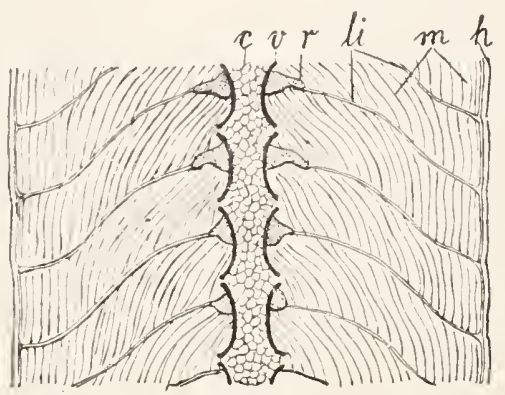

FIG. 523 .

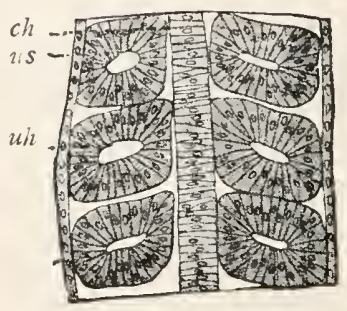

Fic. 524 .

FIG. 523.-Horizontal section through the anterior trunk region of a young Rhodeus amarus at the level of the ventral arches. $c$, notochord; $h$, skin; $l i$, myosepta; $m$, longitudinal muscles; $r$, rib end of the cartilaginous ventral arch; $v$, osseous centrum.

FIG. 524.-Horizontal section of an embryo of Triton (from O. Hertwig). ch, notochord; $u h$, myocœle; $u s$,primary muscle segment (myotome).

musculature. The development of an internal skeleton renders it necessary that the point of resistance of the muscles be transferred from the skin to the interior. A dermal musculature occurs only as an inconspicuous remnant; it is replaced by a body musculature. This latter consists primarily of a system of longitudinal muscle fibres on either side of the vertebral column (fig. 523), which are divided by connective-tissue partitions, the myosepta, into successive segments, the myotomes. Thus when the connective tissue of a fish is dissolved by cooking, the muscles fall into disk-like parts. The myosepta extend from skin to axial skeleton. Since they run obliquely backwards from the skeleton to the skin, they serve to render the skeleton a point of resistance for the action of the muscles.

A segmented trunk musculature occurs in the Myxinoids (and in Amphioxus), in which the axial skeleton consists only of notochord and is consequently unjointed. The segmentation of the muscles is therefore older than that of the skeleton and, as we can further say, is the cause of it. 
The action of the muscles prevents the formation of a cartilaginous or bony vertebral continum such as the notochord and skeletogenous layer are. It produces at intervals joints or flexible parts separating the cartilaginous or bony column into vertebre. Naturally these flexible portions cannot coincide with the boundaries of the muscles, but must lie between them; in other words, muscle segments and skeletal segments - myotomes and sclerotomes-must alternate. Segmentation is lacking in the cranium, since the myotomes here have no locomotor significance, are reduced, and only small remnants of them persist.

In the mammals only a little of this segmental arrangement of muscles is recognizable, a result of the development of the appendages; and the more these gain in importance as the locomotor structures, the more the muscies are modified and grouped for the service of the limbs, so that only the intercostals and a part of the muscular system to the sides of the vertebral column show clearly the primitive metamerism. Iet in all vertebrate embryos the muscles appear at first strictly segmental, in the form of the primitive somites (fig. 524 ), formerly called protovertebre. Parts, like buds, separate from the ventral sides of the protovertebrix and furnish the musculature of the limbs.

Another important point in the musculature lies in the fact that it is dorsal in origin and therefore in fishes is largely dorsal in position throughout life. The muscles which are ventral have largely been transferred from the back, and the cause of the migration is to be recognized to a large extent in the progressive development of the appendages. The dorsal position of the muscles is only a part of a general fact, that the skeletal axis divides the body into a dorsal zone. containing only animal organs, and a ventral zone, chiefly vegetal in character, Besides the muscles, the central nervous system, and the most important sense organs - -yes, nose, ears-belong to the dorsal zone.

The central nervous system of vertebrates consists of brain and spinal cord. Like that of all chordates it is distinguished from that of other segmented animals-annelids, arthropods, in which there is a dorsal brain and a ventral nerve chain-in its purely dorsal position. It is further listinguished from that of all non-chordates by its tubular character, that is, by the presence of a central canal in the axis of cord and brain (fig. 79), lined by a special epithelium, the epend yma, and containing a fluid, the liquor cerebrospinalis. This central canal is the result of the mode of development, the nervous system arising by an in rolling of the ectoderm (irg. 9). A median longitudinal medullary groove arises early in the dorsal ectoderm of the embryo. The floor of this groove, the medullary plate, gradually rolls into a tube, the edges curving upwards and meeting above. In this process there is developed, as in the tunicates (fig. 500), a neurenteric canal connecting the hinder end of the neural canal with the digestive 
tract. Besides the neurenteric canal there long persists at the anterior end an opening to the exterior, the neuropore.

In all vertebrates, in contradistinction to the lower chorclates, the brain is large and sharply marked off from the spinal cord (medulla spinalis). The spinal cord is a cylindrical structure (flattened in Cyclostomes, fig. 5II) which is marked in the middle line above and below by two longitudinal grooves, the dorsal and ventral fissures of the cord ( $p$, sa, fig. 79). The central canal (Cc) is greatly narrowed by the growth of the nervous tissue, in which two layers a re distinguished, one containing almost solely nerve fibres, the other both fibres and nerve or ganglion cells. The arrangement of these layers is contrasted with that of the invertebrates in that the ganglion-cell layer-the gray matter-lies in the centre, the fibrous layer-white matter (II) - on the periphery, a reversed position consequent upon the development by infolding. The distinction in color depends upon the fact that white medullated fibres run in the cortex, while in the gray matter gray non-medullated fibres are present between the nerve cells. The color distinctions fail in the cyclostomes (and Amplioxus), which have no medullated fibres, although the same general structure occurs.

The gray matter surrounds the central canal, but extends on either side dorsally and ventrally into the white matter, so that in section it resembles somewhat the letter $\mathrm{H}$, with its dorsal (fig. 79, $H H$ ) and ventral horns $(\mathrm{I} H)$. These horns and the dorsal and ventral nerve roots arising from them, divide the white matter on either side into three tracts, the dorsal $(H)$, ventral $(s)$, and lateral $(S)$ columns of the cord.

Corresponding to each muscle segment two nerve roots arise from the cord, a dorsal root, with a ganglon (spinal ganglion) at some distance from the cord, and a ventral root, without a ganglion. The dorsal root contains mostly sensory fibres-i.e., those carrying nervous impulses to the cord-and is afferent, while the ventral roots are efferent and contain only motor elements (Bell's Law). 'These roots unite into a mixed root, which then divides into dorsal and ventral branches.

The brain of vertebrates in general corresponds in its fundamental plan (figs. 525, 526), lest seen in development, with the brain of man. At first, in the embryos of a few lower vertebrates there is a stage with two divisions, an anterior archencephalon and a posterior metencephalon which passes into the spinal cord. This condition is transitory and gives place to a brain with three regions by the division of the archencephalon into a fore brain (prosencephalon) and a mid brain (mesencephalon), the metencephalon forming the lind brain. Usually this stage is reached before the closure of the medullary folds. Formerly it was stated that a 
condition with five vesicles followed upon this with three, the mid brain remaining undivided, while the hind brain divides into cerebellum (metencephalon, $(b)$ ) and medulla oblongata (myclencephalon, $m$ ); the fore brain into cerebrum and 'twixt brain. This is not exact so far as the hind brain is concerned, for cerebellum and medulla are roof and floor of the same cavity (fig. 526). The fore brain becomes divided, by lateral

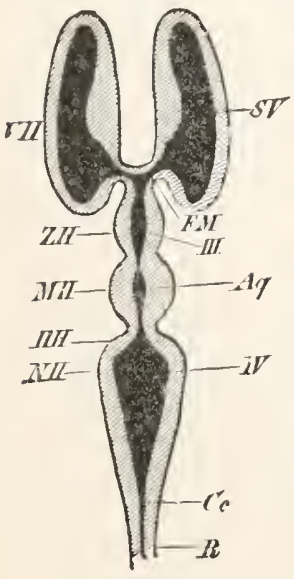

FIG. 525 . outgrowths on either side in front, into three parts, an unpaired portion, the 'twixt brain, and paired anterior parts, the cerebrum.

Introducing the terms of human anatomy for the separate parts of the lrain, the first vesicle consists of the two cerebral hemispheres (telenceplialon) whose dorsal and lateral walls

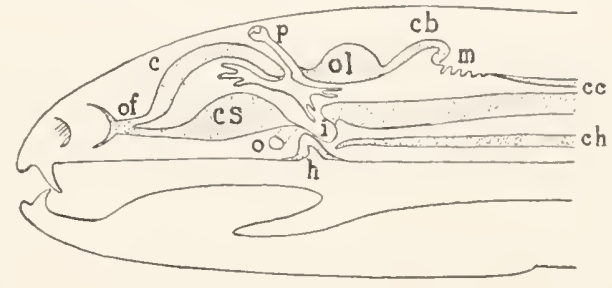

FIG. 526 .

FIG. 525.-Diagram of a vertebrate brain (from Wiedersheim). Aq, aqueduct; $C c$, central canal; $F M$, foramen of Monro (connection of lateral ventricles with each other and with the third); $H H$, cerebellum; $M H$, corpora bigemina (optic lobes); $N H$, merlulla oblongata; $R$, spinal cord; $S V$, lateral ventricles; $V H$, cerebrum; $Z H$, optic thalami ('twixt brain); $I I I, I V$, third and fourth ventricles.

FIG. 526.- Scheme of brain in sagittal section. $c$, cerebrum; $c b$, cerebellum; $c c$, canal of spinal cord; $c h$, notochord; $c s$, corpus striatum; $h$, hypophysis; $i$, infundibulum; $m$, medullary region; $o$, optic chiasma; of, olfactory lobe; ol, optic lobes; $p$, parietal eye.

are called the pallium, while in the floor of each hemisphere is an enlarge. ment, the corpus striatum (cs). The spaces in the hemispheres are the first and second ventricles $(S V)$. From the front of each hemisphere arises a distinct region, the olfactory lobe or rhinencephalon (of), which gives origin to the olfactory nerve. Since the organ of smell is frequently at some distance from the brain, the olfactory nerve must elongate, as in Amphibia (fig. 568), or the olfactory lobe must lengthen, as in many Elasmobranchs (fig. 549). In the latter case the swollen end of the lobe, called the bulbus olfactorius, is close to the olfactory epithelium and is connected with the brain by a long stalk, the tractus. Both, as parts of the brain, must be distinguished from the olfactory nerve.

Only the lateral walls of the second vesicle become thickened, producing the optic thalami or diencephalon, directly adjoining the corpora 
striata; the roof of this vesicle develops no nervous substance, but remains a thin layer of epithelium closing in the third ventricle above $(I I I)$. The floor is also thin-walled between the thalami and is pushed downwards, forming a funnel-like pocket, the infundibulum (i). The mesencephalon is divided on its dorsal side by a longitudinal groove into a pair of optic lobes, divided in the mammals by a transverse groove into four corpora quadrigemini. In the same group the ventricle of the mid brain is reduced to a narrow canal, the aqueduct, so that the fourth rentricle is the cavity of the hind brain.

This last region is called the medulla oblongata (myelencephalon); it is a prolongation of the spinal cord, and in many respects shows a similar structure. It is distinguished from the cord externally in that it gradually increases in size in front, while its roof is reduced to a thin epithelium (chorioid plexus), often torn away in dissection, learing an opening, the fossa rhomboidalis, into the ventricle. In front of this fossa is the cerebellum, often a thin transverse nervous lamella, but usually is a considerable part of the brain, composed of a median vermis and two lateral cerebellar hemispheres.

Although these five parts are present in all vertebrates, the appearance of the brain in the various classes is very different, because their relative size and form vary greatly. In the lower vertebrates optic lobes and medulla oblongata are disproportionately large, while the cerebrum, and often the cerebellum, are insignificant in size. In the higher vertebrates, on the other hand, the cerebrum and cerebellum far surpass the other parts, the increase in size of the cerebrum being proportional to the increase in intelligence. The cerebral hemispheres grow backwards, in man and the apes covering the other parts, while a similar growth forwards carries the olfactory lobes to the lower surface. Since the capacity of the skull is limited, the cortex of the cerebrum, the seat of intelligence, is increased in amount by the development of folds ( $g$ yri), separated by grooves (sulci). Somewhat similar conditions exist in the cerebellum, which in mammals and birds is, next to the cerebrum, the largest part of the brain.

Connected with the 'twixt brain are two problematical organs, one, the cpiphysis (pinealis), being dorsal; the other, the hypophysis (pituitary body), ventral. The hypophysis arises like a gland by an outgrowth from the embryonic mouth. This pocket cuts off from its source, increases by budding, and fuses with parts derived from the end of the infundibulum to a single two-lobed body. It has been compared with the subneural gland of the Tunicata (p. 445). The epiphysis is an outgrowth from the roof of the brain, from which develops in many vertebrates the pariftal organ. In cyclostomes and many reptiles this has the structure of an eye (parietal eye), and in these, separated from the brain, 
but connected with it by a nerve, it lies in a special cavity in the parietal bones, which occurs not only in recent but in fossil forms. Ibove this eye the skin may be transparent.

Most of the nerves which come from the brain (cranial nerves) arise between the mid brain and the spinal cord, especially from the meclulla oblongata. The olfactory and optic nerves are an exception, the one arising from the cerebrum, the other from the "twixt brain, but both, and especially the optic, differ so much from the peripheral nerves that they can hardly be classed with them. Development shows that the optic nerve is a part of the brain. Following custom, howerer, and including these two, the pairs of cranial nerves may be enumerated in the terms of

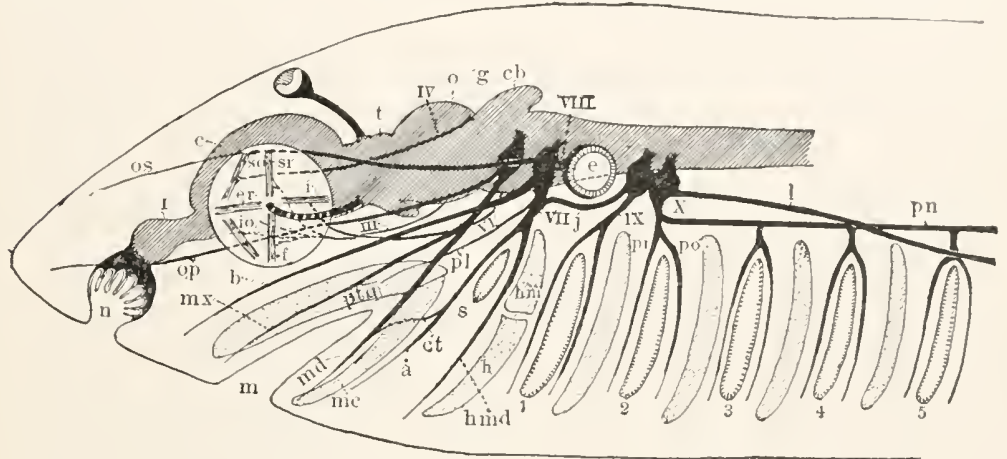

Fig. 527.-Diagram of cranial nerves (shark). $a$, alveolaris; $b$, buccalis; $c$, cerebrum; $c b$, cerebellum; $c t$, chorda tympani; $e$, ear; $e r$, external rectus muscle; $f$, inferior rectus muscle; $g$, Gasserian ganglion; $h$, hyoid cartilage; $h m$, hyomandibular; $i$, internal rectus muscle; $i o$, inferior oblique muscle; $j$, Jacobson's commissure, $l$, lateralis of vagus; $m$, mouth; $m c$, Meckel's cartilage; $m d$, mandibularis; $m x$, maxillaris superior; $n$, nose; $o$, optic lobes; op, ophthalmicus profundus; os, ophthalmicus superficialis; $p$, pinealis; $p l$, palatine; $p o$, posttrematic branches; $p r$, pretrematic branches; $p n$, pneumogastric (intestinal) of vagus; $p t g$, pterygoquadrate; $s$, spiracle; so, superior oblique muscle; $s r$, superior rectus muscle; $t$, 'twixt brain; $I-\mathrm{X}$, cranial nerves; $I-5$, gill clefts.

human anatomy as follows: I, $\mathrm{N}^{\top}$. olfactorius; II, $\mathrm{N}$. opticus; III, $\mathrm{X}$. oculomotorius; $\mathrm{IV}, \mathrm{N}$. trochlearis (patheticus); $\mathrm{V}, \mathrm{N}$. trigemimus; $\mathrm{II}$, N. abducens; VII, N. facialis; VIII, N. acusticus; IX, N. glossopharyngeus; X, N. vagus (pneumogastricus); XI, N. accessorius; XII, N. hypoglossus. To these must be added the recently discovered N. terminalis, first found in fishes and recently in man. It arises from the cerebrum and supplies the wall of the nasal organ. The accessorius in fishes and amphibia is a part of the vagus; the hypoglossus, strictly speaking, belongs to the spinal nerves and only secondarily is associated with the cranial nerves, which explains its course, outside the skull, in cyclostomes and amphibia. 
The relations of these nerves are the most primitive in the Ichthyopsida (fig. 527). Of these, three (I, II, and VIII) are purely sensory, going to the olfactory organ, eye and ear respectively. The third, fourth and sixth are as purely motor, supplying the eye muscles. The remainder are mixed in character. The glossopharyngeal nerve is typical. This leaves the medulla and, at the top of the first gill cleft, divides into two branches, one of which (pretrematic) goes in front of the opening; the other (post-trematic) passing behind it, both innervating the muscles of the region and supplying a sense organ above the gill opening. The vagus, in the same way, has pre- and post-trematic branches for the posterior gill clefts, a condition which leads to the view that this is a compound nerve. In addition the vagus sends a branch to the stomach and heart. In the Amniotes, with the development of lungs, this branch also supplies the lungs, hence the name pneumogastric. The vagus also gives off a nerve which supplies the sense organs of the lateral line of the trunk.

The facial nerve divides over the spiracle in the same way as the ninth over the first gill cleft. It also supplies the lateral line organs of the head. The fifth nerve also clivides at the angle of the mouth, a branch going into each of the jaws, a third going towards the tip of the head. With the loss of gills and lateral line organs in the terrestrial vertebrates the seventh and tenth nerves lose the corresponding branches. 'The facial nerve, which in the Ichthyopsida is largely sensory, becomes almost exclusively motor in the mammals, as a result of the great development of the muscles of the face, which are practically lacking in the lower forms.

Since the head undoubtedly consists of several coalesced body segments (at least as many as there are visceral arches, and apparently more), the question arises whether the cranial nerves are as evidently segmental as are those of the trunk. 'To this is allied the further question whether Bell's Law that a mixed nerve consists of dorsal sensory and ventral motor components is applicable here. In answering this question the olfactory and optic nerves, arising from the prosencephalon, must be excepted. The latter, especially, is not a peripheral nerve, but is a tract connecting two parts of the brain, since the retina, as we shall see, is but an evagination of a part of the brain towards the periphery. With this cerebral character is connected the peculiarity of the crossing or chiasma of the optic nerve. In the teleosts the whole right nerve goes to the left eye, the whole of the left to the right eye, the nerves either simply crossing or passing through networks in each other. In most vertebrates only a part of the fibres cross outsicle of the brain. If all of the fibres cross inside the brain (as is the rule for true cranial nerves) the result is the appearance as in the cyclostomes, that each eye is supplied by the nerve from its own side.

Of the remaining nerves which spring from the hind brain and, with the exception of the trochlearis, from its side and base, the oculomotor and abducens (possilyly also the trochlearis) develop like ventral roots. The eye muscles which they supply are the remains of the somatic musculature arising from the myotomes, which has largely degenerated. All of the other cranial nerves (the hypoglossus excepted) arise like dorsal roots, are sensory, and are provided with the equivalents of spinal ganglia (geniculate ganglion of the facial, Gasserian ganglion of the trigeminal, vagus and glossopharyngeal ganglia), but they also contain numerous motor fibres. It is important to note that the muscles innervated by these motor fibres do not arise from the myotomes and hence do not belong to the somatic musculature, but are a part of the 'visceral muscles.' Now, since the dorsal roots in Amphiowus contain motor fibres which supply visceral muscles, and visceral motor nerves occur in the dorsal roots of the vertebrates, it is necessary to modify Bell's Law and to say that the ventral roots 
innervate the somatic musculature, the dorsal roots supply both the sensory apparatus and the visceral muscles.

One of the most striking facts in the question of the segmental nature of the cranial nerves is the relation of these to the visceral arches, the trigeminal in front of the mandibular, the facial in front of the hyoid arch, and the glossopharyngeal and ragus with similar relations to the gill arches.

Besides the nervous system of the body already outlined, the vertebrates have a special nervous system supplying the viscera, the sympathetir system, ant in this a special central orman consisting of right and left cords beneath the vertebral column, in which ganglia are incorporated. 'The last of these ganglia lies at the base of the caudal vertebre, the most anterior at the beginning of the neck. From the latter nerve cords extend in to the head and are connected with ganglia (otic, sphenopalatine). This system sends out nerves in the form of delicate networks (plexus sympathetici) which usually accompany the blood-ressels 10 the vegetative organs (intestine, sexual apparatus, etc.). It is also connected with the spinal nerves.

The space between the central nerrous system and the surrounding skeleton is large in fishes and is filled with a loose fatty tissue which, close to the nerrous mass, is thickened to a richly vascular envelope (meninx) of the brain and spinal cord. In the groups from amphibia to birds this primitive meninx is divided by the development of a subdural lymph space into two layers, the dura mater and the pia mater. The pia, which in mammals differentiates a delicate arachnoid membrane by the formation of numerous lymph spaces, is next to the nervous system and is the vascular layer, while the dura is a tougher membrane which approaches and fuses with the periosteum lining the skull in proportion to the extent that the brain fills the cranial cavity. In the vertebral column the dura and the periosteum remain distinct.

Regarding the sense organs of the vertebrates we stand on firmer ground than with the invertebrates, since their great similarity to those of man supports the ideas of their functions derived from studies of their structure. Of the sense organs of the skin, the tactile organs of mammals consist of nerve fibres which extend into the epidermis, lose the medullary sheath, and branch among the epithelial cells where they may terminate in special tac- ope; $K H$, its nuclei; .2 , tile discs (Eimer's organs of snout of pig and mole).

Besides these interepithelial nerve endings are others in the subepithelial mesoderm (tactile corpuscles) (fig. 528), which are specially modified and receive special names (Krause's and Grandry's corpuscles). They consist of a few or many cells, probably of epithelial origin, enclosed in a connective tissue envelope. The nerve fibres branch within the organ, the 
branches ending in tactile discs. Though long known among terrestrial vertebrates, these have recently been found in fishes.

The club-shaped corpuscles (also known as V'ater-Pacinian corpuscles, fig. 8I) are allied to the tactile corpuscles by form and mesodermal position, although they differ materially in structure and are not easily to be regarded as ectodermal in origin, since they occur not only in the skin but in deeper structures like the mesentery of the cat. The latter position renders their function problematical.

While in the terrestrial vertebrates typical sensory epithelium is lacking, it attains a high development in the skin of fishes. The dermal nerves pass into the epidermis and end in oral corpuscles, which, while imbedded in a stratified epithelium, consist of a single layer of sense and supporting cells. According to structure, nerve hillocks (neuromasts) and nerve-end buds are distinguished. The first are the specific organs of the

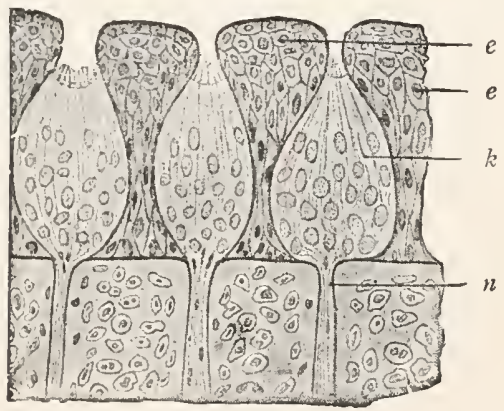

FIG. 529 .

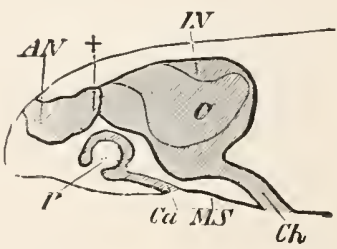

FIG. 530 .

FIG. 529.- Section of olfactory epithelium of a fish Belone (from O. Hertwig, after Blaue). $e$, epithelium; $k$, olfactory buds; $n$, nerves.

Frg. 530.-Diagrain of nose of lizard (after Wiedersheim). $A N$, outer nasal cavity; $C$, olfactory sac: $C a$, Stenson's duct: $C h$, choana; $I N$, inner nasal cavity; $M S$, roof of mouth; $P$, Jacobson's organ; $\dagger$, connexion between nasal cavities.

lateral line, to be mentioned later, of fishes and branchiate amphibians and amphibian larva, and therefore appear to subserve special and important sensations connected with aquatic life. The end buds are especially collected in the neighborhood of the mouth, on the lips and barbels. Since they also occur in the mucous membrane of the mouth, especially in the palatal regions, they connect with the taste organs. The taste buds have the same structure as the end buds of fishes. They occur in all classes of vertebrates, and are most abundant in man in the walls of the circumrallate papilla at the base of the tongue; in rodents on the large foliate papillix, etc.

Whether the end buds are related to the olfactory organs is questionable. 
The olfactory epithelium of many tishes and amphibia is a stratified epithelium with closely arranged end buds (fig. 529). It is conceivable that by disappearance of the isolating parts of ordinary epithelium the ent buds would form a continuous sensory epithelium, which is the rule in most vertebrates, yet this view meets difficulties in the finer structure of the olfactory cells.

The olfactory organ, the sose, lined with its sensory epithelium, acquires a special interest both from its grade of development and from the important systematic distinctions it affords. Except the cyclostomes, which have an unpaired nasal sac, all vertebrates have paired olfactory organs. In adult fishes and in the embryos of higher forms there are two pits which lie in front of or dorsal to the mouth; they are either distinct from it or only connected with it by an oronasal groove in the skin (fig. 556). If the animal be terrestrial and replace branchial by pulmonary respiration, a respiratory canal is developed in connection with the nose. The oronasal groove closes to a tube which begins with a nostril (naris) on the surface and ends with a second opening (choana) in the mouth carity. The olfactory sac proper is included in the wall of this tube, usually on its dorsal surface (fig. 530). In Amphibia, lizards, snakes, and birds the choana is far forward, behind the upper jaw; in alligators, turtles, and mammals it is far back, in crocodiles and some mammals (edentates) nearly to the vertebral column. This is brought about by the development of the hard palate, a parting wall which divides the primitive mouth cavity into two portions, a lower, the persistent or secondary mouth cavity, and an upper, which, as a secondary nasal cavity, continues the passage backwards. The bones of the maxillary and palatine series contribute to the hard palate, sending out horizontal processes which meet in the middle line. In mammals and crocodiles this partition is continued back by the soft palate.

A further increase in the nasal cavity is brought about by complicated folds in the walls supported by special skeletal parts, the turbinal bones, and also by the outgrowth of chambers, lined with mucous membrane which extend into the neighboring bones. Again, a part of the primitive chamber lined with olfactory epithelium can be cut off from the rest and form an accessory nose, Jacobson's organ (fig. 530, $P$ ), which opens into the mouth behind the premaxillaries by Stenson's duct. This organ is best developed in lizards, monotremes and ungulates, but of ten occurs in a reduced condition in other terrestrial vertebrates.

In all vertebrates, except $M y x i n e$ and a few forms living in the dark, the eyes are composed of all the principal constituents which occur in the human eye and which have already been briefly described (p. I I9, fig. 86). In most vertebrates it is nearly spherical, the optic nerve entering it from behind, its interior occupied by transparent, refractive substances (lens, 
vitreous body) and a fluid, the aqueous humor, and its walls of three concentric layers. The outer of these is the tough protecting sclera (sclerotic), a usually fibrous, but in many fishes a cartilaginous, layer, which in front is transparent and strongly curved, forming the cornea. The second layer, the chorioid coat, is richly vascular and pigmented; at the boundary between sclerotic and cornea it is changed to the iris. The inner layer is the retina, the structure and arrangement of which are characteristic of the vertebrates.

From the developmental standpoint the retina (fig. 85) consists of two parts, the retina proper and the tapetum nignum (pigmented epithelium), formerly regarded as part of the chorioid. In the retina the following layers are distinguished: (1) the limitans interna; (2) nerve-fibre layer; (3) ganglionic layer; (4) inner molecular layer; (5) inner granular layer; (6) outer molecular layer; (7) outer granular layer; (8) limitans externa; and (9) layer of rods and cones. The limitans externa is the bounding membrane of the embryonic retina, which is later penetrated by the rods and cones. Between the two limiting membranes Müller's fibres ("nn) extend, large supporting cells, the nuclei of which lie in the inner granular layer, and which are aided in their supporting function by the fine horny framework of both molecular layers. The nervous elements which are imbedded in this support are best understood by beginning with the optic nerve. This spreads out in the nerve-fibre layer, and on its way to the end apparatus comes twice into relation with ganglion cells; first in the ganglionic layer, second in the inner granular layer, the 'granules' being largely the nuclei of bipolar ganglion cells. Thus a great part of the retina (layers 1 to 6) are to be considered as an optic ganglion, such as occurs in molluscs and arthropods, but which there lies outside the sensory apparatus. The sensory epithelium (the retina in the sense this term is used in invertebrates) consists of but two layers, the outer granular layer and the rods and cones. The outer granules are the nuclei of the extremely slender epithelial cells which bear the rhabdomes (rods and cones) on their peripheral ends. The pigment so necessary for the visual function is supplied by the tapetum nigrum already mentioned." This is a layer of hexagonal epithelial cells which lies on the tips of the rhabdomes and sends pseudopodia-like processes between them, and since it is rich in black pigment granules, the rods and cones are enveloped in a close pigmenit mantle.

Although in this relation of pigment and in the union of the optic ganglion with the sensory cells important differences are to be noted from the eyes of the invertebrates, even from the closely similar cephalopod eye (p. 337), the most striking difference remains to be mentioned. The retina abuts with its limitans interna and nerve-fibre layer against the vitreous body; with its rhabdomes and tapetum against the chorioid. Hence the incoming light must traverse the optic ganglion and pass through the layer of sense cells before reaching the end organs, the rhabdomes. In nearly all invertebrates, for example the Cephalopoda (fig. 349), the light falls directly on the peripheral end of the rhabdome. The rhablomes in cephalopods, as in most invertebrates, are turned towards the light, in the vertebrates away from it. 
This peculiar and functionally purposeless inversion of the vertebrate retina is explained by the development of the eye. 'This can be dividerl, according to origin, into two parts, a cerebral (optic nerve, retina, tapetum) and a peripheral (all other parts). Is the eye in tunicates and Amphioxus is permanently a part of the brain, so is the retina of vertebrates genetically, coming from the first cerebral vesicle. An outgrowth occurs on either side (fig. $53 \mathrm{I}, B$ ) of the 'twixt brain and becomes expanded distally to an optic vesicle which is connected with the brain by an optic stalk. 'The vesicle extends out to the periphery and, coincidentally with the development of the lens, is folded into a double-walled optic cup with outer or tapetal, inner or retinal layers. If the position of the epithelial cells be followed (fic. 53 $\mathrm{I}$ ), it will be seen that the peripheral ends rest upon the tapetum, and when these ends develop the rhabdomes, these must grow in to the tapetal layer.
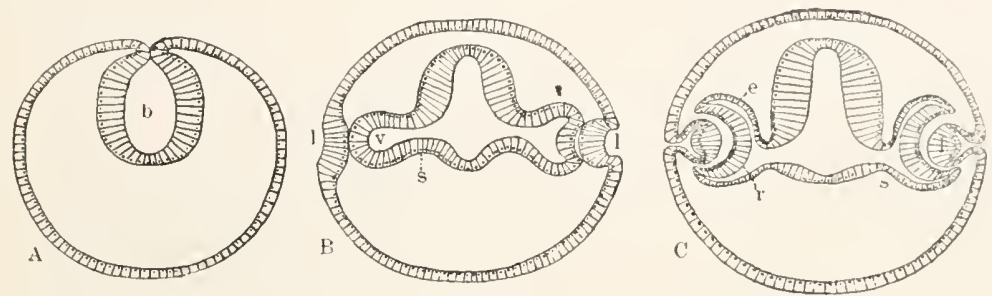

FIG. 531.-Diagram showing the inversion of layers in the formation of the retina (orig.). The nuclei are placed in the (morphologically) deeper ends of the cells. In $A$ the brain $(b)$ has been closed in; in $B$ the optic vesicle $\left(r^{\prime}\right)$ has reached the lens $(l)$ and on the right is being converted into the double-walled optic cup with, as shown in $C$, an outer tapetal $(e)$ and an inner retinal layer $(r)$.

In contrast to the retina, the lens develops as an invagination from the epithelium of the body $(l)$; sclera, cornea and vitreous body from connective tissuc. Thus the important part of the eye arises from the hrain and is later provided with accessory apparatus which arise from peripheral parts The invertebrate eye, on the other hand, with all its parts arises from the skin.

The vertebrate eye is furnished with secondary structures: with muscles which move it, with lids which protect the cornea from injury and drying. 'I he lids are dermal folds which extend over the eyeball from above and below. The lids enclose a conjunctival sac filled with lacrimal fluid, and bounded by the epithelium (conjunctiv'a) which covers the cornea. To these a third licl, the nictituting membrane, may be added. It arises as a conjunctival fold from the inner angle of the eye, and can extend over the cornea beneath the upper and lower lids. A special lacrimal gland, which occurs at the outer angle of the eye. provides the fluid to moisten the cornea, while a second or Harder's glend occurs at the inner angle when a nictitating membrane is present. Both are lacking in the fishes; there is a single gland in the Amphibia.

The ear, at the level of the medulla oblongata, has one point in common with the invertebrate otocyst-it arises as an ectodermal pit which is usually completely cut off from its parent layer, and only in elasmobranchs (fig. 532, II) remains connected with the exterior by a tube, the elsewhere closed endolymphatic duct. In the cyclostomes (fig. 532, I) it consists of a single vesicle with a single patch of sensory cells, the macula arustica; from the fishes upwards the vesicle becomes constricted into an 
upper utriculus and a 'ower sacculus, the connecting utriculo-saccular duct being narrow in the mammals. Both utriculus and sacculus receive a part of the macula acustica. Outgrowths from the vesicle occur, giving the whole the name of labyrinth. From the utriculus arise three semicircular canals, connected at either end with this cavity, each swollen at one end to an ampulla, containing a special nerve termination, the crista acustica. 'These canals stand at right angles to each other in the three dimensions of space and without doubt subserve the function of equili-

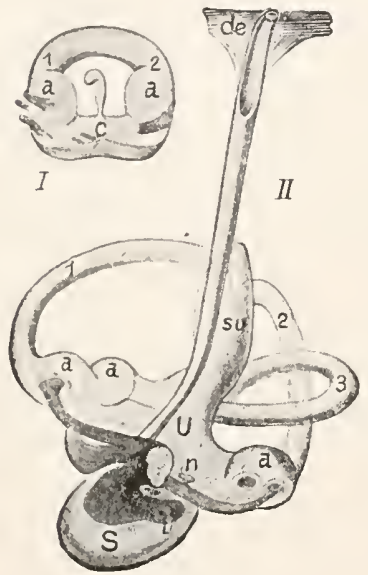

FIG. $53^{2}$.

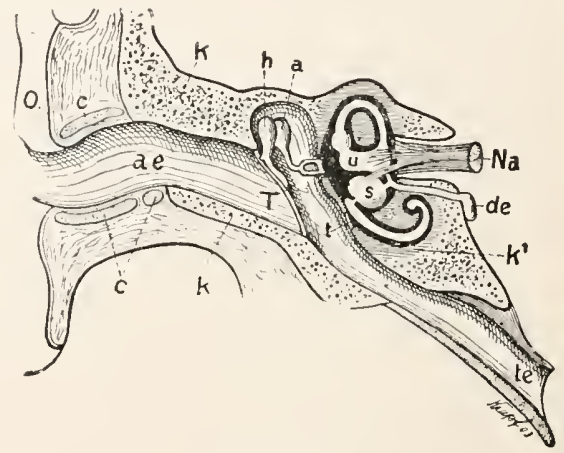

FIG. 533 .

FIG. 532.-Membranous labyrinths of (I) Myxine; (II) Chimara (after Retzius). $a$, ampullie; $c$, undivided vesicle with endolymph duct; de, endolymph duct; $l$, anlage of lagena; $n$, macula neglecta; $S$, sacculus; $s u$, sinus utriculi; $U$, utriculus; $I$, anterior, 2 , posterior, 3, outer semicircular canals. Auditory nerve and its branches dark.

FIG. 533.- - Human ear (baserl on Schwalbe). $a$, incus and stapes; ae, external meatus; $c$, cartilage; de, endolymph duct: $h$, malleus: $K^{\prime}$, temporal bone; $\mathrm{K}^{\prime}$, bony labyrinth; $N a$, nerve; $o$, base of conch; $s$, sacculus; $T$, tympanic membrane; $t$, fenestra rotunda; te, Eustachian tube; $u$, utriculus.

bration (p. II7). They are an outer horizontal, an anterior vertical (nearly sagittal), and a posterior vertical (nearly transverse). The nonampullar ends of the two vertical canals unite, a condition which is understood when it is recalled that in cyclostomes these canals alone are present, and in $I / y x i n e$ (fig. $532, I$ ) form a single canal with two ampullæ. A late formation is an outgrowth from the sacculus, which appears even in the fishes as a sriall pocket, the lagena, containing a part of the macula acustica; in the reptiles and birds the lagena becomes much larger, and in the mammals is spirally coiled and is known as the cochlea. A part of the macula acustica of the lagena develops into a special nerve-end apparatus, the organ of Corti. 
This membranous labyrinth is partially or entirely enclosed in the otic capsule of the skull, which may ossify to the otic or petrosal bones (p. 458$)$. In the birds and mammals the enclosure is such that the structure is duplicated in bone, so that the membranous labyrinth lies in a bony labyrinth, the two being separated by lymph spaces. These spaces are developerl in the cochlea into two tubes, the scala tympani and scala restibuli, the two connecting only at the tip, being separated elsewhere in part by the membranous cochlea (the ductus cochlearis or scala media). 'The spaces of the bony labyrinth are filled by two different fluids: inside the membranous labyrinth an endolymph, and between this and the walls of the bony labyrinth a perilymph.

Accessory structures may be added to this auditory apparatus proper, their purpose being to bring sound waves to it. Such structures are rarely present in fishes (it is not certain that all hear), since the sound waves are easily carried by the water to the tissues and thence directly to the ears. On the other hand, with the change to terrestrial life a sound-conducting apparatus is necessary on account of the differing densities of the air and the tissues. So we find from Amphibia onwards an air space closed by a vibrating tympanic membrane, which receives the sound vibrations from the air and carries them to a chain of ear bones (ossicula auditus), which in turn transmits them to the inner ear or labyrinth. These structures are not always functional (cetacea), and they may be wholly or in part rudimentary (urodeles, snakes, Amphisbarnids).

To understand this apparatus it must be recalled that the ear lies between the hyoid and mandibular arches in the neighborhood of a canal which leads from the surface to the pharynx. In many fishes this canal is the spiracle, a reduced gill cleft. In the Anura and amniotes it consists of an air chamber closed externally by the tympanic membrane, stretcher on a tympanic anmulus, while the opening to the pharynx is retained. The part next the nembrane becomes expanded into the tympanic cavity, this with the membrane forming the tympanum or drum. The part connecting with the pharymx is usually narrowed and is called the Eustachian tube. The membranous labyrinth touches the wall of the tympanic cavity at one or two places where the bony auditory capsule is interrupted. One of these openings (foramen oiale) is always present and is occupied by the inner end of the ear bones.

As the mandibular arch lies just in front of the spiracle, and the hyoid close behind it, it is readily understood how parts of these arches can enter the tympanum and produce the ear bones. In Anura, reptiles, and birds a columella has one end attached to the stapedial plate, which lies in the fenestra ovale, while the other is inserted in the drum membrane, the whole conveying the waves across the tympanum to the labyrinth. In the mammals the structure is different. since the columella is replaced by two bones, the malleus, which is attacherl to the drum membrane, and the incus, which articulates with the stapes (fig. 534 ). Most students believe incus and malleus to be parts (quadrate and articulare) 
of the mandibular arch-a view which has its opponents, who believe these to be a divided columella

The tympanic membrane is usually flush with the surrounding skin or only slightly below its level. In the mammals it is protected by being placed at the bottom of a deep tube, the external auditory meatus. The ear conch, a fold of skin supported by cartilage, is also confined to the mammals.

The more important vegetative organs of the body are enclosed in a large body cavity or calom beneath the vertebral column. The colom has degenerated in the head and tail region, and in the amniotes in the neck as well. The cœlom is, as development shows, an outgrow th from the primitive digestive tract, an enterocale (pp. I 47 and 263), lined with epithelium. Since it arises, as in other cœlomate animals, by paired

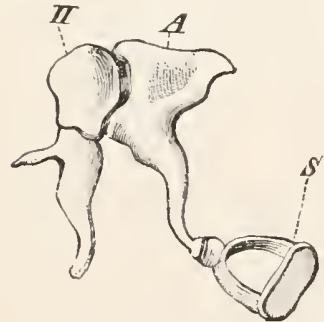

FIG. 534 .

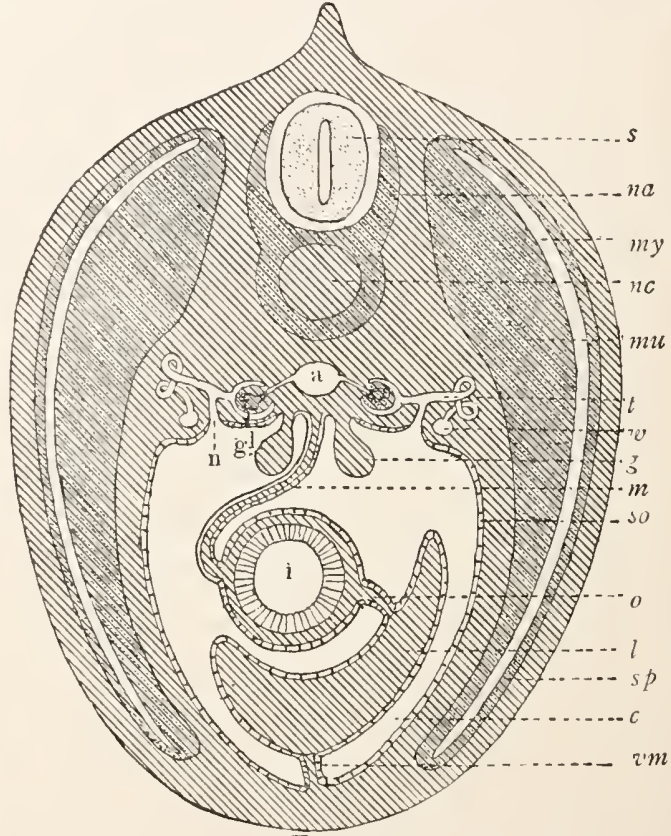

FIG. 535 .

FIG. 534.-Ear bones of man (from Wiedersheim). .1 , incus; $H$, malleus, $S$, stapes.

FIG. 535.-Section of vertebrate in abdominal region. $a$, dorsal aorta; $c$, colom; $g$, gonad; $g l$, glomerulus; $i$, digestive tract; $l$, liver; $m$, mesentery; $m u$, muscular part of mytoomes; $m v$, its coelom (myocole); o, omentum; $s$, spinal cord; so, $s p$, somatic and splanchnic epithelia; $t$, nephridial tubule; $z ' m$, ventral mesentery, w, Wolffian duct.

outgrowths from the archenteron, it follows that at first the two cavities must be separated by a partition which also encloses the intestinal tract (fig. 535). This partition furnishes the mesentery which usually supports the intestine dorsally in its whole length from the vertebral column, but ventral of the digestive tract only reaches as far back as the liver, so that right and left coloms unite behind. Some other organs are atso suspended in the body cavity by membranes: the testes by the mesorchium, the ovary by the mesozarium. In most fishes and in many reptiles the 
coelom is connected with the exterior by one or two openings (pori abdominales) beside or behind the anus.

The body cavity is frequently called the pleuroperitoneal cavity, since in mammals it is divided by a partition, the diaphragm, into an anterior or pleural and a posterior or peritoneal (abdominal) cavity. The lining membranes of these cavities are called pleura and peritoneum respectively. 'The pericardial cavity is also a derivative of the coetom, and the lining, the pericardium, but a part of the pleuroperitoneal membrane. Hence it is that in many fishes (sharks, sturgeon) a communication persists between the pericardial and the other coelom.

Of the regetative organs, the alimentary tract possesses the greatest systematic interest for it not only is concerned with digestion, but furnishes, as in all chordates, the respiratory organs (gills and lungs) as well, these arising in the non-chordates from the ectoderm. It begins with the anterior ventral mouth and ends ventrally with the anus, some distance in front of the tip of the tail; it is almost wholly entodermal in origin, there being but slight ectodermal portions at either end, which are lined with the soft 'mucous membrane' much the same in character as the entodermal part of the canal.

The first division is spacious and consists of the ectodermal mouth cavity and the entodermal pharynx, two spaces which, in most vertebrates, are not sharply marked off, but in alligators and mammals are separated by the soft palate. Then begins the narrow asophagus, widening behind to the stomach. From the hinder or pyloric end of the stomach begins the small intestine, which enlarges into the large intestine, separated from the small intestine in the higher vertebrates by a valve and one or two creca. The terminal portion in most vertebrates is called the cloaca because it receives the urogenital ducts. The lizer is the only" gland constantly present; it is a large compact organ, generally provided with a gall bladrler. Usually a smaller gland, the pancreas, occurs. The ducts of the liver (bile duct, ductus choledochus) and pancreas empty into the small intestine near the pylorus. The mouth cavity may have salivary glands connected with it, while the rectal region occasionally has blind sacs and glands.

A striking vertebrate characteristic occurs in the dentition. In the cyclostomes there are horny teeth-strongly cornified epithelial products totally distinct from the true teeth of dentine and enamel of the higher groups, which occur in places where the underlying skeleton affords them a firm support, especially on the upper or lower jaws, but they may occur on other bones of the mouth and plaryngeal cavities (roof of the mouth, gill arches). They have apparently arisen from a diffuse dentition, recalling the scales of the skin, since many elasmobranchs possess, besides 
the ordinary teeth, rudimentary teeth in mouth and pharyns. Where teeth are lacking (birds, turtles, baleen whales) they have been lost.

The respiratory organs arise from the pharynx. In the fishes and some Imphibia its walls, right and left, are perforated by gill clefts, each of which lies between two successive visceral arches (fig. 527). These are canals which open internally into the pharynx, while the outer gill openings are on the outer surface. The anterior and posterior walls of the clefts bear delicate vascular folds of mucous membrane, the gill filaments. These are the internal gills, in contrast to the external gills of larva of Amphilia and scveral fishes which are dendritic external ectodermal growths arising above and between the gill slits (figs. 4, 5). It is important for the phylogeny of the vertebrates to note that reptiles, birds, and mammals, which never breathe by gills, have gill clefts outlined and later lost with the exception of the Eustachian cleft (fig. 3).

Two problematical organs, the thymus and the lateral lobes of the thyreoid gland, develop from the epithelium of the gill clefts. The middle unpaired part of the thyreoid has been regarded as a modification of the endostyle of the Tunicata (p. 443). The thyreoid, which produces iodine compounds, is doubtless very important; disease or extirpation of it causes serious nervous disturbances.

The lungs also arise as two sacs (one occasionally remaining rudimentary), which grow downwards and backwards from the pharynx. 'They retain their opening into it either directly or by means of a trachea or windpipe, which just before its entrance into the lungs usually divides into two tubes (bronchi) (figs. 573, 589). At the opening into the pharynx (glottis) the supporting cartilages (remnants of the visceral skeleton, p. 460) are strong and form the larynx, which in mammals may be closed from the pharynx by a valve, the epiglottis. The lungs and trachea have their counterparts in the fishes in the swim bladder (a hydrostatic apparatus) and its duct.

The swim bladder of fishes and the lungs of most amphibia are smoothwalled sacs, but in some have greater respiratory surface since folds extend into the central space. In this way the bladder may become respiratory (Lepidosteus, Amia, Dipnoi). There is also a small increase of respiratory surface in the Amphibia (fig. 536, I). In the reptiles the peripheral folding increases at the expense of the single air chamber and grows inwards. The more the central chamber is divided and restricted, the more it takes on the character of a canal, lengthening the bronchus (fig. $53^{6}, 2$; turtles and crocodiles). In the mammals (3) there is no longer a central space, since the bronchus divides again and again, to fine bronchioles, which communicate with in fundihula or 'air cells' $(4, c)$ by means of an alveolar duct which is lined with vesicles (alveoli) like those of the infundibula. This comparative conception of the lung needs modification since development shows that the higher stages are not formed by the division of an air chamber by the ingrowth of walls, but by lateral outgrowths, as in a 
gland, from a growing air tube (primary bronchus) If the outpushings remain rudimentary, and the primary bronchus widens, the result is the amphilian lung. If the bronchus remain a canal and continue to develop new outgrowths of the second and third order, the complicated lung forms $(2,3)$ result.

The circulatory apparatus is easily deriverl from that of annelids, and, like it, is completely closed. In the annelids (p. 272) there is a longitudinal blood-vessel and another below the digestive tract, these being connected in each somite by loops which pass around the intestine. The vertebrate scheme varies in the development of a heart in the ventral trunk (dorsal of the annelid). In the fishes (figs. 66, 554), the heart lies close behind the gills and sends to them the blood which it receives from the body. Hence, like the whole ventral trunk, jt carries venous bloorl. Since the
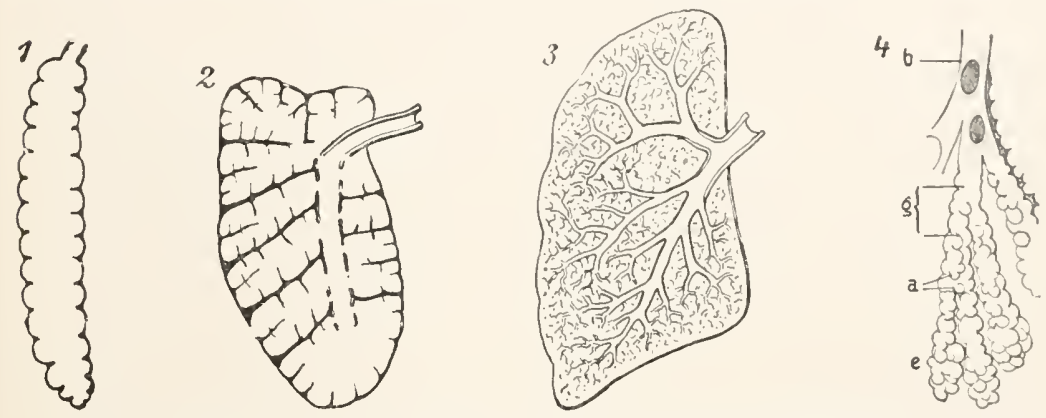

FIG. 536.-Diagrammatic long section of lungs (1) of a young salamander; (2) turtle; (3) $\operatorname{man} ;(4)$, a bronchiole $(b)$, giving off several alveolar ducts $(g)$, lined with alveoli $(a)$, and connecting with infundibula $(e)$.

anterior loops, the gill arteries, pass through the gills, the dorsal trunk, which collects from these, must contain oxygenated blood, which is sent by the carotid arteries to the head, and by the dorsal aorta and the vascular loops to the body. It there becomes venous and flows back into the ventral trunk and so to the heart.

This scheme of circulation in fishes needs further description. The heart, a strong muscular organ enclosed in the pericardium, consists of two parts, auricle (atrium) and ventricle, separated by valves. The trunk (ventral aorta) arising from the ventricle is arterial and corresponds to the ascending aorta and pulmonary artery of man. The arterial arches of the gill region which arise from it pass directly into the dorsal vessel only in young fishes (fig. 554); later they furnish the branchial circulation of gill arteries, gill capillaries, and gill veins (fig. 66). The dorsal trunk is the dorsal aorta (aorta descendens); the posterior ventral trunk, which only occurs in the embryo, is the subintestinal vein, from which the portal. vein, going to the liver, arises. To this are added a system of paired 
veins, consisting of Cuvierian ducts and jugular and cardinal zeins, the latter with growth encroaching more and more into the territory of the subintestinal rein.

The circulation of the fish type undergoes a great modification with the loss of gills and the appearance of pulmonary respiration in the higher vertebrates. Gills and gill capillaries disappear, and the branchial circulation is reduced to arterial arches leading direct from the ventral to the dorsal aorta. The swim bladder received its blood from the body (systemic) circulation, but with the functioning of the lungs pulmonary arteries and veins come into existence, while the arterial arches in part

I

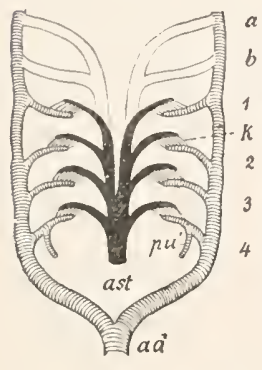

II

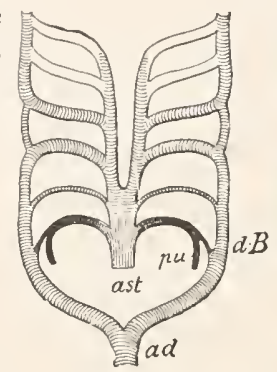

III

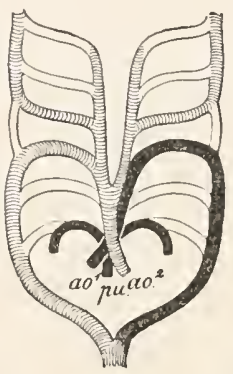

$7 V$

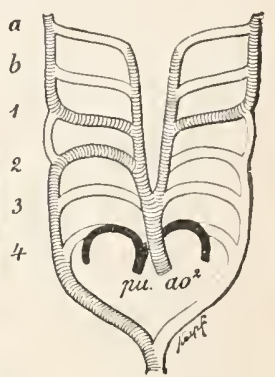

FIG. 537.-Diagram of modification of arterial arches in various vertebrate classes. White, vessels which degenerate; cross-lined, vessels containing arterial blood; black, vessels containing venous blood. I, Dipnoi; $I I$, Urodeles with pulmonary respiration; $I I I$, Reptiles; $I V$, Birds (in mammals the left instead of the right aortic arch persists). $a o^{2}$, venous aorta of reptiles; $a o^{2}$, arterial aorta; ast, arterial trunk; $a, b$, arches which usually disappear; $a d$, dorsal aorta; $d . B$, ductus Botalli; $k$, gill capillaries; pu, pulmonary artery; $\mathrm{I}-4$, persistent arterial arches.

disappear, in part are divided between the pulmonary and systemic circulations (fig. 537). Of the six arches which usually appear in the embryo, the first and second, and the fifth in animals with lungs, usually degenerate. The last arch (4), which even in the Dipnoi supplies the swim bladder, becomes a pulmonary artery, the other arches ( $\mathrm{I}$ and 2) furnish the systemic portions-the dorsal aorta (2) and the carotids supplying the head ( $\mathrm{I}$ ). Since special pulmonary veins, distinct from the systemic circulation, carry the blood from the lungs to the heart, the heart becomes divided by a septum which separates it into right and left halves. The right half retains the renous character of the fish heart; since the right auricle receives the systemic veins, the right ventricle gives off the pulmonary artery. The left half is purely arterial, receiving arterial blood by the left auricle from the lungs and sending it out through the aorta ascendens to the body. This complete separation of pulmonary and systemic circulation, and the corresponding division of 
the heart, occurs only in birds and mammals. Reptiles and amphibia show how the modification has been accomplished. In these the separation begins in the venous system and extends to the auricle, in the reptiles the septum arises in the ventricle. In the arterial

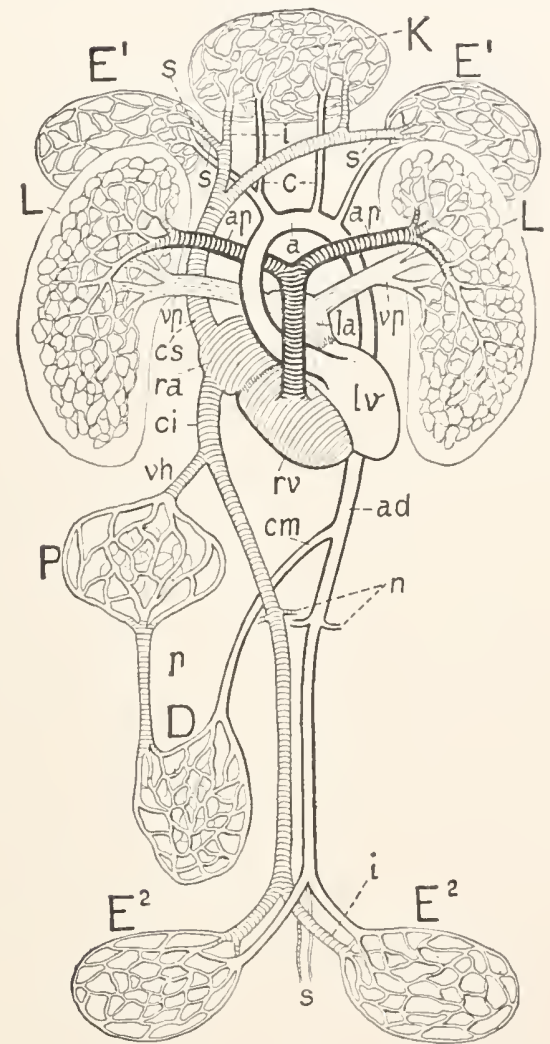

FIG. 538.-Diagram of mammalian circulation. IIeart: $r a, l e$, right and left auricles; $r v, l v$, right and left ventricles: $L$, cavillary system of lungs: $K$, cajillary system of head; $E^{\prime}, E^{2}$, of anterior and posterior extremities: $D$, of intestine; $P$, of liver (portal system). Arteries: $a p$, pulmonary arterv; $a$, ascending aorta; $a d$, descenting aorta; $c$, carotirl : $s$, subclavian; $\mathrm{cm}$, visceral (cœeliac, mesenteric); $n$, renal; $i$, iliac; $s$, sacral (cauclal). Veins: $x p$, pulmonary; $c s, c i$, pre- and postcave (precava paired in most vertebr.tes); $i$, jugular; $s$, subclavian; $z$, hepatic; $p$, portal; $n$, renal; $i$, iliac; $s$, sacral.

system remnants may persist, such as a connection (ductus Botalli) of the pulmonary with the aorta $(I I, d . B)$, or one aortic arch may arise with the pulmonalis from the right side of the heart $(I I I$, ao $)$.

As a result of this in the pulmonate vertebrates systemic blood enters the right auricle by the pre- and postcave, then passes through the right ventricle 
and pulmonary artery to the lungs. Becoming arterial there, it flows by the pulmonary vein into the left auricle and ventricle, and thence by the aorta into the systemic circulation. For details see fig. $53^{8}$.

Besides blood-vessels, lymph vessels occur as complements of the venous system. The fluids which collect in the spaces of the connective tissue are taken by them and carried (thoracic duct) into the large venous trunks. Usually the action of the heart and the movements of the body are sufficient to cause the flow of this lymph, but special lymph hearts may occur. The lymph vessels distributed to the digestive tract play an important rôle, since they serve in the resorption of digested food. They are called chyle ducts because their contents, the chyle, rendered white by oil globules at the time of digestion, distinguishes them from other lymphatics. The most important features of lymph and blood have already been noticed (p. 78 ). In special places small bodies, the lymph glands, are inserted in the course of the lymph vessels, in which lymph corpuscles arise. Among these from its structure is to be enumerated the spleen, colored bright red by its rich blood supply.

The scxual and excretory organs are so closely associated that they are generally united as the urogenital system. The sexual products are formed in the embryo in a special region of the peritoneal epithelium on either side of the vertebral column. These primordial cells arise from the entoderm, migrate into the epithelium and then early leave this position, and sink into the underlying connective tissue (fig. 34 ), forming in the male glandular tubes, in the female cords which break up into numbers of round follicles, each containing a single larger cell, the ovmm. In the male the gonads thus formed are compact and frequently oval testes; in the female they are looser and follicular or'aries.

In many fishes the sexual cells pass out by way of the body cavity and the abdominal pores, and in this case a part of the colom may be cut off as a special z'as deferens or oziduct. In most vertebrates the ducts are formed from a part of the nephridial system. The urogenital system thus formed recalls that of the annelids and in both there is the same origin of gonads and nephridia from the colomic epithelium. As the lower vertebrates (Amphibia and Elasmobranchs) show, the first stage of the excretory system is furnished by segmentally arranged canals (nephridial tubules) which open into the body cavity by ciliated funnels, the neplurostomes. In many forms the nephrostomes persist throughout life, in others they legenerate. In the higher vertebrates they are usually not formed, a fact comnected with the loss of the excretory function of the corlom and its assumption by the blood system. This has entered into connexion in a characteristic way with the nephridial tubules, and especially by the formation of glomemli, networks of blood capillaries which, pushing the wall of the tubules before them, produce the Malpighian bodics. Rarely there is a rich blood vascular development in the wall of the body cavity, 
in close proximity to the nephrostome. This may consist of several net works or they may be united to a single large glomus, situated in a special capsule of the coclom.

The excretory system of the vertebrates is farther distinguished from that of the annelids in that ( $\mathrm{I}$ ) the nephridial tubules do not open separately to the exterior but by a common canal on either side; (2) the segmental arrangement is largely obliterated by the development of secondary, tertiary, etc., tubules which produce a compact glandular body. 'The accurate explanation of these relations requires a knowledge of the different kinds of excretory organs in the vertebrates, for which we are indebted to comparative embryology. Of these there are three kinds: (1) pronephros or head kidney, (2) mesonephros or II olffian body, (3) metanephros, or true kidney, the relative positions of which along the rertebral column are indicated by their Greek names.

The pronephros is greatly reduced in all vertebrates with the possible exception of some cyclostomes; it is functional but rarely in the larra (amphibians) and is confined to the anterior segments. Whether it formerly extended farther back, as has been suggested, is questionable. Its rascular system is a glomus, belonging to the coclom and its short (pronephridial) tubules unite distally to form the pronephric duct, which grows back from its point of origin to the cloaca, into which it opens. In the elasmobranchs a so-called Miiller's duct splits from the pronephric duct, retaining its connection with the pronephros. In the other vertebrates the Müllerian duct develops from the pronephros as a canal growing from in front backwards, and opening in front into the coelom by large ciliated funnel, the ostium tubce abdominale, behind into the cloaca. Hence Miiller's duct belongs to the pronephric system. It derelops in the female into the oviduct and uterus, degenerates in the male. Whether the pronephros is an individual excretory organ, or a part of the same system is the meso- and metanephroi is disputed.

The mesonephros is a considerable organ in all vertebrates, functioning in amniotes only in foctal life, and later being replaced functionally by the metanephros and degenerating to a few inconsiderable remains in the male sexual organs. Its tuluules bear Malpighian hodies and unite secondarily with the pronephric duct, which now is called the Il olffian duct. The organ has a compact character because additional tubules are added to those first formed, these being more numerous farther back. These later tubules do not empty directly in to the duct but into the primary tubules, the terminal section of which thus becomes a collecting tulute. In the ciccilians there is a modification in that the secondary canals connect with branches from outpushings from the Nolftian duct, this 
type occurring most abundantly in the posterior part of the mesonephros, and leading some to regard this portion as a metanephros.

The metanephros, in its typical form as it appears in the amniotes, consists of two anlagen. A canal grows out from the Wolffian duct, extends cranialward, and branches richly at its anterior end. The canal is the ureter, the tubules arising from it are the so-called straight tubules B
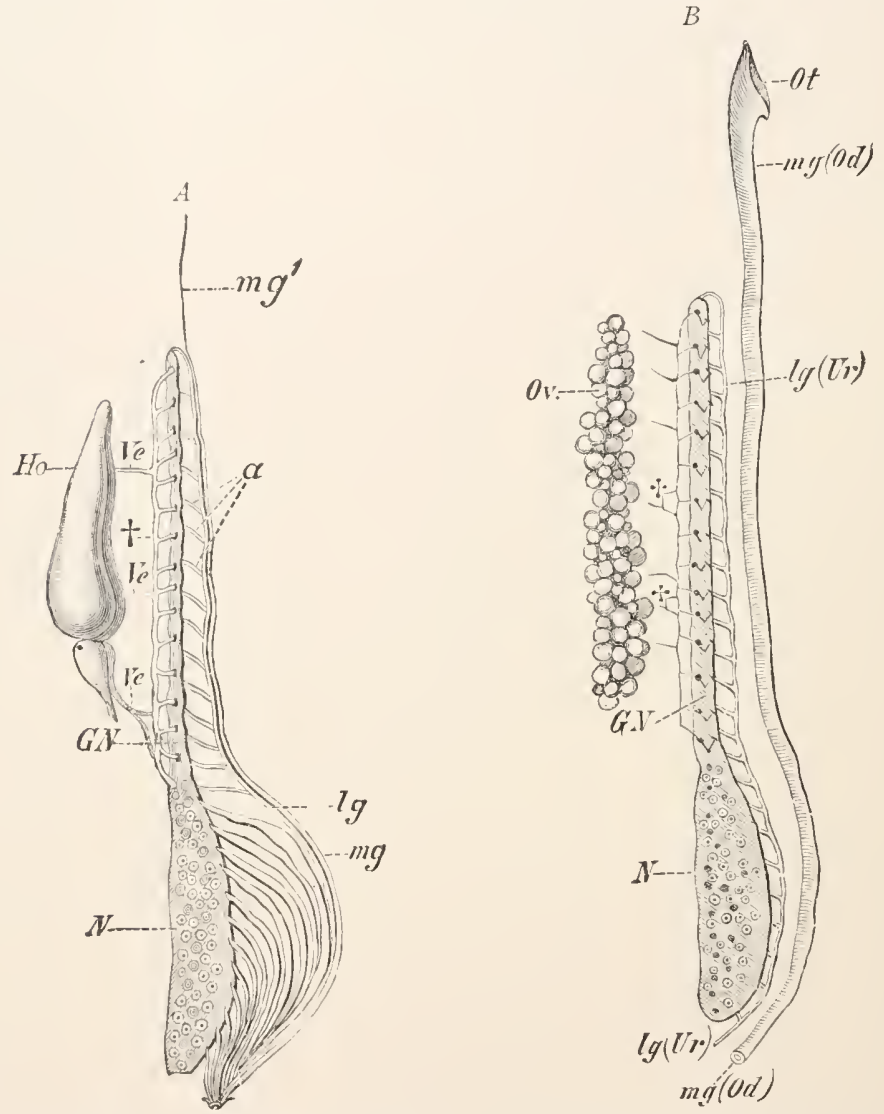

Fig. 539.-Scheme of urodele urogenital system based on Triton (from Wiedersheim, after Spengel). $A$, male; $B$, female. $a$, excretory ducts; $g h$, sexual part of mesonephros; Ho, testis; $l g$, Leydig's duct (ureter); $m g$, Müllerian duct (oviduct); $m g^{\prime}$, its restigial end in male; $N$, functional part of mesonephros; $O v$, ovary; $O t$, ostium tuba ; $V e$, vasa efferentia; *, collecting duct of vasa efferentia (rudimentary in $B$ ).

of the kidney. The other part of the organ consists of tubules, which arise from 'nephrogenic tissue,' have no nephrostomes, but include a Malpighian body at the end. The other end connects with the straight tubules. They form the convoluted tubules of human histology. 
The metanephros has the farther peculiarity of appearing late. The mesonephros functions during fotal life and degenerates accordingly as its work is taken by the metanephros. It last only a part persists, and that in the male, as a few tubules which have united with the testis and now serve as sperm conduits (epididymis); farther the Wolffian duct, which forms the was deferens, carrying the sperm; and a rudimentary structure, the paradidymis, remnants of nephridial tubules which do not connect with the testis. In the female the whole Wolffian body disappears with the exception of epoophoron and paroophoron, corresponding to the epi- and paradidymis, and remains of the Wolffian duct (Gaertner's canal).

In close connection with the kidneys, usually in contact with them, are the problematic organs, the suprarcnals. They consist of a cortical portion, derived from the peritoneal epithelium, and a central portion, developmentally connected with the sympathetic system. In the sharks the medullary portion is widely separated from the olker.

The ducts of the urogenital system open behind the anus in most fishes on a urogenital papilla; in the elasmobranchs, amphibians, birls, and most reptiles dorsally in to the hinder part of the digestive tract, which thus becomes a cloaca. In turtles and mammals the urogenital canal opens into the urinary bladder, a ventral diverticulum of the rectum which first appears in the Amphibia. Urinary and sexual ducts then either open into the urogenital sinus, the lowest part of the bladder leading to the cloaca (turles, monotremes), or this part receives only the genital ducts, while the ureters enter the base of the bladiler. The urogenital sinus remains in connection with the cloaca in the turtles and monotremes; in the other mammals a cloaca occurs only in embryonic life. Later, by formation of the perineum, the cloaca is divided into a hinder digestive and an anterior urogenital canal.

Asexual and parthenogenetic reproduction are unknown in the vertebrates. The impregnation of the eges in the lower groups is usually external and occurs during oviposition; in the higher internal copulation is effected by apposition of the genital orifices or by the development of an intromittent organ, the penis. The fertilized egg may undergo a part or the whole of its development in specialized parts of the oviduct (uterus). Accordingly viviparous and oviparous forms are distinguished, and between these extremes those that are ovoviviparous ( $f f$. p. I $5 \mathrm{r}$ ). Most elasmobranchs are viviparous, but many are oviparous. In the teleosts oviparous forms predominate, but there are viviparous exceptions. So, too, among the reptiles and Amphibia there are some viviparous species among the egg-laying majority. The birds and mammals are most constant, the first being exclusively ovoviviparous, while all the mammals with the exception of the ovoviviparous monotremes bring forth living young.

Three embryonal appendages may occur in the development, the rolk sac, the amnion, and the allantois. The yolk sac is small in those rertebrates which have some yolk, but not enough to cause merololastic segmentation (Amphibia), yet it is everwhere present and is best developed in those groups (fishes, fig. 540; reptiles and birds (fig. 54I, do) with 
discoidal segmentation, and is the result of the accumulation of the material in the digestive tract, which forces out its ventral wall like a hernia. Its presence in the mammals, which have small eggs lacking in yolk, is an indication that these have descended from large-yolked forms, such as the monotremes yet are. 'The embryo either lies directly on the yolk or is connected with it by a yolk stalk.

While the yolk sac is widely distributed, the amnion and allantois are restricted to reptiles, birds, and mammals, which are ccnsequently spoken of as Amniota or Allantoidea, in contrast to the fishes and Amphilia, which are frequently caled Anamnia or Anallantoidea, from the absence of these structures. The ammion (fig. $54 \mathrm{r}, \mathrm{am}$ ) is a sac which envelops

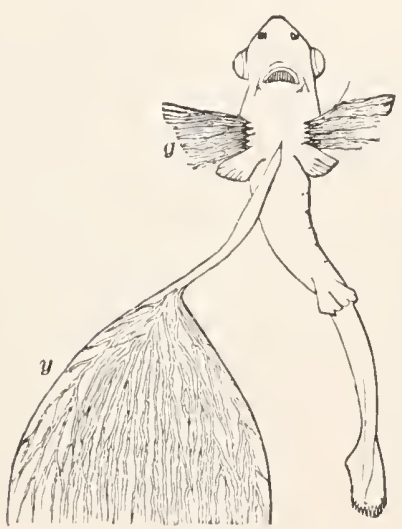

FIG. 540.

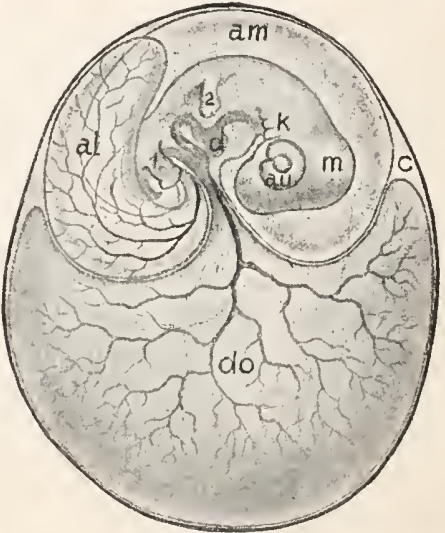

FIG. $5+1$.

FIG. 540.- Shark embryo (from Boas). $y$, part of yolk sac; $g$, external gills in front of pectoral fins.

FIG. 5+1.-Embryonic envelopes of chick (schematized, after Duval). al, allantois; am, amnion; au eye; $c$, extraembryonic colom; $d$, digestive tract; $d o$, yolk sac; $k$, gill clefts; $m$, mid-brain; I, 2 , hinder and anterior extremities.

the whole embryo and is connected with the rest only at the umbilicus, that is, the point where the yolk sac projects from the ventral wall. In this sac is an albuminous amniotic fuid. The amnion is genetically a part of the ventral surface; it develops ventrally as a circular fold-lateral, anterior, and posterior-which grows up over the back on all sides and unites above the embryo.

The allantois (al) is an enlargement of the urinary bladder. This grows out from the borly cavity at the umbilicus and extends between yolk sac and amnion and then grows in all directions until its folds meet above the hack carrying with it blood-ressels and connective tissue. The blood-vessels are the most important, for the allantois forms the respiratory 
apparatus of the embryo, and in the mammals it develops the placcula, by which nourishment as well is conveyed to the young. Yolk sac, amnion, and allantois are enveloped in a common coat, the serosa.

Aristotle recognized four divisions of vertebrates, and these were retained by Linné and Cuvier under the names Pisces, Reptilia or Amphibia, Aves, and Nammalia. Blainville divided the second of these into two classes, retaining the name Reptilia for one, Amphibia for the other. Nilne Edwards showed that this division corresponded with one between the higher and lower groups, the amniote and the anamniote divisions. Later Haeckel separated the Cyclostomes from the other fishes as a distinct class, while Huxley pointerl out the close resemblances between reptiles and birds, uniting them as Sauropsida. Another convenient division contrasts the fishes with all other forms, the Tetrapoda, so called because they have legs rather than fins.

\section{Series I. Ichthyopsida (Anamini, Anallantoida).}

Vertebrates respiring for a time or throughout life by means of gills; neither amnion nor allantois present in the embryo.

\section{Class I. Cyclostomata (Marsipobranchii, Agnatha).}

The class of Cyclostomes contains but few species of lamprey eels and slime or hag fishes. In shape they are eel-like. They are distinctly vertebrate in the possession of large liver and nephridia; of a muscular heart with auricle and ventricle, lying in a pericardium; olfactory lobes, epiphysis and hypophysis, and the higher sense organs. In the brain, cerebrum and cerebellum are not so prominent as are the optic lobes and medulla. The inner ear (fig. 532, I) is not divided into utriculus and sacculus, and it has but one or two semicircular canals, but always two ampullæ. The stin (fig. 27) consists of corium and a stratified epidermis.

The cyclostomes are distinguished from the true fishes by the lack of a vertebral column. The axial skeleton of the trunk consists either of the notochord alone or of it and small cartilaginous arcs, representing neural arches and intercalaria. A cranium and a basket-like gill skeleton are present, but so different are these from those of other vertebrates that homologies are difficult. The absence of paired fins is important. The median fins are supported by horny threads alone, and the cartilaginous appendicular skeleton-alone of importance-is entirely wanting. 'Then the skin lacks scales, and the mouth true dentine teeth, for the pointed teeth, arranged in circles in the mouth of the lamprey (fig. 5+2), and the fewer teeth of the myxinoids, are purely epidermal products and cannot be compared with the teeth of other vertebrates.

The name Cyclostomata refers to the circular mouth, which rests on the important fact that the jaws are absent (Agnatha) or extremely 
rudimentary, and clo not close on each other as do the jaws of other vertebrates. This cyclostome condition is of value to the animals, as it aids them in sucking on to other animals. At the base of the dome-like mouth cavity is the so-called tongue, which is the sucking apparatus, since it can be drawn backwards like, a piston.

The name Marsipolranchs refers to the form of the gills, usually six or seven in number, but in Bdellostoma may be twelve or fourteen on either side. Fach gill cleft consists of three parts, the gill sac (marsupium),

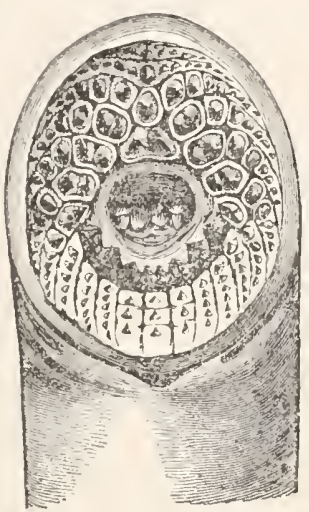

FIG. 542 .

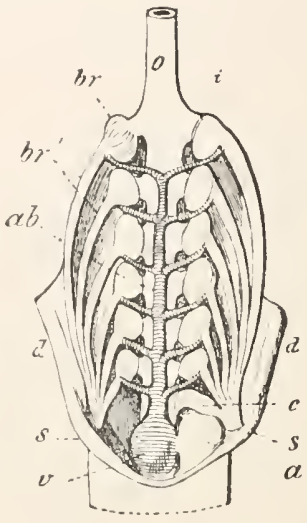

Fig. 543 .

Fig. 542.- Nouth of Petromyzon marinus with horny teeth and tongue (from (jegenbaur).

Fin. 543-- Gill apparatus of Myxine glutinosa (after J. Müller). a, atrium; $a h$, gill artery and gill arch; $h r$, gill sac (the lines show the gills); $b r^{\prime}$, efferent canal; $c$, u'sophageo-cutaneus duct; $d$, skin turned away; $i$, afferent gill canal; $o$, cesophagus; $s$, mouth of atrium; $v$, ventricle of heart.

which contains gills, and the afferent and efferent ducts (fig. 5+3). These ducts develop separately, and may continue so (Bdellostoma), but in Pctromyzon the afferent ducts unite to a single tube which opens ventrally in the pharynx. In Myxine (fig. 543) the conditions are reversed, the efferent canals of a side uniting to empty through a single external opening.

1 third name, Nionorhina, has been given, since these forms, in contrast to all other vertebrates, have an unpaired olfactory organ. The single nostril, lying in the mid line of the head, opens into a nasal sac, from the bottom of which a canal descends towards the roof of the mouth, ending blindly in Petromyzontes (Hyperoartia), or penetrating the palate in the Myzontes (Hyperotretia), so that an inner nasal opening (choana) into the pharynx occurs. A paired olfactory nerve supplies the organ. 
Sub Class I. Myzontes (IIyperotretia).

Semiparasitic cyclostomes with cirri around the mouth, very primitive nephridia, eyes rudimentary. From the large amount of mucus they are known as slime eels. They bore into fishes and eat the flesh. Wyrine, * east coast; Bulllostoma,* west coast.

\section{Sub Class. II. Petromyantes (Hyperoartia).}

The lampreys (Petromyzon*) liave well-developed dorsal fins, and seven gill openings. They occur in salt and fresh water, some marine species ascend-

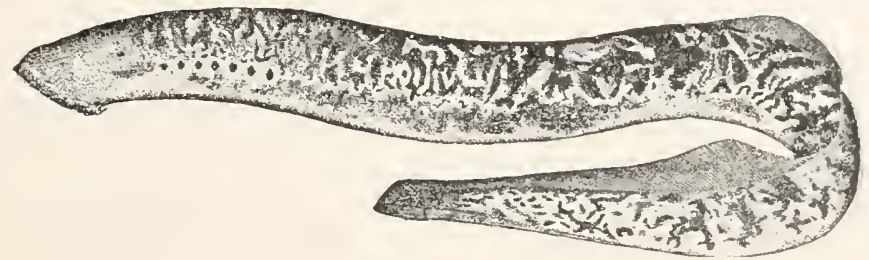

FIG. 544.-Petromyzon marinus, * sea lamprey (after Goode).

ing streams to lay their eggs. The young have a larval (Ammocotes) stage with rudimentary eyes and slit-like mouth. Many species live on mucus and blood which they rasp from fishes.

Here may be mentioned a group of paleozoic fossils, the OSTRACODERMII, of uncertain position. They have fish-like bodies, but no skeleton or jaws are known. Pteraspis, Cephalaspis, Pterichthys.

\section{Class II. Pisces (Fishes).}

The term fish is used in a wider and a narrower sense. In the first is includes any aquatic vertebrate swimming by means of fins and breathing by gills; in the strict sense, as used here, it means aquatic branchiate forms with vertebral column, cranium, and well-developed visceral skeleton; with paired and unpaired fins, supported by a cartilaginous or bony skeleton; with double nasal pits; with a skin and oral mucous membrane which can produce ossifications, the scales and teeth. The fishes are the best adapted of all vertebrates for an aquatic life, and their whole organization must therefore be considered from this standpoint.

The epidermis consists of numerous layers of cells with an extremely thin cuticle. Cornifications of this epidermis are lacking under ordinary conditions, except a thin portion of the external subcuticular layer. It the time of sexual maturity cornifications increase greatly in most Cyprinoids and many Salmonids, producing hard bodies in the skin, the pearl organs. Enormous numbers of large slime cells give the fishes their well-known slippery skins. All protective structures arise from the corium, which is composed of many layers of dense connective tissue and 
furnishes the characteristic dermal skeleton, the scales. These lie at the boundary of epidermis and corium, commonly imbedded in pockets of the latter, and are, on account of their different structure, of systematic value, although the classification based entirely upon them is no longer retained.

The placoid scales (figs. 510, 545, 4) have already been mentioned, because they form the starting point for dermal ossifications and teeth (p. 45r). They are rhombic bony plates, usually close together like a mosaic, but not overlapping. In the centre of each is a spine, directed
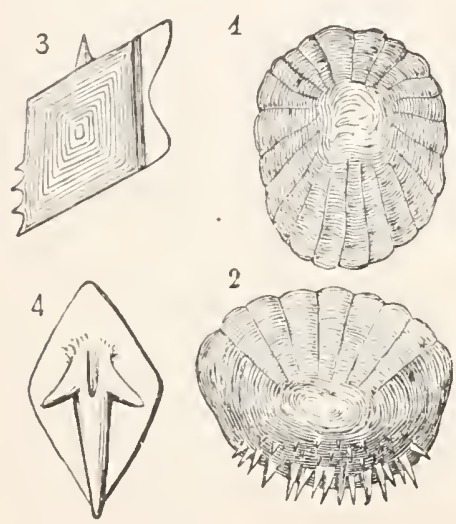

FIG. 545.- Scales of fishes. I, cycloid; 2, ctenoid; 3 , ganoid; 4, placoid. backwards, in which is a pulp cavity, while the tip of the spine is covered with a cap of hard substance, variously called enamel or vitrodentine.

The ganoid scales (3) are usually rhomboid and arranged like parquetry. In the early stages they may bear teeth, but these are lost in the adult. The outer surface is always covered with a thick layer of ganoin, which gives, even in fossils, an iridescent effect, a most characteristic feature. The ganoin is no longer regarded as enamel, but the most superficial layer of dentine (vitrodentine).

Cycloid and ctenoid scales are closely related. They are always more loosely placed in the pockets, from which they are easily withdrawn as in 'scaling' a fish. They are arranged in oblique, transverse, and longitudinal rows, and overlap like shingles, one scale covering the parts of two scales behind. The cycloid scales (I) are approximately circular, marked on one side by concentric lines, while on the other numerous lines radiate to the periphery. The ctenoid scale (2) has the radial and concentric lines of the cycloid, but has the hinder edge truncate and the free portion bearing small spines or teeth, processes of the concentric ridges.

Besides these types of scales many fishes bear considerable spines (strongly developed single scales) and larger bony plates, these last usually resulting from the fusion of numerous scales.

The coloration of fishes may have three sources. The silvery lustre is due to crystals of guanin which occur in the skin and in the peritoneum and pericardial walls. In some fishes (Alburnus lucidus) from their iridescence these crystals become of commercial value, forming the important part of essence of pearl, used in making artificial pearls. The other colors of fishes are due in part to the numerous strongly pigmented fat celis, in part to chromatophores in 
the corium, which, under control of the nervous system, can alter their form and extent and thus produce color changes in the fish, thus adapting them to their surroundings. Destruction of the eyes results in loss of power to change color. Many deep-sea fishes possess phosphorescent organs (P. I I I, fig. 87), possil)ly of use in the assemblage of the sexes.

The axial skeleton shows many conditions unknown outside the class, and varies in character from group to group, the most important differences consisting in its cartilaginous or bony character. The vertebre are nearly always amphicoelous, the notochord persisting in the cavities between the successive centra (fig. 513). Neural and hxmal arches, completed by the unpaired spinous processes occur. The neural arches extend throughout the column; the hamal are complete only in the tail; in the trunk the hremal spines are absent and the hrmal processes, divided into basal processes and ribs, surround the viscera. Ithen ossification is lacking or is incomplete, two paiss of arches may occur in each segement, the anterior being the stronger and alone persisting in fishes with ossified vertebre; the second is much smaller, so that its elements are not called arches, but intercalaria (figs. 512, 546). No fish has a sternum.

The great number of visceral arches, and their independence from the cranium, are characteristic of fishes. After removal of these the cranium in all cartilaginous fishes is very simple (fig. 546), but in the teleosts, with the appearance of ossification, it becomes very complicated, since the bones are numerous. There are also great differences between the different families of fishes, some having bones which are lacking in others (figs. 5 I 6,547$)$.

The large membrane bones of the cranial roof (parictals, $p$, frontals, $f$, and nasals, na) and the large ventral parasphenoid ( $p s)$ are especially constant. The vomer $(v o)$, in front of the parasphenoid, is unpaired, while in all other vertebrates it is paired. Most constant of the cartilage bones are the ethmoids (the paired ectchmoids, ec, and the sometimes paired mescthmoid), and the four occipitals. On the other hand the otic and optic regions vary considerably; the otic region usually has five bones (fig. 547 ): plerotic, pto, often called squamosal; sphenotic, spho, frequently called posifronlal; cpiotic, epo; prootic, pro; and opisthotic, oo, the last sometimes lacking. In the region of the eve the cartilaginous sphenoids are rarely well developed, the large parasphenoid taking their place. The same is true of the ali- and orbitosphenoids, these sometimes forming an interorbital septum (fig. 5I6) or leaving a more or less wide interorbital fenestra (fig. 547).

The character of the visceral skeleton is related to the aquatic life. All fishes have numerous gill arches (five to seven, mostly five), which, since their function-gill supporting-is similar, are similar in structure. So far as they are not degenerate they consist each of four parts and are connected below by unpaired copula, these often being fused. The 
upper ends are frequently toothed and, in chewing are opposed by the rudimentary last arch, on which account these are spoken of as the superior and inferior pharyngeal bones. The anterior visceral arches are greatly different in cartilaginous and bony fishes. In the former (fig. 546) the pterygoquadrate $(p q)$ and the Meckelian cartilage $(M d)$ bear teeth and oppose each other in biting. In the bony fishes (fig. 547) the teeth of the lower jaw oppose the tooth-bearing elements, premaxillary and maxillary, of the maxillary series, while the pterygoquadrate elements-the palatine and the series of pterygoids-are the antagonists of the hyoid. In the elasmobranch type the two halves of the pterygo-

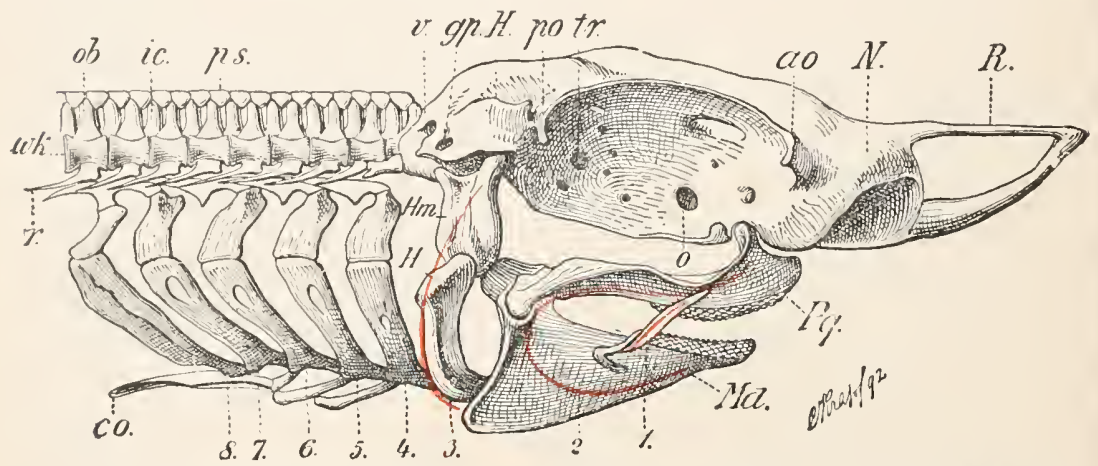

FIG. 546.-Cranium, visceral arches, and part of vertebral column of IIustelus 2'u'saris. ao, antorbital process; co, copula; $g p$, foramen for glossopharyngeal; $H$, otic capsule and hyoid; $H m$, hyomandibular; $i c$, intercalare; $M d$, mandible (Meckel s cartilage); $N$, nasal capsule; $c$, optic foramen; $o b$, neural arch; $p o$, postorbital process; $P q$, pterygoquadrate; $p s$, spinous process; $R$, rostrum; $r$, ribs; $t r$, trigeminus foramen; $v$, vagus foramen; $1-8$, visceral arches; $x$, labial; 2 , mandibular; 3 , hyoid; $4-8$, gill arches.

quadrate meet by symphysis in the middle line; in the others they are separated by the floor of the skull.

A second characteristic of the bony fishes is already outlined in the cartilaginous fishes: the modification of the hyomandibular to a suspensor of the jaws. In the elasmobranchs (especially the skates) the parallelism of hyoid and mandibular arches is lost, the hyomandibular separating from the hyoid and attaching itself to the hinge of the jaws. In the teleosts the hyomandibular is thus brought in connexion with the quadrate, and lies between it and the cranium, the joint being thus indirectly supported from the cranium, a bone, the symplectic (known only in fishes) helping out the suspensor, while another bone, the interhyal, connects this with the hyoid, which itself divides into two, so that the hyoid arch, like a gill arch, consists of four elements. 
The opercular appara!us does not occur in all fishes. It consists of a number of bony plates and processes which arise from the hyoid arch and extend backwarks over the gills, protecting them. It arises in part (opercular bones-O, Pro, So, Io, fig. 5+7) from the hyomandibular, in part (branchostegal rays) from the hyoid bone. The significance of this apparatus will be spoken of in connection with the gills; it gives the fish skull a definite character, but hides its structure, on which account it, like the infraorbital ring, is shown in red in the figure.

$$
\text { epo ple r pro sptho fro of me na. }
$$

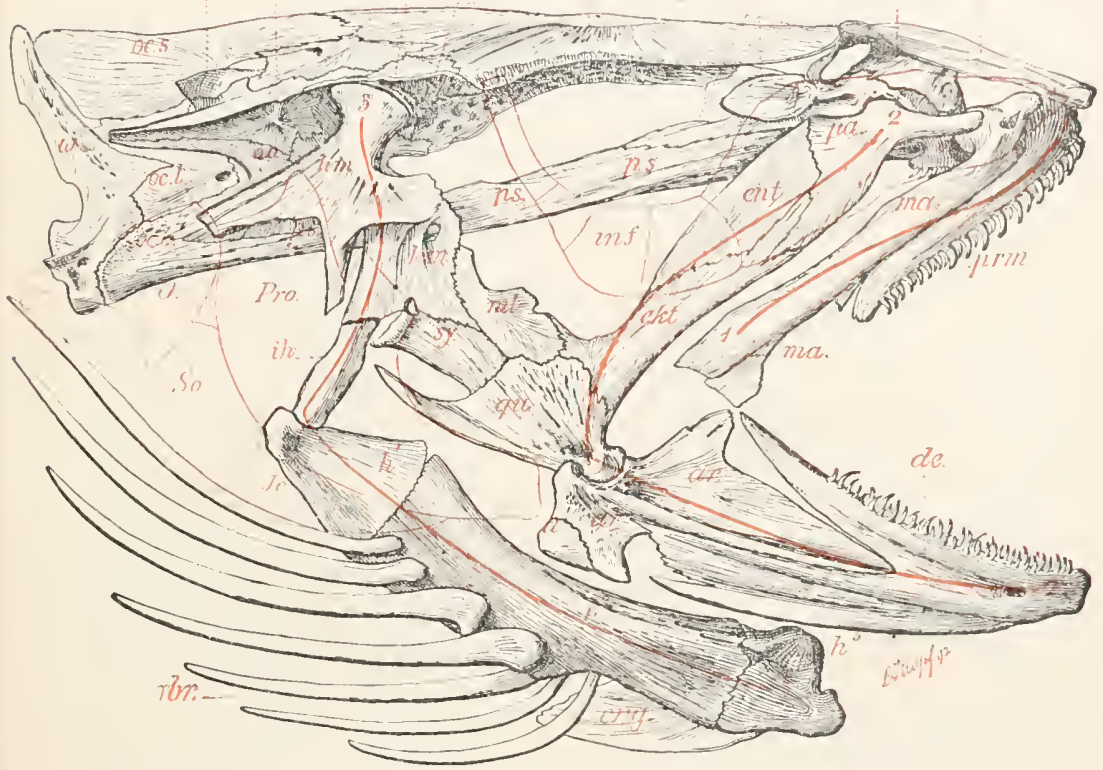

FIG. 547.-Skull of haddock. Infraorbital ring and operculum outlined in red. $a$, angulare; ar, articulare; as, alisphenoid; de, dentary; ee, ectethmoid; eht, ectopterygoid ; eng, os entoglossum; ent, entopterygoid; epo, epiotic; $f r$, frontal; $h^{1}-h^{3}$, hyoid elements : $h m$, hyomandibular; $i h$, interhyal; ma, maxilla; me, mesethmoirl; mt, metapterygoid; $n a$, nasal; $o c b, o c l$, ocs, basi-, ex-, and supraoccipital; oo, opisthotic; $p$, parietal; pu, palatine; prm, premaxillary; pro, prootic; $p s$, parasphenoid; plo, pterotic; qu, quadrate; rbr, branchiostegals; spho, sphenotic; sy, symplectic; t'o, vomer; w', rertebra. Bones outlined in re l; inf, infraorbital; Jo, interoperculum; O, operculum; Pro, preoperculum; So, suboperculum; 1, 2, 3, axes of labial, mandibular, and hyoid arches.

The appendages are also influenced by the aquatic life. In contrast to the cyclostomes, there are two pairs of paired fins, the thoracic or pectoral and the pelvic, ventral, or abdominal fins; in contrast with Amphibia, reptiles and mammals, which occasionally have fin-like structures, the fishes have three unpaired fins, dorsal, caudal, and anal fins. Only rarely, as in the eels, the ventral fins are lacking; more rarely (Muranidix) the pectorals 
are lost. The function of the fins in swimming and in balancing makes it necessary that they be broad and well-supported plates. Hence numerous skeletal parts are present; besides those preformed in cartilage, numerous horny or bony rays; further, that all parts should be similar and closely, even if flexibly, bound to each other. Joints are unnecessary except at the base where the fins join the supports and move upon the body. The supports of the paired fins are the girdles, pectoral and pelvic, arched skeletal parts, which in the sharks are held only by muscles, a statement which is true for the pelvic girdle of all fishes. This is why the ventral fins so readily change their place. Their primitive position is at the hinder end of the body cavity (Pisces abdominales, fig. 559). From this point they can move forward to beneath the pectorals (Pisces thoracici, fig. 560 ), or may even come to lie in front of them (Pisces jugulares) in the throat region (fig. $5^{61}$ ). The pectoral arch is united to the vertebral column in the skates; in the teleosts it is covered by a large membrane bone (clavicle or cleithrum), and connected to the epiotic region of the skull by a chain of small bones.

The dorsal and anal fins are supported by elements, preformed in cartilage, which rest upon the neural or hxmal spines and in turn support the fin rays. In the caudal fin the rays rest directly upon the spinous processes. Three types of caudal fin are recognized (fig. 10)-distinctions of great importance. The primitive type is the diphycercal, in which the vertebral column extends directly into the middle of the fin, dividing it into symmetrical halves. In the heterocercal type the vertebral axis binds upwards at the base of the fin, so that the dorsal part is reduced, the rentral greatly enlarged, the result being extremely asymmetrical, as seen from the exterior. The homocercal fin is symmetrical externally, but in reality is extremely asymmetrical. The end of the vertebral column is bent abruptly upwards, and hence the fin is almost entirely formed of the rentral portion, which is usually divided by a terminal notch into upper and lower halves. The homocercal fin begins with a diphycercal and passes through a heterocercal stage in development.

In correspondence with the simple motions the musculature is simple and consists largely of longitudinal muscles divided into myotomes, which are conical with the apex in front, and are so inserted in each other that a cross-section gives concentric circles. In a section there are at least two such systems, the muscles being divided by a lateral partition into dorsal and ventral halves. There are also smaller groups of muscles related to fins, gill arches, jaws, etc., but of much smaller size, derivatives from the larger mass. Reference has already been made (P) III) to the modification of muscles into electric organs.

The brain shows the low position of the class in the slight development of the cerel)rum. This is especially true of the telensts (fig. 548), in which, in place of a cortex, there is only a thin epithelial layer (Pall), what was formerly 
called cerebrum being only the corpora striata $(B G)$. The independent olfactory lobes lie either close to the cerebrum (most teleosts, Lol) or are separated from it by an olfactory tract (fig. $549, \mathrm{Lol}$ ). The optic thalami are small $(d)$, but below them are enlargements characteristic of fishes, the lobi inferiores, and between them the saculus vasculosus. Both optic lobes and cerebellum are greatly developed.

The nose consists of two preoral pits, the opening being divided by a bridge of skin into anterior and posterior nostrils. In many selachians the nostrils are connected with the mouth by a oronasal groove covered ly a fold of skin, and in the Dipnoi there is a choana. The eye has several peculiarities. The

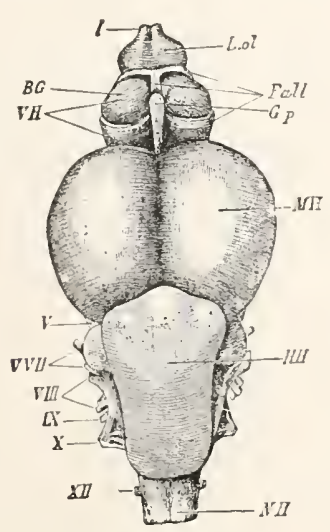

FIG. 54 S.

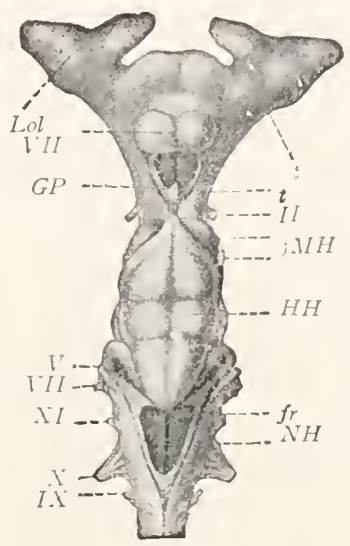

F1G. 549 .

Fig. 548.-Brain of trout (after IViedersheim). $B G$, corpus striatum; $G P$, epiphysis; $H H$, cerebellum; $L o l$, olfactory lobes; $/ H$, optic lobes; $N H$, medulla oblongata; Pall, pallium, in part cut away; I" $H$, cerebrum; $I-\mathrm{I} I I$, nerves. (See p. $47 \mathrm{I}$ ).

FIG. 549.--Brain and nasal capsules of Scyllium catulus (from Wiedersheim). fr, fossa rhomboidalis; $t r$, olfactory tract.

lens is very convex, almost conical, and the eye is very short-sighted because light is so absorbed by water that objects forty feet away are invisible. With this is connected the campamula. Halleri. 'The processus falciformis, a sickleshaped outgrowth of the chorioid, extends from the entrance of the optic nerve into the vitreous body as far as the lens, swelling out into the campanula; this contains a muscle which draws back the lens and so is an apparatus of accommodation. Chondrifications and ossifications of the sclera are common. Lirls are weakly developed or absent, and only some elasmobranchs have a nictitating membrane.

The ear has a relative size found in no other vertebrates, the labyrinth (fig. 532) has the sacculus and utriculus separated, the sacculus with a diverticulum, the lagrena, the beginning of a cochlea. In the labyrinth of the teleosts there are two large otoliths. Experiments show that the ear is especially an organ of equilibration, and in some an organ of hearing to a limited extent. I few species have the power of making a noise, usually by the rubbing of parts on each other.

Of all sense organs of the skin, the most noticeable are those of the latcral line, which occur elsewhere only in cyclostomes and aquatic amphibia. In fishes a line on either side usually begins at the tail and extends to the head, where 
it divides into several curved lines (fig. $559, S l$ ). It is caused by a groove or a canal bencath the scales which opens to the exterior by numerous canals through the scales. The special sense organs (neuromasts) lie in the epithelium of the canals They may also occur in special pits in the skin (ampulle of Lorenzini, Sari's vesicles of the elasmobranchs), or they may occur separated in several rows (Amphibia). Those of the head are irnervated by branches of the facial nerve, those of the trunk by the lateralis branch of the vagus. These nerves have their cerebral connections in close association with the auditory nerve, which would imply a similarity of function. Since these organs are lacking in mammals and man it is difficult to determine their function. Experiment scems to show that they are for the preception of water cu"rents and indirectly the proximity of solid bodies. Mention has already been made of the nerve end luds (taste) which, besides occurring in the skin, are especially abundant in the mucous membrane of the mouth as well as on the lips and barbels

The alimentary tract is spacious only in the oropharyngeal region. Then it narrows to a slightly convoluted tube in which the various regions
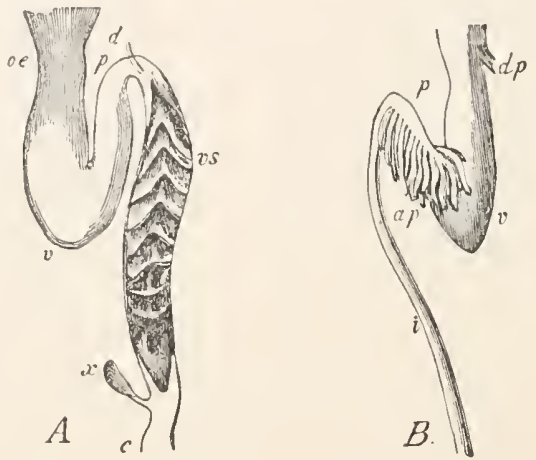

FIG. 550.-Digestive tracts of (.1) Squatina inlgaris (partly opened) and (B) Trachinus radiatus (from Gegenbaur). ap, pyloric ceca; $c$, rectum; $d$, bile duct; $d p$. duct of air blardder; $i$, intestine; oe, cesophagus; $p$, pylorus; 2 , stomach; $v s$, spiral gland; $x$, rectal gland.

are not sharply marked off from each other. Mouth and pharynx frequently bear teeth. In the teleosts the bones of the roof of the mouth and the risceral arches may be covered with coalesced heckel-like teeth. In the elasmobranchs the lining of the mouth, like the external skin, is frequently covered with small dermal scales; the large chewing teeth are confined to the edges of the Meckelian and pterygoquadrate arches, where they are implanted in several rows behind one another. Since the teeth are held only by membrane and are easily torn out, they may be replaced indefinitely. Liver and spleen are always present; pancreas and gall bladder usually occur. In many fishes blind sacs, the pyloric caca, occur at the junction of stomach and intestine (fig. $550, B$ ); others have a spiral valie $(A)$, a fold of mucous membrane, which extends like a spiral 
stairway into the lumen of the intestine, increasing the digestive surface. Caca and spiral valve rarely occur in the same fish (Lamargus, ganoids).

Gills of two types occur (fig. 55I). In both the gill clefts, which lie between successive branchial arches, begin by openings in the pharynx, but differ in their external openings. In the elasmobranch type ( 1$)$ the
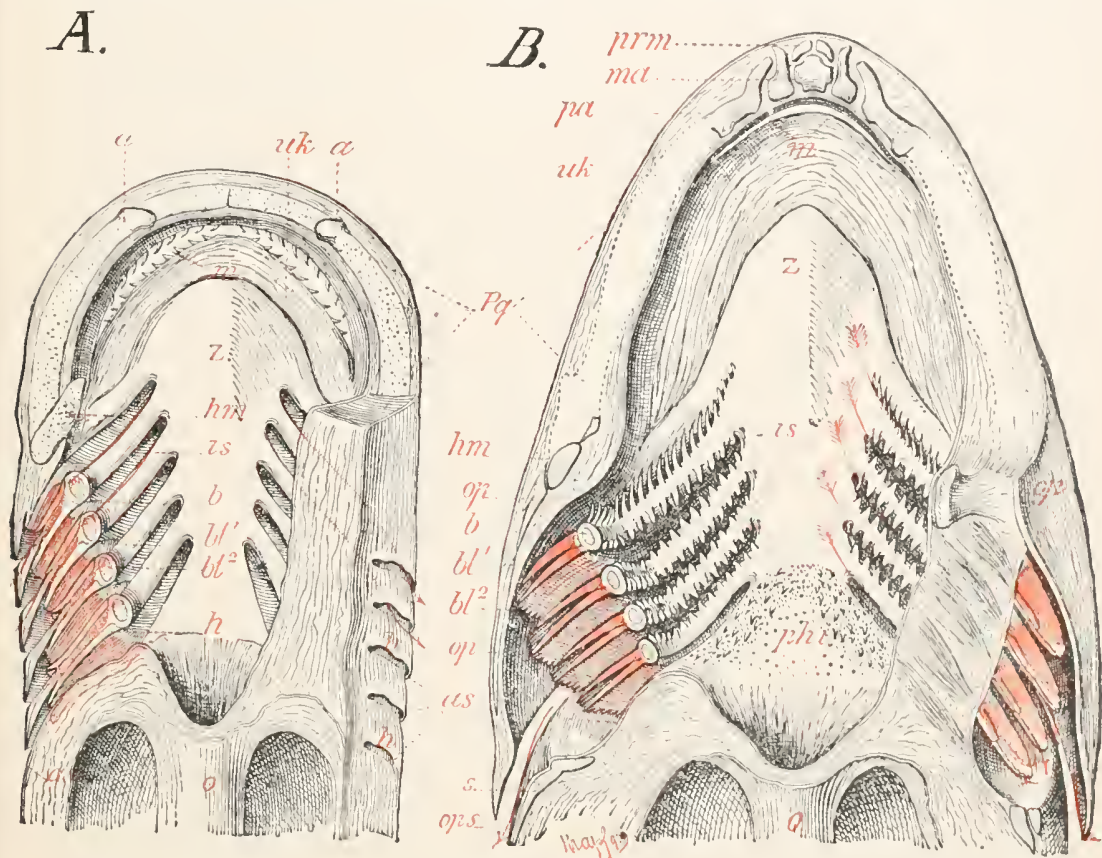

FIG. 55 I - Pharynges of (A) Elasmobranch (Zvgrena) and (B) Teleost (Gadus), the skull removed and on the left the gill slits cut across. $a$, attacnment of upper jaw to cranium; $a s$, outer gill slit; $b$, gill arch; $b l^{1}, b l^{2}$, anterior and posterior gills (demibranchs); $h$, dermal projection; $h m$, hyomandibular; $i$ inner gill cleft; $m$, mouth; ma, maxillary; $o$, nesophagus; $o p$, operculum; ops, opercular opening; $p a$, palatine; $p h i$, inferior phary $n$ geal bones; $p q$, pterygoquadrate; $p r m$, premaxilla; $s$, shoulder girdle; $u k$, lower jaw; $z$ tongue.

external openings are a series of slits separated by broad dermal bridges which cover the gills (fig. 555). The gills are vascular folds of mucous membrane with secondary folds distributed on anterior and posterior sides of the cleft. Each arch except the last, as the sections (figs. 55I, 552) show, bears two rows of gill folds (demibranchs) which belong to different clefts and are separated from each other by tissue containing the cartilaginous gill rays.

In the second type $(B)$, which occurs in all other fishes, the dermal bridges are lacking, and the septum between the demibranchs has more 
or less completely disappeared, so that the demibranchs of one arch become connected, their free ends projecting into the water like the teeth of a double comb. Ifere, on account of their very delicate structure, they would be exposed to serious injury were they not protected by the operculum $(o p)$ or gill cover. This is a fold of skin arising from the hyoid arch and extending back over the gill region. It is supported by two groups of bones, the opercular bones proper (fig. $547, \mathrm{O}, \mathrm{So}, \mathrm{Io}, \mathrm{Pro}$ ), attached to the hyomandibular, and the branchiostegals $(r b r)$ from the hyoid, these latter supporting the branchiostegal membrane. Between the free edges of the operculum and the branchiostegal membrane and the skin of the body behind is the opercular cleft (fig. 55 $\mathrm{I}$,ops), which is

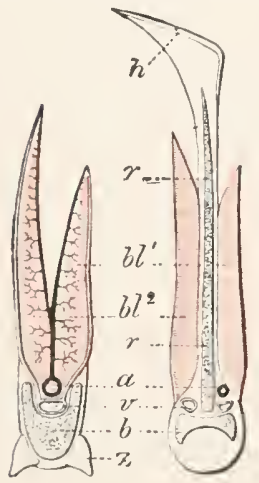

FIG. 552.- Sections of gill arches of Gadus (left) and Zyg ena (right), slightly enlarged. $a$, artery; $b$, gill arch; $b l^{1}$, $b l^{2}$, demibranchs; $h$, dermal projection; $r$, cartilage ray; $v$, vein; $z$, tooth. obviously not identical with a gill cleft, but leads into an atrium into which the gill clefts empty.

In many elasmobranchs and ganoids there is a rudimentary cleft, the spiracle, between the pterygoquadrate and hyomandibular, in which a rudimentary gill, or pseudobranch, may occur, this often persisting when the spiracle is closed. The gills proper develop from that part of the cleft derived from the skin, they are therefore ectodermal in origin, agreeing with the external gills of the amphibian larvæ, a matter in which the two groups were thought to differ. This explains the existence of external gills in Protopterus and the larvie of Lepidosiren and Polypterus.

Besides gills, fishes, with the exception of elasmobranchs and some teleosts, have a swim bladder, usually regarded as the homologue of the lungs. It is often shaped like an hour glass, filled with air, and may open into the oesophagus by a pneumatic duct (Physostomi), or this, appearing in development, may be lost in the adult (Physoclisti).

In the physoclisti there is a spot, the 'oval,' in the region of which is a richly vascular network. Apparently this is for the resorption of the gases of the bladder when the pressure is reduced in going to a higher level, a matter accomplished in the physostomes by passage of the gases through the duct. When a fish is rapidly brought to the surface from great depths, neitler process is sufficient, and in order to accommodate the expansion of the bladder, the viscera are frequently forced from the mouth. As the fish can resorb the gases from the swim bladder, it can reform them, they being secreted from the blood the 'red spots' or 'gas glands,' spots rich in blood-vessels and covered with a special cpithclium. The possibility of this gas exchange shows how the swim bladder can function as a respiratory organ, not only as long known in the Dipnoi, but in other forms like Lepidosteus and A mia.

Regarding the chief functions of the swim bladder there are two views which are not incompatible. (I) The swim bladder is a hydrostatic apparatus, since the ability to regulate the amount of the contents makes it possible to compensate 
for the differences in pressure due to different depths, so that the fish may remain without muscular action at any desired level. (2) The bladder is a hydrostatic sense organ for the recognition of water pressure and thus the depth and, by reflex, for the regulation of the muscular action and muscle tonus. Support for this view is found in the existence in many fishes of structures adapted for convering the variations in pressure to the ear, which is an organ for equilibration. This may be accomplished, as in the Clupeidx, by means of processes of the bladder which extend into the region of the ear, or by the IVeberian apparatus, a system of levers formed by appendages of the vertebræ and extending from bladder to ear.

The heart, enclosed in the pericardium, lies immediately behind the gill region, and is protected by the shoulder girdle. It always consists of auricle and ventricle (fig. 553), separated by a pair of valves to prevent back-flow of the blood; it sends the blood to the gills by the arterial trunk (ventral aorta), and receives it from the body through a thin-walled sac,
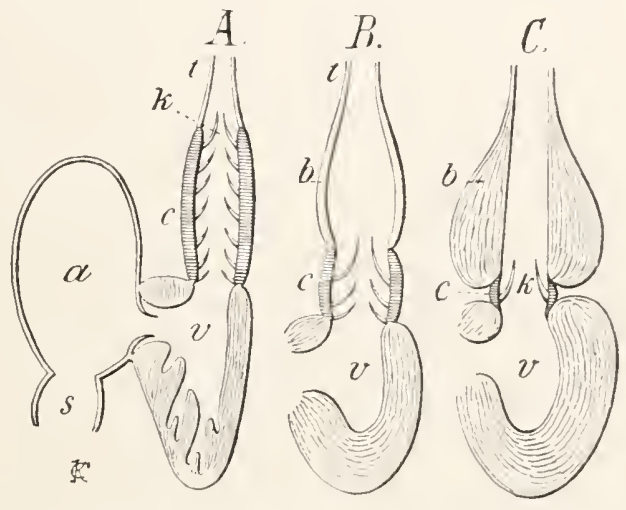

FIG. 553.-Forms of hearts of fishes in schematic long section (after Boas). A, selachian and most ganoids; $B$, A mia; $C$. teleost. $a$, auricle; $b$, bulbus arteriosus; $c$, conus arteriosus; $k$, valves; $s$, sinus venosus; $t$, truncus aorte; $v$, ventricle.

the venous sinus, in which the hepatic reins and the Cuvierian ducts (formed by union of jugular and cardinal reins) empty (figs. 66, 554).

The most important differences lie in the derelopment of conus and bulbus arteriosus. These are muscular accessory organs, the first arising from the heart, the other from the arterial trunk; and correspondingly the conus has striped, the bulbus smooth muscle fibres. The anterior end of the heart contains 'semilunar' ialies, which prerent the back-flow of the blood. When, by increase in the number of valves, this part becomes elongate, a conus arteriosus (fig. $553, A$ ) is formed. The bulbus (C) is a muscular swelling in front of the valves in the arterial trunk.

The connexion of rentral and dorsal aortie is effected in young fishes (fig. 55t) by the gill arteries directly; later by means of the complicated 
loops of the gill circulation. When these are developed, affercnt branchial arteries, gill capillaries, and efferent arteries can be recognized, the latter uniting to form the dorsal aorta and also giving off the arteries (carotids), which go to the head.

The nephridia are a pair of large reddish-brown organs lying olitside the body cavity to the right and left of the vertebral column, usually extending from heart to anus. 'Their ducts empty behind the anus or in the dorsal wall of the intestine and are often provided with enlargements called, from their functions, urinary bladders, although totally different

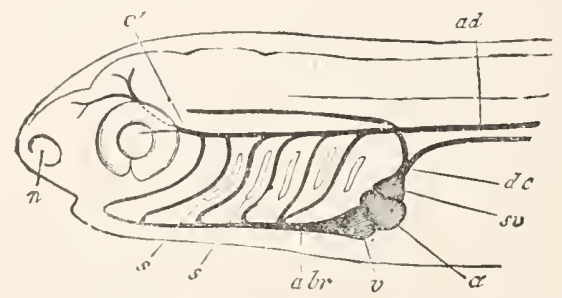

FIg. 554.-Thearl of embryo teleost (diagram from Gegenbaur). $a$, auricle; $a b r$. ventral aorta with arterial arches; $a l$, dorsal aorta; $c$, carotid; $d c$, Cuvierian duct, formed by union of jugular and posterior cardinal veins; $n$, nostril; $s$, gill clefts; $s v$, sinus venosus; $v$, ventricle.

morphologically from the urinary bladder of the higher vertebrates. The gonads, suspended by mesorchia or mesovaria, are large and project into the borly cavity. They are rarely unpaired. In the elasmobranchs and most ganoids their products pass out by the urogenital system (p. 487), in other forms by the pori abdominales or by special ducts. With the exception of the Dipnoi and ganoids which have an unequal total cleavage, the eggs of fishes have a discoidal segmentation.

\section{Sub Class I. Elasmobranchii (Plagiostomi, Chondropterygii).}

The elasmobranchs, the shark-like fishes, are almost wholly marine, varying in length from a foot and a half to sixty feet, living largely on other vertebrates, and noted for their voracity. Sometimes slender and cylindrical (sharks, fig. 555), sometimes flattened dorsoventrally (skates, fig. 556), they agree in that the head is prolonged into a snout, supported by a cartilaginous prolongation of the cranium, the rostrum (fig. $5+6, R)$. The mouth is ventral, at some distance from the anterior end, and is transverse, (Plagiostomi-transverse mouth). This makes it necessary that a shark approaching its prey from below must turn on its back before biting. The tail is heterocercal or is drawn out in a long 
filament. The skin is covered with placoid scales, usually close together, these being so small in some cases that the skin (shagreen) is used instead of sandpaper. More rarely the scales are larger, and the spines, which project from the skin, justify in size and form the term dermal teeth. Such strong spines occur especially at the front of the dorsal fins (ichthyodolurites of paleontologists). The skeleton is cartilaginous, frequently calcified on the outside. The calcification can also extend into the vertebræ, producing star-like figures (Asterospondyli) or concentric circles

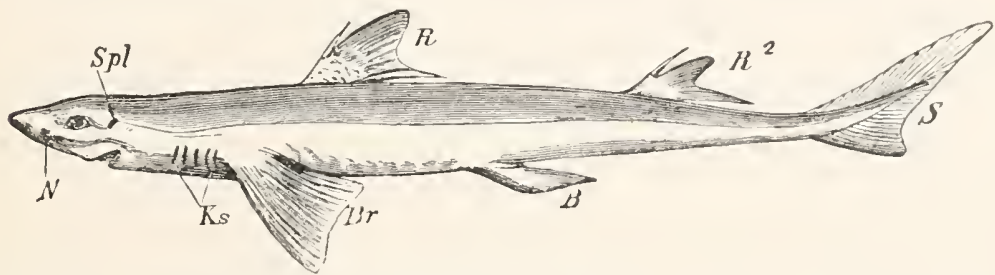

FIG. 555--Acanthias inlgaris, $*$ dogfish (from Claus). $B$, ventral fin; $B r$, pectoral fin; $K$ s, gill clefts; $n$, nostril; $R^{1}, R^{2}$, dorsal fins; $S$, heterocercal caudal fin; $S p l$, spiracle.

(Cyclospondyli). Since bone is lacking, the sharks have no upper jaws, but bite with the pterygoquadrate. The amphicolous vertebre (lacking in Holocephali and several extinct groups,) have neural arches, small ribs, and intercalaria. The number of gill arches and clefts varies between five and seven, the first cleft lying between the hyoid and the first branchial arch. Besides, most elasmobranchs have a spiracle and pseudobranch (fig. 555, Spl). Except in the Holocephali the gill clefts open separately, the hyoid arch being without an operculum.

In the visceral anatomy these points are of importance as distinguishing elasmobranchs from Teleostomes. (1) The heart has a large conus, with several rows of valves (fig. $553, A$ ), but lacks a bulbus. (2) The alimentary tract (fig $55^{\circ}, A$ ) has a spiral valve, but lacks swim bladtler and pyloric caca. (3) The sexual products are carried to the exterior by the urogenital ducts except in Lamargus which has abdominal pores. The eggs escape from the follicles of the orary (occasionally unpaired) by dehiscence into the body cavity, and from thence by the unpaired ostium tube and the paired Müllerian ducts to the exterior. 'The spermatozoa traverse the anterior part of the Wolffian borly ('kidney'). Sexual and urinary ducts open dorsally into the cloaca.

Male elasmobranchs are distinguished by the presence of a copulatory structure (mixipterygium) developed by enlargement of some radii of the ventral fin (fig. $556, c$ ). The large eggs, rich in yolk, are fertilized in the oviducts and usually develop in uterine enlargements of the ducts. The embryos (fig. 540), with long gill filaments protruding from the gill slits, are nourished by the yolk 
in the yolk sac. In Mustehs and Carcharias, as Aristotle knew, there is the formation of a placenta, which differs from that of the mammals in that the embryonic blood supply arises from the blood-ressels of the yolk sac and are not allantoic. There are oviparous elasmobranchs, and in these the egg is surrounded by albumen and a shell, but these egres differ from those of birds in that the shell is horny and is usually drawn out at the four corners, sometimes with threads for attaching the egg to plants, etc.

\section{Order I. Selachii.}

With the notochord more or less completely replaced by vertebral centra.

Sub Order I. DIPLOSI'OND YLI. Gill slits lateral, six or seven in number, a single dorsal fin. Chlamydoselachus with terminal mouth. Hexanchus, * mouth normal, six gill slits; Heptanchts, seven gill slits.

Sub Order II. SQU.ALI (Euselachii). Normal sharks, with cylindrical bodies, free thoracic fins, heterocercal tail, lateral gill slits. Most are fast

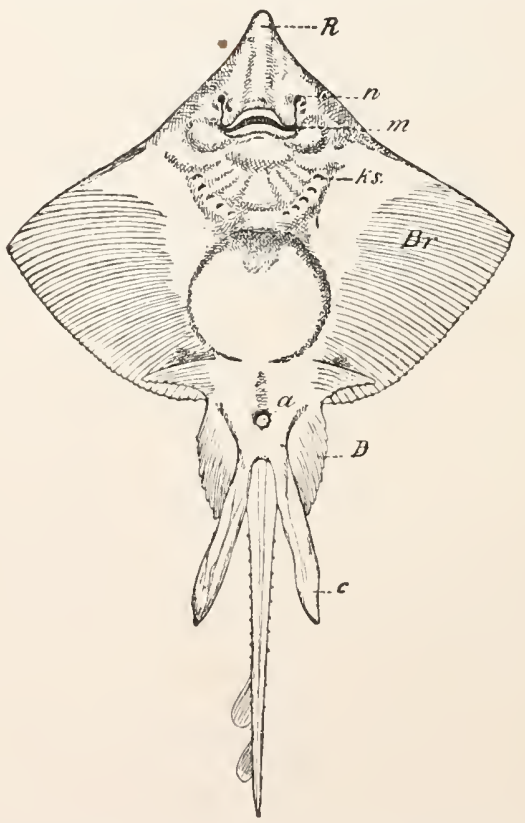

Fic. 556.-Raia batis, male, ventral view (after Möbius and Heincke). $B$, ventral, $B r$, pectoral fin; $R$, rostrum; $a$, anus; $c$, copulatory part of ventral; $k s$, gill clefts; $m$, mouth; $n$, nostril; between them the oronasal groove.

swimmers and rapacious, the teeth usually pointed, but in some the teeth are pavement-like. Numerous families, distinguished by vertebral characters, number of dorsal fins, presence of nictitating membrane, etc. GALEID nictitating membrane present; lesides the dog-sharks (.Mustelus* and Galeus*), the largest sharks, Carcharimus, * some with man-eating reputations. The hammer heads (Zygena*) are closely allied. The mackerel sharks (Lamna*) and the great white 'man-eater,' Carcharodon,* lack nictitating membranes. 
The foregoing are asterospondylous. The dog-fishes (1canthins vulgaris, * fig. 555) are cyclospondylous; spine in front of each dorsal fin.

Sub Order III. RAIE, skates; body flattened horizontally (fig. 556); the pectoral fins, also flattened, united to sides of body, the union usually extencling to tip of snout, and frequently back to the pelvis, giving the body a rhombic appearance from above. The animals swim by undulating motions of these fins. The union of fins to the side has resulted in transfer of the gill slits to the lower surface, the spiracles to the upper. The teeth are usually pavement-like. PRISTID E, sawfishes, snout prolonged, the edges with teeth. Pristis.* RAIDE; the typical members of the group; Raia.* TRYGONIDæ, sting rays, whip-like tail with one or two spines at base; Dasyatis.* Torpedinid. electrical organs between gill arches and pectoral skeleton. Torpedo*

\section{Order II. Holocephali.}

These forms, which have no common names, differ from the selachii in having the pterygoquadrate arch, which bears a few large chisel teeth, fused with the cranium; in having a dermal fold (operculum) which covers the gill

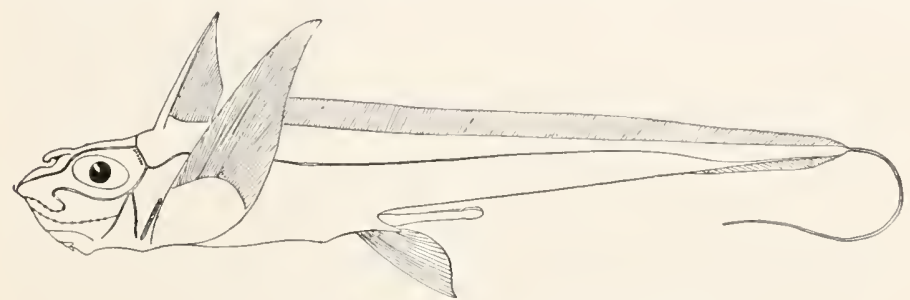

FIG. 557--Chimara monstrosa (f rom Kingsley).

slits; and corresponding with this, the gills more on the teleost type (p. $50 \mathrm{I}$ ). Lastly, the vertebral centra are not developed. Chimera.* Fossils appear in the Devonian.

Cladoselachit, Ichthyotomi (Pleuracanthus), and Acasthodidz, paleozoic; vertebral centra lacking.

\section{Sub Class II. Ganoidei.}

The ganoids form a transition group in which elasmobranch and teleost characters are mingled. They have the spiral valve of sharks, the swim bladder of teleosts; the heart with conus is selachian, the rcspiratory structures - the comb-like gills and the operculum-are as distinctly teleostean. With the development of the operculum the hyoid arch has not entirely lost its respiratory function, since in garpike and sturgeon it bears an opercular gill, and often there is a pseudobranch in the spiracle. The skeleton is always ossified in certain parts; large membrane bones lie on the shoulder girdle, on the roof and floor of the skull (parasphenoid); the horny threads of the fins are bony rays. In general the skeleton ranges between two extremes-an extremely primitive cartilaginous condition with persistent notochord, and one with considerable ossification, Lepidos- 
teus having opisthocole vertebrx. There are no characters in all ganoids which occur only in the group. The ganoid scales are often absent, since the sturgeon has bony plates free from ganoin, while the paddle bill (Polyodon) has almost no dermal skeleton, and Amia has cycloid scales. Most recent and fossil forms possess fulcra, bony plates with forked ends lying shingle-like in front of the fins (fig. Iо, $B$ ), but these are frequently absent, e.g., in 1 mia and Polypterus (fig. Io, $A$ and $C$ ). The group is largely American. The few recent ganoids fall into three distinct groups.

\section{Order I. Crossopterygii.}

Largely extinct, two genera persisting. Tails diphycercal or heterocercal; pectoral fins scaled at base; broad gular plates beneath jaws in place of branchiostegals; skeleton well ossified. Polyptcrus and Calamoichthys, Africa. Probably ancestral to the Amphibia.

\section{Order II. Chondrostei.}

These resemble sharks externally in heterocercal tail, spiracle, ventral position of mouth; internally in cartilaginous skull and (except Polyodon) in pterygoquadrate serving as upper jaw. Vertebral column primitive, centra lacking, neural and hæmal arches and intercalaria resting on notochordal sheath

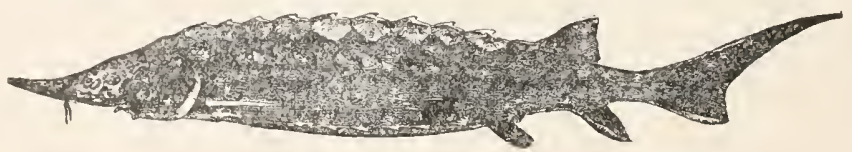

FIG. 558.-Acipenser sturiv,* common sturgeon (after Goode).

(fig. 512). Acipenseride, large bony dermal plates. Acipenser, $*$ sturgeon. Polyodoxtidi, naked skin and long paddle-like snout, toothed maxillaries present. Polyodon,* paddle fish.

\section{Order III. Holostei.}

Skull is ossified as in teleosts; maxillary and premaxillary bones present, pterygoquadrates reduced and not meeting in front, mouth terminal. Body with sanoid or cycloid scales. The living forms (the group appears in the trias) have no spiracle and diphy- or homocercal tails. LEPIDoste1dæ. Scales rhomboid, branchiostegal rays present, vertebre opisthocole. Lepidosteus, * garpike. AmID.e, distinctly teleostean in appearance with cycloid scales, amphicolous vertebrx, and heart with reduced conus (fig. 553, B). Amia,* bow fin.

\section{Sub Class III. Teleostei.}

The teleosts owe their name to the extensive ossification of the skeleton, which consists, in the trunk, of amphicœlous vertebræ bearing large ribs (p. 454), and in front a skull with numerous primary and secondary bones, already enumerated (p. 495, fig. 547). Maxillaries and premaxillaries are present, but these are frequently without teeth, since other bones of the mouth (vomers, palatines, para- 
sphenoid, hyoid, gill arches, superior pharyngeals-the latter alone in Cyprinoids) may bear teeth. Frequently there are present small bones, epipleurals, usually forked, lying in the intermuscular septa above the ribs, which are not preformed in cartilage. In the fins both cartilage and dermal rays are ossified, the former remaining small, the rays forming most of the support. 'These rays may either be soft and flexible (Malacopteri) or hard and spine-like (Acanthopteri), a matter of classificatory value. In the first case they consist of numerous small threads (fig. 559 , Fr,

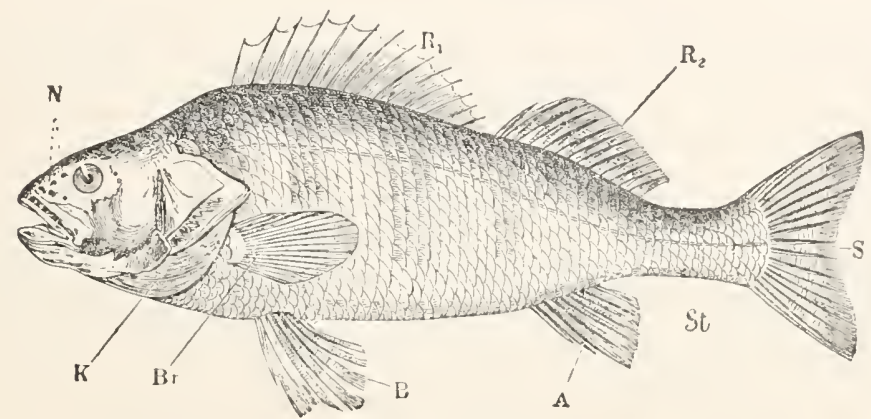

FIg. 559.-Perca fuviatilis (from Ludwig-Leunis). $A$, anal fin; $B$, ventral fin; $\mathrm{Br}$, pectoral fin; $K$, operculum; $N$, nostrils; $R_{1}, R_{2}$, spinous and soft dorsal lins; $S$, caudal fin; $S l$, lateral line.

$\left.A, B, R_{2}\right)$, in the other the parts of a ray are fused to a spine which, sometimes connected with poison glands (Scorpana, etc.), are good defensive weapons. The tail is usually homocercal ; the diphycercy of eels and other fishes is secondary. The dermal skeleton consists of ctenoid or cycloid scales, sometimes of spines or bony plates, these with true dermal teeth in the armored Siluroids. In rare instances the skin lacks scales.

The hyoid arch always bears an operculum and branchiostegal membrane; there is no spiracle and the opercular gill is rudimentary or absent. The gills of the comb-like type are confmed to the four anterior gill arches, but they may be reduced to two and one-half pairs of demibranchs. Instead of a conus (present in Butrimus), the bulbus arteriosus is well developed; a spiral valve is lacking, but pyloric appendages are common. A swim bladder is usually present, but its duct is frequently closed.

The teleosts are distinguished from all vertebrates except the cyclostomes and perhaps some ganoids in that the nephridial system does not form part of the sexual ducts. The eggs and milt are deposited through the abdominal pores or by special canals developed from the body cavity. Copulation occurs in only a few viviparous forms (Embiotocidæ, Gambusia, etc.). The rule is that males 
and females deposit their reproductive products in the water at the same time. Many species have brighter colors or develop pearl organts (p. 493), at the time of oviposition. In rare instances the males care for the young (sticklebacks); more noticeable are the conditions in the lophobranchs (sea horses and pipe fish), where the males receive the eggs in a brood pouch on the ventral surface. A metamorplosis is known only in the eel-like fishes, the larra of which (Leptocephalus) are flat, transparent, with colorless blood, enormous tails, and extremely small trunk. These larwa normally occur in the sea at the depth of some hundred fathoms. The fresh-water eels go to the ocean for propagation. On the other hand, many salt-water fish go to fresh water for reproduction.

The classification of the fishes is yet in an unsettled state. Most European writers recognize the six divisions below. American authorities separate the Ostariophysi from the Physostomi, the Pediculati and Hemibranchii from the Acanthopteri, and unite the Anacanthini and some of the Pharyngognathi with the Acanthopteri and make a distunct group, Synentognathi, of the others. The characters on which these divisions are based are less convenient for the tyro than those adopted here.

\section{Order I. Physostomi.}

The character to which this name refers is not readily seen without dissection, the persistence of the duct of the swim bladder. This is, however, correlated with the soft character of the fin rays (few exceptions) and the abdominal position of the ventral fins. The Ostariophysi are remarkable in having a chain of bones (Weberian apparatus, p. 503) connecting the swim bladder with the ear. More than a third of the food fishes and nearly all of the fresh-water fishes belong here.

The Ostariophysial families are SILURIDE (I000 species), or cat-fish; Cýprinide, or carp (iooo species); the suckers, Catostomide. Grinoti, electric eel of South America. The other families are true Physostomes. The Salionide, trout and salmon (Salmo*) Osmerus,* smelt; Coregonus, * white fish; Clupeide, herring, shad; Anguillide, eels, Esocide, pike and pickerel. Amblyopside, blind fish of Mammoth Cave.

\section{Order II. Pharyngognathi.}

The inferior pharyngeal bones (i.e., the last rudimentary gill arch) fuse to form a single bone. Some have spiny fins; LABRIDE, Ctenolabrus, $*$ the cunners

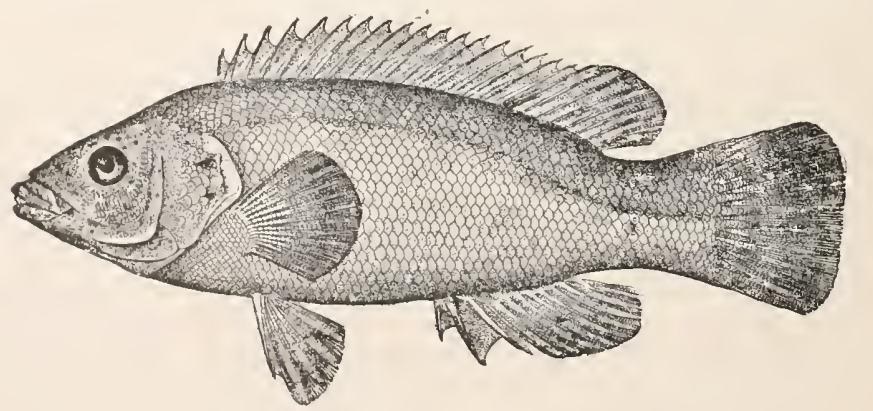

FIG. 560.-Citcnolabrus coruleus,* cunner (after Goode).

These are placed among the Acanthopteri by American authors. Others have only soft fin rays. These are the Synentognathi and include the ExOCETIDE,
some flying fishes. 


\section{Order III. Acanthopteri (Acanthopterygii).}

The largest group of fishes, its members usually having the ventral fins thoracic in position and more than three rays spiny in dorsal, anal, and rentral fins. Sticklebacks (GASTERostime) and some other forms have the pharingeal bones reduced, the ventral fins farther back, and form the group Hemibranchii. Gasterosteus.* The perch (PERCIDA), and the marine SERRANID.玉 have ctenoid scales. ScombriD E, mackerel, XiphID., sword fishes; snout prolonged into a long sword; LoRICATI, sculpins (Cottus, * Hemitripterus*). 'The EMBIOTOCID E, surf perches of the Pacific, viviparous. The suck fishes, Remor $l, *$ first dorsal modified to a sucker on top of head.

\section{Order IV. Anacanthini.}

Soft-finned fishes with ventral fins in front of pectorals; descended from Acanthopteran forms. With few exceptions (Lota* burbot), marine. GaDID.F,

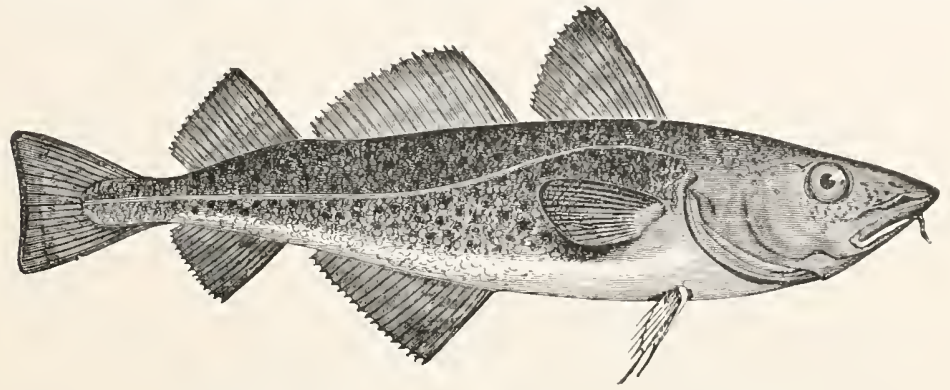

FIG. 561.-Gadus morrhua, * cod (after Storer).

cod and haddock; Pleuronectid $£$ (flat fishes), halibut, flounders, sole. The Pleuronectidæ, from their asymmetry, need a word. The young are perfectly symmetrical, but the animals turn on one side, the lower becoming white. 'The eye of this side shifts to the upper side, twisting the bones of the skull in its progress.

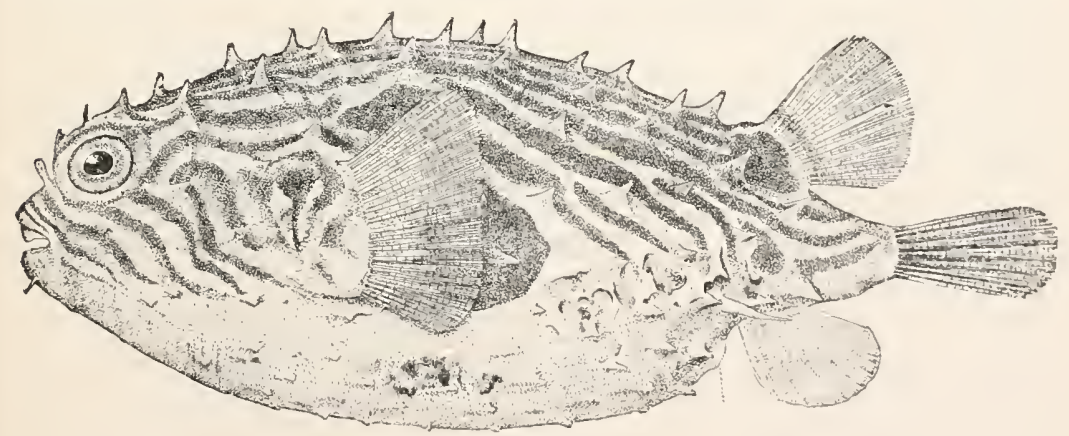

FIG. 562.-Chilomycterus geometricus,* swell fish (after Goode).

\section{Order V. Lophobranchii.}

Marine species, having gills composed of rounded tuits, body covered with a segmented armor of hony plates and peculiar breeding (p. 5 ro); sea horses, Hippocambus,* pipe fishes, Syngnathus.** 


\section{Order VI. Plectognathi.}

Peculiar compact fishes, in which the bones in each jaw are coossified, the upper jaw fused with the cranium, the ventral fins reduced or absent. OstriconER uI, the body is enclosed in a firm angular box of bony plates. (ivmononta, swell fishes (fig. 562), can inflate the body to a spherical sac; flesh is poisonous.

\section{Sủ Class II:. Dipnoi (Dipnensti).}

The lung fishes have the form of true fishes, with scales and paired fins; supported by a single or a doubly pinnate archipterygium. The median fin is not separated into dorsals, caudal and rentral, and the caudal part is diphycercal. The skeleton is very primitive, consisting largely of cartilage, the notochord being retained to a great extent. The pterygoquadrate fuses with the cranium. The animals live in fresh water and, under ordinary conditions, breathe by gills which are covered by an operculum. P'rotopterus and the young of Lepidosiren have external as well as internal gills, recalling the Amphibia. The resemblances are strengthened by the

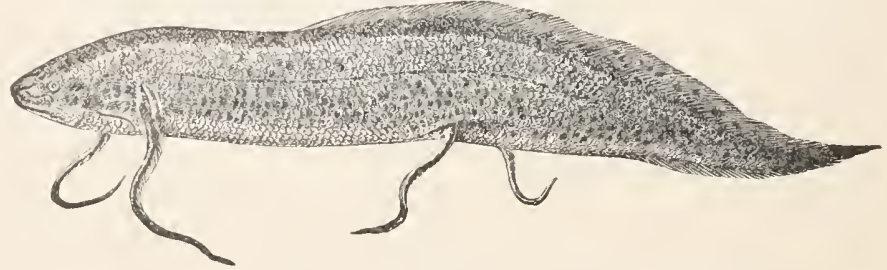

FIG. 563.-Protopterus annectens, lung fisin (from Boas).

periorlic appearance of pulmonary respiration. The lung fishes live in pools and swamps which, during the hot season, may be more or less completely dried up. When the water becomes too foul for branchial respiration or dries up the swim bladder is used. This is a paired or unpaired sac with a duct leading to the osophagus, and has its respiratory surface increased by the development of air cells. Protopterus indeed can live out of water; it burrows in the mud at the dry season, and builds a cocoon lined with mucus in which it remains quiescent until the wet season. The nose is respiratory, with choanx opening into the mouth cavity. The last gill vessels give off pulmonary arteries, and there are veins carrying the blood back to the heart, pulmonary and systemic circulations being lifferentiated. The heart shows the beginning of division into arterial and renous halves, especially in the regions of the conus and auricle.

The fer species now living have a wide and discontinuous distribution, and are the remnants of a much richer group which appeared in the paleozoic. 
Monopneumonla, with one swim bladder: Ceratodus, Australia. DipneuMONIA, with two bladders: Protopterus, Africa; Lepidosiren, South America. The larve of both have four pairs of gills, three of which are retained in Protopterus. Possibly the paleozoic ArThrodra, some of gigantic size (Dinichthys), belong here.

\section{Class III. Amphibia.}

There are two views as to the origin of the Amphibia. According to one they have descended from Crossopterygian ganoids; the other is that they have come from the Dipnoi. The class is distinguished at once from the fishes by the absence of fins. There is, it is true, a median fin in larval life, and this may persist (Perennibranchs, Triton), but it is never divided into dorsal, caudal, and anal, and it lacks any skeletal support (figs. 4, 5). The paired fins are replaced by toed feet (p. 464). These are often webbed and are used for swimming, for creeping and leaping, and are consequently jointed between the separate skeletal elements (fig. $5_{4}$ ). Besides the shoulder and hip joints, which alone occur in fishes, there occur also elbow (knee), wrist (ankle), and finger joints. The number of digits is not always five, for a reduction to four, three, or eren two occurs.

The connexion of the girdles with parts of the axialeskeleton (lacking in most fishes) is of importance. The pelvic girdle is connected with the vertcbral column by means of the ilium, which arti ulates either directly or by a sacral rib with the single sacral vertebra. Ventrally the two halves of the girdle fuse, and usually the limits of ischium and pubis cannot be traced.

The attachment of the pectoral girdle is less firm (fig. $52 \mathrm{I}, A$ ). The dorsal portion, the scapula, ends free in the muscles; the ventral, differ-

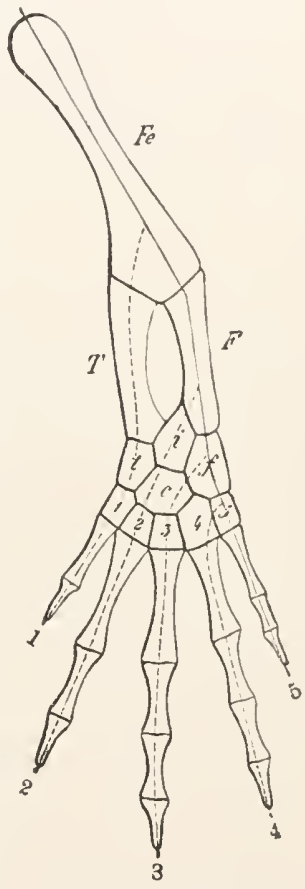

F1G. 564.-Skeleton of hind leg of Salamandra maculosa larva (from Gegenbaur). c, centrale; $F$, fibula; $f$, fibulare; Fe, femur; $i$, intermedium; $T$, tibia; $t$, tibiale; $\mathbf{I}-5$, carpalia and corresponding metacarpals and digits. entiated into coracoid and clavicle, either meets the opposite side in a symphysis (firmisternous Anura) or the two sides overlap (arciferous Anura and urodeles). A connexion with the vertebral column does not exist since the sternum (lacking in urodeles) is not connected with the rilss. The sternum extends forward to the clavicle and coracoid, and in the firmisterna is continued forward by an episternum (fig. 52 I, $A$ ). 
The vertebral column often (Perennibranchs, Derotremes, Cæcilians, and many Stegocephali) resembles that of fishes in amphicoelous centra and persistence of notochord. The notochord may disappear, there then occurring opisthocœlous (Salamandrina) or procœlous centra (most Anura). There is also an articulation of skull with vertebral column, rare in fishes lut characteristic of land animals, by which the first vertebra (atlas) becomes distinct from the rest.

The skull is remarkable for the extent to which the chondrocranium is retained and the consequent small number of primary bones (figs. $5^{6} 5$, 566). The bones of the orbital region are represented by a pair each of ali-and orbitosphenoids in the urodeles, by a ring of bone, the sphenethmoid, in the anura. The auditory region usually contains only prootics, the

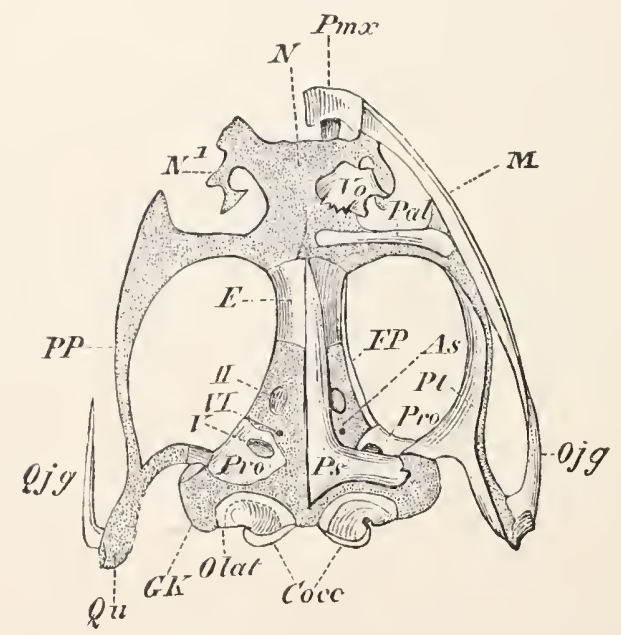

FIG. 565.-Frog skull from below (from Wiedersheim). For letters see fig. $5^{66 .}$

occipital only exoccipitals. The absence of other occipitals is often of value in distinguishing between amphibian and reptilian skulls, since in the former the articulation with the atlas is consequently by double occipital condyles. Of secondary cranial bones are to be mentioned the nasals, frontals (in many prefrontals also), and parietals, the latter two fused in anura to frontoparietals; ventrally the large parasphenoid.

The cranium is increased by the addition of the large quadrate cartilage, which becomes applied to the otic capsule and (Anura) fuses with it, while the rest of its arch (pterygoid) extends forward in a more or less complete condition, reaching the nasal capsule in the Anura. The quadrate cartilage is covered externally by the squamosal and supports the lower jaw, composed of Meckel's cartilage surrounded by membrane bones (dentary, splenial, angulare, etc.); its 
articular portion, like the quadrate, being rarely incompletely ossified. V'omers, palatines, and pterygoids appear in the lase of the skull, all three forming a continuous arch in the Anura; in front of them lie the premaxillaries, and in most cases maxillaries. Between the hinder end of the maxillaries and the quadrate there may be a gap or it may be bridged by a jugal. By the modifica-

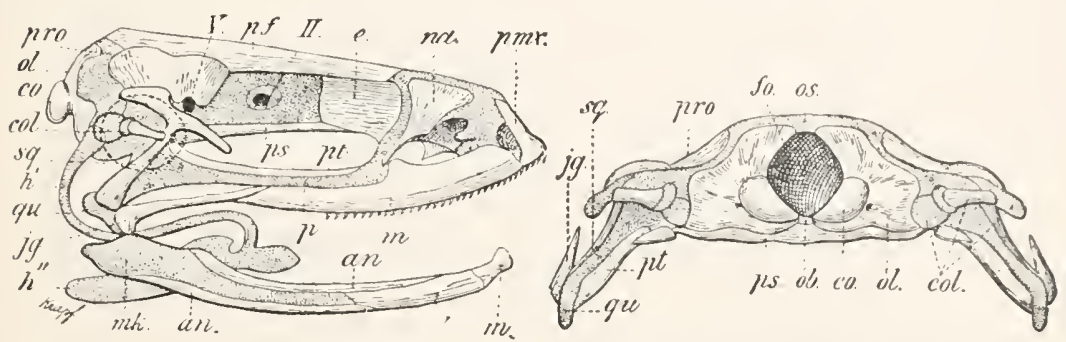

FIG. 566.-Lateral and hinder views of frog skull (after Parker). Letters for this and 565: $a n$, angulare: $A s$, alisphenoid cartilage; co (Cocc), occipital condyles; col, columella; $d$, dentary; $E(e)$, sphenethmoid; $f o$, foramen magnum; FP. frontoparietal; $G k$, otic capsule; $h^{\prime}, h^{\prime \prime}$, hyoid and copula; $j g$, jugal; $M(m)$, maxillary (in lower jaw mento-Meckelian); $m k$, Meckel's cartilage; $N, N^{1}$, nasal capsule; na, nasal; $o b, o s$, cartilages from which basi- and supraoccipitals arise elsewhere; ol (Olat), exoccipital; $p f$, frontoparietal; $P a l$, palatine; $p(P P)$, palatine arch; $P m x$, premaxillary; Pro, prootic; $P s$, parasphenoid; $P t$, pterygoid; $Q u$, quadrate; $Q j g$, jugal; $s q$, squamosal; $V o$, vomer. Cartilages dotted.

tion of the quadrate into a suspensor the hyomandibular loses its function, and if represented at all, it is as part of the columella. 'The character of the remaining risceral skeleton depends upon the respiration (fig. $5^{6}{ }_{7}$ ). Where gills occur, the copula and hyoids-representing body and cornua-as well as four gill arches are present, but with pulmonary respiration the hyoid apparatus is reduced to a hyoid with anterior and posterior cornua, the gill arches being contained in the posterior horns. To understand the conditions in the higher vertebrates it must be remembered that the reduced fifth arch becomes a support of the larynx and that in many anura the hyoid extends to the skull and fuses with the otic capsule.

With the assumption of a terrestrial life changes occur in the sense organs. The organs of the lateral line, which occur in all larve and are persistent in the aquatic perennibranchs, and the

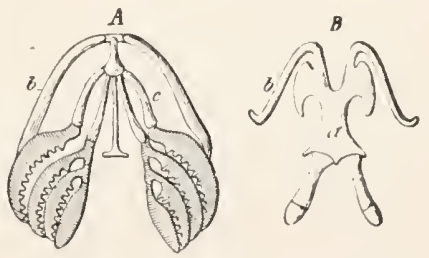

FIG. 567.-Hinder visceral skeleton of (A) larva of a salamander; (B) of toad (from Gegenbaur). $a$, body of hyoid; $b$, anterior horn (hyoid); $c$, rest of branchial skeleton. nerves which supply them, disappear; the eyes in the Salamandrina have upper and lower lids; in the frogs an under lid (really nictitating membrane). The nose becomes respiratory and is provided with choanx opening into the mouth. Especially noteworthy is the auditory apparatus. This, in the urodeles and cacilians, is very primitive, even the tympanum being absent, but in the Anura a sound-conducting appa- 
ratus appears. 'The spiracular cleft persists as a canal, opening into the pharynx by the Eustachian tube, its outer end expanded into the tympanum, closed externally by the tympanic membrane, supported by the tympanic annulus (dotted circle in fig. 566). The connexion of the labyrinth with the tympanum is by an opening in the otic capsule, the fenestra ovalis, in which is the stapes (? part of capsule), the columella extending from this to the tympanic membrane

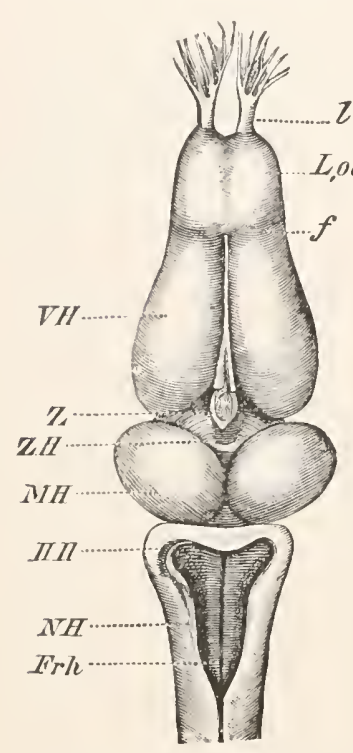

Fig. 568.-Brain of frog. $f$, line between olfactory lobes and cerebrum; Frh, fossa rhomboidalis; $H H$, cerebellum; $l$, olfactory nerve; Lol, olfactory lobes; $\boldsymbol{H} H$, optic lobes; I $H$, cerebrum: $Z$, epiphysis; $Z H$, 'twixt brain. and carrying the sound waves across to the inner ear. The brain (fig. 568) has advanced above that of the fishes in the stronger develepment of the cerebrum, but remains behind in the small size of the cerebellum, which is but a thin lamella.

The respiratory organs afford important characters, since both gills and lungs occur. The larve of the anura, as a rule, have internal gills, but the dorsal part of these, as in many fishes, develop early and very strongly and extend out through the clefts to the skin above them as three pairs of external gills. These, however, are absorbed with the development of the other gill leaves, which become enclosed in a special chamber (atrium) by the backward growth of an opercular fold from each hyoid arch. The atrium usually opens to the exterior by a spiracle on the left side, but occasionally there are a pair of spiracles. The external gills are strongly developed in the larval urodeles and crecilians, and the internal gills are correspondingly undeveloped. The paired lungs open into the hinder part of the pharynx, either directly through the glottis or more rarely by a short trachea. Cartilages, the remnants of gill arches, may support trachea and glottis, and on the latter support vocal cords (lary $n x$ ). Breathing is accomplished by a kind of swallowing, the air being forcel into the lungs by the muscles of the floor of the mouth and the pharynx. Persistent gills and lungs are found together only in the Perennibranchs. U'sually the young breathe by gills, the adults by lungs, the origin of the metamorphosis to be described below.

Besides gills and lungs the skin is an important respiratory organ, as are pharynx and mouth cavity, in which the air must remain for 
some time on account of the respiratory mechanism. This renders intelligible the fact that many Salamandrina (Spelerpes, Desmognathus, Plethodon, Gyrinophilus, etc.) have neither gills nor lungs, lut have only pharyngeal and cutaneous respiration. The capillary network in these parts is greatly developed and may extend into the epithelium. Thus, also, it happens that in the Anura the skin receives as large an artery as the lungs (fig. $570, \mathrm{cu}$ ).

The skin is thin and slimy from the numerous mucous glands, which not infrequently produce a poisonous secretion (so called parotid gland in the ear region). The epithelium bears a thin horny layer which at intervals is molted as a continuous sheet. The corium in the Anura is

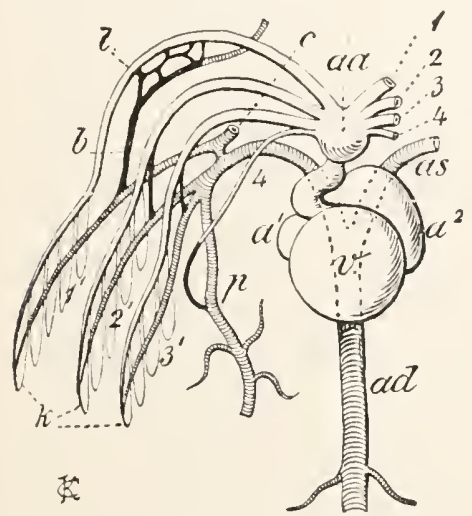

FIG. $5^{69}$.

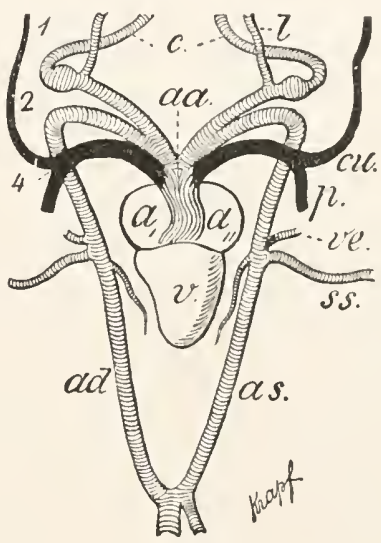

FIG. 570 .

Fig. 569.-Heart and arterial arches of salamander larva (after Boas). $a^{1} a^{2}$ right and left auricles; $a a$, arterial trunk; $a d$, dorsal aorta; $a s$, left aortic arch; $b$, direct connection between afferent and efferent arteries; $c$, carotid; $l$, afferent artery; $p$, pulmonary artery; $v$, ventricle; $\mathrm{I}-4$, afferent arteries; $\mathrm{I}^{\prime}-3^{\prime}$, gills.

Fig. 570.- Heart and arches of frog (diagram). $a, a_{\mu}$, right and left auricles; $a a$, ventral aorta; $a d, a s$, right and left aortic arches (radices aortax); $c$, carotid; $c u$, cutaneus; $l$, lingualis; $p$, pulmonary artery; ss, subclavian; $v$, ventricle; ve, vertebralis; $1,2,4$, persisting arches.

undermined by large lymph spaces, the presence of which makes the skinning of a frog such an easy matter. Ossifications in the skin-enormously developed in the fossil Stegocephali-occur but rarely (Gymnophiona) in recent Amphibia. On the other hand, the abundance of chromatophores is noticeable, these, under the influence of the nerves, changing their shape and thus producing color changes in many Amphibia.

The heart (figs. 569, 570) has two auricles, distinctly separated in Anura, a right with renous blood, a left which with pulmonary respiration receives arterial blood. There is, however, but a single ventricle, and the 
arterial trunk is, at least externally, single. The arterial arches show different relations and have different fates. With branchial respiration the first three afferent and efferent arteries are connected in two ways, the one by the capillaries of the gills, the other direct $($ fig. $569, b)$. In the fourth arch there is no gill system, but on the other hand this arch gives off the pulmonary arteries $(p)$ to the lungs (compare also fig. 537, II).

With the loss of gills (fig. 570) the third arch frequently disappears entirely (Anura), as well as the gill circulation of the others, while the direct circulation persists, at least in part. The first arch gives rise to the carotids $(c)$ supplying the head, the second unites with its fellow of the opposite side to form the dorsal aorta; the fourth forms the pulmonary artery, and in the Anura, gives off a cutaneons artery (cu) to the skin. A longitudinal fold inside the arterial trunk is so arranged that the venous blood from the body coming to the heart through the right auricle is mostly sent out through the fourth arch to the lungs and the skin, while the blood returned from the lungs by the pulmonary vein passes through the left auricle and then through the first and second arches (carotid and aortic arches). So there is a separation of pulmonary and systemic circulations, although the blood all passes through a common ventricle. In the urodeles the primitive part of the last arch, connecting the pulmonary artery with the dorsal aorta, may persist as a ductus Botalli.

The sexual organs (fig. 539) are similar to those of selachians. The eggs pass from the ovary to the oviducts (Müller's duct), and in this are enveloped with a gelatinous layer. 'The spermatozoa, on the other hand, pass through the anterior part of the Wolffian body ('kidney') and thence out through the ureter. The distinction from the selachians lies in the fact that a urinary bladder, lying ventrally to the rectum, is present, at some distance from the urinary ducts, which open dorsally into the cloaca. Besides sexual organs fat bodies frequently occur, lobed and often brightly colored structures, best developed between the reproductive seasons.

A sort of copulation occurs, and internal impregnation is effected in many urodeles and in the Gymnophiona, but not in the Anura. The Anura and most other forms are oviparous, but occasionally, as Salamandra maculosa and $S$. atra of Europe, viviparous species occur. The male of Alytes obstetricans wraps the cords of egrgs about his legs and crawls into a hole until the young are hatched, while the females of Amphinma and Ichthyophis watch over the eggs. The male of Rhinoderma daminii has a large sac arising from the pharynx in which the eyrs and young are cared for until the completion of the metamorphosis. In Pipa americana the male places the eggs on its back, the skin thickening around them so that each lies in a separate pocket, from which the young escape at length in nearly the adult form. In Nototrema and Notodelphys there are dermal sacs upon the back for the reception of the eggs. 
The development of the Amphibia possesses special interest, since it affords the only easily observable instances of a metamorphosis among the vertebrates. This metamorphosis is the more marked the more the adults differ from the fishes. In the Anura a larva, the tadpole (fig. 4) escapes from the egg. It lacks lungs, but has three pairs of external gills, no legs, but a swimming tail with a fin-like fold. In the metamorphosis the external gills and tail-larval organs-are absorbed, while lungs and legs are formed. A complication is introduced into the metamorphosis in that, for a time after the loss of the external gills, internal branchia, lying in gill slits, occur (P. 5I6). In the tailed forms the metamorphosis is simplified, usually consisting in the loss of the external gills and sometimes in the change of form of the tail, which may lose its fin fold and become cylindrical. The last traces of a metamorphosis disappear in the perennibranchs, where lungs occur and the gills persist (Siren is said to lose the external gills and then re-form them). In the Anura the metamorphosis is lost when, as in Hylodes martinicensis, Pipa and several other forms, the whole development occurs in the egg, the young hatching in the adult form.

\section{Order I. Stegocephali.}

Extinct forms with well-developed tail, numerous membrane bones in the skull, and frequently a bony armor, at least on the ventral surface. Some were of gigantic size, and some from the folded condition of the enamel of the teeth are known as Labyrinthodonta. The group appeared in the carboniferous (footprints in the Devonian), and died out in the trias.

\section{Order II. Gymnophiona (Cæciliæ, Apoda).}

These are the nearest of living forms to the Stegocephali, but fossils are entirely unknown. The group is exclusively tropical, occurring in Ceylon, African islands, and America, a discontinuous distribution indicative of great age.

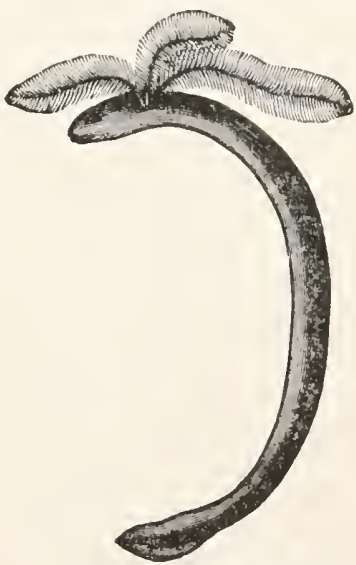

FIG. 57.--Larva of Ichthyophis glutinosus (from Boas, after Sarasins).

They are burrowing animals and feed on small invertebrates. As a result of this subterranean life the eyes are small and concealed under the skin, the legs are entirely lost, so that the animals are snake-like in appearance. In the skin there are usually small bony scales; the drum of the ear is lacking; the vertebre are amphicolous. Inside the egg many species have three pairs of feathered gills (fig. $57 \mathrm{I}$ ), a proof of their pertinence to the Amphibia. Later, there is an external gill opening which finally closes. Ichthyophis, Ceylon; Hypogeophis, Seychelles; Cacilia, America.

\section{Order III. Urodela (Gradientia).}

Of recent Amphibia the urodeles are the most fish-like. The vertebral column consists of numerous vertebre, and of these a large part belong to the 
tail. Ribs are present, but so short that they do not reach the sternum, which is weakly developed or is entirely absent. Tympanum and Eustachian tube are entirely lacking, as are the vocal cords and the production of sound.

Sub Order I. PERENIIBRANCHIATA. Two or three gill slits, three bushy gills, and a swimming tail persist throughout life. Nechurus, * mud puppy, with legs and two gill slits. Siren,* three gill slits, hind legs lacking. Typhlomolge, * Typhlotriton, * both blind, from cares and underground waters of U.S. Sub Order II. DEROTREMA. External gills lost, but an opening in the neck leading to the gill slits. Menopoma* (Cryptobranchus), hellbender, legs strong; 1 mphiuma, * legs rudimentary. Sub Order III. SALAMANDRINA. After the loss of gills the gill slits close. Amblystoma* (fig. 5), A. tigrinum and the Mexican axolotl breed in the larval stage. This and other facts leads to the riew that possibly the Perennibranchs are all neotenic (p. I50). Plethod.m, * Spelerpes, * Salamandra.

\section{Order IV. Anura.}

The anura have the compact bodies familiar in frogs and toads, with a small number $(7 \div 9)$ of trunk vertebre and. complete absence of tail, the caudal vertebre being represented by a long bone, the urostyle. Ribs are sometimes distinct, sometimes fused to the transverse processes; the limbs are larger than in other Amphibia, and are frequently used for leaping and climbing. Ear drum and tympanic membrane are lacking only in the Pelobatide; their presence is correlated with the existence of vocal cords and the production of sound. The metamorphosis includes a tadpole stage.

Sub Order I. AGLOSS.A. Toad-like anura with degenerate tongue and unpaired opening of the Eustachian tube. Pipa (p. $\left.5^{\mathrm{I}} \mathrm{S}\right)$, South America; Dactylethra, Africa. Sub Order II. ARCIFERA. Tongue present, Eustachian tubes widely separate, coracoids of the two sides overlapping. BufoNide, toads, toothless; Bufo, * the dermal glands poisonous. Pelobatide, with teeth. Scaphiopus,* burrowing toad, with tympanum. Hylide, tree toads, toothed; tips of toes with sucking discs; Hyla,* Acris.* Sub Order III. FIRMISTERNIA. Tongue present, Eustachian tubes distinct, coracoids firmly united in the middle line. RAxide, frogs. Rana.*

\section{Series II. Ainiota.}

Vertebrates with amnion and allantois (p. 490) in embryonic life; with the pro- and mesonephros functional only in the embryos, and replaced in the later stages by the true kidney (metanephros); ducts of the embryonic excretory system retained only so far as they have genital functions; gill slits appearing as transitory structures, but without gills and never functional. There are two great divisions of the Amniotes, the Sauropsicla and the Mammalia. The Sauropsida include the Reptilia and the Aves, which agree with each other and differ from the mammals in having a single occipital condyle, the quadrate acting as suspensor of the jaws; ankle joint between the first and second rows of tarsals; the presence of epidermal scales, nucleated red blood corpuscles, and a cloaca. 


\section{Class I. Reptilia.}

On account of similarity of form, the reptiles and Amphibia were long untied. They form parallel groups: urodeles and lizards, frogs and turtles, cacilians and snakes. Hence the points of distinction must be emphasized. The most important are two: the reptiles belong to the Amniota and, as such, have the embryonal features of the group; second, although often aquatic, they are, in the entire absence of branchial respiration, in character of skin and skeleton, in their entire structure, like the true land animals.

The skin, the better to withstand desiccation by the air, is strongly cornified, so that in the epidermis a many-layered stratum corneum and a many-layered stratum Malpighii can be distinguished. At the tips of the toes the stratum corneum develops strong claws on the dorsal side, the claw plate being better developed than the rentral claw sole. Further protection is afforded by the thick corium in which not infrequently bony plates occur. Dermal glands are very rare, the femoral pores of lizards (fig. $577, b$ ), which appear like the ducts of glands, being produced by the ends of cornified epithelial cones. Here must be mentioned the dorsal, anal and musk glands of the crocodiles.

The axial skeleton, both skull and vertebral column, is nearly always ossified; only exceptionally (Sphenodon and the amphicœle Ascalabotæ) are considerable parts of the notochord retained. The vertebre are usually procoelous. In the skull of reptiles (as in the allied birds) are many

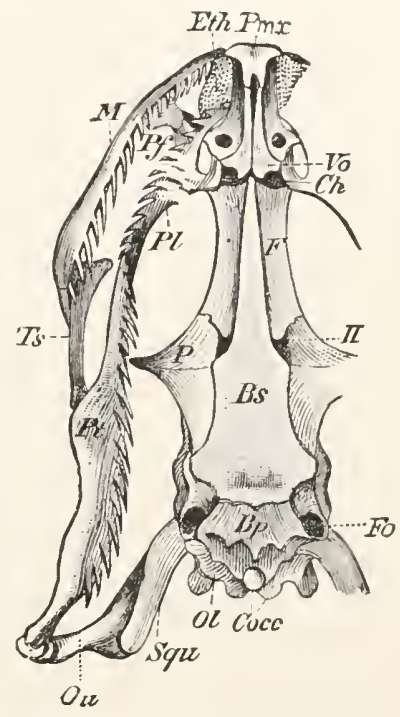

FIG. 572.- Ventral view of skull of Tropidonotus (from Wiedersheim). Bp, basioccipital; $B s$, basisphenoid (in front also parasphenoid): ( $h$, choana: Cocc, occipital condyles; Eith, ethmoid cartilage; $F$, frontal; $F_{0}$, fenestra ovalis; $M$, maxillary; ol, exoccipital; $P$, parietal; $P f$, prefrontal; $P l$, palatine; $P m x$, premaxillary; $P t$, pterygoid; $Q u$, quadrate; Squ, squamosal; Ts, transtersum; lo, vomer; $I 1$, optic foramen. characters which they share with Amphibia and which distinguish them from mammals. This is especially the case with the visceral skeleton. As in the Amphibia, the hinder end of the pterygoquadrate is attached to the otic capsule; the quadrate is ossificd and affords the articulation for the lower jaw, which is composed of many bones. 'The squamosal lies at the base of the quadrate and, in the Squamata, is intercalated between 
it and the cranium. Behind it is the columella, its inner end inserted in the fenestra ovalis. From the quadrate the palatine series of bonespterygoid, palatine, romer-extends forward, these being frequently toothed; and in front of and parallel to it the premaxillaries and maxillaries. Extremely characteristic of the reptiles, the turtles excepted, is an os transiersum, which appears in no other vertebrates. It extends from the hinder end of the maxillary to the pterygoid (figs. $572,578,579,58 \mathrm{I}$, $T s, t r) . \quad 1$ jugal is also frequently present. Of the other visceral arches, since gills are lacking, only the hyoid bone and laryngeal cartilages persist.

In the cranium the complete ossification of the occipital region is noticeable, the four occipital bones being present. The basioccipital forms the larger part of the single occipital condyle, in which parts of the exoccipitals participate, the single condyle being the sharpest distinction between the reptilian and amphibian skull. The basisphenoid, which lies in front of the basioccipital, has an anterior process or rostrum, representing the rudimentary parasphenoid (possibly presphenoid). Above, the skull is roofed in with membrane bones: parietals (frequently fused and perforated by the parietal foramen for the parietal eye), frontals, nasals, as well as pre- and postfrontals and postorbitals, and usually lacrimals as well.

The ethmoidal region is largely cartilaginous; ali- and orbitosphenoids are small and variable. Only the prootic is constant in the otic region; epiotic and opisthotic usually fusing with the occipitals, the opisthotic being large and distinct only in the turtles. The zygomatic arch (lost in snakes) is formed of jugal and quartratojugal, while above it may be a second arch formed of postorbital and squamosal. The zygomatic arch bounds a gap in the skull, the orbitotemporal fossa; the other arch a supratemporal fossa. The first fossa may be subdivided by a process from the zygomatic to the postorbital or postfrontal bones, thus giving separate orbital and temporal fossæ.

The convex occipital condyle forms, with the concave surface of the first vertebra (atlas), an articulation for motion in the vertical plane and lateral motions, while a twisting around the long axis of the body is permitted by the joint between the atlas and the second vertebra, the axis or epistropheus. The atlas is a bony ring, its centrum having separated and united with the body of the axis, forming a pirot around which the atlas turns. There are two sacral vertebre, and the vertebre of the trunk are divided into thoracic and lumbar, the former bearing long ribs which reach to the sternum, while the shorter ribs of the neck end freely.

Limbs are lacking in snakes and some lizards. When present the number of digits varies between three and five. In the pelvis ischium and pubis are separated by an obturator foramen and are united with the cor- 
responding bones of the opposite side by a double symphysis. In the shoulder girdle scapula and coracoid alone are constant, a clavicle occur-

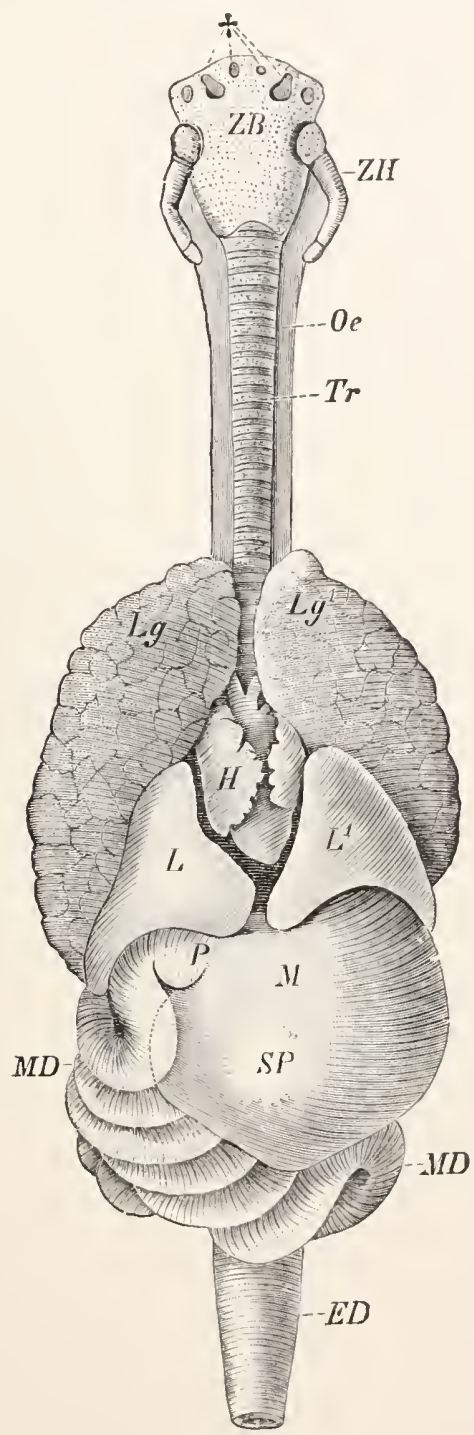

FIG. 573.-Viscera of Alligator (from Wiedersheim). IEI), rectum; $I I$, heart: $L$, liver; $L g$, lung; $M$, stomach; $M D$, intestine; $O e$, osophagus; $P$, pylorus; $T r$, trachea; $Z B$, body of hyoid; $Z H$, its cornua; *, perforations of hyoid.

ring in turtles and lizards, in the latter an episternum (fig. 52I) as well. Of considerable systematic importance is the position of the ankle joint. 
This is intertarsal, occurring between the first and second rows of tarsal bones (fig. $587, C$ ).

Since reptiles lack even transitory gills, the gill slits are completely degenerate before the young escapes from the egg. Dermal respiration is far less important than with the Amphibia, lungs, as in birds and mammals,

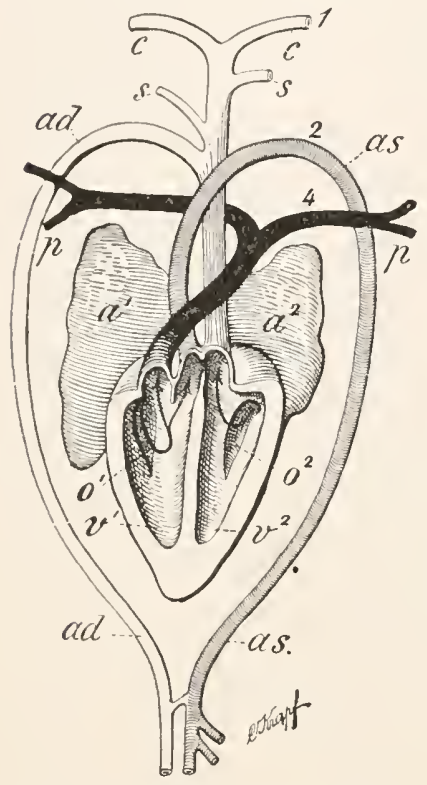

FIG. 574 - - Heart of crocodile with arteries (diagram). $\quad a^{1}, a^{2}$, right and left auricles; $a d$, as, right and left aortic arches; $c$, carotids; $o^{1}, o^{2}$, right and left auriculo-ventricular ostia; $P$, pulmonary artery; $s$, subclavians; $v^{1}, v^{2}$, right and left rentricles (the arrows show the direction of the blood flow); $1,2,4$, arches comparable with those of amphibia. Notice the origin of the vessels from the heart, and the connection (foramen I'anizza) between the arterial trunk and the left aortic arch, just in front of the heart. carries venous blood, and takes over the fourth arterial arch, which gives off the pulmonary arteries $(4, p)$. A second vessel arises from the left ventricle, is purely arterial and connects with most of the remaining arterial arches, the first, which gives off the carotid, and the right half (aortic arch, ad) of the second arch. The third vessel connects on the 
one hand with the remaining (left, second) arch and on the other with the right or venous half of the heart. The foramen l'anizza occurs between this and the right aortic arch. The renous character of the left aortic arch and the incomplete ventricular septum (or presence of foramen Panizze) prevent a complete separation of systemic and pulmonary circulations. In the turtles a third element enters, the persistence of a ductus Botalli (as in Lrorleles, fig. 537, $I I, d B$ ).

'To the foregoing adaptations to a terrestrial life may be added indications of higher development. 'The brain shows two advances, The cerebellum, especially in turtles and alligators, has become larger, and the cerebrum grows over the 'twixt brain and forms the temporal lobes of the hemispheres. The parietal organ is developed as nowhere else. In many lizards it forms an unpaired dorsal eye lying beneath the skin in the parietal foramen. The paired eyes possess lids (usually upper and lower as well as a nictitating membrane), and frequently (turtles, lizards, and many fossils) a ring of bony plates (sclerotic bones) in the sclera. A new opening in the petrosal, the fenestra rolunda, places the tympanic cavity and the labyrinth in close relations.

In the excretory system amniote characters prevail. The Wolffian body is functional in the embryo. Later the permanent kidney (metanephros) with the ureter arises behind it, while the embryonic structures disappear, except those retained as accessory to the genital apparatus. Thus in the male the vas deferens and epididymis are formed from the Wolffian duct; in the female the Mïllerian duct (early lost in the male) becomes the oviduct. Usually the urogenital canals open dorsally in the cloaca, rarely in an elongation of the urinary bladder (Chelonia). The bladder is lacking in snakes and crocodiles.

Almost all reptiles lay eggs; only in some snakes and lizards are viviparous or ovoviviparous forms present. The eggs much resemble those of birds, in that the large yolk is surrounded with a layer of albumen and enclosed in a fibrous, often calcified shell; the eggs undergo a discoidal (meroblastic) segmentation. To open the egg the embryo has an egg tooth on the tip of the snout; this consists of dentine in the Squamata, but elsewhere, as in birds, is horny. As the egg has a shell it follows that internal impregnation must take place. Copulatory organs to accomplish this occur, and these are of classificatory value since they differ in character in the Squamata on the one hand, the turtles and crocodiles on the other. These differences are correlated with differences in the form of cloacal opening and in structure of skull and skin, so that all living species may be divided into two groups, the Squamata, containing the lizards, snakes and Sphenodon, and the Hydrosauria with turtles and crocodiles. 'This, 
however, ignores the fossil forms. When these are taken into consideration another grouping must be adopted.

\section{Order I. Theromorpha.}

Extinct reptiles from the Permian and triassic, closely related to the stegocephala; with amphicuelous vertebre, immovable quadrate, and from two to six sacral vertebrx. Anomodontia, with partial or complete loss of teeth, near the turtles; the Thlorodonta, in which a heterodont dentition is developerl, resemble mammals, which, by many, are supposed to have descended from them.

\section{Order II. Plesiosauria.}

Extinct aquatic forms from the mesozoic, some of them forty feet long. They had long necks; limbs were swimming paddles recalling the flippers of whales; quadrate immovable; jaws long, with numerous teeth in sockets.

\section{Order III. Ichthyosauria.}

Resembled the Plesiosaurs in swimming feet, elongate jaws, and quadrate, but had teeth (sometimes absent) in groores, and short necks. At least some species were viviparous. Mesozoic.

\section{Order IV. Chelonia (Testudinata).}

The turtles form in external appearance a sharply circumscribed group, with the short and compact body enclosed in a bony case, from which only head, tail, and legs protrude (figs. 575, 576). The case consists of a convex dorsal portion, the carapace and a flattened ventral plastron, the two being united in most forms at the margins. Each consists of bony plates, the positions and names of which may be learned from the adjacent cut. It only needs mention that the neural plates are united with the spinous processes, the costals
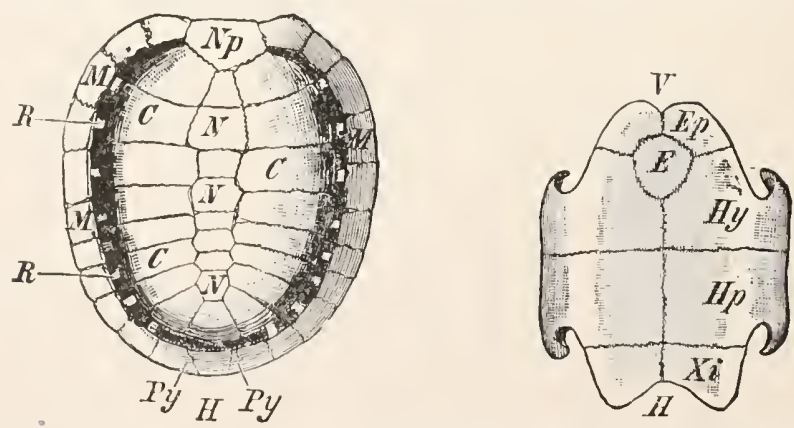

FIs. 575.-Carapace (A) and plastron $(B)$ of Testudo greca (from Wiedersheim). $C$, costal plates; $E$, entoplastron; $E p$, epiplastron; $H$, posterior; $H p$, hypoplastron; $H y$, hyoplastron;.$M$, marginal plates; $N$, neural plates; $N p$, nuchal plate; $P y$, pygal plate; $R$, ribs; $V$, anterior; $X i$, xiphisternum.

with the ribs, and that the entoplastron is regarded as an episternum. It is not connected with the internal skeleton, since the sternum is lacking. The pelvis is only rarely fused with the plastron. This bony case is usually covered with horny shields, their number and arrangement usually agreeing with the bony plates, although without their contours exactly coinciding.

More important are the great firmness of the skull and the immovable 
condition of the quadrate, the lack of an os transversum and of any but basisphenoid of the sphenoidal bones (Dermochelys has a parasphenoid), and the growth forwards and backwards by which the girdles are brought inside the rilss. The teeth are entirely lost, and, as in bircls, the jaws are enclosed in sharp horny beaks, in many cases efficient weapons against larger vertebrates. 'The cloacal opening is oval, its major axis corresponding to that of the body, and in its anterior end is an unpaired erectile penis used in copulation. Turtles appeared in the Permian.

Characters of armor and legs serve to contrast sharply the land and sea turtles; the first with well-developed legs, five-toed in front, four-toed behind, the toes with claws; the carapace arched, into which legs, head, and tail may be retracted. In the sea turtles the feet are flipper-like (fig. 576), claws mostly absent, and the carapace weakly united to or free from the plastron, flat and incapable of covering head or appendages. The fresh-water species are intermediate in position.

Sub Order I. ATHECA. Carapace of numerous mosaic scales not connected with ribs and vertebræ; skin leathery. Dormochely's, ${ }^{*}$ leather-back tortoise, reaches a weight of 5500 pounds. Sub Order II. TRIONYCIIIA. Fresh-water forms with poorly ossified carapace, but ribs and vertebre connected with it. Leather turtles (Amyda*) and soft-shelled turtles (Aspidonectes*).

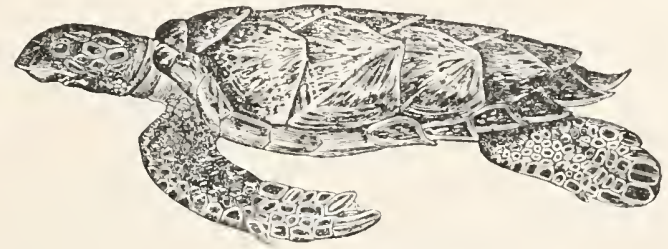

FIG. 576.-Eretmochelys imbricata, tortoise-shell turtle (from Hajek).

Sub Order III. CRYPTODIRA. Carapace well developed and united with ribs and vertebræ, but the pelvic arch free. Species numerous, including terrestrial, fresh-water, and marine forms. CHELYDRIDe, fresh water, tail long. Chelydra serpentina,* snapping turtle; Machrochelys lacertina,* alligator turtle. Chelonid.e, marine, paddle-like feet. Thalassochelys,* loggerhead; Chelone mydas,* green turtle; Eretmochelys imbricata, whose horny shields furnish 'tortoise shell.' Testudinide, terrestrial, including Xerobates, * the 'gopher turtle' of the South, the giant Testudo of the Galapagos Islands, and the fossil Colossochelys atlas of India, I 8-20 feet long, 8 feet high. Other families contain our mud turtle (Kinosternon**), box turtles (Cistudo*), and terrapins (Malaclemmys*). Sub Order IV. PLEURODIRA. Pelvis united to carapace and plastron; southern hemisphere.

\section{Order V. Rhynchocephalia.}

These resemlle the lizards not only in body form (four five-toed feet) and in scaly skin, but in lack of hard palate, presence of epipterygoid, transverse cloacal opening, and heart, lungs, and brain. On the other hand, they recall crocodiles in having two postorbital arches and immovable quadrate. The large abdominal sternum and abdominal ribs are noticeable as well as the uncinate processes of the true ribs. The notochord is but incompletely replaced, the vertebræ amphicolous. The group appears in the Permian and is one of the 
oldest of reptilian types; usually regarded as ancestral to all the orders yet to be mentioned. 'The only living species, Sphenodon punctata, belongs to New Zealand.

\section{Order VI. Dinosauria.}

This Mesozoic order included some of the largest land animals which have ever existed. Some were from forty to one hundred feet long and twelve to twenty feet high (Amphicalias, Camarasaurus). In some there was an exoskeleton, some of the plates of which in the stegosaurs measured a yard across. Among the characters are the fixed quadrate, jugal and postorbital arches, three to ten sacral vertebre, and ilium elongate in front of and behind the acetabulum. Some (Orthopoda) in pneumaticity of bones, in having the pubic bones directed backwards, and in the formation of an intratarsal joint, resembled the birds, and have been regarded as the ancestors of that group.

\section{Order VII. Squamata (Lepidosauria, Plagiotremata).}

One of the characters which unite lizards and snakes and which has given the nante Plagiotremata is the transverse cloacal opening (fig. 577), behind which, in the male, are the paired copulatory organs, each lying in a sac from which they can be everted like the finger of a glove. The names Squamata and Lepidosauria refer to the scaly condition of the skin. The corium forms flattened papilla which resemble the scales of fishes in that in many species they contain bony plates (fig. 509). Since

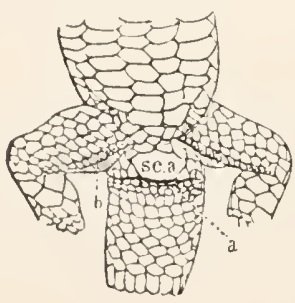

FIG. 577 .

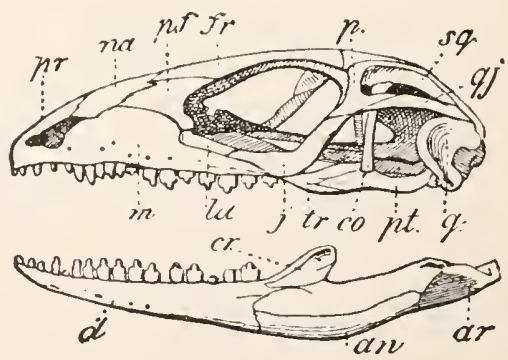

FIG. 578 .

FIG. 577.-Hinder trunk and hind limbs of a lizard (from Ludwig-Leunis). a, cloacal slit; $b$, femoral pores; $s c a$, anal shield.

FIG. 578. - Skull of A meiva v'ulgaris. an, angulare; ar, articulare; co, epipterygoid; er, coronoirl; $d$, clentary; fr, frontal; $j$, jugal; la, lacrimal; $m$, maxillary; na, nasal; $p$, postorbital, above and behind it the parietal; $p f$, prefrontal; $p r$, premaxilla; $p t$, pterygoid; $q$, quadrate; $q j$, quadratojugal; $s q$, squamosal; $t r$, transversum.

the stratum corneum is thick on the top of the papillæ and thinner between them, rhomboid and oval plates occur, which either lie flush with each other (shields) or overlap like shingles (scales). The rule is that the head is covered with regularly arranged shields, each with its name, the trunk with scales in longitudinal, transverse, and oblique lines. Outside these is a layer of cornified cells, the psendocuticula, and outside of all an 
inconspicuous true cuticle. Since all cornified cells are dead and require periodic removal, the horny layers are cast yearly and replaced by new. During this periodic molting, which recalls that of arthropods, the animals are sickly and apt to die in captivity.

All Squamata are characterized by the slenderness of the cranial bones (figs. $572,578,579$ ), which, especially in the Lacertilia, incompletely close in the cranium. The quadrate is movable, and the squamosal is intercalated between it and the cranium. A hard palate is lacking, and the choanx, as in the amphibia, lie far forward (fig. $57^{2}, C h$ ). 'There is a wide gap in the partition between the two rentricles of the heart.

Sub Order I. LACERTILIA (Saurii). The lizards are usually distinguished from the snakes by the possession of limbs, but a few undoubted lizards (glass snakes and Amphisbænæ,) lack limbs. These are distinguished by the existence of the scapula and the iliac bone united to the vertebra, and the presence of a sternum, which never occurs in snakes. In the skull is a peculiar bone (lacking only in Chameleons and Amphisbrene), found nowhere else, the epipterygoid (fig. $578, c 0)$; it reaches from the pterygoid to the parietal, and is sometimes called columella (not to be confounded with the true columella of the

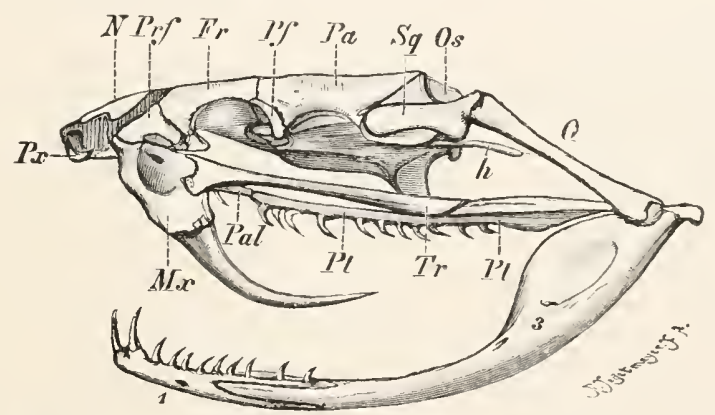

FIG. 579.-Skull of rattlesnake (from Boas). Fr, frontal; $h$, hyomandibular (columella); $M x$, maxillary; $N$, nasal; $O s$, supraoccipital; $P a$, parietal; $P a l$, palatine; $P f$, postfrontal; Prf, prefrontal; $P t$, pterygoid; $P x$, premaxilla; $Q$, quadrate; $S q$, squamosal; $T r$, transversum; I, dentary; 3 , articulare.

ear). The bones of the jaws are firmly united, so that the mouth has no special capacity for opening widely. The jugal-quadratojugal arch is present. In external appearance the presence of eyelids, nictitating membrane, tympanic membrane, and Eustachian tube are noticeable, these being absent only in the Amphisbænæ. In the Ascalabotæ, as in snakes, the lids grow together, forming a transparent covering over the eyes. The group dates back to the cretaceous.

Section I. Ascalabotæ (geckos). Skeleton incompletely ossified, notochord persistent, a mphicole vertebræ; skin granular, usually adhesive discs on toes, $P h y l$ lodactylus.* Section II. Crassilingera. Tongue thick, fleshy, not protrusible. IGUANIDE; American, often a comb of spines on back, teeth pleurodont. i.e., united to inner side of jaw. Anolis, * Sceleporus, * Phrynosoma,* 'horned toads.' AGamid x; Old World, teeth acrodont, i.e., on the edge of jaw. Chlamydosaurus, Draco. Section III. Fissilinguia. Tongue long, thin, divided at tip, protrusible. 
TEJIDE; American, teeth acrodont; Cnemidophorus, ${ }^{*}$ Tejus.* HelodermaTID.E, pleurodont; Heloderma, * 'Gila monsters,' only poisonous lizards. LaCertilide (Lacerta) and VARANide (I'aramus, monitors) Old World, Lacerta vivipara bringing forth living young. Section IV. Brevilinguia. Tongue short, slightly notched at the tip, slightly protrusible. SCINCID E, with tendency to reduction of the limbs. Eumeces,* Oligosoma.* Anguis and Typhline, legs absent. ZoxUrin.玉, Old World except Ophisaurus ventralis,* glass snake, limbless, with brittle tail. Section V. Axxulata. Snake-like; legs and epipterygoid, tympanum, and movable eyelids lacking and usually girdles; tropical or subtropical. Chirotes, Amphisbana. Section VI. Vermilinguia; Old World chameleons, long fleshy tongue, ring-like eyelids functioning as an iris, the climbing feet with toes united into two opposable groups; epipterygoids, clavicle, sternum, and tympanic membrane lacking. Celebrated for color changes produced by alterations of chromatophores.

Sub Order II. PYTHONOMORPHA. Large, extinct, extremely elongate reptiles with four flipper-like limbs and strong swimming tail. Flourished in the cretaceous. Mosasaums, Clidastes.

Sub Order III. OPHIDIA. Snakes are distinguished from most lizards by the absence of limbs, and connected with this the similar vertebra in which only trunk and caudals can be distinguished. The caudals lack ribs, but these are present and long in the trunk region, serving for locomotion and supporting the body on their distal ends. Since there are legless lizards, it is further necessary to say that in the Ophidia the girdles and sternum are lost, only the Peropoda having remnants of the hindler appendages and pelvis, but these are not connected with the vertebral column. The ventral side of lizards is covered with scales, of reptiles with transverse scutes.

Further distinctions exist in sense organs and jaws. The columella is indeed present, but tympanum and Eustachian tube are lacking. 'The eyelids

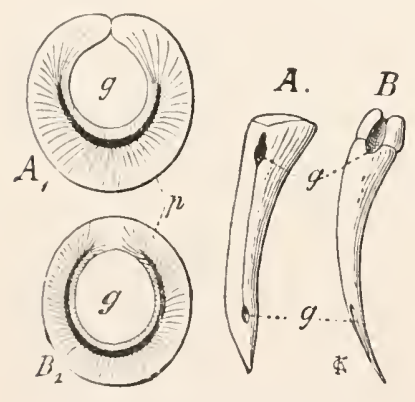

FIG. 580.-Poison fangs. $A$, $A_{1}$, proteroglyphic (grooved) tooth of cobra, and section of same; $B$, $B_{1}$, solenoglyphic tooth (tubular) of rattlesnake; $g$, poison canal; $p$, pulp cavity. by the elasticity of its walls and the great mobility of the ribs, which are not united ventrally by a sternum.

In the non-poisonous snakes the dentition is similar on jaws and palate bones (fig. 572). The vomer and, usually, the premaxilla are toothless. In the poisonous serpents poison fangs apnear on the maxilla (fig. 579), distinguished from the other teeth by their greater size and connexion with a 
large poison gland, the duct of which opens at the base of the tooth; the poison is led to the tip of the tooth either by a groove (proteroglyphic tooth, fig. 580 , A) or, when the groove is closed to a canal (solenoglyphic looth, B), through this canal which opens at base and tip of the tooth.

The asymmetrical character of the lungs is interesting. In the Peropoda the left lung is much smaller than the right; in the poison snakes and some others it is rudimentary or even absent. In the 'Typhlophidx, on the other hand, the right is degenerate. The urinary bladder is always absent. 'The excreta, chiefly uric acid, accumulate as a solid mass and form the chicf part of the excrement; the fæces, on account of the extraordinary digestive powers, being small in amount.

Section I. Opoterodorta (Angiostoma). Burrowing blind tropical snakes. Typhlops. Section II. PeropodA. Large snakes, viviparous or ovoviparous, have paired lungs and rudiments of hind extremities; lack poison fangs, and kill their prey by muscular power. Python, Asia and Africa; Boa and Emmertes (anaconda), South America. Secion III. Colubriforma. Ordinary snakes (500 species) with numerous teeth in upper jaw, appendages entirely absent. Some poisonous, some not, but no structural lines can be drawn between them. AgLYPHA, no grooved teeth. Tropidonotus, ${ }^{*}$ water snakes; Bascanion, * black snakes; Eutainia, * garter snakes. The Proteroglypha, with grooved teeth, permanently erect, poisonous. Most are brightly colored. Elaps, * the coral snake; Naja tripudians, the cobra of India; N. haje, Cleopatra's asp. Here belong the pelafic sea snakes (HyDropHID $\approx$ ) of the Indo-Pacific, viviparous. Section IV. Solenoglypha. Maxilla reduced and serving as a socket for the single large tubular tooth with one or more reserve teeth (fig. 579). VIPERIDA, Old World, no pit between nostril and eye. Crotalide, New World and Asia, a pit between nose and eye. Crotalus, * tail ending in a rattle formed by remnants of cast skins; Agkistrod $n$ contortrix, ${ }^{*}$ copperhead, and $A$. piscivorus, moccasin, lack the rattle.

\section{Order VIII. Crocodilia (Loricata).}

The crocodiles, alligators, etc., agree with turtles in the oval cloacal opening with single copulatory organ, immovable quadrate, and the bony plates in the skin. In shape they are lizard-like, but in structure they differ from all other living reptiles and approach most nearly to the

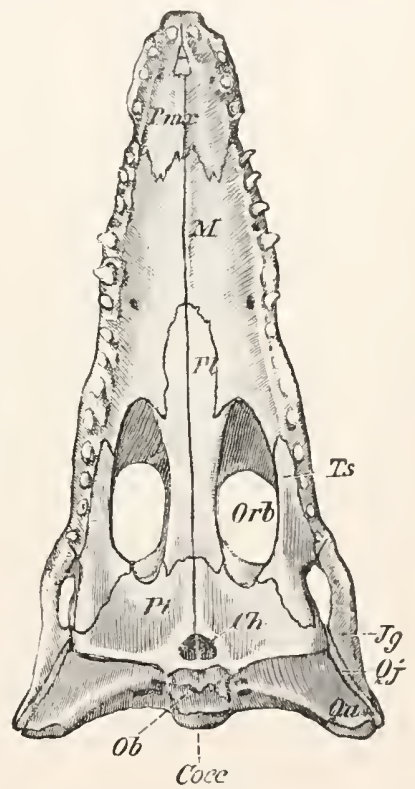

Fig. 58r.-Ventral surface of skull of crocodile (from Wiedersheim). Cocc, occipital condyle; $C h$, choana: $j g$, jugal; $M$, maxillary; $O b$, basioccipital; $O r b$, orbit; $Q i$, quadratojugal; $Q u$, quadrate; $P l$, palatine; Pmx, premaxilla; $P t$, pterygoid; $T s$, transversum.

Theromorphs. The maxillaries, palatines, and pterygoids are united in the midlle line, forming a hard palate and forcing the romers uprards into the nasal region, and carrying the choana (fig. $58 \mathrm{I}, \mathrm{Ch}$ ) to the 
back of the skull. Some of the ribs have two heads; the ears and nostrils are provided with valves. A sternum is present and, farther back, abdominal ribs (gastralia), and an abdominal sternum. The jaws are extended into a long snout, and the teeth, which occur only on the margins, are placed in sockets or alveoli (thecodont). The four-chambered heart has already been described (p. 52-t). The animals move slowly on land, but are very active in the water. They have a strong smell, owing to musk glands in the cloaca and on the under jaw. The group appeared in the trias, and of the three sub orders two, the Pseudosuchia and Parasuchia, are extinct.

Sub Order EUSUCHIA. External nostrils united, choana posterior; five toes in front, four behind. Gavialis, India, snout long and slender. Alligator lucius,* alligator; Crocodilus, $*$ most species Old World, one, C. americanus, * occurring in our southern waters.

\section{Order IX. Pterodactylia (Pterosauria).}

Extinct Mesozoic reptiles, adapted for flight. The bones were hollow, the wings were broad membranes, supported, like those of a bat, by the body and

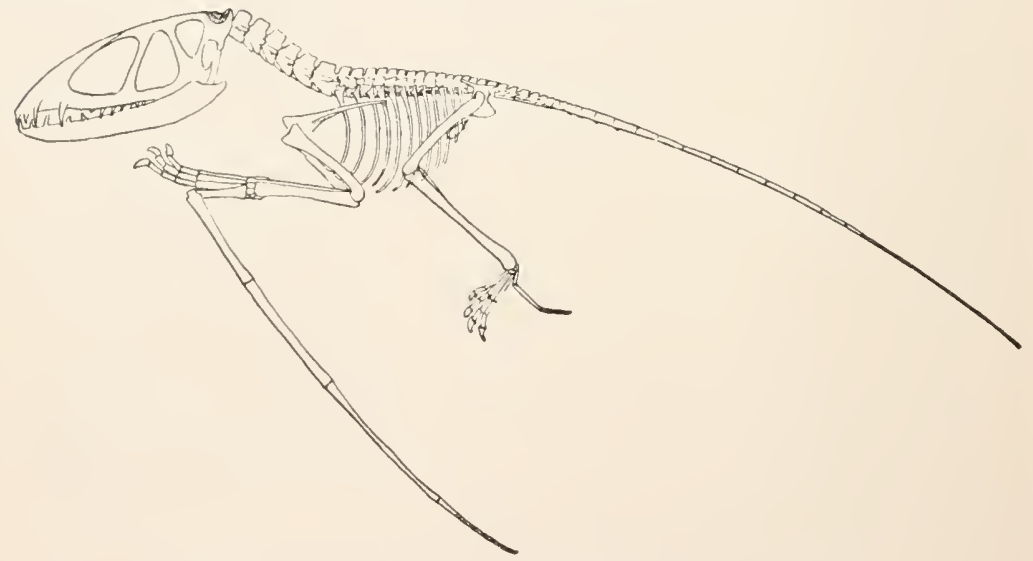

Fig. 5S2,-Dimorphodon, a pterodactyle (after Woodward).

the very long fifth finger. Some were sparrow-like in size and some, Pteranodon, had a wing expanse of twenty feet. One of these large forms, had its pelvis so small that its egres could not have been more than half an inch in diameter.

\section{Class II. Aves.}

While structurally the birds are near the reptiles, yet by the development of wings and the feathering of the body the group is strictly circumscribed. The skin in some places, as the lower part of the legs, is covered with horny scales and shields; on the toes and occasionally on the fingers 
are claws, but as a rule the fingers are feathered. On most places the skin is soft and thin, since corium and stratum corneum are poorly developed. Periodic molts of the integument do not occur, since the horny layer, as in mammals, undergoes a constant renewal. These peculiarities of the skin are correlated with the appearance of the protecting plumage.

The feather, like the hair of mammals, is exclusively epithelial in character, but is a much more complicated structure. The cornified epithelium forms a firm axis, the scape, from which, right and left arise branches, or barbs. The scape is solid as far as the barbs extend (rachis, or shaft), while below it is hollow (quill, or calamus). The quill is inserted deep in a follicle in the corium and is provided with muscles for its movement. Its hollow in most fully developed feathers is empty

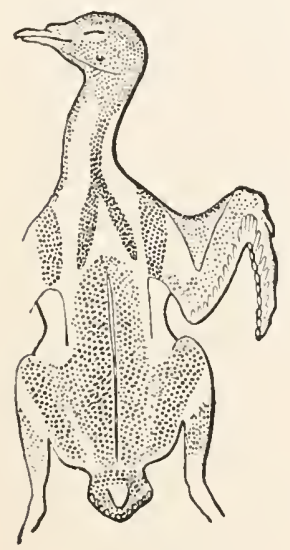

FIG. $5^{8} 3$.

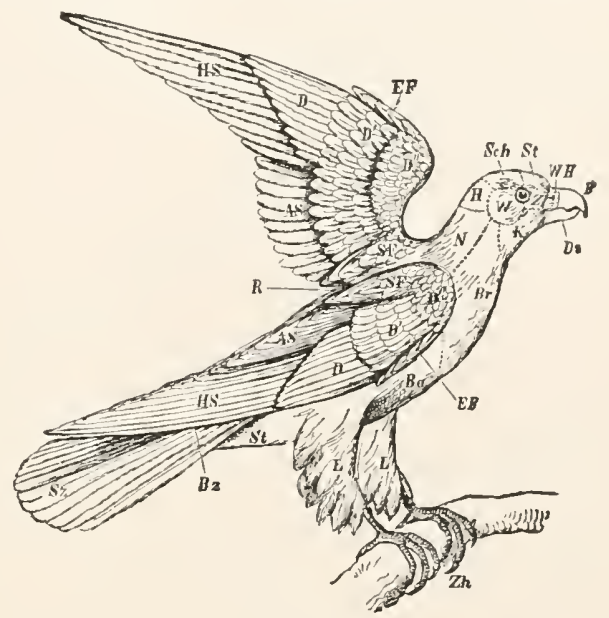

Fig. 584. Leunis).

FIG. $5^{8} 3$.-Feather tracts and apteria of pigeon, dorsal view (from Ludwig-

FIG. 58.4-Regions and feathers of Falcc lanarius (from Schmarda). As, secondaries; $B a$, belly; $B r$, breast; $B z$, rump; $D^{\prime}-D^{\prime \prime}$, wing coverts; $D i$. gonys of bill; $E F$, alula; $F$, culmen of bill; $H$, occiput; $H S$, primaries; $K$, throat; $L$, legs; $N$, neck; $S c h$, crown; $S F$, parapterium; $S t$, forehead, lower tail coverts; $S z$, rectrices; $W$, cheek; $W H$, cere with nostril; $Z h$, toes.

save for the 'pith,' a small amount of clried tissue. In young growing feathers it is occupied by a richly vascular feather papilla, an outgrowth of the corium for purposes of nourishment. The feather may therefore be regarded as a cornified outgrowth from the skin which has arisen on a papilla of the corium, a view which accords with its development and shows its homology with the scales. In many birds (cassowaries) two welldeveloped feathers arise from the same follicle-a fact which explains the 
existence in many feathers of a rudimentary feather, the hyporachis, or after-shaft, attached to the scape below.

In contour feathers the barbs are, to a great extent, united into a vane. They lie close together and parallel, right and left of the shaft, each repeating in miniature the entire feather, the barb having branches or barbules, which, overlapping the barbules of adjacent barbs, give the vane its close texture. The vane is held together by minute hooks on the barbules of one barb interlocking with those of the next. Down feathers (plumes) differ from contour feathers in the absence of hooks and the loose arrangement of the barbs. Since feathers consist of cornified epithelium and these cells are held firmly (only in powder down is there a gradual loss), they, like the scaly coat of the snakes and lizards, must be molted yearly and replaced by new.

Young birds or embryos have only down feathers. Later the contour feathers arise in regularly arranged feather tracts, or pteryla, between which are apteria in which no contour feathers appear (fig. 583). Since the contour feathers overlap like shingles, they form a firm coat beneath which the down and semiplumes form a warm coat. Besides these covering feathers (coverts, or tectrices, fig. $584, D$ ) there are the longer feathers of the wing, the remiges, and the tail feathers, or rectrices $(S z)$. The larger remiges form the chief part

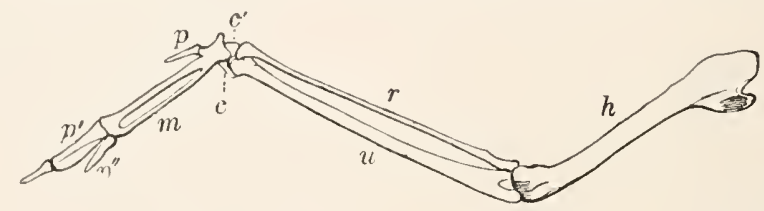

Fig. 585.-Wing skeleton of stork (from Gegenbaur). $c, c^{\prime}$, carpalia of first row; $h$, humerus; $m$, fused metacarpals and carpals of second row; $p-p{ }^{\prime \prime}$, phalanges of first three fingers; $r$, radius; $u$, ulna.

of the wing; they spring from the part of the limb corresponding to the hand (carpus, metacarpus, phalanges) and are known as primaries $(H S)$, while the secondaries $(A s)$, arising from the forearm, are shorter. These are overlapped at the base by the coverts $\left(D, D^{\prime}, D^{\prime \prime}\right)$ and by the parapterium $(S F)$ springing from the shoulder. A few feathers arising from the first finger remain distinct from the remiges and form the alula $(E F)$. In the water birds especiaily the feathers are oiled by the secretion of a pair of glands at the base of the tail above the coccyx.

Since the feathers are not only for protection, but give to most birds the power of prolonged flight, they predicate a special mode of life, under the influence of which all of the other organs exist. The character of the skeleton, the respiratory organs, and in part the sense organs and brain, are connected with the power of flight.

As the feathers of the wings, like the fins, form what may be called a paddle working as a whole, the skeleton of these limbs is simplified (fig. $\left.5^{8} 5\right)$, first, by the reduction of the fingers, of which only three with a small number of phalanges persist $\left(p, p^{\prime}, p^{\prime \prime}\right)$; second, by fusion of the corre- 
sponding metacarpals $(m)$ with each other and with the arljacent carpal bones. On the other hand, in order that there may be the necessary energy and the most complete transfer of the same to the borly, the connexion with the skeletal axis is strengthened byspecial (levelopment of the parts. In the shoulder girdle (fig. 586) all three elements are firm, a sword-shaped scapula $(s)$, a columnar coracoid $(c)$, and clavicles which are usually united to a 'wish-bone,' or furcula $(f)$. Clavicles and furcula are united directly or by ligaments to the broad sternum, the anterior face of which is developed into a strong keel, the carina, in order to give the largest surface for attachment of the large muscles of flight. Usually the greater the powers of flight the more developed the carina, yet in some cases (albatross) the weak carina is compensated for by the enormous width of the sternal plate. In running birds (ostriches, etc.) the carina is entirely gone. The thoracic framework is rendered more firm by the development of uncinate processes from the vertebral parts of the ribs (u) which overlap the succeeding ribs.

Since the fore limbs are no longer used for walking, the support of the body depends upon the hinder extremities, only the toes reaching the ground, the middle foot (metapodium) increasing the length of the leg. This has brought

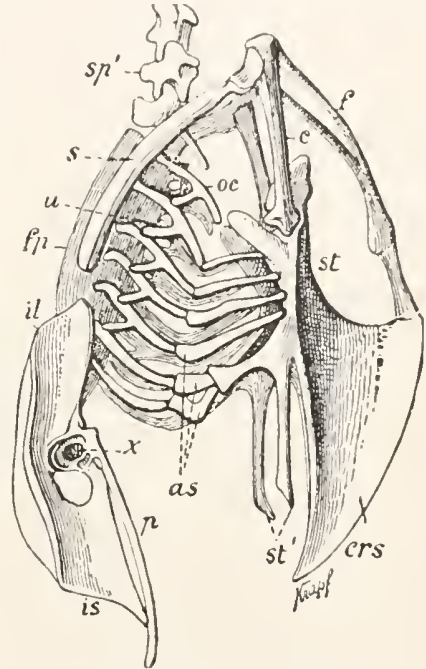

Fig. 586.-Trunk skeleton of stork (from Gegenbaur). as, sternal part of rib; $c$, coracoid; $c r s$, keel; $f$, furcula (fused clavicles); $f p$, fused spinous processes of thoracic vertebre; il, ilium; is, ischium; $o c$, vertebral part of ribs; $p$, pubis; $s$, scapula; $s p$, spinous process; st, st ${ }^{1}$, sternum and abdominal processes; $u$, uncinate processes; $x$, acetabulum. about two striking characteristics-the broad union of the pelvis with the vertebral column, and the simplification of the leg skeleton. In the embryo the ilium (fig. $586, i l$ ) is connected only with the two sacral vertebre present in most reptiles, but later it extends forward and back, uniting with from nine to twenty-three vertebra; while the iliac bones of the two sides may meet dorsal to the vertebral column. This extensive union of pelvis and axial skeleton is understood when we recall that the vertebral column is not vertical as in man, but is inclined. Ischium and pubis are peculiar in that they extend backwards, parallel to each other, from the acetabulum, and that only exceptionally (ostrich) are the bones of the two sides united by a symphysis. 
In the hind limbs occur conditions similar to those in the ungulates. The weight of the body makes it necessary that the simplification found in the wing should be repeated in the lower leg and foot, and that the numerous bones usually occurring in these regions be replaced by one

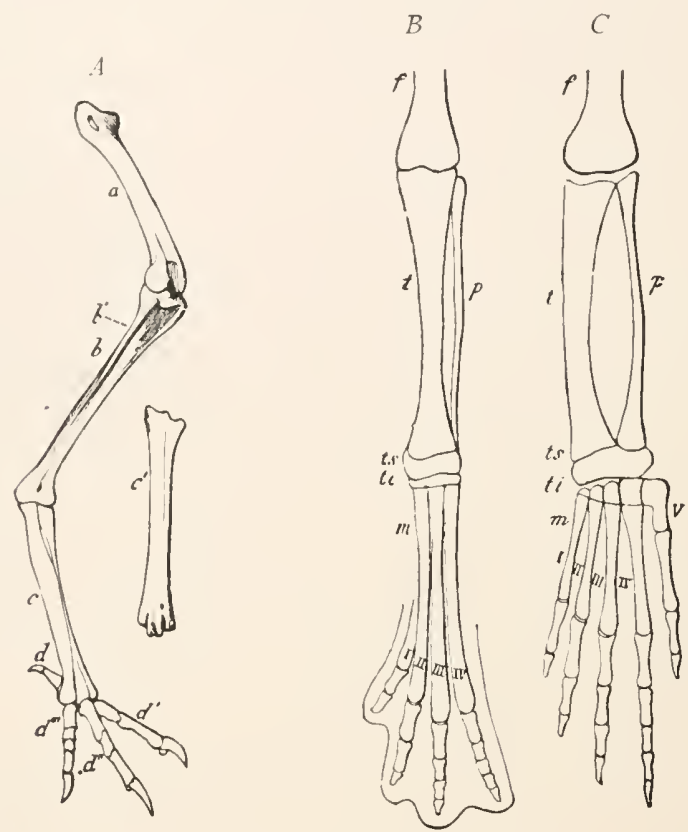

FIG. $5^{8} 7 .-A$, leg of Buteo vulgaris. $a$, femur; $b$, tibio-tarsus; $b^{\prime}$, remains of fibula; $c$, tarso-metatarsus, $c^{\prime}$, same, front view; $d-d^{3}$, toes. $B$, lower leg of bird embryo; $c$, of lizard. $f$, femur; $t$, tibia; $p$, fibula; $t s$, tarsales of first row (talus); $t i$, tarsales of second row; between these intertarsal joint; $I-V$, digits (from Gegenbaur).

to support the pressure (fig. $5^{8} 7$ ). Therefore the fibula, well developed in the embryo $(B)$, becomes reduced to an inconspicuous rudiment; the metatarsals, distinct in the embryo $(B)$, fuse to a single tarso-metatarsus $(A, C)$, which has below as many articular surfaces as there are toes (since the fifth toe only appears in the embryo, at most four, in some three or even $\left(w o, d-d^{\prime \prime \prime}\right)$. At the same time the tarsals disappear by fusion with adjacent parts. Even in reptiles $(C)$ a part of the tarsals unite with the bones of the shank, and the remainder with the metatarsals; in the birds the union is completed, the proximal series fusing with the lower end of the tibia to form a tibio-tarsus, the distal with the metacarpus to form the tarso-metatarsus, in this way producing the intertarsal joint so characteristic of birds. 
In respect to the vertebral column, it only needs mention that the vertebrx articulate with each other by a so-called saddle-joint, that (in living birds) only a few caudal vertebre persist behind the pelvis, that these are partially fused to a single bone, the pygostyle, which supports the tail feathers, and that, corresponding to the well-developed neck, there are many cervical vertebre, among them an atlas and an axis or epistropheus, all except the last two fused with the corresponding cervical ribs.

The skull (fig. 588 ) resembles that of the lizards in the presence of a single occipital condyle, in the movable condition of the quadrate upon the cranium, and in the presence of a slender columella, but an os transversum is lacking. The cranium, as a result of the increase in size of the brain, is more

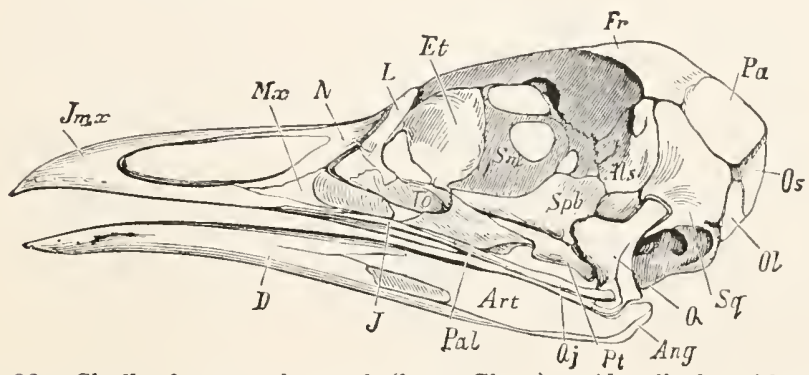

FIG. 588.-Skull of young bustard (from Claus). Als, alisphenoid; Ang, angulare; Art, articulare; $D$, dentary; $E t$, mesethmoid; Fr, frontal; $J m x$, premaxillary; $J$, jugal; $L$, lacrimal; $M I x$, maxillary; $N$, nasal; $O l$, exoccipital; $O s$, supraoccipital; $P a$, parietal; $P a l$, palatine; $P t$, pterygoid; $Q$, quadrate; $Q j$, quadratojugal; $S m$, interorbital septu'n; $S p b$, basi- and presphenoid.

spacious; the bones of its walls fusing early so that the sutures between them are obliterated. The occipital condyle is on the under surface, so that the skull is carried at nearly right angles to the axis of the vertebral column. Teeth are lacking in living birds, although they occurred in some fossil forms. In their place are hard horny sheaths covering the jaws which are frequently carried back on the outside into a softer cere (fig. $584, W^{\prime} H$ ).

The cranium consists of four occipitals, a basisphenoid prolonged in front by a rostrum (possibly a part of a parasphenoid, possibly presphenoid); above, the parietals and frontals; and on the sides prootics, alisphenoids and orbitosphenoids, while the broad squamosals also enter its wall. The large mesethmoid lies in the interorbital septum; the nasal cavity is roofed by the nasals and beside them are the lacrimals. The quadrate articulates with the squamosal, and from it extend forward internally the pterygoid, palatine, and romer, externally a zygomatic arch of quadratojugal and jugal to the maxillaries and premaxillaries. The maxillaries are hinged in the ethmoidal region, so that in opening the mouth there is besides the depression of the lower jaw an upward motion of the upper jaw. 
The pneumaticity of the bones is an important feature. In place of marrow and bony tissue, the cavity of the bones in strong flying birds is more or less completely occupied by air spaces. This gives the greatest possible lightness and strength to the skeleton. In Buceros and Palamedea all bones are pneumatic; in others (Pelecamus, Sula, etc.) only the phalanges of the toes contain marrow, while in the penguin and $A$ pteryx, as in mammals, air spaces occur only in some of the cranial bones.

The air spaces of the bones are in part (skull) connected with the nose and tympanum, but most of them, by means of the air sacs, communicate

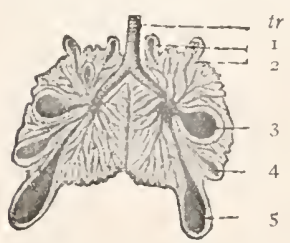

Fig. 589.--Development of trachea, lungs, and air sacs of a chick (after Selenka). tr, trachea; $1-5$, lung sacs. with the lungs. The long trachea forks at its lower end into two bronchi. At its upper end is a larynx, as in other vertebrates, but this is not rocal; the notes of birds are produced by the $\operatorname{syrinx}$, which lies at the division of trachea into bronchi. It is usually formed of both trachea and bronchi, but more rarely of either trachea or bronchi alone. Its vocal cords are regulated by special muscles, which in the singing birds have a complicated arrangement. The relatively small lungs send out from their surface air sacs, especially well seen in embryos (fig. 589, I-5). These later become large, thin-walled spaces, easily torn away in dissection, leaving large openings on the surface of the lungs (fig. 590, I-5). Usually five pairs of these air sacs are present, largely in the colom, but extending in between the muscles (breast and axillary region), and also into the bones.

The spongy lungs lie on either side of the vertebral column and are attached to the ribs. On entrance to the lung the bronchus (fig. 590, br) Ioses its cartilage supports and enlarges into a vestibule $(v)$ and extends thence as a mesobronchus $(\mathrm{b} m)$ backwards, terminating in the abdominal air sac (5). A side branch supplies the hinder subcostal sac (4). Secondary bronchi arise from the restibule and mesobronchus; of these there are three to five entobronchi $(I-I V)$ supplying the remaining air-sacs and six or more ectobronchi. Arising from the mesobronchi and secondary bronchi are tertiary bronchi, parabronchi, or lung pipes, running parallel to each other and anastomosing frequently. Each air pipe has a thick spongy wall (fig. 59r).

Inspiration is effected by raising the framework of the chest, thus causing an increase of the sternovertebral diameter; expiration by the reverse motion. $B y$ this the lungs, attached to the ribs, are alternately enlarged and contracted in spite of their slight elasticity. This is also true of the air sacs, which, on account of their poor blood supply, are not respiratory but serve as accessory air pumps. It is probable that in flight this air-pump action occurs especially with the subpectoral and axillary air sacs, drawing air through the lungs and rendering other respiratory motions superfluous, thus enabling the thorax to remain quiet, an important matter. If the trachea be closed and the air canal in the humerus opened, the bird can breathe through the latter. 
The circulation in the birds has arisen from that of the reptiles by complete separation of systemic and pulmonary systems. Of the three great arterial trunks present there (fig. 574), the pulmonary artery and the right aortic arch, arising from the left ventricle, are retained, the left venous arch being lost. The septum between the ventricles is complete. The striking features of the alimentary canal (fig. 6I) are the crop (not always present), a glandular stomach or proventriculus (c), and a muscular chewing stomach or gizzard (d), as well as two long, rarely rudimentary, cxca $(k)$ at the junction of small and large intestine. Liver and

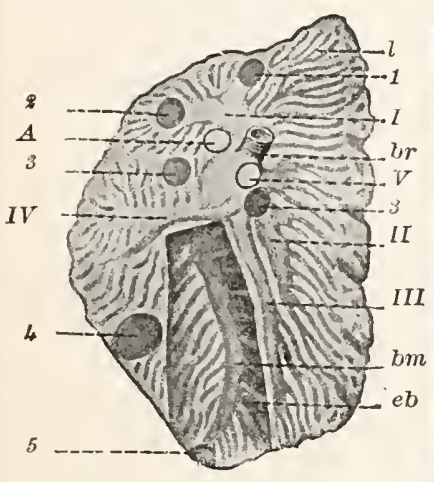

FIG. 590 .

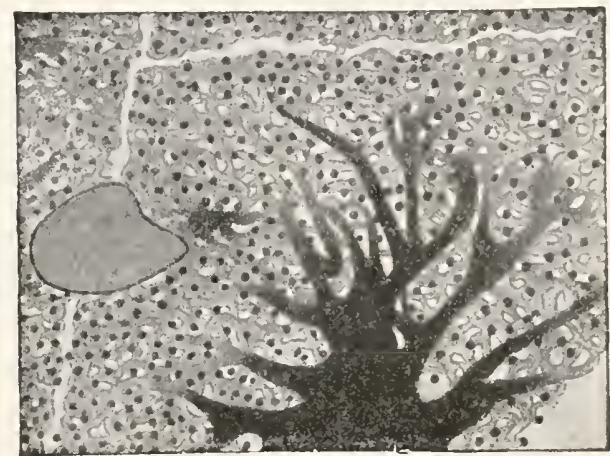

FIG. 59 I.

FIG. 590.-Right lung of hen, somewhat diagrammatic. A window shows a mesobronchus with its branches. $A$, artery; $b m$, mesobronchus, arising from the vestibule; $b r$, bronchus swelling to vestibule; $e b$, ectobronchus; $l$, lung pipes; $I-I V$, entobronchi; $V$, vein; $\mathrm{I}-5$, ducts of lung sacs.

FIG. 591.-Cross-section of a lung pipe with parts of an arljacent pipe; between the two a blood-vessel; the air space injected (after Fischer).

gall bladder $(e, f)$, pancreas $(g)$, and spleen are present. A blind sac (the bursa Fabricii), the paired ureters $(m)$, and the sexual ducts $(n)$ open into the cloaca. The sexual ducts are peculiar in that the right oriduct and ovary are degenerate. Since copulation occurs the large eggs (the ' $y o l k$ ') are fertilized in the oviduct (fig. I02). As they pass slowly through the duct, they become enveloped first with a thick layer of albumen ('white,' w), then with a double egg membrane (ism, sm), the two parts being separate and enclosing an air chamber at the larger end of the egg. Lastly comes the shell. During the passage down the oviduct the first phenomena of development (segmentation, gastrulation) occur; after oviposition the development stops and again starts when the necessary warmth is supplied.

The care for the young, the sexual life connected with reproluction, and the complicated conditions of existence connected with flight have 
resulted in an intelligence far superior to that of the reptiles, which finds its expression in the better development of sense organs and brain. In the brain (fig. 592) the cerebellum, the central organ for the coordination of the action of parts, is strikingly developed. 'The cerebral hemispheres are correspondingly large, the frontal lobes of which begin to cover the olfactory lobes, the temporal lobes in like manner extending back over the 'twixt brain and optic lobes. Corresponding to the vocal apparatus,

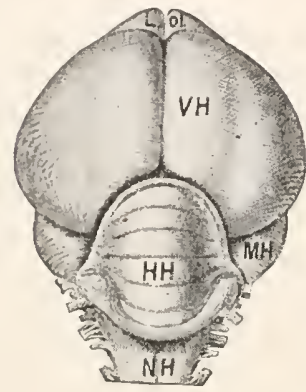

FIG. 592 .

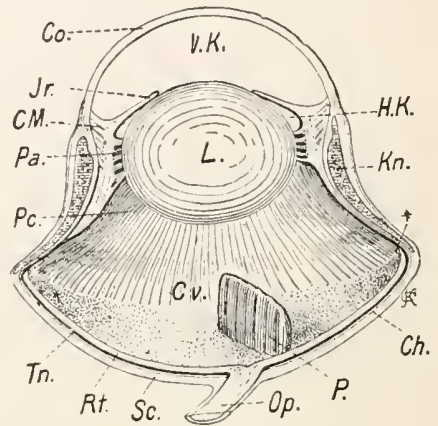

FIG. 593 .

FIG. 592.-Brain of pigeon (from Wiedersheim). HH, cerebellum; Lol, olfactory lobes; $M H$, mid brain; $N H$, medulla oblongata; $V H$, cerebrum.

FIG. 593.-Eye of owl (after Soemmering and Franz). Ch, chorioid; $\mathrm{Cm}$, ciliary muscle; $\mathrm{Co}$, cornea; $\mathrm{Cr}$, vitreous body; $H K$, posterior chamber; $J r$, iris; $K n$, sclerotic bone; $L$, lens; $O P$, optic nerve; $P$, pecten; $P c$, ciliary process; $R t$, retina; $S c$, sclera; $T n$, tapetum nigrum; $V K$, anterior chamber.

the ear is highly organized, the lagena of the labyrinth being greatly enlarged and the sound-conducting apparatus (columella, tympanum, etc.) well developed. The beginnings of an external ear are seen in the deeper position of the drum membrane. Since the power of flight necessitates vision at great distances, most birds have exceedingly sharp sight, and the eye itself (fig. 593) has a special apparatus (Crampton's muscle) for different distances. Peculiarities of the bird's eye, already weakly developed in the reptiles, are the pecten $(P)$, a comb-shaped growth of the chorioid into the vitreous body, the scleral ring, a circle of bones developed in the sclera and supporting the outer part of the eye, and a nictitating membrane besides the ordinary eyelids.

Among birds there is spirited rivalry for the females, especially among polygamous species. At the time of mating the males seek to win the favor of the females either through striking motions (dances), by singing, or by beauty of plumage. All of these peculiarities are confined to the male and frequently lead to a marked sexual dimorphism. 'The distinction in plumage is commonly strengthened at this time, the male receiving the brilliant wedding dress. 
The reason for the dull plumage of the female is due to the fact that she usually sets on the nest, at which time inconspicuous colors protect her from destruction by enemies. In only a few instances is the heat necessary for incubation produced by other causes, such as the heat of the sun on eggrs buried in the sand, or the increase of temperature caused by fermentation of decaying vegatation (Megapodes) The rule is that both sexes build the nest. When the clutch of eggs is complete the female (rarely the male) begins the incubation, at this time in some instances losing the feathers from certain regions the better to warm the eggs. Many birds, like hens and ducks, are so far advanced when they leave the nest that they can follow the mother and feed themselves. Such birds are called Precoces - in contrast to the Altrices, which hatch with incomplete coat of feathers and therefore need the warmth of the nest and the protection and care of the parents.

The migrations of birds possess great interest. We distinguish permanent residents and migratory species which, in orker to obtain food, take long journeys. At the approach of cold weather these seek the south, following regular paths in their travels. They cannot hibernate like reptiles and amphibians, when insects and fruit are scarce, because their more energetic vital processes demand a more rapid metabolism and a continuous food supply. Hence they, like the mammals, in contrast to the 'cold-blooded' vertebrates, maintain, under all extremes of external temperature, a body heat of $38-40^{\circ}\left(44^{\circ}\right.$ ?) C. (100-104 $\mathrm{F}$.).

The classification of birds is in a state of change. The older system based upon adaptive characters is not in harmony with the results of anatomical study, which would divide the whole class into many small groups. Here it has been thought best to retain the old, larger, easily recognized divisions, and to call attention to the differences from later results.

\section{Order I. Saururæ.}

The view that birds are closely related to reptiles received considerable support by the discovery of fossil birds with teeth. The most reptilian of these (Archaopteryx) occur in the Jurassic of Bavaria, and only two specimens have been found. In these carpals and metacarpals have not fused, the three fingers are well developed and clawed, and the caurlal vertebræ, although bearing feathers, form a long slender tail like that of a lizard (fig. 2).

\section{Order II. Odontornithes.}

These forms, from the cretaceous of Kansas and Colorado, also had teeth. The Odontorif (Ichthyornis) had a keeled sternum and normal pygostyle. In the ODONтоноцсж (Hesperomis) the wings were reduced (only the humerus persisting), the sternum lacked a keel, and the caudal vertebre formed a broad paddle.

\section{Order III. Ratitæ.}

Here are included several families, very different in structure, which agree in having the feathers not arranged in feather tracts; and in that, together with the lack of flight, many structures normally connected with it are absent. The bones are but slightly pneumatic, the sternum has no keel, and a furcula is not formed, the clavicles being rudimentary (Dromaus) or not present as clistinct bones. The wings are small and lack primaries and secondaries adapted for flight, for typical contour feathers with close vanes, as well as typical down feathers, are absent.

Since several structures apparently adapted for flight occur here (fusion of hand bones and often of caudal vertebrx; arrangement of wing muscles), 
it is probable that the Ratites have descended from carinate forms by loss of power of flight. The anatomical distinctions between the various families lead one to believe that they have arisen from different groups of carinates and hence do not form a natural assemblage.

Section I. STRUTHIONES, long humerus, legs and neck. STruthionID.E, two-toed ostriches of Africa, Struthio. RHEIDe, South American threetoed ostriches, Rhea. Section II. CASUARINA; three toes, humerus short. Dromaus, emus; Casuarius, cassowaries. Section III. APTERYGES, bill long, nostrils near tip, rudimentary wing skeleton; four toes. Apteryx, New Zealand. The Dinornithide, three toes, wing skeleton absent; giant birds of New Zealand; now extinct, apparently contemporaneous with man. Epiornis, a gigantic bird of Madagascar, possibly allied. Skeletons and eggs holding two gallons found in alluvium.

\section{Order IV. Carinatæ.}

The name refers to the presence of the keel to the sternum, correlated with the powers of flight possessed by most species. Other characters are the presence of rectrices and remiges on tail and wings, and the fusion of clavicles to a furcula. Some strong fliers, like the raptores and albatrosses, have but a small

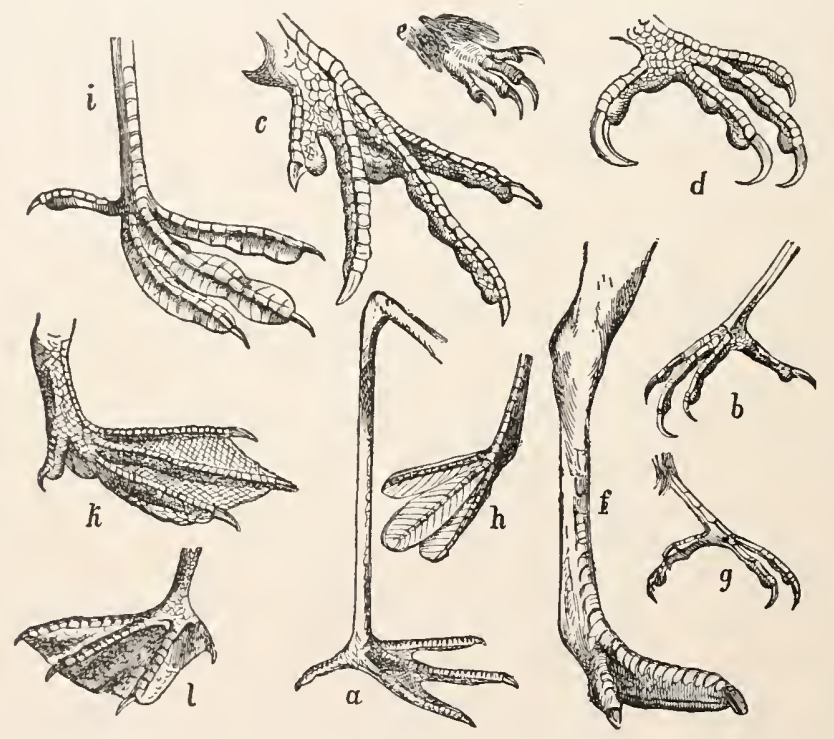

Frc, 594.-Foot forms (from Schmarda). a, semi-palmate, wading of Ciconia; b, perching of Turdus; $c$, rasorial of Phasiamus; $d$, raptorial of Falco; $e$, adherent of ( $y$ pselus; $f$, cursorial of Struthio; $g$, zygodactyl (scansorial) of Picus; h, lobate of Podiceps; $i$, lobate and scalloped of Fulica; $k$, palmate of Anas; $l$, totipalmate of
Phacthon.

carina; in many poor fliers the carina may be entirely absent. The furcula is not always present, the clavicles not uniting (many parrots and toucans) or being absent (Mesites). The remiges are also degenerate in some, as in the penguins (which are flightless, although they have a strong carina), where they 
appear like small scales. Thus the distinctions between ratite and carinate birds vanish in places.

Sub Order I. GALLINACEA. The hen-like birds are praecoces with compact bodies and well-developed wings and legs, so that they run and fly well without excelling in either direction. The feet have three toes in front, usually connected by a membrane at the base (fig. 594. c); the first toe is behind and at a higher level. Above this in the male is usually the spur, a process of the tarso-metatarsus, covered with horn; scratching claws. Naked, richly vascular lobes form comb and wattles which are specially large in the more elegantly plumaged males. I'HASIAxidDE, Phasianus, pheasants; Gallus bankiz'a of the Sunda Islands, ancestor of domestic fowl. Meleagris, ${ }^{*}$ turkeys. TETRAoxID.E, Coturnix,* quail; Perdix,* partridge; Bonasa, * grouse. The incubation of the Megapodes has been referred to (p. 54I).

Sub Order II. COLUMBIN玉. Pigeons are distinguished from Gallinacese by the more slender bodies, shorter legs, free toes, and the longer wings capable of prolongerl flight. They are altrical; the crop produces a milky secretion used in feeding the young. Columba ${ }^{*}$ According to Darwin domestic pigeons come from $C$. livia, the blue rock pigeon. Allied was the dodo, Didus ineptus, of Madagascar, exterminated in the eighteenth century.

Sub Order III. NATATORES. The swimming birds, with webbed feet, are excellent swimmers and divers. Either all four toes are connecterl by the web (fig. $594, l$ ), or only the three anterior toes are webbed $(k)$, or the three toes are each bordered with a swimming membrane $(h)$. Thus the foot structure gives distinctions which forbid a closer association of the families, and this is strengthened by differences of wing and beak. On the other hand palatal structures show that here, as in the Grallatores, very diverse forms are associated.

Section I. LAMEllirostres (Anseriformes), feet palmate; the beak softskinned up to the hard tip, its margins with transverse horny plates. Anas boschas, * wild duck, source of domestic breeds. Anser, $*$ goose (domestic derived from A. ferus). Cygnus, * swans. Section II. Tubinares (Longipennes), predaceous birds with strong beak, tubular nostrils, palmate feet, and long wings capable of rapid and prolonged flicht. Diomedea, albatross; Larus, ${ }^{*}$ gulls; Sterna, * terns. Section III. URINATORES. Birds with small wings, sometimes reduced to flippers, and upright position owing to position of the legs far back.

The Alcide (Alca imponnis, * the great auk.) Antarctic Imperres (Aptenodytes-fig. 595, penguin), ColymBIDE: Urinator, * loons, Colymbus, * grebes. Section IV. Stegaxopodes, totipalmate fect. Pclccanus, * pelicans; Phalarocorax,* cormorants; Phacthen, * tropic birds.

Sub Order IV. GRALLATORES. Wading lirds; legs lengthened, chiefly by elongation of the tarso-metatarsus, the feet semi-palmate (fig. 594, $a$ ), and the feathers only on the upper parts, the lower with horny plates. Correlated is the striking length of neck and beak. These features have appeared in 
groups which are very different in anatomical characters. Section I. CIconIFORxiEs. Beak with a strong horny coat. Ardea, ${ }^{*}$ herons; Ibis; Ciconia, storks; Phanicopterus, * flamingo. Section II. Gruiformes. Beak always with soft skin at the base, often extending to the tip. Grus, * cranes; Rallus, * rails; Otis, bustards, terrestrial. Section III. CharadrIformes. Allied to auks and gulls. Scolopax,* woodcock; Charadrius, * plover.

Sub Order V. SCANSORES. Climbing birds; zygodactyle feet (fig. 59., g), toes 2 and 3 directed forwards, $x$ and 4 backwards. The forms differ much in structure and their association does not rest on blood-relationship. Section I. Cuculifories. The Psittaci, parrots, brightly colored tropical birds with short, compressed, and strongly bent beak and fleshy tongue. But one species (Conurus corolinensis*) in the U. S. Cacatua, Plictolophus, cockatoos; Melopsittacus, F'sittacus, parrots. CUCuLI, bill slightly arched or straight; outer toe usually versatile; Coccygus, * cuckoos. Section II. PICARIE, woodpeckers; long, straight, conical beak and long, protrusible tongue; Picus. * Nearly allied are the toucans (Rhamphastos).

Sub Order VI. PASSERES. By far the richest in species of the groups of birds. They are altrices of moderate size, with slender feathered tarsi and strong, horny beak without cere. Of the three anterior toes the two outer are either united or separated to the base (fig. $594, b$ ), the hind toe is at a level with the rest. In some, noticeable for the powers of song of the males, there are special muscles to the syrinx which are lacking in other birds. These are called Oscines, in contrast to the crying birds, or Clamatores. These groups are further distinguished by a large, freely movable hind toe in the Oscines, while in the Clamatores it is restricted in its motions.

Section I. Oscines. All our song birds belong here: FrINGIlLIDE, finches; Passer domesticus, * English sparrow; Loxia,* crossbills; IcterIDÆ; Icterus, * orioles; Dolichonyx, * bobolink; Alaudide, Alauda, * sky-lark; Sylvicolide, Dendreca, * Helminthophaga, * warblers; TuRDIDE, Turdus, * thrushes; Siala,* bluebirds; Hirundisid.玉, Hirundo, * swallows; Troglodytide, wrens; CORvid $\approx$, Corvus, * crows; Cyanocitta,* jays. The PARADISEID $\approx$, or birds of paradise (fig. I5), with marked sexual dimorphism, are closely related to the crows. Section II. Cualcatores. Here are frequently included a few groups (Cotingide, Tyrannide) best developed in South America and the lyre birds (MENURIDE) or Australia. Earlier other forms were regarded as allied, but now are separated as Cypselomorphæ, or Coraciformes, and united with the owls and Picariæ. Cypseled.e; Chatura,* chimney 'swallow', with adherent feet (fig. 594, c). TROCHILIDE, humming birds, best developed in tropical America; Trochilus.* CAPRIMulgide, night hawks; Antrostomus vociferus, * whippoorwill. Alcedinide, kingfishers, Ceryle** Bucerontide, horn bills, tropical.

Sub Order VII. RAPTORES, birds of prey; strong birds of considerable size; tarso-metatarsus feathered and four strongly clawed toes of the raptatorial type (fig. 594, $d$ ). The beak is strong, the upper half, strongly hooked at the tip, extending over the lower. The two groups probably are not closely related. Section I. Falconiformes. Slender birds with close plumage and extraordinary sight; related structurall $v$ to the herons. CATHARTIDA, buzzards; Cathartes aura, * turkey buzzard. PANDIONID Falconide: Aquila, * Halicius, * eagles; Buteo,* buzzards; Falco, * falcons; Accipiter, * hawks. Section II. STrIges, owls; compact birds with loose, fluffy plumage, large eyes in a circle of feathers; related structurally to the Caprimulgidie. Bubo,* horned owls; Scops, * screech owls; Strix,* gray and brown owls; Speotyto,* burrowing owls. 


\section{Class III. Mammalia.}

The mammals occupy the highest place among the vertebrates, and consequently in the animal kingdom; they also possess a special interest for us, for man, in structure and derelopment, belongs to the group, although separated in intelligence from the most highly organized of the members by a wide gap.

The most striking characteristics of the mammals are furnished by the skin. In fact one may, with Oken, call them hair-animals, since hair is as diagnostic as feathers are for birds. The hairs (fig. 596, $H$ ) are cuticular structures which are seated on papillie of the corium, and are nourished by blood-ressels in these. The lower end, the root of the hair, lies in a pit in the epidermis, the llair follicle, and is surrounded by a

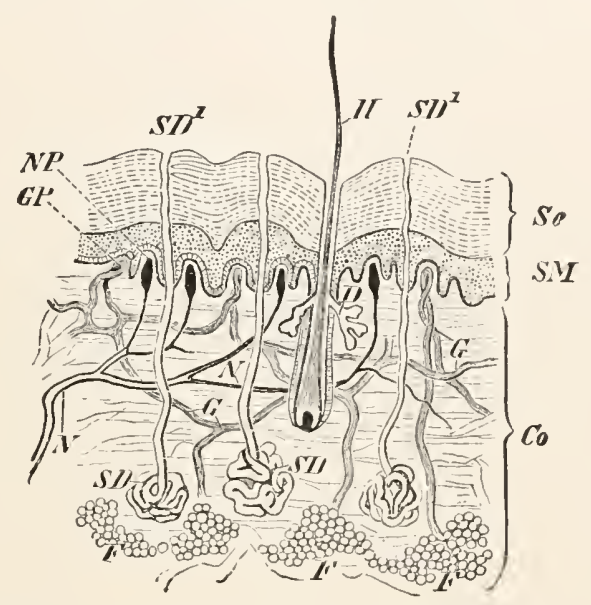

FIG. 596.-Section of skin of man (from Viedersheim). Co, corium (derma); $D$, oil gland; $F$, fat; $G$, blood-vessels; $G P$, vascular papilla; $H$, hair; $N$, nerves; $N^{\top} p$, nerve papilla; $S c$, stratum corneum; $S D, S D^{\prime}$, sweat gland and duct; $S . M$, stratum Malpighii.

double envelope, the epithelial root sheath, formed by an inpushing of the epidermis and an outer connective-tissue follicular sheath. Small muscles attached to the base of the larger hairs serve for their erection. Since side branches are lacking, the structure of the hair is more simple than that of feathers, and the forms fewer. Wool is characterized by its spiral turns; then there is straight hair which, by increase in size, forms the 'whiskers' (vibrissa) on the upper lip of many mammals, bristles (swine), and lastly the spines of hedgehogs and porcupines. In the pelts of many animals two kinds of hair may occur, wool below and straight hair outside. Histologically hair consists of cornified cells, often arranged in medullary 
and cortical layers. On the outside there may be another layer recalling the pseudocuticula of reptiles. In most mammals there is a periodic sherkling and renewal of the hair, the new hair arising from the old follicle (?from the old papilla). Ordinarily this occurs only in spring. Besides hair some mammals have true scales. Constant horny structures are the armatures of the tips of the digits, which, according to form, are divided into claws (ungues), hoofs (ungule), and nails (lamne). See p. $45^{\circ}$.

The old view that the hair, like feathers, corresponds to the scales of reptiles has recently found both defenders and opponents, the latter thinking it probable that the hair has arisen from the neuromasts of aquatic vertebrates, which in the transition to a terrestrial life, became functionless and cornified. Where scales are present, the hair is regularly grouped around them. The same sort of grouping occurs in the scaleless ma'nmals, often only in the embryo, a fact that leads to the view that the scales are an inheritance from the ancestral mammal, which possessed them in addition to the hair, a view that is opposed to any homology between hair and scale.

The skin of mammals is further characterized by its richness in glands, of which there are two kinds, sebaceous and sweat glands. The first are acinous, and usually open in the hair follicles, giving the hair the required oiliness (fig. $596, D$ ). The sweat glands, except in the monotremes, are entirely independent of the hairs, and are simple tubes, coiled at their deeper ends $(S D)$, secreting a fluid, sweat, which is of great value in the preservation of a constant temperature, its evaporation cooling the body. Under the influence of sexuality the glands in certain regions, and especially the sebaceous glands, develop great activity and form considerable glanclular pouches or pockets: caudal and anal glands of many carnivores, hoof and suborbital glands of ruminants, musk and castor glands of musk deer and beaver (fig. 603,a). More important than these are the modifications of dermal giands into mammary or milk glands, which indeed are so characteristic that they have given rise to the name mammalia. In the monotremes the milk glands are very large branched sweat glands; and the same is true for the other mammals, in which they were formerly regarded as modified sebaceous glands, on account of the somewhat vesicular ends. In Ornithorhynchus the glands open on a circumscribed area of the skin, which in Echidna is sharply set off from the surroundings (areola mamme). In the higher mammals this area may be elevated directly to a papilla, the true nipple (most mammals, fig. 597, $A$ ), or the skin around the gland openings may be elevated in a tubular form as in the teats of the cow $(B)$. The mammx are always symmetrically arranged upon the ventral surface, sometimes in the breast region, but more frequently in the inguinal region. There are at least two, usually more $(22$ in Centetes). In general the number corresponds to the maxi- 
mal number of young at a birth. Although the mamme are present in both sexes, they are functional only in the female, and here only after the birth of the young.

A dermal skeleton occurs in few species (e.g., the firm bony plates of the armadillos); on the other hand the axial skeleton shows many features not occurring elsewhere. In the skull many of the bones already referrerl to are eviclent only as centres of ossification, fusing early with their neighbors to form larger bones. As the temporal bone shows, parts of diverse origin may fuse-parts of the visceral skeleton and parts of the cranium; membrane and cartilage bones-so that a sharp line between cranial and
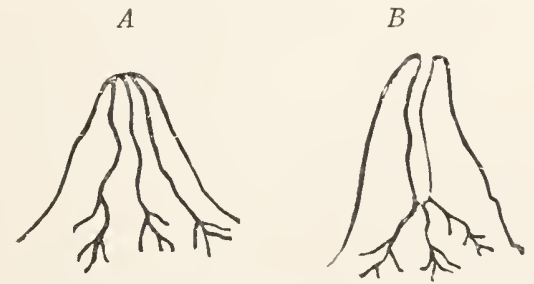

FIG. 597.- $A$, true; $B$, false nipple (after Gegenbaur).

facial portions cannot be drawn. So it becomes necessary in describing the skull not to follow exactly the model adopted so far, but to take that of human anatomy.

In the hinder region of the skull is a large occipital bone (figs. 5 $\boldsymbol{y}^{-}$ $5^{\text {I } 9)}$, jointed to the atlas by double occipital condyles, and arising by the fusion of the four occipitalia, p. 458. Besides it includes usually a membrane bone, the interparietal, which occurs only in mammals. This is, strictly speaking, a paired bone, arising in the angle between the parietals and the supraoccipital and fusing with the latter. In front of it lie, in the roof of the cranium, as in other vertebrates, the parietals (fused with the interparietals in many ruminants and rodents), the frontals and nasals, the lacrimals being always associated with them. In the floor of the cranium the sphenoid bone lies in front of the basioccipital portion of the occipital. In many mammals this consists of an anterior and a posterior portion throughout life; in man this condition occurs at least in the embryo. Each of these parts in development consists of three elements, the posterior with the basisphenoid as the body, and the paired alisphenoids (greater wings); the anterior is similarly composed of the presphenoid and the paired orbitosphenoids (lesser wings) (fig. 518, Spb, Ps, Als, Ors). In front of the sphenoid lies the ethmoid, Eth, likewise formed from three parts, the mesethmoid, which forms a partition between the two 
nasal carities, and the paired ectethmoids, which form part of the lateral walls of the nasal cavities. These last have complicated folds on their inner surface, the superior and middle turbinal bones, which support the olfactory membrane, thus greatly increasing its surface. With these is associated the os turbinale, a distinct bone, the inferior turbinal bone of human anatomy.

The temporal bone, which is intercalated between the roof and floor of the skull, can only be understood by its embryonic relations and its connexion with the first and second visceral arches (fig. 598). Its centre

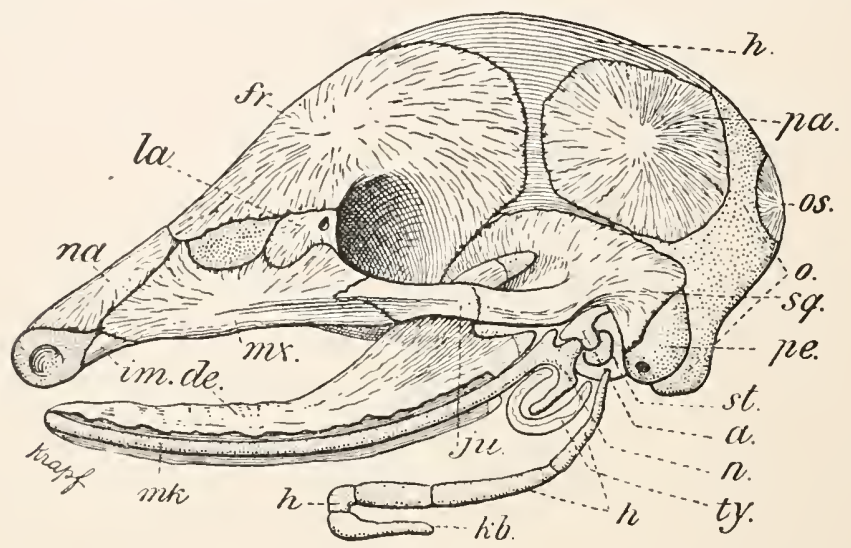

FIG. 598.- Skull of embryo Tatusia (after Parker, from Wiedersheim). Cartilage dotted, membrane and membrane bones lined. $a$, incus (quadrate); de, dentary; fr, frontal; $h$, (above) membrane over anterior fontanelle, (below) hyoid bones; $i m$, premaxillary; $j u$, jugal (malar); $k b$, remnants of gill arch; la, lacrimal; $m k$, Meckel's cartilage; $m x$, maxillary; $n$, malleus (articulare); $n a$, nasal; o, occipital cartilage; os, supraoccipital; $p a$, parietal; pe, petrosal; $s q$, squamosal; st, stapes; $t y$, tympanic.

is formed by the petrosal ( $p e)$, developed in the walls of the otic capsule, to which, as elsewhere in the vertebrates, are attached: (I) the cartilaginous jaw arches, the quadrate $(a)$, and mandibular $(n$ and $m k)$; $(2)$ the cartilaginous hyoid arch, the stapes (in part equalling the hyomandibular, $s t$ ), and the hyoid proper ( $h$ ) (compare with the visceral skeleton of the selachian, fig. 546). To these are added the membrane bones, the squamo$s a l(s q)$, at the base of the quadrate, which increases as the latter loses in size, and below the squamosal the tympanic $(t y)$. With ossification of the cartilaginous parts several centres form the petrosal, which fuses with the squamosal, and frequently with the tympanic, which in some forms enlarges to a conspicuous auditory bulla. Petrosal and squamosal on the one side, tympanic on the other, enclose a space, the tympanic cavity, into which the upper parts of both visceral arches extend, ossify- 
ing into the ear bones, the quadrate to the incus, the hyomandibular possibly to stapes (fig. 534).

The tympanic in uniting with the squamosal encroaches on the mandil)ular cartilage so that the upper end $(n)$, which is homologous with the articulare of other vertebrates, is encloserl in the tympanic cavity anil, along with a second bone, the goniale, ossifies to form the malleus, while the lower portion, Meckel's cartilage proper ( $m k$ ), is pinched off, to form the axis of the lower jaw. Meckel's cartilage gradually disappears; on the other hand the surrounding membrane bone, the dentary $(d e)$ increases and alone forms the lower jaw, which now forms a new articulation with the squamosal. It will be noticed that the old articulation was between cartilage bones, the new between membrane bones dereloping around the cartilages. (There is, however, some evidence to show that the mammalian lower jaw consists of several bones, some of them preformed in cartilage, and that one of these forms the articulation with the squamosal.)

The transformation of the articulare and quadrate of the lower rertebrates into the malleus and incus and the consequent view that the articulation of the jaws is not homologous is strongly opposed by some, who see difficulty in the transfer of the hinge. It must be remembered that the transfer is necessary upon any hypothesis, and loses some of its difficulties, if we suppose a time when both articulations were functional.

The lower part of the hyoid arch, the hyoid, remains outside the ear and often fuses with the petrosal. Its upper end (styloid process) may then become entirely separate from the lower, which becomes attached to the copula (body of hyoid) as the anterior horn, the connecting cartilage being reduced to a stylohyoid liganent. In the hyoid of mammais there is also included a remnant of a gill arch as the posterior horn.

As the quadrate, by its modification into the incus, becomes strikingly reduced, the rest of the arch-romer, palatine, and pterygoid-is poorly developed in contrast to the large maxillary bones. Premaxillaries and maxillaries (fused in man to a single bone on either side) form an important element in the face, and send backwards and inwards palatine processes into the roof of the mouth. These encroach upon the lones of the palatal series; the vomers of the two sides are pressed together to a single bone lying vertically entirely within the nasal partition; the palatine and pterygoid are forcel backwards. The palatines contribute to the hard palate, the pterygoirls only exceptionally (Cetacea, many edentates); the latter usually lose their independence and fuse with the nearest bone of the base of the cranium, the basisphenoid (more accurately with a process of the basisphenoid, the lamina externa of the pterygoid process, 
the pterygoid forming the lamina interna). Thus the hinder sphenoid, like the temporal, contains cranial and visceral elements.

The vertebre are completely ossified and are connected by intervertebral ligaments, discs of fibrous cartilage. The cervical and rib-bearing thoracic vertebre are always distinct, and except in the cetacea, lumbar, sacral and caudal vertebre as well. These regions are little variable in extent. The cervicals, including atlas and axis (epistropheus) number seven except in Bradypus tridactylus (9), Cholopus hofmanni and Manatus (0). In the formation of the sacral region two steps are distinguishable. There is the union of the true sacral vertebre with the os ilium and then the fusion of these and with them certain of the caudal vertebre (pseudosacral vertebre) to form the os sacrum. The number of true sacral vertebre is frequently (Marsupials, ungulates, rodents, lemurs) one, as in

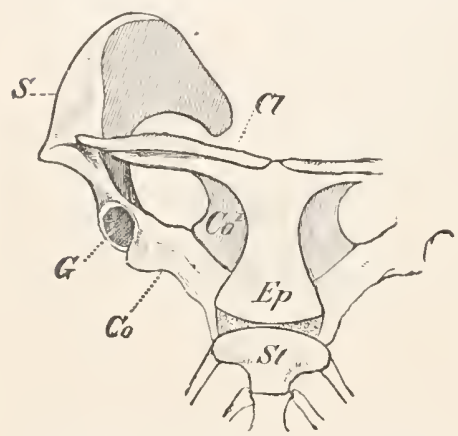

FIG. 599.--Sternum and shoulder girdle of Ornithorhyn hus paradoxus (f rom Wiedersheim). $\mathrm{Cl}$, clavicle; $\mathrm{Co}$, ( ${ }^{\prime}$ ', coracoid; Ep, episternum; $G$, glenoid fossa for humerus; $S$, scapula; $S !$, manubrium sterni (anterior element of sternum).

of interarticular cartilages (once regarded as a rudimentary episternum, now called preclavia). In the pelvis all three elements are fused to a single os innominatum; pubis and ischium unite rentrally with each other, enclosing between them the obturator foramen (fig. 606). The pubes of the two sides unite by a symphysis which can extend back to the ischia.

The great differentiation of the appendages is also highly characteristic of the mammals. In the climbing species the thumb and great toe become opposable, resulting in a grasping hand or foot; but usually they are used to support the body on the ground. Occasionally the whole hand or foot rests on the ground (plantigrade) but usually the basal joints of the feet contribute to the len'th of the leg, the phalanges alone forming the 'sole' (digitigrade) or (unguligralc) the weight being supported on the tips of the toes. 
Since the mammals in general are distinguished from other vertebrates by their intelligence, the brain is characterized by the size of cerebrum and cerebellum (fig. 600). In contrast to birds and fishes, the cerebellum (II) is differentiated into a median vermis and lateral cerebellar hemispheres. In the cerebrum the frontal lobes of the pallium grow forwarls over the clfactory lobes, which consequently lie farther and farther back on the lower surface. The temporal lolses extend right and left over the optic lobes and down to the floor of the cranium; the occipital lobes cover successively the mid brain, cerebellum, and medulla oblongata. Since there is a great range of intelligence in the mammals, the cerebra may be arranged in an ascending series. In the monotremes, marsupials, insectivora, and rodents (fig. $600, A$ ) the olfactory lobes are visible in
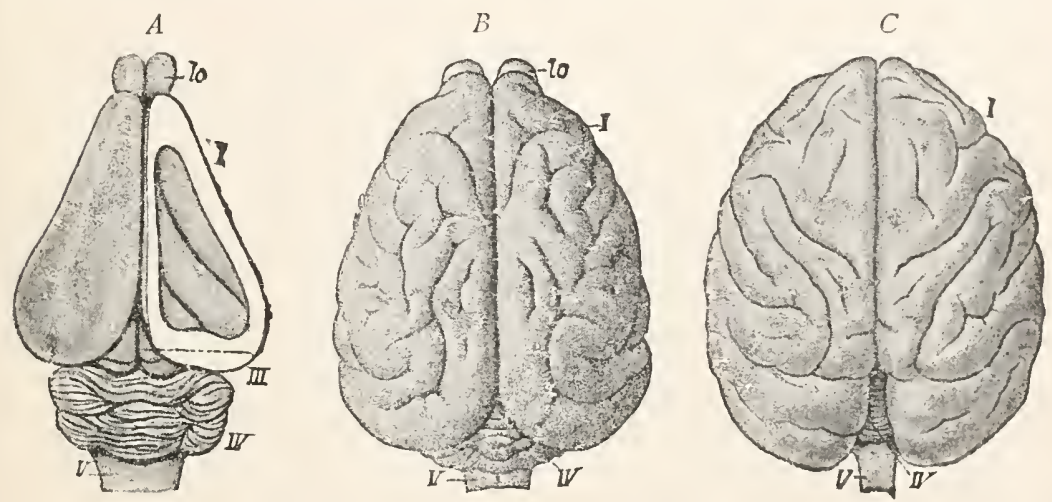

FIG. $600 .-A$, brain of rabbit (after Gegenbaur). $B$, of fish otter. $C$, of pavian monkey (after Leuret and Gratiolet). $I$, cerebrum; $I I I$, optic lobes; $I V$, cerebellum; $V$, medulla oblongata; lo, olfactory lobes.

front, usually the mid brain behind $(I I I)$. In the lemurs, carnivores $(B)$, and ungulates the olfactory lobes are nearly, the cerebellum partly, covered. In man and the anthropoid apes (C), on removing the roof of the skull, only the two cerebral hemispheres are visible.

Further, it is to be noted that in the first group the surface of the cerebrum is smooth, while in the others the cortex is increased by infolding and the formation of convolutions (gyri and sulci) which reach their greatest complication in the anthropoid apes and in man. A consequence of the increase in size of the brain is the great development of the connecting nerve tracts, which become more and more prominent as parts of the brain. Thus the two halves of the cerebrum are connected by two transverse tracts, the corpus callosum and the fornix; two solid cords, the crura rerebri, run back from the cerebrum to the other parts, while a 
transverse commissure, the pons I'arolii, passes below, connecting the two sides of the cerebellum. These connexions in the other vertebrates are small, and even in the lower mammals, like monotremes and marsupials, are but slightly developed.

The increase of cerebrum and cerebellum, which occurs chiefly in the dorsal portion, has resulted in flexures in the axis of the brain, already indicated in the reptiles, increased in the birds, and reaching their maximum in the mammals. Instead of continuing in the direction of the spinal cord, the axis of the brain bends ventrally in the medullary region (cervical flexure), then in the region of the pons again dorsally (pontal flexure), and at the level of the optic lobes again ventrally (cephalic flexure). By its increase in size the brain has influenced the skull in an interesting way; for, while even in birds the brain is almost entirely confined to the region behind the eyes, in the higher mammals it has extended forward to the olfactory region. Thus there comes an increase of the cranium at the expense of the face.

Of the sense organs the nose is characterized by three features. An outer nose, supported by cartilage and often extended as a proboscis, has been formed. Its cavity has been increased, since by the formation of hard and soft palate a part of the primitive mouth cavity has been included in it. Its upper portion, the olfactory region, has been complicated by the formation of olfactory folds, supported by the turbinal bones already referred to (p. 548). The eye has the upper and lower lids, besides the nictitating membrane in a more or less reduced condition. The ear, except in monotremes, cetacea, sirenia, and some seals, has a conch supported by cartilage, while the external auditory meatus is always present. Internally the ear is much modified, since the three bones, malleus, incus, and stapes (p. +79), occur nowhere else, while the lagena has been greatly lengthened, coiled into a spiral with two to four turns (figs. 83, 533), inside of which is the wonderful organ of Corti.

Of digestive structures, the teeth, restricted to maxillary, premaxillary, and dentary bones, need special mention, because of the distinctions they afford from all other vertebrates, and because of their importance in differentiating the various orders. If we omit the monotremes, edentates, and whales, in which there is marked degeneration in the dentition, there are four particulars which show the dentition of mammals more developed than that of other vertebrates. (1) The number of teeth is constant for the species, usually for the genus, and often for the family. As man normally has thirty-two teeth, so the dog has forty-two, the anthropoid apes thirty-two, the platyrhine apes thirty-six, etc. (2) The teeth are more firmly fixed. The body of dentine is divided, by a slight constriction, into a crown covered with enamel, and a root enveloped in cement (bony tissue). The roots are placed in separate sockets (alveoli) 
in the jaws, and in those cases where continuous growth is necessary the pulp persists and the teeth (incisors of rodents, tusks of elephants and pigs) grow indefinitely. (3) In consequence of their greater hardness the teeth are not used up so fast and lo not require rapid replacement. There occurs at most only one change, in which the dentition present at birth or developed soon after-the milk, or lacteal, dentition or, better, first dentition - is replaced by the second or permanent dentition (diphyodont mammals). In some cases (monophyodont mammals) there is no change, the first dentition being permanently retained (marsupials, perhaps toothed whales), or the first dentition is more or less rudimentary (edentates, many rodents, bats, seals, some insectivores). Besides the two typical dentitions traces of a third or even a fourth may occur. A prelacteal dentition of calcified germs which are never functional is best seen in marsupials, and is rare in placental mammals. A post-permanent dentition following the permanent one is outlined in many placentalia, and some of its teeth may exceptionally come into function. (4) Among the teeth a division of labor has brought about change of form (heterodont dentition). The teeth of the premaxillaries and their antagonists in the lower jaw are singlerooted and usually have more or less a chisel shape, hence they are called incisors even when, as in insectivores, the crowns are needle-like and there are two roots (some insectivores, marsupials, lemurs) (fig. 6r2). Behind the incisors (in the maxillary bone in the upper jaw) is the canine tooth (fig. 60I, c), which is singlerooted and has usually a conical crown (probably a modified premolar). Following the canine come the molars, broad teeth mostly with two roots and tubercular crowns. Only the anterior ones appear in the milk dentition, while the others appear only in the permanent dentition and are not replaced. On this developmental basis the molars are divided into premolars (bicuspids of dentists), which appear in both dentitions, and the true molars, which occur only in the last.

As stated above, every species of mammal has a characteristic dentition; its features may be expressed by a short formula. It is only necessary to place the number of each of the four kinds of teeth mentioned in their regular order, those of the upper jaw separated from those of the lower by a horizontal line, to express this. Since the two sides of the body 
are symmetrical, only those of one side need be enumerated, and in case that one kind be absent the deficiency is indicated by a zero. Thus the dental formula of man is $\frac{2}{2} 1 \frac{2}{2} 3$; of the reindeer, in which in the upper jaw incisors and canines are absent, $\frac{0}{3} 1 \frac{3}{3}$. The different formula, by comparison, give us a fundamental formula from which they have been derived by reduction. This was probably 4144.

The molars undergo, according to the food, the greatest modification of form. Is a starting point the bunodont tooth of ommivorous mammals may-be taken, which has the crown with several blunt projections or cones. With animal food (figs. 60I, 608) the cones are sharper and cutting (secodont dentition of carnivores and insectivores), and when the cutting angle becomes very sharp, with a special prominence on the inner side, it is spoken of as a flesh or carnasial tooth. In vegetable feeders the cones become connected by crests (lophs) or are half-moon-shaped (lophodont or sclenodont). Since the cones and lophs become in part worn away and the grooves between them are filled with cement, there arise broad grinding surfaces strengthened by the harder and more resistant enamel of the cones and lophs; this extends inwards as folds from the outer enamel wall of the tooth; the folds may become cut off and form islands of enamel on the grinding surface (dentes comlicati of ungulates). When the folds extend in regular order from the outside and inside and meet in the middle they form numerous successive plates bound together by cement (compound teeth of elephants, fig. 6 r8, and many rodents).

Paleontological investigation, with which the more recent embryological results are in accord, has shown that a great regularity prevails in the formation of the cones of the molars. Triconodont and tritubercular teeth are recognized, in which the three cones are either arranged in a line or in a triangle, as well as multitubercular teeth with more numerous cones irregularly arranged. The triconodont type develops farther by the formation of secondary cones. The development of these occurs in different ways in molars and premolars. Since the latter are the more simple, their distinction from the molars does not rest alone upon the existence of a milk dentition, but upon structure as well. This is important, because it happens that there are premolars which are not replaced (marsupials, many insectivores and rodents) and, on the other hand, beneath the molars the anlagen of replacing teeth may be found. The latter fact shows that the molars, strictly speaking, belong not to the permanent but to the milk dentition. They are late in formation and are therefore parts of the first dentition carried over into the second.

The lips, bounding the opening of the mouth, differ from those of all other vertebrates by their soft character and the mobility caused by special muscles. 'The mouth carity, which contains tongue and teeth, is separated from the next division of the alimentary tract, the pharynx, by the urula. The pharynx narrows behind into the sesophagus, the entrance of which into the stomach is marked by a constricting cardia. At its other end the stomach has a similar constrictor, the pylorus, separating it from the intestine. In the latter small and large intestines (the latter consisting of colon and rectum) are differentiated by the diameter of the lumen. The small intestine opens laterally into the colon anct at the junction arises a blind diverticulum, the cacum, which is small in mammals with animal food, but in herbivores (especially rodents) is always large and forms a conspicuous part of the alimentary tract. The vermiform appendix (primates, rodents) is a narrower part of the cacum with numerous lymph follicles. Three pairs of salivary glands empty into the mouth, the liver and pancreas into the small intestine (duodemum). 
Most important of respiratory peculiarities is the diaphragm, which separates the body cavity into thoracic and abdominal carities. This occurs only in its beginnings in other vertebrates (perhaps even in Amphibia). In the thoracic cavity are the osophagus, heart with its pericardium, and especially the trachea, bronchi, and lungs; the remaining vegetative organs are in the abdominal cavity. The diaphragm is a muscular dome, its convex side towards the thoracic cavity; by contraction it flattens and increases the size of the cavity, in consequence of which air is drawn into the lungs (inspiration). On relaxation the lungs contract from their own elasticity and force out a part of the air (expiration). The intercostal muscles, which raise and lower the framework of the chest, also play a part, as in birds. The respiratory ducts begin with the larynx (with rocal cords), supported by the thyreoid cartilage occurring only in mammals, which can be closed from the pharynx by the epiglottis; this is followed by the trachea, which divides into right and left bronchi. Each bronchus divides again and again, and the finest of these divisions, the bronchioles, are continued as alveolar ducts to small chambers, the infundibula, both these and the alveolar ducts being lined with small respiratory pockets, the alreoli. (See p. 482 .)

The heart, with two auricles and two ventricles, is completely separated into systemic and pulmonary halves. In early embryonic life the arterial trunk, which at first is simple, is divided into a pulmonary artery, arising from the right half of the heart and carrying venous blood, and an aorta ascendens, with arterial blood, connected with the left half. In contrast with reptiles and birds, the right aortic arch is lost, the left persisting.

The urogenital system is of great importance in the separation of the group into smaller divisions. In both sexes this consists of practically the same parts in early embryonic life (fig. 602). 'These are the early formed Wolffian body $\left(\mathrm{II}^{*}\right)$; the permanent kidneys, which appear later and are not shown in the diagram; the urinary bladder (4), a part of the allantois which extends (5) into the fotal appendages; the three ducts, the Muillerian $(m)$, the Wolffian $(w)$, and the ureter (3). These ducts no longer empty into the intestine, but in to the allantoic structures, the ureters, except in monotremes, into the base of the urinary bladkler, the Wolffian and Muillerian ducts into the urogenital sinus $(u g)$, the lower continuation of the bladder. In the anterior wall of the urogenital sinus is a mass of highly vascular tissue $(c p)$, from which and a surrounding fold the external genitalia are developed. Since the urogenital sinus opens from in front into the intestine, there is always a cloaca $(c l)$ in the embryonic stages. The entodermal section of the cloaca is separated in all mammals into rectum and urogenital sinus. In the monotremes there is added a deep 
ectodermal cloacal pit, which is less distinct in the female marsupials, many insectivores, rodents and edentates. In all other mammals anus and urogenital openings are separated by the formation of a partition, the perineum.

From this indifferent condition the male apparatus is derived, the structures being closely similar in most species (fig. 603). The Müllerian

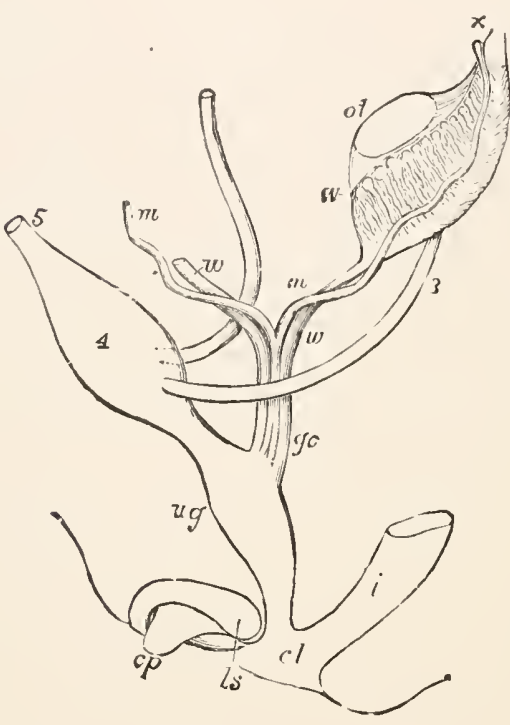

FIG. 602.

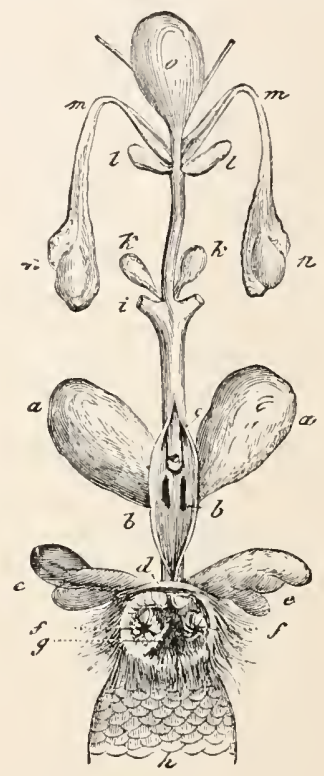

FIG. 603 .

FIG. 602.-Diagram of embryonic nammalian urogenital system (from Balfour, after 'Thompson). $c l$, cloaca; $c p$, genital process; go, genital cord; $i$, rectum; $l s$, ridge for formation of labia or scrotum; $m$, Müllerian duct; ot, gonad; $u g$, urogenital sinus; $\mathrm{I}^{r}$, Wolffian body; $w$, Wolffian duct; 3 , ureter; 4, urinary bladder; 5, continuation of latter to allantois (urachus).

FIG. 603:- - Lrogenital system of male beaver (from Blanchard). a, castoreum sacs; $b$, openings of their ducts into preputial canal; $c$, tip of penis; $d$, preputial opening; $e$, anal glands; $f$, their ducts; $g$, anus; $h$, base of tail; $i$, corpora cavernosa; $k$, Cowper's glands; $l$, seminal resicles; $m$, vasa deferentia; $n$, testes; $o$, urinary bladder with ureters.

duct vanishes, while the Wolffian duct becomes the vas deferens and its accessories, serving as a canal for the genital products, while the external genitals arise from the other parts mentioned, these forming an intromittent organ (penis). In the female the Wolffian body and duct degenerate, the Müllerian ducts become the reproductive canals. The modifications of these become of great systematic importance. In the monotremes both ducts open separately into the urogenital sinus and become differentiated into two parts (fig. 60.4, A), anterior oviducts (od) with wide 
openings into the body cavity $(t)$ and the uterus $(u)$. The ureters open into the sinus between the uterine openings. In the marsupials ( $B$ and (') there are three divisions, oviduct, uterus, and vagina; besiles, the two Müllerian ducts may approach, near the uterus $(B)$, or fuse in this region $(C)$ in some species, forming an unpaired blind sac $(v b)$, which may even

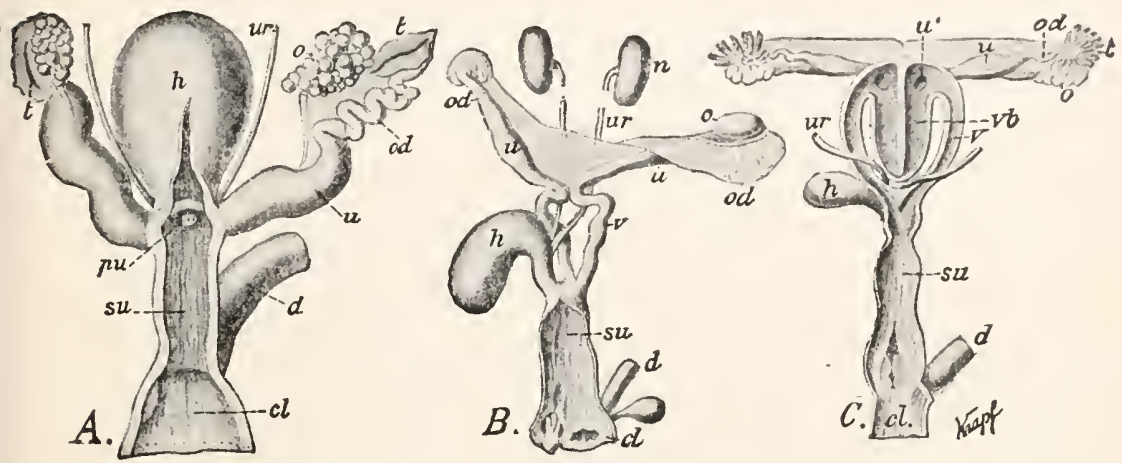

FIG. 604.-Female genitalia of (A) Echidna aculeata; $(B)$ of Didelphys dorsigera; $(C)$ Phascolomys wombat ( $B$ and $C$, after Wiedersheim). $c l$, cloaca; $d$, rectum; $h$, urinary bladder; $n$, kidney; $o$, ovary; od, oviduct; $p u$, mouth of ureters; su, urogenital sinus; $t$, ostium abdominale tube; $u$, uterus; $u^{\prime}$, opening into vagina; $u r$, ureter; $\tau$, vagina; $v b$, vaginal blind sac.

open into the urogenital sinus as a third vagina. This partial fusion of the vaginæ of the marsupials is completed in the placental mammals, the single vagina and the sinus forming a single canal (fig. 605). Here the uterine portions may remain distinct (uterus duplex or rodents, $A$ ), or they may fuse partially (uterus bicornis of insectivores, whales, ungulates, and
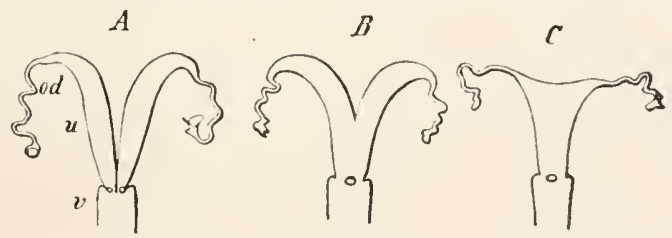

FIG. 605.-A, uterus duplex; $B$, uterus bicornis; $C$, uterus simplex (from Gegenbaur). od, oviduct; $u$, uterus; $z$, vagina.

carnivores, B), or they may be completely fused (uterus simplex of apes and man, $C$ ).

Thus there are three different types of the female genitalia, in which the vagina is not differentiated (Ornithodelphia), or is double (Marsupialia), or is single and unpaired (Monodelphia). To these correspond three types of development. The Ornithodelphia are oviparous, the 
others viviparous, lut are distinguished by the duration of pregnancy. The eggs of the viviparous species are so small (about .o inch) that they have a total, nearly equal segmentation. Such esgs require nourishment from the mother in order to produce an animal with the complicated structure of a mammal. Since in the Didelphia the uterine nourishment is usually very incomplete, the period of pregnancy is very short, in comparison with the Monodelphia, in which a placenta, a complicated apparatus for the nourishment of the young, appears; hence the marsupials, with their small imperfectly formed young, are ofen called Aplacentalia; the Monodelphia, Placentalia.

All mammals care for the young, this being chiefly or wholly done by the mother, who not only supplies them with milk but protects them in nests. Most mammals are monogamous, some polygamous, while in others there is no permanent association of the sexes. The body temperature is constant and ranges from $36^{\circ}$ to $41^{\circ} \mathrm{C}$. $\left(98^{\circ}\right.$ to $106^{\circ} \mathrm{F}$ ) ; in Echidna it is only $26^{\circ}$ to $34^{\circ} \mathrm{C}$. $\left(79^{\circ}\right.$ to $83^{\circ} \mathrm{F}$.). In most, continual feeding is necessary for existence; from this rule there are a few exceptions, like the bears, marmots, badgers, etc., which hibernate during the winter, taking no food. At this time there is a fall in the temperature (in the marmots nearly to freezing) due to the diminished metabolism.

\section{Sub Class I. Monotremata (Ornithodelphia, Prototheria).}

A few mammals, confined to Australia and New Guinea, are the only living representatives of the group. They are distinguished from all

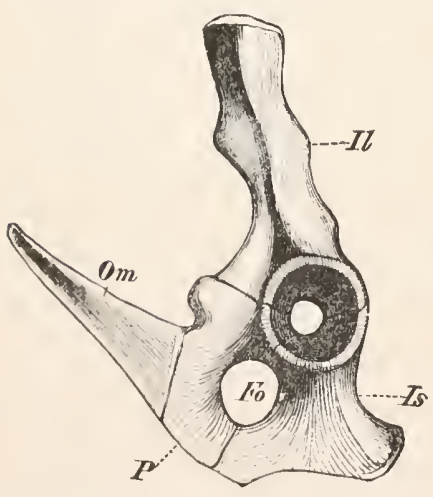

Fig. 605.-Pelvis (left side) of Ornithorhynchus paradoxus (from Wiedersheim). Fo, obturator foramen; Il, ilium; $I s$, ischium; $O m$, marsupial bone; $P$, os pubis. other mammals by laying eggs about half an inch long, rich in yolk and with soft shells. These undergo in the uterus a discoidal (meroblastic) segmentation and are then incubated by Ornithorhynchus in a nest, by Echidna in a temporary pouch (marsupium) on the ventral surface of the body. On hatching the young are nourished by the secretion of enormously enlarged sweat glands, which form two large masses to the right and left of the mid-ventral surface. Each opens on a special region of the ventral surface, which is slit-like in Ornithorhynchus, a flattened pocket in the others.

Other distinctions from other mammals, which are also points of resemblance to reptiles and birds, are the strong development of the episternum and the extension of the coracoid to the sternum (fig. 599), the 
termination of the ureters in the urogenital sinus (fig. 60.4), the existence of a cloaca in both sexes, and the specifically bird-like character of the female sexual organs, in which the large left ovary is alone functional, and uterus and vagina are not differentiated. But with all this it must not be forgotten that the monotremes have the hair, the skull (ear bones, articulation of jaw), the urogenital sinus of true mammals; and in the presence of marsupial bones connected with the pelwis (fig. $606, \mathrm{~m}$ ) show a close relationship with the marsupials. The jaws are toothless and enclosed in horny sheaths, yet in the young of Ornithorhynchus there are in each jaw three pairs of multitubercular molars, which are later replaced by four broad horny plates. In the embryo Echidna tooth germs are formed and also a dentine egg-tooth for opening the shell.

ECHIDNIDE. The spiny ant-eaters have the body covered with spines, snout with a worm-shaped tongue used in catching insects; Echidna, Australia, feet five-toed, with digging claws; Proechidna, New Guinea, three-toed. ORNithORHYNCHIDE. The duckbills are toothless, close-haired animals with horny jaws which resemble those of a duck; the five-toed feet with a swimming web

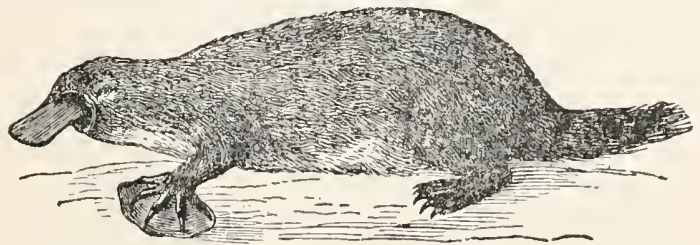

FIG. 6o7.-Ornithorhynchus paradoxus, duckbill (from Schmarda).

especially well developed on the fore feet. Ornithorhynchus paradoxus of Australia; male with a spine and gland on hind feet which fits in a corresponding pit on the thigh of the female and apparently plays a rôle in copulation.

The oldest known fossil mammals are possibly related to the monotremes. They appear in the trias and form an imperfectly known group, MULTITUBERCULATA (Tritylodon, Microlestes, Plagianlax). Their multitubercular teeth resemble the temporary ones of Ornithorhynchus, while there are indications that the coracoid existed as a distinct bone. Less certain are the Protononta (Dromatherium, Microconodon) of the American Jurassic, of which only the lower jaws are known.

\section{Sub Class II. Marsupialia (Didelphia).}

These, like the remaining mammals, are viviparous. They have small eggs which undergo a total segmentation in most species, and develop in the maternal uterus, being nourished by a secretion from its walls. Only in Perameles is there a placenta in which the allantois is so intimately connected with the uterine wall that an exchange of fluids between the two 
is possible (to a less extent in Phascolarctos and Halmaturus). In Dasyurus there is a similar connection of the yolk sac. Yet the ressels do not extend with the villi into the uterine tissues as is the case with the placenta of all Placentalia. In all there is insufficient nourishment and the young are very immature when loorn. They are therefore carried a long time by the mother in the marsupium, a pouch formed by a fold of skin on the posterior ventral surface, into which the nipples open. The ventral surface is supported by the marsupial bones, slender rods articulated, right and left, at the pubic symphysis. Other characteristics of the marsupial skeleton are the inflected posterior angle of the lower jaw (fig. 6o8, $a$ ) and the rudimentary replacement of teeth, only premolar 3 being replaced. In front of the functional teeth is a row of dental anlagen, which never develop. These are usually regarded as indicating a prelacteal dentition, and the functional dentition as the milk dentition; but they may be the milk dentition and the functioning teeth the permanent dentition. The cloacal and sexual apparatus has already been described (p. 557).

Marsupials occur in the Jurassic and tertiary of Europe and America. They were apparently then spread over the earth, but were crowded out by the placental mammals and persisted onl $v$ as remnants (Canolestes and the opossums) in America, but as a richly developed fauna in Australia; yet no fossils are known

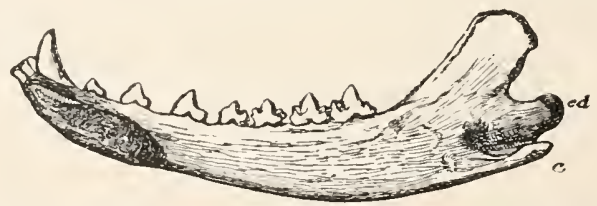

FIG. 608-Lower jaw of Thylacinus cynocephalus (from Flower), showing (a) the inflected angle characteristic of marsupials; $c d$, articular surface.

there earlier than the pleistocene. In the latter region they continued because, on account of the early separation of this continent from the rest of the world, no development of Placentalia occurred (p. I62). In Australia, in adaptation to similar conditions they have undergone a development analogous to that of the placentals in other parts of the earth, so that they present groups parallel with the carnivores, rodents, insectivores, and ungulates.

\section{Order I. Polyprotodonta (Zoophaga).}

Nany marsupials, among them the oldest, have a dentition adapted to animal food. 'They have numerous incisors (up to five in each upper half-jaw), strong canines, and sharp-pointed molars (fig. 608). Some, in teeth and in borly form, resemble the Insectivora, others the carnivores. DAsyuride, Carnivorous: Dasyurus; Sarcophilus, the Tasmanian 'devil,' dangerous to larger mitmmals; Thylacinus, pouched wolf. Peranelide, insectivorous; Perameles, bandicoot. Didflphyid, opossums, confined to America (chiefly South) are more carnirorous in dentition and recall the apes with their opposable thumb. Didelphys.* 


\section{Order II. Diprotodonta (Phytophaga).}

The herbivorous habits are correlated with the degeneration of canines, usually lacking in the lower jaw and at least very small in the upper. There are two large incisors in the lower jaw, while the middle two of the upper are much larger than the one or two lateral which may be present. The fact that young phalangers and kangaroos are polyprotodont makes it probable that the Diprotodonts are descended from the Polyprotodontia. The Phascolomide are the rodents of the marsupials with one chisel-like incisor in each half of each jaw. Phascalomys, wombat. The MACropodide, or kangaroos, resemble ungulates in their large herds on the grassy places. The fore legs being very small, the animals leap with the strong hind legs and tail. Macropus. The Phalangistide have very variable teeth. They resemble in habits the squirrels, Petalirus having the same parachute folds as does our flying squirrel. Many fossil Diprotodonta in Australia, a few in South America. Some Australian fossils were large, Diprotodon australis larger than a rhinoceros.

\section{Sub Class III. Placentalia (.Monodelphia).}

The first reason for associating the mammals of the Old World and most of those of the New together as Placentalia is an embryological one, the presence of a placenta. When serosa, amnion, and allantois (p. 490)

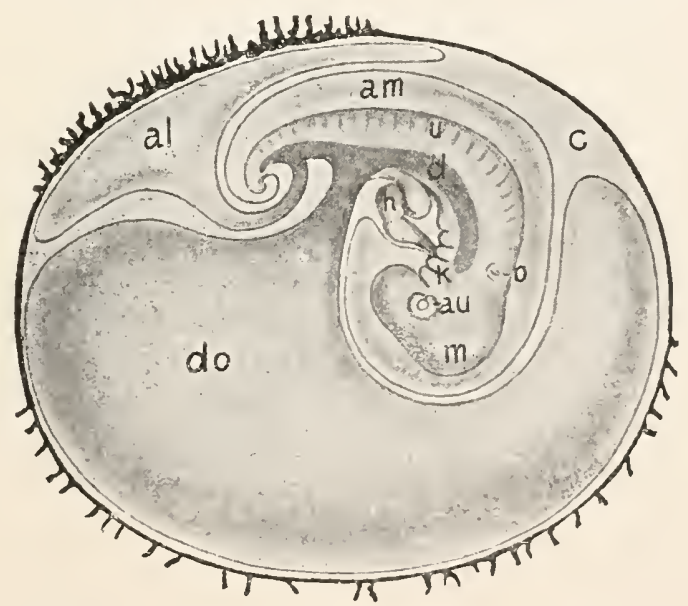

FIG. 609.-Rabbit embryo with envelopes (after Van Beneden and Julin). al, allantois; $a m$, amnion; $a u$, eye; $d$, digestive tract; $h$, heart: $k$, gill clefts: $m$, mid brain; u, protovertebra; external black, chorion with villi from which the placenta develops.

have developed in the embryo, the ressels of the allantois spread out beneath the serosa and form with this the chorion, which sends small processes or villi into the now highly vascular mucous membrane of the uterus in order to obtain nourishment somewhat as a tree obtains food by its roots. These villi (sometimes poorly developed, as in the pig) may be distributed over the greater part of the surface (fig. 609), producing the 
chorion frondosum, or diffuse placenta, which occurs in whales, perissodactyles and many artiodactyles. On the other hand, the villi may be restricted to certain places, becoming very strong there. This gives rise to cotyledonary, discoidal, or zonary placente. To these correspond portions of the uterine lining which are distinguished from the rest by becoming extremely vascular (uterine placenta). The cotyledonary placenta (fig. 6ro) consists of many small placentar patches, the cotyledons (most

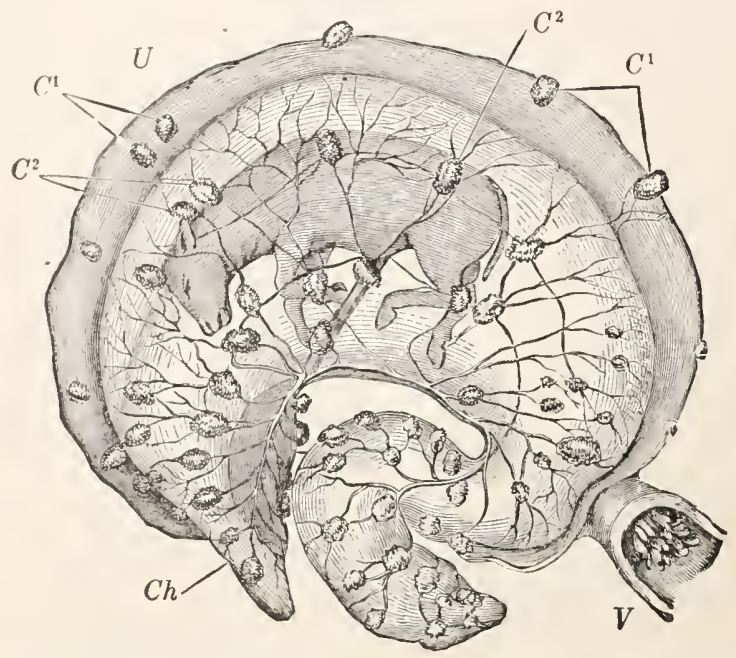

FIG. 6ro.-Cotyledonary placenta and embryo of cow (from Balfour, after Colin). $C^{1}$, cotyledons of uterine; $C^{2}$, of fotal placenta; $C h$, chorion; $U$, uterus; $V$, vagina.

ruminants). In the zonary placenta the villous area takes the shape of a girdle or barrel (carnivores, Sirenia), while the discoidal (other mammals) is, as its name indicates, disc-like. By the restriction of the nutrient structures to certain regions of the placenta, the corresponding part of the uterus (the zonary or discoid uterine placenta) is more modified than is the case with the diffuse or the cotyledonary type. While in the latter the placental villi are withdrawn from the uterine walls without injury to them (Indeciduata), in the case of the zonary or discoidal placenta the superficial part of the mucous lining of the uterus, the decidua, separates (I)eciduata), leaving the uterine placenta as a bleeding wound, the hemorrhage being stopped by the energetic muscular contractions of the uterus.

Besides the placental structures the higher mammals are characterized by the disappearance of the cloaca, the unpaired vagina, and absence of 
marsupial bones and inflected angle of the jaw. The dentition, on the other hand, has undergone a progressive, divergent development, so that the distinctions are much more pronounced than in the marsupials, and hence of importance in differentiating the orclers.

\section{Order I. Edentata.}

A few families, poor in species, are united as Edentata because teeth are absent or, more usually are markedly degenerate. Persistent functional incisors are lacking, canines but rarely occur (Bradypus); molars may be present, sometimes in great numbers (Priodon gigas, the large armadillo, has about a hundred molars), but they are poorly rooted, prismatic, without enamel, and usually monophyodont. Since the aardvark (Orycteropus) and Tatusia have a heterodont milk dentition in embryonic life in which incisors occur, and fossil edentates (Entelops) with complete dentition are known, the absence of a replacement of the teeth is to be explained by degeneration. The great number of sacral vertebre is striking, being as many as thirteen in some armarlillos. The order is essentially tropical, but one species entering the United States. The oldest fossils occur in Patagonia (eocene or oligocene).

Sub Order I. NOMARTHRA. Old World. FOIIENTIA. Animals with strong digging claws, long tail, and long, sticky tongue used in catching insects. Orycteropus, aardvark, long snout, sparse bristly hair. SQUAMITA. Toothless, body covered with overlapping scales. Manis, pangolins of Asia and

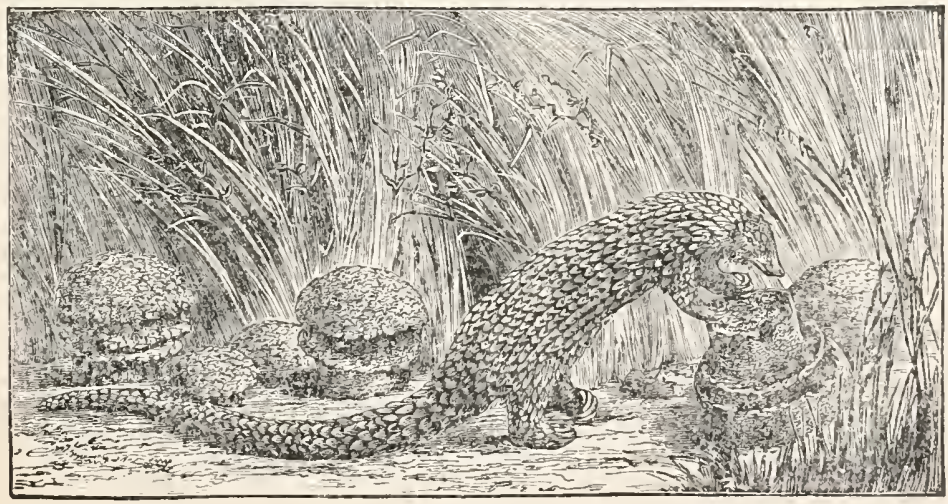

FIG. 6I1.-Manis longicaudutu, pangolin (from Monteiro).

Africa (fig. 6II). Sub Order II. XENARTHRA. New World. VERMILINGUI.1, ant eaters. Resemble manids in toothless jaws, tongues, and claws, but are hairy and lack scales. Myrmecophaga. TARDIGRADA, sloths. Hairy, rudimentary tail, and few teeth, long strong claws by which they hang back downwards from limbs of trees. Bradypus, nine cervical vertebre; Clolupus, six cervicals. Fossils allied are Megatherium, as large as an elephant, $M$ ylodon, Megalonyx, extending to Pennsvlvania. Grypotherium, as large as a cow, has not long been extinct. LORICATA, armadillos. Body with armor of bony plates, insectivorous. In the extinct Gurptonontide of South America the plates fused to a continuous armor. One species twelve feet long. D.ASYPODIDE; dermal armor in movable transverse plates; nocturnal. Genera based upon the number of bands; Dasypus; Tatusia novemcincta*. 


\section{Order II. Insectivora.}

Of all living mammals the insectivores stand nearest to the primitive type, the order clating back to the eocene. The sharp-pointed teeth (sectorial), arlapted for insect food vary greatly in number in the different families (moles,

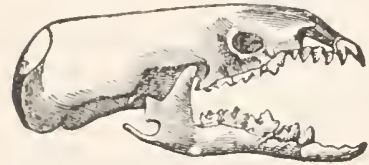

I16. 612.- Skull of Sorex (from Ludwig-Leunis). $3 \frac{1}{1}+3$; shrews, $\frac{2113}{2} 13$ ), as also does the replacement, since many forms retain the milk dentition for a long time or never lose it, while the shrews lose it very early. They have the process at the angle of the lower jaw, a primitive brain, a uterus bicornis or one divided throughout. There are frequently scales on the tail among the hairs; the claricle is present; usually fire clawed toes. The animals which as a rule are small, with soft hair, usually have a proboscis-like, tactile snout.

The ERINACIDE, Old World hedgehogs, are spined; the SORICID., or shrews $\left(\right.$ Sorex; $*$ Blarina ${ }^{*}$ ), are mouse-like, as are the allicd TALPID

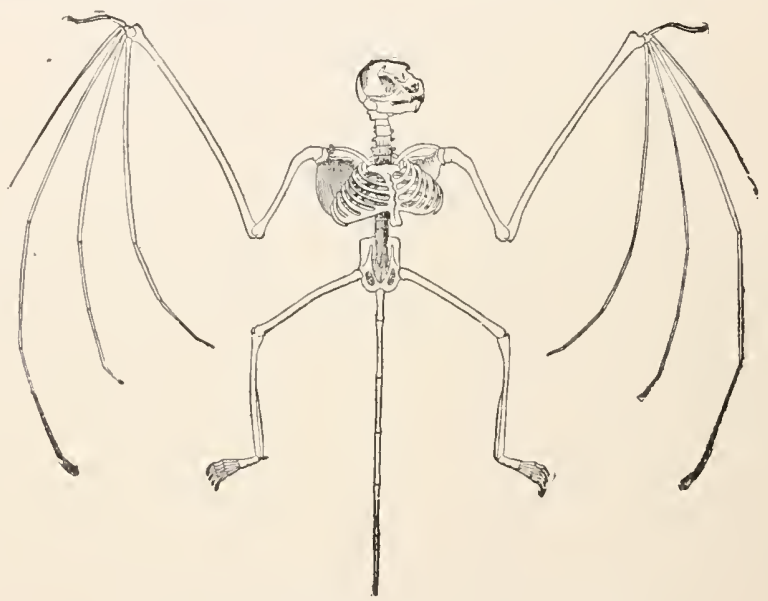

FIG. 613.- Skeleton of bat (after Brehm).

Condylura,* star-nosed mole), which burrow in the earth and have the eyes more or less ruclimentary. Some authors place here Galcopithecus of the East Indies, which has a similar membrane and sailing powers as the flying squirrels. It also resembles the bats and lemurs.

\section{Order III. Chiroptera.}

The bats are the only mammals which actually fly, and this at once characterizes them. The flying membrane (patagium), a thin fold of skin, begins at the tail, includes the lower extremities to the foot, and extends thence to the fingers, leaving the thumb free. Fingers $2-5$ are enormously clongated and support the membrane. Since flight requires strong muscles, the sternum develops a small keel for the attachment of the large pectoral muscle. In connexion with the flying powers the clavicle is strong. The patagium is rery sensitive; blinded bats can fly among all kinds of obstacles without disturbing them. The enormous ear conchs and a noticeable nose leaf, widely distributed through the group, also have marked tactile powers. In the pectoral position 
of the mammary glands and in the diseoidal placenta these animals resemble the primates. The dentition is variable, often $3213 \frac{3}{3}$. Fossils occur in the eocene.

Sub Order I. MICROCHIROP'ERA, insectivorous dentition, onty the thumb clawed. Vespertilionide, tail long, no nose leaf; Vesperugo, *Atalapha.* Phyllostomide, with nose leaf, tropical America; Desmorius, bloodsucking or vampyre bat. Sub Order 1I. MACIIROCIIIROP'TER.1 (Frugivora), smeoth-crowned molars, claws on thumb and first two fingers. Includes the flying foxes, Pteropus, of the East Inchies.

\section{Order IV. Carnivora.}

The carnivores live chictiy on the flesh and blood of other vertebrates, which they catch by craft, by coursing, or by pouncing upon them. With this mode of life correspond the high development of the brain (fig. $600, B$ ) and sense organs, as well as structure of teeth and claws. Especially striking is the shining of the eyes, especially in the cats (p. r.2I). Since the predaceous character increases from the bears to the cats, and again tends to disappear in the aquatic species, there is a great variation in structure. For greater mobility the clavicle is reduced or lost, ulna and radius well developed. In the structure of the feet there is a gradual transition from the plantigrade bears, in which the whole sole rests upon the ground, to the digitigrade cats, which tread on the toes. In the latter the claws, which occur in all carnivores, are kept from injury by being retracted into pockets on the penult joint, from which they are extended by strong muscles. In dentition (fig. 6or) the striking features are the almost constant three incisors, and the great size of the canines; the molars, on the other hand, vary with the different families, the cusps assuming more of the shearing character (secodont teeth). The last premolar of the upjer jaw and the first molar of the lower jaw become carnassial teeth (sectorial tecth), and acquire a dominating position, while the others become smaller and tend to disappear at either end of the series. Further characters are the possession of a penis bone, the abdominal position of the milk glands and the uterus bicornis; the placenta is zonary. Anal glands, furnishing a strong, even ofiensive smelling secretion, are common.

Sub Order 1. FISSIPEDIA. Terrestrial animals with well-developed toes usually cleft to the base. Frequently five digits on all feet, but often reduced to four on the hind feet (Felidæ, Canidx), rarely on the fore feet (Hyænida); but in these cases (domestic dog) the reduced toe may bear a claw. URSID ; Ursus, * bears; Procyon lotor, * raccoon. Mustelid a; many species of Mustcla* and Putorius* (minks, martens, sable, ermines, and weasels) are valuable for their fur; Lutra, * otter; Enhydris, * sea otter; Mephitis, * skunk; anal glands common, in this family. Fossils (Arctotherium, etc.) connect the bears and the CANIDE five toes in front, four behind, claws not retractile; Canis* dogs, foxes and wolves. The FELID玉 have retractile claws. Felis domestica, our domestic cat. $F$. leo, lion; F. tigris, tiger; $F$. concolor, * puma or cougar. Hrexid 5 , all feet four-toed; Hyana of Africa. VIVERRIDe; IViverra, civets; Herpestes, ichneumons. Sub Order II. PINNIPEDIA. Aquatic carnivores with the limbs flattened to broad flippers, the five toes long and webbed, nails frequently rudimentary; the dentition differs from that of the terrestrial forms in the similarity of molars and premolars (absence of carnassial); the milk clentition degenerates, without being functional. PHOCIDж, seals, no external cars; Phoca vitulina, * harbor seal. OTARID 2 , with external cars; Otaria, ${ }^{*}$ sea lions; Callorhimus ursimus, fur seal of Alaska. TRICHECHIDE; incisors reduced, up per canines developed into large tusks; Trichechus, walrus. The first carnivores 
appear in the eocene in the order CREODONTA, plantigrade forms with slightly differentiated dentition; they resemble marsupials, insectivores, as well as the Condylarthra. True carnivores appear in the upper eocene.

\section{Order V. Rodentia.}

The rodents unite great similarity in appearance with a characteristic dentition. The canines are absent, the molars separated by a large gap (dias. tema) from the incisors (fig. 6I.4). The latter are strong, chisel-like, have persistent pulps and grow at the root as they are worn away at the cutting edge. Since only the front surface has enamel, wear keeps them constantly sharp. Usually there is but a single incisor; only the Duplicidentata have a second in the upper jaw. The molars are cuspidate or have enamel folds and frequently continue to grow throughout life. Their number is frequently reduced, the formulie varying between $-203 \frac{3}{1} 02$ and 11002 . Many species have an inflected angle

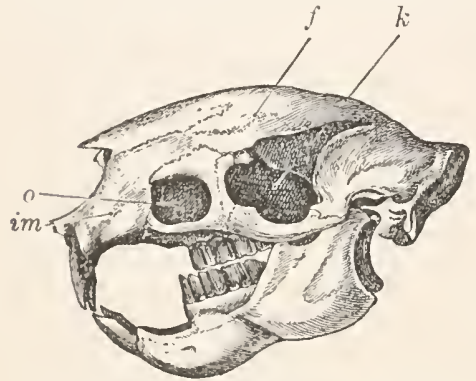

lirg. 6I.4.-Skull of porcupine (from Schmarda). $f$, frontal; $i m$, premaxillary; $k$, temporal fossa continuous in front with orbit; $o$, infraorbital foramen, enormous on account of the portion of the masseter muscle which passes through it.

of the jaw like that of marsupials. The infraorbital canal is a striking feature in Muridæ and Hystricidæe (fig. 61 4,o), a large opening in front of the orbit for a part of the masseter muscle.

The rodents are distinguished from the ungulates by the smaller size, the possession of claws, five toes (sometimes reduced to three), the occurrence usually of a clavicle, and a discoid placenta. The mammx are inguinal in position and are very numerous. The occurrence of glands with a strong-smelling secretion, which open near the anus, is common (fig. 603). Over one thousand living species are known, occurring in all regions except the Australian. The order appears in the eocene.

Sub Order I. DUPLICIDENTATA (Lagomorpha), two upper incisors, includes hares, Lepus, * and picas, Lagomys.* Sub Order II. SCIUROMORPHA. The squirrels, ScIURIDe, have soft fur and bushy tail. Sciurus, * squirrels; Cynomys, * prairie dogs; Sciuropterus, ${ }^{*}$ flying squirrels. The CastorID.E have soft fur and scaly tail. Castor fiber,* beaver. Sub Order III. MYOMORIHA, rats and mice. Hus musculus, * common mouse; Mus rattus, ${ }^{*}$ house rat, once abundant but now replaced by the gray rat, 1 . decumanus, * an immigrant from Lsia. White rats are albinos of $M$. raltus. Fiber zibethicus, * musk rat; Arvicola, ${ }^{*}$ field mice. Sub Order III. HYSTRICOMORPHA. The porcupines (Hrstricid w) have spines; the Old World forms, Hystrix, are terrestrial, ours (Erethyzon) arboreal. The Cavind 2 , South America, hooflike claws. Caria cobaya, guinea pig. Hydrocharus, capybara, largest existing rodent.

\section{Order VI. Ungulata.}

The Ungulata, or hoofed animals, include two groups of living animals in which the body is supported on hoofs on the tips of the toes, and which are sharply marked off from other forms. If, however, the fossils be included, the limits of the group must be extended so that it includes the elephants and conies of the existing fauna as well as several extinct forms, for these so intergrade that sharp lines cannot be drawn. 
The ungulates, which arise from the Condylarthra of the eocene (Phenacodon), are preeminently herbivorous; the canines are rarely well developed, the molars numerous and adapted to grinding the food, more or less flattened and frequently with folked enamel. The namme are inguinal, the uterus bicornuate, and the placenta either diffuse or (most ruminants) cotyledlonary (fig. 6ro). The legs are exclusively locomotor structures and the clavicles are absent. Since the metacarpals and metatarsals are greatly elongate, the wrist and ankle are raised from the ground so that they are frequently confounded with elbow and knee. With this exclusively supporting character of the limbs there is a tendency to reduction and fusion of bones. There is a constant increase in the development of radius and tibia to the chief supports of the body, the fibula becoming rudimentary, the ulna being developed sometimes throughout its whole extent, sometimes only in its upper part, and is more or less fused with the radius. The same tendency to simplification prevails in the feet, but is ex-

A

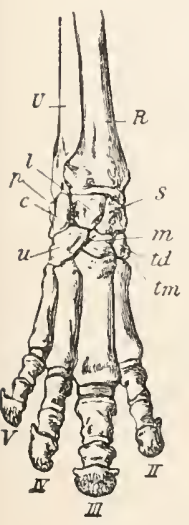

$B$

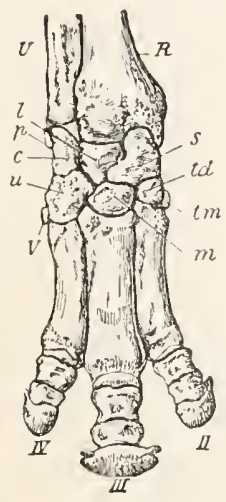

c

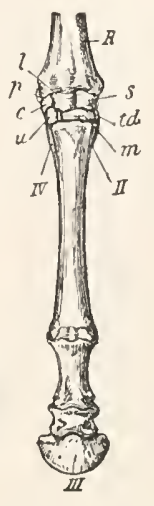

$D$

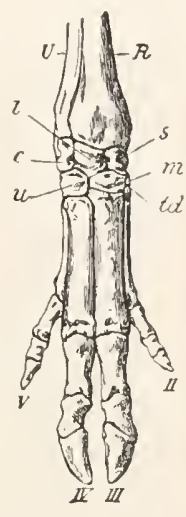

E

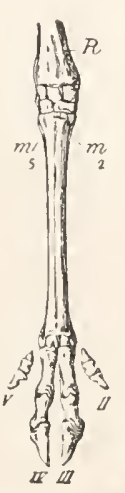

F

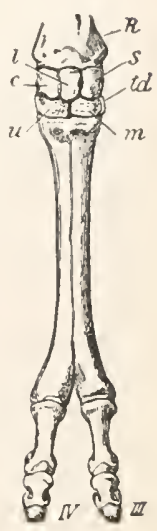

FIG. 6I5-Fore feet of ungulates (after Flower). $A-C$, perissodactyle; $D-F$, artiodactyle. $A$, tapir; $B$, rhinoceros; $C$, horse; $D$, pig; $E$, deer; $F$, camel. $c$, triquetrum (ulnare); $l$, lunatum (intermedium); $m$, capitatum; $m^{2}-m^{5}$, rudiments of metacarpals II and $\mathrm{V}$; $p$, pisiforme; $R$, radius; $s$, scaphoid (radiale); $t d$, trapezoid; $t m$, trapezium; $L$, uln $; ; u$, hamatum; II-V, digits.

pressed differently in the odd-toed (perissodactyle) and even-toed (artiodactyle) forms. In the Perissodactyla the axis of pressure passes through the middle toe (fig. 6I5, A-C, III), while the other toes disappear symmetrically around this. Since the first toe is early lost, toe $V$ is next to disappear $(B)$, and then toes II and IV $(C)$, so that at last there remain only the skeleton of the middle toe (horse), the rucliments of toes II and IV persisting as the small splint bones.

In the Artiodactyla the axis of pressure falls between toes III and IV $(D)$, which support the body, are equally developed and frequently fuse, at least so far as the metacarpals are concerned $(E, F)$. The figures $D-F$ show how the other digits disappear, digit I being lost still earlier. Since the weight of the body rests more upon the hind legs than upon the front ones, the former are the first to become modified. Since we are able, with the fossils, to follow in detail the lines of descent of both artiodactyles and perissodactyles, it is certain that these form diverging series, distinct from the beginning. In each series 
most of the common characters enumerated above have been independently acquired so that the uniformity in appearance is in great part the result of convergence.

Sub Order I. PERISSODACTYLA. Molars and premolars (with more or less pronounced enamel folds) of equal size; predominant development of the middle toe, the others reduced to different degrces. TAPIRID E, fore feet fourtoed, hind feet three-toed; teeth ${ }_{3}^{3} \frac{1}{3} 33$; nose elongate into a proboscis. Tapirus, tapirs, tropical America and India. RHINocERoTIDE, three toes on all feet, teeth $20+\frac{3}{1}$; one or two horns on the nasal bones; slin thick, hairless (hence these were formerly united with elephants as Pachydermata). Rhinoceros, single horn, India; Ceratorhimus (Asia), Atelodus (Africa), two horns. EQuide, a single functional toe (fig. 6r $5, C$ ); teeth $\frac{3}{3} \frac{1}{3} \frac{4}{3}$; Equms caballus, * horse, a native of Isia; E. asinus, ass; E. Zebra.

Sub Order II. ARTIODACTYLA. Besides the features of the feet, these have the three or four premolars, smaller than the molars. Species much more numerous than of perissodactyles. Section I, NON-RUMINANTIA (Bunodontia); omnivorous and have a bunodont dentition, $\frac{2}{1}-31+43$, the canines frequently developed into tusks; stomach usually simple, but occasionally divided into three chambers (Dicotyles, Itippopotamus), although rumination does not occur. The leg skeleton is little modified (fig. $61_{5}, D$ ), ulna and fibula not reduced, metacarpals and metatarsals separate. НIPpopotAMID reach the ground; skin thick ('pachyderm'), body heary; African. Hippopotamus. SuID e; two functional toes, skin with bristles, snout proboscis-like. Sus scrofa, swine; Dicotyles, ; peccarics.

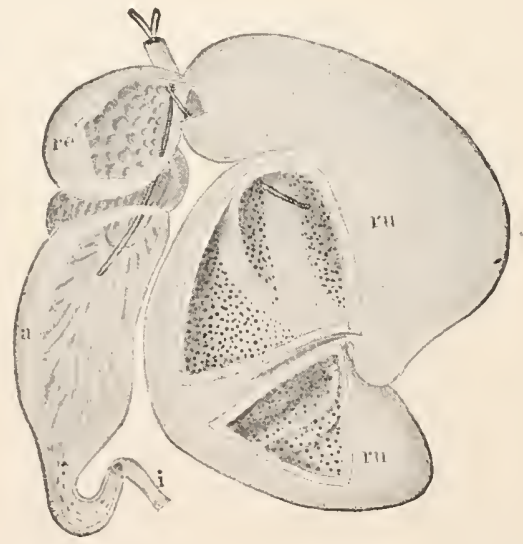

FIG. 6I6.- Stomach of sheep (after Carus and Otto). a, abomasum (true stomach); $o$, omasum (manyplies); re, reticulum (honeycomb); $r$, rumen (paunch).

Section II. RUMINANTIA (Pecora); teeth and stomach adapted to the exclusively herbivorous dict. The stomach (fig. 616) is divided into two portions, each again subdivided. The first of these, the mumen, or paunch $(r u)$, receives the food as it is eaten; then at a time of quiet it is regurgitated into the mouth and ground by the molars ('chewing the cud'). It then passes back, this time into the second division, the honeycomb, or reticulum (re), thence to the manyplies (psalterium) or omasus $(o)$, and lastly to the abomasus, or true stomach $(a)$. Usually canines and incisors of upper jaw degenerate; incisors of the lower jaw strong and the canines have form and position of incisors. 
The molars are selenodont. With few exceptions Ruminants are of large size and many bear horns on the frontal bones, larger in the males and may occur exclusively in that sex. In the giraffes these are cones free from the frontals and covered with skin. In others (Caricornia) the horn cores fuse secondarily with the frontals and are covered with a sheath of horn. Lastly, the horns are outgrowths of the frontal bone, in which usually the outer coats of skin and hair (velvet) are soon lost and only the bone projects freely (antlers). These are shed yearly, the new antler which takes its place being larger and consisting of a larger number of branches or tines, thus constituting an index of age (Cervicornia). Canielopardalide (Devexa), giraffes, long-legged forms from Africa with persistent horns; teeth $\frac{0033}{31}$, Giraffa, Okapia. Cervide, deer. deciduous horns in the male. Cerv'ts," common deer; Alces, " moose; Rangifer, * reindeer; Moschid.玉, horns lacking, males with enlarged upper canines and a musk gland; Moschus, central Asia. Tragulid. The CAVICORNIA include many species, some of great cconomic importance; teeth $\frac{003 \frac{3}{3}}{3}$. BovID.E: Bos tuurus, domestic cattle, probably descended from three distinct stocks ( $B$. primigenius, aurochs, $B$. longifrons and $B$. frontosus): Bison, * including $B$. europeus, bison proper, and B. americumus* our 'buffalo.' Bubalus, the true buffalo of the Old liorld. Ovide: Oris aries, sheep; $O$. montana, * big horn; Capra hircus, goat; Oribos moschatus, ; musk ox. AхтাLOPIDE: including a host of Old World forms (Antilope, Gasella, Rupicapra tragus, the chamois, etc.) and Antilocapra americana, ${ }^{*}$ prong horn, which sheds its horns, and Hoploceras montanus, * Rocky mountain sheep.

Section III. TYLOPOD A, stomach without manyplies, no frontal horns, difuse placenta. Camelus, Old World camels; Auchenia lama, A. alpaca of South America.

\section{Paleontology of the Ungulata.}

Abundant paleontological material, especially from the tertiary of our western states, has cleared up many lines of ungulate descent and has shown it probable that the CONDILARTHRA of the eocene, with fire-toed plantigrade feet, well-developed ulna and fibula, and omnivorous dentition, formed the stock from which the artiodactyles and perissodactyles descended, and

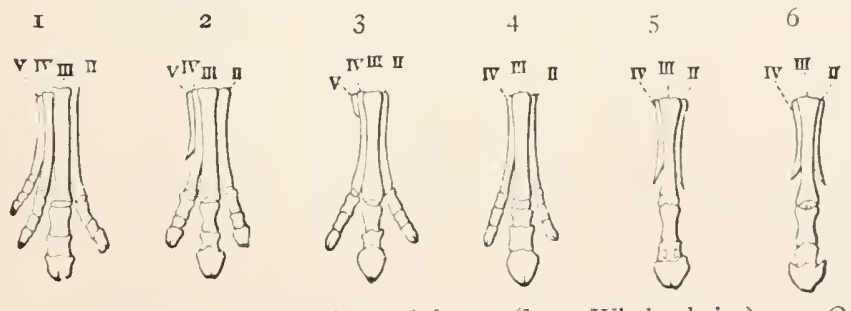

FIG. 6r7.-Evolution of fore font of horse (from Wiedersheim). I, Orohippus (eocene); 2, Mesohippus (lower miocene); 3, Miohippus (miodene); t, Protohippus (upper pliocene); 5, Plivhippus (pleistocene); 6, Equus.

possibly carnivores and primates as well, the ungulate line extending through the Amblypoda. From one group of these (PHENacodontide:) the lines of rhinoceros and tapir have come, and in an almost complete series we know the ancestry of the horse. Hyracotherinm (Eohippus) and Orohippus of the eocene had the fore feet four-toed (fig. 617, I); Palantherium and Wesohippus (2) of the lower miocene and Miohippus of the later miocene were three-toed, while. Meryhippus and Hipparion (Pliohippus, 4) of the pliocene were near the horse in 
tooth structure. The single-toed horses appeared in the pleistocene with Pliohippus (5) and then Equms itself (6). It is a pecullar fact that the horse entirely died out in America, although the chief part of its history was enacted here. The AMBLIPODA, mentioned above, were semi-plantigrade pentadactyle forms, appearing in the lowest eocene, and reaching, in Uintatherium (Dinocerus) an elephantine size. The TOXODONTIA of the South American tertiaries combined perissodactyle, rodent, hyracoid, and proboscidian features, while the TILLODONTIA of the eocene recall both carnivores and rodents.

\section{Order VII. Proboscidia.}

The elephants and their allies, with their hoofs and herbivorous dentition and lack of clavicle, are closely related to the ungulates. They are characterized by their thick skin ('pachyderm'), the large, massive, five-toed legs, and especially by the nose drawn out into a long proboscis, lastly by the dentition.

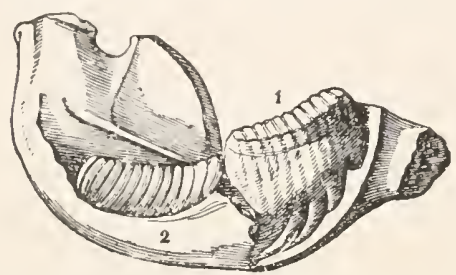

FIG. 6I8.- Inside of left lower jaw of Elephas indicus, the alveoli opened (after (wen). I, functional molar; 2, its successor.

Canines are lacking, but the incisors of the upper jaw continue to grow throughout life, forming the well-known tusks. In the living elephants there are but a single pair of tusks, but in some extinct Mastodons there were a second smaller pair in the lower jaw, while Dinotherium had only the lower incisors, these projecting downwards. The molars (in Mastodon and Dinotherium with normal replacement and cusps) consist of numerous plates of enamel and dentine united by cement, and undergo a horizontal displacement. Of the three large molars and premolars only one at a time is functional (fig. 6r8, I); when worn out the next one behind (2) takes its place. Further features are a uterus bicornis, a zonary placenta, and two pectoral mamme.

Elephantide: Elephas indicus, small ears; $E$. africanus, large ears. $E$. primigenius, mammoth, pleistocene; specimens found frozen in ice in Siberia have close woolly hair, in some places three fect long. Mastodon, with tuberculate teeth, miocene and pliocene. Dinotherid.玉, Dinotherium, Old World miocene.

\section{Order VIII. Hyracoidea.}

The single genus Hyrax, including species from western Asia and Africa, with four-toed front feet, hind feet with three toes, the digits with nails, the placenta zonary, and the dentition $\frac{1}{2} 0+3$, forms this group, no fossils being known. Hyrax syriacus is supposed to be the 'coney' of the Bible.

\section{Order IX. Sirenia.}

This order consists of a few aquatic whale-like mammals, with the fore limbs fin-like, hind legs lacking, and a horizontal caudal fin. They live in shallow seas or in the mouths of rivers, where they feed on the vegetation, which they chew with jaws corered with horny plates. The teeth (in the fossil Prorostomus $3 \frac{3}{2} 1+\frac{4}{4}$ ) are reduced or entirely lacking. The fore legs are pentadactyle, often have rudimentary nails and always a flexible elbow. The two pectoral mamme are possibly the germ of truth in the mermaid myth. Manatus americamus, * manatce, six cervical vertebre, eight to ten molars; Halicore dugong, Indo-Pacific; Rhytina stelleri, northern Pacific, exterminated in $\mathbf{1} 768$. 


\section{Order X. Cetacea。}

In form the whales resemble the sirenians, a result of an aquatic life, but the resemblance ends here. 'The whales are so fish-like that every one speaks of the whale fishery. Head and trunk are scarcely distinguished, the cervical vertebre being very short and more or less completely fused. 'The hind limbs are absent, of the pelvic girdle only a small ilium remains, and no sacral vertebre are developed. The caudal fin differs from that of a fish in being horizontally flattened; the skin is thick and is sparsely haired or completely naked, some lacking hair even in the embryo. Most of the species inhabit the high seas, Inia boliviensis and Platanista gangetica occur in rivers.

The fore limbs are modified into flippers, the bones of which are of nearly equal size and are jointed only at the shoulder. A dorsal fin ('fin backs') occurs in some. The lack of hair is compensated by the thick layer of subcutaneous fat (blubber). In order that the animals may breathe while feeding, the larynx is prolonged into a tube which extends up through the pharynx to the choanx, from which the nostrils extend directly upwards to the single (Denticetes) or paired (Mysticetes) external opening. Since the air expelled contains much moisture and this is condensed on contact with the cooler external air, the impression was natural that the animals in 'blowing' spouted water. As the olfactory membrane is degenerate and the olfactory lobes are reduced, the nose is an organ of respiration only.

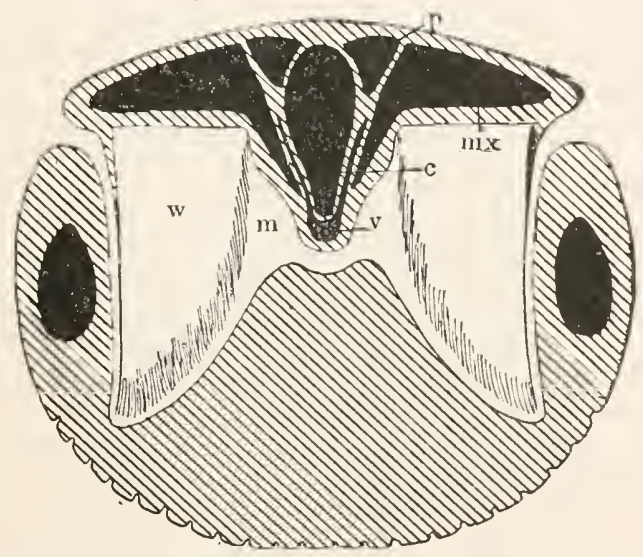

FIG. 6I0.-Section through jaws of whalebone whale (after Delage). c, septum of nose; $m$, mouth cavity; $m x$, maxillary bone; $p$, premaxillary (hinder end); $v$, vomer; $w$. baleen.

The eyes are small, external ears are lacking, the mammæ are close to the sexual opening. The teeth are either present in large numbers, similar and conical, and, since the second dentition is rudimentary, are monophyodont (Denticetæ) or they are outlined early and then resorbed and replaced by plates of baleen (Mysticetx), composed of large horny plates (whalebone) in large animals a dozen feet long (fig. 619, w), of which hundreds are arranged in close succession extending inward to the tongue. They correspond to the transverse palatal folds which occur in other mammals. As they are fringed on the inner edges they form a strainer which retains the small animals on which these whales feed. The origin of the whales is one of the unsolved problems. 'That they had a terrestrial, quadrupedal ancestry is beyond question, and the little 
evidence would seem to point to the ungulates, creodonts, or carnivores. The toothed and whalebone whales may have had different ancestries, their rescmblances being the result of convergence.

Sub Order I. ZEUGLODONTA. Extinct (eocene) forms with heterodont dentition, the posterior teeth two-rooted. Sub Order II. DENTICET E, toothed whales, carnivorous, some having but two teeth. Delphinus, ${ }^{*}$ dolphins; Clobiocephalus, black fish; Monodon, narwal, male with a long maxillary tusk. Physeter macrocephalus, sperm whale, pursued for the spermaceti, an oily mass situated in the 'chair' between the cranium and the snout, as well as for a mbergris, formed in the intestines. Sub Order III. MYSTACETI, whalebone whales, with baleen. Balcnoptera, * rorquals and fin backs. B. sibbaldi, * the largest whale, eighty-five feet long. Balcna, right whale.

\section{Order XI. Prosimiæ (Lemuroida).}

Linne united with the true apes a small group of animals known as lemurs, because of similarity in body form and climbing habits, because they had grasping hands and fect (opposable thumb and great toe), and frequently nails on some of the toes. To-day many set them aside as a separate order on account of their lower organization. They have a less-developed cerebrum, uterus bicornis, and a diffuse placenta. Further peculiarities are the peculiar and

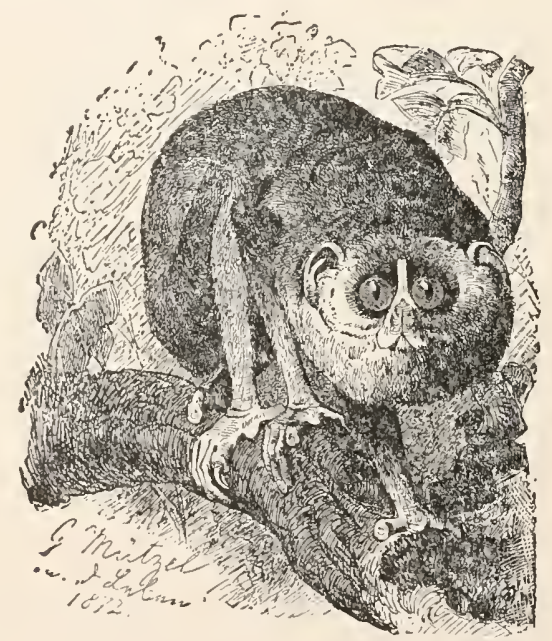

FIG. 620.- Stenops gracilis, slender loris (from Brehm).

variable dentition (Chiromys $\frac{1013}{1003}$, Lemur $\frac{2}{2}+\frac{3}{3} 3$ ). Nocturnal habits have resulted in large eyes, which give these animals a most striking appearance. A distinction from the primates is the connexion of orbital and temporal cavities bencath the osseous postorbital ring. Usually there are a pair of pectoral mamme, to which are added in many species a pair in the abdominal or inguinal region, the latter alone occurring in Chiromys. All come from the Indian and Malagassy regions.

Cinromvin: digits long, all except the great toe with claws; Chiromys, ayc-ayc. 'TARSIDE, second and third hind toes clawed. Tarsins, East Indies, differs in closed orbits and discoidal placenta. LEMURIDe, second hind toe 
alone clawed. Lemur; Stenops, loris. The tertiary PACHYLATRID. and AxAPTOMORPHIDE are close to the most primitive mammals, creodonts, and insectivores. The Galeopithecidie (p. 564) are often placed here.

\section{Order XII. Frimates.}

The most highly organized mammals, the monkeys, apes, and man, are united in a single order because of their great agreement in features of classificatory value. If we here, as elsewhere, ignore grades of intelligence and regard alone greater or lesser anatomical resemblances, we are forced to the conclusion that the anthropoid apes are much closer to man than to the lower monkeys. The primates have, in common, nails on all the fingers and toes (except Hapalidx), orbits separated from the temporal fosse by a bony wall, and a cerebrum which covers the other parts of the brain (fig. $600, c)$. They
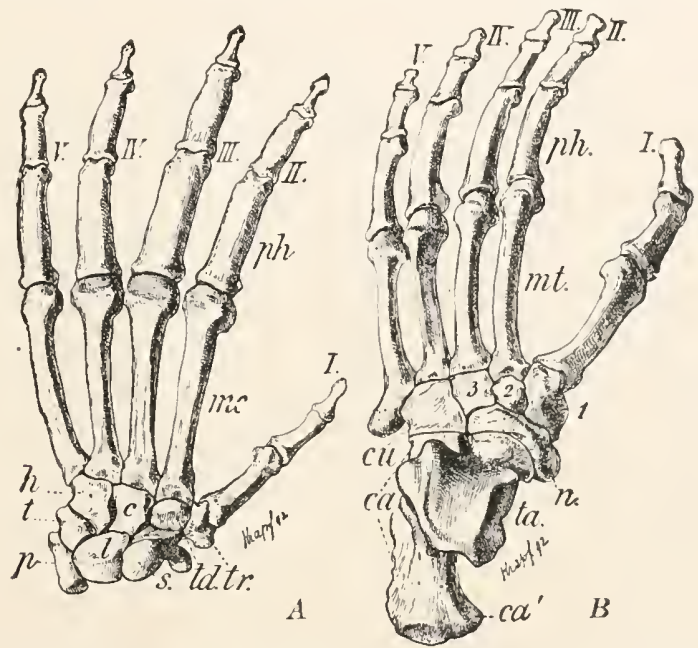

FIG. 62I.-Hand and foot of gorilla. c, capitatum; ca, calcaneus; cu, cuboid; $h$, hamatum; $l$, lunatum; $m c$, metacarpals; $n: t$, metatarsals; $n$, naviculare; $p$, pisiforme; $p h$, phalanges; $s$, scaphoid; $t$, triquetrum; $t a$, talus; $t d$, trapezoid; $t r$, trapezium; $I-l$, digits; $I-3$, cuneiformia.

have a single pair of pectoral mammæ, uterus simplex, and discoidal placenta. The dentition is essentially the same throughout; in the Platyrrhine $\frac{13}{2} 133$, in the Hapalidæ $\frac{2}{2} 1 \frac{3}{2}$, in the Catarrhinæ and in man $\frac{21}{2} 1 \frac{23}{2}$. Yet there is a tendency to variation, since in the chimpanzee and in man the third molar (wisdom tooth) is in process of degeneration, while in the orang a fourth molar often occurs. The molars are bunodont.

As in the lemurs and opossums, the thumb and great toe can be opposed to the other digits, so that an ape can grasp objects with either hand or foot. In man this opposability of the thumb is increased, but that of the great toe, in consequence of the upright position, is only retained to a slight degree by children and primitive people. On this peculiarity rest the names Bimana, for man, and Quadrumana, for the apes and monkeys. But it must be emphasizerl that the apes do not have a hand, but rather a grasping foot, on the hinder 
extremities. The grasping foot (fig. 62I) has the same bones, similarly arranged and of about the same shape as in the foot of man, while the musculature is essentially the same. On the other hand, the same distinctions between hand and foot $(A$ and $B$ ) occur as are found in the hand and foot of man. The separation of Quadrumana and Bimana is without anatomical basis; it rests solely upon functional peculiarities and egotism.

Sub Order I. PLAT'YRRIINE, New World monkeys. Nostrils separated by a wide septum so that they open laterally; tympanum not extended by an outer bony meatus. CEBID $\approx$, tail frequently prehensile, long. Cebus, sapajous; Ateles, spider monkeys. The HAPALIDE, or marmosets, are aberrant with teeth 132 and claws on all digits except the relatively small great toe, thumbs not opposable. Hapale, Midas. Sub Order II. CATARRHINE, Old World apes; internasal septum small, the nostrils directed forwards and downward; since the large canines interlock in the opposite row of teeth, there is a more or less evident diastema in each jaw; tympanum prolonged as in man into a hony meatus. Section I. CYNOMORPHE, with naked places on the buttocks (ischial callosities), usually a long tail and hairy face, and usually only two sacral vertebre. Cynocephalus, baboons, mandrils; Macacus, macaques; M. ecuudatus, entering Europe at Gibraltar. Section II. ANTHROPOID E (Simiidx), man-like apes, usually without ischial callosities, face, fingers and toes without hair, no tail, five sacral vertebre (three in Hylobates) fused to an os sacrum. Hylobotes, gibbons, very long arms; Simia satyrus, Sumatra and Borneo, orang-utan; Gorilla engena; Troglodytes niger, chimpanzee, Africa.

Sub Order III. ANTHROPINE, man. Degeneration of hair on most parts of the body; upright position and as a result slight mobility of great toe (non-opposable); development of articulate speech; high intelligence; large cerebrum and consequent increase of the cranium at the expense of the face, are the most prominent characters of mankind. Dentition as in the Catarrhinx, canines smaller, no diastema. It was long a question whether there was a single species of man (Homo safiens) with several races or whether there were several species. Since crosses between the different races are fertile, the first view receives general acceptance, although the differences are constant and point to the sccond alternative. 'The answer to these questions, which in the light of evolution have lost most of their significance, and the characterization of the various races, belong to a special branch of science, anthropology.

Since an arboreal life was unfavorable for fossilization, the palcontological material for the history of the primates is so far very scanty. Of these the greatest wei sht has been laid on a 'find' in the pleistocene of Java. 'This consisted of a top of a skull, a femur, and two molar teeth which were found near each other. These fragments were regarded on one side as a connecting link, Pithecanthropus crectus, between apes and man, on another as belonging to a true ape, and from the third as true man. The latter is now to be regarded as out of the question. Most probably it was an extinct gibbon-like animal of extraordinary size, an enormous cranial capacity (about $\$ 00 \mathrm{ccm}$.) and correspondingly a very large brain. In these respects no Anthropoid now living could compare with Pithecanthropus. Not less interesting than Pithecanthropus are the numercus remains of man, mostly found in the caves of Europe, dating from the diluvium. Most of these are portions of skulls, which differ so from the living European that the first one discovered at Neanderthal was regarded as pathologic (microcephalic). As the number of these increased, they were regarded ats a distinct species, Homo primigenius, but this has difficulties, since many low races, and especially the Australians, show marked resemblances to them. It is certain that diluvial man of Europe was very low. This is shown by the slight arch of the skull, the prominence of the face, and the massive lower jaw which lacked a chin. 


\section{Summary of Important Facts.}

r. The CHORD ITA possess an axial skeleton, the notochord lying between the nervous system and the alimentary tract; a central nervous system entirely on one side of the digestive canal, and gill slits extending from the pharynx to the exterior.

2. The Chordata are subdivided into Leptocardii, Tunicata, Enteropneusta, and Vertebrata.

3. The LEPTOCARDII are fish-like in form, have a notochord extending the length of the body, but lack heart, auditory organs, skull and vertebral column; the brain is rudimentary, the gill slits numerous.

4. The TUNICATA have a notochord only in the caudal region. The young is tadpole-like, but in most forms there is a metamorphosis in which tail and notochord are lost.

5. The body is usually enclosed in a tunic or mantle containing cellulose: gill slits and an endostyle are present in the pharynx, the heart changes in the direction of the flow of blood. The nervous system in its derelopment is tubular. In the Salpida there is a typical alternation of generations between a solitary asexual and a sexual chain form.

6. 'The ENTEROPNEUSTA are worm-like, with collar and proboscis; a diverticulum of the digestive tract is compared to the notochord; gill slits occur in the pharynx; some undergo a metamorphosis in derelopment, the larva resembling those of Echinoderms. The pertinence of the Enteropneusta to the Chordata is not certain.

7. The VERTEBRATA are segmented animals without external ringing of the body, but with metameric arrangement of internal parts (myotomes, neurotomes, sclerotomes).

8. A cuticular skeleton is absent, but there may be cornifications of the epithelium or ossifications in the derma (scales of fishes, etc.).

9. An axial skeleton is present, consisting of skull and vertebral column, which more or less completely replace the notochord.

ı. There are two kinds of appendages supported by an axial skeleton: the unpaired fins, occurring only in fishes and Amphibia, and the paired appendages (anterior and posterior), which are usually present.

I I. The central nervous system (brain and spinal cord) are tubular and dorsal in position. The brain consists of five parts-cerebrum, 'twixt brain, optic lobes, cerebellum, and medulla oblongata.

I2. Eyes and ears are the most highly developed sense organs.

I3. The respiratory organs arise from the entoderm (pharynx); gill slits are present at least in the embryo, extending from the pharynx to the exterior. In all terrestrial groups these are later replaced by lungs, developed from the hinder end of the pharyn. 
I 4. The heart, consisting of auricle and ventricle, lies ventrally in a pericardium. In gill-breathing species it contains only renous blood, but with pulmonary respiration it is divided into renous and arterial halves. The circulation is closed.

I5. The sexes are usually separate. In most species the excretory (nephridial) system forms the ducts for the reproductire products (urogenital system).

I6. The reproduction is strictly sexual.

I7. In the Crclostomata there is a primitive skull; but rertebræ, paired fins, true scales, and teeth are lacking. The gills are saccular and the nose is unpaired. There is no skeleton to the mouth (no jaws).

IS. The true fishes (PISces) have jaws (Gnathostomata). The fishes are further distinguished from the Cyclostomes by the rertebral column (usually amphicoele vertebre), by paired pectoral and ventral fins, scales, and paired nestrils. They breathe by sills, and have a venous heart with auricle and ventricle.

I 9. The fishes are divided into Elasmobranchii, Ganoidei, Teleostei, and Dipnoi.

20. 'The Elasmobranchii have a cartilaginous skeleton, usually a heterocercal tail, placoid scales, usually a ventral mouth, gills covered, heart with arterial cone, spiral valve in the intestine, no swim bladder.

2 I. They are divided into Selachii (subdivided into Squali, sharks, and Raix, skates) and Holocephali.

22. The Teleostei have bony skeleton, usually a homocercal tail, usually cycloid or ctenoid scales, comb-like gills and operculum, bulbus arteriosus, usually pyloric appendages, and a swim bladder; no spiral valve.

23. They are subdivided into Physostomi, Pharyngognathi, Acanthopteri, Anacanthini, Lophobranchii, and Plectognathii.

24. The Ganoidei form a connecting group; they resemble the elasmolranchs in the presence of a conus arteriosus and spiral valve, and usually in the heterocercal tail; they are like the teleosts in operculum and comb-formed gills, swim bladder, and pyloric appendages. They usually have fu'cra and ganoid scales.

25. The ganoids are divided into Chondrostei, with cartilaginous, and Crossopterygii and Holostei, with bony skeletons.

26. The Dipnoi have gills; occasionally the swim bladder serves as lungs; heart with beginning division; nose with choana.

27. The Aupinisid, in contrast to the fishes, have pentadactyle appentages; in contrast to the reptiles, double occipital condyles. They have bushy external gills and lungs, either persisting together or succeeding 
each other, the young (larve) lreathing by gills, the adult by lungs (metamorphosis!). The heart consists of two auricles and one ventricle. 28. The Amphibia are subdivided into Gymnophiona, Lrodela, and Anura; to these are added the extinct Stegocephali.

29. The Gymnophiona are blind and have lost the limbs.

30. The Urodeles have many vertebre and a well-developed tail. They retain the gills permanently (Perennibranchia), or at least a gill slit (Derotrema), or they lose the branchial apparatus completely in development (Salamandrina); the metamorphosis is not pronounced.

31. The Anura have few vertebre, no tail nor gills in the adult, and a marked metamorphosis (the larva, tadpoles, are furnished at first with external, then with internal, gills, and with swimming tail, but at first lack appendages and lungs).

32. Cyclostomes, fishes, and Amphibia are grouped as Anamnia because of the lack of amnion and allantois; they are also called Ichthyopsida, because of their branchix and aquatic habit. They are poikilothermal (cold-blooded).

33. The reptiles, birds, and mammals are called Amniota on account of the embryonal organs, the amnion and allantois. They never respire by gills (although gill clefts occur in the embryo), and the appendages are based on the pentadactyle type.

34. Reptiles and birds are united as Sauropsida from the similar scales and single occipital condyle.

35. The REPtiliA are poikilothermous, have a strongly ossified skeleton, with unpaired occipital condyle and usually an os transversum in the skull; a strongly cornified skin, two auricles, and usually two incompletely separated ventricles in the heart.

36. Recent reptiles are divided among the Chelonia, Rhynchocephalia, Squamata (including Lacertilia and Ophidia), and Crocodilia. To these are added the extinct groups Theromorpha, Plesiosauria, Ichthyosauria, Dinosauria, and Pterodactylia.

37. The Chelonia are compact, have a skeletal capsule (carapace + plastron) composed of bone and horny plates, an immorable quadrate and hard palate, no os transversum or teeth, but hormy plates in the place of the latter; the cloacal opening elongate.

38. The Squamata have horny scales periodically renewed, a transverse cloacal opening, with behind it paired penes, and a morable quadrate.

39. The Lacertilia have the ventral surface scaled, usually movable eyelids, tympanic membrane, four appendages or their rudiments, and all but invariably a sternum.

40. The Ophidia lack appendages, sternum, and tympanum; the 37 
eyelids are fused; the mouth is usually extensible; poison fangs are frequently present. The ventral surface has scutes.

4. The Rhynchocephalia resemble the Lacertilia in form, but differ in having a fixed quadrate.

42. The Crocodilia are elongate, have bony plates in the skin, elongate cloacal opening, fixed quadrate, teeth placed in separate alveoli, and a long swimming tail.

43. The Aves (birds) are distinguished by feathers, and by the heart completely divided into right and left halves.

44. Other characters are homoiothermy (warm-blooded), pneumaticity of bones, fusion of bones of manus, formation of tibio-tarsus and tarso-metatarsus (intertarsal joint).

45. The birds are divided into Ratita, which lack a furcula and a keel to the sternum, and the Carinata, in which the sternum is keeled and the clavicles are united to a furcula. To these are added the extinct Saurure and Odontornithes, with teeth.

46. The Mammala have a double occipital condyle, hairy skin, and milk glands in the female for the nourishment of the young. Other characters are homoiothermy, the complete separation of the heart, the morlification of parts of the visceral arches into the ear bones, high development of the dentition (formation of roots, usually heterodont and diphyodont).

47. The mammals are divided into Monotremata, Marsupialia, and Placentalia.

48. The Monotremata are egg-laying mammals with persistent cloaca; they have a distinct coracoid and an episternum.

49. The Marsupialia are viviparous, but the young, on account of imperfect nourishment (usually no placenta), are born early and usually carried in a marsupium (marsupial bones).

50. In the skeleton the inflected angle of the lower jaw is characteristic; uterus and vagina are double.

5I. The Placentalia have well-developed young, nourished in the uterus by a placenta; they have no marsupium nor marsupial bones. The vagina is single (Monodelphia), the uterus simple or paired.

52. The clawed Edentata and the Cetacea and Sirenia, which have flippers, have a degenerate dentition (teeth monophyodont or lacking).

53. The hoofed Ungulata (Perissodactyla and Artiodactyla), the Proboscidia, and the clawed Rodentia are herbivorous.

54. The Chiroptera, which have a flying membrane (patagium), are partly herbivorous, partly insectivorous.

55. The small Insectivora (with small canines and no carnassial) 
and the Carnivora (with strong canine and carnassial molar) are carnivorous. The Carnivora are subdivided into the terrestrial Fissipedia and the aquatic Pinnipedia.

56. The Prosimie and Primates have a more or less indifferent dentition. They have partly or entirely replaced claws by nails, and are largely provided with grasping hands and feet. The Prosimic are lower, the Primates more highly organized.

57. The Primates are subdivided, according to the position of the nostrils, the development of the tail, and the dentition and the feet, into the Platyrrhinx, or New World monkeys, the Catarrhinx, or Old World apes, and the Anthropinx, or man. 



\section{INDEX.}

Aardvark, 563

Abalone, 326, 333

Abdominal cavity, 555

Abdominal fin, 462

Abdominalia, 374

Abdominal pores, 48 I

Abducens nerve, $47 \mathrm{I}$

Abiogenesis, 127

Abomasus, 568

Acanthia, 428

Acanthias, 507

Acanthin, 176

Acanthobothrium, 254

Acanthocephala, 268

Acanthocystis, 175

Acantholerus, 39

Acanthodidix, 507

Acanthometra, 177

Acanthopleura, 3 I 5

Acanthopteri, 510

Acanthopterygii, 5I I

Acaridæ, 397

Acarina, 396

Accessory chromosome, 73

Accessory nerve, $47 \mathrm{I}$

Accipiter, 544

Acephala, 3 I 7

Acerata, 387

Acerentomon, 4I9

Acetabulum, 248

Achæta, 28I

Achatina, $33^{6}$

Achoreutes, 4 I9

Achromatin, 56

Achtheres, 28, $37^{\circ}$

Acineta, 198

Acinetaria, 197

Acinous glands, 63

Acipenser, 50 ;

Acmea, 333

Acontia, 226

Acrania, 43 ?

Acridium, 42 I

Acris, 520

Acrodont, 529
Actinaria, $23 \mathrm{I}$

Actinophrys, 175

Actinopoda, 308

Actinosphærium, I73

Actinotrichia, $46_{3}$

Actinotrocha, 287

Actinozoa, 224

Aculeata, 426

Aculeus, 4r 3, 425

Acustic nerve, $47 \mathrm{I}$

Adambulacral plates, 206

Adamsia, 227

Adductor muscle, 228, 318

Ega, 356

Agina, 2 IS

Eginopsis, 2 I 4

Eolidæ, 335

Eolidia, 335

Apiornis, 542

Aquoria, 2I4, 2 I 8

Eschna, 420

Asthetes, 3 I 5

Æthalium, IS I

After-shaft, 534

Agalmia, 219

Agamidie, 529

Agelacrinoidea, 302

Agelacrinus, 303

Agkistrodon, $53 \mathrm{I}$

Aglaophenia, 2 I $\mathrm{S}$

Aglossa, 520

Aglypha, 531

Agrion, 420

Air bladder, 502

cells, 482

pipes, $53^{8}$

sacs of bircls, $53 \mathrm{~s}$

Alie, 408

Alie cordis, $4 \mathrm{r} 2$

Alauda, 544

Albatross, 543

Albumen gland, 249

Alca, 543

Alcedinidæe, 544

Alces, 569
Alciopidæ, 277

Alcyonaria, 230

Alcyonidæe, 230

Alcyonidium, 287

Alcyonium, 22\%, 230

Alecithal, r43

Aleppo evil, I84

Aletia 433

Alima, $37^{6}$

Alisphenoid, $45^{8}$

Allantois, 490

Allelomorphs, ${ }_{3} \mathrm{~S}$

Alligator, $53^{2}$

Alligator turtle, 527

Allobophora, 279

Alloposus, $3+7$

Alosophila, 433

Alpaca, 560

Alpheus, 380

Alternation of generations, I3 I

Altrices, $54 \mathrm{r}$

Alula, 534

Alveolar duct, 482, 555

Alveoli, ${ }_{4} \mathrm{S2}, 532$

Amaroucium, $44^{6}$

Ambergris, 572

Amblyopsirlie, 5 то

Amblyporla, $57^{\circ}$

Amblystoma, 27, 520

Ambulacral system, IIr, 292,296

Ameiva, 528

Ametabolous, $4^{\mathrm{I}} 4$

Amia, 32, 508

Amicula, 3 I 6

Ammocoetes, 493

A mmoniticlic, 346

Am:ion, $4 \mathrm{I} 4,4 \mathrm{Sg}$

Amniota, 520

Amniotic fluid, 490

Amoba, 53, I7I, I72

Amobina, 172

Anceboid motion, 53, III

Amphiaster, 60 
Amphibia, 5I3

Amphibiotica, 420

Amphicole, 454

Amphicolias, $52 \mathrm{~S}$

Amphicliscs, 205

Amphigony, I 29

Amphilina, 247

Amphimixis, I 49

Amphineura, 3I 5

Amphinuclei, 57

Amphioxus, 439

Amphiporla, $3^{8} 3$

Amphiporus, $25^{8}$

Amphisbena, $53^{\circ}$

Amphistomum, 247

Amphitrite, 276, 278

Amphiuma, 520

Amphiura, 299

Amphoride, 303

Ampulle, 203, 292, $47 \mathrm{~S}$ of Lorenzini, 500

Ampullaridæe, 334

Amyda, 527

Anabrus, 42 I

Anacanthini, 5I I

Anaconda, 53 I

Anærobic organisms, OI

Anal fin, 462, 497

Anallantoidea, 490

Analogy, 10, 90

Analytic species, I+I

Anamnia, 490

Anaptomorphide, 573

Anas, 543

Anaspides, $3 S_{2}$

Anaxial animals, 123

Anelasma, 373

Angiostoma, 53 I

Anguillidie, 510

Anguillula, 265

Anguis, 530

Angulare, 549

Animalcula, I 70

Animal organs, 90, I 10 pole, I 33, I 42

Animals and Plants, I 59

Anisomyaria, 323

A nisoporla, 386

Ankylostoma, 266

Anlage, iii (preface)

Annelida, 260

Annulata, 530

Anodonta, 324

Anolis, 529

Anomodontia, 525
A nopheles, I 90, 430

Anoplocephala, 255

Anser, 543

Anseriformes, 543

Anteater, $5^{6} 3$ spiny, 559

Antedon, 302

Antennae, 352

Antennulæ, 377

Antheomorpha, 225

Anthomedusæ, 217

Anthozoa, 224

Anthropinæ, 574

Anthropoidea, 574

Antilocapra, 569

Antilope, 569

Antimere, 126

Antipathes, 23 I

Antlers, 560

Ant-lion, 422

Ants, 417,426 white, $4 \mathbf{I} 9$

Antrostomus, 544

Anura, 520

Anurida, 4I9

Aorta, 483

Aphaniptera, 43 I

Apes, 573

Aphida, 430

Apiariæ, 426

A pis, ${ }_{22} 6$

Aplacentalia, $55^{8}$

Aplacophora, 3 I 6

Aplysia, -334

Aplysina, 203

A poda, 308, 374, 5 I9

Apodidæ, 366

Apophysis, 454

Aporosa, 23 I

Appendicularia, 443

Appendix, vermiform, 554

Aprophora, $42 \mathrm{~S}$

Aptenodytes, 543

Aptera, 430

Apteria, 534

Apterygota, 4 I 8

A pteryx, 542

Apus, 366

Apyreme, 73

Aqueduct, 470

Aquila, 5.4

Arachnida, 380

Arachnoid membrane, 473

Araneina, 395

Arbacia, 305
Arcella, 179

A rchæopteryx, 25, 54 I

Archegony, 127

Archencephalon, 468

Archenteron, 94, I 46

Archianellida, 278

Archicoele, I45

Archigetes, 247, 253

Archiptera, 4I9

Archipterygium, 464

Architeuthis, 347

Arcidae, 323

Arcifera, 520

Arciferous, 5 I 3

Arctogæa, I6 3

Arctotherium, $5^{6} 5$

Arcyria, I8 I

Ardea, 544

Arenicolidæ, 278

Areolæ mammæ, 540

Areolar connective tissue, 76

Argas, 397

Argina, 323

Argiope, 291, 396

Argonautidæ, 347

Argulus, 370

Arion, 336

Arista, 43 I

Aristoile's lantern, 305

Armadillidium, 386

Armadillos, $5^{6} 3$

Armata, 28I

Army worm, 433

Artemia, 366

Arterial arches, $4 S_{4}$

Arterial blood, $\mathrm{IO}_{2}$

Arteries, IOI

Arthrodira, 5I3

Arthrogastrida, 39I

Arthropoda, 349

Arthrostraca, $38_{3}$

Articulare, 46I

Articular process, 455

Articulata, 302, 349

Artificial parthenogenesis, I 37

selection, 35

Artiodactyla, 568

Arvicola, 566

Ascalabotæ, 529

Ascaridæ, 266

Ascaris, 26.4, 266

Ascidiæformes, 443

Ascidians, 443 
Ascones, 204

Ascons, 201

Ascyssa, 203

Asellidre, 386

Asiliidæ, 43I

Asiphonate, 3 I 9

Asp, 53I

Aspidobranchia, 333

Aspidochirotx, 308

Aspidocotylidx, 244

Aspidonectes, 527

Aspidotus, $43^{\circ}$

Assimilation, .9 I

Astacoidea, 380

Astarte, 324

Aster, 60

Asterias, 298

Asteridæ, 298

Asteriscus, 298

Asterinidæ, 298

Asteroidea, 295

Astrophyton, 299

Asterospondyli, 506

Astræa, 232

Astrangia, 23I

Astroides, 232

Ass, 568

Asymmetrical animals, I23

Asymmetron, 44I

Atalapha, $5^{6} 5$

Atavism, I4I

Atax, 397

Ateles, 574

Atelodus, 568

Atheca, 527

Atlantidæ, 334

Atlas, 514, 522

Atoke, 275

Atolla, 224

Atriopore, 440

Atrium, 445, 516 of heart, IоI, $48_{3}$

Atrypa, 290

Attus, 396

Atypus, 396

Auchenia, 560

Auditory bulla, 548

organs, I I 6

ossicles, I I6, 461, 479

Auks, 543

Aulophorus, 278

Aurelia, 2I 4, 223

Auricle, ror, $48_{3}$

Auricularia, 294

Aurochs, 569
A utoflagellata, I \& I

Autogamy, I 74

Autoinfection, I 86

Autolytus, 277

Aves, 532

Avicularia, 286

Aviculidæe, 323

Axial skeleton, 9I

Axis, 522

Axis cylinder, 85

Axolotl, 520

Axon, 84

Aye-Aye, 572

Azygobranchia, 333

Babesia, Igo

I3aboons, 574

Bretisca, 420

Balæna, 572

Balænoptera, $57^{2}$

Balancers, 430

Balaninus, 424

Balanoglossus, 448

Balantidium, I 96

Balanus, 374

Baleen, $57 \mathrm{I}$

Bandicoot, 560

Barnacles, $37 \mathrm{I}$

Basalia, 292, 300, 463

Bascanion, $53 \mathrm{I}$

Basioccipital, $45^{8}$

Basipodite, 97, 360

Basisphenoid, $45^{8}$

Bassomatophora, 336

Bats, 564

Bdellostoma, 493

Bdellura, 242

Beach fleas, $38_{4}$

Bears, 565

Beaver, 566

Bedbug, 42S

Bee louse, 43 I

Bees, 426

Beetles, 423

Belemnites, $34 \mathrm{I}$

Bell's law, 468, 472

Belosepia, 34 I

Belostoma, 428

Bicidium, 230

Bilateral symmetry, 124

Bile duct, 48 r

Bilharzia, 247

Binomial nomenclature, 7

Biogenesis, law of, 26

Biology, 50
Biometric methorl, 4

Bipalium, 242

Bipinnaria, 204

Bipolar cells, $8_{4}$

Biradial symmetry, I 24

Bird lice, 420

Birds, 532

Birds of Paradise, 4I, 544

Birgus, $38 \mathrm{I}$

Bison, 569

Bittacus, 422

Bivium, 291, 296

Black fish, $57^{2}$

Black flies, 430

Black snakes, 53 I

Bladder worm, 247

Blarina, $5^{64}$

Blastoderm, I 43

Blastodermic vesicle, I 45

Blastoidea, 303

Blastomeres, I $4 \mathrm{I}$

Blastopore, I 46

Blastostyle, 2 I 8

Blastula, I 45

Blatta, 42 I

Blepharoplast, IS2

Blind fish, 5 iо

Blissus, 428

Blister beetles, 424

Blood, 78

arterial, 103

Blood-vascular system, IO3

Blood, venous, 103

vessels, 100

Blow flies, $43 \mathrm{I}$

Bluebirds, 544

Boa, 53I

Bobolink, 544

Body cavity, 99

Bojanus, organ of, 32 I

Bolina, 235

Bombycina, 433

Bombyx, 433

Bone, 77

Bones, membrane, 45 $\mathrm{I}$

Bonasa, 543

Bonellia, 28 r

Book lice, 420

Bopyridx, 3 s6

Bos, 560

Bosmina, 366

Botall's duct, $4{ }^{85}$

Bot flies, $43^{\text {I }}$

Bothriocephalus, 249, 254

Botryllus, 446 
Bougainvillea, $\mathrm{I} 3 \mathrm{I}$

Bovida, 569

Bow fin, 508

Brachialia, 300

Brachiolaria, 294

Brachionus, 260

Brachiopoda, 287

Brachycera, 43I

Braconidse, 426

Bradypus, $5^{6} 3$

Brain coral, 232

Brain of vertebrates, 469

Branchiæ, 97

Branchial arches, 460

chamber, $4+2$

clefts, $43^{8}$

heart, $3+3$

tree, 307

Branchiata, 359.

Branchiopoda, 366

Branchiomeric, 460

Branchiostegite, 377

Branchiostegal membrane, 502

rays, 497

Branchipus, 365, 366

Branchiura, 370

Braula, 43I

Brevilinguia, 530

Brine shrimp, 366

Brissus, 306

Bristle tails, +19

Brittle stars, 298

Bronchioles, 482,555

Bronchus, 482, 555

Bryozoa, 284.

Bubalus, 560

Bubo, 544

Bubonic plague, $43 \mathrm{I}$

Buccal cavity, 94

Buccinida, $33+$

Buceronticlæ, 544

Budding, I 28

of cells, 59

and germ layers, $\mathbf{r}_{4} 8$

Buffalo, 569

Bufo, 520

Bugs, 427

bed, 428

June, 424

pill, 386

salve, 386

sow, 386

stink, 428

Bugu!a, 287
Bulbus arteriosus, $5 \circ 3$

olfactorius, 469

Bulimus, 336

Bulla, 334

Bunodes, 230

Bunodontia, 568

Burbot, 5 I I

Bursa, 266, 293, 299

Bustard, 5+4

Buteo, 544

Buthus, 392

Butterflies, 432, 433

Buzzards, 544

Byssus gland, 32 I

Cabbage worm, 433

Cacatua, 544

Caddis flies, 422

Cæca, pyloric, 500

Cacidotea, 386

Ciecilia, $5 \mathbf{I 9}$

Ciecum, 95, 554

Canosarc, 227

Calamoichthys, 508

Calandra, 424

Calamus, 533

Calanide, 369

Calappa, $3^{82}$

Calcispongiæ, 204

Caligus, 370

Callianira, 233

Calliphora, 43 I

Callorhinus, 565

Calosoma, 42.4

Calyconecte, 2 I 9

Calycophoræ, 2 I 9

Calyptoblastea, 2 I 7

Camarasaurus, $52 \mathrm{~S}$

Cambarus, 380

Camelopardidæe, 560

Camelus, 560

Campanella, 2 I 8

Campanula Halleri, 499

Campanularia, 2 Io

Campanulariæ, 2 I 7

Campodea, 4I9

Cancer, 382

Cancridx, 382

Candona, $37 \mathrm{I}$

Canis, 565

Canker worms, 433

Cantharidæe, 424

Canthocamptus, 369

Capillaries, IoI

Capillitium, I 80
Capra, 560

Caprella, $38_{4}$

Caprimulgide, 544

Capybara, 566

Carabidæ, 424

Carapace, 359, 527

Carcharinus, 506

Carcharodon, 506

Carchesium, 196

Cardia, 554

Cardinal vein, 484

Cardium, 324

Cardo, 406

Caridea, 380

Carina, 372,535

Carinariidæ, 334

Carinate, 542

Carinella, 258

Carmine, +28

Carnassial tooth, 554

Carnivora, 565

Carp, 5 Io

Carotid arteries, $48_{3}$

Carpal bones, 465

Carpocapsa, 432

Carpome, iso

Carpopodite, 97

Carpus, 465

Cartilage, 77

bones, 455

Caryogamy, I 68

Caryosomes, 57

Caryophyllæus, 247

Cassowary, $5+2$

Castor, 566

Castration, I Io

Casuarina, 542

Cataclysm theory, I5

Catallacta, 200

Catarrhina, 574

Caterpillars, 432

Catfishes, 5 10

Cathamma, 223

Cathartes, 544

Catocala, 433

Catometopa, $3^{82}$

Catostomidx, 5 ro

Cats, $5^{6} 5$

Caudal fin, 462,498

Caudina, 308

Cavicornia, 569

Cavolinidie, 335

Cavy, 566

Cebus, 5 it

Cecidomya, 430 
Cell, 5I division, 59 nucleus, 55 reticulum, 53 theory, I2, 5 I

Cells, blood, 78 ganglion, $8_{3}$ gland, 68 goblet, 68 sensory, 73 sexual, I30 sizes of, 52 somatic, 130 supporting, 74

Cellular connective tissue, Cheliceræ, 389 75

Chelifer, 394

Chelone, $5^{27}$

Chelonia, 526

Chelura, $3^{8} 4$

Chelydra, 527

Chernes, 394

Chevron bones, 454

Chiasma, 472

Chiastoneury, 328

Chigoe, 43 I

Chilina, 336

Chilognatha, 433

Chilomonas, I 8 I

Chilomycterus, 51 2

Chilopoda, 402

Chilostomata, 287

Chimæra, 507

Chimpanzee, 574

Chinch bug, 428

Chiromys, $57^{2}$

Chiroptera, 564

Chirotes, 530

Chitin, $35^{\circ}$

Chitonidæe, 315

Chlamydosaurus, 529

Chlamydoselachus, 506

Chlamydozoa, I9I

Choana, 475

Choanoflagellata, $18_{4}$

Cholœpus, 563

Chondrilla, 203

Chondrin, 77

Chondrioderma, I8o

Chondrocranium, 455

Chondropterygii, 504

Chondrostei, 508

Chone, 277

Chorda rlorsalis, 438

Chordata, $43^{8}$

Chordodes, 268
Chordotonal sense organs, 410

Chorioidea, I 20

Chorioid plexus, 470

Chorioid coat, 476

Chorion, 134, 413, 561

Chromatin, 56

Chromatophores, 339

Chromidial apparatus, 58

Chromosome, accessory, 73 reduction, 134,138

Chromosomes, 60 conjugation of, I 39 function of, 137

Chrysalis, $43^{2}$

Chrysaora, I 24

Chrysomelidx, 424

Chrysomitra, 2 Io

Chrysomonadina, 184

Chyle, 104, 486

Chyle vessels, $\mathrm{IO}_{4}$

Cicada, 428

Cicindelidx, 424

Ciconia, 544

Cidaridea, 305

Cilia, 65, I I I, 102

Ciliary process, I 2 I

Ciliata, I0I

Ciliated epithelium, $6_{5}$

Cilioflagellata, I 84

Cimbex, 425

Cinclides, 226

Ciona, 444

Circulatory apparatus, 99

Circumvallate papillæ, 474

Cirolana, 386

Cirri, 276,299

Cirripedia, $37 \mathrm{I}$

Cirrus, I09, 243

Cistenicles, 277

Cistudo, 527

Citigrada, 396

Civets, 565

Cladocera, 366

Cladoselachii, 507

Clamatores, 544

Clathrulina, 174

Clava, 217

Clavellinidæ, 445

Clavicle, 464

Claws, 450, 545

Cleavage cavity, 145 of egg, I4 I-I 45 nucleus, 135

Cleithrum, $49 \mathrm{~s}$ 
Clepsidrina, $\mathrm{I}_{7}$

Clepsine, 284

Clibanarius, $38 \mathrm{I}$

Clidastes, $53^{\circ}$

Clione, 335

Clisiocampa, 433

Clitellio, 270

Clitellum, 278

Cloaca, 96, 203, 445, 48 I

Closed blood system, IO3

Clothes moth, 432

Clupeidæ, 5 Iо

Clymene, 278

Clypeaster, 304

Clypeus, 405

Clytia, 2 I 8

Cnemidophorus, $53^{\circ}$

Cnidie, 207

Cnidaria, 206

Cnidocill, 207

Cobra, 53I

Coccida, 428

Coccidix, ISS

Coccidium, IsS

Coccinellidis, 424

Coccus, 428

Coccygus, 544

Cochlea, I I $7,+78$

Cochineal, 428

Cockatoos, $5+4$

Cockroaches, 42 I

Cod, 5 II

Codlin moth, 432

Codosiga, $\mathrm{I}_{4}$

Coelenterata, 206

Coelenteron, 206

Colhelminthes, 260

Coelom, 99 of vertebrates, 480

Crelomic pouches, I 48

Coeloplana, 235

Coeloria, 23 I

Colopleurus, 303, 305

Conosarc, 209

Conurus, 252

Cold blood, I0.4

Cold rigor, 55

Coleoptera, 423

Collaterals, $s_{4}$

Collembola, +10

Collozoum, 177

Colon, ton

Colony formation, I 53

Coloborhombus, 40

Colorado beetle, +24
Coloration, sympathetic, 37

Colossendeis, 399

Colossochelys, 527

Colubriformia, 53I

Columba, $5+3$

Columella, 229, 326, 46I, 529

of mycetozoa, ISo

Columns of cord, 468

Colymbus, $5+3$

Comatulidx, 302

Commissures, I I 3

Complemental males, 373

Compound eyes, 354

Conchiolin, 3 I I

Conchoderma, 3 I6

Condylarthra, $5^{6} 7,5^{6} 9$

Condylura, 564

Coney, 570

Conidxe, 334

Conjugation, I69 of ciliata, I 93

Conjunctiva, I 2 I, 477

Connectives, II 3

Connective tissues, 74

Conocephalus, +2 I

Conocladium, IS4

Conotrachelus, +24

Contractile fibre cells, 82 substance, So vacuoles, I 67

Conurus, 544

Conus arteriosus, 503

Convergent development, I 58

Copelatæ, 443

Copepoda, 366

Copperhead, 53 I

Copula, 460

Copulation, $13+, 169$

Coral, 228

Corallium, 230

Coral reefs, 229

Coral snake, 53 I

Coraciformes, $5+4$

Coracoid, 464

Coracoid process, $55^{\circ}$

Coregonus, 5 Io

Corium, 9I, 450

Cormorant, $5+3$

Cornacuspongia, 205

Cornea, I I 9, 476

Corona, 303

Coronata, 224

Coronula, 374
Corpora quadrigemini, $47^{\circ}$

Corpus collosum, 55 I

Corpus ciliare, 120

striatum, 469

Corpuscles, blood, 78

connective tissue, $7+$

directive, ${ }^{3} 32$

Grandry's, 473

Krause's, 473

lymph, 79

Malphigian, I05

Meissner's, I I 5

Miescher's, I 9 I

muscle, so

Rainey's, I9I

tactile, 473

Vater-Pacinian, I I 5, 474

Corrodentia, 4I9

Corticum, 205

Cortis' organ, $55^{2}$

Corvus, $54+$

Corycæida, 369

Corydalis, 422

Corymorpha, 2 I 7

Costæ, 229

Cotingidæ, $5+4$

Cotton worm, 433

Cottus, 5 I I

Coturnix, 543

Cougar, 565

Covering scales, 2 I 9

Coverts, $53+$

Cowries, $33+$

Coxa, $40_{5}$

Coxopodite, 97

Crabs, 382

Crampton's muscle, $54^{\circ}$

Crane flies, 430

Cranes, $54+$

Crangon, 380

Crania, 290

Cranial nerves, $47 \mathrm{I}$

Cranium, 455

Craspedon, 2 I I

Craspedotella, I 85

Crassilinguia, 529

Crayfish, 380

Creodonta, 566

Cribrellum, 396

Crickets, +2 I

Crinoidea, 299

Crisia, 287

Crista acustica, I I 7,352 , 478

Crocodilia, 53 I 
Crop, 94, 408

Crossbills, 544

Crossing of species, 2 I

Crossopterygii, 50 \&

Cross-striated muscle, i I

Crotalus, 53 I

Crows, 544

Crura cerebri, 55 I

Crustacea, 359

Cryptobranchus, 520

Cryptochiton, 3 I 6

Cryptodira, 527

Cryptoniscus, 386

Cryptotetramera, 424

Crystalline style, 322

Ctenida, 387

Ctenidia, 3 I 2

Cteniza, 396

Ctenodiscus, 298

Ctenoid scales, 494

Ctenolabrus, 5 ro

Ctenophora, 232

Ctenoplana, 235

Ctenostomata, 287

Cubical epithelium, 65

Cubomedusæ, 224

Cuculi, 544

Cucumaria, 308

Culcita, 296, 298

Culicidæ, 430

Cumacea, $3^{82}$

Cunina, 2 Is

Cunner, 5 Io

Cunocantha, 2 I6, 2 I 8

Curculio, 424

Cursoria, 42 I

Cuspidaria, 325

Cutaneous artery, $5 \mathrm{I} 7$

Cuticle, 65

Cuticula, I67

Cuticular skeleton, I I I

Cutis, 9I, $45^{\circ}$

Cuttle bone, 34I, 347

Cuttlefish, 347

Cuvierian ducts, $4 \mathrm{~S}_{4}, 503$ organs, 307

Cyamus, $3^{84}$

Cyanea, 223

Cyanocitta, 544

Cyclas, 324

Cycloid scales, 494

Cycloposthium, I97

Cyclops, 20, 360

stage, 29

Cyclospondyli, 506
Cyclostomata, $287,49 \mathrm{I}$

Cydippidax, 235

Cygnus, 543

Cymothoa, 386

Cynipidæ, 425

Cynocephalus, 574

Cynomorphæ, 574

Cynomys, 566

Cynthia, 445

Cypræa, 330

Cypridina, $37 \mathrm{I}$

Cyprinicle, 5 ro

Cypris, $37^{\mathrm{I}}$

Cypselida, 544

Cyrtophilus, $42 \mathrm{I}$

Cysticercoid, 253

Cysticercus, 247

Cystid, 285

Cystidea, 303

Cystoflagellata, I 84

Cystoneciae, 220

Cytopharynx, I67, 192

Cytopyge, 167, I92

Cytosporida, $\mathrm{I}_{5}$

Cytostome, 167 , I92

Dactylethra, 520

Dactylopodite, 97

Daddy long-legs, 394

Daphnia, 366

Dasypus, 563

Dasyurus, 560

Datames, 394

Decapoda, 347,377

Decidua, $5^{62}$

Deer, 569

Degeneration, I 56

Delphinus, 572

Demibranchs, $50 \mathrm{r}$

Demodex, 397

Dendrites, 84

Dendrochirotx, 308

Dendroccelum, 242

Dendrœca, 544

Dendronotus, 335

Dendrosoma, i 98

Dentary bone, $46 \mathrm{I}$

Dental formula, 554

Dentalium, 325

Dentes complicate, 554

Denticetie, 572

Dentine, 45I

Dentition, 48 I, 553

Derma, OI, $45^{\circ}$

Dermal skeleton, 9 I
Dermanyssus, 397

Dermatobia, 431

Dermatoptera, 42 I

Dermochelys, 527

Dermo-muscular tunic, I I I, 237

Dero, 279

Derotrema, 520

Desmodont, $3 \mathrm{IS}$

Desmodus, 565

Desor's larva, 257

Determinants, 43 , I 37

Deutomerite, I86

Deutocerebrum, 405

Deutoplasm, 7I

Diapheromera, 42 I

Diaphragm, 48I, 555

Diapophysis, $45+$

Diarrhcea, 196

Diastole, Iо I

Diastylis, $3^{8} 2$

Diaptomus, 369

Dibranchia, 347

Dicotyles, 568

Dicyemida, 200

Didelphia, 559

Didelphys, 560

Didus, 543

Diencephalon, 460

Difflugia, I 79

Diffuse nervous system, I I 2

Digenea, 245

Digestive tract, 93

Digitigrarle, $55^{\circ}$

Dihybrids, I 40

Dimorphism, sexual, 40, I69

Dimorphodon, 532

Dimyaria, 323

Dinichthys, $5^{\mathrm{I}} 3$

Dinobryon, I 84

Dinoceras, 570

Dinoflagellata, I 84

Dinornithidæ, 542

Dinosauria, 528

Dinotherium. $57^{\circ}$

Diøecious, Io8

Diomedea, 543

Diopatra, 277

Diotocardia, 333

Diphasia, 2 Is

Diphycercal, 32,498

Diphyes, 220

Diphyodont, 553

Diplocardia, 279

Diplopoda, 433 
I) iplospondylii, 506

1)iplozoon, 154,244

1)ipneumonia, $5^{1} 3$

Dipneumones, 346

I)ipneusti, $5^{12}$

Dipnoi, 5 I2

Diporpa, I 54, 244

Diprotodon, $56 \mathrm{I}$

Diprorlonta, 56 I

Diptera, 430

Dipylidium, 255

Directive corpuscles, 131

Directives, 227

Discina, 200

Discorlernia. 204

Discorrrilidx, 270

Discomedusæ, 223

Disconanthæ, 220

Discophori, 28I .

Dispermy, I35

Distaplia, $44^{6}$

Distomeæ, 245

Distomum, I05, 243, 246

Distribution of animals, 33

Disuse of parts, 47,89

Division of labor, 63,155

Division, reproduction by, I 28

Divaricator muscles, $28 \mathrm{~S}$

Dochmius, 266

Docoglossa, 333

Doctrine of causes, 34

Dodo, 543

Dogfish, 506

Dogs, 565

Dolichonyx, 544

Doliolum, 4.48

Dolomedes, 396

Dolphins, 572

Dominant, $\mathrm{I}_{3} \mathrm{~S}$

Dondersia, 3 I 6

Doris, 335

Dorsal aorta, $48_{3}$

fin, 462,497

root, 468

Dorylina, 427

Doryphora, 424

Double animal, I $5+$

Draco, 529

Dranculus, 269

Drepanirlotienia, 25.5

Dromieus, $5+2$

Dromatherium, 559

I) rones, $4 \mathrm{I} 7$

Duckbill, 559
Ducks, 543

Ducts of cuvier, 484 genital, Ios

Botallii, $4 \S_{5}, 5^{\text {I }} 8$

cochlearis, 479

choledochus, $4^{81}$

ejaculatorius, 109

Dugong, $57^{\circ}$

Duodenum, 554

Duplicidentata, 566

Dura mater, 473

Dysodont, 3 I 8

Dytiscidæ, 424

Eagles, 544

Ear bones, 46r, 479

Ear conch, 4 Eo

Ear drum, 479

Ears, I 16

vertebrate, 477

Earth worms, 279

Earwig, 42 I

Ecardines, 200

Echidna, 559

Echinarachnius, 306

Echinobothrium, 254

Echinocardium, 306

Echinoderide, 260

Echinoderma, 29I

Echinoidea, 303

Echinorhynchus, 269

Echinosphærites, $30_{3}$

Echiuroidea, $28 \mathbf{I}$

Echiurus, 28I

Ecdysis, $35^{\circ}$

Eciton, 427

Ectoblast, I 46

Ectobronchi, $53 \mathrm{~S}$

Ectocyst, 285

Ectoderm, 9I, 94, I46

Ectethmoid, $45^{8}$

Ectoparasites, I 57, 242

Ectoprocta, 285

Ectosarc, 172

Edentata, $5^{6} 3$

Edrioasteroidea, 302

Edriophthalmata, $38_{3}$

Edwardsia, 226, 230

Edwardsiella, 230

Eels, 5 Io

Efferent branchial arteries, 504

Egg cell, 7 I

cleavage of, IfI

fertilization of, $\mathrm{I} 34$
Egg, maturation of, 132 nucleus, $\mathrm{I}_{32}$

sacs, 369

segmentation of, I 4 I

tooth, 525

Eimeria, 188

Eimer's organs, 473

Elaps, 53 I

Elasipoda, 308

Elasmobranchs, 504

Elastic cartilage, 77

Elastic tissue, 76

Elastica externa, $45^{2}$

Elastin, 75

Elaters, I 8 I

Electric organs, I I I

Elephant, 5\%०

Elephantiasis, 268

Elephas, $57^{\circ}$

Eleutheria, 200

Elysiidæ, 335

Elytra, 276, 408

Embiotocidae, 5 I I

Embryo, I 50

Embryology, I 27

Emu, 542

Enamel, 45I, 494

Enchytrxida, 279

Endites, 36r

Endocyst, 285

Endolymph, II6, 479 duct, 477

Endopodite, 360

Endostyle, 443

English sparrow, 544

Enhydris, $5^{6} 5$

Ensatella, 324

Entalis, 325

Entamoba, I73

Entelops, $5^{6} 3$

Enterocole, 99, 480

Enteropneusta, 448

Enteroxenus, 308

Entoblast, I 46

Entobronchi, $53^{8}$

Entocolax, 308

Entoconcha, 308

Entoderm, 94, I.46

Entomostraca, $3^{6} 3$

Entoniscus, 385,386

Entoparasites, 1 57, 242

Entophaga, 425

Entoplastron, 527

Entoprocta, 284

Entosarc, I72 
Entosternite, 389

Entovalva, 308

Entwicklungsmechanik, 3

Environment, influence of, 47

Enzymes, 96

Eohippus, 569

Eozoon, I64

Epaxial muscles, $45+$

Epeira, 396

Ependyma, I I 4, 467

Ephemera, 420

Ephippium, 366

Ephydatia, 205

Ephyra, 22I

Epibdella, 244

Epiblast, I 46

Epidermis, 9I, 450

Epididymis, $28_{3},{ }^{\text {Se }}$

Epigenesis, I 2

Epiglottis, 482, 555

Epitoke, 275

Epimeral plates, $3^{8}+$

Epimerite, I 86

Epiotic, $45^{8}$

Epipharynx, 405

Epiphragm, 327

Epiphysis, 470

Epipleurals, 509

Epipodite, 36I

Epipodium, 325

Epipterygoid, 529

Episternum, 46.4, 5I3

Epistropheus, 522

Epistylis, I97

Epitheca, 229

Epithelial muscle, 8I

Epithelium, 64 germinal, 70, 107

Epizoanthus, I 58, 23 I

Equatorial plate, 60

Equus, 568

Erax, 43I

Erethizon, 566

Ergatoids, 4I 7

Ergatomorphs, 417

Erinacida, 564

Erichthus, 376

Erigone, 396

Ermine, 565

Errantia, 277

Erythroneura, 428

Eschara, $2 S_{7}$

Esocidæ, 510

Esperia, 205
Essence of pearl, 494

Estheria, 366

Ethmoidalia, $45^{8}$

Ethmoid bone, 459

Euconous eyes, $35^{6}$

Eucopepoda, 360

Eucrinoidea, 302

Eucyrtidium, 177

Eudendrium, 2 IO, 2 I 7

Eudoxia, I 55

Eutlagellata, I $\delta_{4}$

Euglena, I $8+$

Eugyra, 446

Euisopoda, 386

Eumeces, 530

Eunectes, 53I

Eunice, 275

Eunicidie, 277

Eupagurus, $38 \mathrm{I}$

Euphausiiclæe, 376

Euplectella, 205

Euplexoptera, 42 I

Eupyreme, 73

Euryalidx, 299

Eurypterida, 388

Eurypterus, $3^{88}$

Eusclachii, 506

Euspongia, 205

Eustachian tube, 479

Eusuchia, 532

Eutainia, 53 I

Evadne, 366, 368

Everyx, 433

Evolution, convergent, ${ }_{5} \mathrm{~S}$ theory of, It

Excreta, $6_{5}$

Excretory organs, I0.4 of vertebrates, 486

Exites, 36I

Exoccipital, $45^{8}$

Exocœtidx, 5 io

Exopodite, 360

Extracapsulum, I 75

Exumbrella, 2 I I

Exuviation. $35^{\circ}$

Eye-muscle nerves, 472

Eyes, II9, 354, 475

Facial nerve, $47 \mathrm{I}$

Fairy shrimp, 366

Falciform spores, I $8 \mathrm{~S}$

Falcons, 54t

Farrea, 205

Fasciolaria, 246

Fat body, 409, 5r8
Faunal regions, 160

Favia, 231

Favositiclix, 230

Feathers, 533

Feather tracts, 534

Fecampia, 24I

Fecundation, I34

Felis, 565

Femoral pores, $52 \mathrm{I}$

Femur, 405, +65

Fenestra rotunda, 525

Ferments, 06

Fertility of hybrids, 22

Fertilization, I34

Fertilization and reproduction, I 49

Fiber, 566

Fibrin, 78

Fibrous connective tissue, 75

Fibula, 465

Fibulare, 465

Fiddler crab, $3^{82}$

Filaria, 268

Filar substance, 53

Filibranch, 320

Finback, $57^{2}$

Finches, 547

Fins, 462,497

Fire flies, +24

Firmisternia, 520

Firmisternous, $5^{\mathrm{I}} 3$

Fishes, 493

Fish hawk, $5+4$

Fissilinguia, 529

Fissipedia, 565

Fissurellid $\mathfrak{3}, 33$

Fissures of cord, 468

Flabellum, $36 \mathrm{I}$

Flagella, 65, I I I

Flagellata, I 8 I

Flagellated epithelium, 65

Flame cells, I $_{5}$

Flaming $3,5+4$

Flat fish, 5 I I

Flat worms, $23 \mathrm{~s}$

Fleas, 43I beach, $3^{84}$

Flesh flies, 43 I

Flies, 430

black, 430

bot, $43 \mathrm{I}$

blow, 43 I

caddis, 422

crane, $43^{\circ}$ 
Flies, dragon, 42C

fire, 424

flesh, 43 I

Hessian, $43^{\circ}$

horse, 430

house, 43 I

May, 420

robber, $43 \mathrm{I}$

saw, 425

Spanish, 424

Flounders, 5II

Flowers of $\tan$, I 8 I

Flustra, 287

Flying fish, 5 Io

foxes, $5^{6} 5$

squirrels, 566

Forlientia, ${ }_{5} 63$

Follicle mite, 397

Fontanelle, 457 .

Food vacuoles, 167, 192 yolk, 7 I

Foot, molluscan, 3 I I

Foramen magnum, $45^{8}$

ovale, 479

Panizze, 524

Foramina repugnatoria, 433

Foraminifera, 178

Fore-brain, 468

Fore-gut, 94

Forficula, 42 I

Formicariæ, 426

Fossa rhomboidalis, 470

Fossores, 426

Fowl, 543

Foxes, 565

Fringillidæe, 544

Frogs, 520

Frons, 405

Frontal bones, 459 plane, I 25

Frontoparietals, 5I4

Fungiacea, 232

Funiculus, 286

Furca, 366

Furcula, 535

Gadus, 5I I

Girtner's canal, 489

Galea, 406

Galeodes, 394

Galeopithecus, 564, 573

Galeus, 506

Galley worms, 402

Gallinacea, 543
Galls, 425

Gallus, 543

Galton's curve, 45

Gammarina, 384

Gammarus, 384

Gamasus, 397

Gametocytes, I 86

Ganglia, 86, I I 3

Ganglion, Gasserian, 472

geniculate, 472

optic, 476

otic, 473

sphenopalatine, 473

stellate, 343

cells, $s_{3}$

Ganglionic nervous system, I 13

Ganoidei, 507

Ganoid scales, 494

Ganoin, 494

Gapes, 266

Garpike, 508

Garter snakes, 53 I

Gas glands, 502

Gasserian ganglion, 472

Gastralia, $53^{2}$

Gastral pouches, 99

Gasteropoda, 325

Gasterosteirla, 5 I I

Gastric cæca, 409

Gastrogenital pockets, 22 I

Gastrophilus, 43 I

Gastrotricha, 260

Gastrovascular cavity, 206 system, 99

Gastrula, I 45

Gazella, 560

Gecarcinidx, ${ }_{3} \mathrm{~S}_{2}$

Gecko, 529

Gelasimus, $3^{8} 2$

Gelatine, 76

Gemmation, 59

Gemmellaria, 287

Gemmulæ, 205

Genæ, 364, 405

Generation, I27 spontaneous, 127

Generations, alternation of, I3 I

Geniculate ganglion, 472

Genital ducts, I08 plates, 304

Genotypic character, 45

Geocores, 428

Geodia, 204
Geographical distribution, 33,160

Geological distribution, I64

Geometrina, 432

Geonemertes, $25^{8}$

Geophilus, 403

Gephyræa, 279

Gerardia, $23 \mathbf{I}$

Germ layers, I 45

and budding, 148

Germinal disc, I 43

Germinal epithelium, 70 , 107

glands, 107

selection, 43

spot, 72

vesicle, 72

Geryonia, 2 I4, 2 I8

Giant cells, 62

Gibbon, 574

Gila monster, 530

Gill arches, 460

arteries, IOI

books, 387

clefts, $98,438,482$

veins, IOI

Gills, 97

tracheal, 402, 4II

of vertebrates, 482

Gigantostraca, $3^{8} 7$

Gipsy moth, 433

Giraffa, 569

Girdles, 463

Gizzard, 95, 409

Glabella, 364

Gland cells, 68

Glands, 68

anal, 96, 565

gas, 502

germinal, Io

green, $35^{8}, 36 \mathrm{I}$

Harder's, 477

lacrimal, 477

lymph, 486

lymphoid, 293

mammary, 546

milk, 546

nidamental, 344

ovoid, 293

paraxon, 293

repugnatorial, 434

salivary, 96

sebaceous, 546

sexual, I07

shell, $35^{8}, 3^{62}$ 
Glands, sweat, 5.6 subneural, $4+45$ thymus, 482 thyreoid, 482 yolk, 1 I 0

Glandular epithelium, 6.3 Glass snake, 530

Glia, I I 4

Globigerina, I 79

Globiocephalus, 572

Glochidia, 322

Glomeridax, 434

Glomeruli, 105, 486

Glomus, ${ }_{4} \mathrm{~S} 7$

Glossa, 406

Glossina, 43 I

Glossobalanus, +49

Glossopharyngeal nerve, $47^{\mathrm{I}}$

Glottis, 482

Glue, 76

Glugea, I9I

Glutin, 76

Glyptodontidæ, 563

Gnathobdellidæ, $2 \mathrm{~S}_{4}$

Gnathochilarium, 402, 434

Goats, 560

Goblet cells, 68

Gonads, 107

Gonapophyses, 408, 4I 3

Goniale, $5+9$

Goniatites, 346

Goniodes, 420

Gonochorism, 107

Gonodactylus, 376

Gonophore, 2 I 6

Goose, 543

Goose barnacle, 373

Gordiaceæ, 268

Gordius, 268

Gorgoniidæ, 230

Gorilla, $57+$

Gradientia, 5I9

Grandry's corpuscle, 4.73

Grallatores, $5+3$

Grantia, 204

Graptolites, 2 I 8

Gray matter, I 14, 468

Gray nerve fibres, $8_{5}$

Grebes, 543

Green gland, $35^{8}, 36$ r

Gregarina, I 86

Gressoria, 42 I

Gribble, 386

Gromia, 54
Grouse, 543

Grus, 5+4

Gryllotalpa, 42 I

Gryllus, 42 I

Grypotherium, 563

Guanin, 494

Guinea pig, 566

Guinea worm, 268

Gula, 405

Gular plates, 508

Gulls, 543

Gunda, 2.2

Gymnoblastea, 2 I 7

Gymnodonta, 5 I 2

Gymnolæmata, 287

Gymnophiona, 5 I 9

Gymnosomata, 335

Gynandromorphism, ros

Gyri, 470

Gyrodactylus, 244

Habrocentrum, 396

Haddock, 5 I I

Hadenœcus, 42 I

Hremadipsa, 284

Hæmal arch, 453

ribs, 454

spine, 453

Hæmamaba, 188

Hæmapophysis, 453

Hæmentaria, $28+$

Hæmocaele, roo, ro3

Hæmocyanin, So

Hæmoerythrin, So

Hæmoglobin, 78

Hæmoglobinuria, I 90

Hæmosporida, I 88

Hag fishes, 49I

Hair, 545

Hair-neck worms, 267

Hair worms, 268

Halcampa, 23 I

Haliætus, 544

IIalibut, 5I I

Halicore, $57^{\circ}$

Halionma, I 24

Haliotis, 327, 333

Halteres, 430

Halyclystus, 224

Hammer head, 506

Hapale, 574

Haploceras, 560

Harder's gland, 477

Hares, 566
Harpactide, 369

Harvestmen, 394

Haustellum, 407

Haversian canals, 77

Hawk moths, 433

Hawks, 544

Hazel weevil, 424

Head kidneys, 105,487

Heari, roo

Heart urchins, 306

Heart of vertebrates, 483

Heat rigor, 55

Hectocotylus, 34.4

Hedgehogs, 564

Heliaster, 208

Helicidæ, 336

Heliconiids, 39

Helioporidæ, 230

Heliozoa, 173

Helix, 336

Hellbender, 520

Hellgrammite, 422

Helminthophaga, 544

Heloderma, 530

Hemerobiidæ, 422

Hemibranchii, 5 II

Hemichordia, 448

Hemimetabolous, 4I4

Hemiptera, $42 \mathrm{~S}$

Hemitripterus, 5 I I

Hen clam, 324

Hens, 543

Hepato-pancreas, 96, 36I

Hepatus, 382

Heptanchus, 506

Heredity, $\mathbf{1}_{3} 6$

basis of, $58, I 37$

Hermaphroditism, 107

Hermit crabs, 380

Herons, 544

Herpestes, 565

Herring, 510

Hesperornis, 54 $\mathrm{I}$

Hessian fly, 430

Heterakis, 266

Heterocercal, 32, 498

Heteroconchæ, 324

Heterocotylea, 244

Heterodera, 266

Heterodont hinge, 3 I 8

Heterogony, I32

Heteromera, 424

Heteromyaria, 323

Heteronemertini, $25^{8}$

Heteronereis, 275 
Heteronomous segmentation, I 26

Heteropleuron, 44I

Heteropodia, 334

Heteroptera, 428

Heterosyllis, 275

Heterotricha, I 96

Heterozygote, I38

Hexacoralla, 230

Hexactinellidæ, 205

Hexanchus, 506

Hexapoda, 403

Hind brain, 468

Hind-gut, 94

Hinge ligament, 3 Is

Hipparion, 560

Hippasteria, 298

Hippidx, $3{ }_{3}$ I

Hippocampus, 5 I I

Hippolyte, 380

Hippopotamus, 568

Hippospongia, 205

Hirundo, 544

Hirudo, 284

Hirudinei, 28 I

Histology, 63

Holoblastic, I 42

Holocephali, 507

Holocystites, $30_{3}$

Holometabolous, 4I 5

Holostei, 508

Holostome, 327

Holothuria, 308

Holothurioidea, 306

Holotricha, 195

Homarus, 380

Homaxial animals, I 23

Homo, 574

Homocercal, 32, 498

Homodont, 553

Homogeneous connective tissue, 75

Homology, 10, 90

Homonomous segmentation, 126

Homoptera, 428

Homothermal, I04

Homozygote, I38

Hoofs, 450,545

Hookworm, 267

Hopworm, 433

Hormones, I Io

Hormophora, 233, 235

Horn bills, 544

Horned toads, 520
Hornera, 287

Horntails, 425

Horns of cord, 468

Horse, 568

ancestry of, 569

flies, $43^{\circ}$

sponge, 205

House flies, 43I

Humerus, 465

Humming birds, $5+4$

Hyæna, 565

Hyaleida, 335

Hyaline cartilage, 77

Hyalonema, 20.4

Hyalospongia, 204

Hyas, 382

Hybrids, 2 I

fertility of, 22

Hydrophyton, 427

Hydra, I 29, 208, 2 I 7

Hydrachna, 397

Hydractinia, 2 I 7

Hydranth, 209

Hydraria, 2 I 6, 2 I 7

Hydrobatidx, 428

Hydrocaulus, 209

Hydrochœrus, 566

Hydroccele system, 294

Hydrocorallinæ, 2 I 7

Hydrocores, 428

Hydroid, 209

Hydroides, 278

Hydromedusæ, 208

Hydrophidæ, 53 I

Hydrophilide, 424

Hydrophobia, I 9 I

Hydropolyp, 207

Hydropsyche, 422

Hydrorhiza, 209

Hydrotheca, 209

Hydrozoa, 208

Hyla, 520

Hylobates, 574

Hylodes, 5 I 9

Hymenolepis, 254

Hymenoptera, 424

Hyocrinus, 300

Hyoid arch, 460

Hyomandibular, 460

Hypena, 433

Hypaxial muscles, 454

Hyperia, $3 s_{4}$

Hyperina, ${ }_{3} S_{4}$

Hyperoartia, 492

Hyperotretia, 492
Hypoblast, I 46

Hypobranchial groove, 440

Hypoderna, 43 I

Hypodermis, 350

Hypogeophis, 519

Hypoglossal nerve, 47 I

Hypopharynx, 405

Hypophysis, $47^{\circ}$

Hyporachis, 534

Hypotricha, 197

Hyracoidea, $57^{\circ}$

Hyracotherium, 569

Hyrax, $57^{\circ}$

Hystrix, 566

Hystricomorphia, 566

Ibis, 544

Ichneumonidæ, 426

Ichneumons, 565

Icterus, 544

Ichthydium, 260

Ichthyobdella, 284

Ichthyodolurites, 505

Ichthyophis, 5 I 9

Ichthyopsida, 49I

Ichthyornis, 54 I

Ichthyosauria, 526

Ichthyotomi, 507

Idioplasm, I 37

Idiothermal, Io4

Idotea, $3^{86}$

Iguanidæ, 529

Ilium, 464

Imaginal discs, $4 \mathrm{I} 7$

Imago, 4I 4

Impennes, 543

Imperforata, 79

Incus, $46 \mathrm{I}$

Indirect development, I $5^{\circ}$

Inermes, $28 \mathrm{I}$

Infrabasal, 300

Infraorbital ring, 497

Infundibula, 482, 555

Infundibulum, $47^{\circ}$

Ingluvies, 94, 408

Inheritance, Mendelian, ${ }_{1} 3^{8}$

Inia, $57 \mathrm{I}$

Inquilines, 425

Insecta, 400

Insectivora, $5^{64}$

Integripalliata, 324

Interambulacral plates, 206

Intercalaria, 453, 495

Intercellular substance, 74 
Interfilar substance, 53

Interhyal bone, 496

Intermaxillaries, $46 \mathrm{I}$

Intermedium, 465

Internal secretions, 110

Interparietal bone, $54^{\circ}$

Interrachii, 124, 293

Interse',tal space, 227

Interve cebral cariilage, 455

ligaments, $55^{\circ}$

Intestine, 95

Intraseptal space, 227

Intratarsal joint, 524

Involuntary muscles, 8 I

Iris, 120,476

Ischiopodite, 97

Ischium, 464

Isodont, 3 Is

Isogametes, I6S

Isopoda, $3^{8} 5$

Isoptera, 4I 9

Itch, 397

Ixodes, 397

Jacobson's organ, 475

Japyx, 4I9

Jassidee, $42 \mathrm{~S}$

Jays, 544

Jigger flea, 43I

Jugal bone, 462

Jugular vein, $4 S_{4}$

Julus, 434

June bugs, 424

Kallima, $3^{8}$

Kang roos, 561

Karyakin esis, 60

Katyclid, 42 I

Keratin, 67, 205

Keyhole limpet, 333

Kidneys, 105, 488

Kingfishers, 544

Kinosternon, $5^{27}$

Knee pan, 465

Konenia, 393

Krause's corpuscle, 473

Labial cartilages, $\$ 60$ palpi, 320

Labium, 405

Labor, division of, 63, I 26

Labrida, 5 io

Labrum, 405

Labyrinth, I I $7,478,479$

Labyrinthodonta, 5 19
Iacerta, 530

Iacertilia, 529

Lace wings, 422

Lacinia, 406

Lacrimal gland, 477

lacunar blood system, Io3

Ladier nervous system, I 3

Lady birds, $\$ 2.4$

Læmodipoda, 384

Lag $\cap$ a, $\operatorname{II} 7,47 \mathrm{~S}$

I agomorpha, $5^{66}$

Lagomys, 566

Lamarckism, 46

Lamblia, I ${ }_{4}$

Lamellibranchiata, $3 \mathrm{I} 7$

Lamellicornia, 42.4

Lamellirostres, 543

Lamna, $451,5+6$

Lamprey eels, $49 \mathrm{I}$

Lampyridæ, 424

Land crabs, 382

Lantern of Aristotle, 305

Larus, $5+3$

Larva, I50, 41 4

Larval organs, $I_{50}$

Larynx, 4S2, $5^{\mathrm{I} 6}$

Laryngeal cartilages, $46 \mathrm{I}$

Lateralia, $37^{2}$

Lateral line organs, 499

Lateral organs, $27 \mathrm{I}$

Latrodectes, 396

Laurer's canal, $3+4$

Lazy worm, 267

Leaf hoppers, $42 \mathrm{~S}$

Leather turtles, 527

Leda, 323

Leeches, 28I

Lemnisci, 269

Lemurs, $57^{2}$

Lens, $x \times 9,477$

Lepas, 159,373

Lepidurus, 366

Lepidonotus, 277

Lepidoptera, 432

Lepidosauria, $52 \mathrm{~S}$

Lepidosiren, 5I3

Lepidosteus, 508

Lepisma, 4 IS

Leptasterias, 298

Leptalis, 39

Leptocardii, 439

Leptocephalus, 510

Ieptochela, 386

Leptoclinum, 446

Leptodiscus, I $_{5}$
Leptodora, 366

Leptomedusx, 2 I 7

Leptoplana, 2.42

Leptostraca, 375

Lepus, 566

Lernxa, 370

Lernæocera, 370

Lernæopodidæ, 370

Leucetta, 203

Leucocytes, $7 \mathrm{~s}$

Leucon, 203

Leucones, 20.4

Leucortis, 20.4

Leucocytozoon, $\mathrm{I}_{3}$

Leucosoidea. $38_{2}$

Leucosolenia, 204

Libellula, 420

Libinia, $3^{82}$

Lice, bee, 43I bird, 420

book, 420

human, 430

plant, 430

Lieberkühn's glands, 69

Lieshmania, ${ }^{8} 8_{4}$

Life, origin of, 127

Ligulidx, 253

Limacidæe, 336

Limacinidæ, 335

Limax, 336

Limbs, skelton of, 462

Limicola, 279

Limnadia. 366

Limnæa, 336

Limnoria, $3^{86}$

Limpets, 333

Limulus, 387

Linckia, 206

Linear nervous system, II 2

Lines of growth, 317

Lineus, 258

Lingual ribbon, 314, 329

Linguatulida, 397

Lingula, 290

Linin, $5^{6}$

Linnean system, 7

Liobunum, 394

Lion, 565

Lips, 554

Liquor cerebrospinalis, 467

Liriope, 216,2 Is

Lithistida, 204

Lithobius, 403

Lithodidie, $3^{82}$

Lithodomus, 323 
Lithoglyphus, 327

Littorinidla, 334

Liver, $96,48 \mathrm{I}$

Liver fluke, 243,240

Lizards, 529

Llama, $5^{69}$

Lobatæ, 235

Lobi inferiores, 499

Lobosa, $\mathbf{7}^{2} 2$

Lobster, $3^{80}$

Locomotor organs, I Io

Locustidæ, 42 I

Locusts, 427

Loggerhead, $5^{2} 7$

Lohmanella, 200

Loligo, 336, 347

Longipennes, 543

Loons, 543

Lophobranchii, 5I I

Lophocalyx, I 23

Lophodont, 554

Lophogastridæ, 376

Lophophore, 287

Lophopoda, 287

Lophopus, 287

Lorenzini, ampullæ 500

Lorica, I 82

Loricata, $380,531,563$

Loricati, 5I I

Loris, $57^{2}$

Lota, 5 II

Loven's larva, 238

Loxia, 544

Loxosoma, 285

Lucernaria, 224

Lucia, 446

Lumbricus, 279 sexual organs, Io7

Lunatia, 334

Lung fishes, $5^{\text {I2 }}$ pipes, 538 sacs, 387

Lungs, $98,48_{2}$ of birds, 538

Lutra, $5^{6} 5$

Lycosa, 396

Lygxislæ, 428

Lymph, 78

glands, 486

hearts, 486

vessels, 103,486

Lymphoid gland, 293

Lyre birds, 5.14

Lyriform organs, 390
Macacus, 574

Machilis, 4 I9

Mackerel, 5II

Macoma, 32.4

Macrobdella, 284

Macrobiotus, 398

Macrochelys, 527

Macrochiroptera, 565

Macrodrili, 279

Macrogametes, I6?

Macronucleus, 192

Macropus, $56 \mathrm{I}$

Macrura, 380

Nactra, 324

Macula acustica, 477 lutea, I20

Madrepora, $23 \mathrm{I}$

Madreporaria, 231

Madreporite, 292

Maioidea, 382

Malaclemmys, 527

Malacobdella, $25^{8}$

Malacoderma, 23 I

Malacopoda, 399

Malacopteri, 5 Io

of, Malacostraca, 374

Malar bone, 462

Malaria, I88, 430

Maldanidx, 278

Male pronucleus, I35

Malleus, 46I

Mallophaga, 420

Malpighian bodies, 486 corpuscles, 105 tubes, $35^{8}, 3^{8} 3,3^{8} 7$, 402

Mammalia, 545

Mammary glands, 546

Mammoth, 5;o

Man, 574 embryo of, 27

Manatee, $57^{\circ}$

Manatus, $57^{\circ}$

Mandibles, 352, 406

Mandibula, 460

Mandibular arch, 460

Mandrils, $57+$

Manicina, 232

Manis, $5^{6} 3$

Mantis, praying, $42 \mathrm{I}$ shrimp, 376

Mantle, 3 I I, 338, $44^{2}$ cavity, 3 I I

Manubrium, 2I I

Manyunkia, 276

Margarita, 333
Margelis, I3I, 2 I 7

Marginal plates, 297

Marmosets, 574

Marsipobranchii, 49I

Marsupial bones, 560

Marsupialia, 559

Marsupium, $55^{8}, 560$

Martin, 565

Mastax, 259

Mastigamoba, 172

Mastigophora, I8 I

Mastodon, $57^{\circ}$

Matrix, 75

Maturation of egg, I32

Maturation divisions, I 39

Maxillæ, 352, 405

Maxillaries, $46 \mathrm{I}$

Maxillary palpus, 406

Maxillipeds, $35^{2}$

May flies, 420

Measuring worms, $43 \mathrm{I}$

Meatus, auditory, 480

Meckel's cartilage, 460

Mecoptera, 422

Medulla oblongata, 469

Medullary plate, 467 sheath, 85

Medullated nerve fibre, 85

Medusa, 208

Megalonyx, $5^{6} 3$

Megalops, 380

Megapodes, 543

Megascolex, 279

Megatherium, $5^{6} 3$

Meissner's corpuscles, I I 5

Melanoplus, $42 \mathrm{I}$

Meleagrina, 323

Meleagris, 543

Mellita, 306

Meloidæ, 424

Melolontha, 424

Melonites, 305

Melophagus, 43 I

Melopsittacus, 544

Membracidæe, 428

Membrana propria, 237

Membrane bone, 45I, 455

Membranellæ, I 96

Mendelism, 45

Mendel's Law, I3S

Meninx, 473

Menopoma, 520

Mentum, 406

Menuridæ, 544

Mephitis, $5^{6} 5$ 
Mermis, 268

Meroblastic, I 42

Merogamy, I37

Meropodite, 97

Merozoites, I 86

Meryhippus, 560

Mesectoderm, 202

Mesencephalon, 468

Mesenchyme, $74, \mathrm{I} 47$

Mesenterial filaments, 226

Mesenteries, 99, 480

Mesenteron, 94

Mesethmoid, $45^{8}$

Mesoblast, I 47

Mesoderm, 94, I.46

Mesoglcea, 208

Mesohippus, 569

Mesonemertini, $25^{8}$

Mesonephros, Io6, 487

Mesonotum, 404

Mesopleuron, 40.4

Mesopterygium, 464

Mesorchium, 480

Mesosternum, 404

Mesothelium, I 47

Mesothorax, 404

Mesotroche, 273

Mesovarium, $4 \mathrm{So}$

Metabola, I9I

Metacarpals, 465

Metagenesis, I3 I

Metamere, I 26, 269

Metamerism, I 26

Metamorphosis, I 5o, 4 I 4

Metanemertini, $25^{8}$

Metanephros, 488

Metanotum, 404

Metapleuron, 404

Metapodium, 325. 535

Metapterygium, 464

Metasternum, 404

Metastoma, 377

Metatarsals, $46_{5}$

Metathorax, 40.4

Metazoa, 201

Metencephalon, 468

Methona, 39

Metridium, 23 I

Miastor, $4 \mathrm{I} 4,430$

Mice, 566

Microcentrum, 42 I

Microchiroptera, 565

Microconodon, 559

Microcotyle, 244

Microdrili, 279
Microgametes, I60

Microlepidoptera, 432

Microlestes, 559

Micronucleus, I92

Micropylar apparatus, 134 , 413

Microthelyphonirlae, 393

Micrura, $25^{8}$

Miclas, 574

Midbrain, 468

Mid-gut, 94

Miescher's corpuscles, I0)

Migration theory, 45

Miliola, I79, I80

Milk glands, 546 teeth, 553

Millepora, 2 II, 2 I 7

Mimicry, 37

Mink, 565

Miohippus, 560

Miracidium, 246

Mites, 396

Mitochondria, $5^{8}$

Mitosis, 60

Mitrocoma, 2 I 4

Mixipterygium, 505

Mnemiopsis, 235

Moccasin, $53 \mathrm{I}$

Mole cricket, 42 I

Moles, 564

Molgula, 446

Molpadia, 308

Molting, 350

Mollusca, 3 Io

Molluscoida, $\mathbf{6} 66$

Monactinellidæe, 205

Monascidiæ, 445

Monaster, 60

Monaxial symmetry, I23

Monera, $\mathrm{I} \mathrm{z}$

Monezia, 248

Monitor, 530

Monkeys, 573

Monocaulis, 217

Monocystis, 187

Monodelphia, $56 \mathrm{I}$

Monodon, $57^{2}$

Monogenea, 244

Monogony, I 28

Monohybrid, 140

Monomyaria, 323

Monophyodont, 553

Monopneumonia, 5 I 3

Monops, 242

Monoscelis, 242
Monospermy, I34

Monothalamia, 179

Monotocarclia, 333

Monotremata, 558

Moose, 569

Morphological limits, 50

Morphology, animal. 5o

Mosaic theory of vision, 357

Mosasaurus, 530

Moschidx, 560

Mosquito, 430

Mother-of-pearl, 319

Noths, 432

Motor roots, 468

Mud crab, 382

Mudpuppy, 520

Müller's duct, $4_{4}{ }_{7}$

Multicellularity, 62

Multinuclearity, 62

Multipolar cells, 84

Multituberculata, 559

Murex, 327

Muricidæe, 334

Mus, 566

Muscariæ, 431

Muscidæ, 43I

Muscle corpuscles, So cross-striated, $\mathrm{S}_{\mathrm{I}}$ epaxial, 454 fibres, 80 fibrils, 63,80 hypaxial, 454 smooth, 8 I visceral, $47^{2}$ of vertebrates, 466

Muscular tissue, so

Mushroom coral, 232

Musk deer, 560

Musk ox, 560

Musk rat, 566

Mussels, 323. 324 .

Mustela, 565

Mustelus, 506

Mutations, 43

Mya, 32.4

Mycetozoa, Iso

Myelencephalon, 460

Mygale, 396

Mygnimia, 40

Mylodon, $5^{6} 3$

Myoccele, 466

Myomeric, 460

Myomorpha, 566

Myopsida, 347 
Myosepta, 466

Myotomes, 466

Myrianida, 275, 277

Myriapoda, 402, 433

Myriotrochus, $30 \mathrm{~S}$

Myrmecophaga, 563

Myrmicida, 426

Myrmecocystus, 427

Mysilla, 205

Mysis, 376

stage, $3^{\text {So }}$

Mystaceti, 572

Mytilida, 323

Myxicola, 277

Myxine, 493

Myxobolus, Igo

Myxomycetes, ISo

Myxospongia, 205

Myxosporida, 190

Myzobdella, 279

Myzontes, 493

Nagana disease, 43 I

Nageli's principle of progression, $4^{8}$

Naididie, 279

Nails, 45o, 545

Nais, $27 \mathrm{I}$

Naja, 53 I

Nanomia, 2 I 9

Narcomedusæ, 218

Naris, 475

Narwal, 572

Nasal bones, 459

capsule, 457

Natatores, 543

Naticide, 334

Natural selection, 35

Nauplius, 28, 362 eye, 362

Nausithoe, 224

Nautilus, 346

Nebalia, 375

Nectocalyces, 2 I 8

Nectonema, 268

Nectonemertes, $25^{8}$

Necturus, 520

Needham's sac, 344

Nematocyst, 207

Nematoda, 263

Nemathelminthes, 263

Nematus, 425

Nemerteans, 255

Nemertini, 255

Nemocera, 430
Nemopsis, 217

Neocranium, 455

Neocrinoida, 302

Ne gaea, I62

Neo-Lamarckism, 47

Neomenia, 3 I 6

Neosporida, 190

Neoteny, 150

Nephelis, 284

Nephridia, I05, 272

Nephridial tubules, 486

Nephrostome, 105, 242, 4 6

Nepidæ, 428

Neptunus, $3^{82}$

Nereis, 276

Neverita, 334

Nerve-cord, ventral, I I3

Nerve-end buds, 474

Nerve fibres, $s_{5}$ hillocks, 474

Nerves cranial, $4 ;$ I eye-muscle, 472 spinal, 468 sympathetic, 473

Nervous system, I I I development of, 30 of vertebrates, 467

Nervous tissue, $8_{3}$

Neural arch, 453 spine, 453

Neurapophysis, 453

Neurenteric canal, 439, 467

Neurites, 84

Neuroblasts, 86

Neuroglia, II 4

Neuromasts, 474

Neurone, 86

Neuropodium, 276

Neuropore, 440,468

Neuroptera, 42 I

Nettle bodies, 192 cells, $207,24 \mathrm{I}$

Nictitating membrane, 477

Nidamental gland, $3+4$

Night-hawks, $5+4$

Nirmus, 420

Noctiluca, $1 \delta_{4}$

Noctuina, 433

Nomarthra, $5^{6} 3$

Nosema, I9I

Nothria, 277

Notochord, 438,452

Notochordal sheath, 452

Notodelphidx, 360

Notogxa, I62
Notonectidæ, $428^{\circ}$

Notopodium, 276

Notum, 404

Nuclear fragmentation, 62 spindle, 60 substance, 56

Nuclein, $5^{6}$

Nucleoli, chromatic, 57

Nucleolus, 57

Nucleus, 55 cleavage, I35 function of, $5^{8}$

Nucula, 323

Nuda, 235

Nudibranchia, 335

Nummulites, I8o

Nurse, I3 I

Nutrition, 55

Nyctotherus, I96

Nymphon, 399

Obelia, 2 I 8

Obisium, 394

Obturator foramen, 522, 550

Occipital bone, $45^{8}$ condyles, 5 I 4

Occipitalia, $45^{8}$

Occiput, 405

Ocellatæ, 2 I 6

Ocellus, 354

Ocneria, 433

Octocoralla, $230^{\circ}$

Octopoda, 347

Octopus, 336,347

Ocular plate, 297, 304

Oculina, $23 \mathrm{I}$

Oculomotor nerve, $47 \mathrm{I}$

Odonata, 420

Odontoholcx, 54I

Odontophore, 329

Odontormæ, 54I

Odontornithes, 54I

Ecanthus, 42 I

Cxcology, 50

Edipoda, 42 I

Edogonium, r6o

Enothera, 44

(E)sophageal nerve ring, I I3

Esophagus, 94

Oigopsida, 347

Oikopleura, 443, 444

Oil bottles, 424

Okapia, 569

Olecranon, $46_{5}$ 
Olfactory lobe, 469 nerve, $47 I$ organs, I I 5

Oligochæ: 278

Oligopyreme, 73

Oligosoma, 530

Oligotrochus, 308

Olividæ, 334

Ommastrephes, 347

Omasus, $56 \mathrm{~S}$

Ommatidia, $35^{6}$

Oncosphera, $25 \mathrm{I}$

Oniscus, 386

Ontogeny, 2

Oocyte, 7I, I 32

Ookinete, $I_{3}$, I9o

Oospores, 169

Ootype, 244

Opalina, I95

Opercular cleft, 502

Operculum, 285, 327 of fishes, 497

Ophidia, 530

Ophioglypha, 299

Ophiopholis, 299

Ophisaurus, 530

Ophiurioidea, 298

Ophryodendron, Igs

Ophryoscolex, 197

Opisthobranchia, 334

Opisthocœele, 455

Opisthopatus, 400

Opisthotic, $45^{\mathrm{s}}$

Opossum, 560 shrimp, 376

Opoterodonta, 53 I

Optic cup, 477 ganglion, I I 9,476

lobes, 470

nerve, $47 \mathrm{I}$

stalk, 477

thalami, 469

resicle, 477

Orange scale, 430

Orang-utan, 574

Ora serrata, I 2 I

Oralia, 292, 30I

Orbitelaria, 396

Orbitosphenoid, $45^{\mathrm{S}}$

Orbitotemporal fossa, $5^{22}$

Orb weavers, 396

Orchestia, 384

Organ-pipe coral, 230

Organs, 88

animal, go, I Io
Organs, assimilation, 9I

auditory, 1 I 6

of Bojanus, $32 \mathrm{I}$

circulatory, 99

of Corti, 478,552

electric, I I I

excretory, $\mathrm{IO}_{4}$

Jacobson's, 475

larval, I 50

of locomotion, I ro

Iyriform, 390

olfactory, I I 5, 474

phosphorescent, I 2 I

respiratory, 96

segmental, I05

sensory, $\mathrm{II}_{4}$

sexual, ro7

of smell, I I 5,474

systems of, 90

tactile, I I 5

of taste, II 5.474

tympanal, I I 7

vegetative, go

visual, I 18

urogenital, Iog

Orioles, 544

Origin of species, IS

Ornithodelphia, $55^{\mathrm{S}}$

Ornithorhynchus, 559

Orohippus, 560

Oronasal groove, 475

Orthis, 290

Orthoceras, 346

Orthonectida, 200

Orthoneurous, 330

Orthoptera, 42 I

Orthopoda, 528

Orycteropus, $56_{3}$

Oscarella, 205

Oscines, 544

Osculum, 202

Os innominatum, $55^{\circ}$

Osmerus, 5 I0

Osphradium, I I6, 3I 2

Ossein, 77

Ossicula auditus, 479

Ostariophysi, 5 Iо

Osteoblasts, $7 \delta$

Ostia of heart, Io3, 357

Ostium tubæ, $4 \delta_{7}$

Ostracoda, 37 I

Ostracodermi, 493, $5^{\text {I } 2}$

Ostracoteuthis, $34^{\mathrm{I}}$

Ostræidæ, 323

Os transversum, 522
Ostriches, 542

Otaria, 565

Otica, $45^{\mathrm{s}}$

Otic capsule, 457

ganglion, 473

Otis, $54+$

Otolith, I I 6

Otter, $5^{6} 5$

Ovaries, 4 S6

Ovary, histology of, 70

Ovibos, 569

Ovicells, 286

Oriducts, 109, 486

Oviparous, I 5 I

Ovipositor, 413

Ovis, 569

Oroid gland, 293

Ovoriviparous, I5I

Ovum, $4^{56}$

Owlet moths, 433

Owls, 544

Oyster crab, 382

Oysters, 323

Ox warble, $43 \mathrm{I}$

Oxy-hæmoglobin, 79

Oxyrhyncha, 382

Oxystomata, 382

Oxyuris, 266

Pachyderm, 570

Pachydrilus, 279

Pachylemuridæ, 573

Paddle fish, 50 s

Pædogenesis, I 29, 4 I4

Paguridea, 3 So

Palæchinoidea, 305

Palæmon, 3 So

Palæmonetes, 380

Palæocranium, 455

Palæocrinoidea, 302

Palæotherium, 569

Palate, 475

Palatine bone, ${ }_{4} 6 \mathrm{I}$

Paleacrita, 433

Palinurus, 380

Pallial line, 3 IS sinus, 3 I 8

Pallium, 3I I, 469

Palolo worm, 275

Palpus, 353, 377, 405

Paludicella, $2 S 7$

Paludinida, 334

Pancreas, 96, 48I

Pandalus, 3 So

Pardicn, 544 
Pangolin, 563

Panopeus, 382

I'anorpirlix, 422

Pansporoblasts, Igo

Pantopoda, 399

Papilio, 433

Parabronchi, 538

Parachordals, $45^{6}$

Paractinopoda, zos

I'aradidymis, 489

I'aradisea, 4 I

I'aradoxides, 364

Paragastric canal, 234

Paraglossa, 406

Paragnaths, 377

Paramecium, I91, I95

Paranuclein, 57

Paranucleus, 192

Parapodium, 276, 325

Parapophyses, 454

Parapterium, 534

Parasita, 360

Parasitism, I 56

Parasphenoid, 459

Parasuchia, 532

Paraxon gland, 293

Parenchyma, 239

Parietal bones, 459

eye, 4;o

organ, 470

Pariots, 544

Parthenogenesis, 129 artificial, 137

Partridge, 543

Passer, 544

Passeres, 544

Patagium, 564

Patella, 327, 465

Patellidae, 333

Pathetic nerve, $47 \mathrm{I}$

Pauropoda, 434

Pavement epithelium, 65

Pearl, 3ro

organs, 493

oysters, 323

Pearls, artificial, $40+1$

Pebrine, I9 I

Peccaries, 568

Pecora, 568

Pecten, 540

Pectinatella, 287

Pectines, 392

Pectinibranchia, 333

Pectinirle, 323

Pectoral fin, 497
Pectoral girdle, $46_{4}$

Pedal ganglia, 3 I 2

Pedata, 308

Pedicellaria, 292

Pedicellina, 285

Pediculus, 430

Pedipalpi, 3 So, 392

Pelagia, 223

Pelagic fauna, I64

Pelagothuria, 292, 309

Pelecanus, 543

Pelecypoda, 3 I 7

Pelican, 543

Pelmatozoa, 299

Pelobatidæ, 520

Peltogaster, 374

Pelvic fin, 462, 497 girdle, 464

Penella, 370

Peneus, 380

Penguins, 543

Penis, ro9, $55^{6}$

Pennaria, 217

Pennatula, 230

Pentacrinus, 302

Pentamera, 42.4

Pentamerus, 290

Pentastomum, I 57, 397

Pentatoma, $42 \mathrm{~S}$

Pentremites, $30_{3}$

Pepsin, 96

Perameles, 560

Perca, 5 II

Perch, 5 II

Perdix, 543

Pereion, 351

Pereiopoda, $35^{2}$

Perennibranchiata, 520

Perfecting principle, 48

Perforata, ISo

Perforatorium, 72

Perla, 4I.4

Peromedusa, 224

Perophora, 445

Peropoda, 53 I

Peribranchial chamber.

Pericardium, IOI, 4 SI

Perichxta, 279

Perichondrium, 77

Pericolpa, 224

Periclinium, $\mathbf{I} 8_{4}$

Perilymph, 479

Perineum, 556

Periosteum, 77

Peripatopsis, 400
Peripatus, 400

Peripharyngeal band, $4+3$

Peripheral nervous system, I 3

Periphylla, 224

Periplaneta, 42 I

Periproct, 303

Perisarc, 2 Io

1 erissodactyla, 568

Peristalsis, 96

Peristaltic motion, 96

Peristome, 208, 303

Peritoneum, 99, 48I

Peritricha, 196

Periwinkles, 334

Perradii, I 24

Petaurus, $5^{6}$ I

Petromyzontes, 493

Petrosal, $45^{8}$

Phacellæ, 220, 22 I

Phenicopterus, 544

Phiethon, 543

Phagocata, 2.42

Phalanges, 465

Phalangida, 394

Phalangistidie, 56I

Phalangium, 394

Phalarocorax, 543

Pharyngeal bones, 496 bulb, 264

Pharyngognathi, 510

Pharynx, 94, 408, 442, 482

Phascalomys, 56I

Phascolion, $28 \mathrm{I}$

Phascolosoma, $28 \mathrm{I}$

Phasianus, 543

Phasmide, $4^{2}$ I

Phasmomantis, $42 \mathrm{I}$

Pheasants, 543

Phenacodon, 567

Phenacodontidæ, 569

Philichthys, 30

Phidippus, 396

Philine, 334

Philonexidæ, 347

Phlegethontias, 433

Phoca, 565

Pholadida, 325

Phoronidea, $2 \mathrm{~S}_{7}$

Phosphorescence, I 2 I

Phototaxis, I 8

Phoxichilidium, 399

Phragmocone, 34I

Phronima, $3 \mathrm{~S}_{4}$

Phryganea, 422 
Phrynoidea, 392

Phrynosoma, 529

Phrynus, 393

Phylactolæmata, 287

Phthirius, +30

Phyllium, 39

Phyllodactylus, 529

Phyllopoda, 365

Phyllosoma, 380

Phyllostomidxe, 565

Phylloxera, 430

Phylogeny, 4, 23 proofs of, 24

Physalia, 220

Physeter, 572

Physonectix, 2 I0

Physophore, 2 I 9

Physopoda, 420

Physostomi, 5 io

Phytoflagellata, ${ } S_{4}$

Phy tophaga, 56r

Pia mater, 473

Pica, 566

Picariæ, 544

Pickerel, 5 Io

Pierids, 39

Pieris, 433

Pigeons, 20, 543

Pike, 5 Io

Pill bug, 386

Pilidium, 257

Pinealis, $47^{\circ}$

Pinnipedia, 565

Pinnotheres, 382

Pinnulæ, 30r

Pinworm, 266

Pipa, 520

Pipe fishes, 5I I

Piroplasma, I9o

Pisces, 493

Piscicola, $28_{4}$

Pithecanthropus, 574

Pituitary body, 470

Placenta, 49I, 558, 56I

Placentalia, 56 I

Placoid scale, 45 1 , 494

Placophora, 3 I 5

Plagiaulax, 559

Plagiostomes, 504

Plagiotremata, 528

Plakina, 202

Planaria, 242

Planipennia, 422

Plankton, I64

Planorbis, 336
Plants and animals, I 50

Plant lice, +30

Plantigrade, $55^{\circ}$

Planulie, I 50, 2 I 5

Plasma, blood, $7 \mathrm{~S}$

Plasmic products, 5,

Plasmodiophora, Is 1

Plasmodium, I 8o, ISo)

Plasmogamy, I68

Plastin, 57

Plastron, 527

Platanista, $5 ;$ I

Plathelminthes, $23 \mathrm{~s}$

Plitodes, $23^{8}$

Platyrrhinx, $57+$

Plecoptera, 420

Plectognathi, 5 I 2

Pleon, 35I

Pleopoda, $35^{2}$

Plesiosauria, 526

Plethodon, 520

Pleura, 364,404

Pleuracanthus, 507

Pleural cavity, 48 I

Pleurobrachia, 235

Pleurocercoid, $25 \mathrm{I}$

Pleurodont, 520

Pleuronectidx, 5 I I

Pleuroperitoneal cavity, 48 I

Plexus, sympathetic, 473

Plictolophus, $5+4$

Pliohippus, 560

Plover, $54+$

Plumatella, $2 \mathrm{~S}_{7}$

Plumes, 534

Plumularia, 2 Is

Pluteus, 204

Pneumatic bones, $53 \mathrm{~S}$ duct, 502

Pneumatophore, 2I 8

Pneumodermon, 335

Pneumogastric nerve, 47 I

Podophrya, 59, igs

Podophthalmia, 375

Podura, +io

Poikil thermal, ro4

Poison teeth, $53 \mathrm{I}$

Polar bodies, I 33

Pole capsules, I 90

fields, 234

Polian vesicles, 202

Polybostrichus, 275

Polychærus, 242

Polychætx, 2;6

Polycladidea, 242
Polyclinum, $44^{6}$

Polyclonia, 222

Polydesmidx, $43+$

Polyergus, $42 z$

Polygordius, 278

Polyhybrids, I to

Polymorphism, I54

Polynoe, 277

Polyodon, 5os

Polyp, 208

Polypid, 285

Polyphemidxe, 366

Polyprotodonta, 560

Polypterus, 508

Polyscelis, $2+2$

Polyspermy, I34

Polystomex, 244

Polystomella, I8o

Polystomium, 24

Polythalamia, 179

Polytroche, 273

Polyzoa, 284

Pond snails, 336

Poneridæe, 426

Pons Varolii, $55^{2}$

Pontella, 369

Pontobdella, 284

Pontodrilus, 272

Population, 44

Porcellana, ${ }_{3} \mathrm{~S}_{\mathrm{I}}$

Porcellio, 386

Porcupines, 566

Pori abdominales, $4^{8}$ I

Porifera, 20I

Porites, 232

Porosa, 232

Porpita, 220

Portal vein, 483

Portuguese man-of-war, 220

Portunidæe, $3^{82}$

Post abdomen, 35 I

Post cava, $48_{5}$

Post frontal bone, 462

Post scutellum, to8

Post trematic, 472

Potato beetle, 424

Powder down, 534

Prairie dog, 566

Praya, I 55, 220

Praying mantes, $42 \mathrm{I}$

Præcoces, $54^{1}$

Precava, 485

Preclaviæ, 550

Precious coral, 230

Prefrontal bones, 462 
Premaxillaries, $46 \mathrm{I}$

I'resphenoid, $45^{\circ}$

Pretrematic, 472

Priapuloidea, 2S r

Priapulus, $28 \mathrm{I}$

Primaries, 534

Primates, 573

Primordial cranium, 457

Priodon, $5^{6} 3$

Pristis, 507

Proboscidea, $57^{\circ}$

Processus falciformis, 499

Procœlous, 455

Procoracoid, 464

Proctodæum, 94, 409

Procyon, 565

Proechidna, 559

Proglottid, 247

Progamic development, ISS

P'ro-legs, 408

Promorphology, I23

Pronephric duct, 487

Pronephros, $4{ }_{7}$

Prong horn, 569

Pronotum, 404

Pronucleus, I33

Prosencephalon, 468

Proostracum, 3.4

Prorostomus, $57^{\circ}$

Prootic, $45^{\mathrm{S}}$

Propodite, 97

Propodium, 325

Propterygium, 464

Prosimiæ, 572

Prosobranchia, 333

Prosopygii, 28r, $2 \mathrm{~S}_{4}$

Prosternum, 404

Prostomium, 270

Protamoba, 172

Protandry, Ios

Proteroglypha, 53 I

Prothorax, 40.4

Protobranchiata, 323

Protocaris, 366

Protocerebrum, 405

Protoconchix, 323

Protodonta, 559

Proteroglyphic, 53I

Protogynacy, 108

Protohippus, 570

Protohydra, 217

Protomerite, I 86

Protonemertini, $25^{8}$

Protonephridia, I05

Protoplasm, 51, 52
Protopterus, 5 I 3

Prototheria, $55^{\mathrm{S}}$

Protovertebra, 467

Protozoa, I66

Protracheata, 399

Protrochula, 238

Protula, 278

Protura, 4 I 9

Proventriculus, 400

Psalterium, 568

Psammonyx, I 79

P'seudobranch, 502

Pseudocœle, 263

Pseudocuticula, $52 \mathrm{~S}$

Pseudonavicellæ, $\mathrm{I}_{7}$

Pseudoneuroptera, 4I9

Pseudopodia, 53, I I I, I7O

Pseudoscorpii, 394

Pseudosuchia, 532

Psittaci, 544

Psocidae, 420

Psolus, 303

Psorosperms, I9o

Pteranodon, 532

Pteraspis, 493

Pierichthys, 493

Pterodactylia, 532

Pteronarcys, 420

Pteropoda, 334

Pteropus, 565

Pterosauria, 532

Pterotic, $45^{8}$

Pterotrachea, 334

P'terygoid, $46 \mathrm{I}$

Pterygoquadrate, 460

Pterygotus, $38 S$

Pteryla, 534

Ptyalin, 96

Pubic bone, 464

Pugettia, $3^{\mathrm{S} 2}$

Iulex, 43 I

Pulmonary artery, IOI, $4 \mathrm{~S}_{4}$ veins, IOI, $4{ }_{4}$

Pulmonata, 335

Pulp cavity, 45 I

Pulvillus, 43 I

Puma, 565

Pupa, 336

Pupa coarctata, 416

libera, 4I 5

obiecta, 4I 5

Pupil of eye, Izo

Pupipara, 43I

Purpura, 334

Putorius, 565
Pycnogonida, 399

Pygidium, 364

Pygostyle, 537

Pyloric cæeca, 500

Pylorus, 481, 554

Pyrosoma, 446

Python, 53I

Pythonaster, 298

Pythonomorpha, 530

Quadrate, 46 I

Quadrula, I 79

Quahog, 324

Quail, $5+3$

Quill, 533

Raccoon, 565

Racemose glands, 68 vesicles, $29 \mathrm{~S}$

Races, 20

Rachis, 533

Radial canals, 2 II, 292 chambers, 225 symmetry, 123

Radiale, 465

Radialia, 292, 300, 463

Radiata, 206, 20I

Radii, 293

Radiolaria, I75

Radius, 465

Radula, 3I.4, 329

Raia, 507

Rails, 544

Rainey's corpuscles, I9I

Rallus, 544

Rana, 520

Ranatra, $4_{2} \mathrm{~S}$

Rangifer, 560

Ranvier, constrictions of, 85

Raptores, $54+4$

Ratitie, 54I

Rats, 566

Rattlesnake, $53 \mathrm{I}$

Rays, 507

Razor clain, 324

Receptaculum seminis, 265 , 4r3

Recessive, $\mathrm{I}_{3} \mathrm{~S}$

Rectal glands, 409

Rectrices, 534

Rectum, 409

Recurrent typhus, $\mathrm{x} 84$

Red coral, 230

Red mites, 397

Redia, 246 
Reduction division, I34, I3s Rocky Mountain sheep, 569 Sauropsida, 520

Reduviidx, 428

Regeneration, I 49

Regions, faunal, I60

Regression, 45

Regularia, 305

Reindeer, 569

Remiges, 534

Remora, 5II

Renilla, 230

Reproduction, 55, I 28 and fertilization, I 49 asexual, r2s sexual, 129

Reptilia, $52 \mathrm{I}$

Residual body, $\mathrm{I} \$_{3}, \mathbf{I} 86$

Respiratory canal, 475 organs, 96

Reticularia, 178

Reticulum, 568

Retina, I Is, $4 ; 6$

Retetelariæ, $39^{6}$

Rhabdites, 24I, 265

Rhabdocoelida, 242

Rhabdom, I I 9

Rhabdopleura, 449

Rhachiglossa, 334

Rhachis, 293, 364

Rhamnites, $24 \mathrm{I}$

Rhamphastos, 544

Rhea, 5.2

Rhegmatodes, 2 Is

Rhinencephalon, 460

Rhinoceros, 568

Rhinosporidium, I9I

Rhizocephala, 374

Rhizocrinus, 302

Rhizopoda, I 70

Rhizostomex, 223

Rhopalonema, 2I2, 2 I 4

Rhopalocera, 433

Rhynchobdellidæ, 284

Rhynchobothrium, 254

Rhynchocephalia, 527

Rhynchrta, I9s

Rhynchonella, 288, 290

Rhynchota, 427

Rhynchophora, 424

Rhytina, $57^{\circ}$

Ribs, +54

Right whale, $57^{2}$

Rigor, 55

Ring canal, 2 II, 292

Roaches, 42 I

Robber flies, 43I
Rodentia, 566

Rods and cones, I I0, $4 ; 0$

Root barnacles, 374

Rorqual, $57^{2}$

Rossia, 347

Rostellum, 249

Rostrum, 341, 372, 407, 504, 537

Rotalia, I 7 I, I So

Rotatoria, 259

Rotifera, 259

Round worms, 263

Rove beetles, +24

Rudistid $x, 324$

Rugosa, 230

Rumen, 568

Ruminantia, 568

Rupricapra, 569

Sabellidæ, 277

Sabinea, 380

Sable, $5^{6} 5$

Sicconereis, 275

Sacculina, 374

Sacculus, 117,478

Sacculus vasculosus, 499

Sagartia, 23 I

Sagit1a, 262

Sagital axis, 124

Salinella, 200

Salivary glands, 96

Salamanders, lungless, $5^{1} 7$

Salamandra, 520

Salamandrina, 520

Salmon, 5 Io

Salpa, $4+7$

Salpæformes, 447

Saltigrada, 396

Salve bug, 386

Saltatoria, 42 I

Sand dollar, 306

San Jose scale, $43^{\circ}$

Sapphirina, 369

Sapajou, 574

Sarcode, $5^{2}$

Sarcocystis, I9I

Sarcolemma, $8_{3}$

Sarcoptes, 397

Sarcosepta, 228

Sarcophilus, 560

Sarcopsylla, 43I

Sarcosporida, I9I

Sarsia, 217

Saurii, 529
Saururx, 54I

Savigny's law, $35^{2}$

Suvis' vesicles, 500

Saw fly, 425

Sawfishes, 507

Saxicava, 324

Scale of ear, 479

Srale insects, 428

Scales of fishes, 494

Scallops, 323

Scalops, 564

Scansores, 544

Scape, 533

Scapharca, 323

Scaphiopus, 520

Scaphognathite, 377

Scaphopoda, 325

Scapula, 464

Scarabæidx, 424

Sceleporus, 529

Schizodont, 3 I 8

Schizogony, I69, I86, 299

Schizopoda, 375

Schizopodal foot, 360

Schizosomi, $3 \mathrm{~S}_{2}$

Schwann's sheath, 85

Scincidæe, $53^{\circ}$

Sciuromorpha, 566

Sciuropterus, 566

Sciurus, 566

Sclera, 120, 457, 476

Scleroderma, 23 I

Sclerodermites, 230

Sclerophylla, 23 I

Sclerosepta, 228

Sclerotica, 120,476

Sclerotic bones, 525

Sclerotomes, 467

Scolex, 247

Scolopax, 544

Scolopendra, 403

heart of, I03

Scolopendrella, 434

Scombridæ, 5 I I

Scops, 544

Scorpions, 39I

false, 394

water, 428

Scorpionida, 39I

Sculpins, 5 II

Scutellum, 408

Scutigera, 403

Scutum, 372

Scyphomedusæ, 220 
Sipphopolyp, 220

Sicyphozoa, 220

Scyphostoma, 220

Sca anemones, 224, $23 \mathrm{I}$

(ucumbers, 306

fans, 230

horses, 5 II

lilies, 299

lions, 565

olter, 565

pens, 230

snakes, $53 \mathrm{I}$

squirts, $4+\mathrm{I}, 443$

urchins, 303

whips, 230

Seals, 565

Secodont, 554, 565

Secondaries, 534

Sectorial, $5^{6} 5$

Secreta, 65

Sedentaria, 277,396

Segmental organs, 105, 272

Segmentation cavity, I 45

of body, 126

of egg, $\mathrm{I}_{4} \mathrm{I}$

Selachii, 500

Selection, artificial, 35 germinal, 43

natural, 35

sexual, to

Selenodont, 554

Semrostomex, 223

Semicircular canals, 117 $47^{3}$

Seminal vesicles, го;

Semilunar valves, 503

Sense organs of vertebrates, 473

Sensory cells, 73

organs, il 4

roots, 468

Sepia, $3+\mathrm{I}, 3+7$

Septa, 225, 270

Septal organ, 293

Septibranchiata, 325

Sericteria, 432

Serosa, $+\mathrm{I}+$, + I

Serpulida, 277

Serripes, $32+$

Sertularia, 2 I 8

Serum, bloorl, 78

Sesiida, 433

Serranidx, 5 II

Sexual cells, I 30

characters, secondary, i io
Sexual gland, 3 , 7

dimorphisn, 40, I69

epithelium, 69

organs, ro7

organs of vertebrates, $4 \varepsilon 6$

reproduction, I 29

selection, 40

Shad, 5 Io

Shaft, 533

Shagreen, 505

Sharks, 504

Sheath of Schwann, 85

Sheep, 560

Sheep bot, 43 I

tick, $43^{I}$

Shell gland, $249,35^{8}, 3^{62}$

Ship worms, 325

Shoulder blade, 464

Shoulder girdle, $46+$

Shrews, 564

Shrimp, 380

brine, 366

fairy, 366

mantis, 376

opossum, $37^{6}$

Siala, 544

Sicyonia, $3^{80}$

Siderone, 38

Silicispongiæ, 204

Silenia, 325

Siliqua, 324

Silk glinnds, 432

Silk worm, 433

Siluricle, 5 ro

Silver fish, +19

Simia, 574

Simuliidie, 430

Sinupalliata, 324

Sinuses, bloorl, 103

Siphon, 305, 319, 339

Siphonaptera, +3I

Siphonate, 319

Siphonoglyphe, 225

Siphonophora, 2 I 8

Siphonostomata, 369

Siphonostome, 327

Siphuncle, 340

Sipunculoidea, 28I

Sipunculus, $28 \mathrm{I}$

Siredon, 27

Siren, 520

Sirenia, $57^{\circ}$

Sirex, 425

Siricide, +25

Sixth sense, $11_{5}$
Skates, 507

Skeleton, 9I

axial, 9I

cuticular, I I I

dermal, 9 I

Skin, 9 I of vertebrates, $67,45^{\circ}$

Skull, 455

Skunk, 565

Sky-lark, 544

Sleeping sickness, I $S_{4}, 43 I$

Slime fishes, $49 \mathrm{I}$

Slime moulds, I 80

Sloths, 563

Smell, I 5

Smelt, 5 IO

Smooth muscles, 8 I

Snails, 325

Snakes, 530

Snapping turtle, 527

Snout beetles, 424

Social animals, ${ }_{5} 6$

Social insects, $4 \mathrm{I} 6$

Sof t-shelled crab, $3^{82}$

Soft-shell turtles, 527

Solasteridæ, 298

Sole, 5 I I

Solemyidie, 323

Solen, 324

Solenoconche, 325

Solenocytes, I05, 272

Solenogastres, 3 I 6

Solenoglypha, 53 I

Solif ugæ, 393

Solpuga, 393

Somatic cells, I 30 layer, I4S, 2 OI

Somatopleure, $\mathrm{I}_{4} \mathrm{~S}$

Somites, 269

Sorex, 564

Sow bugs, 386

Spadella, 263

Spadix, 2 I 6

Spanish flies, 424

Sparrow's, $5+4$

Spatangoidea, 306

Spatangus, 306

Species, analytic, $\mathrm{I}_{4} \mathrm{I}$

crossing of, 2 I

nature of, 15

and varieties, I 8

Spelerpes, 520

Speotyto, 544

Spermaceti, 572

Spermatid, I 34 
Spermatocyte, I 34

Spermatogenesis, 134

Spermatophore, 3.4

Spermatozoa, 72, I34

Sperm nucleus, I35

Sperm whale, $57^{2}$

Spheridia, 292

Spharogastrida, 395

Sphaerophrya, ros

Sphenethmoid, $5^{14}$

Sphenodon, 527

Sphenoidalia. $45^{8}$

Sphenoid bone, 450,546

Sphenopalatine ganglion, 473

Sphenotic, $45^{8}$

Sphingina, 433

Sphyranura, 244

Spicules, sponge, 205

Spider crabs, 382

Spider monkeys, $5 i+$

Spiders, 395

Spinal canal, 453

cord, 468

nerves, 468

Spine-headed worms, 26.3

Spindle fibres, 60 nuclear, 60

Spinnerets, 395

Spinous process, 453

Spiny ant-eater, 559

Spittle insect, 428

Spiracle, 98, 326, 401, 4: r, 502, 5 I6

Spiral valve, 500

Spirochæta, I84

Spirifer, 290

Spirobolus, 434

Spirula, 347

Sphæroma, 386

Splanchnic layer, I48, zoI

Splanchnopleure, 96, I.4

Spleen, 486

Splenomegaly, I $S_{4}$

Sponges, 201

Spongida, $20 \mathrm{I}$

Spongilla, 205

Spongin, 204

Spongioblasts, 205

Spongioplasm, 53

Spontaneous generation, 23 , I 27

Sporangia, I8o

Sporocyst, 246

Sporogony, 169, I86
Sporosac, 216

Sporozoa, I85

Sporozoites, ISS

Sports, 20

Spring-tails, 419

Squali, 506

Squamata, 528, 563

Squamosal bone, $4^{\circ} 2$

Squanous epithelium, 05

Squid, $3+7$

Squilla, 376

Squirrels, 566

Stapes, ${ }_{4} 6 \mathrm{I}$

Starfish, 205

Star-nosed mole, $5^{6.4}$

Siatoblasts, 205,286

Statocysts of medusx, 2 I 4 molluscan, 3 I 2

Statolith, I IS

Stauromedusce, 224

Steapsin, 96

Steganopodes, 543

Stegocephali, 519

Stegomya, $43^{\circ}$

Stegosaurs, 528

Stellate ganglia, 343

Stelmatopoda, 287

Stemma, 354

Stenops, $57^{2}$

Stenostoma, 93

Stenson's duct, 475

Stentor, I06

Stephanocyphus, 224

Stercoral pocket, 390

Sterna, 543

Sternaspis, $2 \vec{i}$

Sternite, 104

Sternum, 404, 454

Sticklebacks, 5 I I

Stigma, 98

Stigmata, foI

Sting, 413

Sting rays, 507

Stink bugs, $42 S$

Stipes, 406

Stolo prolifer, 448

Stomach, 94

Stomatopoda, 376

Stomodxum, 94

Stomolophus, 223

Stone canal, 292

Storks, 544

Stratified epithelium, 66

Stratum corneum, 67,450 Malpighii, 67, $45^{\circ}$
Strepsiptera, 422

Sireptoneury, 328

Stridulating organs, 410

Strix, 54t

Strobila, 22 I, 247

Strobilation, 22 I

Strongylidie, 266

Strongylocentrotus, 305

Strongyloides, 265

Strongylus, 266

Struggle for existence, $3.5,36$

Struthiones, 542

Stylaster, 2 I 7

Stylets, 408

Stylochus, 242

Stylohyoid ligament, 549

Styloid process, 54?

Stylommatophora, $33^{6}$

Stylonychia, 107

Stylopidie, $\$ 22$

Stylopized, 423

Stylorhynchus, $\mathrm{I}_{7}$

Sturgeon, 508

Subcuticula, 263

Subcutaneous tissue, $45^{\circ}$

Subintestinal vein, $4^{8} 3$

Submentum, 406

Subneural gland, $4+5$

Subumbrella, 2 I I

Suckers, 5 ro

Suck fishes, 5I I

Sucking stomach, 408

Suctoria, I97

Suidæe, 568

Sulci, $47^{\circ}$

Suminer eggs, I32, 365

Sun animalcules, 173

Supporting cells, 74 tissues, 74

Supraoccipital, $45^{8}$

Supracesophageal ganglia.

$$
\text { I I } 3,354
$$

Suprarenals, 480

Supratemporal fossa, 522

Surf perch, 5 I I

Sus, 568

Swallows, $5+4$

Swallow tails, 433

Swans, 543

Sweat glands, 546

Swell fishes, 512

Swim bladder, 482, 502

Swimming birds, $5+3$

Swine, 568

Sword fishes, $5^{\text {I I }}$ 
Sycandra, 202, 2

Sycon, 204

Syllis, 275

Sylvicolide, $5+4$

Symbiosis, 158

Symmetry, I23

Sympathetic coloration, 37

nervous system, II 4

plexus, 473

system, 473

Symplectic bone, 496

Symphila, 4I 7, 434

Synapta, 308

Synascidie, 446

Syncarida, $3 \mathrm{~S}_{2}$

Syncitia, 62

Syncoelidium, 2.40, 242

Syncoryne, $2 \mathrm{I} 7$

Synentognathi, 5 Io

Syngamus, 266

Syngnathus, 5 I I

Syphilis, I 84

Syringopora, 230

Syrinx, $53 \mathrm{~S}$

Syrphidx, 43I

Systemic arteries, IoI

heart, 343

veins, IOI

Systole, Ior

'Tabanida, 430

'Tabula, 229

Tabulatx, 229

Tactile corpuscles, 473 organs, I I 5

Tadpoles, 27, 510

Tænia, I 57, 247, 25.4

Trenioglossa, 334

Trniole, 220

Tails of fishes, 32,403

Talpidæe, 564

Tanais, 386

Tanystoma, 430

Tapetum, I $2 \mathrm{I}$

nigrum, 476

Tapeworms, 247

Tapirus, 568

Tarantella, 396

Tarantula, 396

Tarligrada, $398,5^{6} 3$

Tarsal bones, 465

Tarsius, $57^{2}$

Tarso-metatarsus, 536

Tarsus, 405, 465

Tasmanian devil, 560
Taste, I I 5

Taste organs, 474

Tatusia, $5^{6} 3$

Taxodont, 3 I 8

Tectibranchia, 334,346

Tectrices, 534

Teeth, $4^{\mathrm{SI}}$

mammalian, $55^{2}$

'Tejus, 530

Telea, 433

Telencephalon, 469

Teleostei, 508

Tellina, 324

Teloblasts, 273

Telodendron, 84

Telolecithal egg, 143

Telotroche, 273

Temnocephalidxe, 24.4

Temporal bone, $462,54 \mathrm{~S}$

Tendinous tissue, 76

Tenebrionidx, 424

Tentaculata, 235

Tent caterpillars, 433

Tenthredinidie, 425

Terebella, $9 \$, 278$

Terebra, 4I3, 425

Terebrantia, 425

Terebratula, 290

Teredo, 325

Tergite, 404

Tergum, 372

Termes, 4I9

Terminalia, 304

Terminalis nerve, $47 \mathrm{I}$

Termites, 4I 7, 4I9

Termitophiles, 4 I9

Terns, 543

Terrapins, 527

Terricola, 279

Tessellata, 302

Tesseridæ, 224

Test, 443

Testes, 486

Testicardines, 290

Testudinata, 526

Testudo, 527

Tethya, 205

Tethyoidea, $4+3$

Tetracoralla, 230

Tetractinellidae, 204

Tetradecapoda, $3_{3} \delta_{3}$

Tetrads, I34

Tetramera, 424

Tetraonida, 5.43

'Tetraphyllidæe, 254
Tetrapneumones, 396

Tetrapoda, $49 \mathrm{I}$

Tetrarhynchus, 249

Tetrastemma, 258

Tetrasticta, $39^{6}$

Tetraxonia, 204

Texas fever, rgo

Thalamophora, i 78

Thalassema, 28I

Thalassicola, 176

Thalassochelys, 527

Thaliacea, 447

Thaumantia, 2 I 8

Theca, 228, 300

Thecasomata, 335

Thecodont, 532

Thelyphonida, 392

Thelyphonus, 393

Theridium, 396

Theriodontia, 526

Theromorpha, 526

Thoracic cavity, 555

duct, 486

fin, 462, 497

Thoracostraca, 375

Threadworms, 263

Thrips, 420

Thrushes, 544

Thylacinus, 560

Thymus gland, 482

Thyone, 308

Thyreoid gland, $4^{82}$

Thysanoptera, 420

Thysanozoon, 242

Thysanura, 4I9

Tiara, 2 I 3

Ti jia, 405,465

Tibiale, 465

Tibio-tarsus, 536

Ticks, 397

Tick, sheep, 43 I

Tierlemann's vesicles, 292

'Tiger, 565

Tiger beetles, 424

Tillodontia, 570

Tima, 2 I8

Tineidæ, 432

Tipulide, 430

Tissues, $6_{3}$

connective, 74

elastic, 76

epithelial, 64

muscular, 80

subcutaneous, $45^{\circ}$

supporting, 74 
Toarls, 520

'Tobacco worm, 433

'Tocogony, r28

'Tokophrya, ros

Tomato worm, 433

Tongue bone, 460

Tooth-shells, 325

Top-shells, 333

'Tornaria, 449

Torpedo, 507

Tortoise, 527

Tortricila, $43^{2}$

Toucans, 544

Toxiglossa, 334

Toxodontia, 570

Toxopneustes, 305

Trabeculie, 456

Trachea, 482

Trachex, 98,387 , 40I, 4 I I

Tracheal gills, 402, 4 I I

Tracheata, 359

Trachoma, I9I

Trachydermon, 3 I 6

Trachymedusie, 2 I 8

Trachynema, 2 i 8

Tractus olfactorius, 460

Trap-door spider, 396

Tree cricket, 42 I

Tree hoppers, 428

Tree toads, 520

Trematoda, 242

Trepang, 308

Triarthrus, 364

Triaxonia, 205

'Trichechus, 565

Trichina, 267

Trichocephalus, 267

Trichocysts, I 02

Trichodectes, 420

Trichomonas, $\mathrm{I}_{4}$

'Trichoplax, 200

Trichoptera, 422

Trichotrachelida, 267

Tricladidea, 242

Triconodont, 554

Tridacna, $3^{24}$

'Trigeminal nerve, 47 I

Trilobitat, 363

Trimera, 424

Trionychia, 527

Tristicta, 306

Tristoma, 244

Tritocerebrum, 405

Triton embryo, $3 \mathrm{I}$

Tritoniidx, 335
Tritubercular, 554

Tritylodon, 559

Trivium, 292, 206

Trochal disc, 250

Trochanter, 405

'Trochilus, 544

Trochlearis nerve, $47 \mathrm{I}$

Trochophore, 238, 273

'Trochosa, 306

'Trochus, 333

Troctes, 420

Troglodytes, 574

Trombidiida, 397

Trophi, 260

Tropic birds, 543

Tropidonotus, 52 I, $53 \mathrm{I}$

Trout, 5 Io

Trygonida, 507

Trypanosoma, IS 4

Trypsin, 96

'Tubicola, 277

Tubicinella, 37 I

Tubificidre, 270

Tubinares, 543

Tubiporida, 230

'Tubitelaria, 306

Tubular glands, 68 nervous sy'stem, I I4

Tubularia, 2 I 7

'Tubulipora, $2 \mathrm{~S}_{7}$

Tunic, $44^{2}$

Tunicata, 44I

Turbellaria, 240

Turbinal bones, 475,548

Turbinida, 333

Turdus, 544

Turkey buzzard, 544

Turkeys, 543

Turritopsis, 2 I 7

Turtles, 526

"Twixt brain, 460

'Tylenchus, 266

Tylopoda, 560

Tympanal organs, i 7 , 4 I0

Tympanum, 479

Tympanic annulus, 470

bone, 462,548

membrane, 479

Typhline, 530

Typhlotriton, 520

Typhlomolge, 520

Typhlops, $53 \mathrm{I}$

Typhoid, 43 I

Typhus, recurrent, I 84
'Tyrian purple, 334

Tyrannidie, 544

Uca, $38_{2}$

Uintatherium, $57^{\circ}$

Ulmaris, 223

Ulna, 465

Ulnare, 465

Umbilicus, 326, 490

Umbo, 3 I 7

Umbrella, 2 I I

Uncinate processes, 535

Unguis, 450,546

Ungula, 450,546

Ungulata, 566

Unguligrade, $55^{\circ}$

Unio, 324

Unipolar cells, 84

Ureter, 107, 488

Urinator, 543

Urinary bladder, 105, 400, 504

Urnatella, $2 S_{5}$

Urochorda, 44 I

Urodela, 5I9

Urogenital sinus, 4 So, 555 system, IOQ

Urosalpinx, 334

Ursus, 565

Use and disuse, 16, 47 effects of, 89

Uterus, I09, 557

Utriculus, II $\overline{7}, 478$

Uvula, 554

Vagabundæe, 396

Vagina, 557

Vagus nerve, $47 \mathrm{I}$

Valkeria, 28 ;

Vampyre, 565

Vanessa, 433

Varanus, 524, 530

Variations, fluctuating, 44

Variola, I 9 I

Vasa deferentia, ro9, 486

Vascula, 99

Vater-Pacinian corpuscles, I I 5,474

V'egetative organs, 9o, 9I pole, I 33, 142

Veins, ror

Velella, 220

Veliger, 3 I $_{4}$

Velum, 2 I I, 3I 4, 440

Veneridie, 324 
V'enous bloorl, ro2

Ventral aorta, $4 \mathrm{~s}_{3}$

fin, $4^{62}$

nerve-cord, I I 3

nerve-root, 468

Ventricles of brain, 469

of heart, ror, $48_{3}$

Venus, 324

Venus girdle, 235

Venus' flower basket, 204

Vermes, 237

Vermiform appendix, 554

Vermilinguia, 530,563

Vermis, 470

Vermetus, 327

Vertebral column, $45^{2}$

Vertebrata, 449

circulation of, $4^{\mathrm{S}_{3}}$

digestive organs, $48 \mathrm{I}$

ear, 477

eye, I20, 475

respiratory organs, $4 \delta_{2}$

skeleton, 453

skin, $45^{\circ}$

sense organs, 473

urogenital system, $4 \& 6$

Vertex, 405

Vesicularia, 287

Vesparix, 426

Vespertilionidæ, 565

Vesperugo, $5^{6} 5$

Vestibule, 538

Vibracularia, 286

Vibrisse, 545

Vicarious species, 46

Vinegar eel, 265

Viperidx, 53 I

Visceral arches, 460 ganglia, 312

muscles, $47^{2}$

sac, 3 Io

skeleton, 459

Vision, I 8

Yisual cells, I 8 purple, I I 9

Vital force, 3

Vitellaria, I Io, 240

Vitreous body, I 20

Vitrodentine, 494

Viverra, 565

Iiviparous, I 51

'Voluntary muscles, 8 I

Volutidee, 334

Volvocina, $\mathrm{I} \mathrm{S}_{4}$

Volvox, I82, I84
Vomer, 46 I

Vortex, I09, 242

Vorticella, 197

Wading birds, 543

Waldheimia, 288

Walking leaf, 37

Walking stick, $42 \mathrm{I}$

Wallace's Line, 34, I62

Walrus, $5^{6} 5$

W'arblers, 544

Warm blood, IO4

Wasps, 426

Water beetles, 424

bears, 398

mites, 397

scorpion, $42 \mathrm{~S}$

snakes, $53 \mathrm{I}$

Water-vascular system, I05, Xenarthra, 563 292

IVeasel, $5^{6} 5$

Weberian apparatus, $5 \circ 3$

Weevils, 424

Whalebone, $57 \mathrm{I}$

Whales, $57 \mathrm{I}$

Wheel animalcules, 259

Whelk, 334

Whippoorwill, 544

White ants, 4 I9

White-fish, 5 ro

White matter, II 4,468

White nerve fibres, 85

Wind pipe, $4 \delta_{2}$

Wings of insects, 408

Winter eggs, 132,365

Wish bone, 535

Wolffian body, 487 duct, 487

Wolves, $5^{6} 5$

Wombat, 56 I

Woodcock, 544

Woodpeckers, 544

Wool, 545

Worms, 237

army, 433

bladder, 247

cabbage, 433

canker, 433

cotton, 433

earth, 279

flat, 238

galley, 402

guinea, 268

hair, 268

hair-neck, 267
Worms, hook, 267

hop, 433

lazy, 267

measuring, 433

nemertean, 255

palolo, 275

pin, 266

round, 263

ship, 325

silk, 433

spine-headed, 268

tape, 247

thread, 263

tobacco, 433

tomato, 433

tube, 277

Wrens, $54+4$

Xenophyophora, 180

Xenos, 423

Xerobates, 527

Xiphiidæ, 5I I

Xiphosura, $3^{87}$

Yellow fever, 430

Yoldia, 323

Yolk, 7I

cells, ro9

and cleavage, $\mathrm{I}_{42} 2$

glands, I ro

membrane, I 35

sac, $4 \mathrm{I} 4,4 \mathrm{Sg}$

Zebra, 568

Zeuglodontia, 572

Zoantharia, 230

Zoantheæ, 23 I

Zoea, 362

Zonula zinnii, I20

Zonuridxe, 530

Zoology, general, $5^{\circ}$ history of, 5

Zoophaga, 560

Zoophytes, I3, 206

Zoospores, I69, I 72

Zoothamnion, I97

Zooxanthellix, I58, I 78

Zygapophysis, 455

Zygeupolia, 258

Zygobranchia, 333

Zygodactyle, 544

Zygomatic process, 462

Zygote, I38, I39 




